\title{
THE PERMEABILITY OF GRANULAR BEDS EMPLACED IN VERTICAL DRILL HOLES
}

Stewart K. Griffiths

Frank A. Morrison, Jr.

February 23, 1979

Work performed under the auspices of the U.S. Department of

Energy by the UCLLL under contract number W-7405-ENG-48.

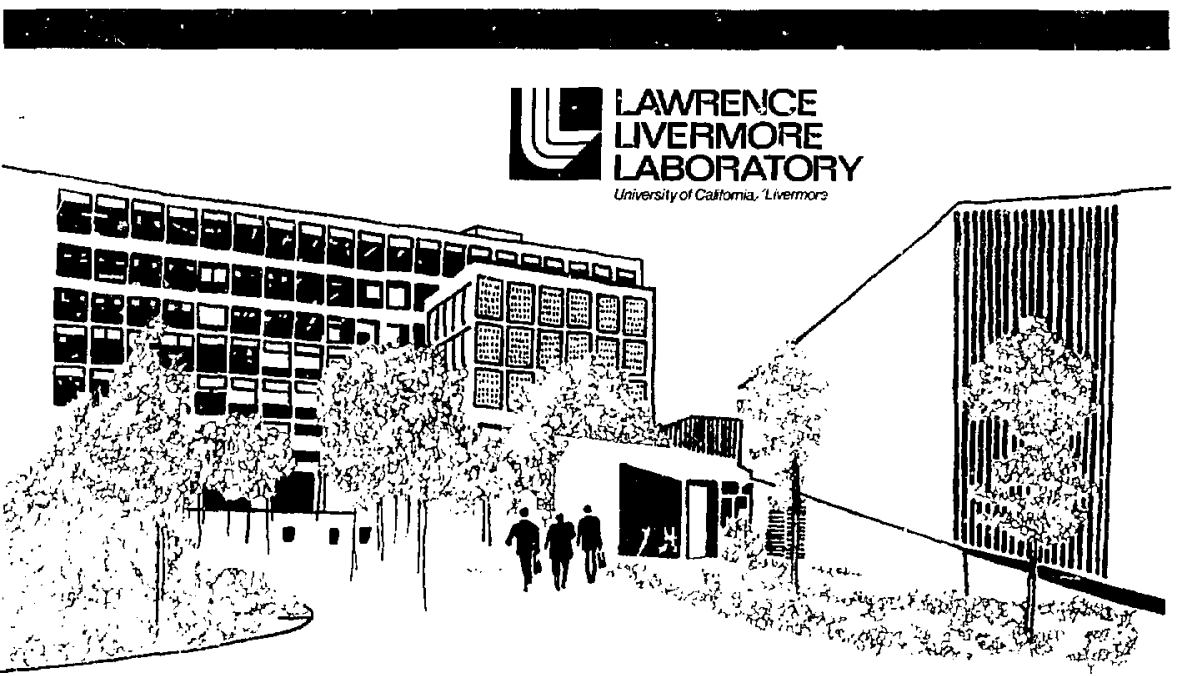




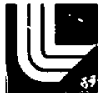

\section{LAWRENCE LIVERMORE LABORATOAY}

Universityor Calfornia - Livermore, Calitonia 94550

UCRL-52674

\section{THE PERMEABILITY OF GRANULAR BEDS EMPLACED IN VERTICAL DRILL HOLES}

Stewart K. Griffiths

Frank A. Morrison, Jr.

MS. date: February 23, 1979

\begin{tabular}{|c|}
\hline 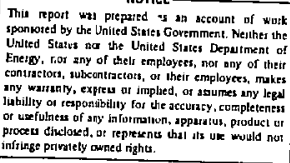 \\
\hline
\end{tabular}




\section{CONTENTS}

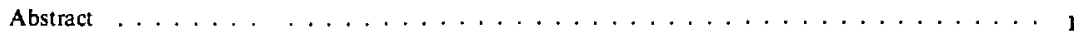

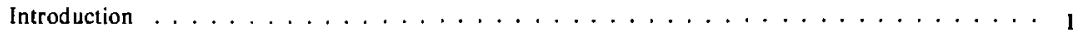

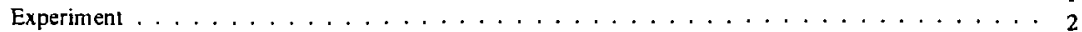

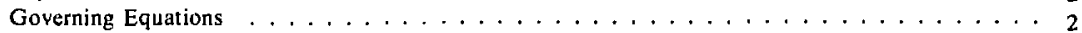

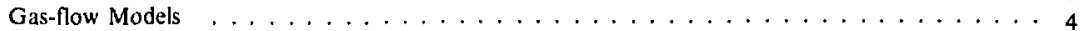

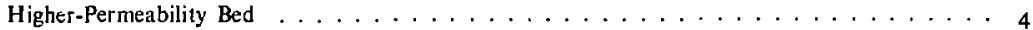

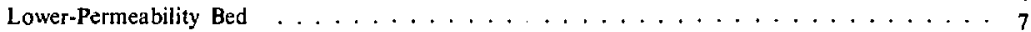

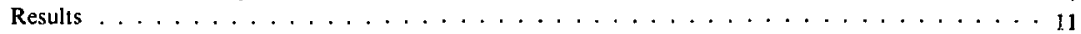

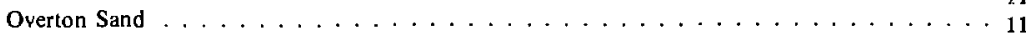

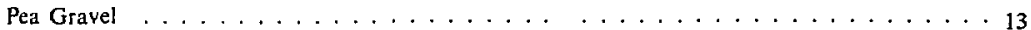

$\operatorname{LLL} \operatorname{Mix} \ldots \ldots \ldots \ldots \ldots \ldots \ldots \ldots \ldots \ldots \ldots \ldots$

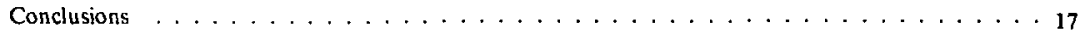

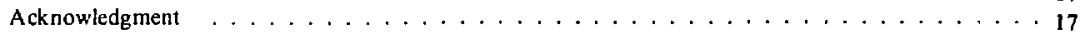

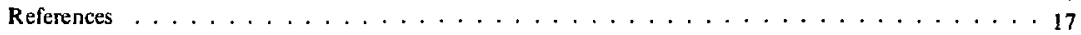




\title{
THE PERMEABILITY OF GRANULAR BEDS EMPLACED IN VERTICAL DRILL HOLES
}

\begin{abstract}
To determine the permeabilities of granular materials emplaced in vertical drill holes used for underground nuclear tests, we conducted an experiment at the USDOE Nevada Test Site (NTS). As the hole is being filled, falling material increases pressure above and within the granular beds beneath. When the filling operation starts or stops, a transient pressure response occurs within the beds; we measured this response in beds of various compositions. The permeabilities after emplacement were found by matching analytical predictions of the response to these data. This information is useful in assuring the containment of nuclear tests conducted in such drill holes.
\end{abstract}

\section{INTRODUCTION}

Underground nuclear tests are usually conducted in deep vertical drill holes. After emplacement of the nuclear device and before defonation, the hole is stemmed (filled). Most of the fill is granular material meant to prevent radioactive gas from reaching the sufface; the ability to impede fluid flow is thus a criterion for selection of stemming materials.

Stemming materials used by lawrence Ljvermore Laboratory (LLL) include pea gravel, Overton sand, and il heterogeneous mixture called LLL mix. The LLL mic provides mosi of the resistance to fluid transport. It is composed of pea gravel and fines from the NTS, zombined with !ly ash. The pea gravel and fines are biended und emplaced by a monitored conveyor as $h_{y}$ ash is added from a hopper; the material is LLL mix as it enters the hole. When it arrives at the botlom, however, it may have different proportions and, consequently, different properties because of the varied sedimentation velocities of the constituents.

For several years, LLL containment design has used LLL mix almost everywhere above the predicted nuclear cavity. Recently, a layered design has been adopted; layers of pea gravel are used so that the amount of LLL mix emplaced is about $20 \%$ of that formerly needed. These shorter layers of LLL mix may have properties significantly different from li:ese of longer layers and those of laboratory mixtures.

To evaluate the efficacy of this layered design for the containment of nuclear tests. we must know the permeabilities of the materials after emplacement. We can then make calculations of the multiphase, multicomponent flow through these porous media. ${ }^{1.2}$ To determine the permeabilities, we conducted a stemming experiment.

Transient downhole tressures produced during the stemming operation wete used to determine permeabilities - no auxiilary source of pressure was needed. The drag on falling particles creates a pressure rise that, at any depth, is proportional to the mass of material suspended above that depth. This pressure increase occurs throughout the depositing layer and penetrates underlying layers.

When stemming of a layer begins or ends, the pressure at the upper bed surface changes. This causes il trinsient response in that layer and all layers beneath. We measured this response with pressure transducers located in different layers; these measurements and models of gas fiow were used to determine the permeabilities of the materials. 


\section{EXPERIMENT}

The stemming experiment was conducted in drill hole U8e at the NTS, shown in Fig. 1. The hole was filled from its total depth of $1947 \mathrm{ft}$ to a depth of $1434 \mathrm{ft}$ with layers of pea gravel, LLL mix, and Overton sand. It was lined between 1846 and $161 \mathrm{ft}$; tuff, the surrounding lithologic unit, served as a barrier to radial flow above the liner because it is considerably less permeable than the stemming materials being investigated. Water was standing in the hole, so pea gravel was emplaced until a dry tag was obtained at a depth of $1809 \mathrm{ft}$. The saturatcd gravel bed ulso acted as an impermeable barrier. The materials investigated were emplaced above this level.

Four semiconductor pressure transducers (denoted as PX) were suspended downhole on a wire rope. They provided high-level output (about $200 \mathrm{mV}$ at full scale, from 0 to $25 \mathrm{psia}$ ) and moderate linearity over the temperature range encountered during the experiment. A three-wire system, insensitive to wire length, transmitted the transducer signals to the analog input panel of a Mod Comp II data acquisition and analysis computer. There the signals were low-pass filtered, converted to digital floating-point format, and buffered before storage. The system operator controlled sampling rates; 10 samples/minute were taken from each input channel near the beginning and ending of stemming, and 1 sample/minute was taken during other times.

The data analysis and output routines of this system are extensive. The operator may introduce scaling factors to correct for effects such as offset in tine original transducer signal and temperature-induced drift. Excessive noise was eliminated from the data, in some cases, using filtering functions and wild-point editing. Edited data are readily printed as single- or multi-channel plots of pressure vs time.

\section{GOVERNING EQUATIONS}

The relations describing gas flow through porous media are well known. ${ }^{3}$ Low Reynolds-number isothermal flow of an ideal gas is governed by a nonlinear diffusion equation. In one dimension, it is

$$
\frac{\partial}{\partial x}\left(\frac{k}{\mu} p \frac{\partial p}{\partial x}\right)=\epsilon \frac{\partial p}{\partial t}
$$

where

$$
\begin{aligned}
& \mathbf{x}=\text { spatial coordinate } \\
& \mathbf{t}=\text { temporal coordinate } \\
& \mathbf{k}=\text { permeability of the medium } \\
& \epsilon=\text { porosity: void volume fraction of the bed } \\
& \mu=\text { gas viscosity (constant in an isothermal flow) } \\
& \mathbf{p}=\text { pressure of the gas. }
\end{aligned}
$$

We are interested here in small deviations from the larger ambient pressure. This restriction permits us to linearize Eq. (1) using

$$
p=p_{0}+p_{d}
$$

where

$$
\begin{aligned}
& p_{0}=\text { ambient pressure } \\
& p_{d}=\text { deviation from the ambient pressure. }
\end{aligned}
$$




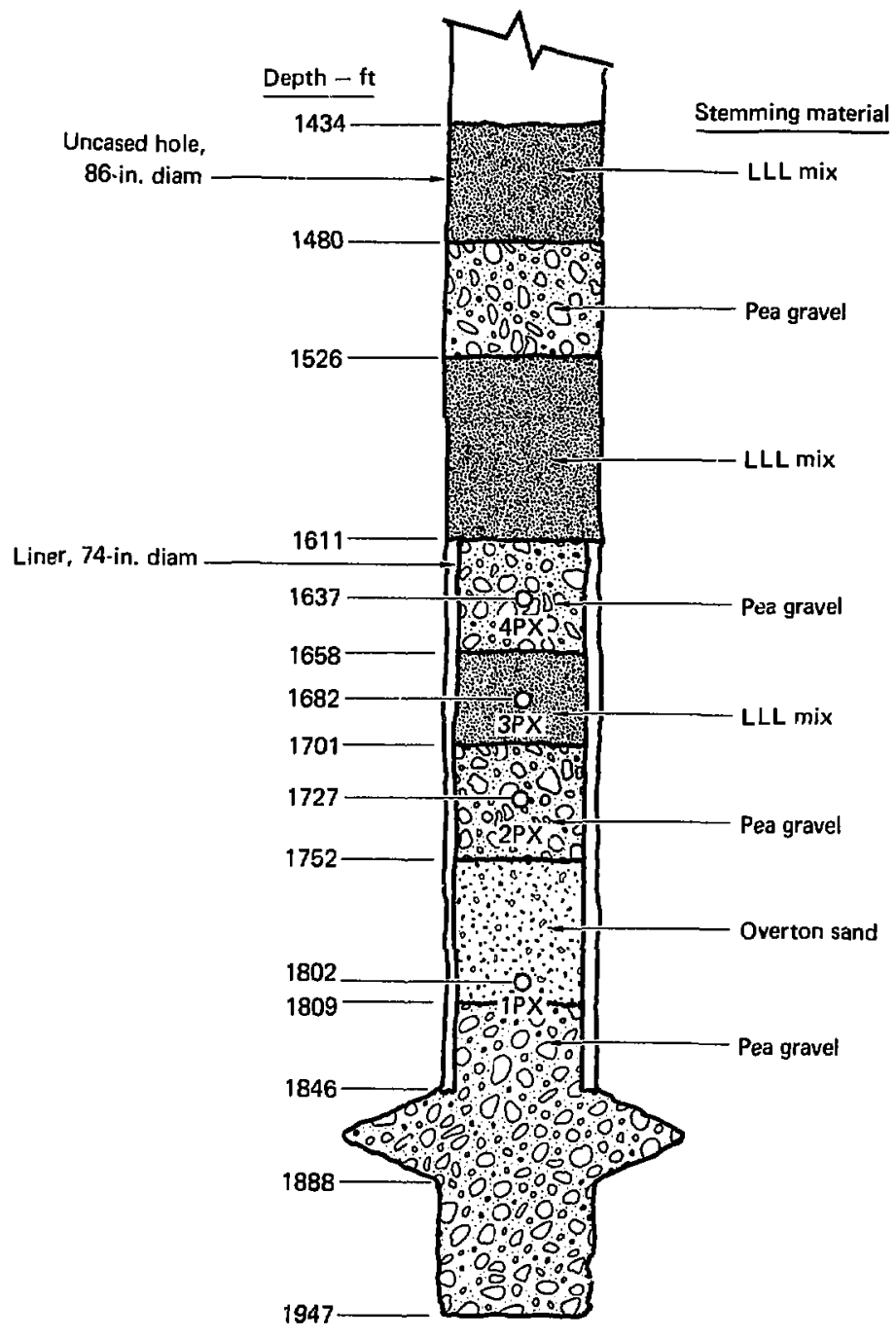

FIG. I. Stemming materials emplaced in drill hote U8e and locations of pressure transducers. 
For sufficiently small $p_{d}$, with constant permeability and viscusity, we obtain the linearized diffusion equation

$$
\frac{\partial^{2} \mathrm{p}_{\mathrm{d}}}{\partial \mathrm{x}^{2}}=\frac{1}{\alpha} \frac{\partial \mathrm{p}_{\mathrm{d}}}{\partial \mathrm{t}}
$$

where the effective diffusivity is

$$
\alpha=p_{0} k / \epsilon \mu
$$

An appropriate value for $\alpha$, and thus $k$, is chosen to match the response predicted by the solution of Eq. (3) to the measured response.

Two cases of flow are pertinent to this study. One, involving what we shall call the higherpermeability bed, is the response in a bed lying above a bed of markedly lower permeability. This applies to the Overton sand layer and all pea gravel layers in UBe. In a higher-permeability bed, the response time within the bed may be comparabie to the time over which the pressure changes at its upper boundary when stemming begins or ends. In such an instance the boundary condition should describe the actual pressure variation.

The second case occurs in a lower-permeability bed-material emplaced over one or more layers of material with higher permeability. All of the LLL mix layers in U8e are instances of lower-permeability beds. The longer response time in such beds allows us to treat the the boundary pressure changes as step changes.

\section{GAS-FLOW MODELS}

\section{HIGHER-PERMEABILITY BED}

Consider a short layer such as that shown in Fig. 2. This bed has jeen emplaced by constant stemming above a comparatively impermeable bed. Gas pressure is uniform through the layer and is higher than ambient by an amount $p_{j}$. This initial condition is

$$
p_{d}=p_{1} \text { when } t=0,0 \leqslant x \leqslant L .
$$

When stemming ceases, the downhole pressure drops linearly while a monodisperse material falls. For fall time $t^{*}$, the condition imposed at the upper boundary is

$$
p_{d}=\left\{\begin{array}{l}
p_{1}\left[1-\left(t / t^{*}\right)\right] \text { when } t<t^{*} \\
0 \text { when } t \geq t^{*}
\end{array} \quad \text { at } x=0\right.
$$

Darcy's law, which was used in the derivation of Eq. (1), relates the apparent fluid velocity $u$ to the local pressure gradient. This law, valid for low Reynolds-number flow, is

$$
\mathrm{u}=-\frac{\mathrm{k}}{\mu} \frac{\partial \mathrm{p}}{\partial \mathrm{x}} \text {. }
$$




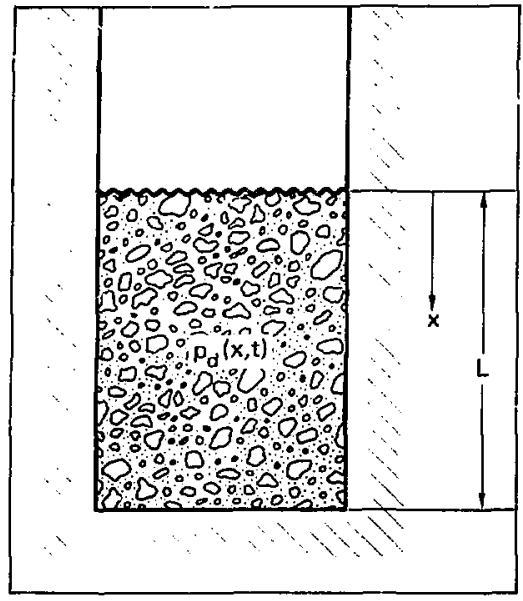

FIG. 2. Schema of the model for a higherpermeability bed. $P_{d}=$ deviation from ambient pressure at time $t$ and position $x ; L=$ length of the layer.

Because no flow crosies the impermeable lower boundary, that boundary condition is simply

$$
\frac{\partial p_{d}}{\partial x}=0 \text { at } x=L
$$

where $L=$ lengih of the layer

The ramp-lype upper boundary condition given by Eq. (6) is most easily sulisfied by applying Duhamel's theoren to the Fourier-series solution of the associated furdamental problem. The fundamental (unit step) problem is given by

$$
\frac{\partial^{2} \mathrm{f}}{\partial \mathrm{x}^{2}}=\frac{1}{\alpha} \frac{\partial \mathrm{f}}{\partial \mathrm{t}},
$$

with the conditiens

$$
\begin{aligned}
& \frac{\partial f}{\partial x}=0 \text { at } x=L ; \\
& f=l \text { at } x=0, t>0: \\
& f=0 \text { when } t=0,0 \leq x \leq L .
\end{aligned}
$$

The solution of this problem is the series

$$
f(x, t)=1-\frac{4}{\pi} \sum_{n=1}^{\infty} B_{n}(x) e^{-\alpha\left(\lambda_{n}\right)^{2} t}
$$


where

$$
\lambda_{\mathrm{n}}=\frac{(2 \mathrm{r}-1) \pi}{2 \mathrm{~L}}
$$

and

$$
g_{n}(x)=\frac{(-1)^{n-1}}{2 n-1} \cos \left[\lambda_{n}(L-x)\right]
$$

According to Duhamel's theorem, the final solution is given by

$$
\mathrm{p}_{\mathrm{d}}=\mathrm{p}_{1}+\int_{0}^{t} \mathrm{f}(\mathrm{x}, \mathrm{t}-\tau) \mathrm{F}^{\prime}(\tau) \mathrm{d} \tau
$$

where $F^{\prime}(\tau)$ is ovtained by differentiating Eq. (6) with respect to time. This yields

$$
F^{\prime}(\tau)=\left\{\begin{array}{l}
-\frac{\mathrm{P}_{1}}{\mathrm{t}^{*}} \text { when } \tau<\mathrm{t}^{*} \\
0 \text { when } \tau ? \mathrm{I}^{*}
\end{array} .\right.
$$

Two conditions must be kept in mind when performing the integration in Eq. (16). The first condition is the case when $t<t^{*}$, the second when $t>t^{*}$. These two cases may be treated simultane ously by changing the upler limit of integration in the equation to a new variable, $t$, defined as

$$
t^{\prime}=\left\{\begin{array}{l}
t \text { when } t<t^{*} \\
t^{*} \text { when } t \geq t^{*}
\end{array}\right.
$$

The final solution satisfying Eqs. (3), (5), (6), and (8) may then be written

$$
p_{d}=p_{1}\left\{1-\frac{1}{t^{*}}\left[t^{\prime}-\frac{4}{\pi} \sum_{n=1}^{\infty} g_{n}(x) \frac{e^{-\alpha\left(\lambda_{n}\right)^{2}}}{\alpha\left(\lambda_{n}\right)^{2}}\left(e^{a\left(\lambda_{n}\right)^{2} t^{\prime}}-1\right)\right]\right\} \text {. }
$$

Responses calculated witi this relation will be compared with experimental results to determine $F$ : meabilities.

Measurements of the pressure response induced by subsequent stemming can be used to contirm these permeabilities. In this case, the initial condition for pressure distribution is

$$
p_{d}=0 \text { when } t=0
$$

in place of Eq. (5). The upper boundary condition given by Eq. (6) now becomes

$$
\mathrm{P}_{d}=\left\{\begin{array}{l}
\mathrm{p}_{1}\left(\mathrm{t} / \mathrm{t}^{*}\right) \text { when } \mathrm{t}<\mathrm{t}^{*} \\
\mathrm{p}_{\mathrm{l}} \text { when } \mathrm{t} \geq \mathrm{t}^{*}
\end{array} \text { at } \mathrm{x}=0\right.
$$


The rise time $t^{*}$ is the time for the material being stemmed to fall from the top. The pressure distribution in this case is

$$
p_{d}=\frac{p_{1}}{t^{*}}\left[t^{\prime}-\frac{4}{\pi} \sum_{n=1}^{\infty} g_{n}(x) \frac{\left.e^{-\alpha\left(\lambda_{1}\right.}\right)^{21}}{\alpha\left(\lambda_{n}\right)^{2}}-\left(e^{\alpha\left(\lambda_{n}\right)^{2} t^{\prime}}-1\right)\right] .
$$

\section{LOWER-PERMEABILITY BED}

Consider the transient pressure response in a layer overlying material of relatively higher permeability, as shown in Fig. 3. The luyers undernetith constitute a finite fluid source, or "bottle," with known volume and pressure. The material in the bottle must be highly permeable, for these calculations, so that spatial pressure gradients are snall and the pressure derivative with respect to time is uniform.

When the overlying layer is being stemmed. pressure in the bottle increases as air enters. At the time stomming ceases, this pressure mily be either less than or equal to the maximum downhole pressure rise.

The governing equations for pressure in the bottle and layer of interest are coupled in their boundary conditions at the common interface. We will decouple these and write a single equation for the layer, with a

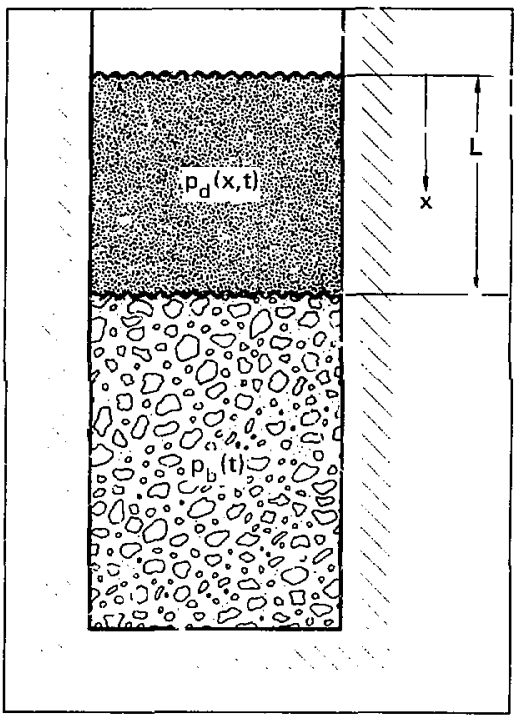

FIG. 3. Schema of the model for a lower-permeability bed. $P_{d}$ and $L$ are the same as in Fig, $2 ; P_{b}=$ irstantaneous pressure in the underlying bed (bottle) at ime t. 
mixed boundary condition at the interface. The initial pressure distribution within the layer is assumed to be uniform except at the lower boundary:

$$
p_{d}=\left\{\begin{array}{l}
p_{1} \text { for } 0 \leq x<L \\
p_{2} \text { at } x=L
\end{array} \text { when } t=0\right.
$$

where $\mathrm{p}_{2}=$ initial pressure in the bottle.

As stated earlier, the pressure at the upper bed surface drops when stemming stops and the mass of suspended material dicreases. The time span of this pressure drop is typically very small compared to the resfonse time in a lower-permeability bed. Therefore, we can adequately describe the pressure at the upper boundary by a step function:

$$
p_{d}=\left\{\begin{array}{l}
p_{1} \text { when } t=0 \\
0 \text { when } t>0
\end{array} \text { at } x=0\right.
$$

At the interface between the layer and the bottle, continuity for gas in the bottle and Darcy's law give the mixed boundary condition:

$$
\frac{\partial p_{\mathrm{d}}}{\partial t}=-\frac{\boldsymbol{\alpha} \beta}{\mathbf{L}} \frac{\partial p_{\mathbf{d}}}{\partial \mathbf{x}} \text { at } \mathbf{x}=\mathbf{L}
$$

where $\beta=$ ratio of void volume in the layer to that in the bottle.

The real-lime Fourier analysis of this problem, via separation of variables, leads to non-orthogonal eigenfunctions; this causes difficulty when classical techniques are used. The most expedient means of solution is through Laplace transforms. The governing equation thus becomes

$$
\frac{\partial^{2} \phi}{\partial x^{2}}=\frac{1}{\alpha}\left(s \phi-p_{1}\right)
$$

where

$$
\begin{aligned}
& \phi=\text { iransform of } \mathbf{P}_{\mathbf{d}} \\
& s=\text { Laplace parameter. }
\end{aligned}
$$

The appropriate boundary conditions are now

$$
\phi=0 \text { at } x=0
$$

and

$$
s \phi-\mathrm{p}_{2}=-\frac{\alpha \theta}{\mathrm{L}} \frac{\partial \phi}{\partial \mathrm{x}} \text { at } \mathrm{x}=\mathrm{L} .
$$

The solution of the transformed equation satisfying the boundary conditions is

$$
\phi=\frac{p_{1}}{s}-h(s)
$$


where

$$
h(s)=\frac{p_{1}\left\{\frac{\alpha \beta}{L} \sqrt{\frac{s}{\alpha}} \cosh \left[\sqrt{\frac{s}{\alpha}}(x-L)\right]-s \sinh \left[\sqrt{\frac{s}{\alpha}}(x-L)\right]+m s \sinh \left(\sqrt{\frac{s}{\alpha}} x\right)\right\}}{s\left[\frac{\alpha \beta}{L} \sqrt{\frac{s}{\alpha}} \cosh \left(\sqrt{\frac{s}{\alpha}} L\right)+s \sinh \left(\sqrt{\frac{s}{\alpha}} L\right)\right]}
$$

where

$$
m=1-\frac{p_{2}}{p_{1}}
$$

By replacing the first term of Eq. (28) with its real-time counterpart, we may write the inversion of $\phi$ in terms of the fundament al operation. That is,

$$
p_{d}=p_{1}-\frac{1}{2 \pi i} \int_{\sigma_{0}-i \infty}^{\sigma_{0}+i \infty} h(s) e^{s t} d s
$$

where

$$
i=\sqrt{-1}
$$

If $h(s)$ is analytic within and on a closed curve $C$, except at a finite number of poles $-s_{0}, s_{1}, \ldots, s_{n}$, all inside $C$-then by the residue theorem:

$$
\frac{1}{2 \pi i} \int_{C} h(s) e^{s t} d s=\sum_{j=0}^{n} R_{j}(x) e^{s_{j} t}
$$

where $R_{j}(x)=$ the residue at the $j$ th polv of $h(s)$.

Examining Eq. (29), we find these poles located at

$$
\mathrm{s}=\mathrm{C} \text { (simple pole) }
$$

and

$$
\sqrt{\frac{s}{\alpha}}=i \lambda_{j} \text { (simple pole) }
$$

where $\mathbf{j}=1,2, \ldots, \mathrm{n}$,

such that

$$
\lambda_{\mathrm{j}} \mathrm{L} \tan \lambda_{\mathrm{j}} \mathrm{L}=\beta
$$

We limit our attertion to the first $n$ roots of the transcendental equation, Eq. (36). The 0 th residue, at $s=0$, is $p_{l}$. When $h(s)$ is written in the rational form

$$
h(s)=\frac{g(s)}{z(s)}
$$


the remaining residues at the simple poles $s_{1}, s_{2}, \ldots, s_{n}$ become

$$
R_{j}(x)=\frac{q\left(s_{j}\right)}{z^{\prime}\left(s_{j}\right)}
$$

This, with Eqs. (35) and (36) and after some simplification, yields

$$
R_{j}(x)=\frac{-2 F_{1}\left[\beta^{2}+\left(\lambda_{j} L\right)^{2}-\frac{m\left(\lambda_{j} L\right)^{2}}{\cos \lambda_{j} L}\right]}{\lambda_{j} L\left[\left(\lambda_{j} L\right)^{2}+\beta^{2}+\rho\right]} \sin \lambda_{j} x .
$$

Because the $O$ th term in the inversion of $h(s)$ cancels the first term in the right-hand-side of Eq. (31), the final solution may be written

$$
p_{d}=-\sum_{j=1}^{\infty} R_{j}(x) e^{-\alpha\left(\lambda_{j}\right)^{2} t} .
$$

In the preceding analysis we assumed that the initial pressure in the layer was un:iorm. Unless the initial pressure in the bottle equals that in the layer (i.e., the botlle is full), this assumption is probably erroneous. This problem does not, however, preclude finding a soluiion. For imes greater than the relaxation time for transients within the layer, a quasi-steady-state condition exists. 1 he pressure in the layer decays slowly as air empties from the bottle. At this point the rate of decay depends only on the instantaneous pressure in the bottle and the permeability of the overiying layer. Quasi-steady-state behavin - is independent of initial conditions. the layer:

By taking the time derivative of $\mathrm{Eq}$. (40) we obtain an exprission for the rate of pressure decay within

$$
\frac{\partial p_{d}}{\partial t}=\alpha \sum_{j=1}^{\infty}\left(\lambda_{j}\right)^{2} R_{j}(x) e^{-\alpha\left(\lambda_{j}\right)^{2} t}
$$

At the lower interface, Eq. (41) describes the decay in the bottle:

$$
\frac{d p_{b}}{d t}=\frac{\partial p_{d}}{\partial t} \text {, evaluated at } x=L \text {, }
$$

where $\mathrm{P}_{b}=$ instantaneous pressure in the bottle.

By matching this analytical prediction of the response to the measured response, we can determine an effective permeability for the overlying layer.

A somewhat simpler expression for explicitly determining the effective permeability of material in a layer/bottle configuration can be derived. The nonlinear governing equation for the local pressure is Eq. (I). We now approximate the quasi-steady-state value of $a p / \partial t$; the first-order relation

$$
\frac{\partial p}{\partial t}=\frac{d p_{b}}{d t} \frac{x}{L}
$$


is satisfactory. The boundary conditions are

$$
\mathrm{p}=\mathrm{p}_{0} \text { at } \mathrm{x}=0
$$

and

$$
p=p_{b} \text { at } x=L
$$

By substituting Eq. (43) into Eq. (I) and assuming constant permeability and viscosity, we obtain an ordinary differential equation. After integration and application of the boundary conditions this yields

$$
\mathrm{p}^{2}=\frac{\epsilon \mu}{3 k} \frac{d \mathrm{p}_{\mathrm{b}}}{\mathrm{dt}} \frac{\mathrm{x}^{3}}{\mathrm{l}}+\left(\mathrm{p}_{\mathrm{b}}{ }^{2}-\mathrm{p}_{0}{ }^{2}-\frac{\epsilon \mu}{3 \mathrm{k}} \frac{\mathrm{dp_{ \textrm {b } }}}{\mathrm{dt}} \mathbb{1}^{2}\right) \frac{\mathrm{x}}{\mathrm{L}}+\mathrm{p}_{0}{ }^{2}
$$

Using continuity of mass for the bottle and Darcy's law in the layer, we obtain

$$
\frac{d p_{b}}{d t}=-\frac{\beta k}{2 \epsilon \mu \mathrm{L}} \frac{\partial p^{2}}{\partial x} \text {, evaluated at } x=L
$$

After finding the gradient of $p^{2}$ from Eq. (46), substituting it into Eq. (47), and rearranging, we find the permeability of the material in the layer:

$$
k=-\frac{2(1+\beta / 3) \epsilon \mu L^{2} \frac{d p_{b}}{d t}}{\beta\left(p_{b}^{2}-p_{0}^{2}\right)} .
$$

\section{RESULTS}

\section{OVERTON SAND}

A 57-ft layer of Overton sand was emplaced in U8e, as shown in Fig. 1. Because Overion sand is known to be a relatively high-permeability material, and no other layers were under it (the saturated bed beneath acted as an impermeable barrier), we apply the analysis for a higher-permeability bed.

When stemming ceased, the boundary condition above the bed was determined from pressure transducer 2PX; this pressure variation and the linear ramp fit to it, described by Eq. (6), are shown in Fig. 4. The value of $t^{*}$ is $138 \mathrm{~s}$. The response within the bed was measured with transducer IPX. This response is plotted in Fig. 5 , with the response predicted by Eq. (19) using a permeability of 36 darcys (D). The agreement between the two is quite satisfactory.

After decay of pressure in the Overton sand layer, stemming of the next layer of NTS pea g:avel began. The resulting pressure rise at the upper boundary of the emplaced layer of Overton sand and the pressure response within the layer were again measured with $2 \mathbf{P X}$ and $1 \mathrm{PX}$. The value of $t^{*}$ in Eq. (21) is associated, in this case, with the terminal velocity of the pea gravel. A permenbility of $36 \mathrm{D}$ provides equally good agreement between these predicted and measured responses in the Oycrton sand layer.

This value of $36 \mathrm{D}$ is considerably higher than that indicated by a previous laboratory investigation, ${ }^{4}$ which found the permeability of rodded Overton sand to be near 25 D. On the other hand, estimation of permeability on the basis of particle size gives a still higher value. Sieve analyses ${ }^{4}$ show Overton sand to be nearly 

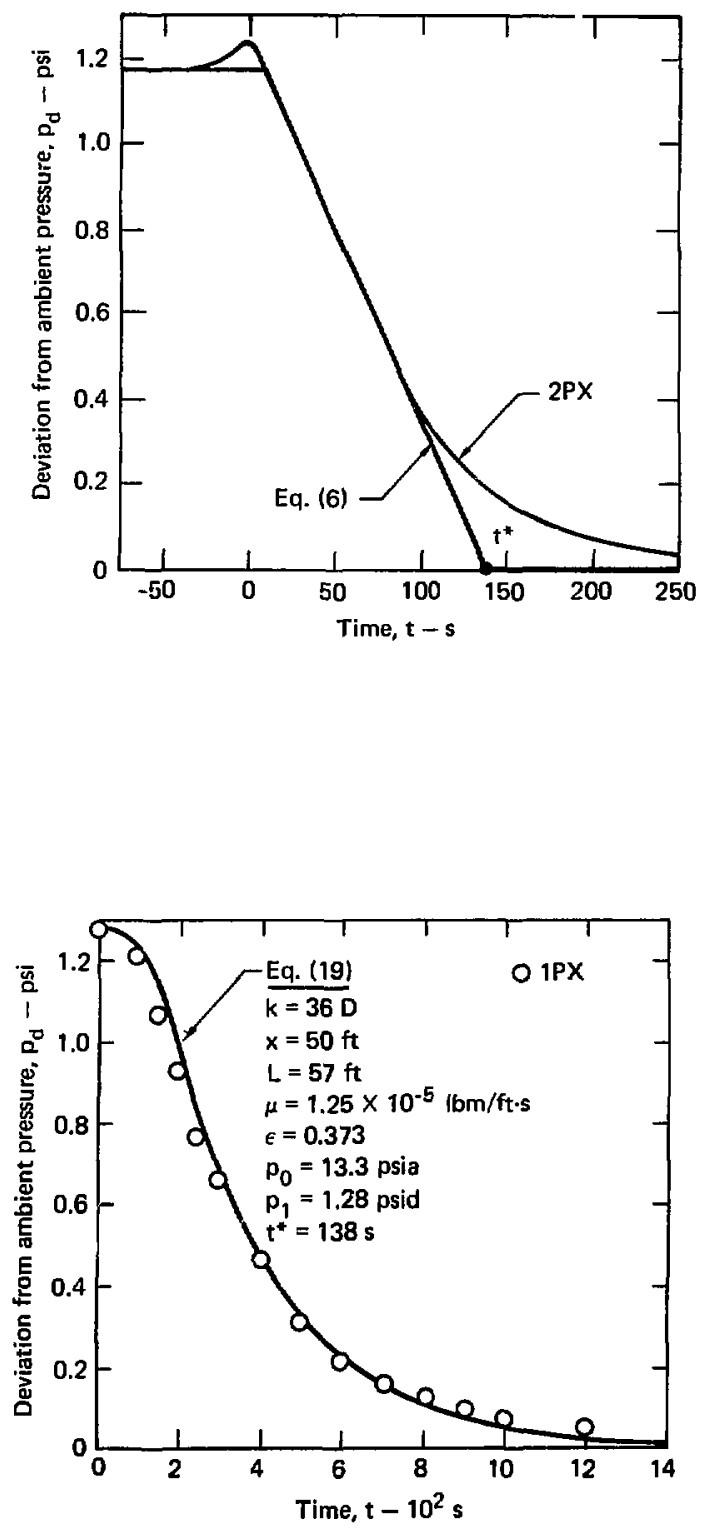

FIG. 4. Pressure variation at upper boundary $(x=0)$ of the Overton sand layer, neasured with transducer $2 \mathrm{PX}$. Issing the fall time $t^{*}, \mathrm{Eq}$. (6) describes the variation.
FIG. 5. Pressure response within the Orerton sand luyer, measured with transducer IPX, and the response predicted by Eq. (19) using permeability $=36 \mathrm{D}$. 
monodisperse, with a natr ow diameter range centered about $0.010 \mathrm{in}$. Use of this diameter, $d$, in the empirical expression 5

$$
k=\left(6.54 \times 16^{-4}\right) d^{2}
$$

yields AS D. Naturally, the presence of smaller particles would tend to reduce permeability. The permeability of emplaced Overton sand, as determined here, is in reasonable agreement with these previous estimates.

\section{PEA GRaVEL}

When stemming of the pta gravel layer stopped, pressure measurements made with 2.PX and 3PX provided an opportunity to estimaie pea gravel permeability. The responses within and above the layer were so nearly identical, however, that an accurate estimate was not possible. Sample calculations, using the model for a higher-permeability bed, convinced us that we easily could have distinguished differences in responses if the permeability were less than $2500 \mathrm{D}$. It must exceed $2500 \mathrm{D}$, perhaps considerably. The diameter of the pea gravel ranges from about 0.15 to $0.36 \mathrm{in}$. A rough calculation, using uniform 0.28 -in. spheres in Eq. (49), gives a permeability of about $30,000 \mathrm{D}$.

Containment calculations $\mathbf{s}^{\mathbf{1}, 2}$ are insensitive to large values for pea gravel permeability. Because flow is controlled by the other, lower permeability, layers, any high permeability of pea gravel gives essentially equivalent results.

\section{LLL MIX}

The U8e experiment offers two layers of emplaced LLL mix that are suitable for analysis. These are shown in Fig. 1. Whereas the model for higher-permeability beds sulficed for treatment of the preceding two, progressively more permeable layers, the model for lower-permeability beds is appropriate for analysis of the LLL mix layers.

The lower 43-ft layer was emplaced in the lined portion of the hole above the pea gravel and Overton sand. The saturated bed beneath the sand layer acted as an impermeable lower boundary fur the bottle. Both the sand and pea gravel contribute to the bottle's total volume.

Pressure in the bottle, measured with $1 \mathrm{PX}$ and 2PX, had reached the maximum possible, as measured with 3PX, before stemming ceased. Hence the initial pressure distribution is known: it is uniform in the layer and bottle. Moreover, as we had assumed, pressure in the bottle was nearly uniform in space throughout the decay.

Measurements with 4PX, just above the emplaced LLL mix layer, provide a description of the boundary condition at the upper interface. The observed decay time, $t^{*}$, of $14 i$ s was very small compared with the total response time. Therefore, we considered this boundary condition to be a step change from $p_{l}$ to zero, as expressed in Eq. (23).

The response measured with $1 \mathrm{PX}$ and $2 \mathrm{PX}$ is plotted with the response predicted by Eq. (40) in Fig. 6. In this instance the permeability determined is the effective permeability of the layer, i.e., the permeability of an equivalent amount of homogeneous material. Using a value of $8.1 \mathrm{D}$ provides good agreement between the analytical prediction and the measured response.

At 4000 s the rate of dectay measured with IPX or $2 \mathrm{PX}$ is $-1.07 \times 10^{-4} \mathrm{psi} / \mathrm{s}$, as indicated in Fig. 7. At that time the instantaneous pressure in the bottle and the lucal ambient pressure are also known. Using these values, in Eq. (48), with 

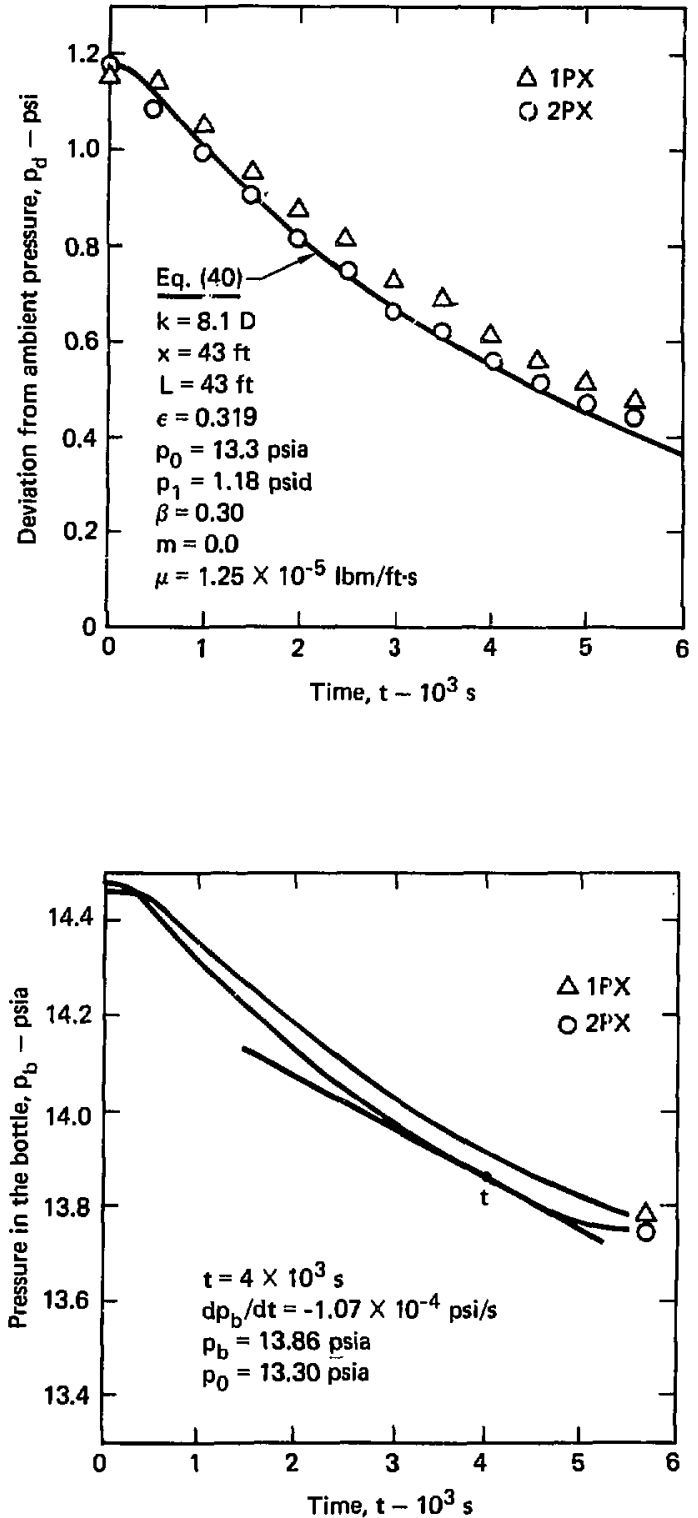

FIG. 6. Pressure response in the underlying bed (bottle) for the 43-ft LI.L mix layer, measured with transducers; $1 \mathrm{PX}$ and $2 \mathrm{PX}$, and the response pr:dicted by Eq. ( 40 using permeability $=$ $8.1 \mathrm{D}$.
FIG. 7. Rate of desay of pressure $\left(\mathrm{dp}_{\mathrm{b}} /\right.$ $\mathrm{dt}$ ) in the underlying bed (bottle) for the 43-ft LLL mix layor, determined from measurements with transducers IPX and 2PX. 


$$
\begin{aligned}
& L=43 \mathrm{ft} \\
& \beta=0.30 \\
& \epsilon=0.319 \\
& \mu=1.25 \times 10^{-5} \mathrm{lbm} / \mathrm{ft} \cdot \mathrm{s},
\end{aligned}
$$

gives an effective permeability of $7.6 \mathrm{D}$, which aprees well with the results from Eq. (40).

Nonuniformity of material in this layer of LLL mix is illustrated by the pressure response measured with 3PX, shown in Fig. 8. At all times, the measured pressure is higher than that predicted for the same location using Eq. (40) and an effective permeability of 8.1 D. The response measured with $3 \mathrm{PX}$ is nearly identical to that predicted $10 \mathrm{ft}$ closer to the lower interface when homogeneity within the layer is assumed. This indicates that the permeability of the material between the transducer and the lower interface is much higher than the effective value for the layer. Such behavior is consistent with the expected stratification of constituents in emplaced LLL mix.

When stemming of LLi mix begins, coarse material with a high terminal velocity 9rrives downholr before the finer constituents. This forms a section of high-permeability material at the low's boundary of a.1 emplaced layer. Similarly, when stemming stops, fine low-permeability material builds up at the upper boundary. In the interim, equilibrium may be established if the stemming interval is sufficiently long.

The available means of analyzing the 85-ft layer of LLL mix are more limited than those for the 43-ft layer-air pressure in the bottle was not uniform at the time stemming ended, so the initial conditions are not known. Also, stemming of a subsequent layer of pea gravel started soon after this layer was emplaced, so only a short time existed during which quasi-steady-state behavior was displayed. These constraints restrict our analysis to the explicit relation given by Eq. (48).

Pressure in the bottle was measured with all four transducers. Although the pressure was not uniform in this region, the rate of pressure decay was. Figure 9 shows the measured rate. Using the measured values for $\rho_{b}$ and $p_{0}$ in Eq. (48), with

$$
\begin{aligned}
& \mathbf{L}=85 \mathrm{ft} \\
& \beta=0.45 \\
& \epsilon=0.319 \\
& \mu=1.25 \times 10^{-5} \mathrm{lbm} / \mathrm{ft} \cdot \mathrm{s},
\end{aligned}
$$

yields an effective permeability of $6.8 \mathrm{D}$ for this layer.

An important aspect of this result is that the emplaced 85-ft layer does not lie in a cased portion of the hole. The medium surrounding the layer is tuff, a porous yolcanic rock; therefore we might have expected to find a much higher effective permeability because of flow through the wall of the hole. Such flow apparently we not significant. In this instance, tuff behaves as if it were impermeable. Measurements in other drill holes, however, have shown the opposite behavior-rapid pressure decay as the result of radial flow into the surrounding rock.

The observed effective permeabilities of $\sim 8$ and $\sim 7 \mathrm{D}$ for the LLL mix layers are, as we found for the Overton sand layer, somewhat higher than determined by laboratory tests. These tests ${ }^{4}$ found the permeability of the rodded material to be 1.21-1.71 D. The differences probably arise from the pronounced nonuniformity in these short layers.

The quasi-steady-state analysis presented here was also applied to data obtained in a 224-ft layer of LLL mix, which was emplaced above a depth of $1922 \mathrm{ft}$ in a hole designated U2ar. The effective permeability of 1.3 D determined for this longer layer was, as expected, closer to the laborat ory value for rodded LLL mix and considerably lower than those found for the $43-$ and $85-\mathrm{ft}$ layers. This probably results from the diministed effect of nonuniformity in the longer layer, which would reduce its effective permeability. 

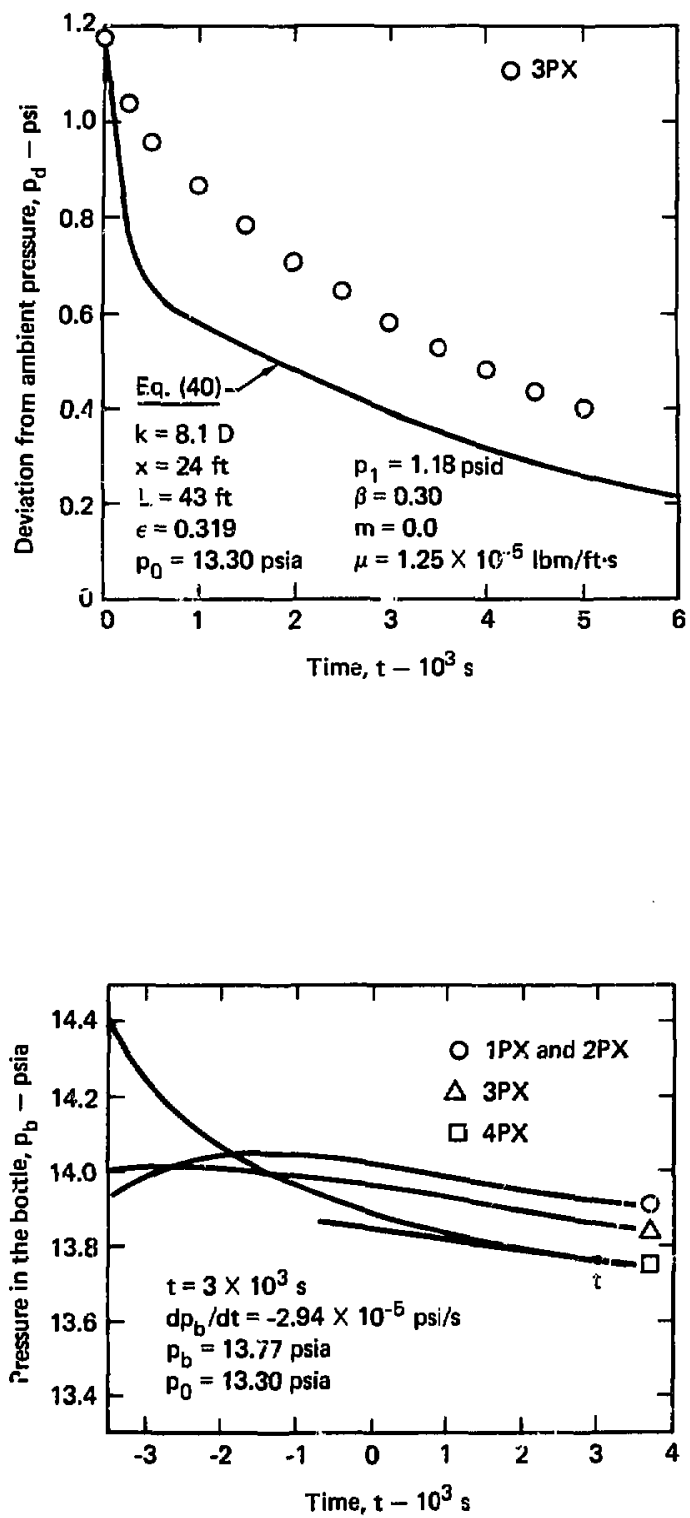

FIG. 8. Nonuniformity in the 43-ft LLL mix layer: the pressure measured with (ransducer $3 \mathrm{PX}$ within the layer is higher than that predicted by Eq. (40) using the effective permeability of $8.1 \mathrm{~L}$.
FIG. 9. Rate of decay of pressure ( $\left.d p_{b}\right)$ di) in the underlying bed (bottle) for the 85-ft LLL mix layer, determined from messurements with transducers IPX, 2PX, 3PX, and 4PX. 


\section{CONCLUSIONS}

To describe the fluid flow through a granular layer following the cessation of stemming, we developed two gas-flow models. One deals with the flow through a higher-permeability bed-one lying above a layer of relatively low-permeability materal. The other applies to flow through a lower-permeability bed lying above a layer of relatively high-permeability material. To determine the permeabilities of LLL stemming materials, we made pressure measurements in layers of Overton sand, NTS pea gravel, and LLL mix; by matching an analytical prediction of the pressure response to the data, we determined the effective permeability of the emplaced materials.

The permeability of emplaced Overton sand was found to be near $36 \mathrm{D}$. This value is higher than that deternined in laboratory tests on rodded sand, but lower than the permeability estimated on the basis of uniform particle size.

The analysis of pressure response in a layer of NTS pea gravel indicated its permeability to be at least $2500 \mathrm{D}$. This value is only a lower bound; we expect that the actual permeability is much higher. An estimated permeability of $30,000 \mathrm{D}$ based on a typical particle size for NTS pea gravel supports this expectation.

Three layers o: LLL mix were analyzed. The effective permeabilities range from about $8 \mathrm{D}$ for a short layer to $1.3 \mathrm{D}$ for a longer layer. These values are consistent with the expected deviations from laboratory results. In each case, the permeability of emplaced LLL mix is greater than or approximately equal to the value found in laboratory tests on rodded LLL mix.

The differences in emplaced LLL mix permeabilitics reflect the non uniformities in these layers. The constituents of LLL mix fall at different speeds, the larger falling faster. The result is nonuniformity at the top and bottoin of each layer; pressure responses within the layers verified this. The effects of LLL mix nonuniformity on fluid flow are expected to be greatest in deep, short layers.

\section{ACKNOWLEDGMENT}

We wish to thank Tom Phillips and Larry Starrh for their constant assistance and Hugh Watling for his continuing support of this project. We are also grateful for the invaluable help of many CREMINO management team members and of the EG\&G special measurements personnel.

\section{REFERENCES}

1. F. A. Morrison, Jr., "Transient Multiphase Multicomponent Flow in Porous Media," Int. J. Heat and Mass Transfer 16, 2331 (1973).

2. F. A. Morrison, Jr., "Flow Through Porous Media from a Nuclear Cavity," American Society of Mechanical Engineers (ASME) Paper 77.FE-23, presented at the ASME Joint Applied Mechanics, Fluids Engineering and Bioengineering Conference (New Haven, Cona, 1977).

3. F. A. Morrison, Jr., "Transient Gas Flow in a Porous Column," Ind. Eng. Chem. Fundam. 11, 19l (1972).

4. J. Pitts and W. Kleck, Jr., Permeability and Porosity of Some Stemming Materials, Lawrence Livermore Laboratory, Livermore, Calif, UCID-15876 (1970).

5. R. J. M. DeWiest, Flow Through Porous Media (Academic Press, New York, 1969). 
$\infty$

SAND79-0264

Unlimited Release

Seismic Reflection Data Report

Waste Isolation Pilot Plant (WIPP)

Site, Southeastern New Mexico

\section{Volume II}

Decembar 1978

John L. Hern, G. J. Long \& Associates, Inc.

Dennis W. Powers, Lawrence J. Barrows, Sandia Laboratories

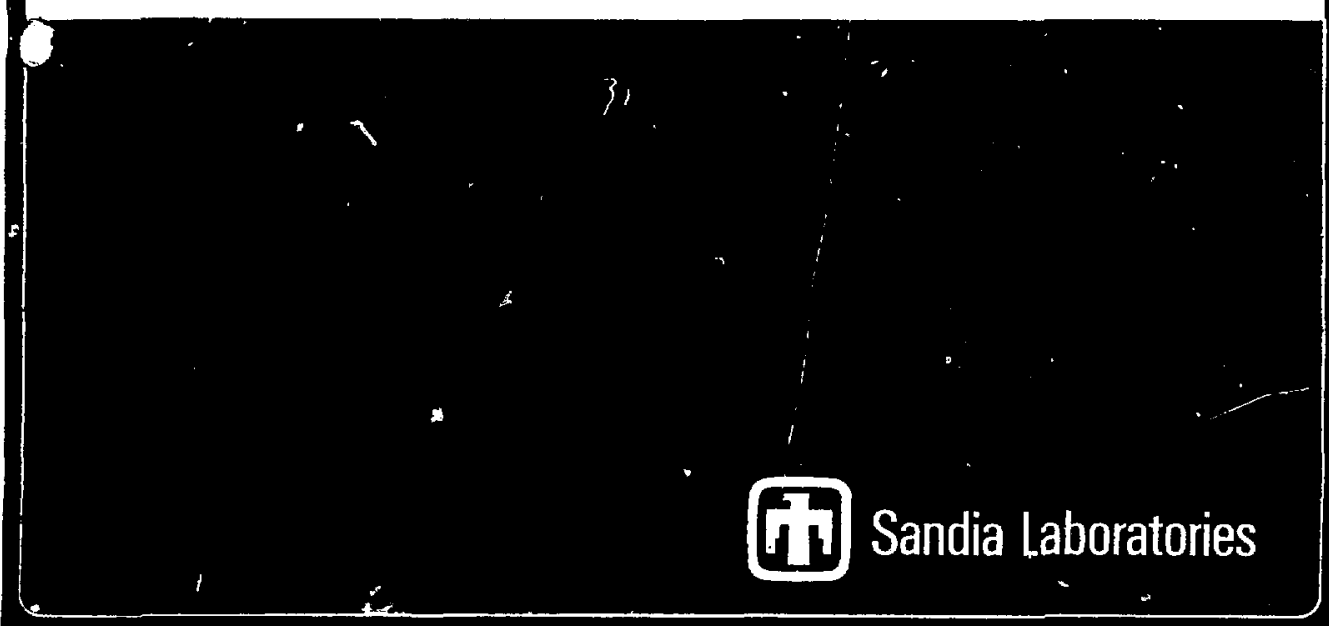

SF 2900 017-73) 


$$
\begin{gathered}
\text { SAND79-0264 } \\
\text { Unlimited Release } \\
\text { Printed May, } 1979
\end{gathered}
$$

SEISMIC REFLECTYON DATA REPOR'

\title{
Waste Isolation Pilot Plant (WIPP) Site, Southeastern New Mexico
}

\author{
VOLUME IT \\ John L. Hern \\ G. J. Long \& Associates, Inc. \\ Houston, Texas \\ Dennis $\%$. Powers \\ and \\ Lawrence J. Barrows \\ Division 4511 \\ Sandi a Laboratories \\ Al buquerque, New Mexico
}

DECEMBER， 1978 


\subsection{INDEX TO ENCLOSURES IN POCKETS*}

Combined Vibrose is Program within WIPP Site Area Map

1976 Seismic Program Map

Seismic Line 1

Seismic Line 2

Seismic tine 3

1977 Seismic Program Maps (2)

Seismic Line $\mathbf{X - 1}$

Seismic Line $x-2$

Seismic tine $x-3$

Seismic Line $x-3 A$

Seismic Line $x-4$

Seismic Line $x-5$

Seismic Line $x-6$

Seismic Line $x-7$

Seismic Line $x-8$

Seismic Line $x-9$

Seismic Line $x-10$

Seismic Line $\mathrm{x}-11$

Seismic tine $x-12$

Seismic Line $x-13$

1978 Seismic Program Map

Sejsmic tine $\mathrm{Y}-\mathrm{I}$

Seismic Line $\mathrm{Y}-2$

Seismic Line $Y-3$

Seismic ine $\mathbf{Y}-4$

Seismic line $Y-5$

*Please note that all of the above data were corrected and processed with $\theta$ 3200 foot elevation datum. 


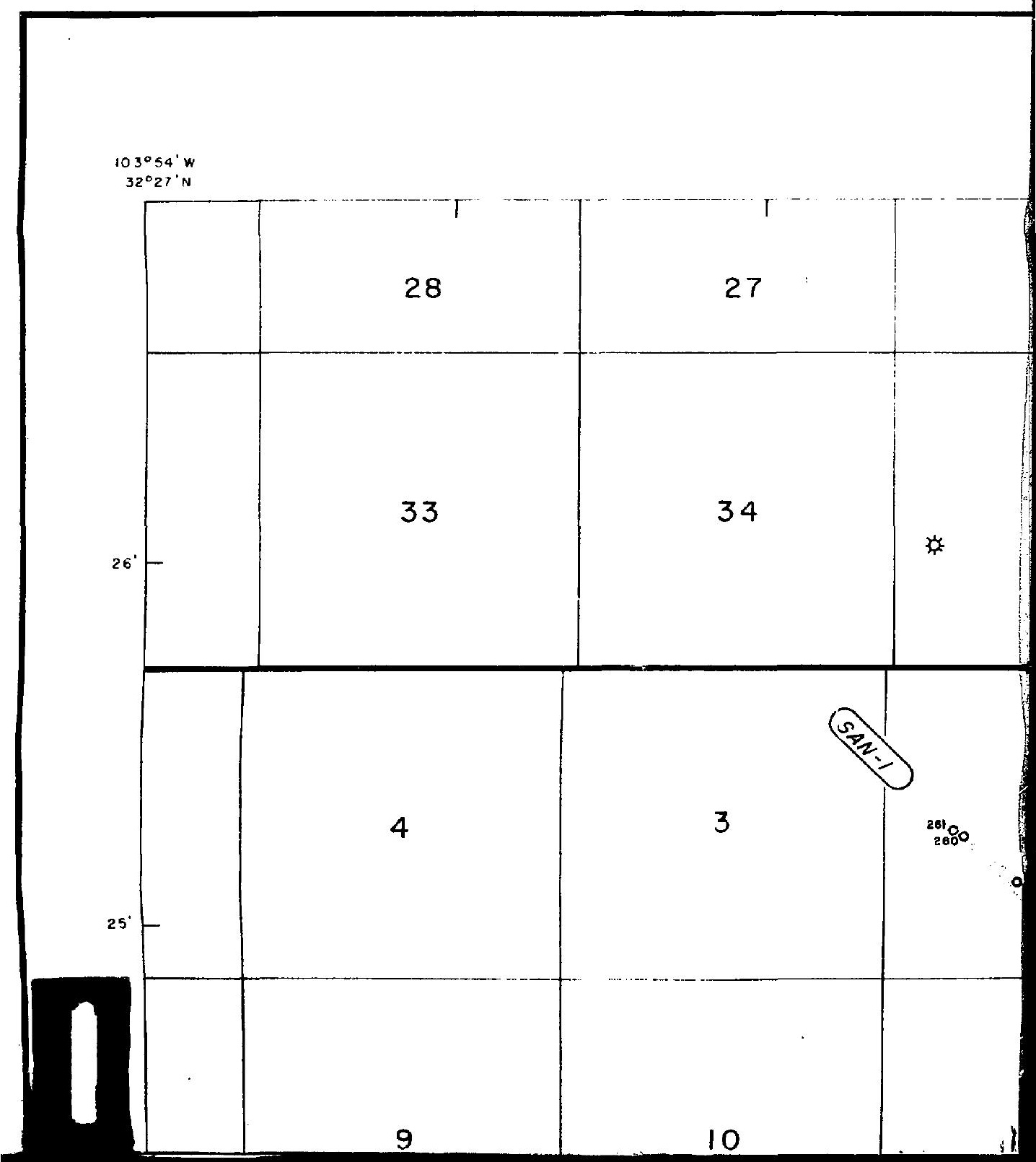




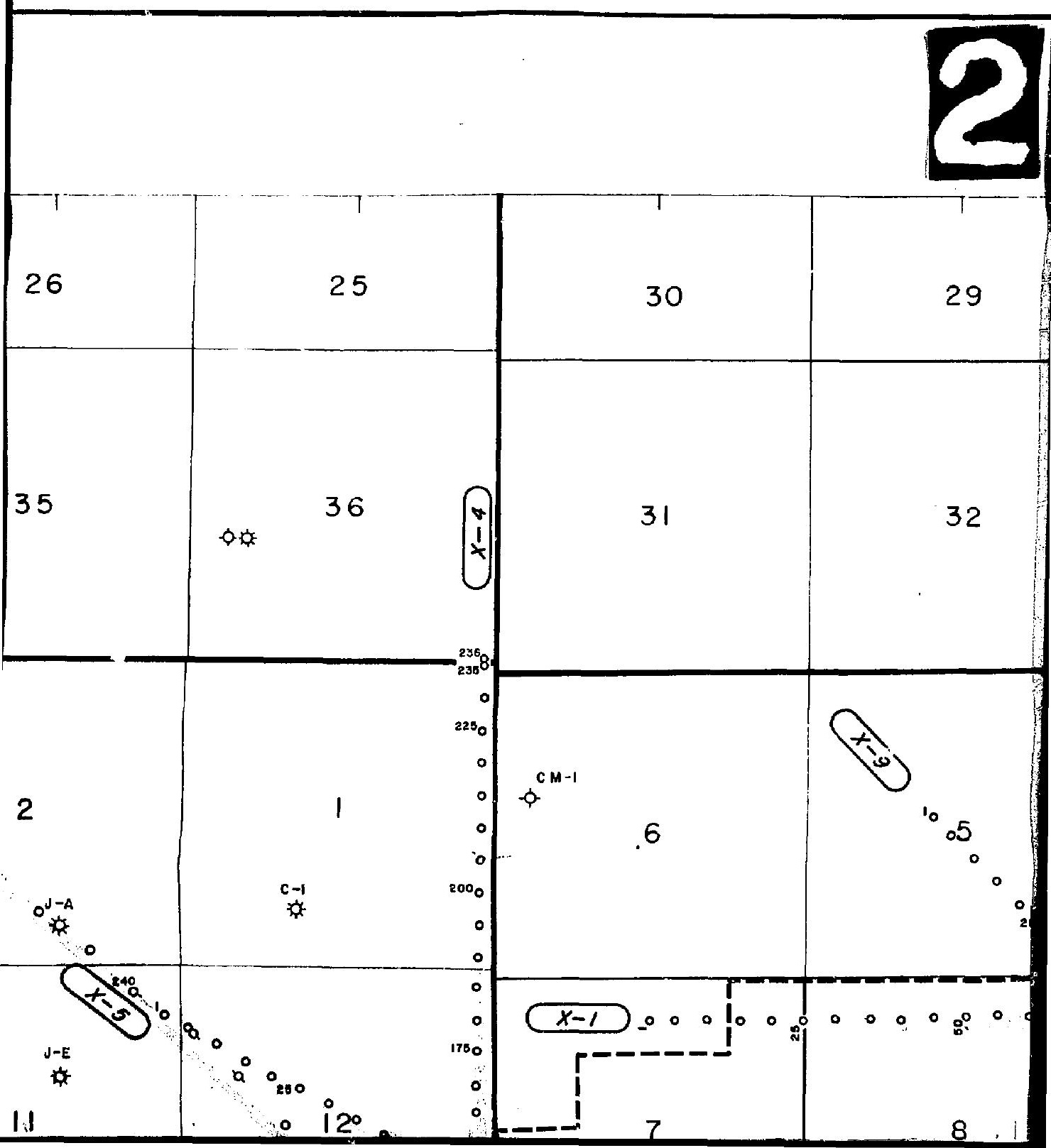




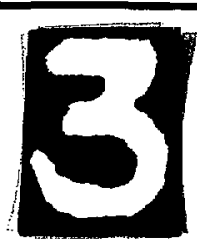

28

27

26

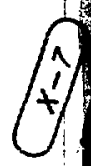

8

33

34

35

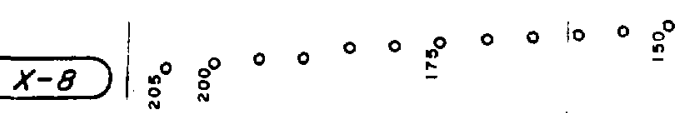

4

3

280

o.
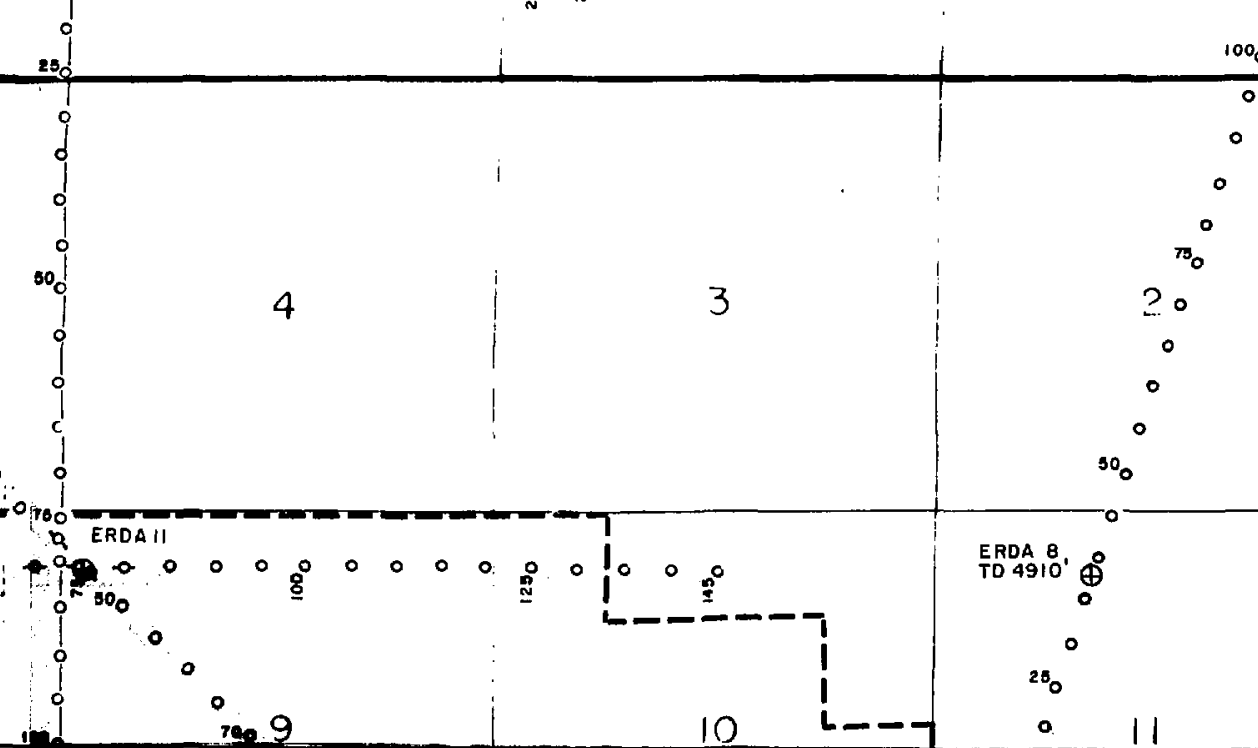

ERDA 8

TD $4910^{\prime} \oplus$

Q ERDAII

$\circ$

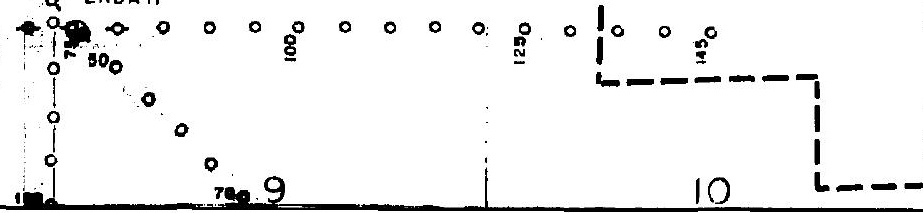




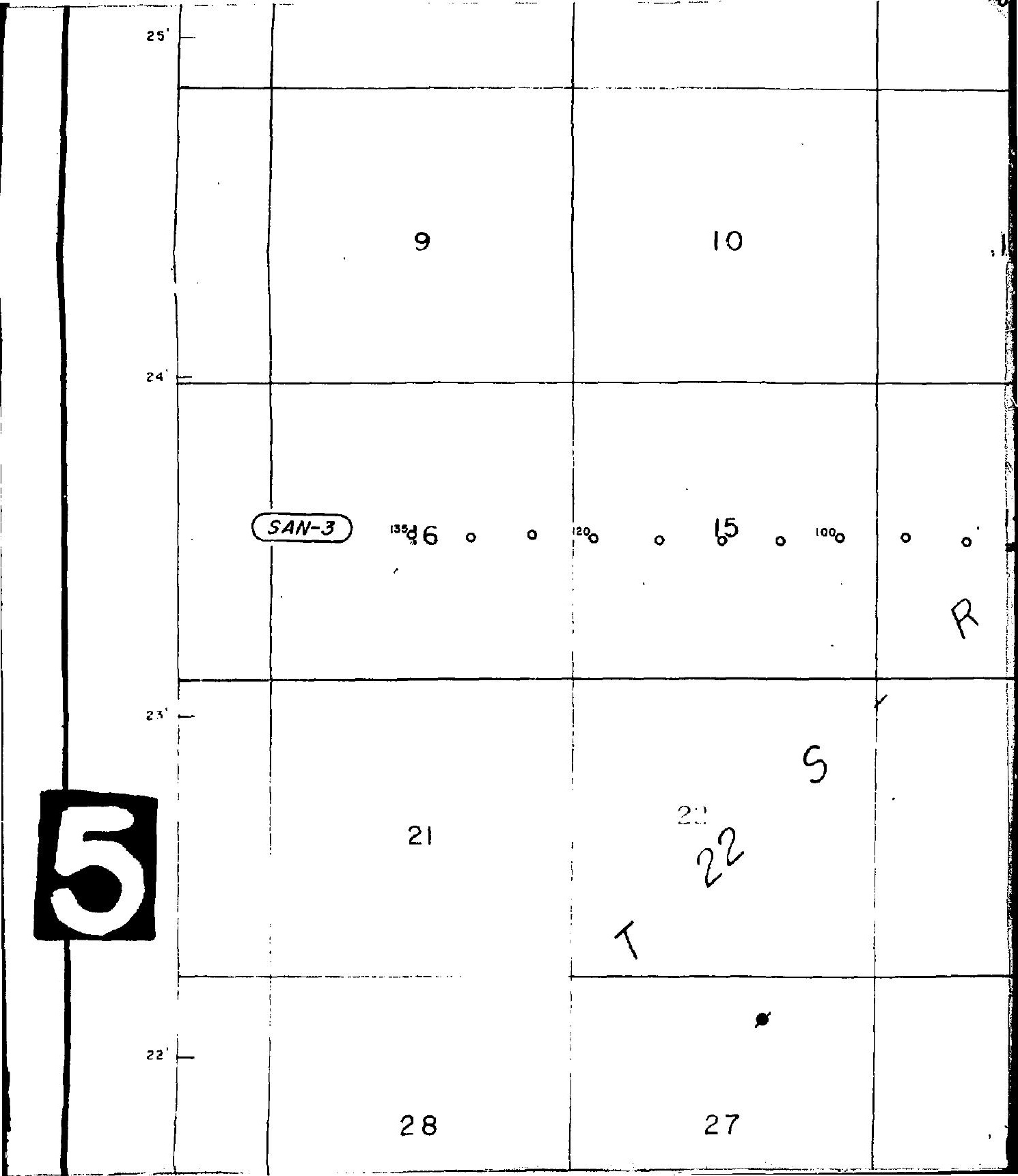




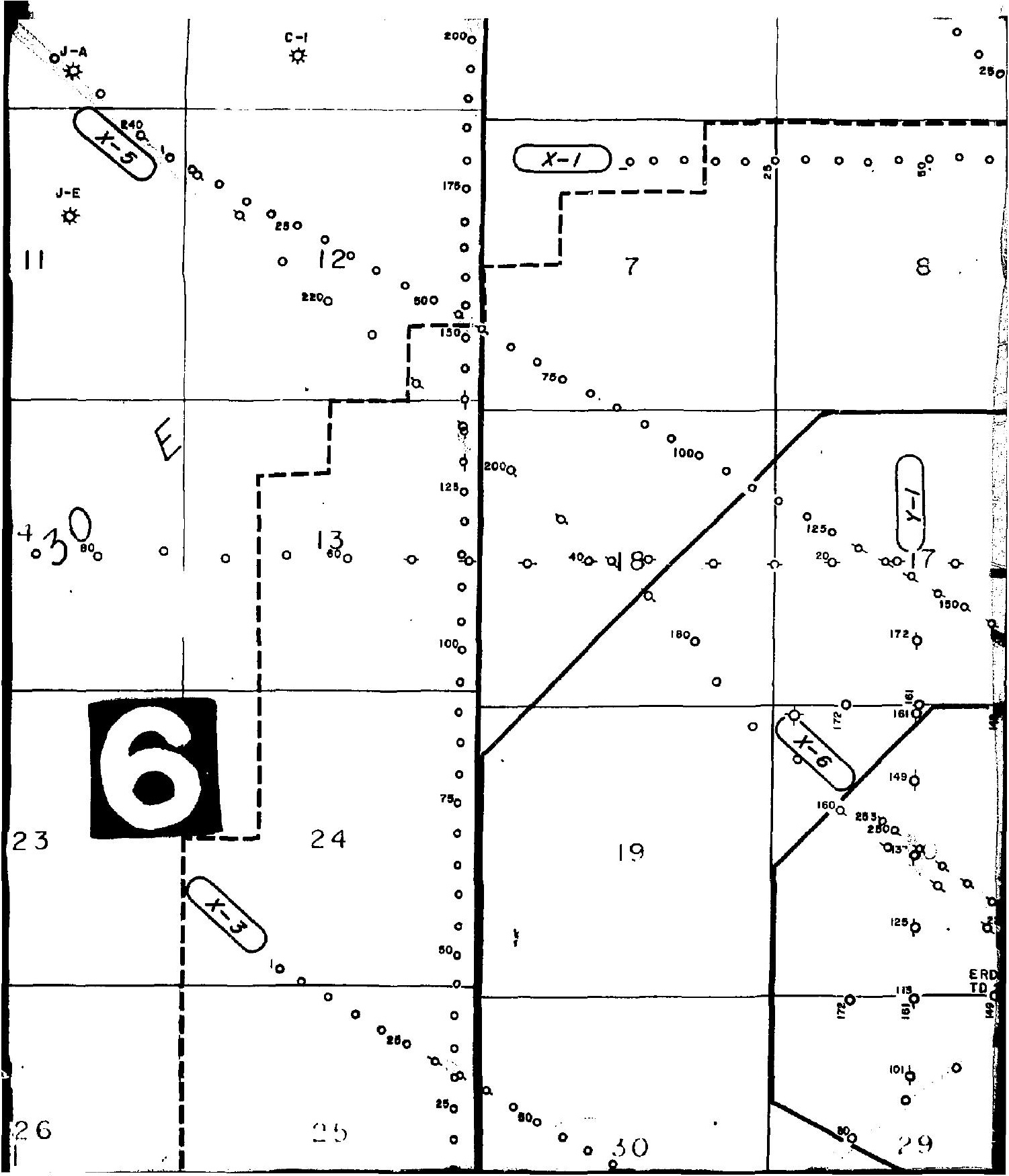




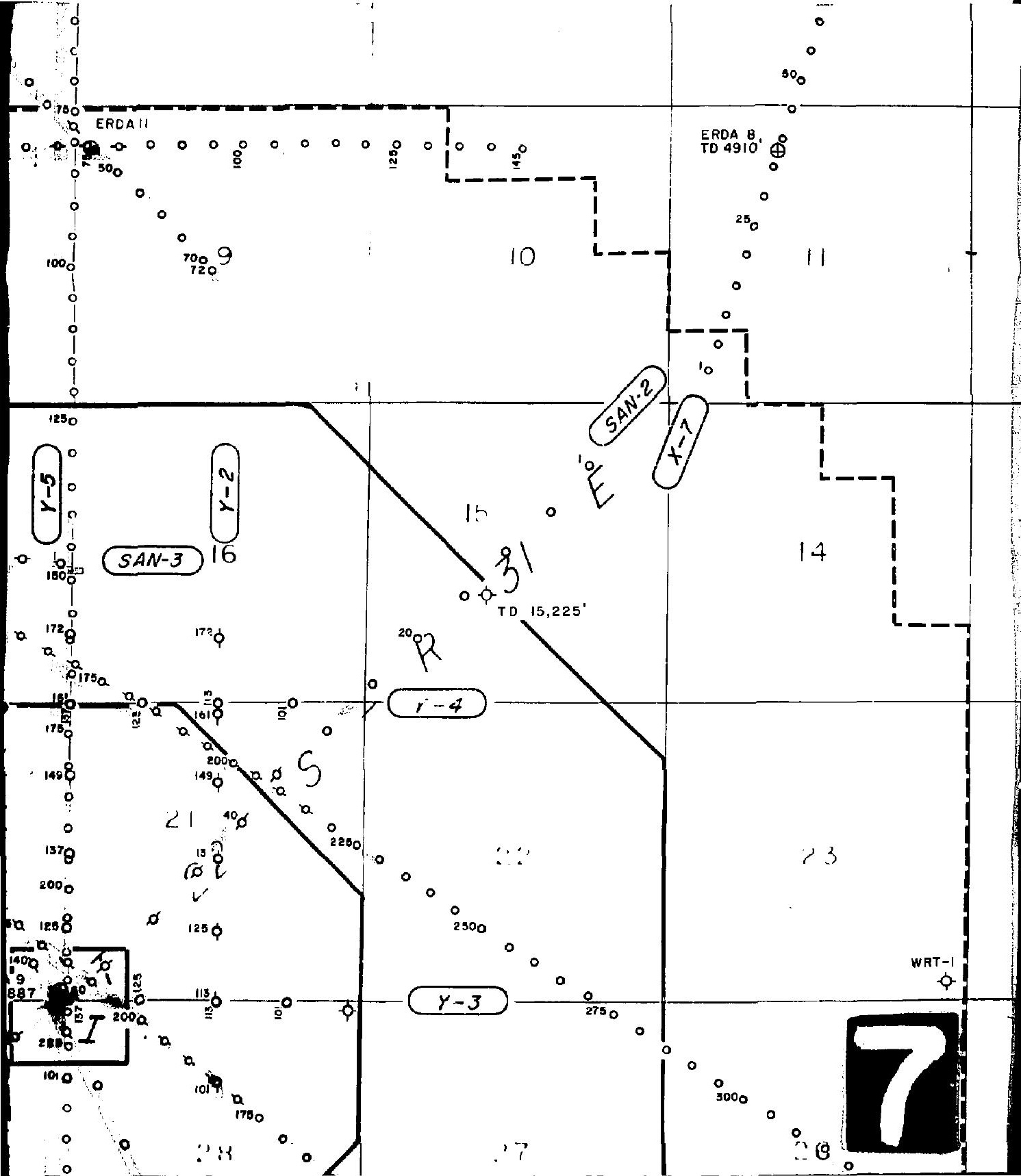




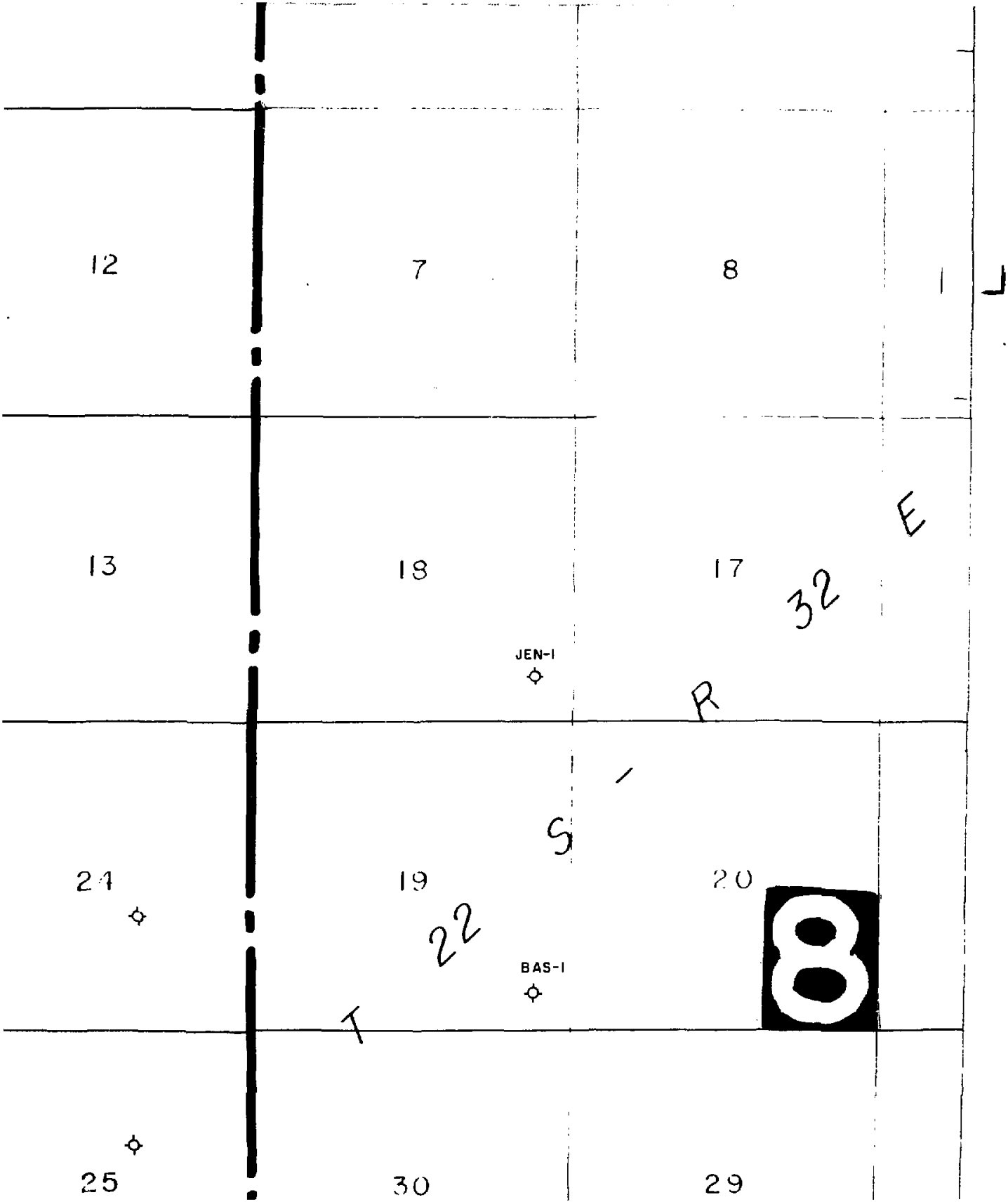




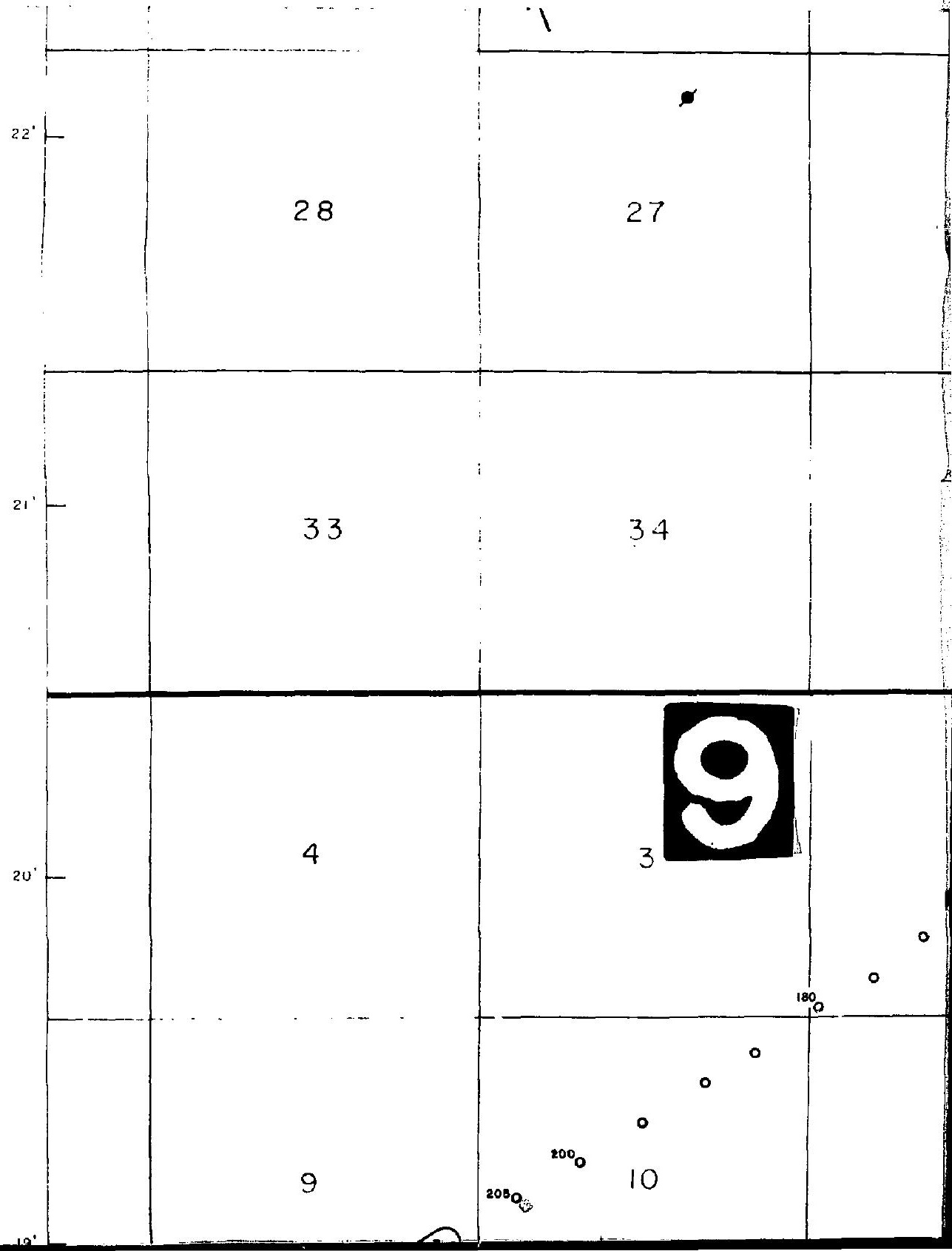




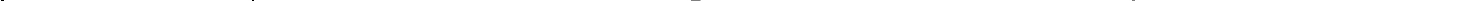




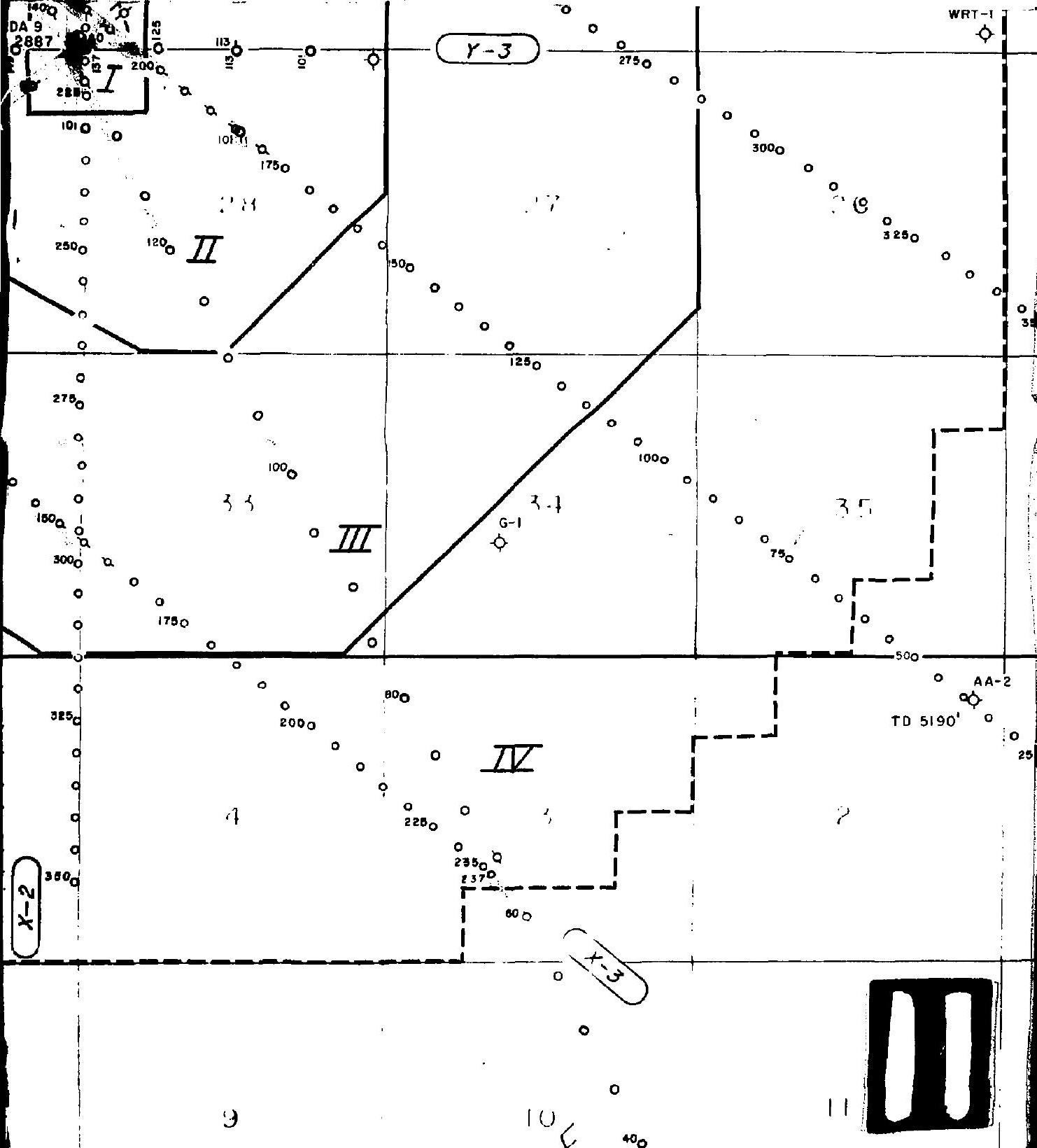




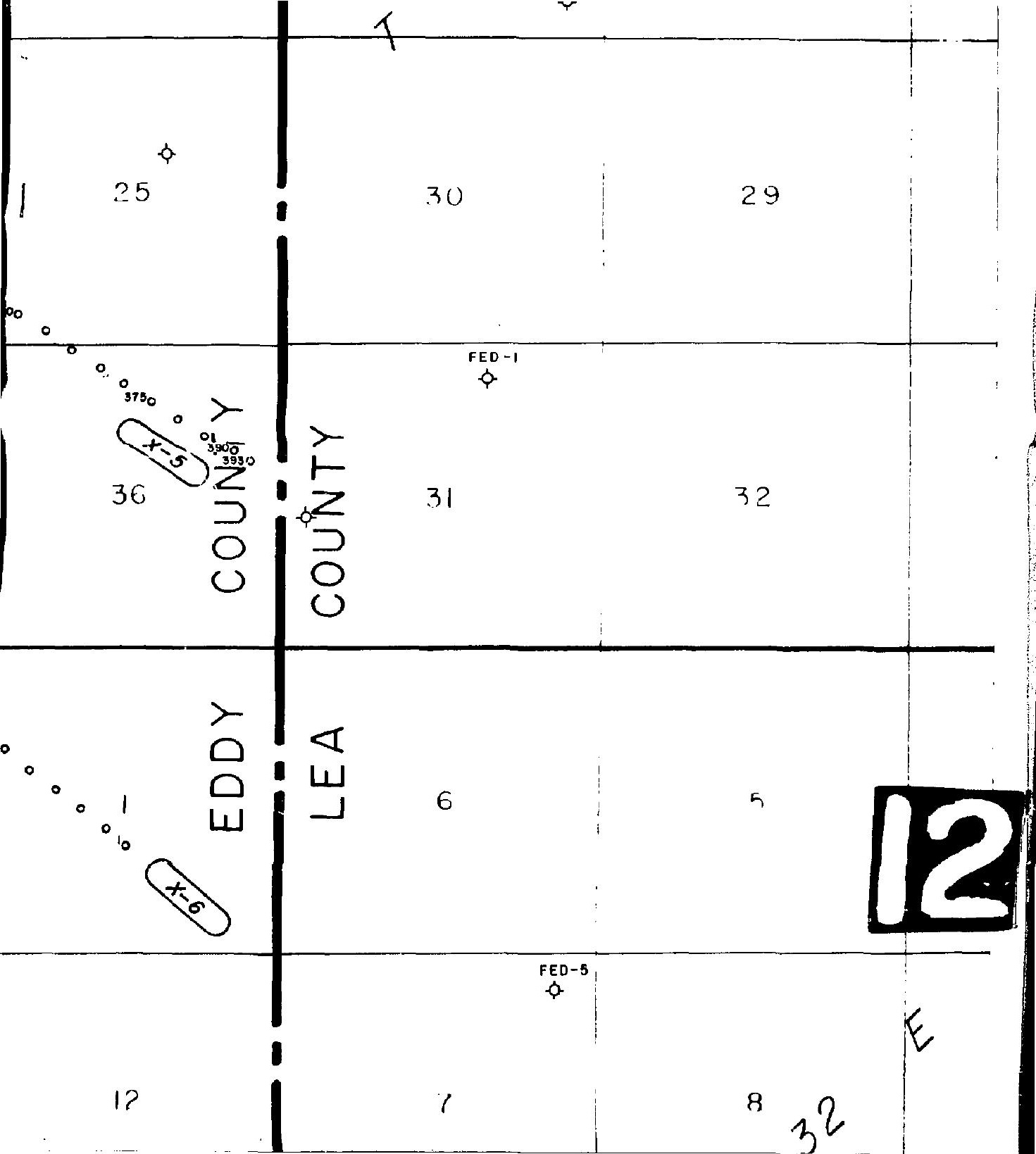




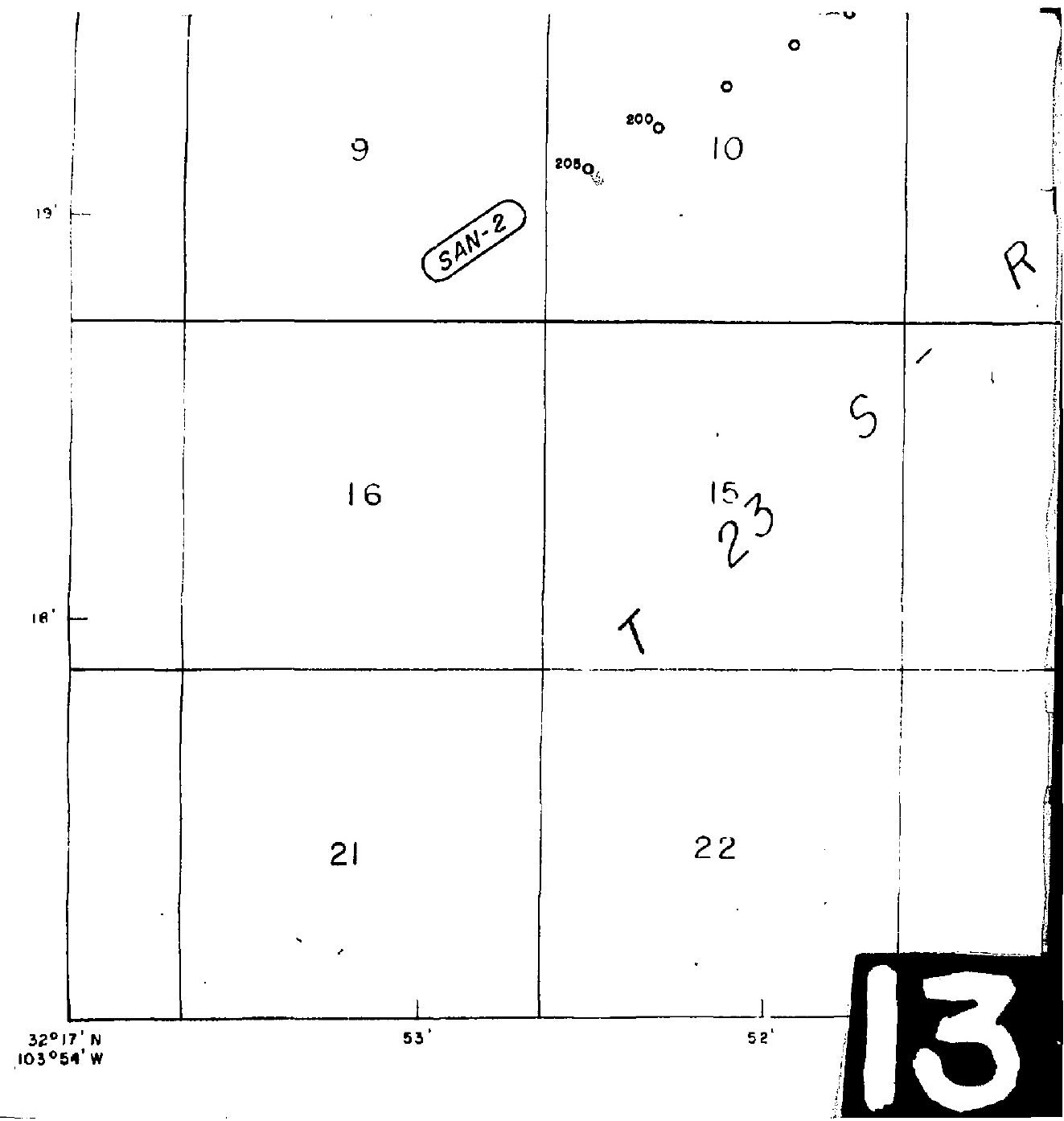




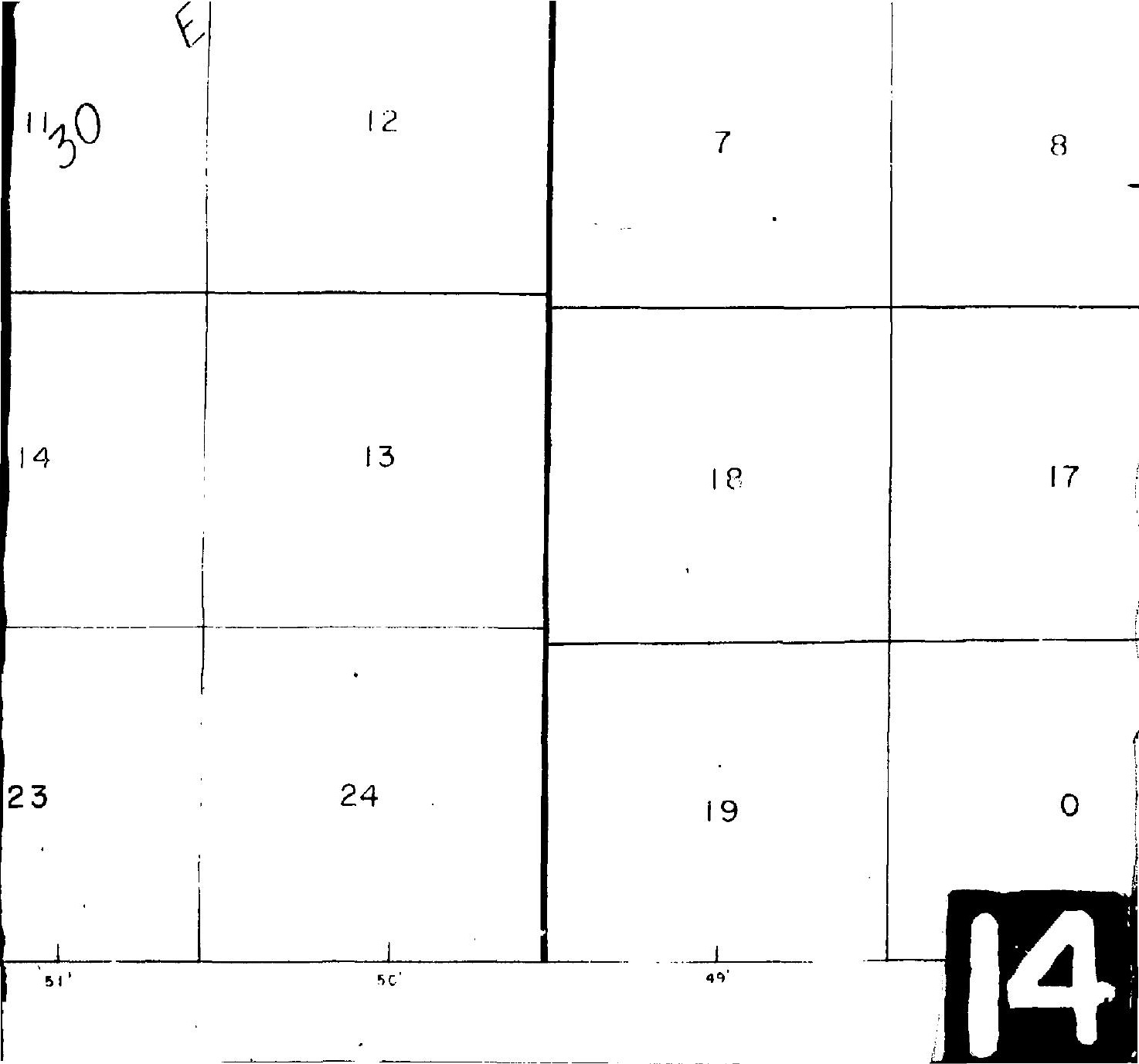




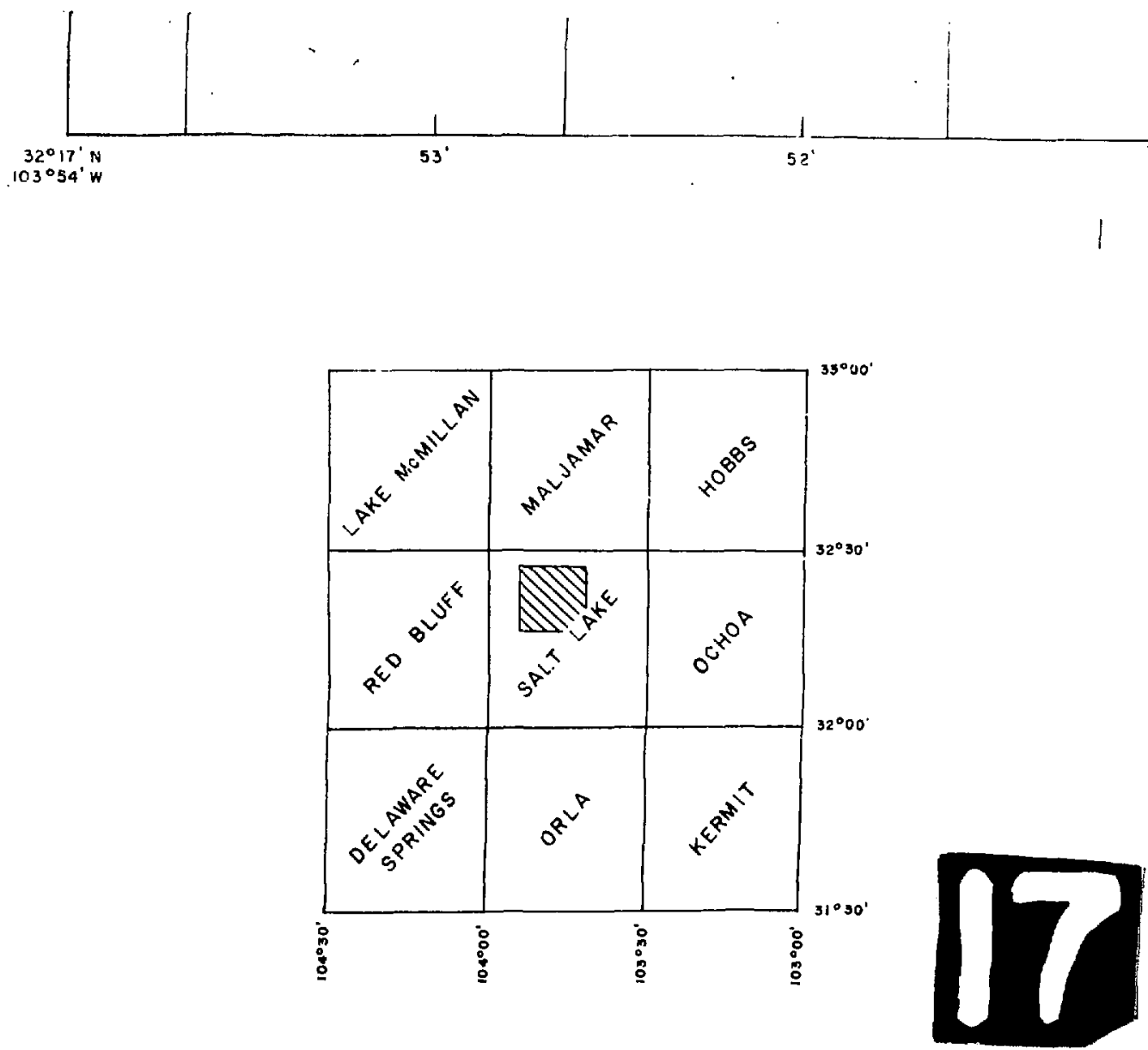
<smiles>C=C[As]=C</smiles> 


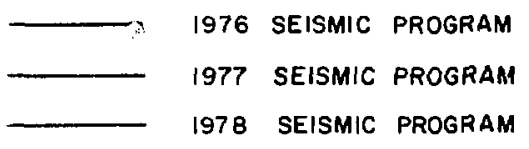

\begin{tabular}{|r|rr|}
\hline ZONE & \multicolumn{2}{|c|}{ AREA } \\
\hline I & 100 & Acres \\
III & 1,818 & Acres \\
III & 6,221 & Acres \\
III & 10,821 & Acres \\
\hline TOTAL & 18,960 & Acres \\
\hline
\end{tabular}

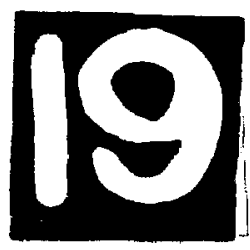



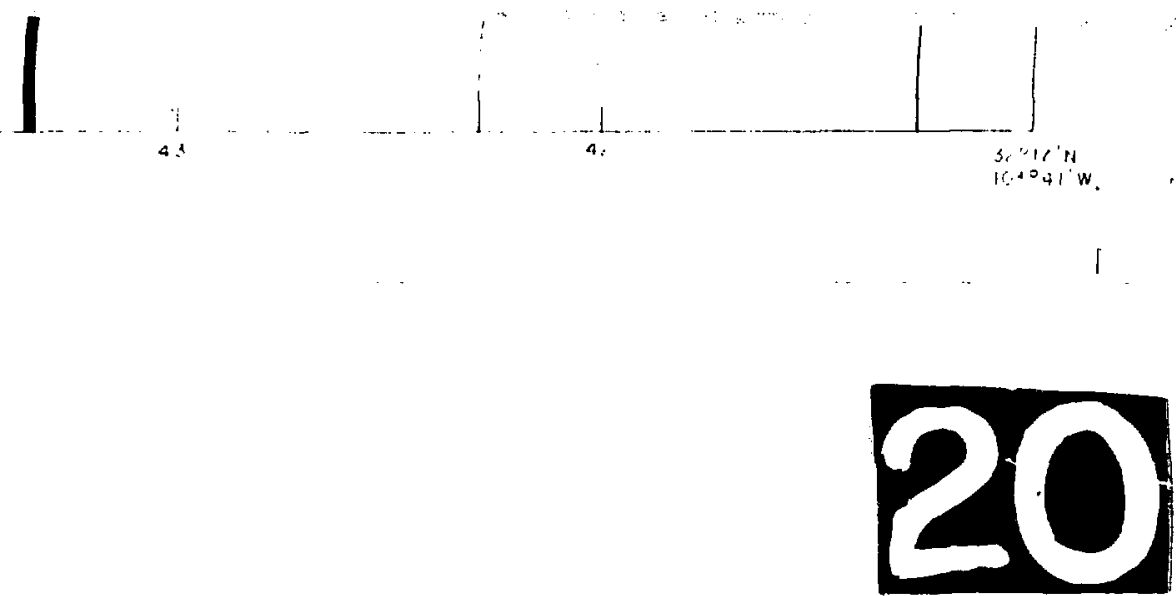

\section{SANDIA LABORATORIES}

SEISMIC SURVEY

LOS MEDANOS SITE AREA

EDOY COUNTY, NEW MEXICO

\section{COMBINEO VIBROSEIS PROGRAM WITHIN WIPP SITE AREA}

IN : EWUHE. ATION GY

G J LONC \& $25 S O C I A T E S$, INC

SEISMO: VG.ST

SCFLE

$1^{\prime \prime}=2,000^{\circ}$
APPROVED

- c. I.




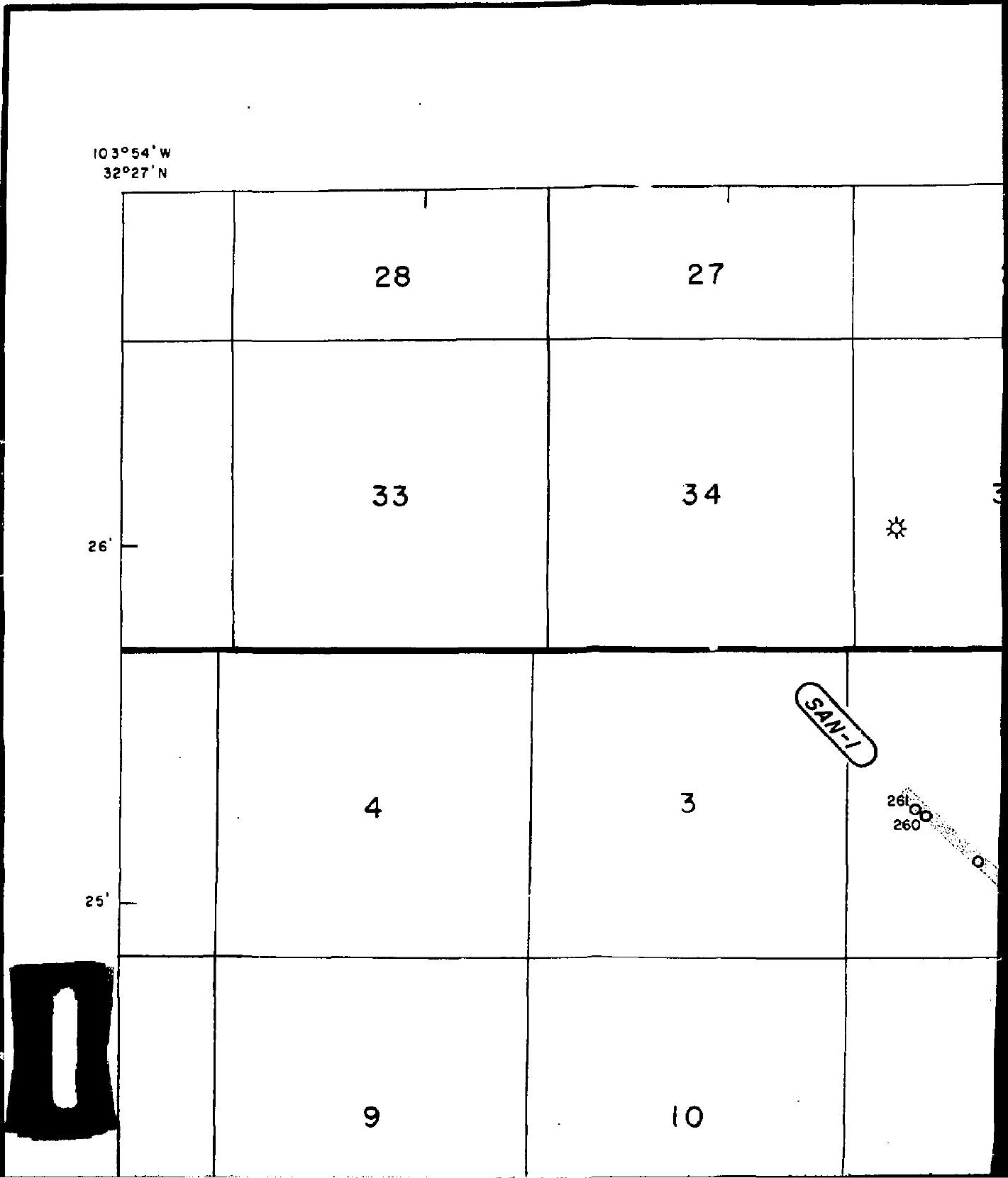




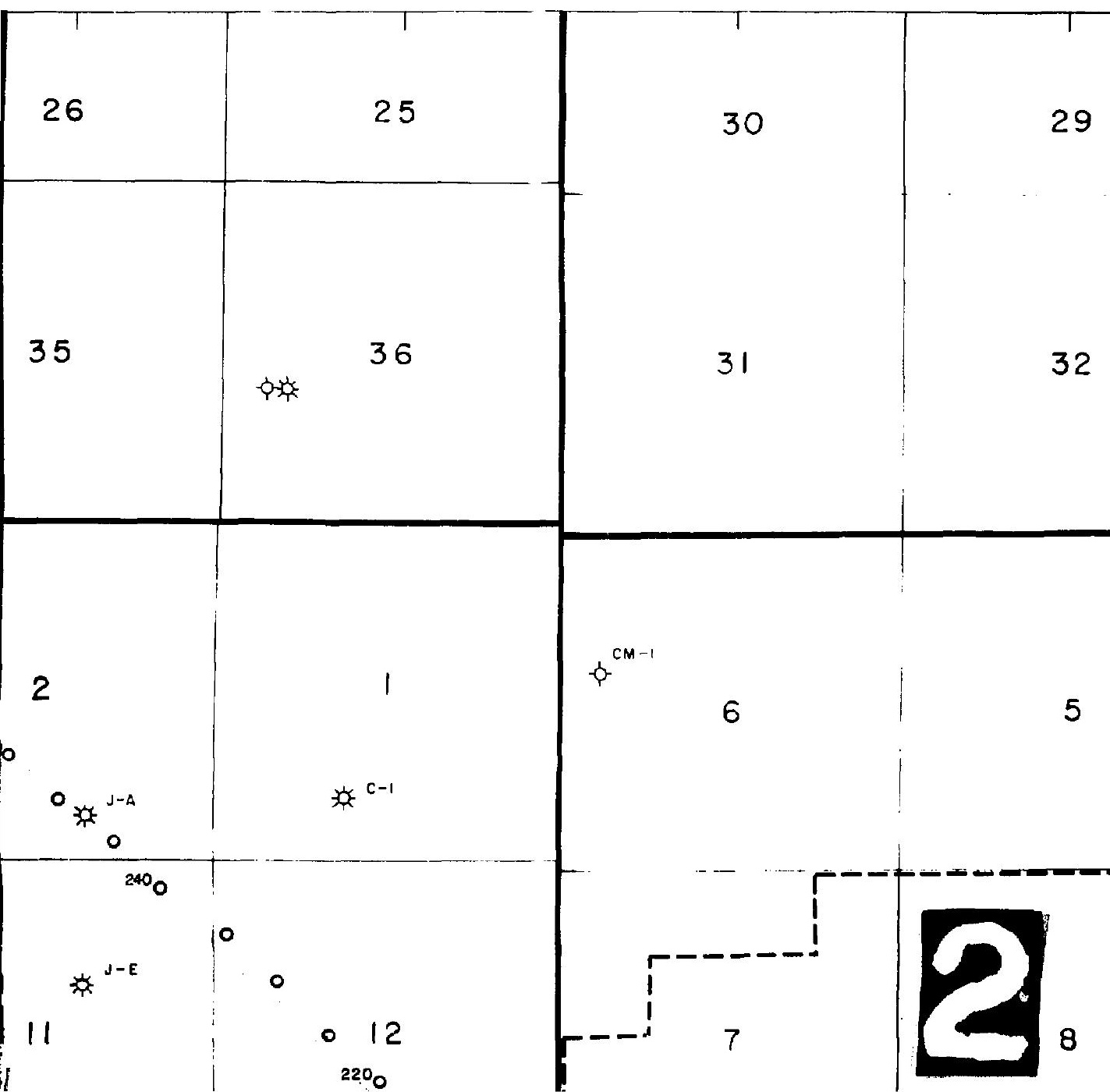




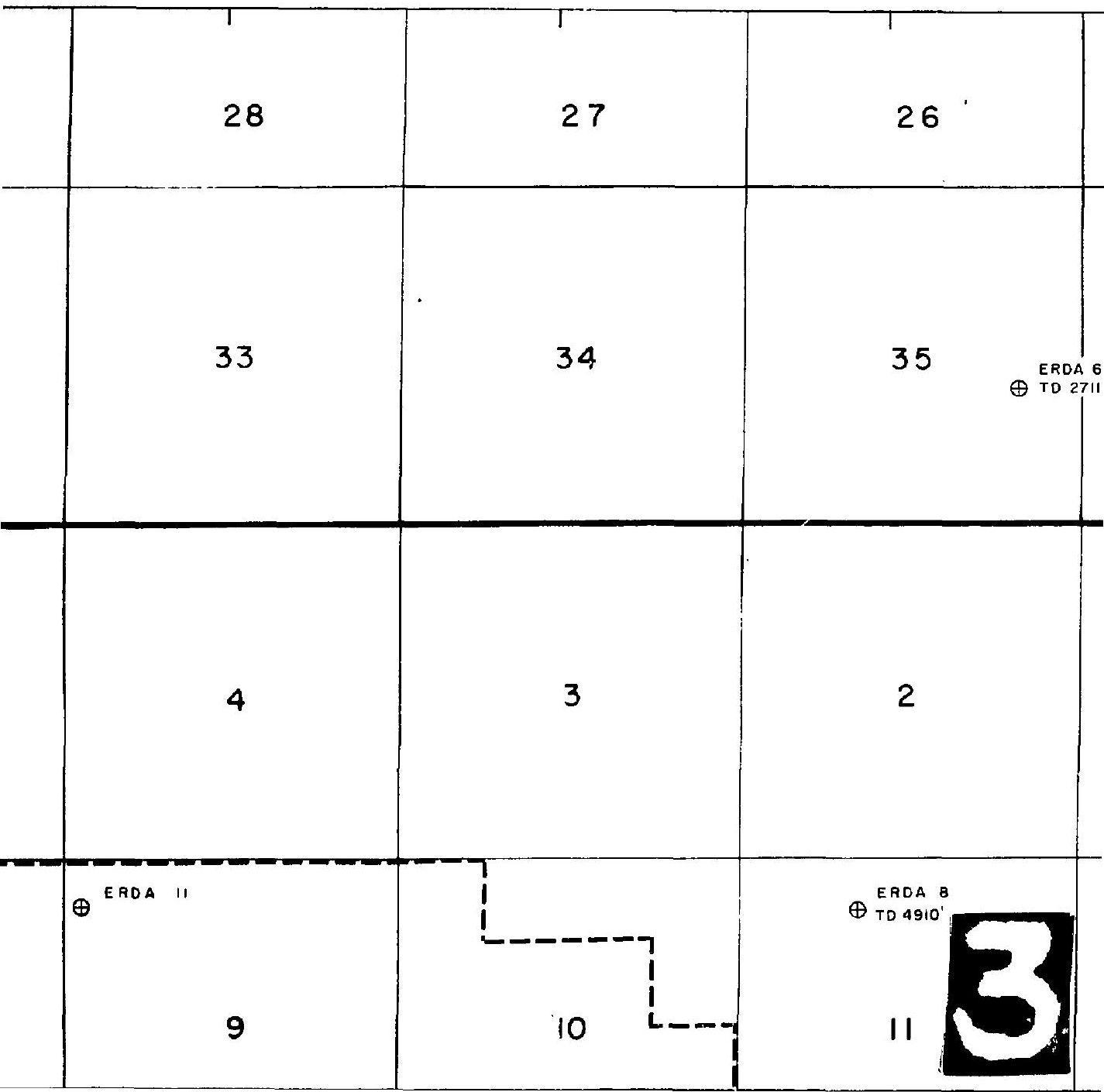




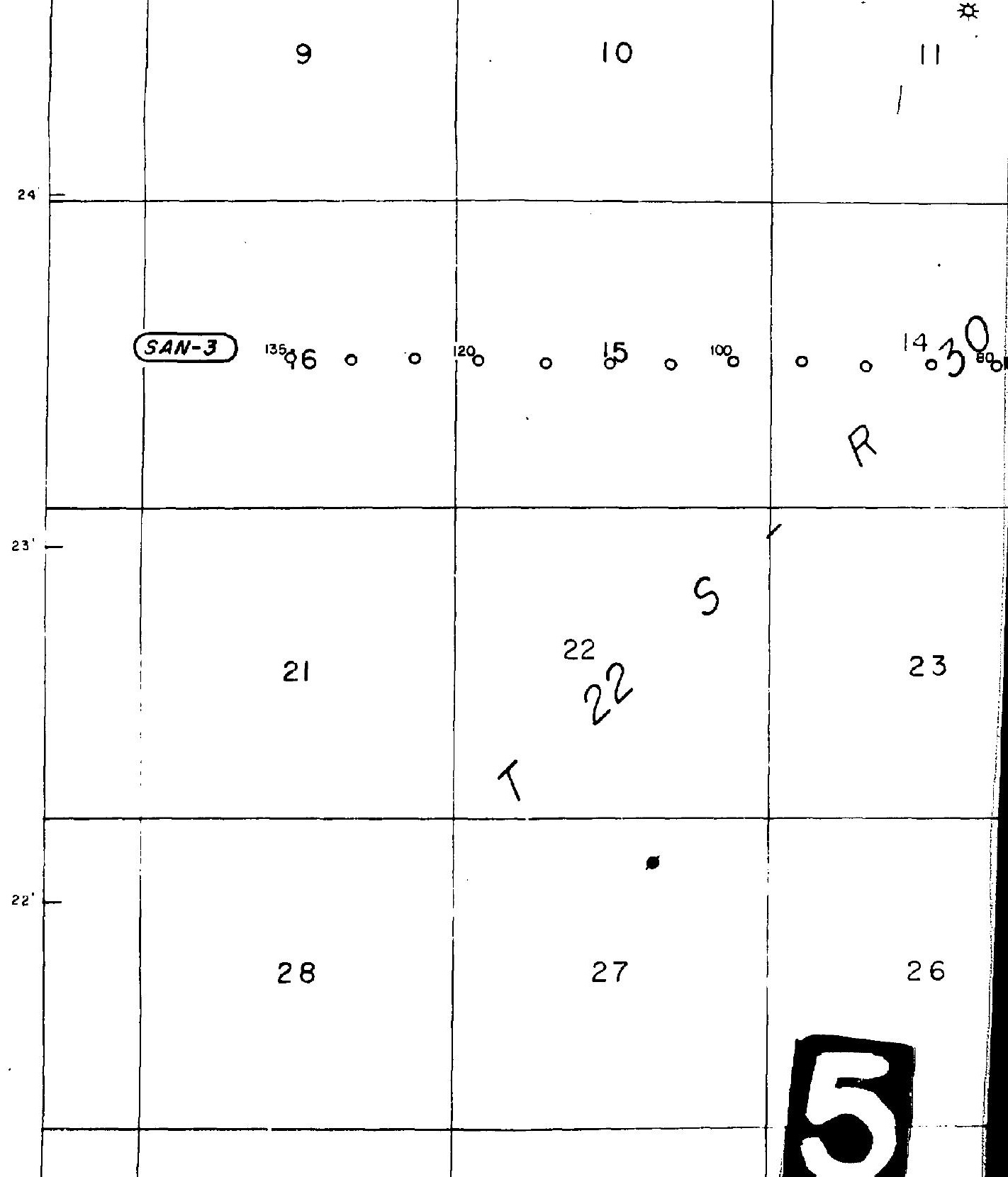




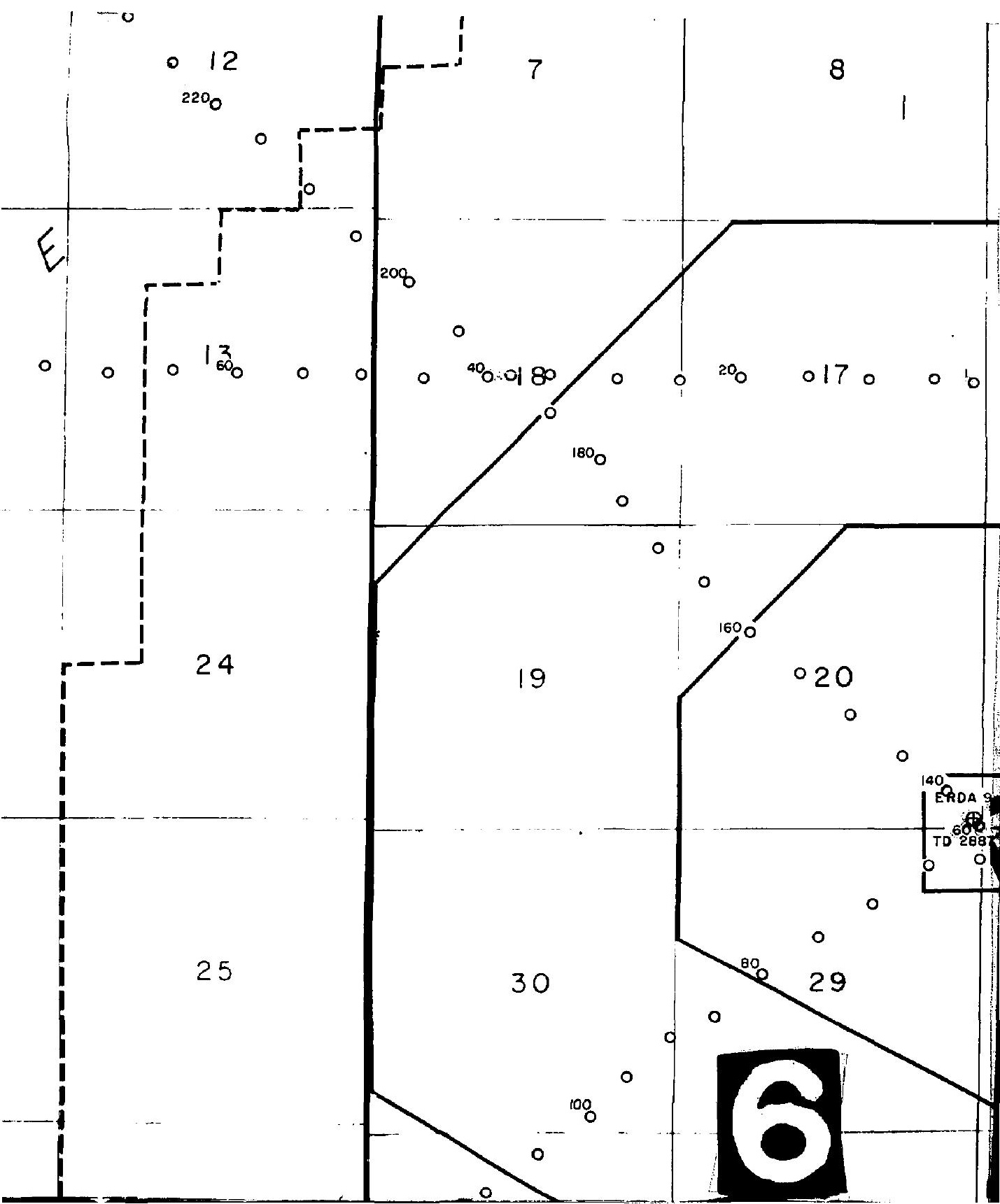




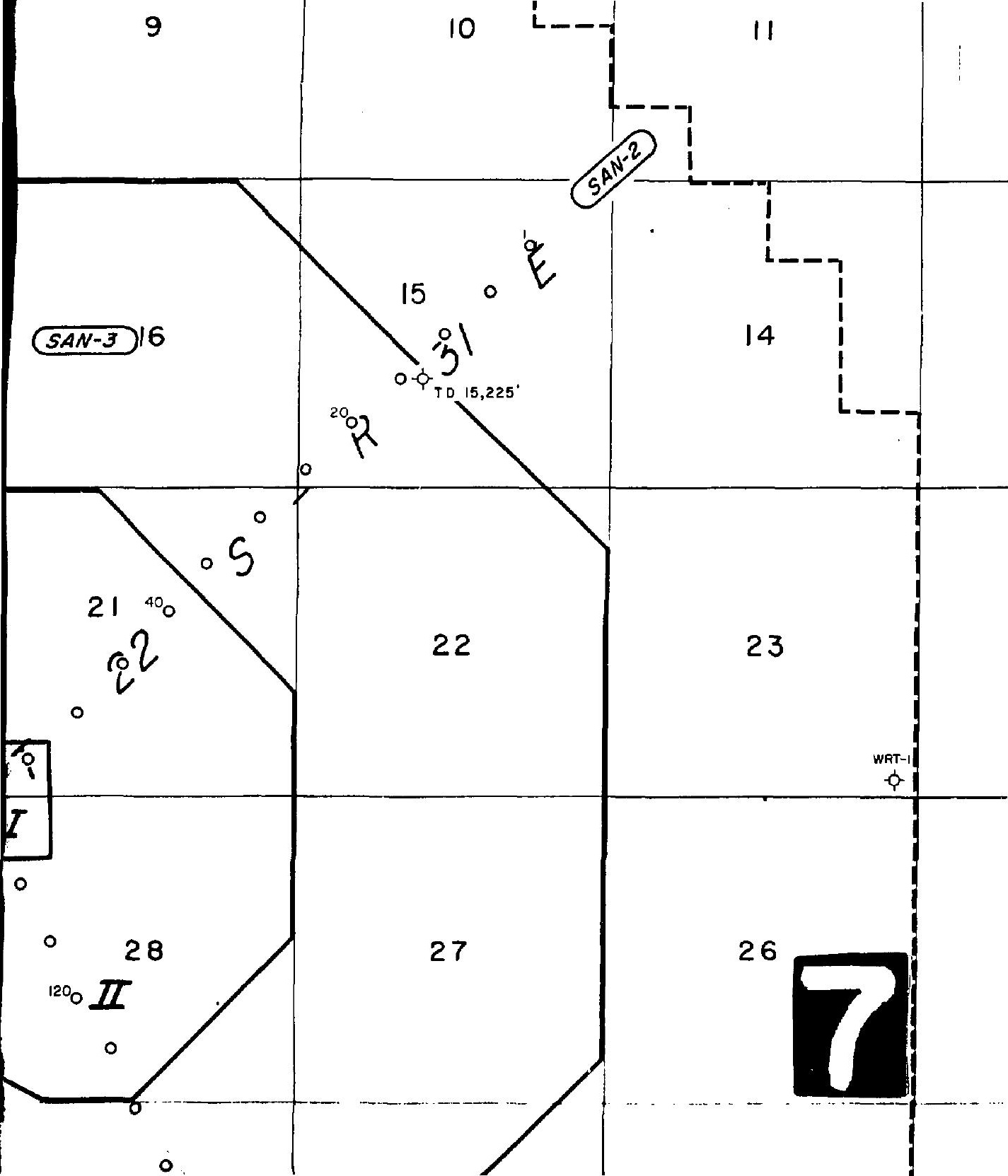


12

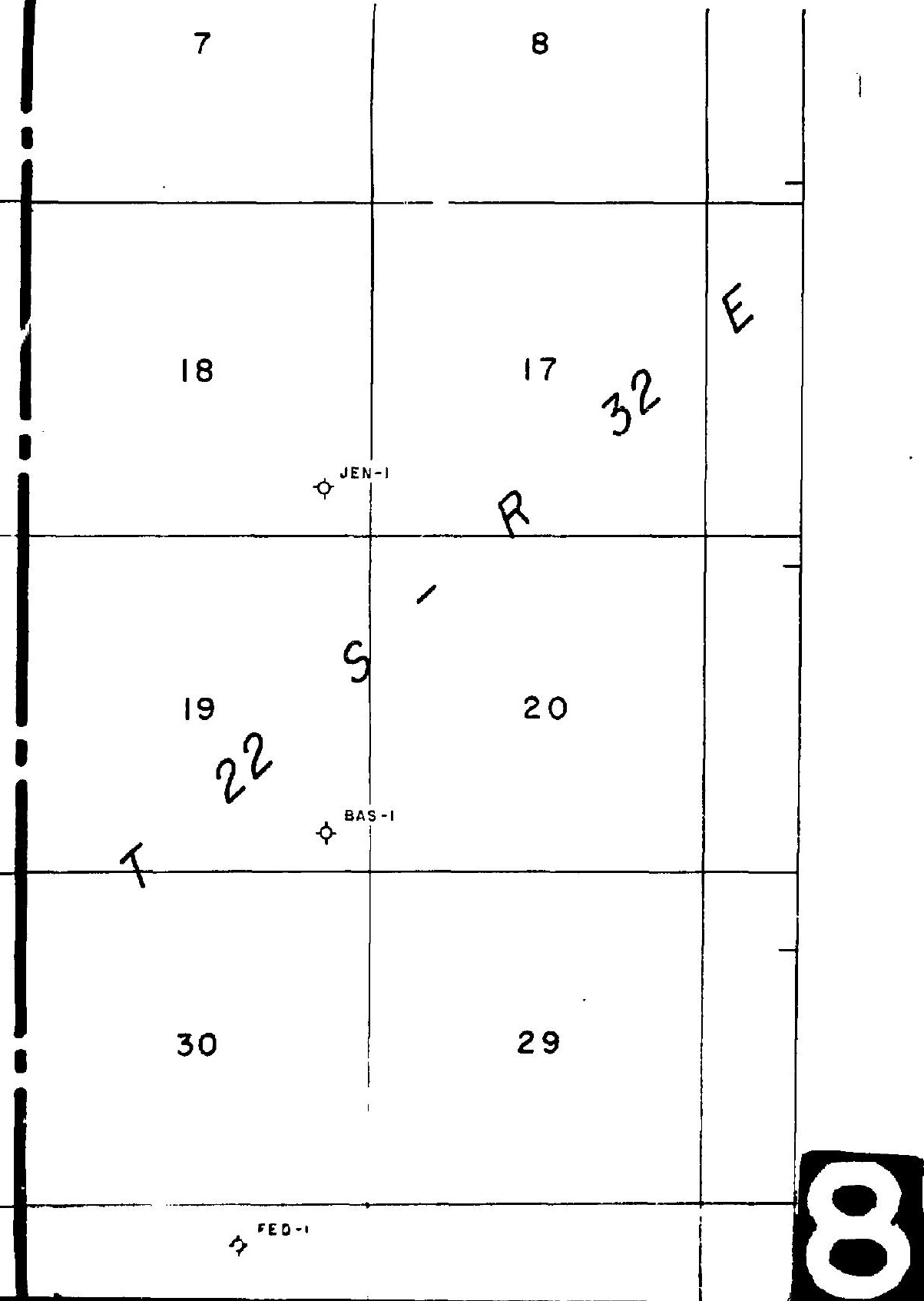




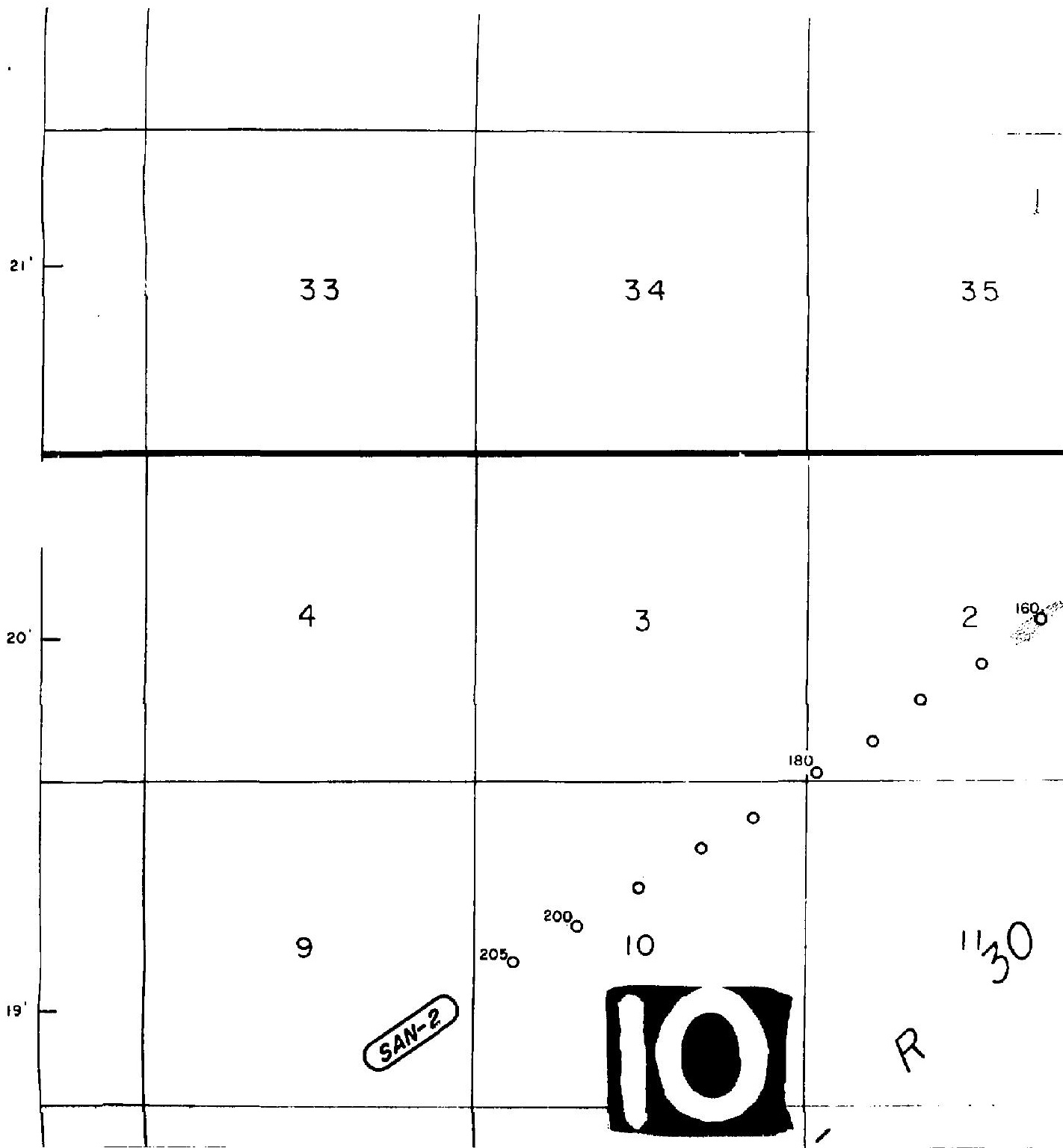




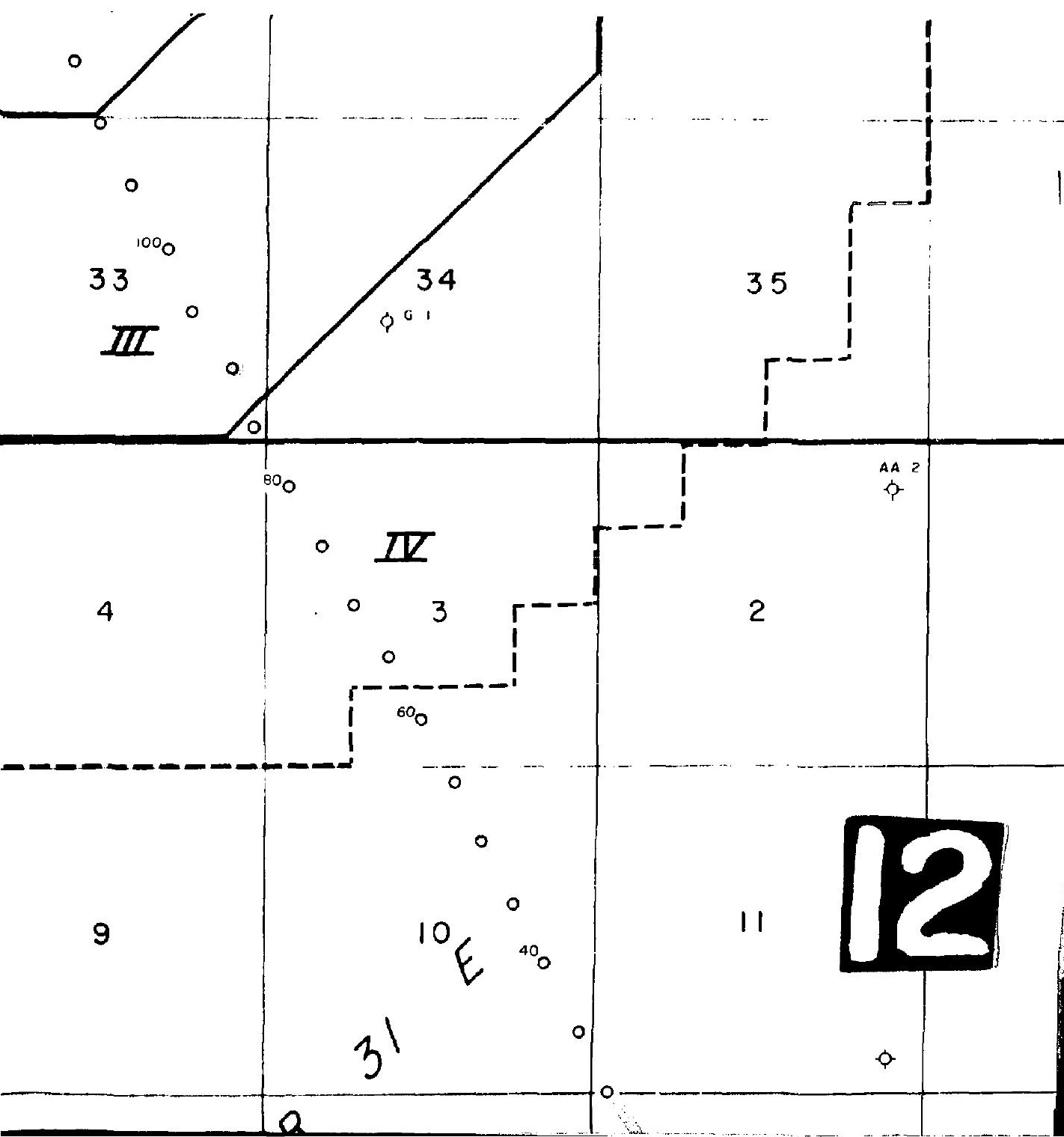




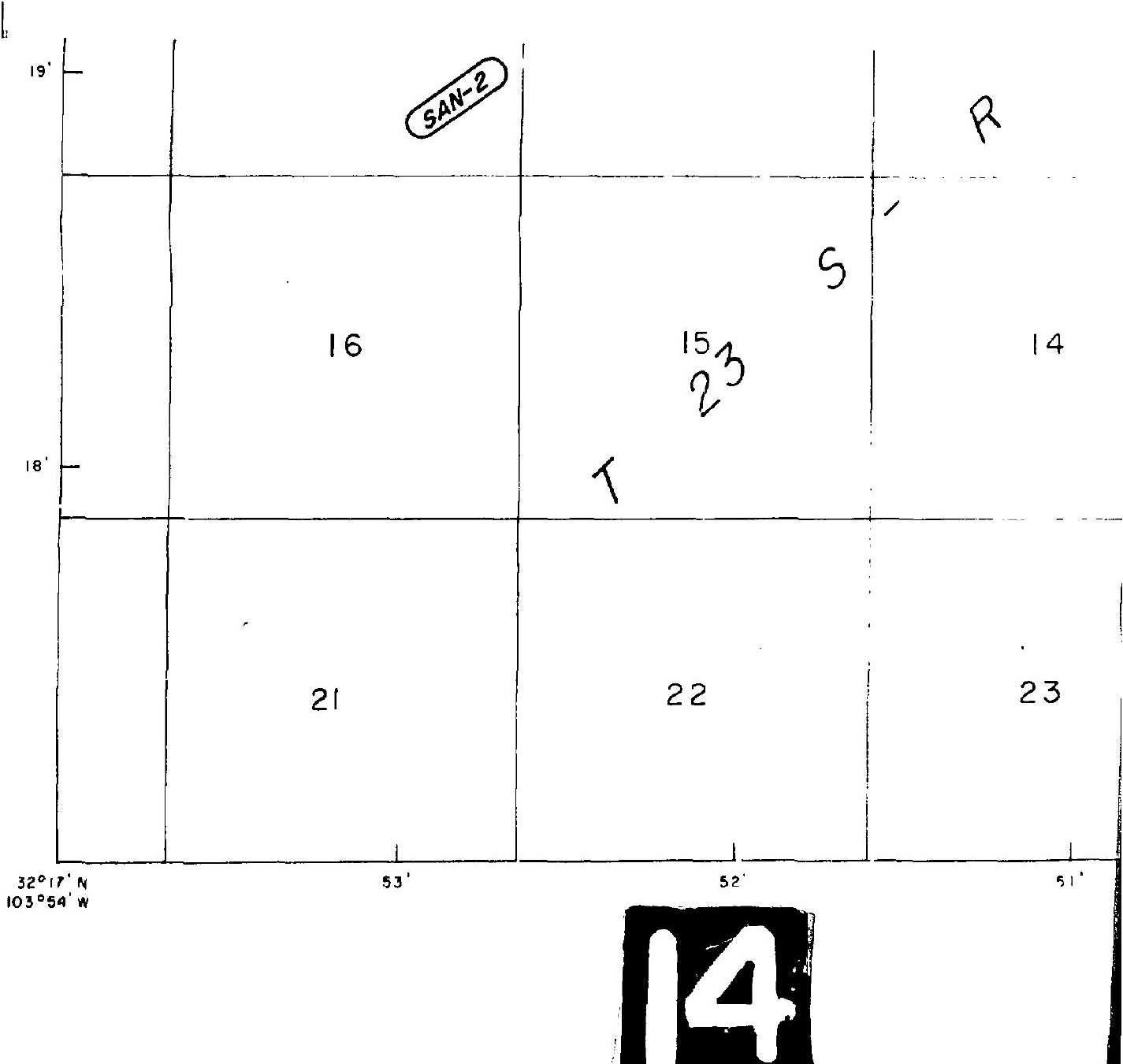




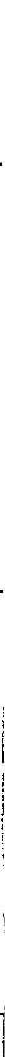




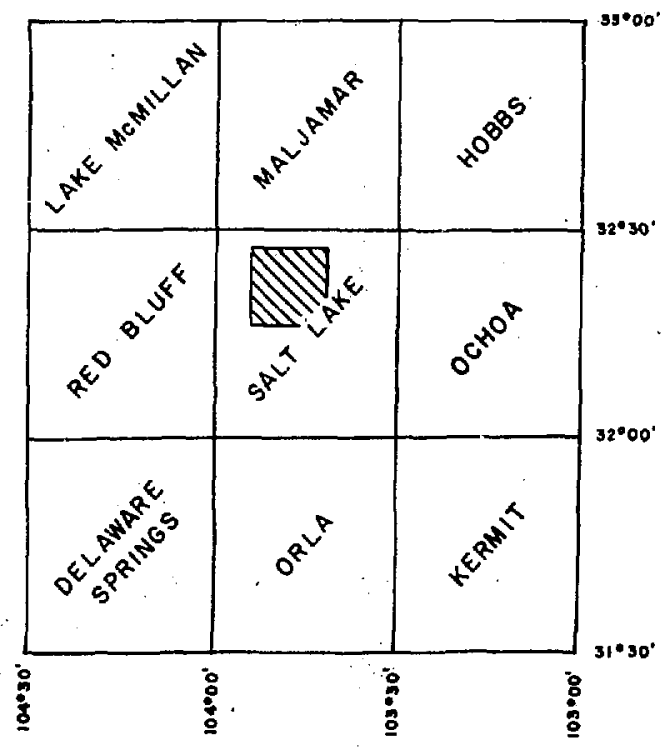

1

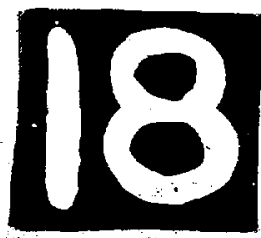


I
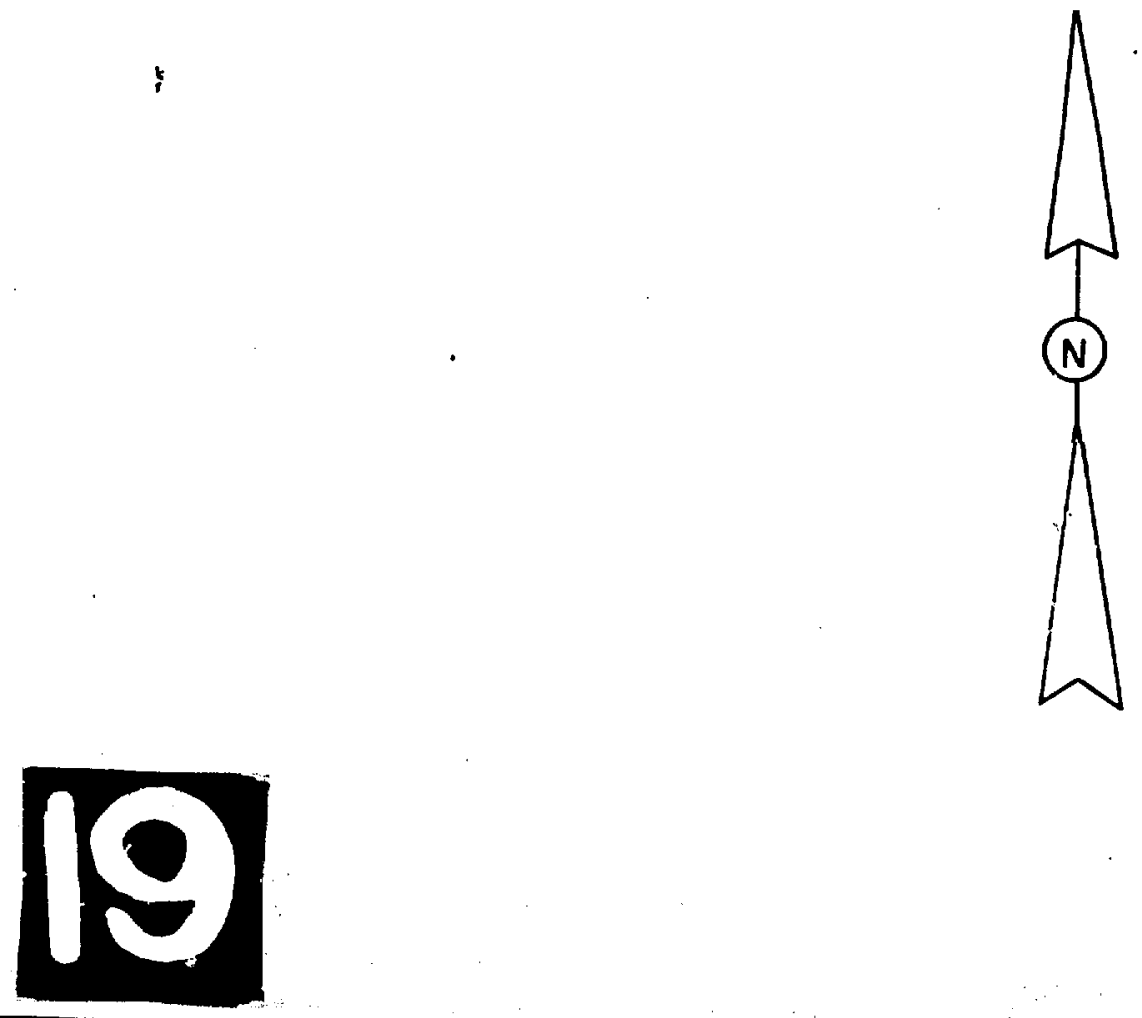


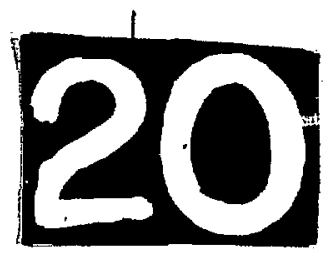

\begin{tabular}{|c|c|c|}
\hline ZONE & \multicolumn{2}{|c|}{ AREA } \\
\hline I & 100 & Acres \\
\hline II & 1,818 & Acres \\
\hline III & 6,221 & Aures \\
\hline II & 10,821 & Acres \\
\hline TOTAL & 18,960 & Acres \\
\hline
\end{tabular}




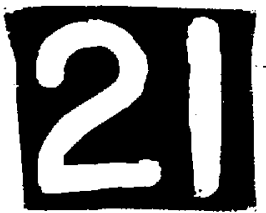

\title{
SANDIA LABORATORIES
}

\author{
SEISMIC SURVEY
}

LOS MEDANOS SITE AREA

EDDY COUNTY, NEW MEXICO

1976 SEISMIC PROGRAM MAP

INTERPRETATION BY:

G. J. LONG a ASSOCIATES, INC. APPAOVEO:

SCALE
C. 1. 


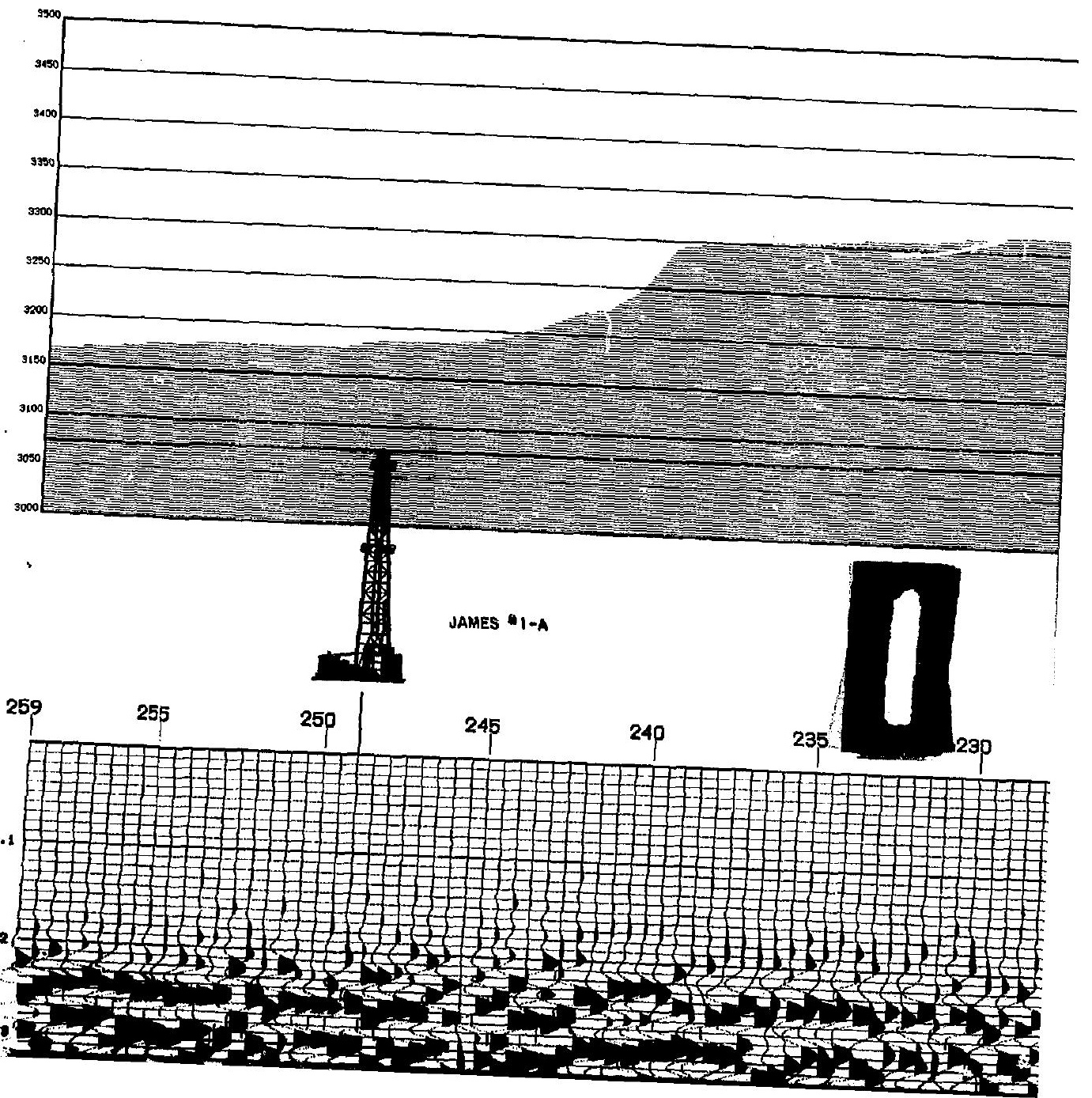




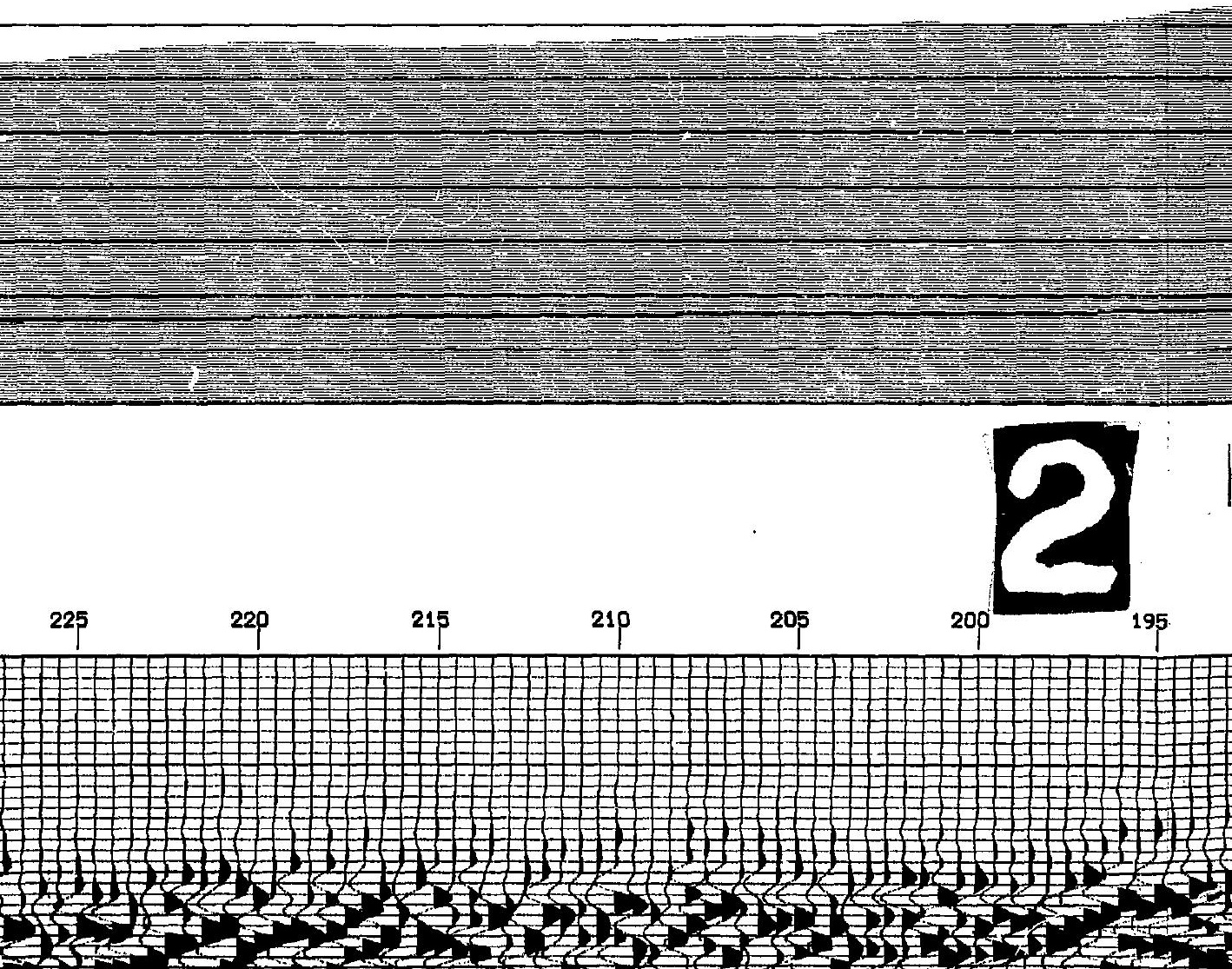




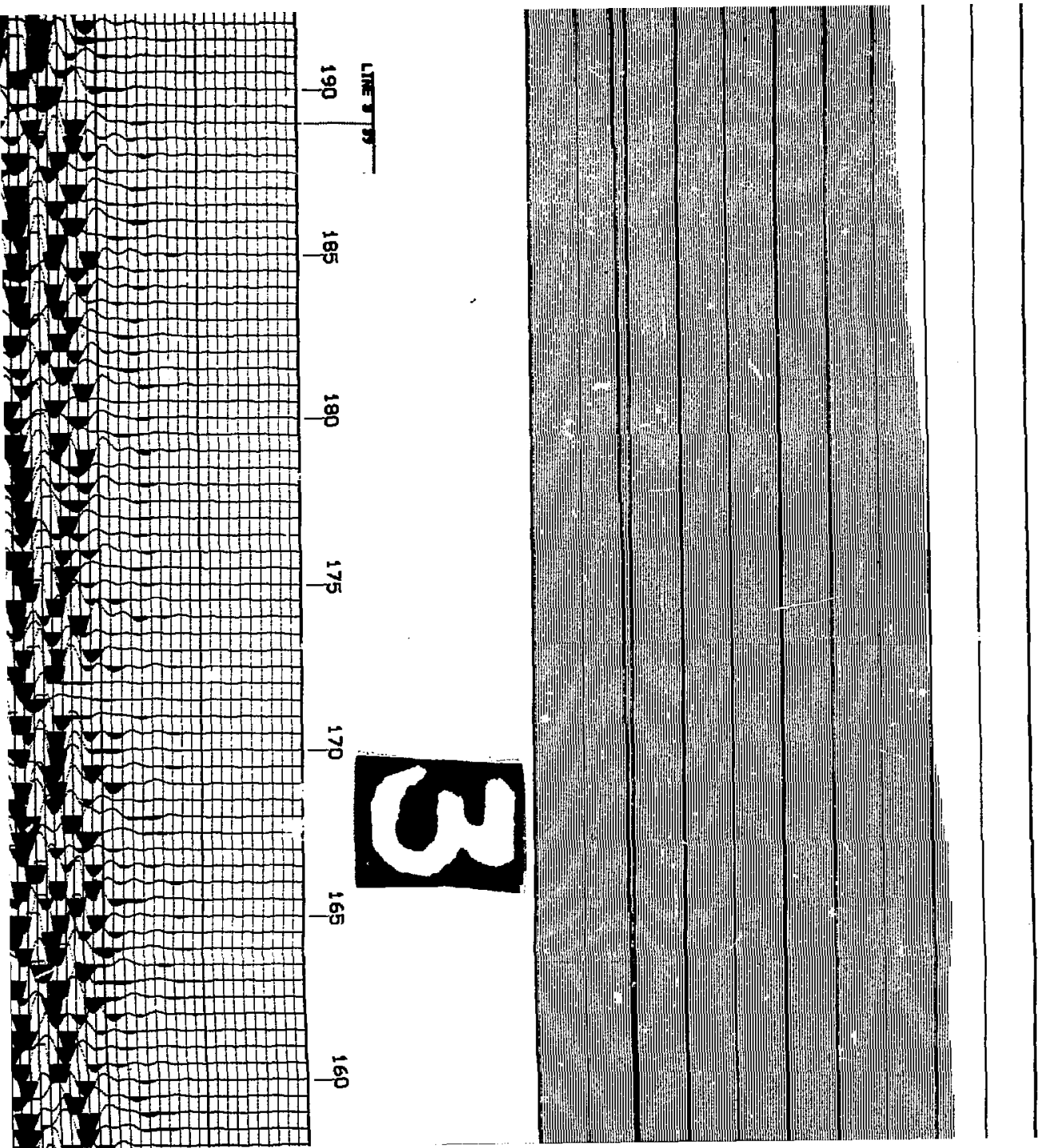




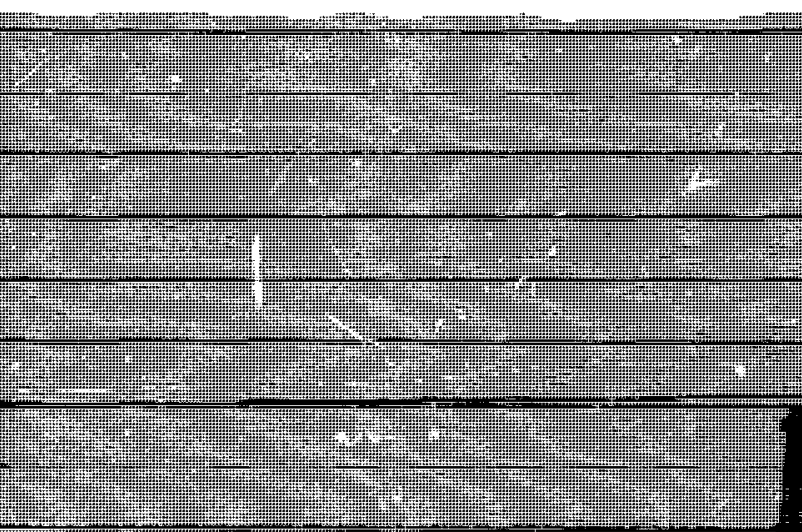

13)

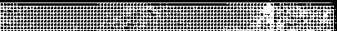

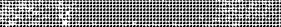
(1) 样 (x) (1)

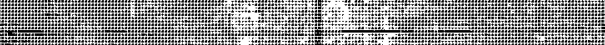
T 廿⿻

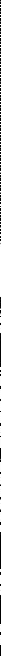




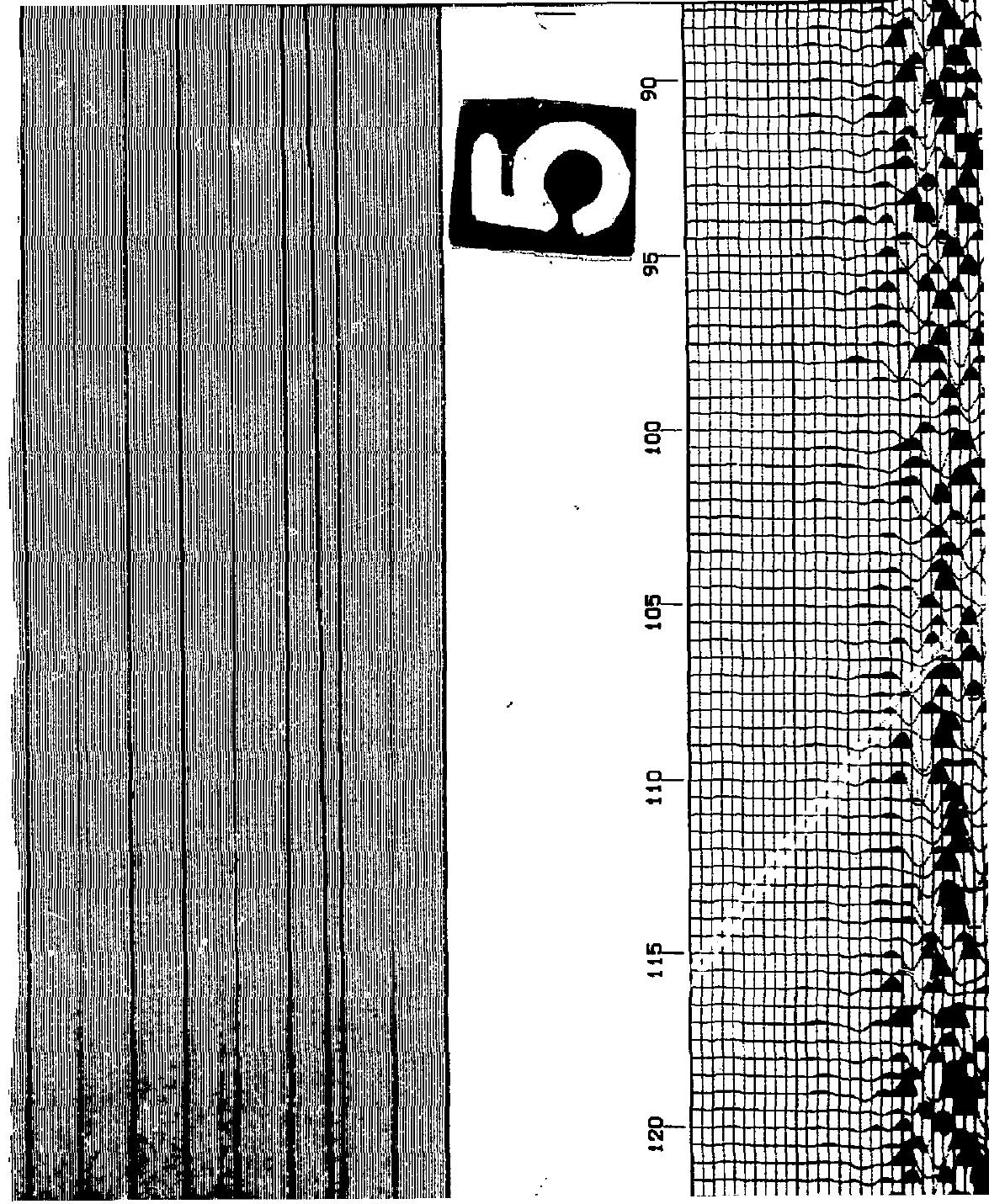




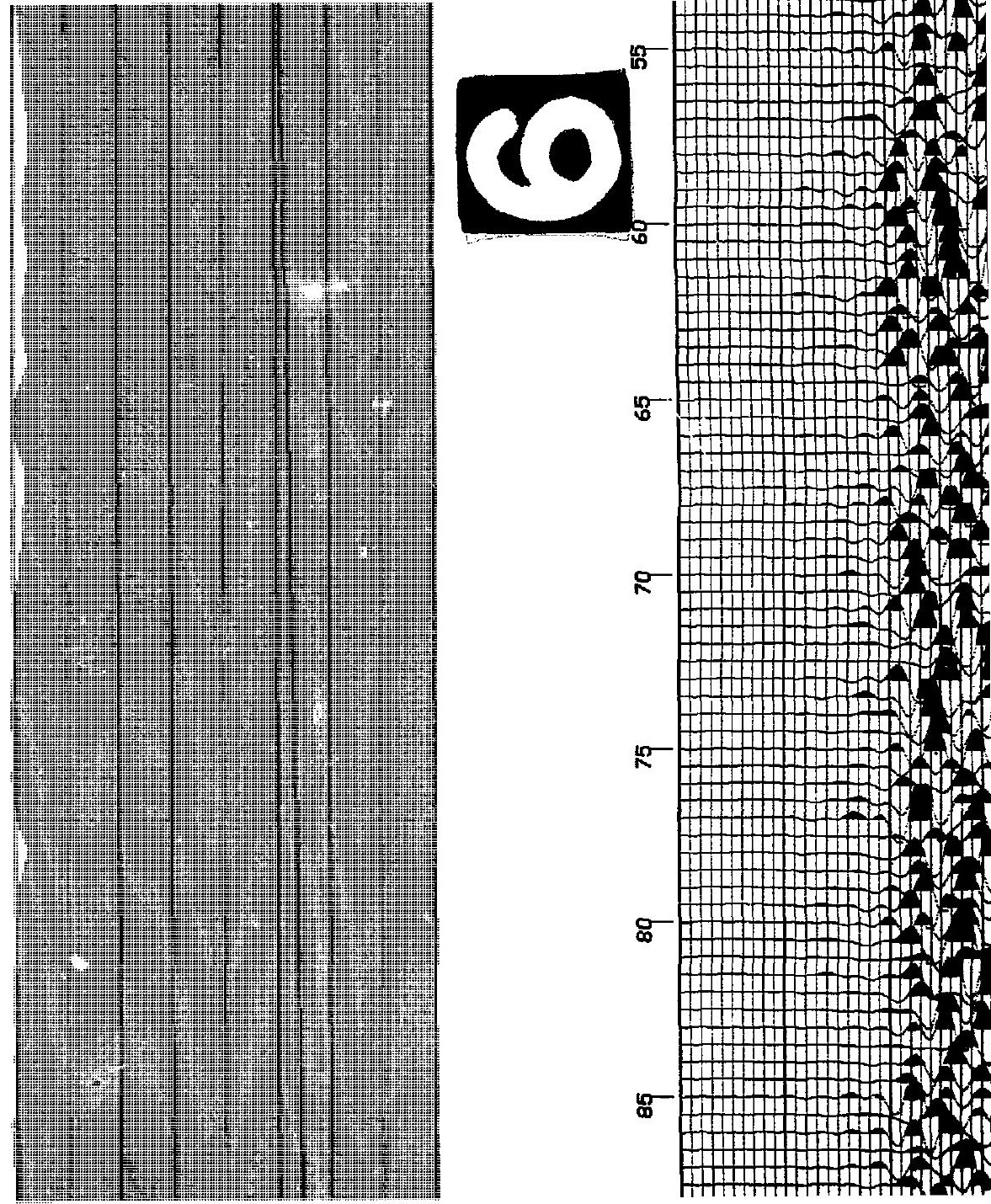




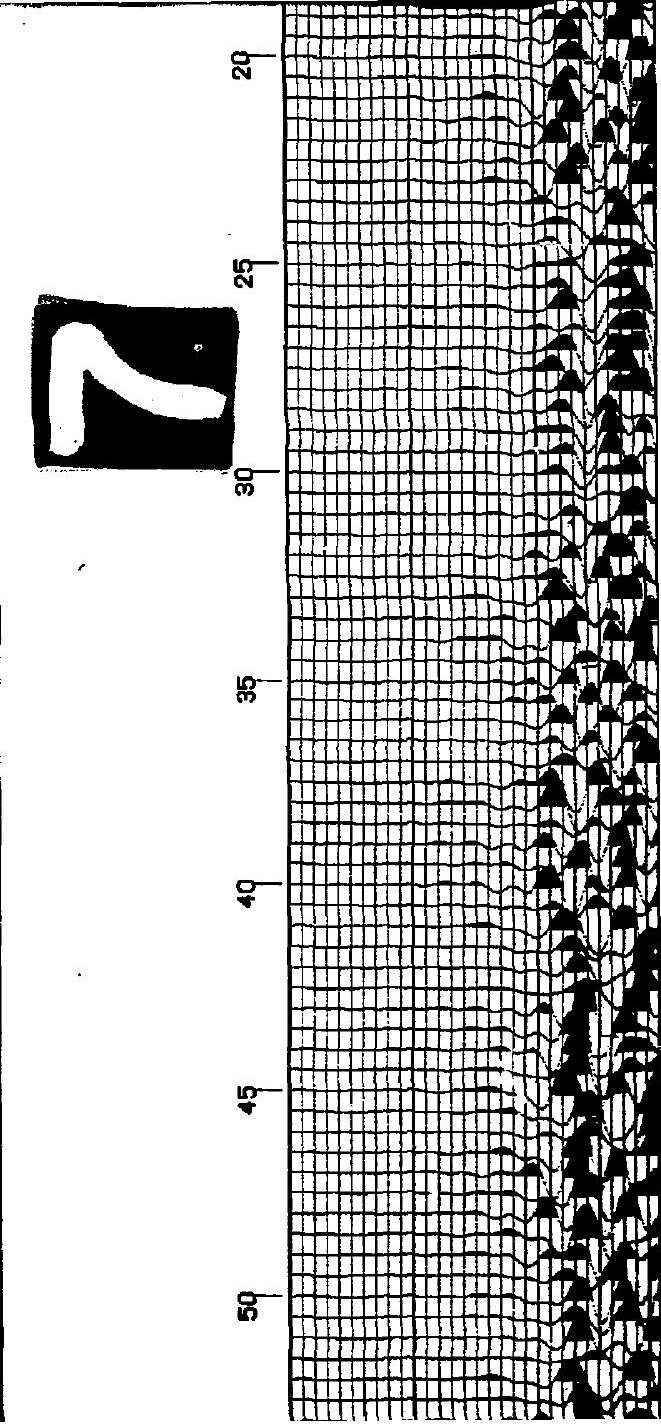




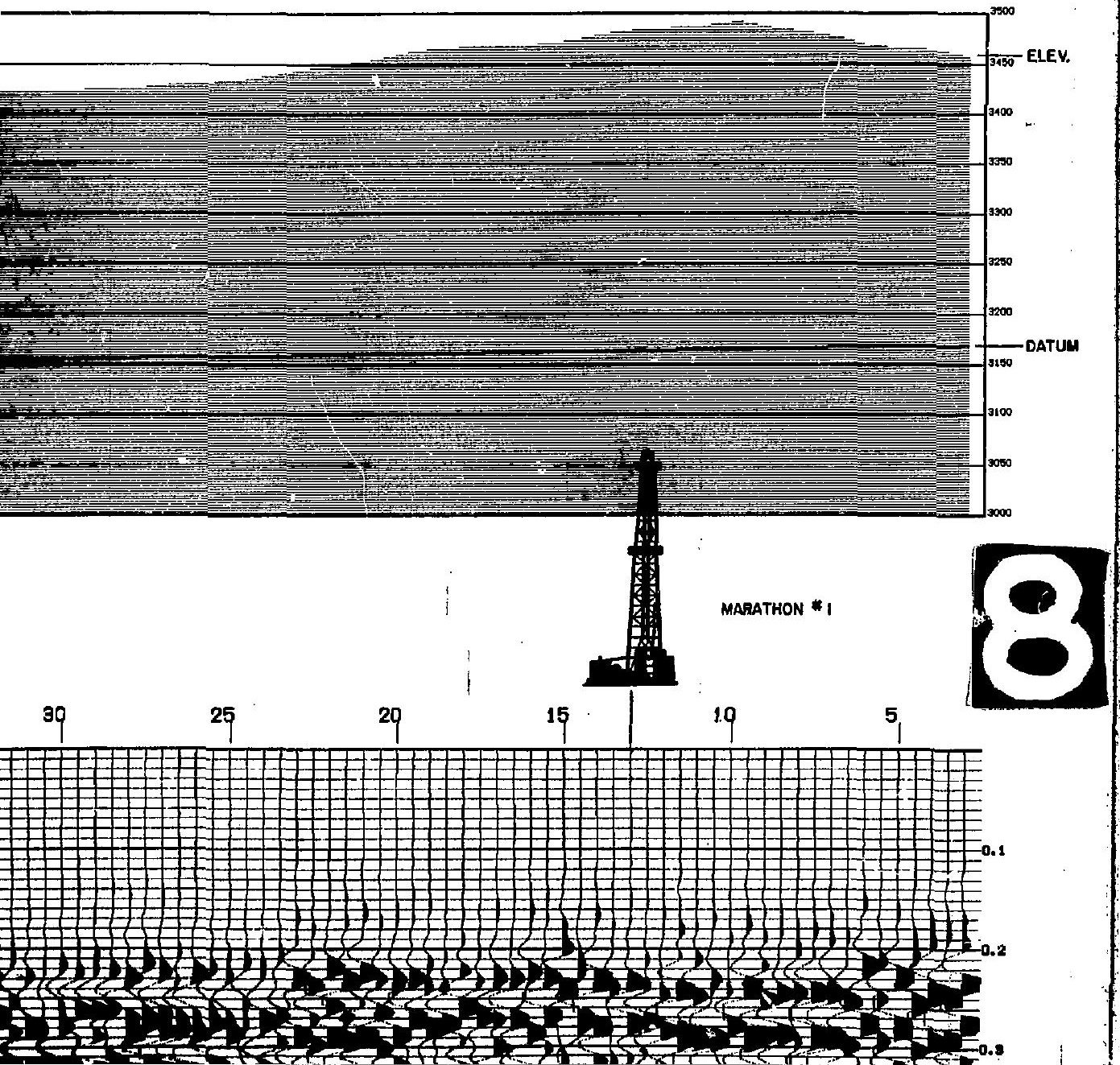



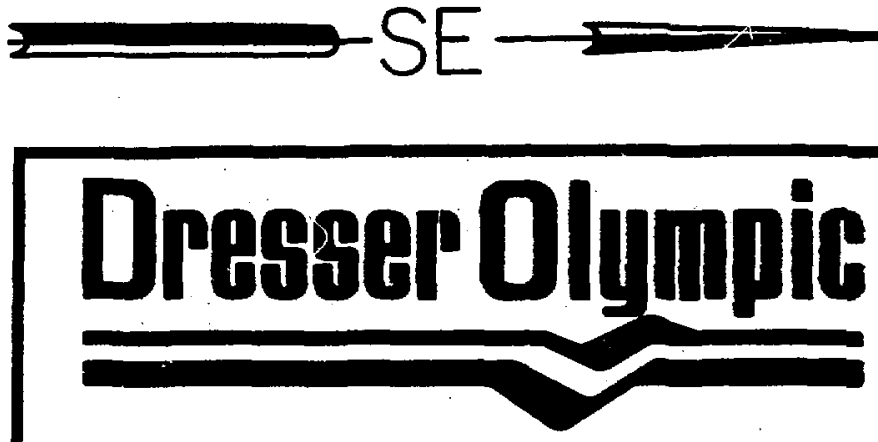

SEISMIC SURVEY

FOR

\section{SANDIA LABS}

PROJECT LQS MEDANOS LME

1

LOCATION EDDY COUNTY, TEXAS

DATE RECORDED $4-28-76$ TO $5-3-76$

DATE PROCESSED $5-17-76$

\section{DATA PROCESSING}

1 DEMULTIPLEX

2 GAIN RECOUVERY

3 COMMON DEPTH POINT GATHER

4 APPLY DATUM STATICS

5 DECONVOLUTION

Pred. Longth AUTO ma. Oper. Leagth 240 



\section{3,5135}

12 125

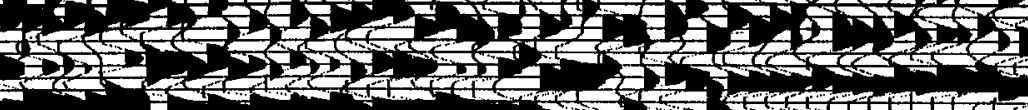

2

(1)

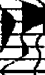

(1)

1)

11

11) 12

1 (1)

tis

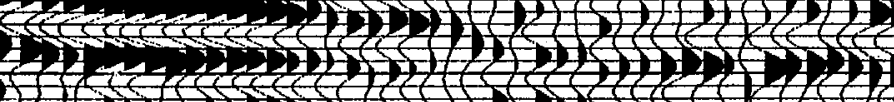

(4)

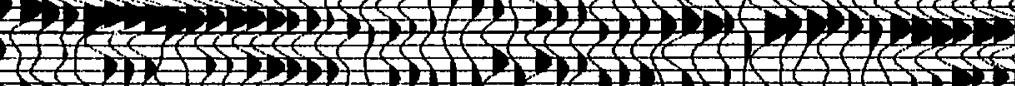

(1)

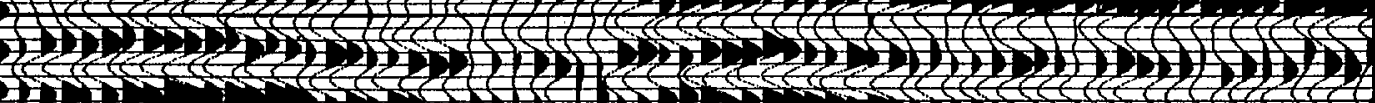

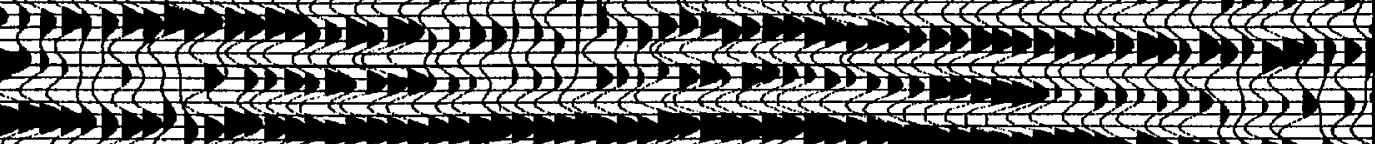

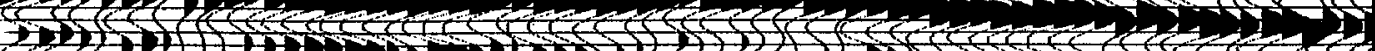

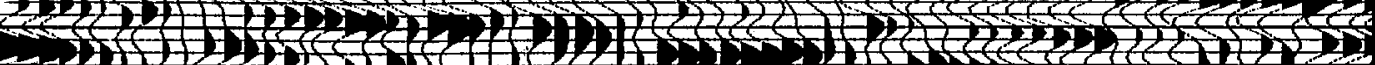

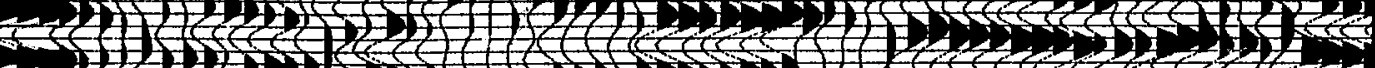

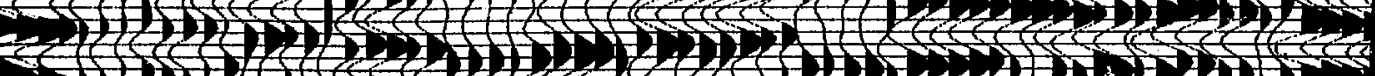

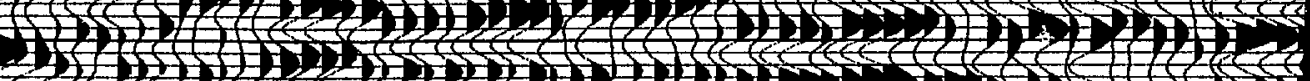

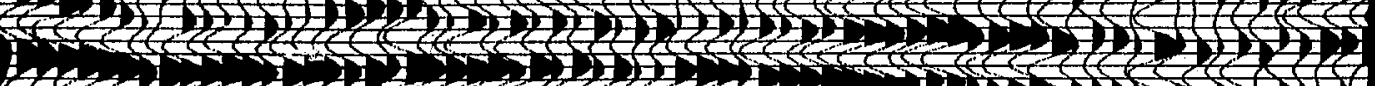

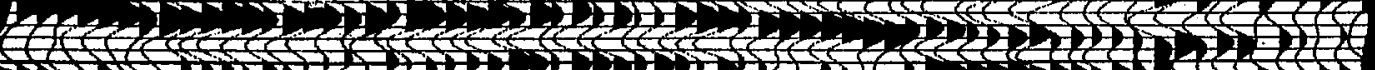

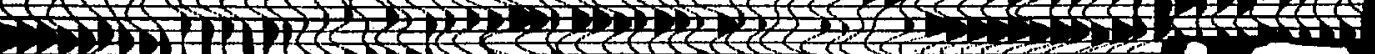

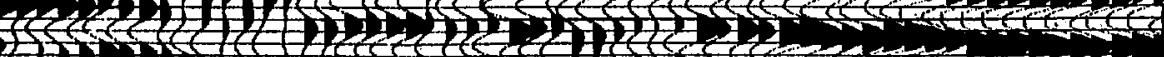

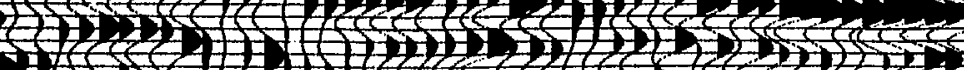

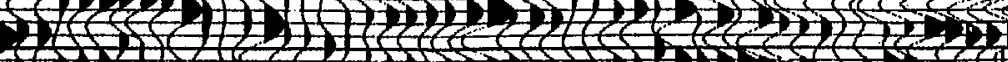
4

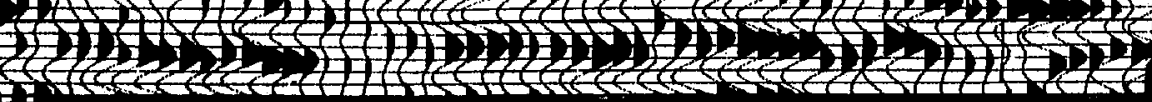


..

(1)

19)

171001

1.5

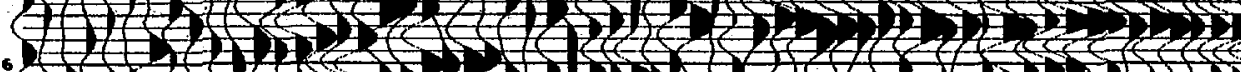

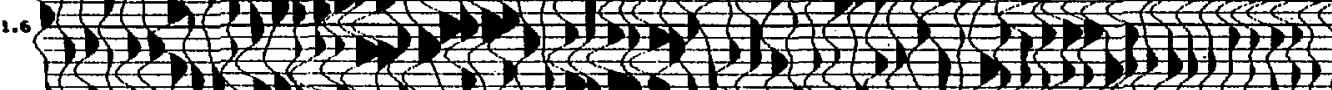

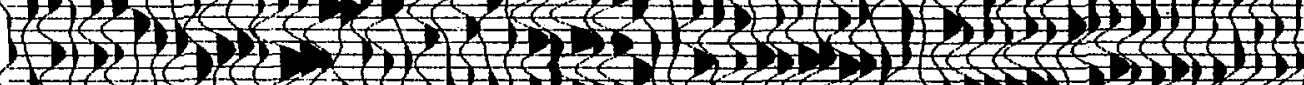

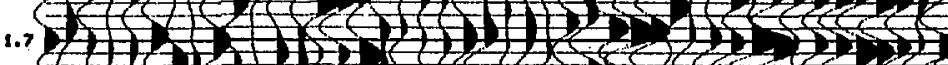
1.8242 (2)

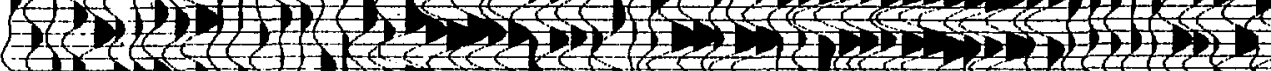

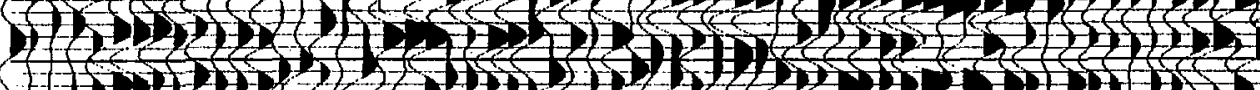
(1.9)

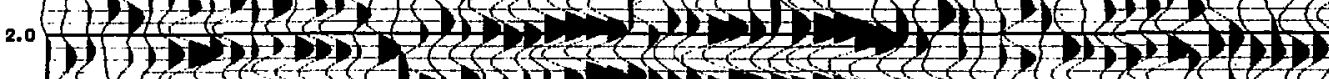

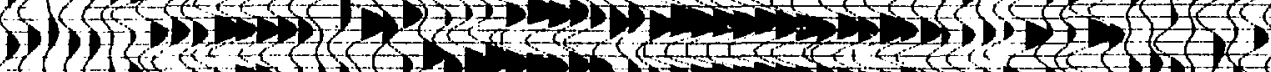

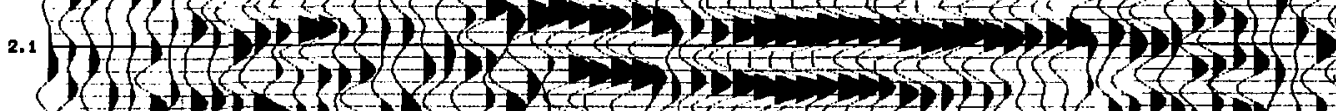

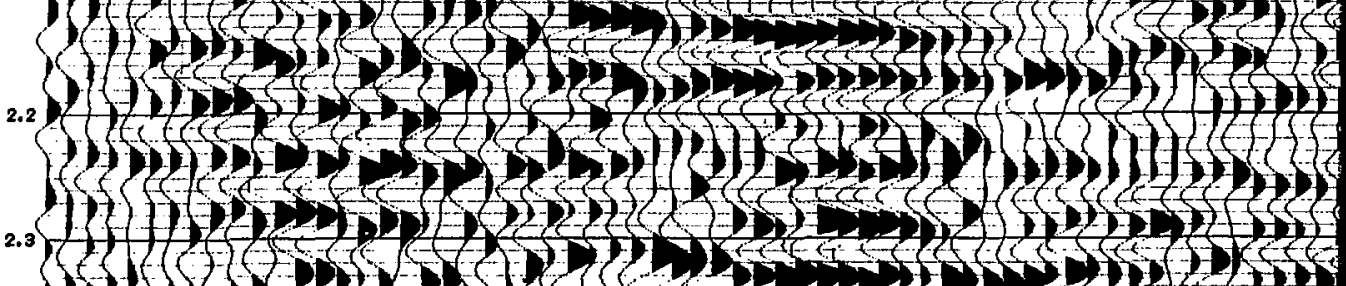

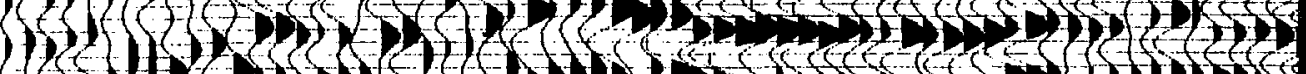

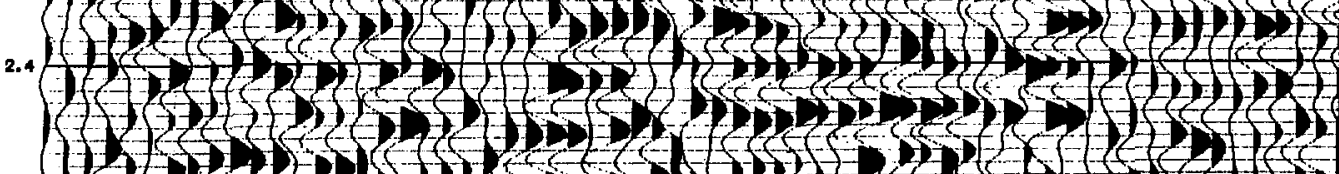

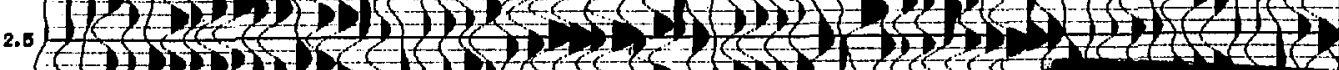

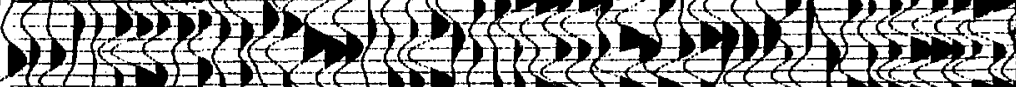
(2)

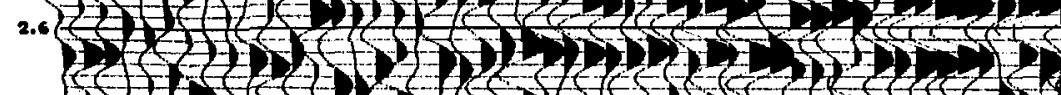
(f) 
12 1) 1121

12 1 1 11 212 W (1) 1 1 12010 12 1) 1121 213

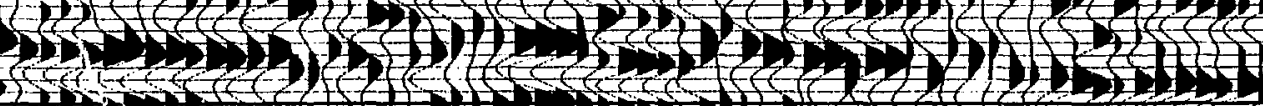

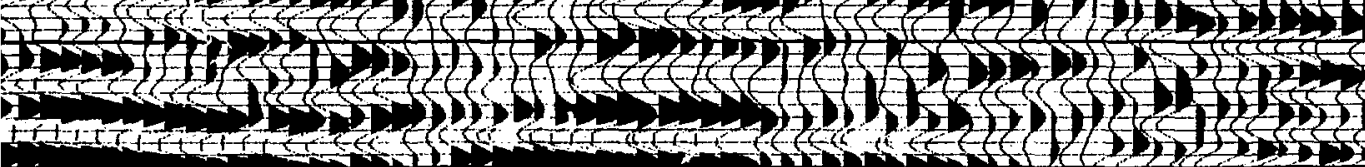

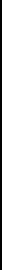

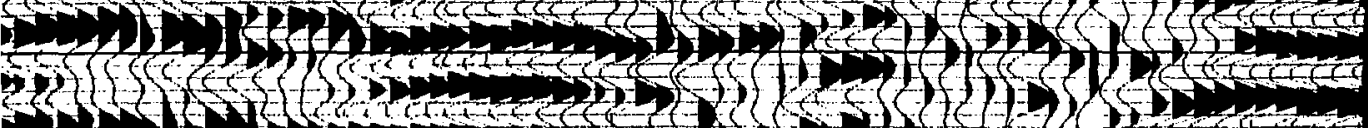
2.

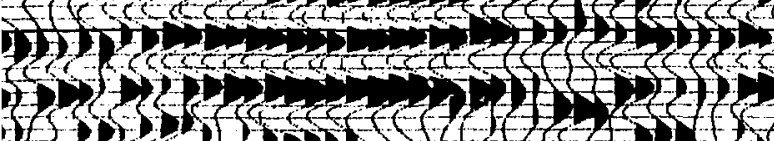
(1)

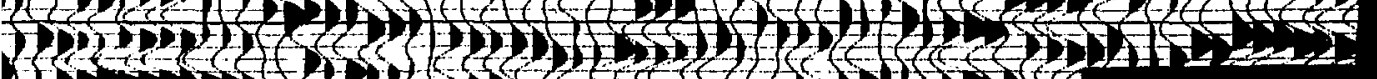

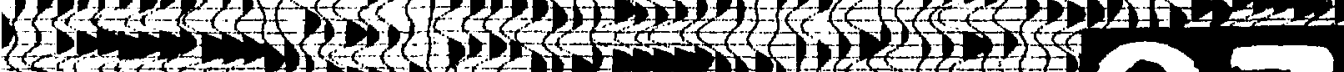

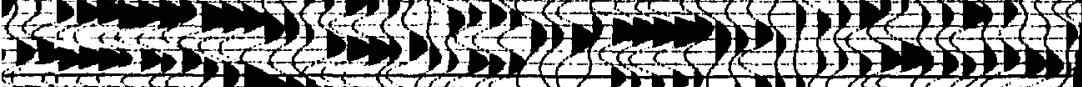

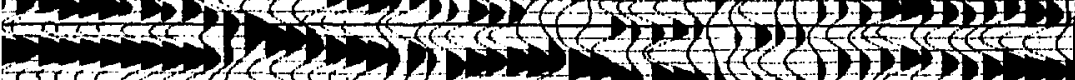

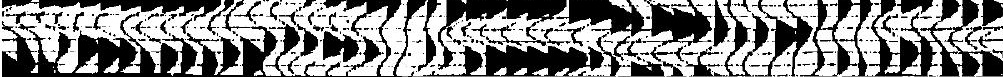


120 1211121 13 (13)

10121 111111 121111 37

1

1)

1)

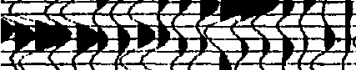

717)

$+2$

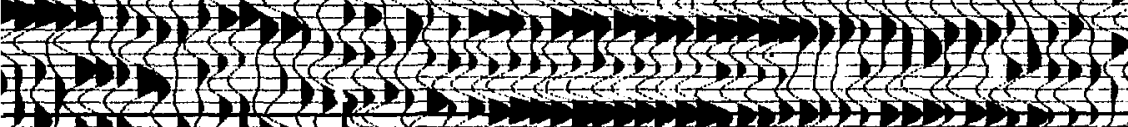

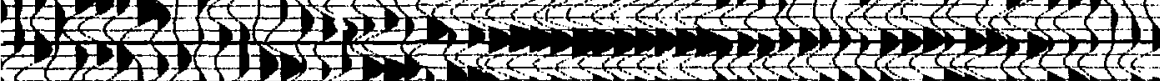

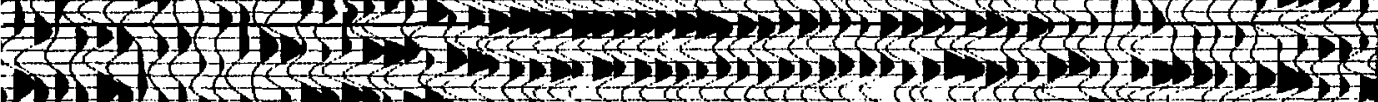
I 1 7) $12 \geq 3$ (a) (1)

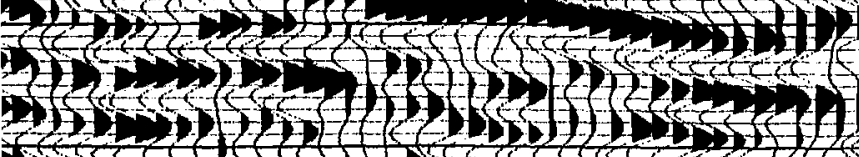
sist

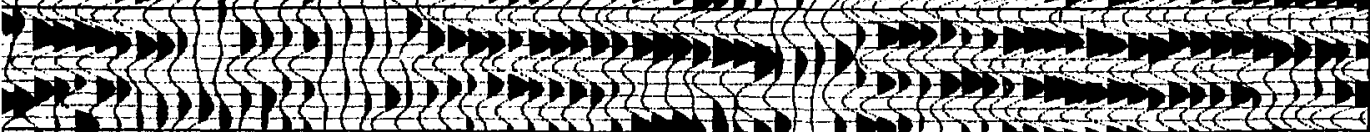
1) II) I. 337,1

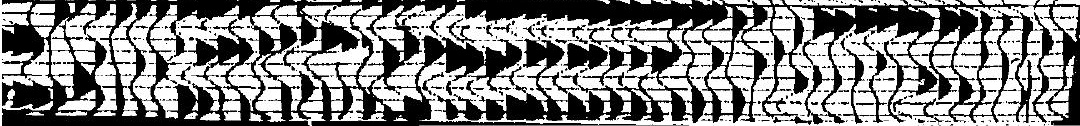




\section{7m}

111

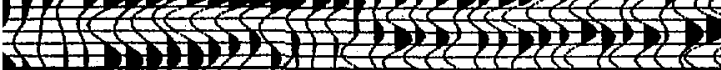

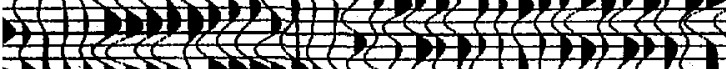

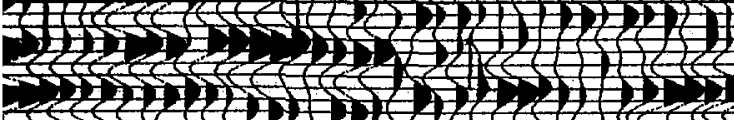
Fut

3f: to 112

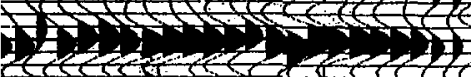

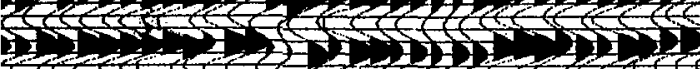

(7) )

1

1

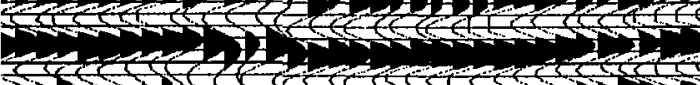

(1)

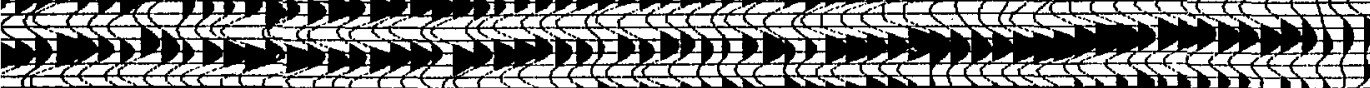
4 ( )

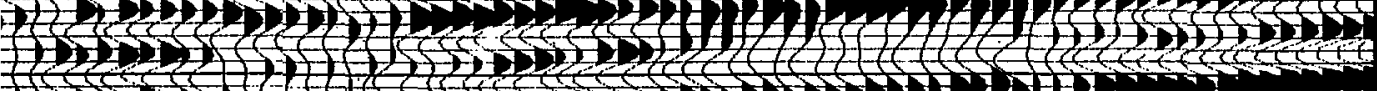
(13)

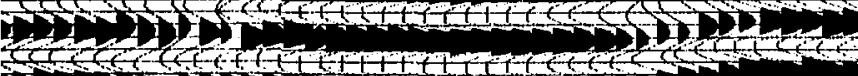

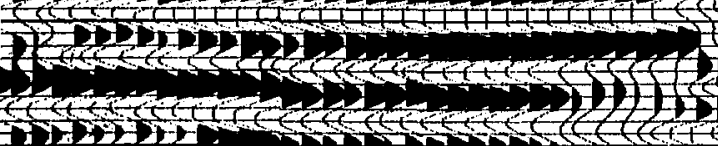

TSt (f)

(1) 31,121

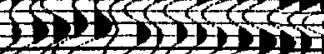

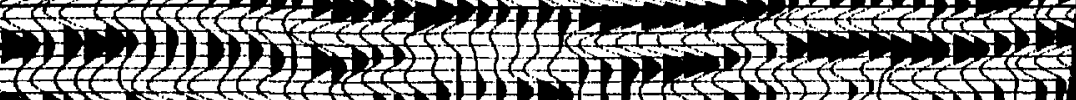
-री 


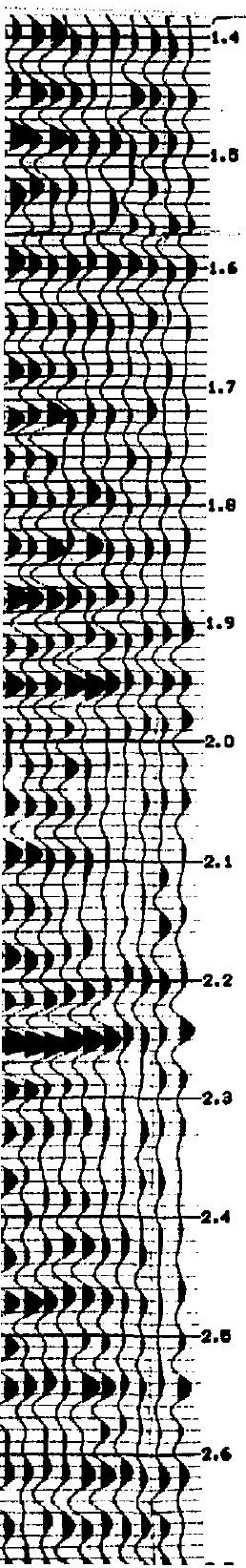

RECORDED OY DRESSER OLYMPIC

INSTRUMENT TYPE_ DES III_RECOFD FILTER \&-18-62Hz NOTCH FILTER INSAMPLE RATE 4 MB RECORD LENOTH 4 ENERGY SOURCE : VIBROSEIS SWEEP FREQUENCY \& - $39 \mathrm{~Hz}$ NO./SWEEPS PER LOCATION _6 LOCATION INTERVAL_220'. MODEL A TYPE GEOPHONES IUHS F FREO \& HE NOKGROUP, 48 TYPE COVERAGE $2400 \%$ SPREAD LENGTH $11880^{\circ}$ NO. TRACES 49 OFFSET $880^{\circ}$ GROUP NTERYAL $230^{\circ}$

SPREAD DIAGRAM

$-24$

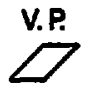

$25---n-1--48$

$1-5060^{\circ} \longrightarrow\left|-880^{\circ} \longrightarrow\right|-880^{\circ} \rightarrow \mid-5060^{\prime} \longrightarrow 1$

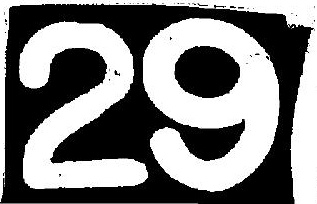




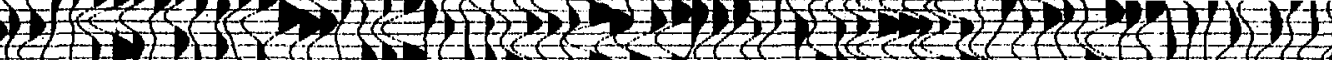

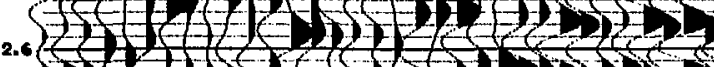

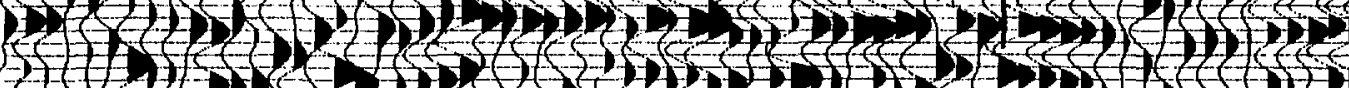

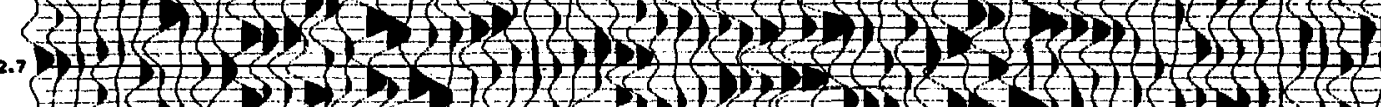

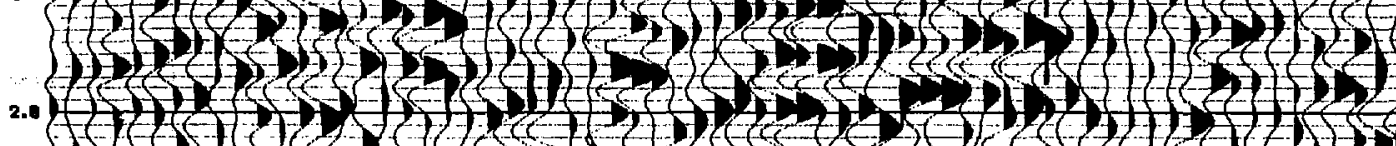

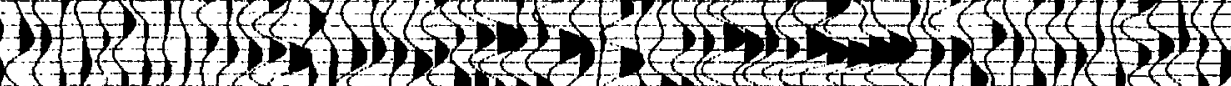

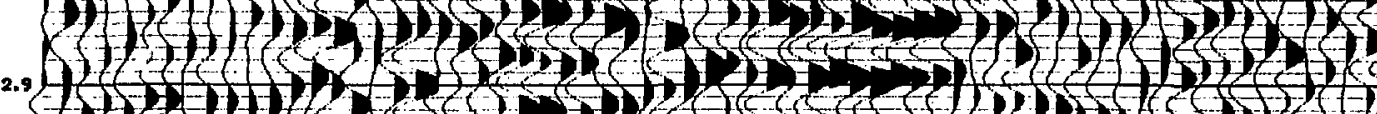

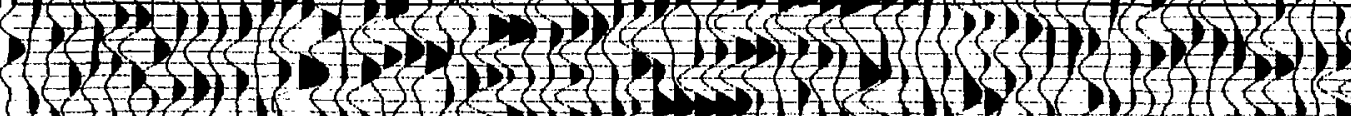

3.0 (1)

$136)$ (5)

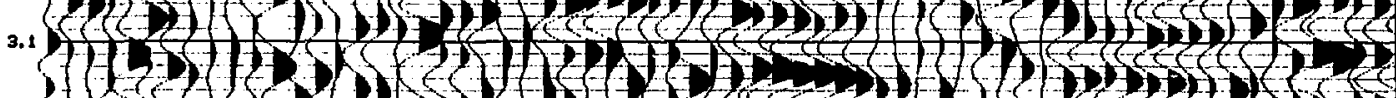

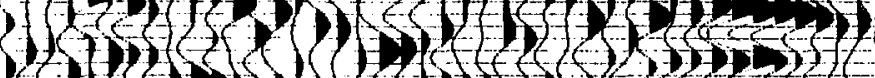

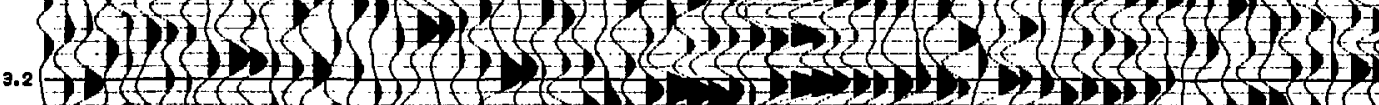

$\left\{\begin{array}{l}3 f(2) \\ 3\end{array}\right.$

3.3

9.4
mis. 1

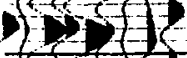

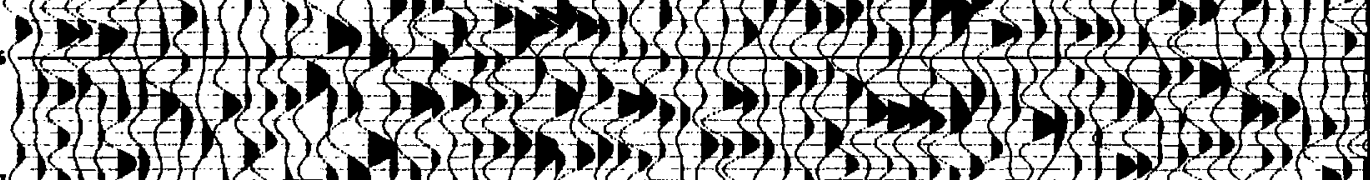

.r. $\rightarrow$ (2)
(t)

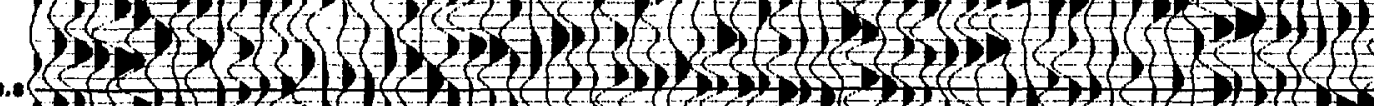

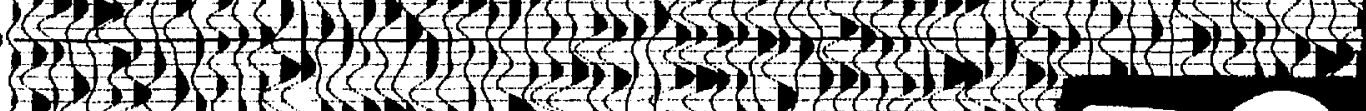

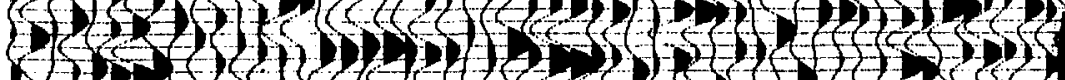

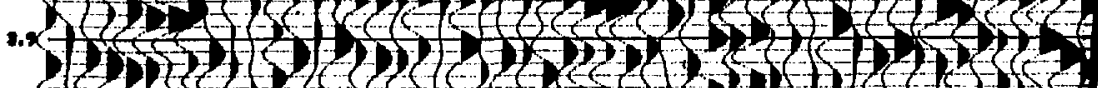

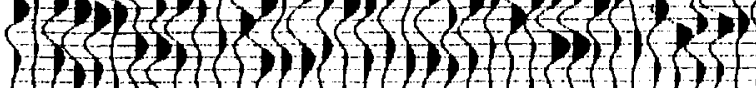



I

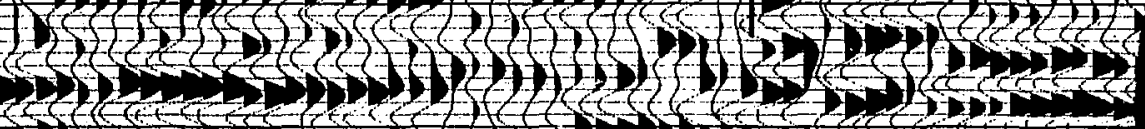

D. 7 (2) (I) 3 (1)

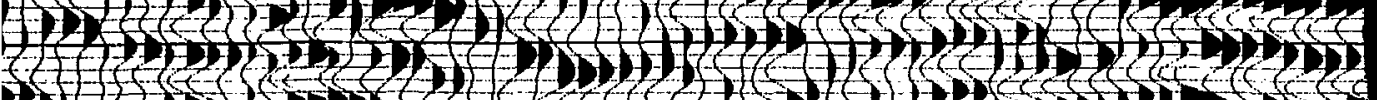
W.

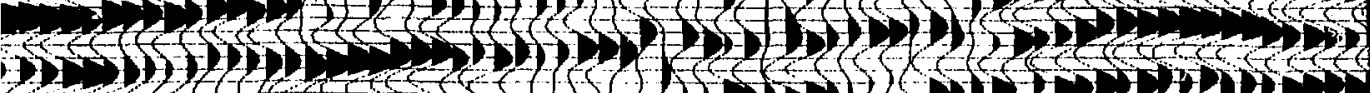
2. (7) 3 . 2.5.

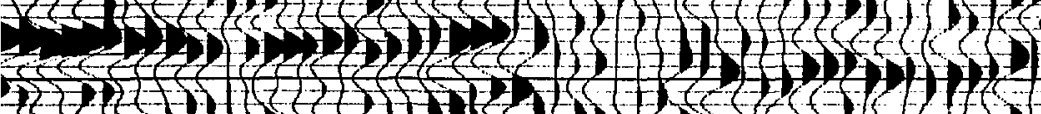
311) $13 \times 13$ 3in (I)

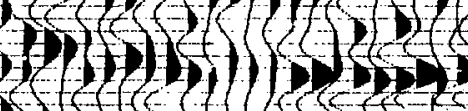

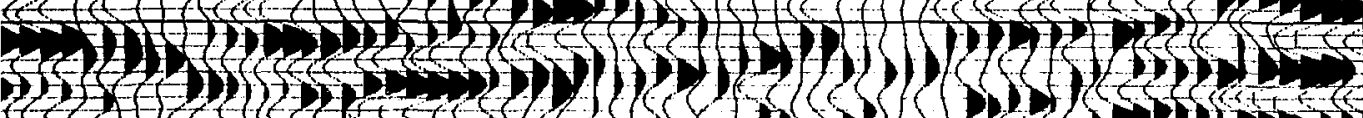

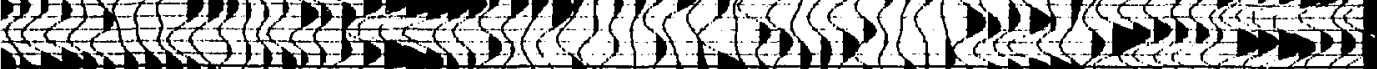

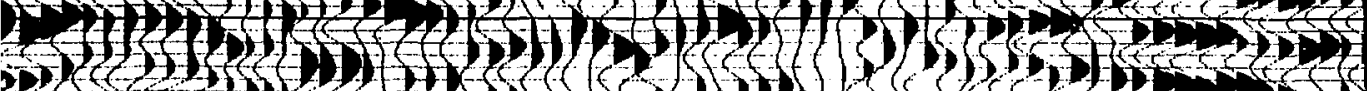

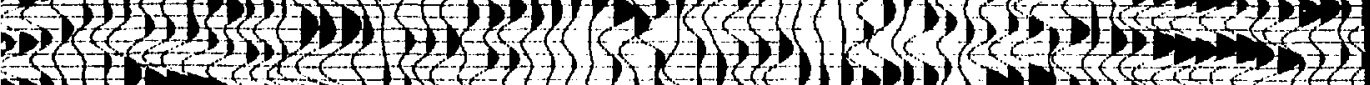
2) 3.

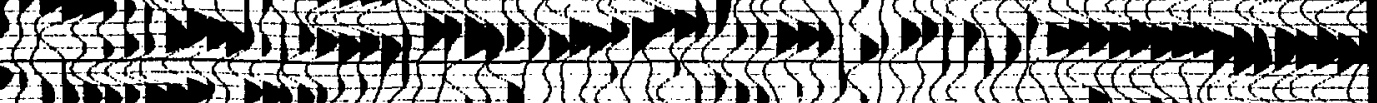

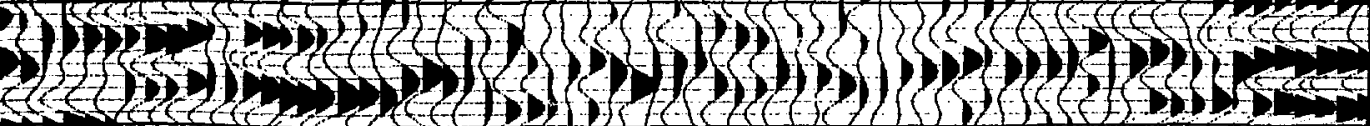

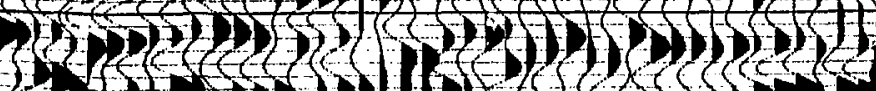

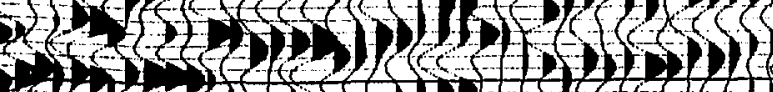




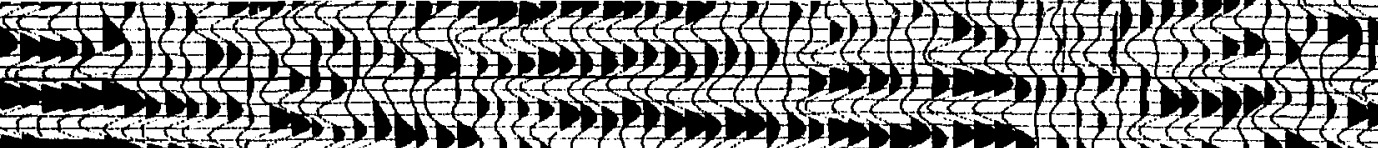
-

$\rightarrow+1+1$

$$
\text { 2. }
$$

7913 3i) $1+1)$

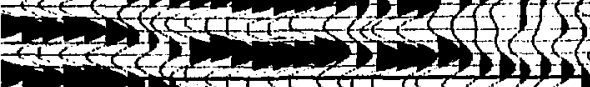

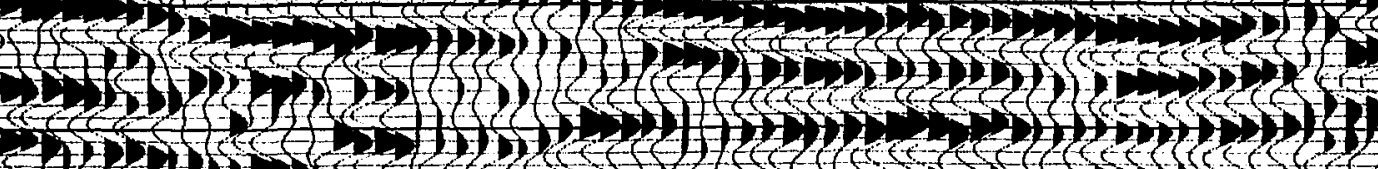

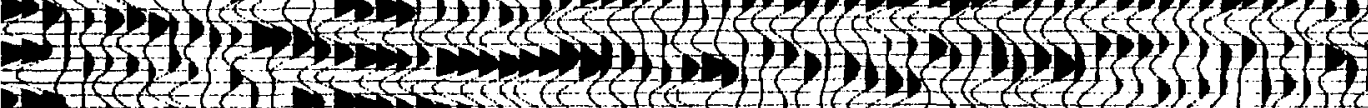

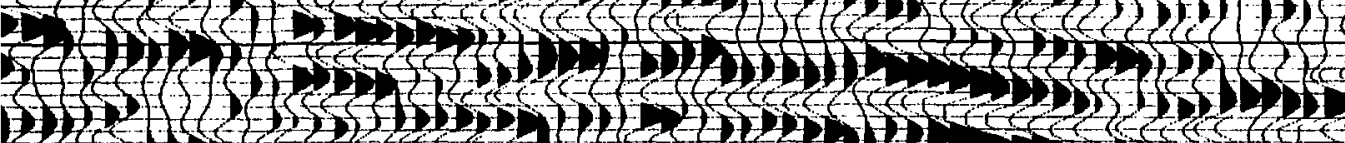
B) D(t) (f) (5) 1)

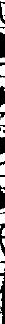

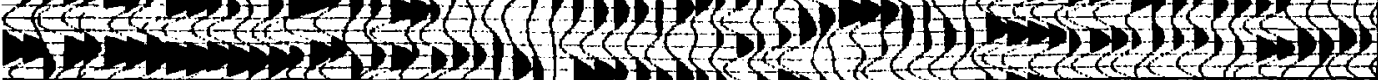
Ff

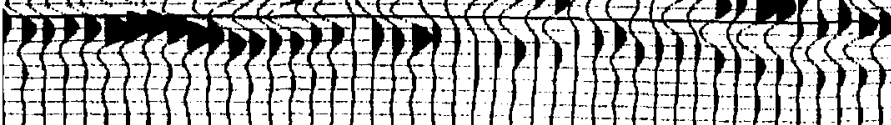




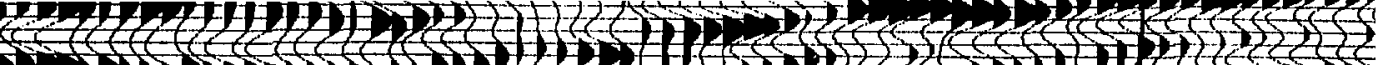
$\checkmark$ -

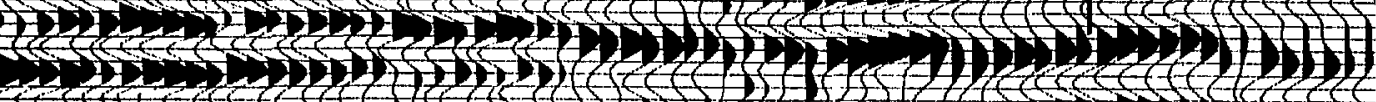

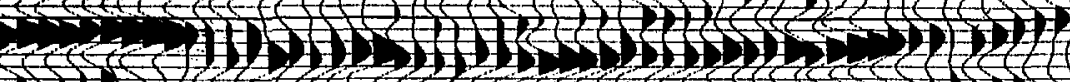

7)

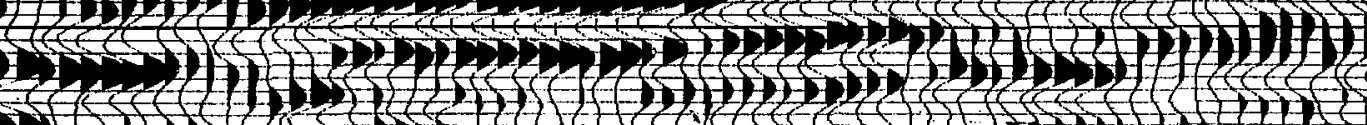
(2)

7) (2)

(1) (3)

II)

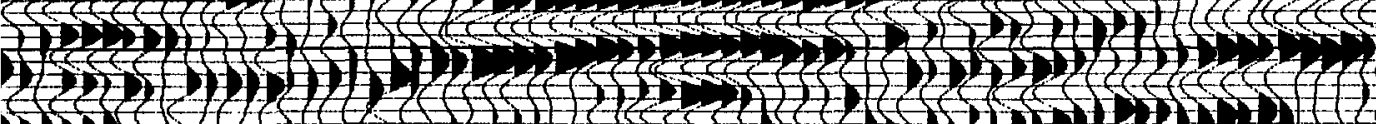

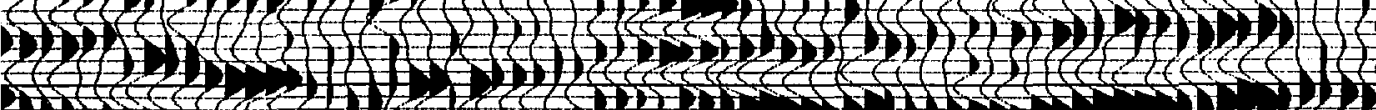

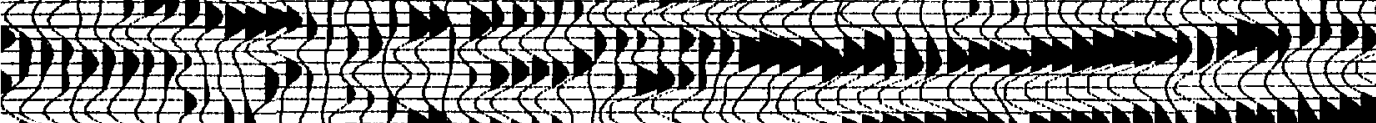
(1) 111211 1319)

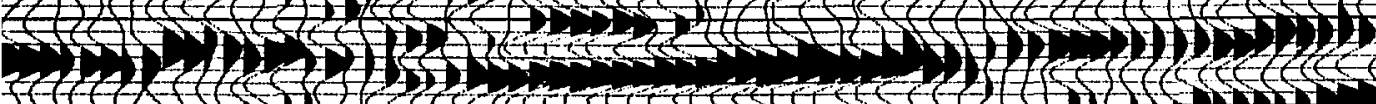

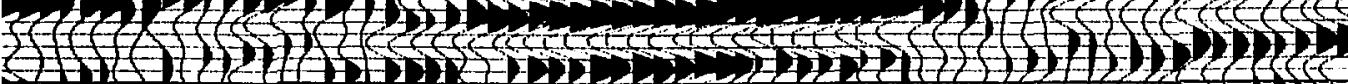

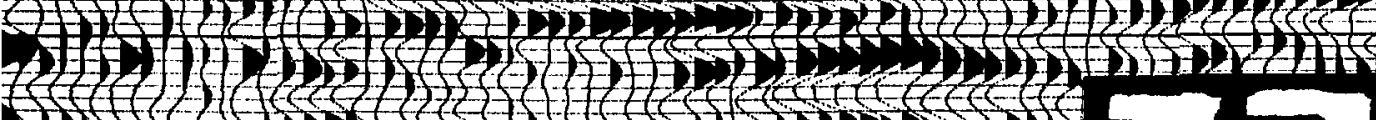

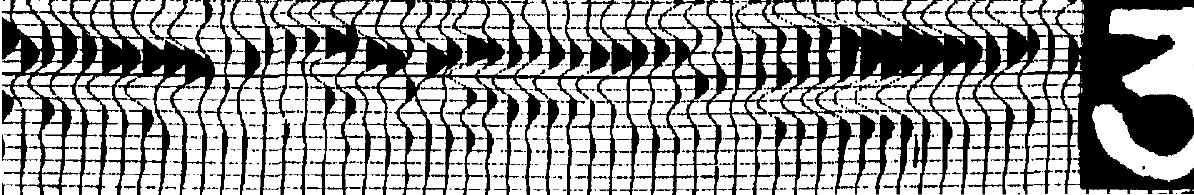


I1) II

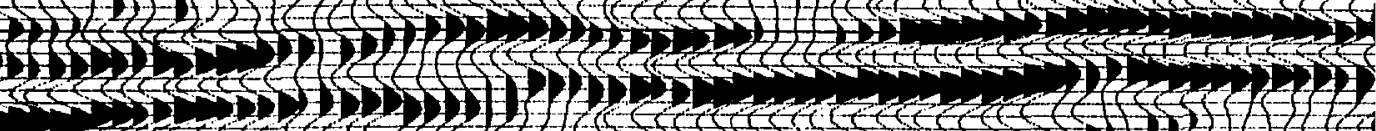

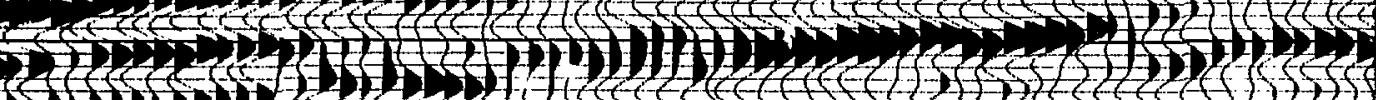
(2)

(1) (1) 1) (1) (1) 12) 13 L 2101 m H.

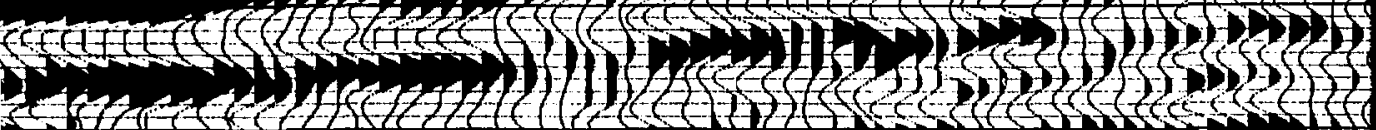
2 -

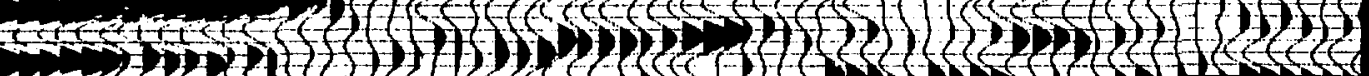
(2) 20 (3) m)

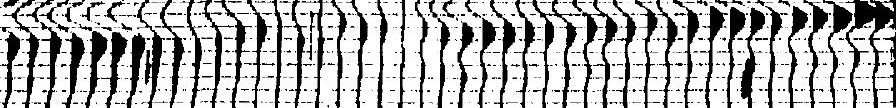




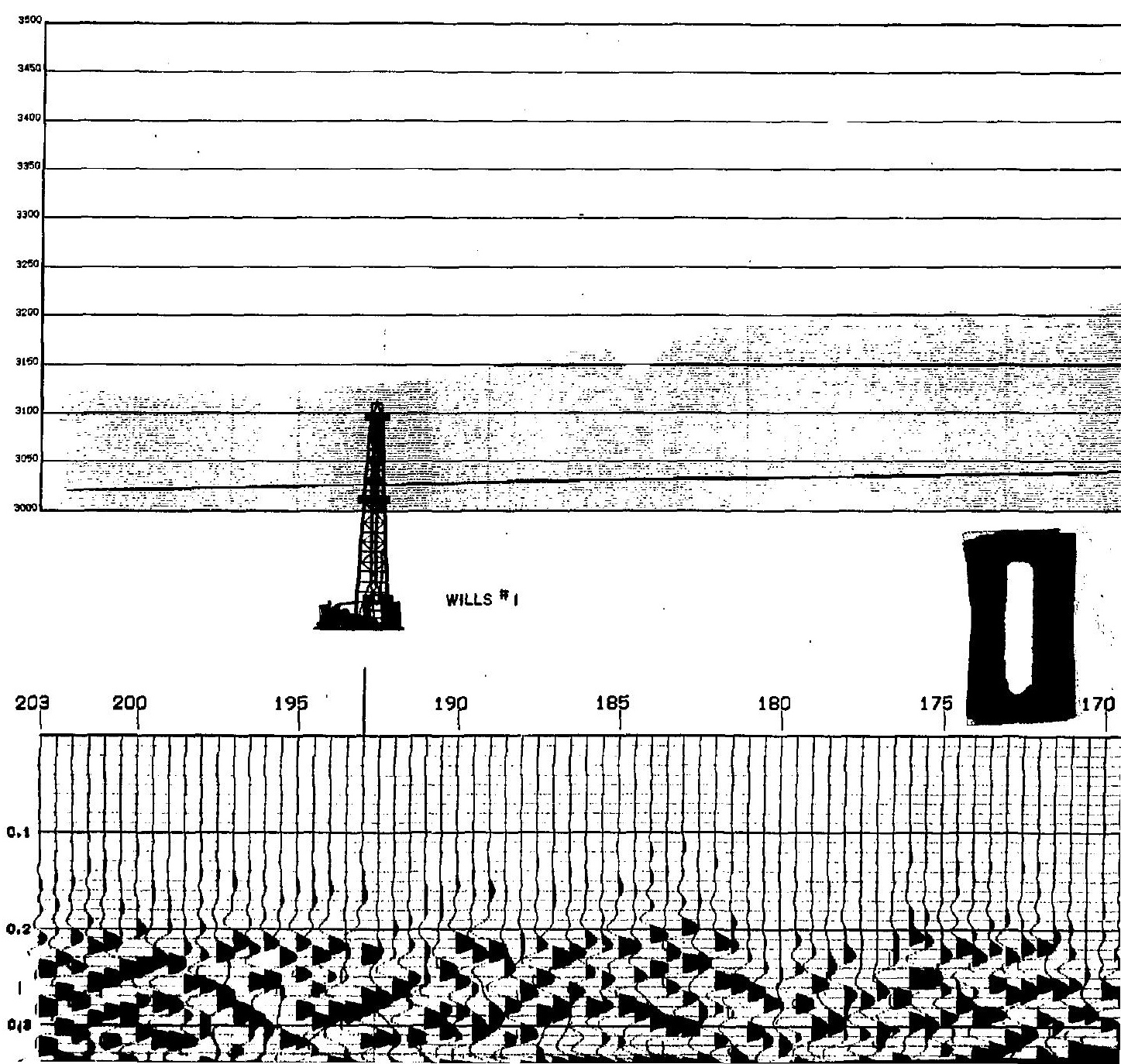



SANOIA CORE HOLE

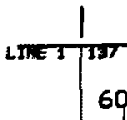

$\because \because$

int

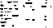

-

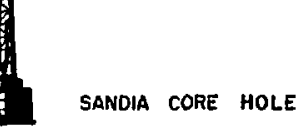

政

60

55

$5 q$

45

40

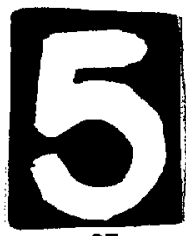

35

30

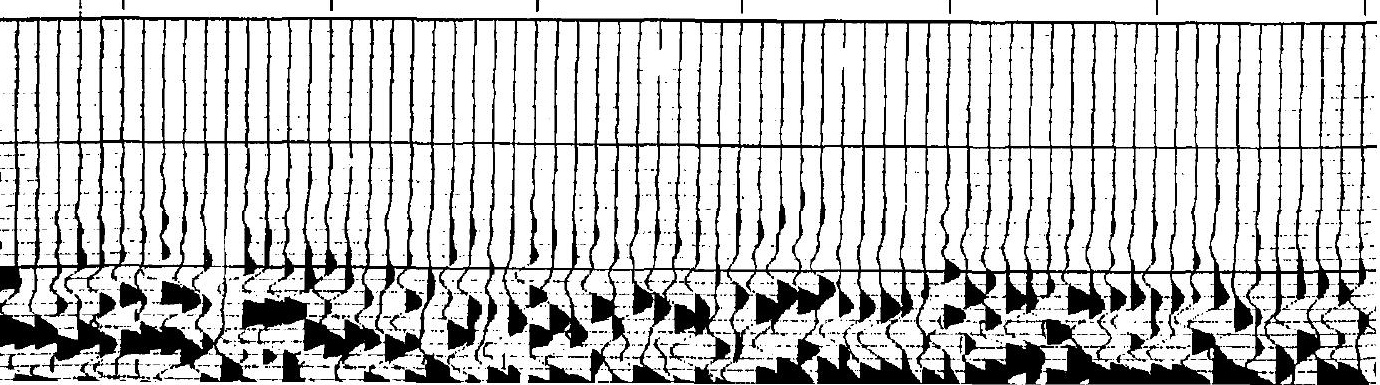




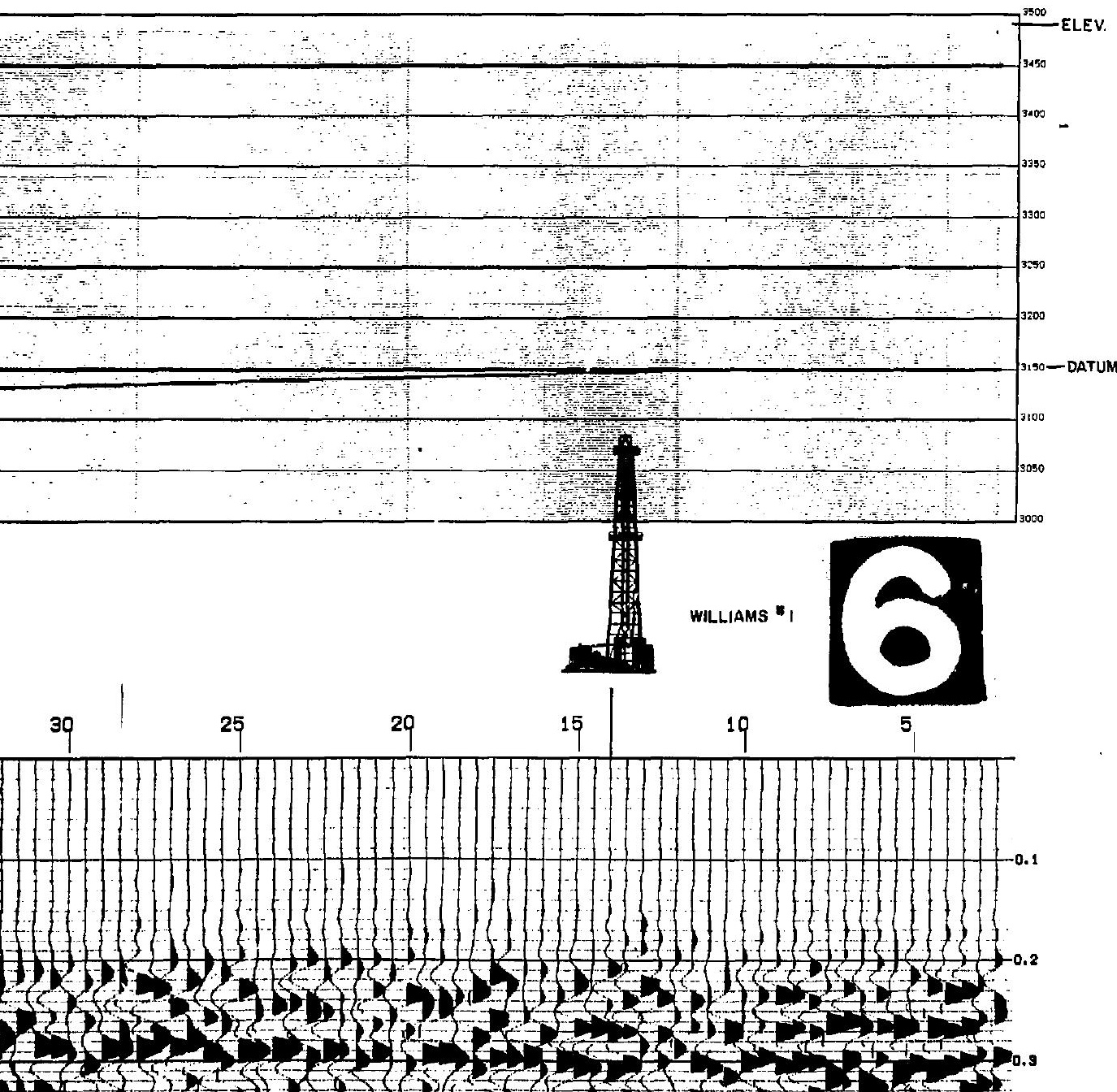



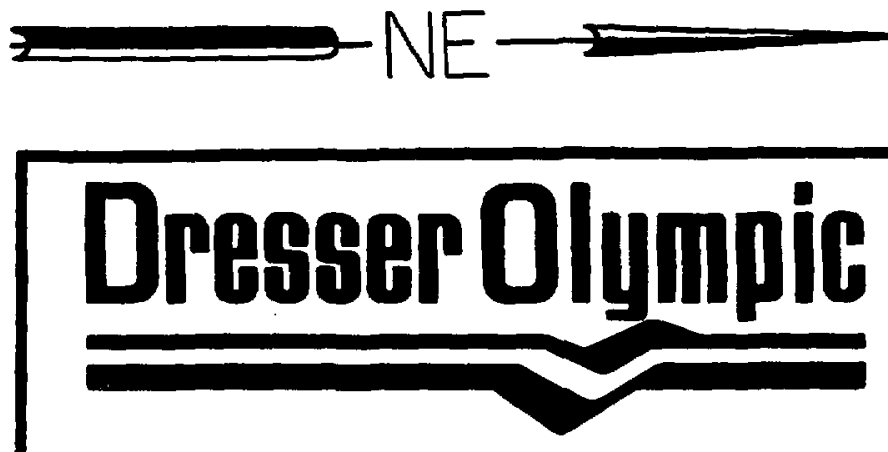

SEISMIC SURVEY

FOR

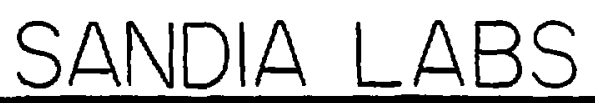

PROJECT LOS MEDANOS LINE

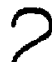

LOCATION EDOY COUNTY. TEXAS

DATE RECORDED $5-4-76$ TO $5-11-76$

OATE PROCESSED $-5-17-76$

DATA PROCESSING

$\perp$ DEMULTIPLEX

2 GAIN RECOVERY

3 COMMON DEPTH POINT GATHER

4 APPLY DATUM STATICS

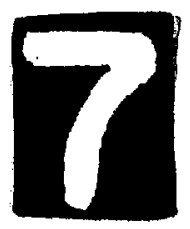

5 DECONVOLUTION

Prod. Longth AUTO Stert Gato For Tr. 800 ma. Oper. Longth 240 ms. ms. Noar Tr. 300 ms. 
西

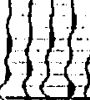

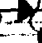

3 o.:1,

(c)

130

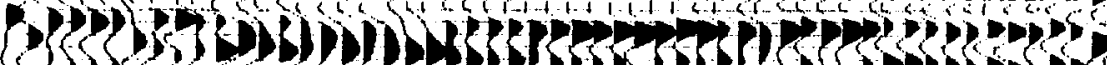

$0.5 \times 1)$ (स)

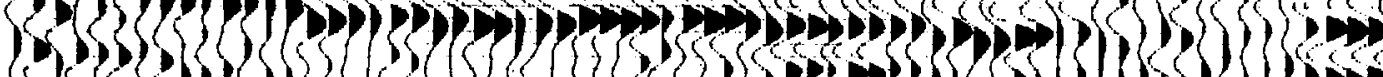

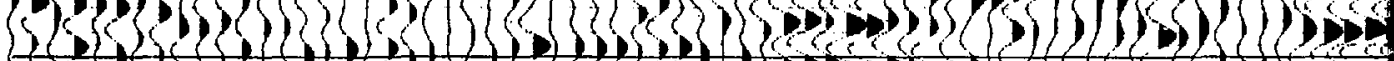

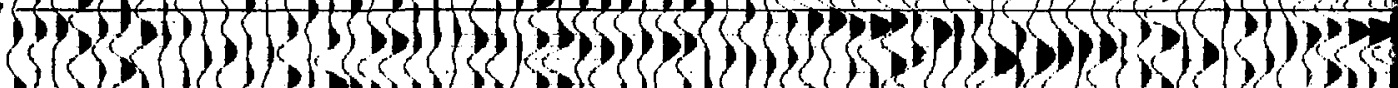

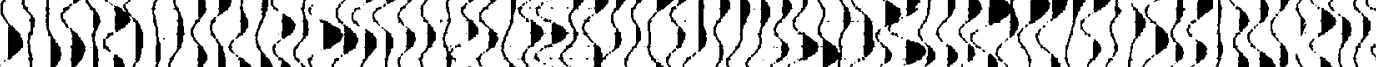

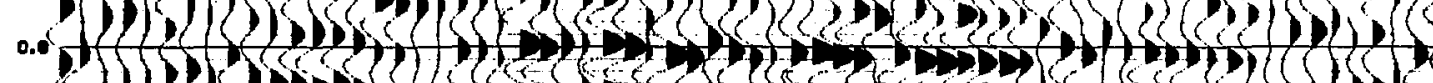

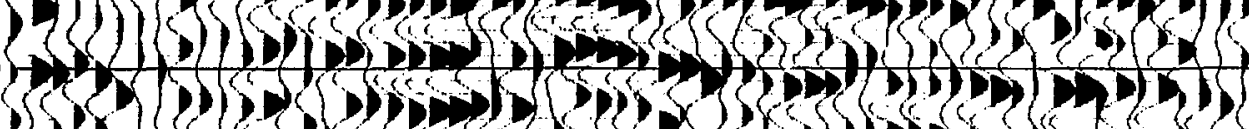

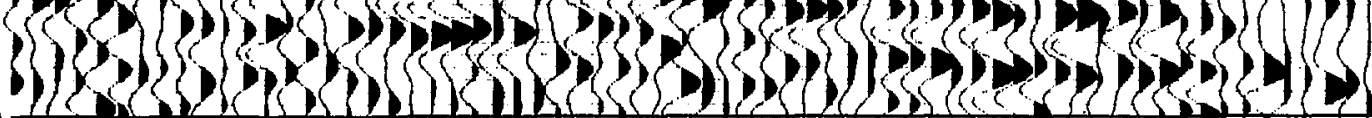

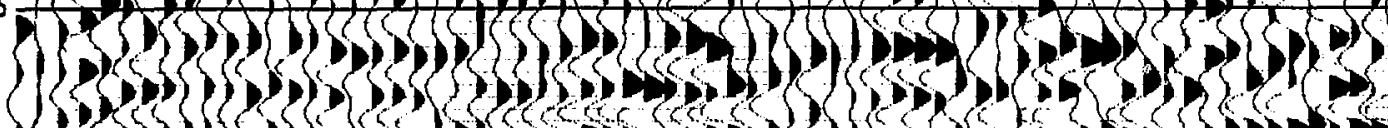

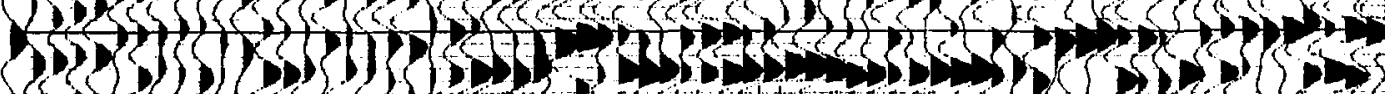

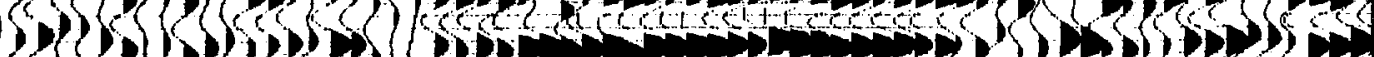

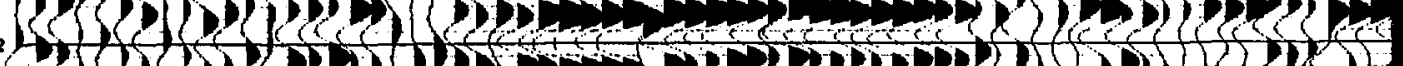

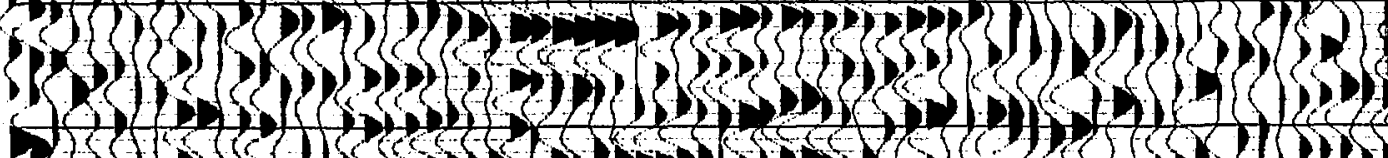

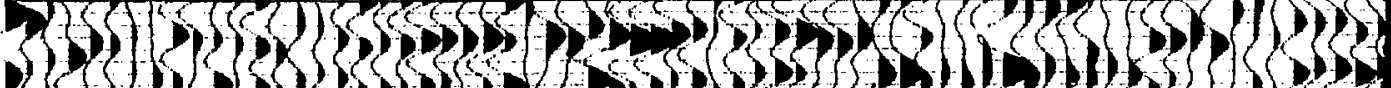

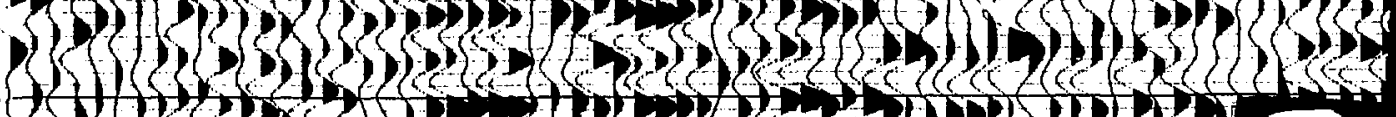
13 (3)

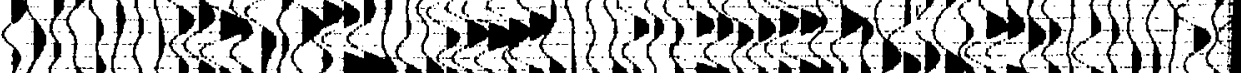

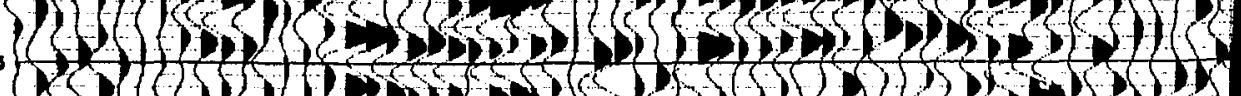

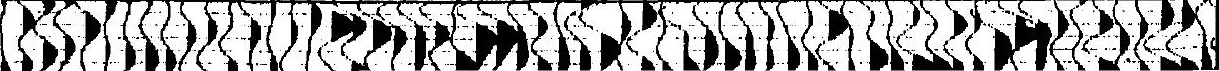


(3)

18

3

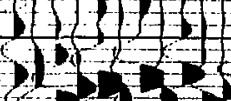

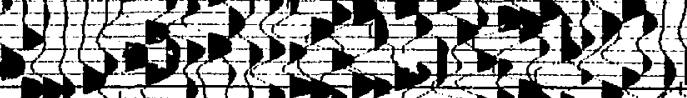

1:3

$x+35 x+2$

723

P.

79

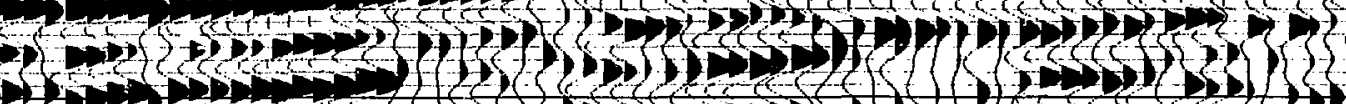
Fton

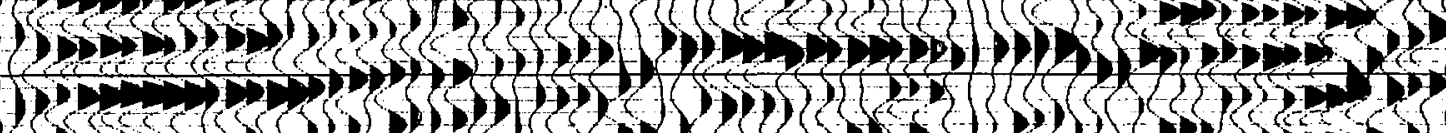

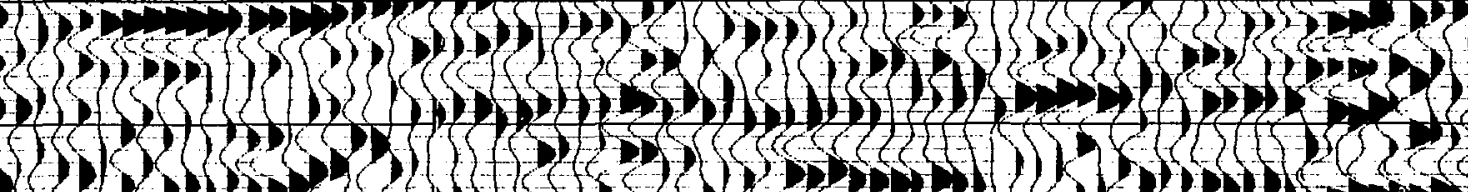

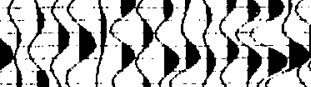

9) (1)

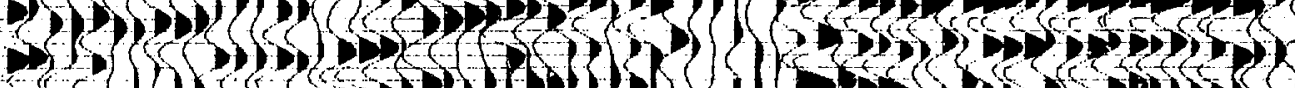

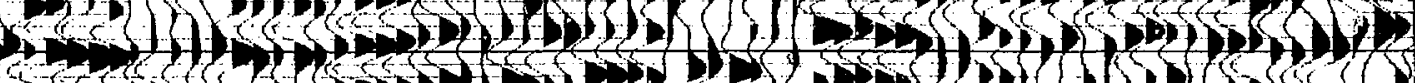
- S m (1)

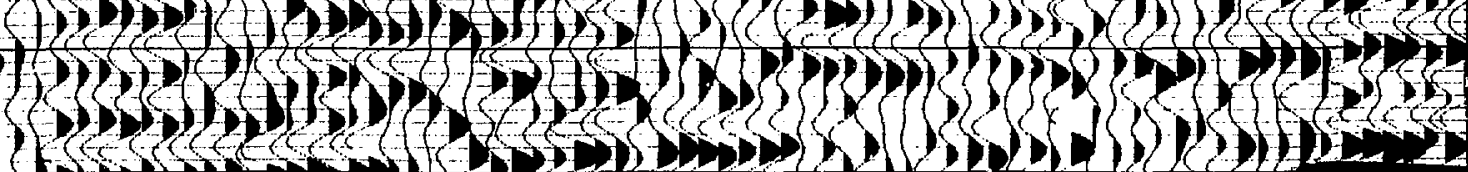

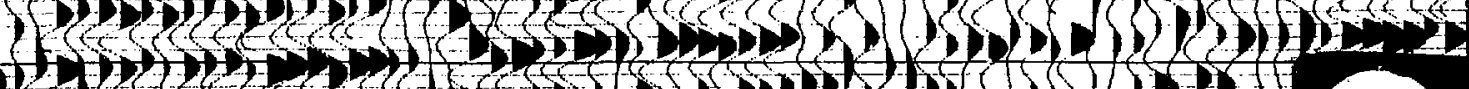

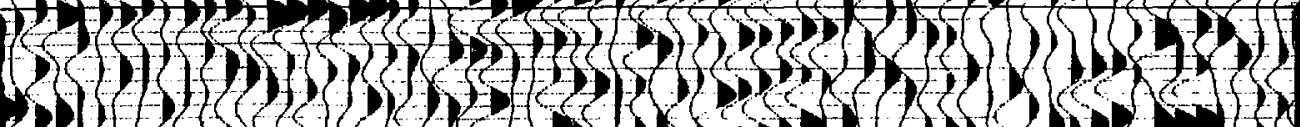
s. 


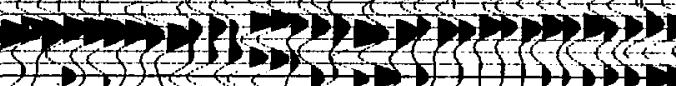

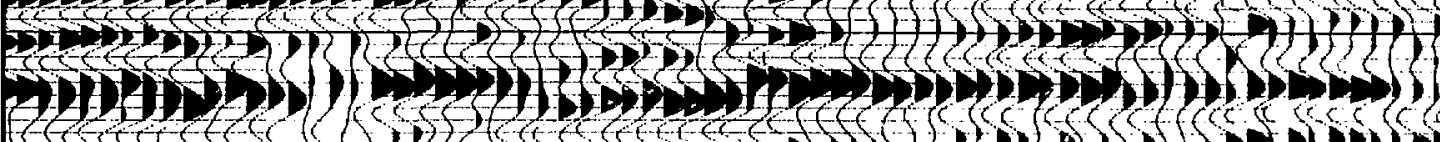
(5)

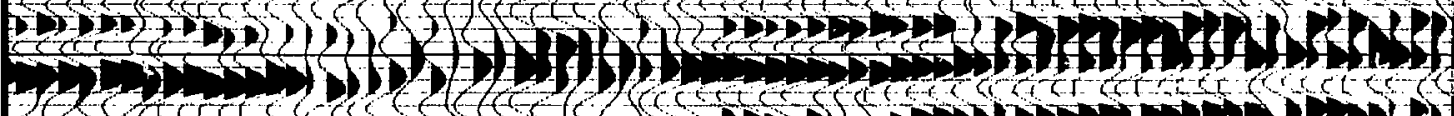

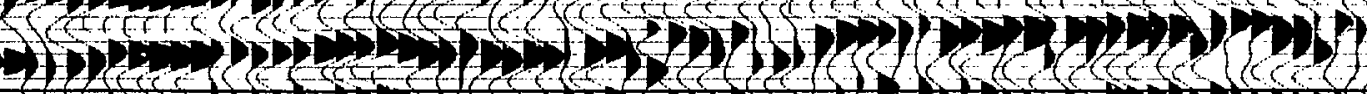
गI)

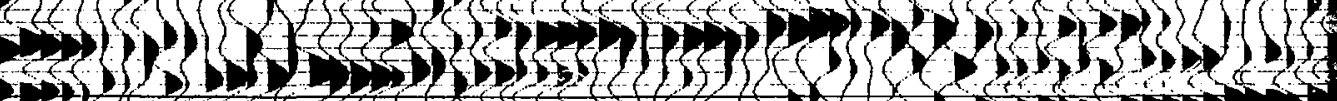

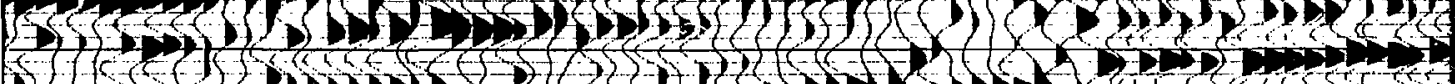

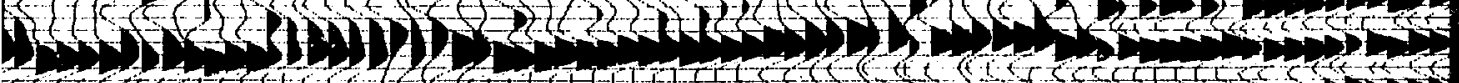

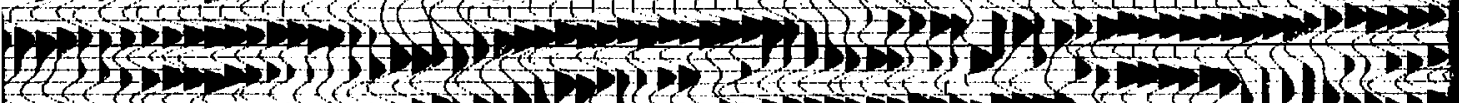

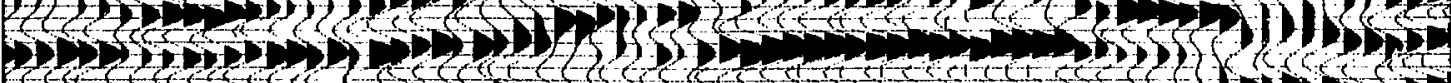

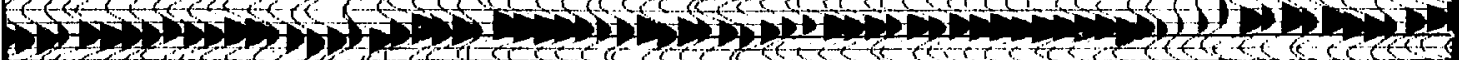

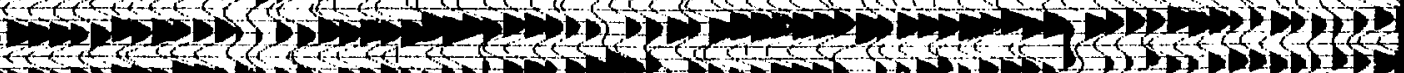

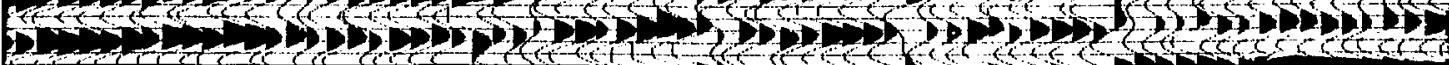

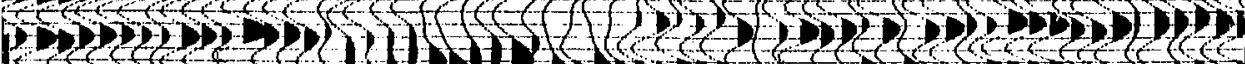

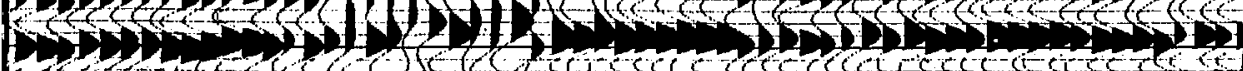
Wh 



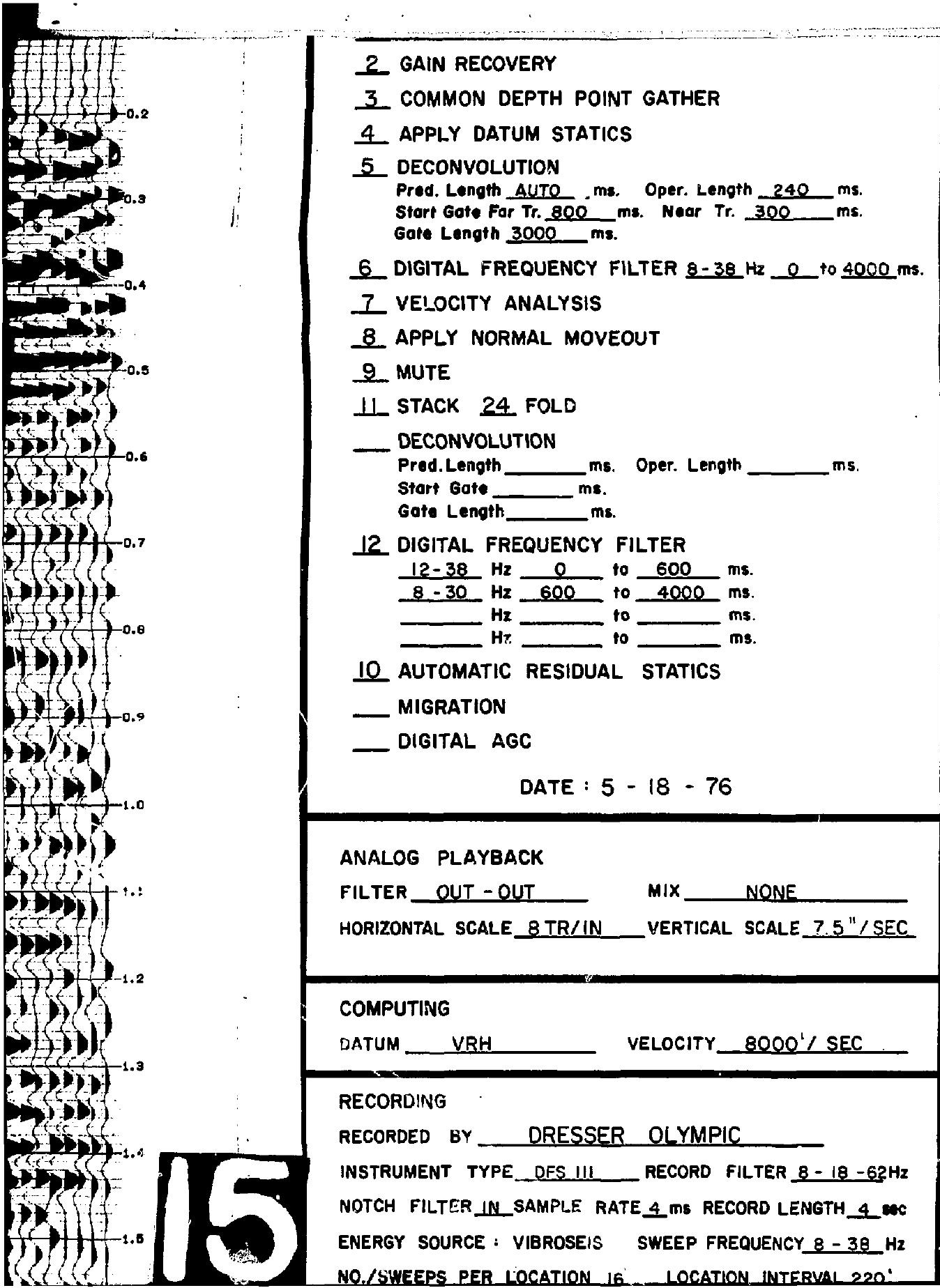




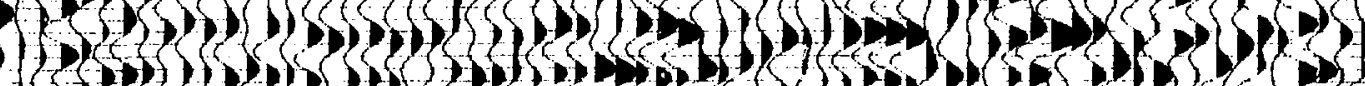
5.m. 17.

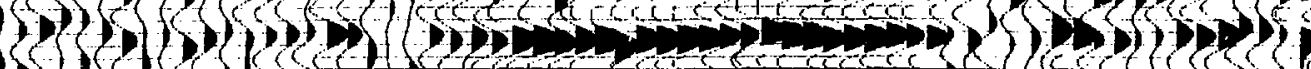
1.

mor. 13

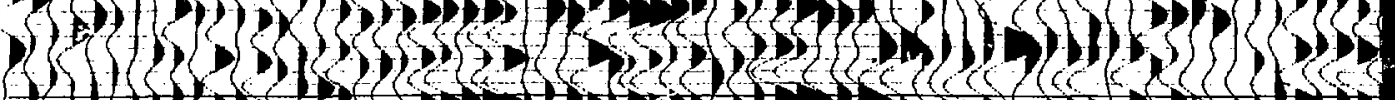
-

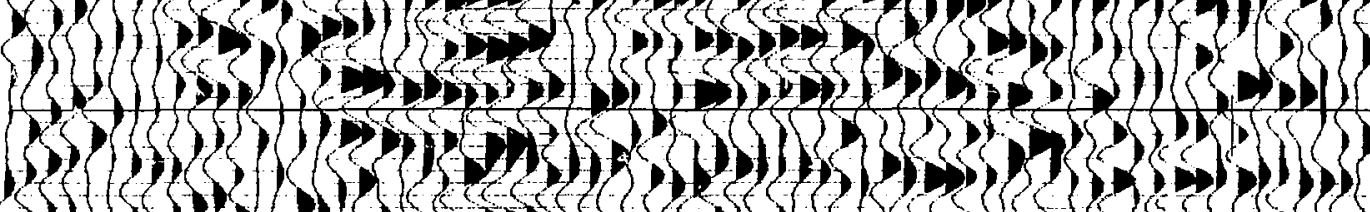

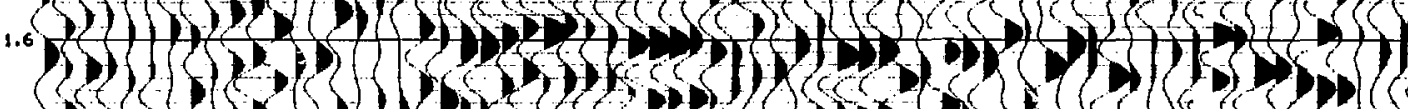
n. 1

9. 2. (1)

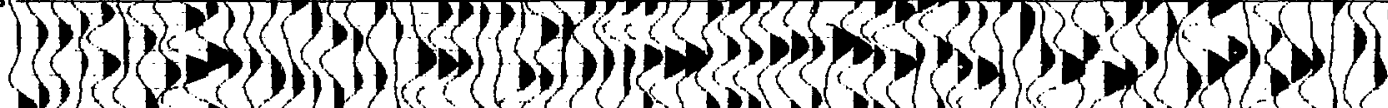
no.

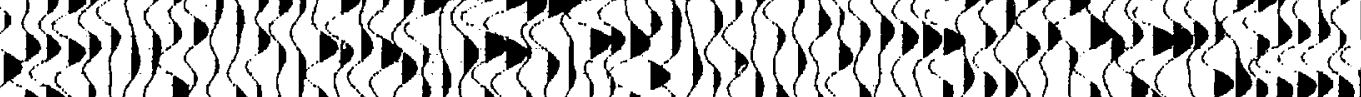

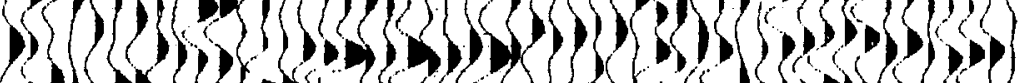

3.

$$
\text { 2. }
$$

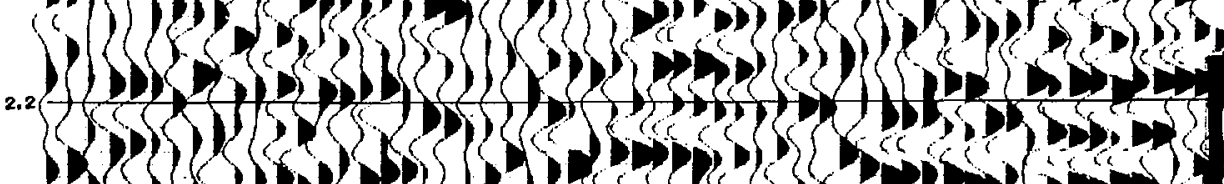
2.39m 


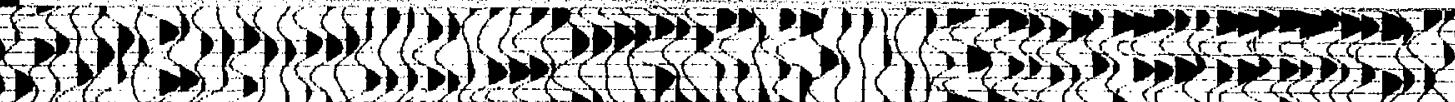
Linm.

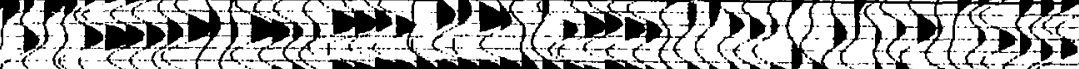

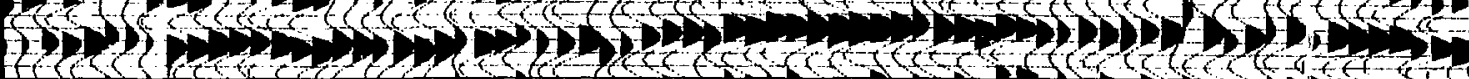

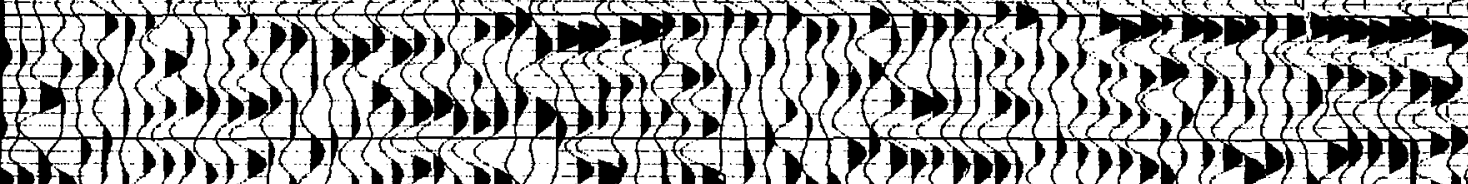

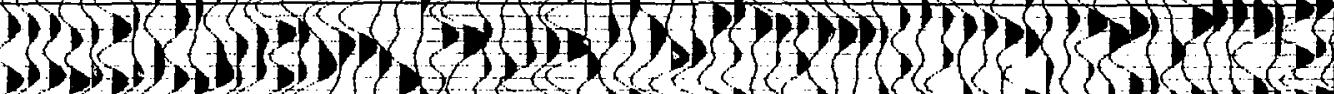

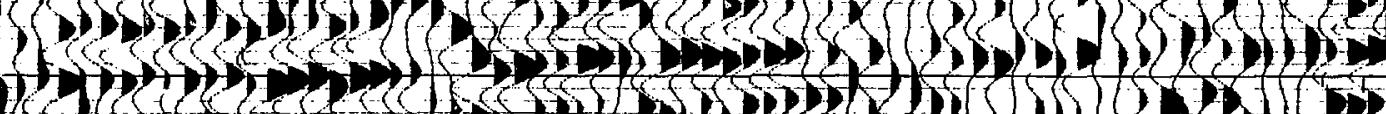
2. 20.1.7. 3.

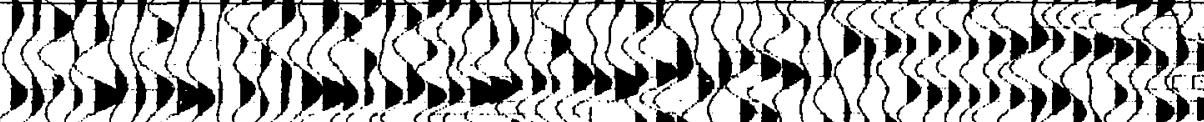

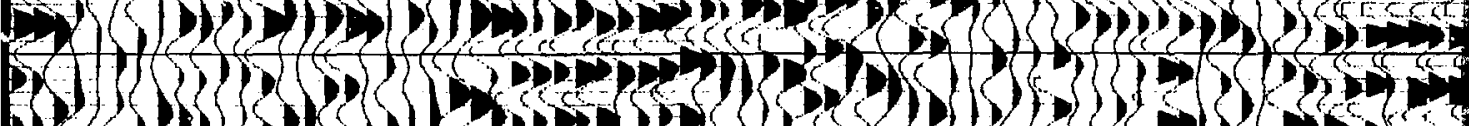
24. n. P. (a)

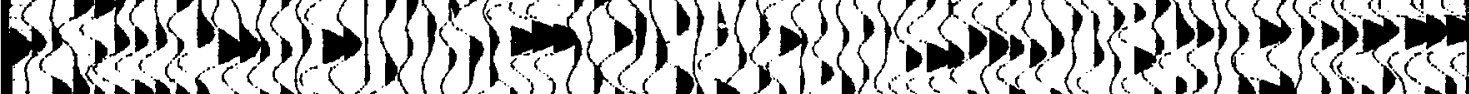

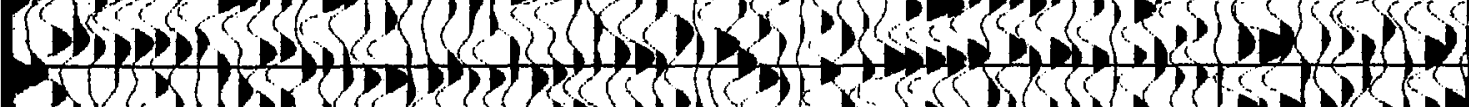

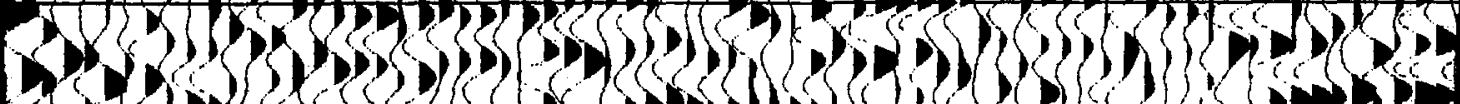

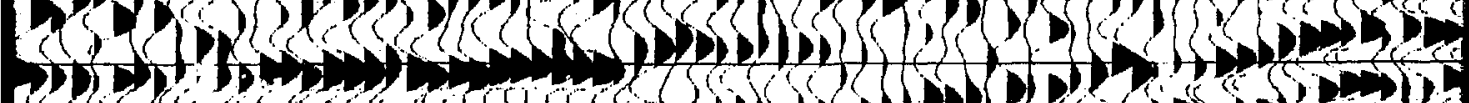

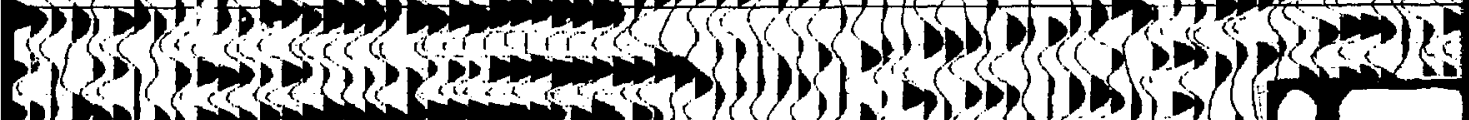
( 303 s

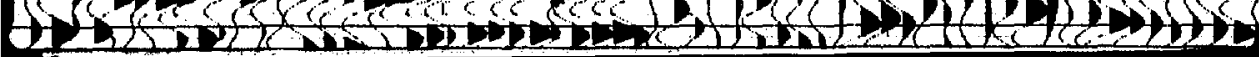




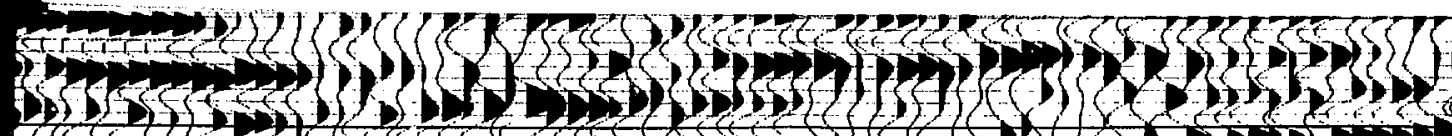

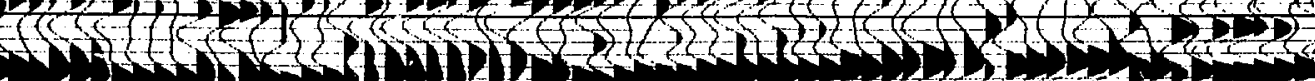

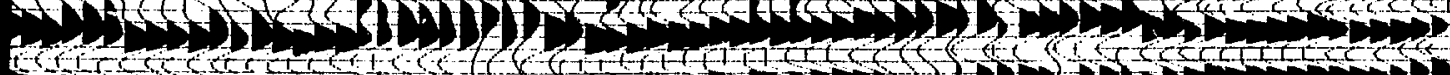

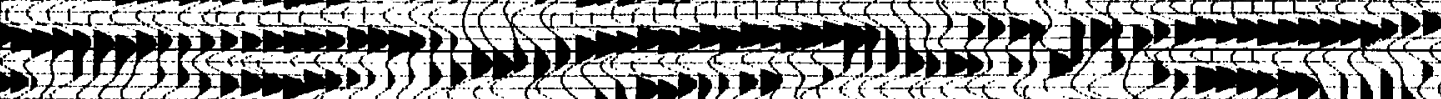
L

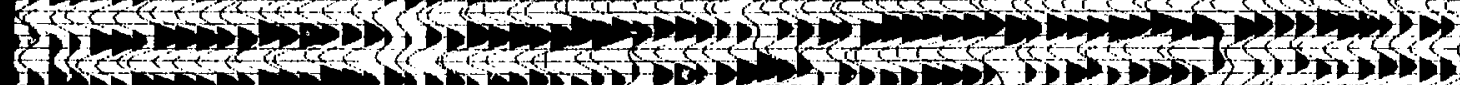

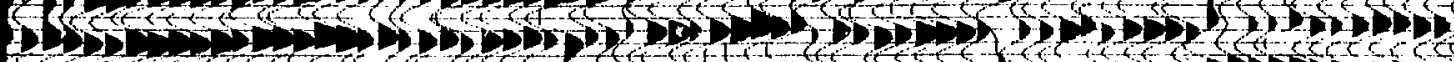
$150,-1,1$

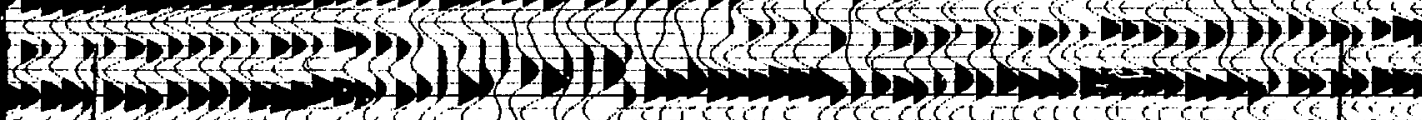

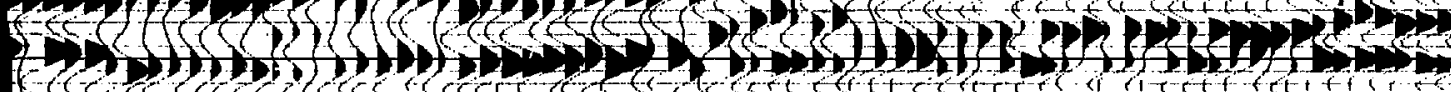
(6)

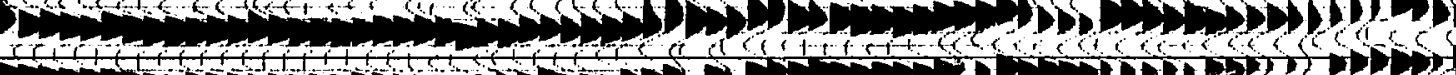

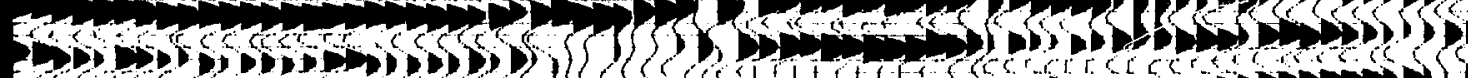
(2)

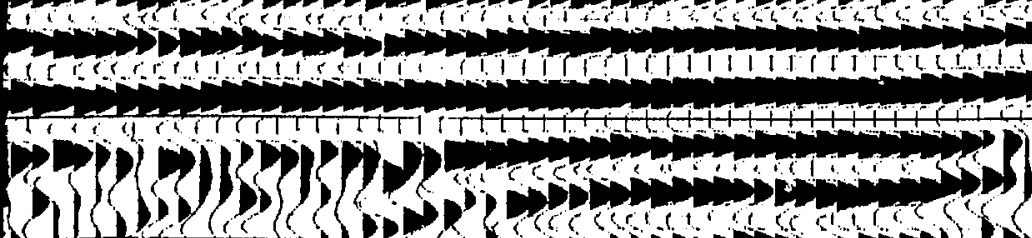
(x)

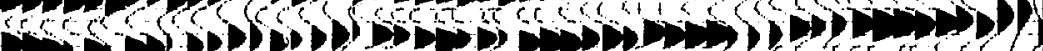

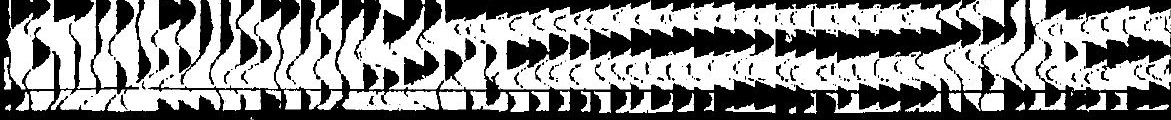


KIE

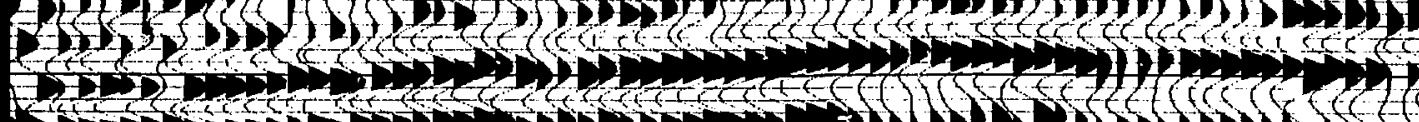

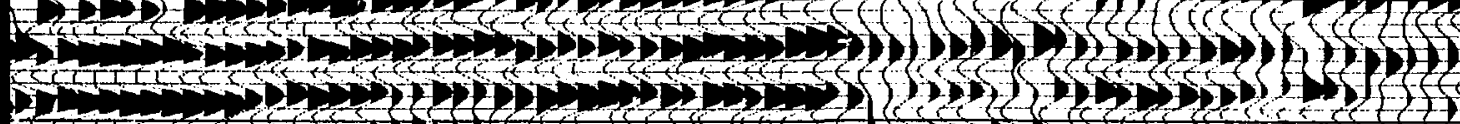

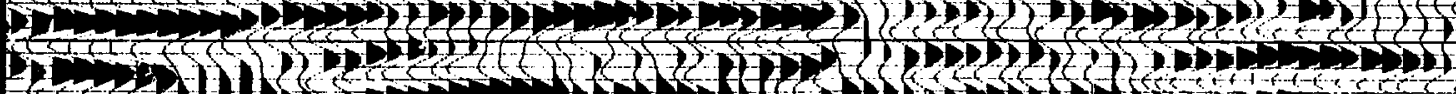
3 In

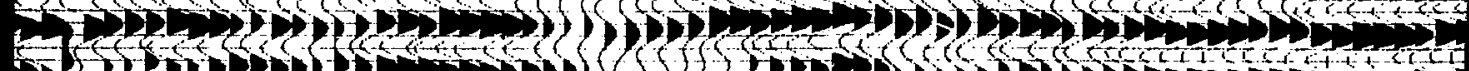

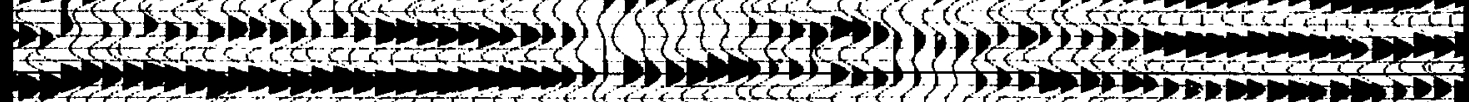

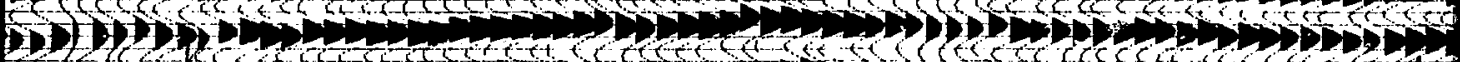
4. D. A

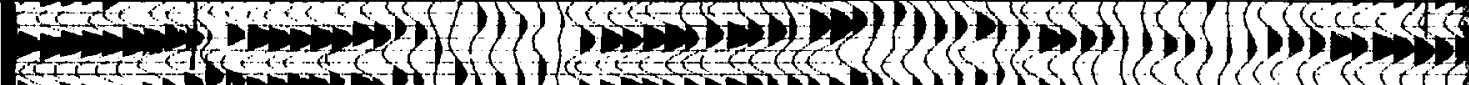

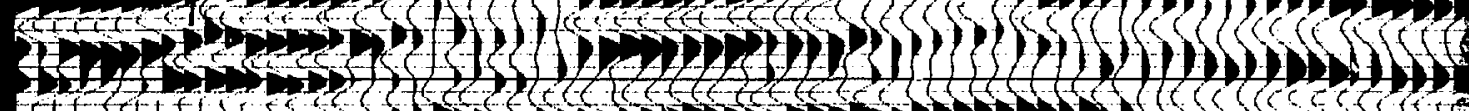

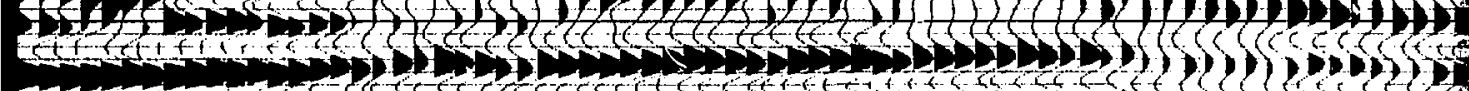

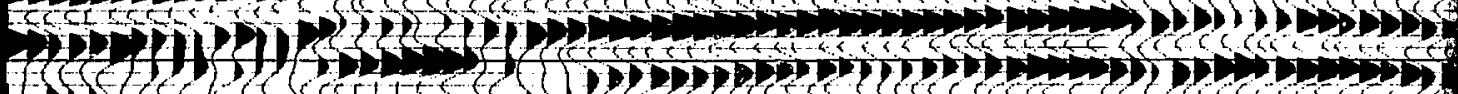

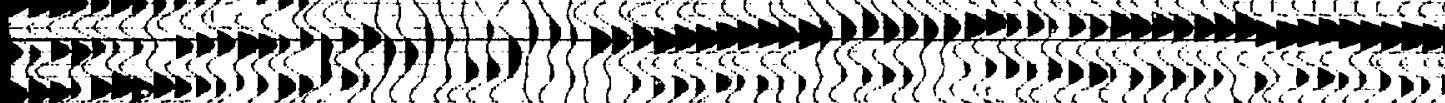

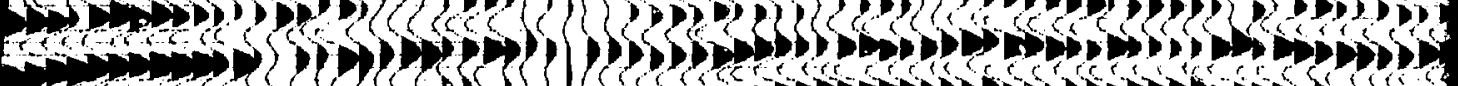

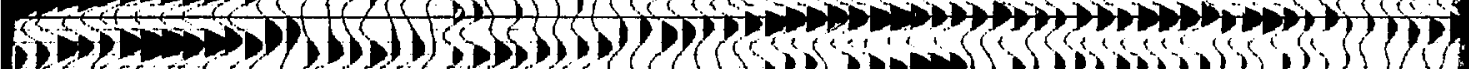

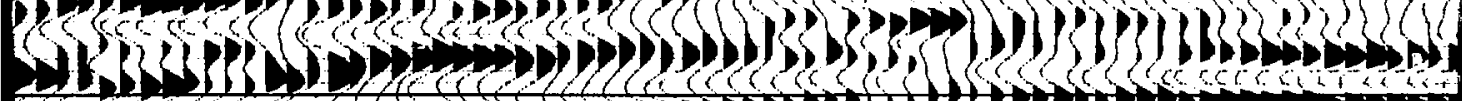

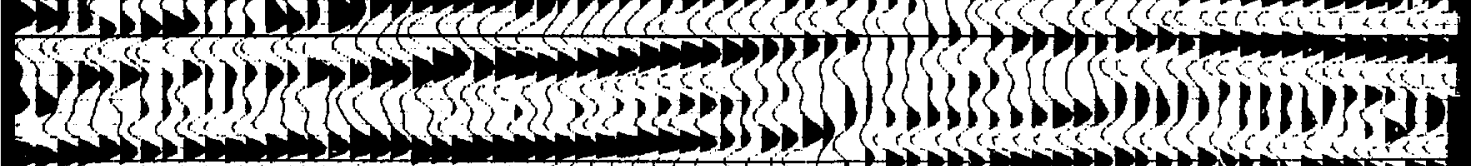

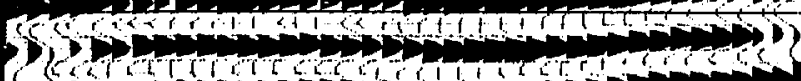

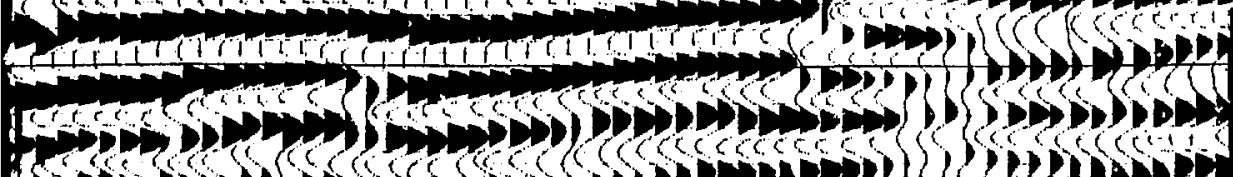
35050, 


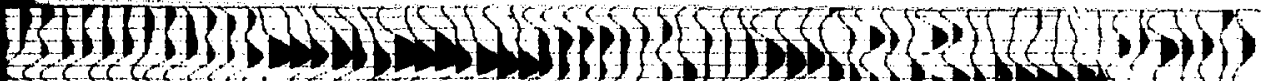

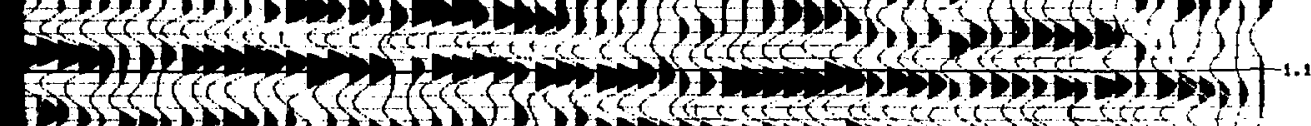

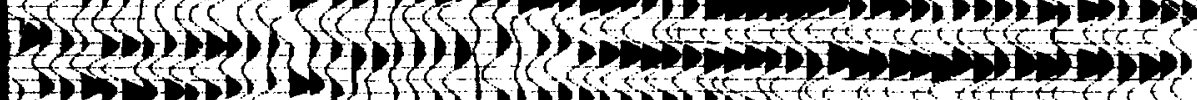

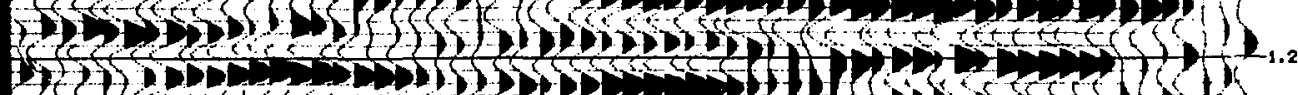

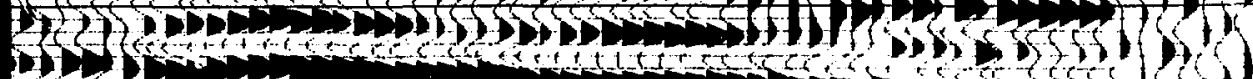

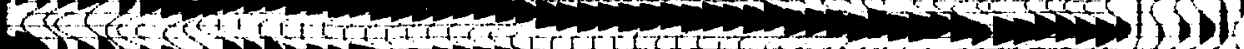

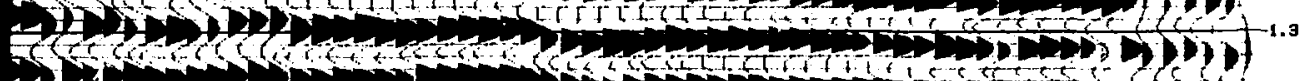

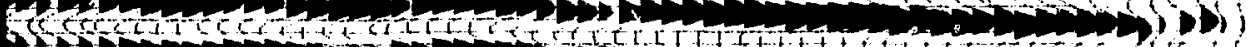

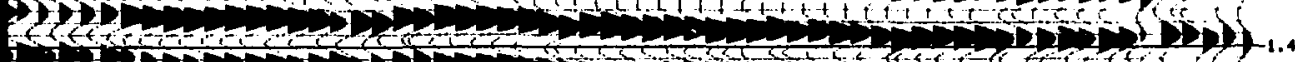

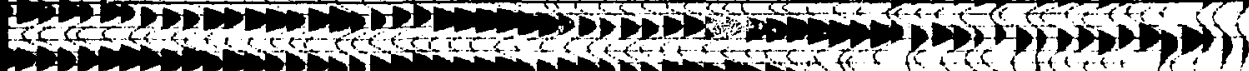

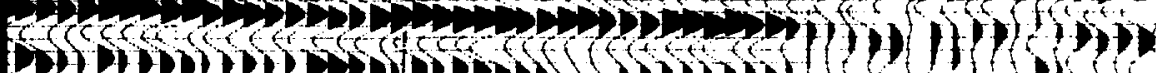

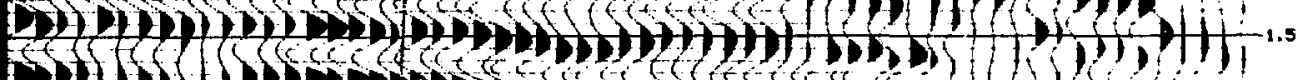

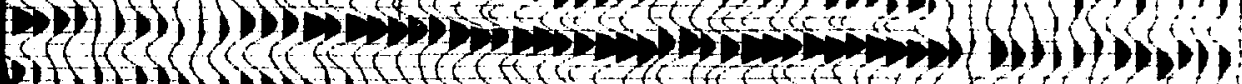

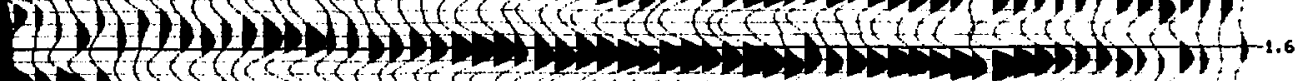

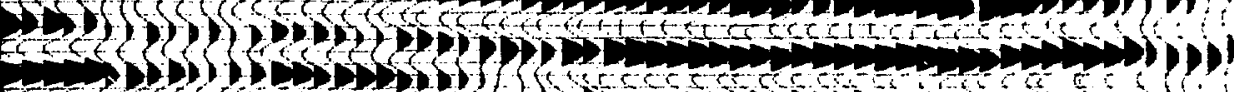

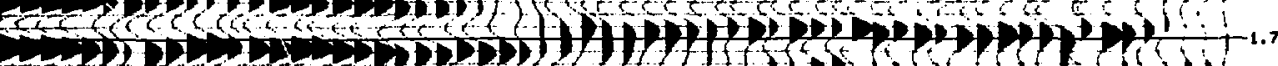

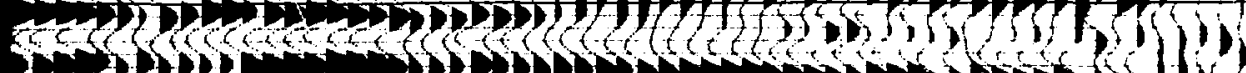

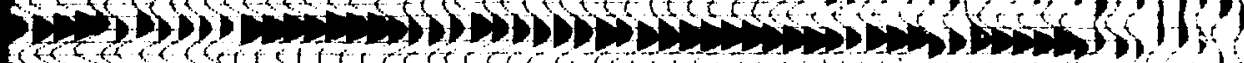
(1) 15 of (2)

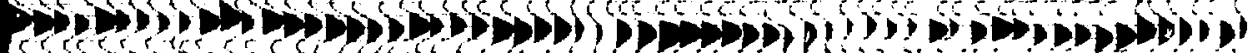

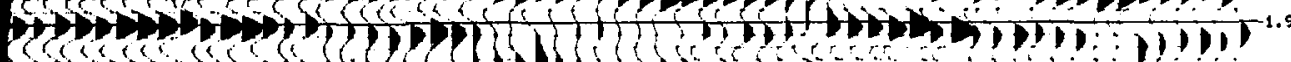

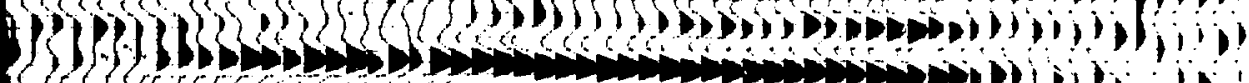

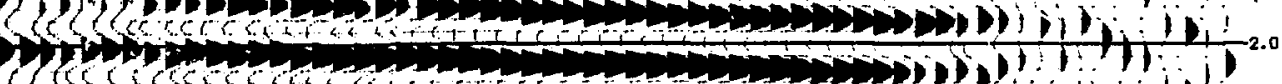

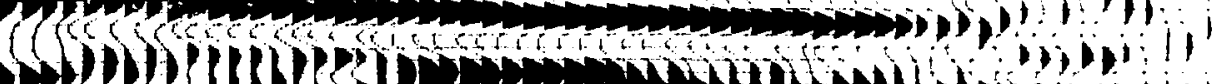

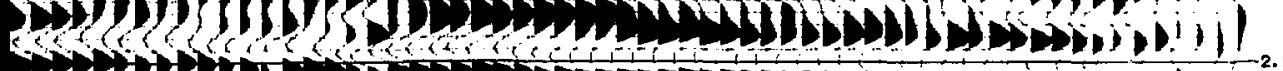

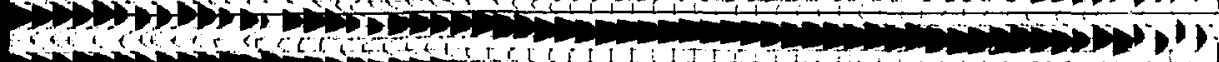

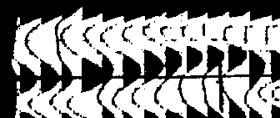

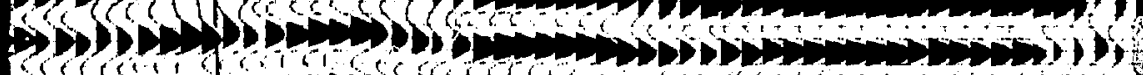

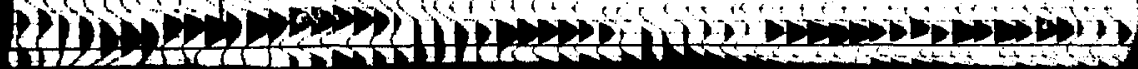

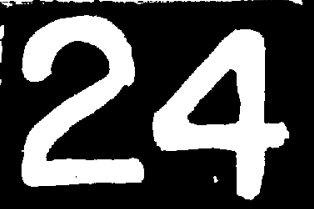


ANALOG PLAYBACK

FILTER OUT - OUT

MIX

NONE

HORIZONTAL SCALE_8TR/IN_VERTICAL SCALE $7.5 \%$ SEC

COMPUTING

DATUM

VRH

VELOCITY $8000^{\prime} /$ SEC

RECORDING

RECORDED BY DRESSER OLYMPIC

INSTRUMENT TYPE DFS III_RECORD FILTER $8-18-62 \mathrm{~Hz}$

NOTCH FILTER IN SAMPLE RATE 4 ms RECORO LENGTH 4

ENERGY SOURCE : VIBROSEIS SWEEP FREQUENCY $8-38 \mathrm{~Hz}$

NO./SWEEPS PER LOCATION 16 LOCATION INTERVAL 220

MODEL Q TYPE GEOPHONES WHS FREQ. 8 HZ NO/GROUP 48

TYPE COVERAGE $2400 \%$ SPREAD LENGTH $11880^{\circ}$

NO. TRACES $48^{\prime}$ OFFSET BBQ $B 0^{\prime}$ GROUP INTERVAL $220^{\circ}$

SPREAD DIAGRAM

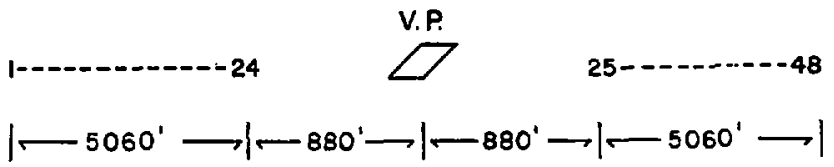

$111)^{1.9}$

(i)

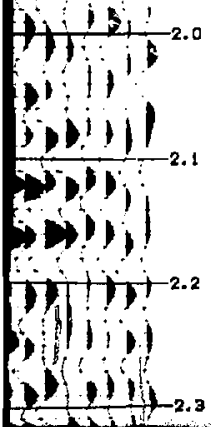

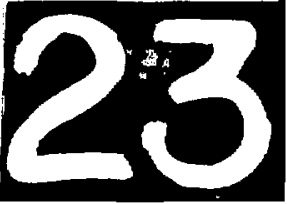


5 3.5

H.7. 3. ...

33.5.3. Hon.

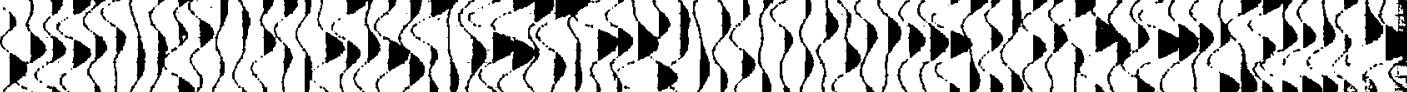
20. (a)

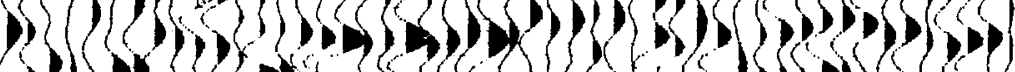

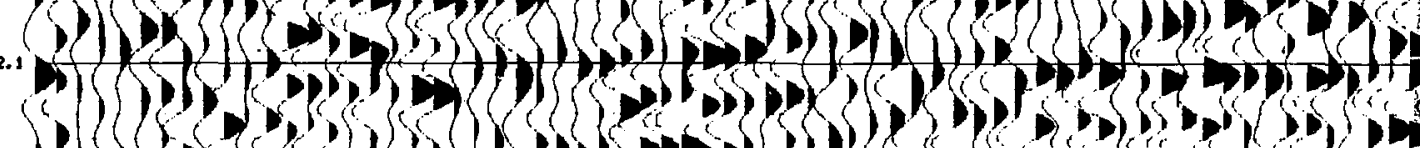

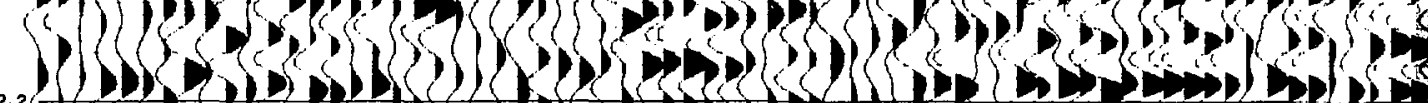
2.25

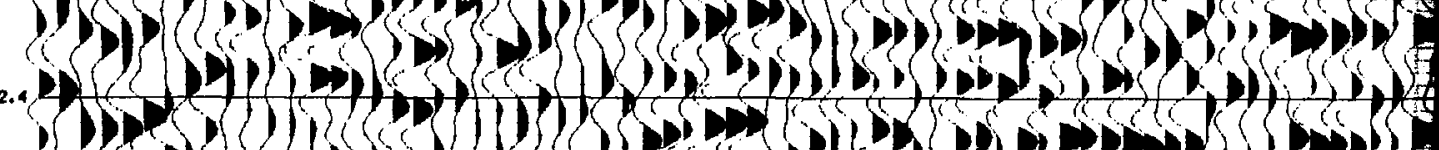
6.m.3.

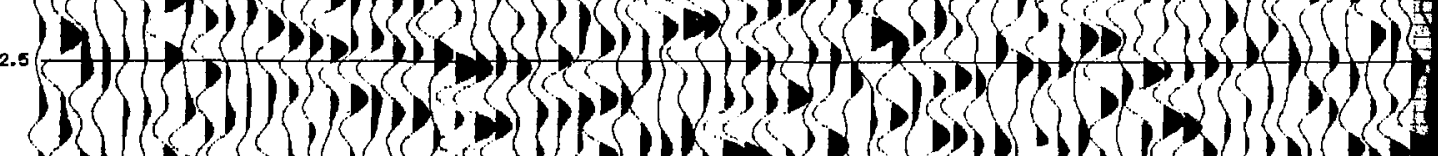
.6.

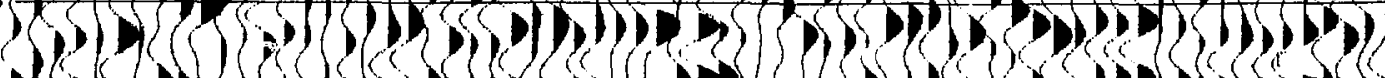

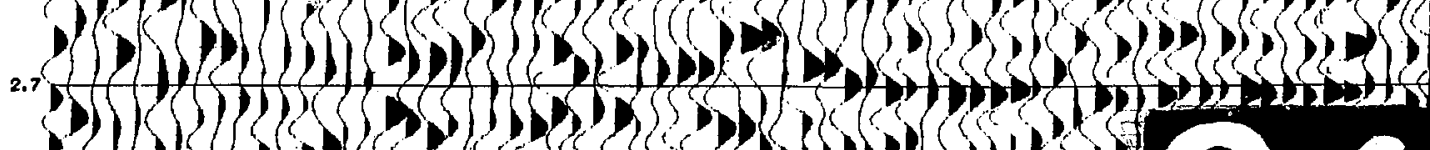

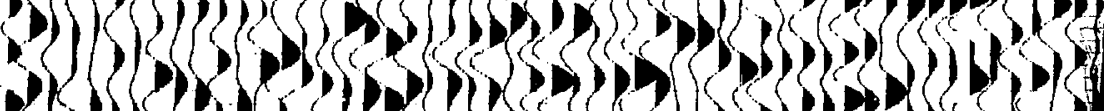

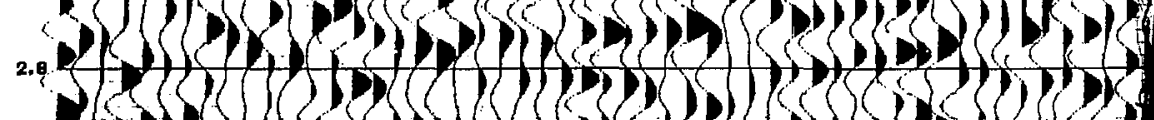

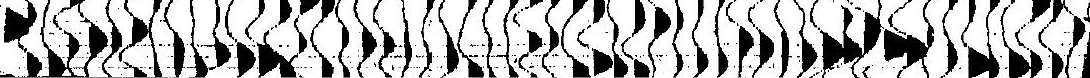




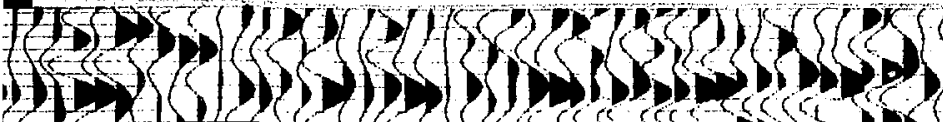

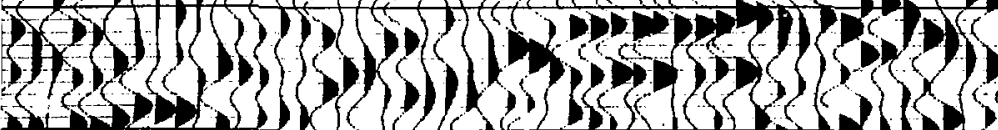

$\rightarrow \rightarrow$.

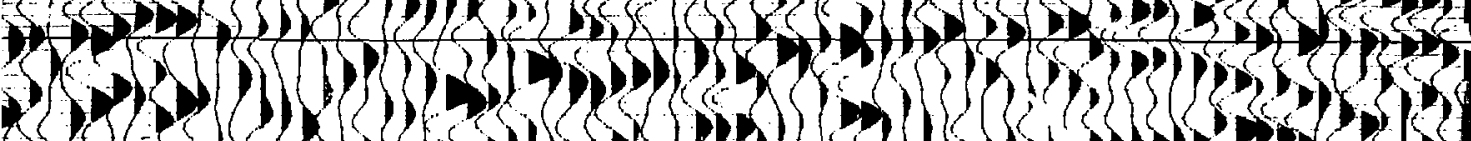

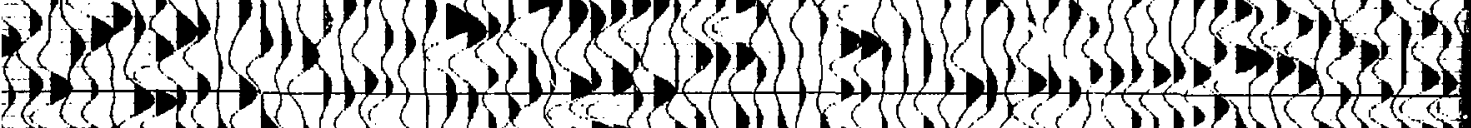

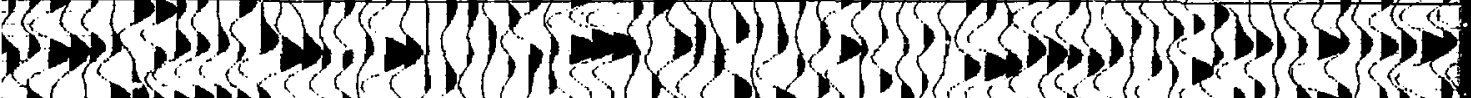

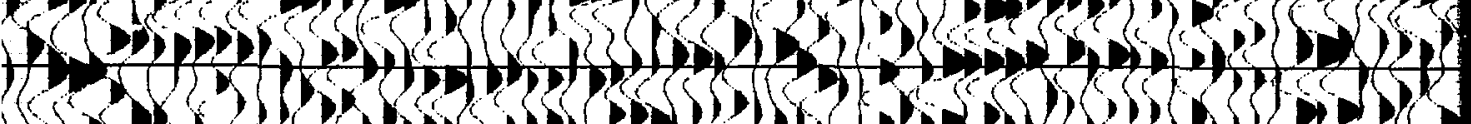
10. 2.3.

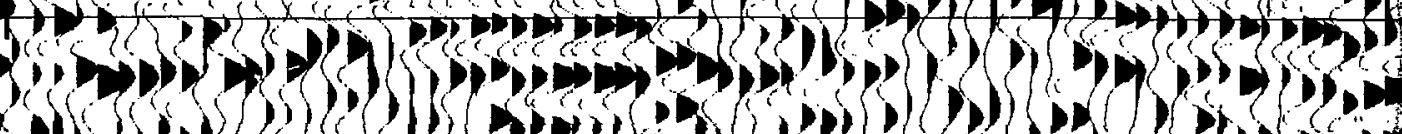
1) in in

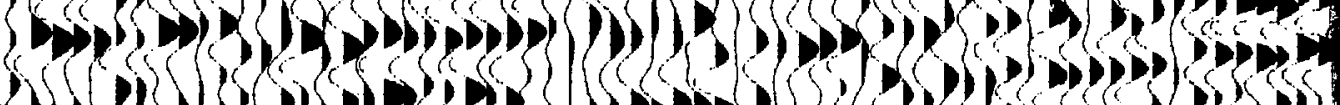

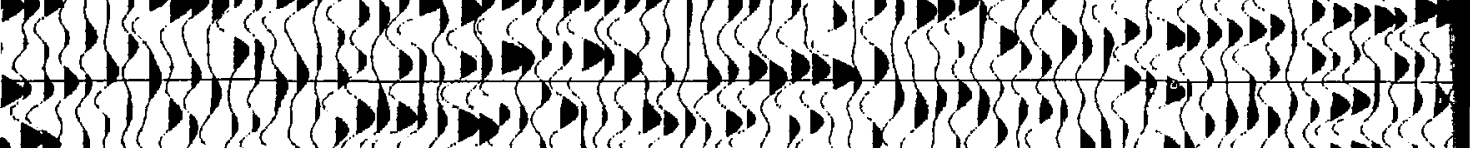

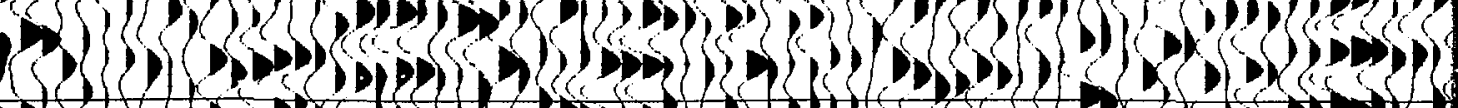

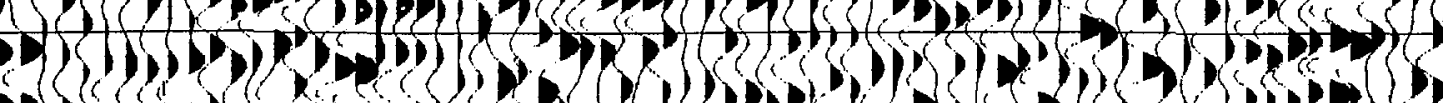

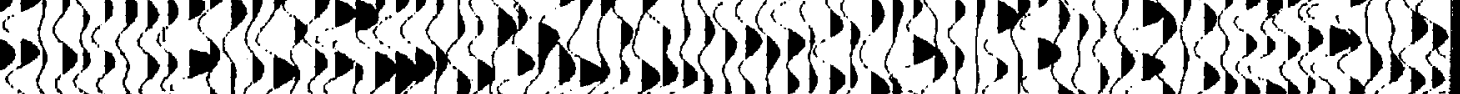

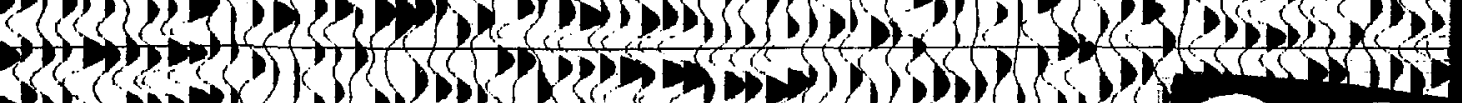

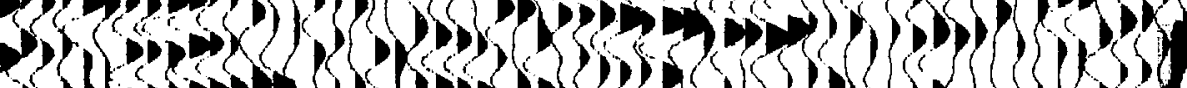

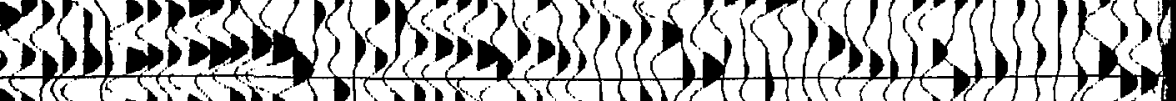
( 


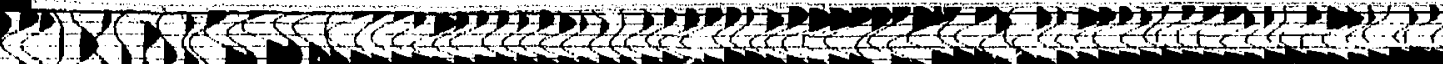

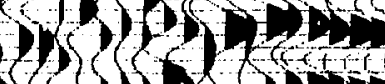

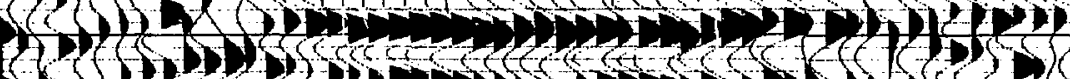

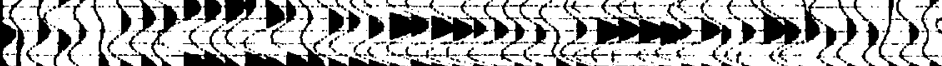

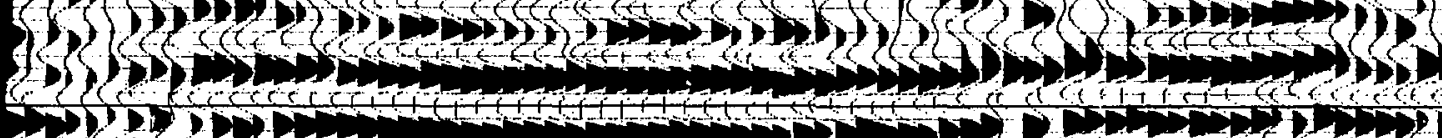

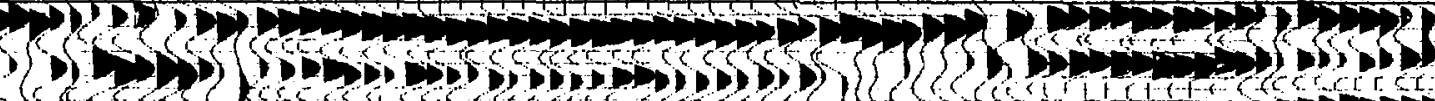

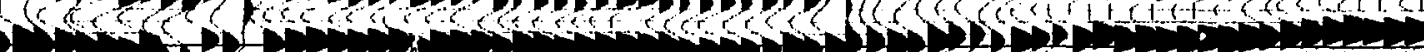

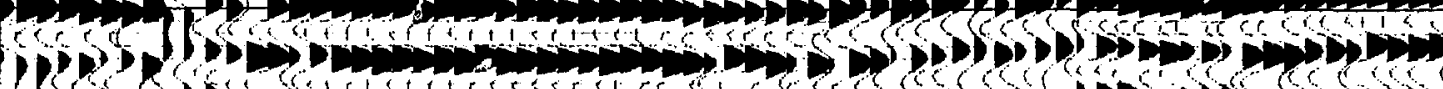

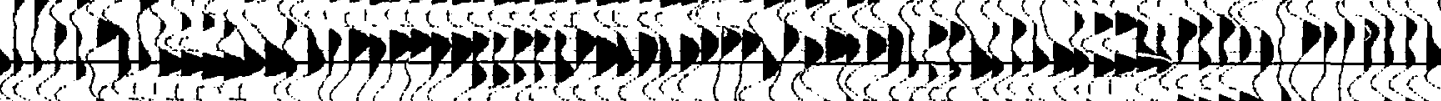

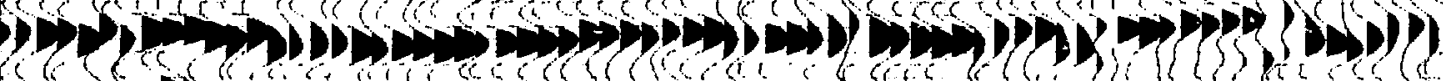

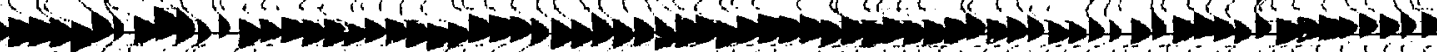
$\lim _{\infty} \rightarrow$

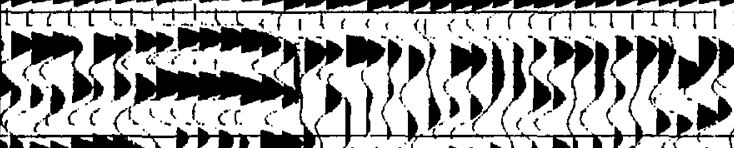
$x_{0}$

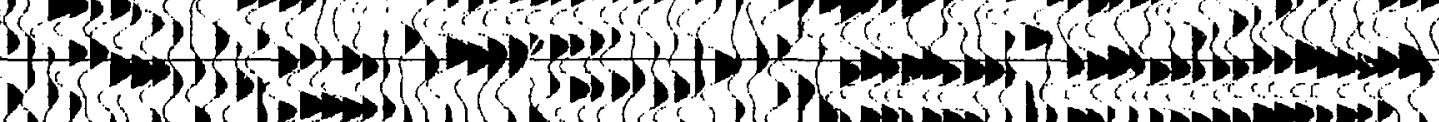
3013 1. P. Pl

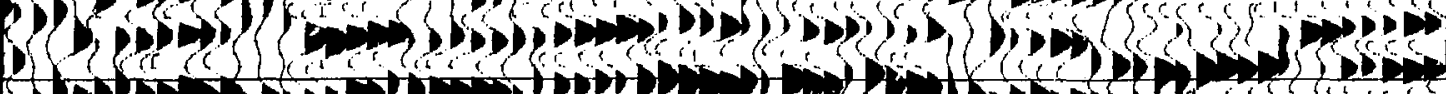

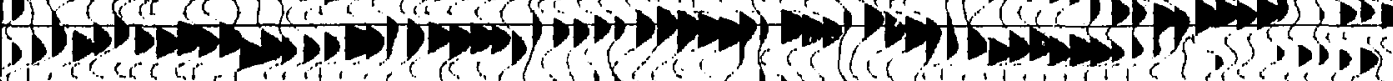

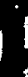

\section{(1)}

(1)

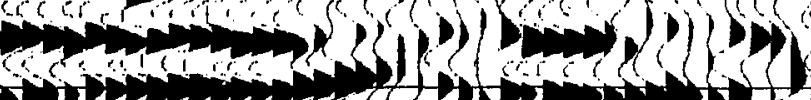

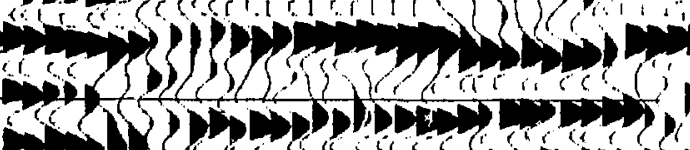

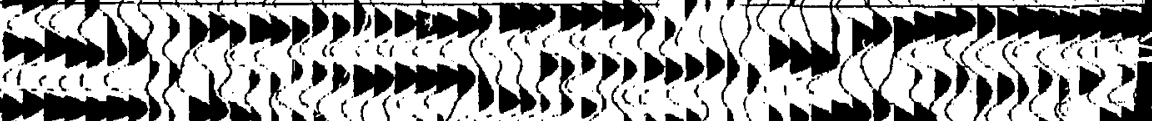

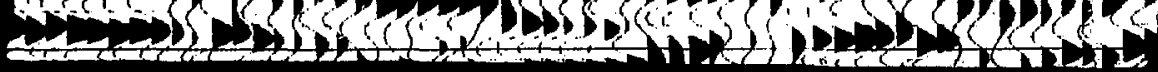




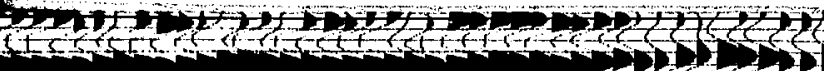

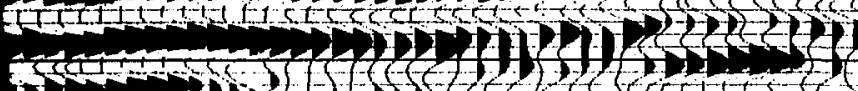

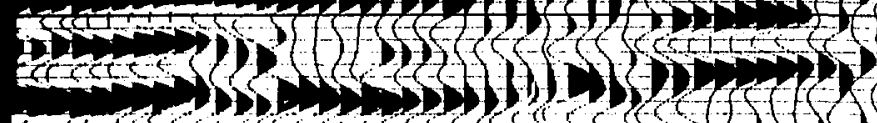

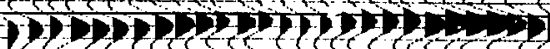

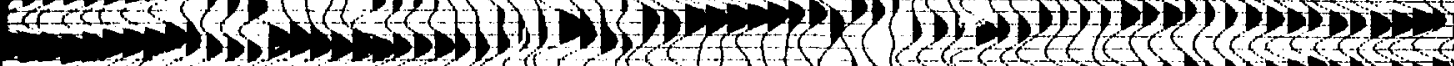

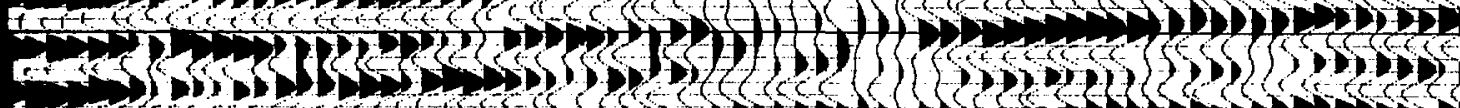

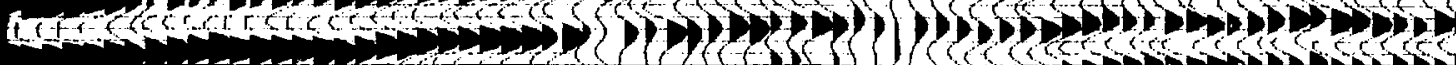
5 s

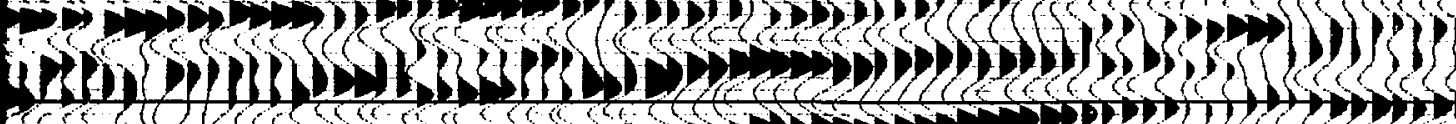

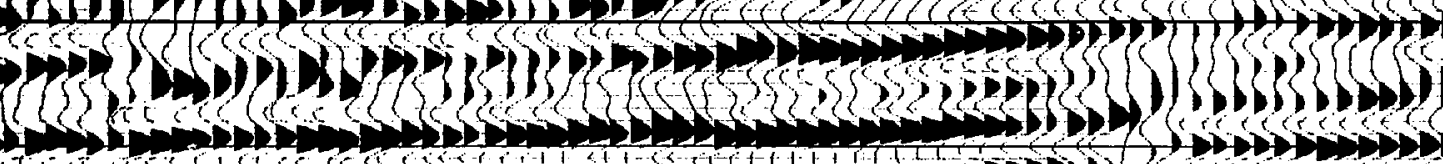

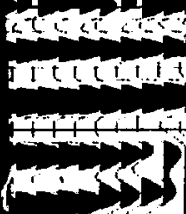

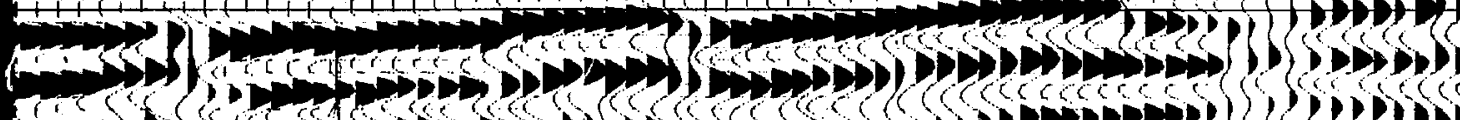

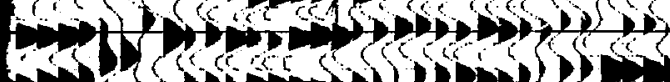

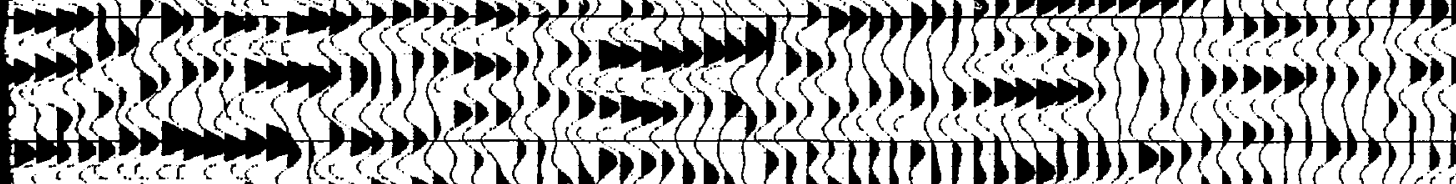

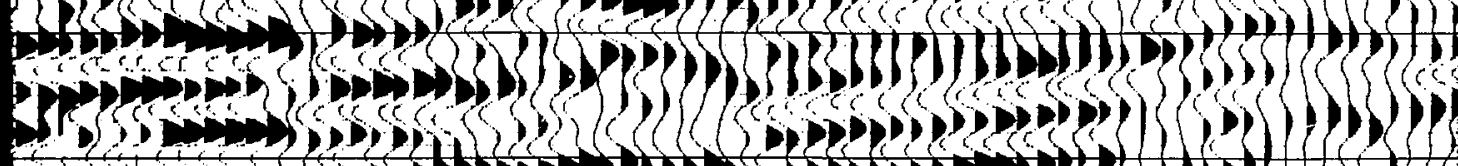
250 s.

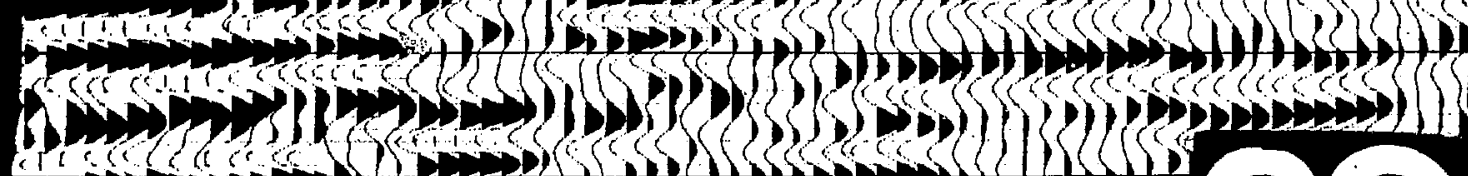

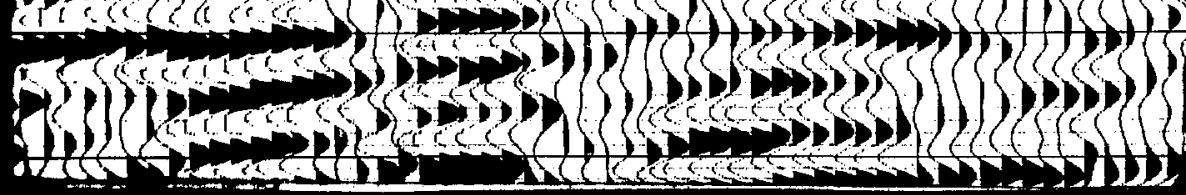


2.8

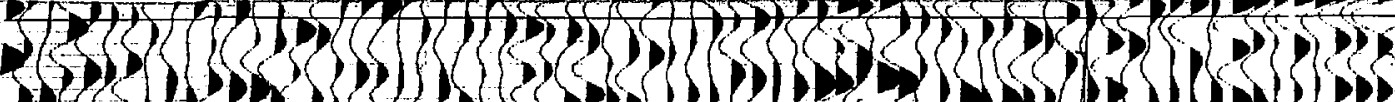

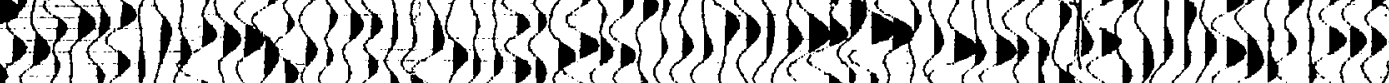
.9.

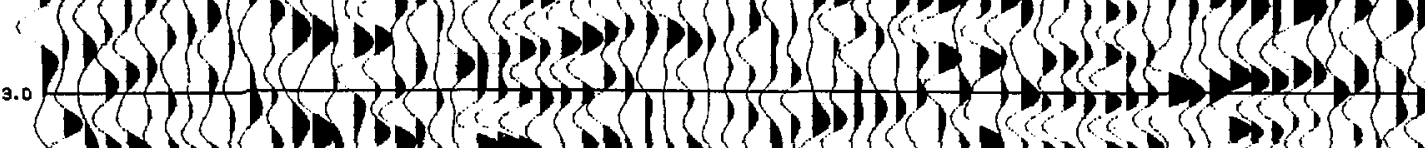
B.

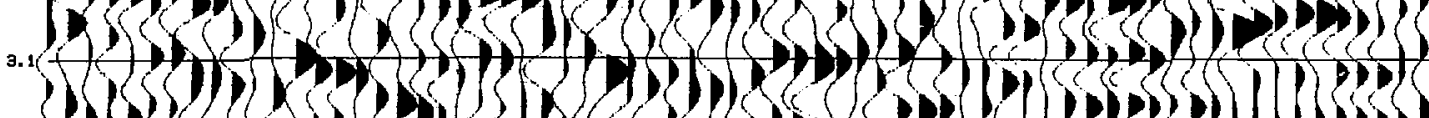

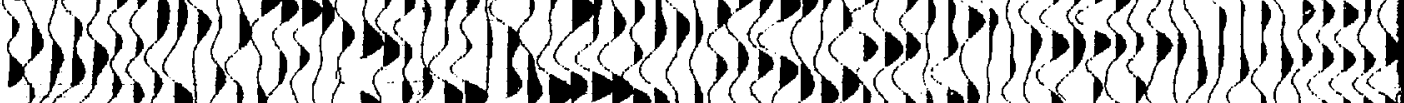

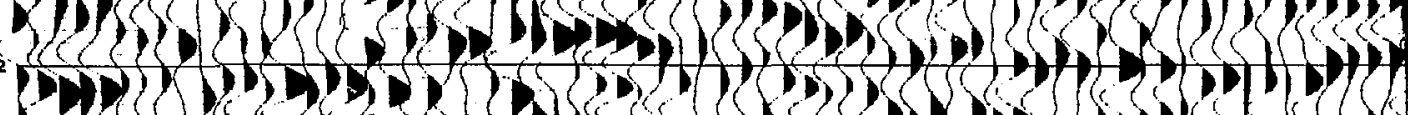

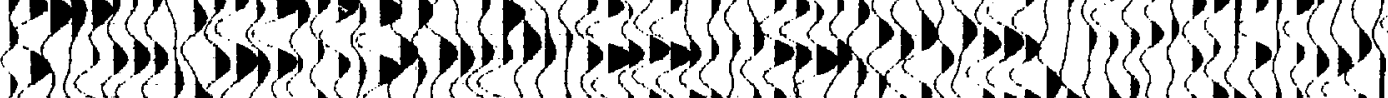

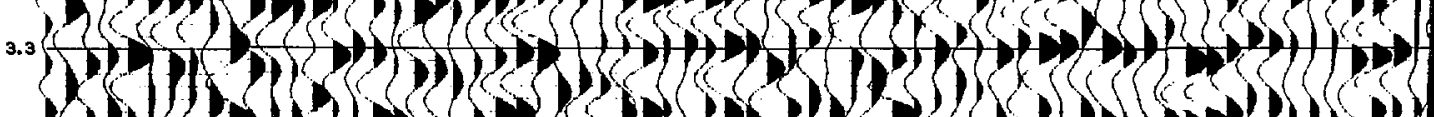

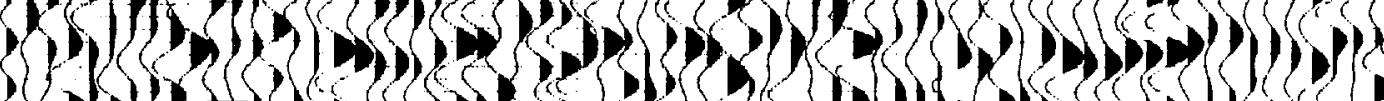
P.

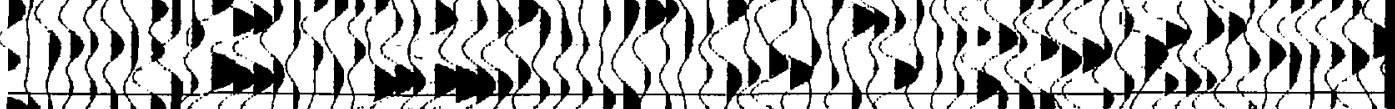
-

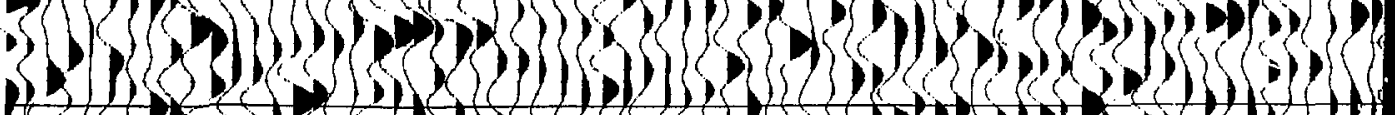

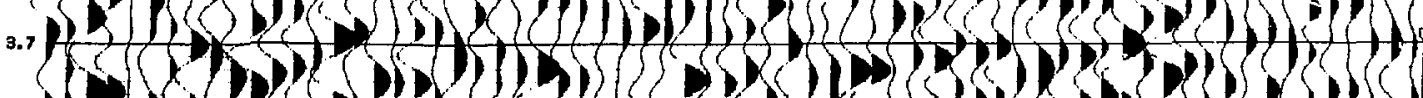

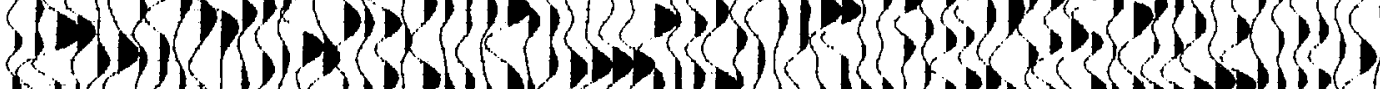
(13)

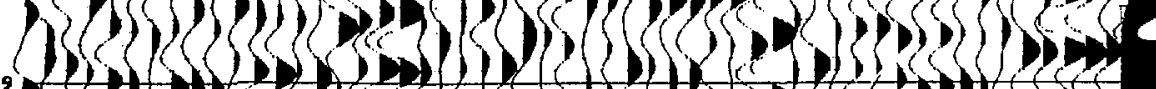
.

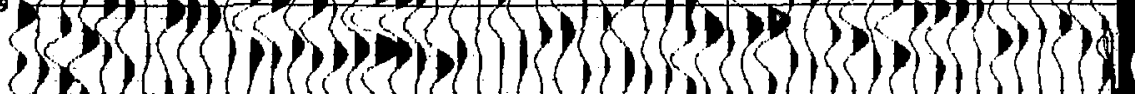




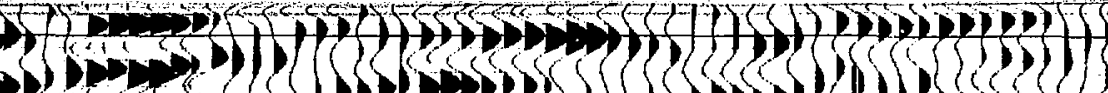

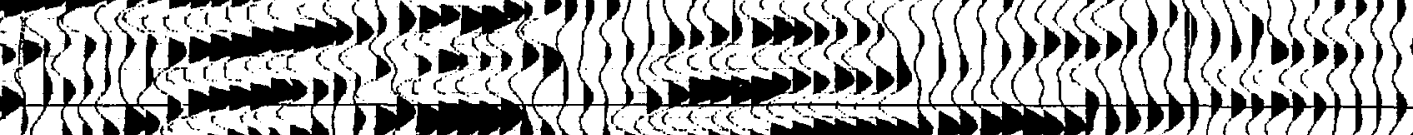

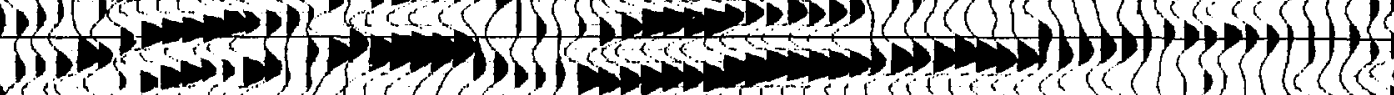

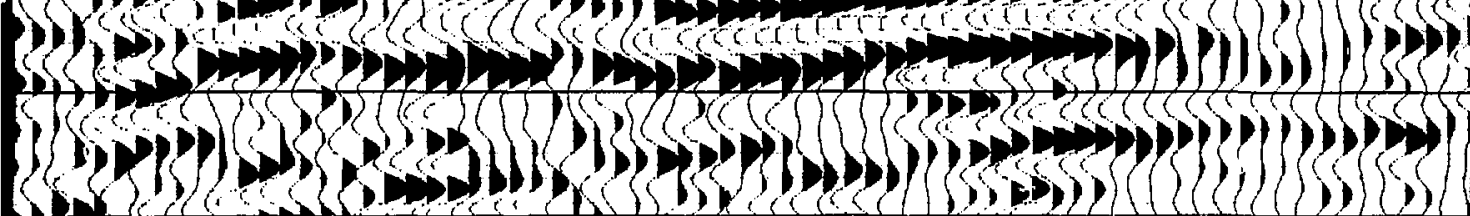

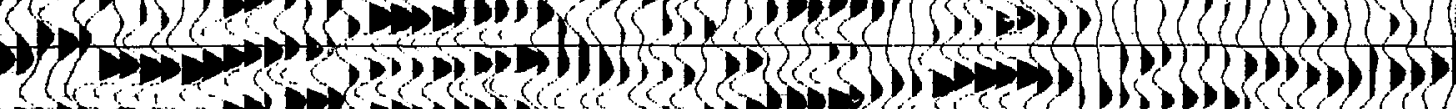
(5) \$3,

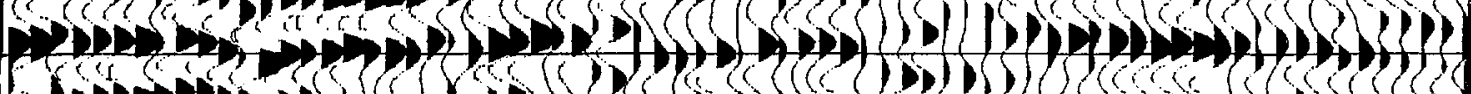

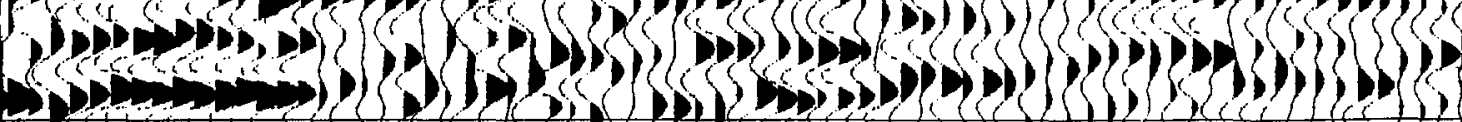

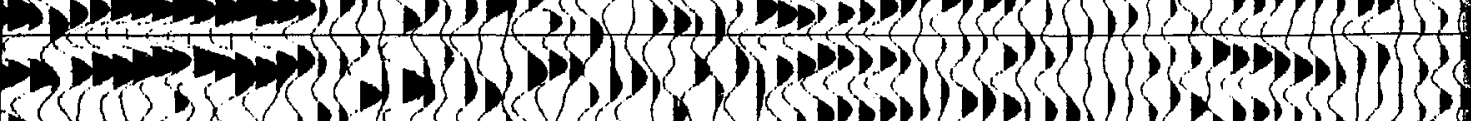

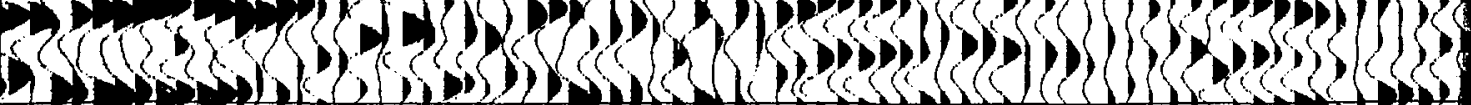
(1) 
ATepo

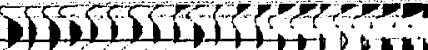

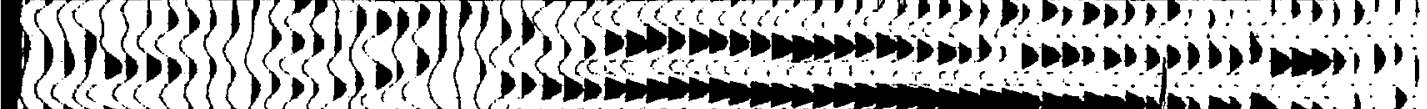

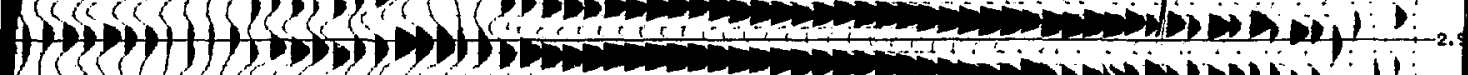
(2)

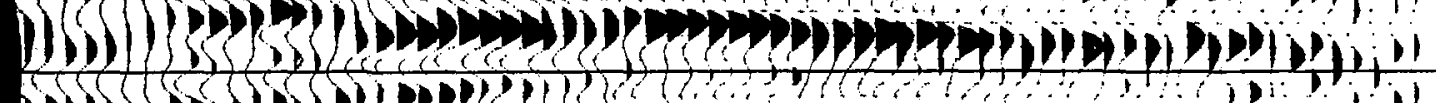

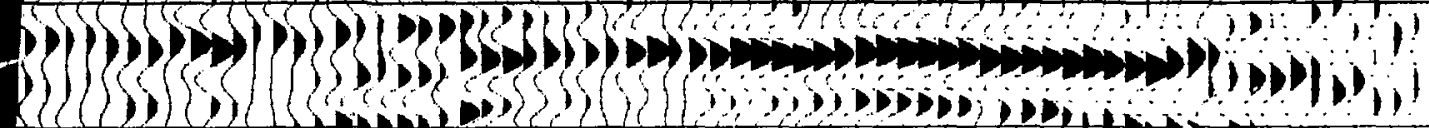

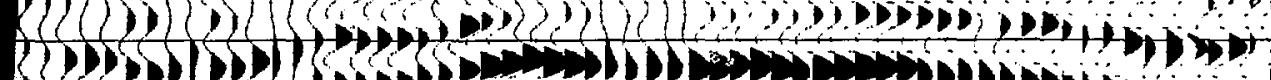

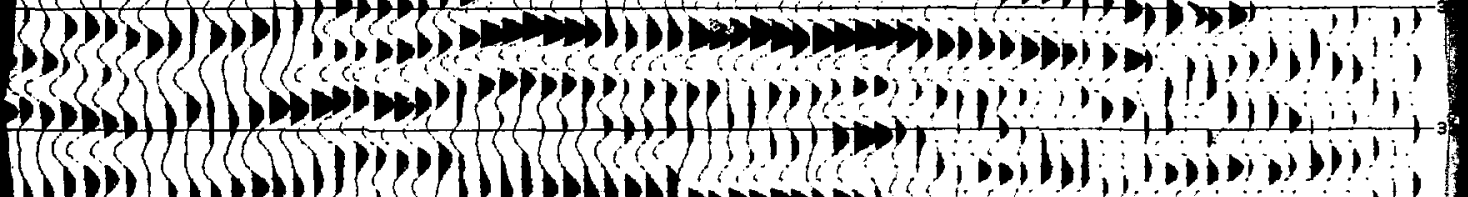

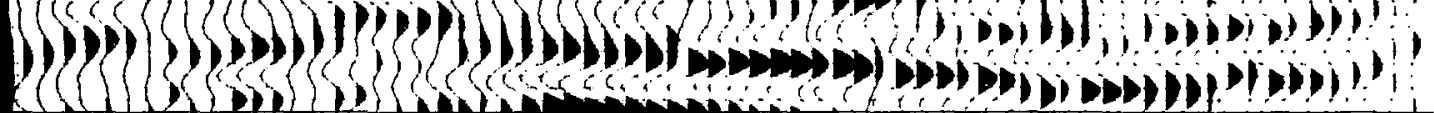

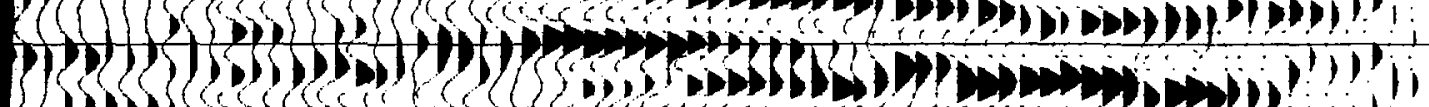

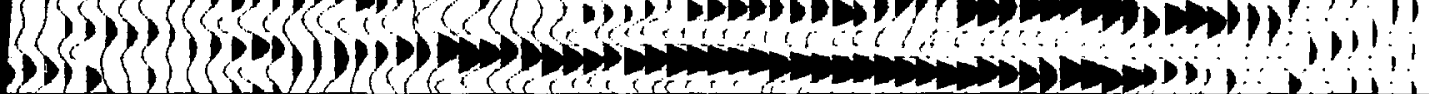

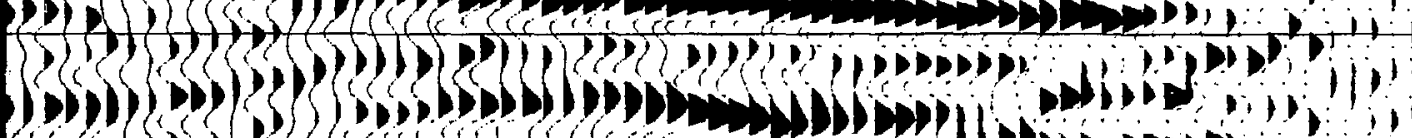
(D) by

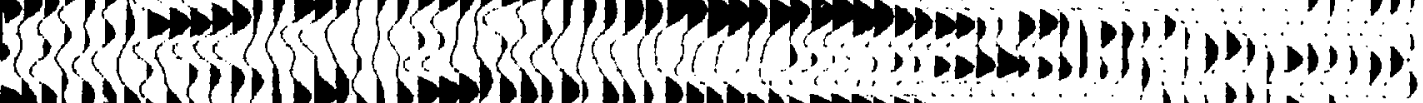

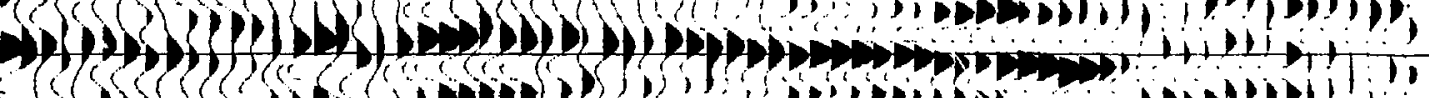

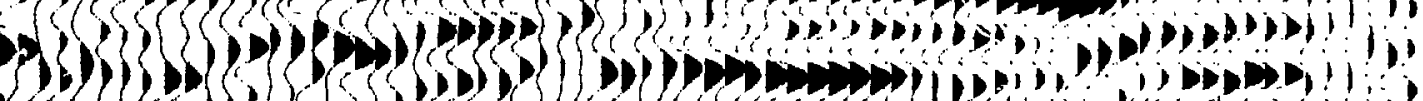

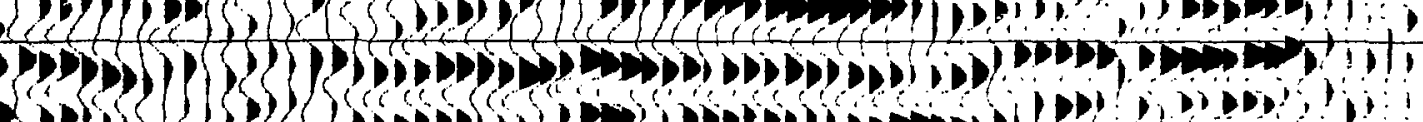

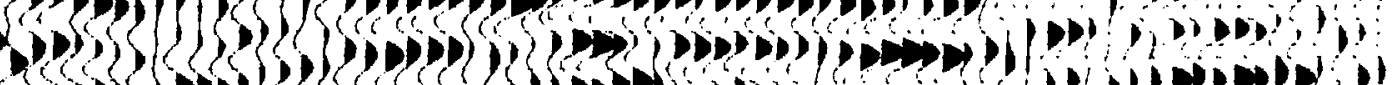

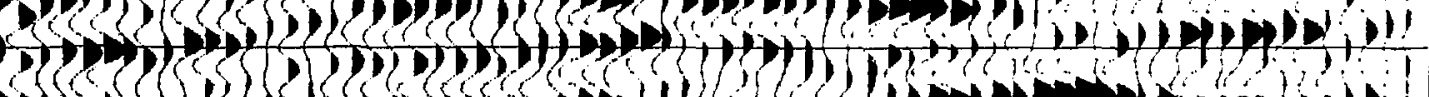

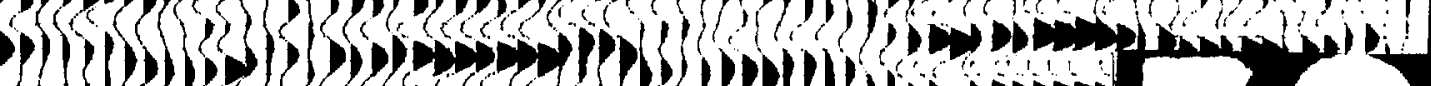
(1) CO 


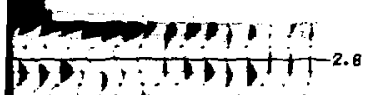

bolidili

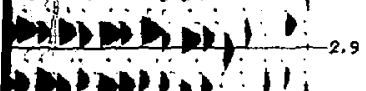

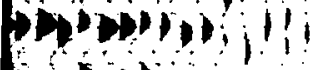

(f)

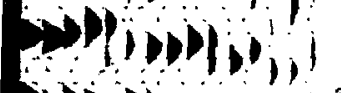

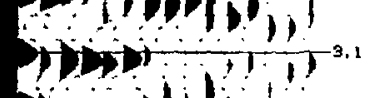

$6(j) j$

$+\infty b i b 11^{+3.2}$

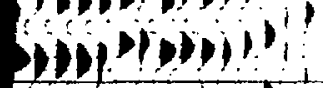

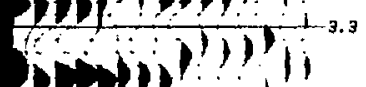

C?!?!?!?

(1) 1)

11;111!

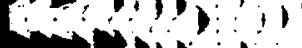

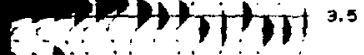

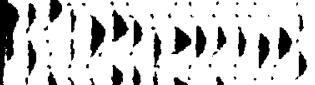
$0+1+1+1 T^{9.6}$ $4+1>1 ! 1 !$

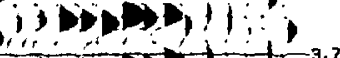
$10+191 !^{3 .}$

popy i

(1) $191,1 !$

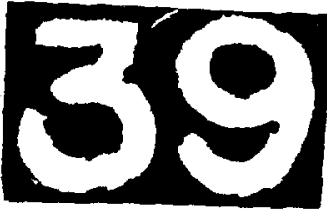

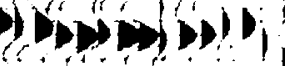

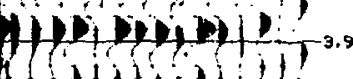




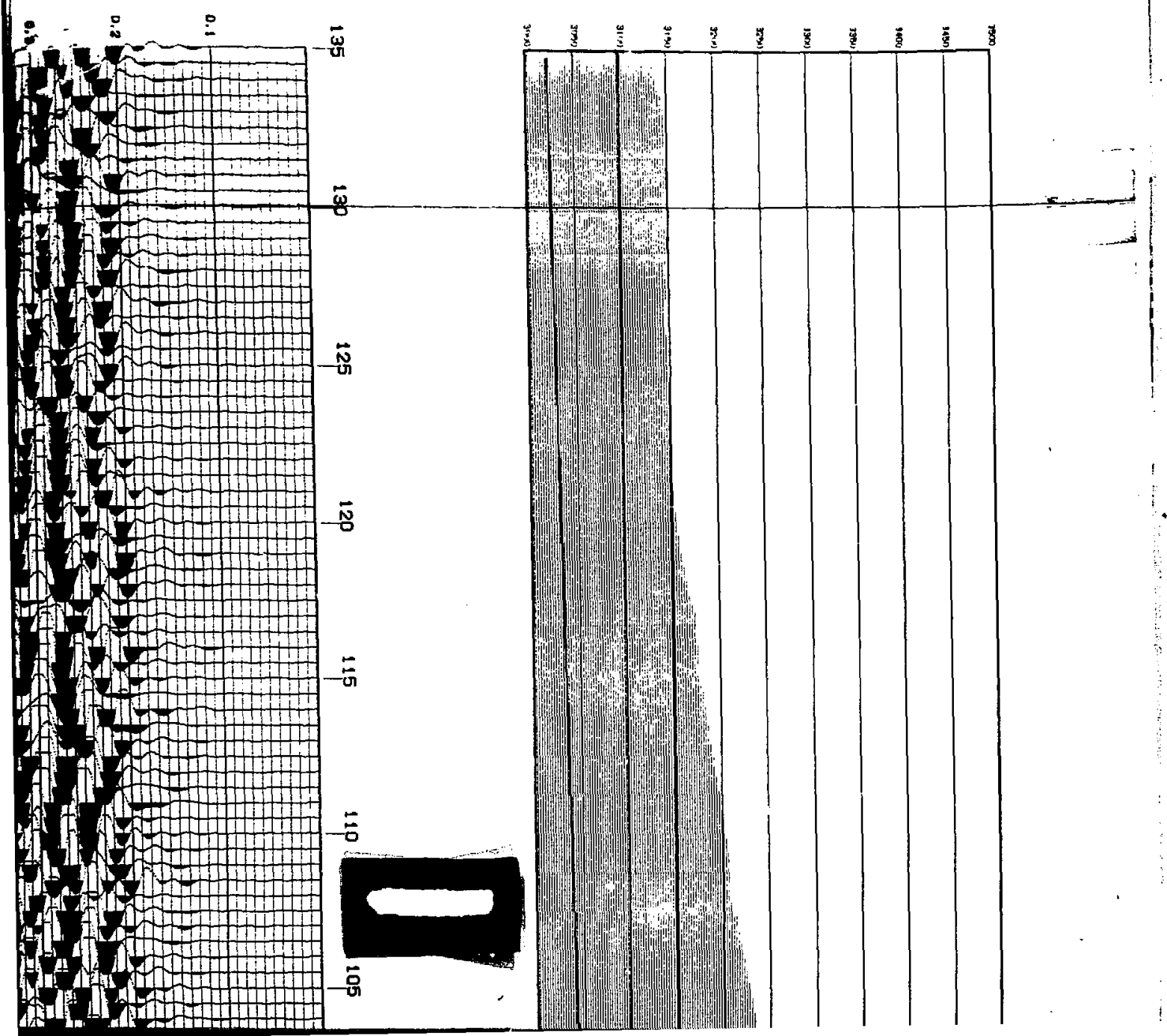




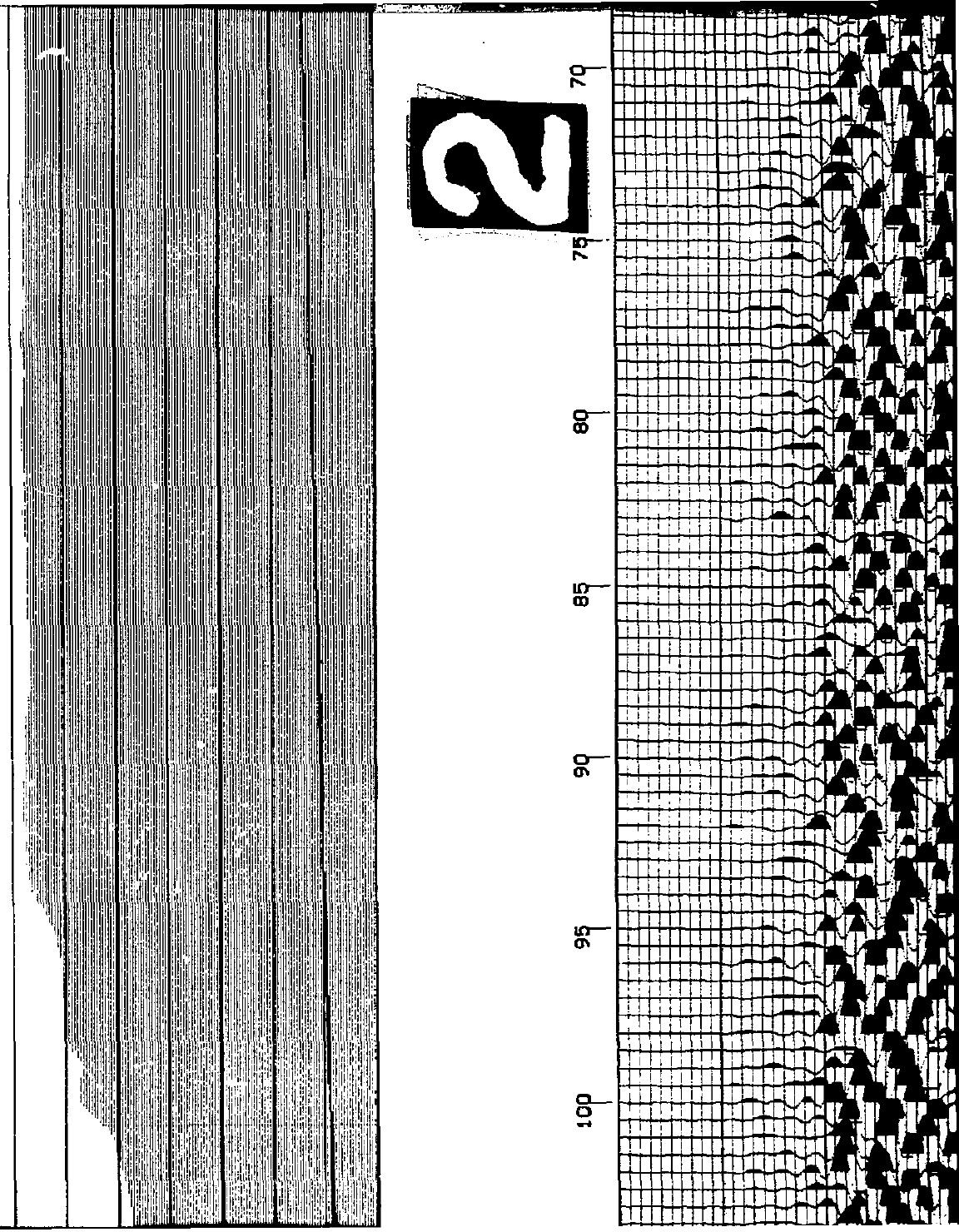





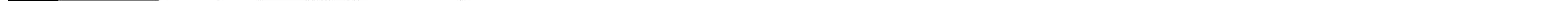



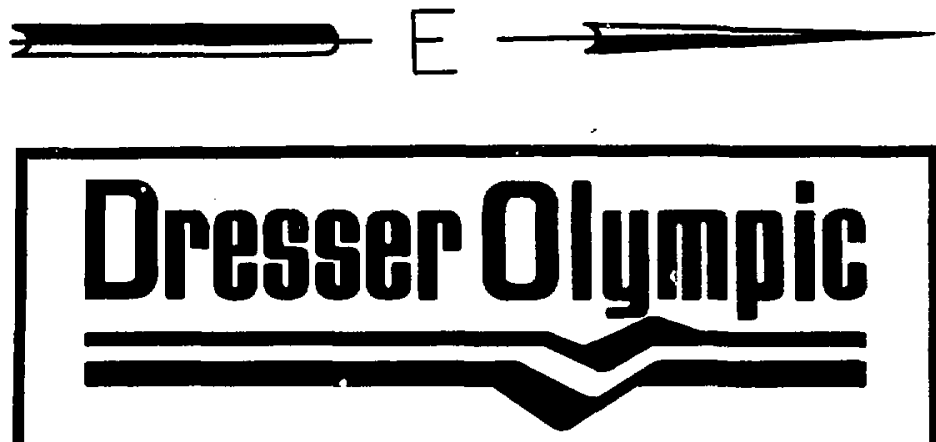

SEISMIC SURVEY

FOR

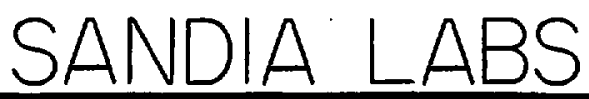

PROJECT LQS MEDANOS LINE

3

LOCATION EDDY COUNTY, TEXAS

DATE RECORDED $5-11-76$ TO $5-14-76$

DATE PROCESSED $5-17-76$

\section{DATA PROCESSING}

$\perp$ DEMULTIPLEX

2 GAIN RECOVERY

3 COMMON DEPTH POINT GATHER

4 APPLY DATUM STATICS

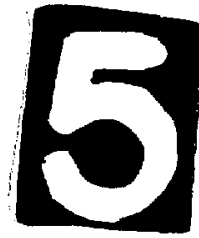

5 DECONVOLUTION

Pred. Length AUTO ms. Oper. Length 240 ms. Start cose Far Tr. 800 ma. Neor Tr. $300 \mathrm{~ms}$. 


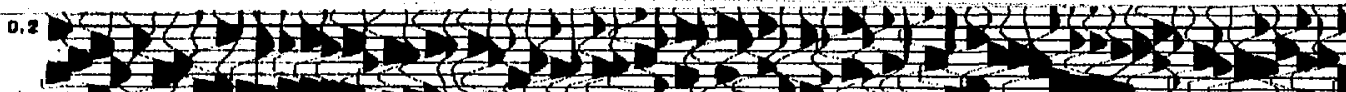

0. $\frac{1}{1}$ $\Rightarrow+1+1+2$

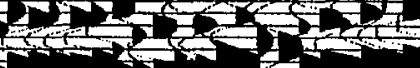

Itita $7+$ 1

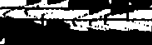

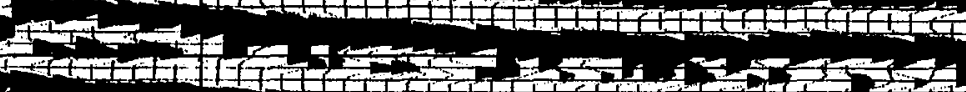

\section{4,1}

\section{tot}

$1+1+1+1-1+1+19$

0.0

$-5$

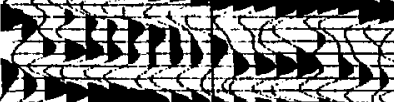

0.6

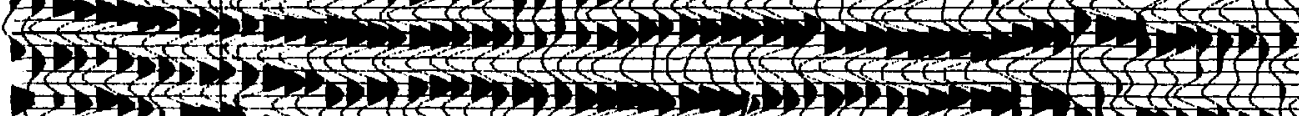

3 (x)

0.7 f(t)

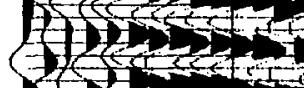

$0 . \alpha 2$

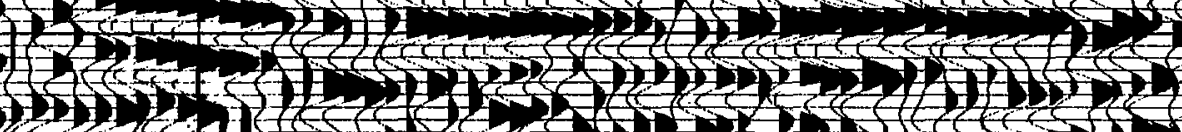

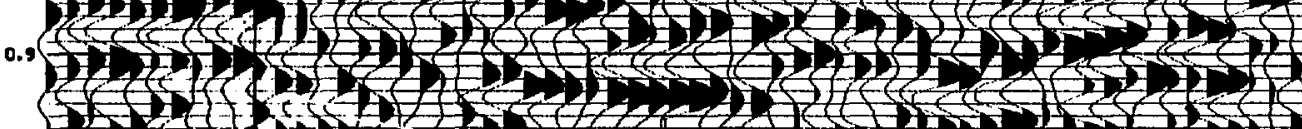

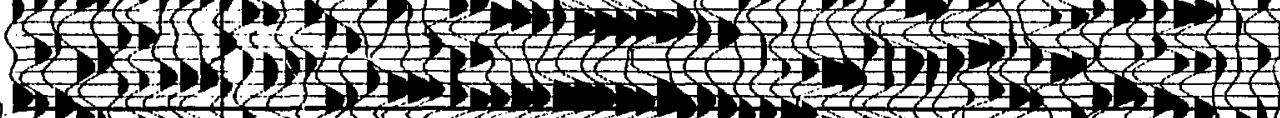

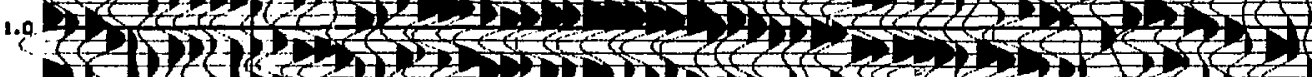

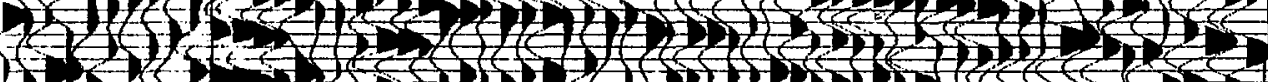

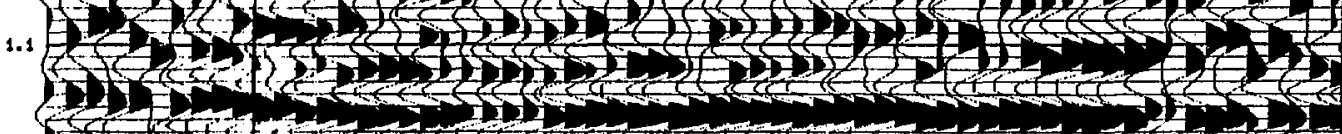
1.2.

$+x_{1}+1$

1.2.

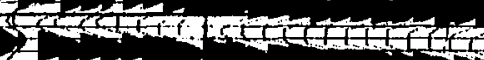

$1.6 \frac{1}{4}$

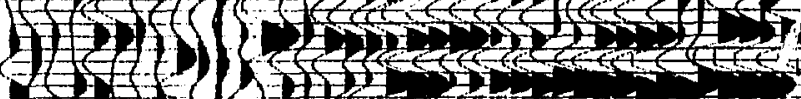

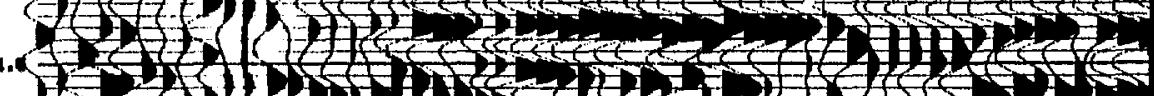

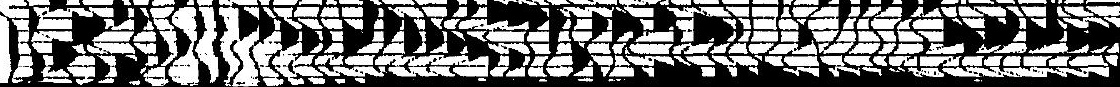



年

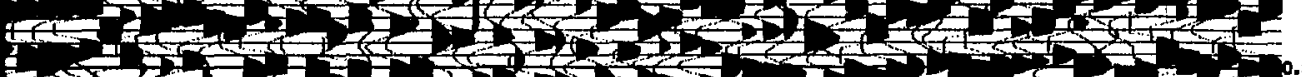
D

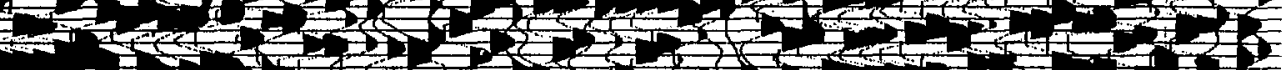

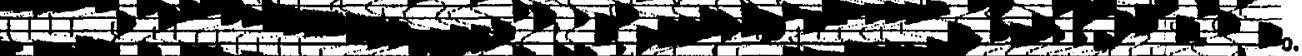

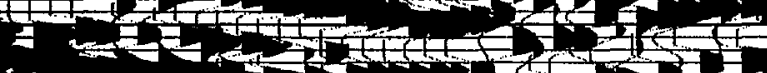

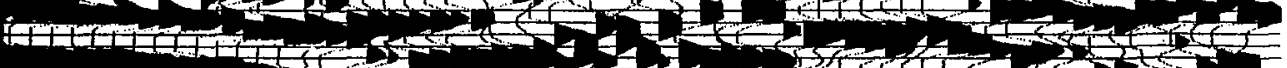
$+1+1+1+13$

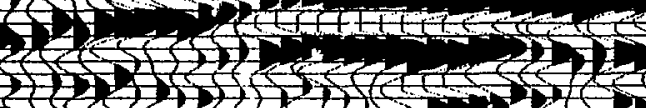

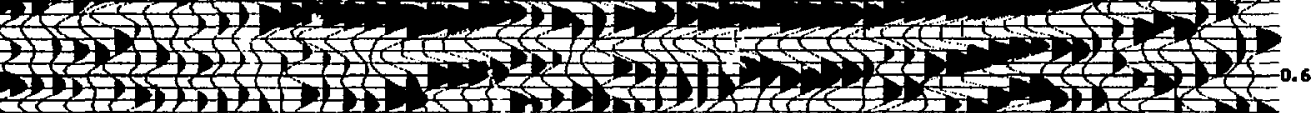

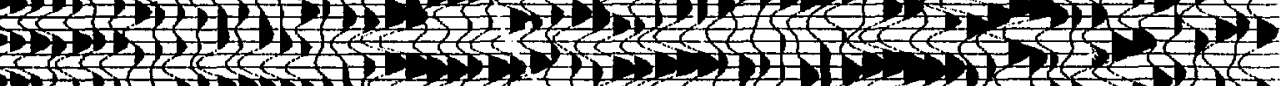

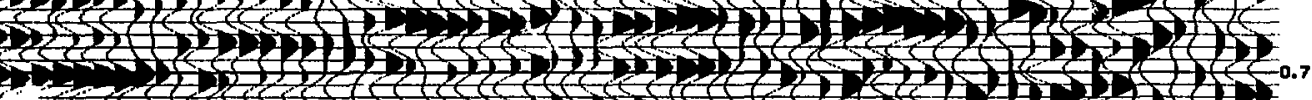
स्य 12 (स) F,

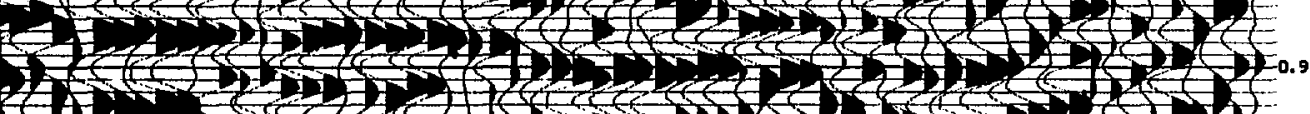

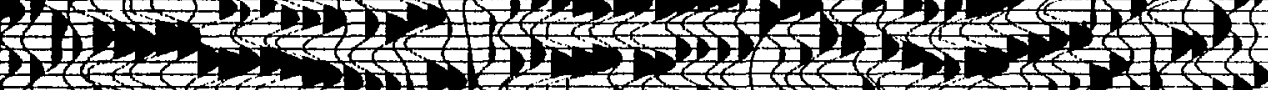

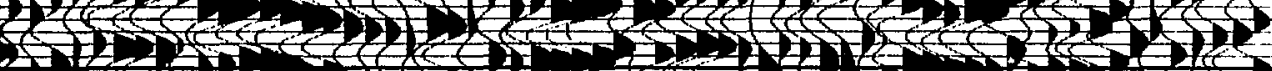

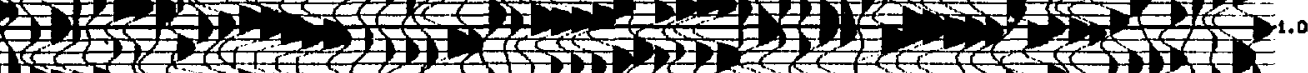

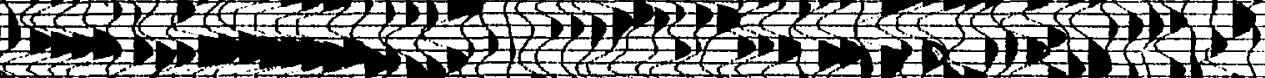

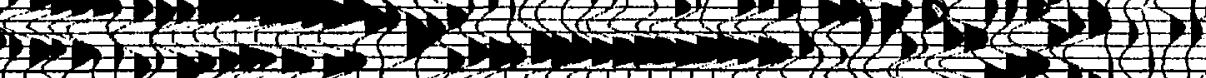

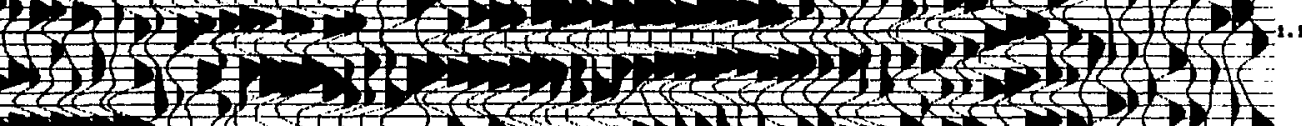
की

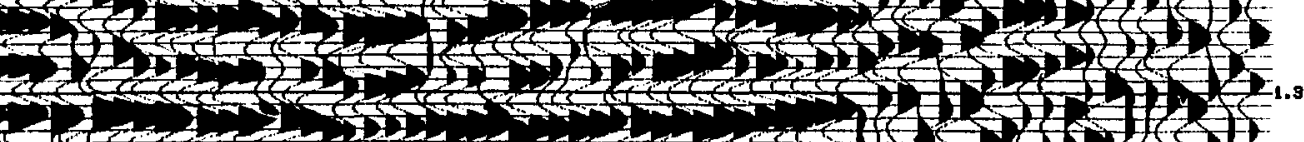

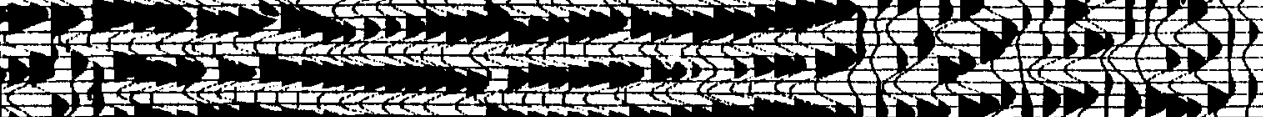

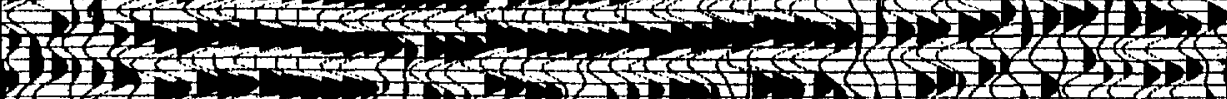

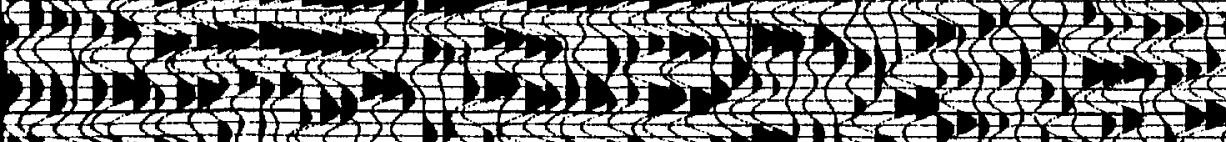

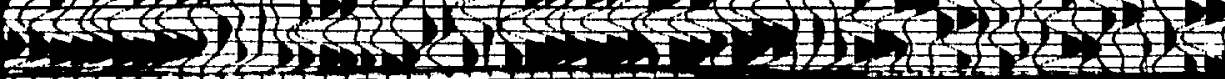




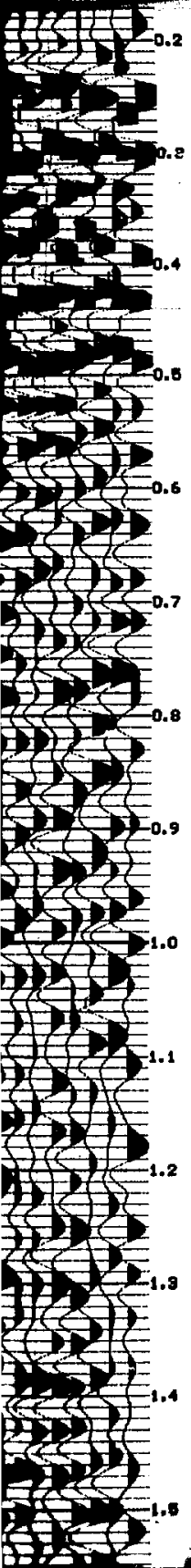

3 COMMON DEPTH POINT GATHER

4 APPLY DATUM STATICS

5 DECONVOLUTION

Pred. Length AUTO Oper. Length 240 ms.

Start Gote Far Tr. 800 me. Near Tr. 300 ms.

Gato Length 3000 ms.

6 DIGITAL FFEQUENCY FILTER $8-38 \mathrm{~Hz} O \mathrm{O}$ to $4000 \mathrm{~ms}$.

7 VELOCITY ANALYSIS

8 APPLY NORMAL MOVEOUT

9 MUTE

II STACK 24 FOLD

DECONVOLUTION

Pred.Length

Stort Gote

ms. Oper. Length ms.

Gote Length ms. ms.

12. DIGITAL FREQUENCY FILTER \begin{tabular}{ll}
$\frac{12-38}{8-30} \mathrm{~Hz} \frac{\mathrm{O}}{600}{ }^{\text {to }} \frac{600}{4000} \mathrm{~ms}$ \\
\hline $\mathrm{Hz}$
\end{tabular}

10 AUTOMATIC RESIDUAL STATICS MIGRATION DIGITAL AGC

DATE : $5-18-76$

ANALOG PLAYBACK

FILTER___ QUT - OUT

MIX NONE

HORIZONTAL SGALE_8TR/IN_VERTICAL SCALE 7.5 " $/$ SEC

COMPUTING

DATUM

VELOCITY $8000^{\prime} /$ SEC

RECORDING

RECORDED BY DRESSER OLYMPIC

INSTRUAENT TYPE_DFS ILI RECORD FILTER 8-18-62 Hz NOTCH FILTER IN SAMPLE RATE 4 ME RECORD LENGTH_ 4 WC ENERGY SOURCE : VIBROSEIS SWEEP FAEQUENGY $8-38 \mathbf{H z}$ 
(3) 101

(1)

(1)

(1)

P3in 13

(

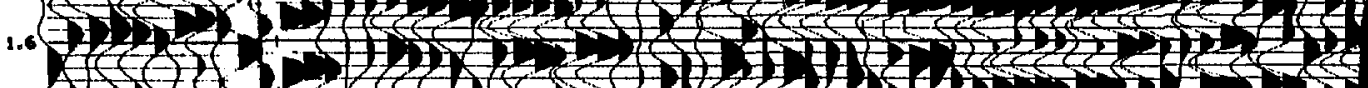

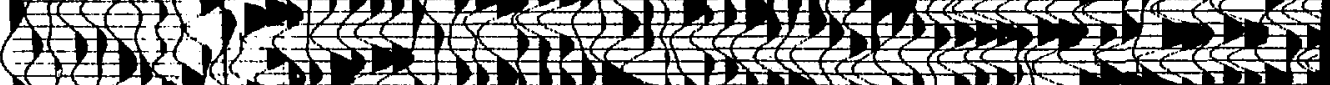
3010

"1)

DII 3 (2)

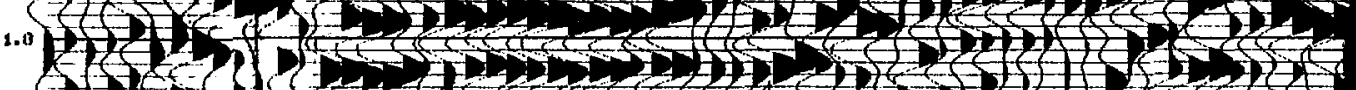

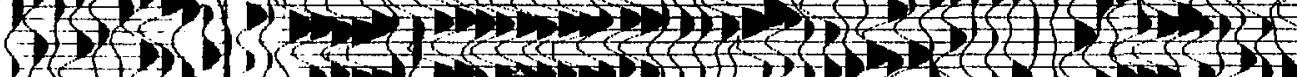
bin.

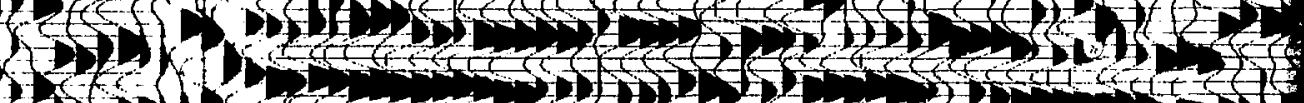
2003

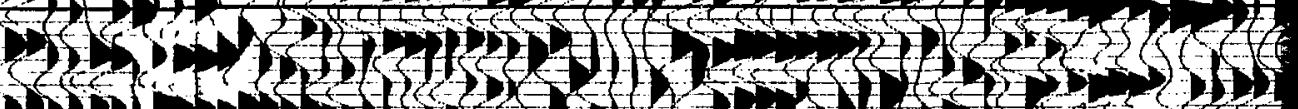

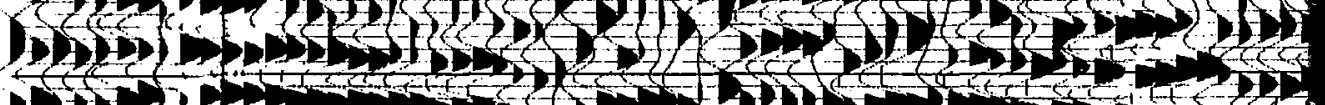
-

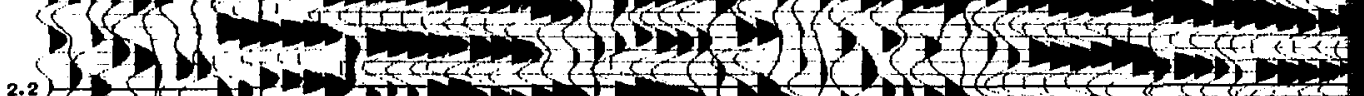

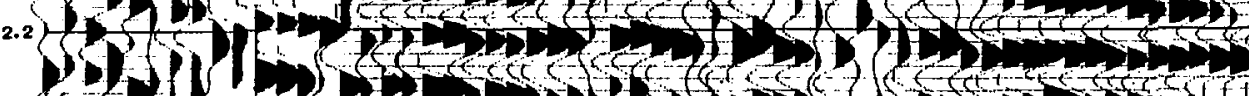

QDil $20-5,5$

M

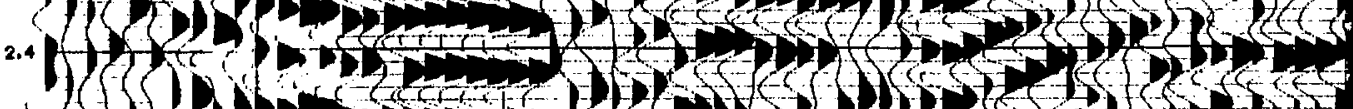

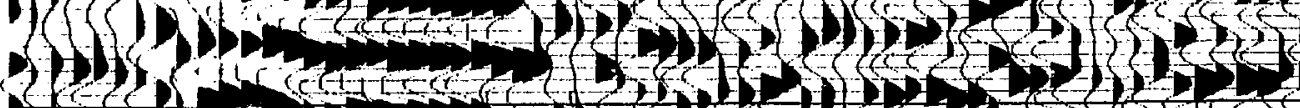
. 17. - 


\section{RECORDING}

RECORDED BY DRESSER OLYMPIC

INSTRUMENT TIPE DFS III RECORD FILTER 8-18-62 $\mathrm{Hz}$ NOTCH FILTER IN SAMPLE RATE 4 ms RECORD LENGTH_ 4 MC ENERGY SOURCE : VIBROSEIS SWEEP FREQUENCY_8-38_ $\mathrm{Hz}$ NO./SWEEPS PER LOCATION_16 LOCATION INTERVAL_220' MODEL \& TYPE GEOPHONES WHS FREQ. \& HZ NO/GROUP 48 TYPE COVERAGE $2400 \%$ SPREAD LENGTH $11880^{\circ}$ NO. TRACES 48 OFFSET_LL80

\section{SPREAD DIAGRAM}

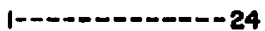

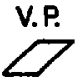

$25---0-----48$

$\left|\longrightarrow 5060^{\circ} \longrightarrow\right|-880^{\circ} \longrightarrow\left|-880^{\prime} \longrightarrow\right|-5060^{\circ} \longrightarrow \mid$

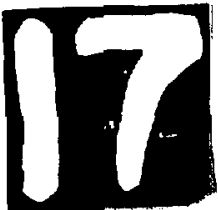


7mom

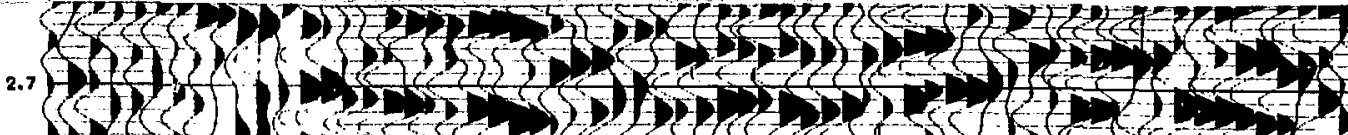

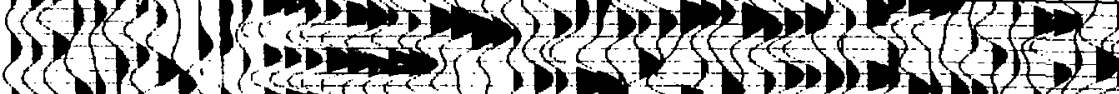

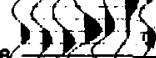

13 (s)

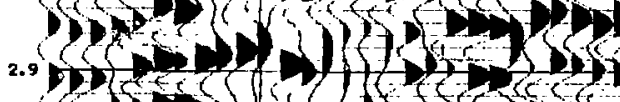

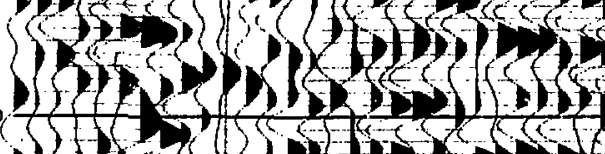

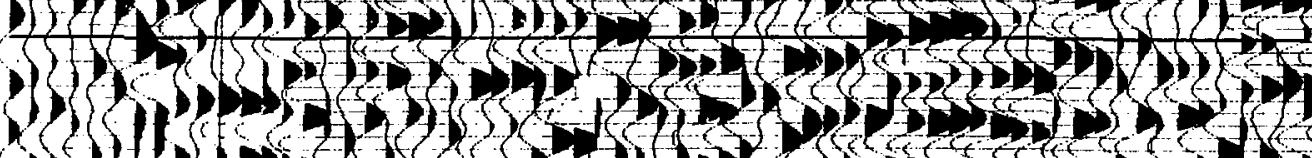

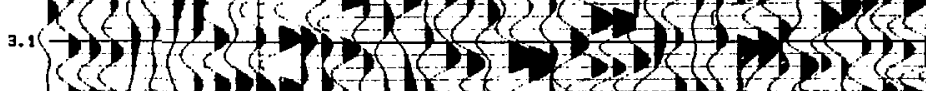

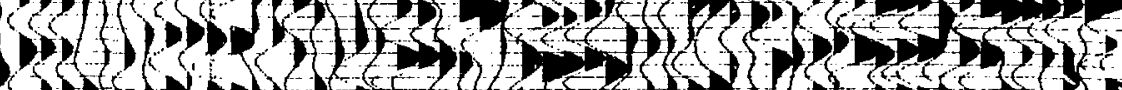

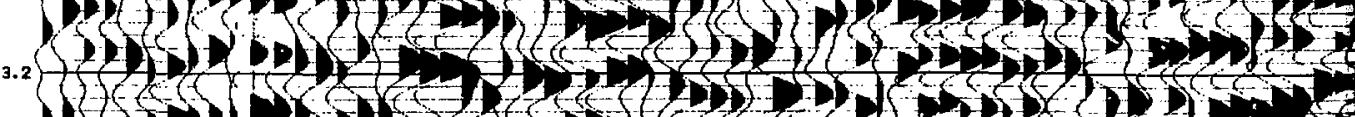

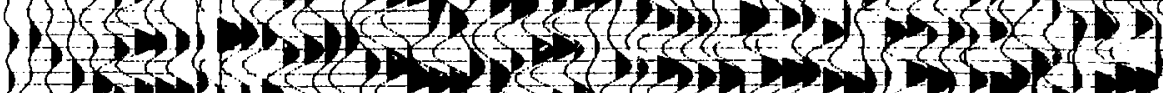

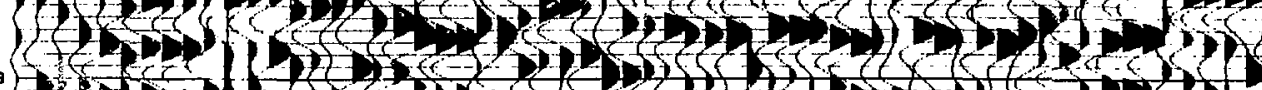

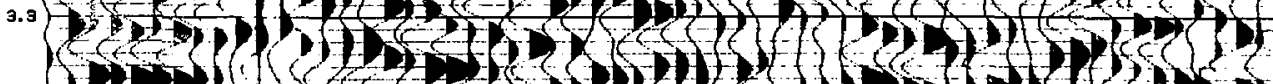

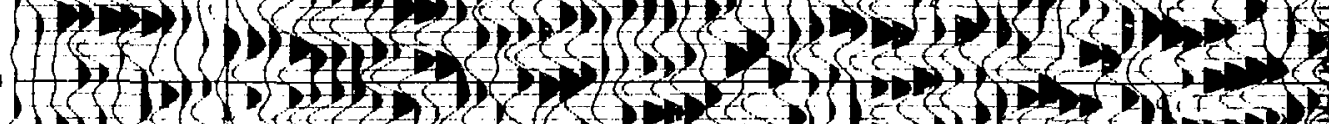

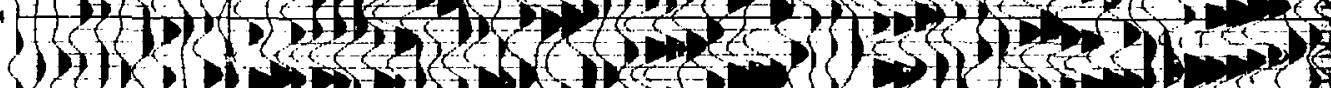

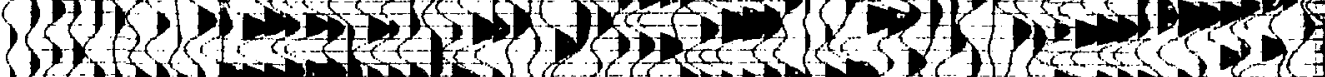

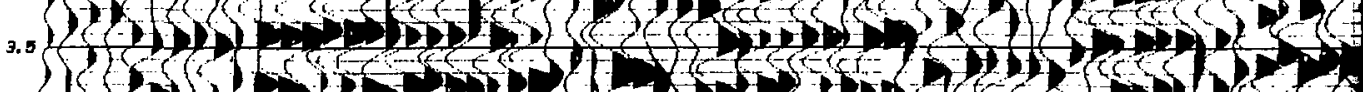

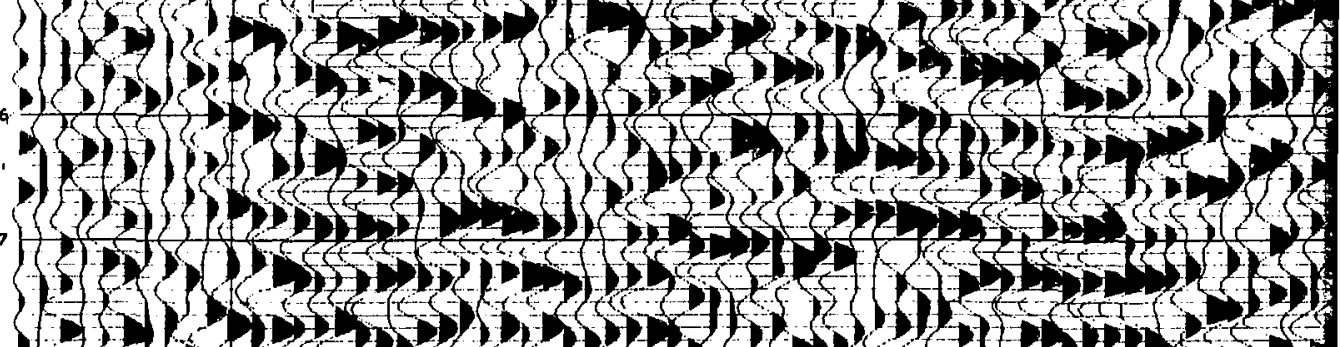

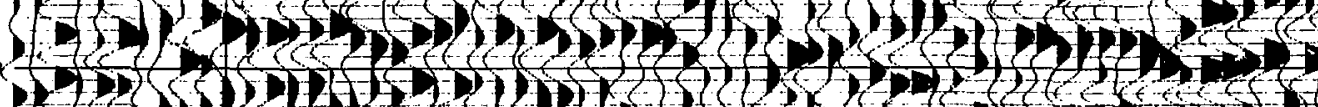

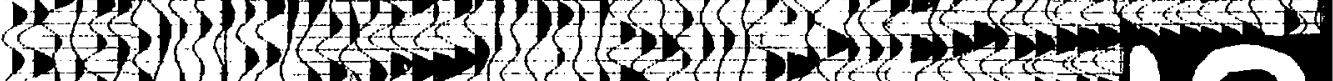

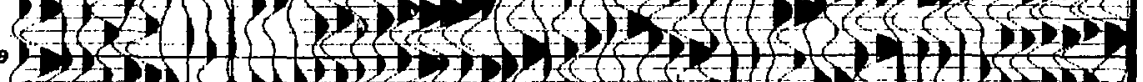

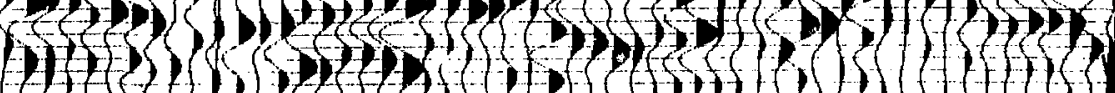

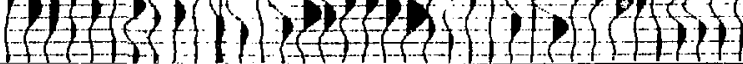


4707 .

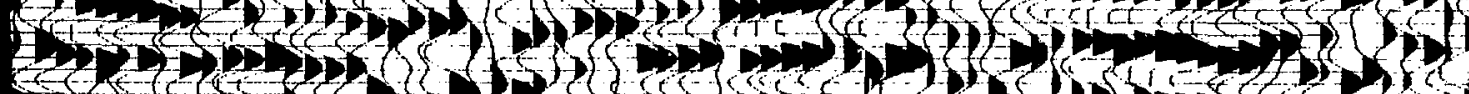
201733. al

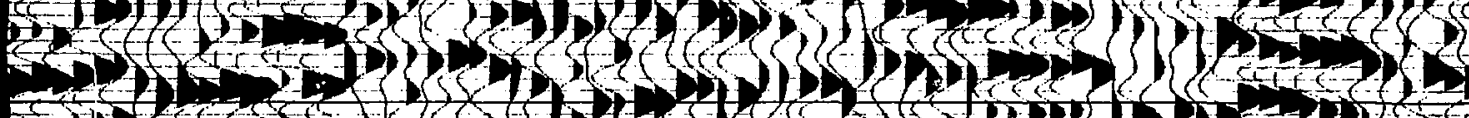

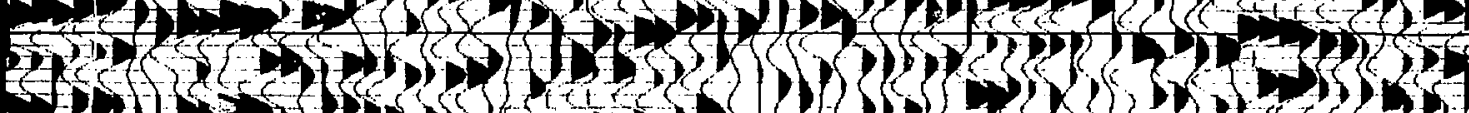

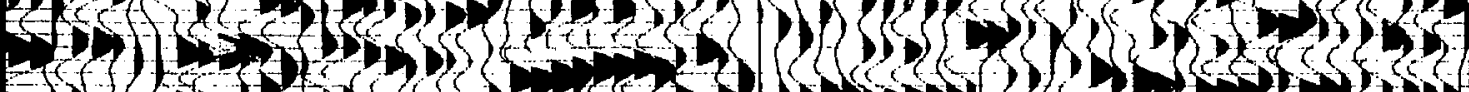
D)

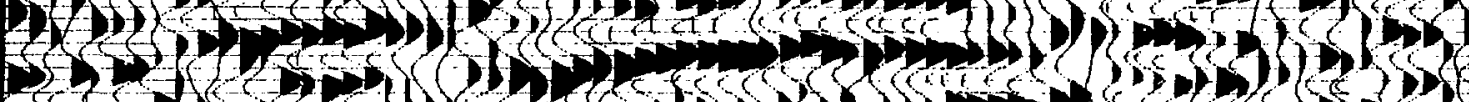
Th

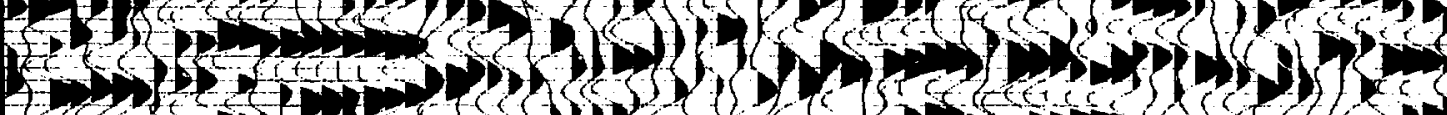

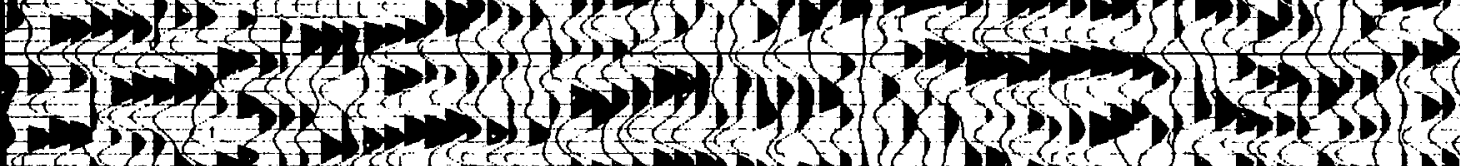

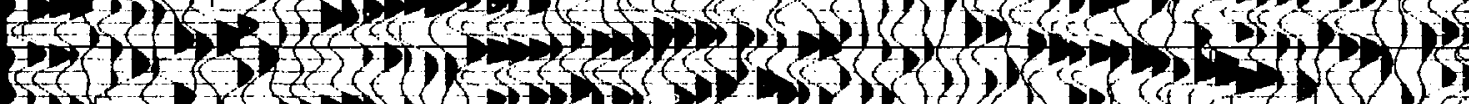

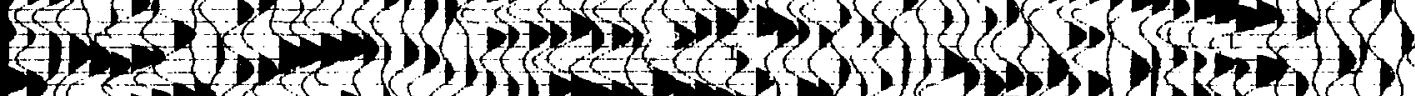

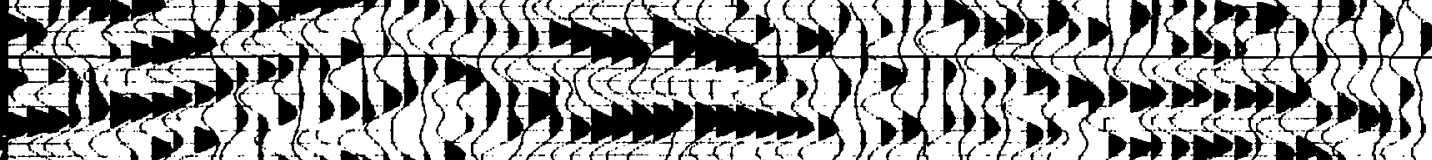
7 in ins 2.1 In

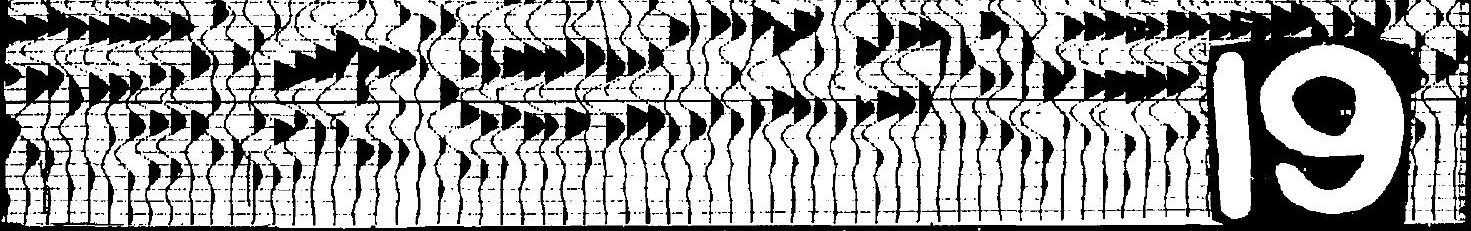


III)

Thon

in 3 in

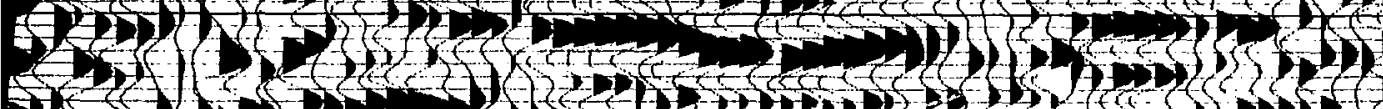

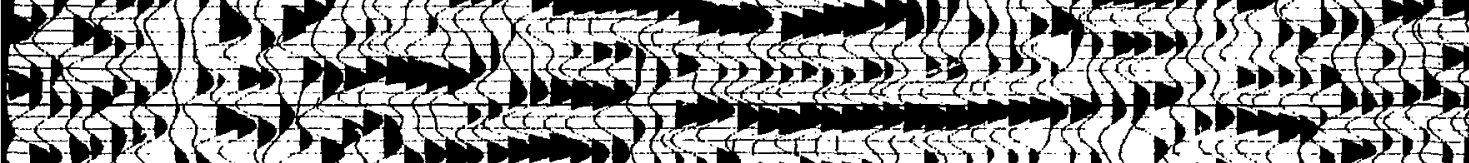

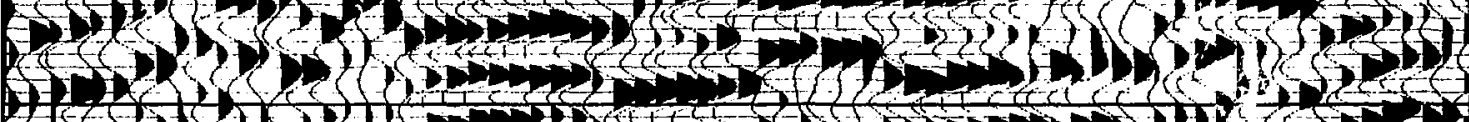
III,

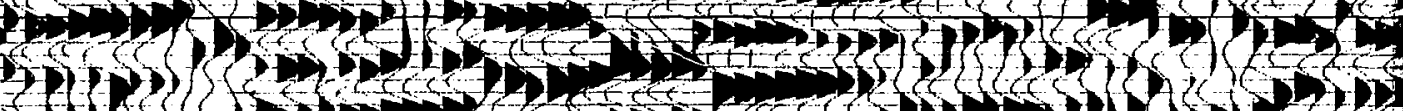

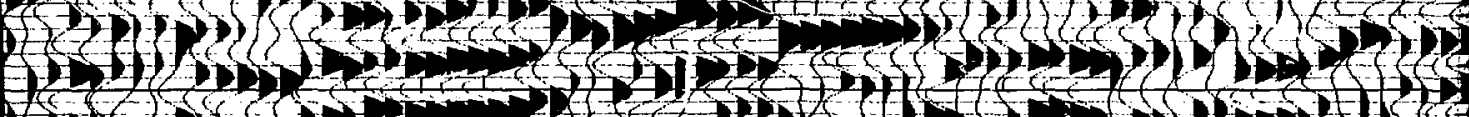

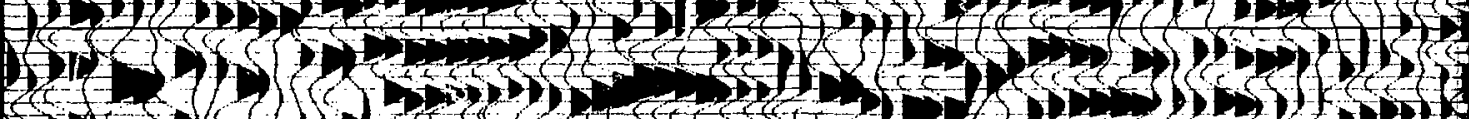
An 7 . 2 .

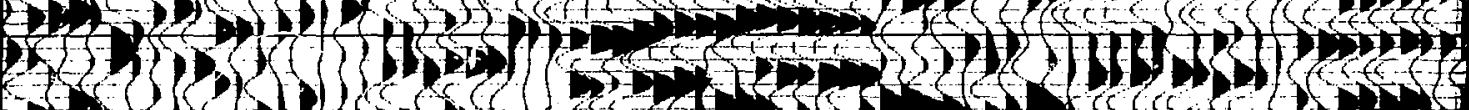

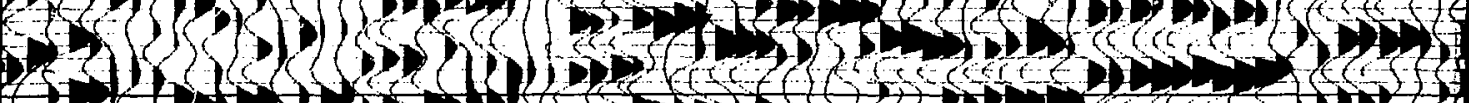
2.1.

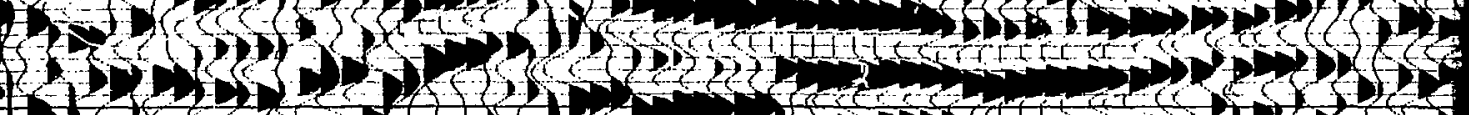

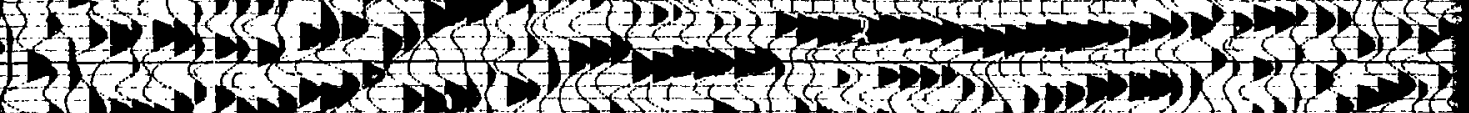

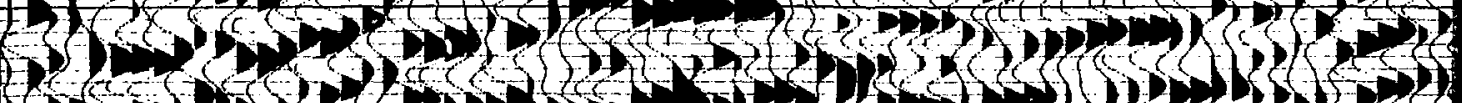

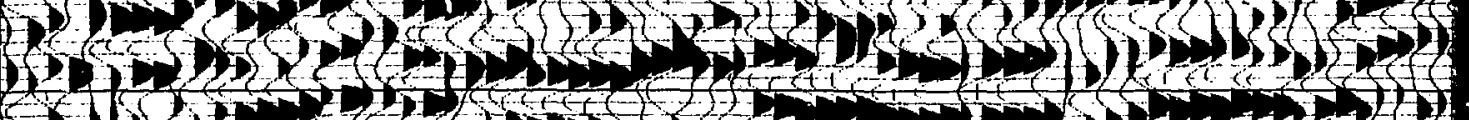
D)

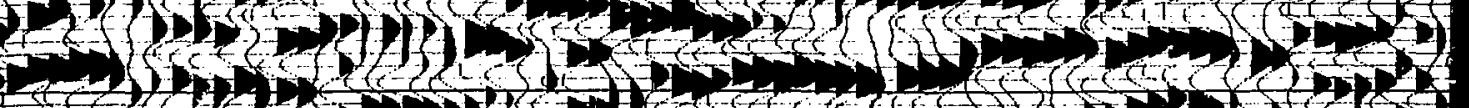

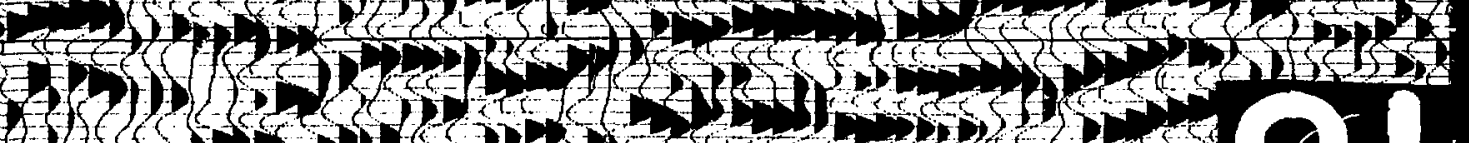

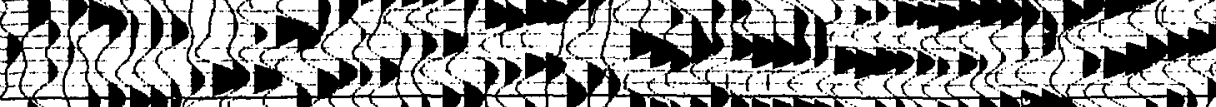
ThM $\rightarrow$. 


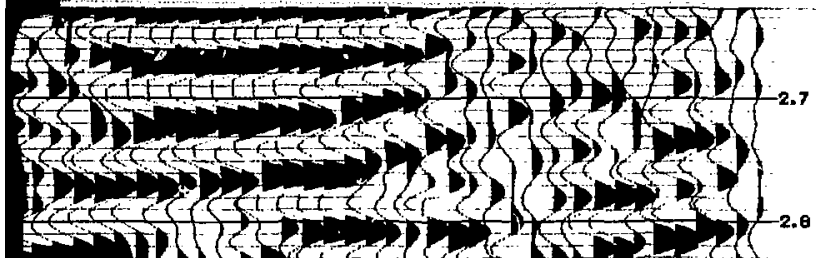

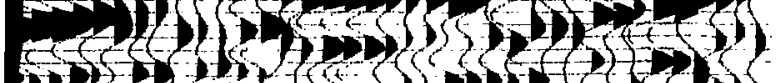

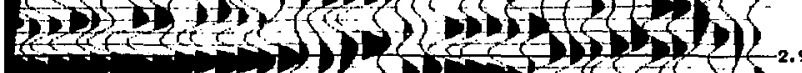

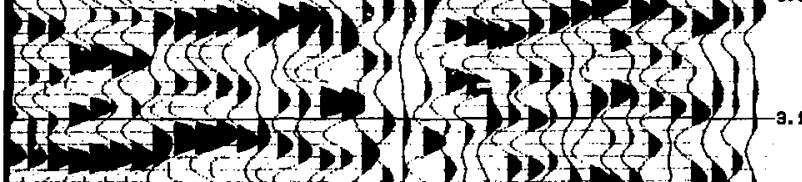

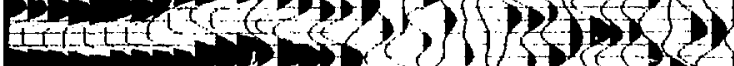

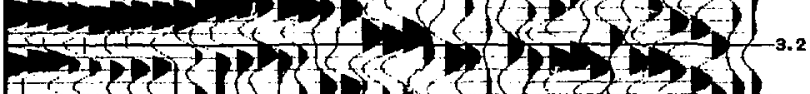

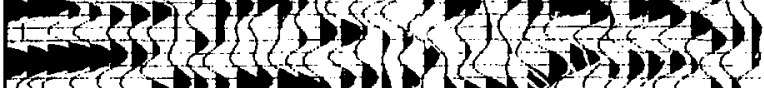

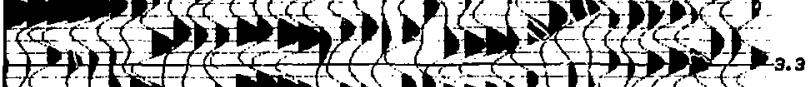

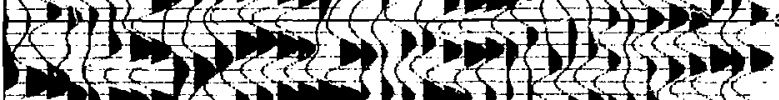

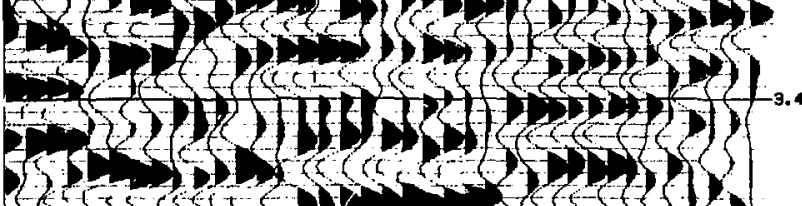

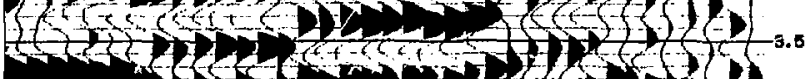

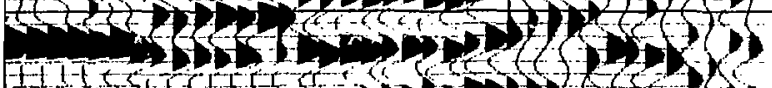

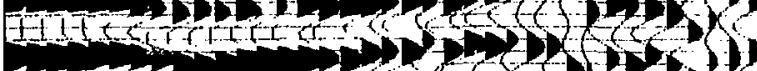

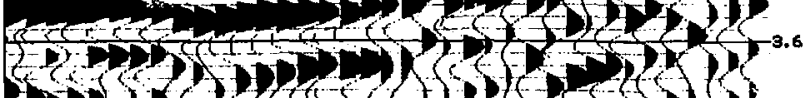

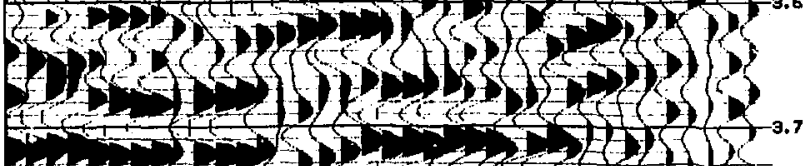

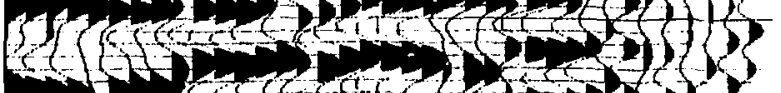
- 1 ere

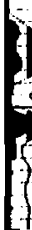

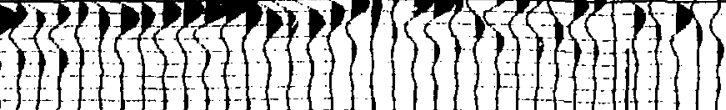
(1)

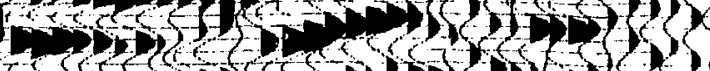

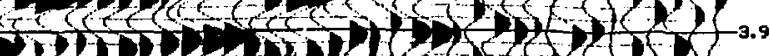

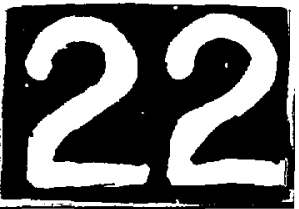




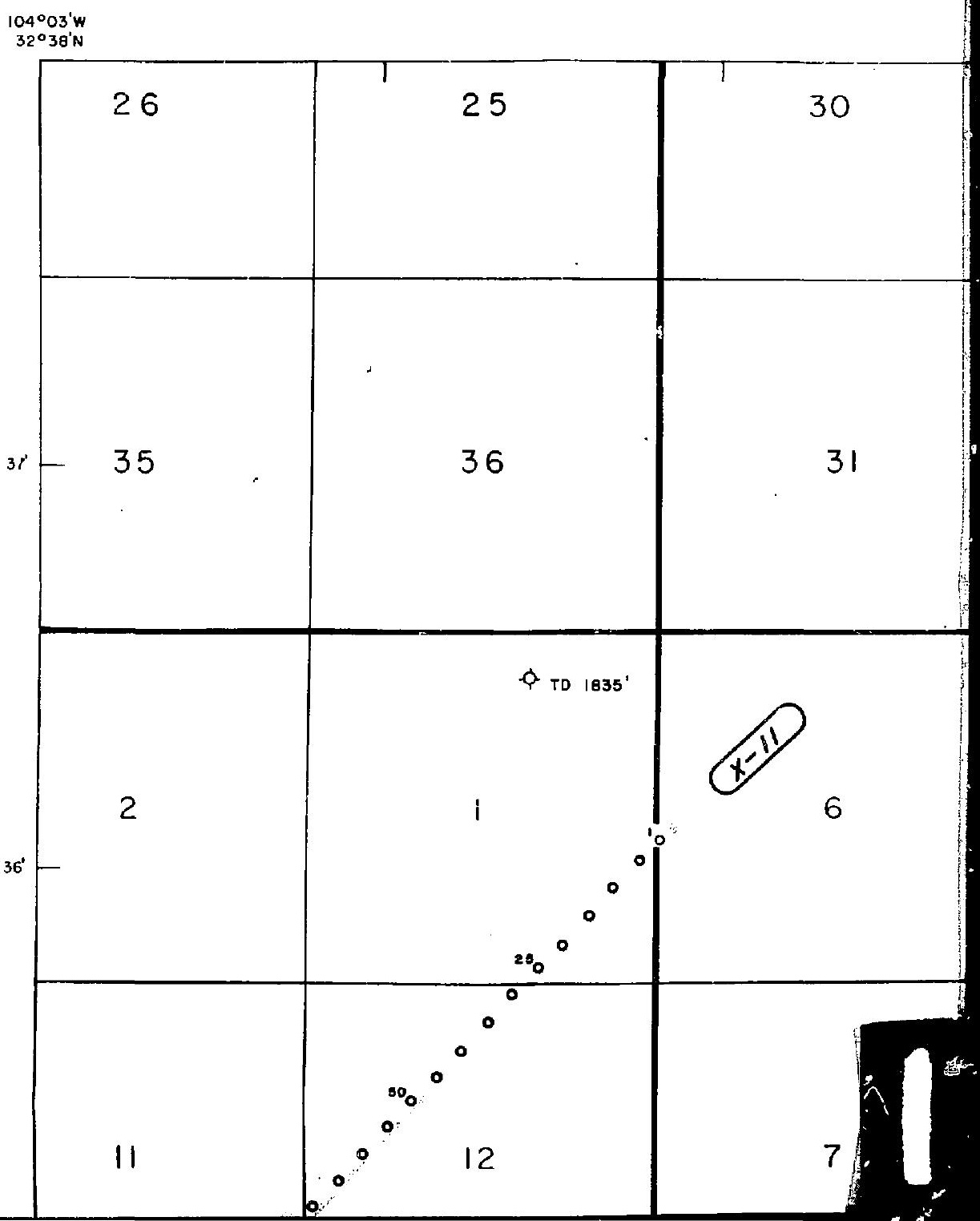


$104^{\circ} 00^{\circ}$

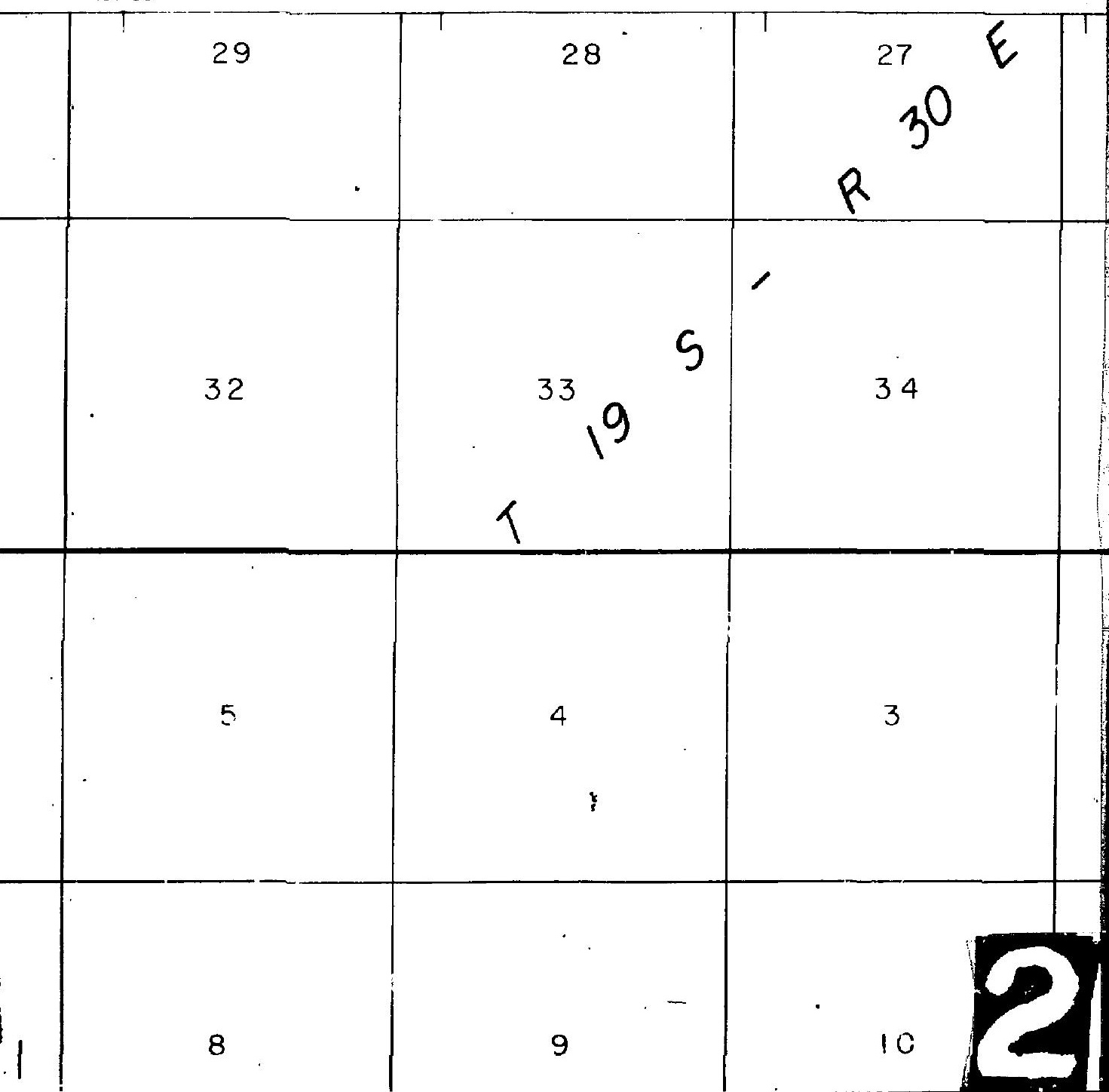




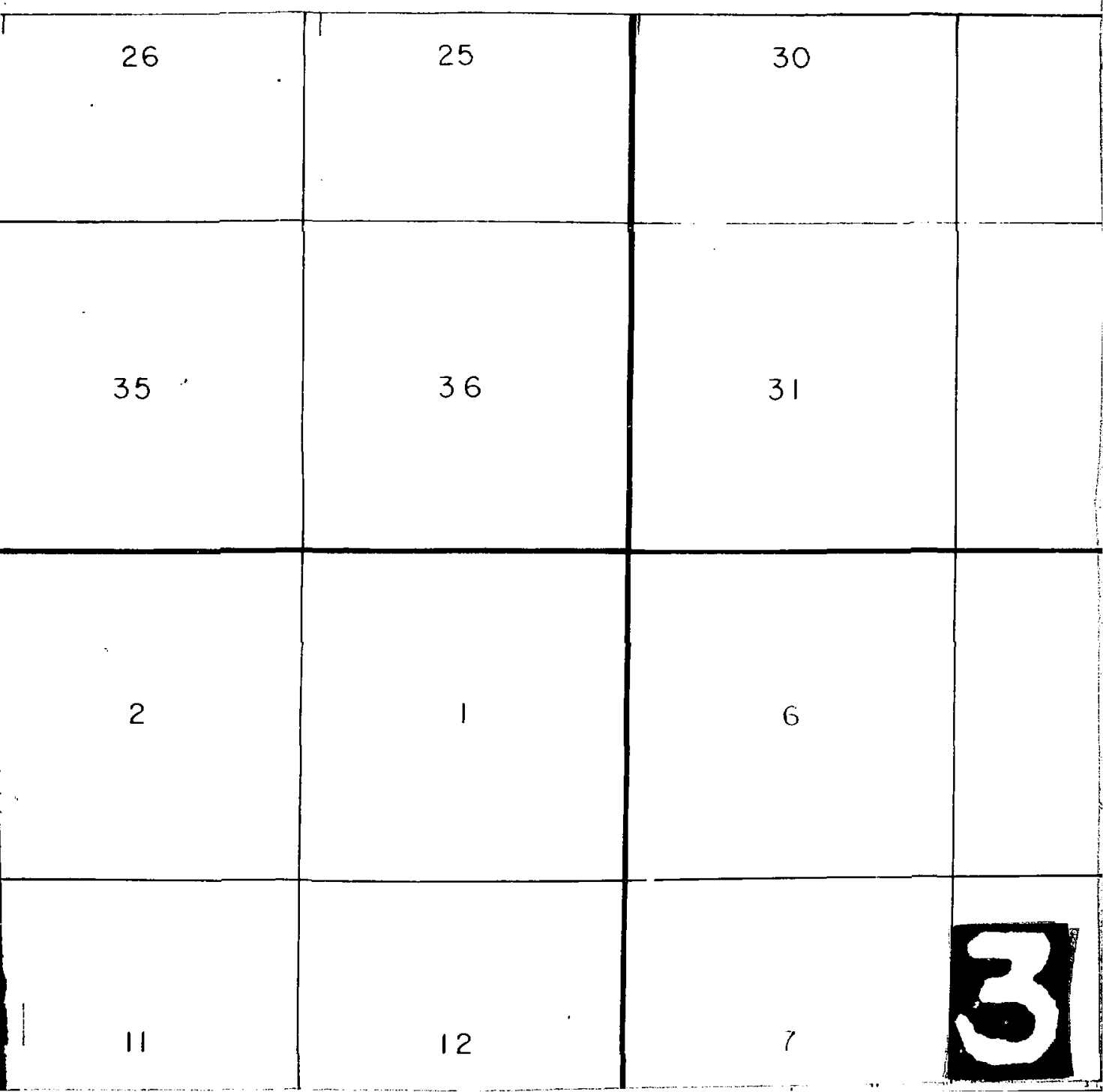




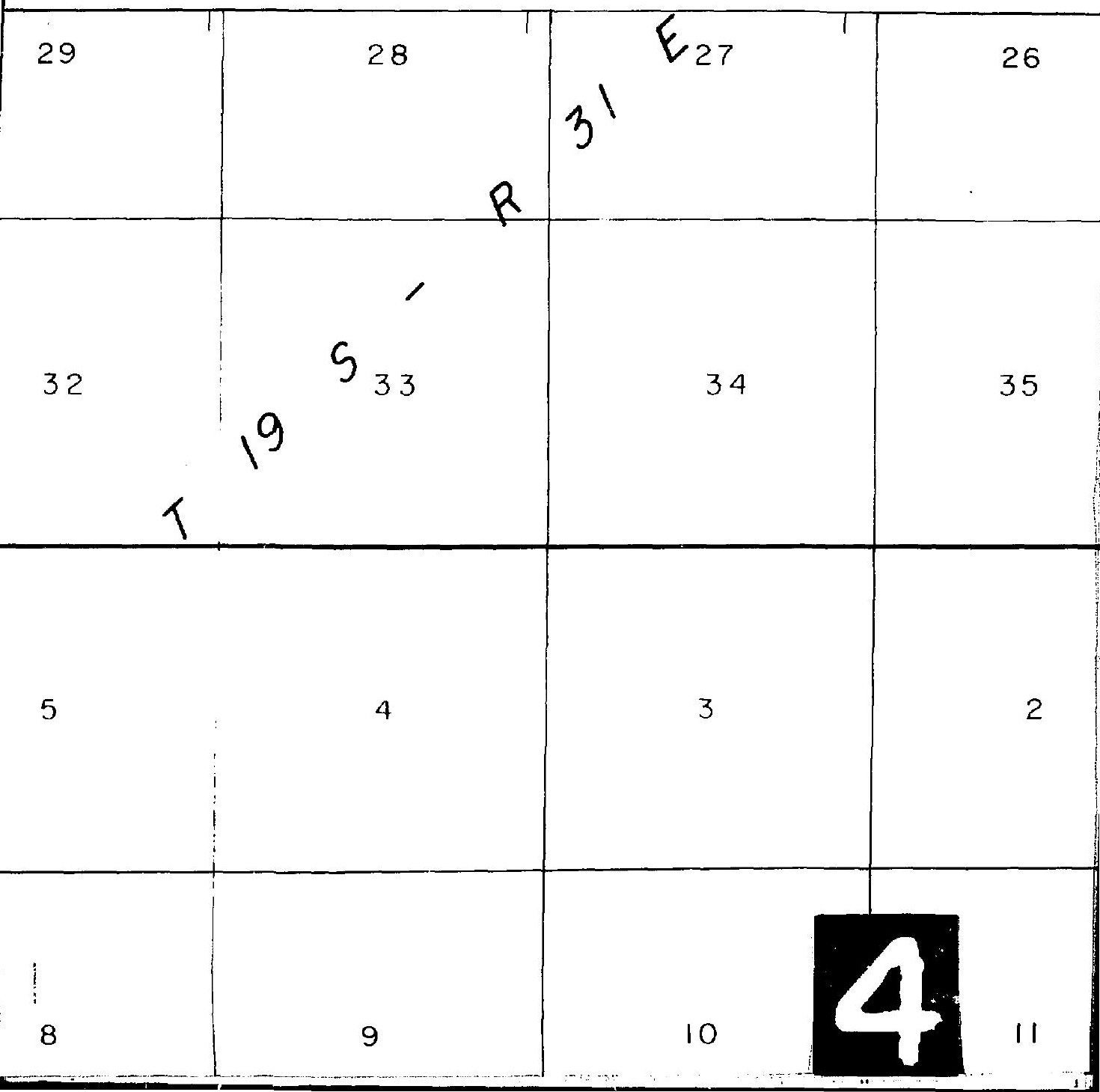


$03^{\circ} 50^{\prime} \mathrm{W}$

$32^{\circ} 38^{\prime} \mathrm{N}$

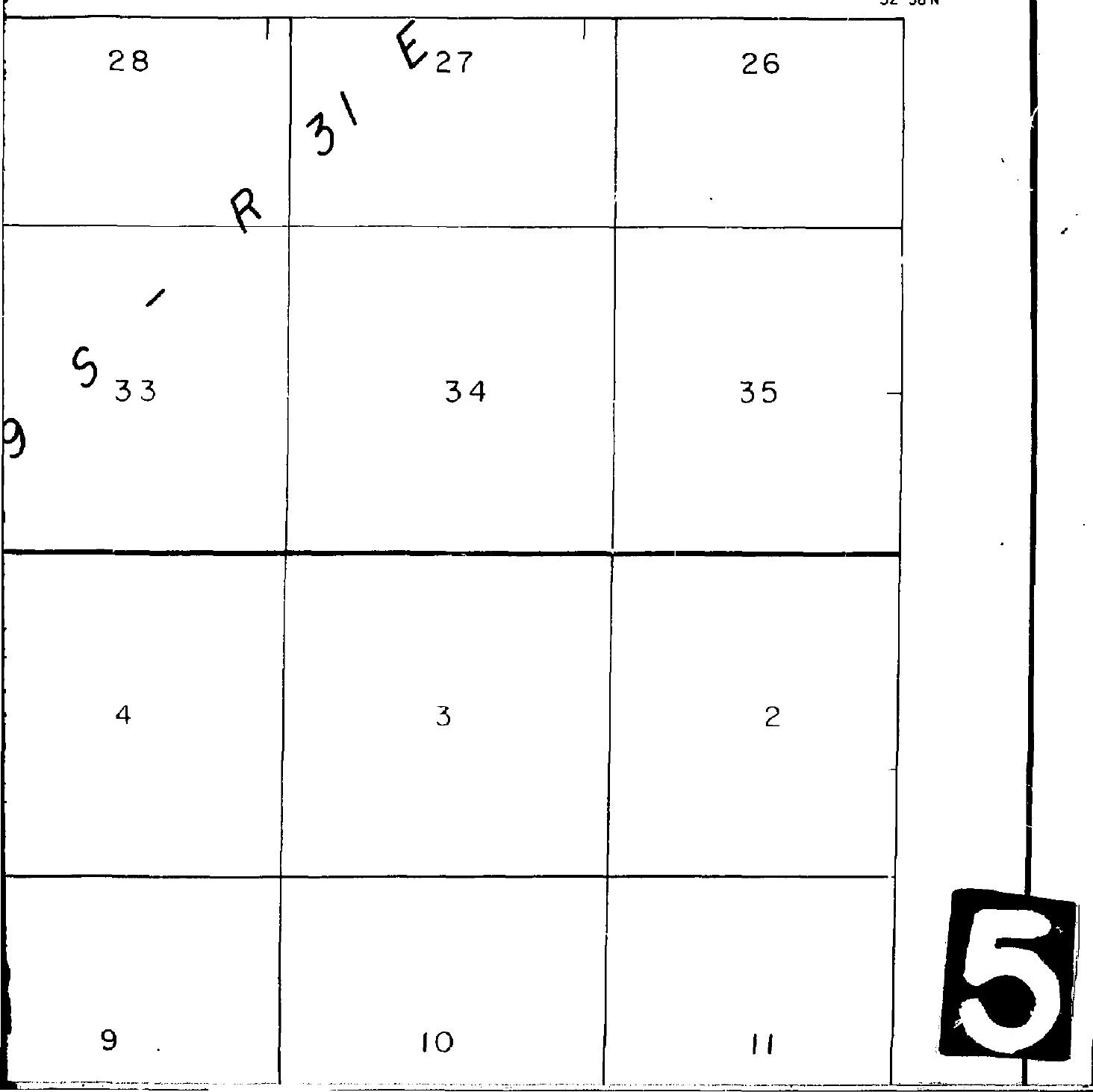




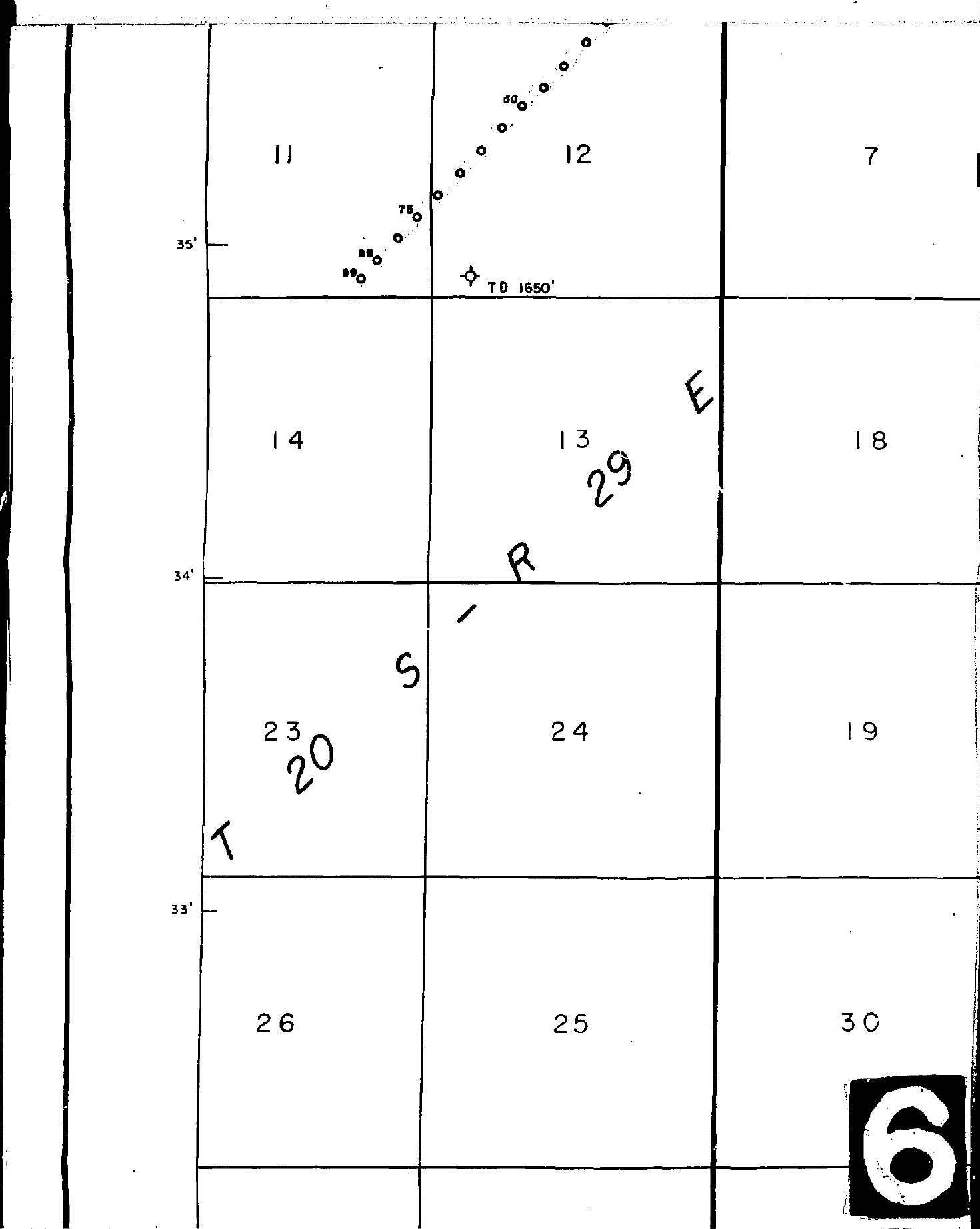




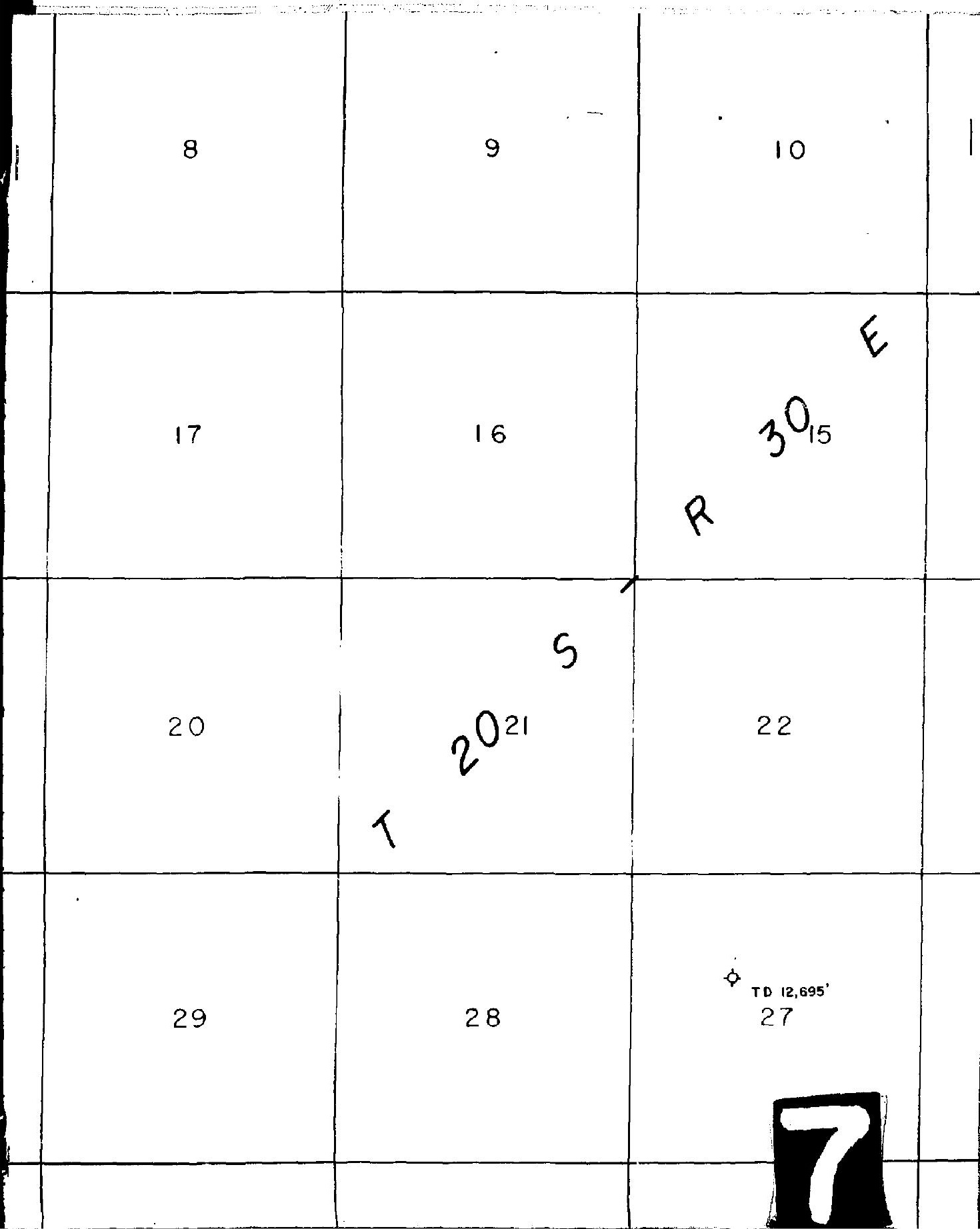




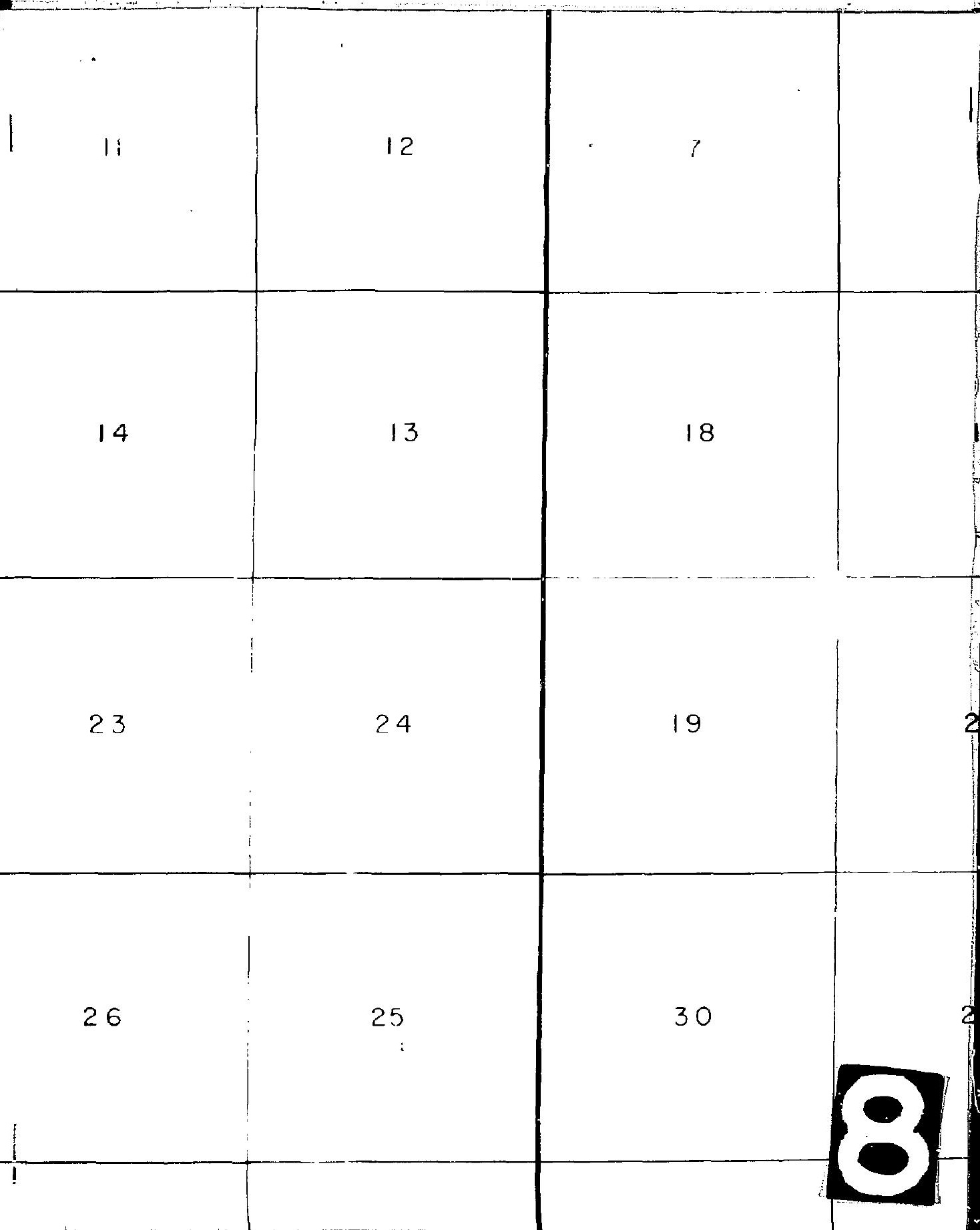




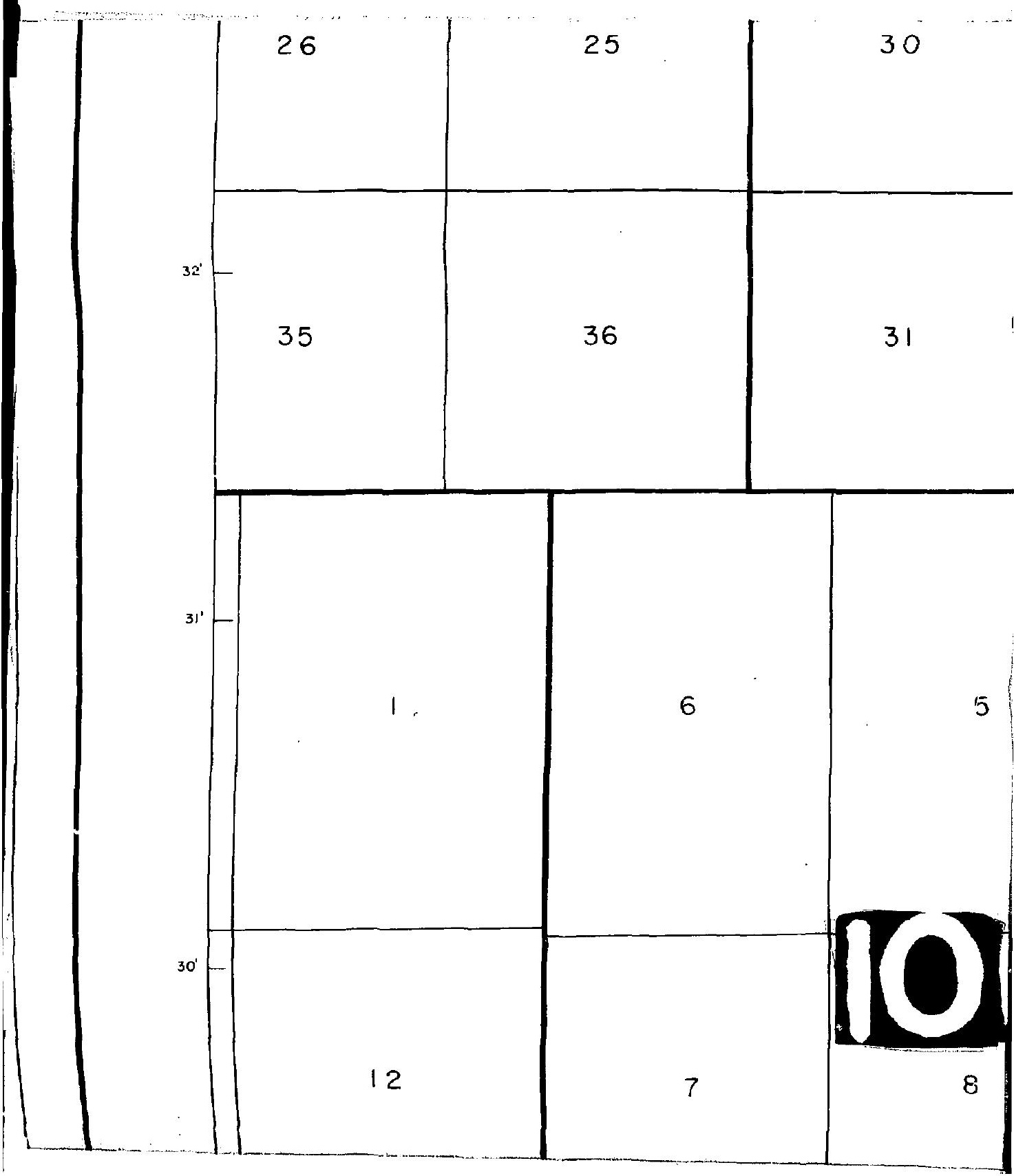




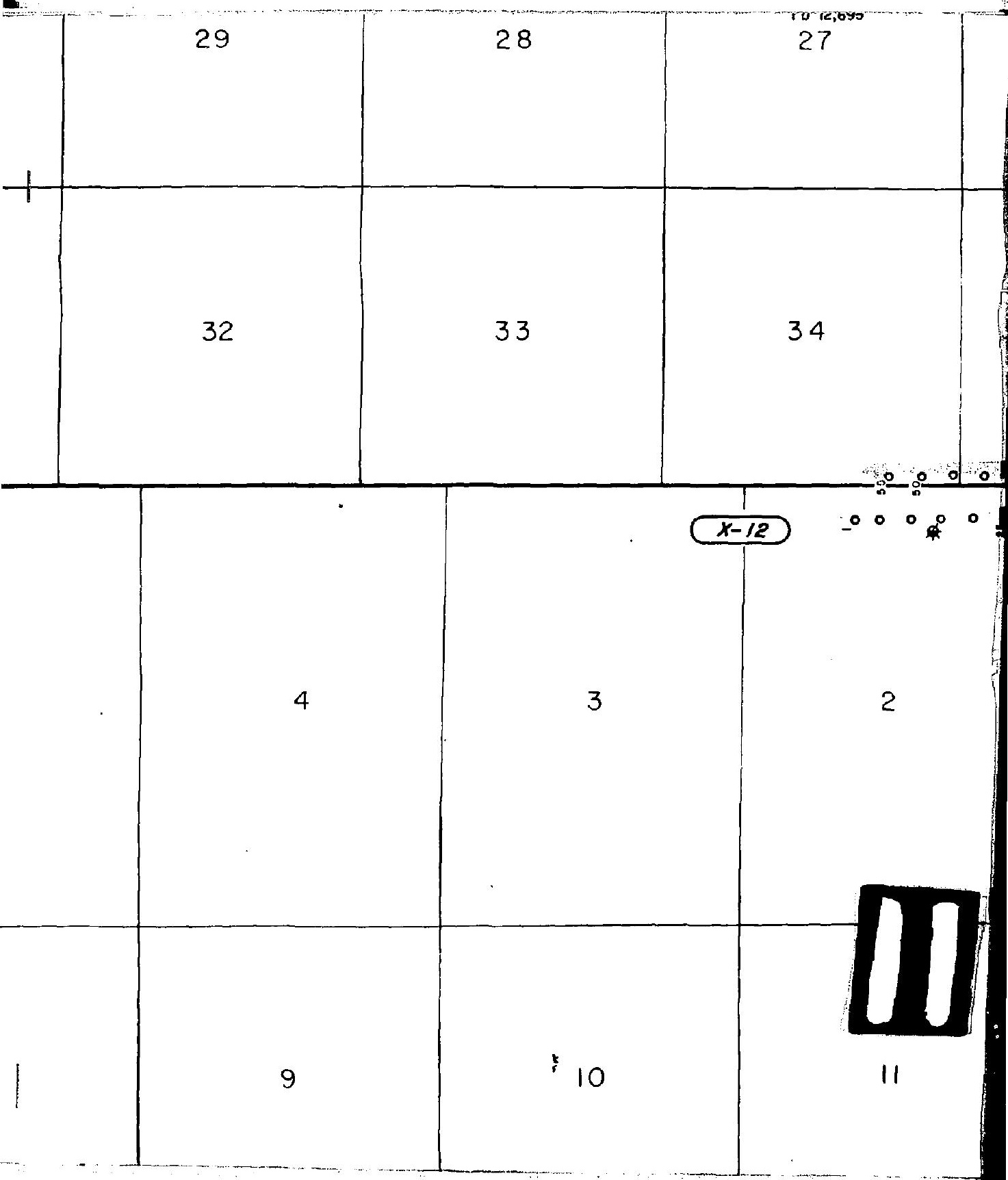




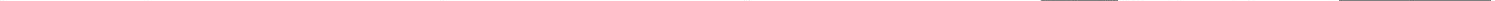




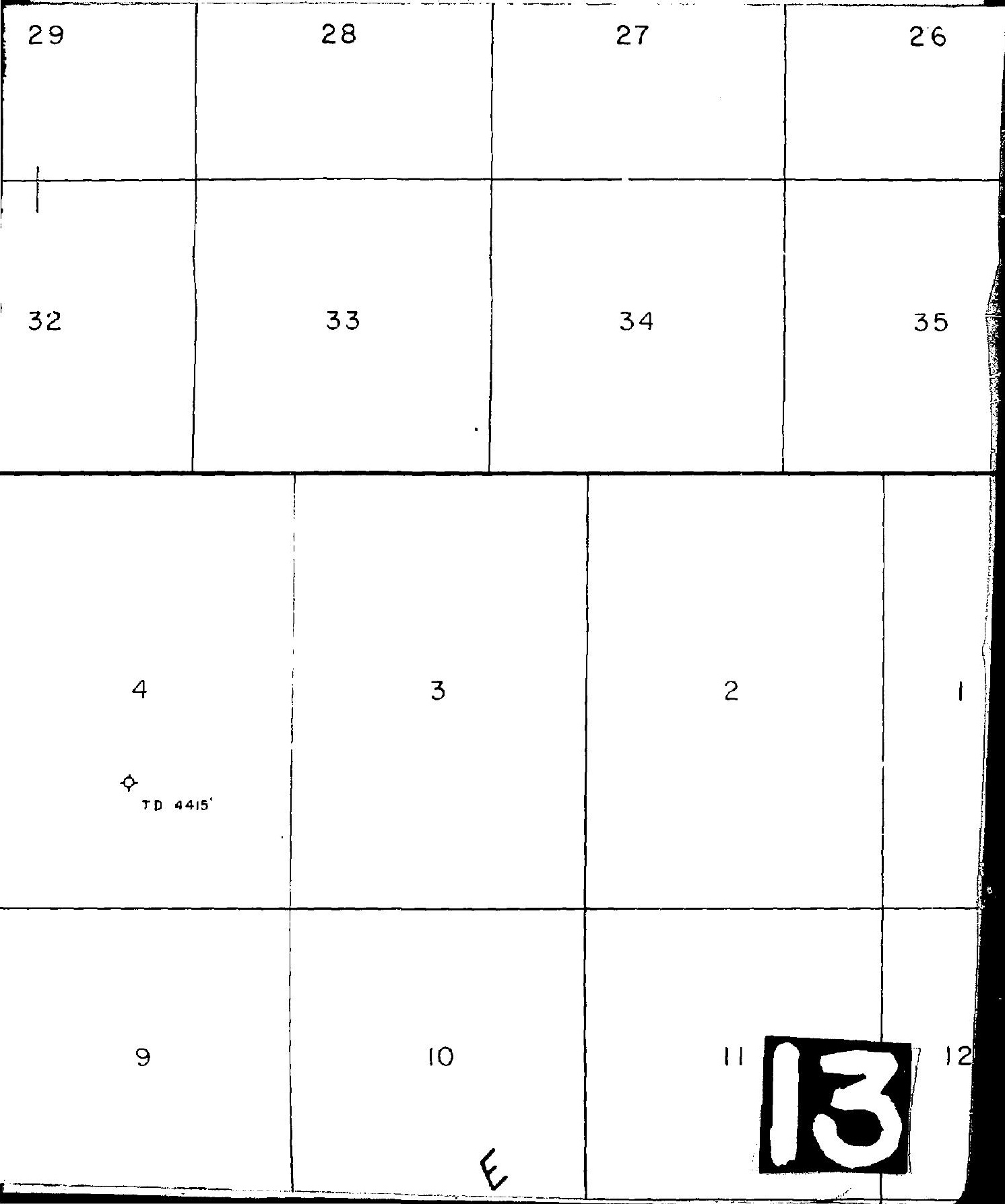




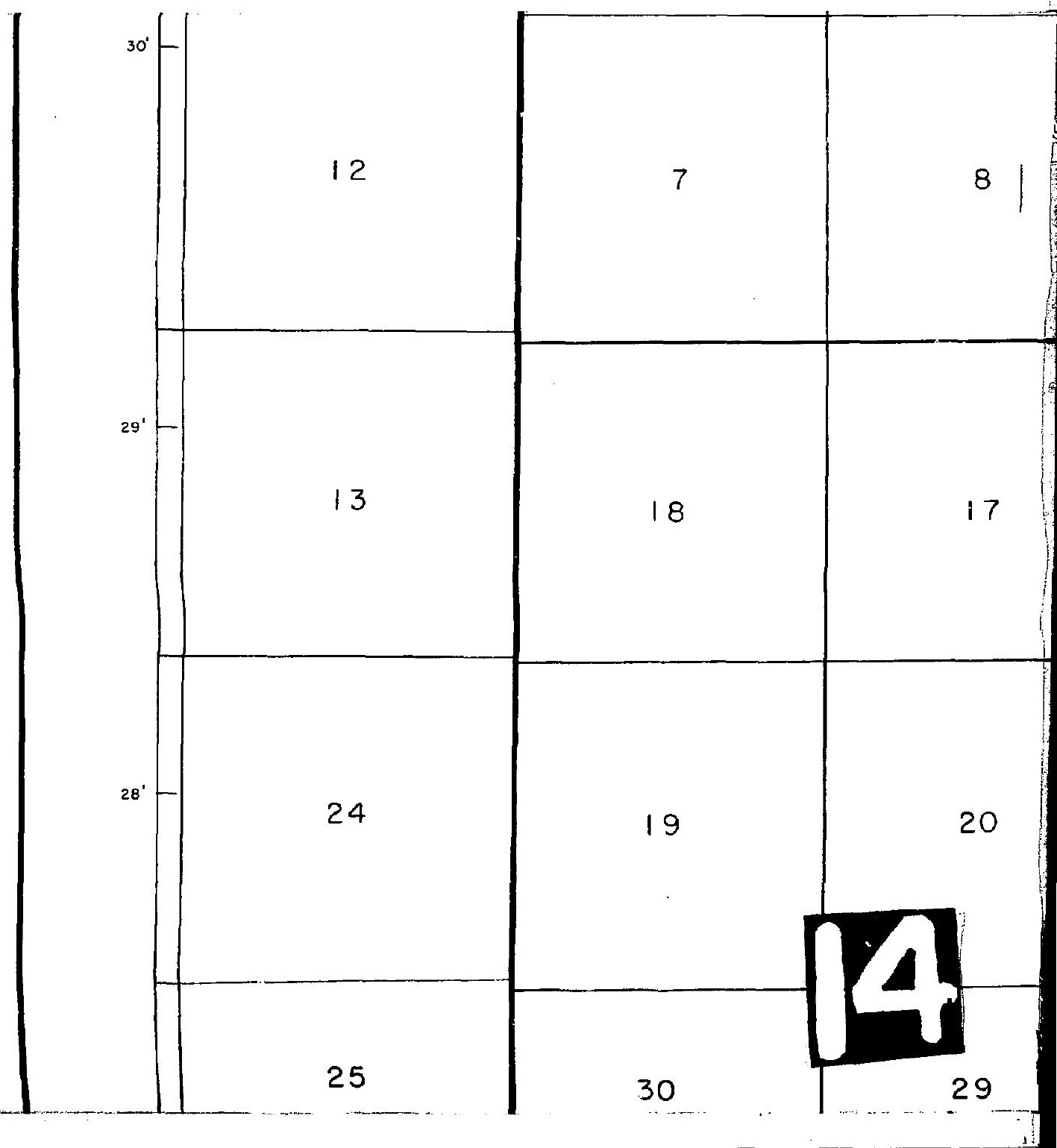




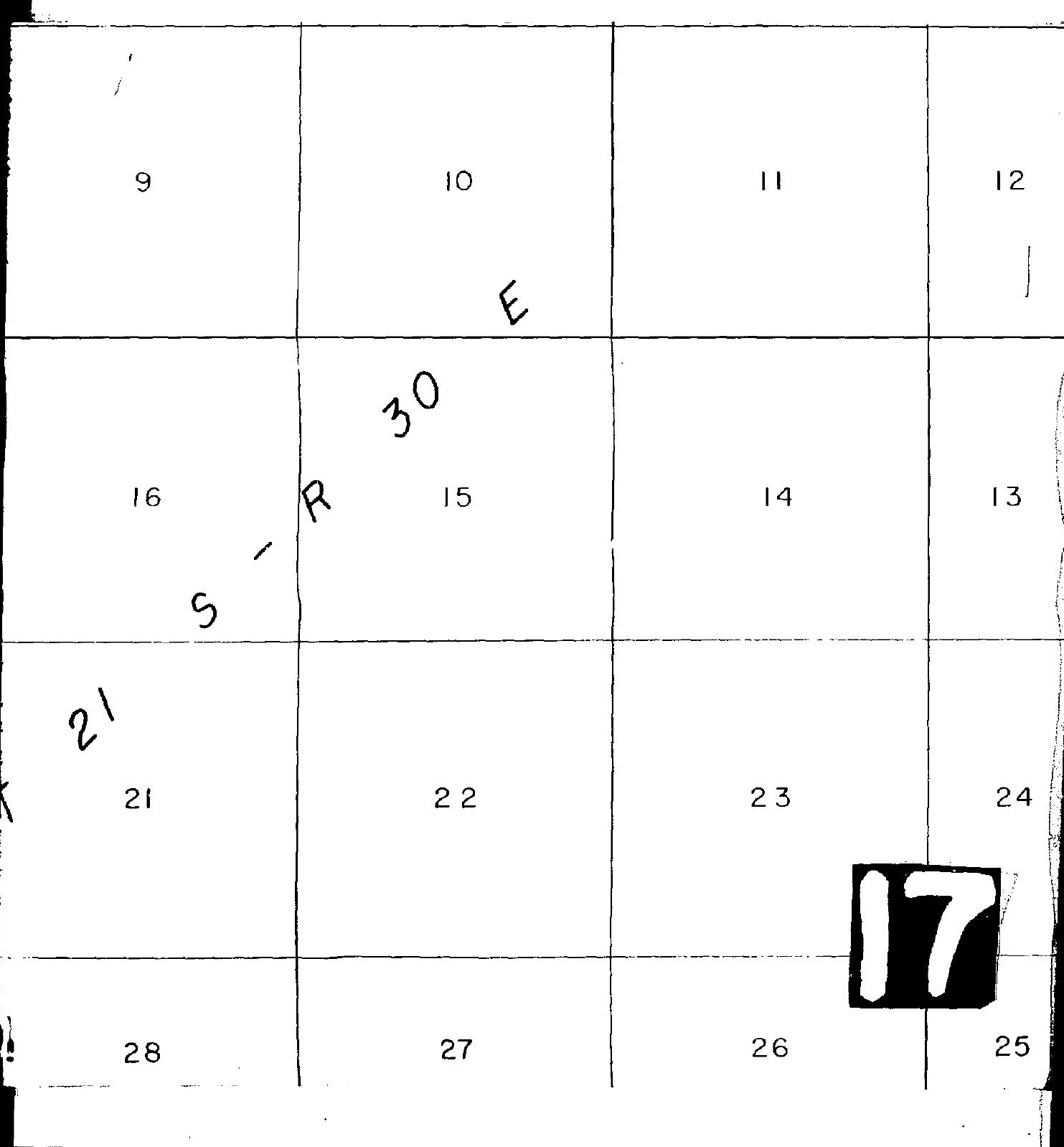



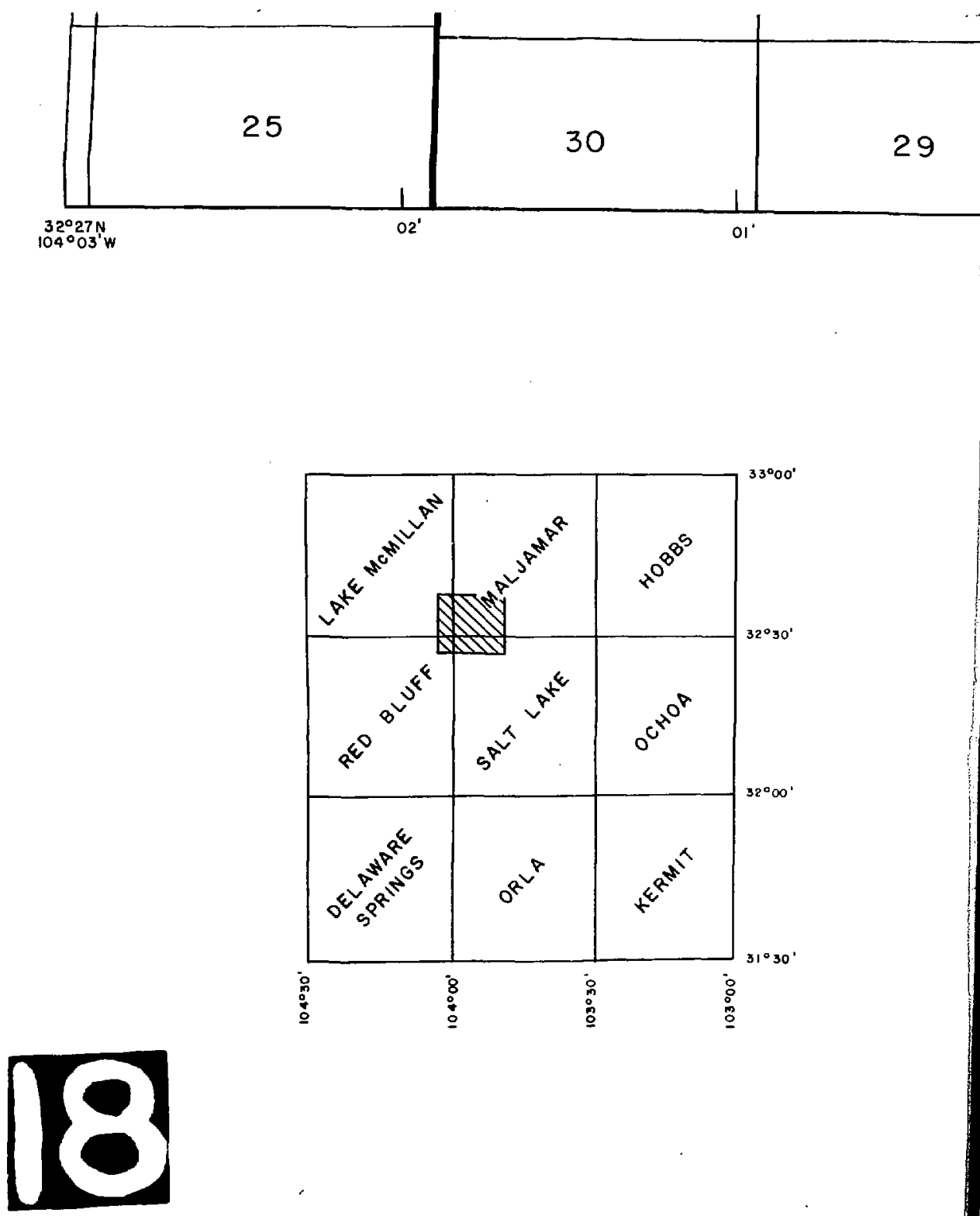


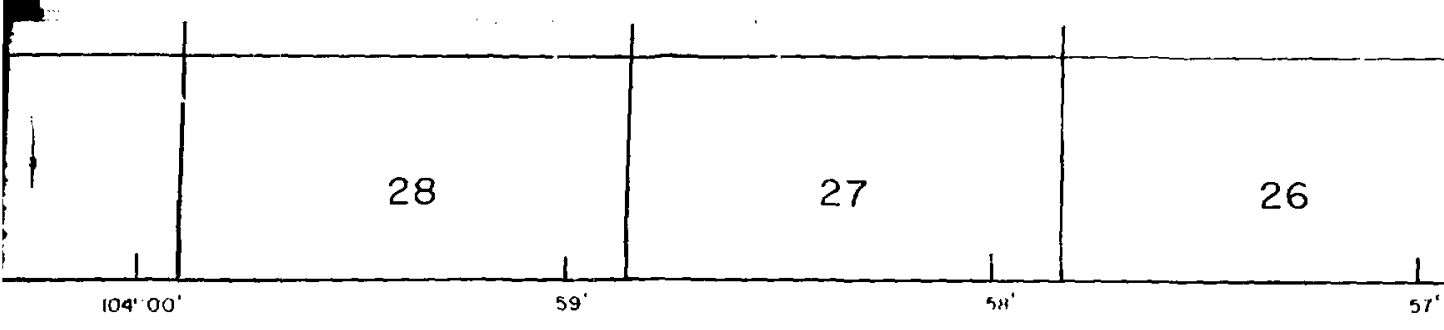

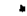

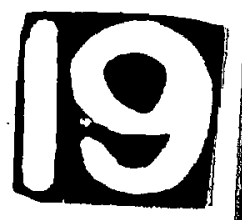



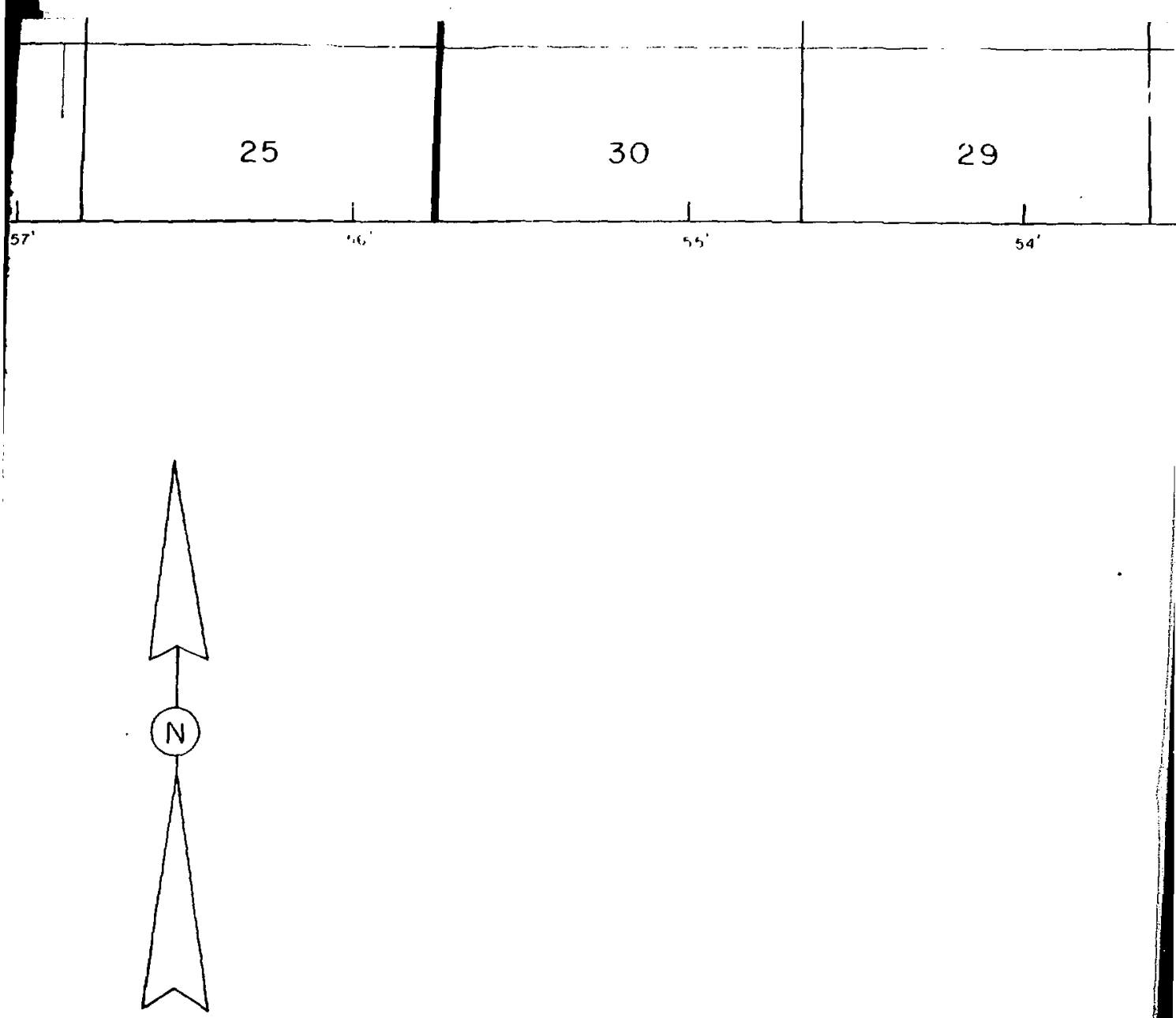

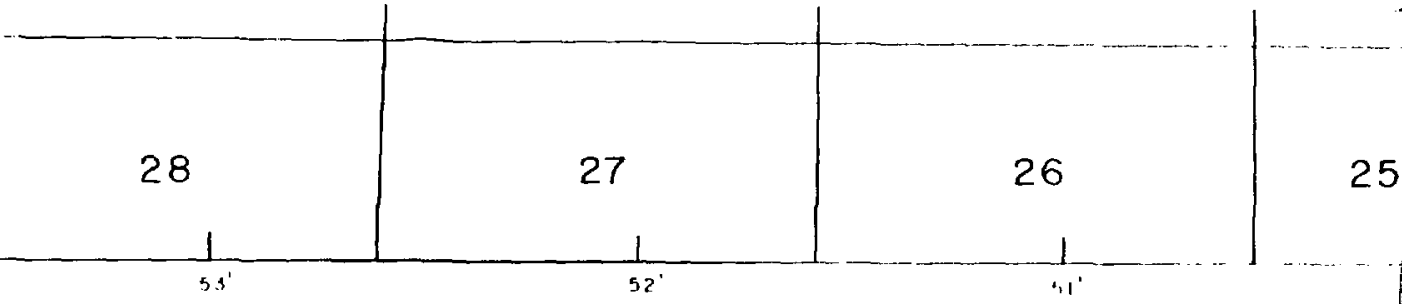

\section{SANDIA LABORATOF}

SEISMIC SURVEY

ADJACENT AREA TO LOS MEDAN EDDY AND LEA COUNTIES, NEW ME

\section{SEISMIC PROGRAM}




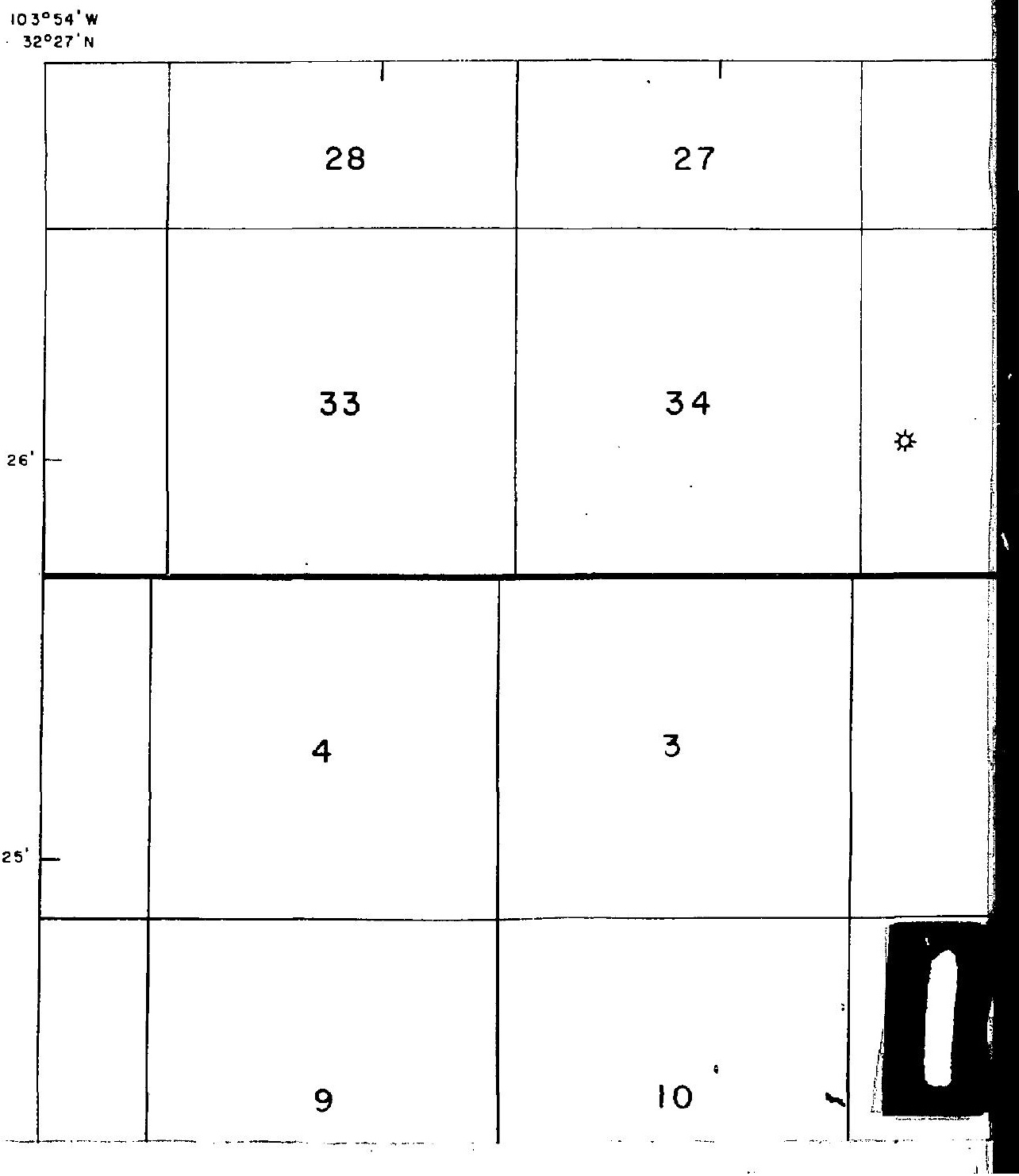




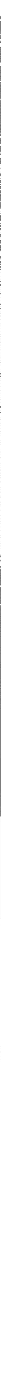




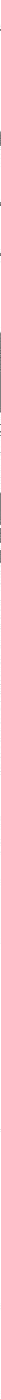




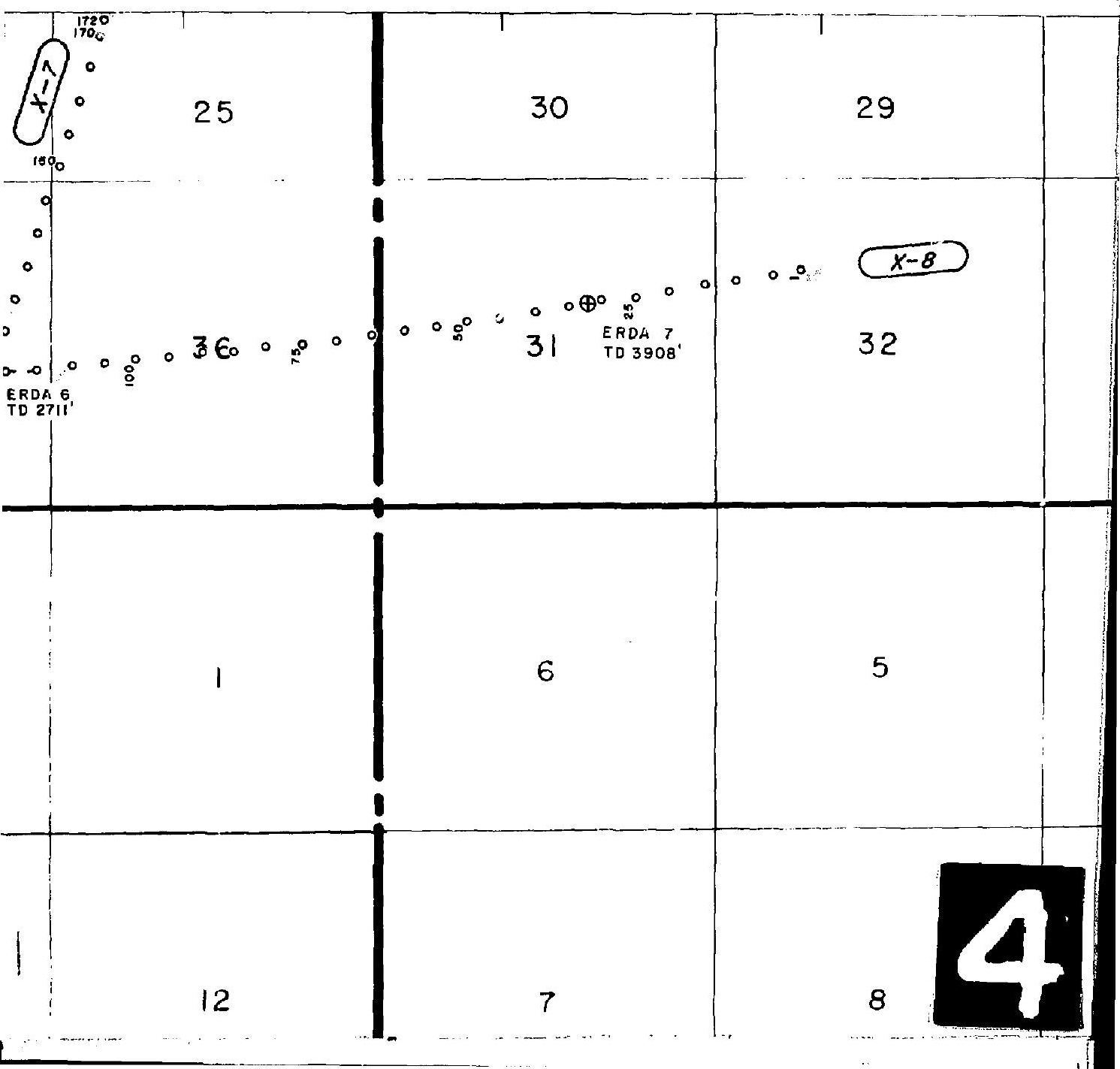




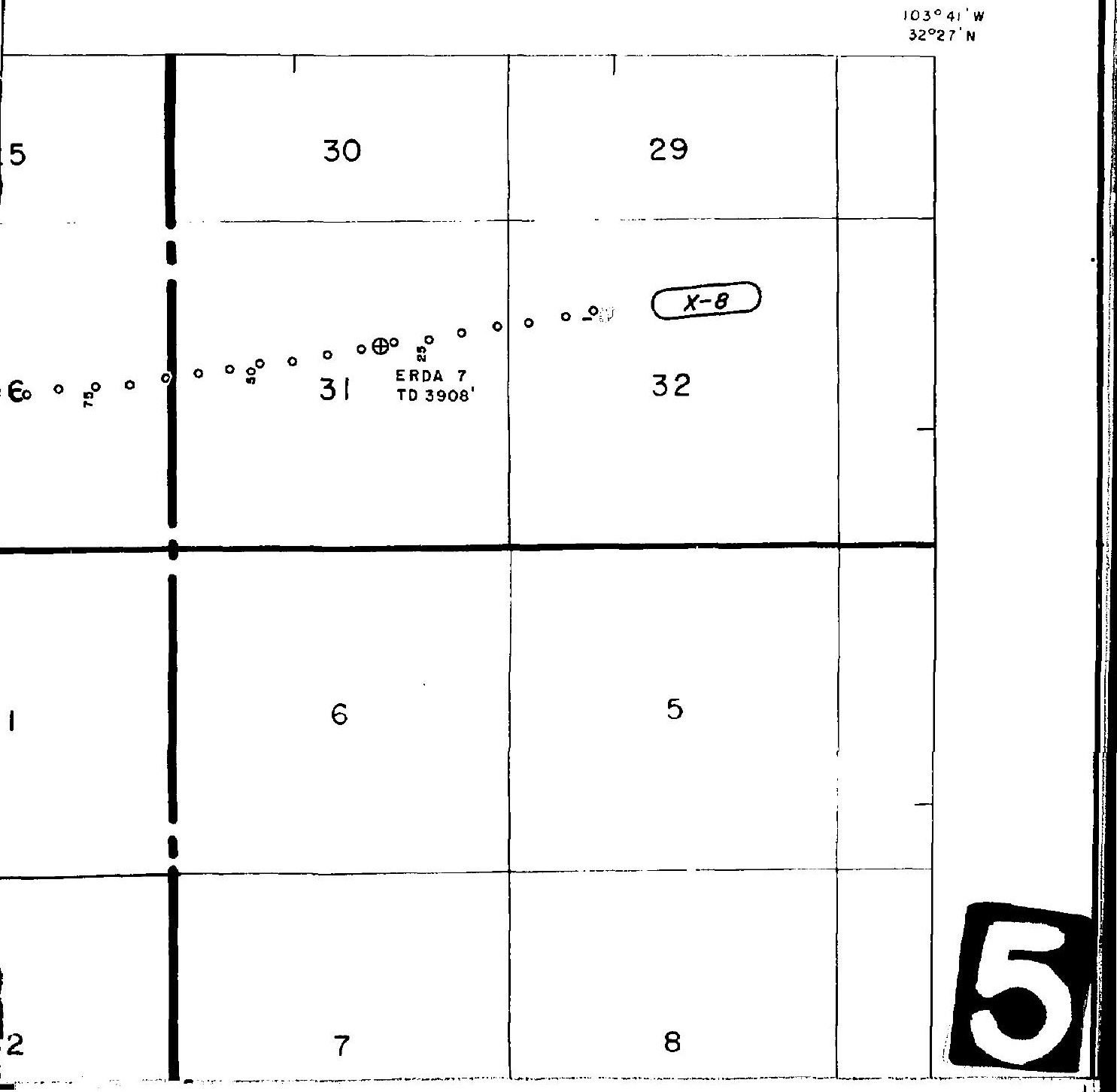




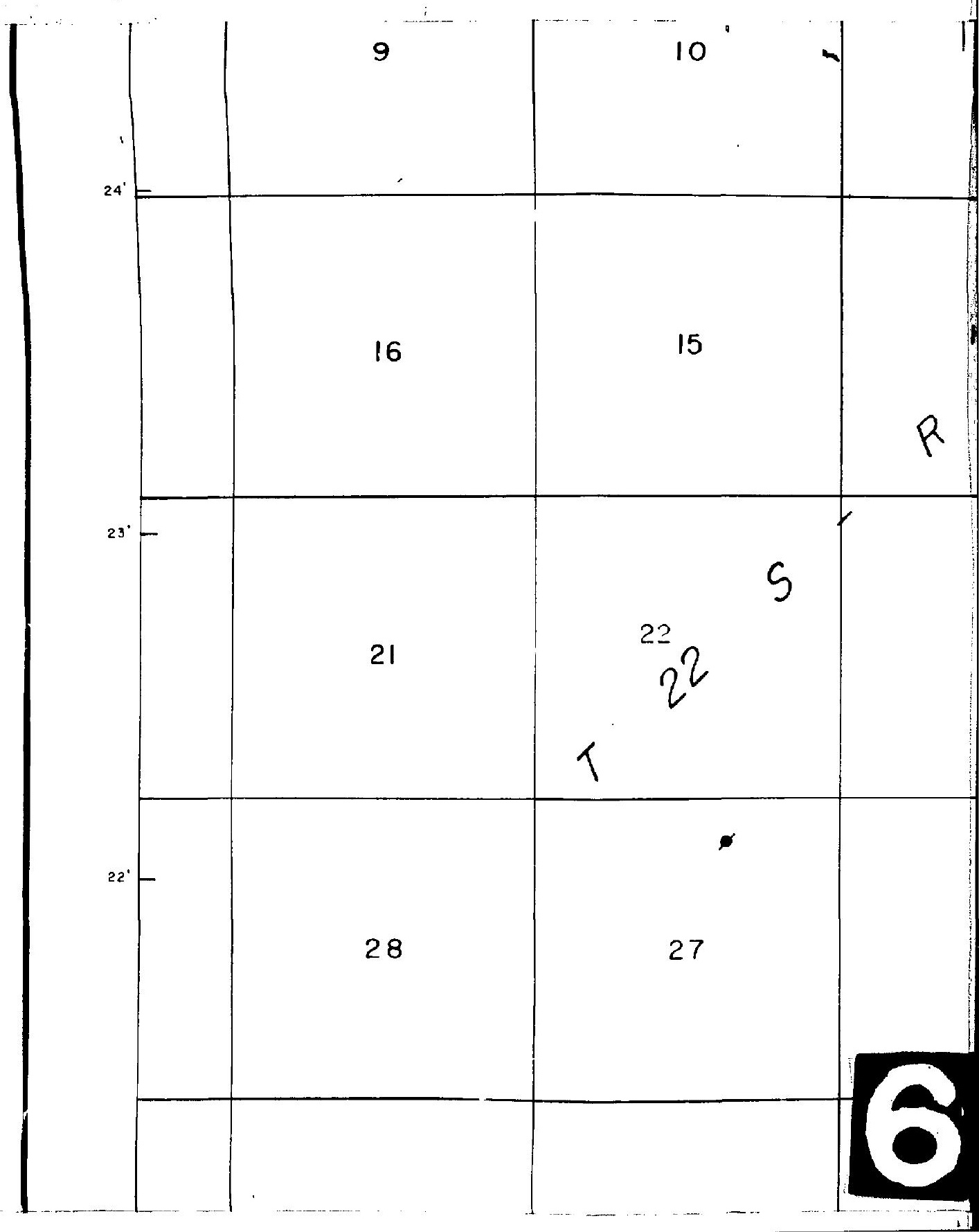




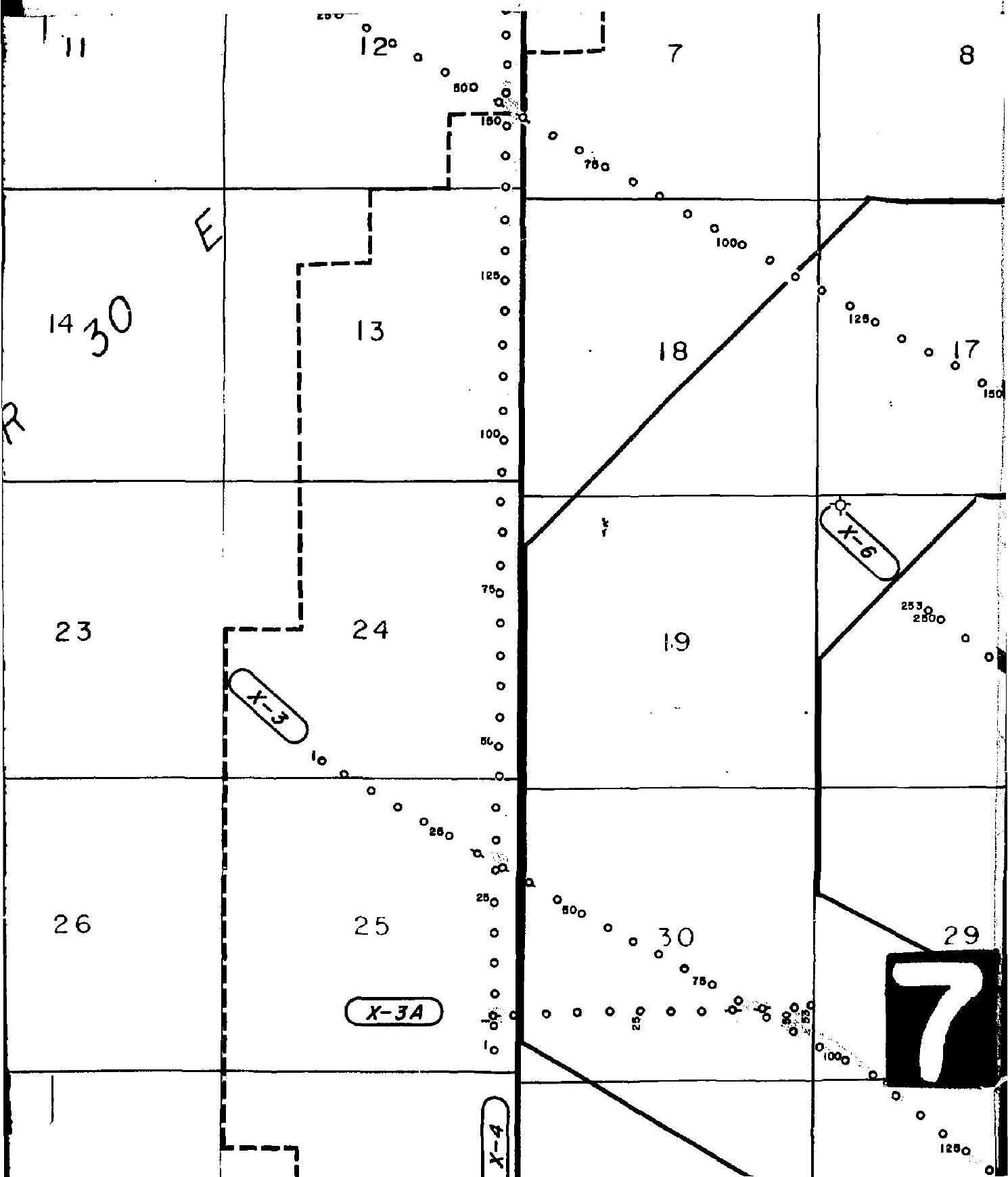




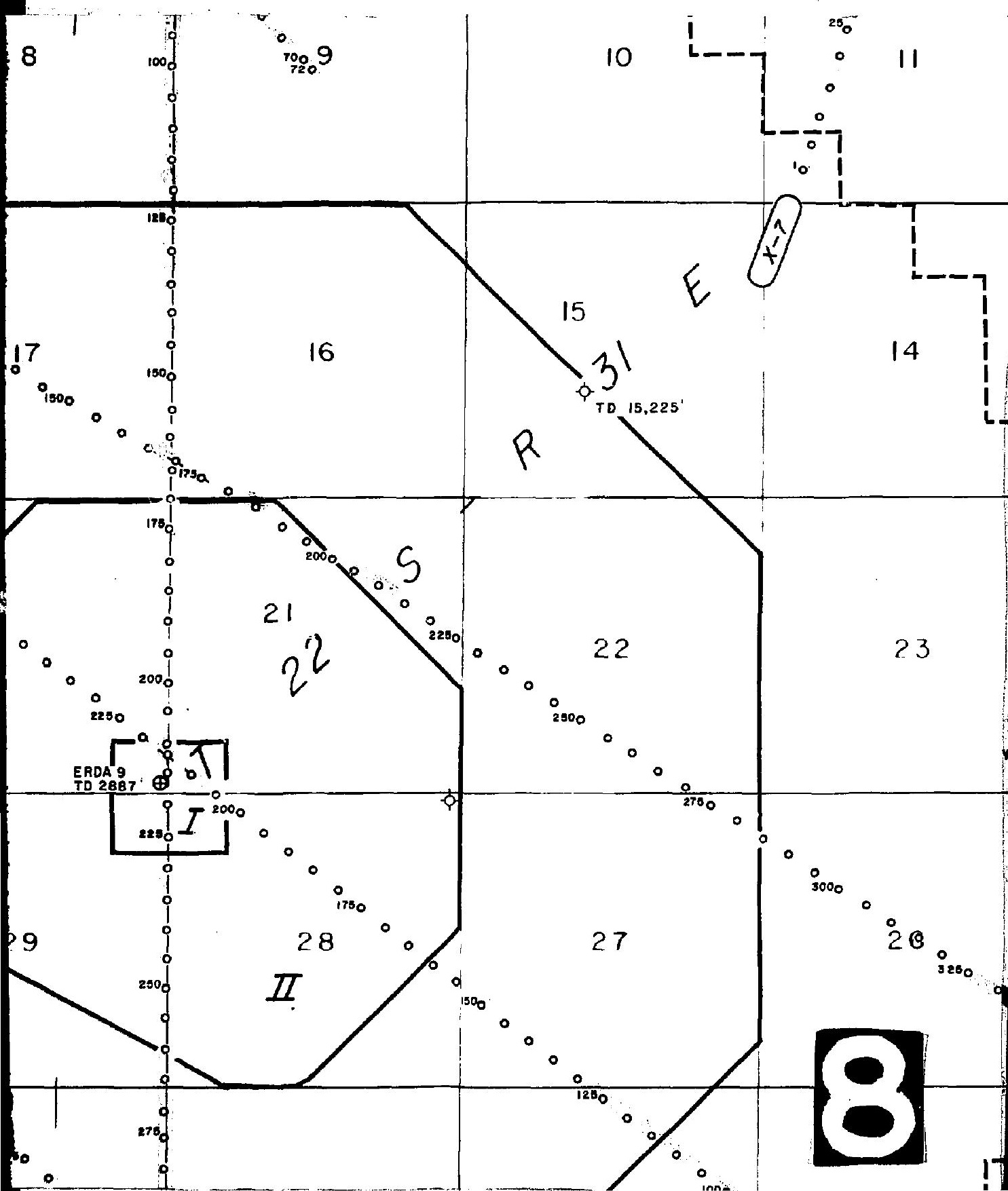




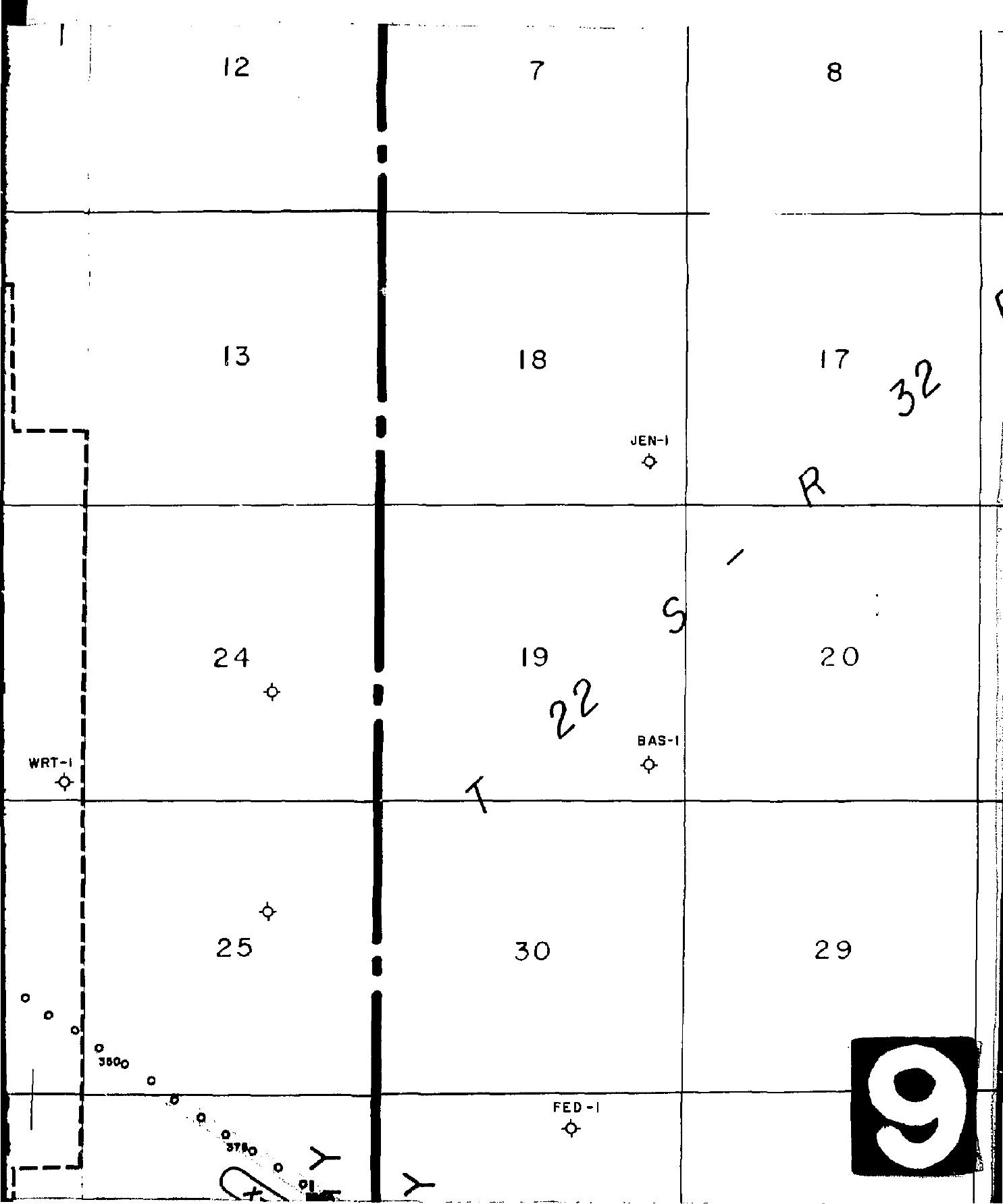




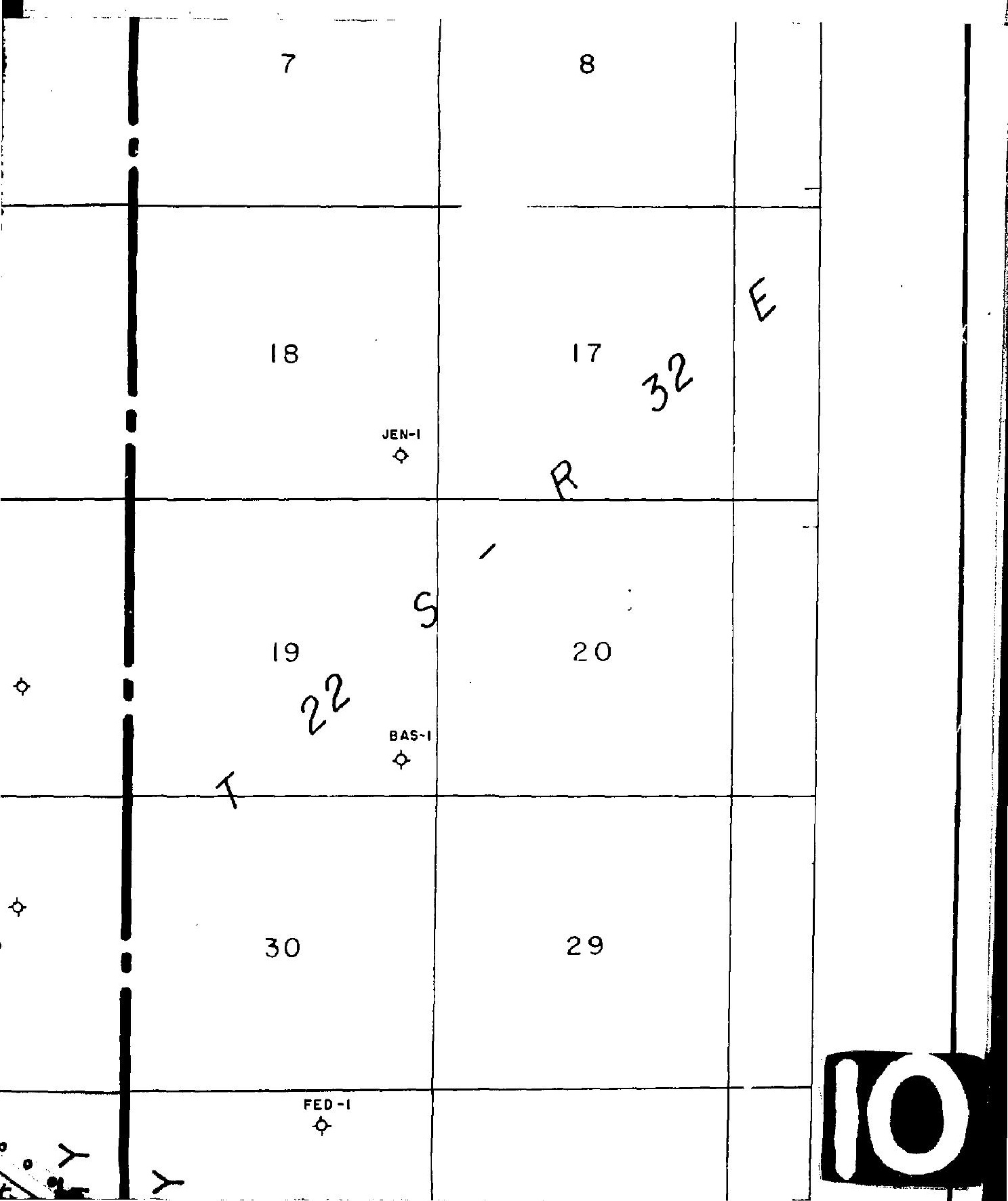




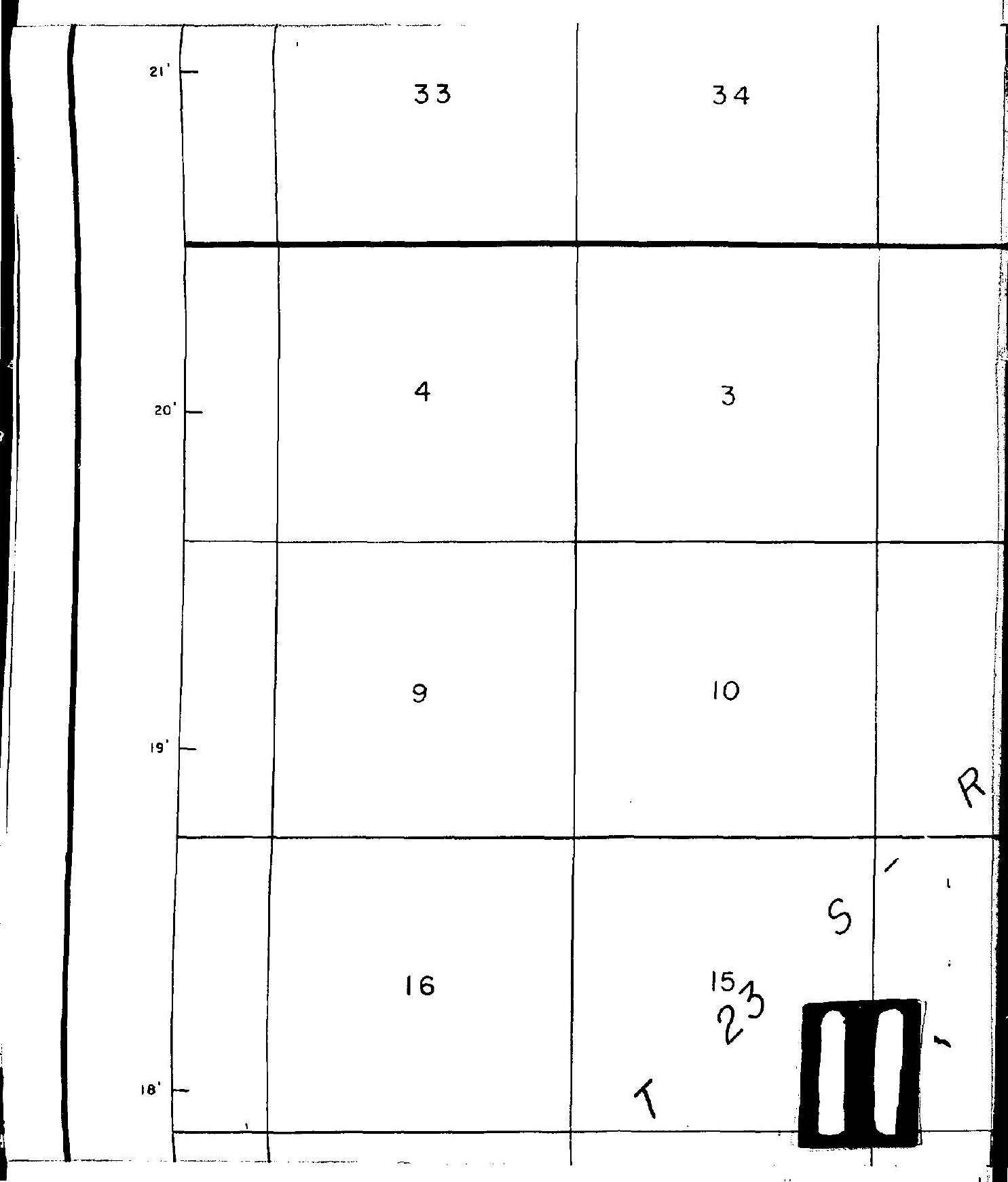




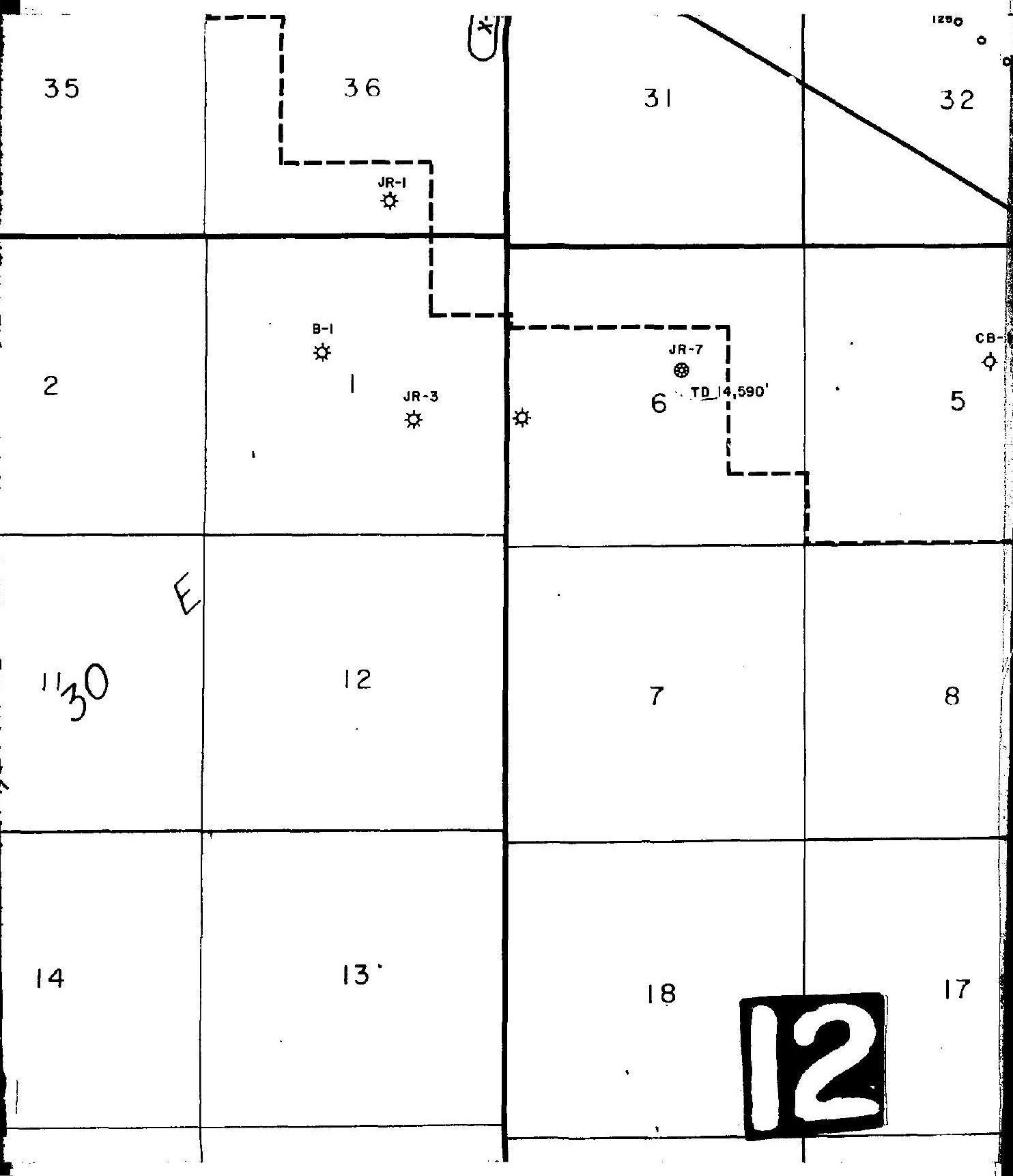




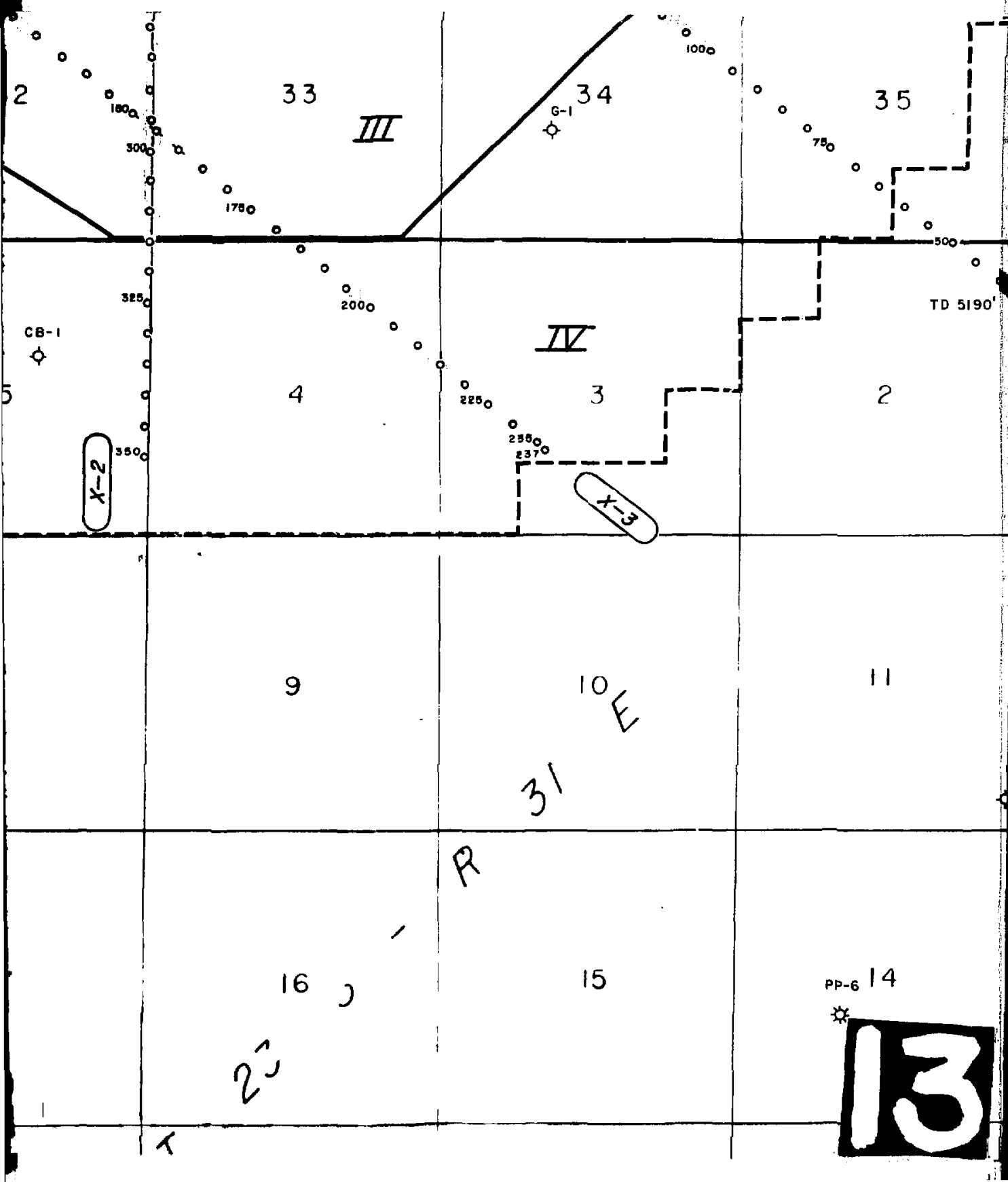




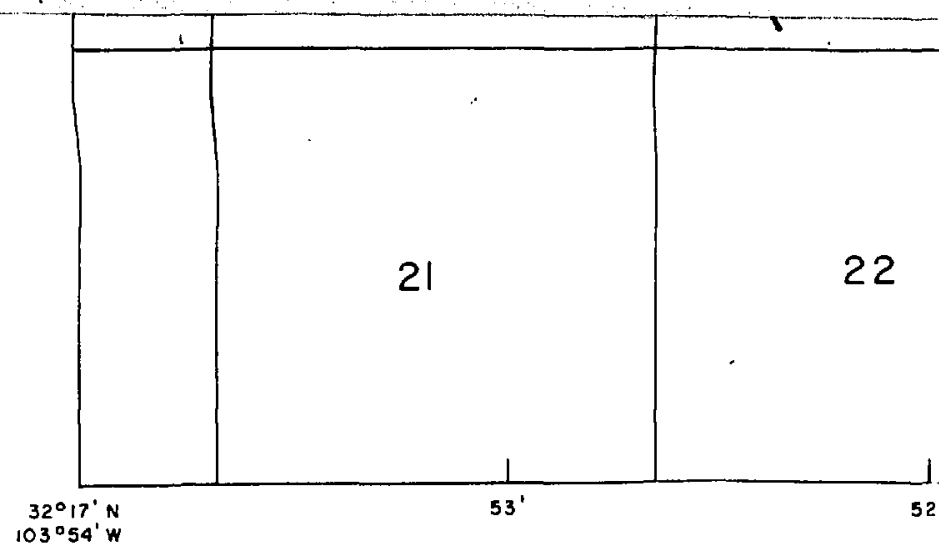

$-$ 


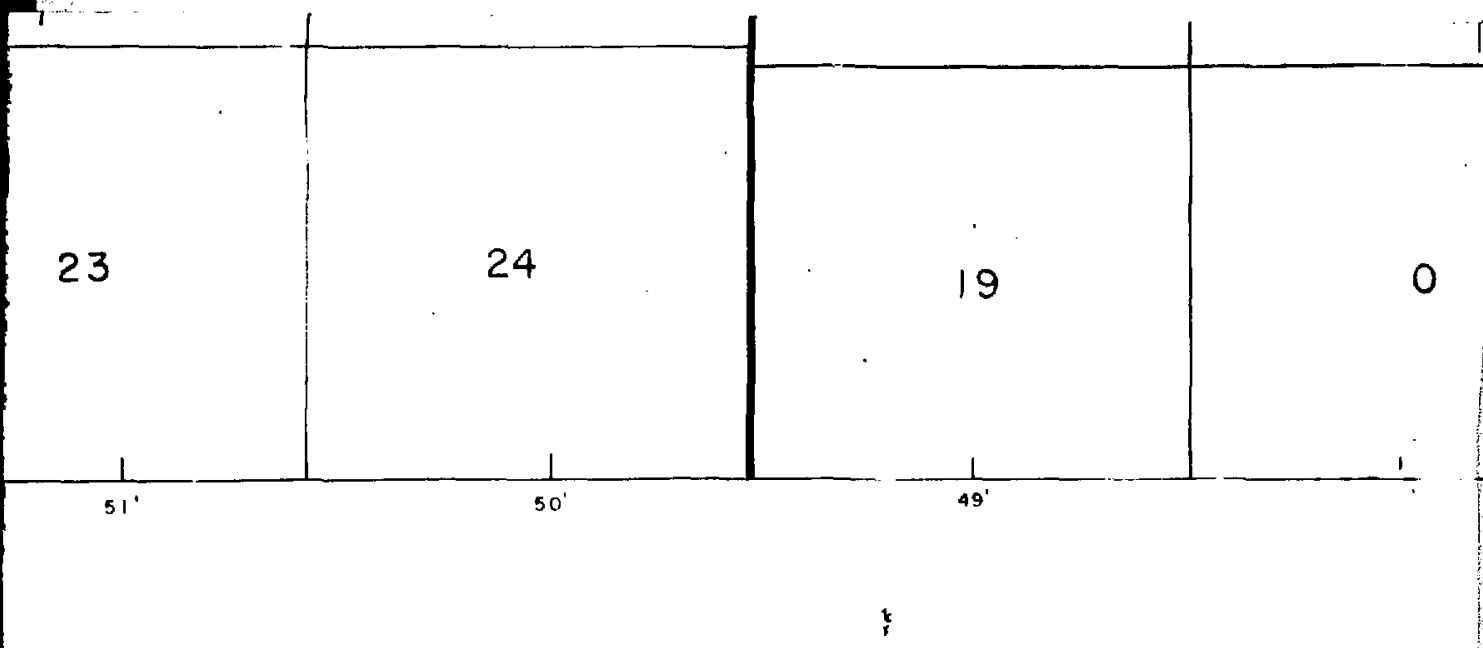




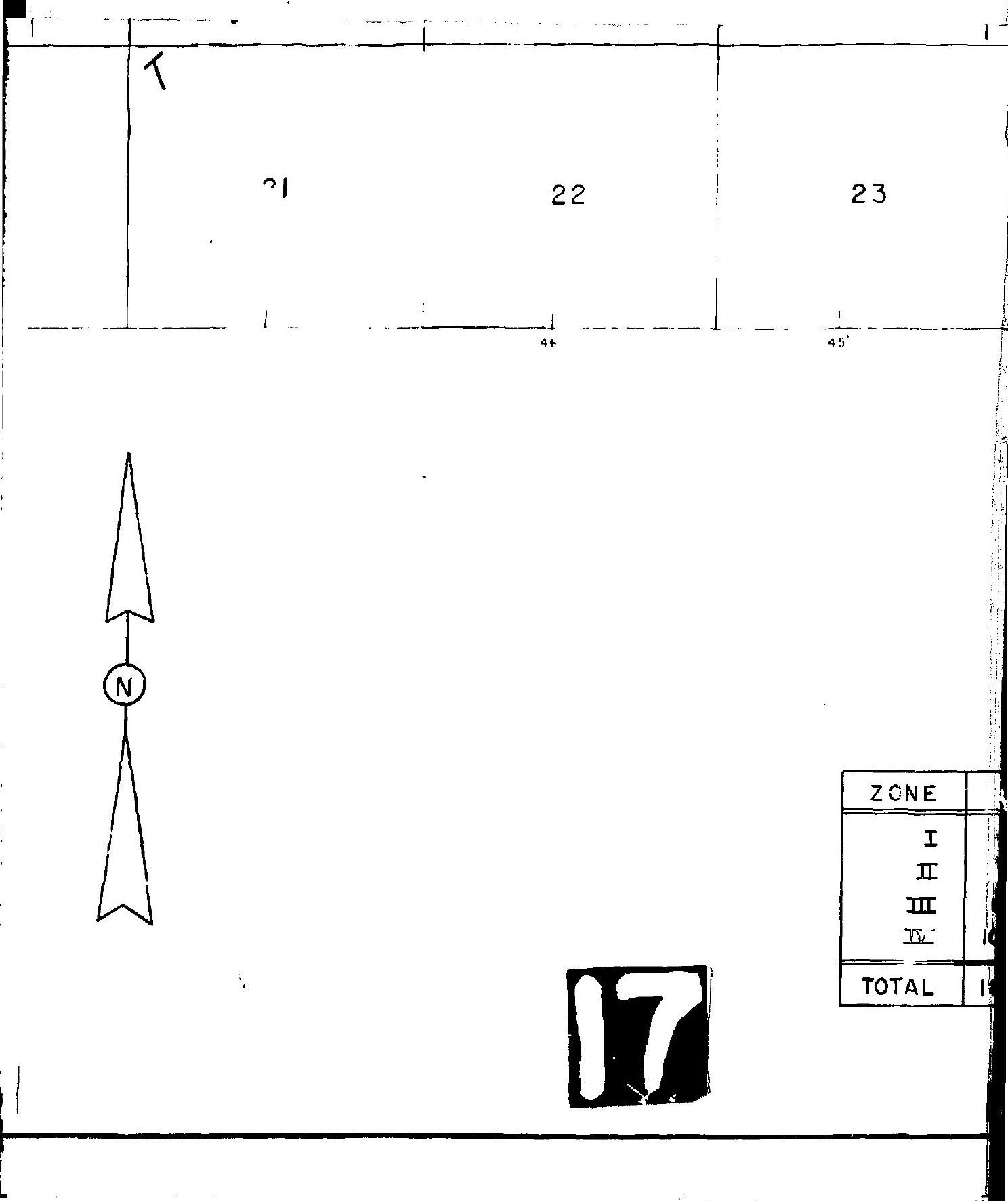




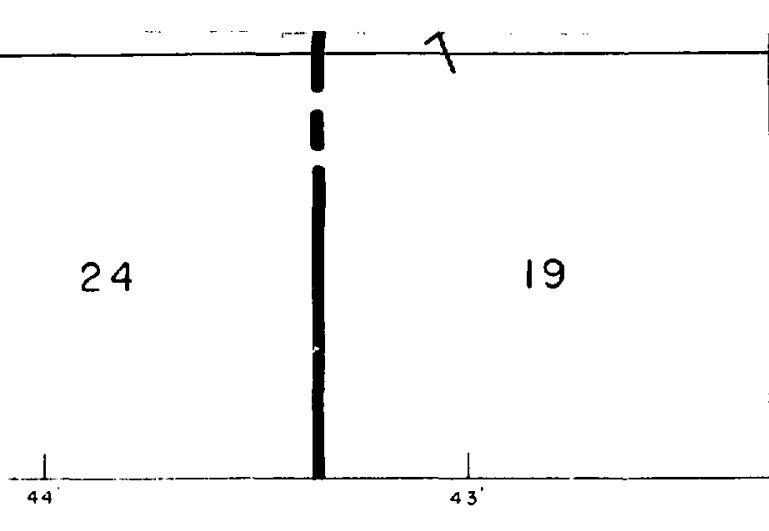

\section{0}

\section{SANDIA LABORATORI}

\section{AREA}

\begin{tabular}{|rl|}
\hline 100 & Acres \\
1,818 & Acres \\
6,221 & Acres \\
10,821 & Acres \\
\hline 18,960 & Acres \\
\hline
\end{tabular}

SEISMIC SURVEY

LOS MEDANOS SITE AREA EDDY COUNTY, NEW MEXICO

1977 SEISMIC PROGRAM MA

INTERPRETATION BY

G. J. LONG \& ASSOCIATES, INC. 


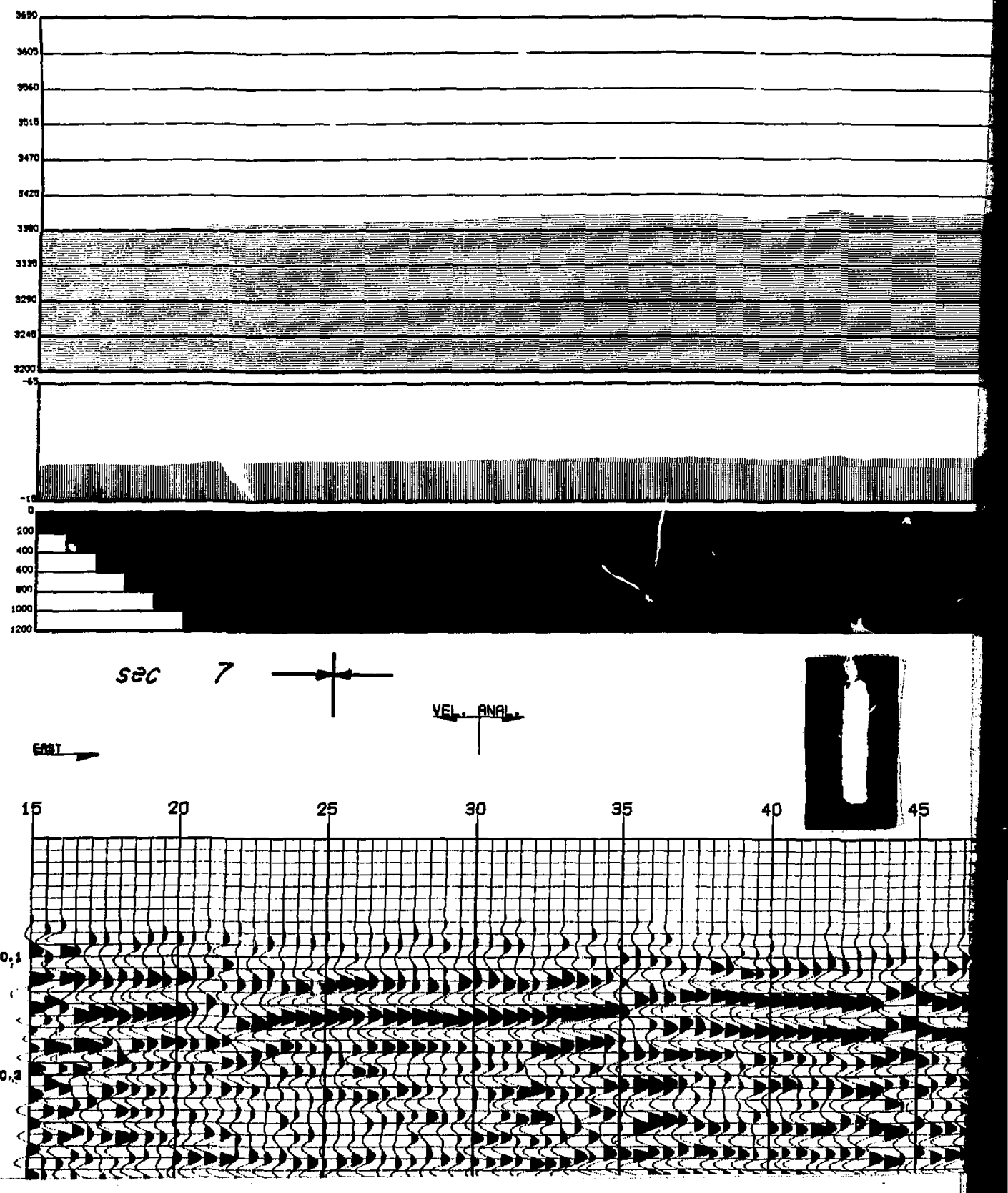




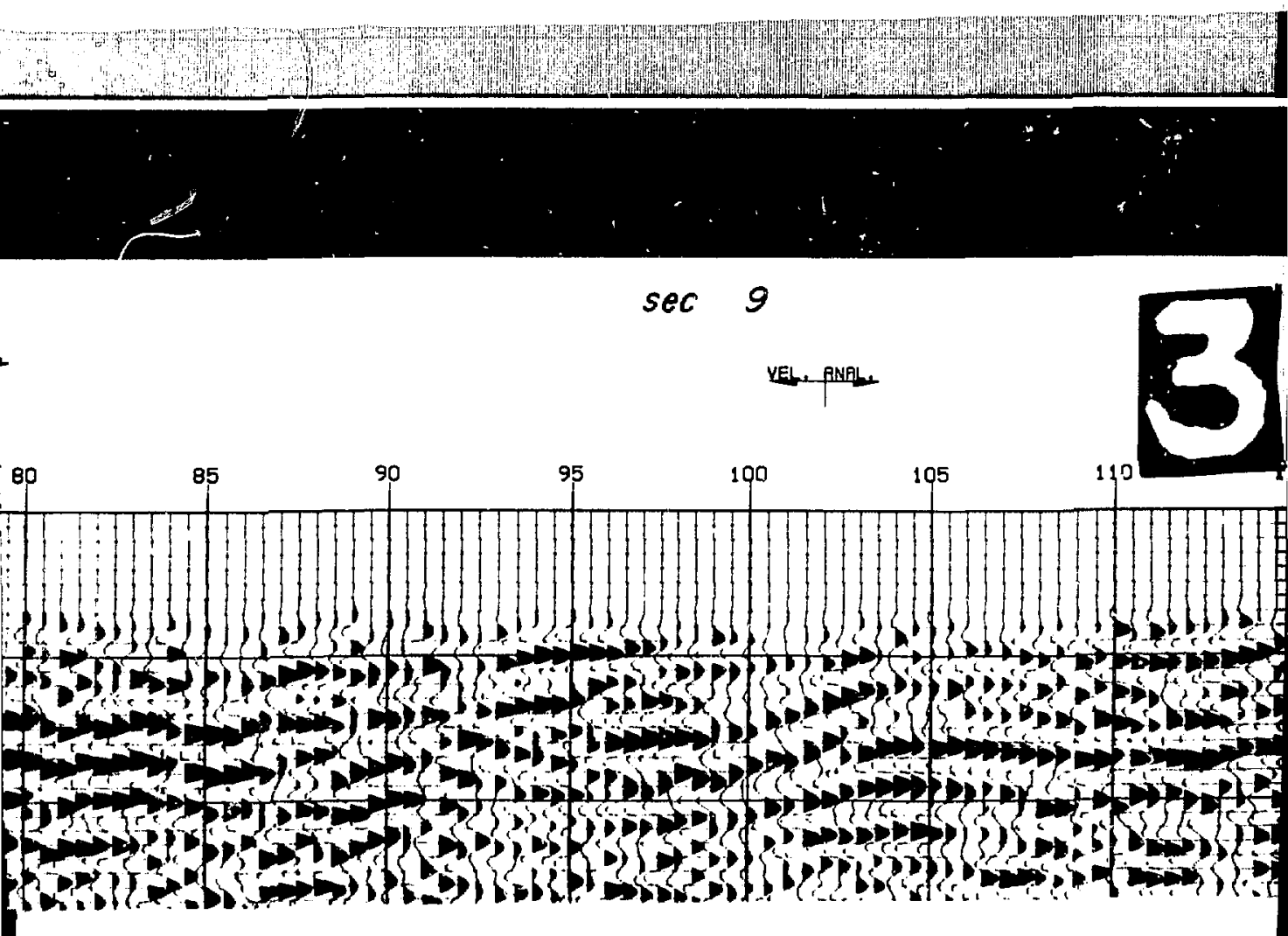



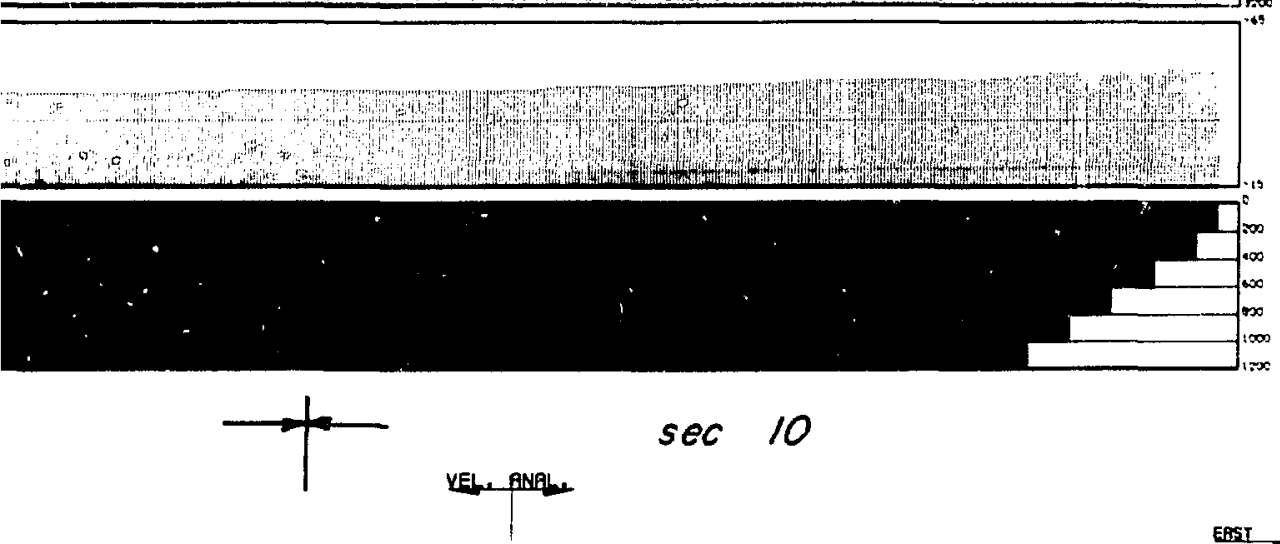


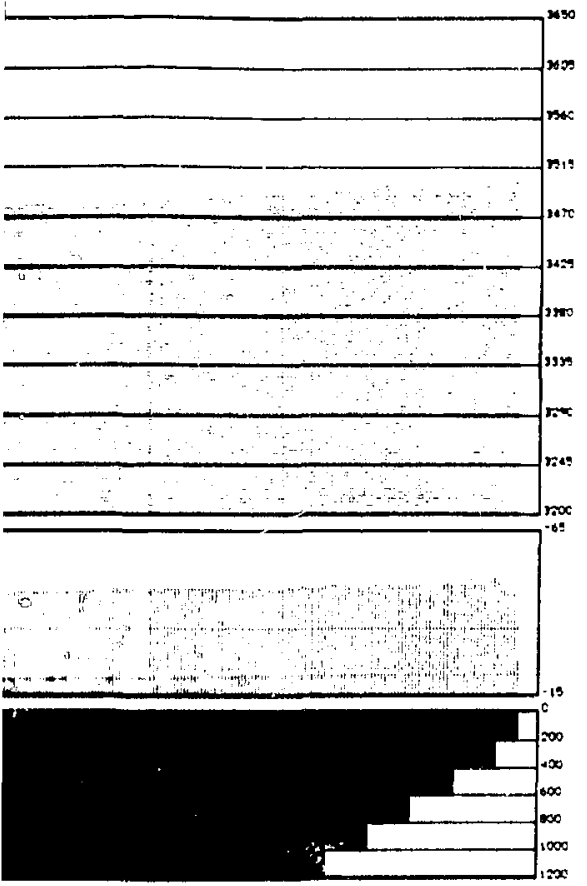

\section{ELEVAT I BNS}

STATICS

FBLD $x$

LINE DIRECTION

VELOCITY FUNCTION

Eas:

OLRECTION

LINE INTERSECTION

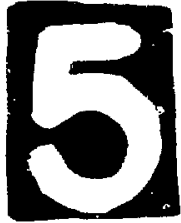

STATIQNS

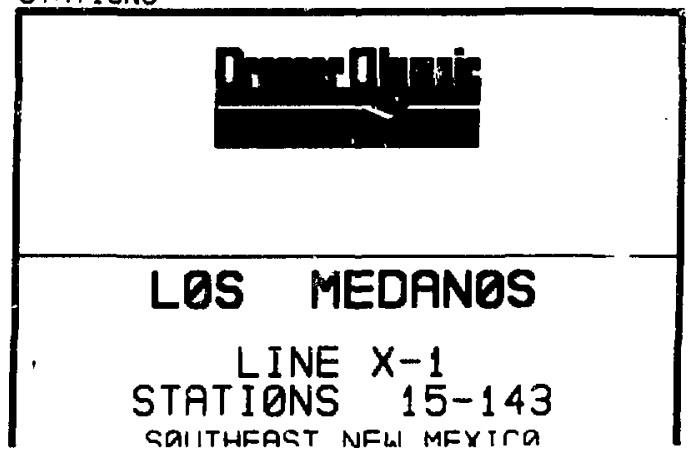

\section{LOS MEDANOS}

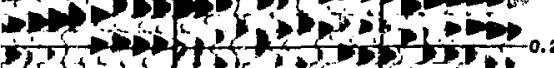

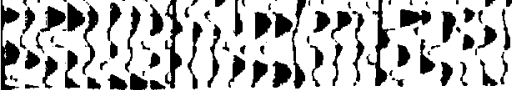




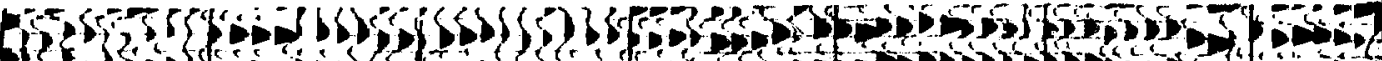

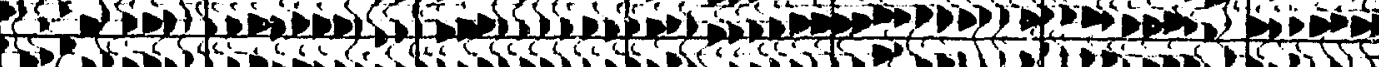

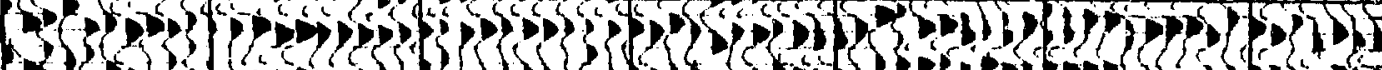

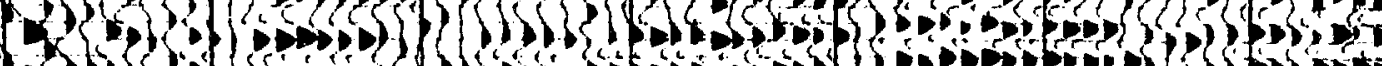

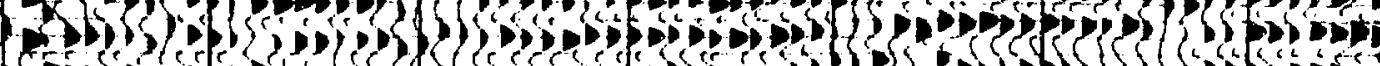

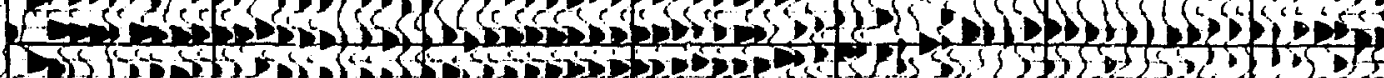

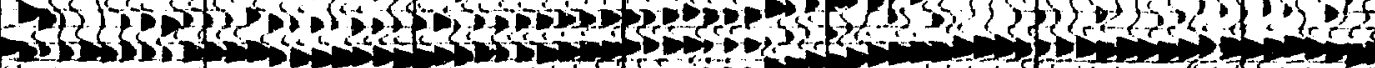

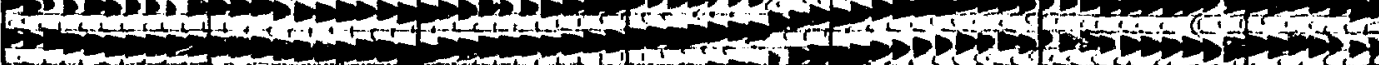

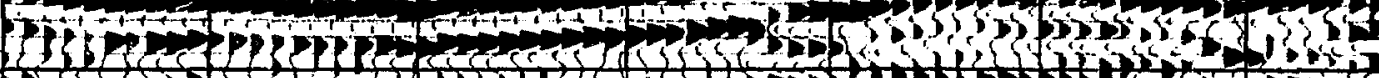

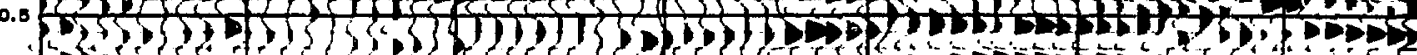

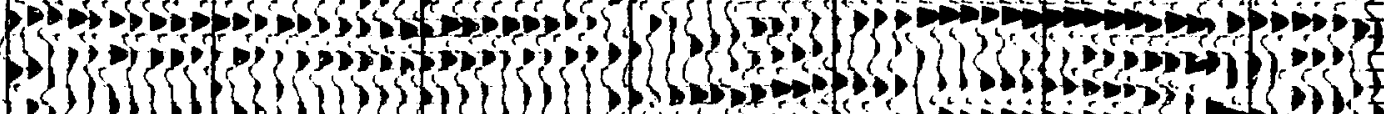

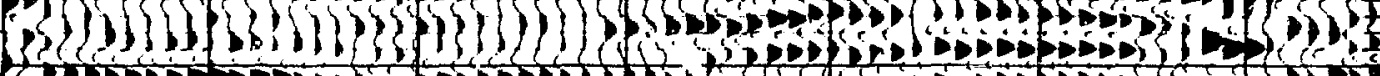

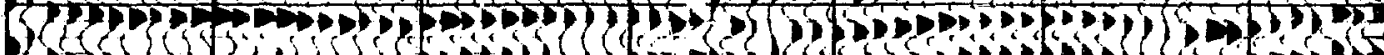

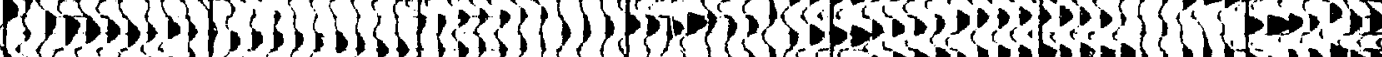

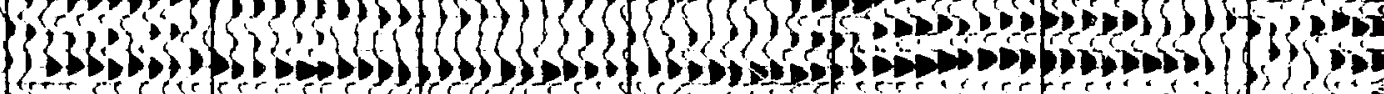

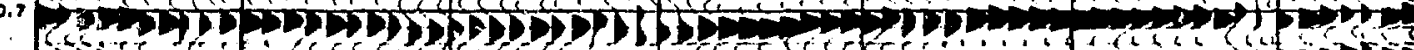

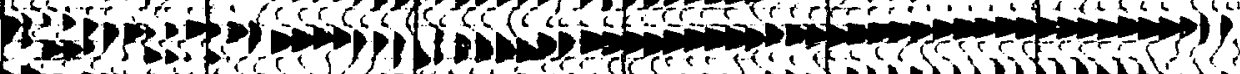

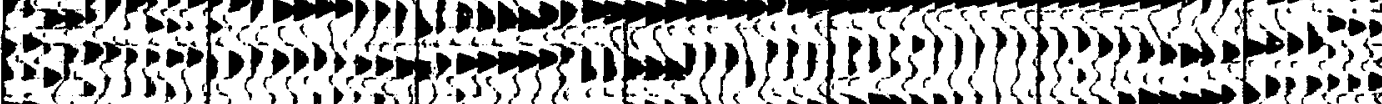

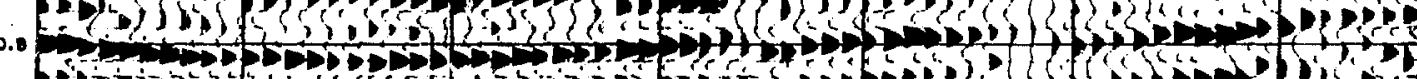

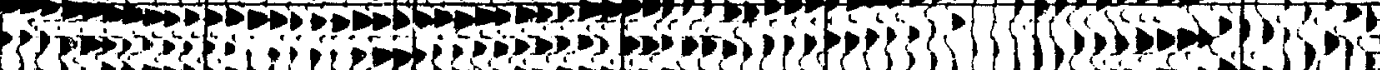

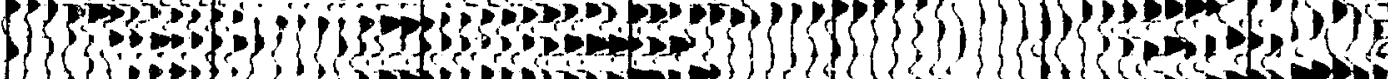

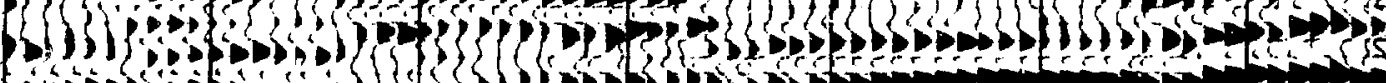

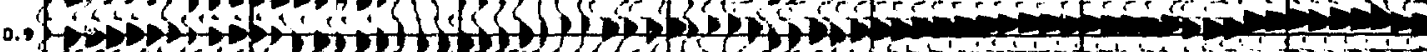

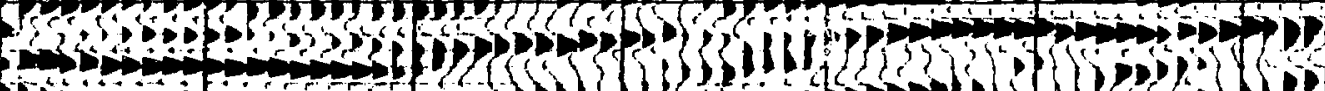

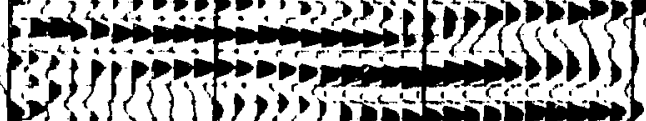

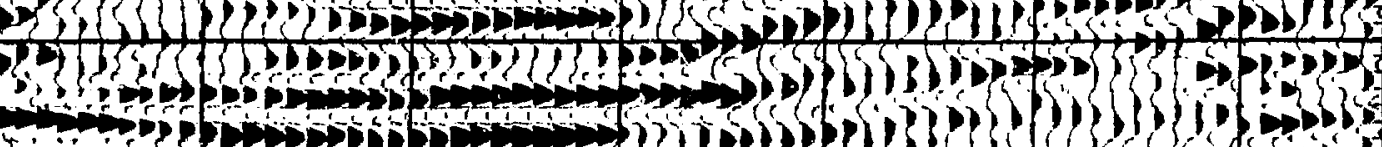

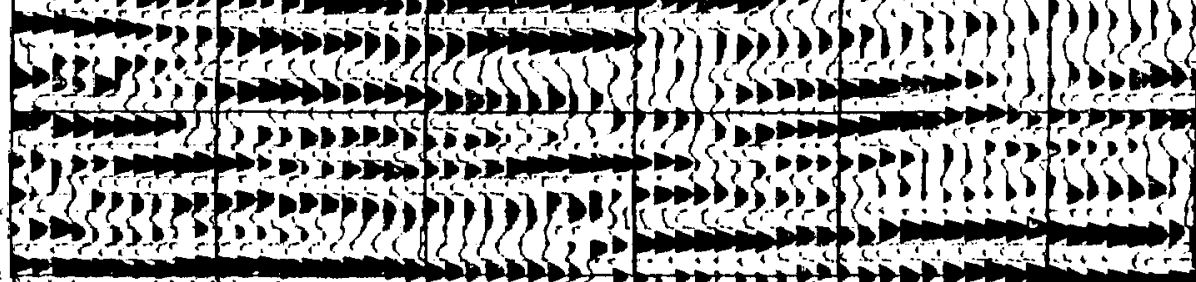




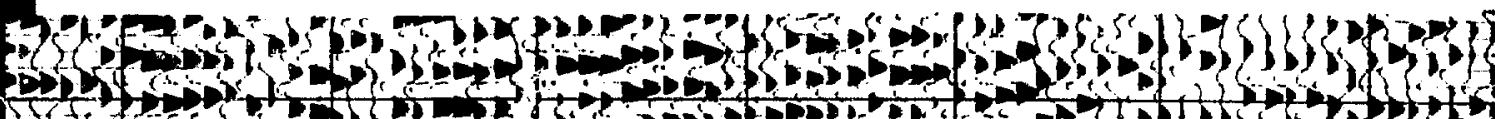

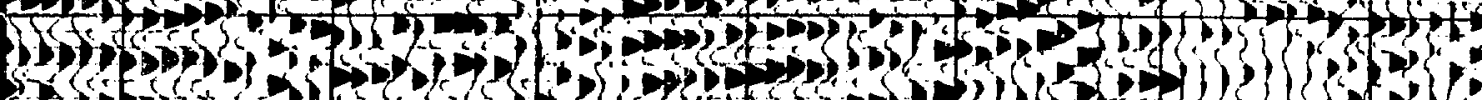

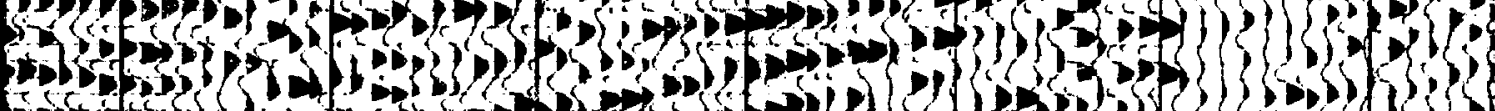

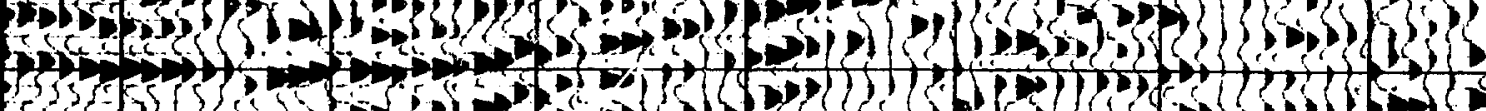
(5)

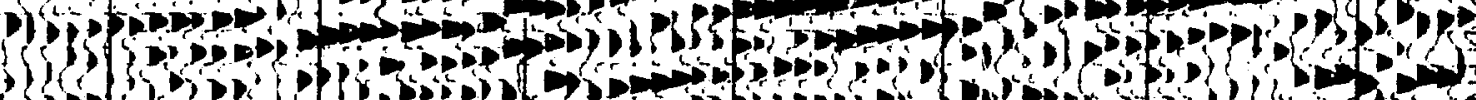

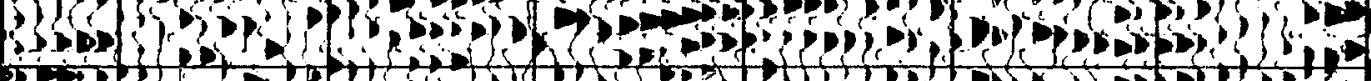

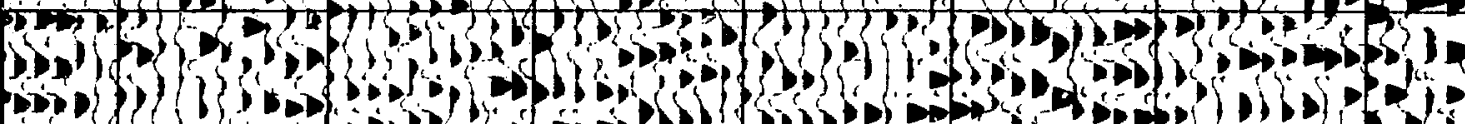

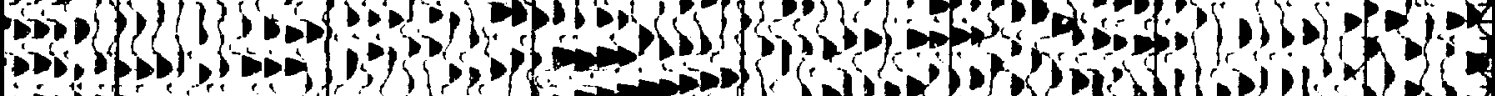

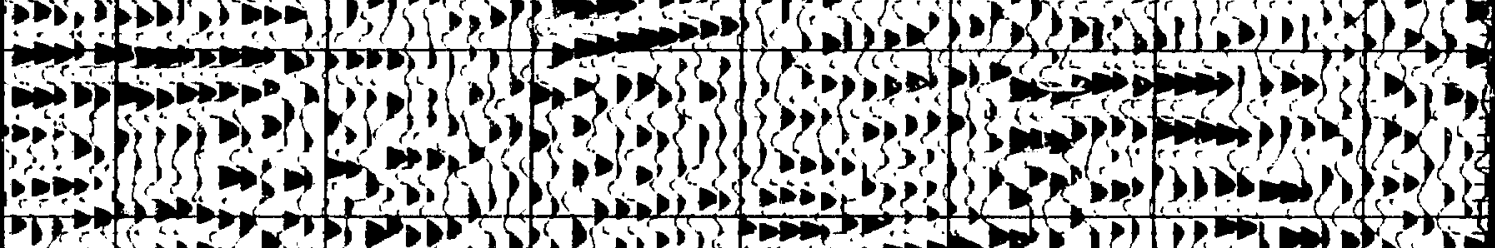
(1)

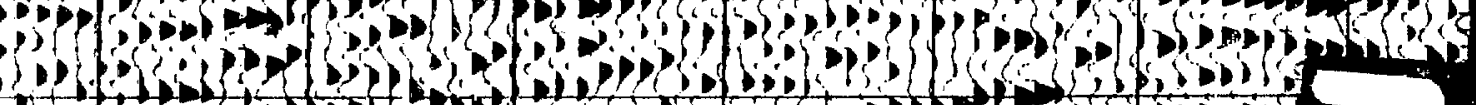

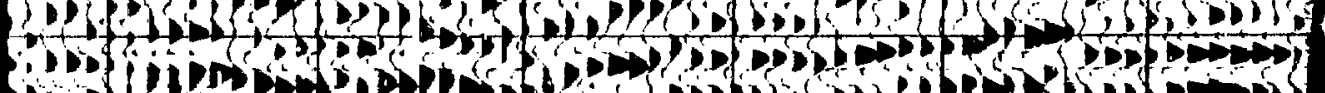

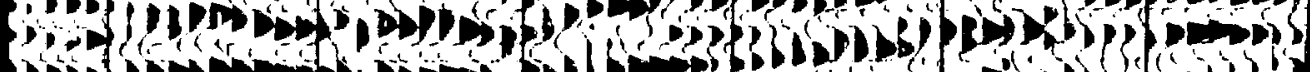

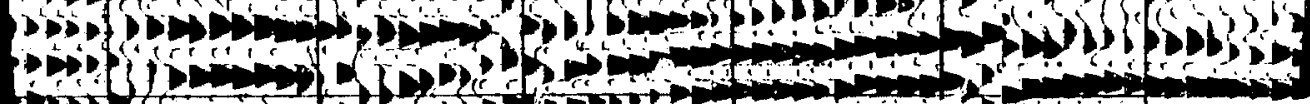
tor 


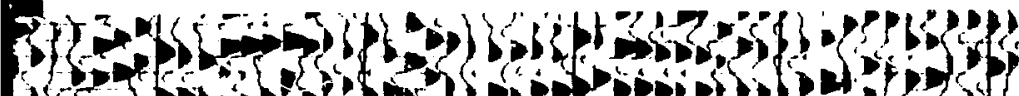

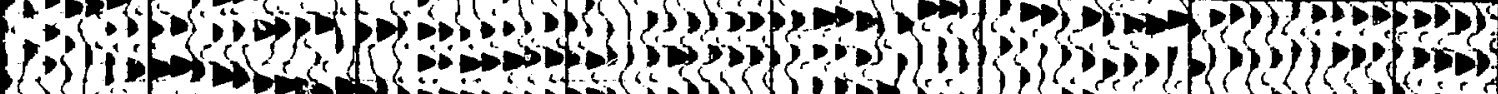

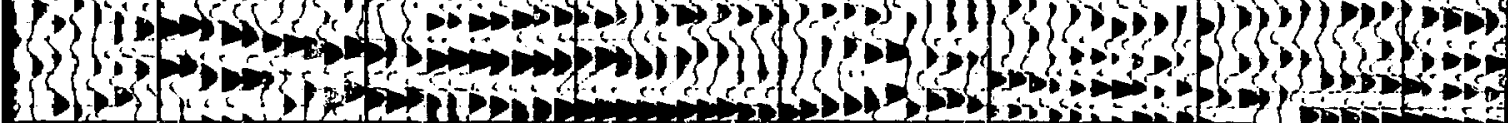
(190 w MPi.m.

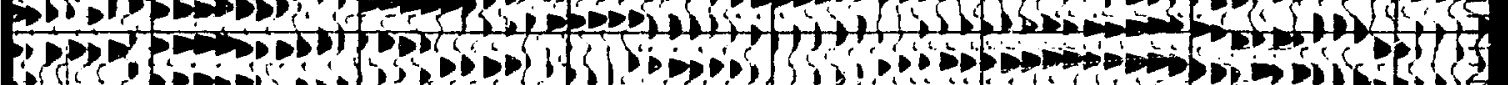

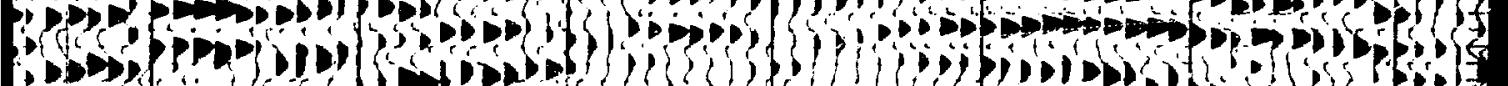

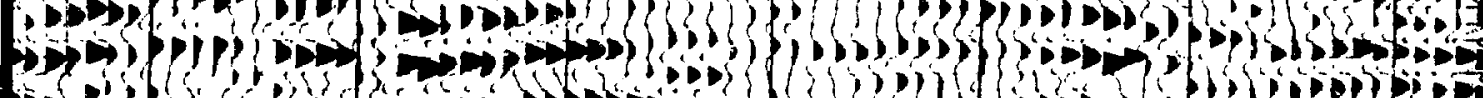

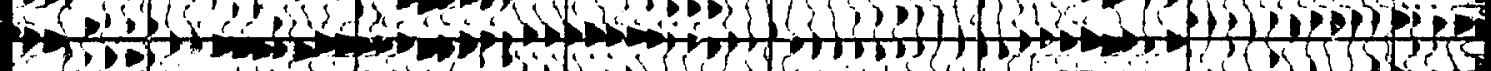

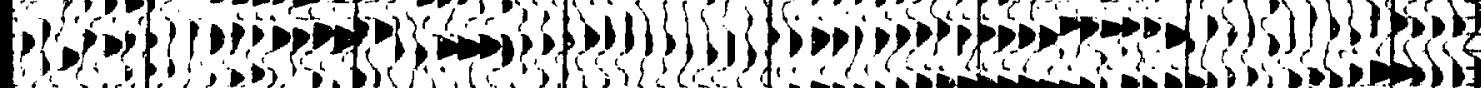

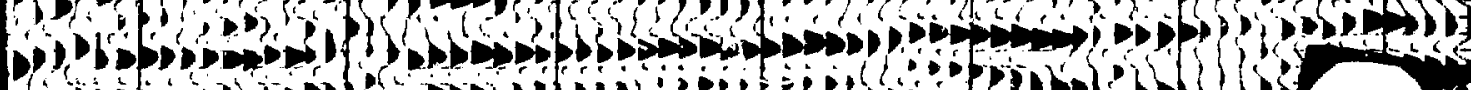
$\rightarrow+0$,

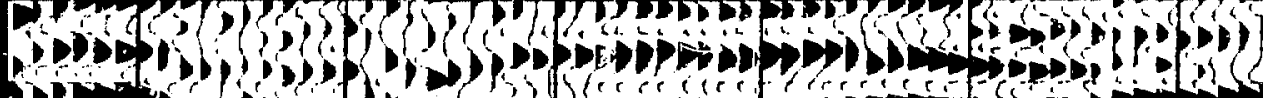

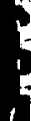

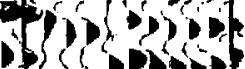
inisivipente 


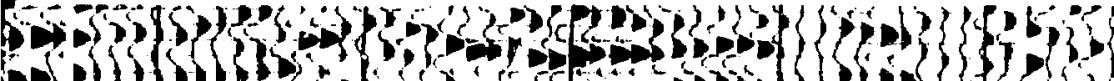

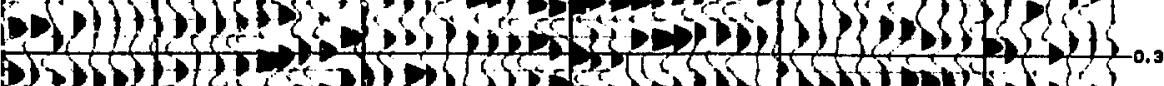

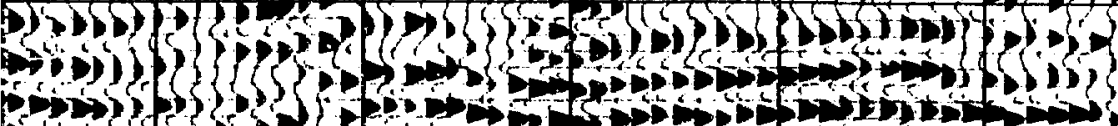

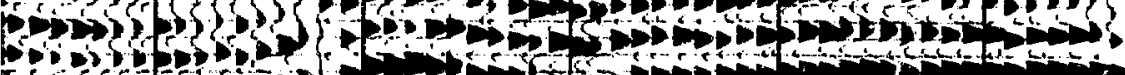
?

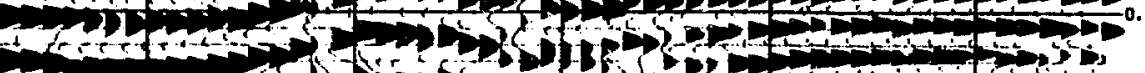
Donases on

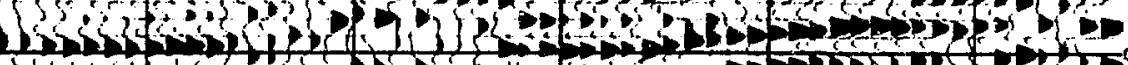

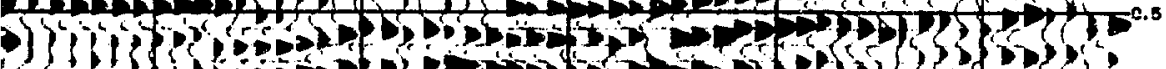

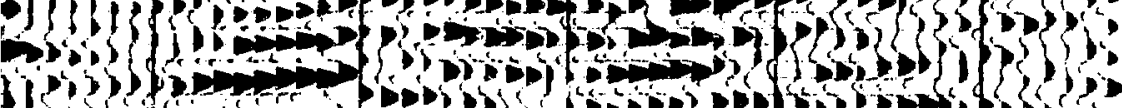

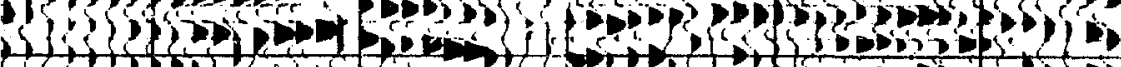

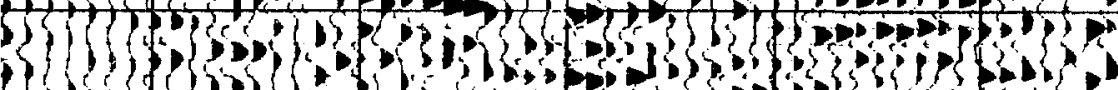

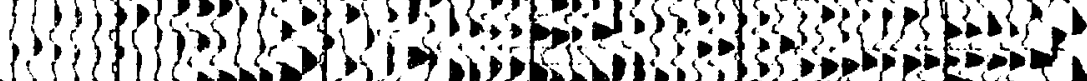

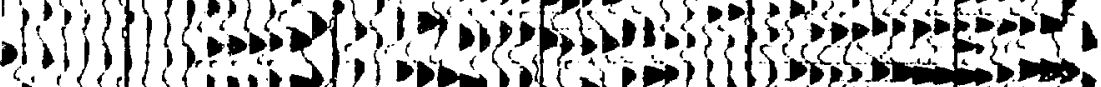
om Stibin,

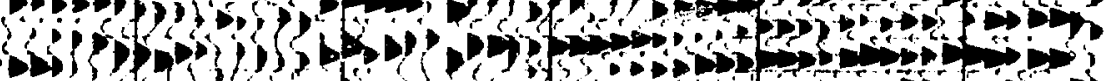

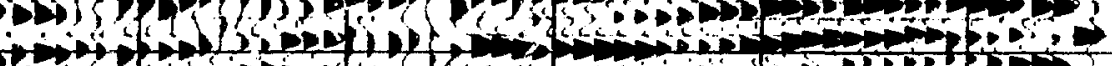

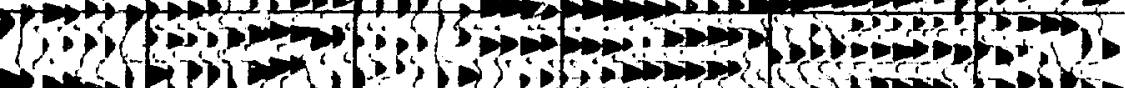

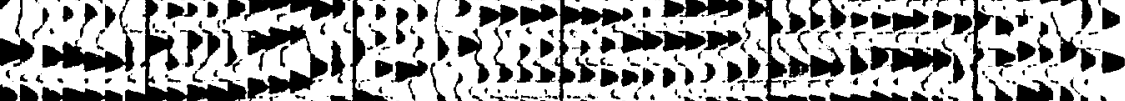

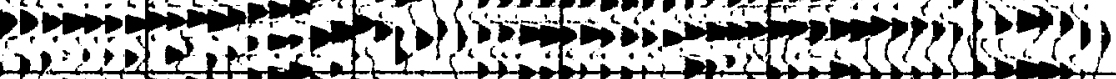

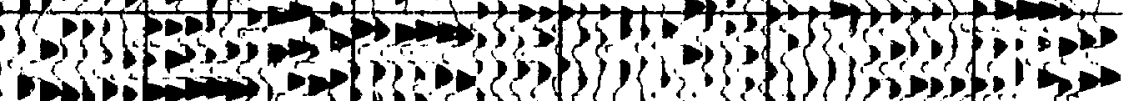
$B$ sos

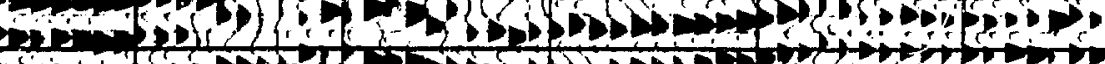

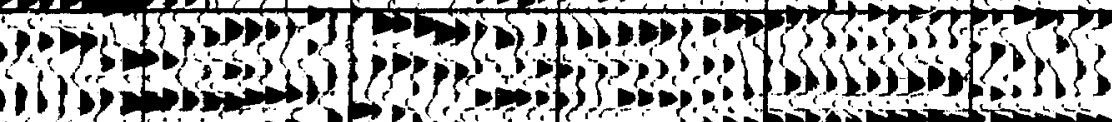

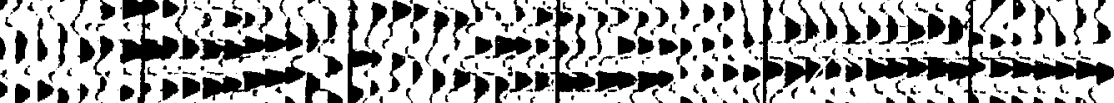

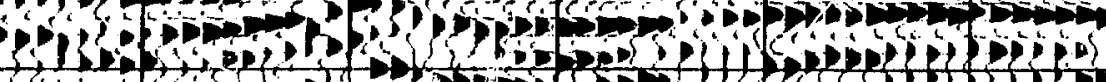

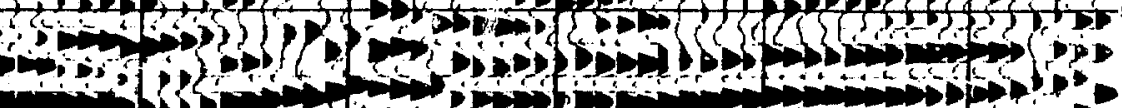

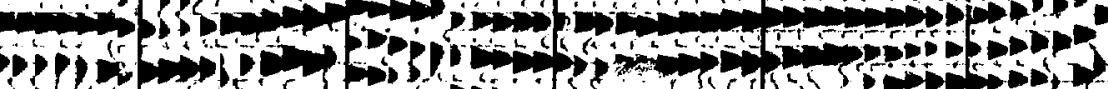

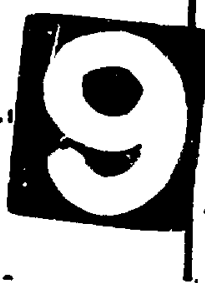

REC

INSTRMENTS: MTCH FILT: MECERO LEV: SIEEP REEO: STN INV: QEO PER STN: nutar TYPE

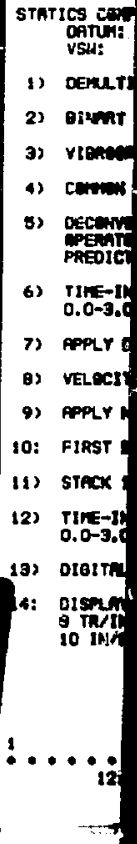




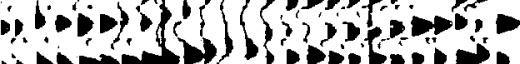
ind on

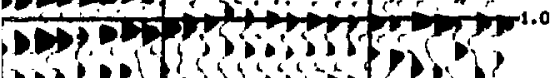

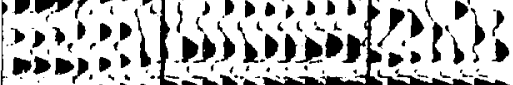

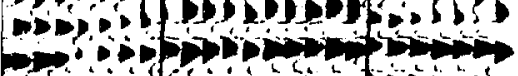

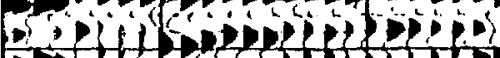
$\Rightarrow\{<\{\{<\{<\}$

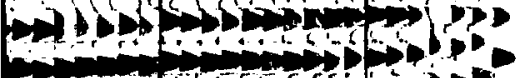

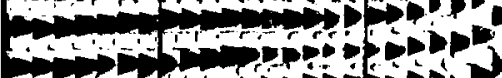

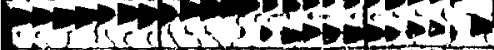

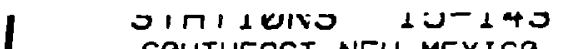
SOUTHEAST NEW MEXICO

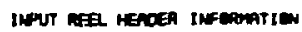

Fa

aile Enito $10 / 19 / 77$

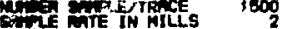

Proces

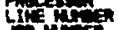

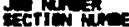

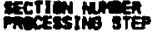

\section{FIELD INFORMATIBN}

hecenoco or: oresser unpis matr: Le. 62

OATE:

INSTRUANTS: CFS : - OFS iv SAMPLE AATE: 2 NS

MTCH FILT: III SEITCE: viluesels

NECEAD LEN: If SEC. GLEE LEN: 12 SEC.

SUEEP FREO: 20-100 HZ MISAUPS 24

STN INV: 110 Ri. V18. INV: IID FT.

GEO PEA STN: 6 GEO TYPE: BSC-200

mint IYPE: IRLINE TYPE COVEA: $1200 \mathrm{menT}$

\section{PROCESSING SEQUENCE}

PATEESSE GY DRESSEA Q MTPIC

STATICS CENOUTATIN

Datlen: go00 FT.

VSU: $\quad 6000 \mathrm{FT}$ ISEC.

1) cenltiplex

2) OImar oatK Recovear

9) VIEANCE Is COPEELAT IEN

4) CENM OEPTM POLNT GATHERS

b) oecenreution

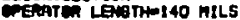

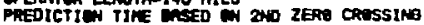

(1) TIGE-IHVMINT DIBITA FREOCHCY FILTER 0.0-a.0 SEC. 20-00 HZ

7 peply tarum statics

b) Velectir amersis

פ) mplr wo

10: Finst exen supasssien (WUTE)

11) $\operatorname{sTa} 12$ Fod

(2) THE-1MNARIANT OIOITR FAECUENCY FILTEA $0.0-9.0$ sec. $20-60 \mathrm{kT}$

1a) Diosta ad

14: OISFLAY

- TR/IN

iD IN/SEC.

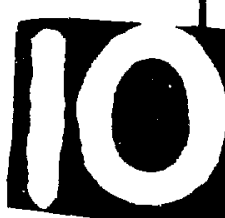

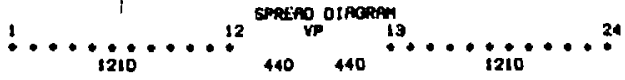




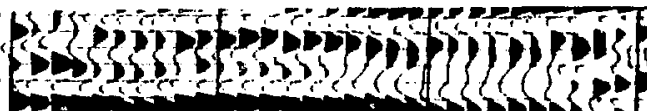

Com

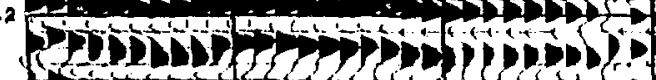

stsiom

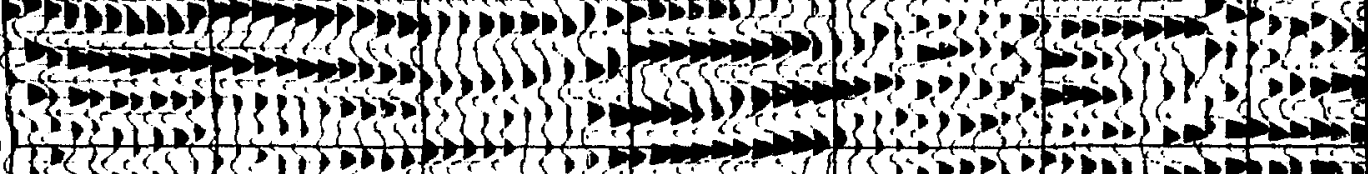
(3.1. B. - .

3

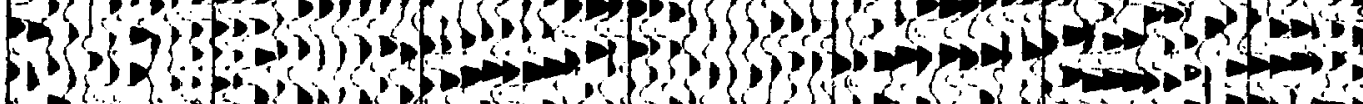

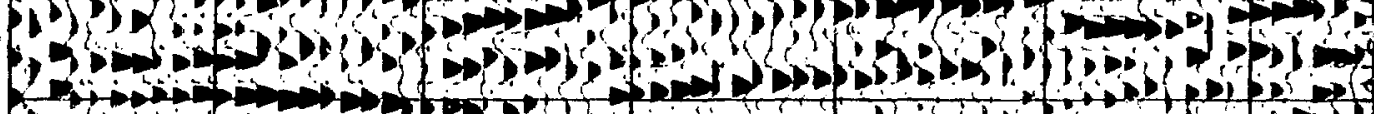

60

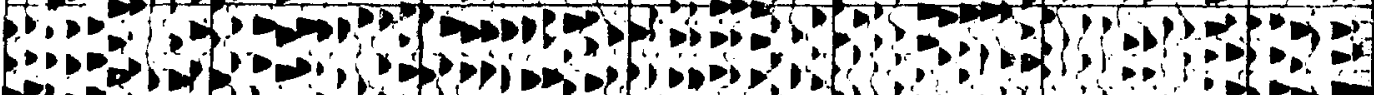

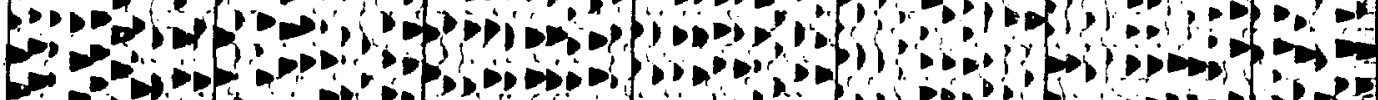

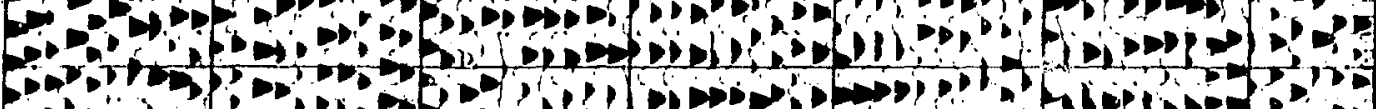
or. Q.2.

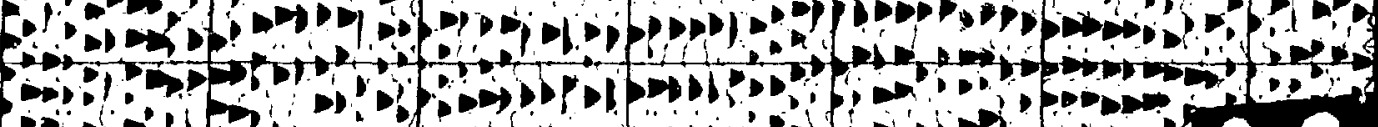

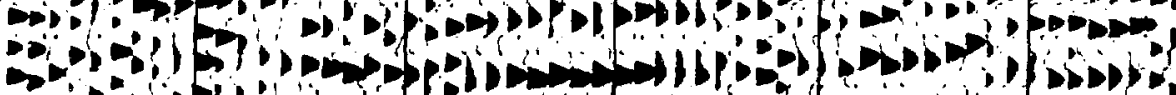
ax:

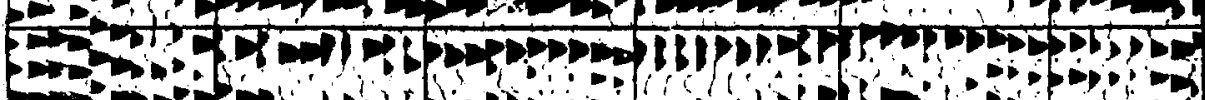

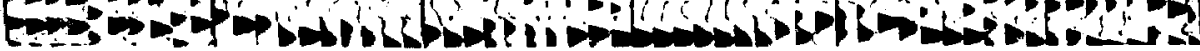


H

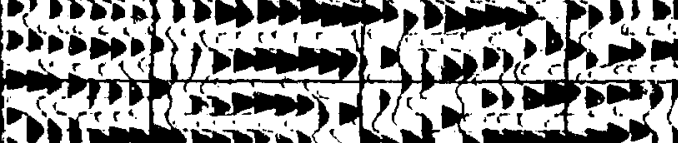

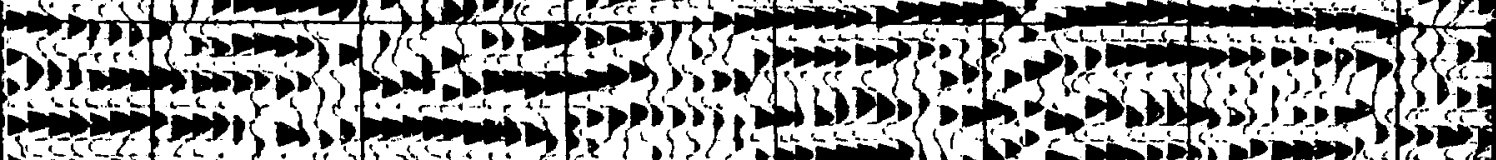

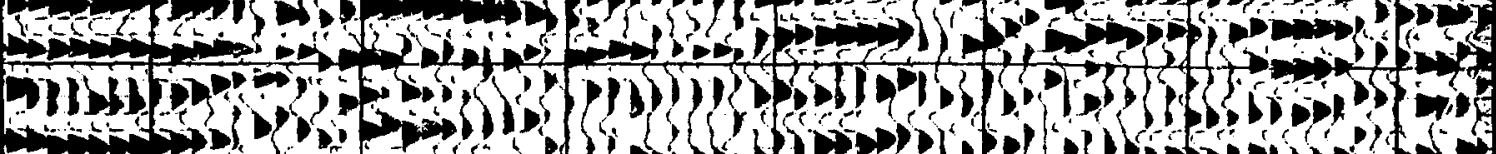

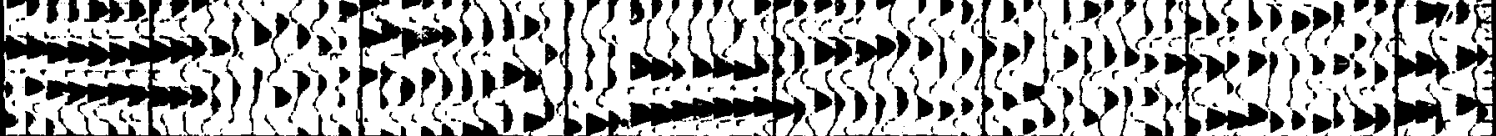

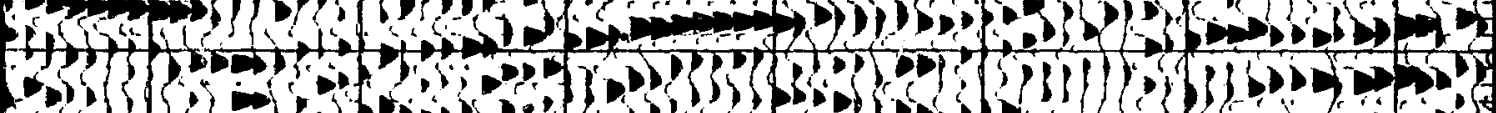
P mons Ans E. H.

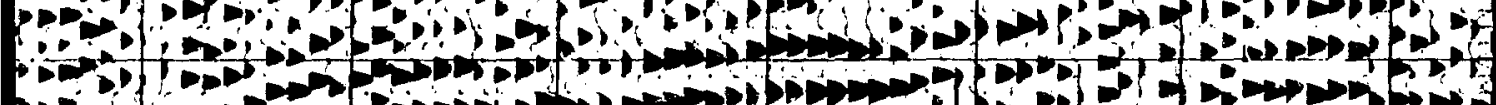

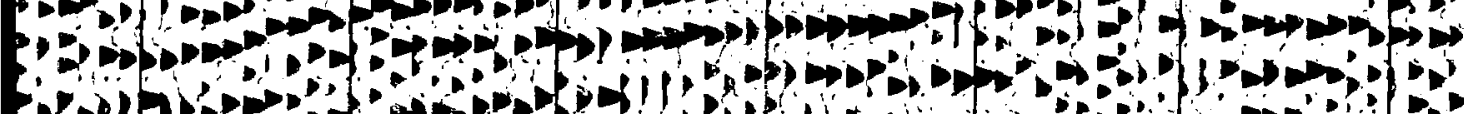

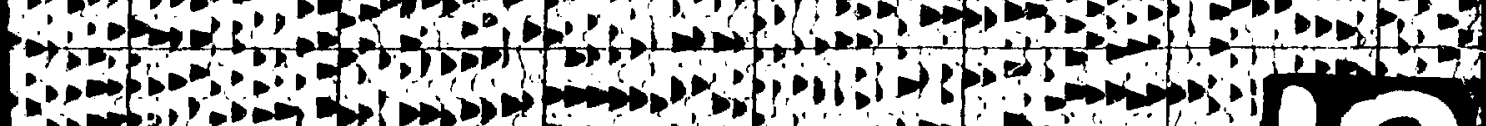

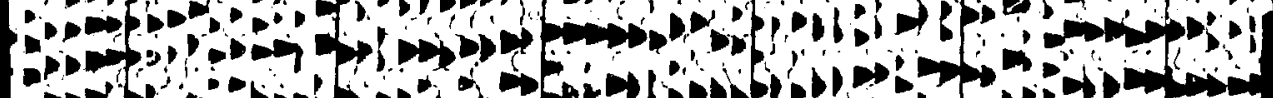

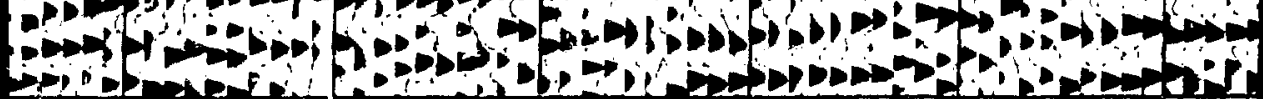

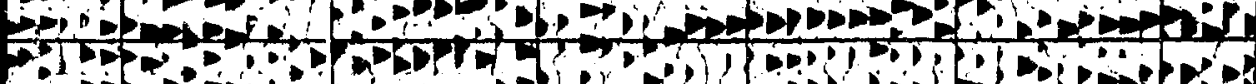

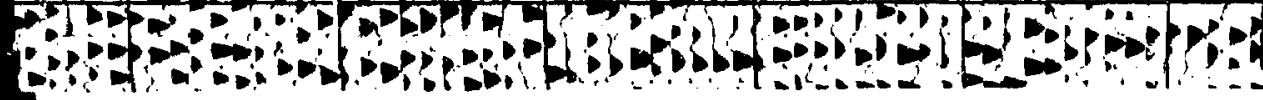

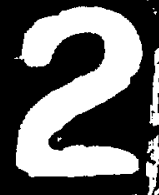



4 D

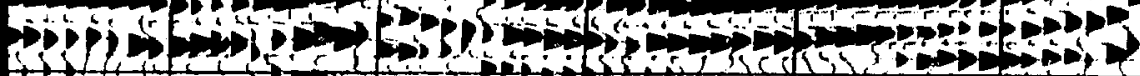

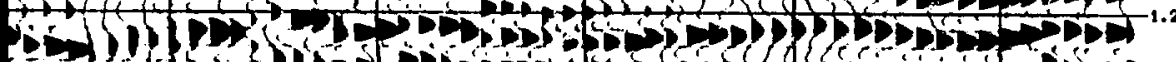
(12)

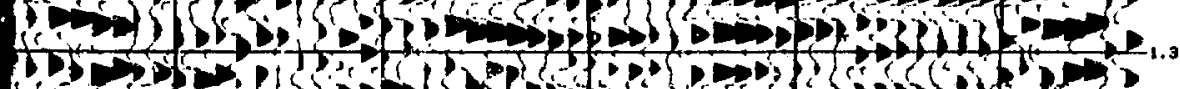

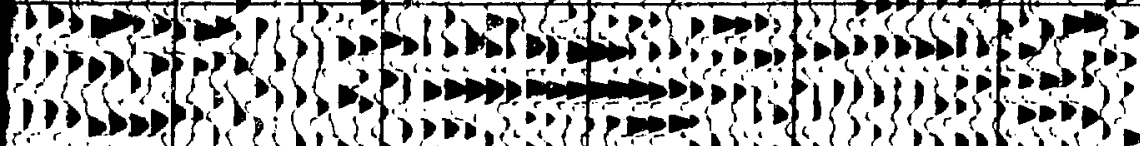

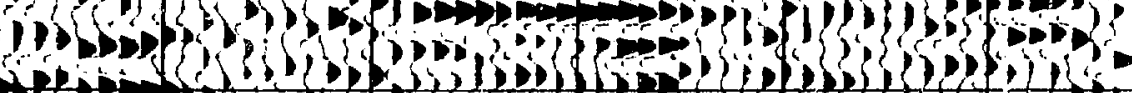

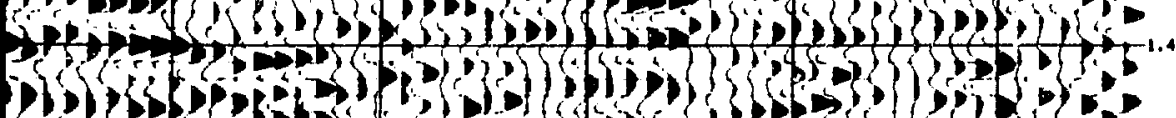

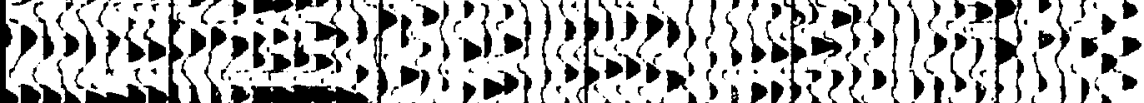
S)

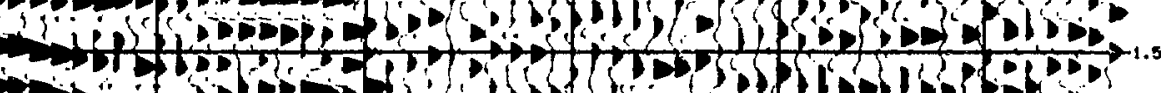

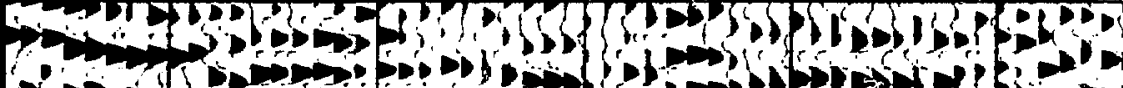

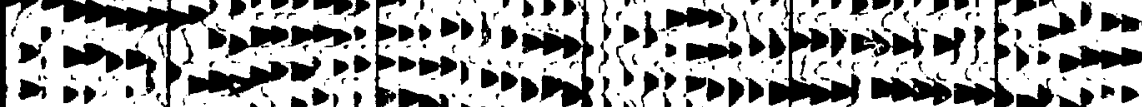

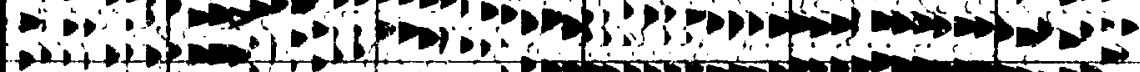

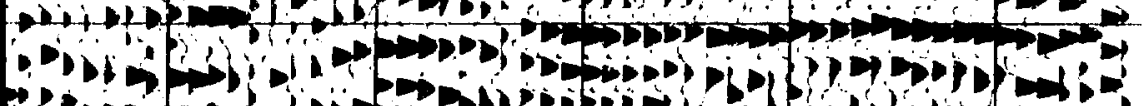
$\Rightarrow$ -

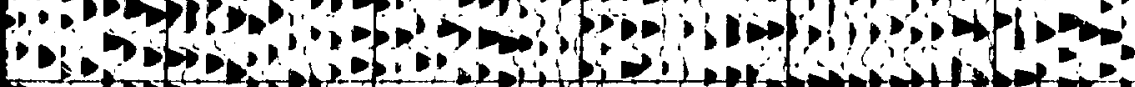

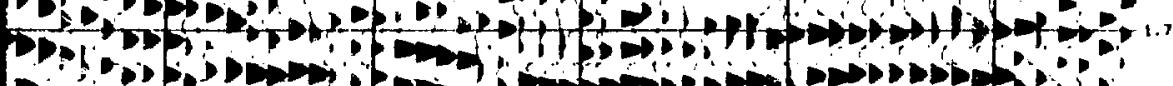

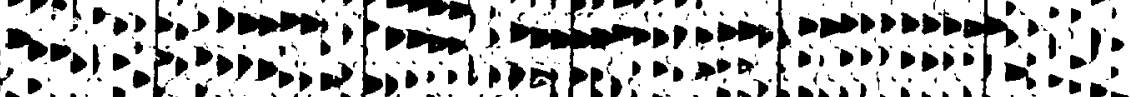
-

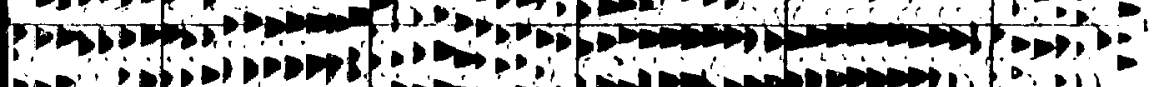

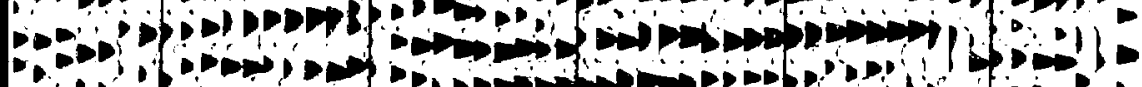

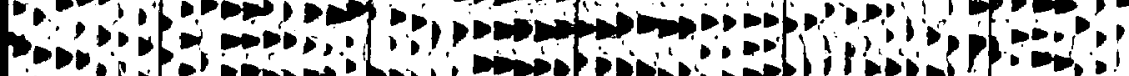
-

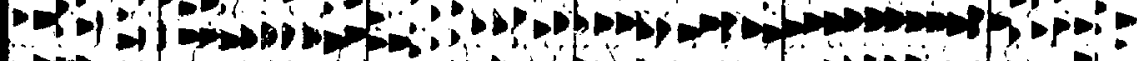

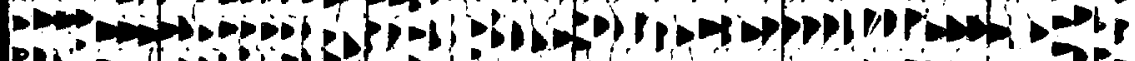

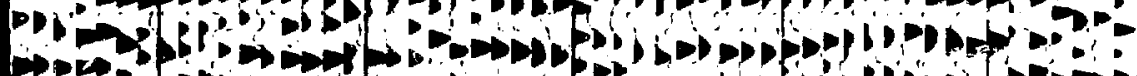

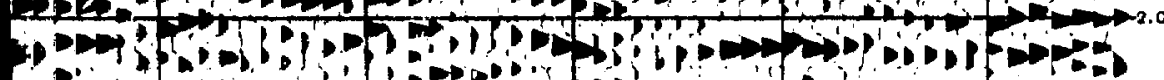

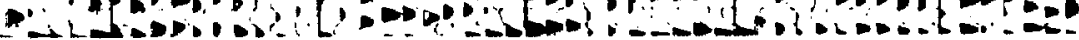

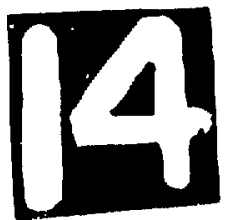




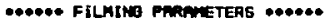

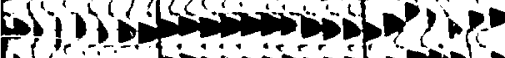

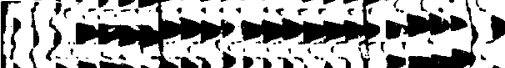

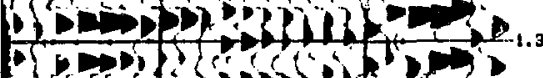

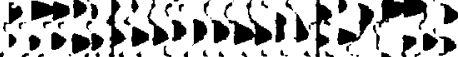

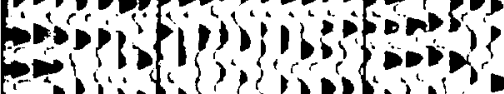

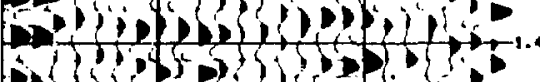

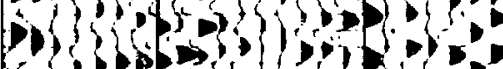

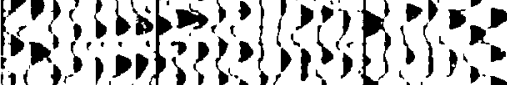

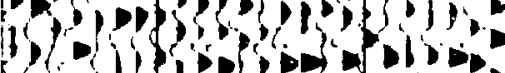

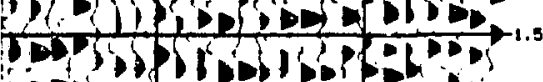

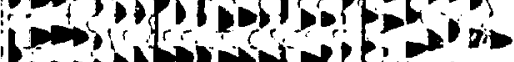
$\Leftrightarrow ;$ 350 1.

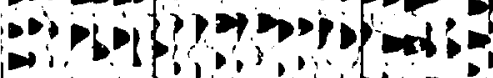

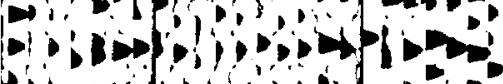

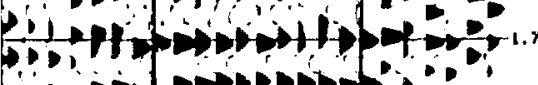
$\rightarrow x_{0 \rightarrow 0}$

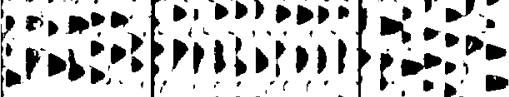
$\rightarrow \lim _{0 \rightarrow 0}$

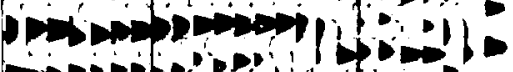

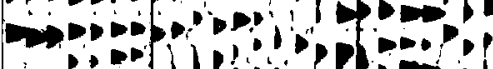

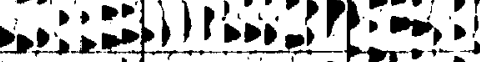
7 ing

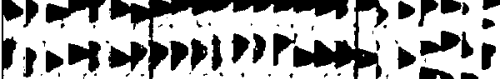

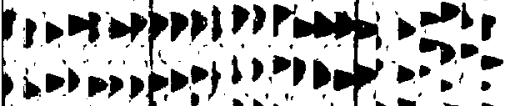
7.7

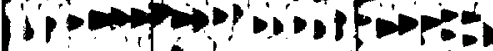

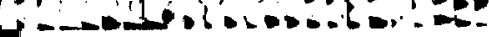

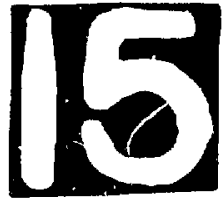




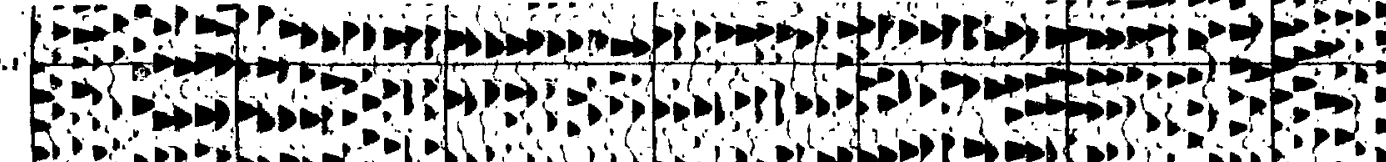

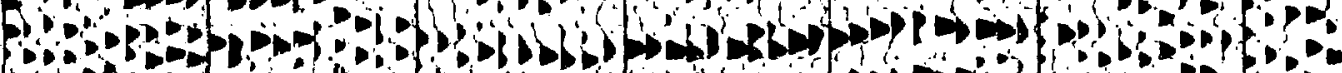
3

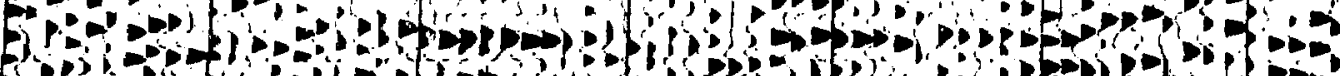

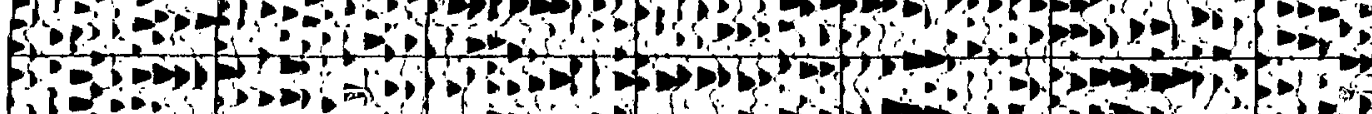
20.1.5. Th . T.

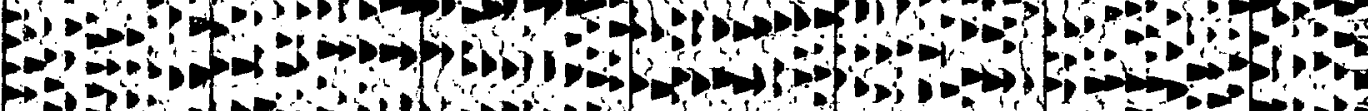

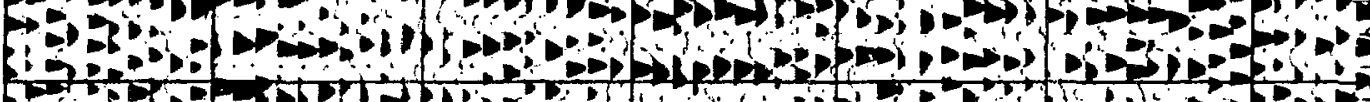
7. (3)

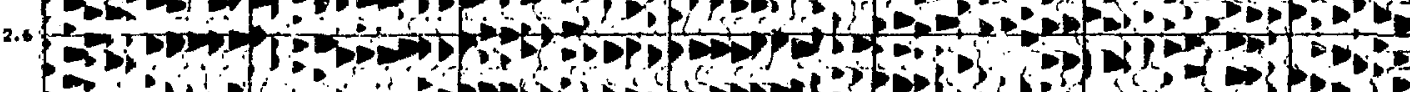

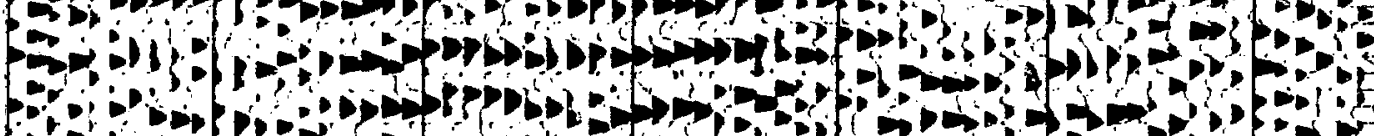

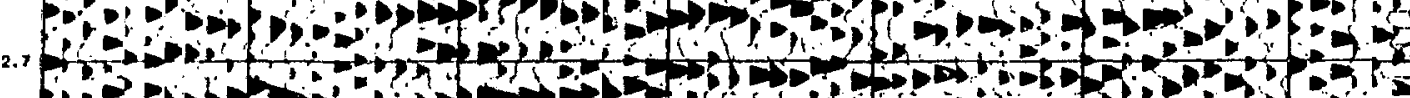

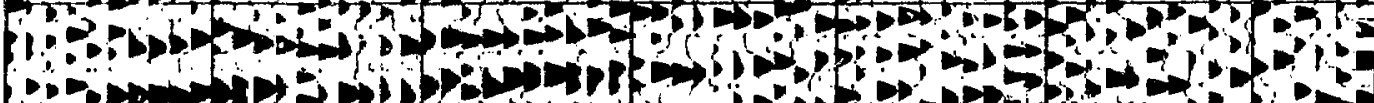

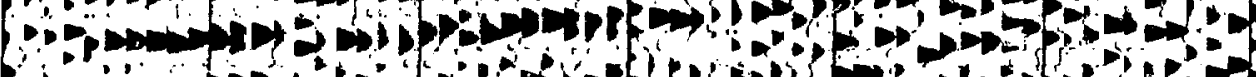

-

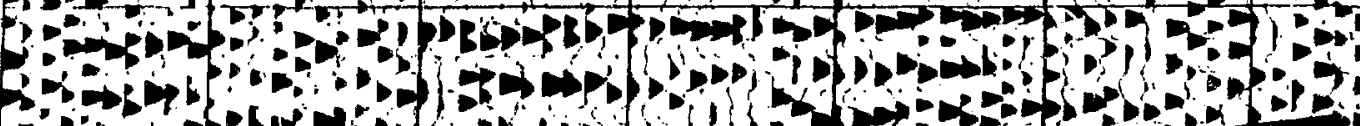

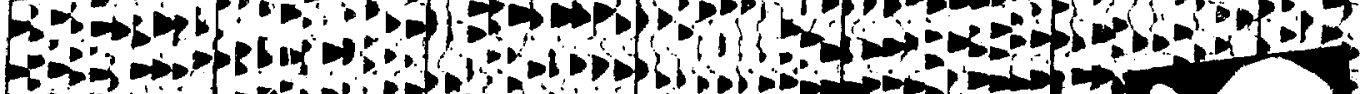

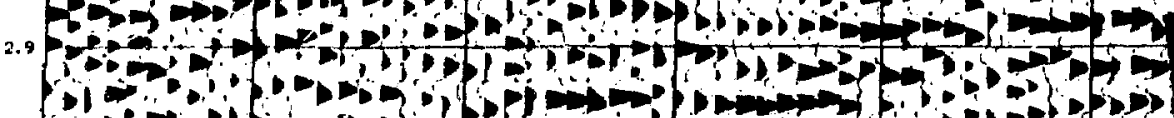

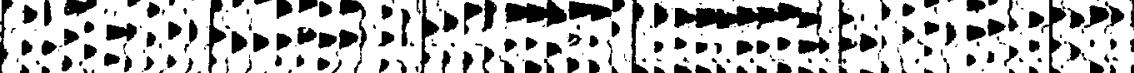
; if?

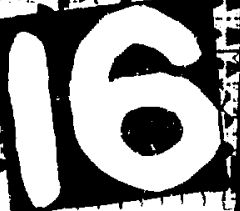




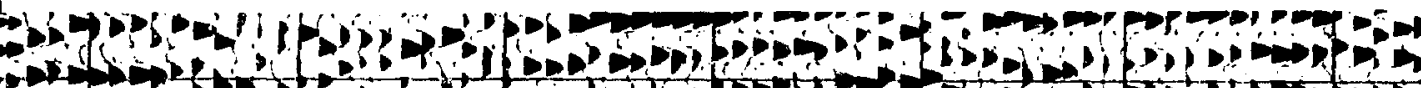
- $-4+2$ o

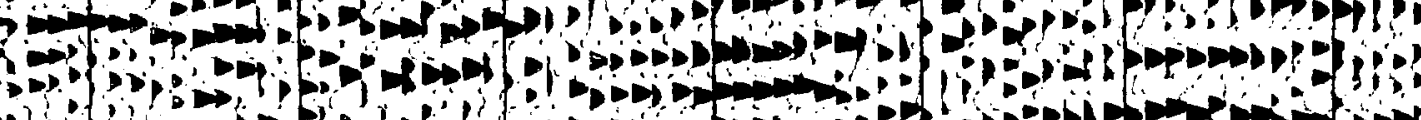

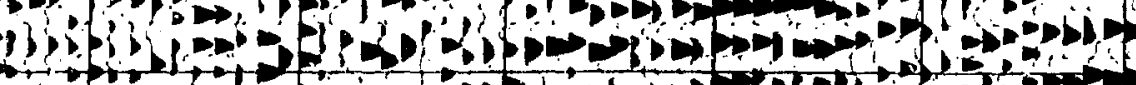

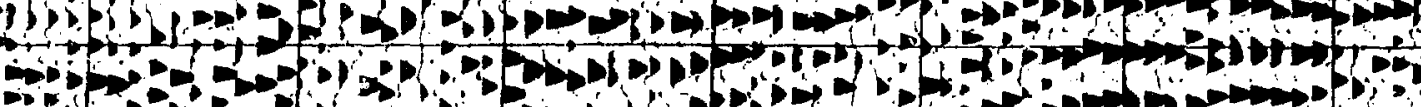

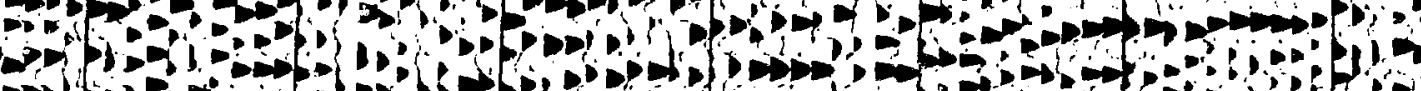

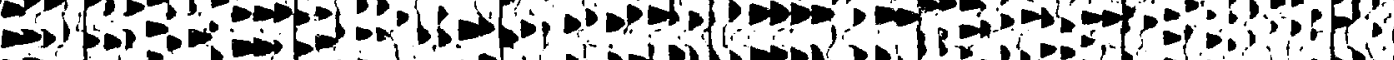

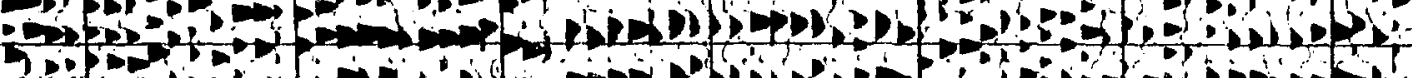

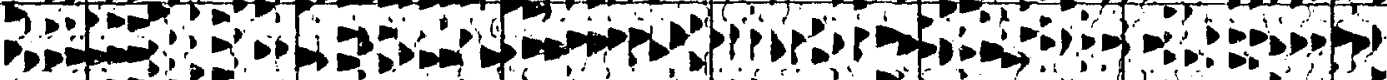

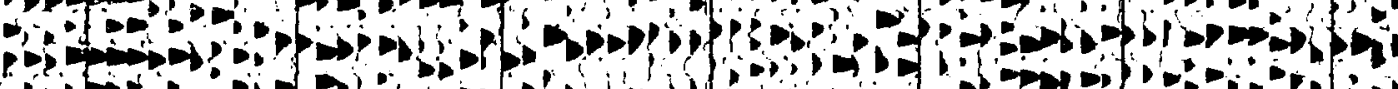

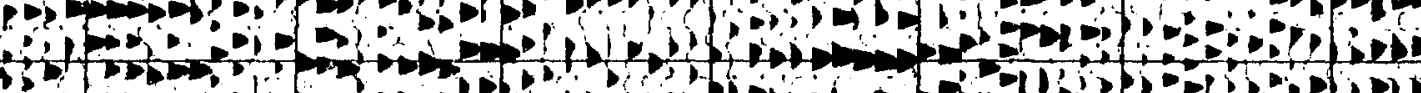

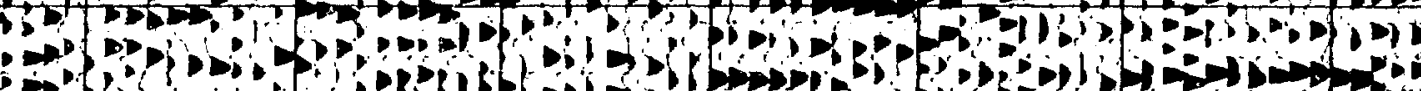
Pin

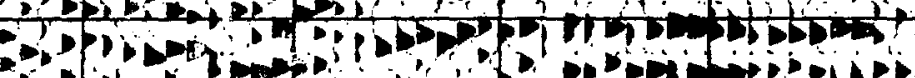
H.... 2.

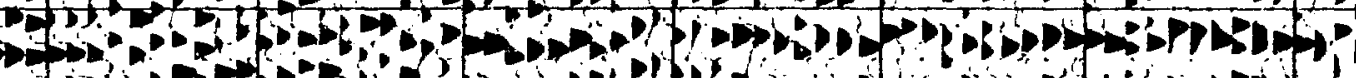

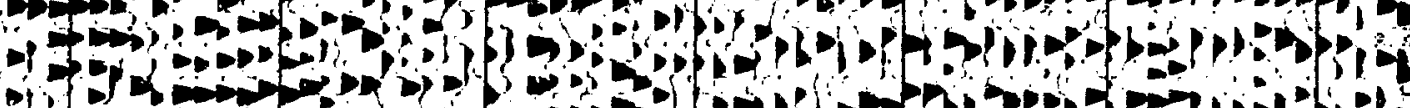

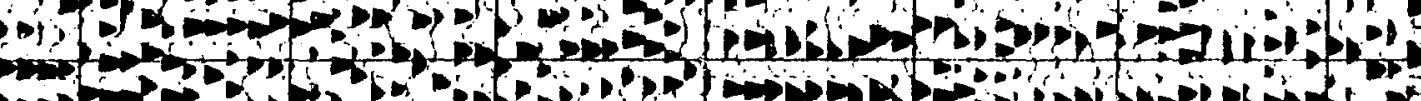
c.s.

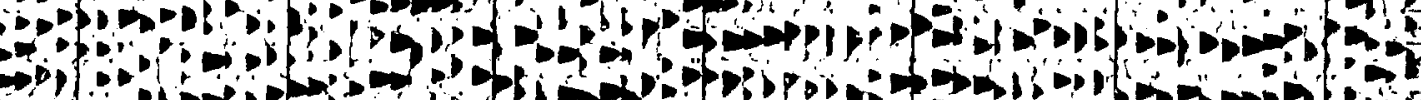

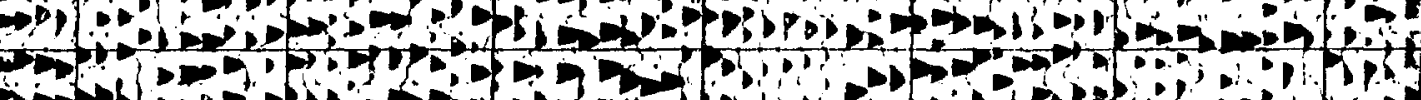

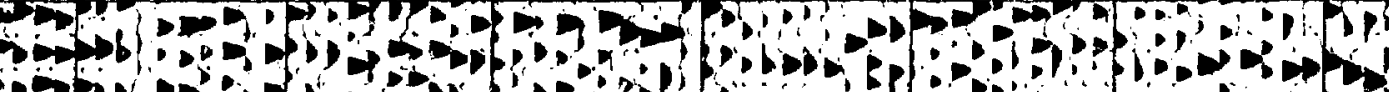

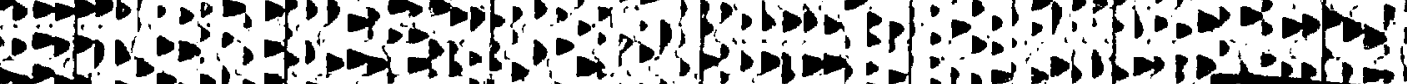

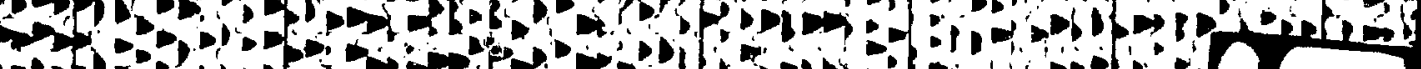

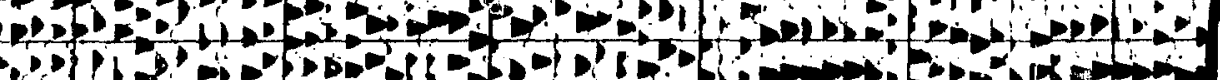

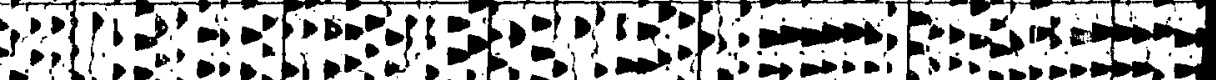

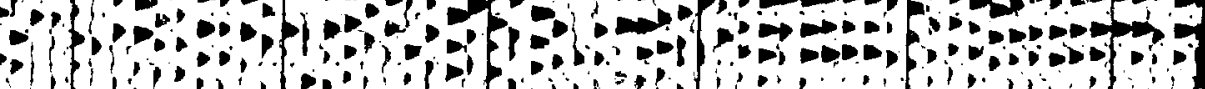




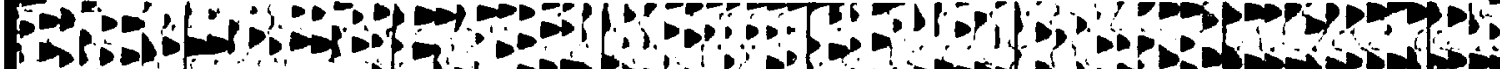

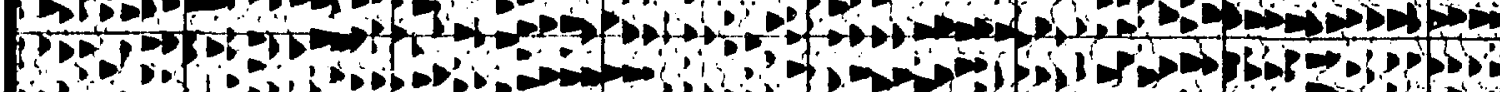

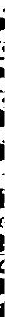

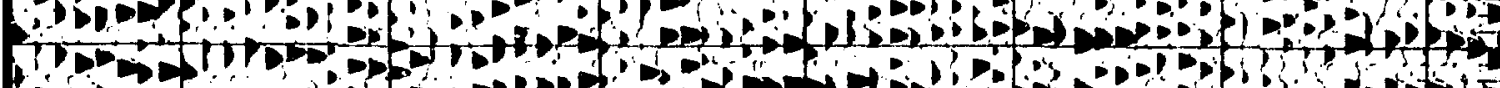

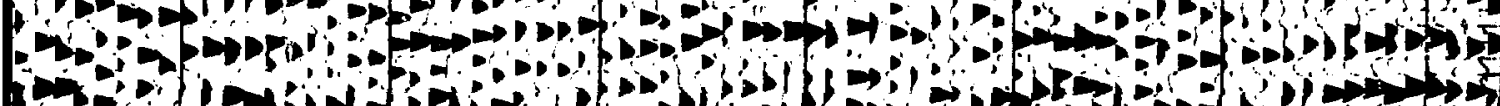

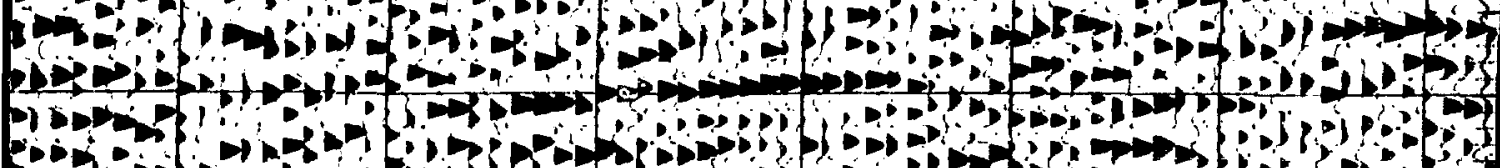

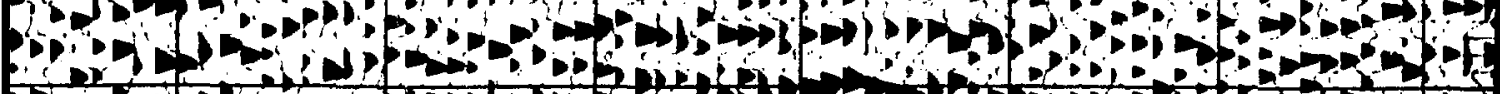
3
3
4 


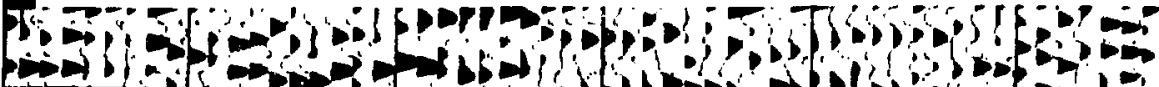

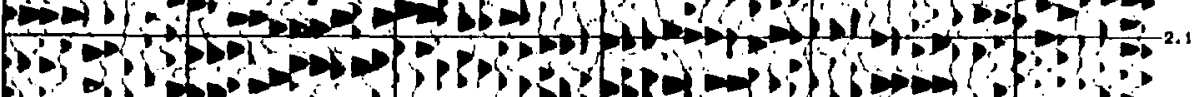

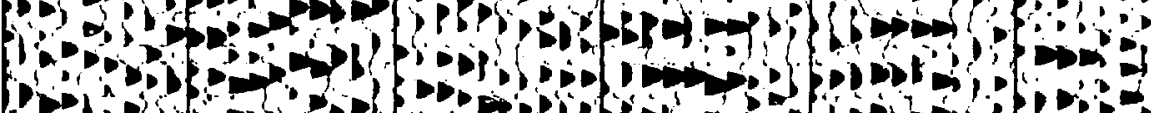

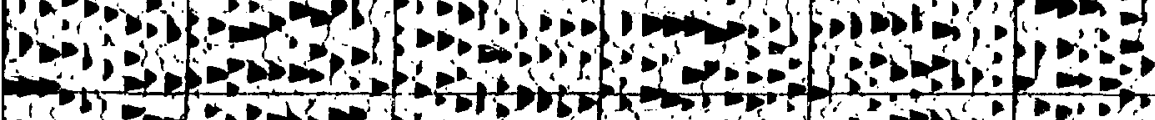
9 D.

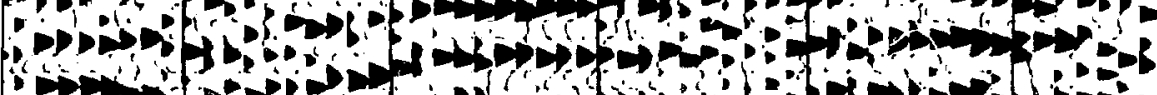

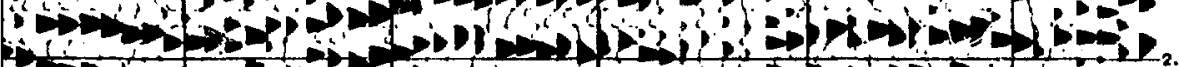

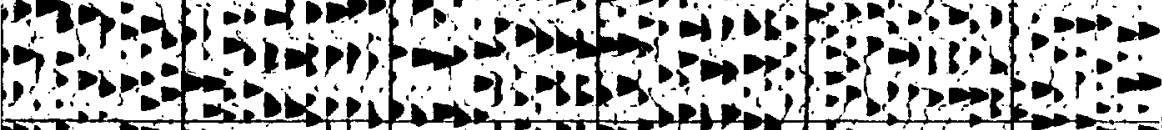

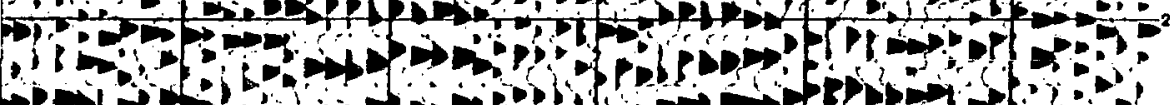
ith BD.

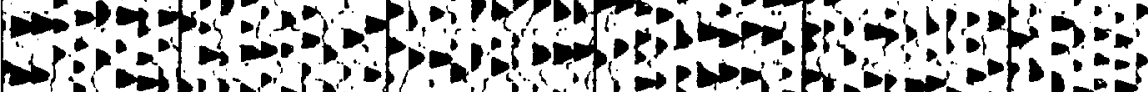

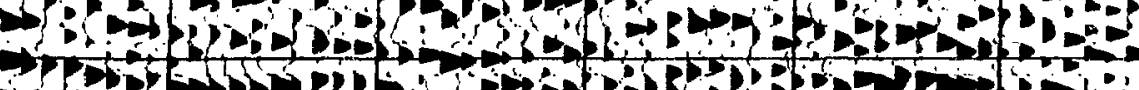
incil

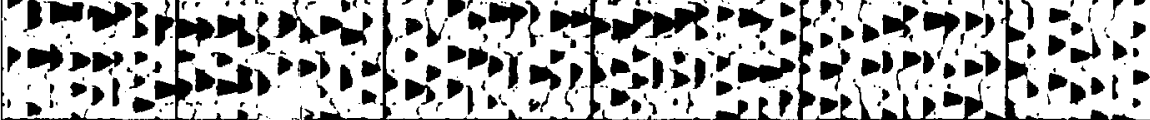
Dof

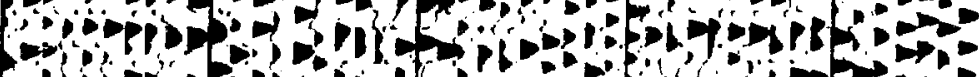

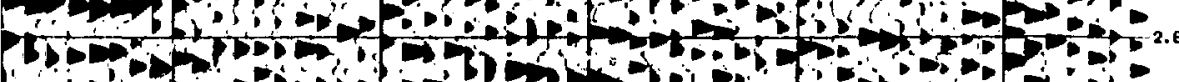

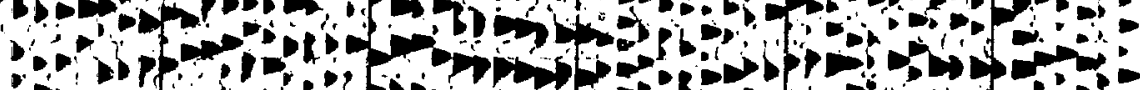

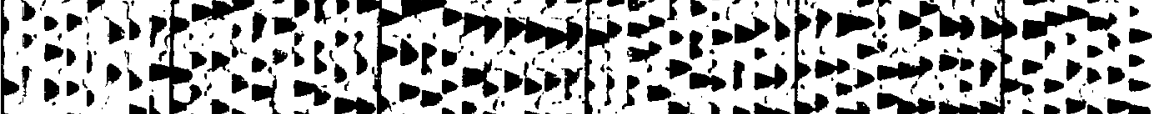
GP... Pub

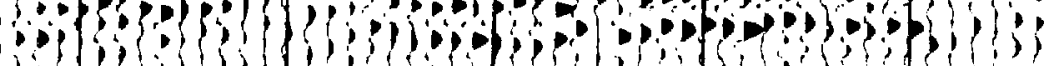

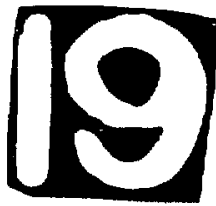



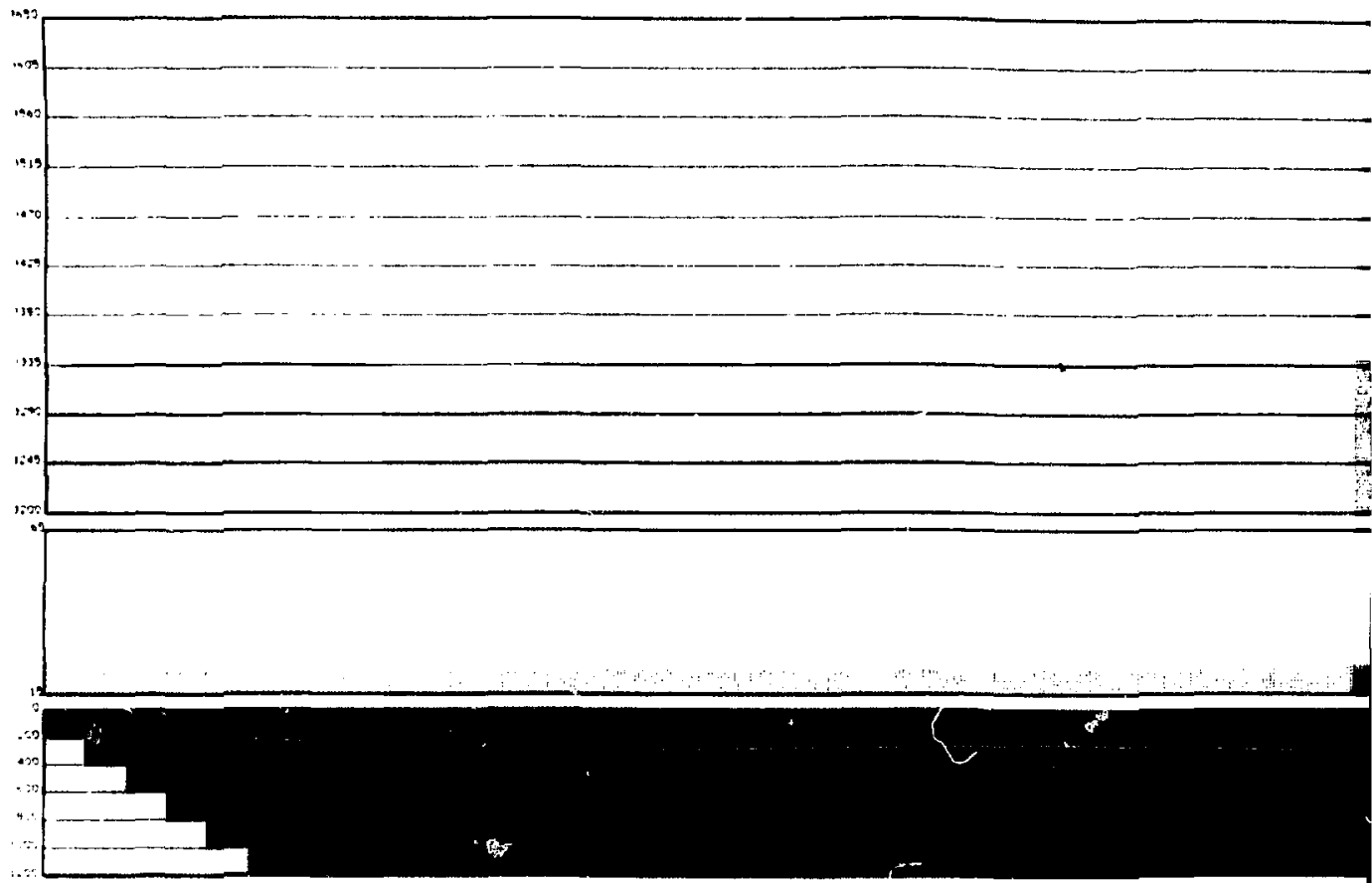

\section{$\sec 5$}

VEL. BNAL1,

MPTH
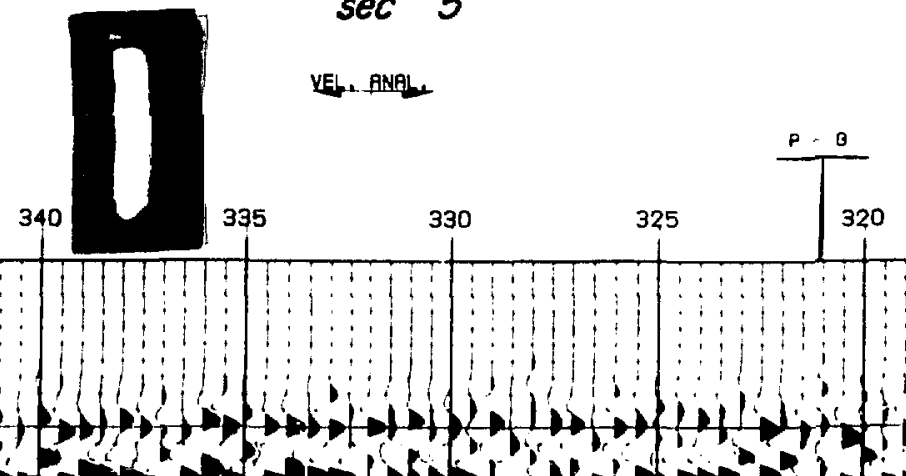

320

348 $0.10 b_{0}$

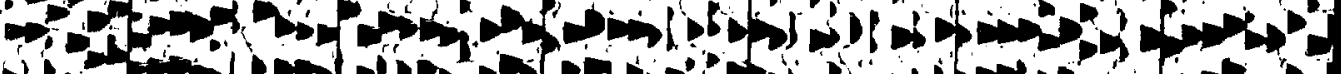

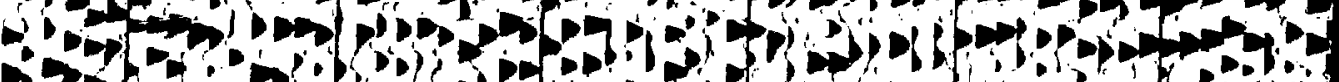

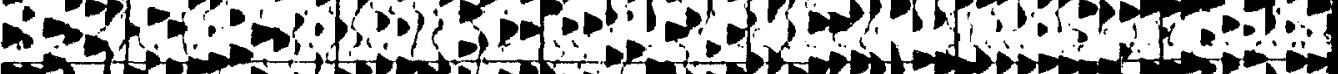

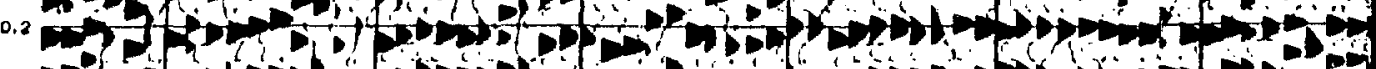
-1 a

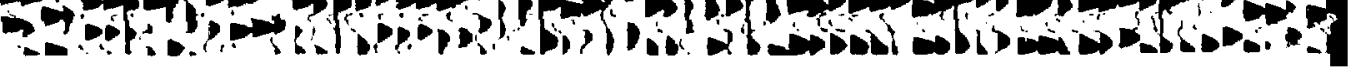


VEL, FNALL

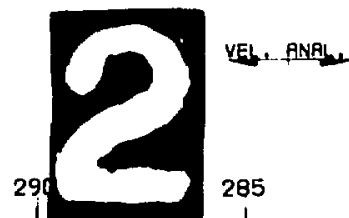

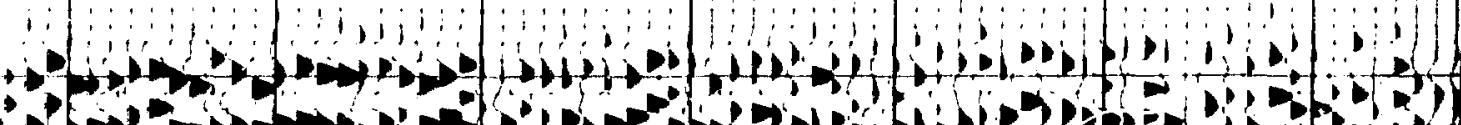

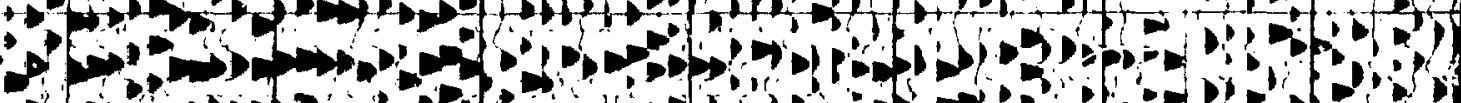

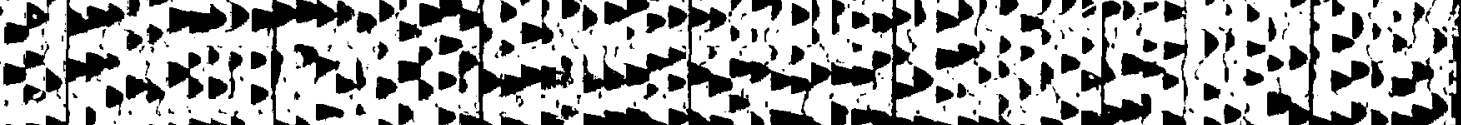

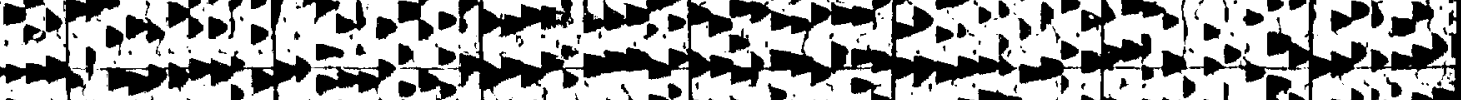

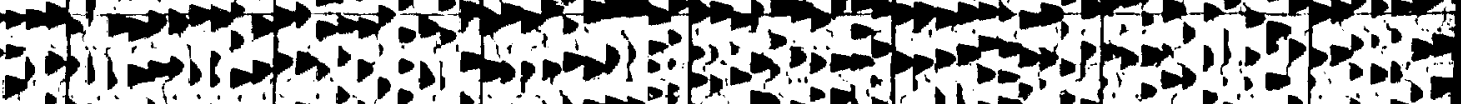
a 


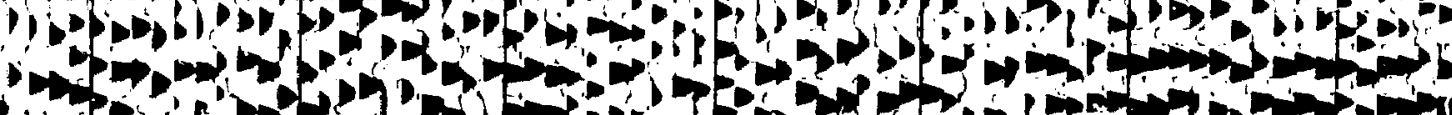

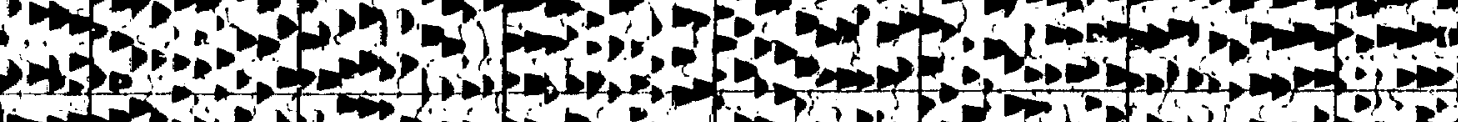

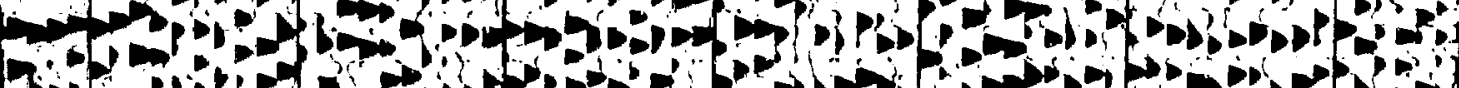

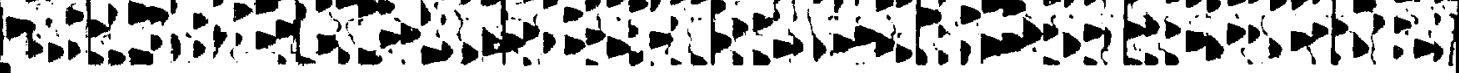


VEL, PNAL,

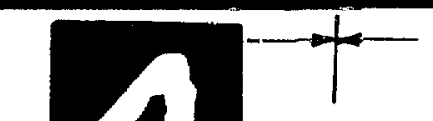

\section{sec 28 a 29}

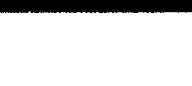


VEl. PNAI,

$\frac{\text { INE } \times 6}{215}$

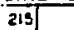

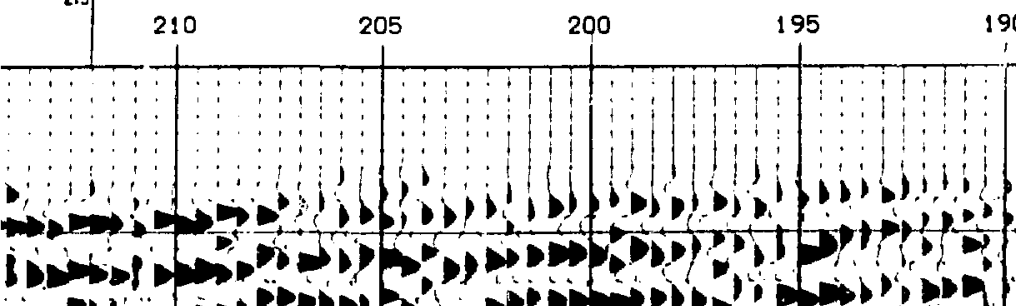

200

195
190
YEL, BNAL,

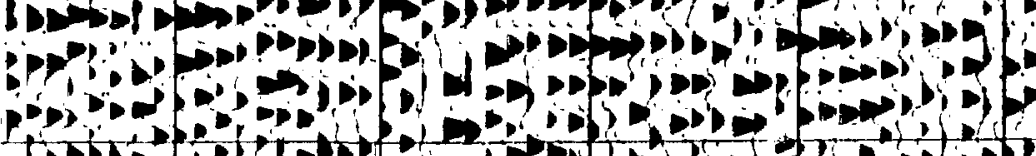
torising i

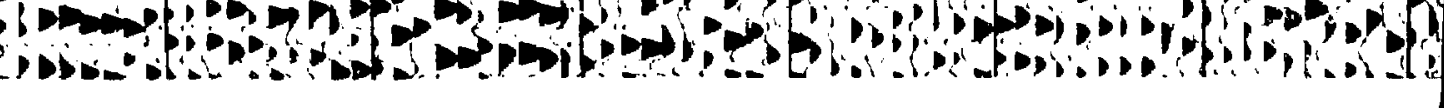
1 Des

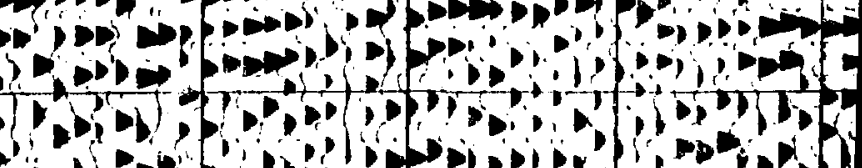
$185 \quad 180$ 
$-$ $T$ $\min$

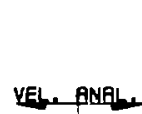

Yan ANALr.

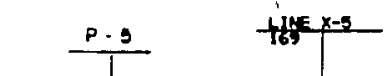

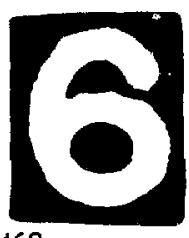

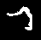

$\sec 17$ a 16

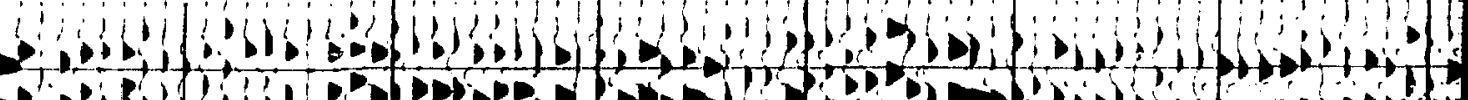

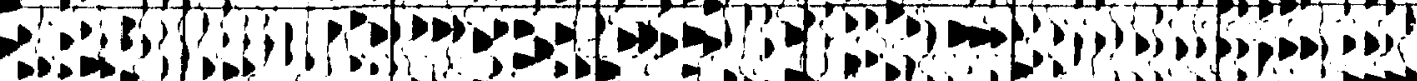

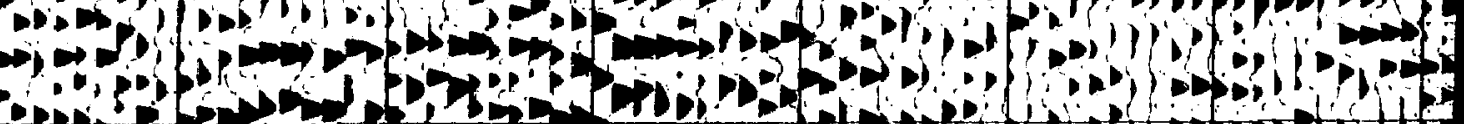
Ders

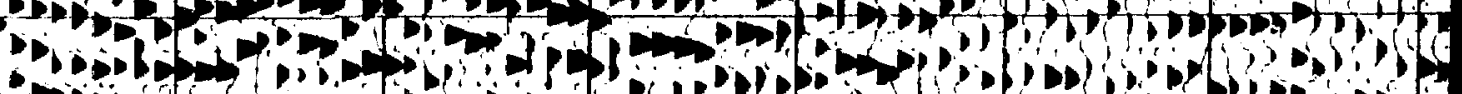
mojof 
VEb RNAL,

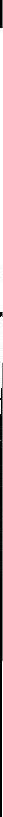


$\sec 8 \& 9$

YEL, ANFIL

110

105

100

95

90

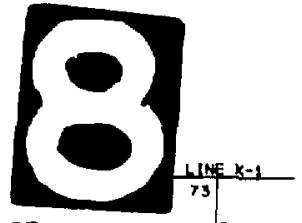

Bp

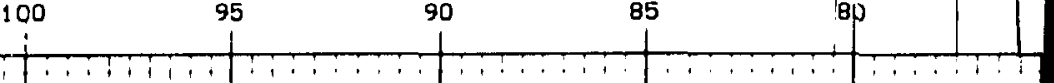

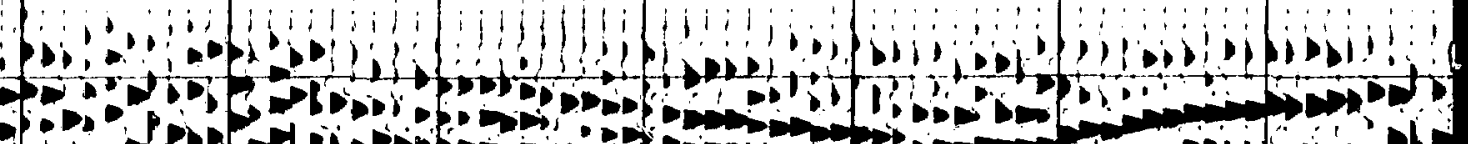
$\left\{\begin{array}{l}0 \\ 6\end{array}\right.$

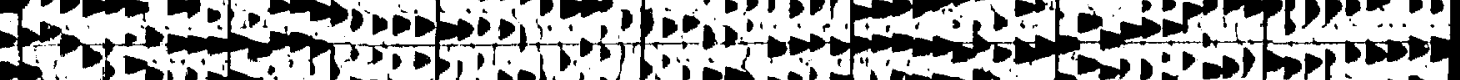

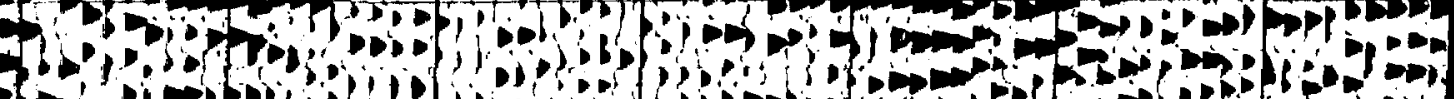

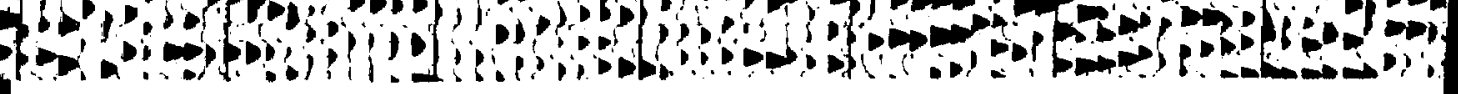


$?$

Lat, ANPL

工n

$y$

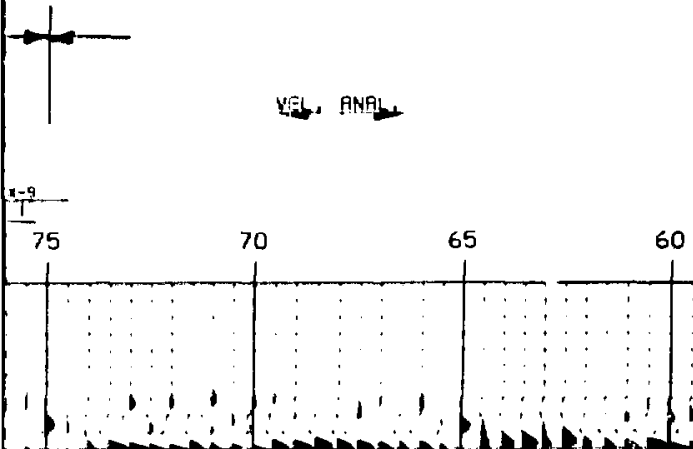

5

tim

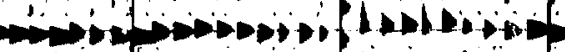

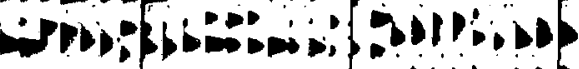

(1):

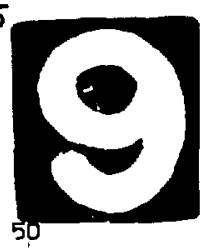

VELE RNAL

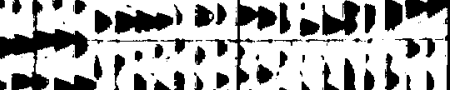

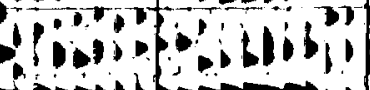




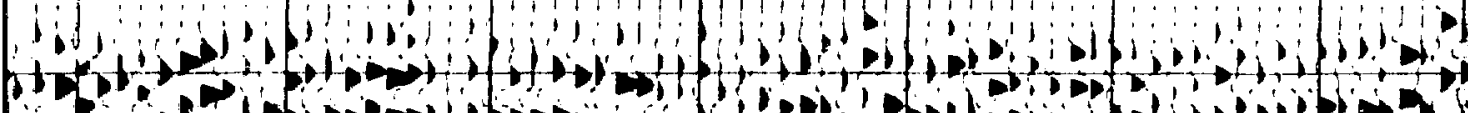

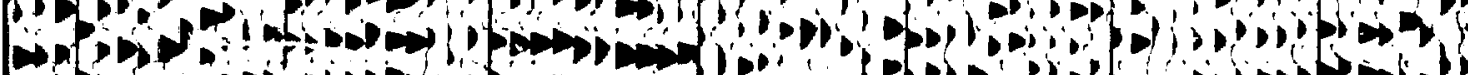

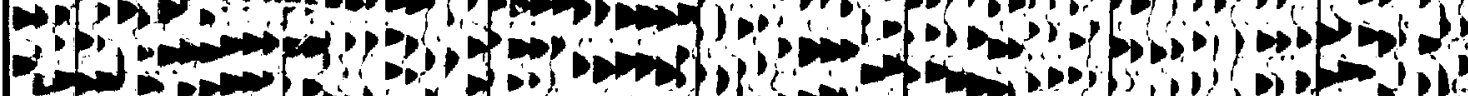

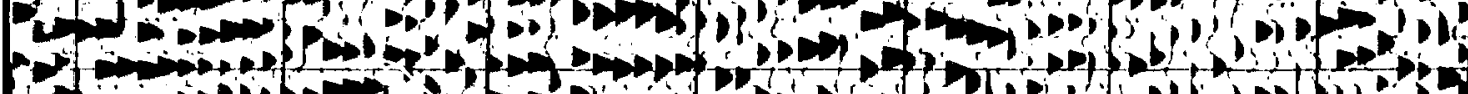
a - 


\section{ELEVATIONS}

STAT ICS

FoLD \%

\section{IINE DIRECTION}

MABTY -

VELOCITY FUNCTION

DIRECTION

LINE INTERSECTION

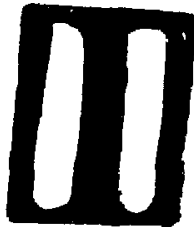

STATIONS

\begin{tabular}{|c|}
\hline LOS MEDANOS \\
\hline LINE $X-2$ \\
STATIONS $3-348$ \\
SOUTHEAST NEW MEXICO
\end{tabular}




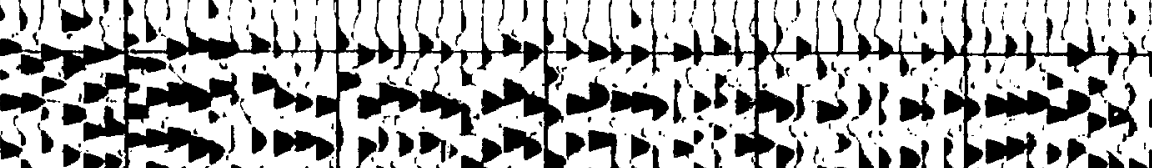

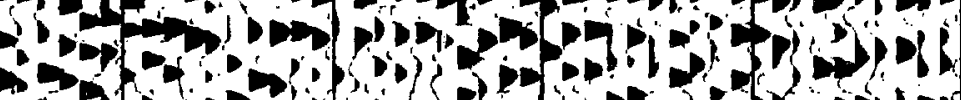

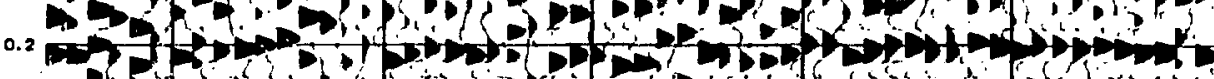

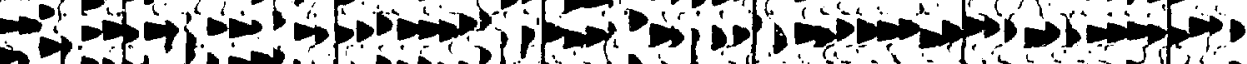

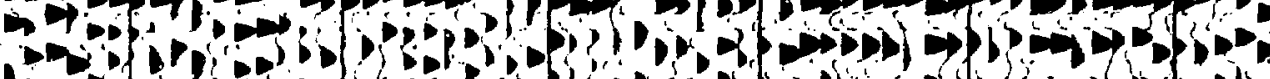

$\Rightarrow$ -

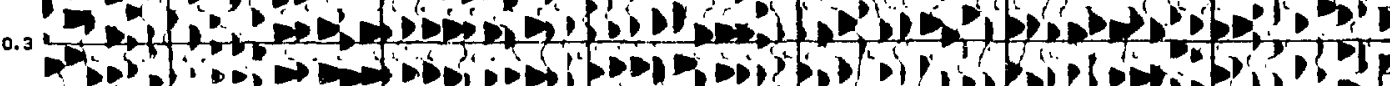

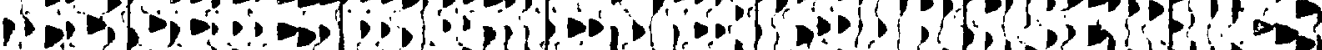
1.

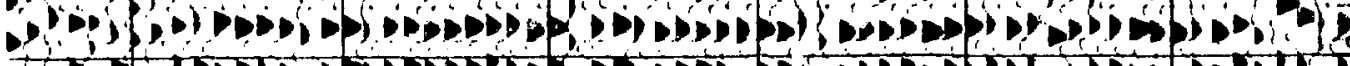

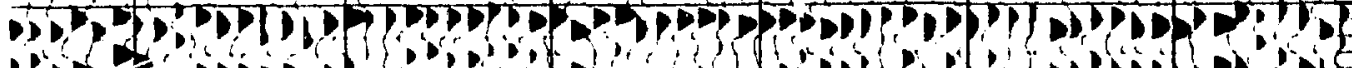

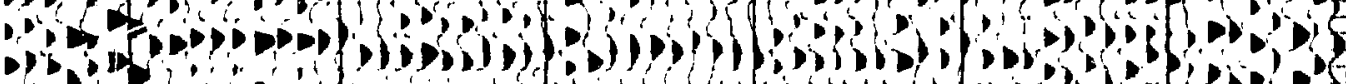

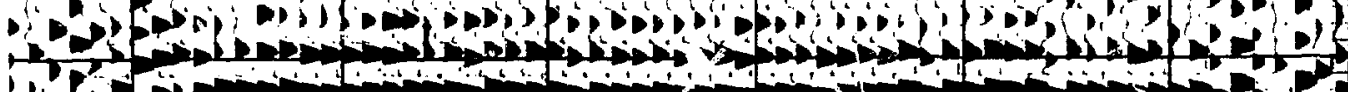

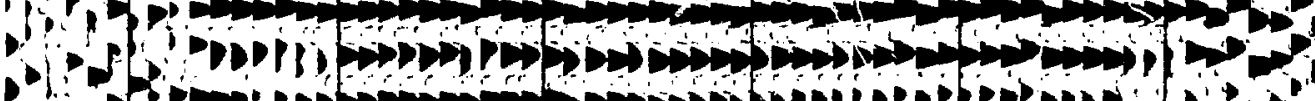

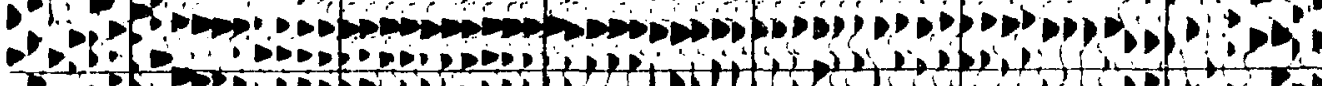

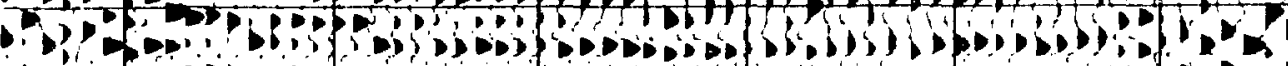

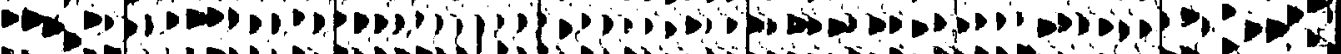
- D

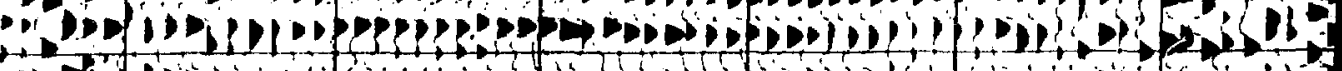

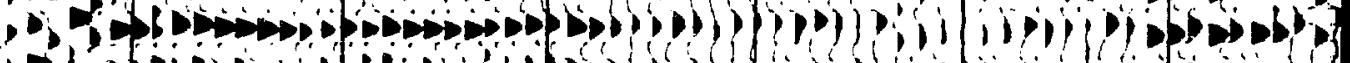

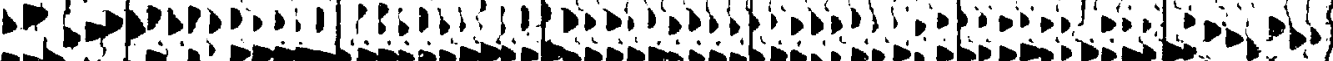

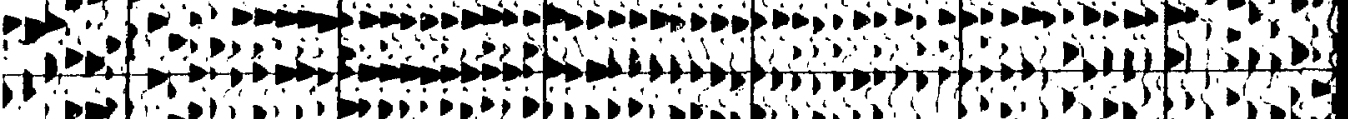
ljipe

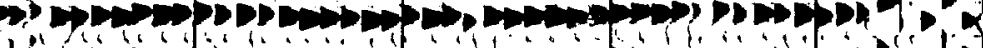

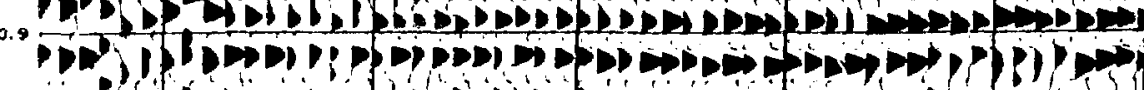

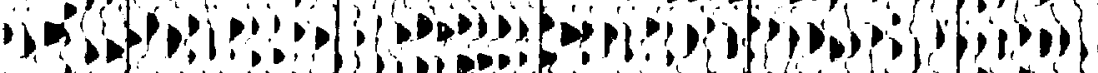

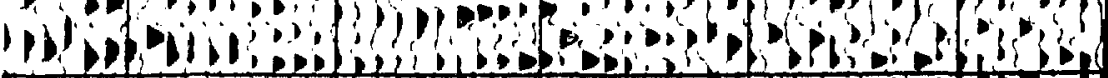

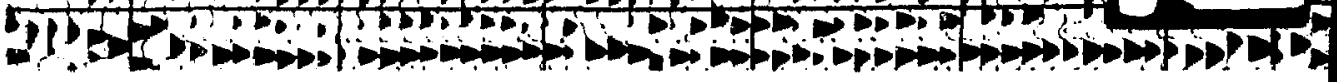




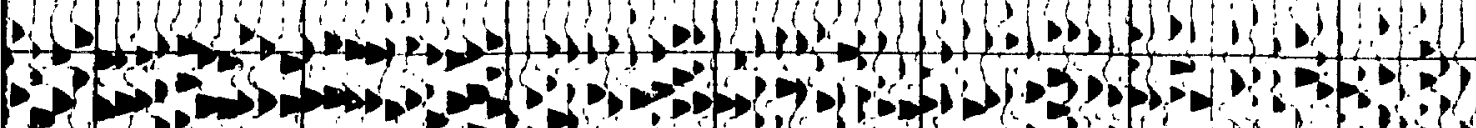

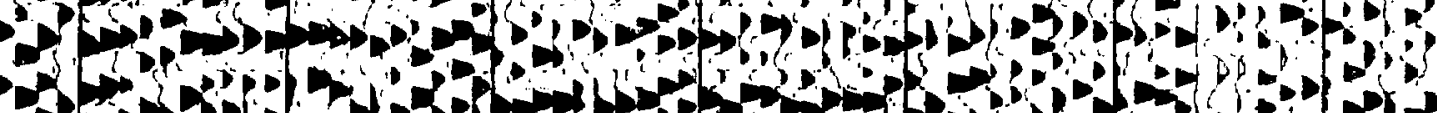
?.

Git

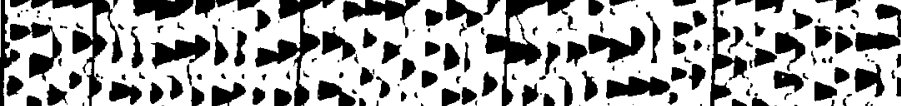

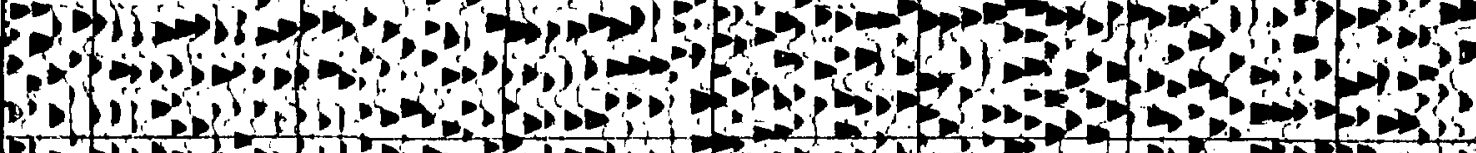
1)

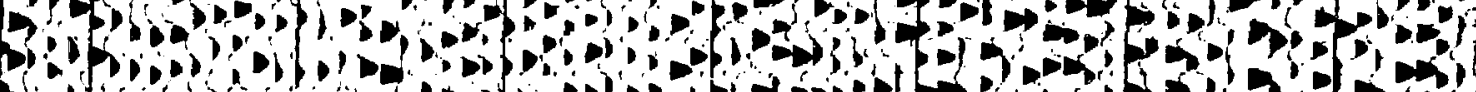

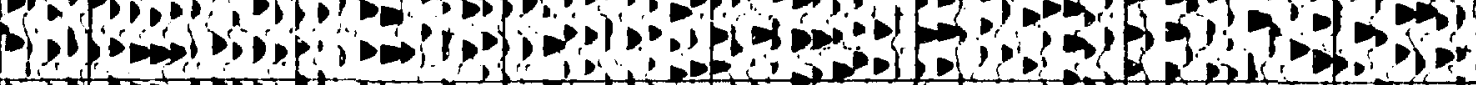

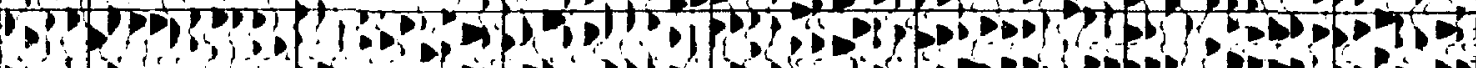

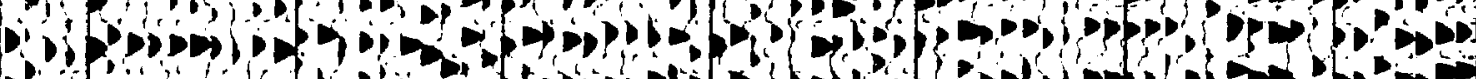

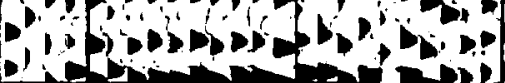

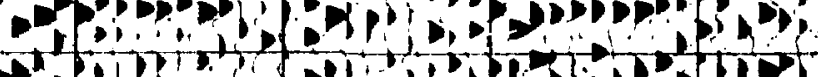

$4 ?$

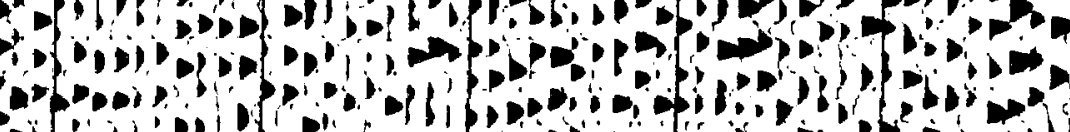

(1)

(1)

(1)

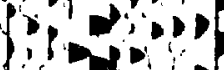

(i)

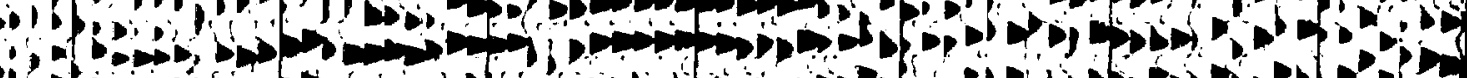

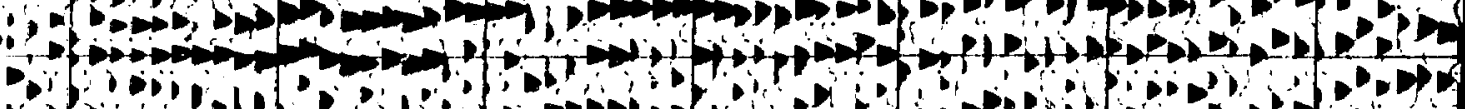

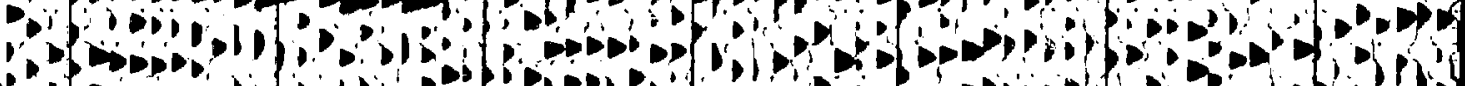

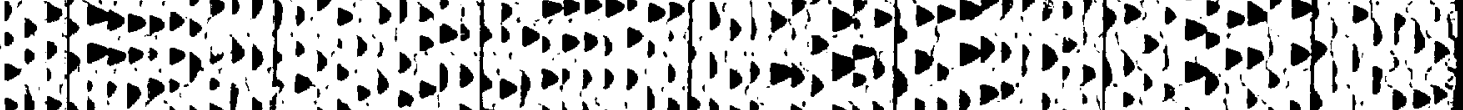
195 bil of

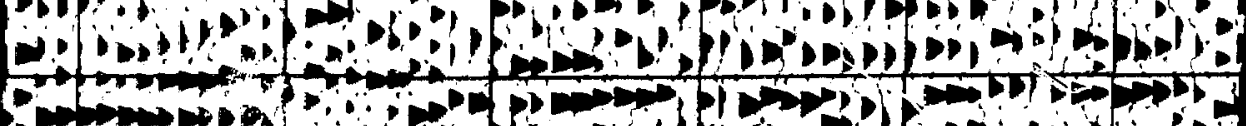

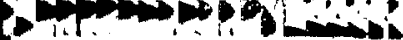

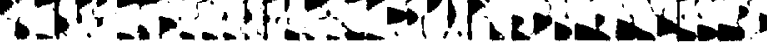


Hin

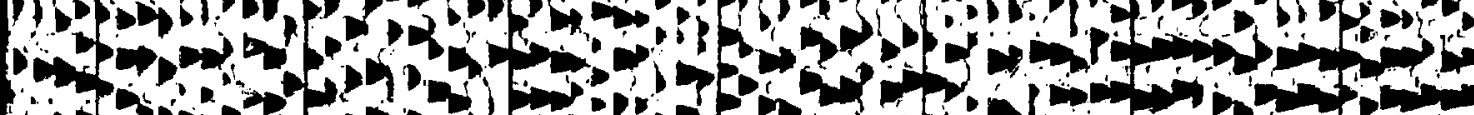

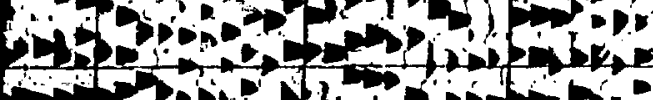

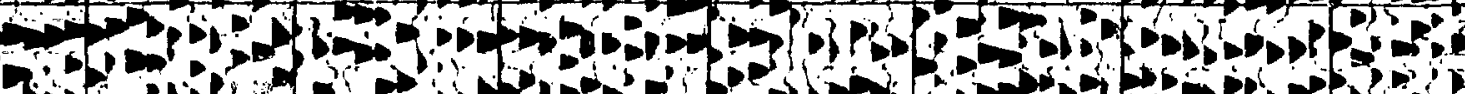

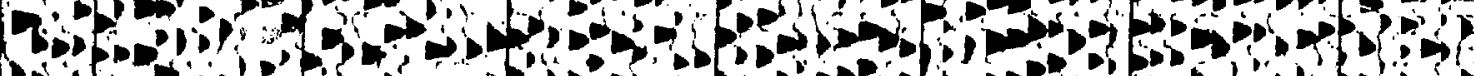

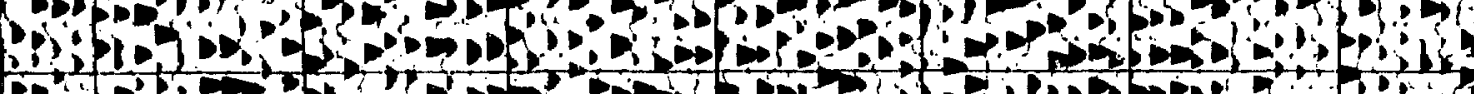

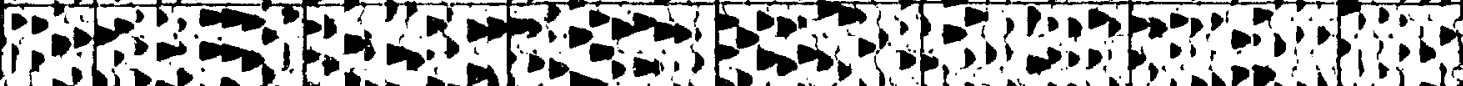
9.9.5.

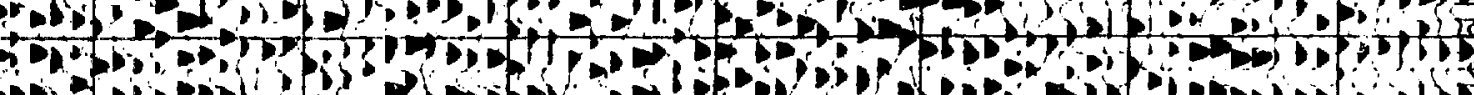

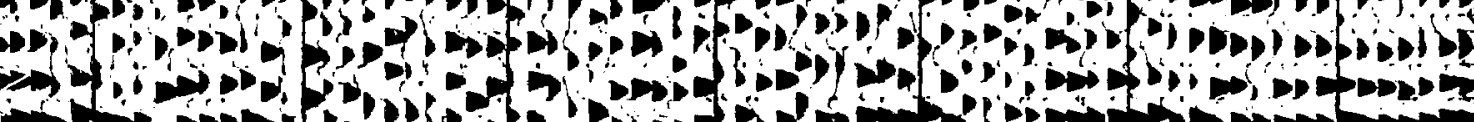
.

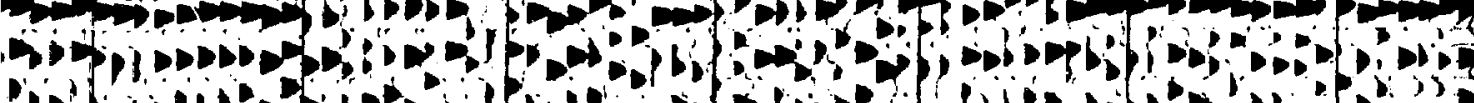
4.

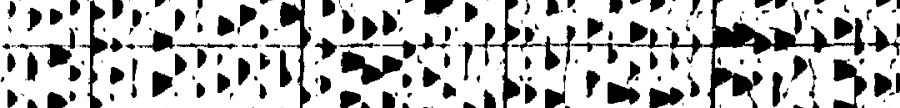

1.5.

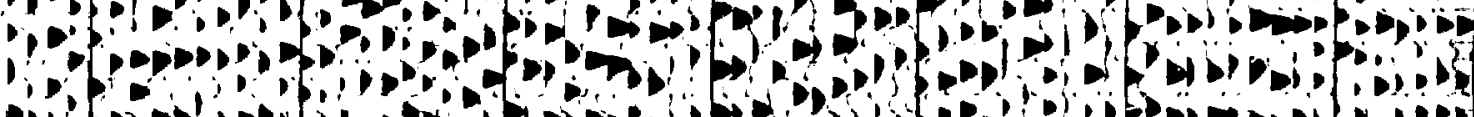
not

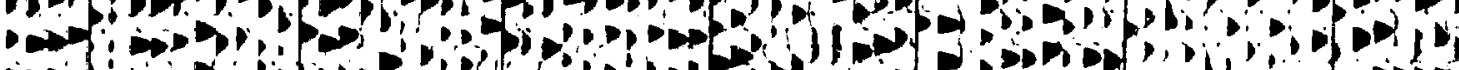

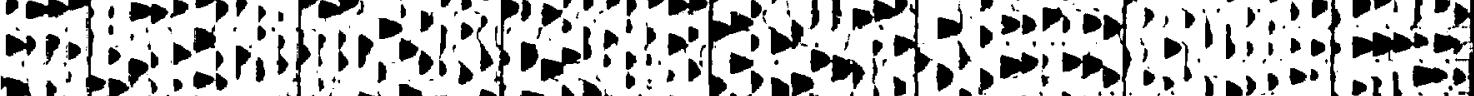

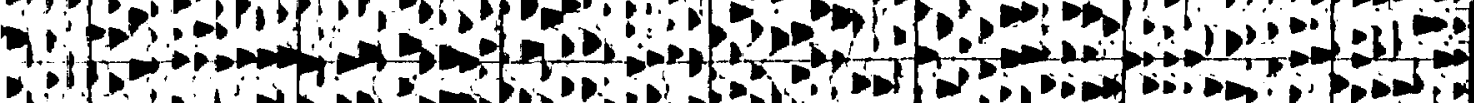

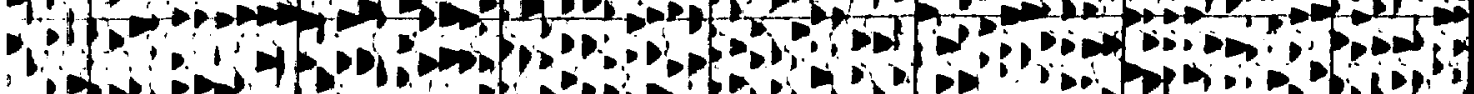

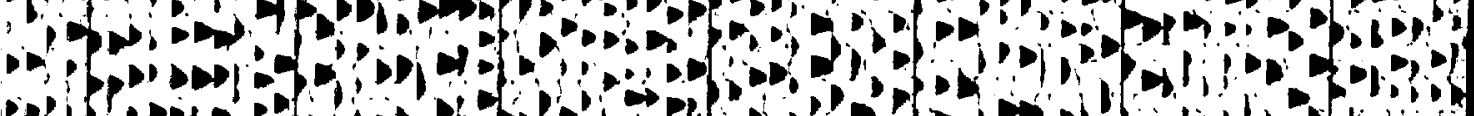

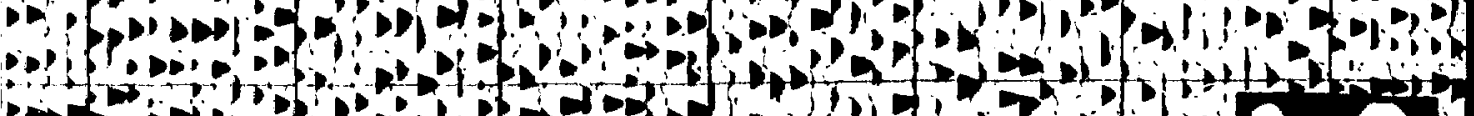

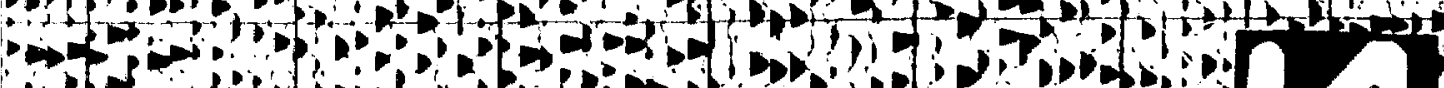

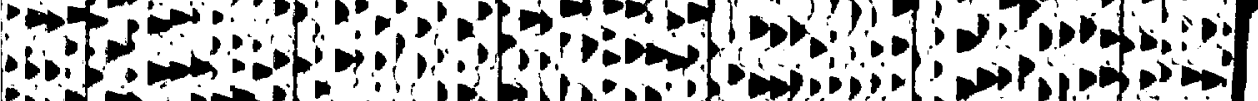

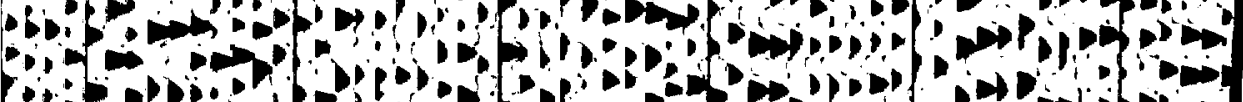
Pen

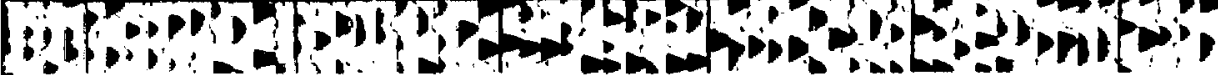




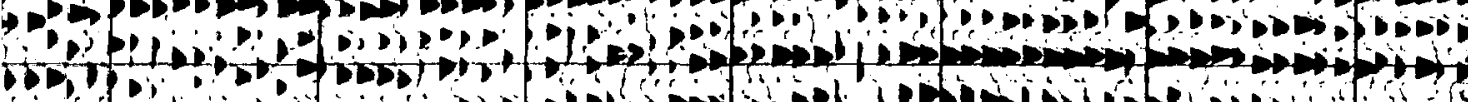

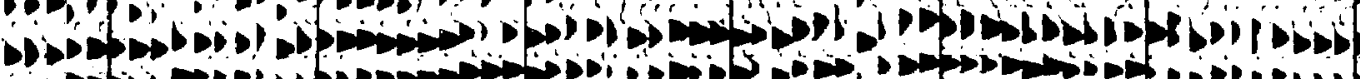

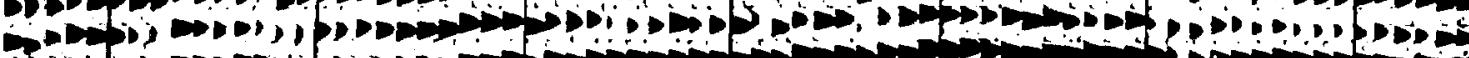

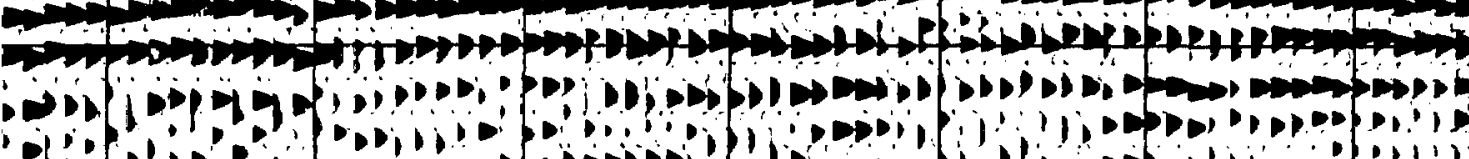

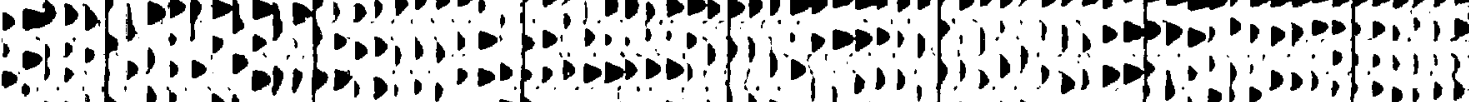

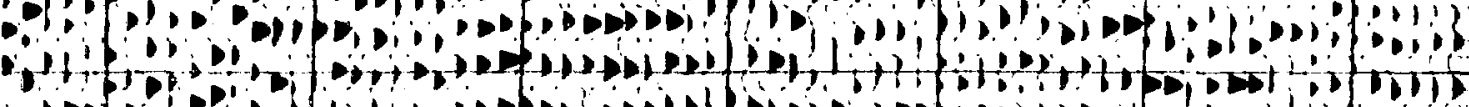

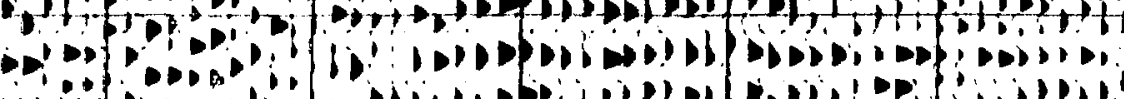

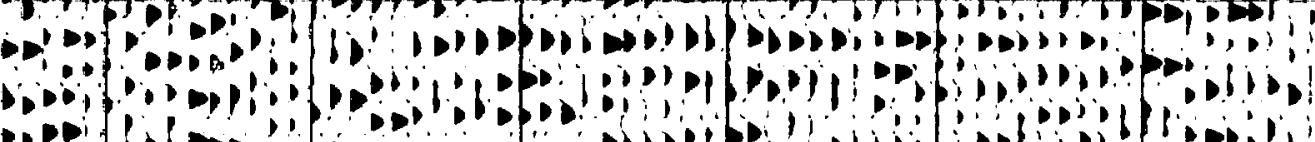

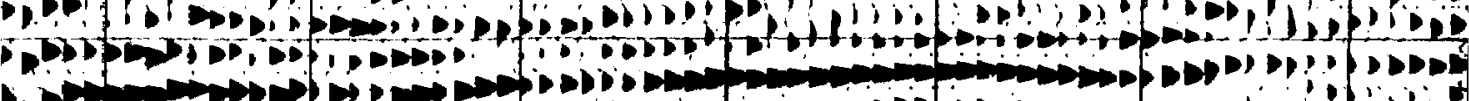

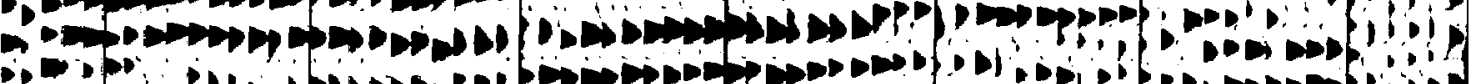

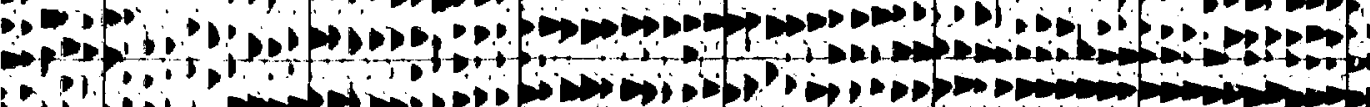
o

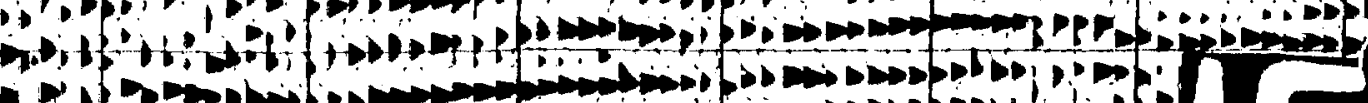

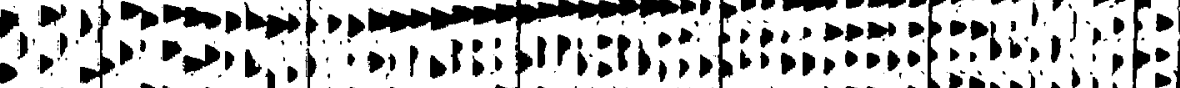

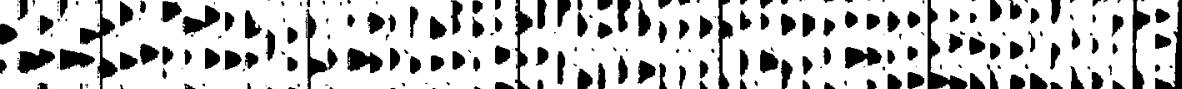

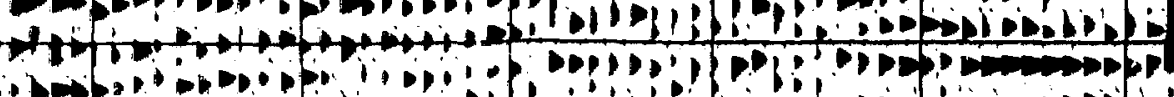
isi.

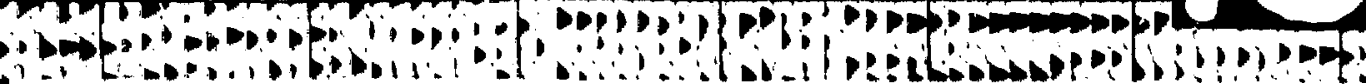




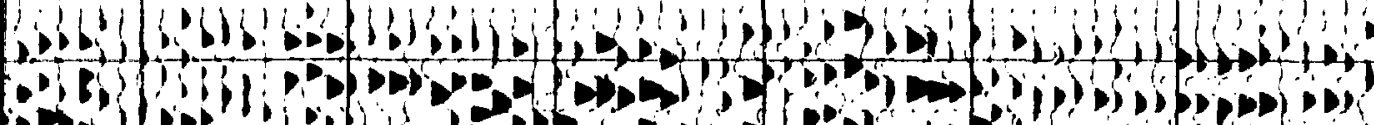

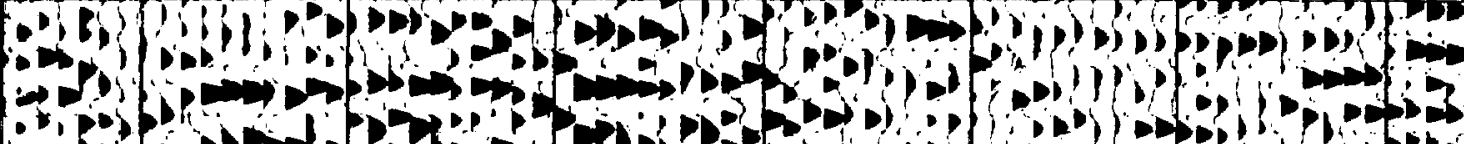

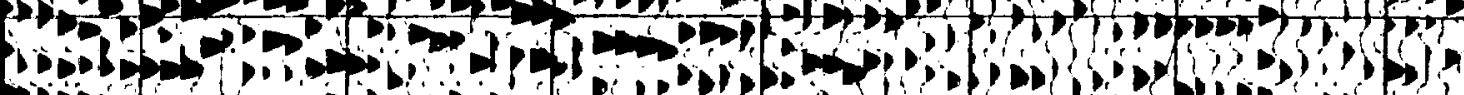
Sis

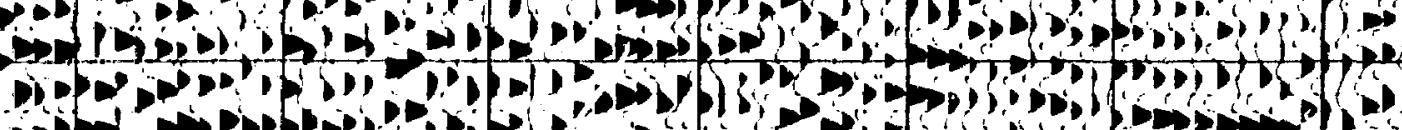

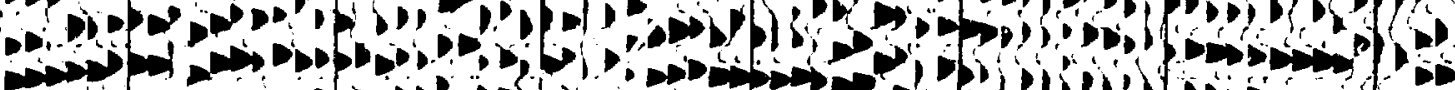

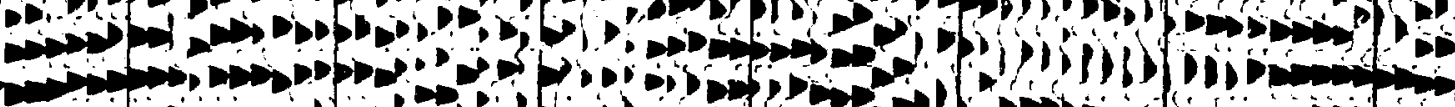

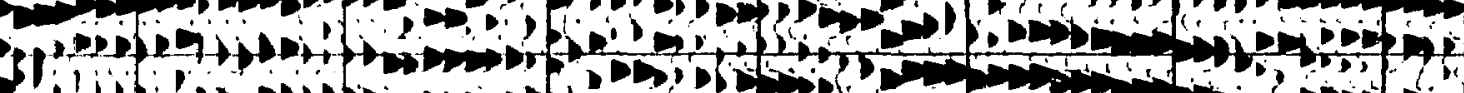
(1)

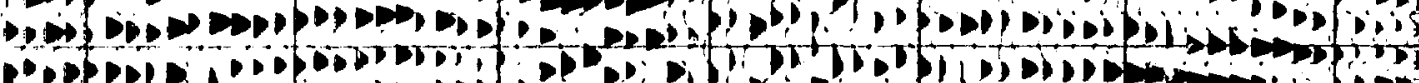
$\because$ of ! : 1

o.

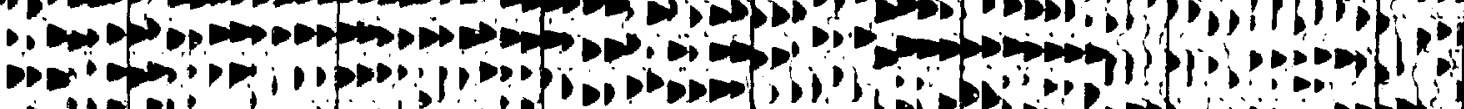

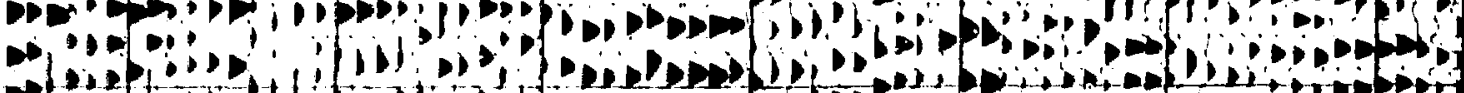

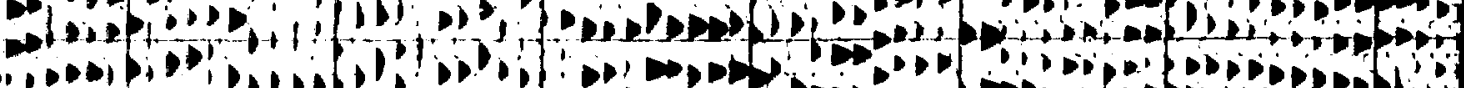

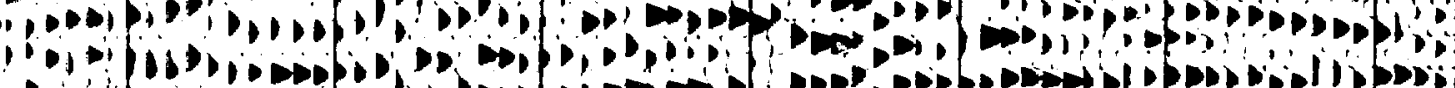

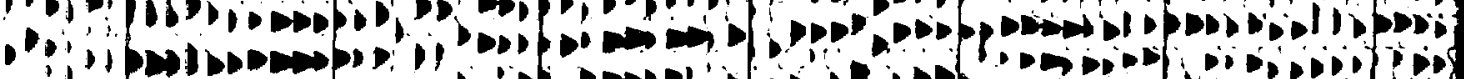

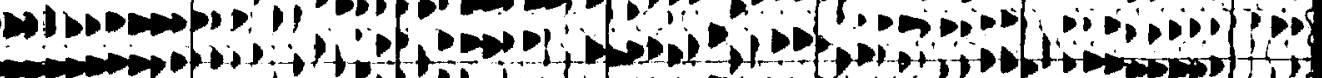

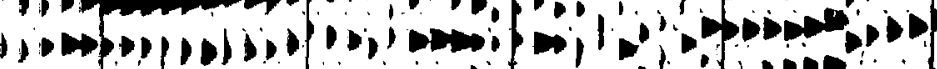

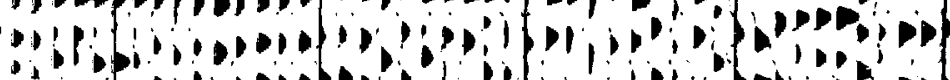

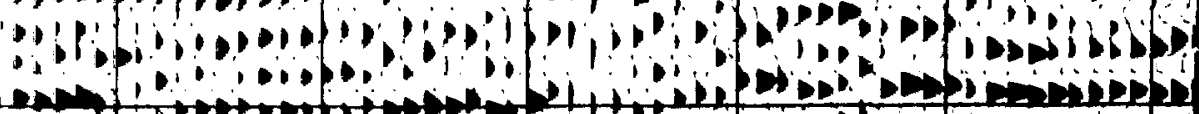

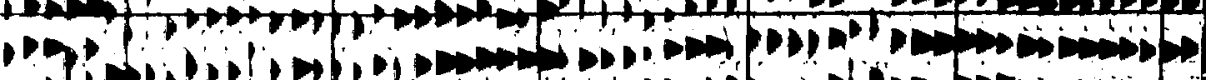

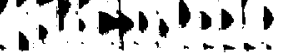


(is

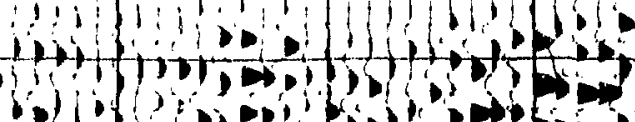

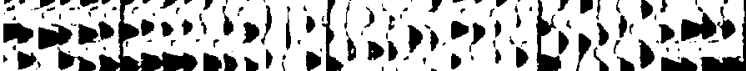

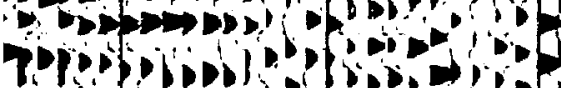

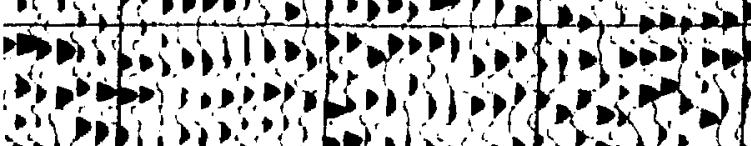
So

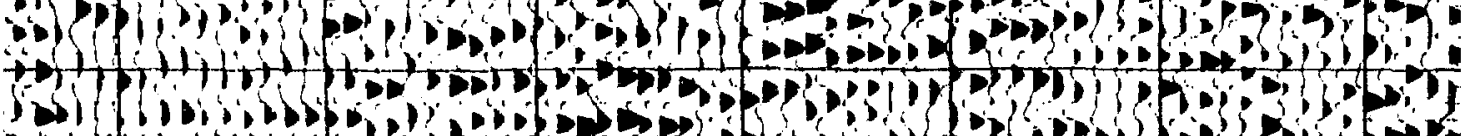

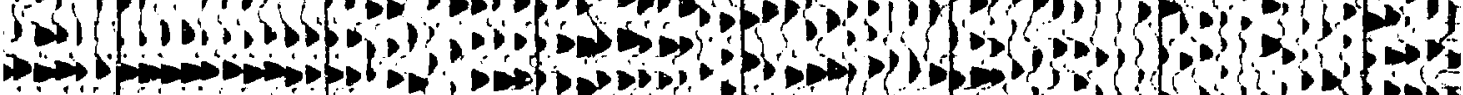

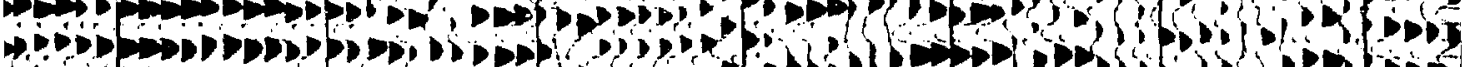

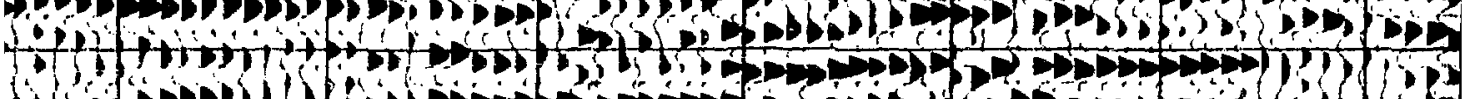

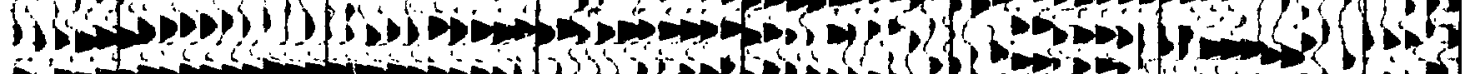

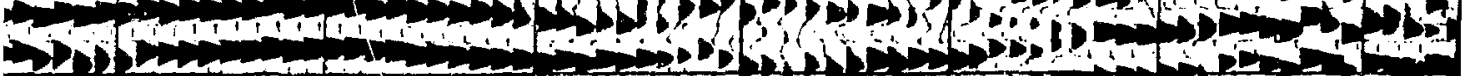

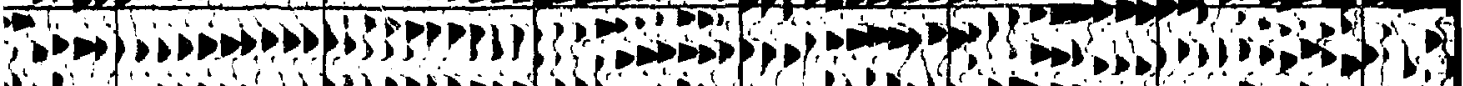

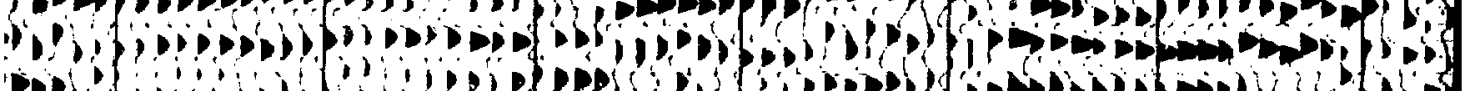

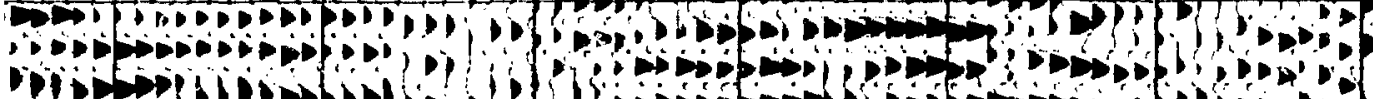

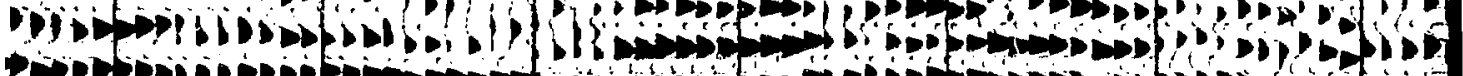

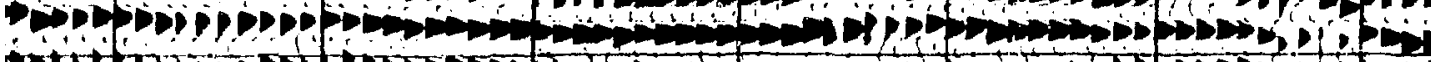

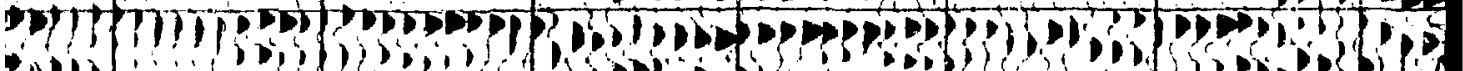

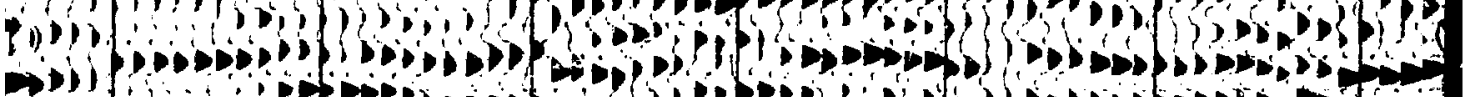
PDil

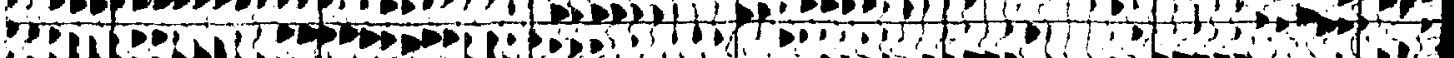
F.

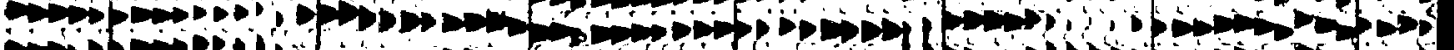

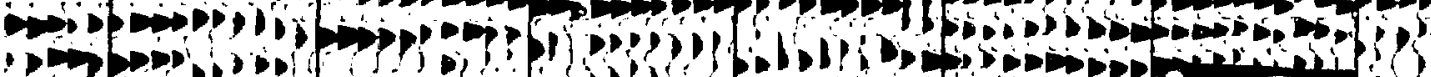

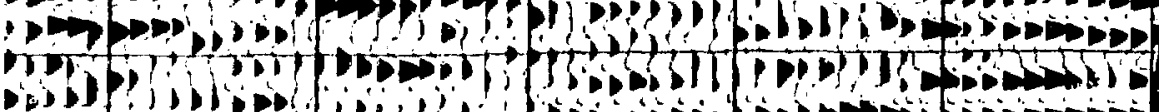

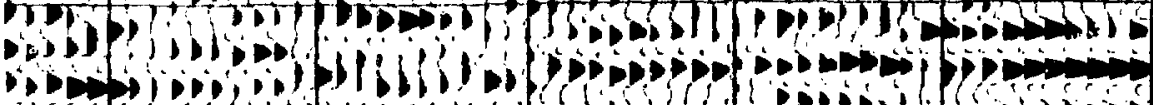

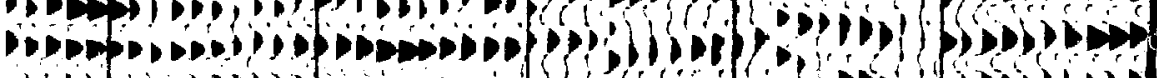

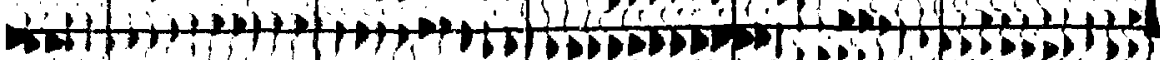
18

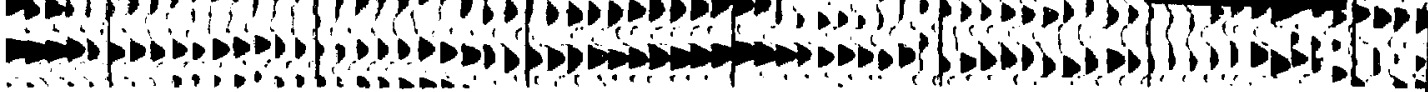




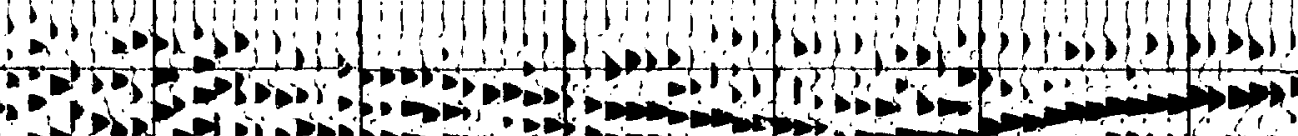

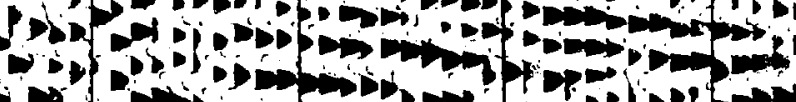

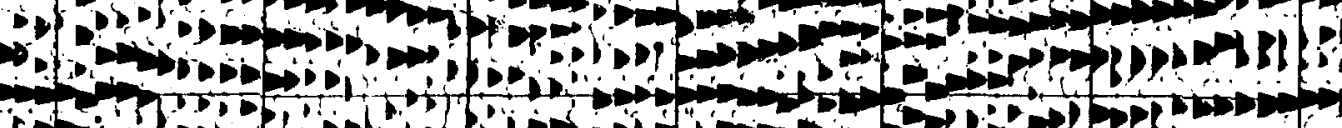

for

fos

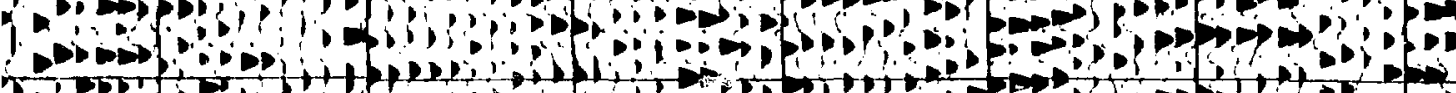
11,

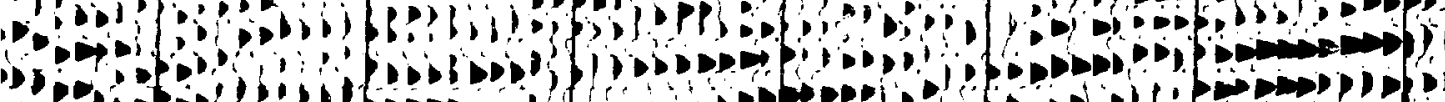

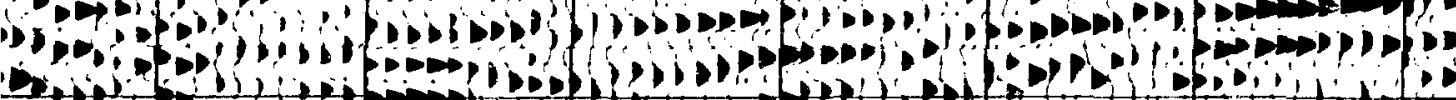

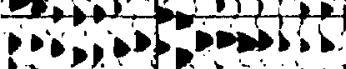

ing

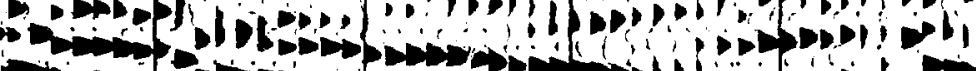

;

;

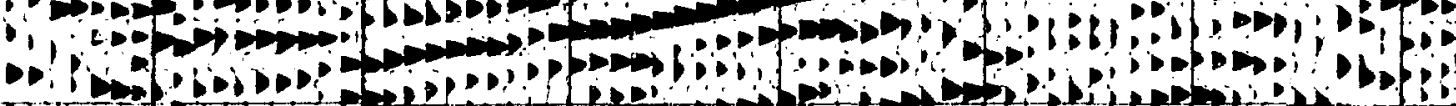

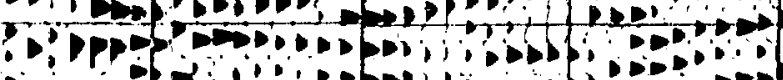

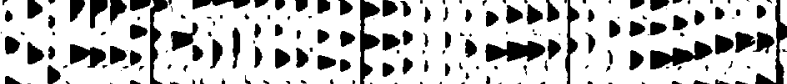

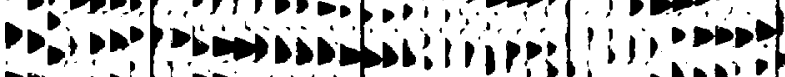

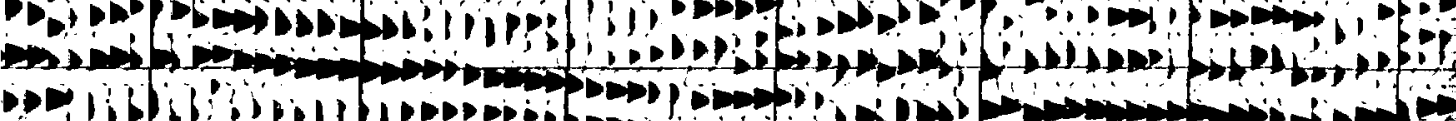

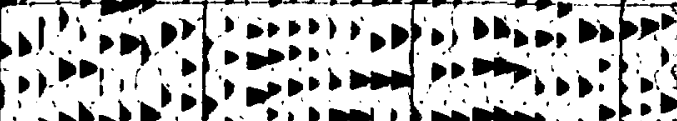

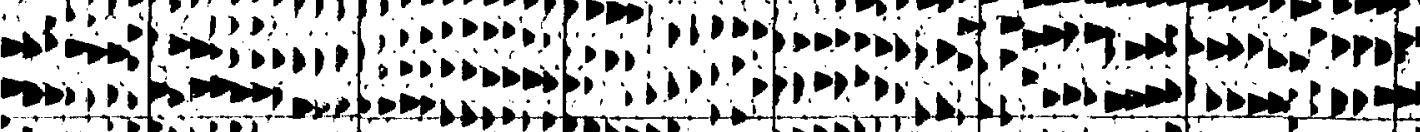

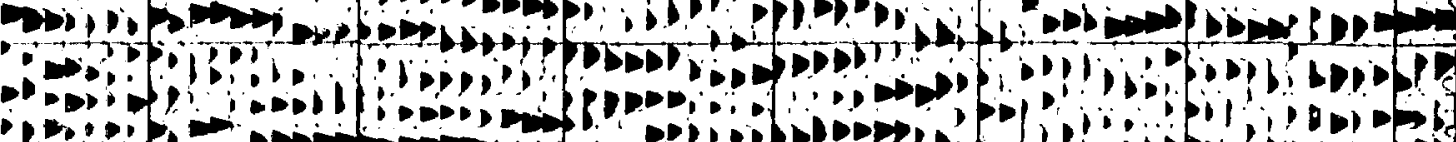
-

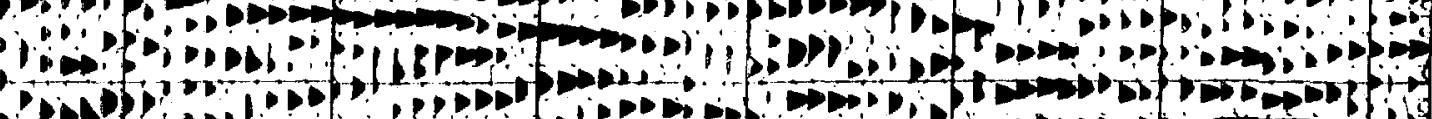

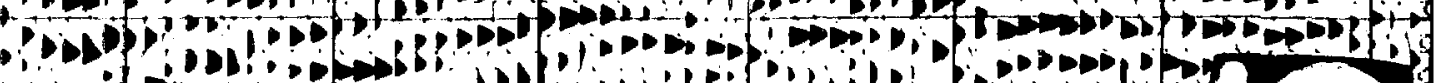

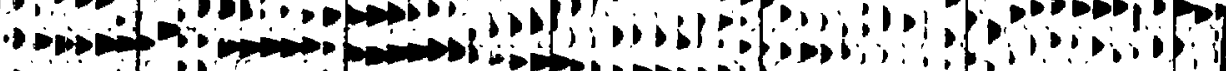

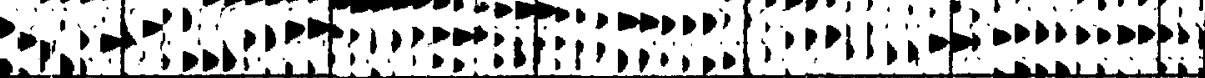
- Ppl - 
150 ,

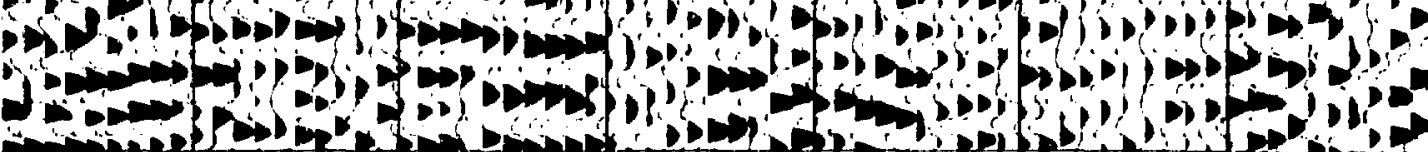

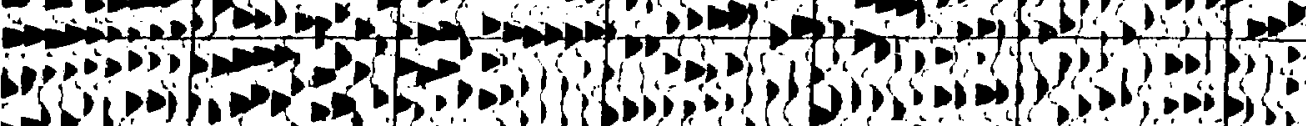

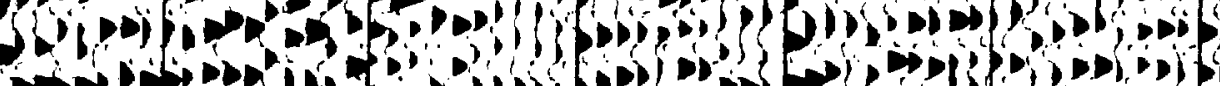

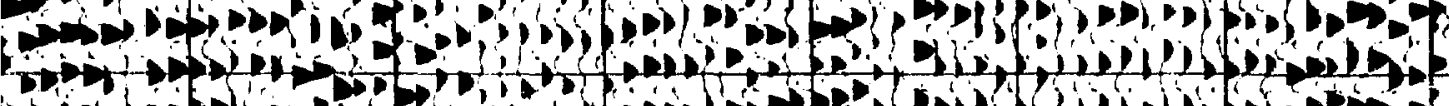

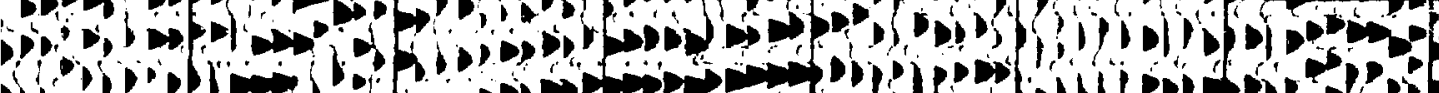

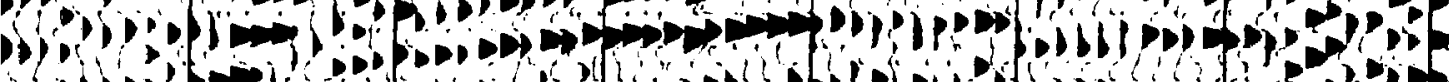

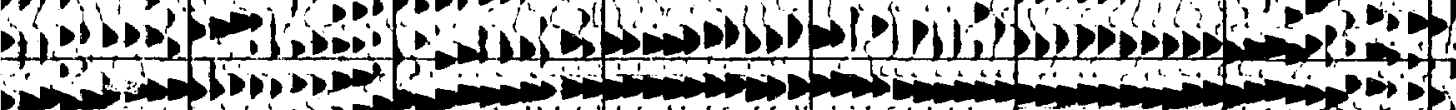

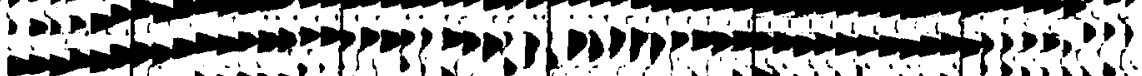

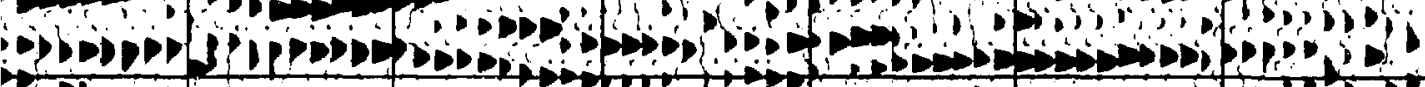

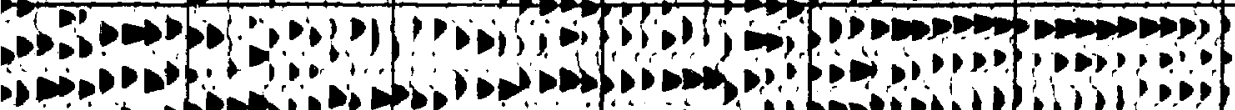

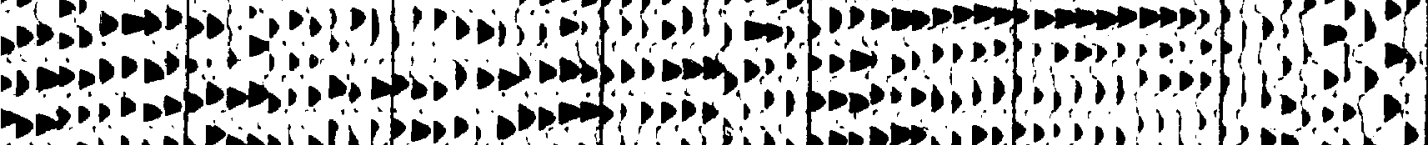
of

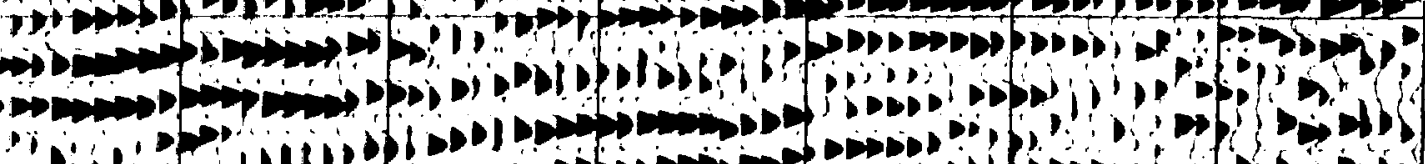

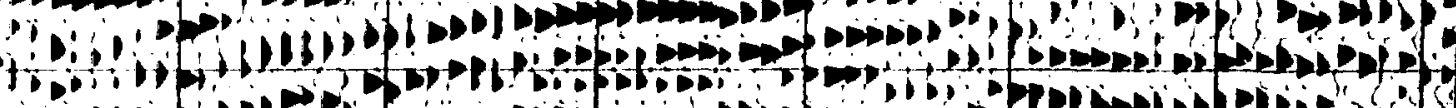
- : pop $\rightarrow \rightarrow l_{0 \rightarrow 01}$

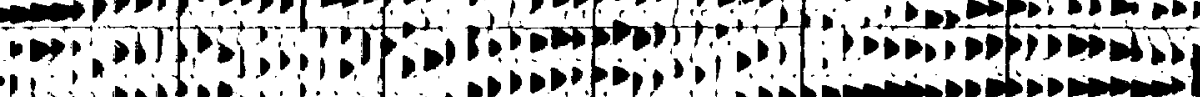

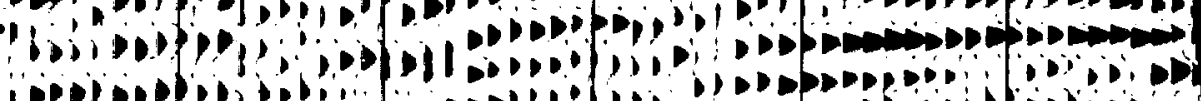

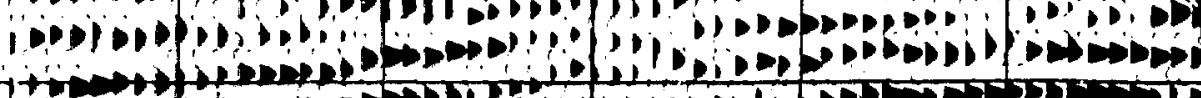

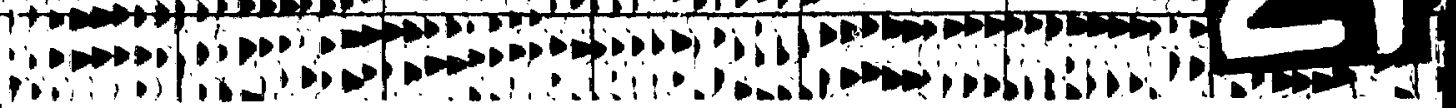




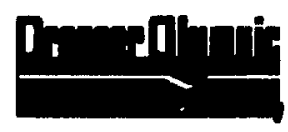

\section{LOS MEDANOS}

LINE $x-2$

STAT IONS $3-348$

SOUTHEAST NEW MEXICO

IMPUT REEL HEMOER IMFLAMAT IEN

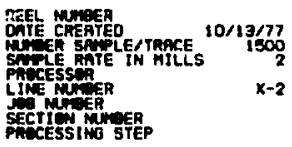

\section{FIELD INFORMAT ION}

AECGADED QY: ORESSER CUYMPIC PAATY: H. 62

OATE: SEPTERAER 22. 1977 FILTER: 10/36-124 HZ

INSTMUMENTS: EFS 1 - OFS IV SAMPLE RATE: 2NS

WOICH FILT: IN IN SERCE: VIBRASEIS

RECENO LEH: 14 SEC. SWEEP LEN: 12 SEC.

SWEEP FREO: $25-100 \mathrm{~Hz}$ MO/GREUPS 24

STH INV: $110 \mathrm{FT}, \quad$ VIO. INV: $110 \mathrm{FT}$.

GEO MER STM: G GEE TYPE: GSE-200

ARRAY TYPE: IMLINE TYPE COVER: 1200 PRCN?

\section{PROCESSING SEQUENCE}

PRECESED \&I OAESSER Q Y YMPIC

STATICS CEPUTATIOF

DATUA: $\quad 3200 \mathrm{FT}$.

YSU: $\operatorname{sOOO}$ FT/SEC.

1) OEMUTIPLFX

2) aimat GAIN RECOVERT

3) VIBROSEIS CORTELATIN

4) COMNIN OEPIH PEIKT CATKERS

b2 oeconvelutien

CPERATE LEHOTHE 140 MILS

PAEDICIION TIME GASED EN 2ND ZERT CRESSIAO

6) IINE-INYAPJAWI DIOITH. FAEQUENCY FILTER $0.0-3.0$ SEC. 25-80 HZ

7) APPLY OAIUN STATICS

D) VELOCITY anPLSIS

9) APPLY TA

10: FIRST GREFK SUPPRESSION (WUTE

11) StFCK 12 FELD

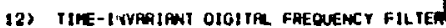
D.0-3.0 5EC. 75-80 HZ

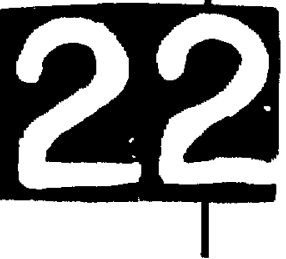




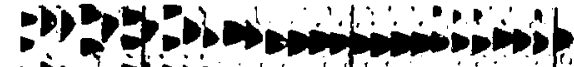

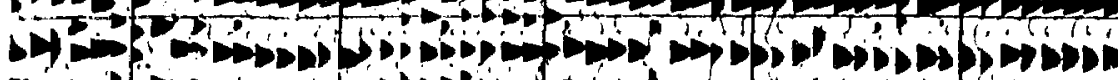

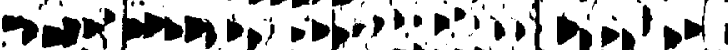

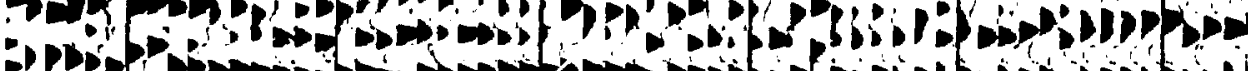

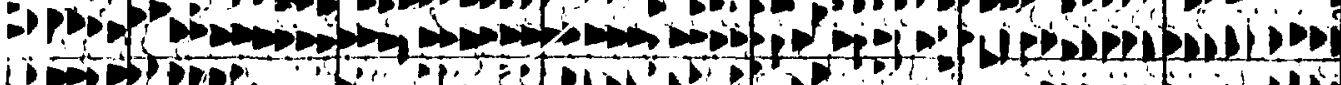

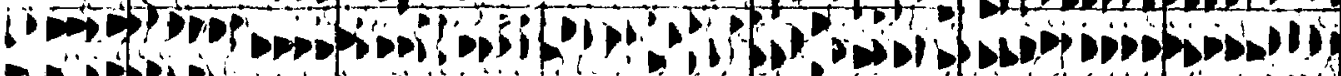

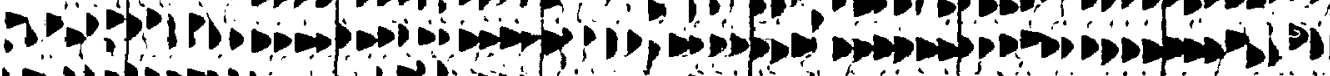

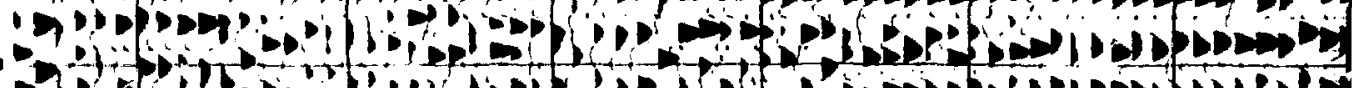

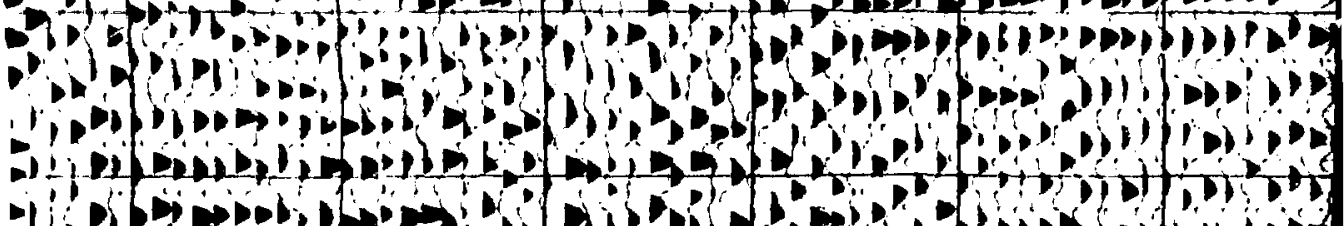

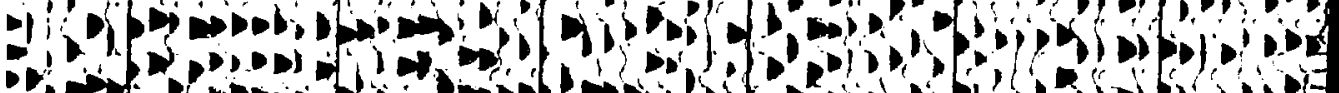

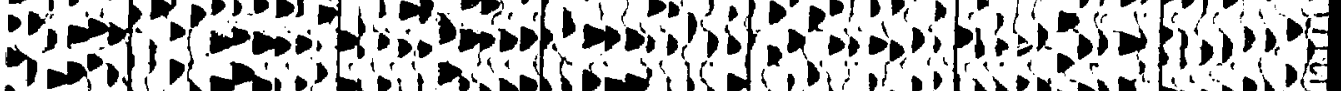
0 - 10 P

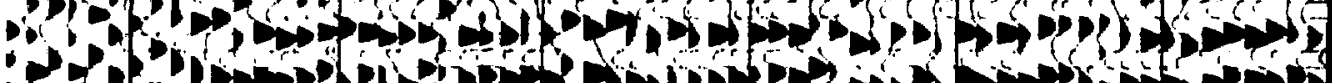

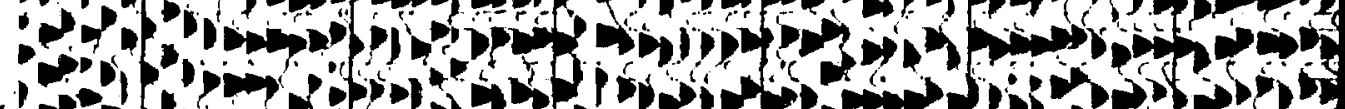

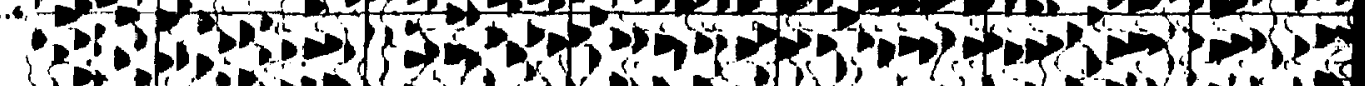

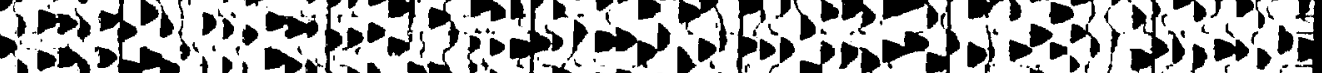
P.

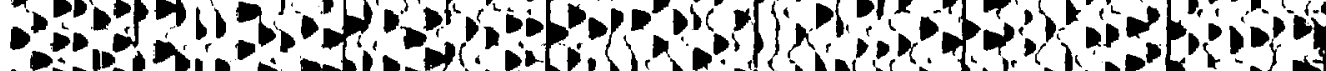

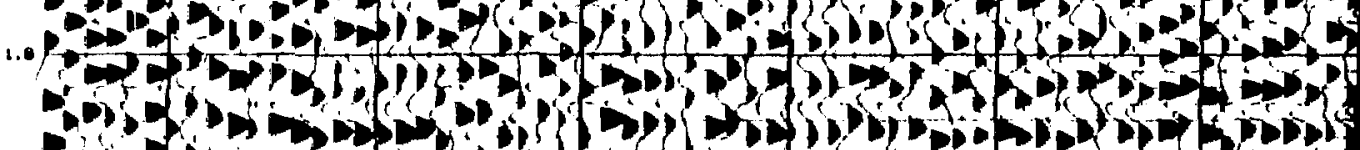

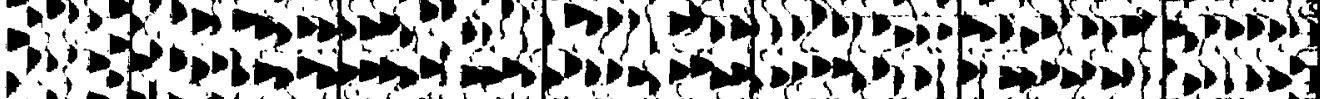

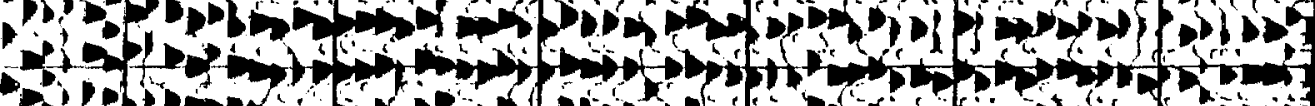

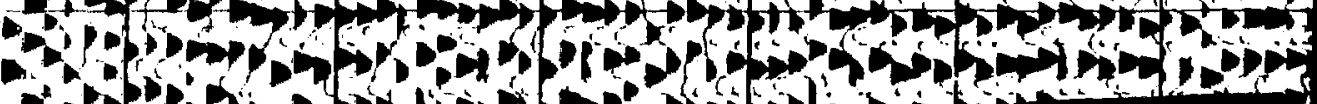

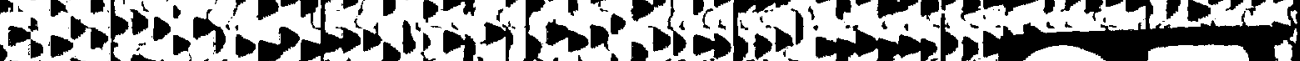




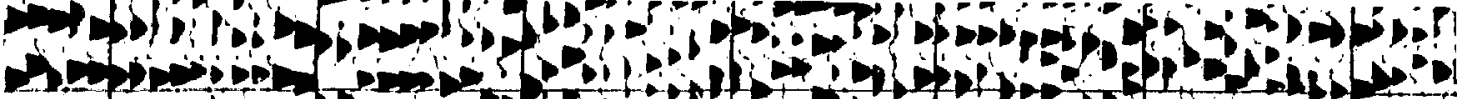
Shatemitio

ind

pinp

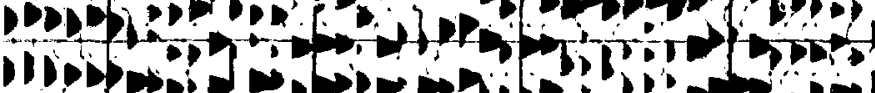

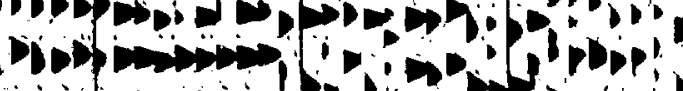

(1)

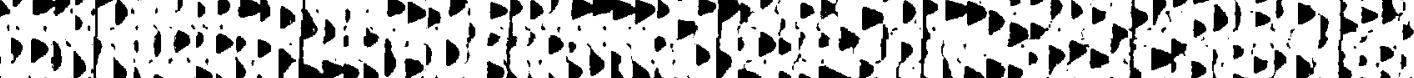

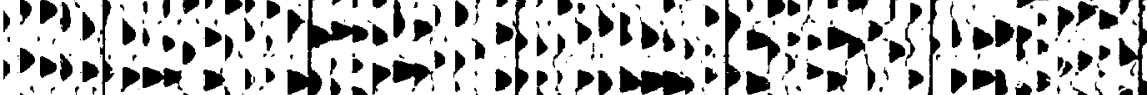
17.

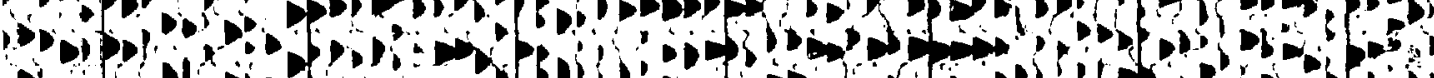

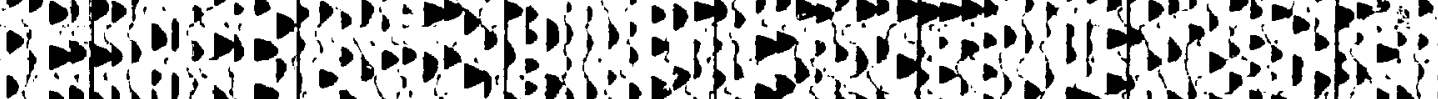

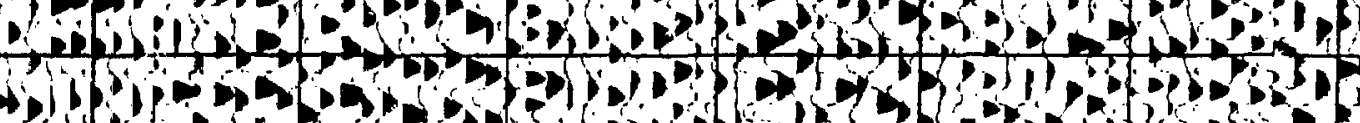
4. abs

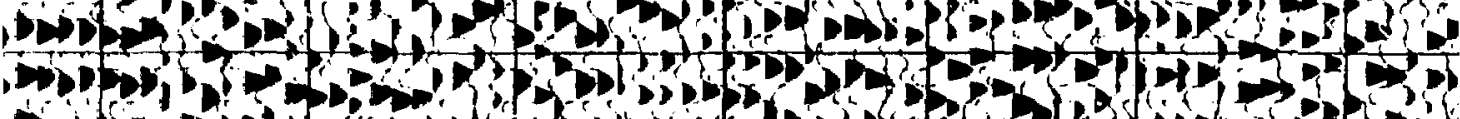

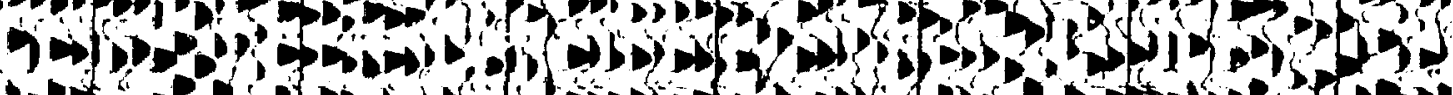

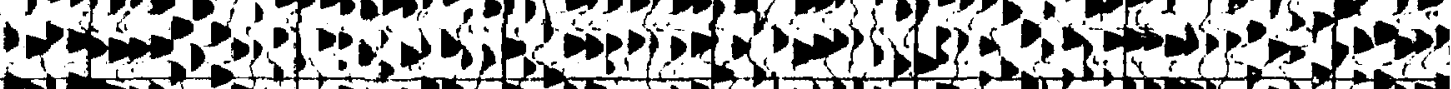

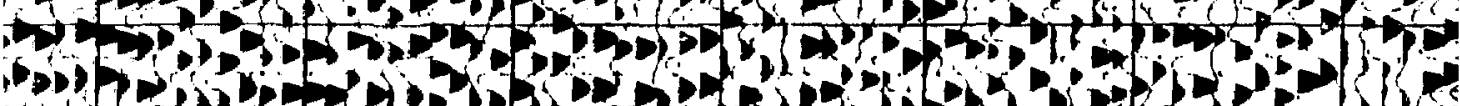

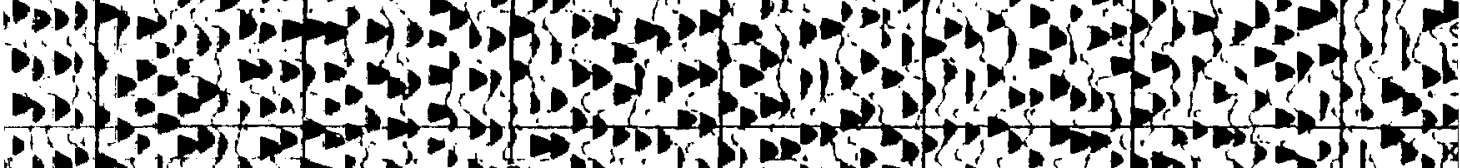

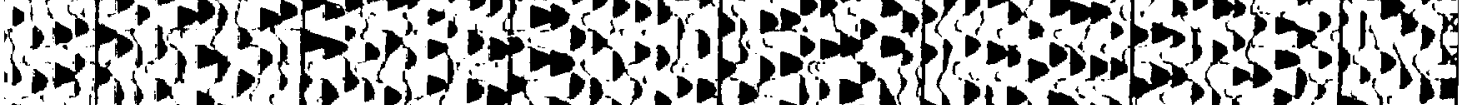
-

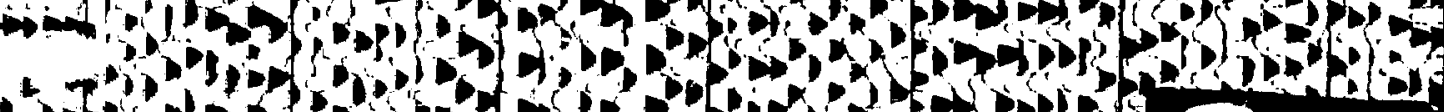

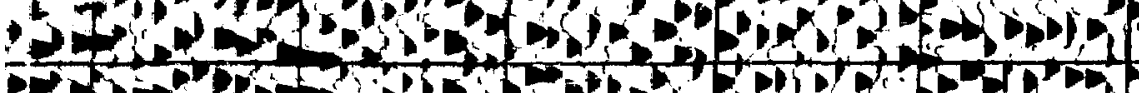

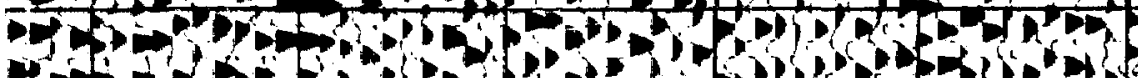

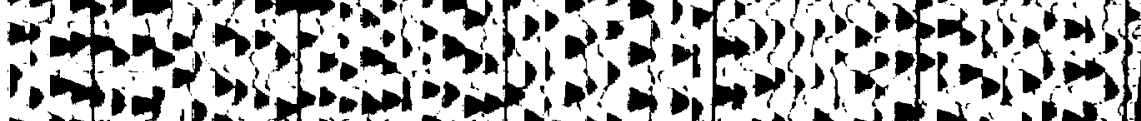

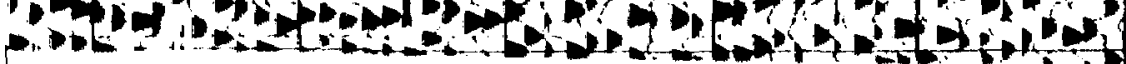




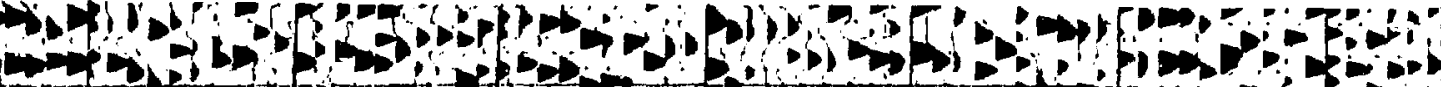
s.tonn

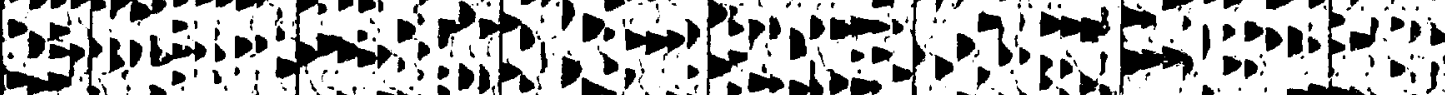

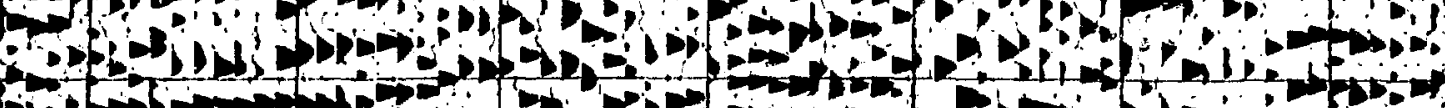
-

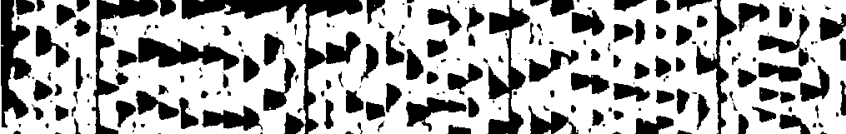

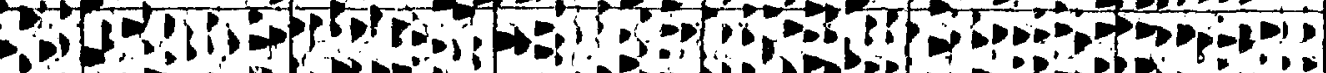

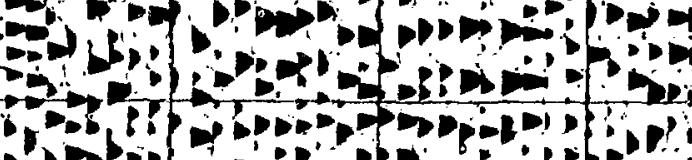
Pos 


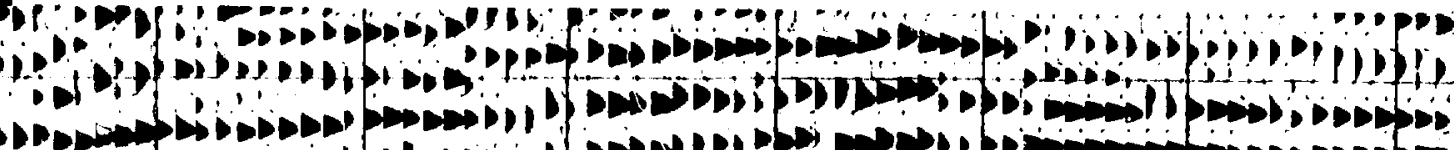

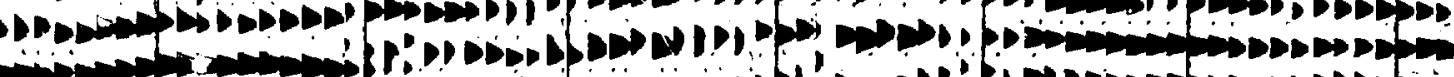

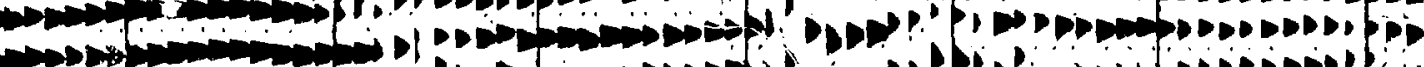

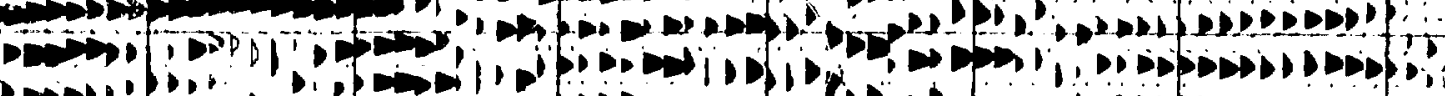

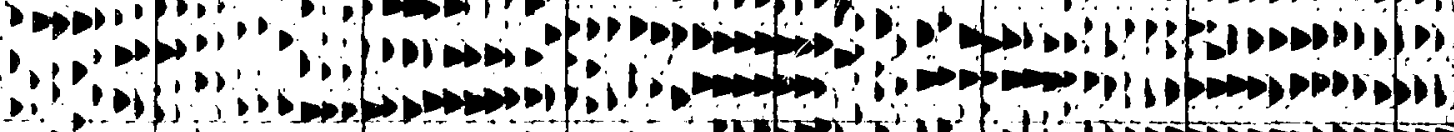

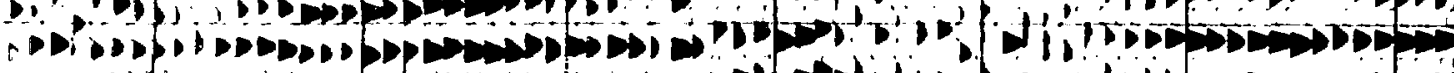

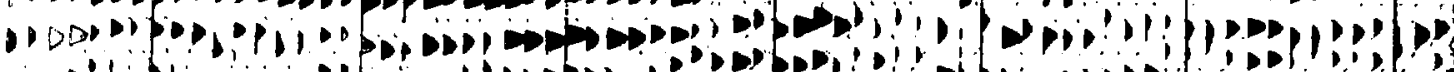

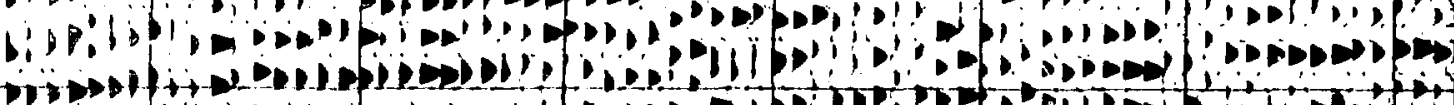

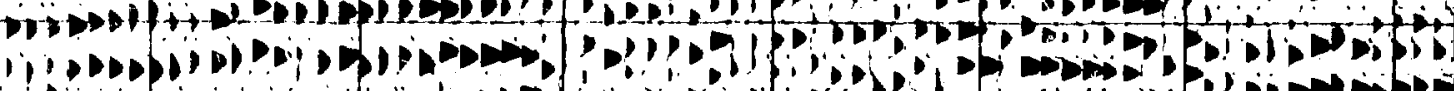

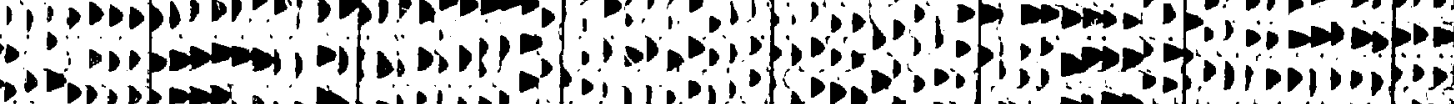

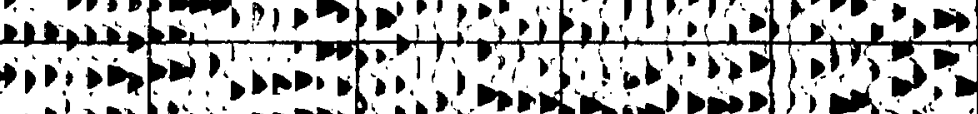

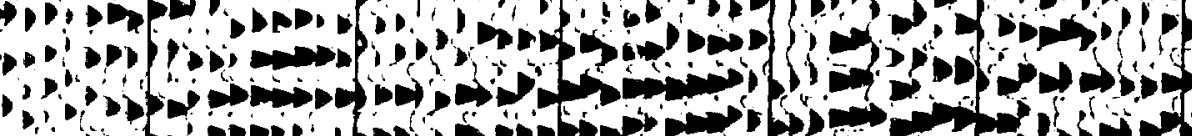

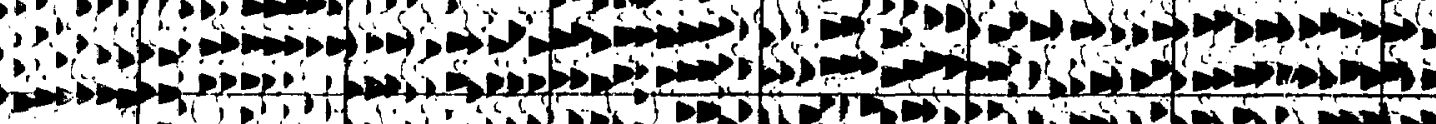

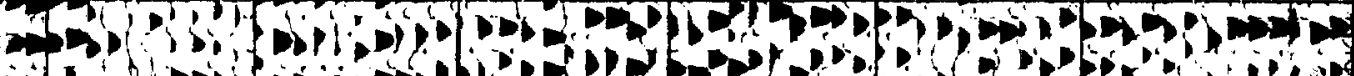

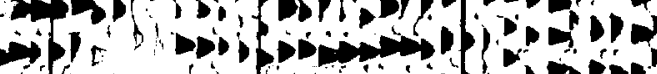

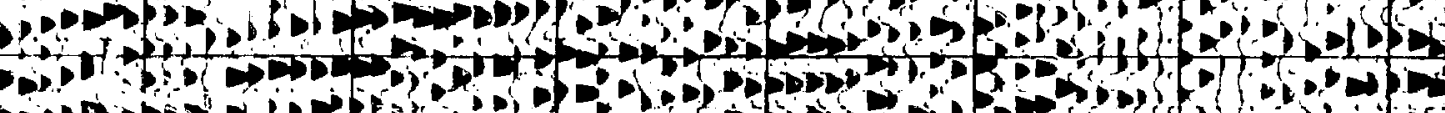

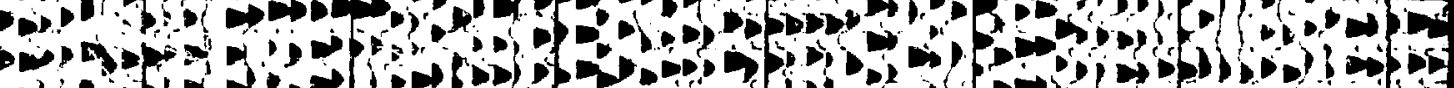

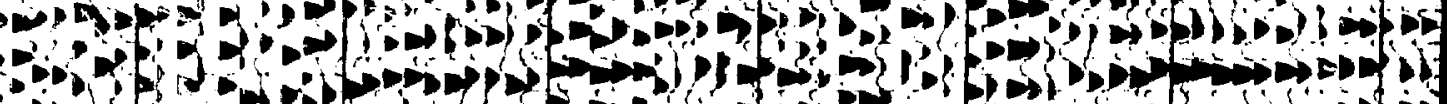

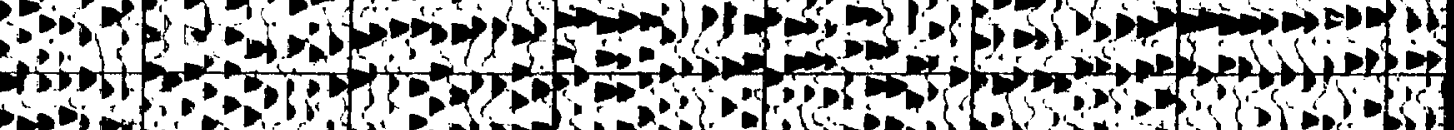

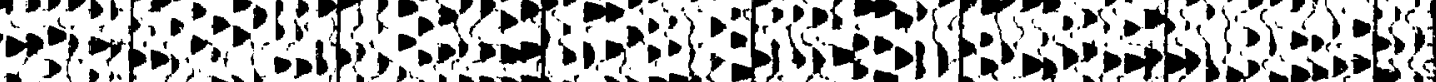
-

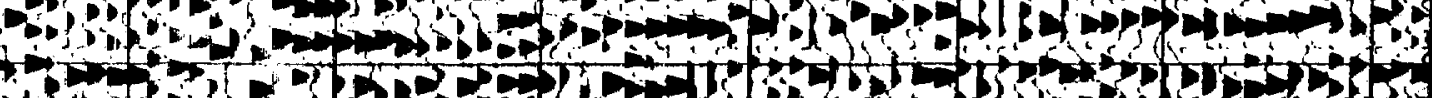

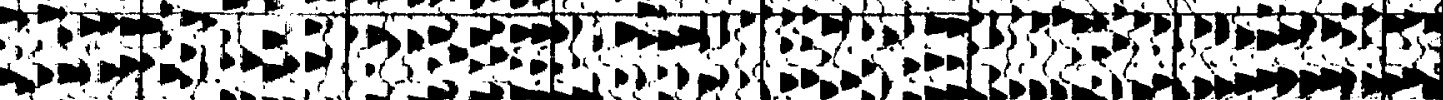

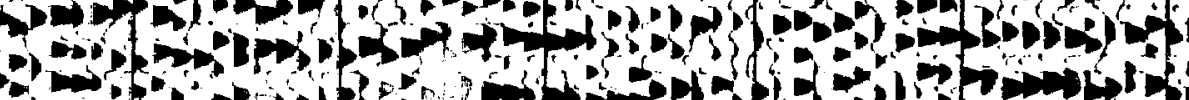
(a) 
tilpip 


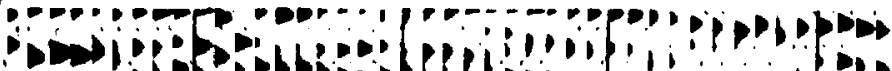

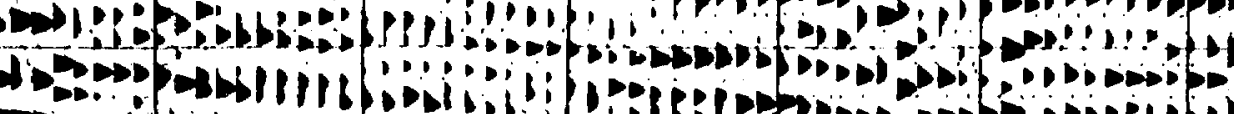

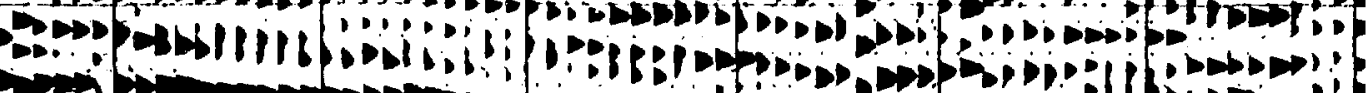

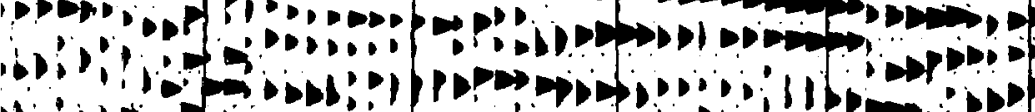

bijis

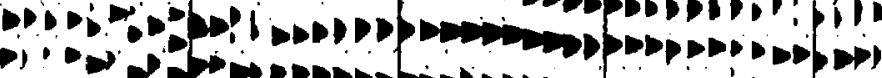

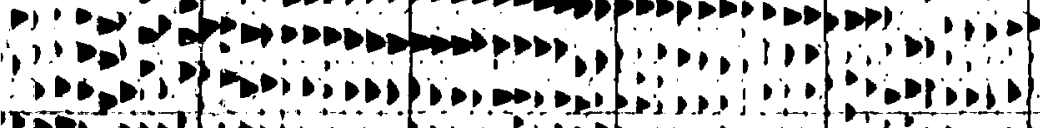

$1: 1 \rightarrow 1$

in

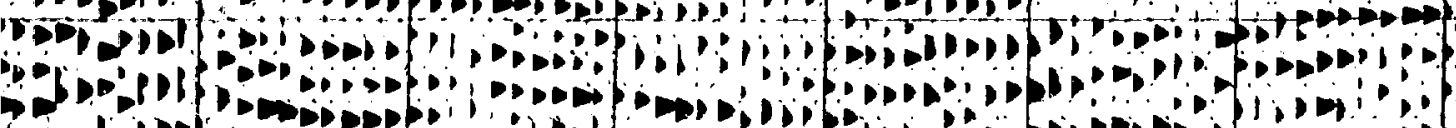

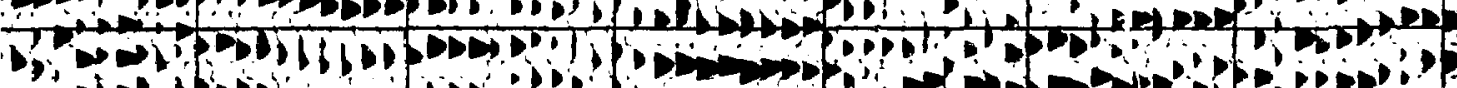
isos Pof

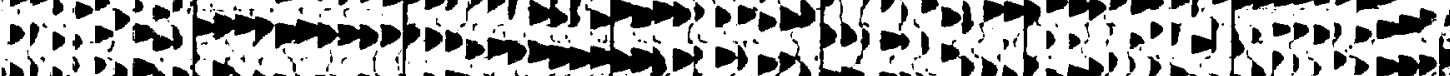

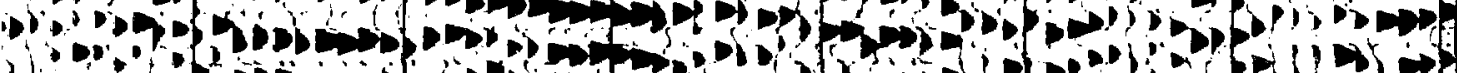

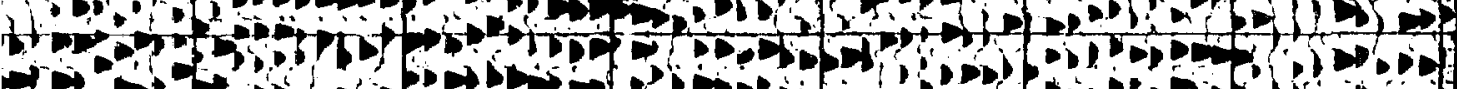

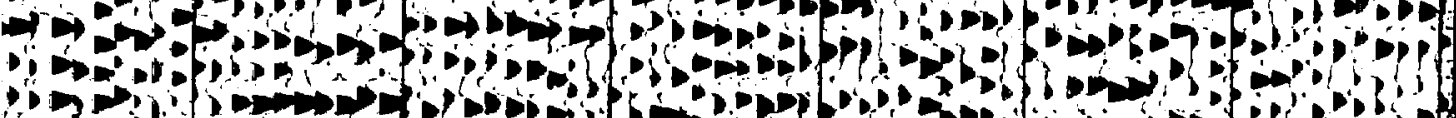

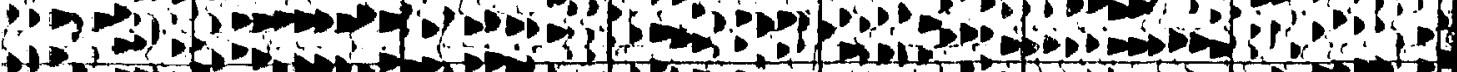

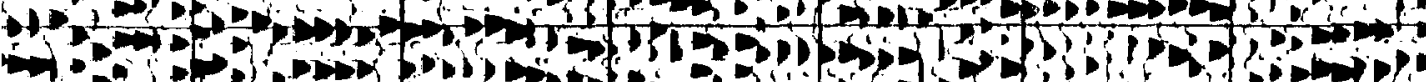

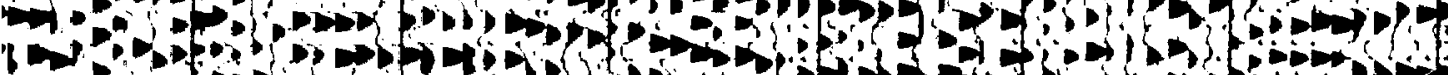

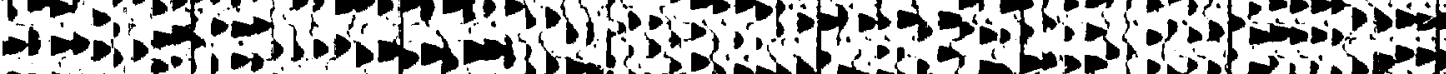
ans

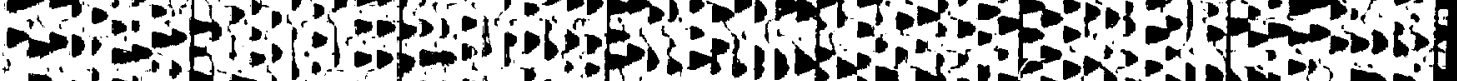

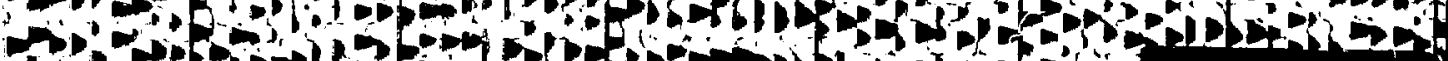
-

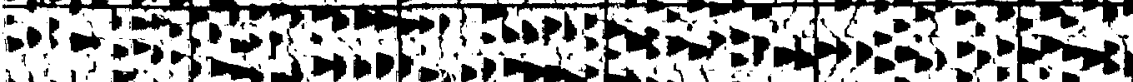

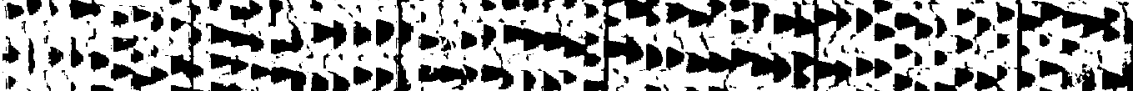

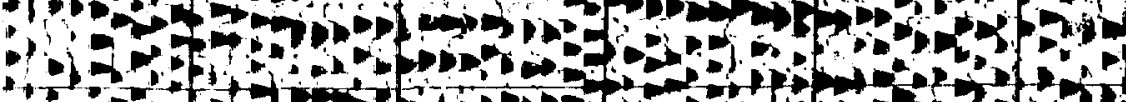

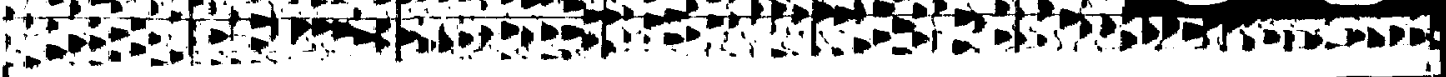




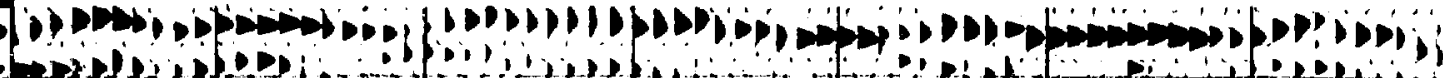

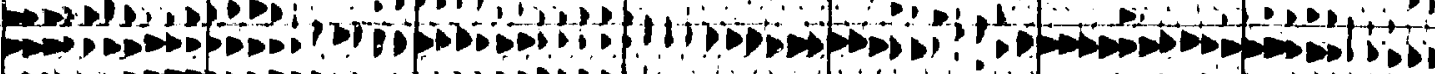

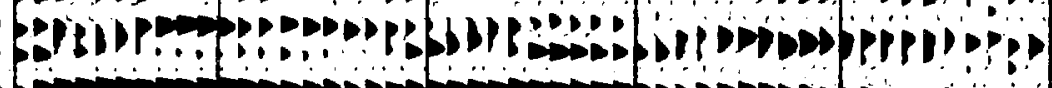

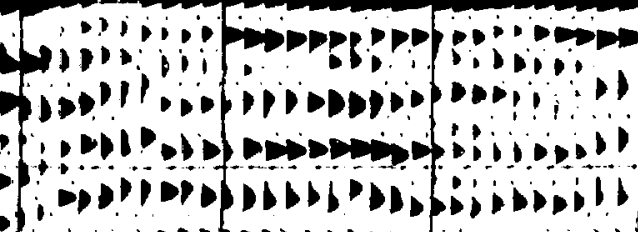

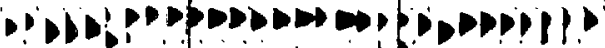

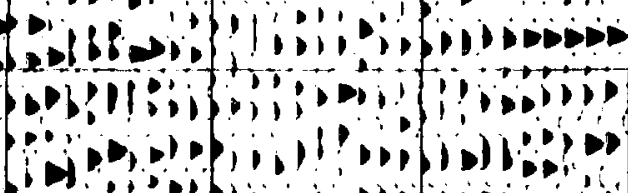
folo $(3,0)$

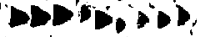

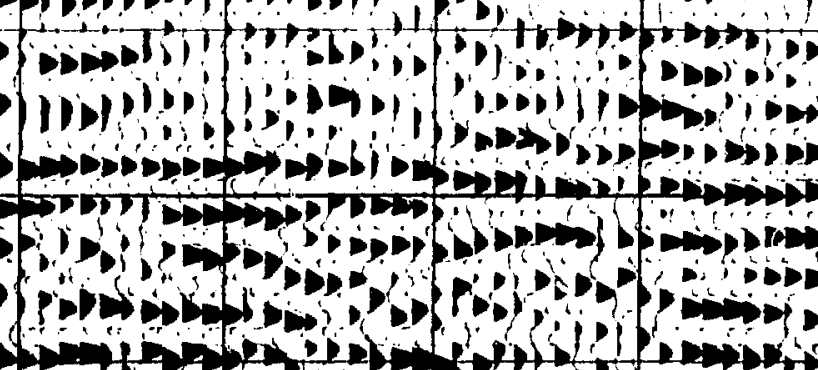
$\rightarrow \rightarrow$

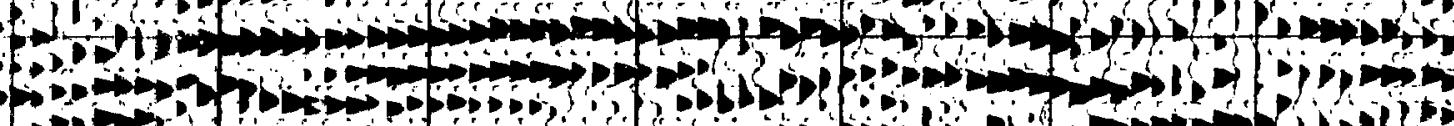

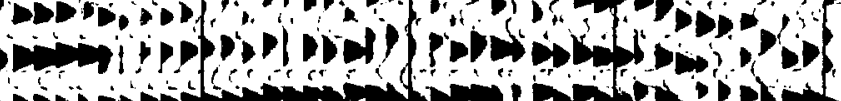

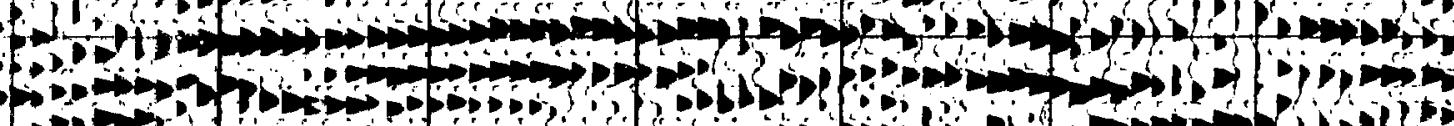

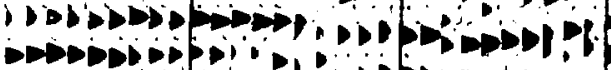
${ }_{10}$

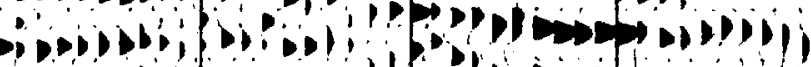

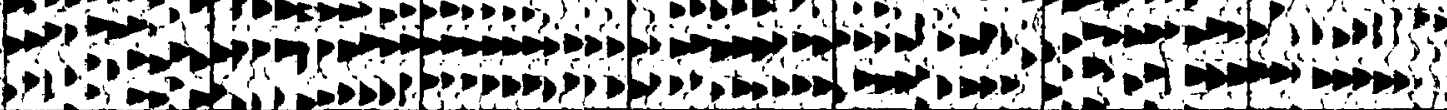

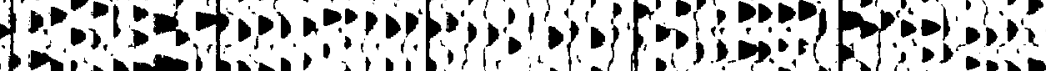

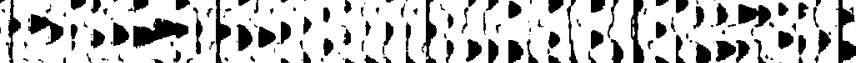
porbion Hilom

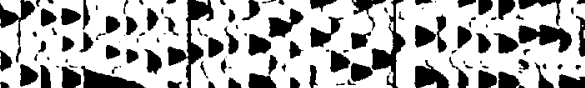

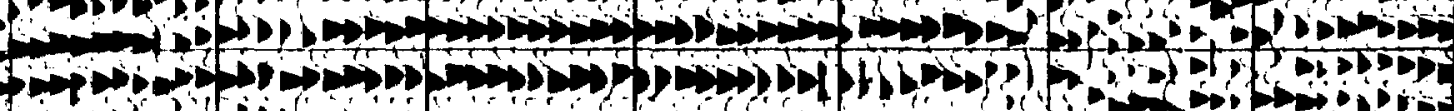

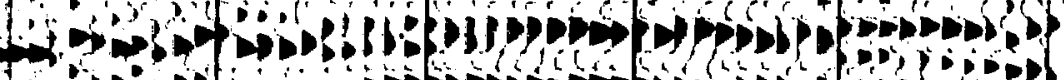

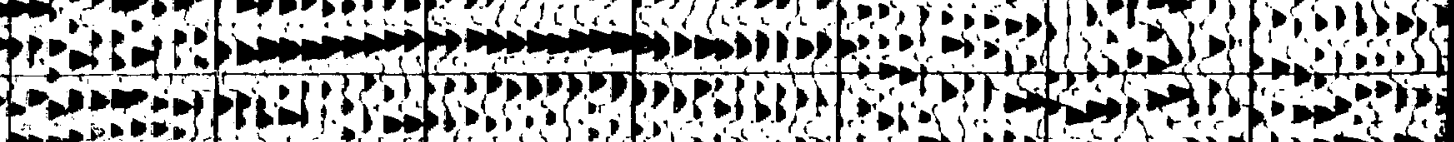

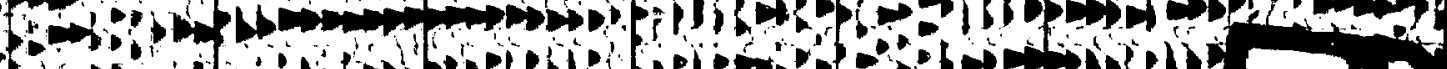
Lien

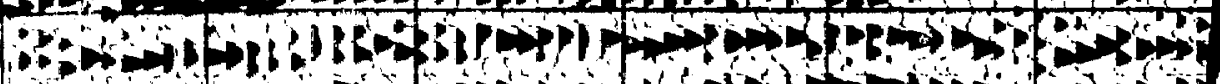

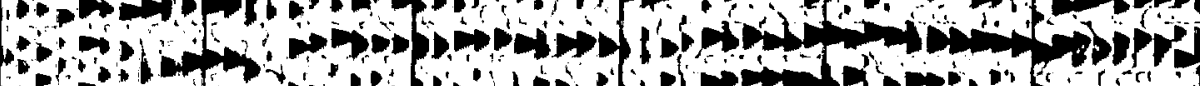

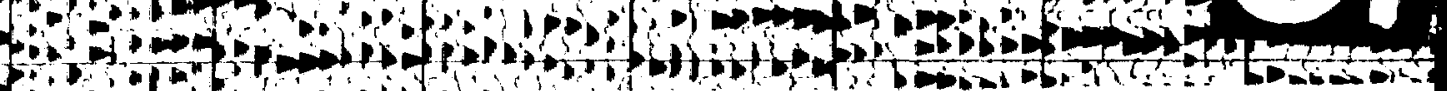




\section{m}

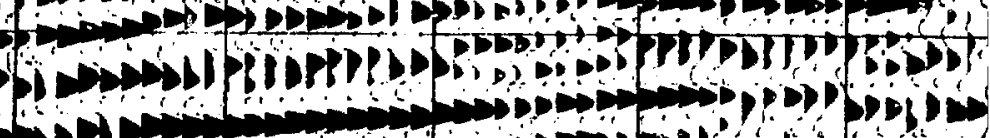
(n)

\section{sibion}

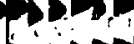

iptistion Bitivition

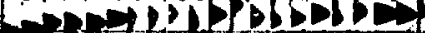

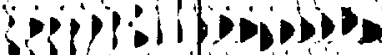

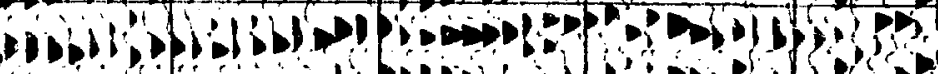

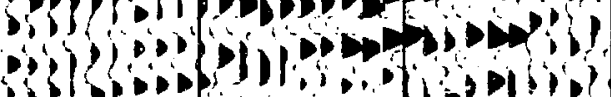
ipipisil plowitosions is iniment

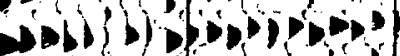

How

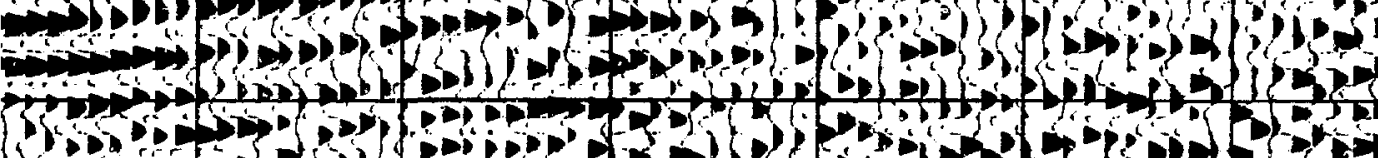

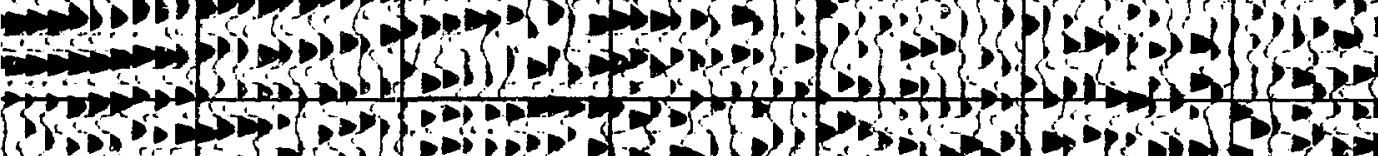

\section{(t)}

(i)

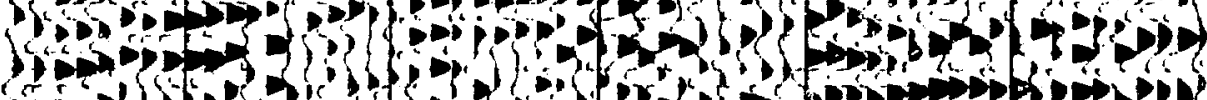

(5)

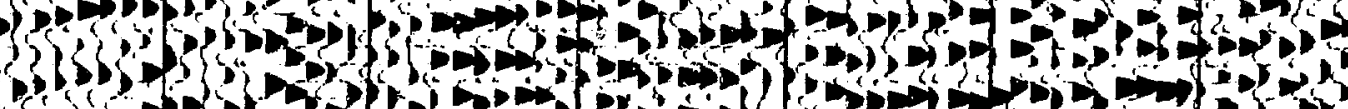

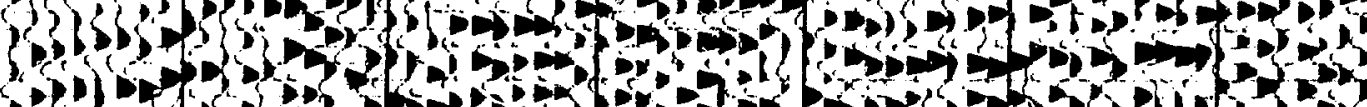

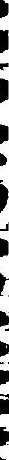

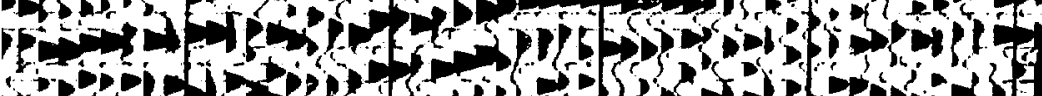

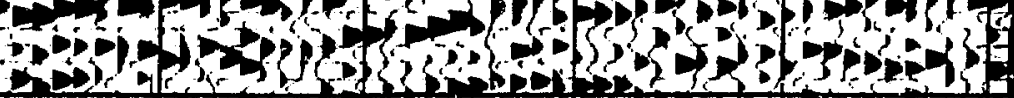

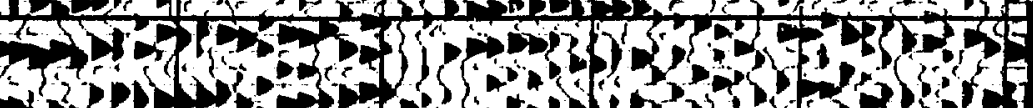

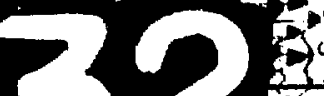

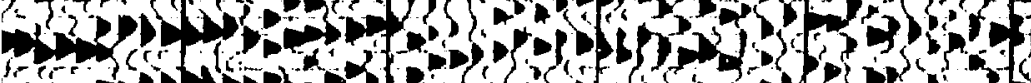

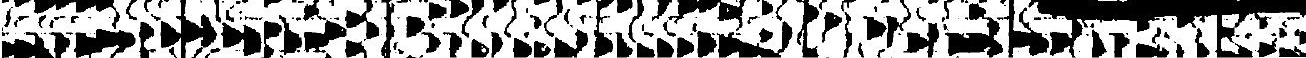




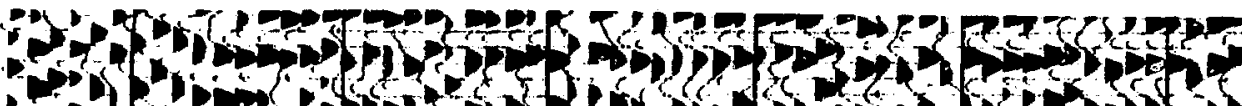

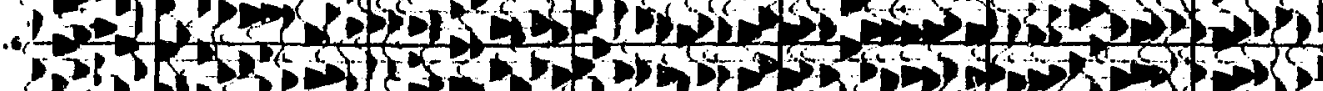

Pin

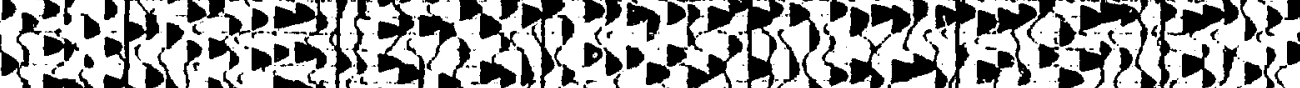

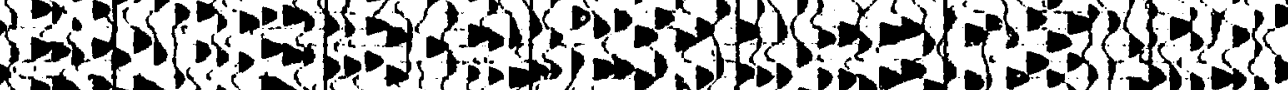

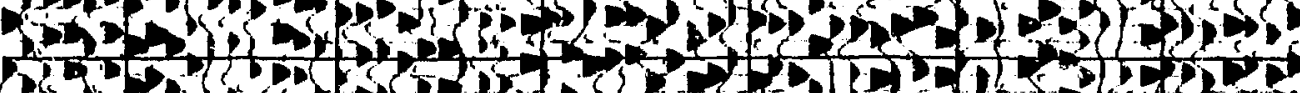
S.

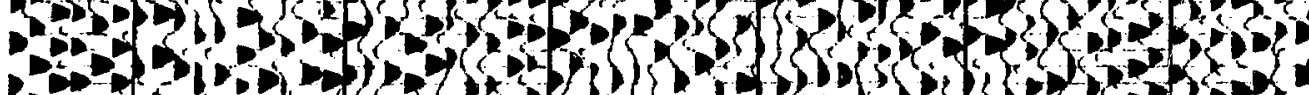

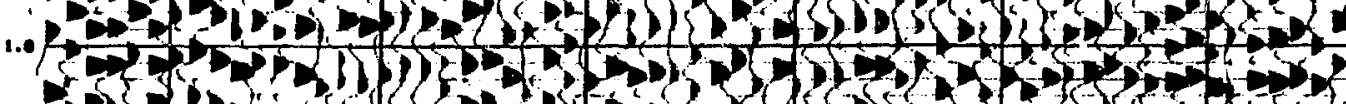

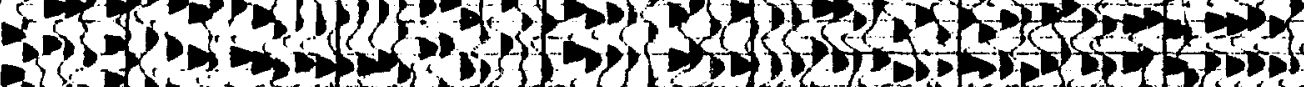

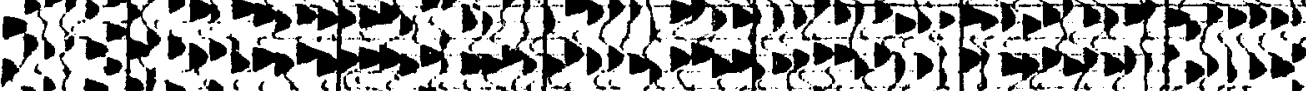

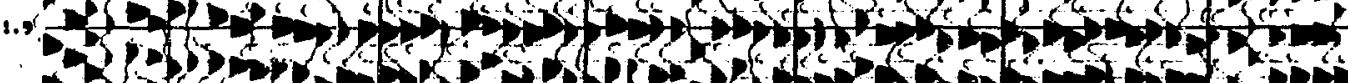

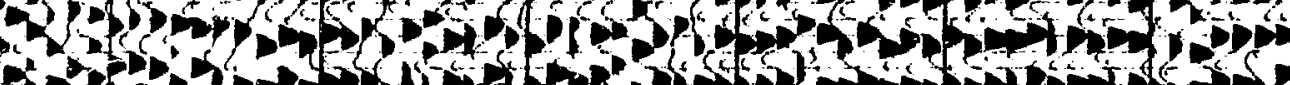

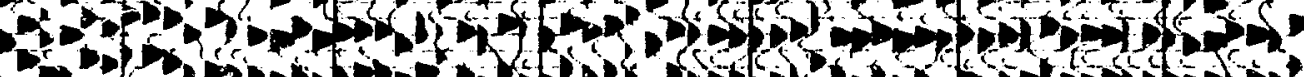

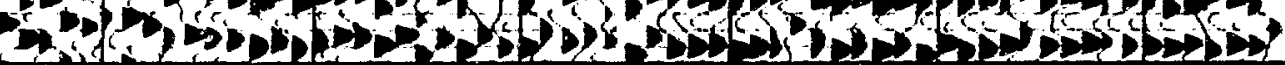

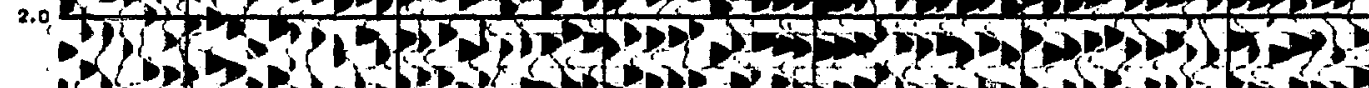

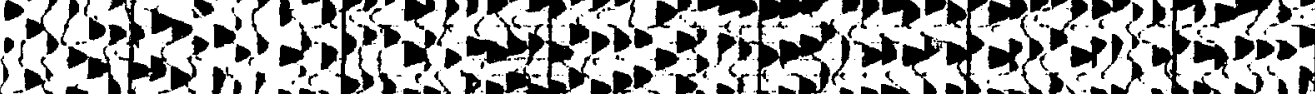

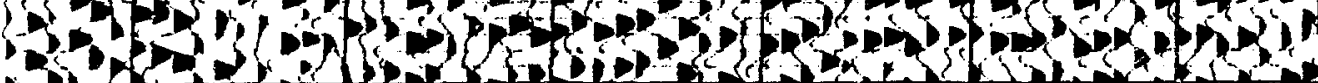

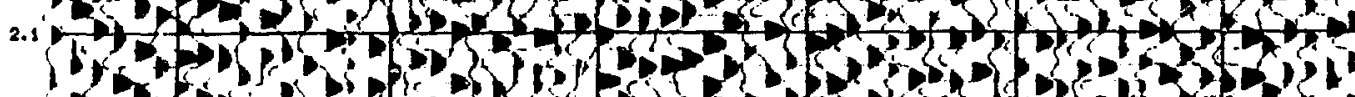

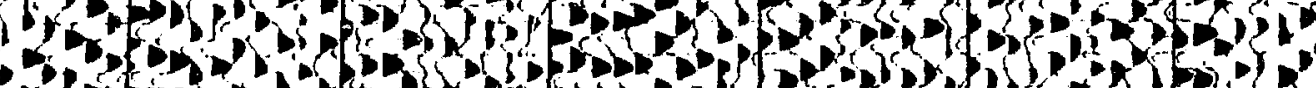
$3 f_{0}$

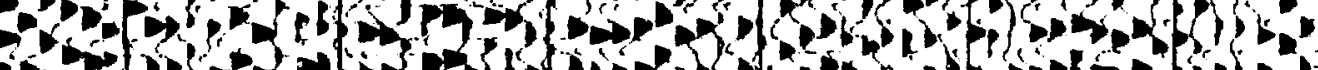

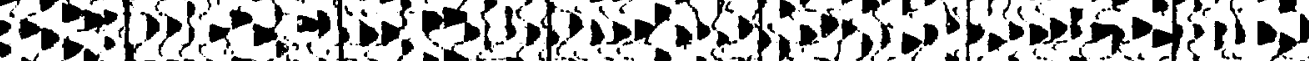

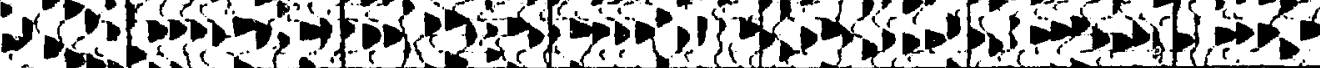

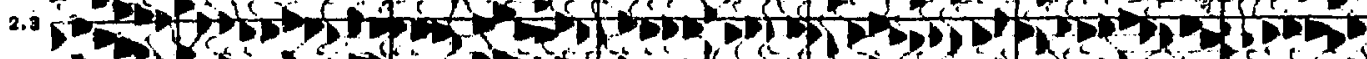

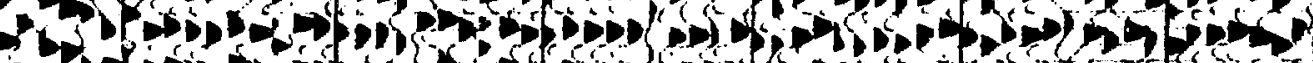
1.4

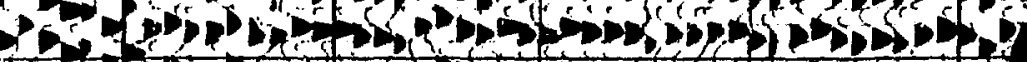

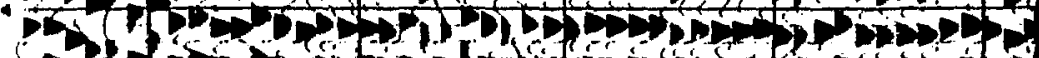

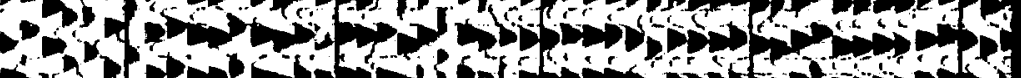

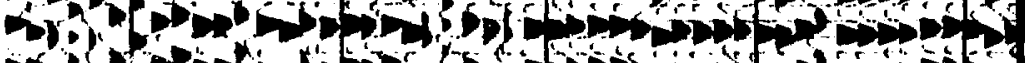

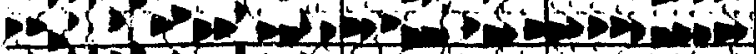

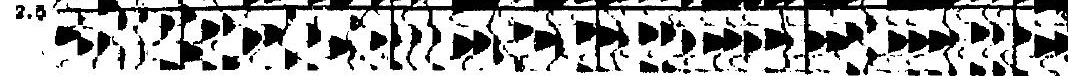




\section{0,565085}

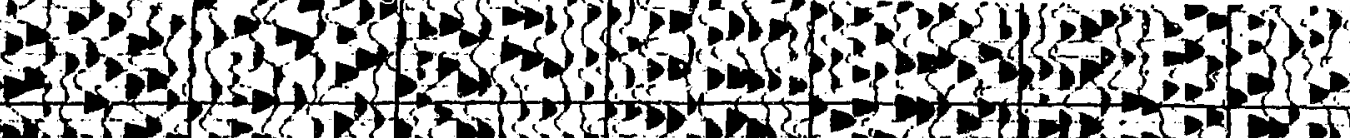

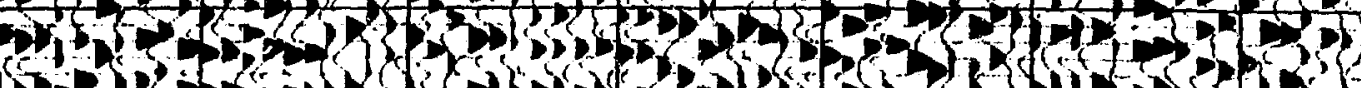

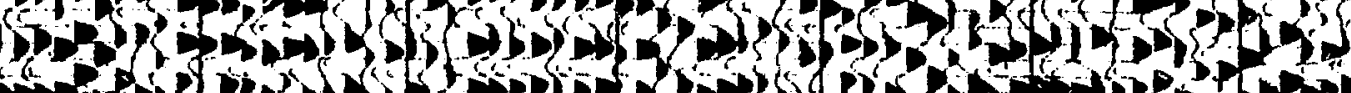

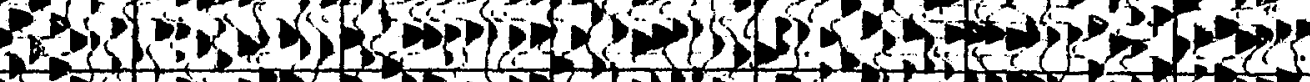
23.53

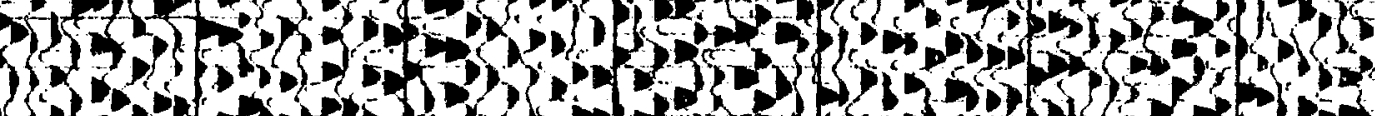

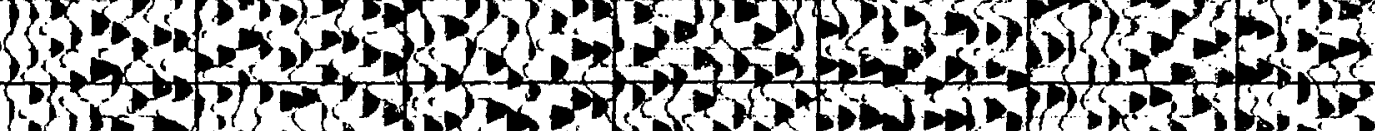

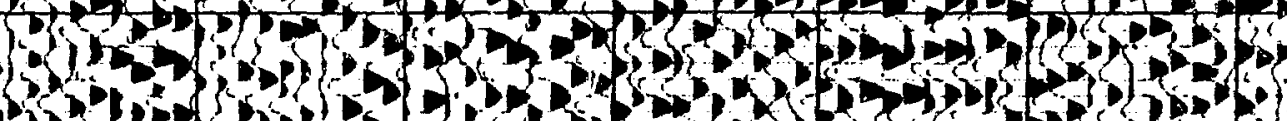

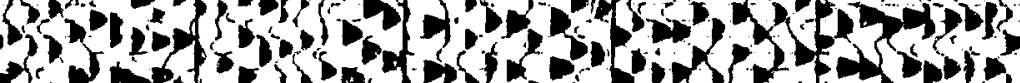

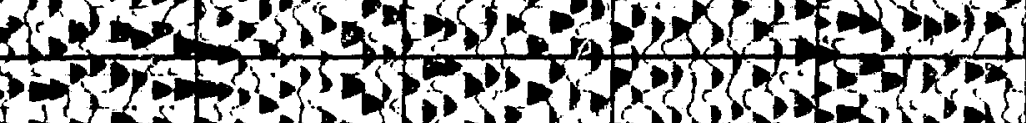

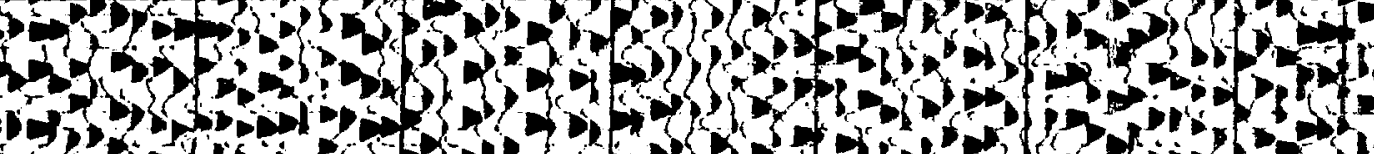

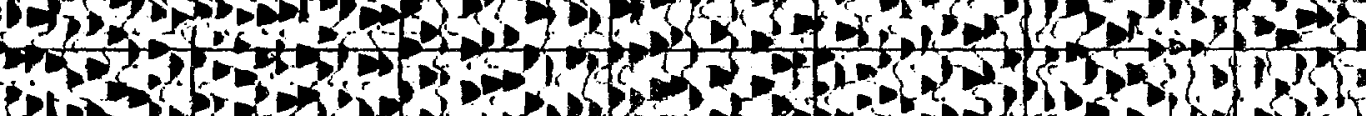

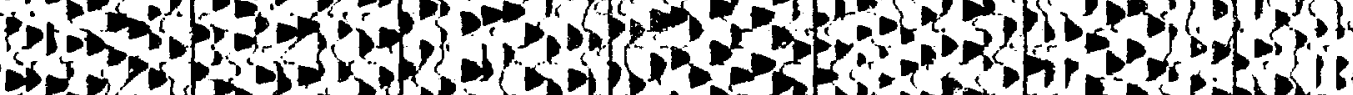

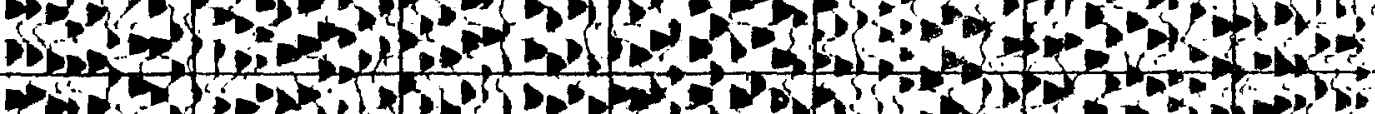

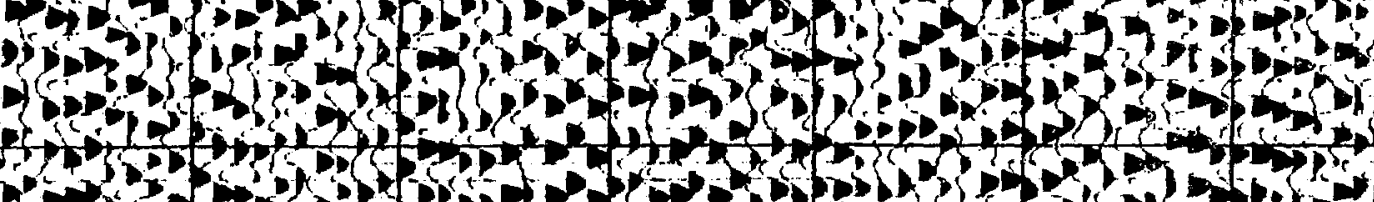

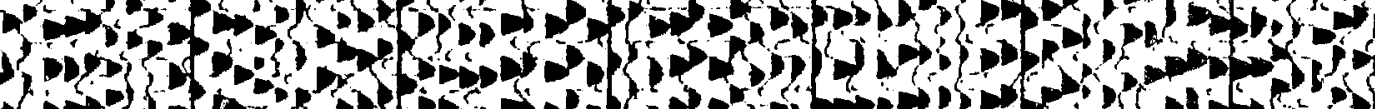

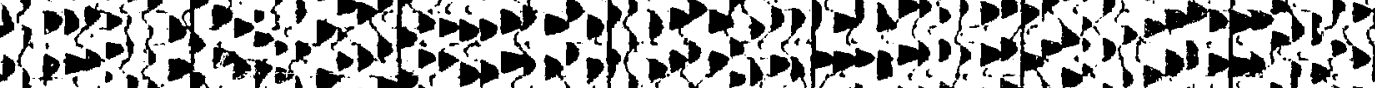
(5) 


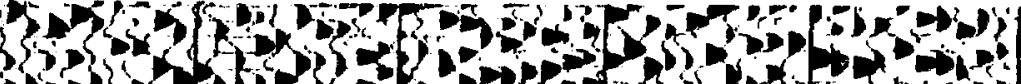

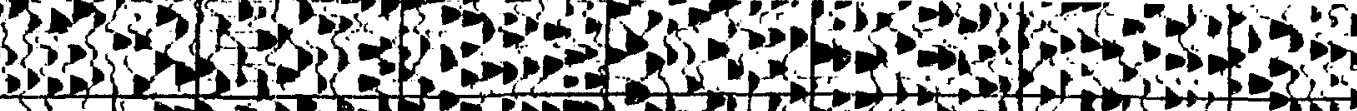

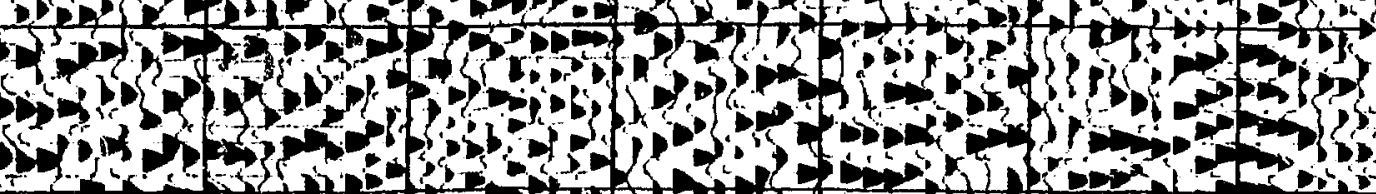

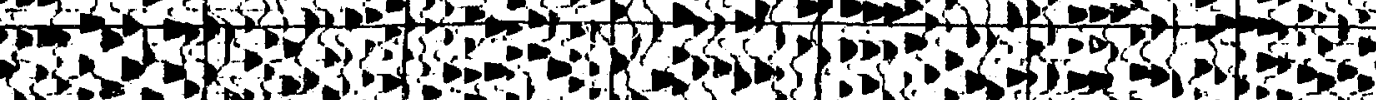

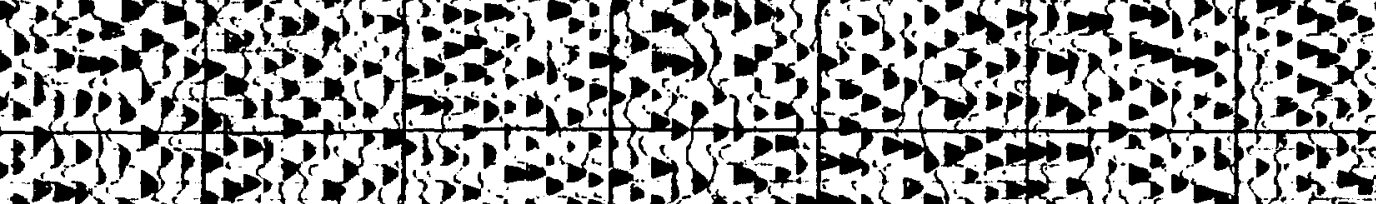

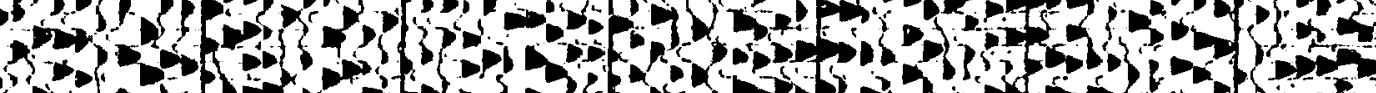

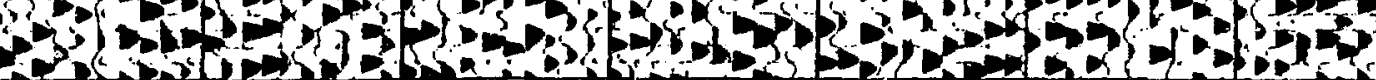

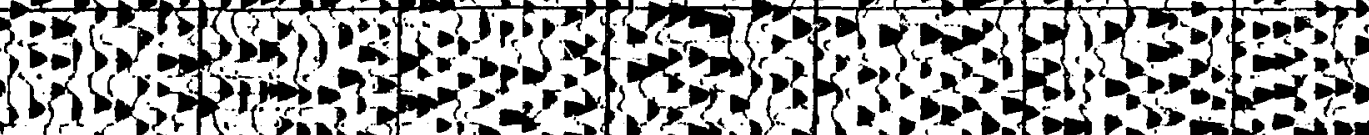

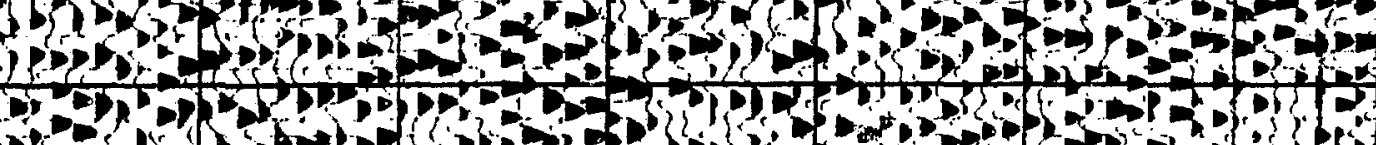
7 P

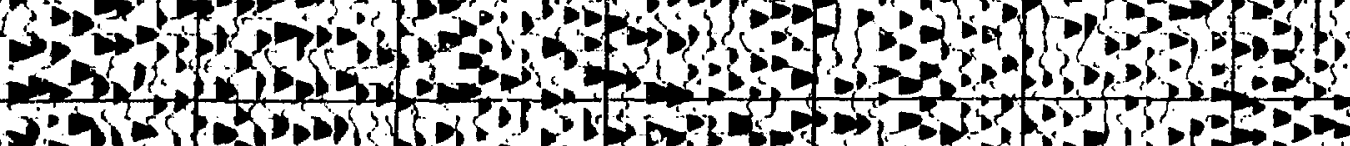

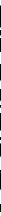

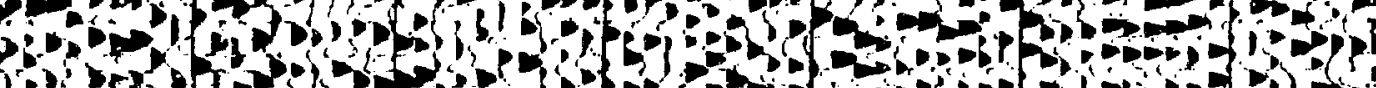

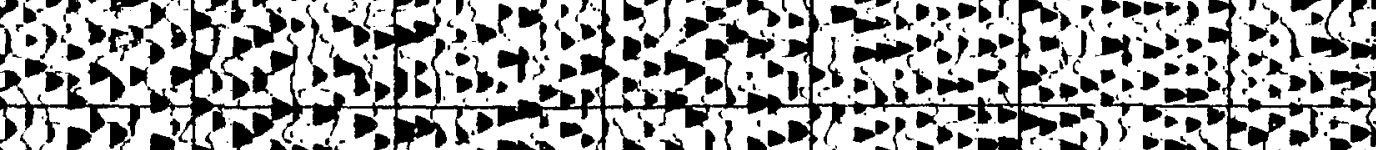

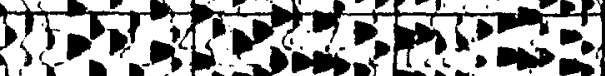

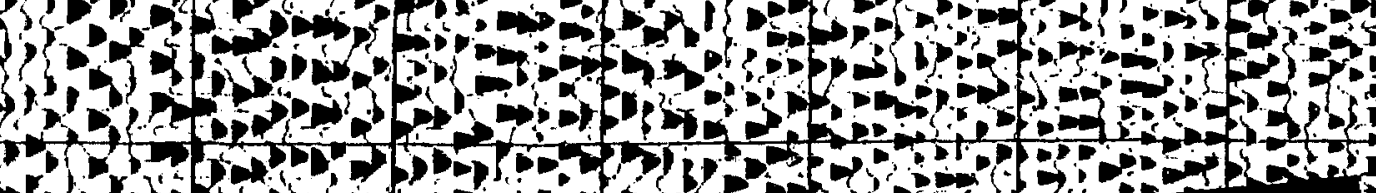

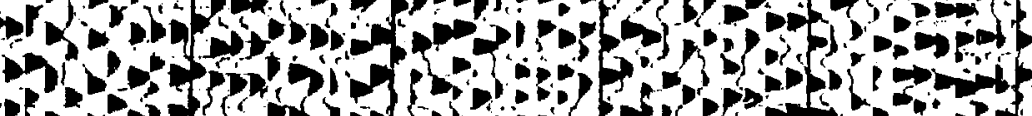

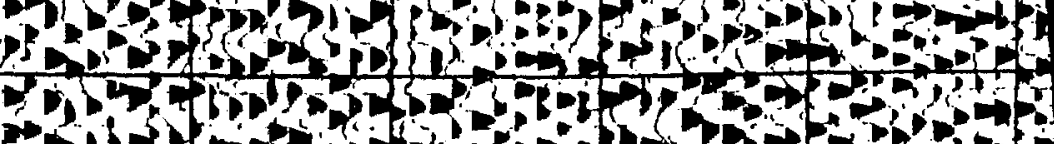

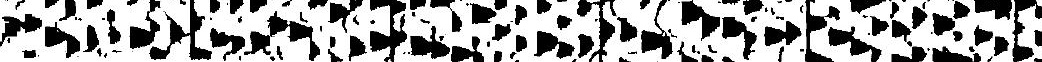




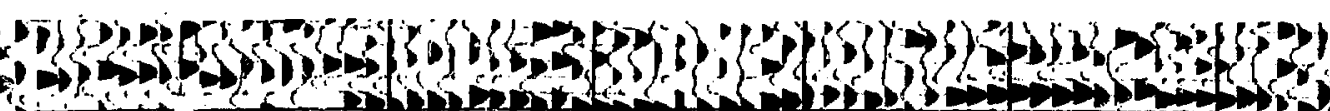

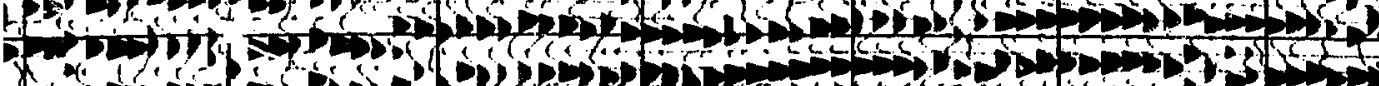

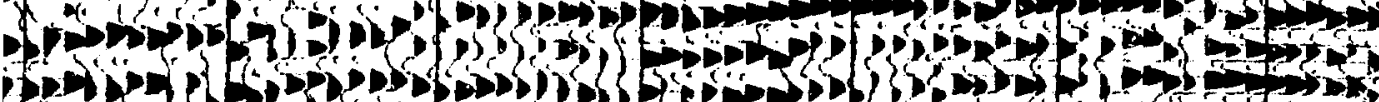

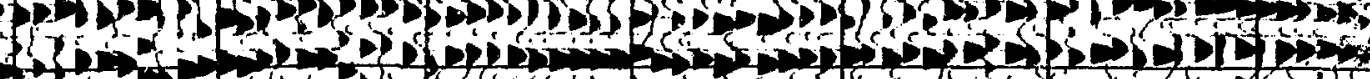
3.3

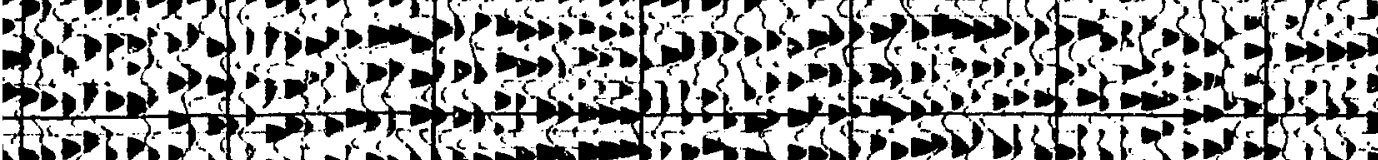
(35 3.

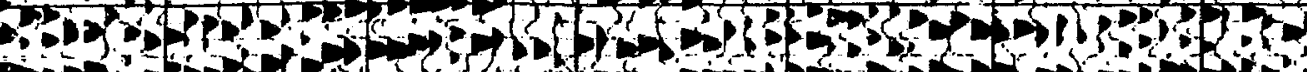

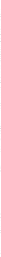

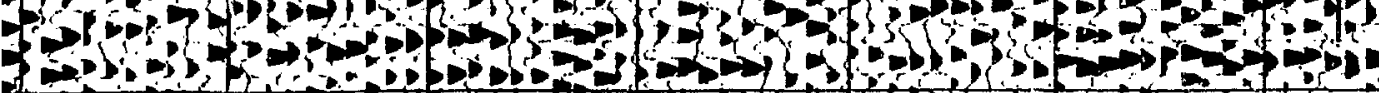

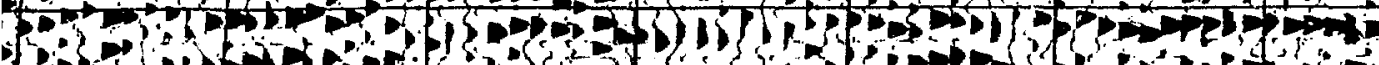

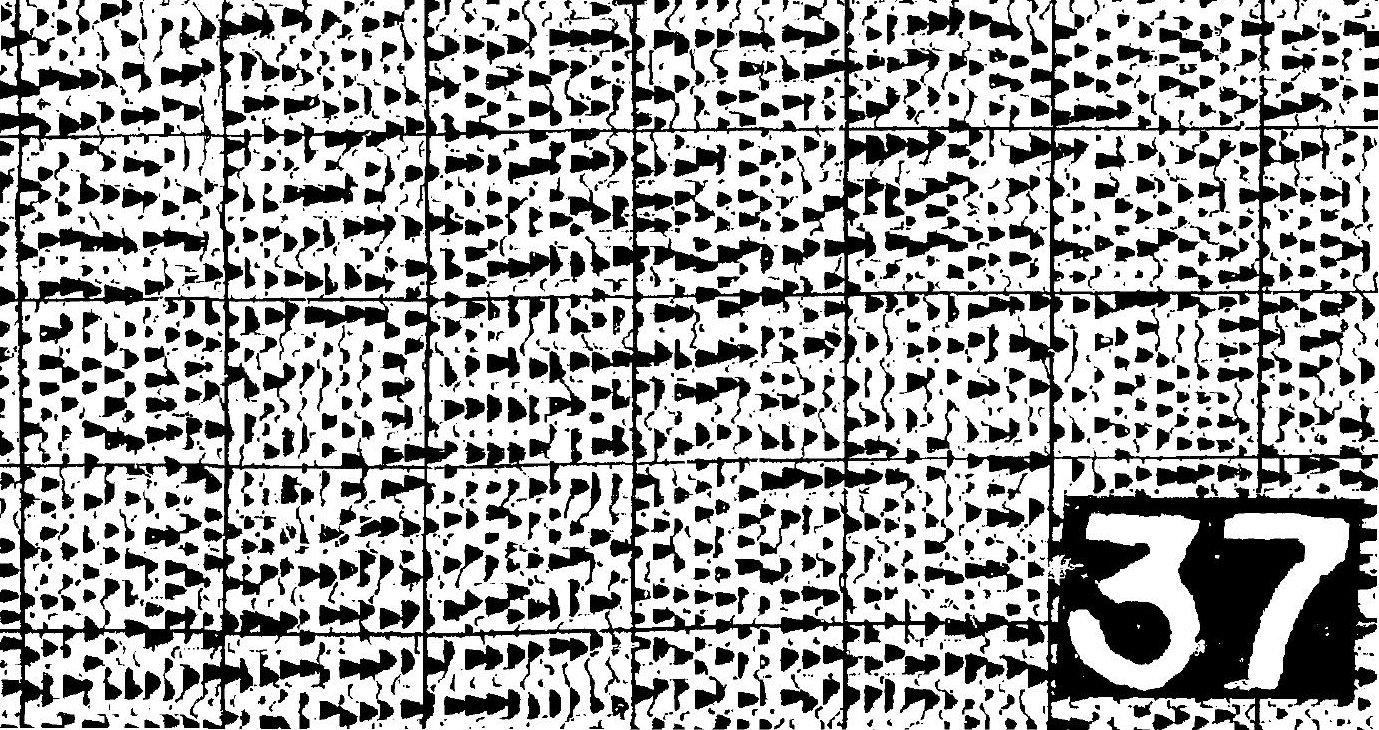




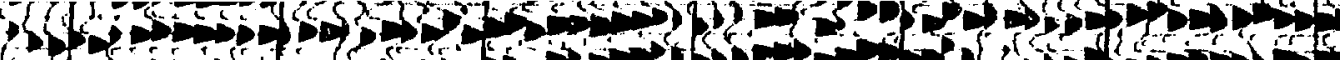
7 H $-23+0.0$

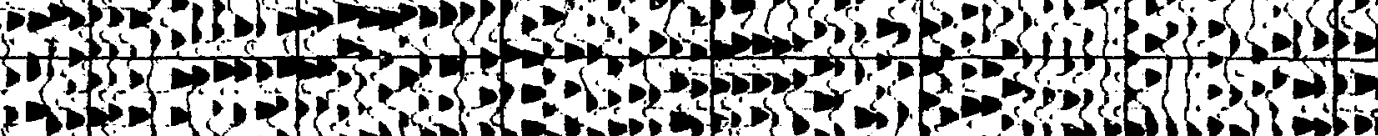
30.045 S.

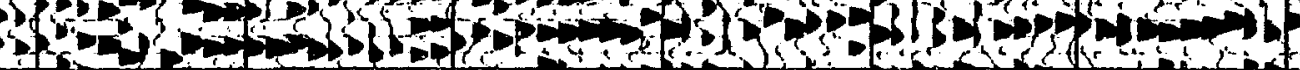

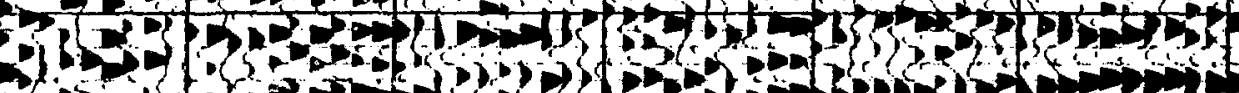
3.7.

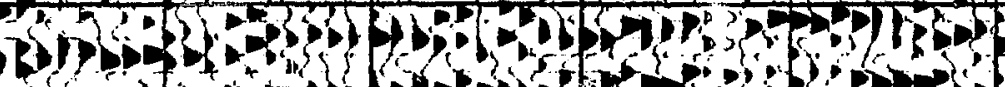

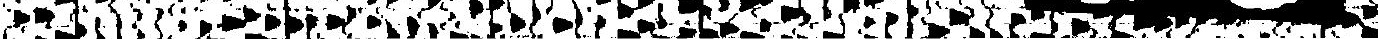





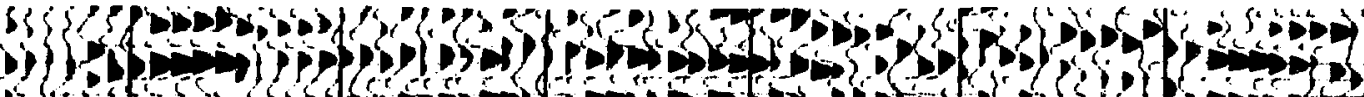
S.

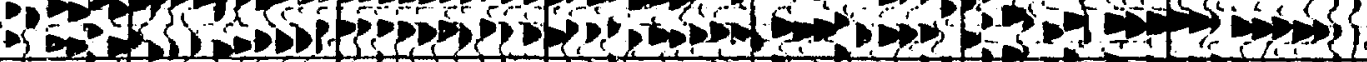

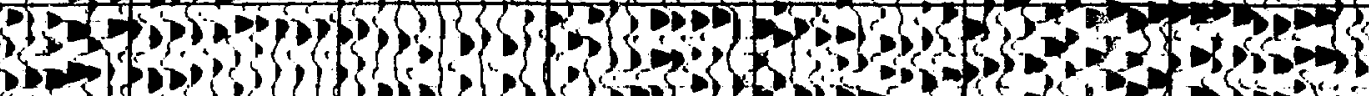

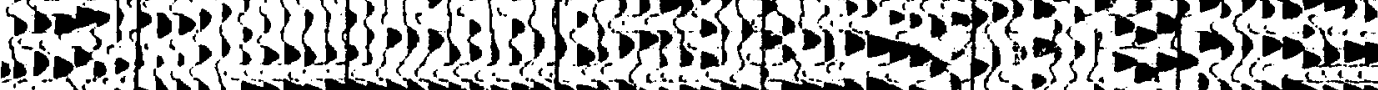

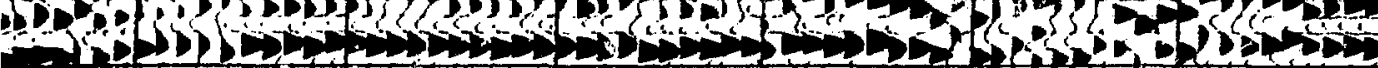

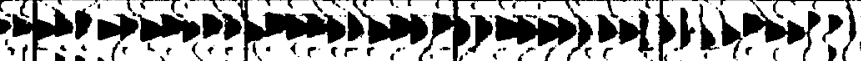
$\left\{\begin{array}{l}3 \\ 3\end{array}\right.$

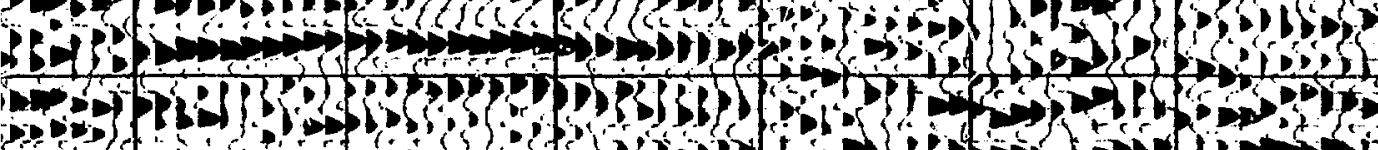

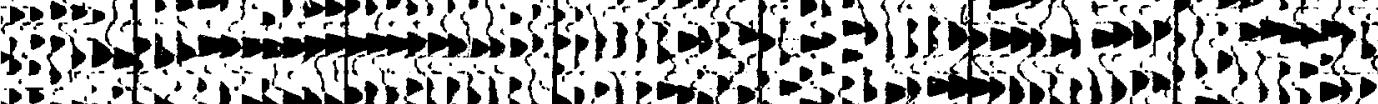

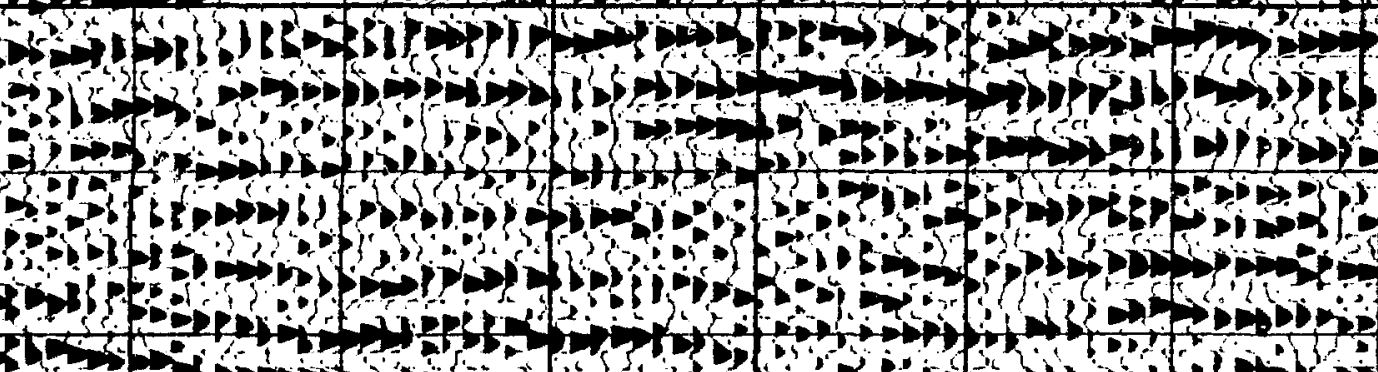
The

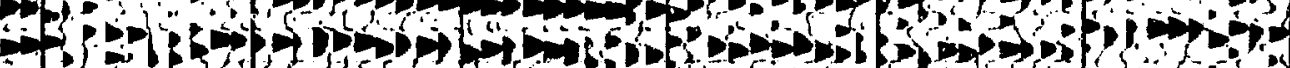

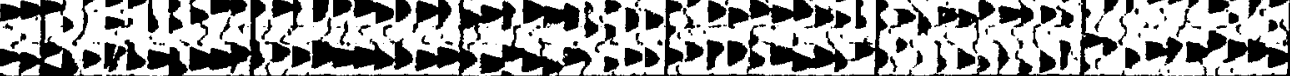

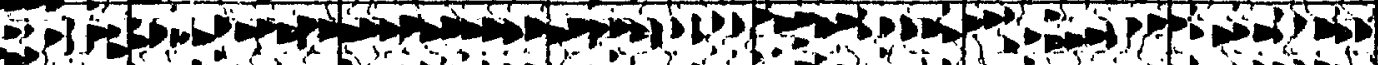

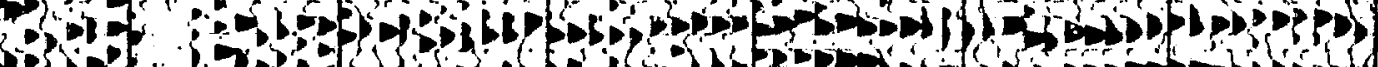

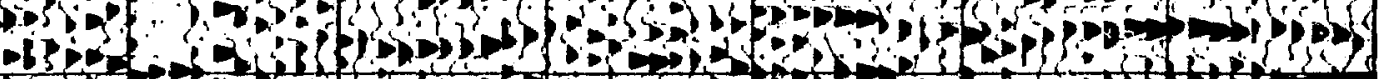
6.1.

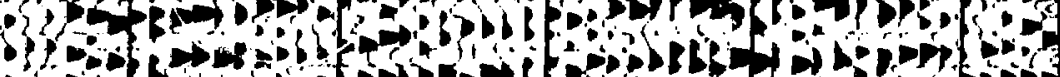

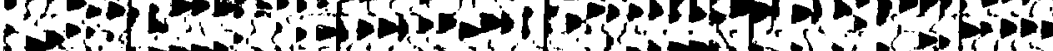
(3)

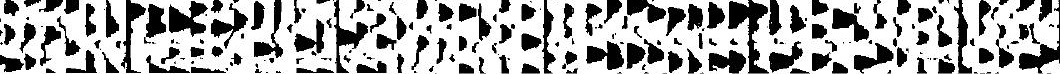

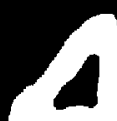




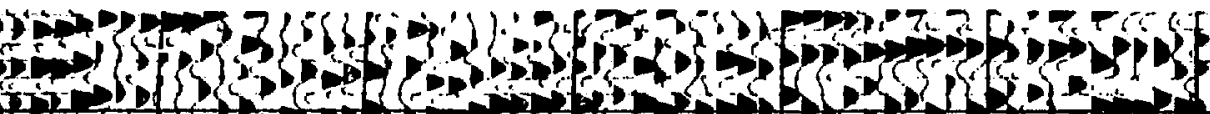
(5)

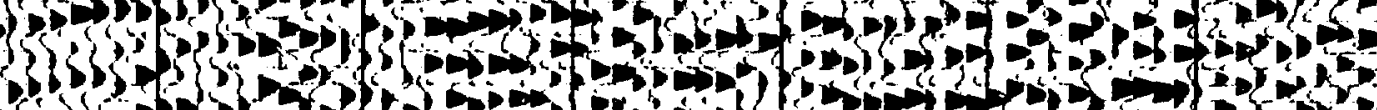

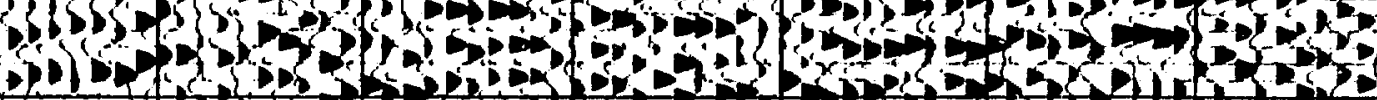

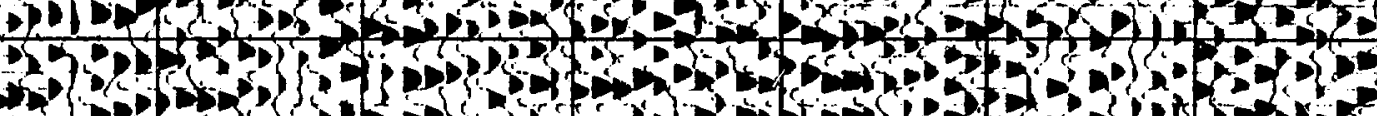
$\rightarrow$

\section{This

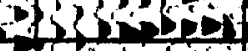

(1)

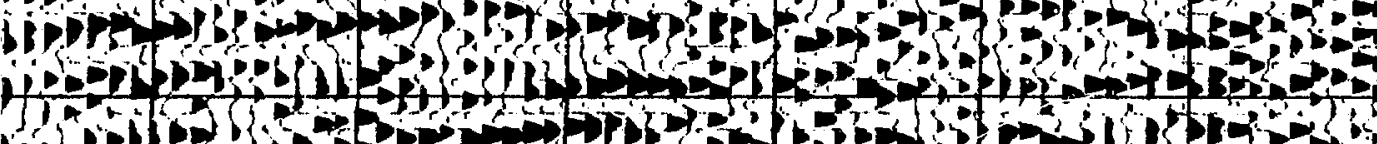

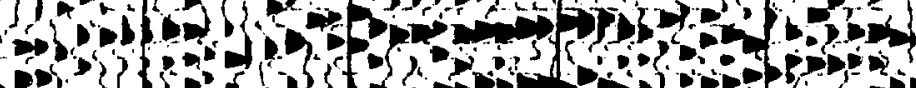

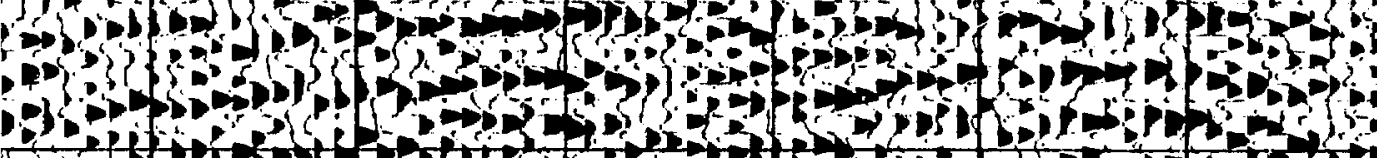
(1) (1)

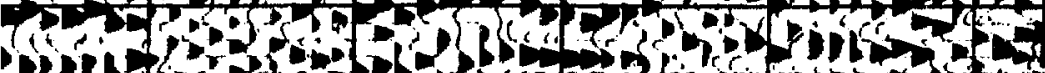
(3) (1) 


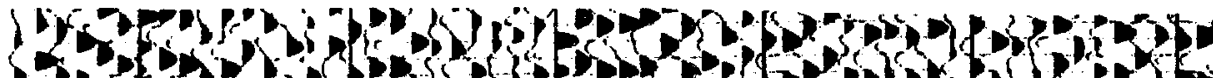

60 (6) 15)

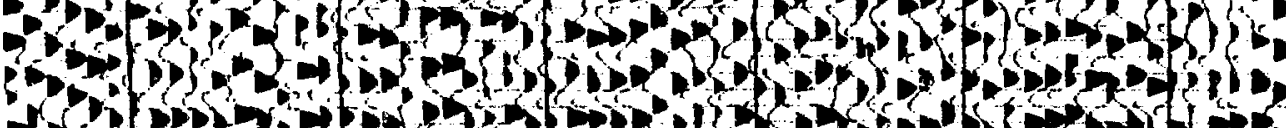

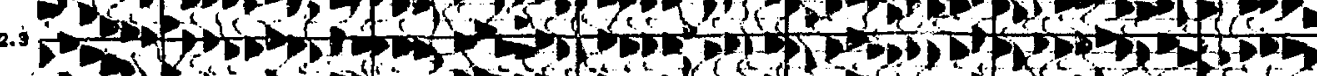

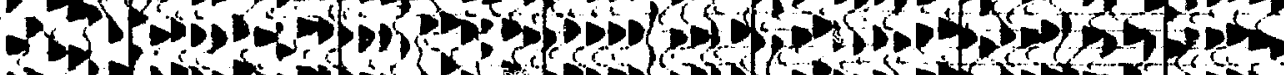

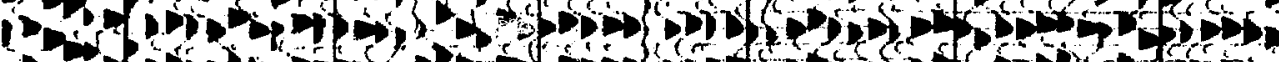

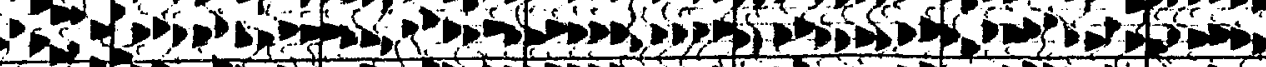

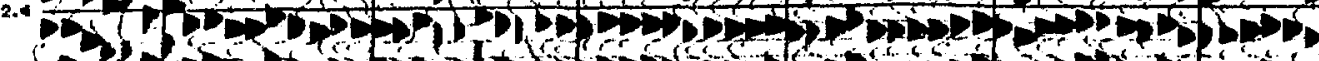

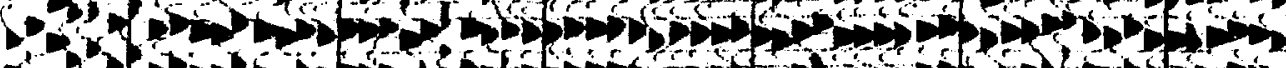

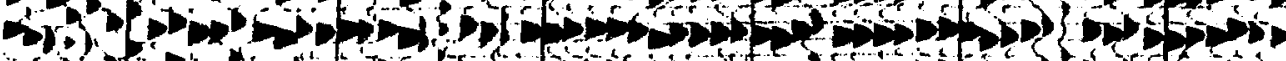

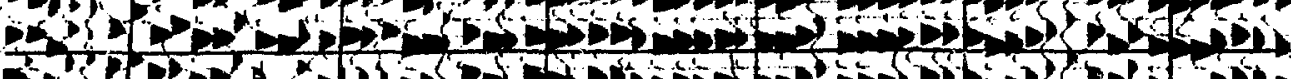
-

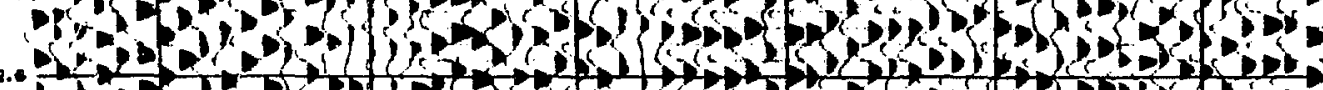

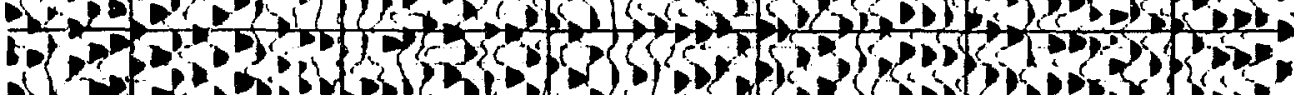

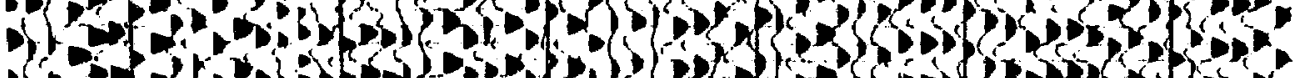

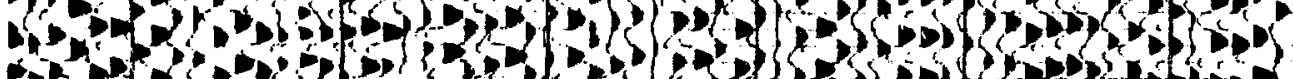

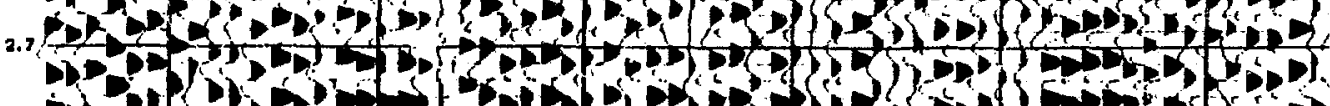

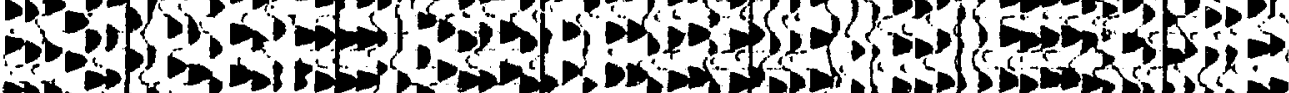

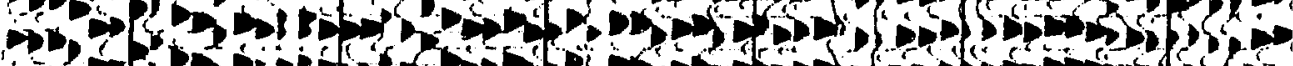

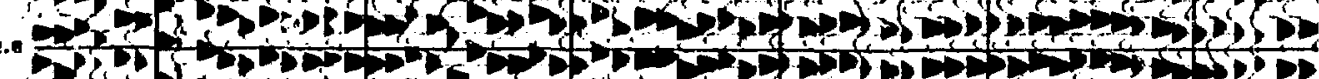

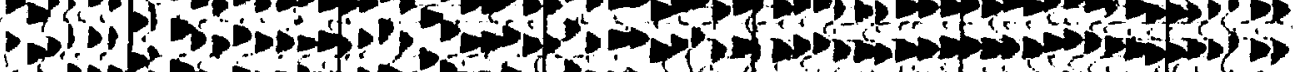
(1) 
3.

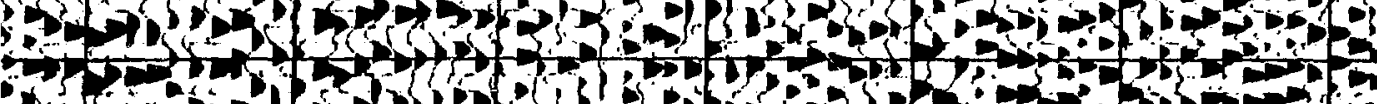

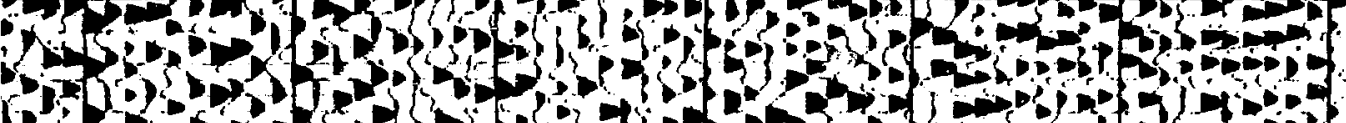

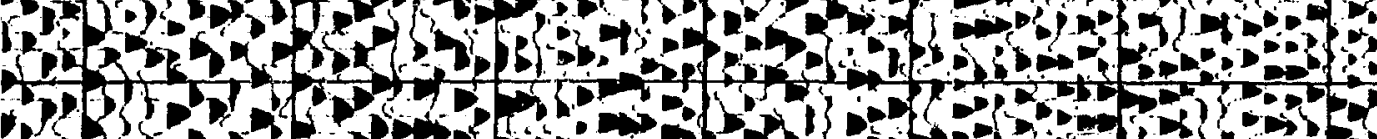

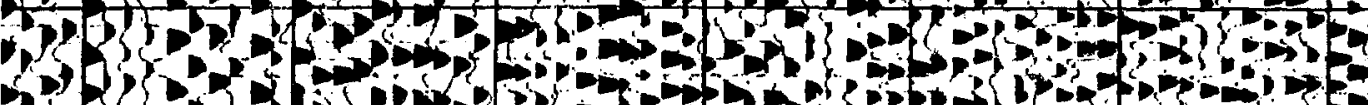

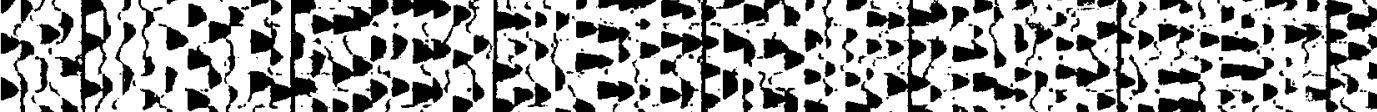

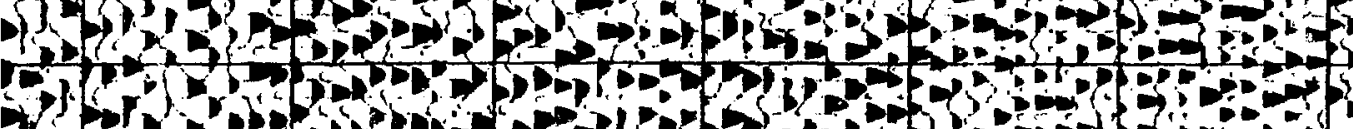

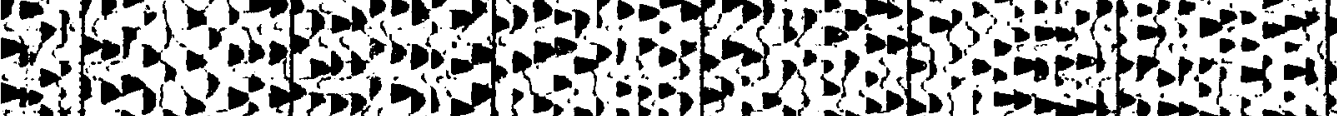

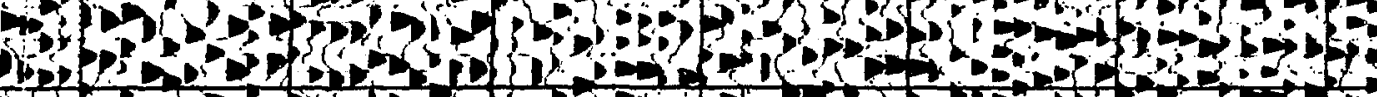

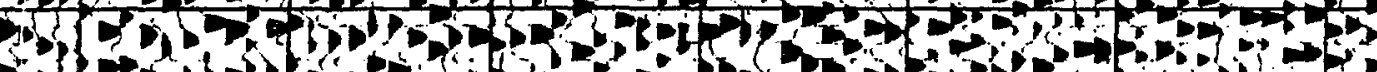

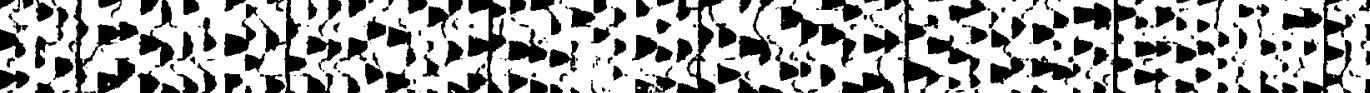

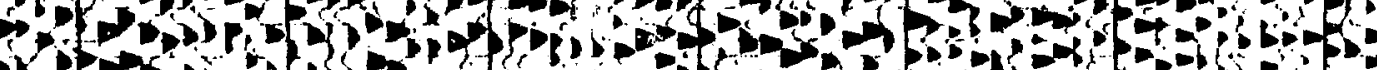

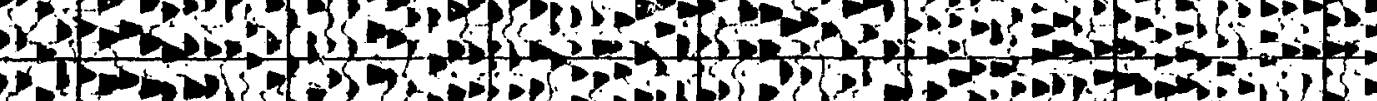

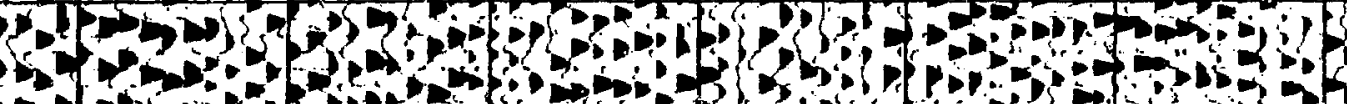

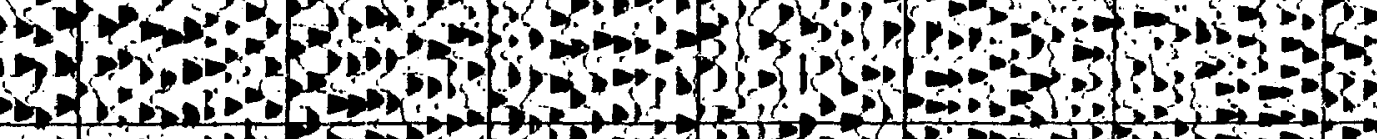
(1)

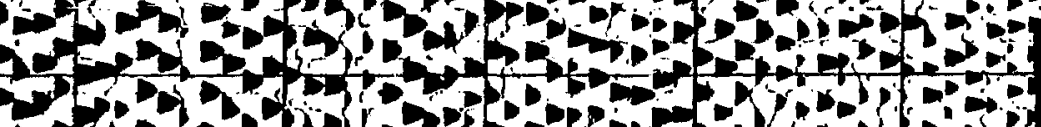
3.9.

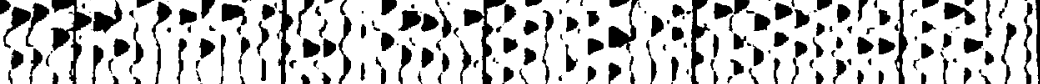

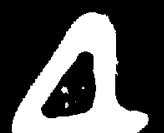


(1)

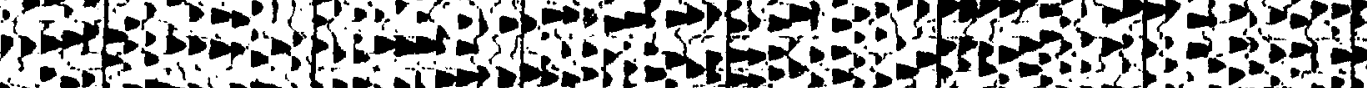

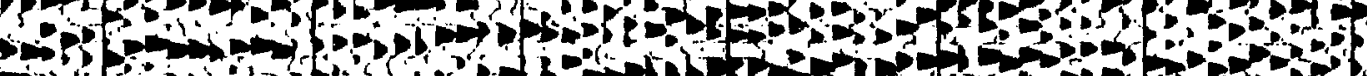

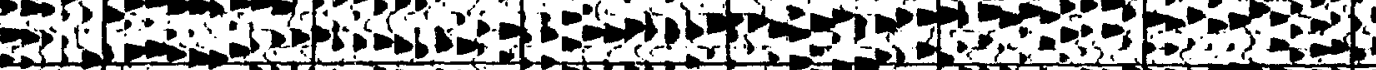
5.5

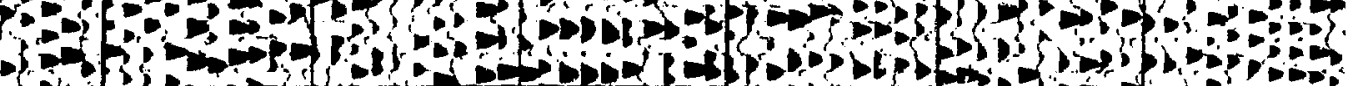

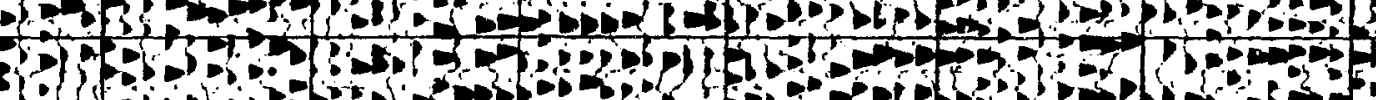

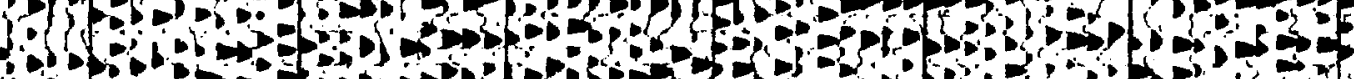

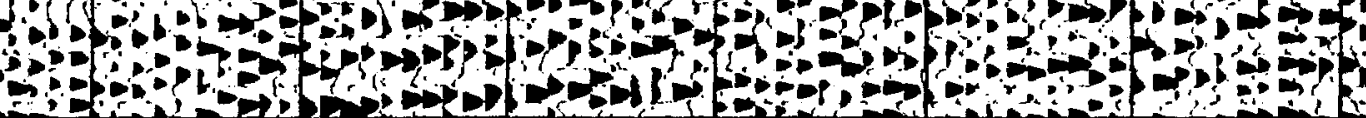

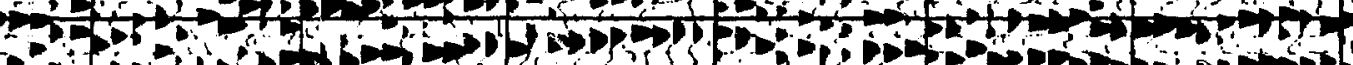

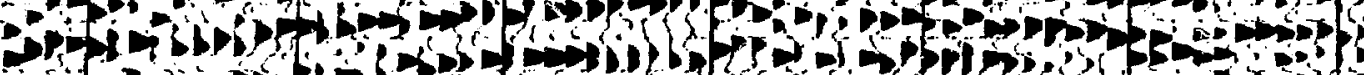

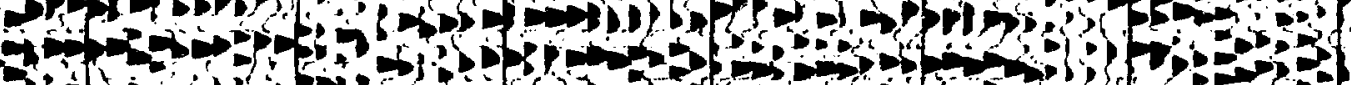
Tof

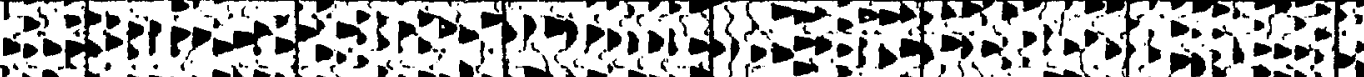

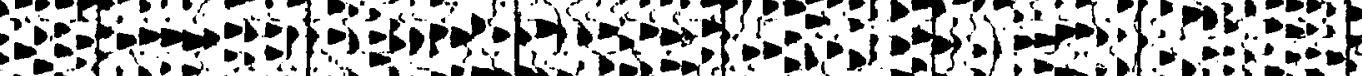
(1)

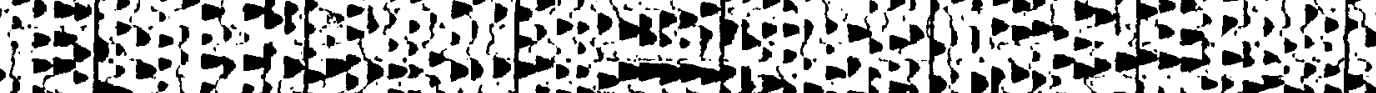

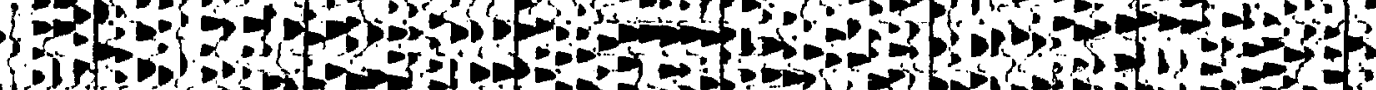

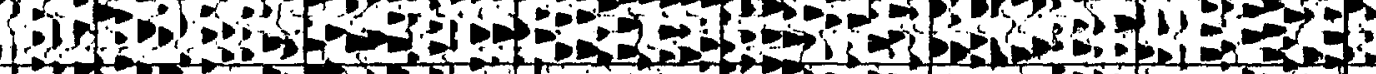

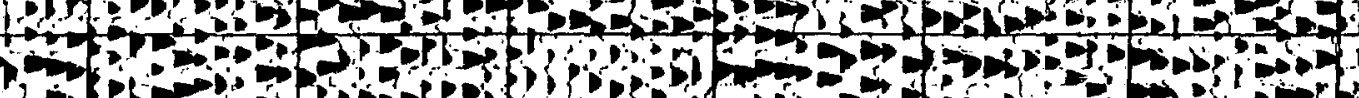
2.

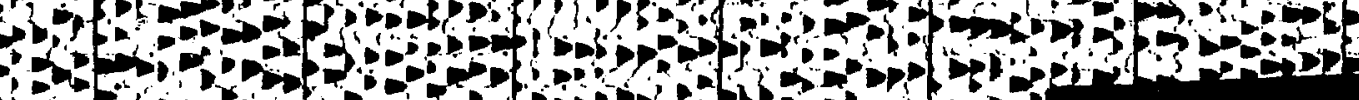

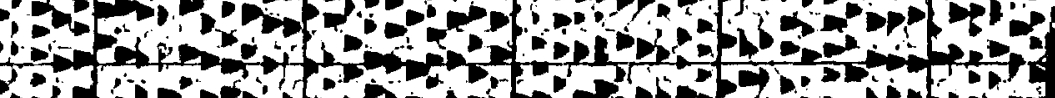

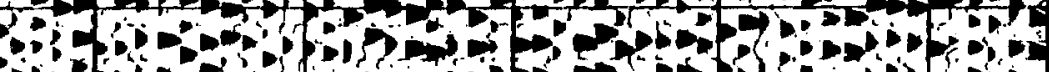

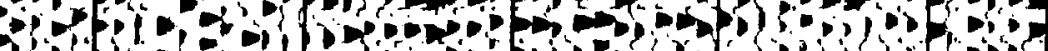

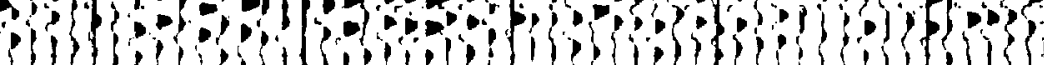




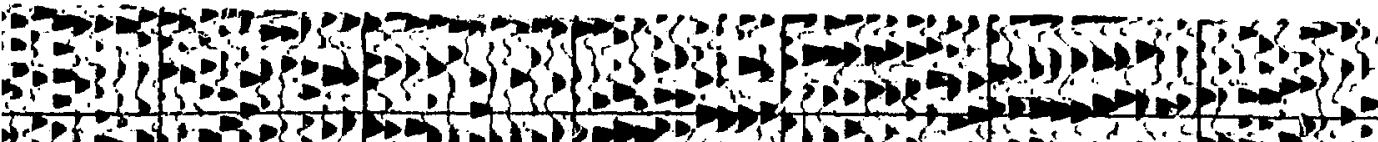

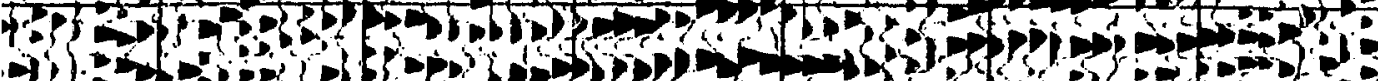

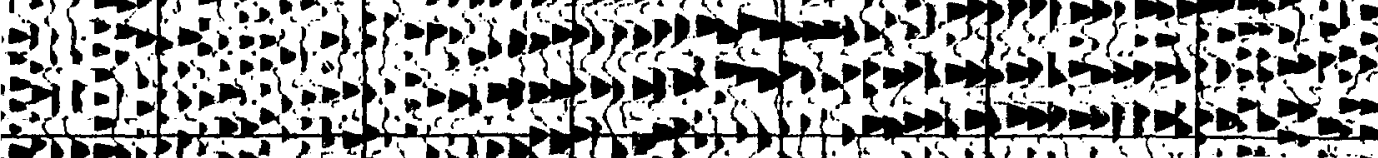
So

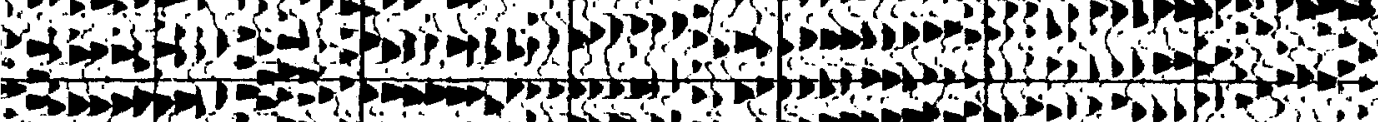

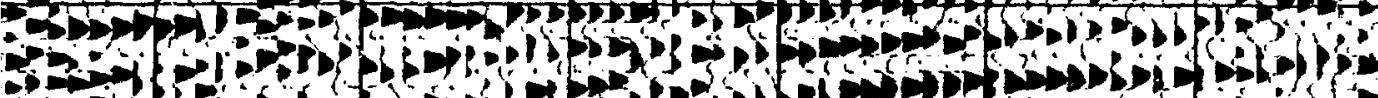

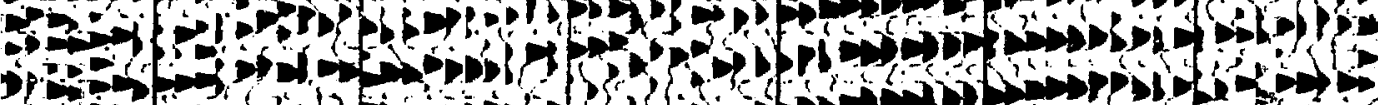

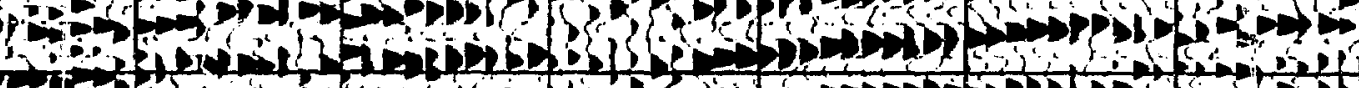

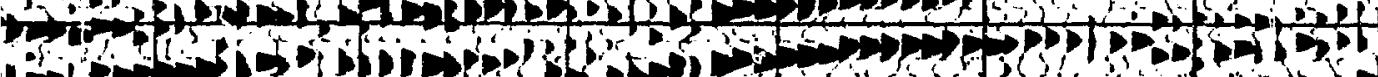
170 is

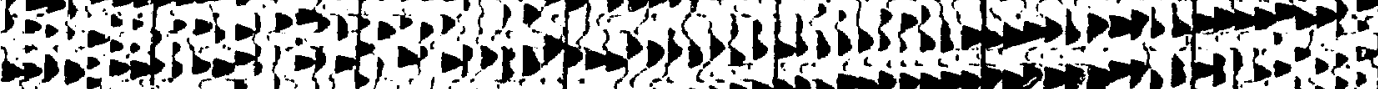

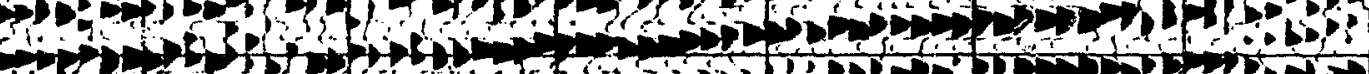

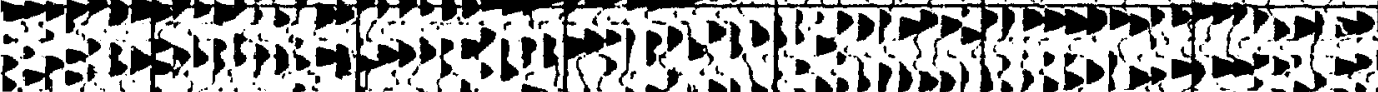

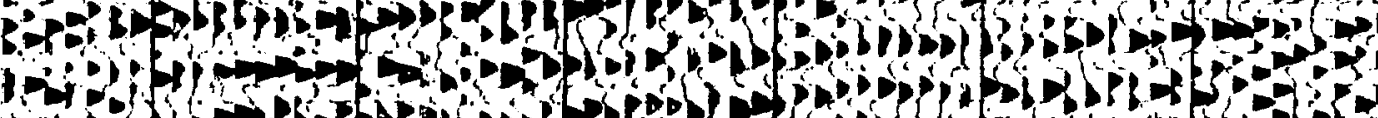

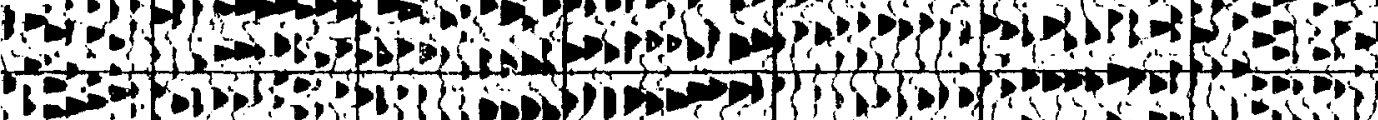

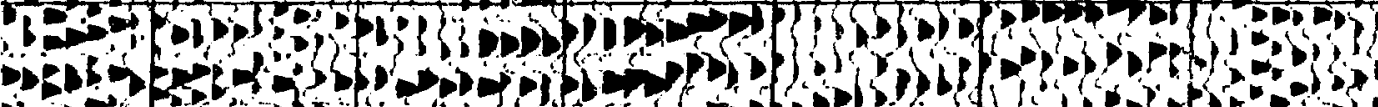

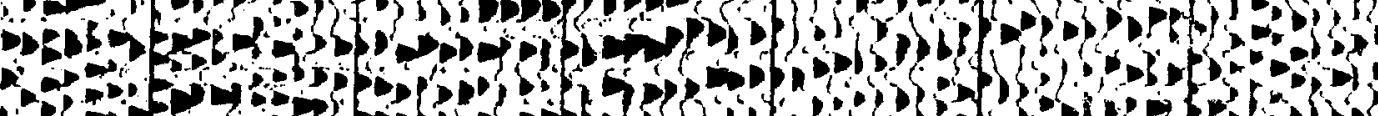

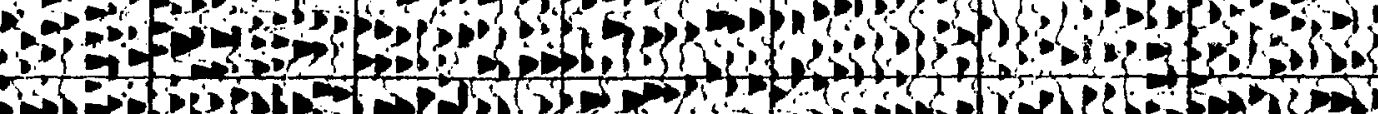

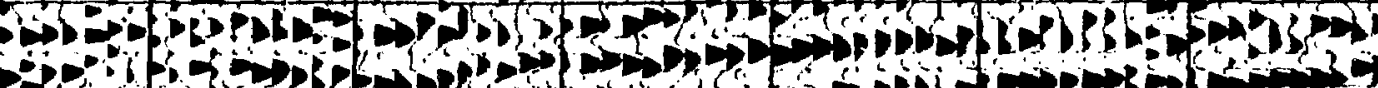

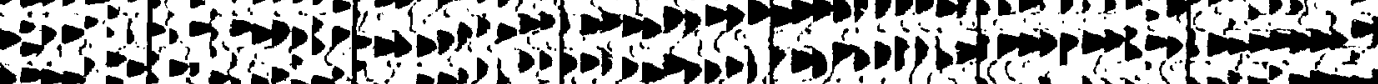

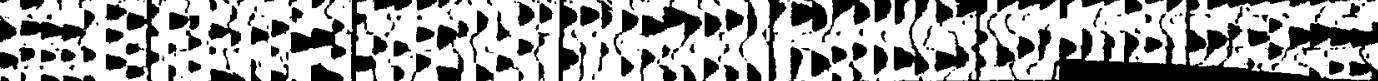

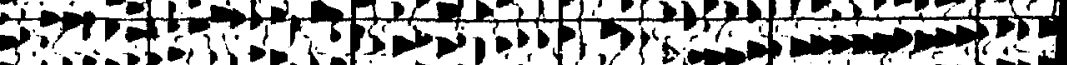
$\therefore$ :

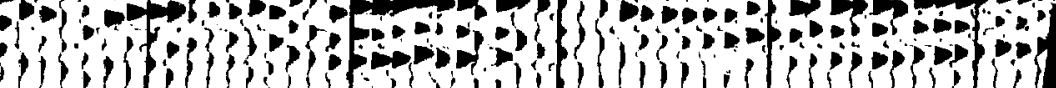

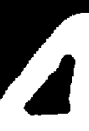




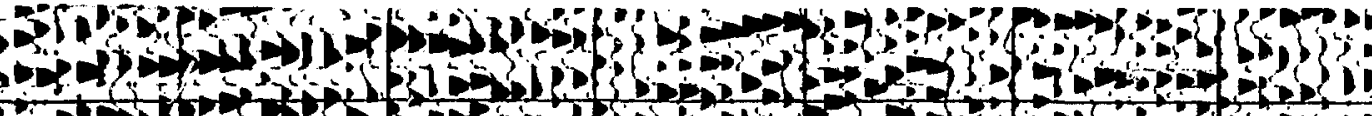

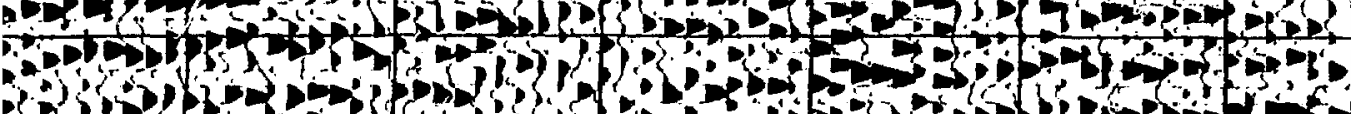

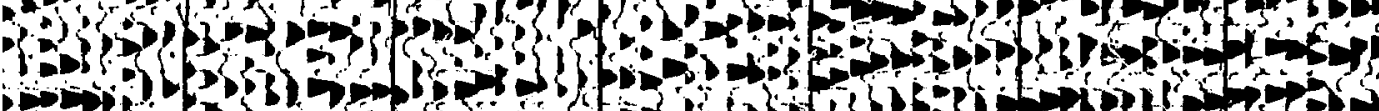

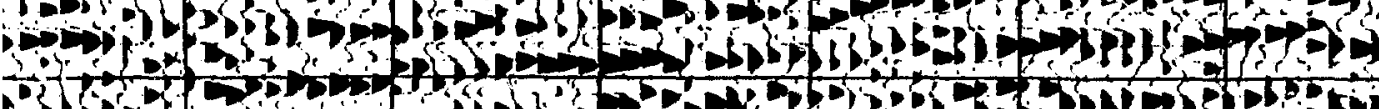

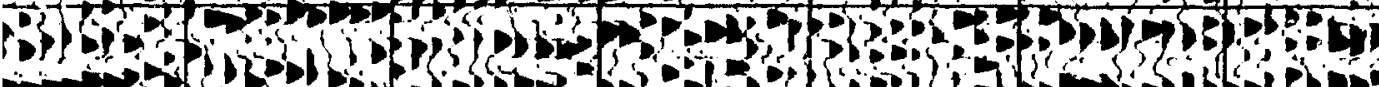

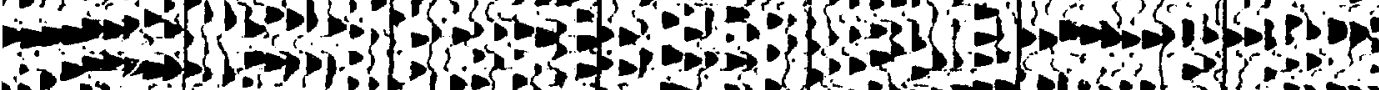

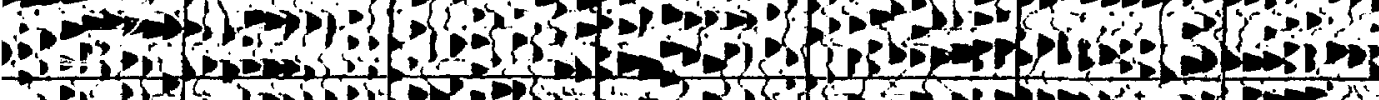
(19

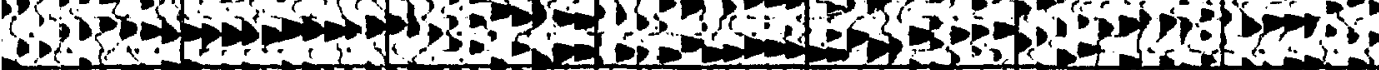

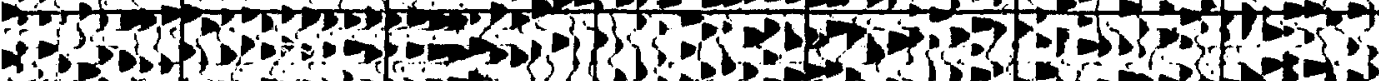

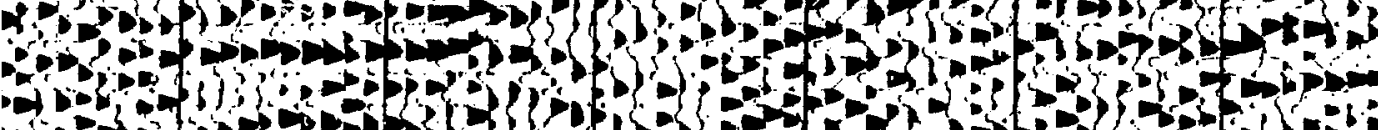

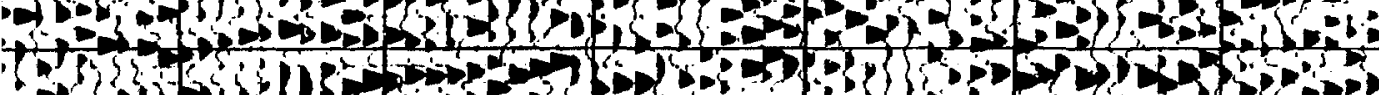

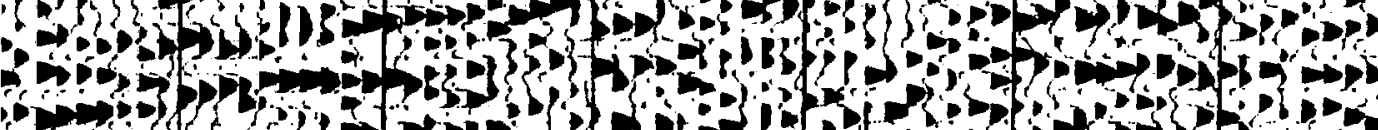

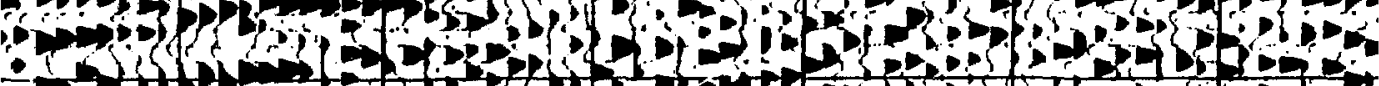

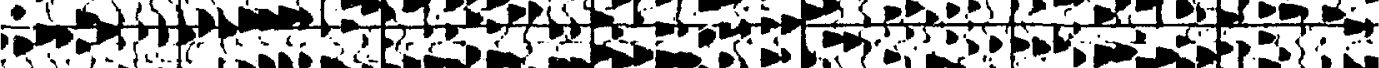

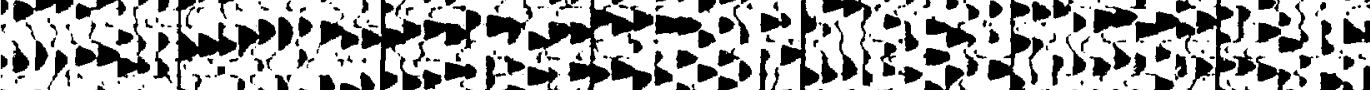

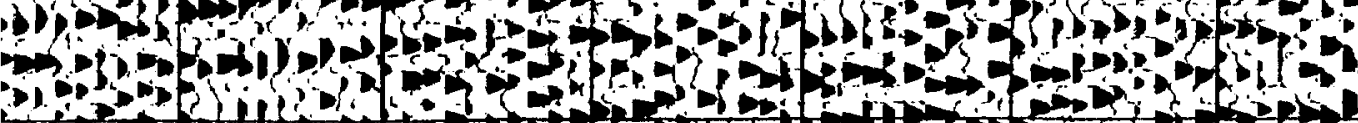

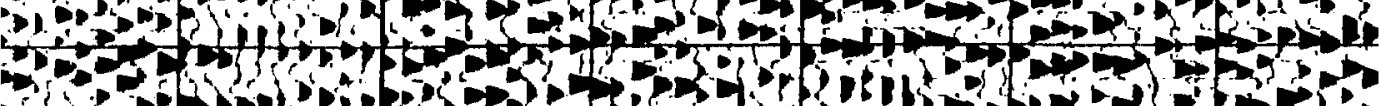

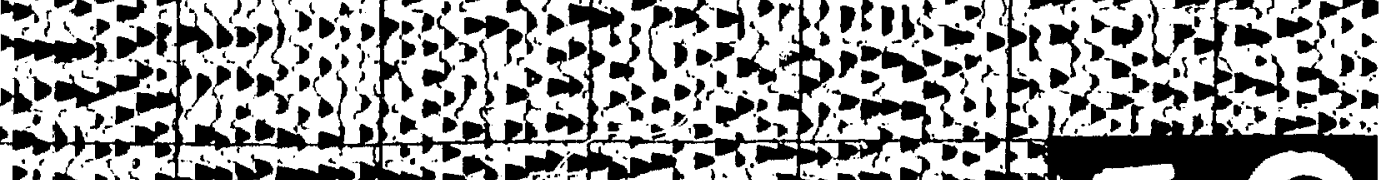
$+2-5+5=5$

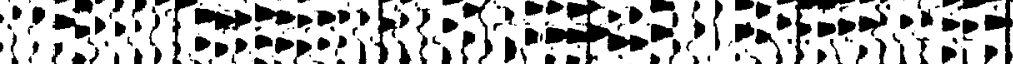

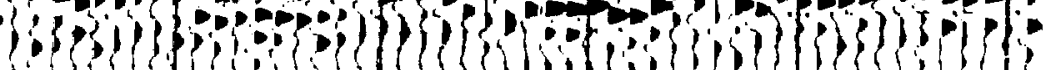


$\left\{\begin{array}{l}3 \\ 3\end{array}\right.$

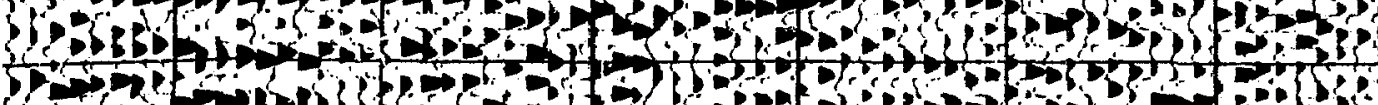

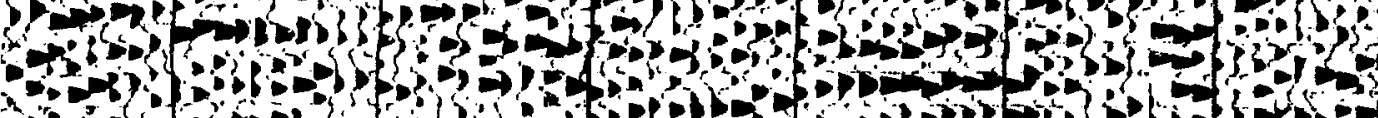

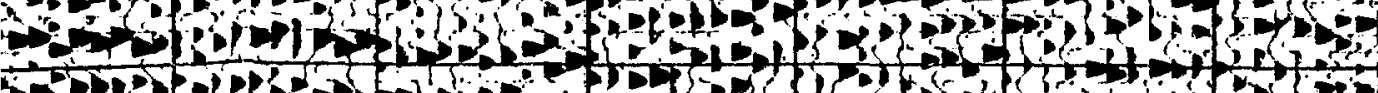

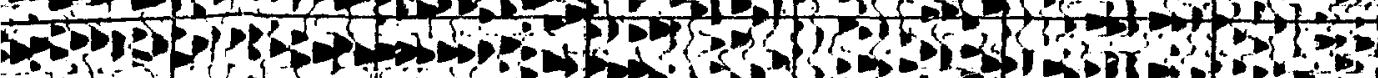

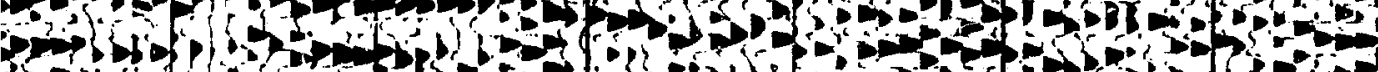

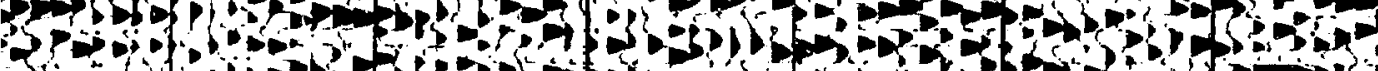
S. 5
$y$

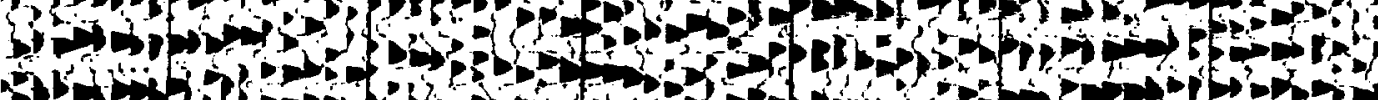

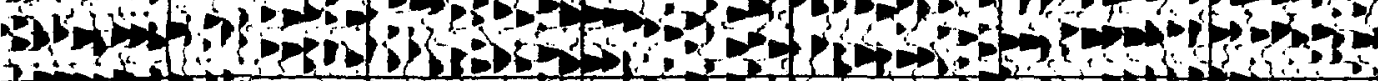

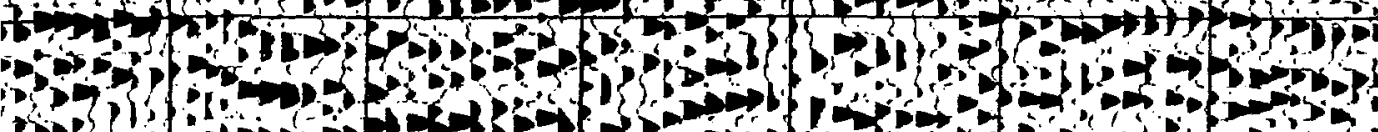

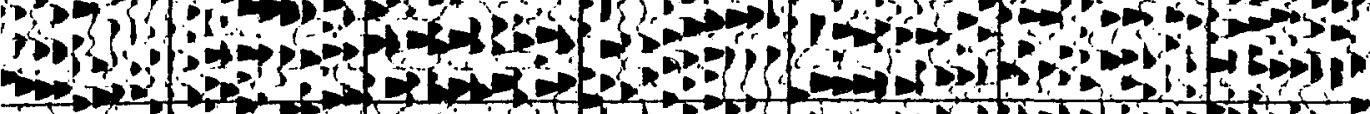

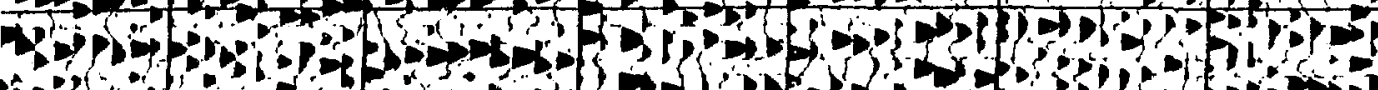

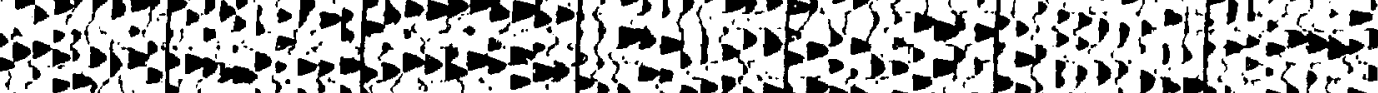

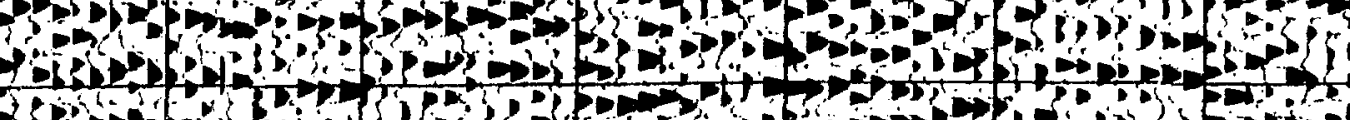

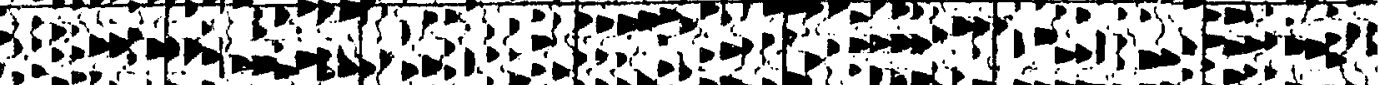

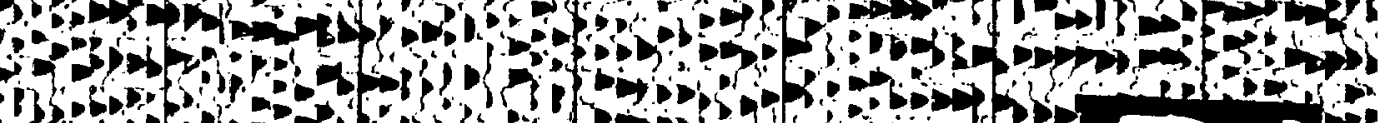
I\}

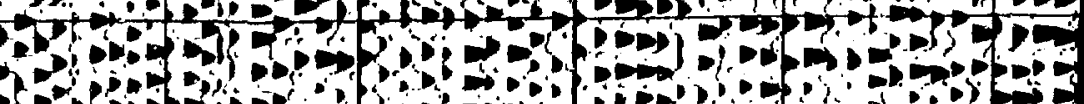

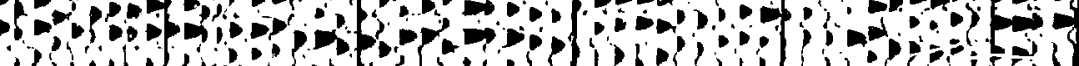
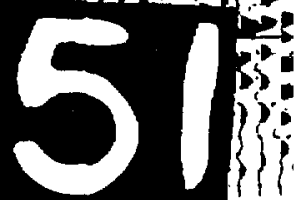


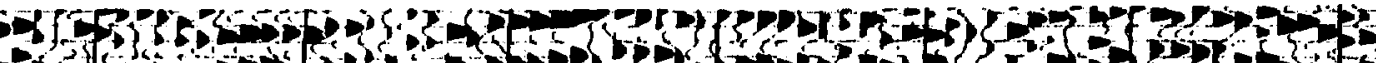

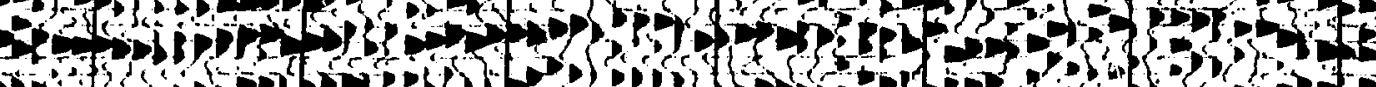

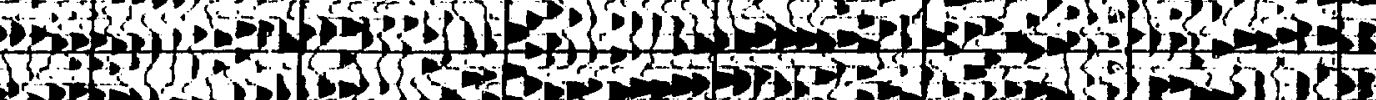

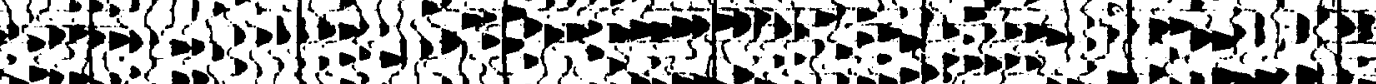

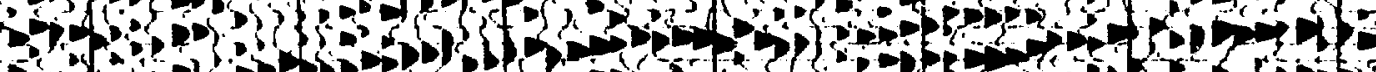

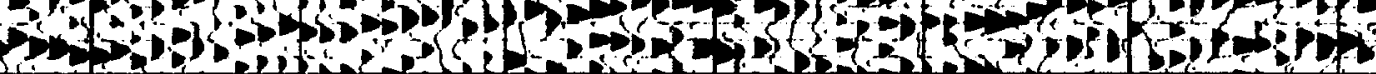

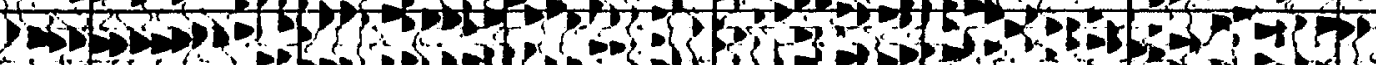

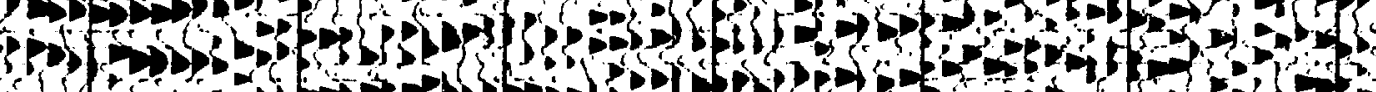

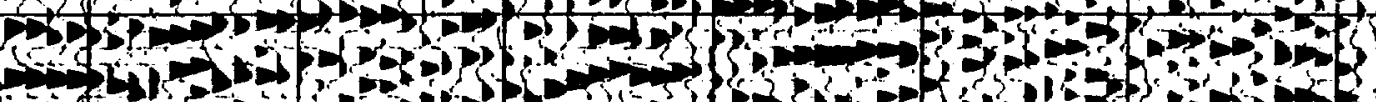

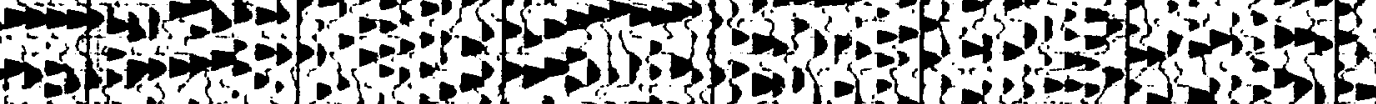

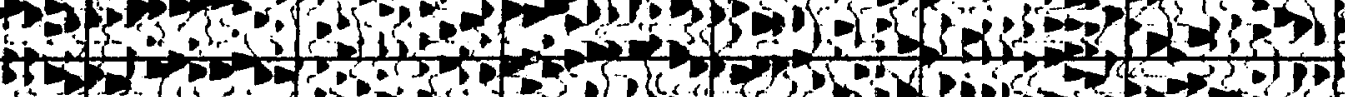

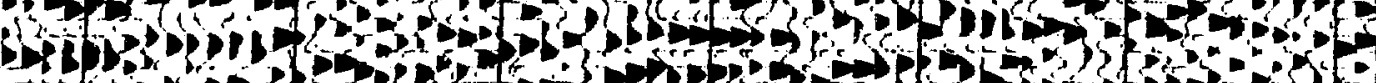

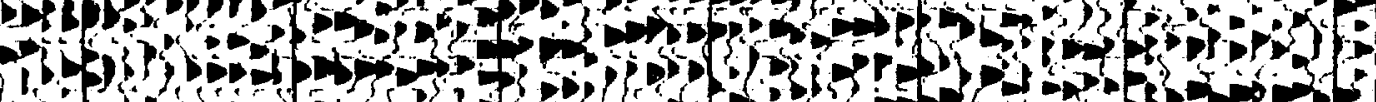

$\left\{\begin{array}{l}5 \\ 5\end{array}\right.$

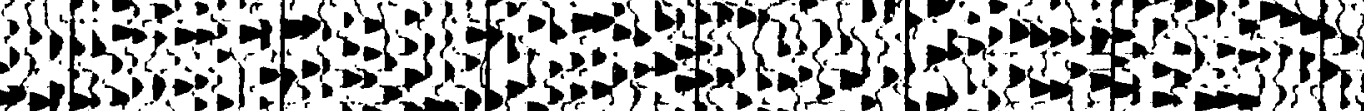

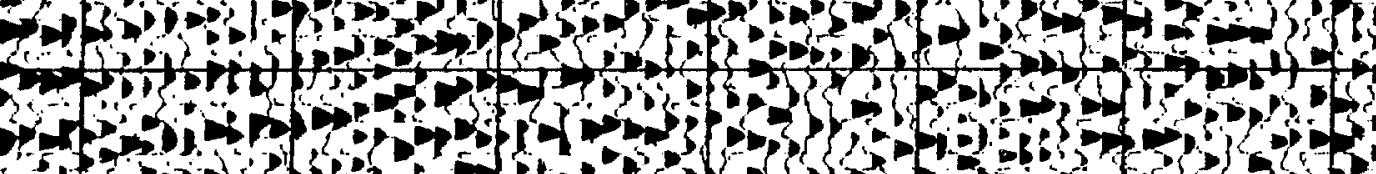

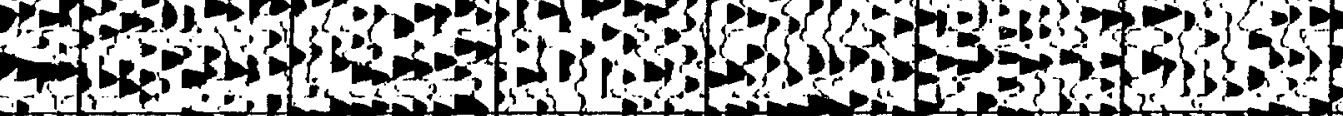
at

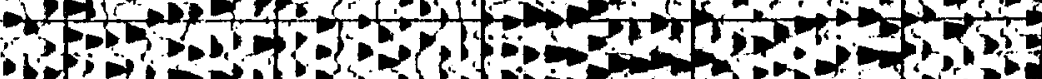

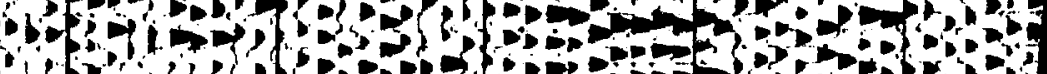

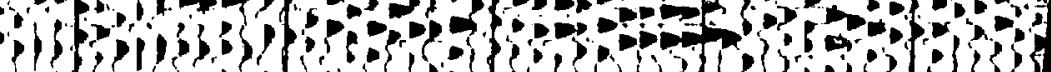




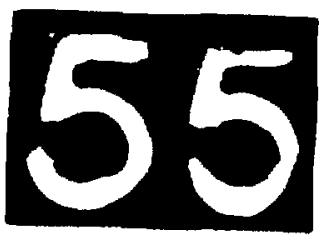




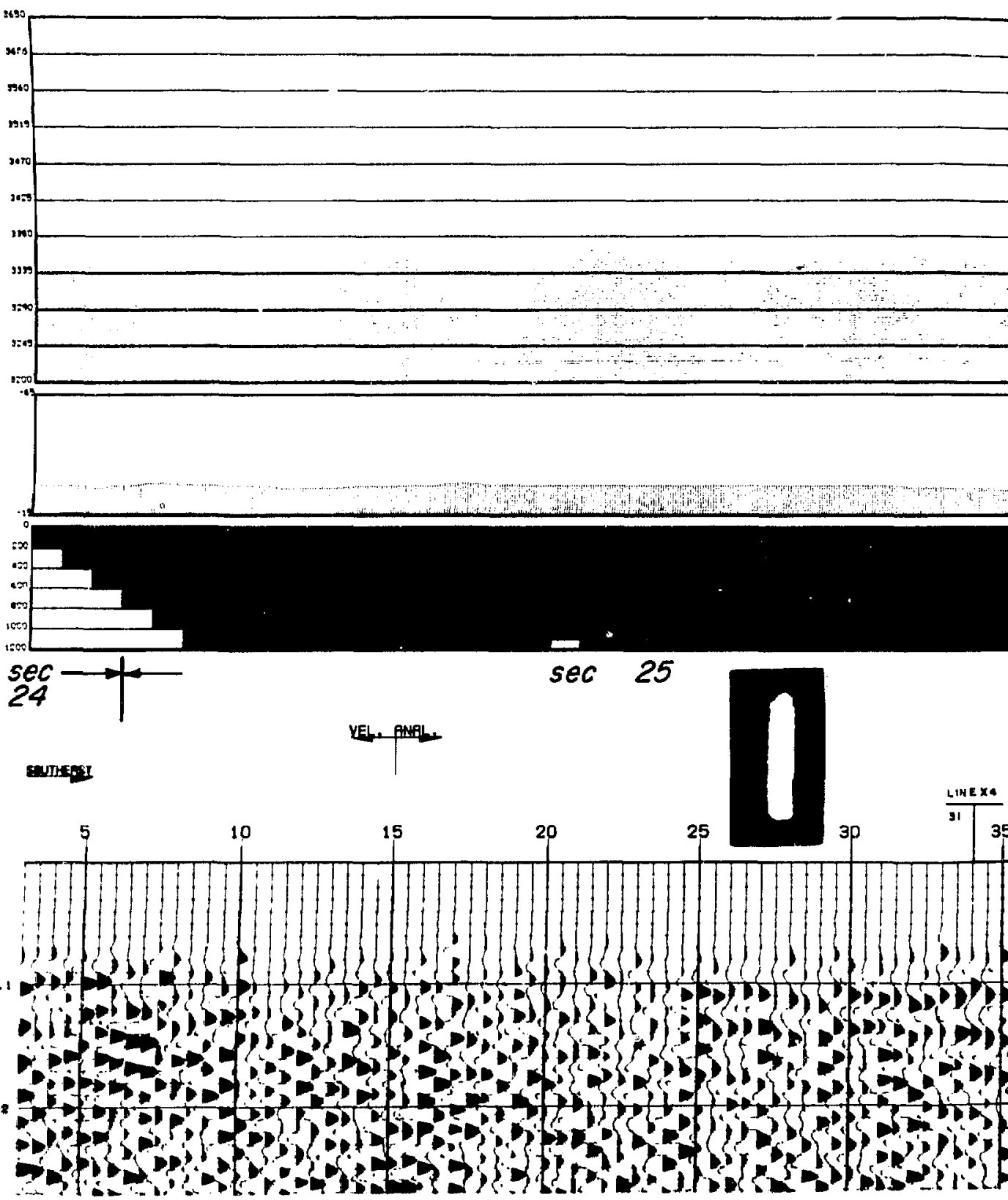




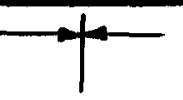

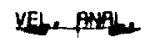

sec 30

VEL, FNPL,

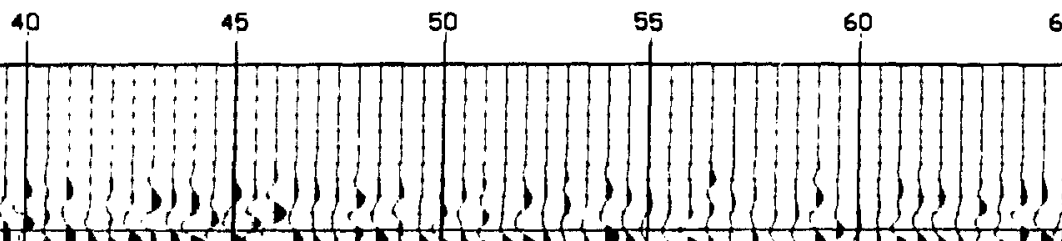

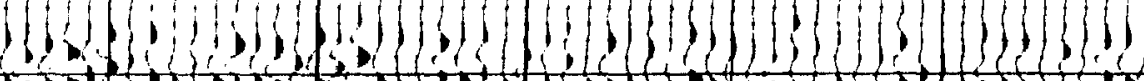
(a) (5)

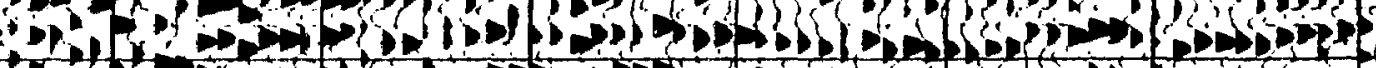

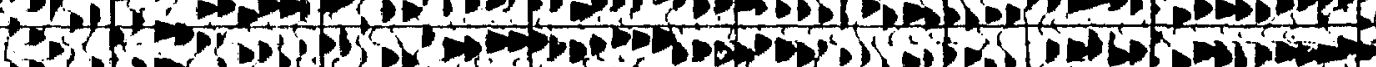

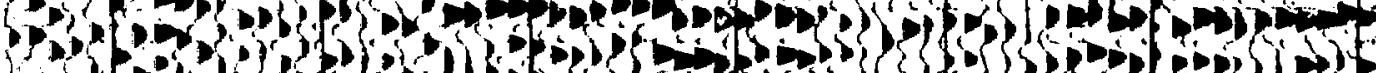

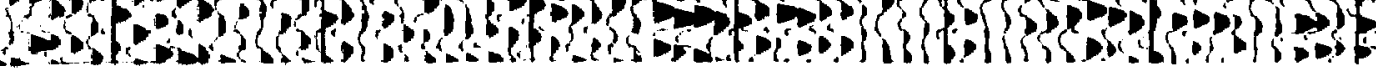




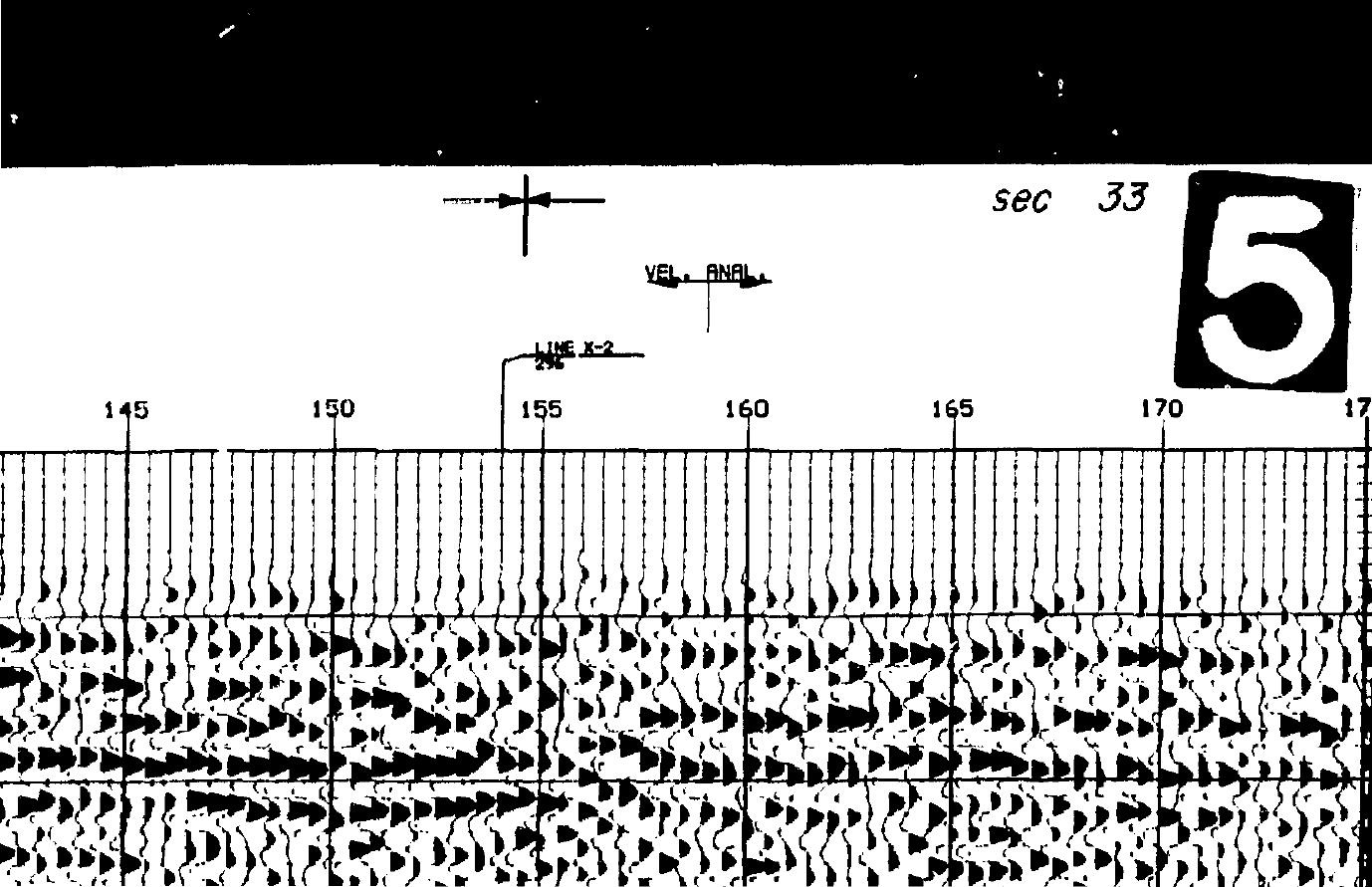

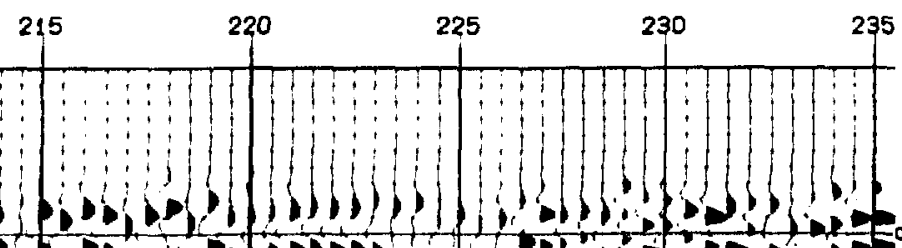

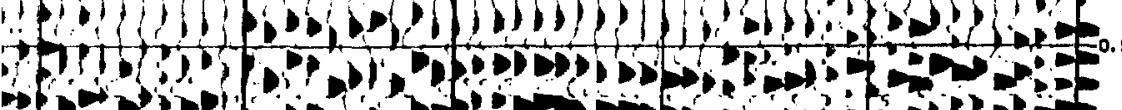

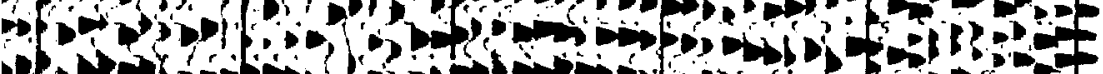

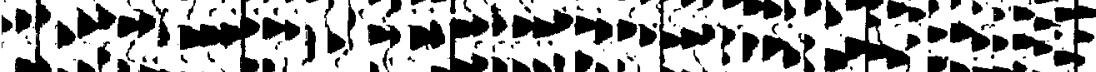

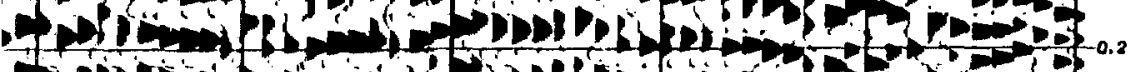

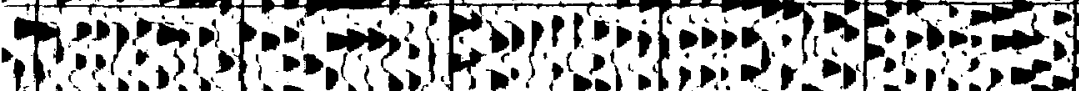
-

LINEDIR

VELOC I TY DIRECTIO LINE INT STATIONS 


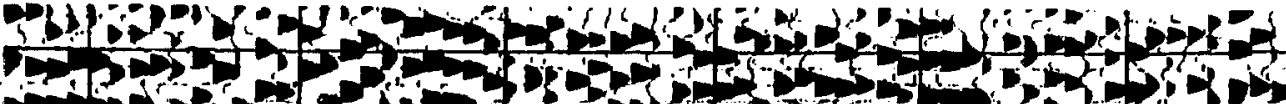
(1)

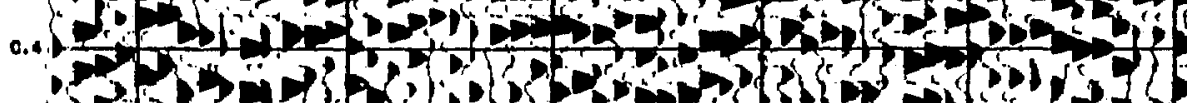

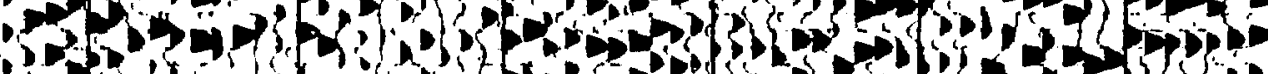

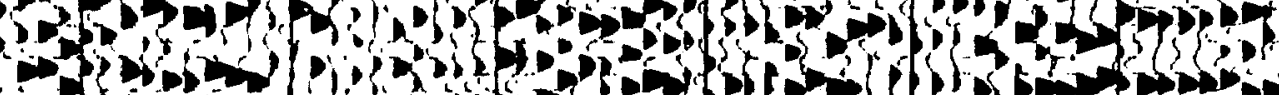

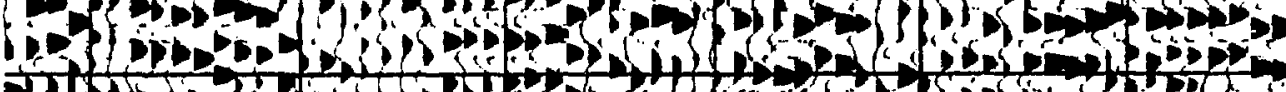
0.8

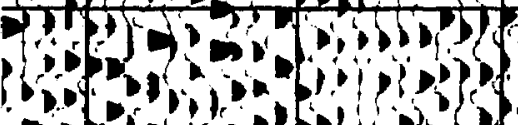

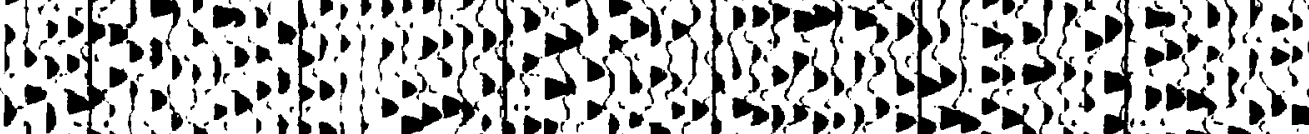
(1)

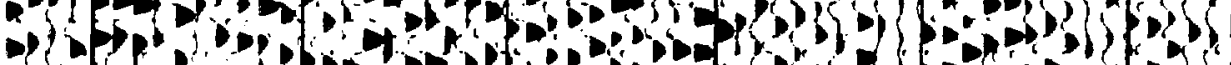

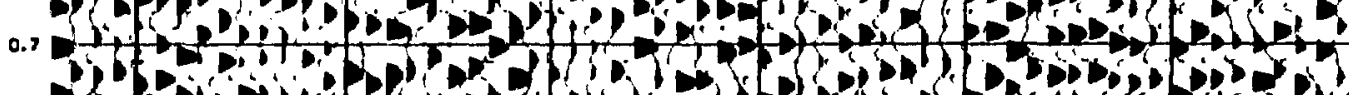
S)

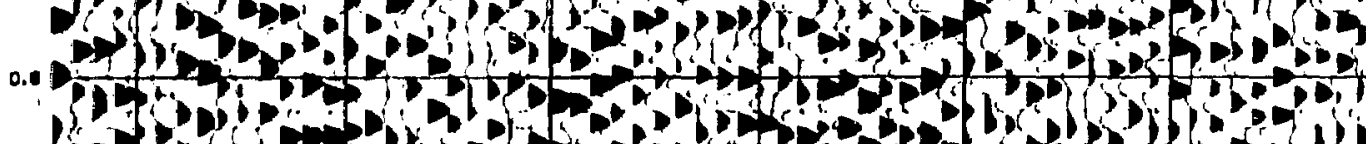
(5)

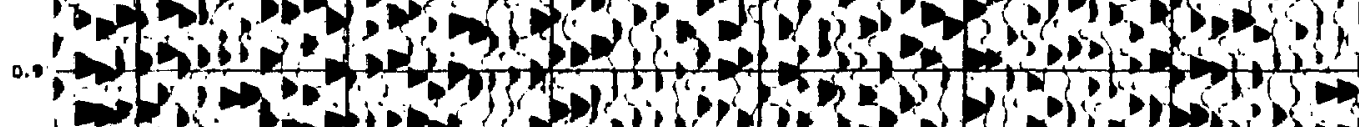
(1)

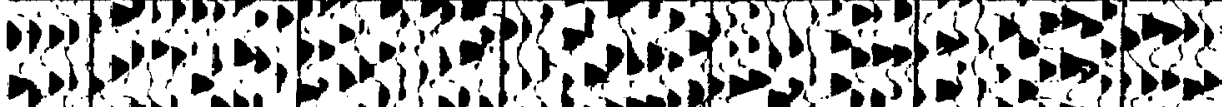
(1) 1.

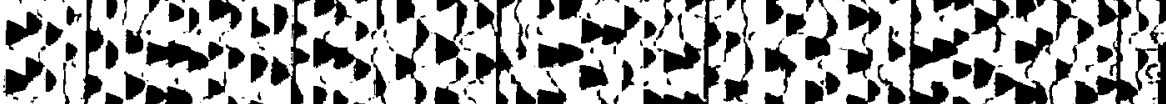

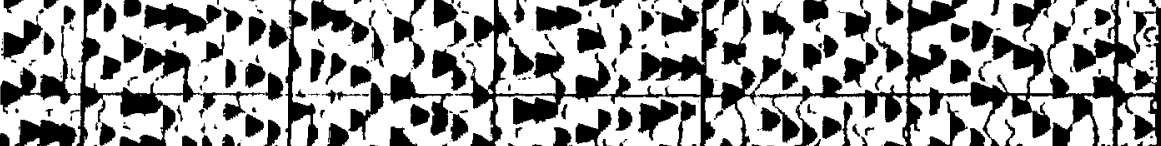
pis 
if (5) (f) 20

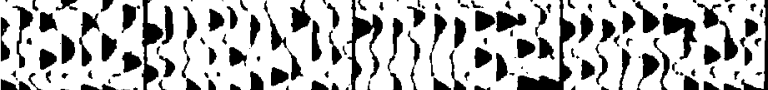

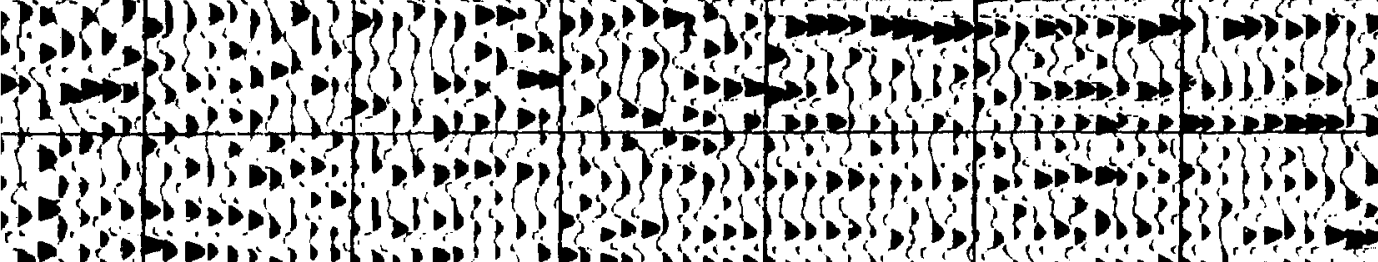

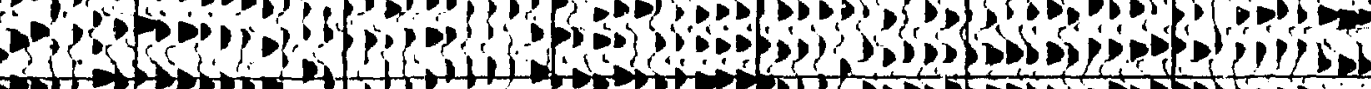
SIf

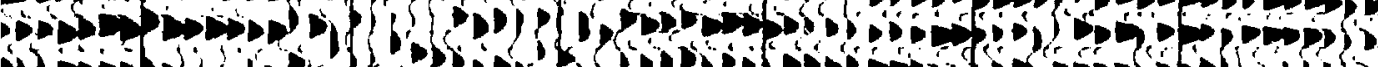

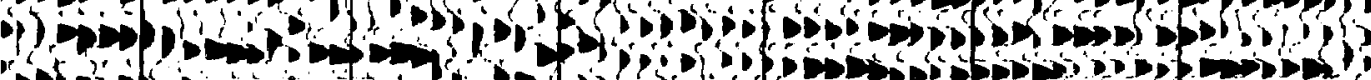

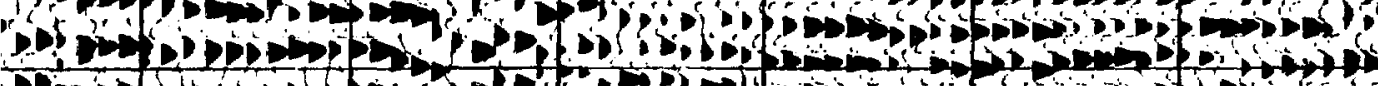

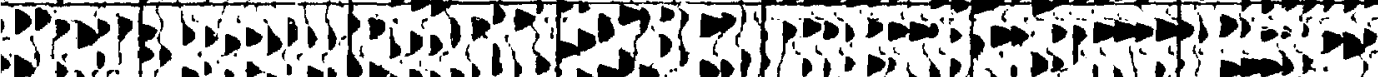
So

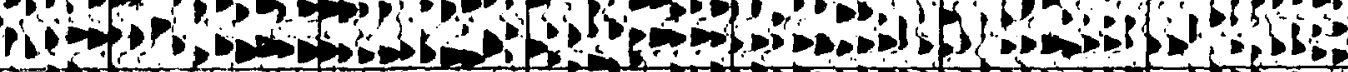
100 (1)

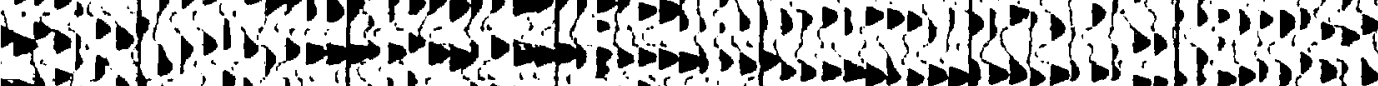

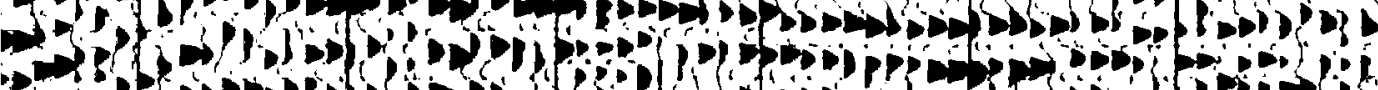
(5)

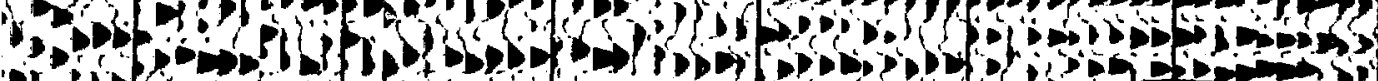
(i) $\left\{\begin{array}{l}10 \\ y\end{array}\right.$

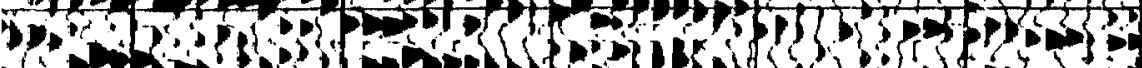

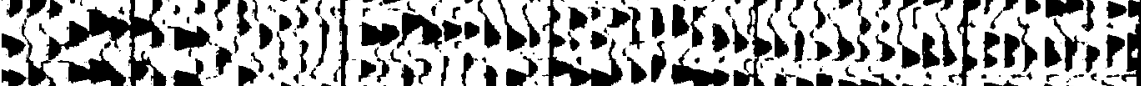

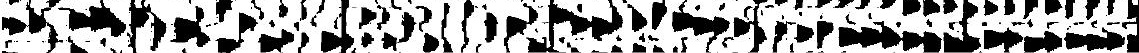





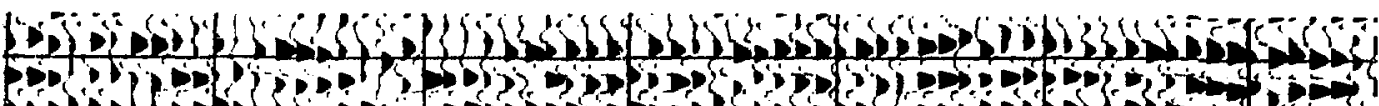

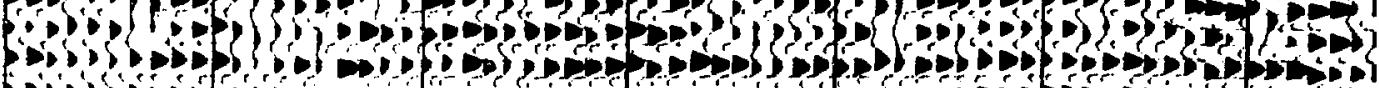

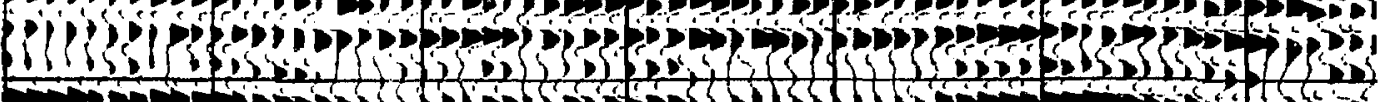

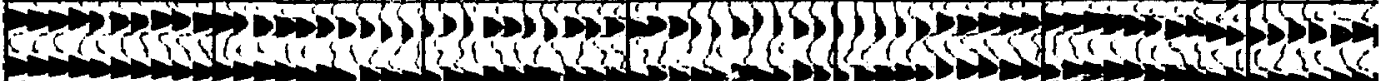
P. (D)

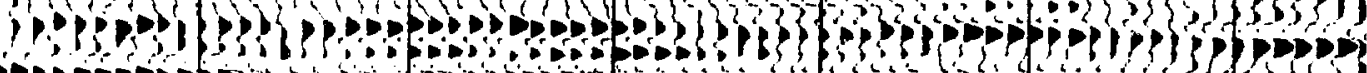
(5)

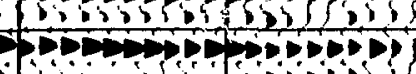
(1)

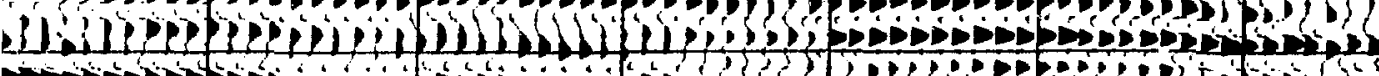

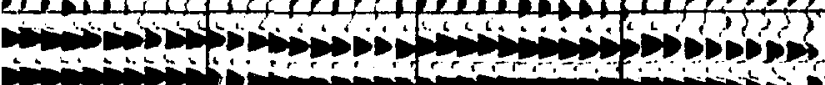

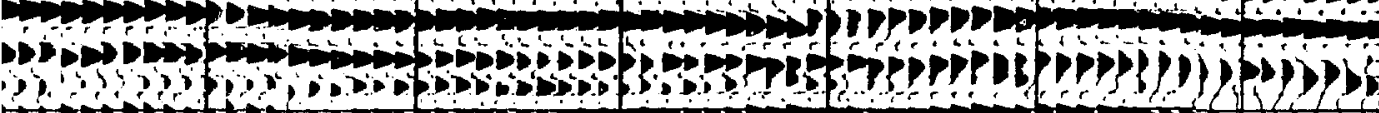

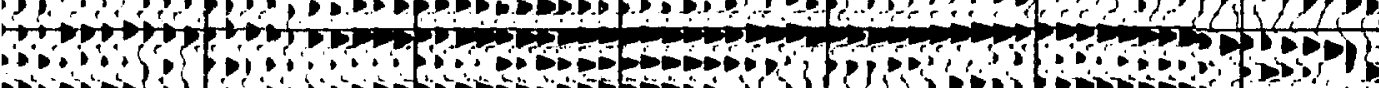

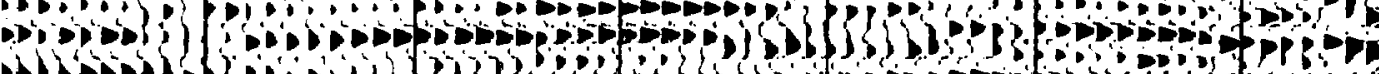

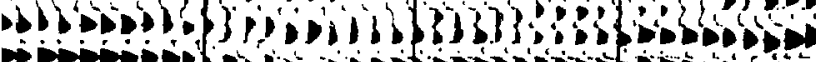

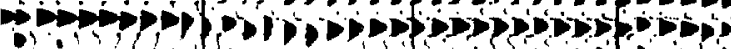

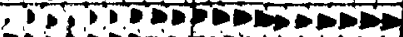

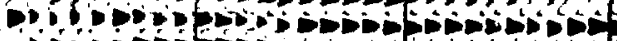

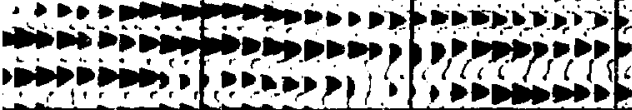
Tा? (2)

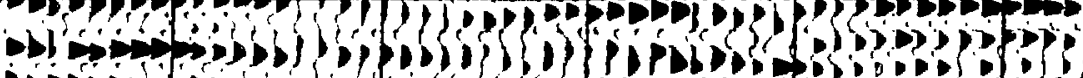

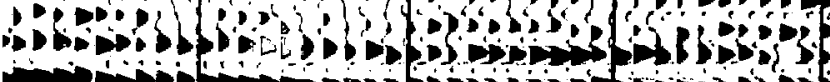
Tophoma

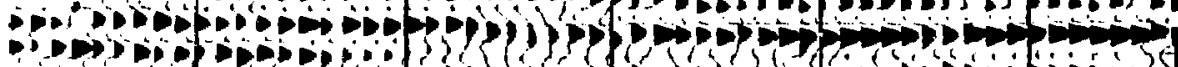

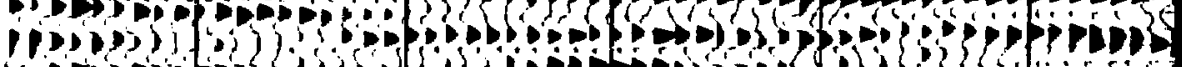

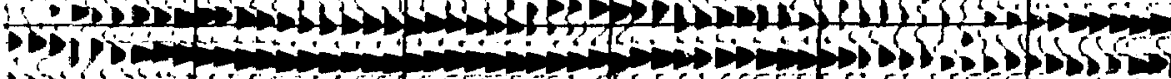

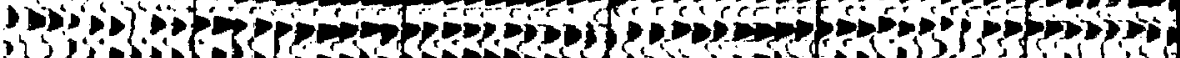

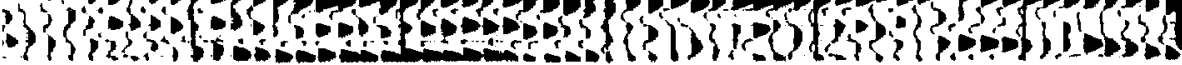




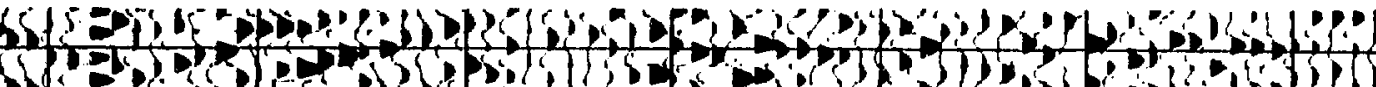

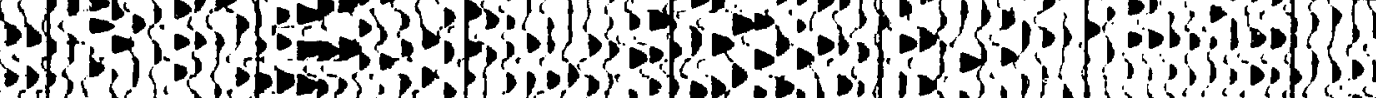

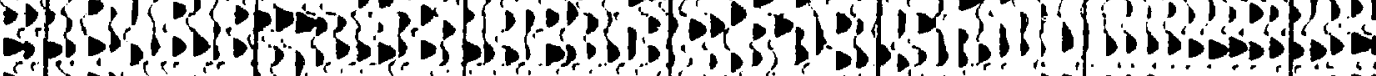

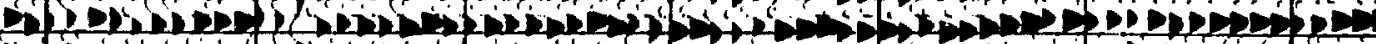

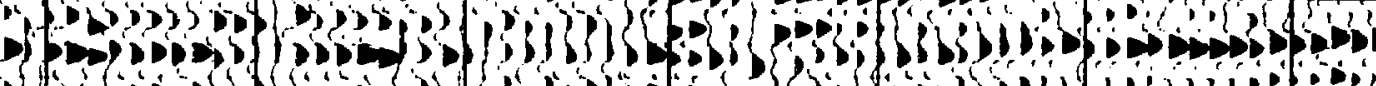

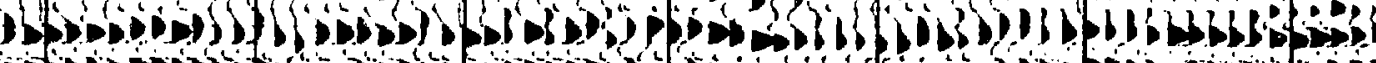

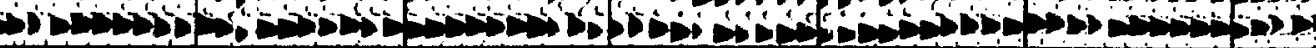

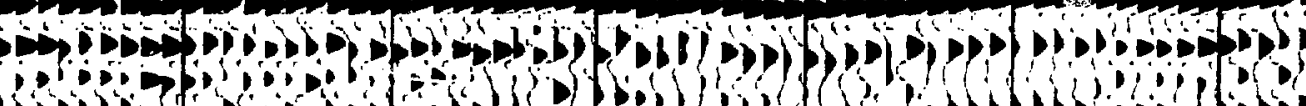

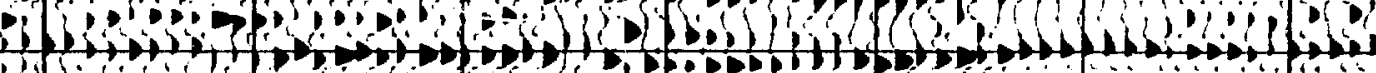

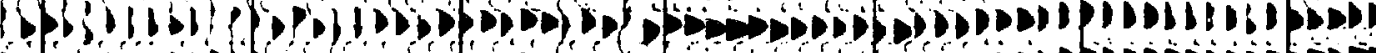

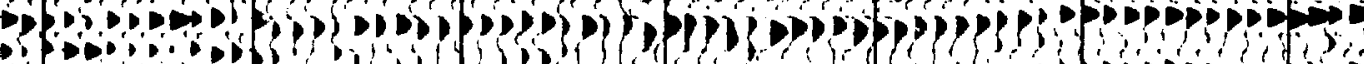

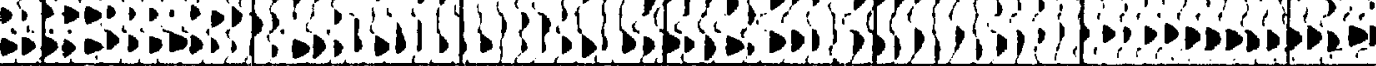

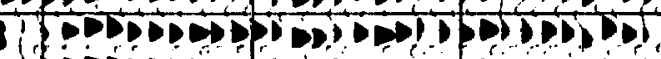

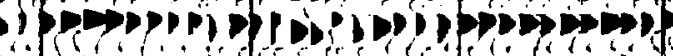

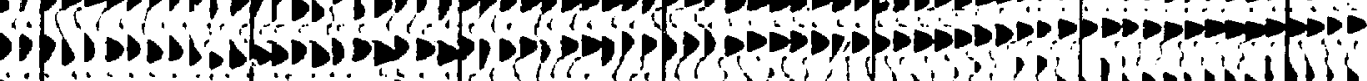
S

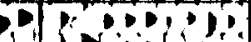
Sipisis (1)

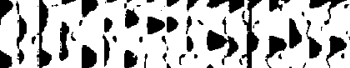
timing $\rightarrow$ $\therefore \rightarrow$ or

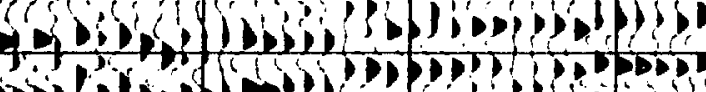
sobsos

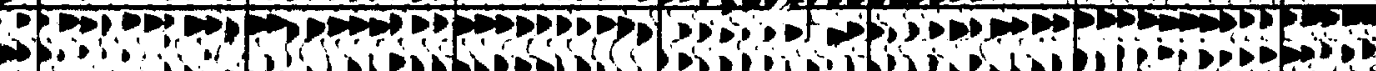

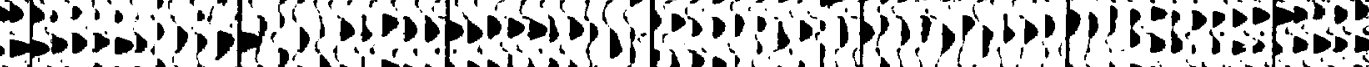
-

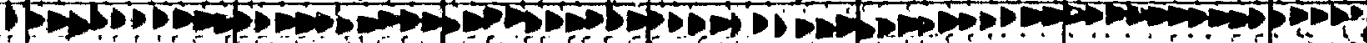

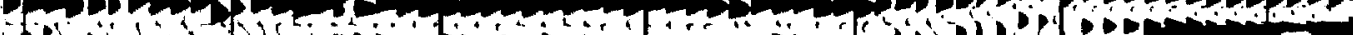

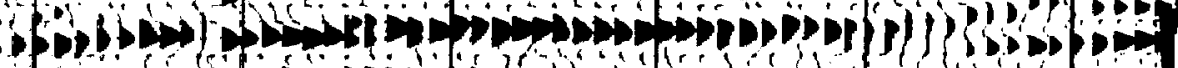

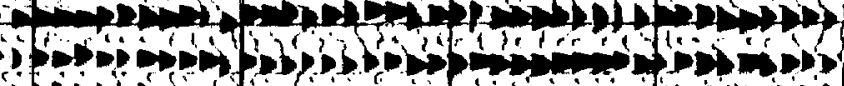

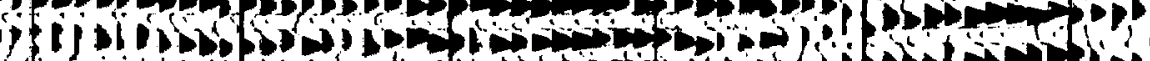

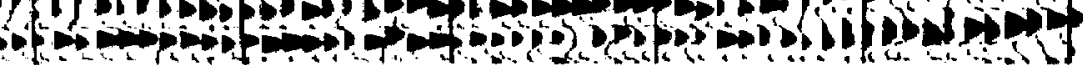


if or 1 (P) H

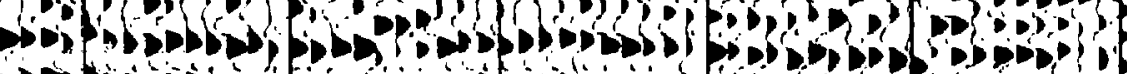

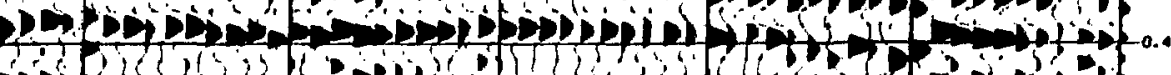

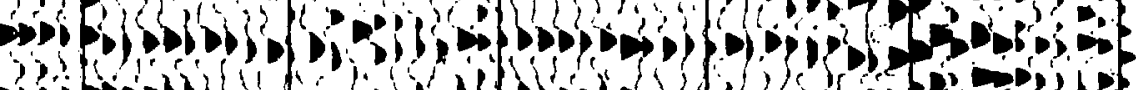

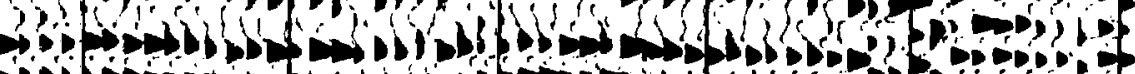

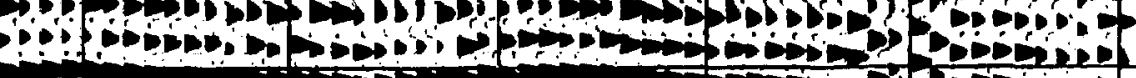

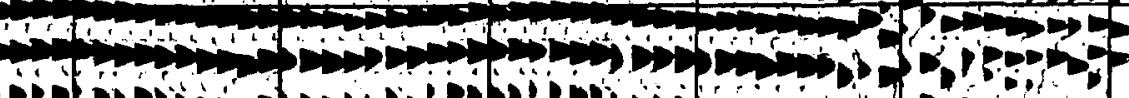

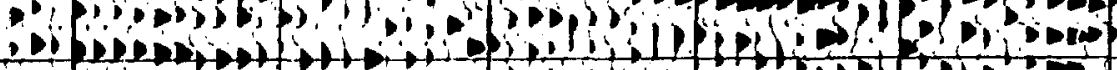

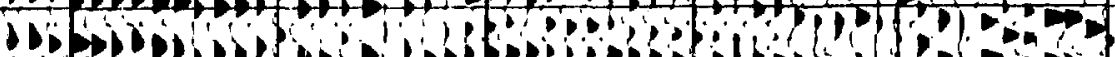
$\therefore$ a b

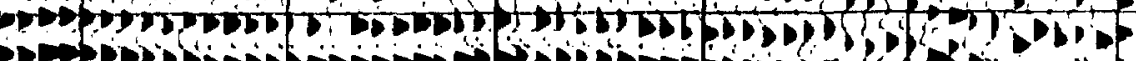

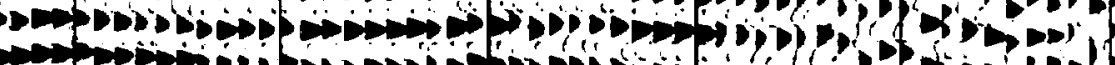

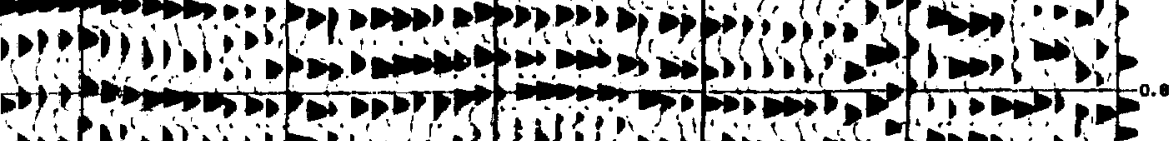

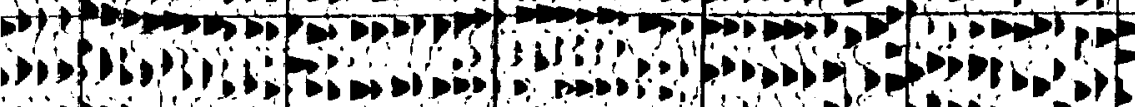

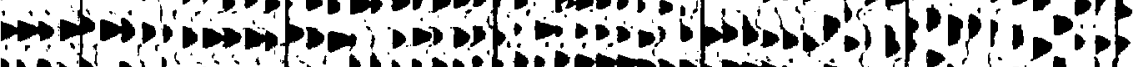

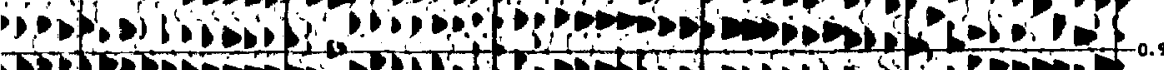

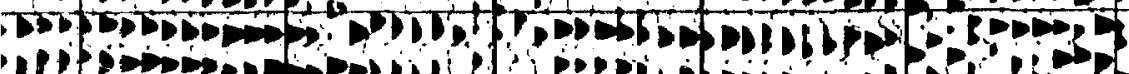

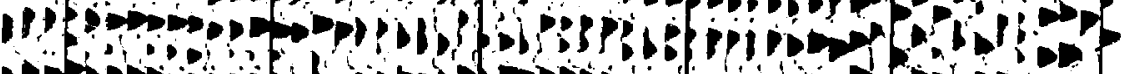

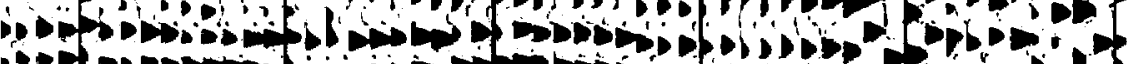

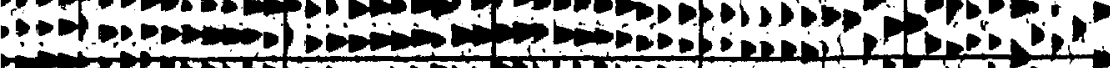

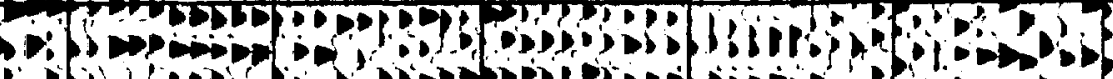

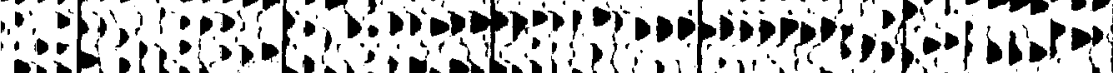

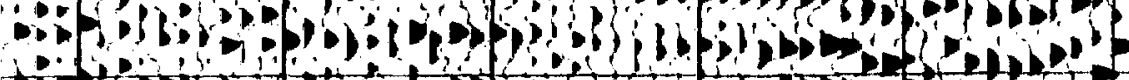
16 of

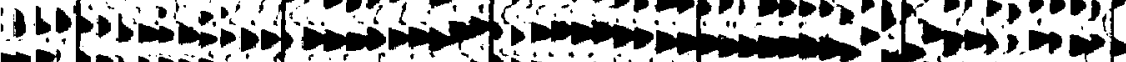

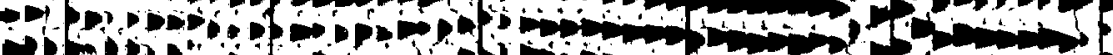

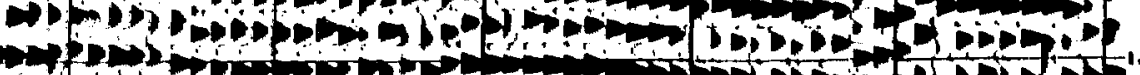

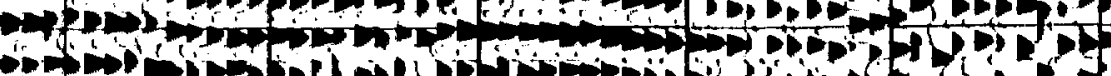

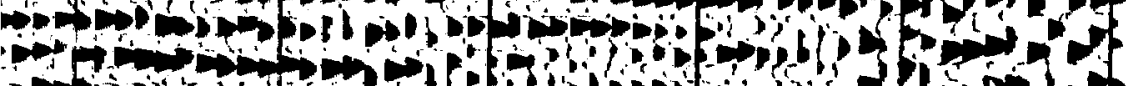

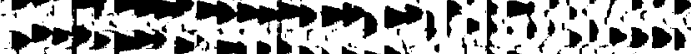
20

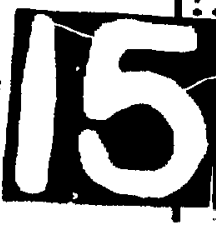




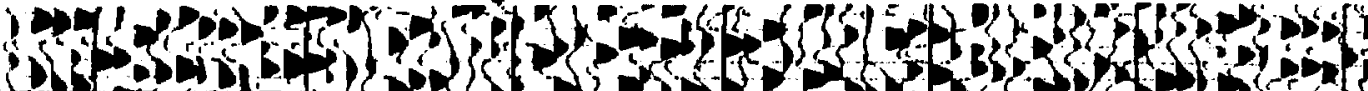

15.

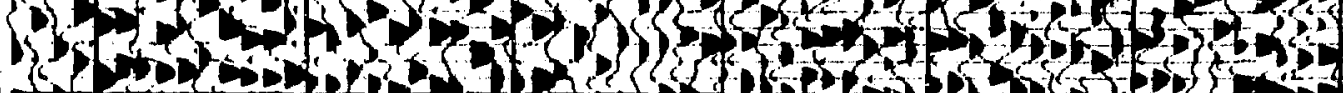

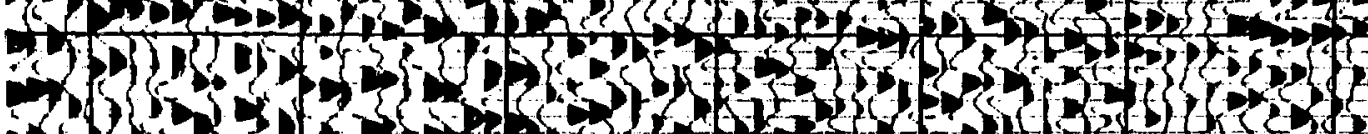

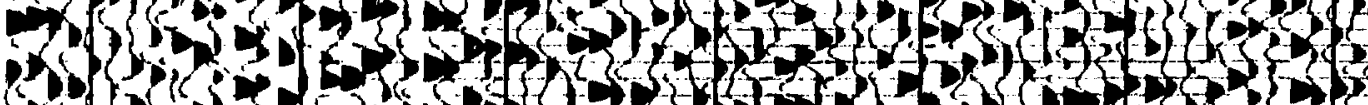

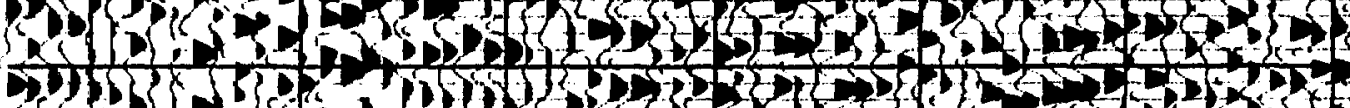

N

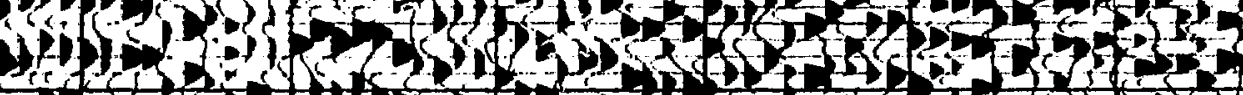

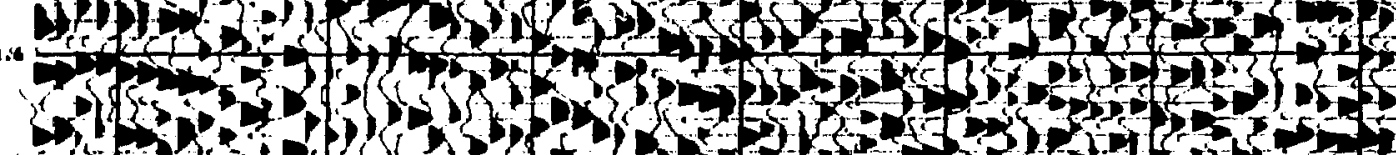

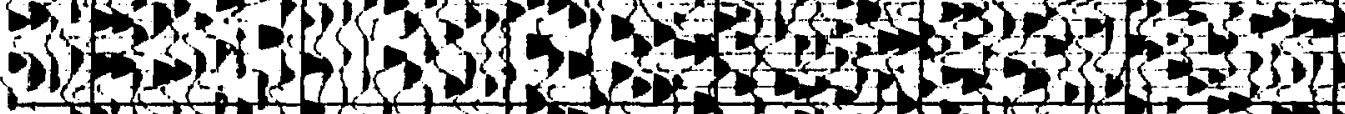

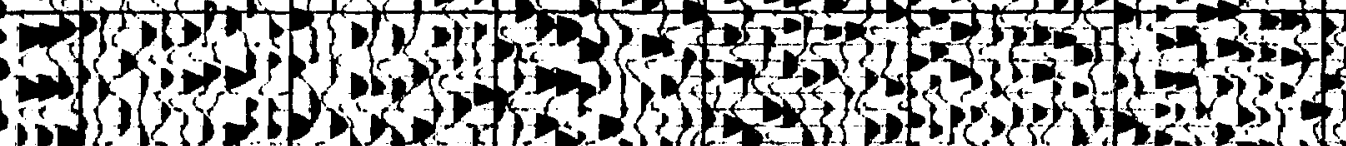

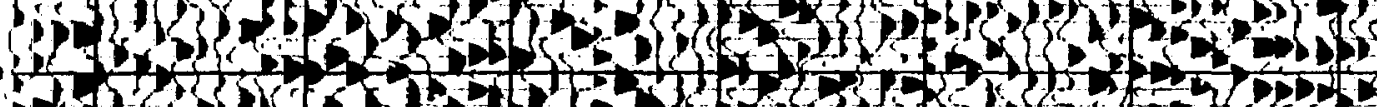

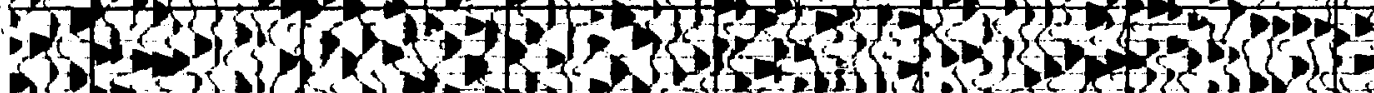

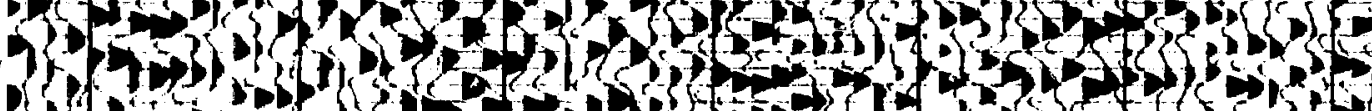

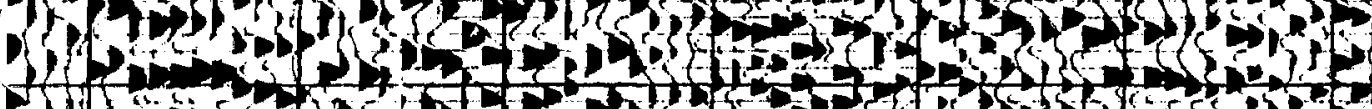
S.P.

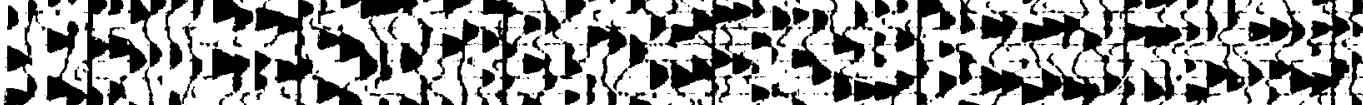

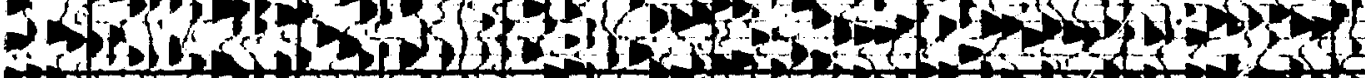
(5) 


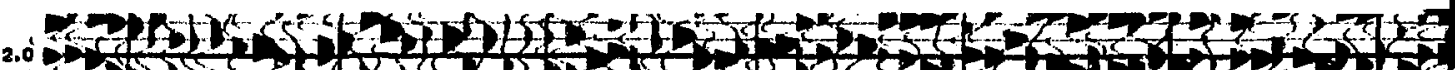

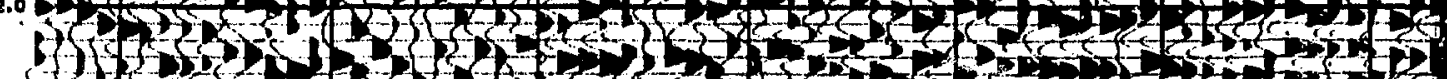

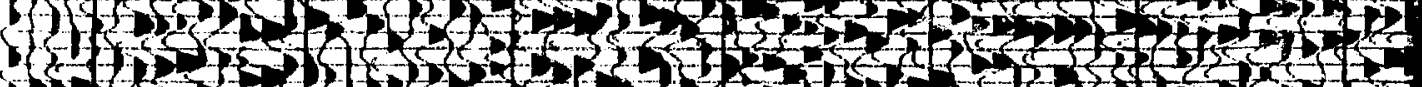

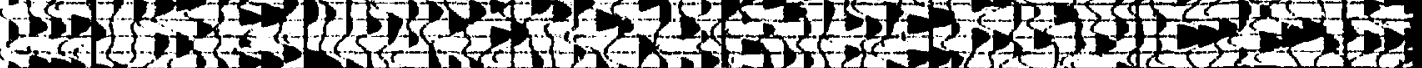

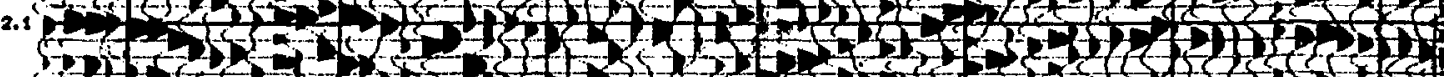

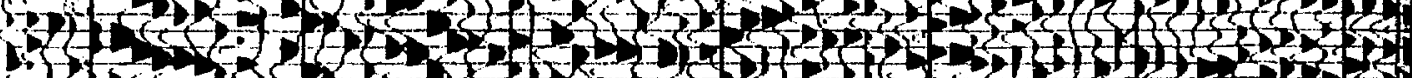

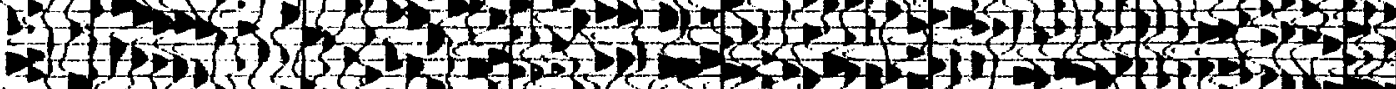

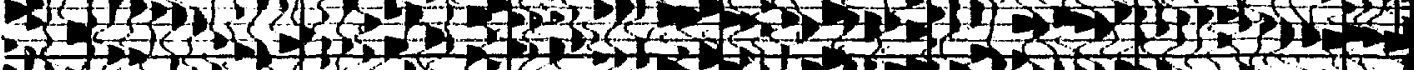

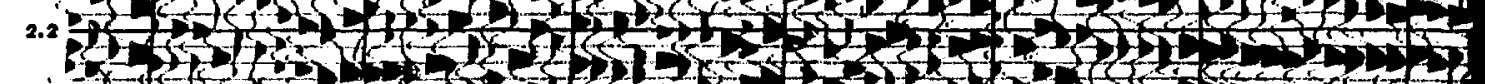

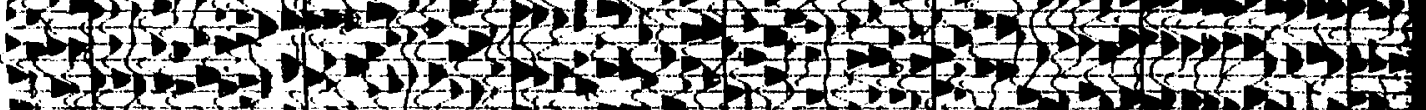

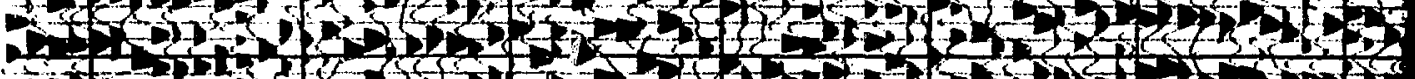

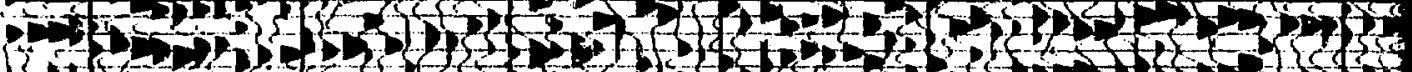

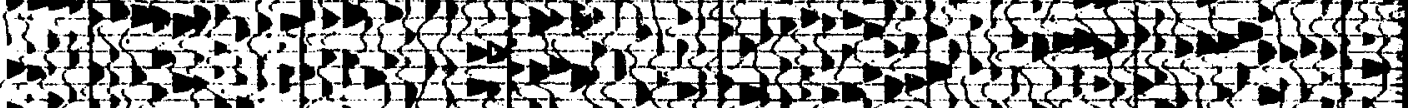

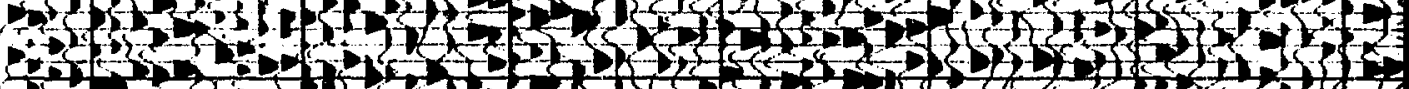

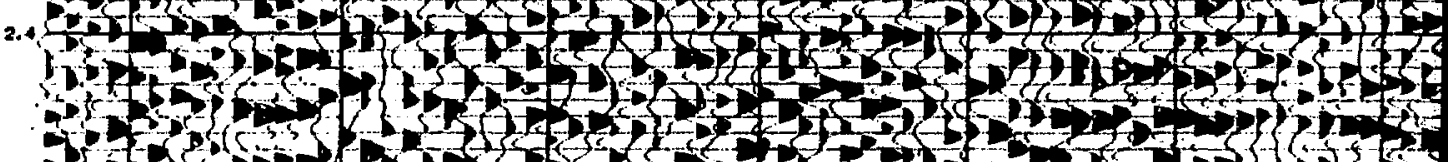

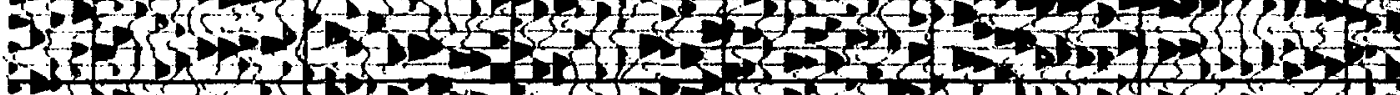

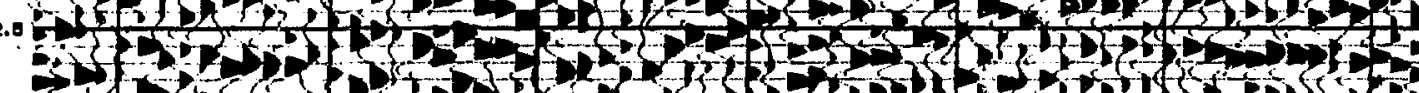

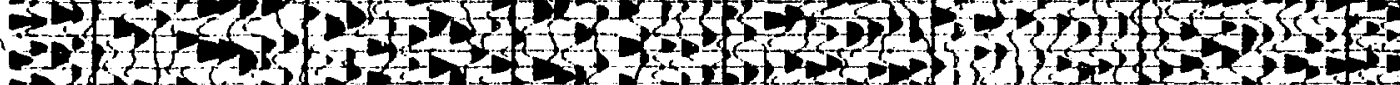

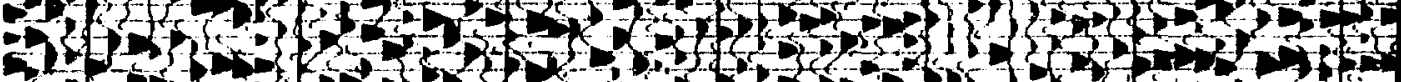

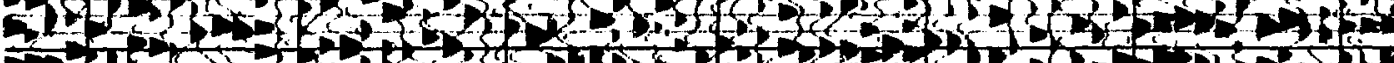

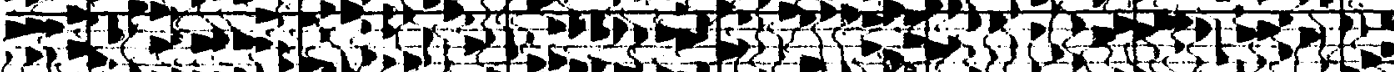

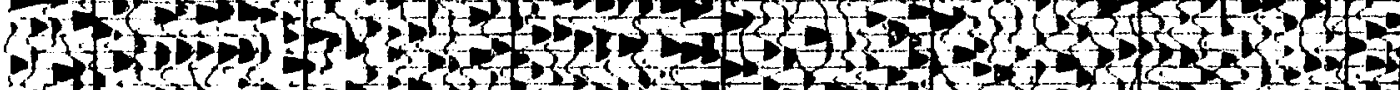

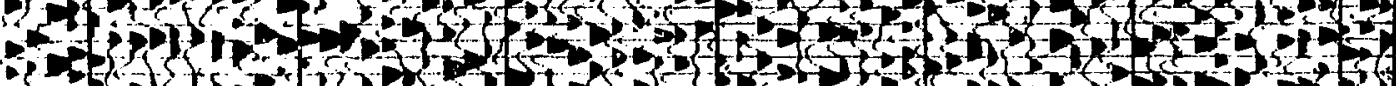
2.7 Lof

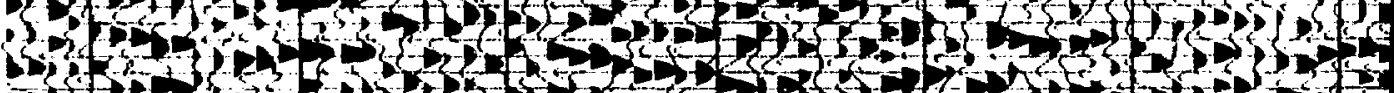

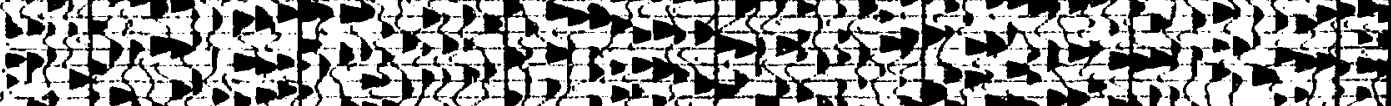
2.0.

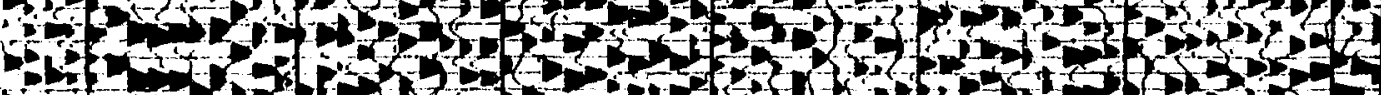

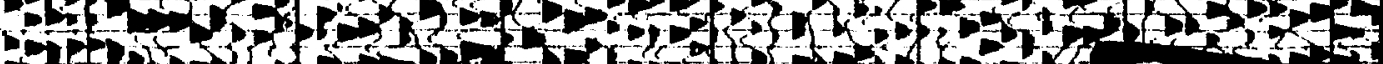

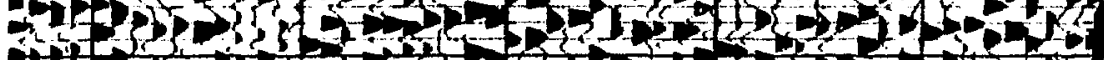

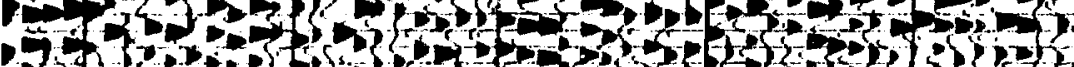

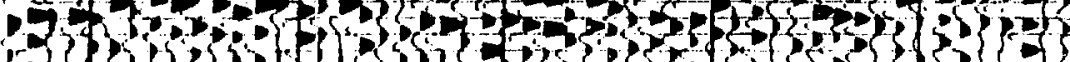

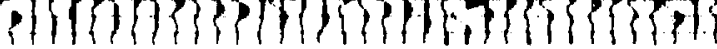


rer

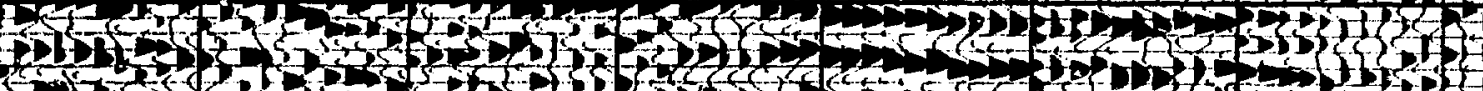
W.

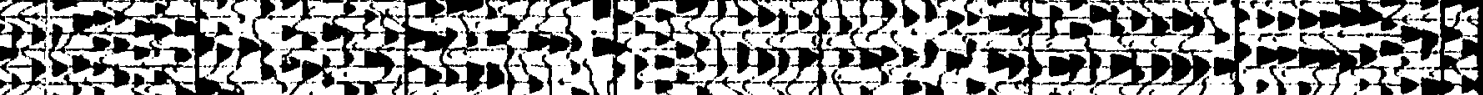
3.5.7.

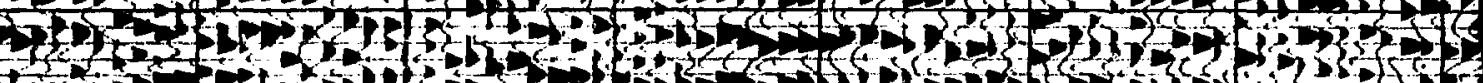

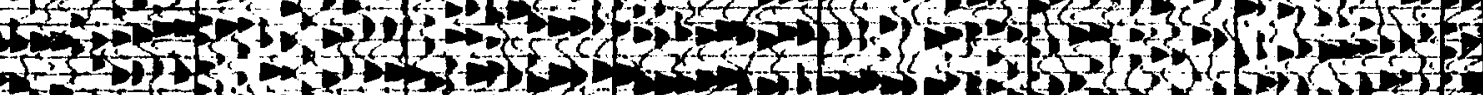

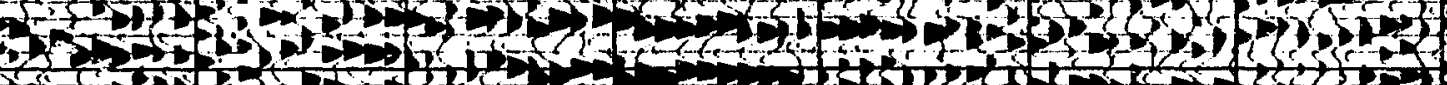

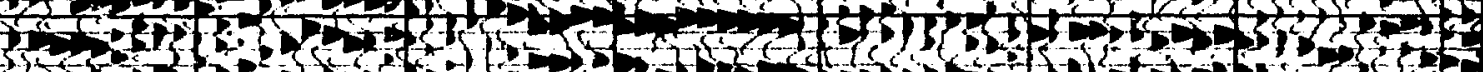

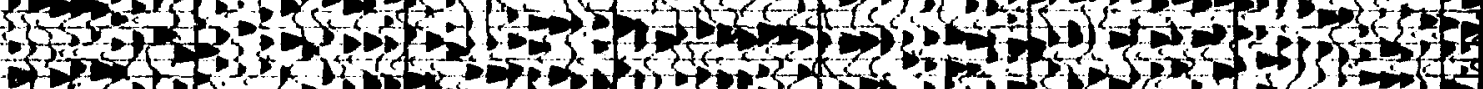

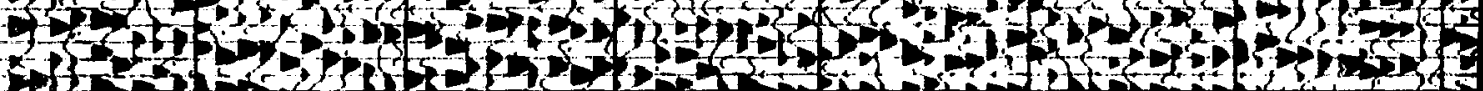

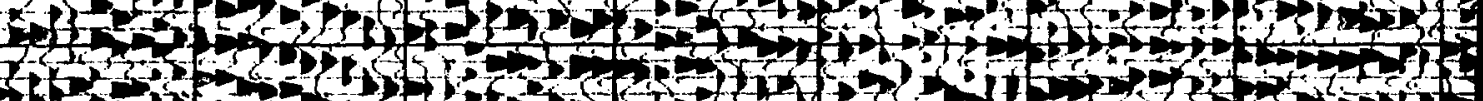

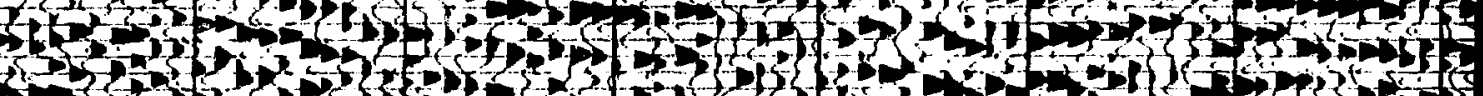

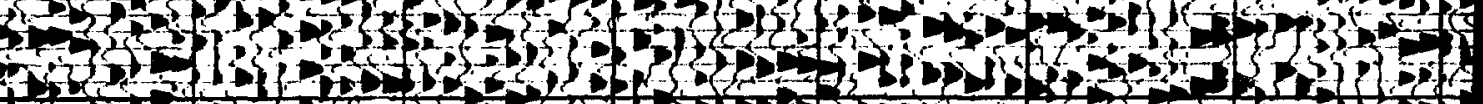

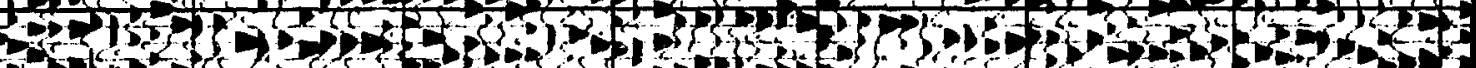

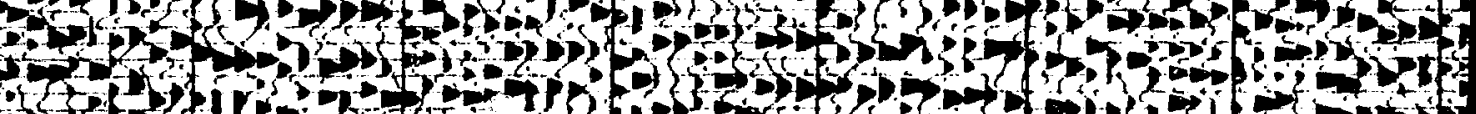

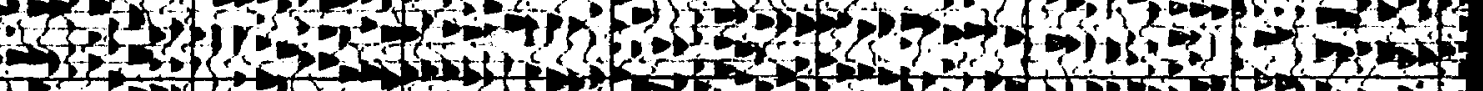

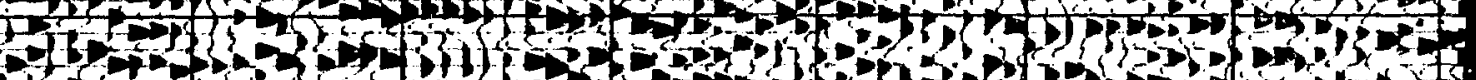

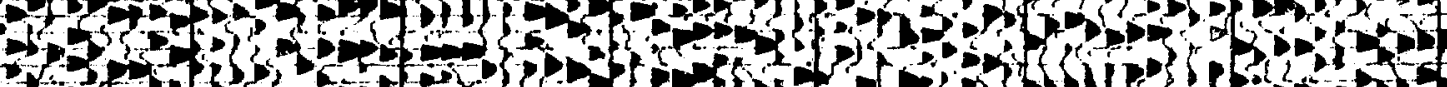
1020303 S m

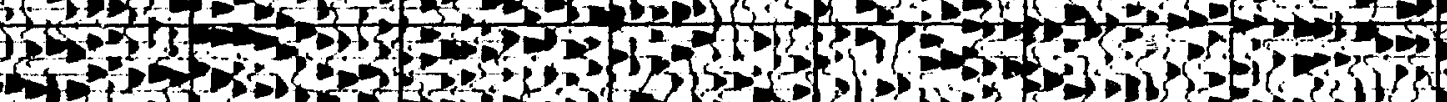

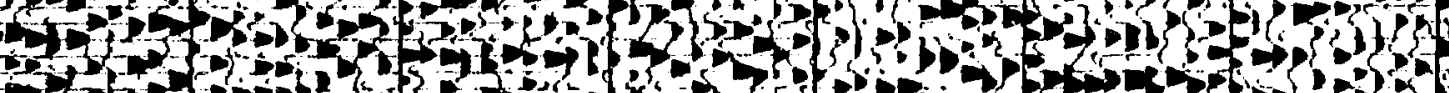

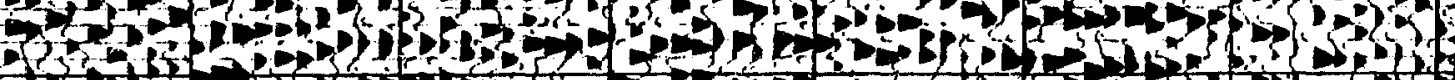

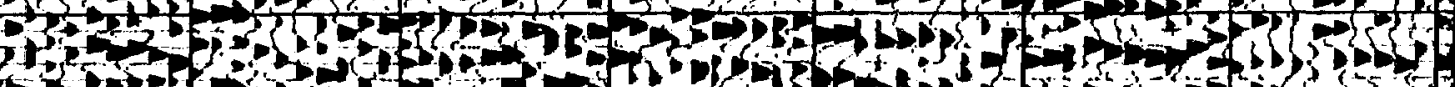

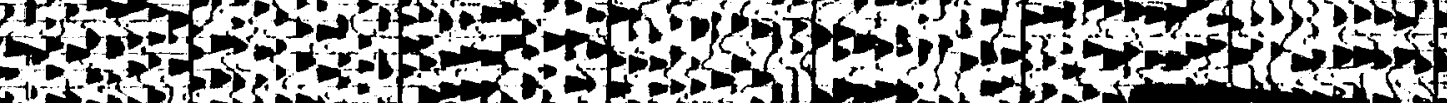

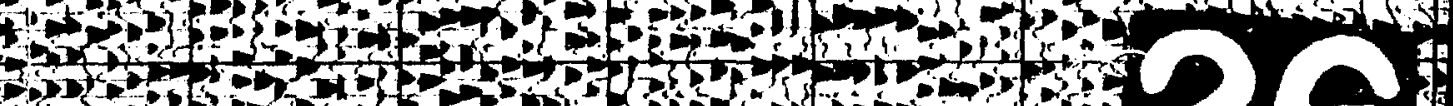

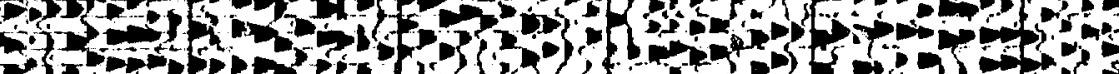
O. 
(1)

3.7.3.

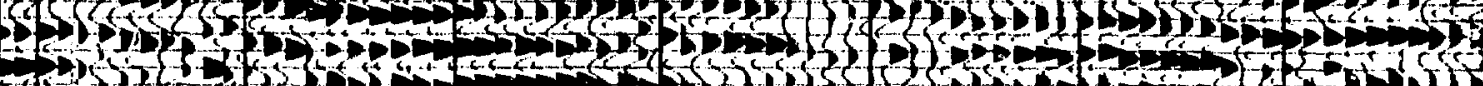

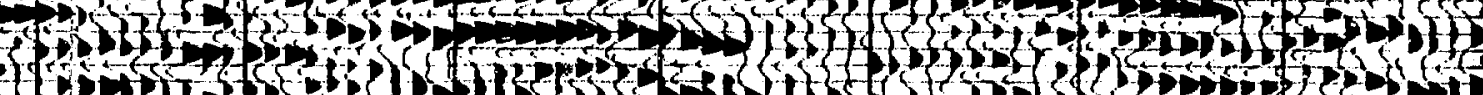

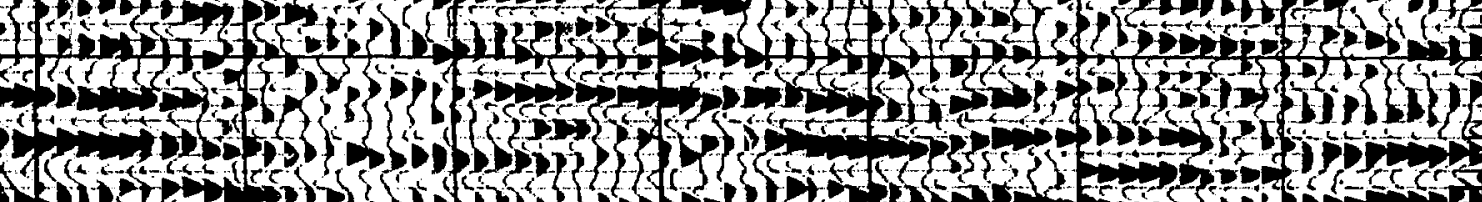

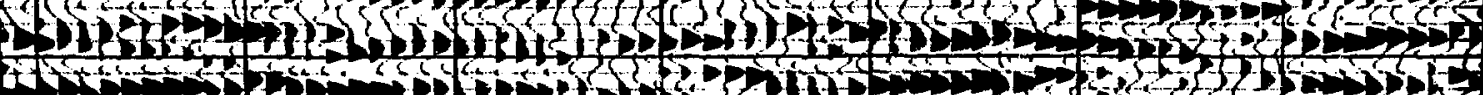

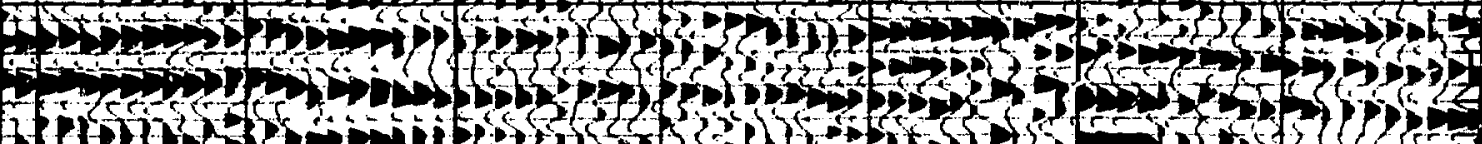

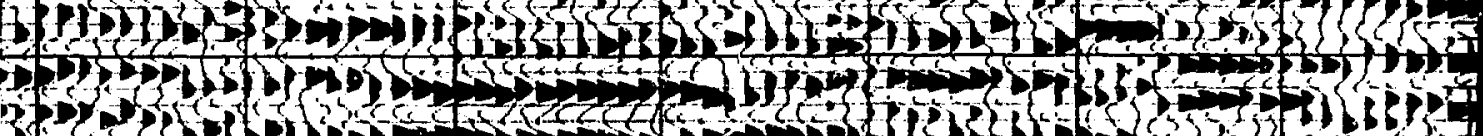
3. H.

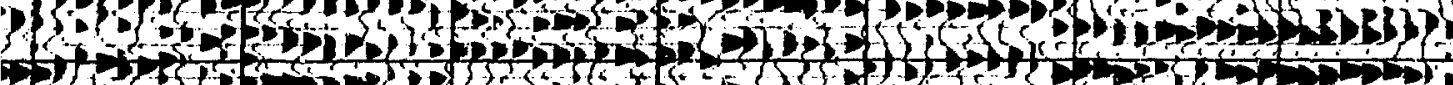

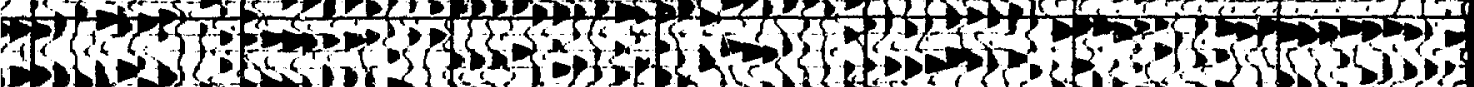

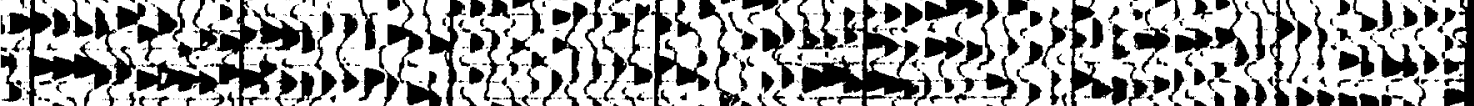

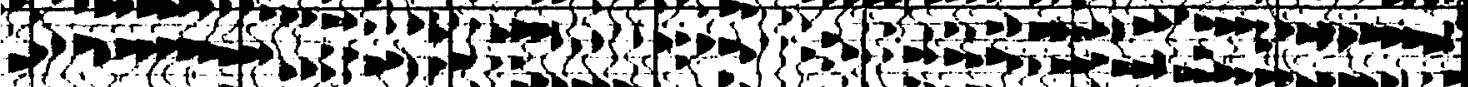

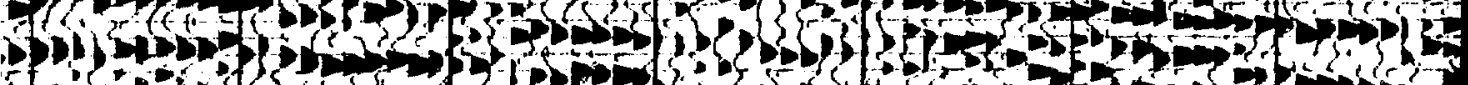

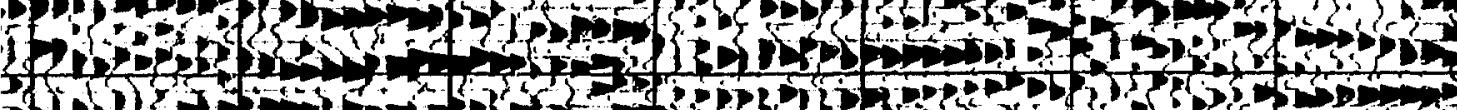

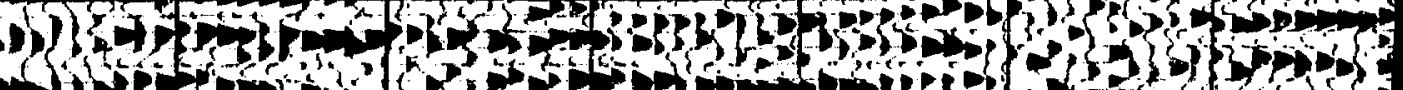

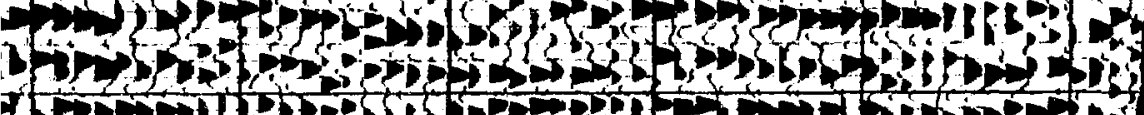

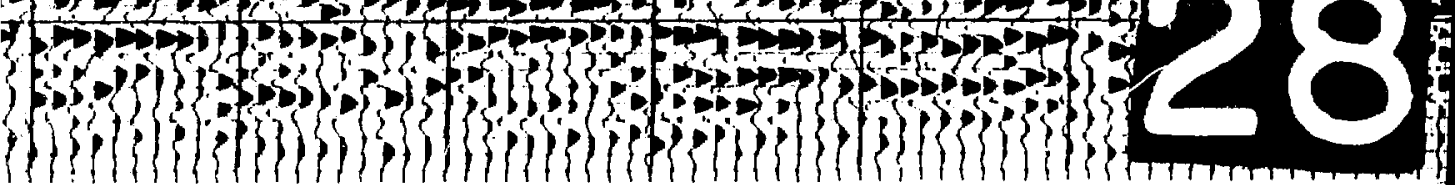




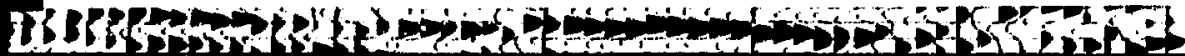
PDda

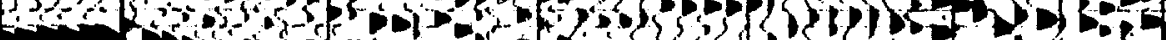
$\therefore-75$ S

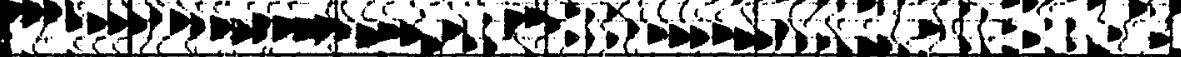

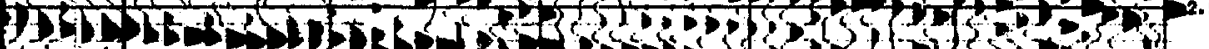

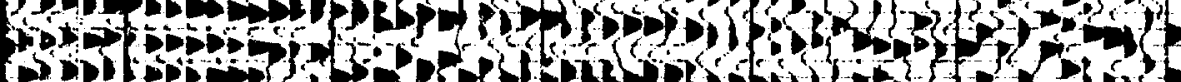

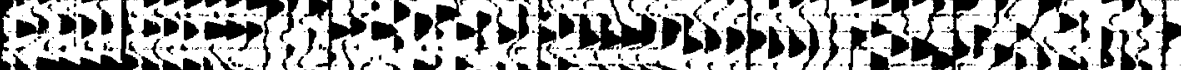

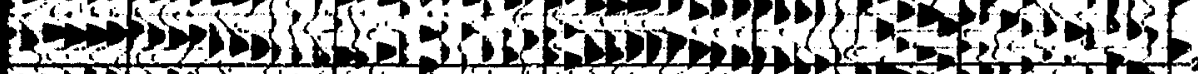

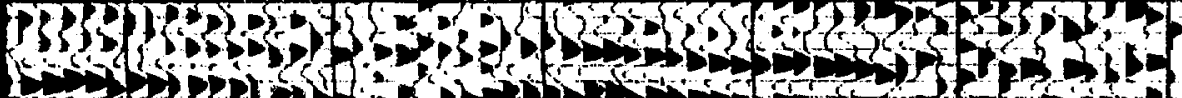

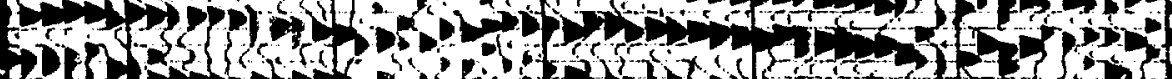

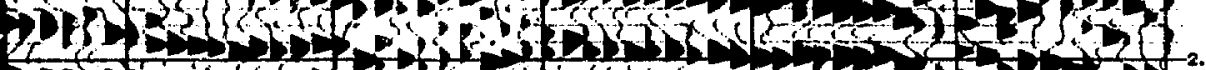

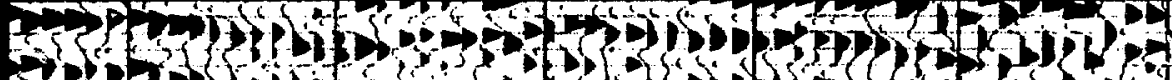

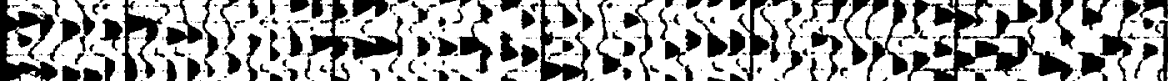

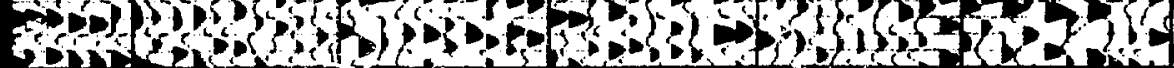

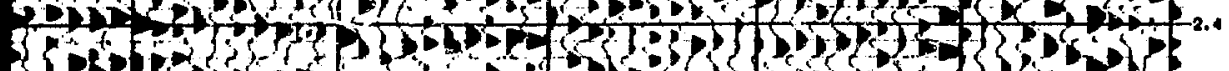

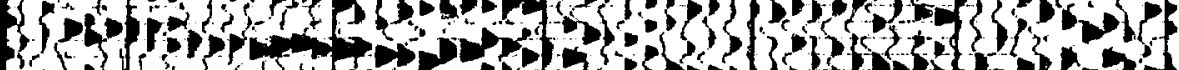

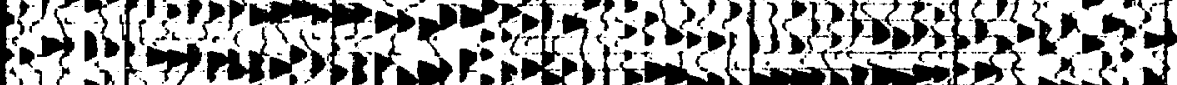

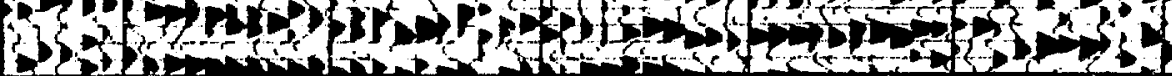

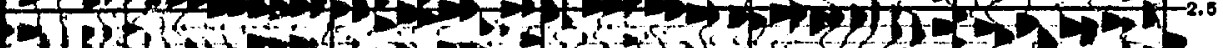

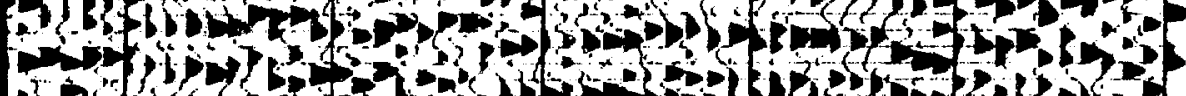

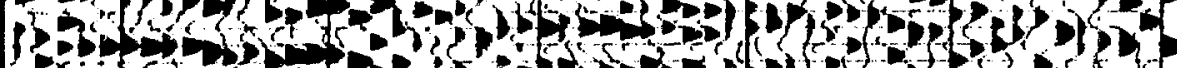

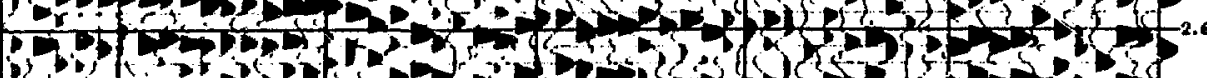

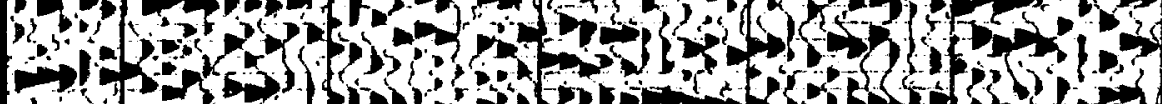

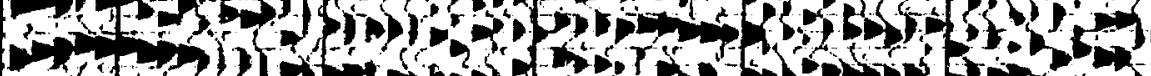
5 सम

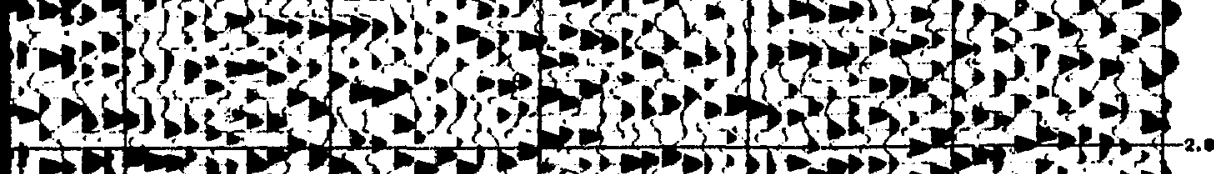

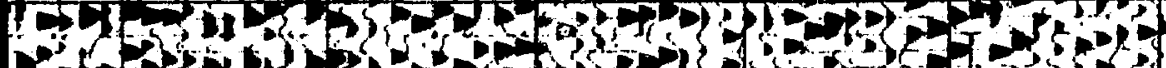

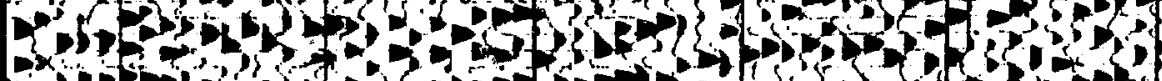

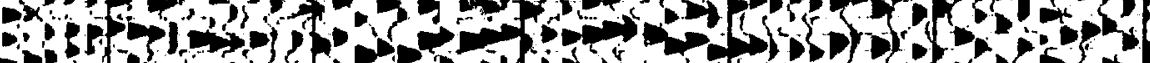

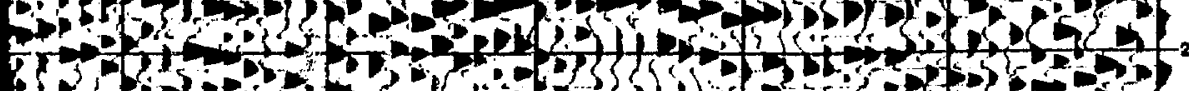

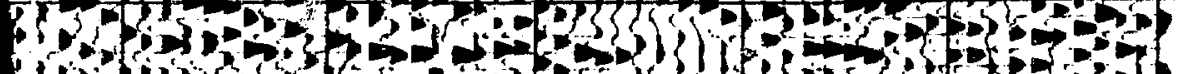

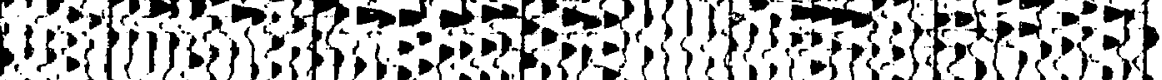

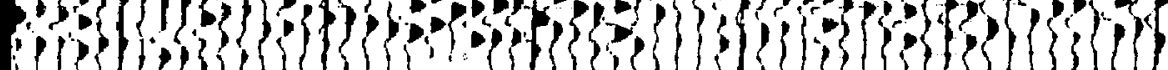

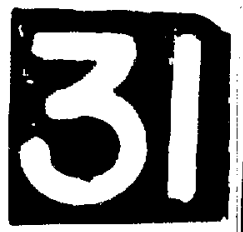




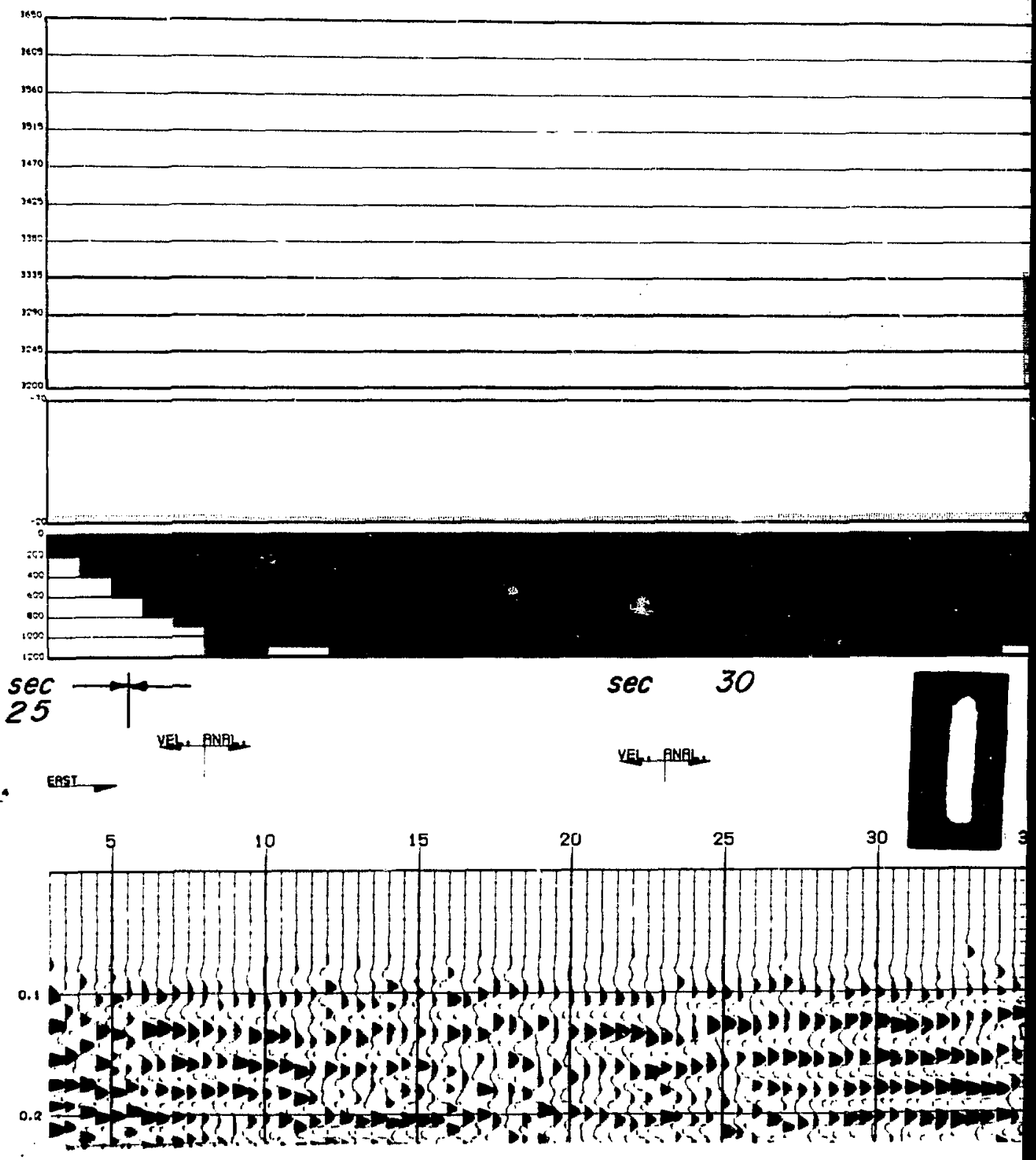




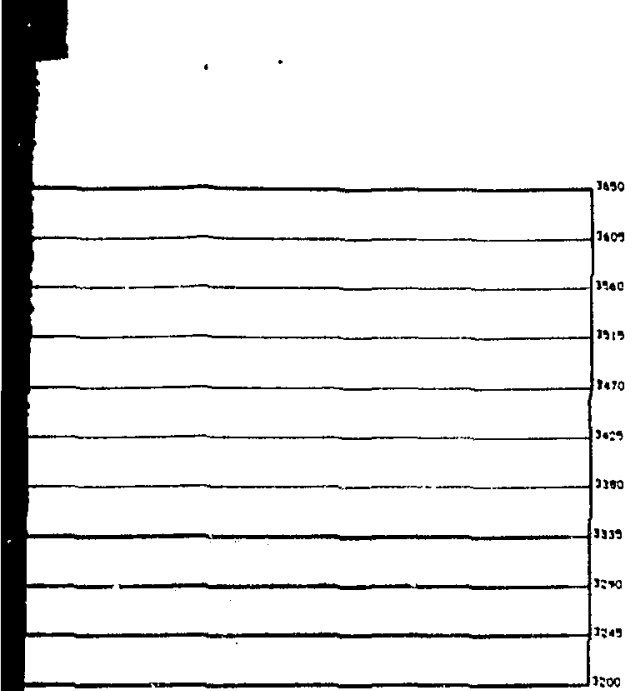

\section{ELEVAT IONS}

STAT ICS

FOLD $x$

WINE DIRECIION

YEL ANefifle

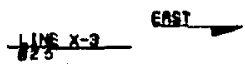

VELOCITY FUNCTION

DIRECTION

LINE INTE:SECTION

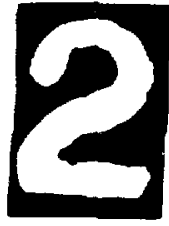

STAT IONS

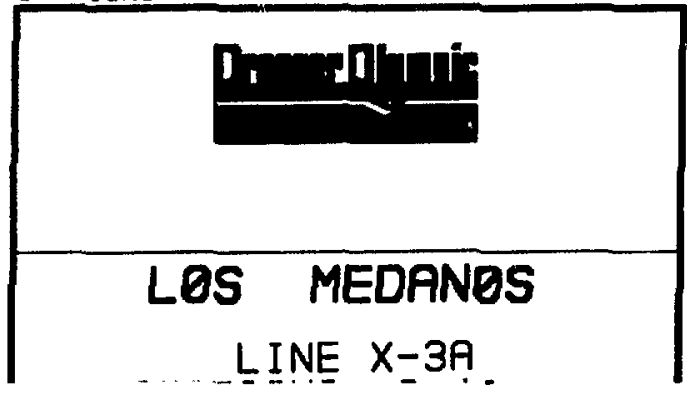




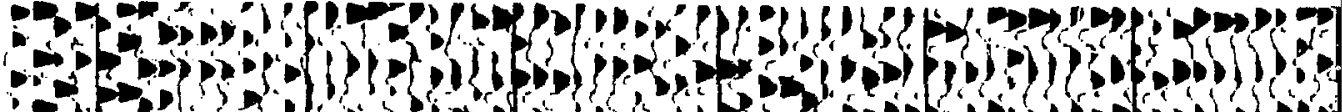

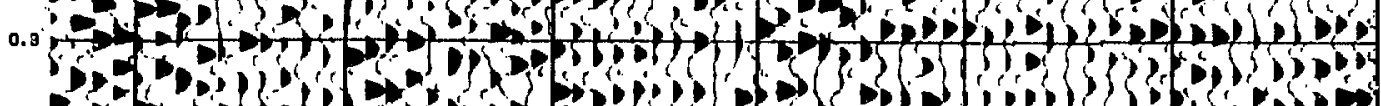

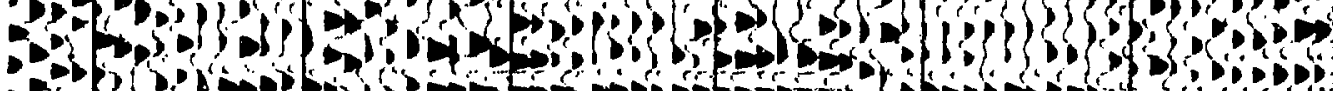

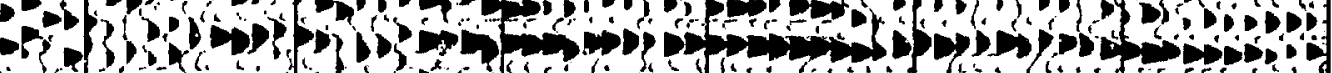

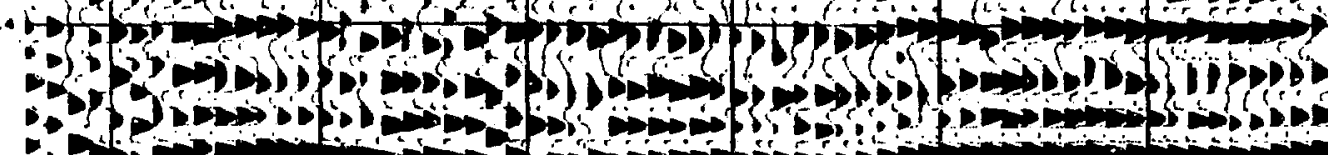

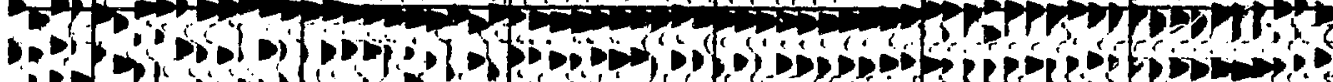

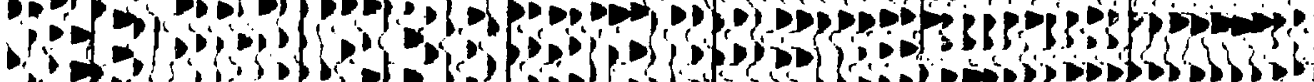

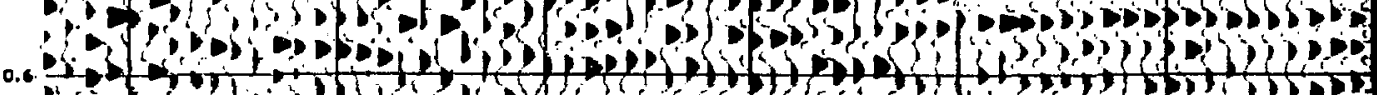

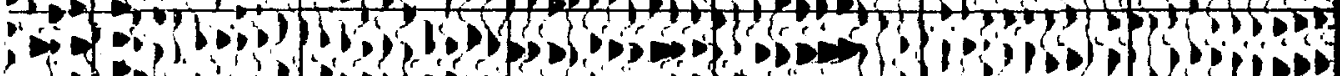

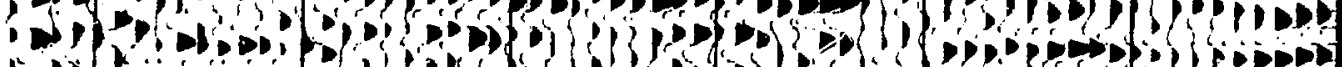

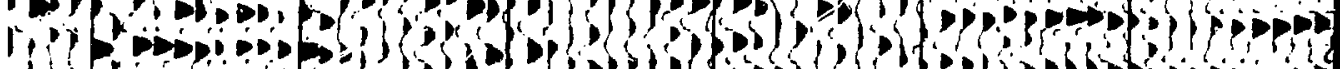

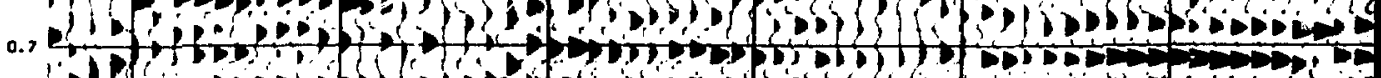

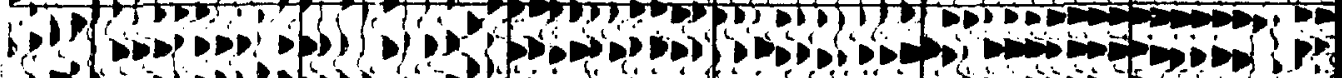

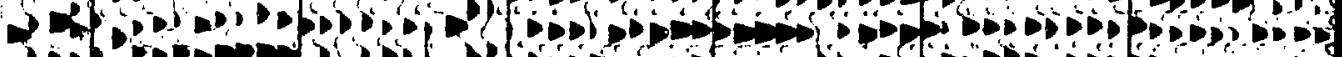

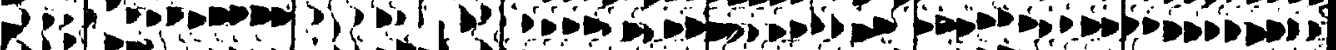

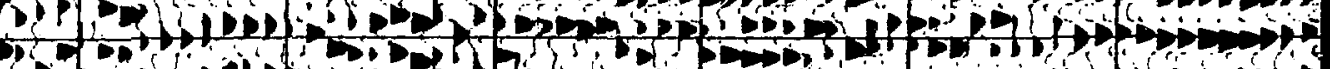
al a

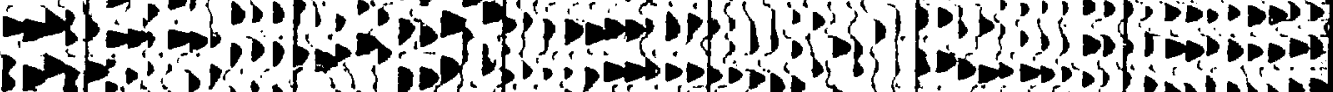

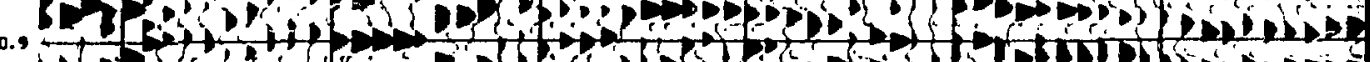

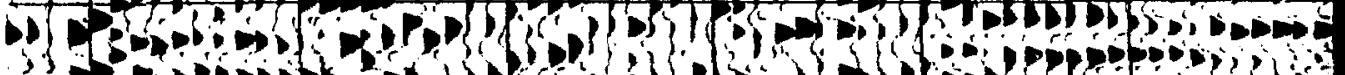

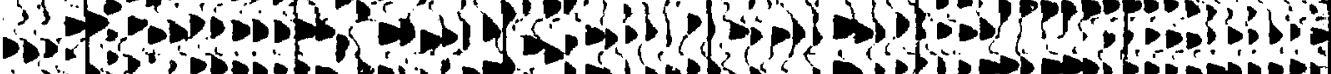

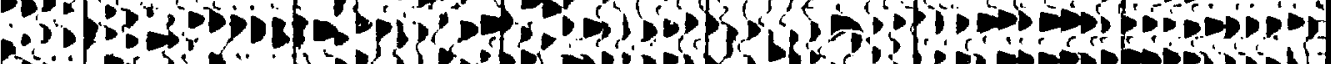

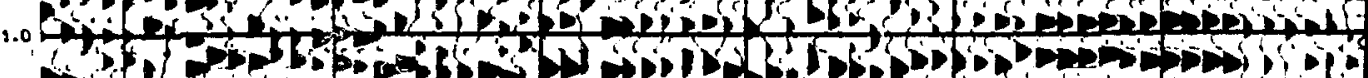

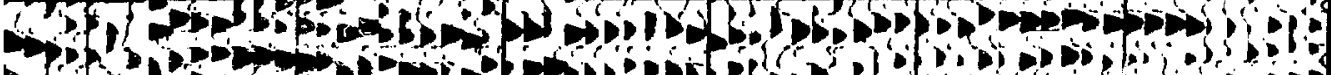

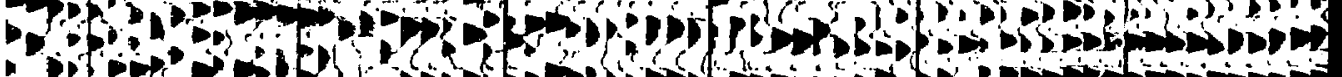

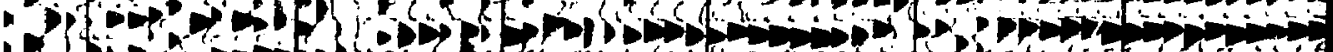

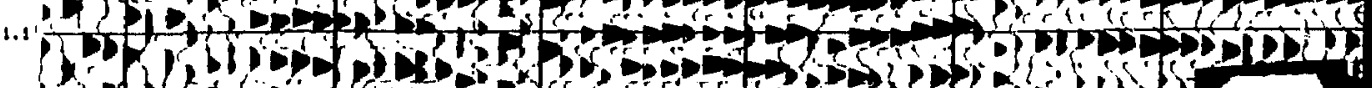
$15\left\{\begin{array}{l}1 \\ 1\end{array}\right.$

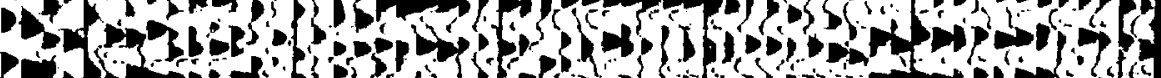
1.2(1) 
INOUT REEL GEROER IMFEMHTIIE

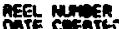

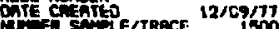

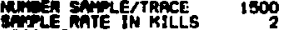

machers

ifre

acrith in

PHCESBINO STEP

\section{FIELD INFERMATION}

\begin{tabular}{|c|c|c|c|}
\hline 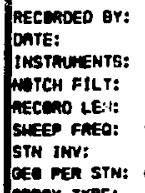 & 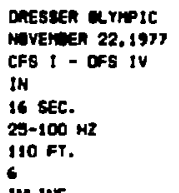 & 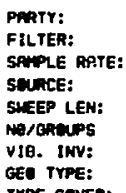 & 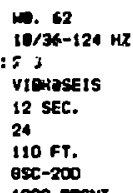 \\
\hline
\end{tabular}

\section{PROCESSING SEGUENCE}

PRACEASED GT ORESSER a MTPIC
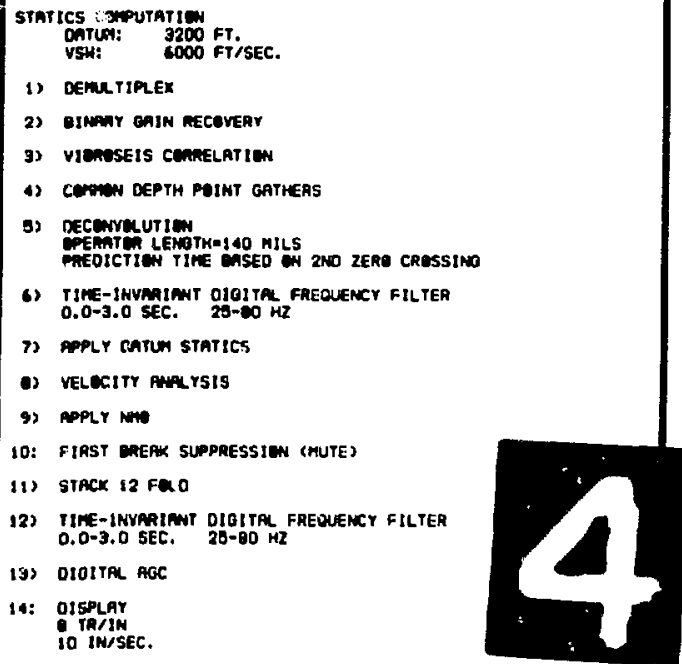

$\cdots \cdots \cdot 10$ 12 GPAERO DJAGRAM ip 19 $440440 \quad 1210$ 


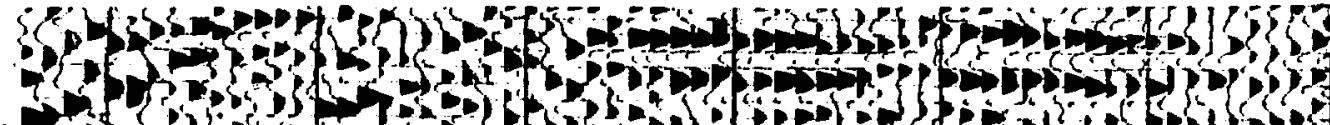

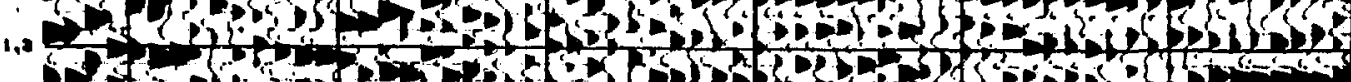
P.

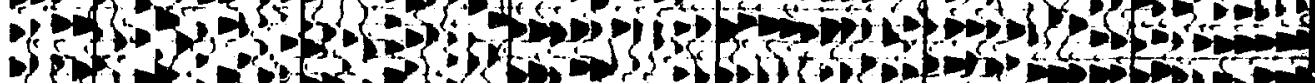

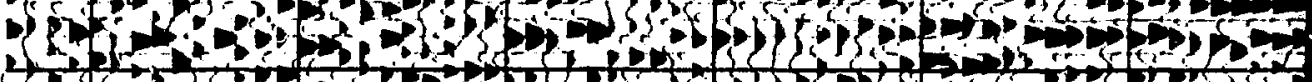

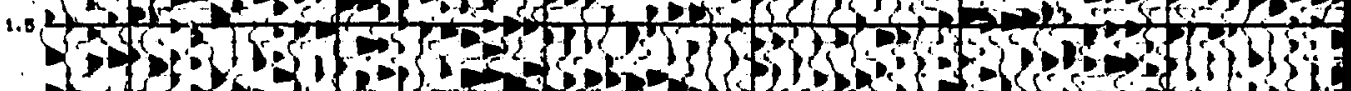

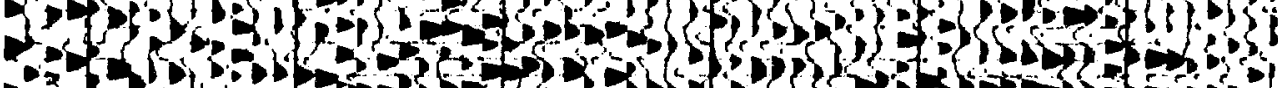

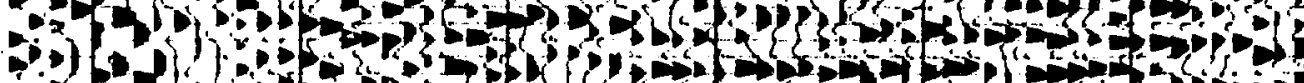

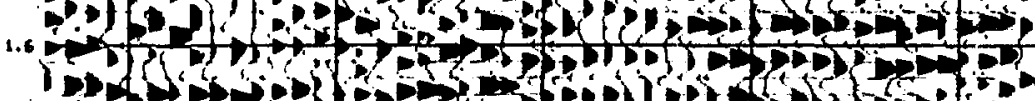

of

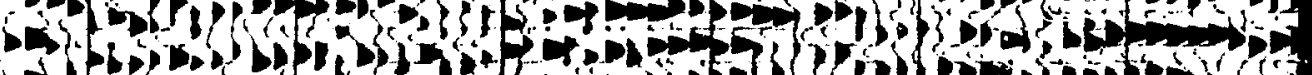

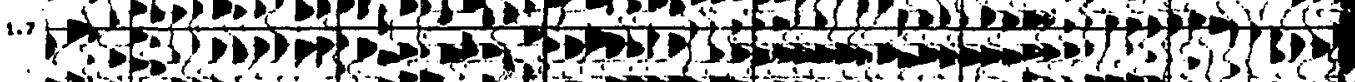
Hof

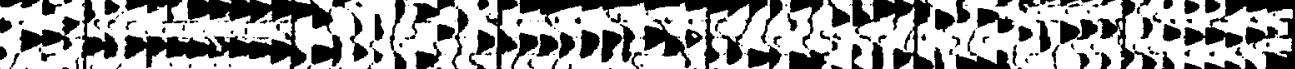

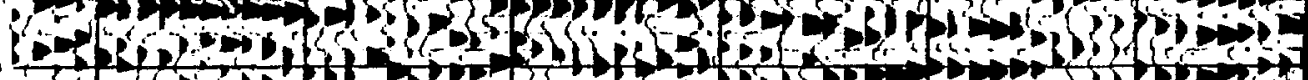

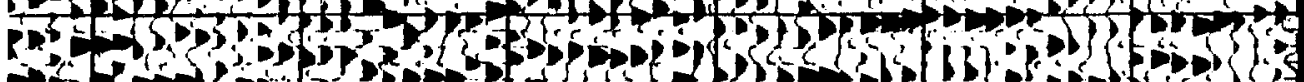

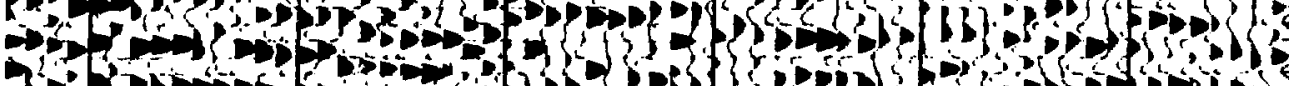

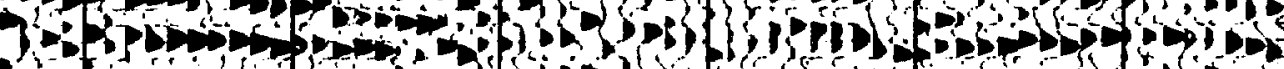

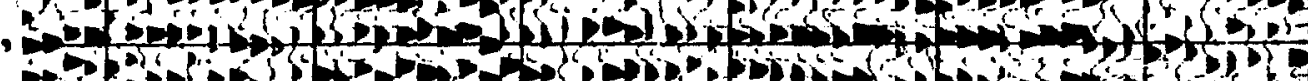

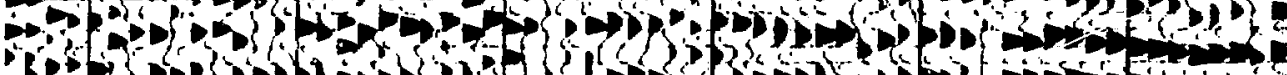

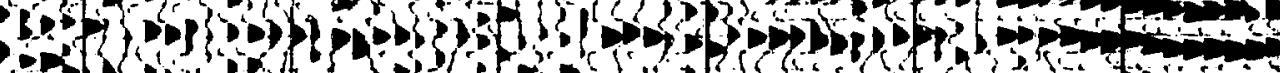

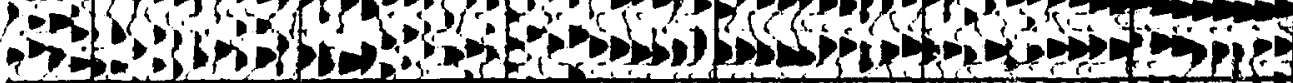

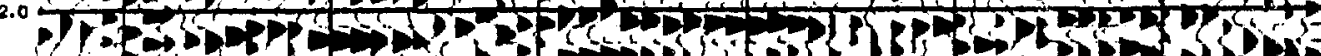
- La

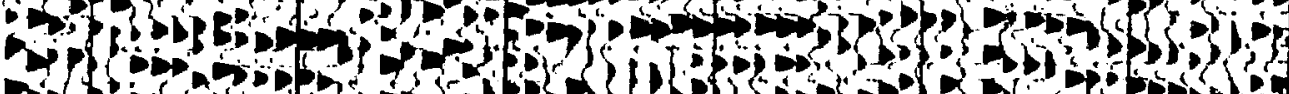

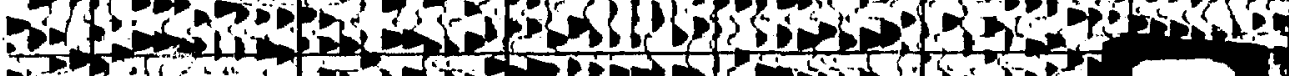
2.1 1.7.

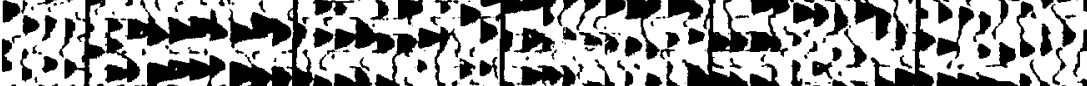

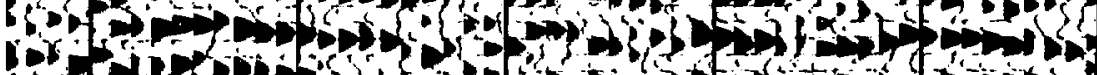

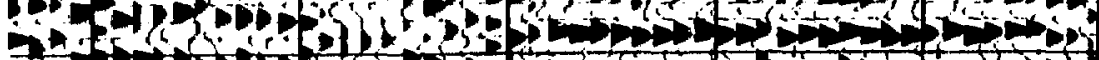

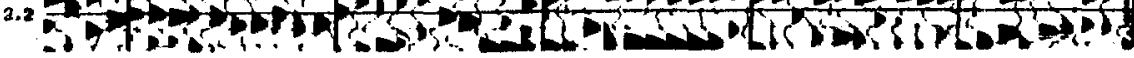

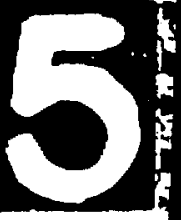




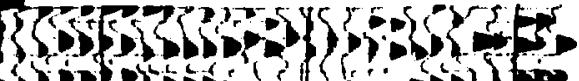
$1,10+16$ inl in S.

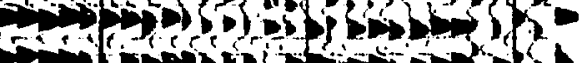

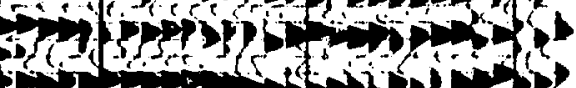

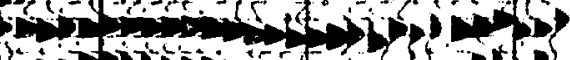

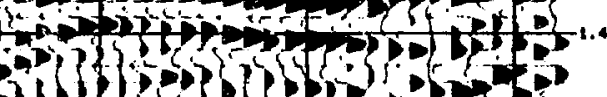
III Is) $-0.05$ 4010 Holobsins 0330193,0005 s.t.

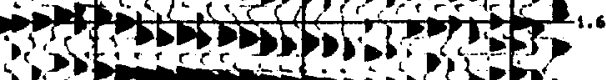
31350 DS 1530 , in

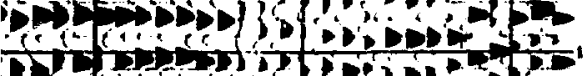

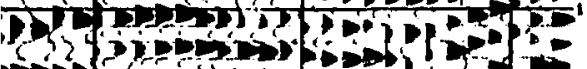
- 20 $75, \mathrm{C}$ . An

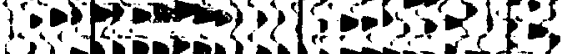

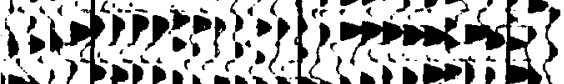

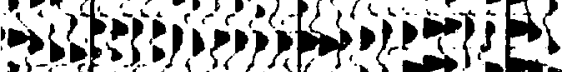
- 35

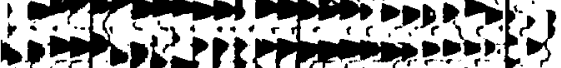

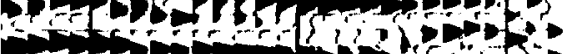
Dif 15,1, Th Sis 40015

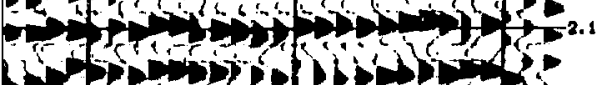

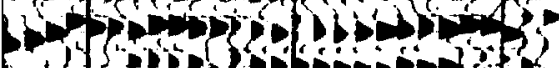

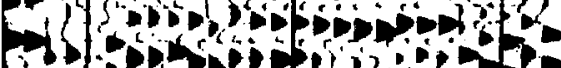

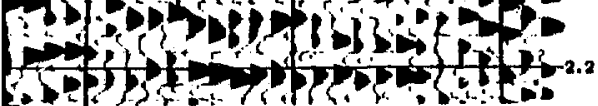
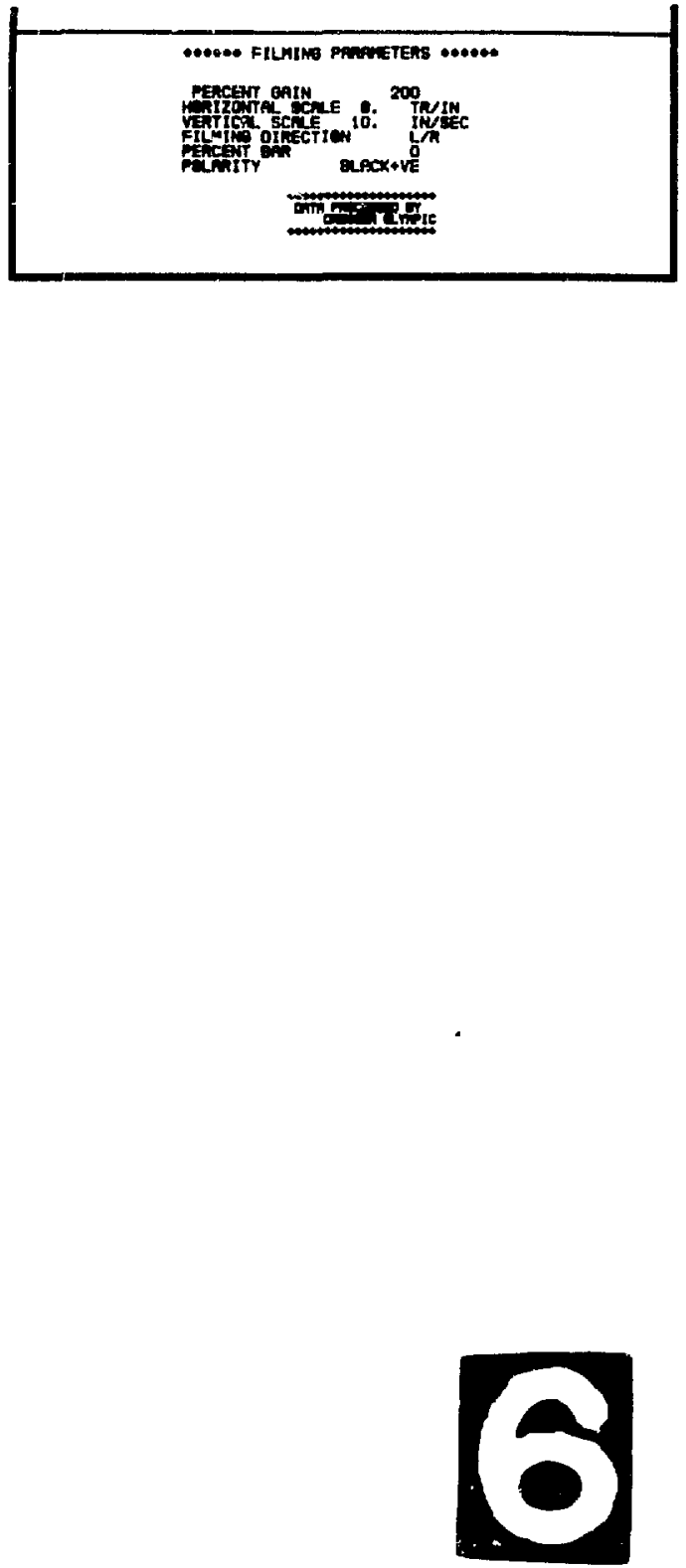
.. 1.

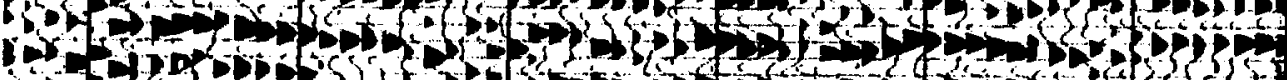

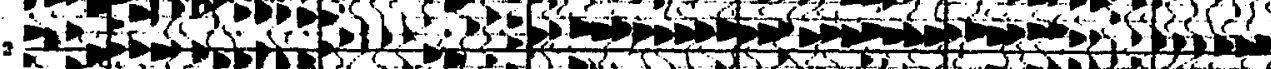

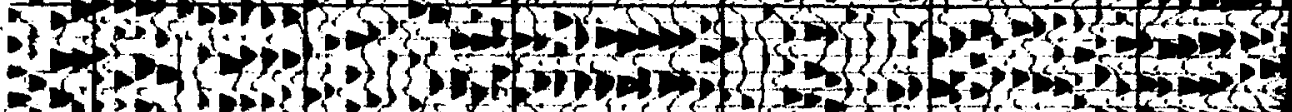

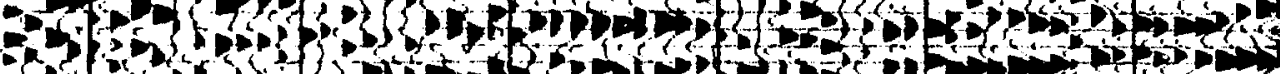

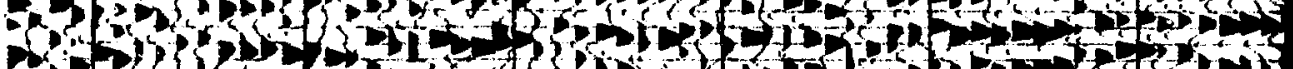

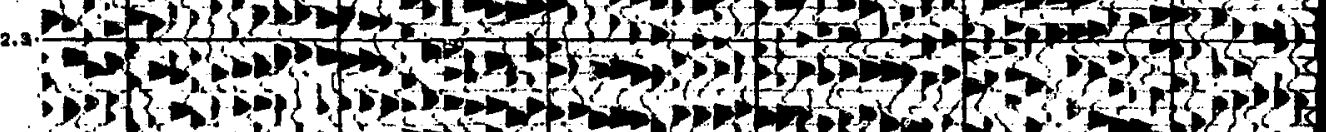

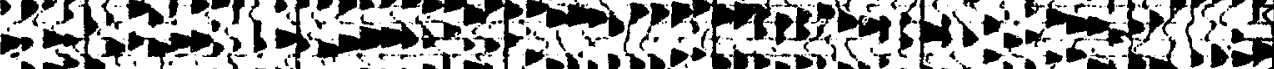

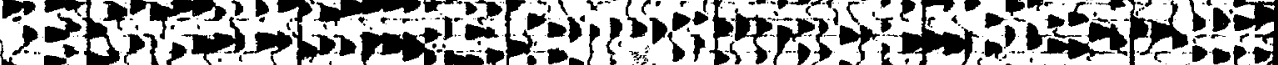

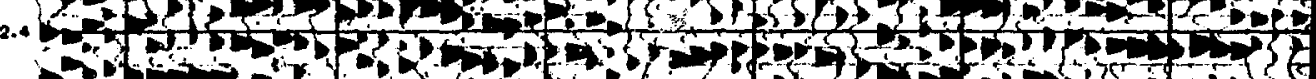

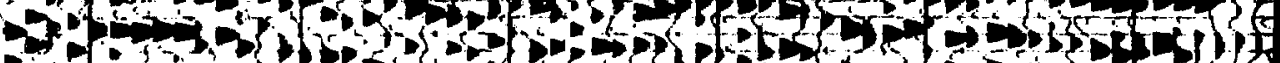

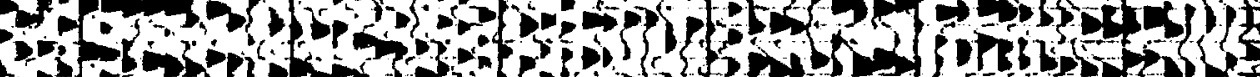

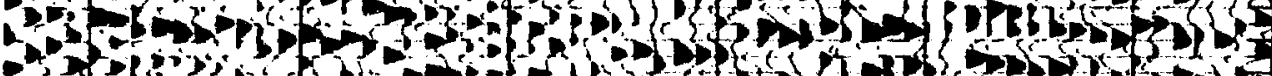

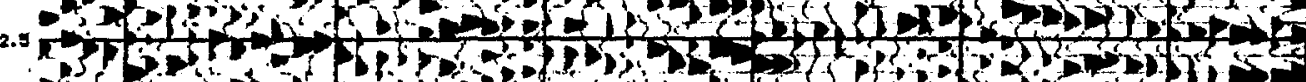

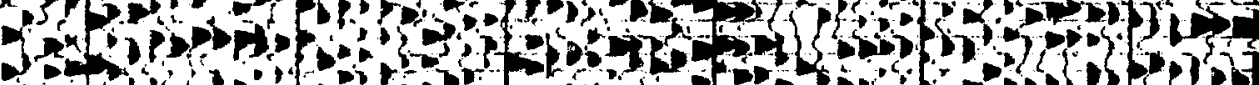

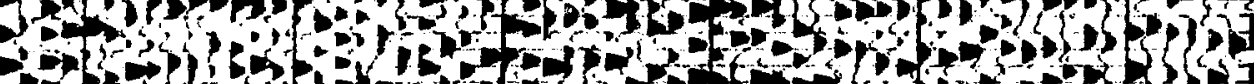
*

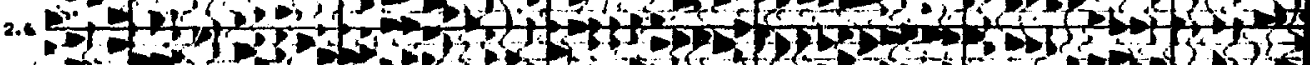

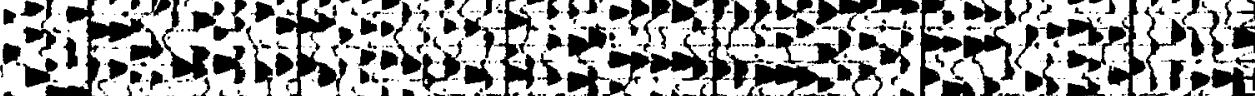

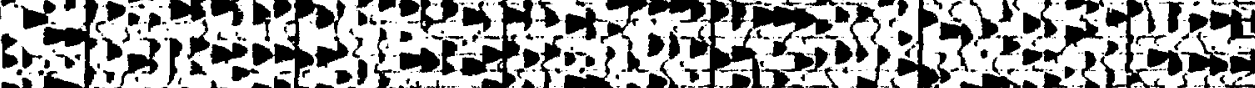

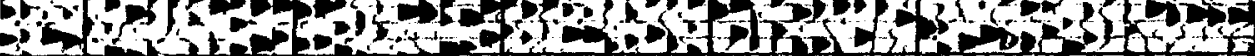

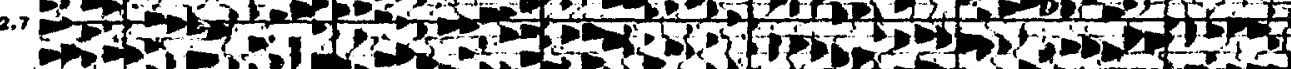

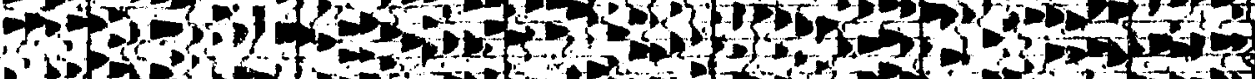

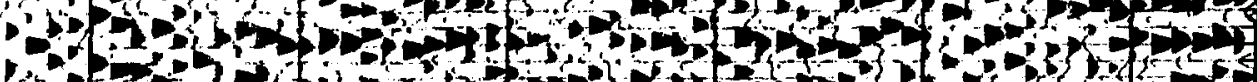

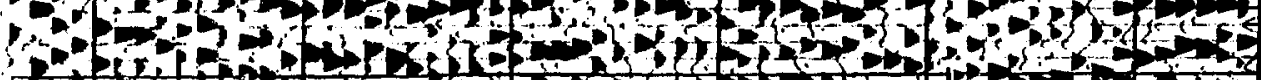
2.0 5 on

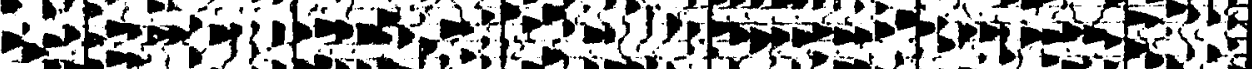

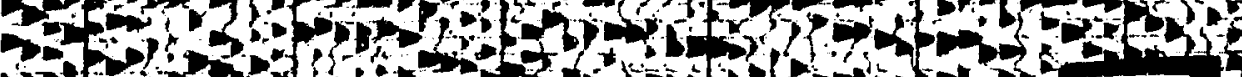

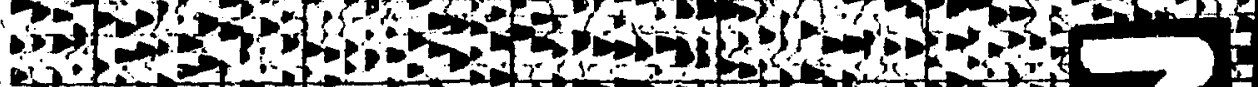
2.3 


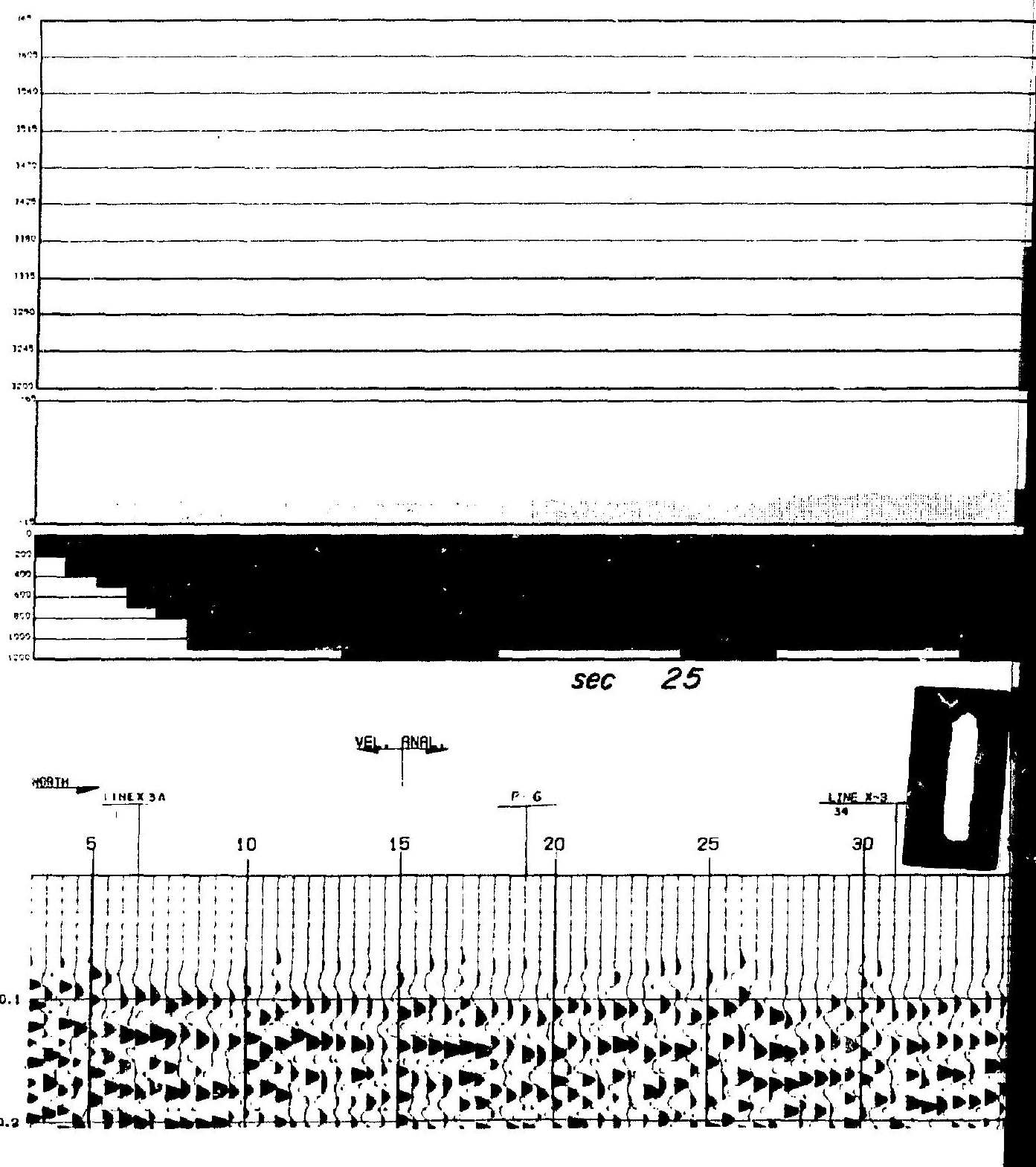




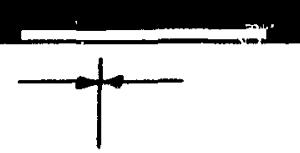

VEL ANPL
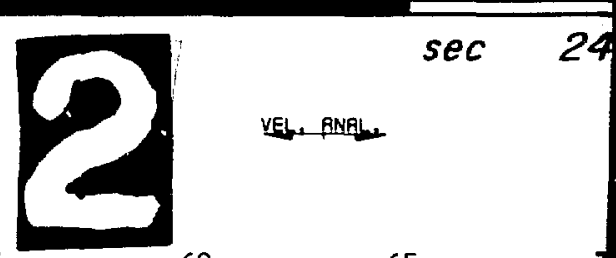

VEL, FNAL

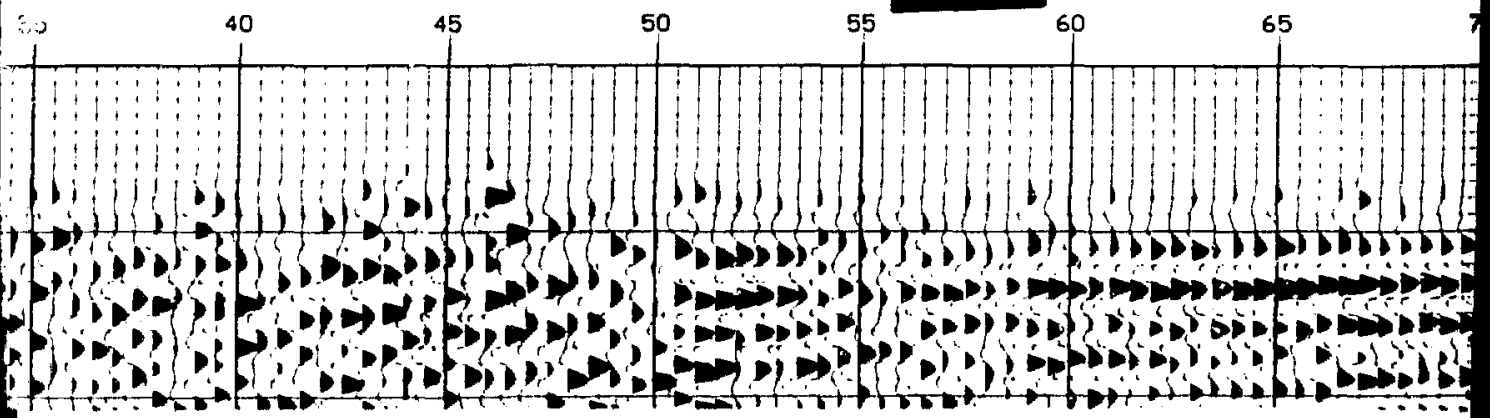


VEl, RNALC

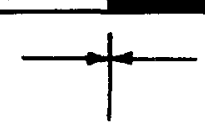

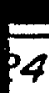
e.

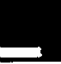




YEth PNAL
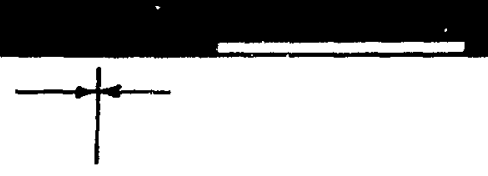

.

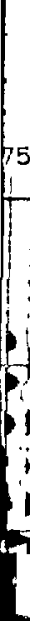
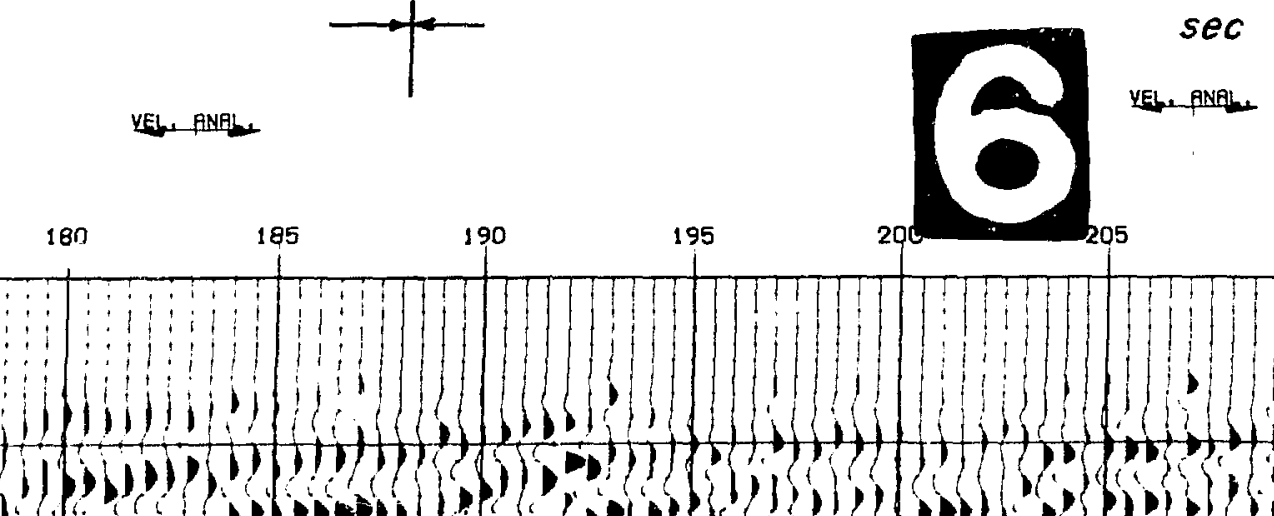

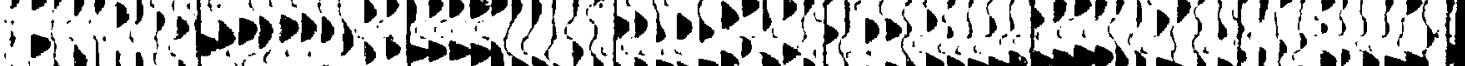

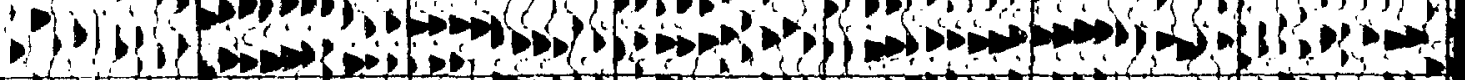

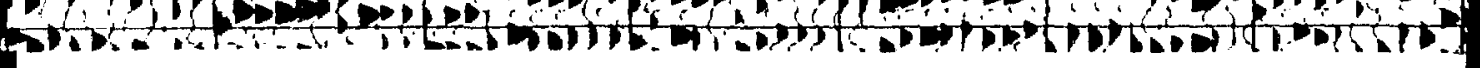




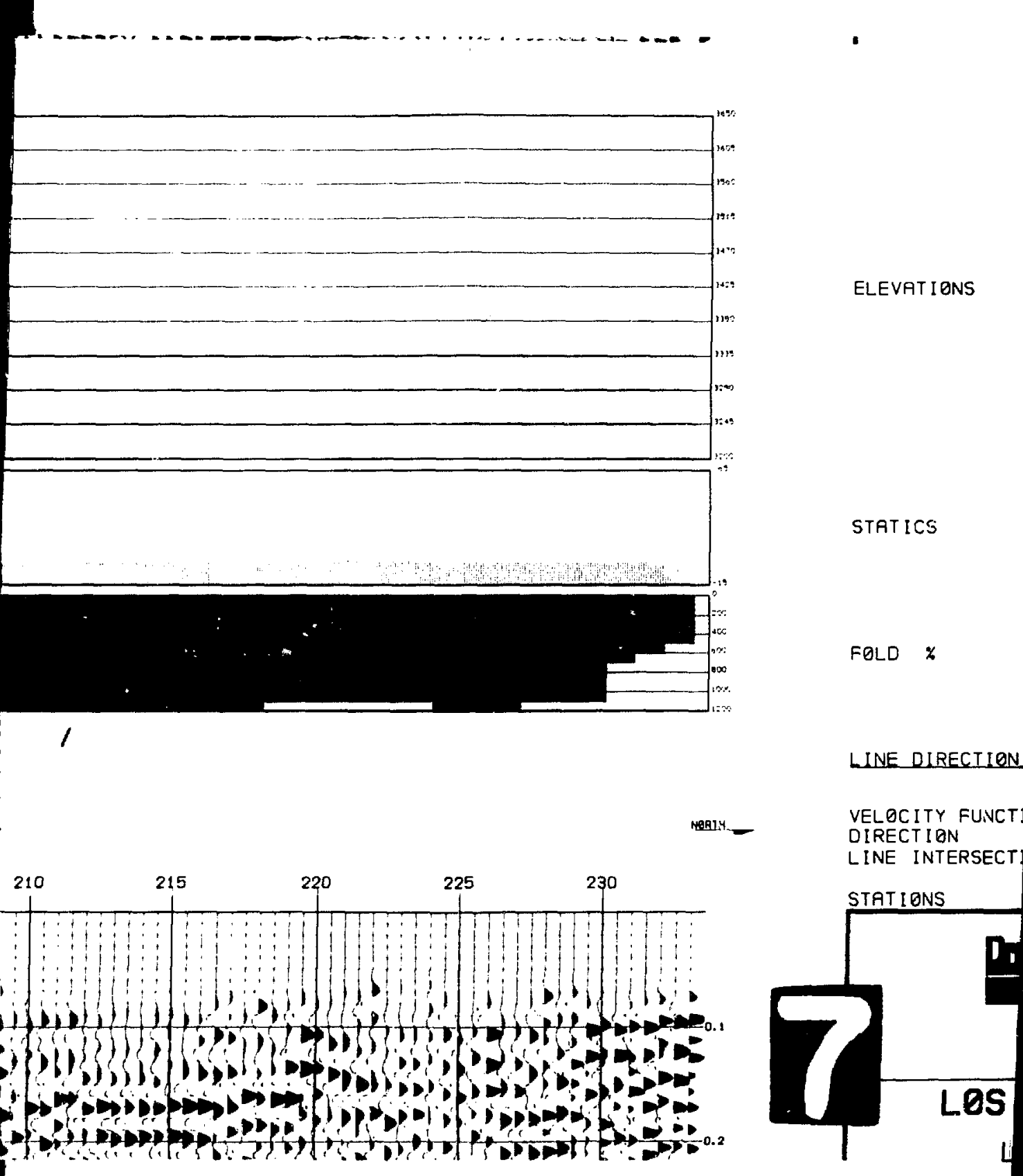




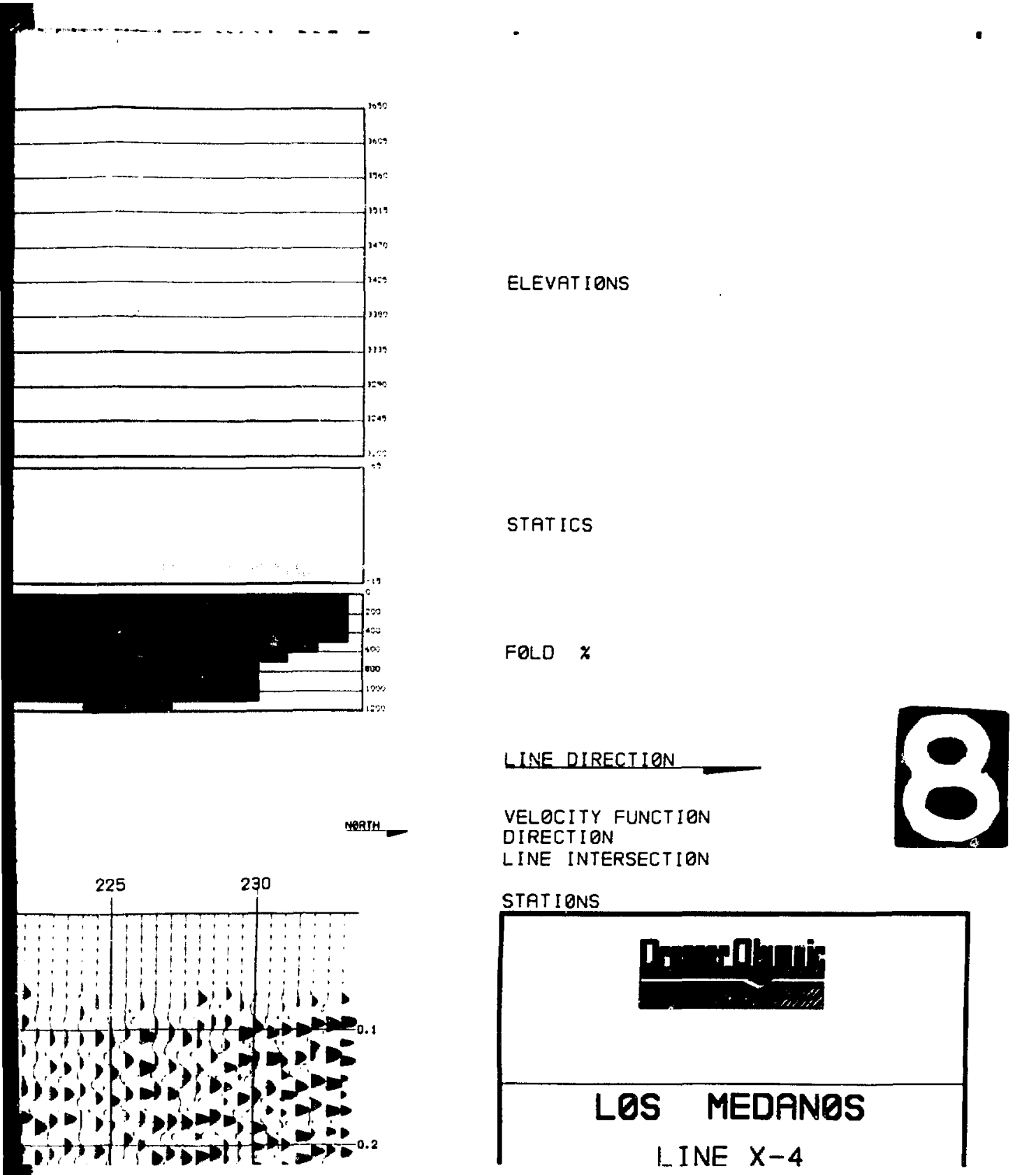




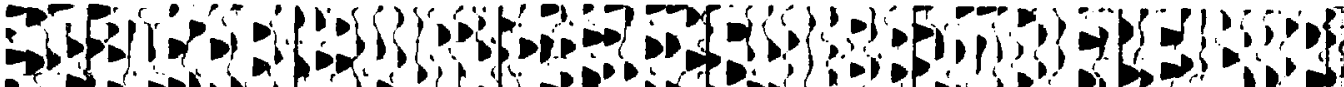

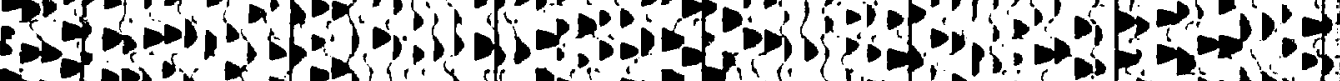

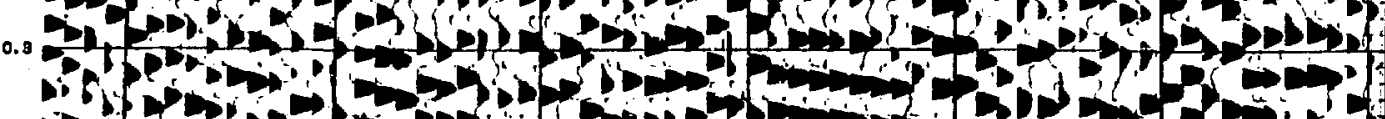
-

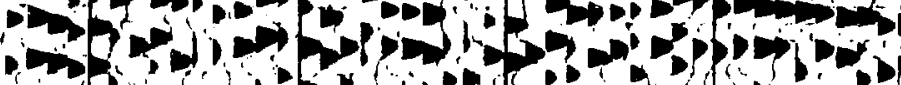

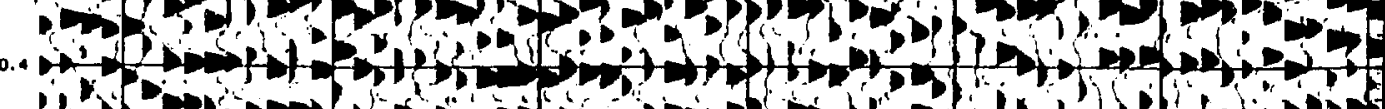

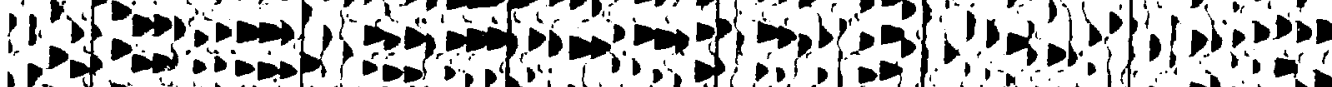

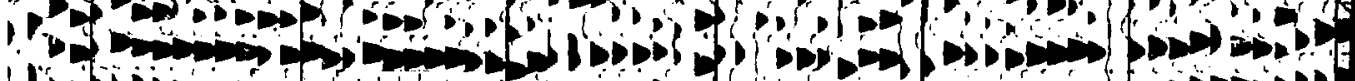
H.

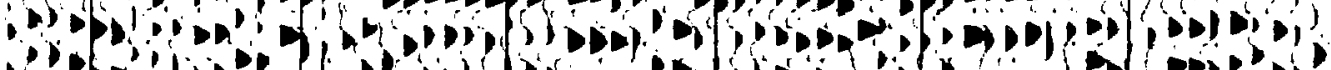

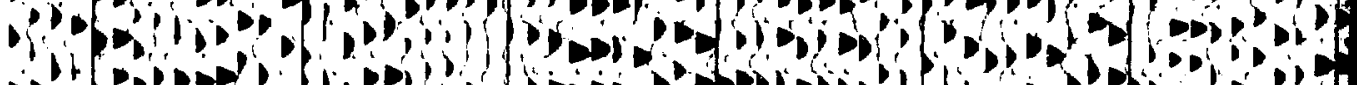

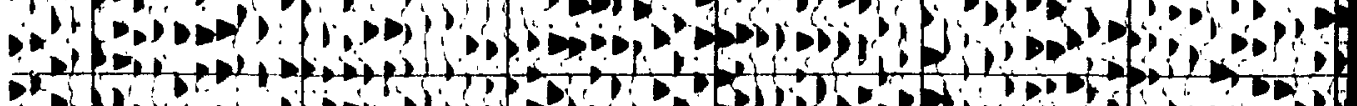

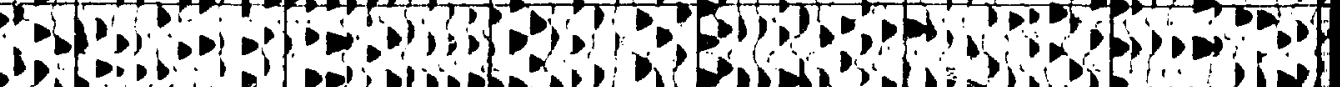

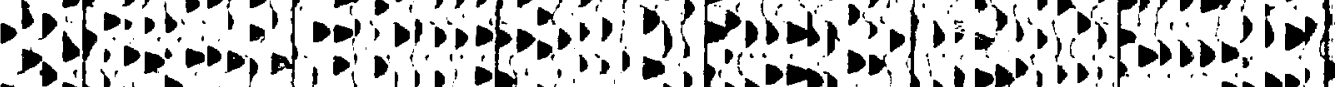

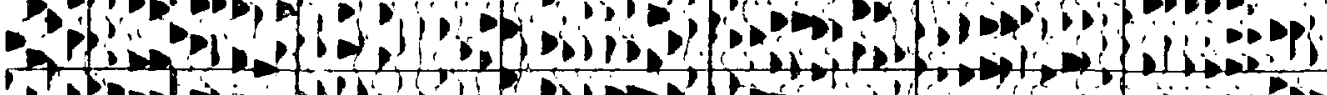

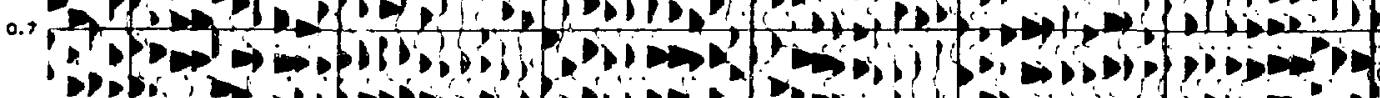
$\therefore$ of

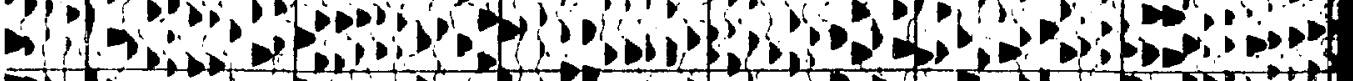
- To

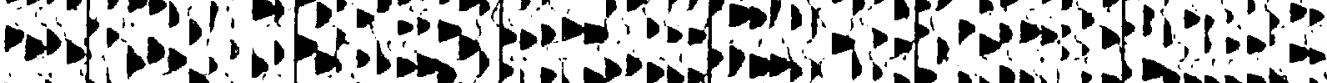

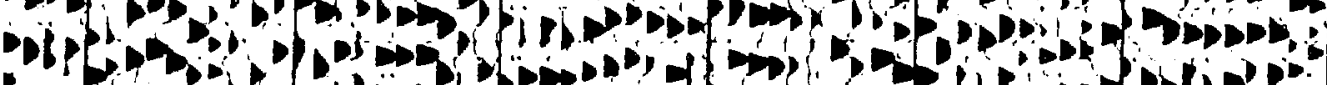

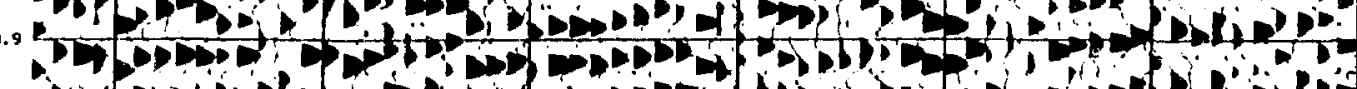

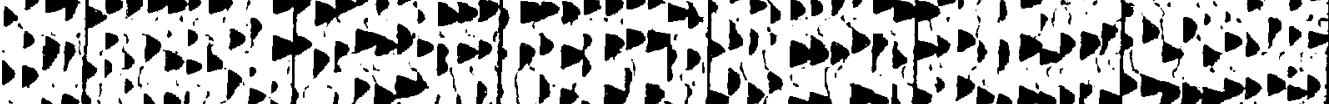
in - :

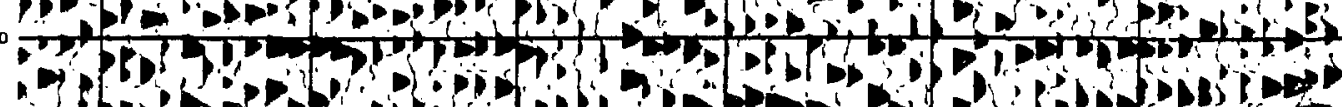

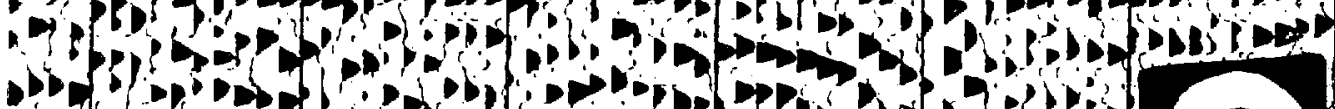

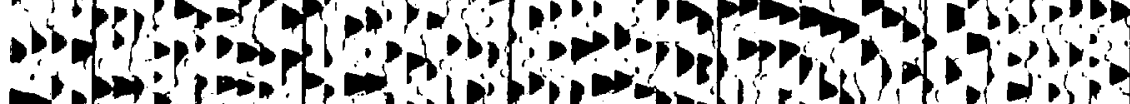

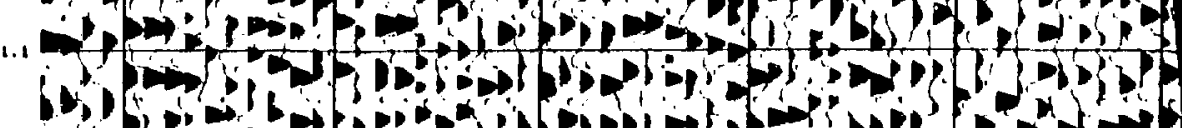
of 
(1)

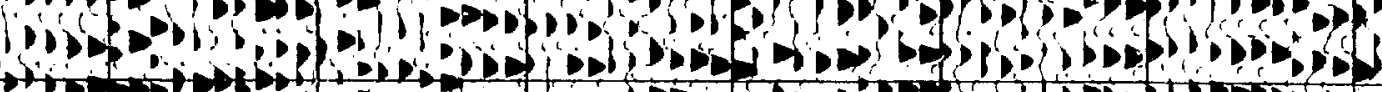
(a)

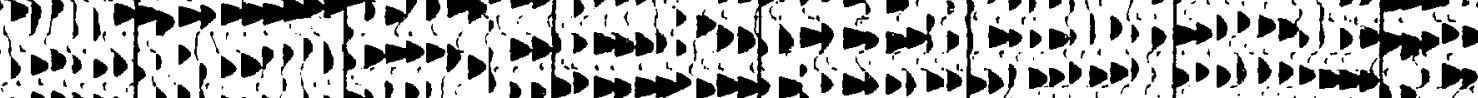

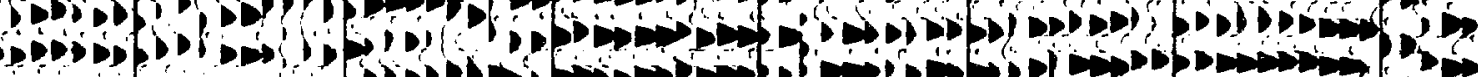
$\because \therefore$ is

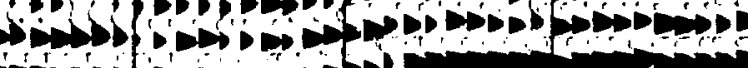

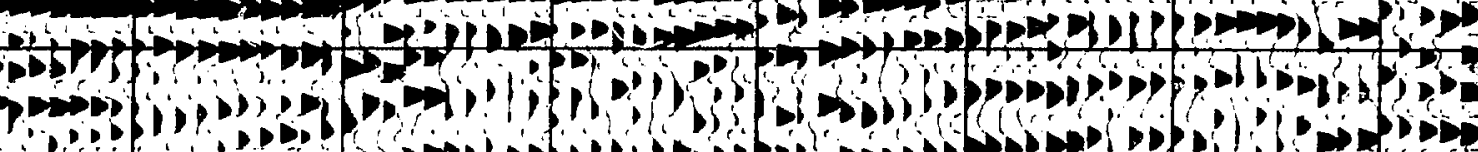

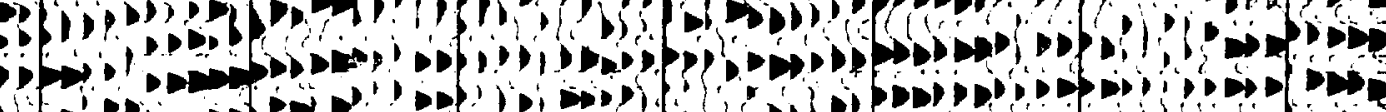

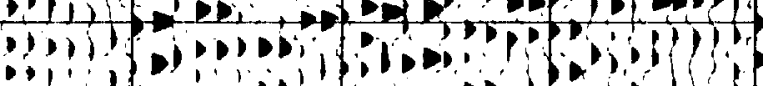

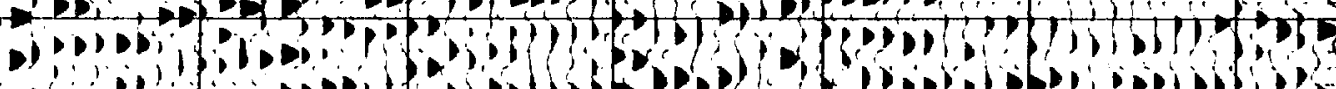
11,

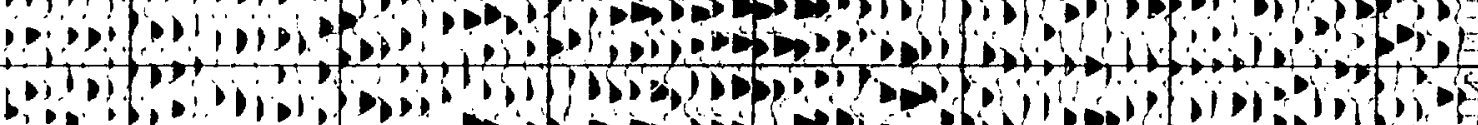

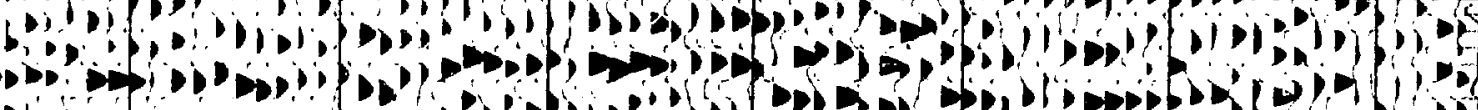
bis

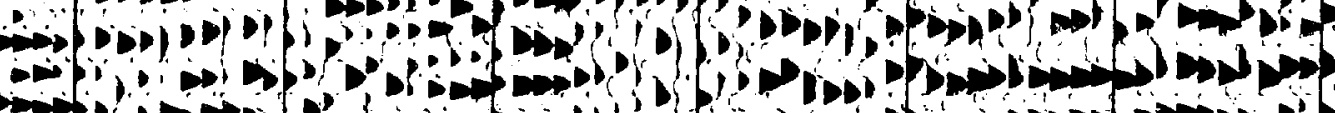

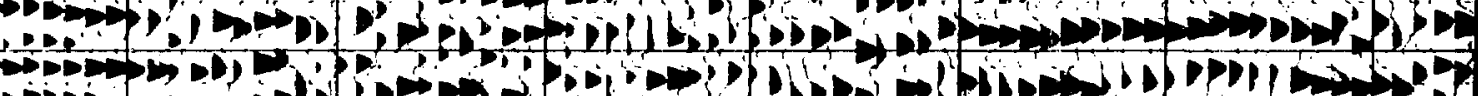

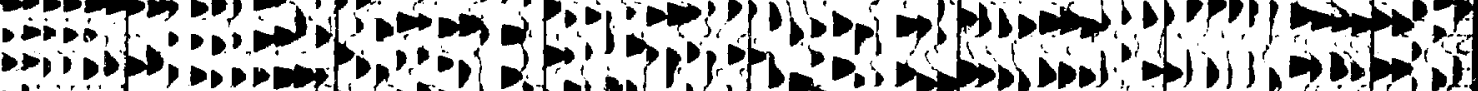

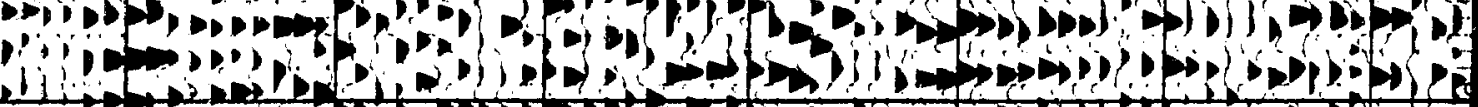
Dofi

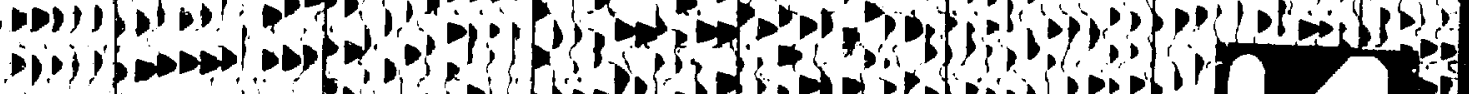
inf

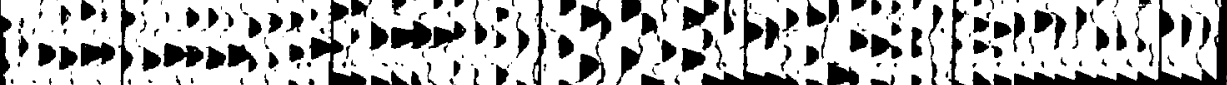

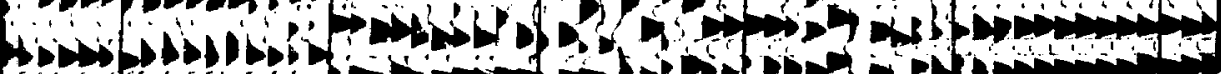




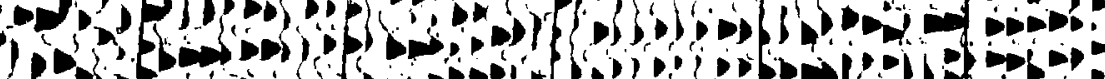
0 . 19450.040

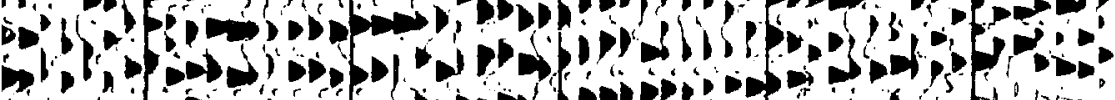
-

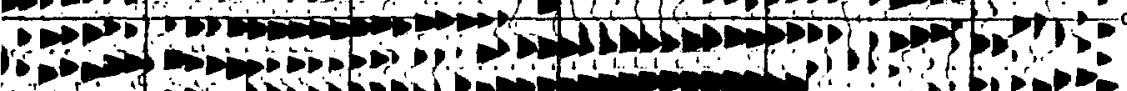

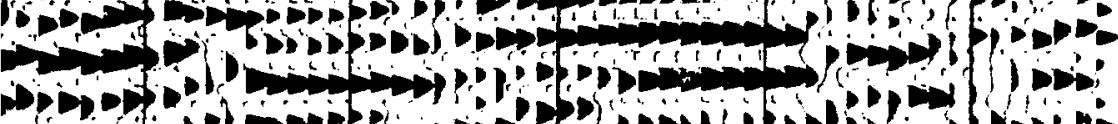

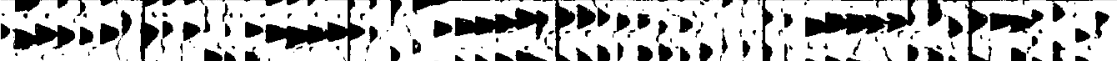
$\therefore$ is

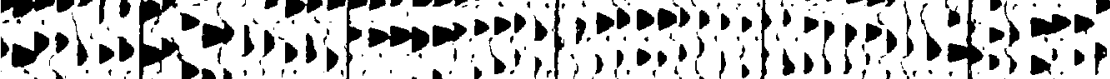
P -

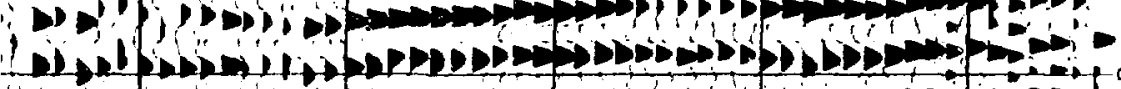

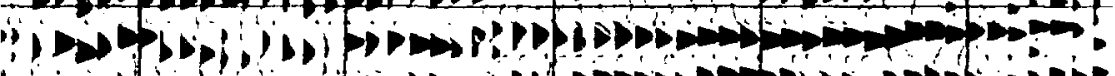

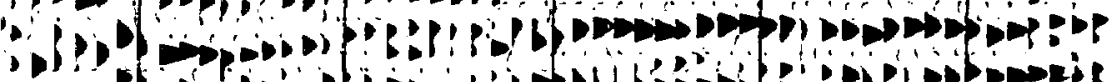

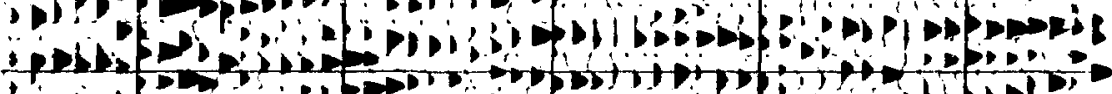

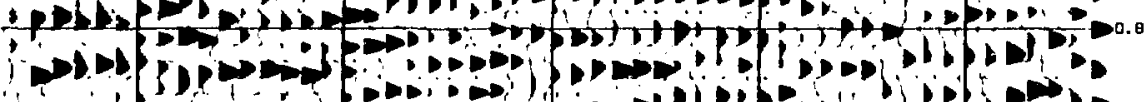

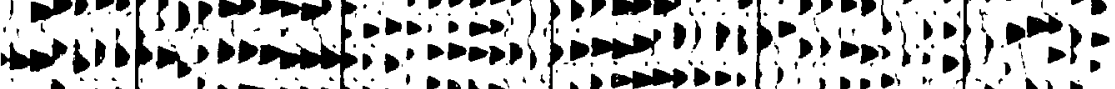

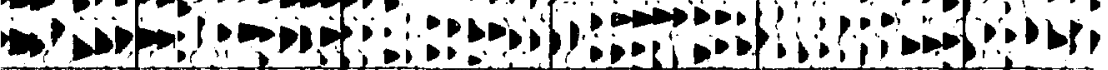

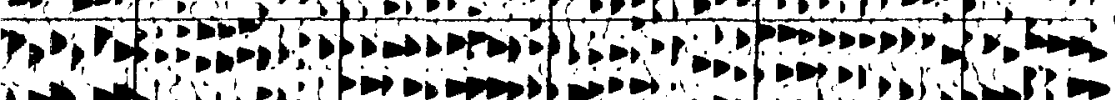
(1)

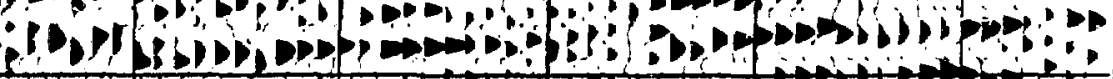

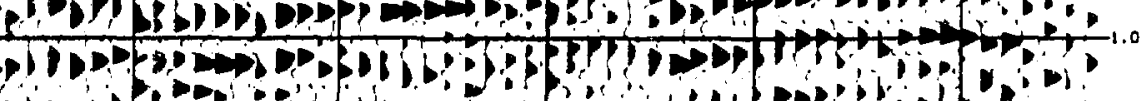

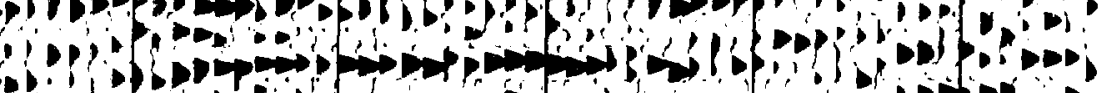

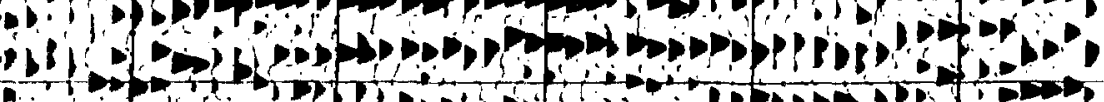

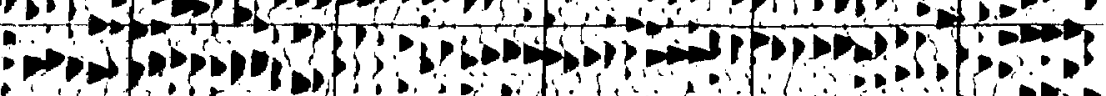

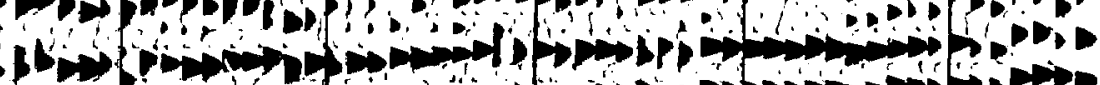

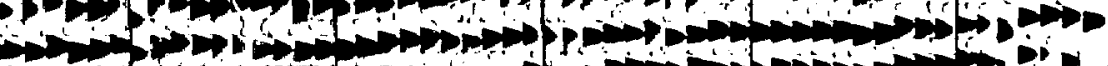




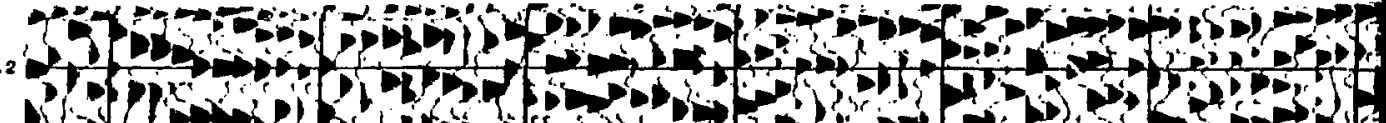

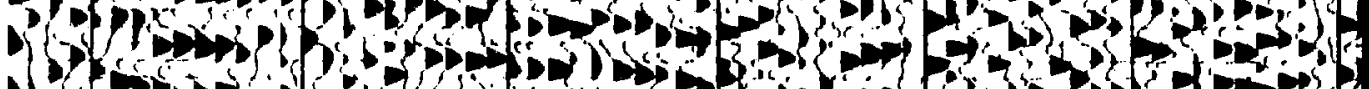

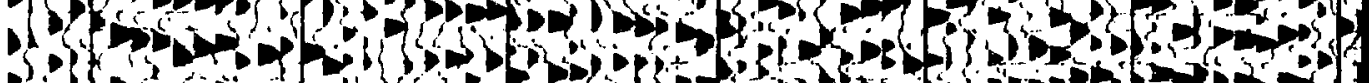

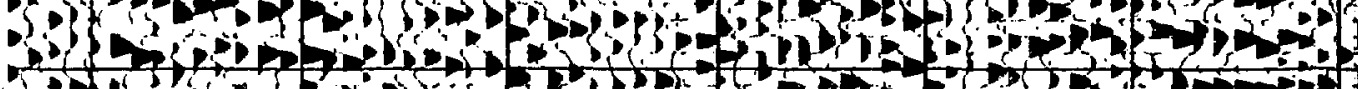
1.30 s.

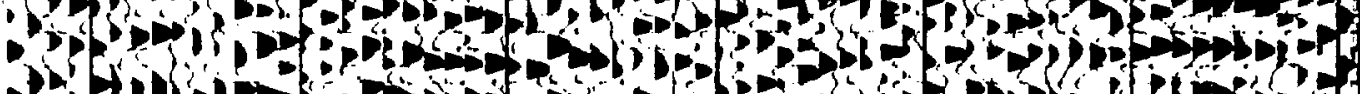

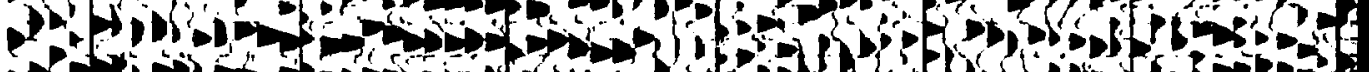

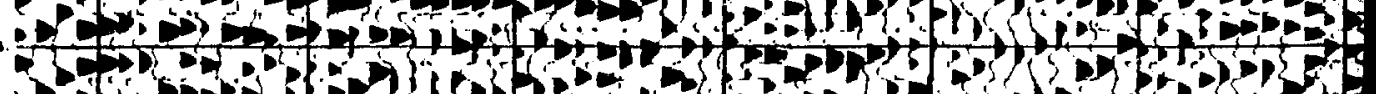

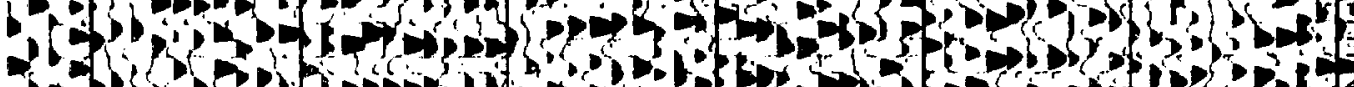

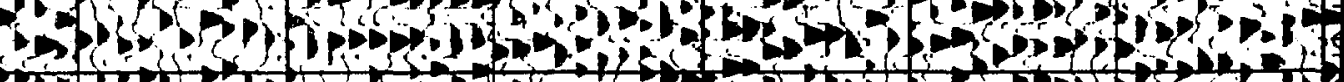

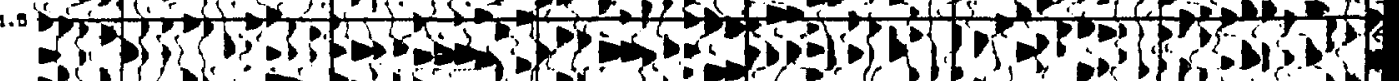

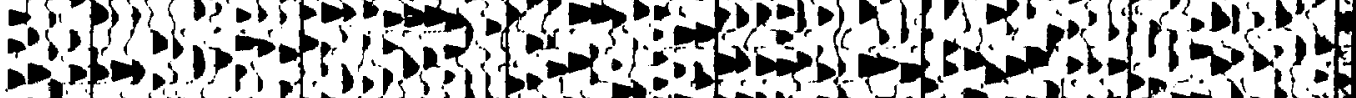

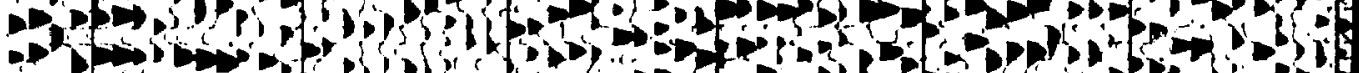

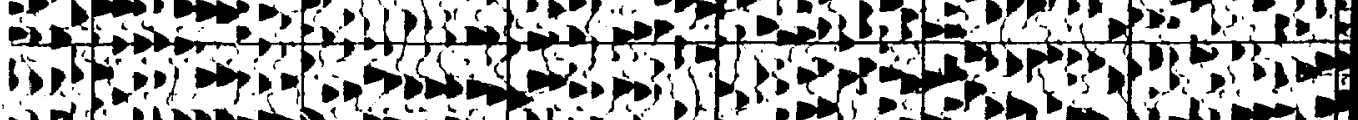

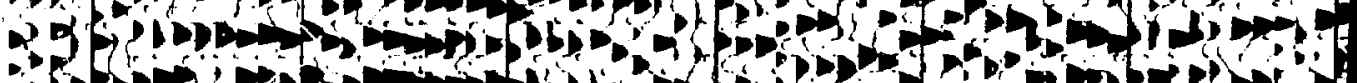
- S

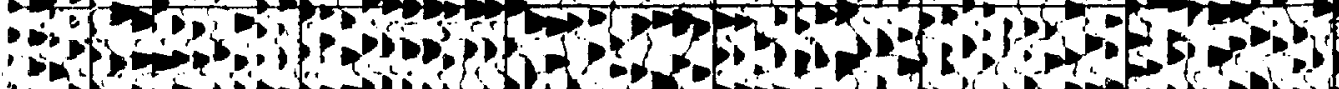

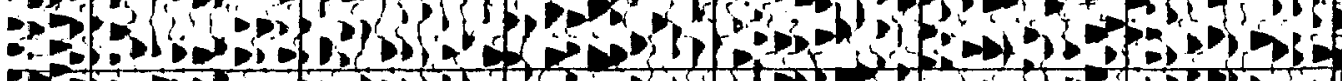

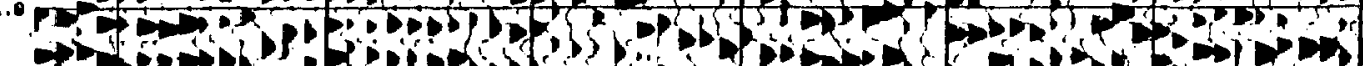

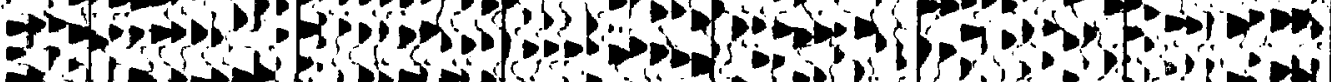

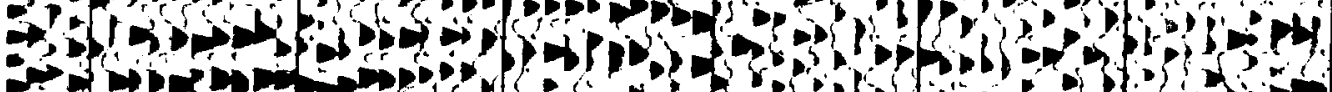
$\rightarrow 9-20$ -

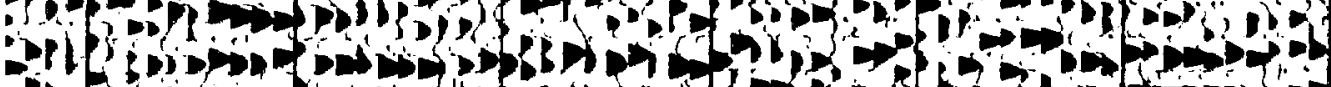

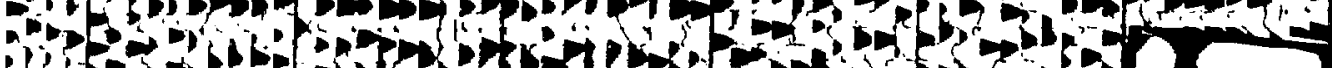

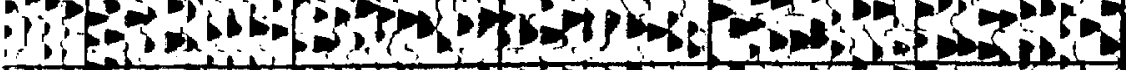

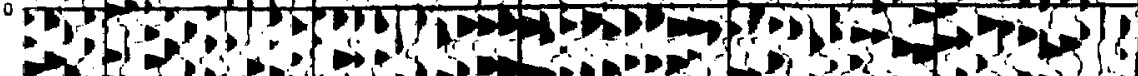

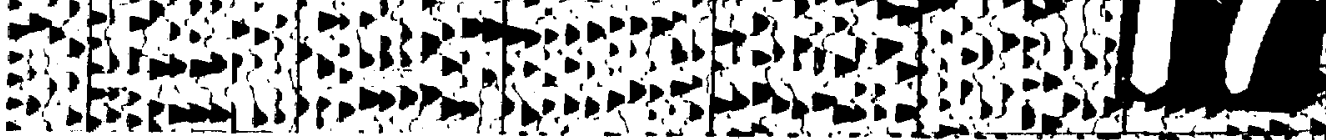




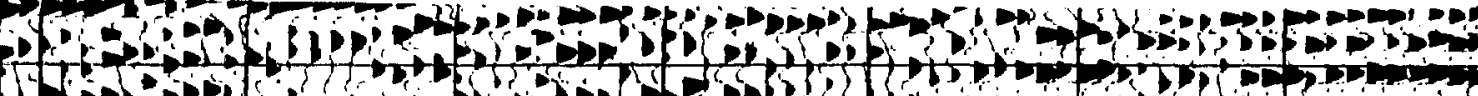

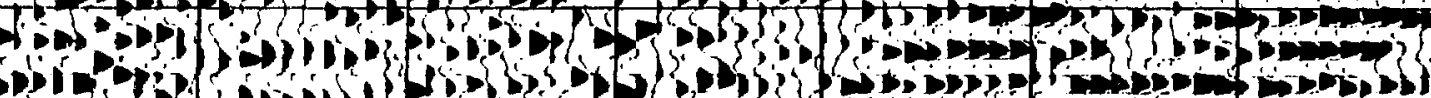

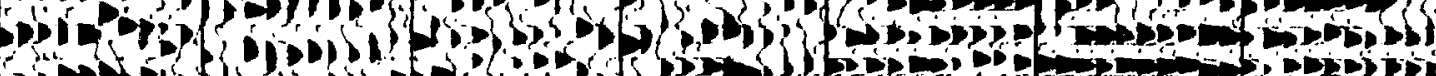

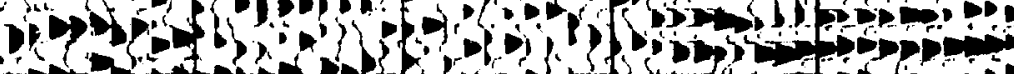

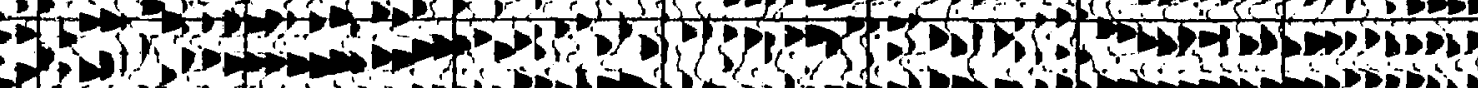
(1)

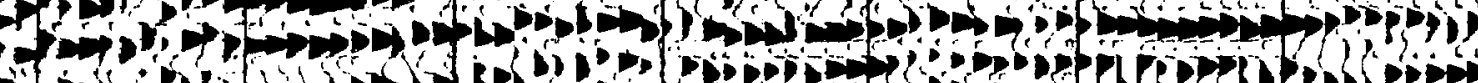

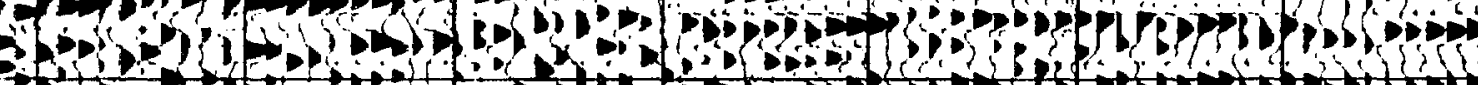
(5)

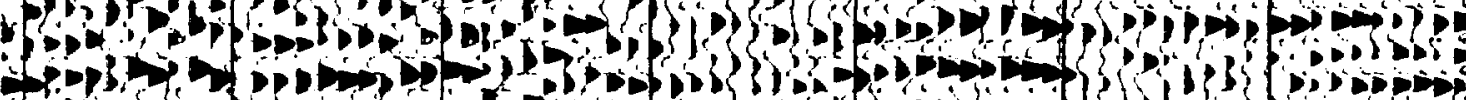

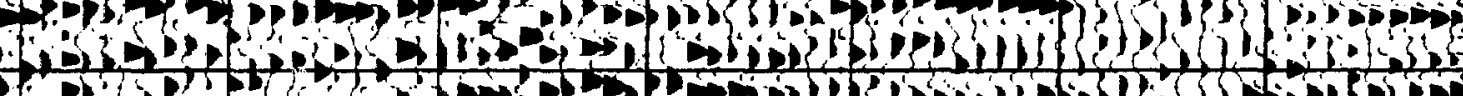

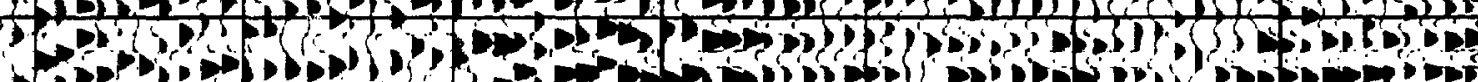

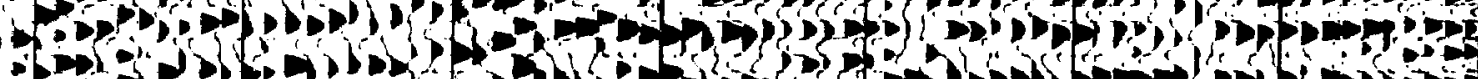

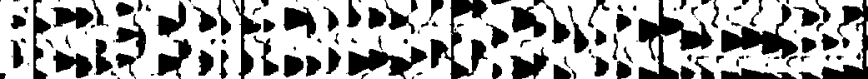

tof

$i$

$\rightarrow$

tion

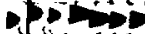

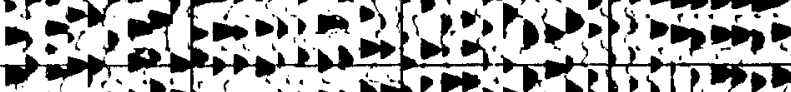
1
1
1
1.

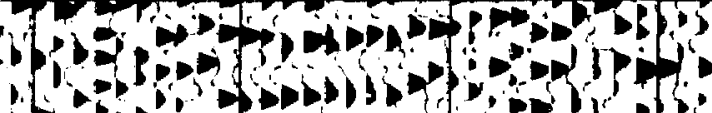

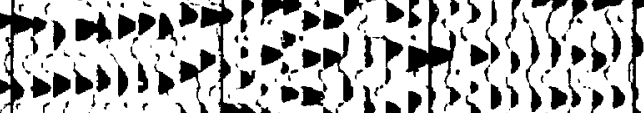
-

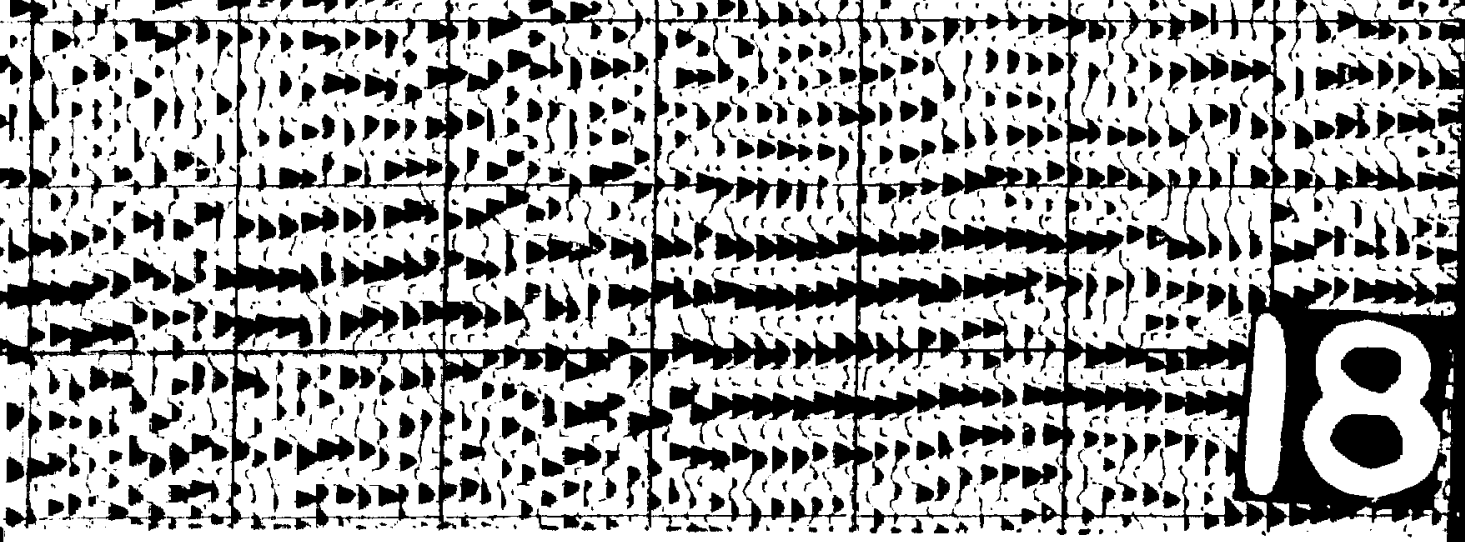




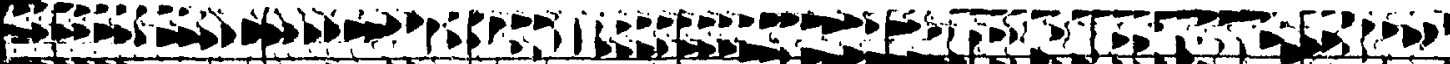
(1)

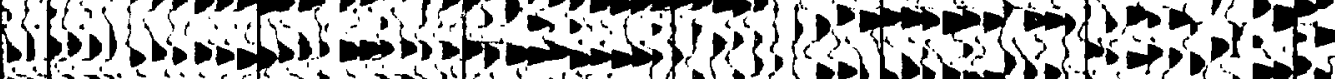

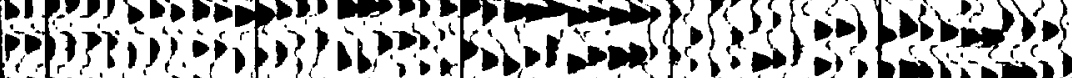

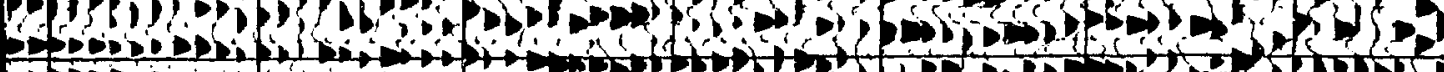

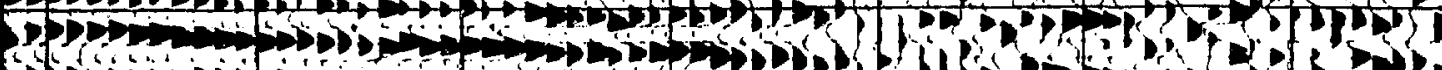

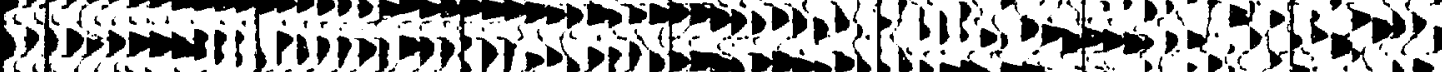

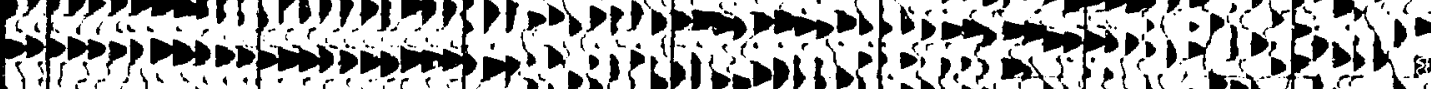

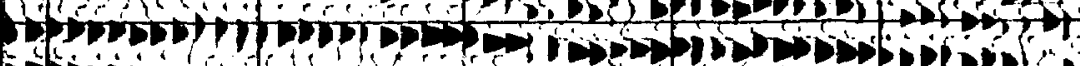

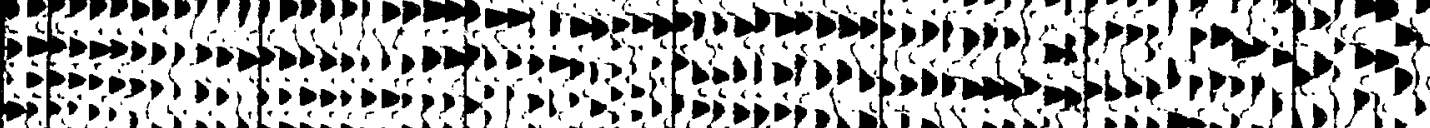

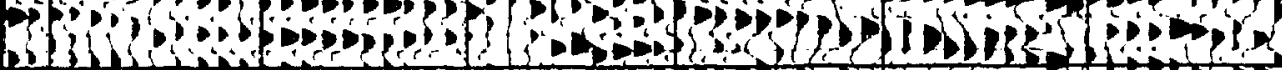
p 1 (1)

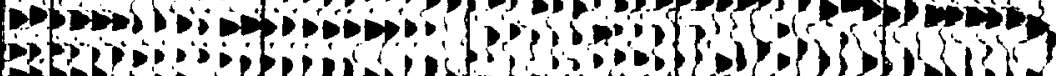

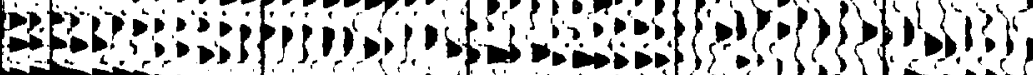

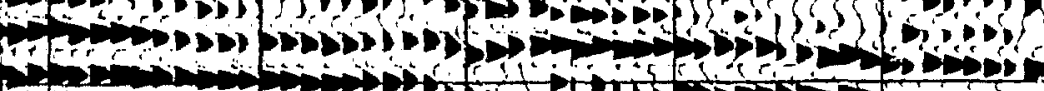

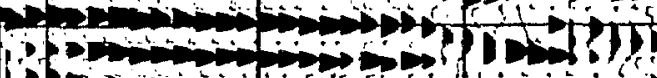

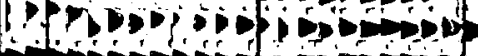

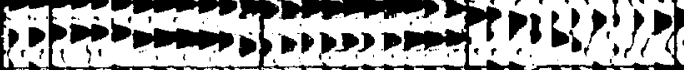

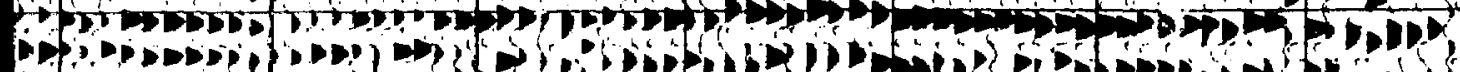
bo 3 il

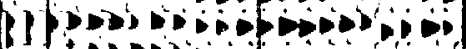
(1)

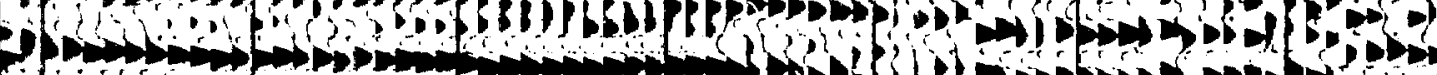

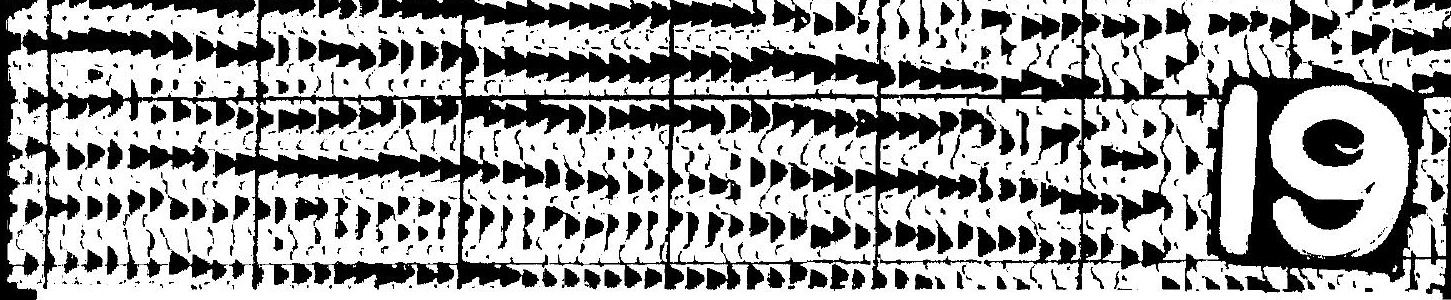




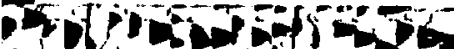

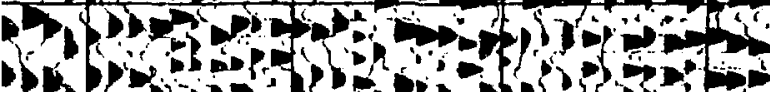

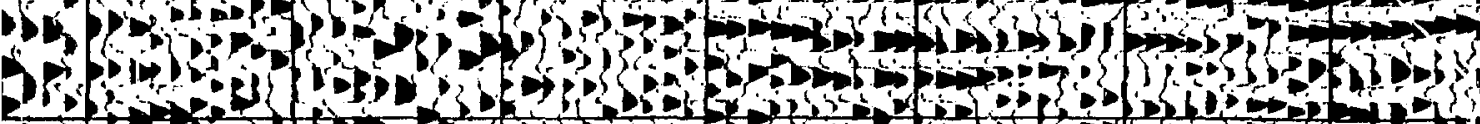

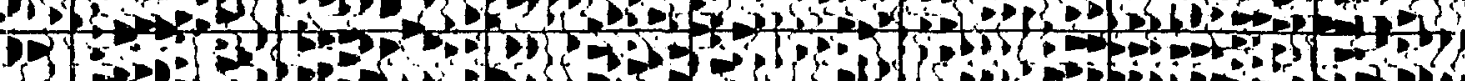

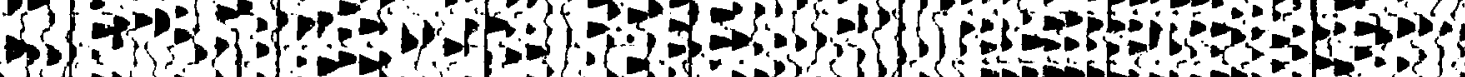

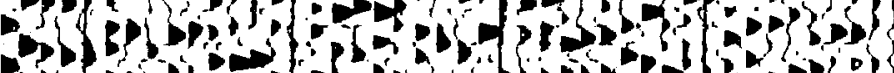

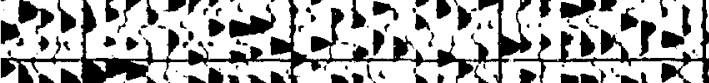

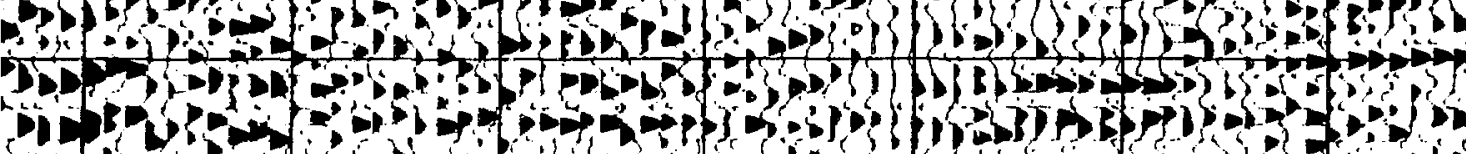

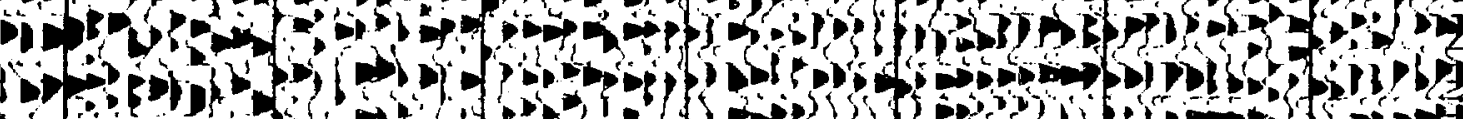

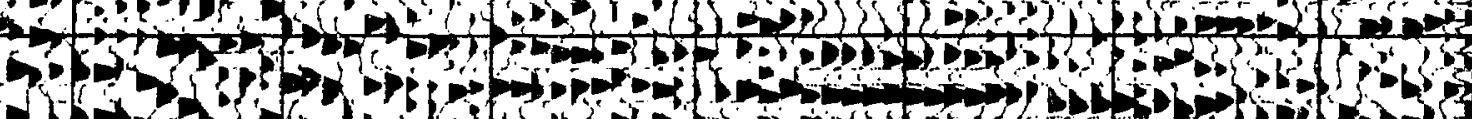

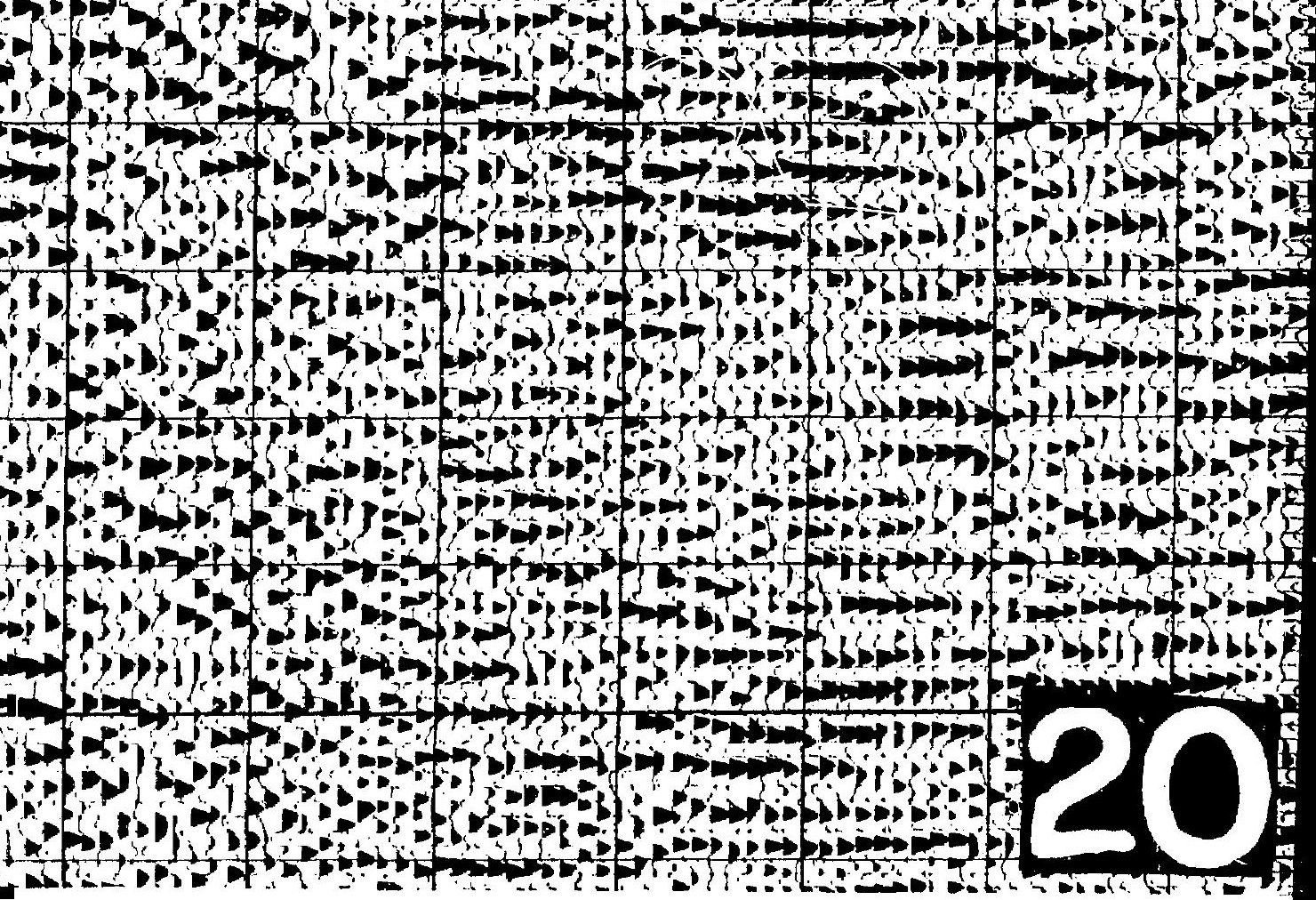




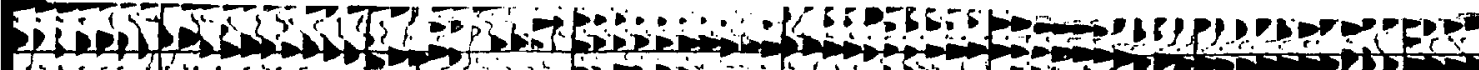

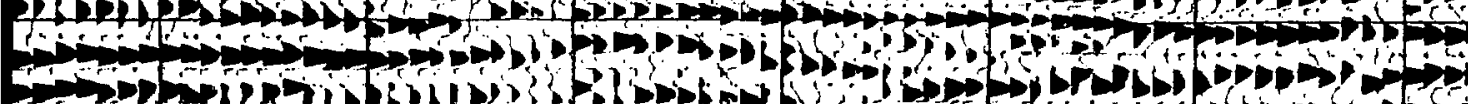
Sbot

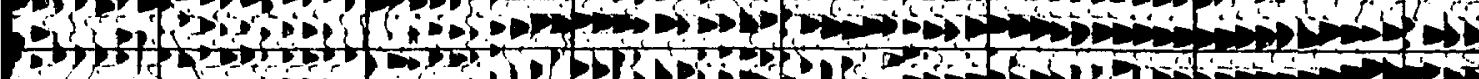

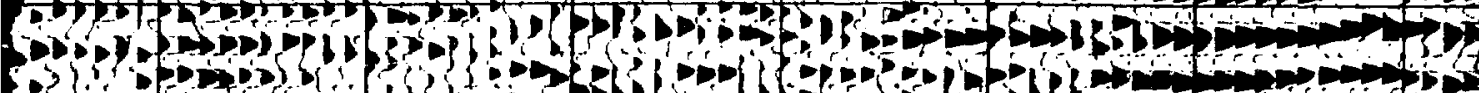

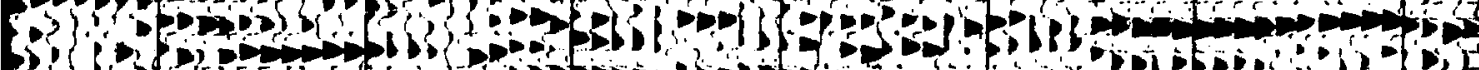

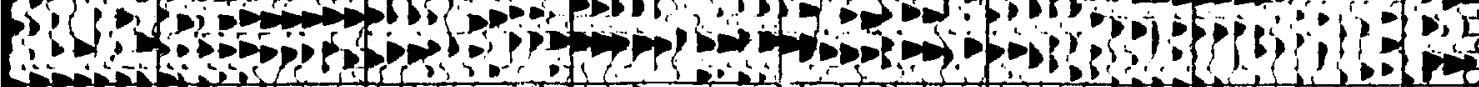

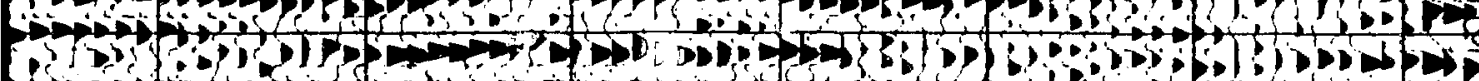

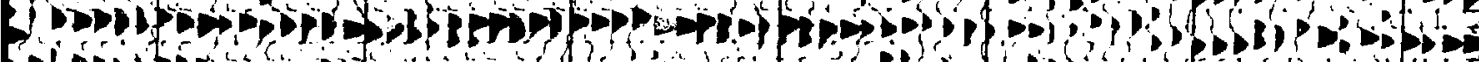

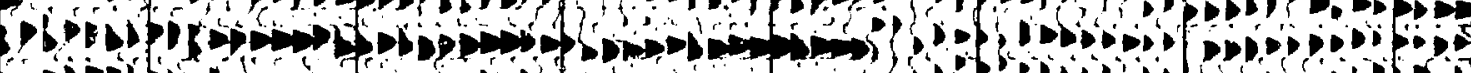

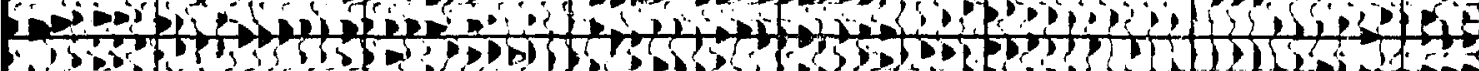
- Soj

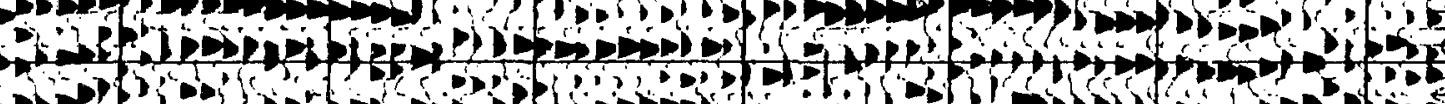

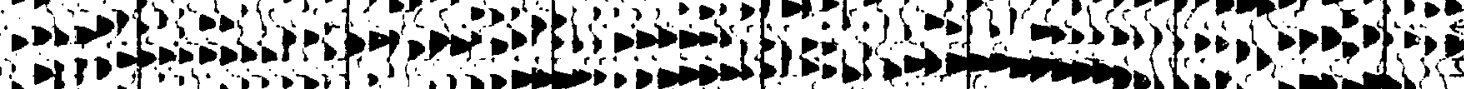

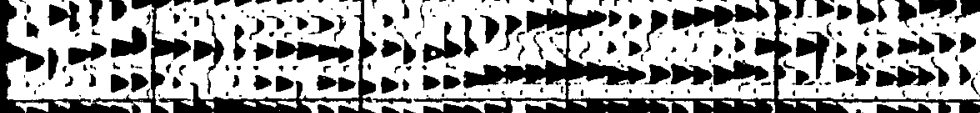

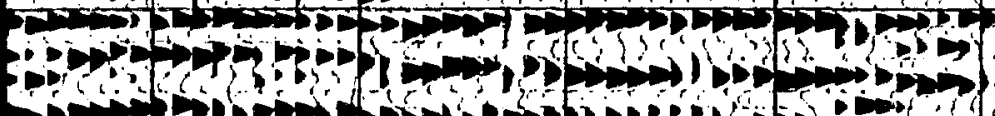

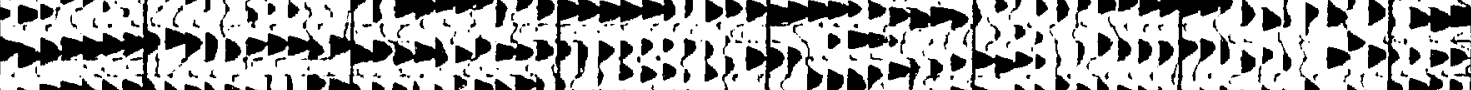

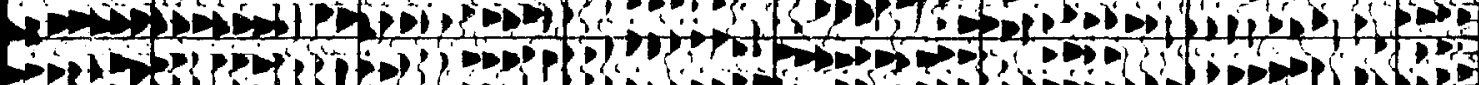

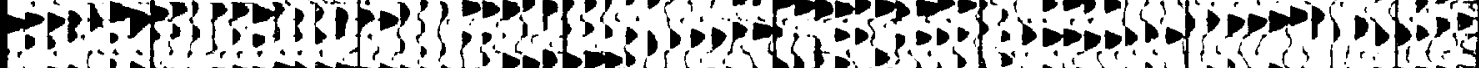
bP is

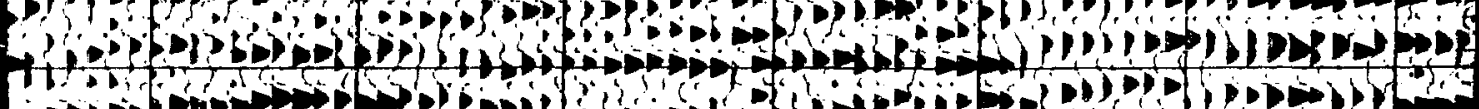

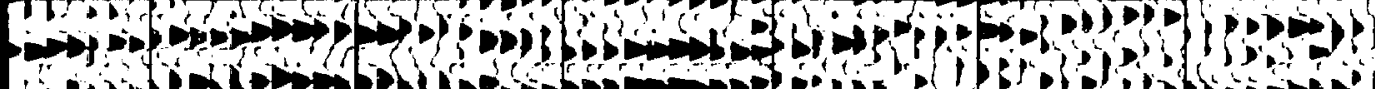

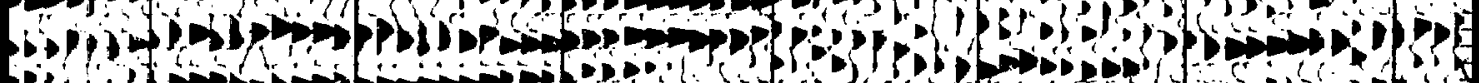

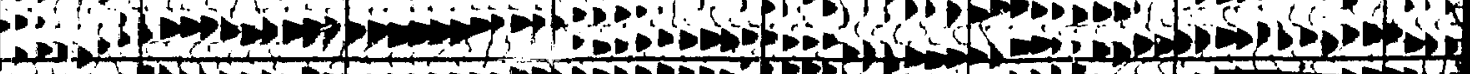

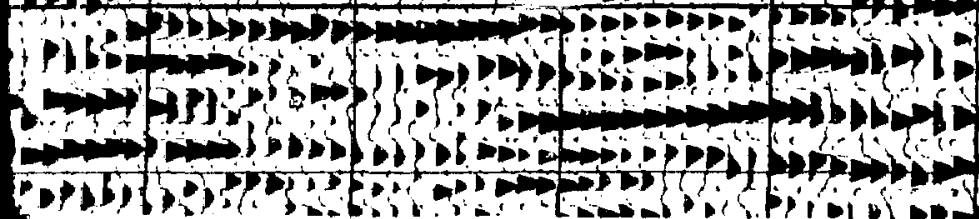




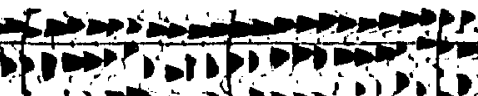

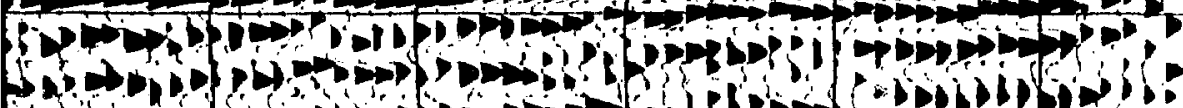

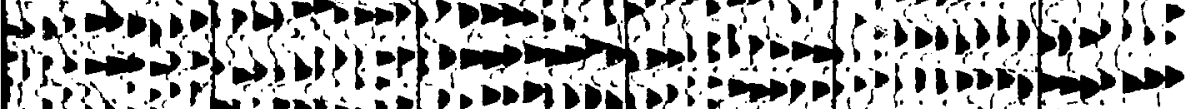
(3) 3 6) 1 of

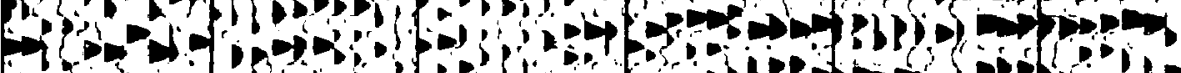

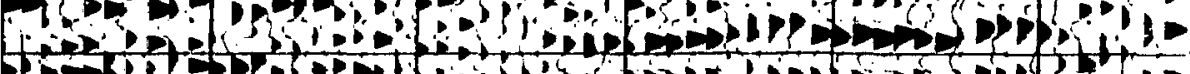

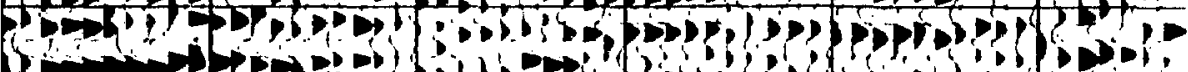

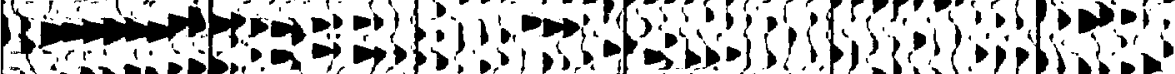

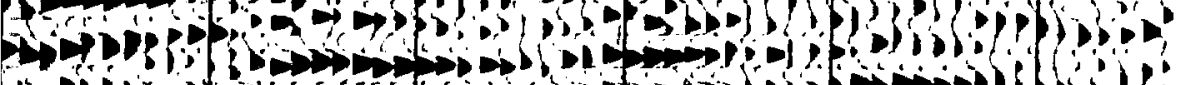
Hoph

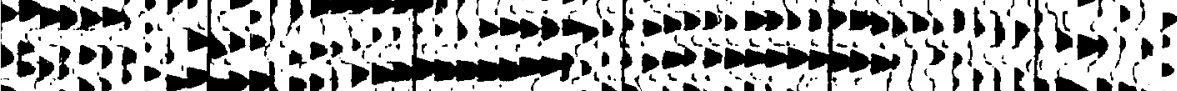

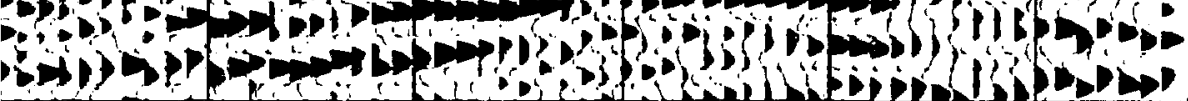

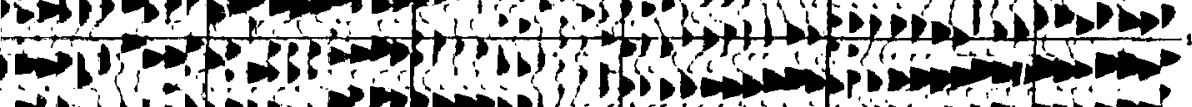

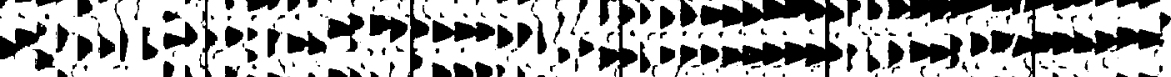

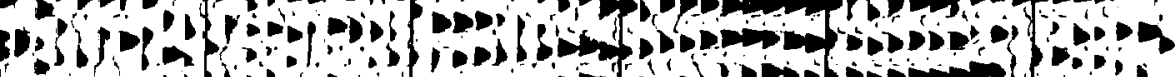

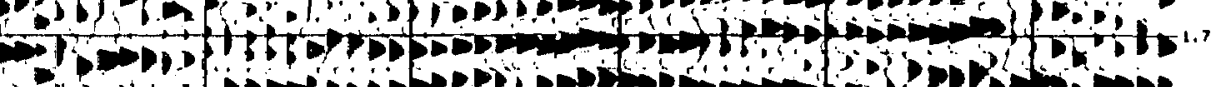
lop

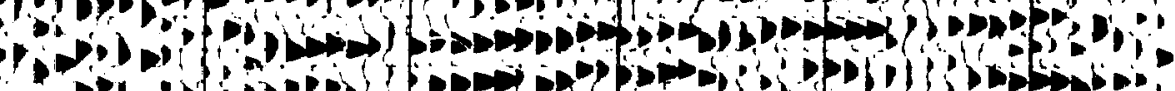

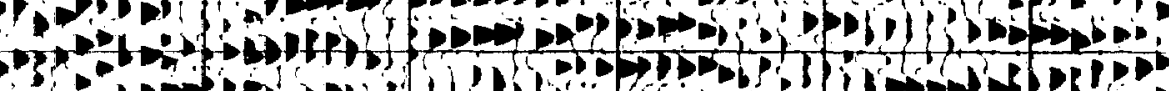
Bbl

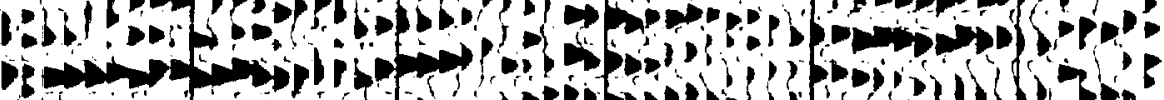

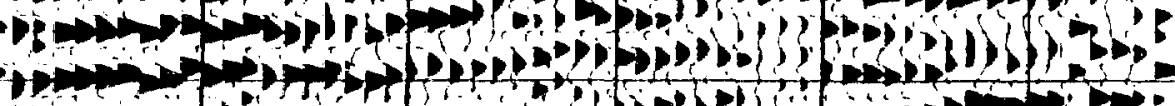

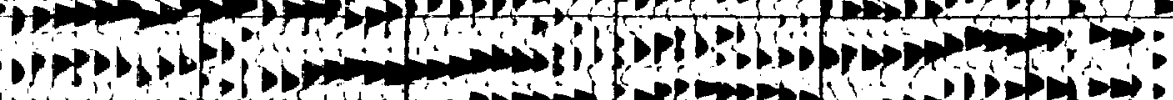

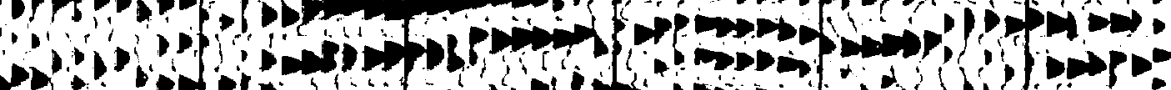

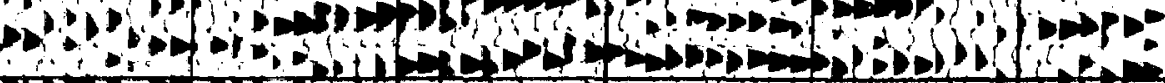

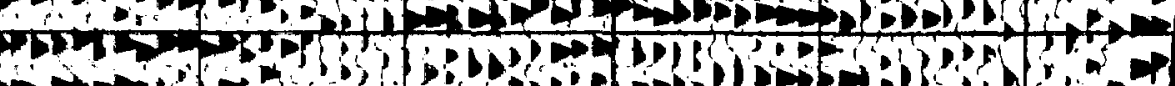
Hof

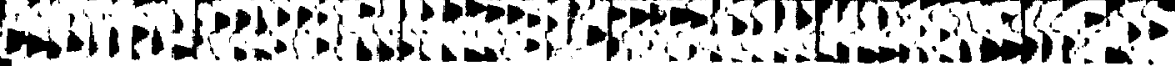


.0.63

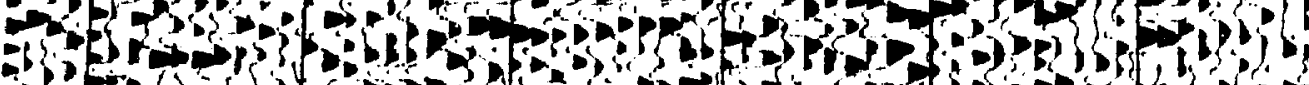

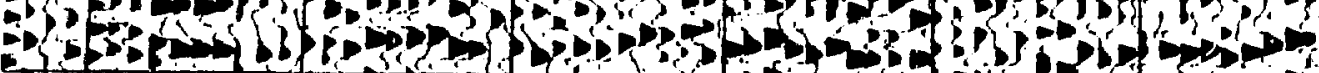

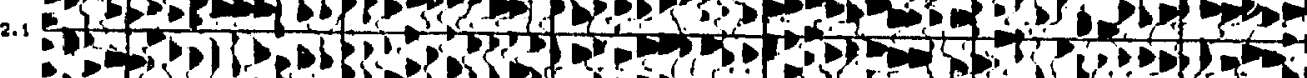

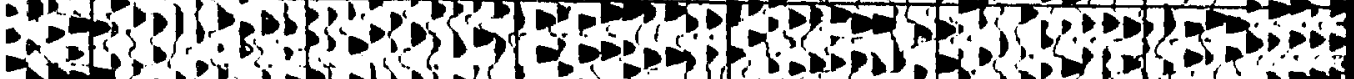

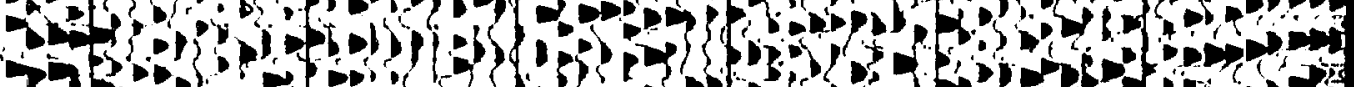
7 a

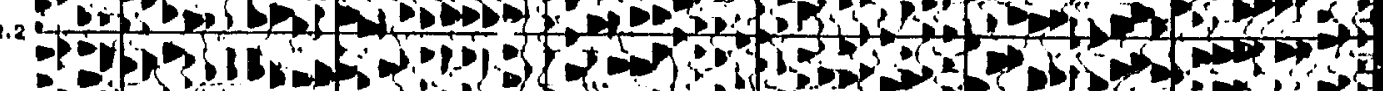

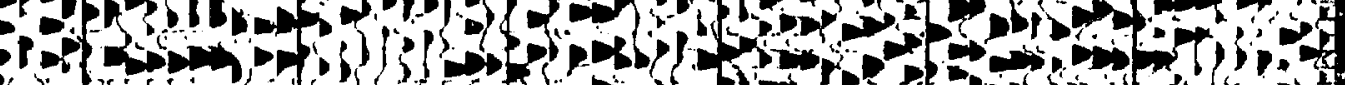

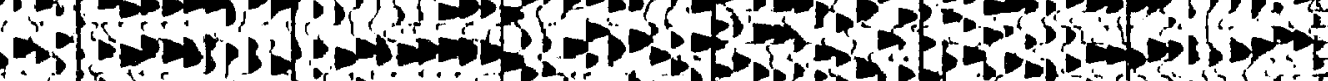

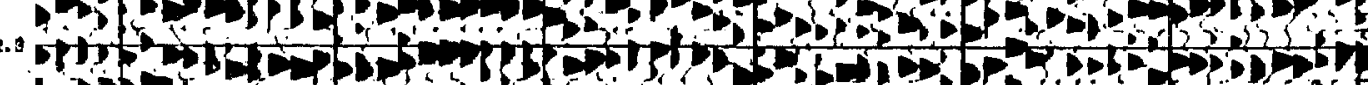

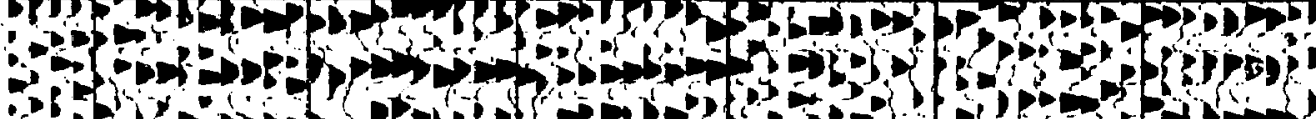

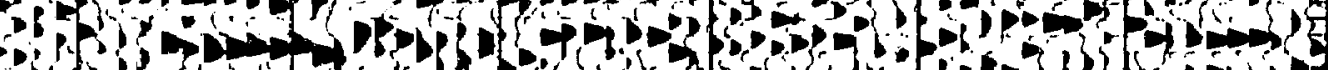

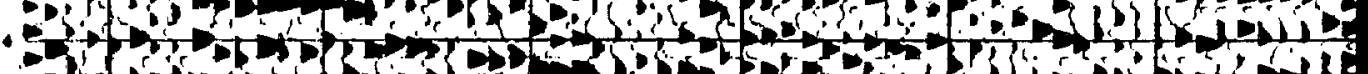

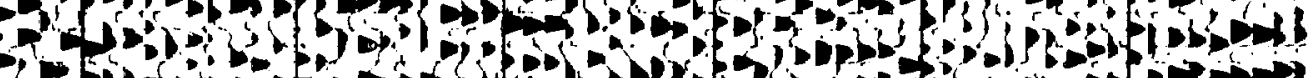

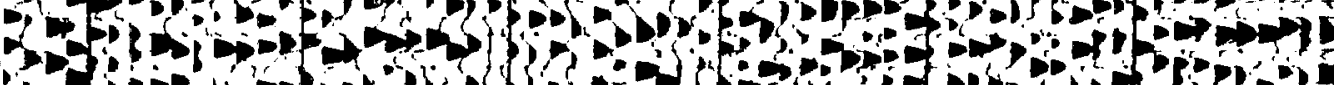

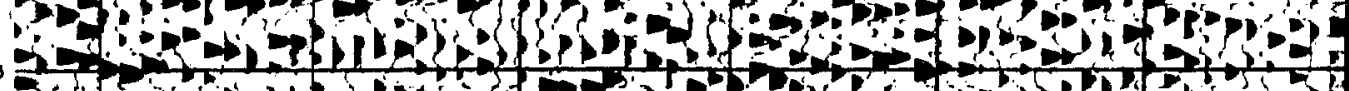

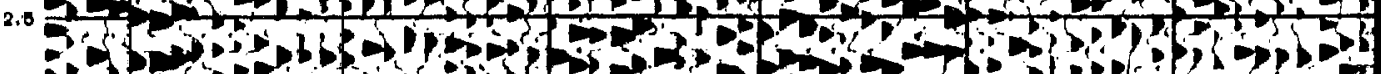

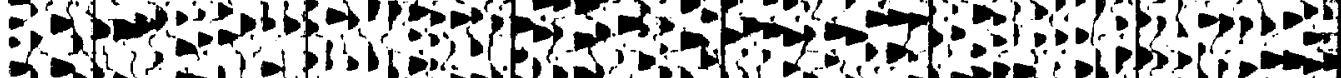

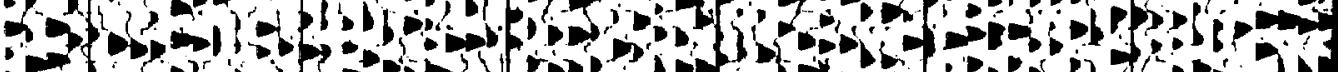

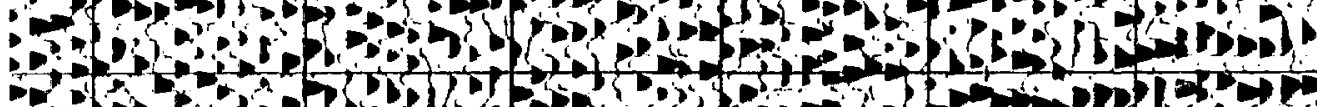

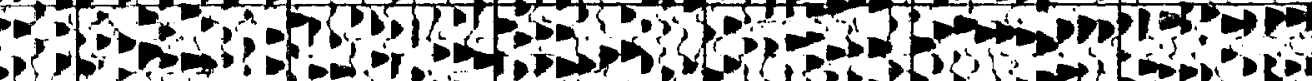

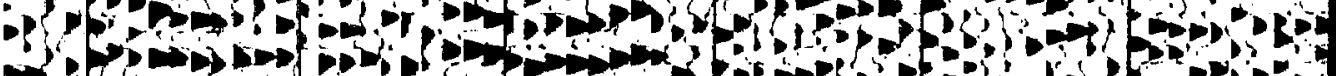

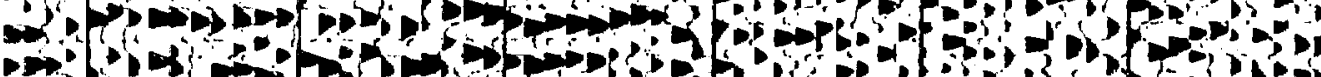
P.

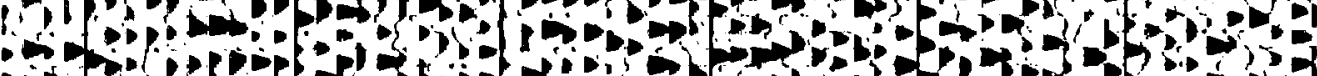

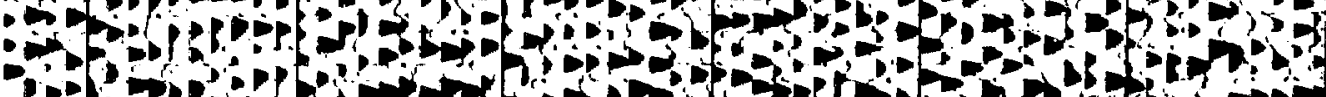

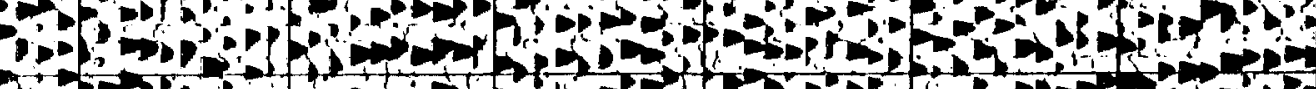

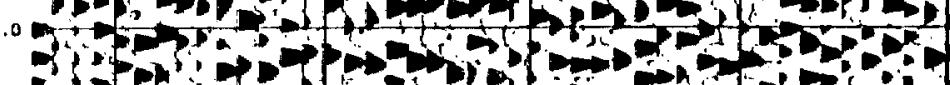

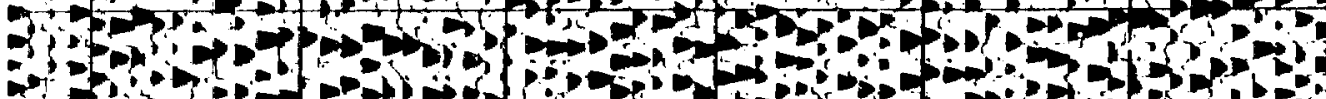

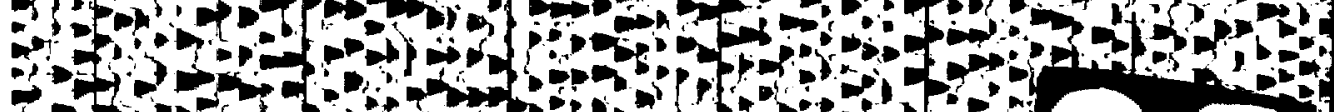

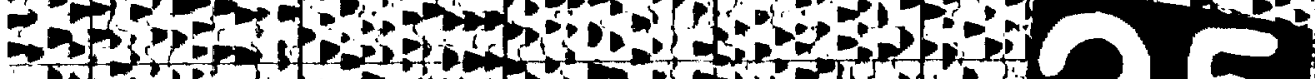

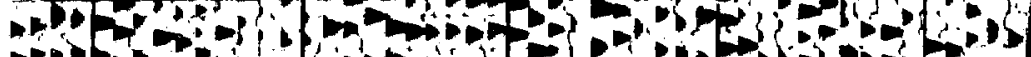

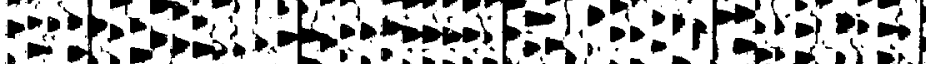
- 
mon M.

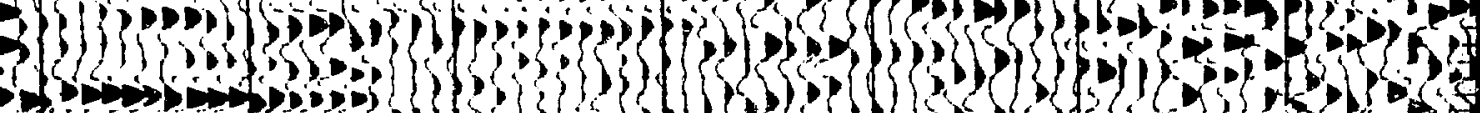

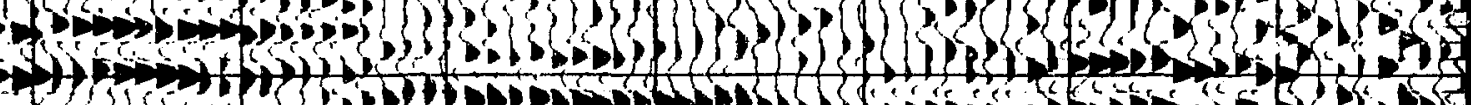
$3+$. No 9.5.5.5.

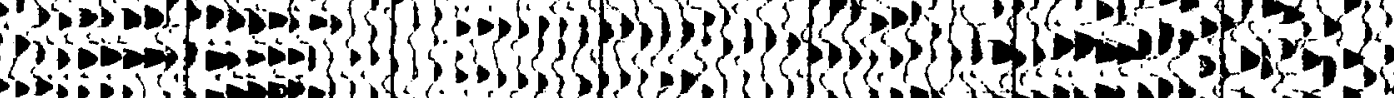

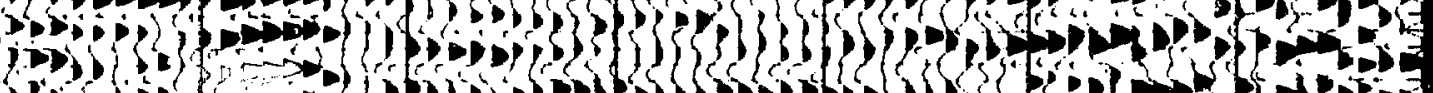
( 17 .

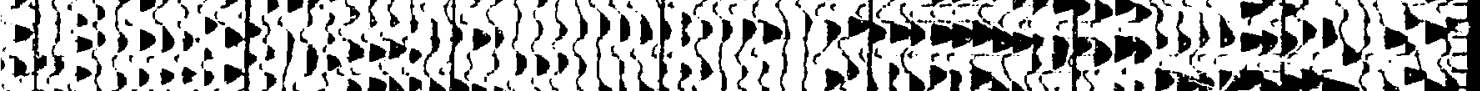
15)

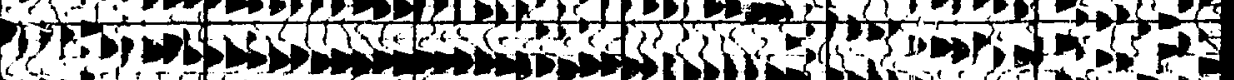

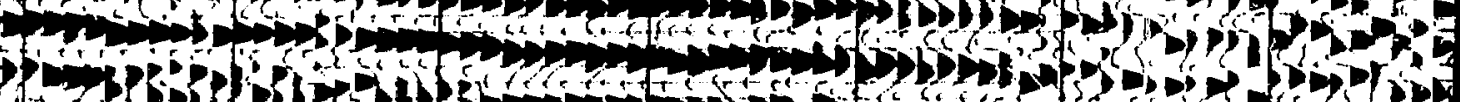

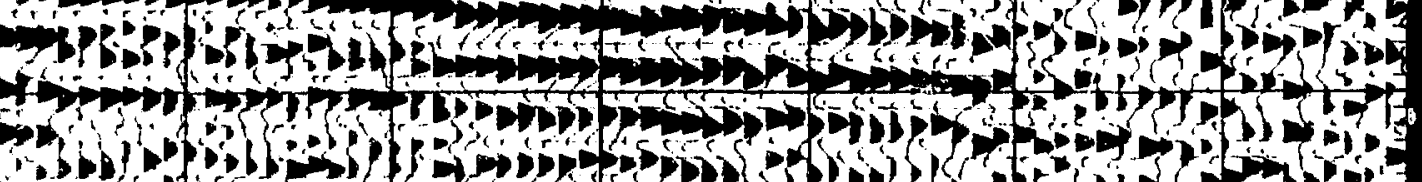

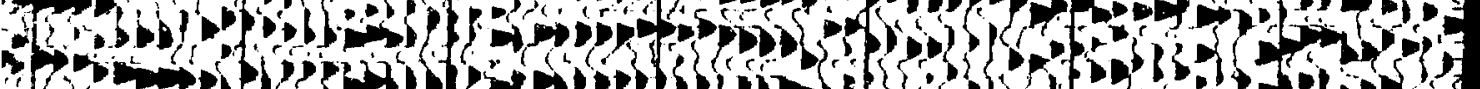

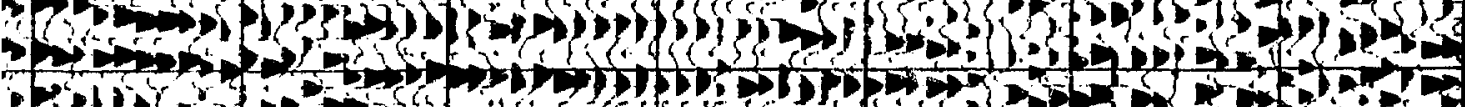
- Hof

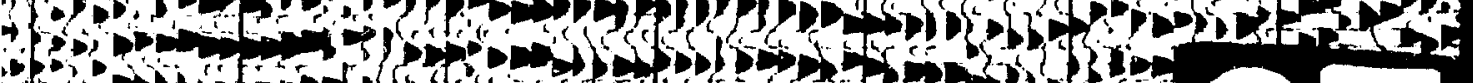

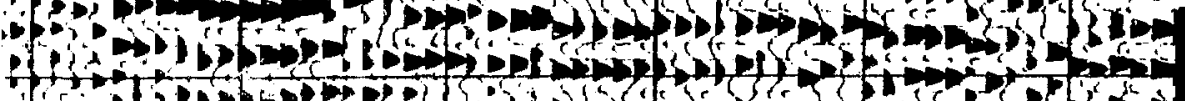

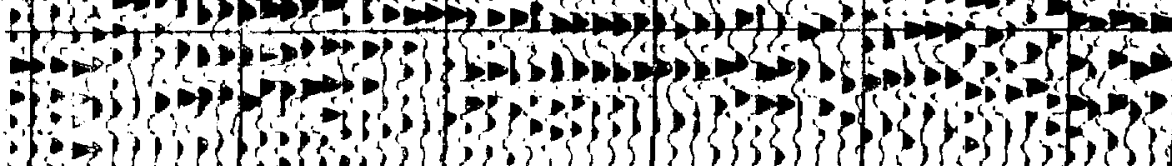




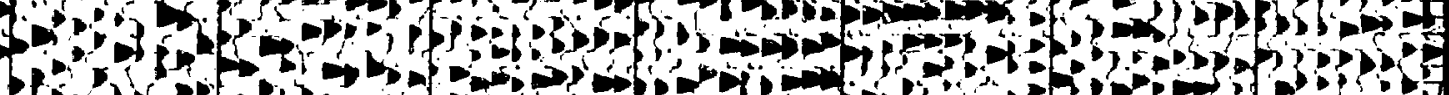

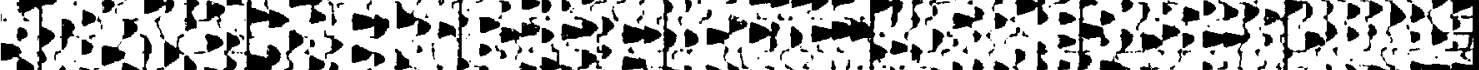
Q 10 of

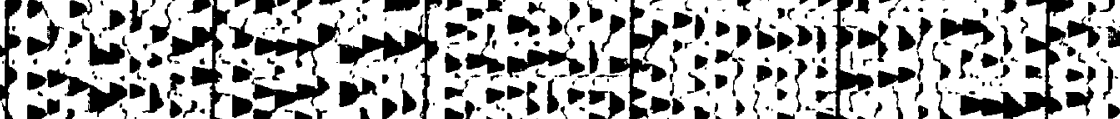
$\rightarrow$

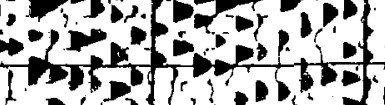

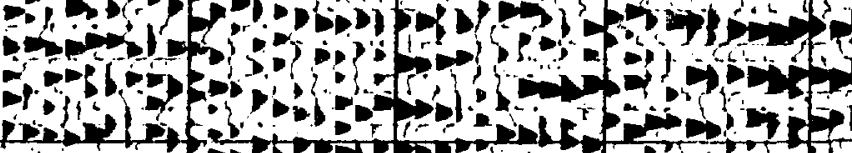

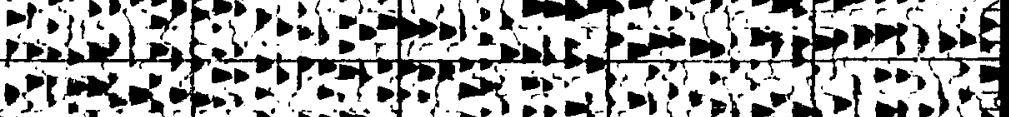

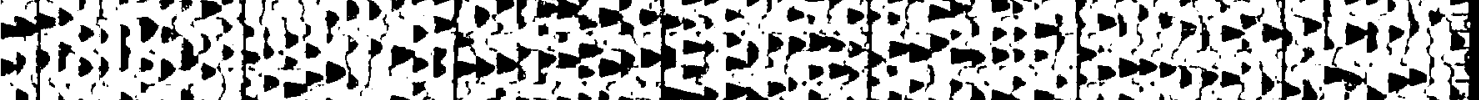
iojes

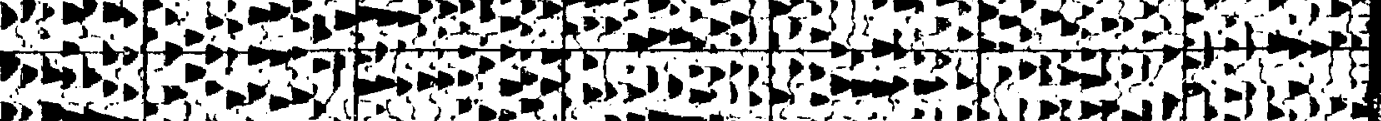

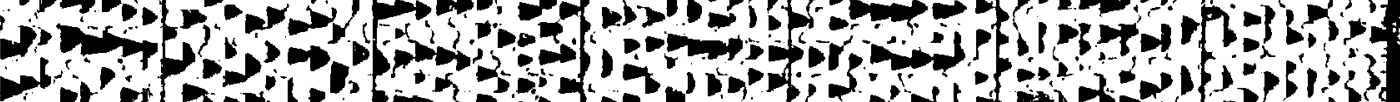

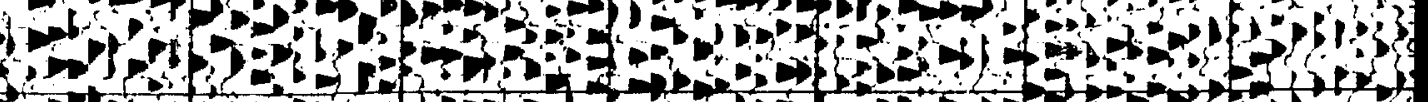

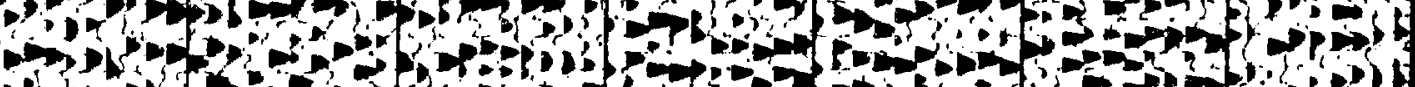

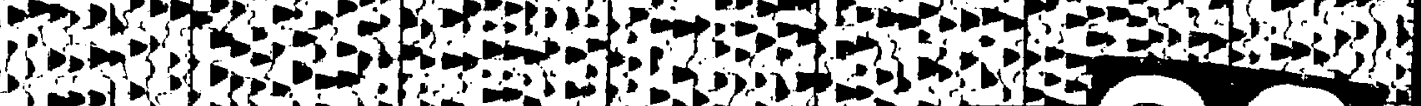

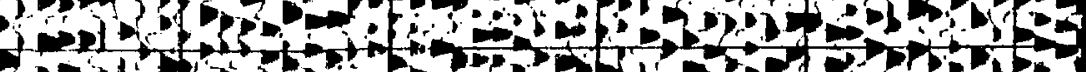

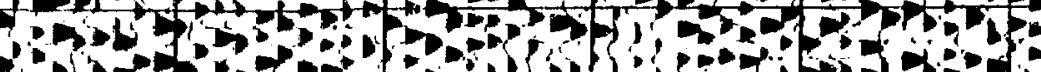

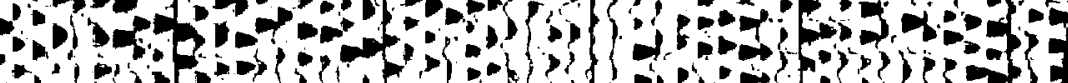

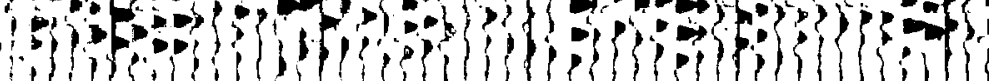


(3in) (7) D.t.

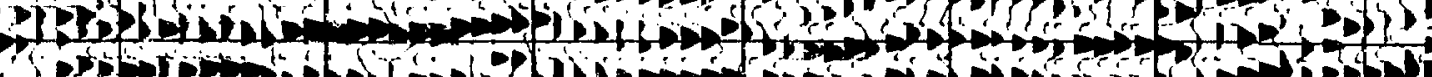
not

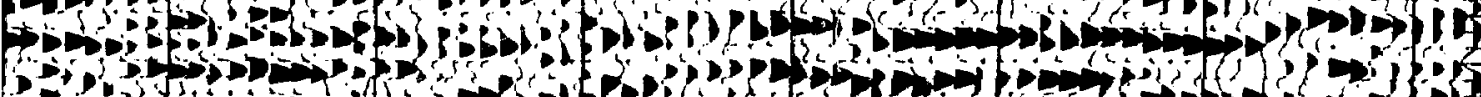
P 1

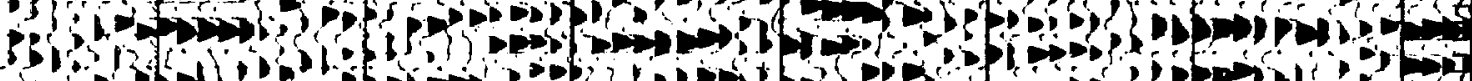

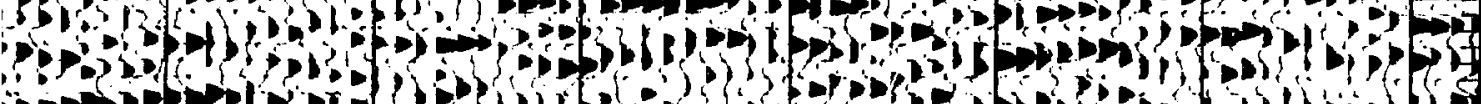

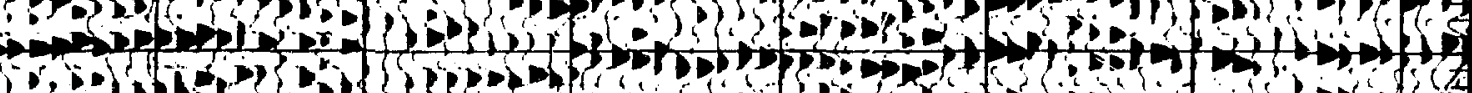

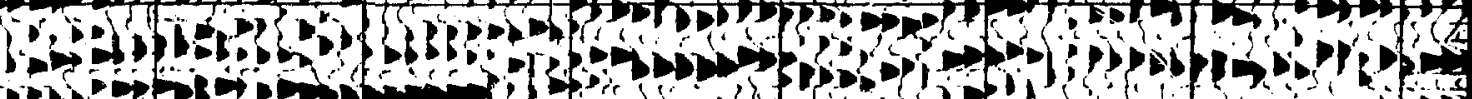

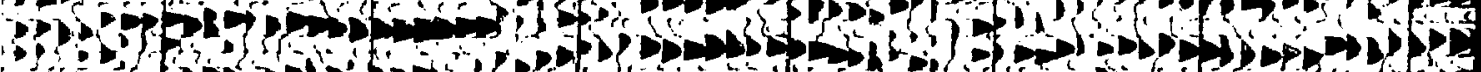

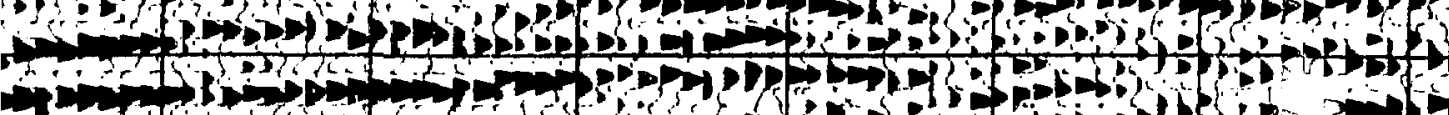

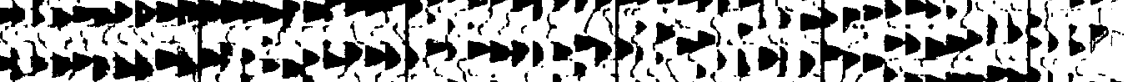

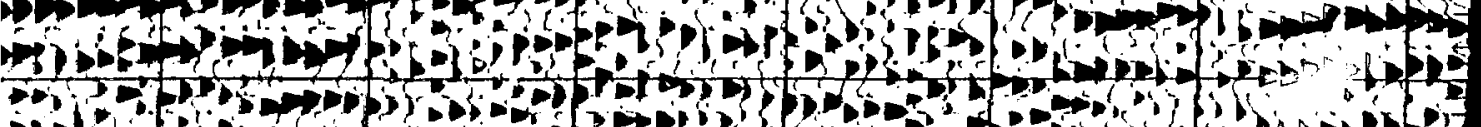

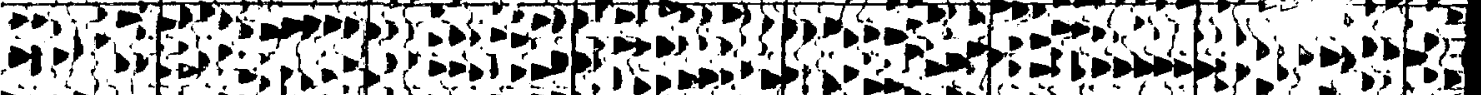

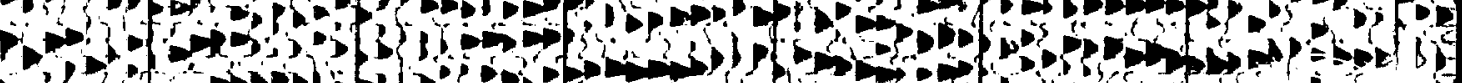

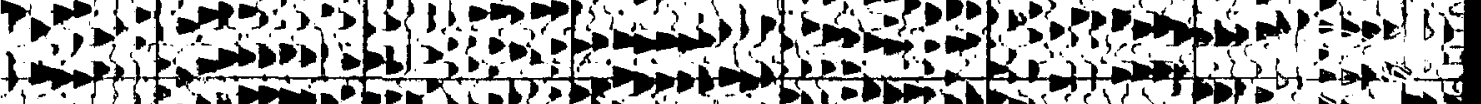

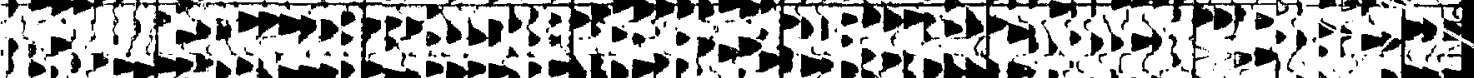

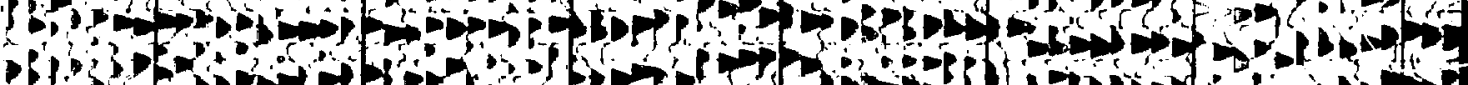

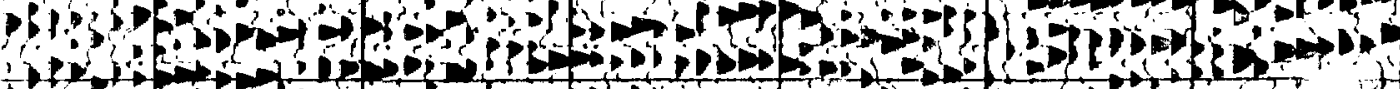

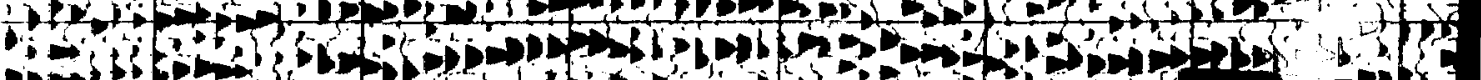

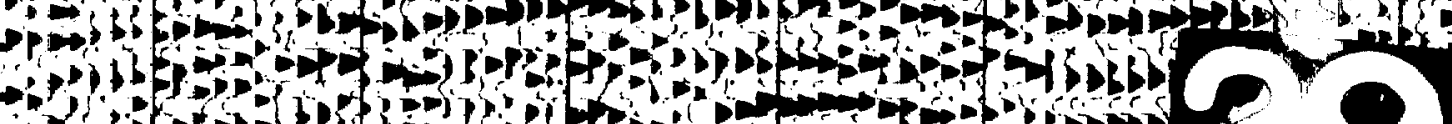

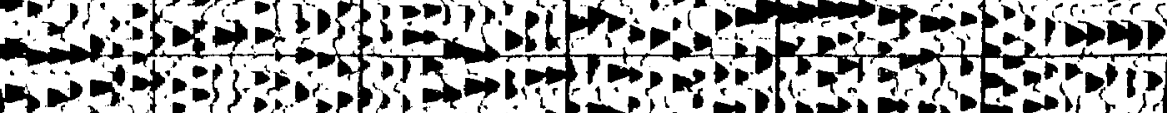

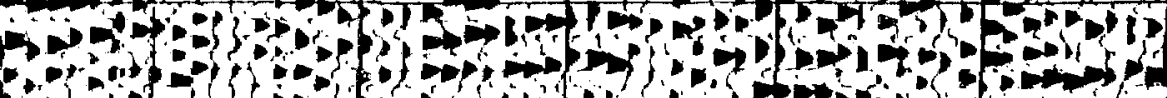




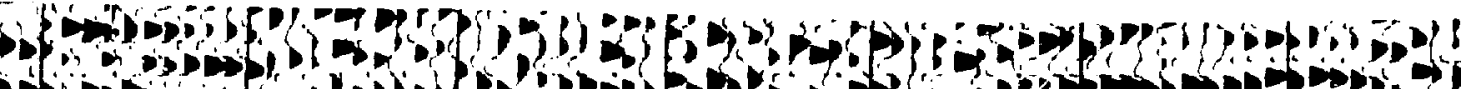
non

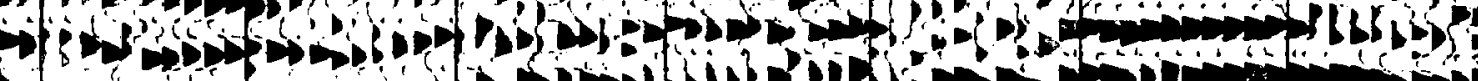

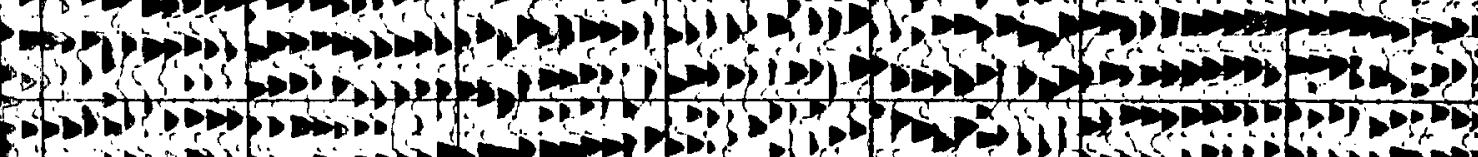

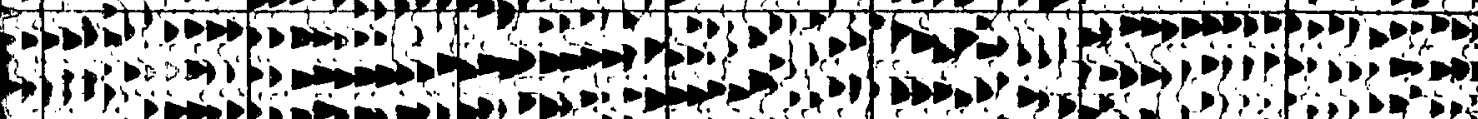
130 -

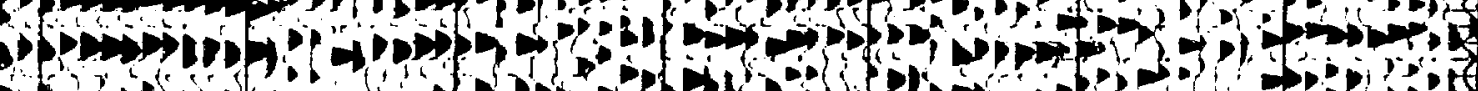

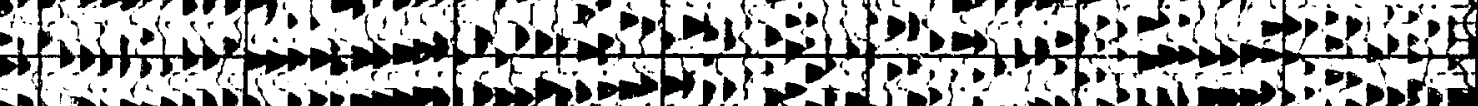

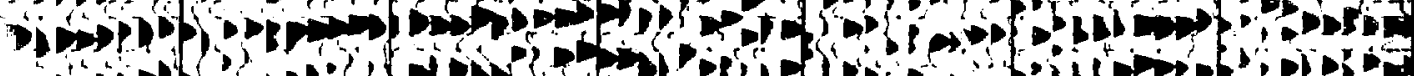

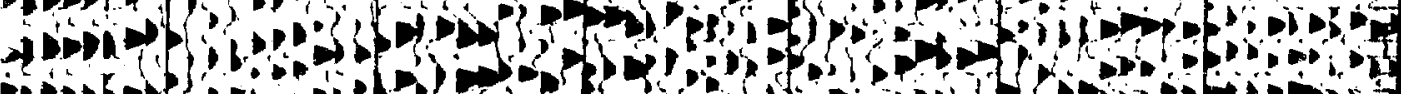

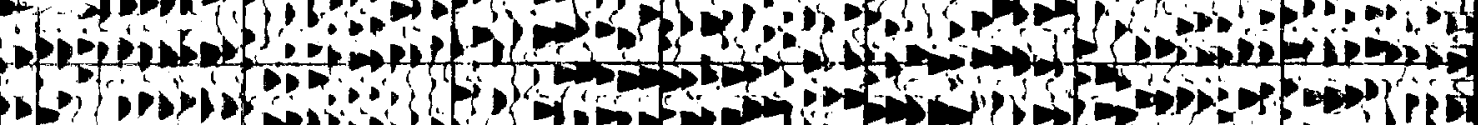

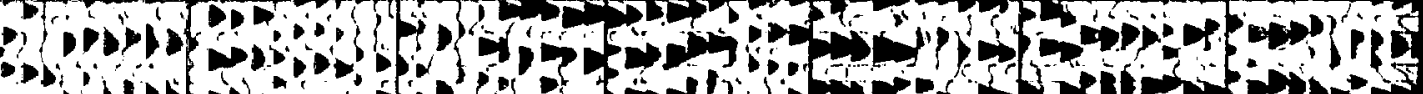

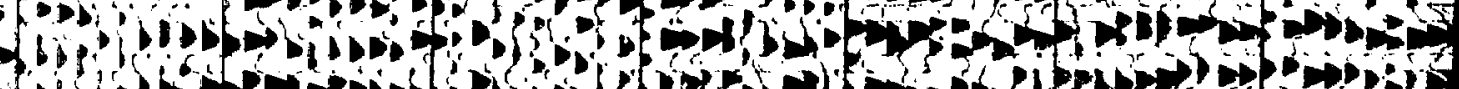

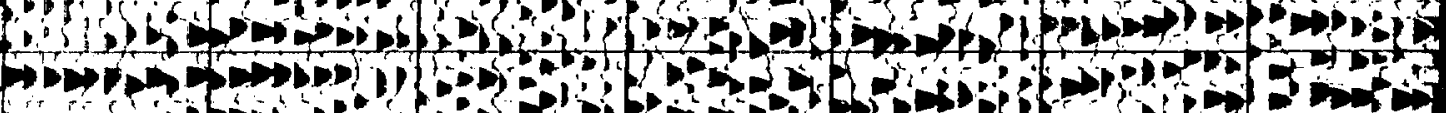

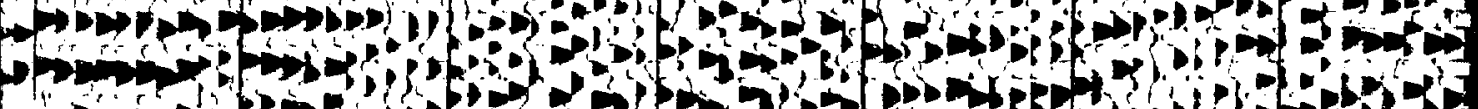

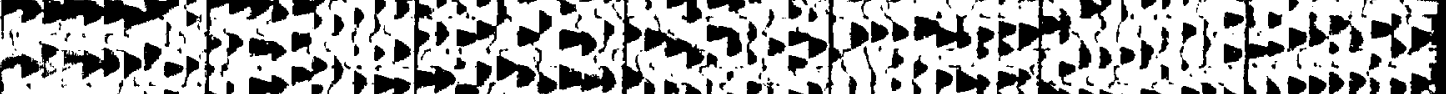
10.

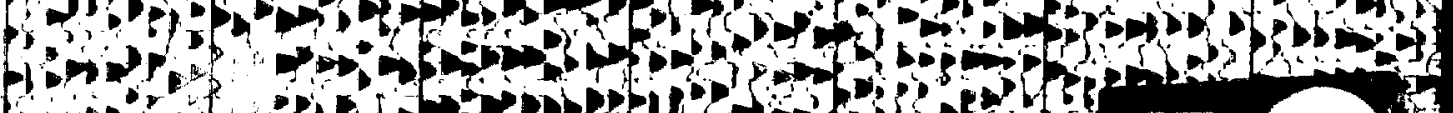

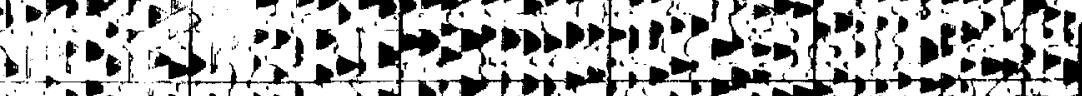

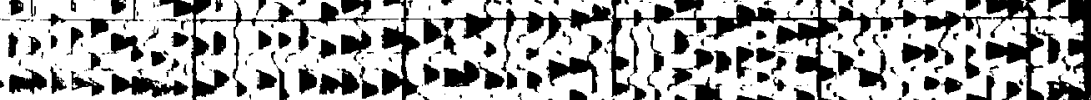

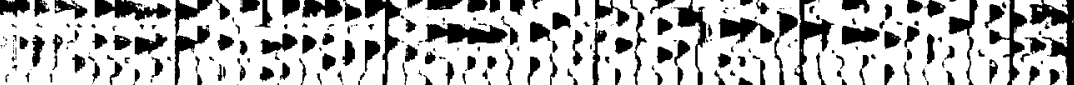




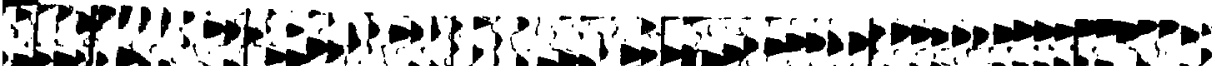
4 S -

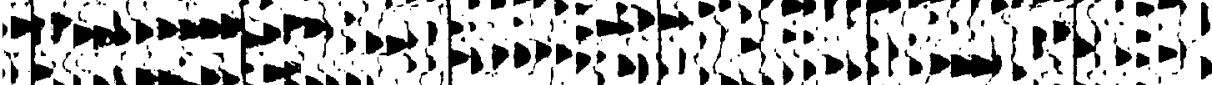

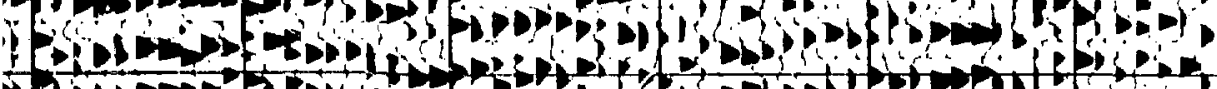

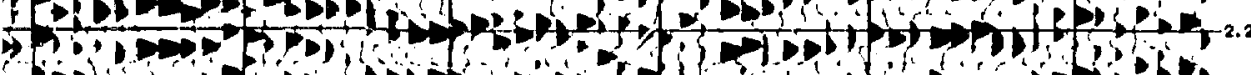
Nobis bos

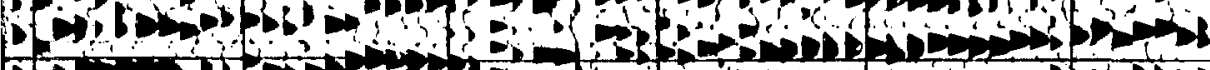
$4 P$ P

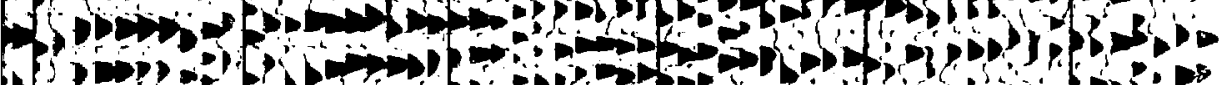

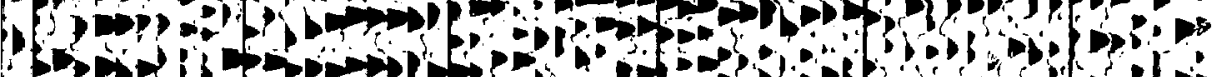

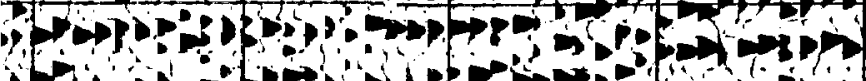

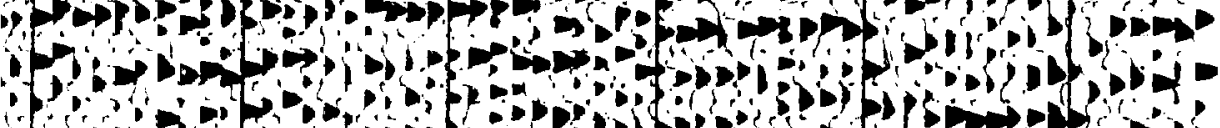

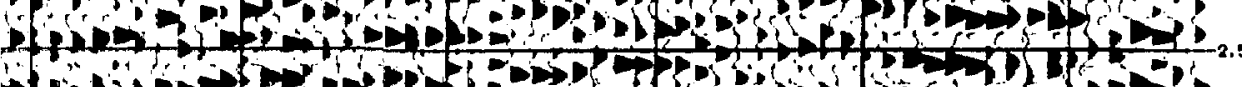

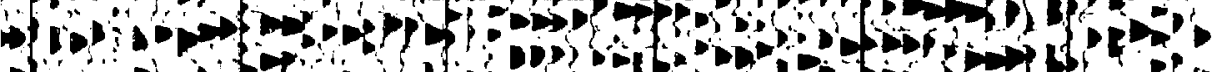

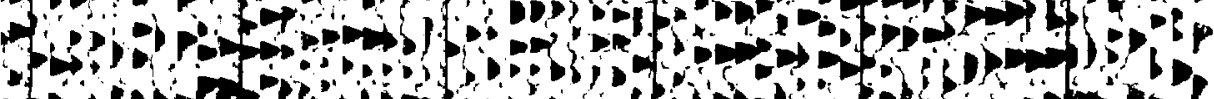

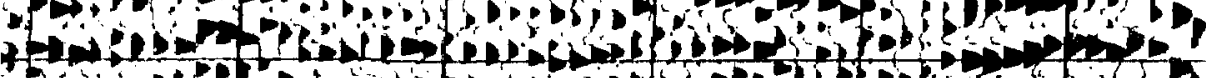

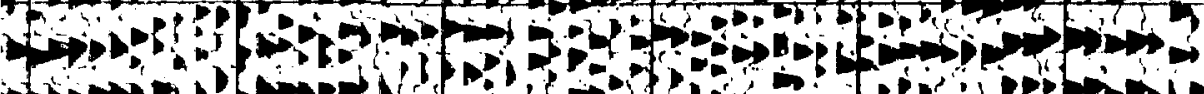

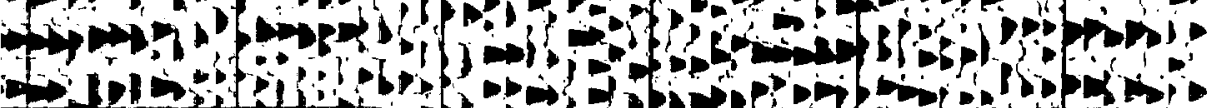

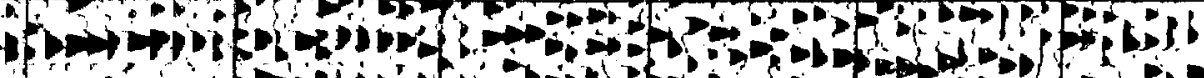

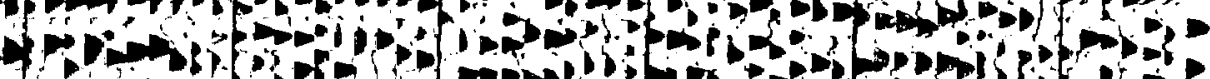

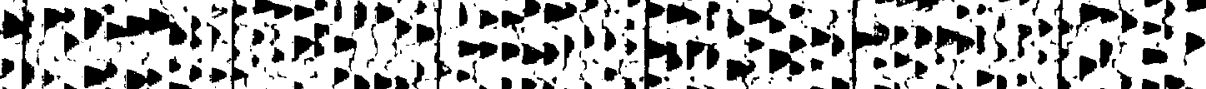

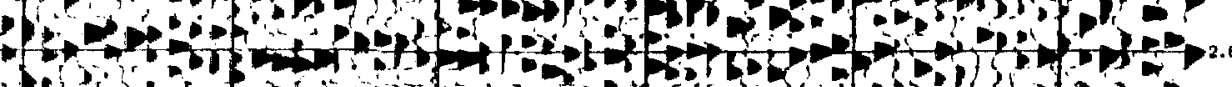
S

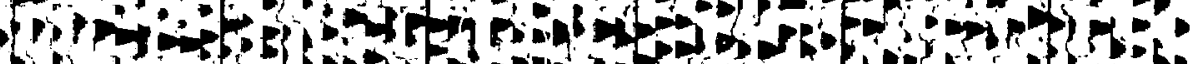

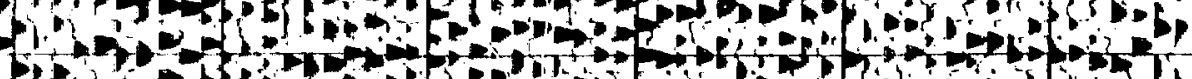

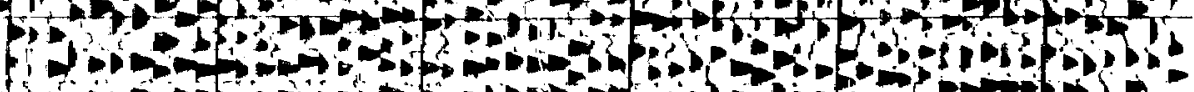

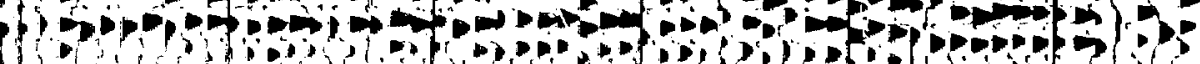

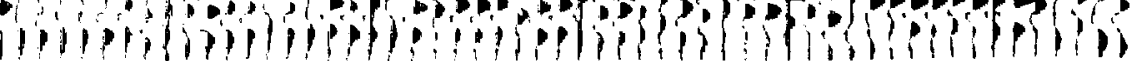

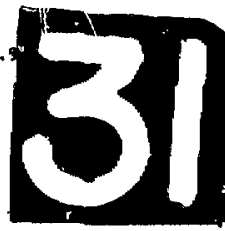




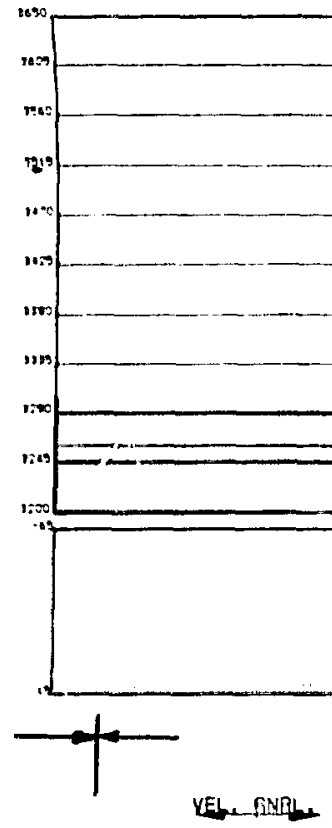

cuthe: I
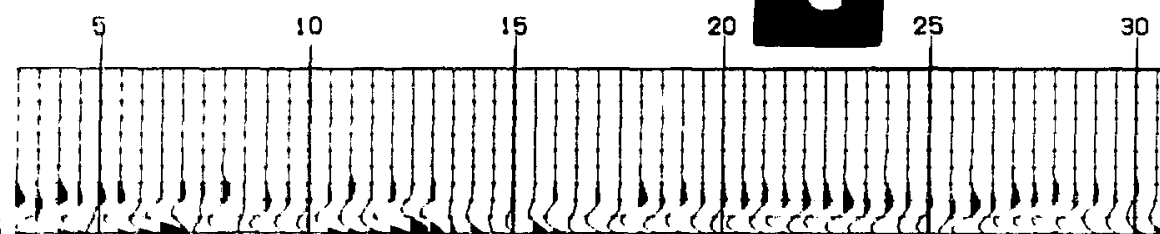

$\sec \quad 12$

VELL. PNALL

ง.i

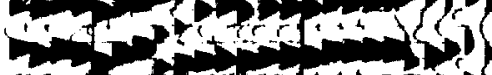

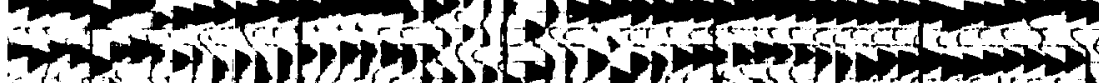

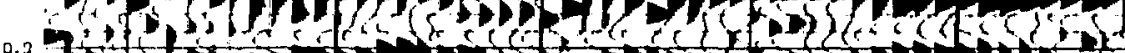

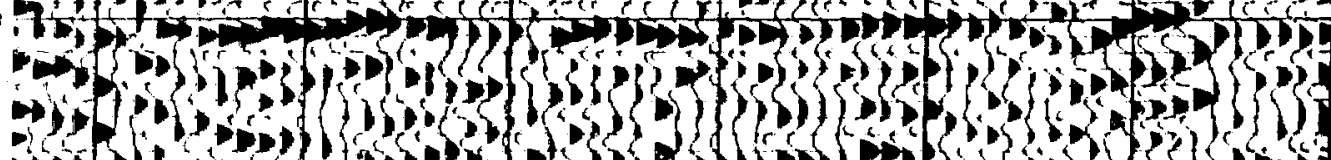

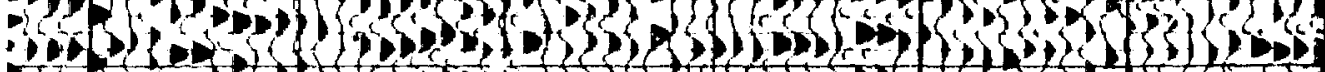
1)

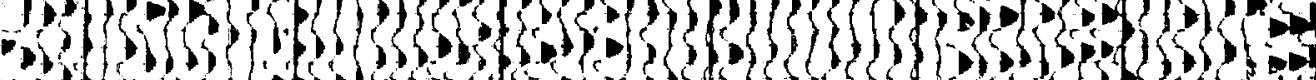

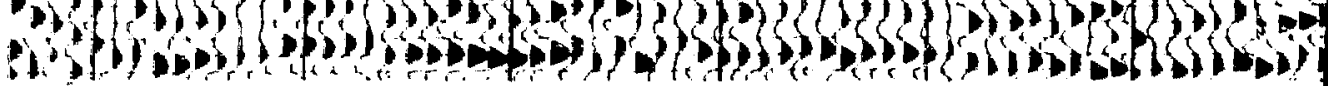



1) phe.

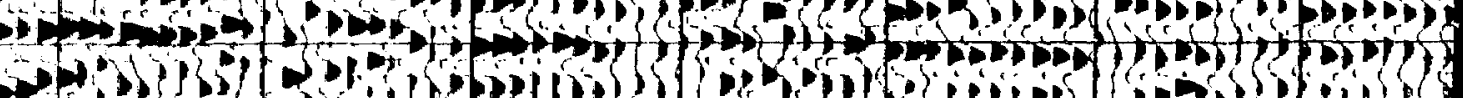
-

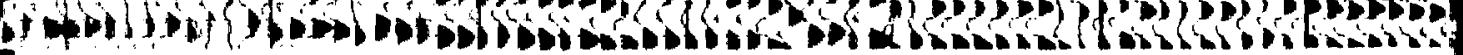




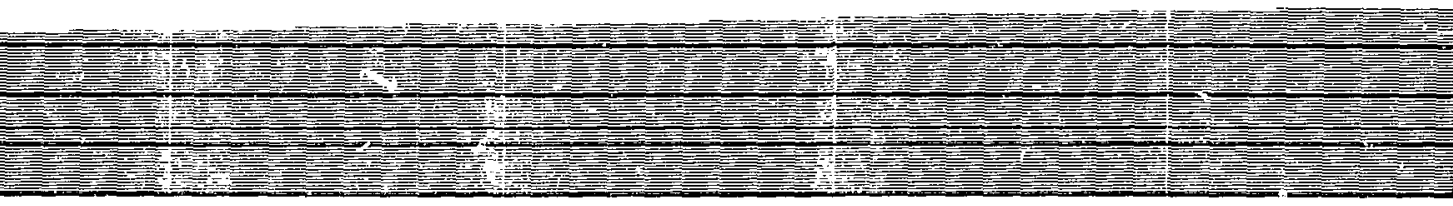

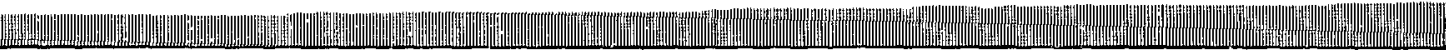

sec 7

YELL, PNALY

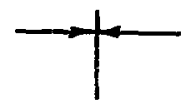

70

80

75
85

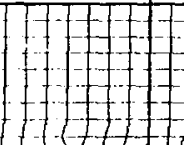

90

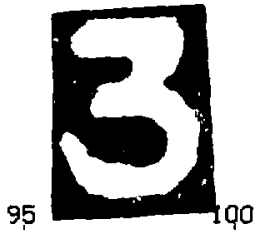

sec

Yatal

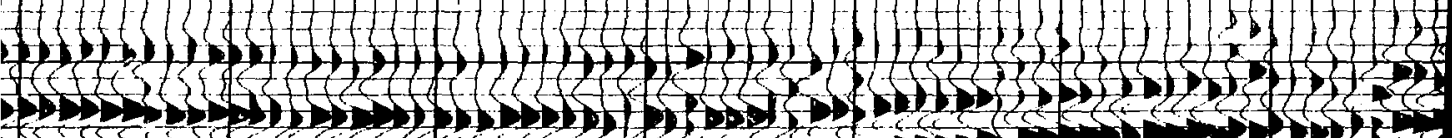

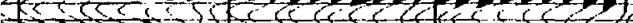

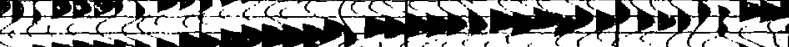

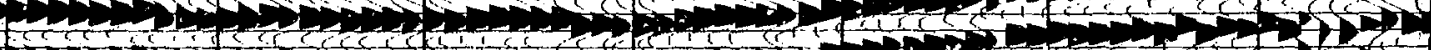

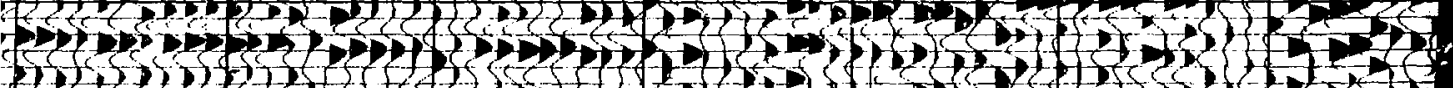

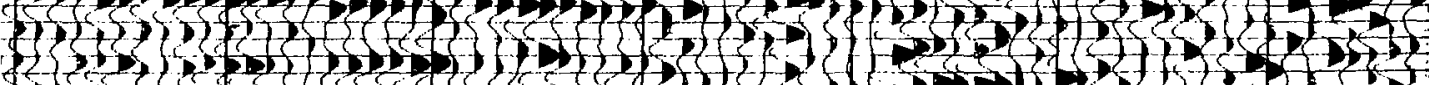

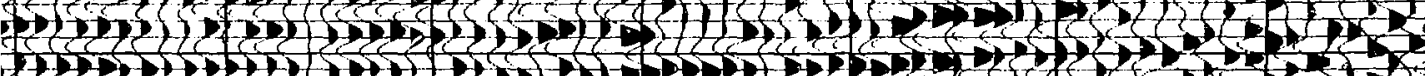

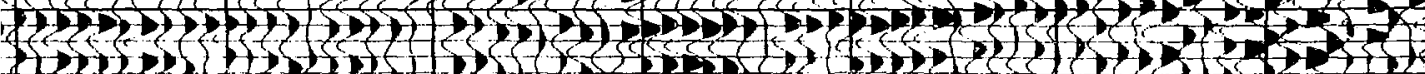

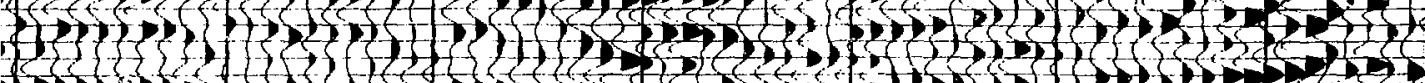

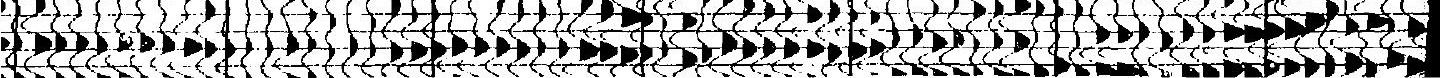



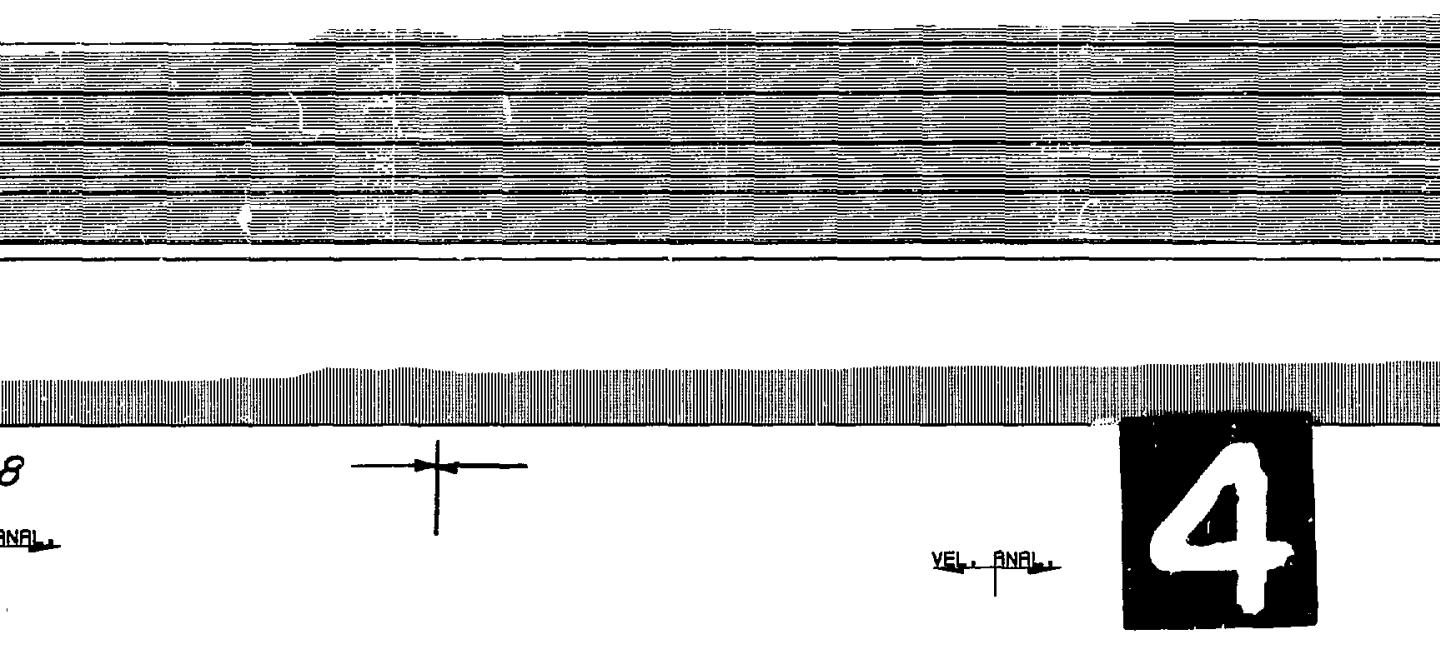

$\begin{array}{lllllll}195 & 110 & 1\{5 & 120 & 125 & 130 & 135\end{array}$

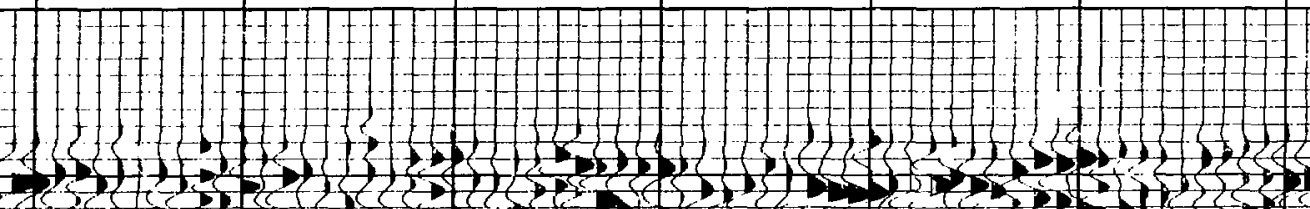

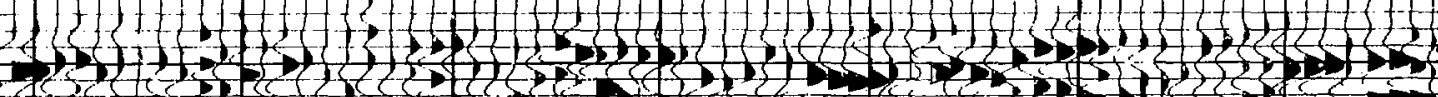

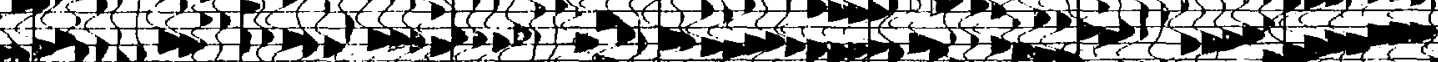

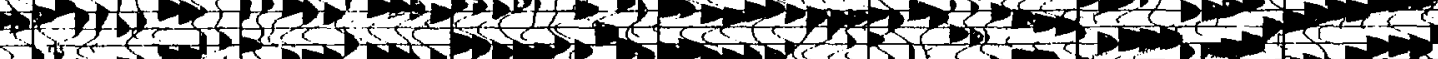

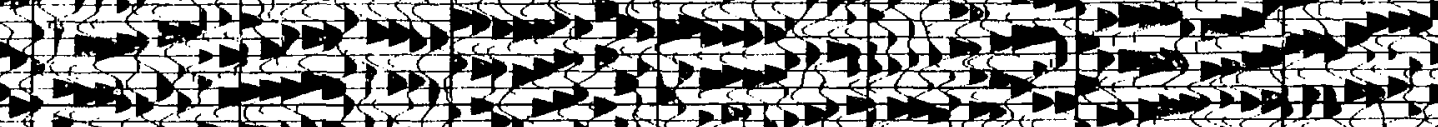

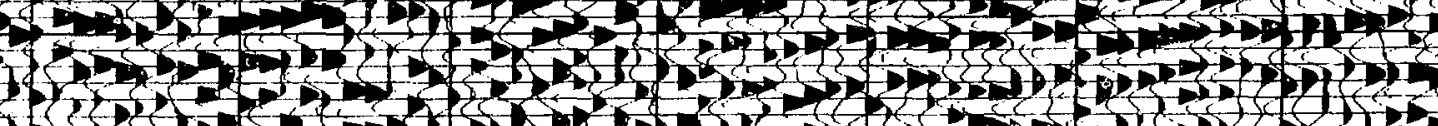

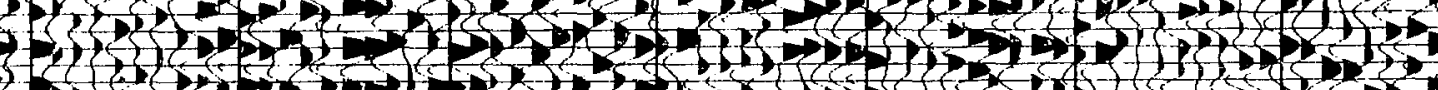

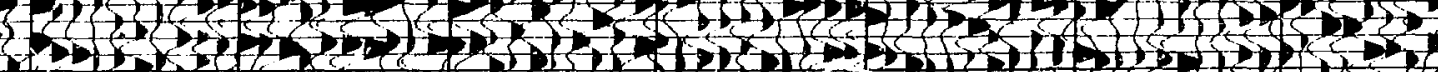

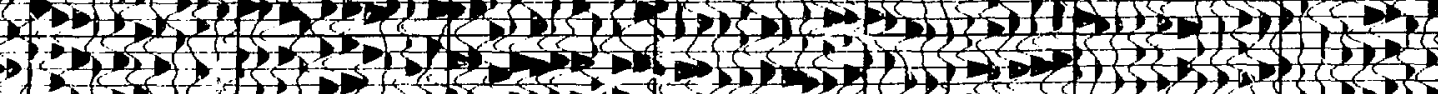

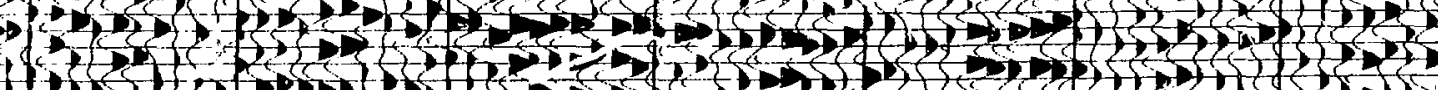

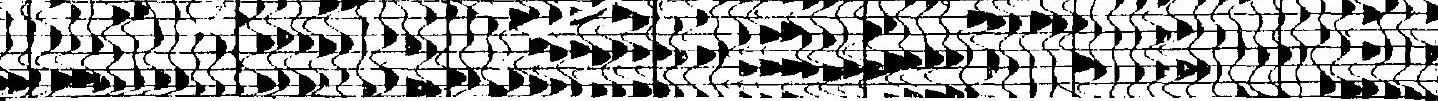




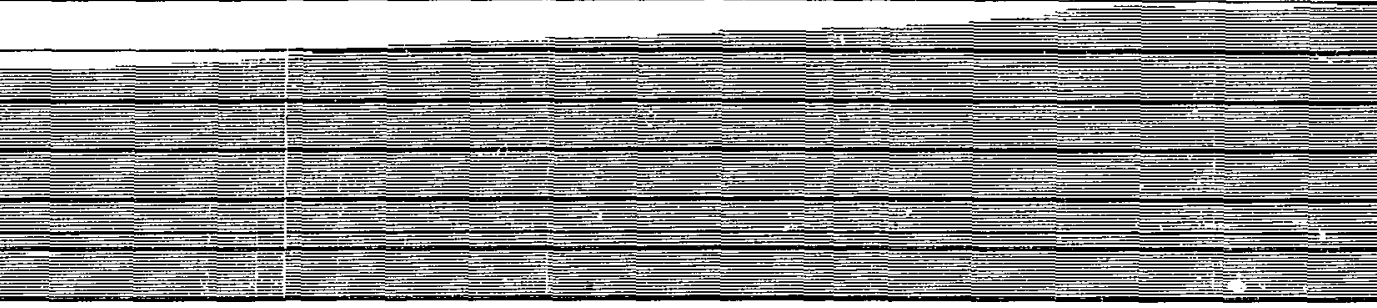

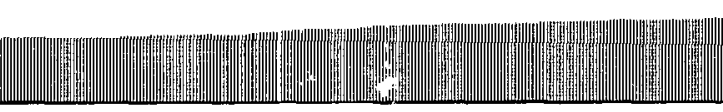

$\sec 17$

VEL, PNRL,
|NA
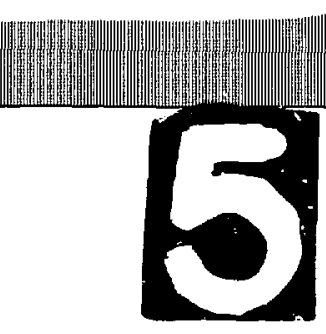

160

155

150

160

165

LINE $x-2$

163

170

$1+1+2=0$

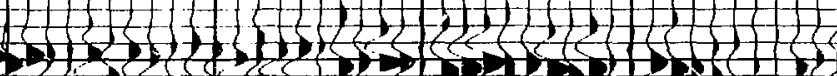

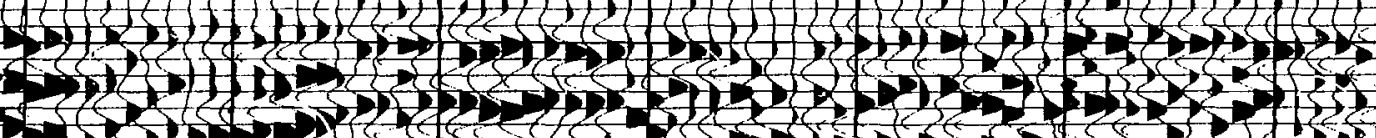
$\rightarrow\{(5), 15$ से

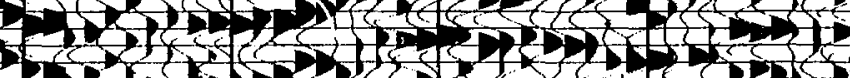
(5)

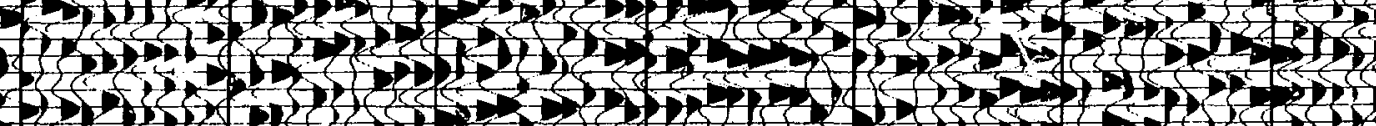

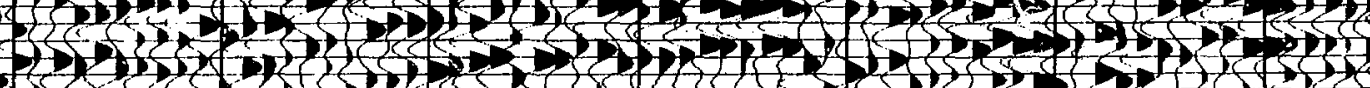

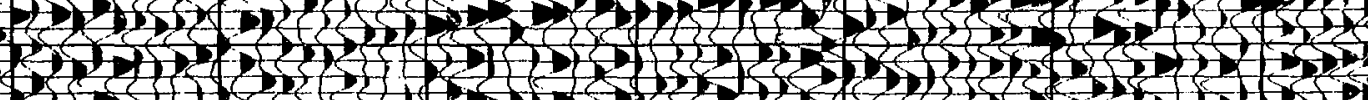

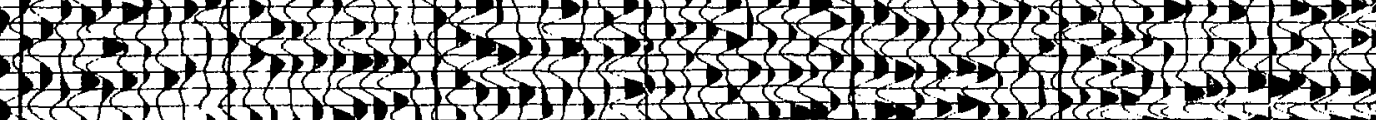

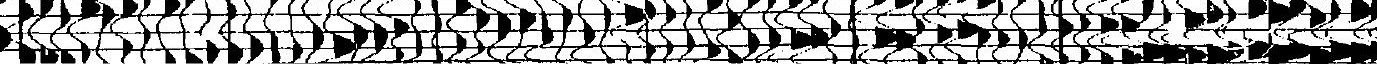



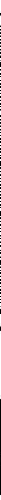

$\sec 16$

VElar. Fikfl,
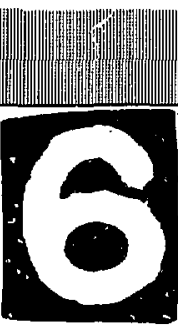

190

195

205

YELLANALL,

sec 21

180

185

190

200

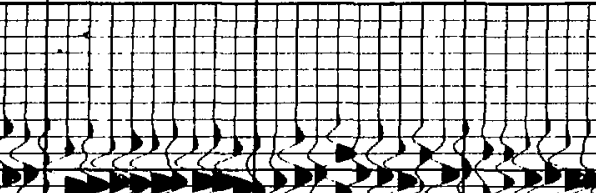

$\operatorname{lig}_{1}$

- 2 दि

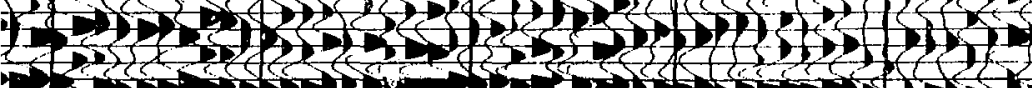

(5)

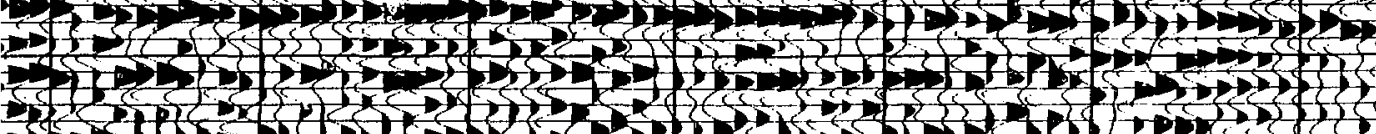

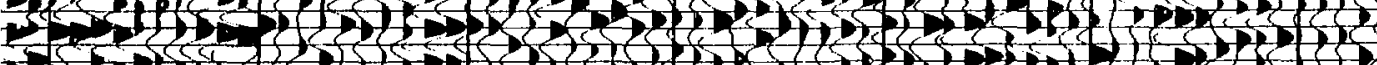

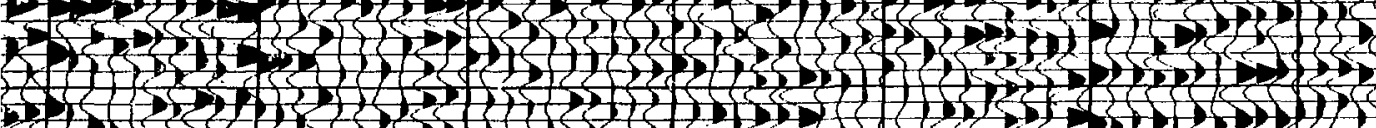

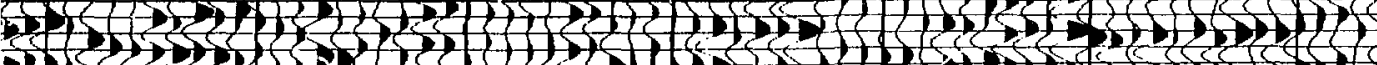

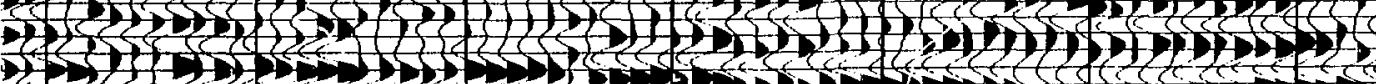

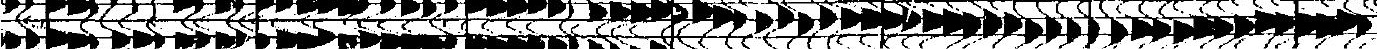




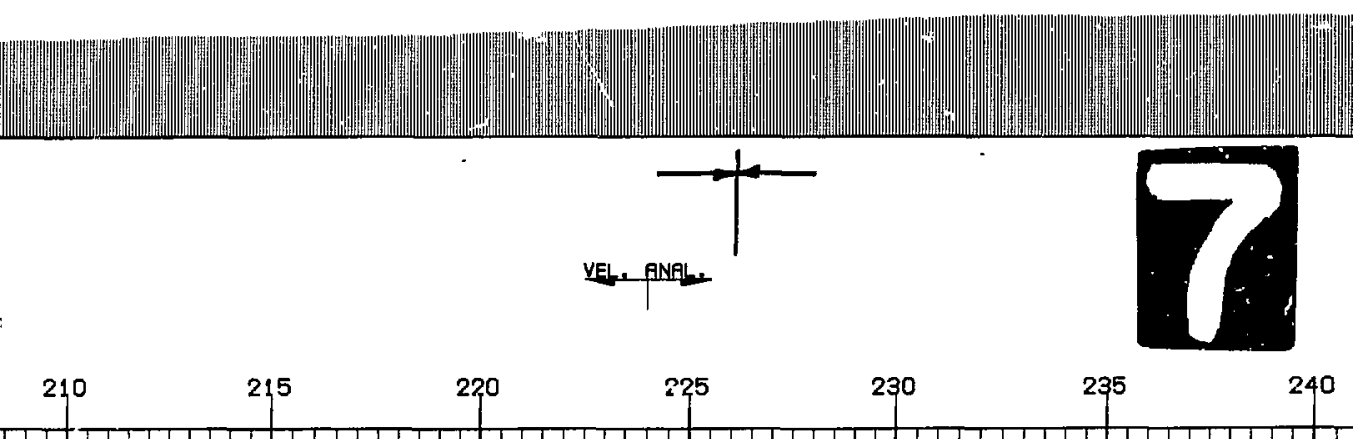



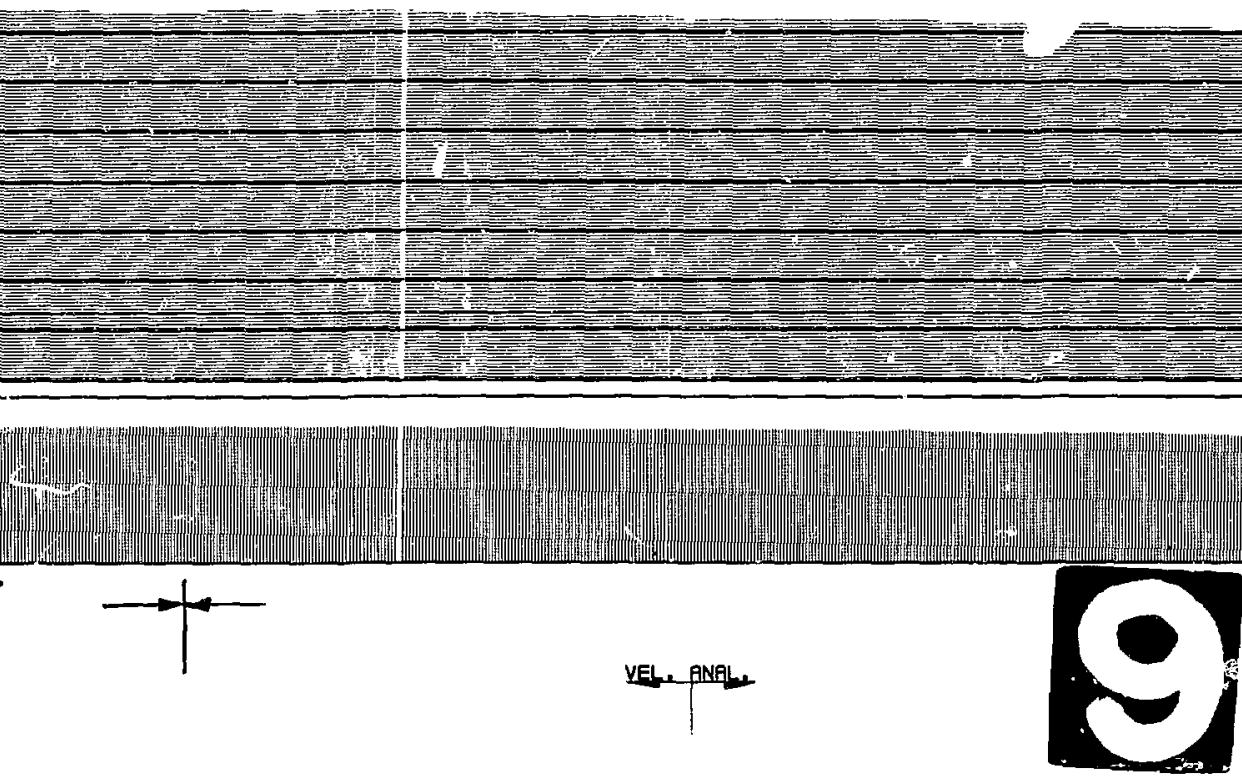

YEl Ge GNALL

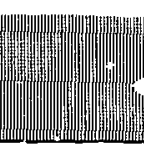

280

285

290

295

300

305

310

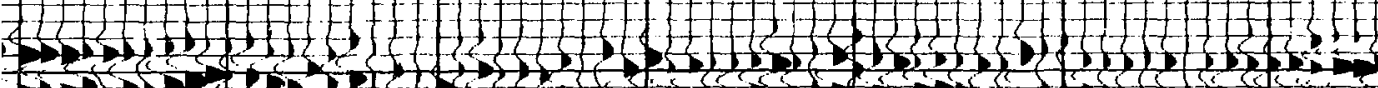

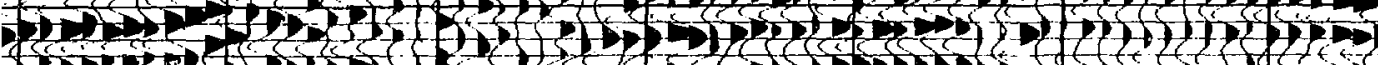

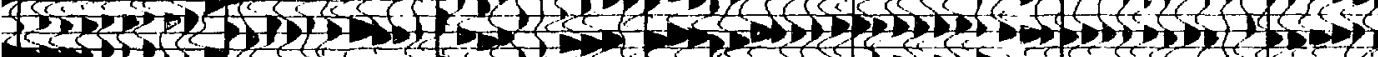

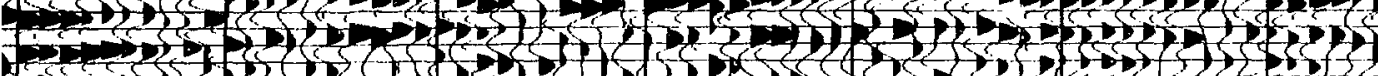

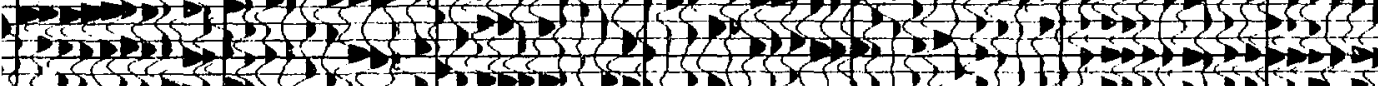
ff

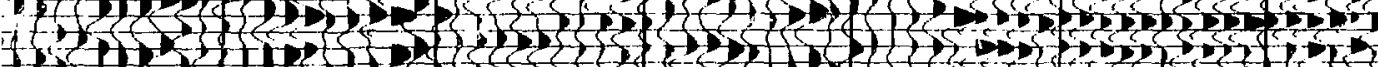

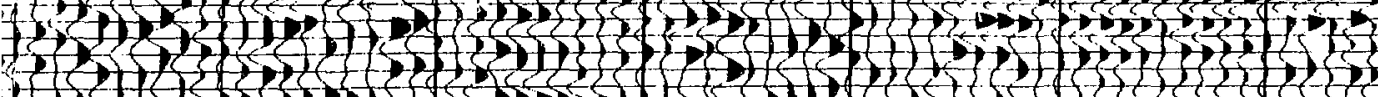

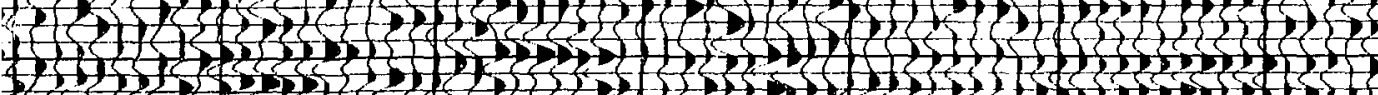

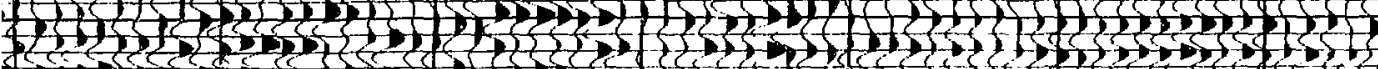

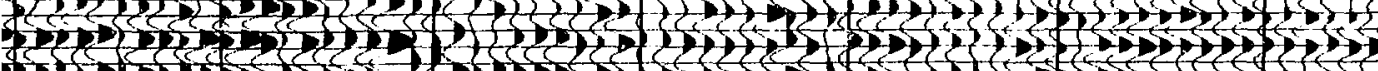
1) 2 री ( 


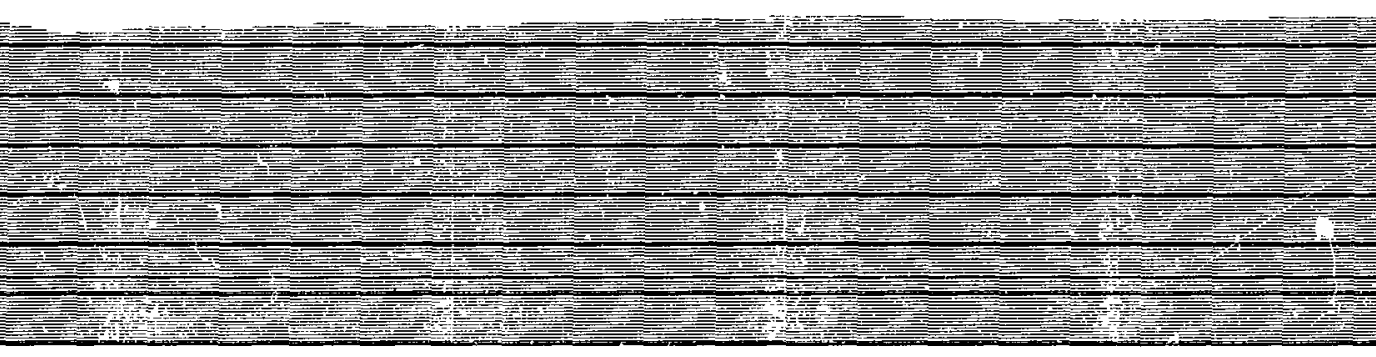

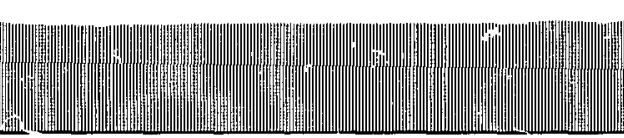

sec 25$$
3
$$

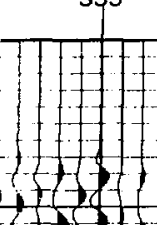

VElat. PNAL,

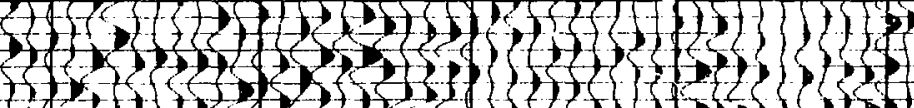

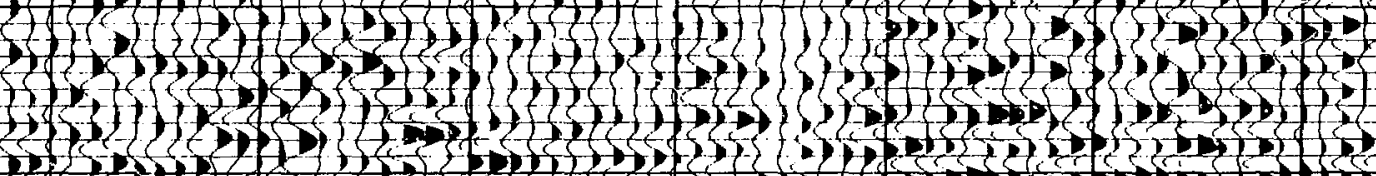

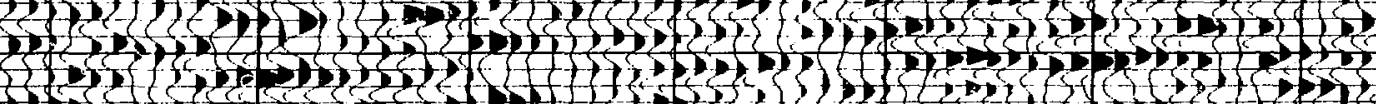

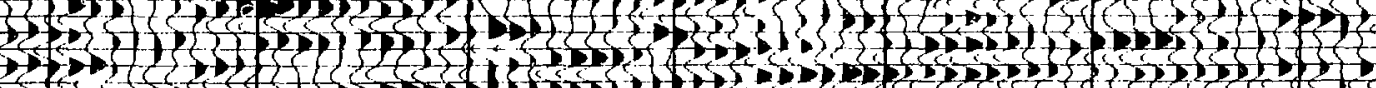
1.7.m. (3)

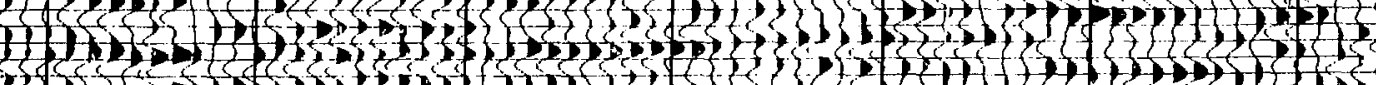

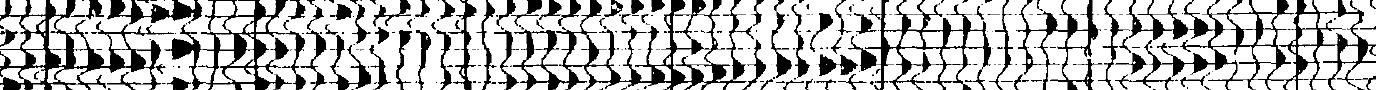




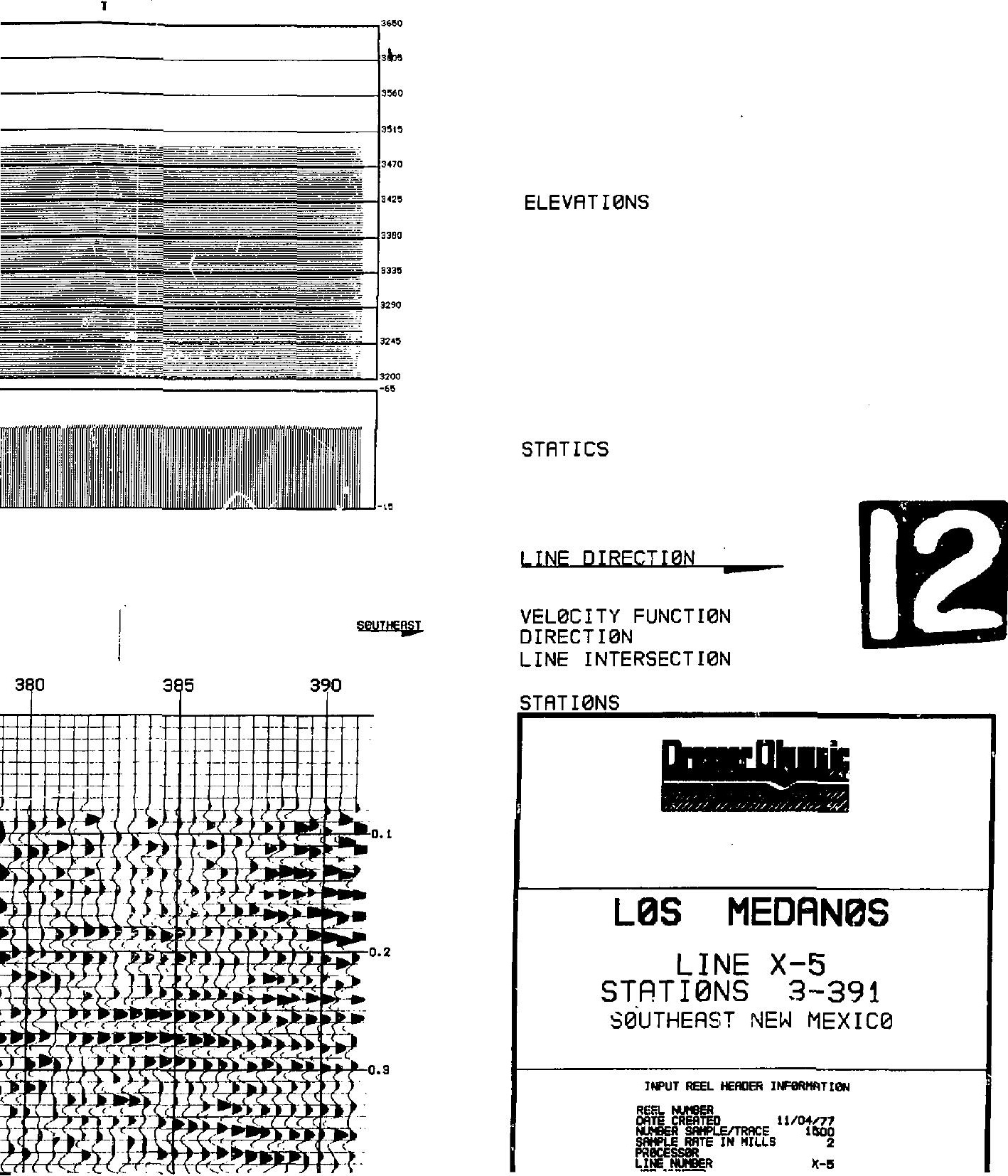


0.450 H

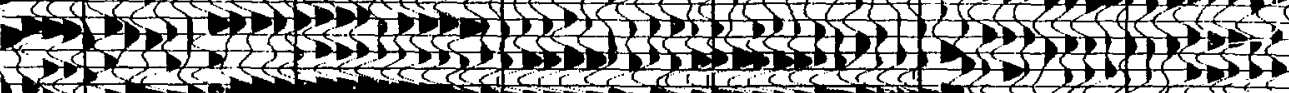

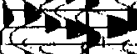

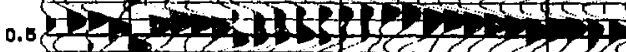

150 (t)

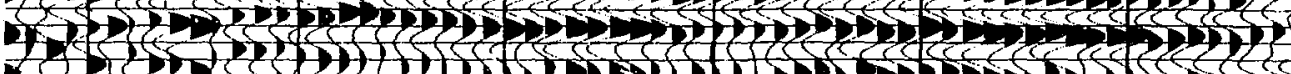

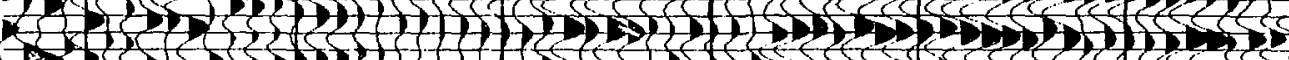

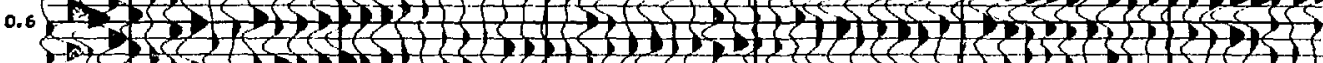
-1) दो

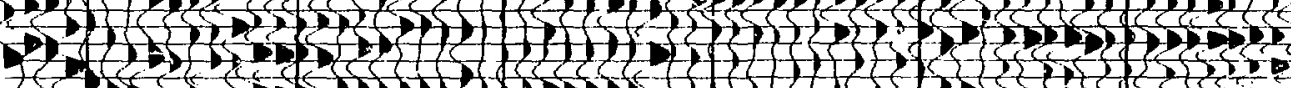

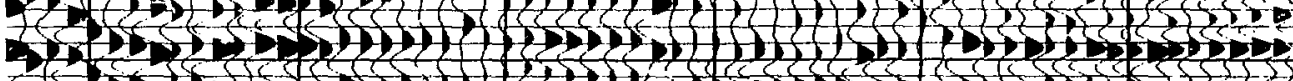

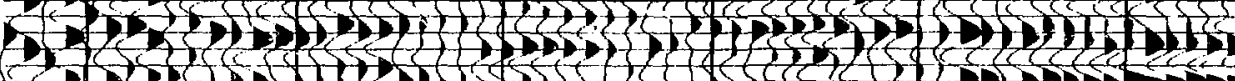

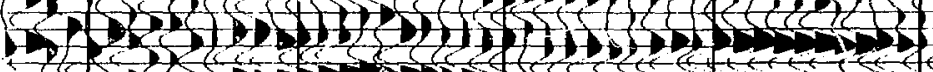

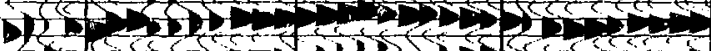

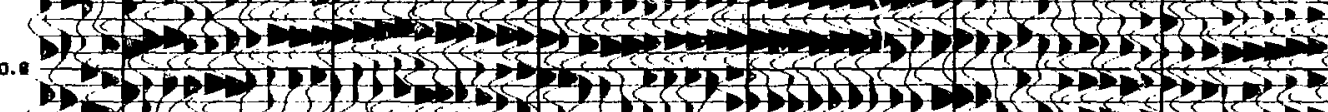

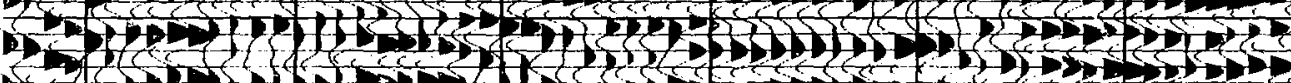

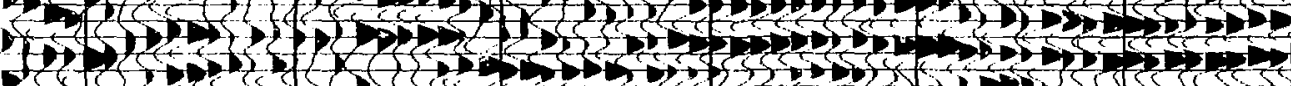

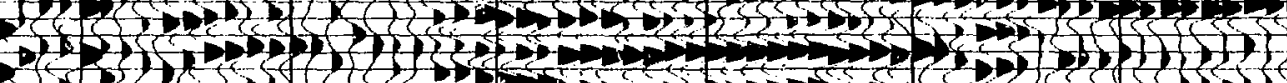

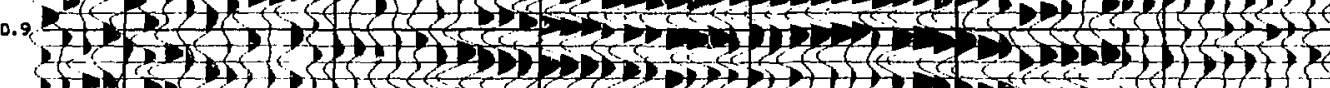

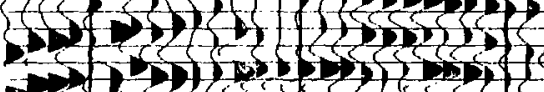

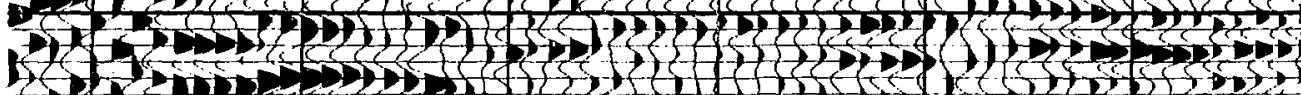

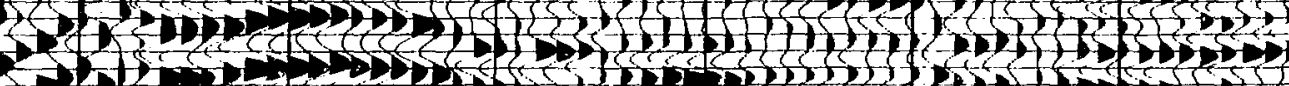

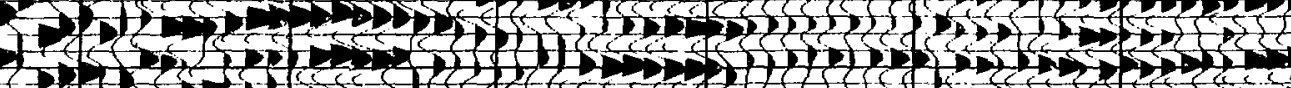

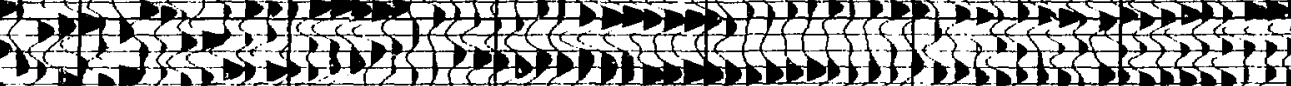

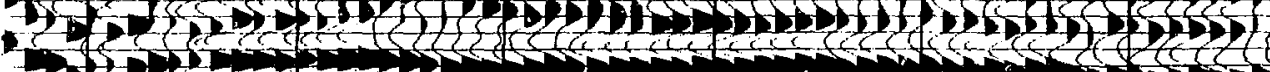

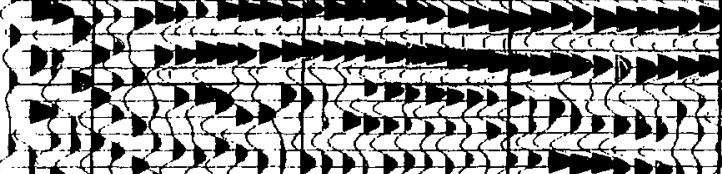

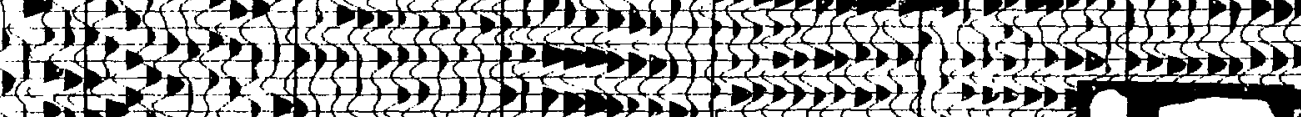

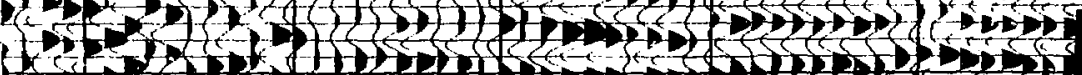

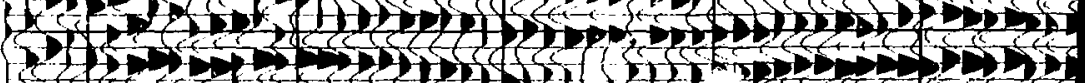

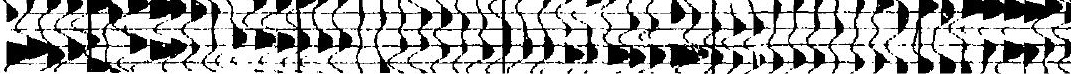


से है?

\section{$7<+2<$

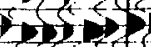

\section{min}

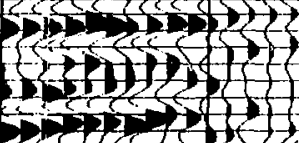

initis

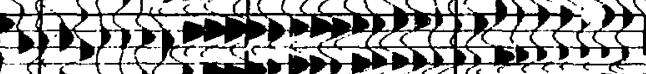

(1) 121$)$

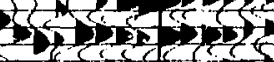

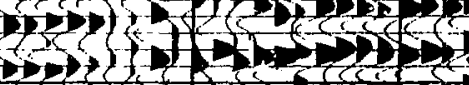

tot

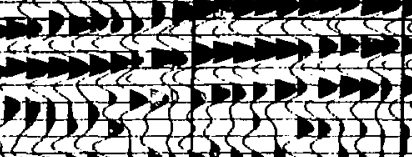

स्यं करें $\rightarrow(2)<2$ 


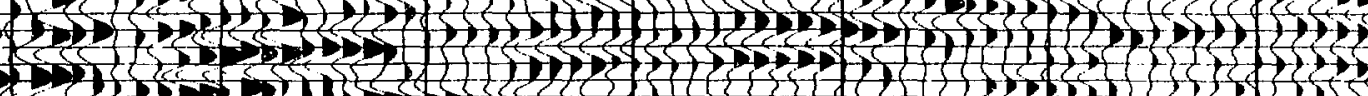

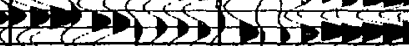

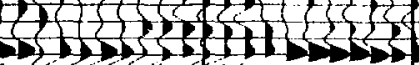

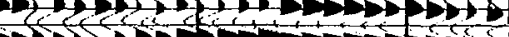

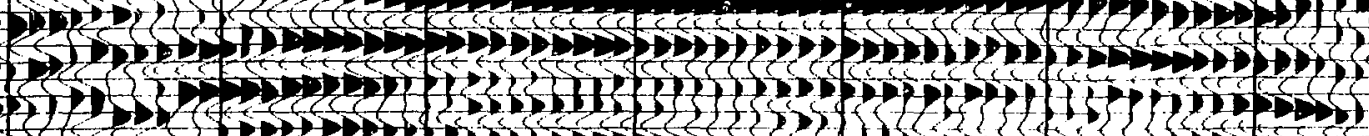

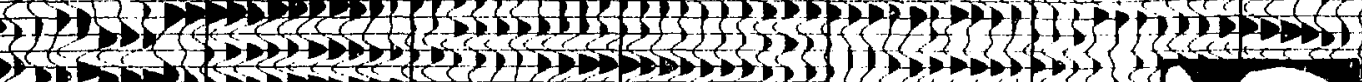

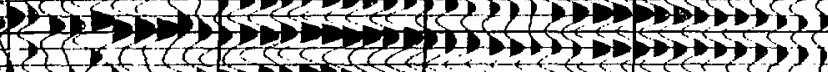

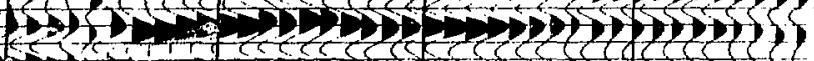

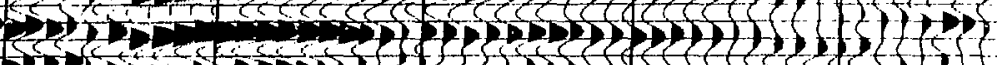

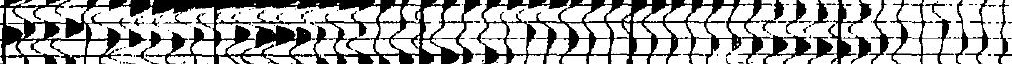

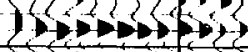
tif 


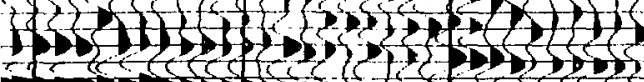

et?

(1)

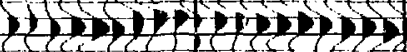

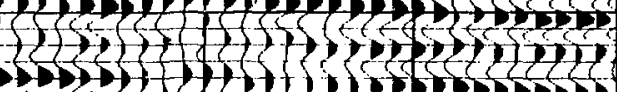

$1\} 2+\infty, 2$

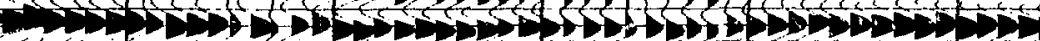
42

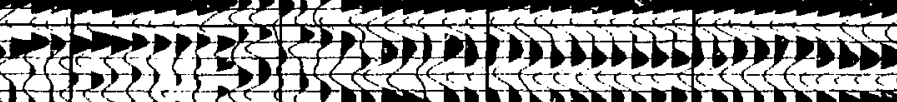

5

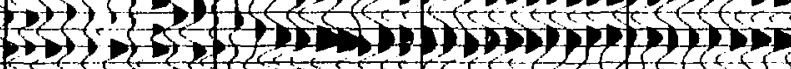

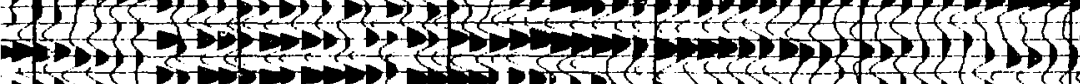

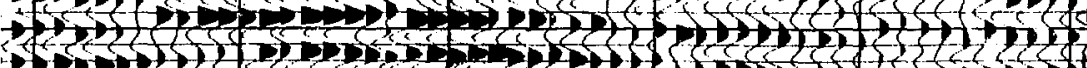

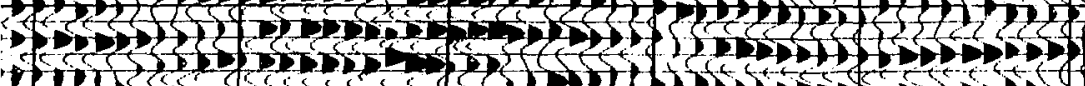
1 1)

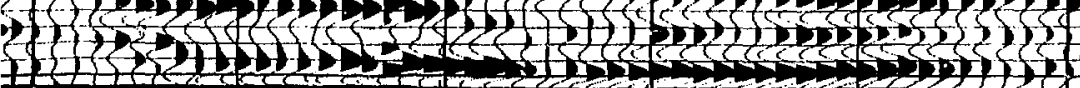

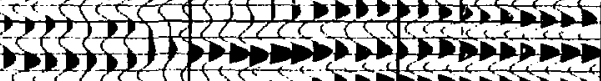





\section{FIELD INFORMAT ION}

\begin{tabular}{|c|c|c|c|}
\hline $\begin{array}{l}\text { RECERDEO BY: } \\
\text { DATE: }\end{array}$ & $\begin{array}{l}\text { ORESSER OLYIC } \\
\text { OCTOSER 3. } 3977\end{array}$ & $\begin{array}{l}\text { PARTY: } \\
\text { FILTER: }\end{array}$ & $\begin{array}{l}\text { 19. } 62 \\
10 / 36-124 \mathrm{HZ}\end{array}$ \\
\hline INSTRLNENTS: & CFS I - DFS IV & SFWLE RATE & 2N6 \\
\hline NaTCH FILT: & & SQUECE: & VIBRESEIS \\
\hline $\begin{array}{l}\text { RECERD LEN: } \\
\text { BEEEP FREQ: }\end{array}$ & $\begin{array}{l}16 \text { SEC. } \\
25-100 \mathrm{HZ}\end{array}$ & $\begin{array}{l}\text { EHEEP LEN: } \\
\text { MO/ERBSPS }\end{array}$ & 12 SEC. \\
\hline $\begin{array}{l}\text { STN INV: } \\
\text { GEO PER STNA } \\
\text { RRRAY TYPE: }\end{array}$ & $\begin{array}{l}110 \mathrm{FT} . \\
6 \\
\text { INLINE }\end{array}$ & $\begin{array}{l}\text { YIB. INY: } \\
\text { GEO TYPE: } \\
\text { TYPE CQVER: }\end{array}$ & $\begin{array}{l}110 \mathrm{FT} \text {. } \\
\text { GSC-200 } \\
1200 \text { Piters }\end{array}$ \\
\hline
\end{tabular}

\section{PROCESSING SEQUENCE}

PRECESSED BY DRESSER QKYMPIC

STATICS CEMPUTATION

DATLM: 3700 FT.

VSH: $\quad 6000$ FT/SEC.

1) DERULTIPLEX

2) BINARY GAIN RECBVERY

9) YJERESEIS CERRELATIEN

4) COMMEN DSPTH POINT EATHERS

5) DELanNaLUI IEN OPERATET LENGTH-140 MILS PREDICTIEN TIHE BASED OA 2ND ZERO CRBSSING

6) TIME-INNARIANT DIBITAL FREGUENCY FILTER $0.0-9.0$ SEC. 20-80 HZ

7) GPPLY OATUM STATICS

B) VELBCITY ALIFLYYSIS

9) APPLY NaO

10: FIRST BREAK SLPPRESSIGN (MUTE)

(1) STACK 12 FQLD

12) TIME-IAVRAIRAYT DIGITAL FREDUEALY FILTER 0.0-9.0 5EC. 25-80 HZ

13) OtGITAL AGC

14: OISPLAY B TR/IN 10 IN/SEC.

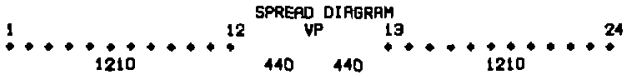

๑**** FILHING PARAMETERS *****

PERCENT GAIN 200 VERTICA SCALE 10. IN/SEC PERCENITY BAR BLACK+VE

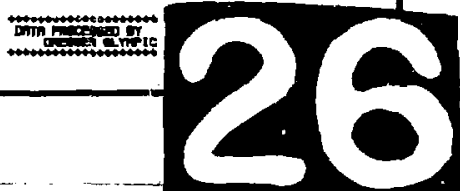


(4)

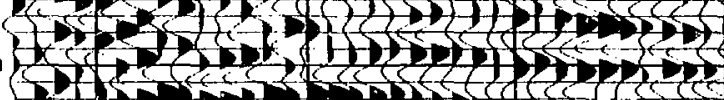

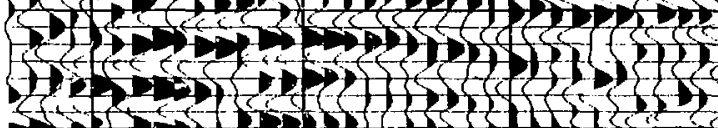

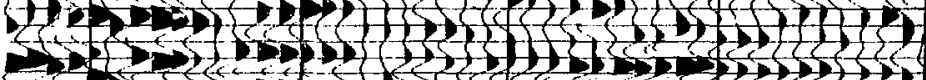

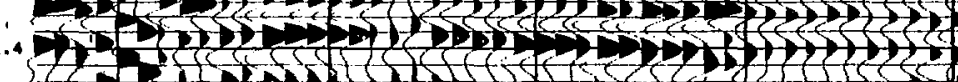

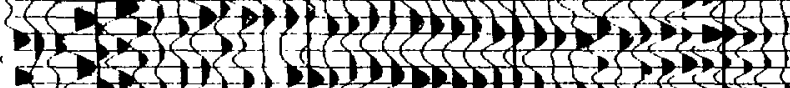

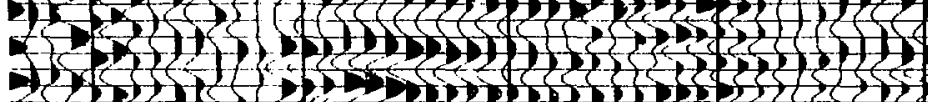

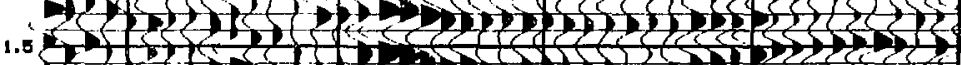
$(2,+2)$ (1)

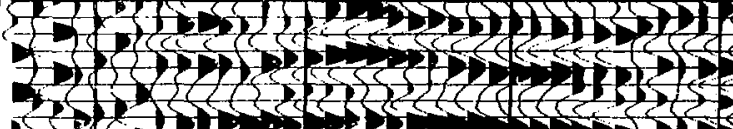

tis?

\section{.}$$
\text { , }
$$

te

$\{, 2,4)$ (2) 1.

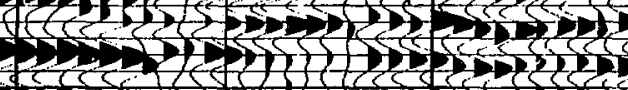

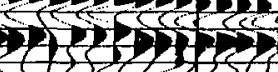

tरी

सरोंगे

$t_{0 \rightarrow 1} \cos _{1}+4$

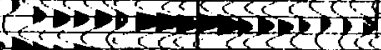
$(1)-3<<<<5\}$

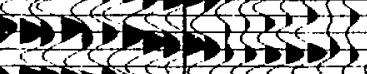

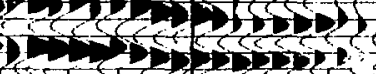

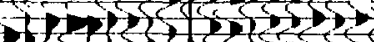
$\{+2,2,12$ if $x+2,2\}$

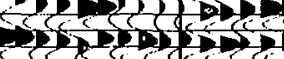

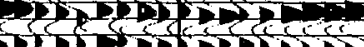

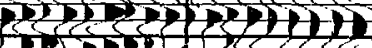

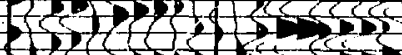






if

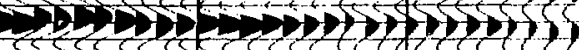

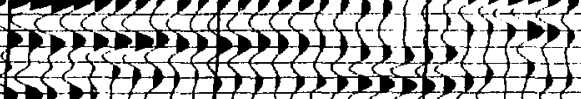

करोंड

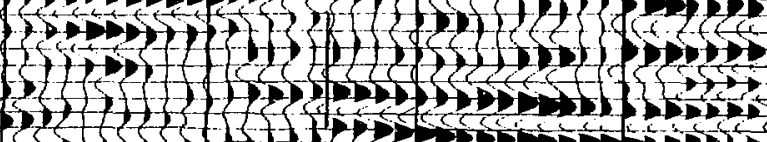

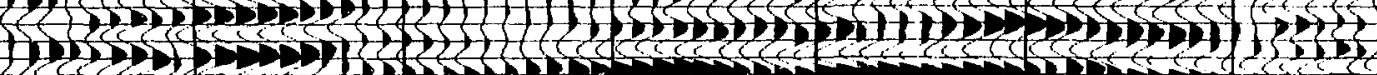

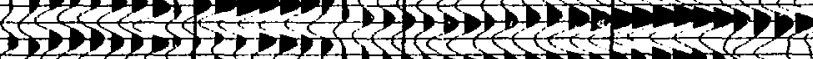

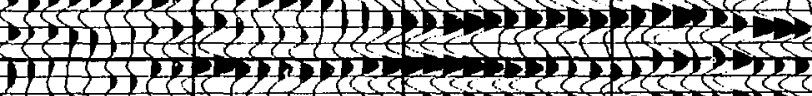

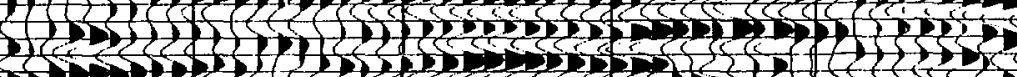

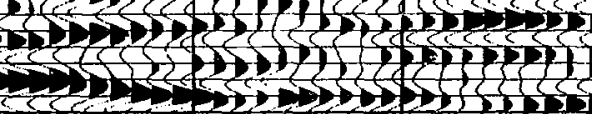

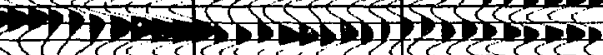

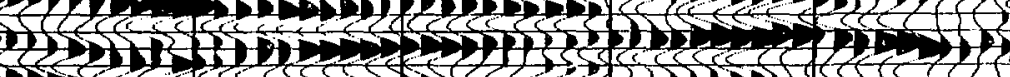
(1) 3 ,

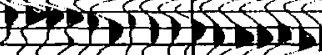
होगी

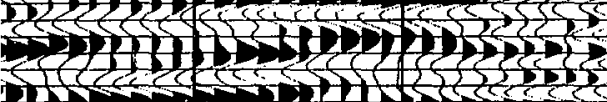
if i)

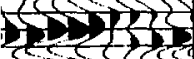

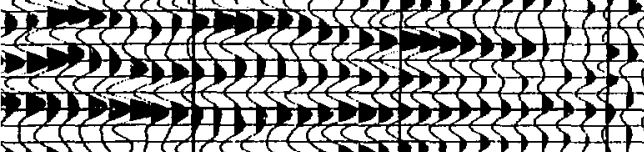
1121 711) 312 11) (17) 37. (1) (2) $4,1,0^{4}$ दो) री रे $11+21$ ) 



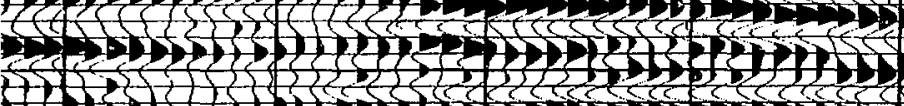

\section{Cरt}

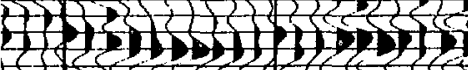
$3(15)+1)$

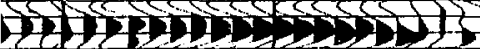

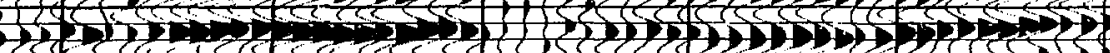

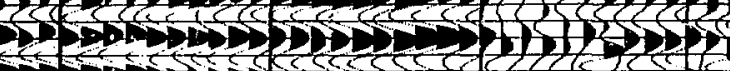

(C)

1 1) 30 री 空

the (1) योin

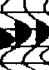
(1) $y 3,25,2,2\}$

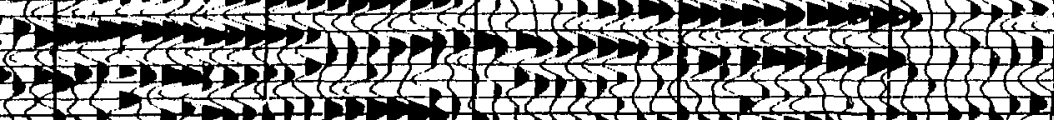
t) (3) 

f

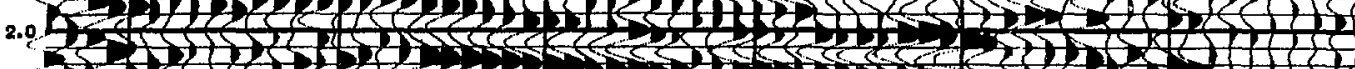

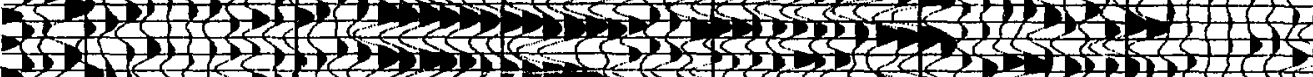

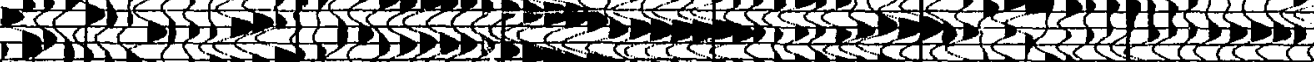

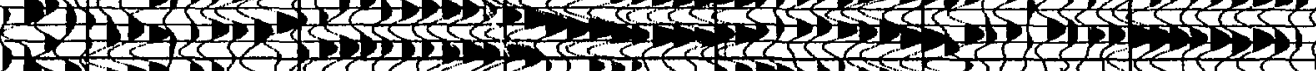

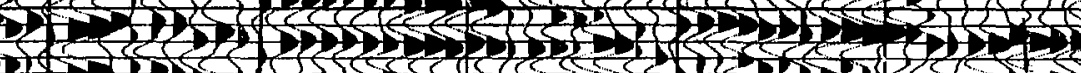

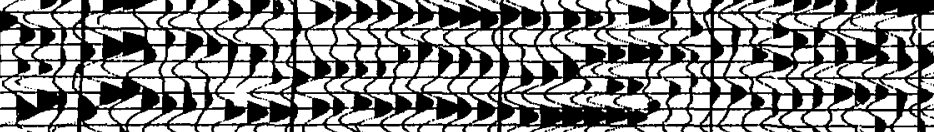

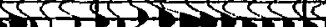
Pte (4)

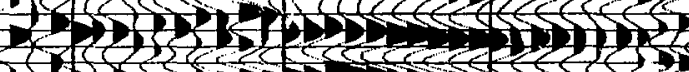
ap $=5<$, $\Leftrightarrow 3\left\{\begin{array}{l}3 \\ 3\end{array}\right.$

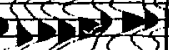

\section{(}

\section{( )}

\section{LC}







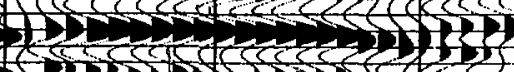



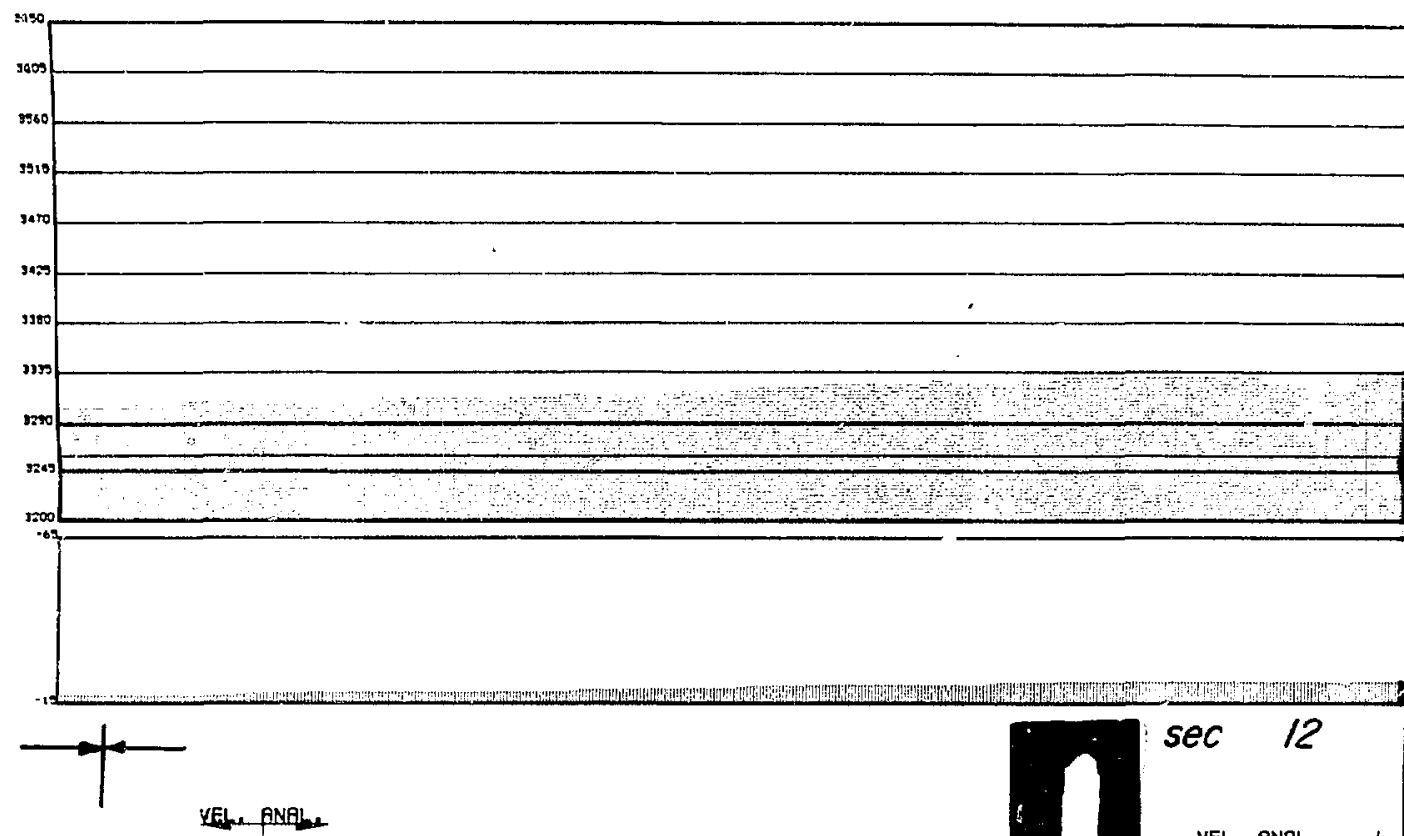

SUPHFAS

$\sec 12$

VEre. BNAl

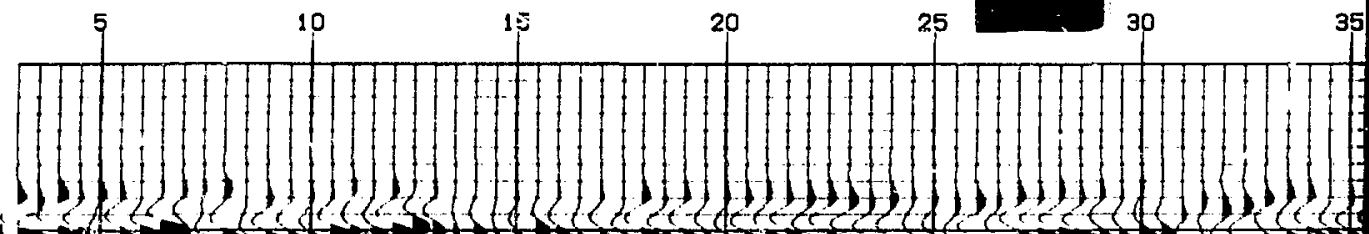

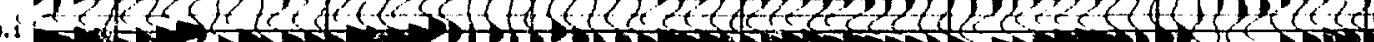

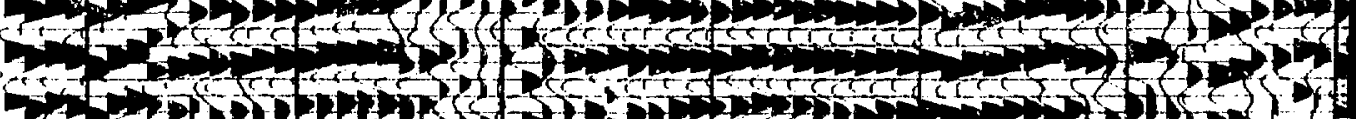

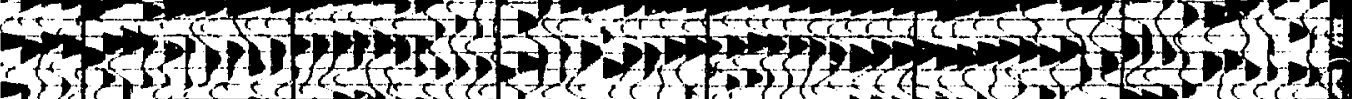

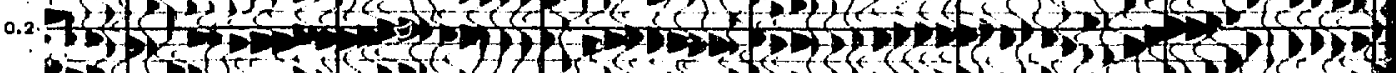

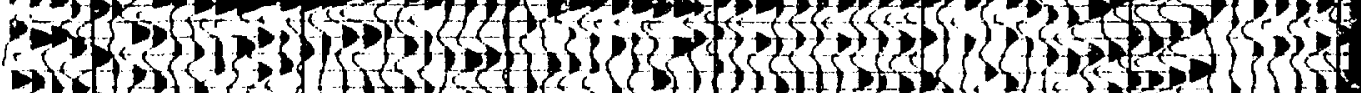

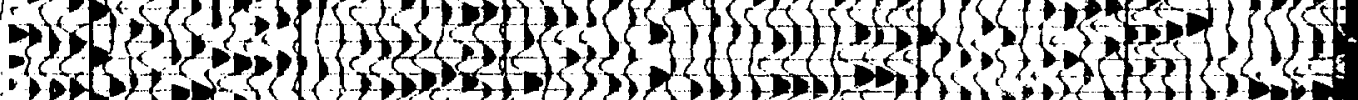

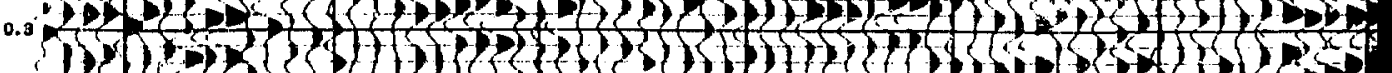

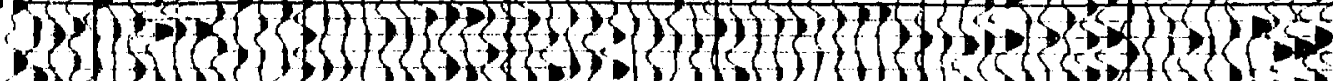

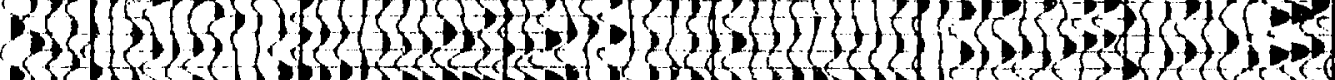

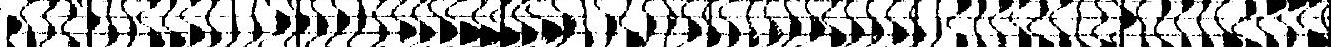




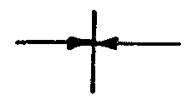

\section{YEA. PNAL,}

WDPDP

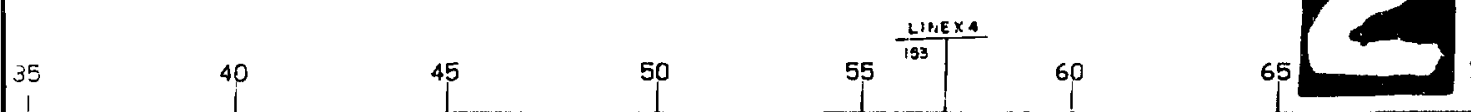

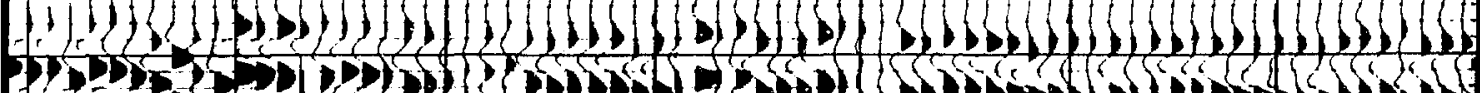

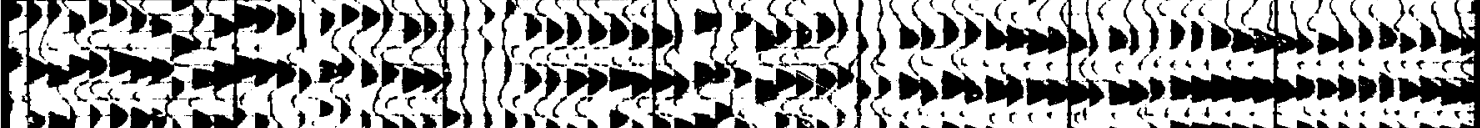

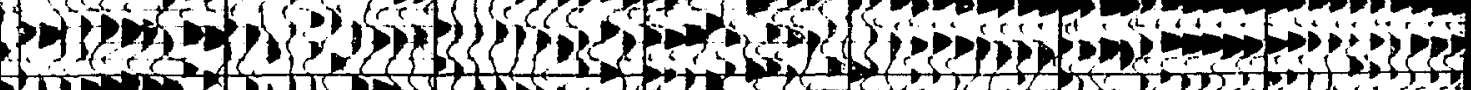
1)

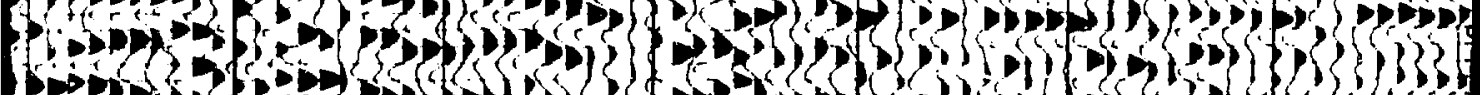
Lif

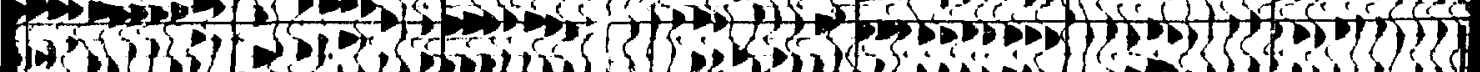

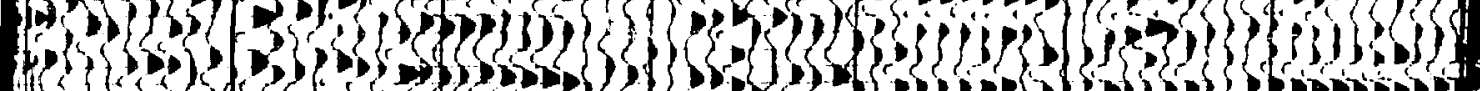

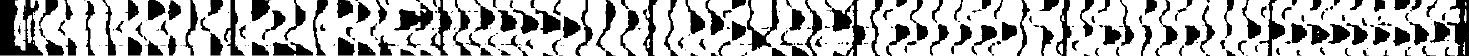


1

$\sec 17$

140

$145 \quad 150 \quad: 55$

VEG PNAL
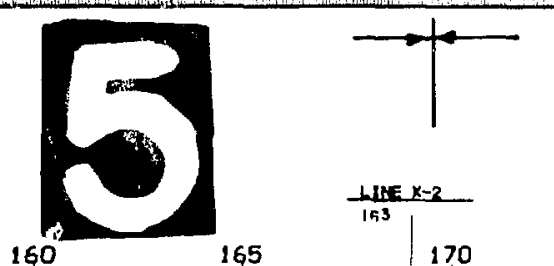

se

LH: $x-2$

170

165

1

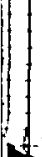

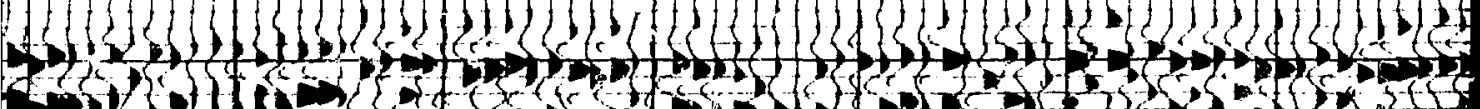

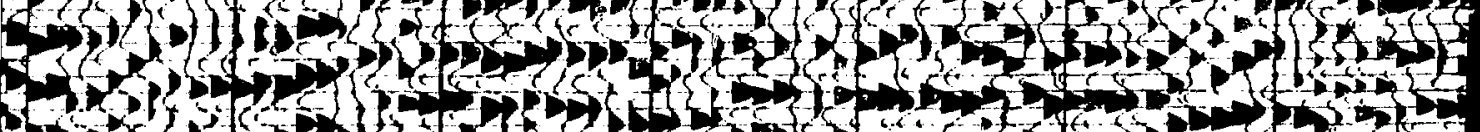

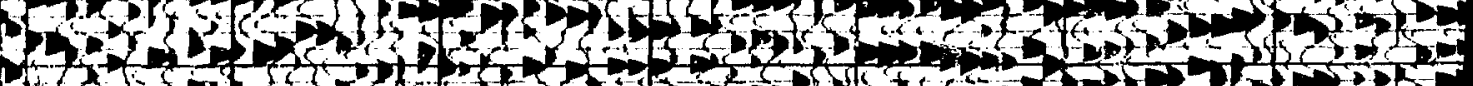

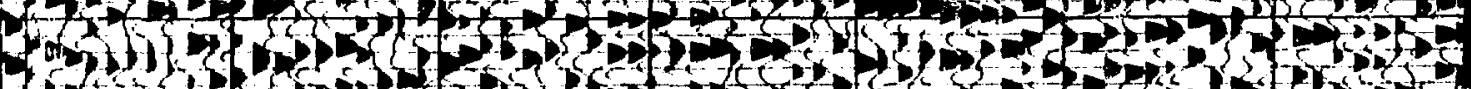

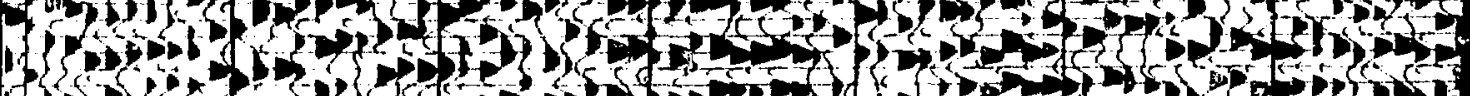

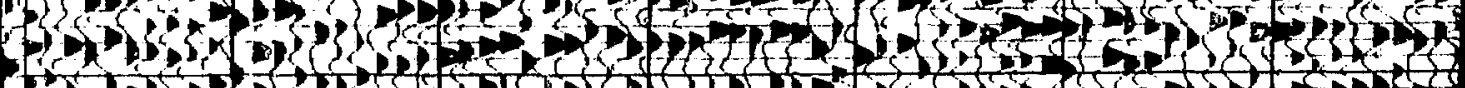
Fof

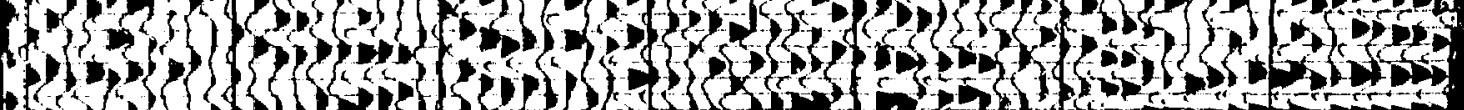

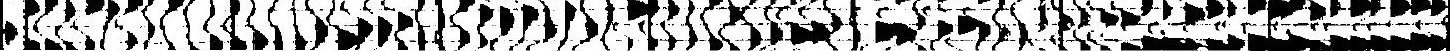



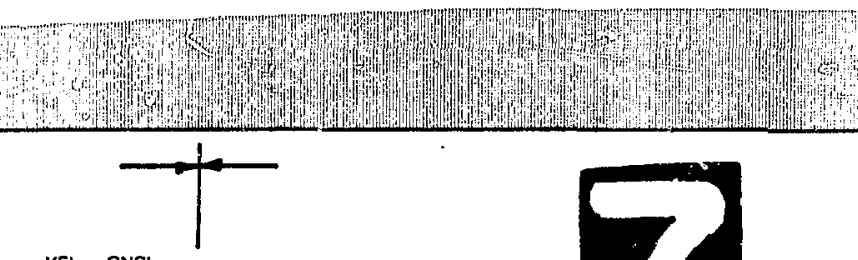

VEl, PNFil.

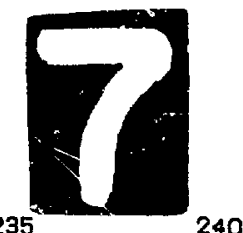

210

215

220

225

230

295

240

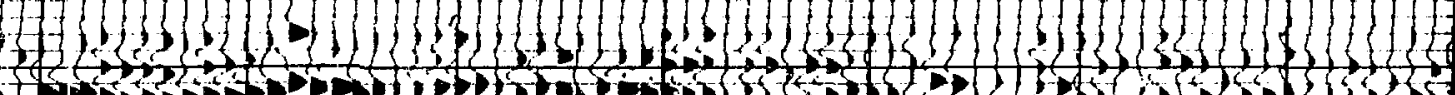

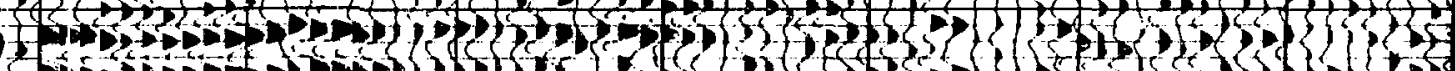

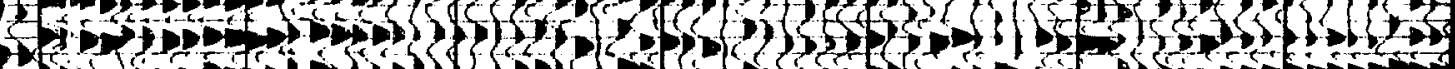

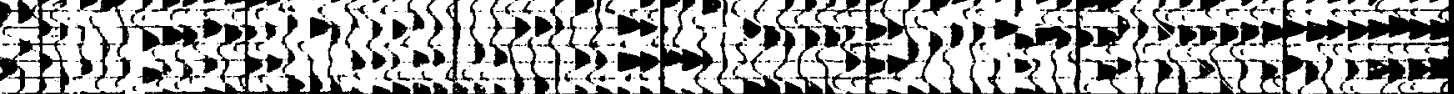

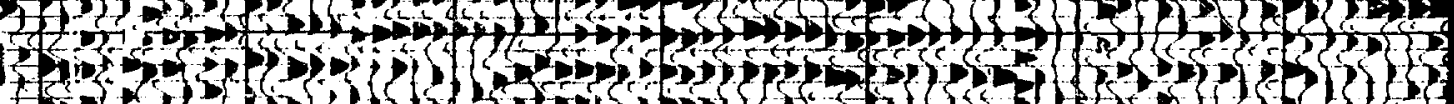

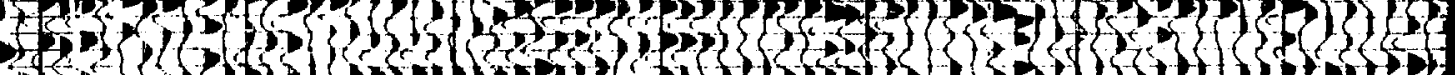

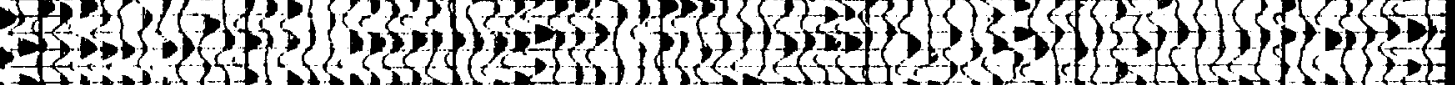

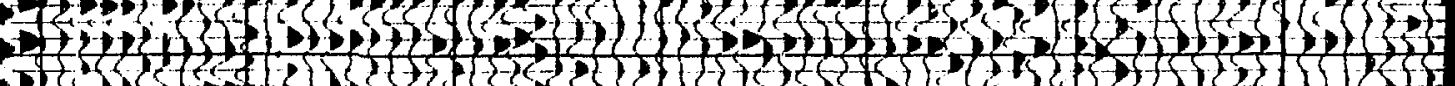
27.

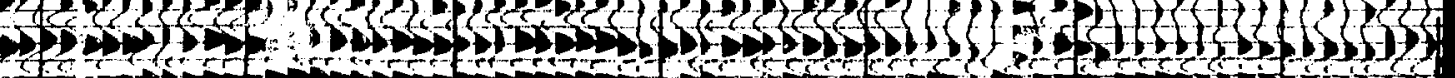

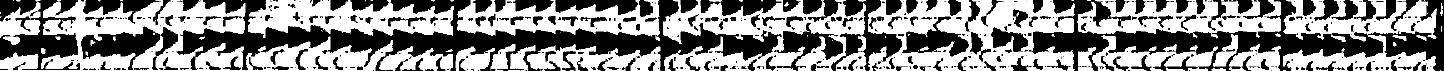




\section{sec 22}

VEL G ANR!
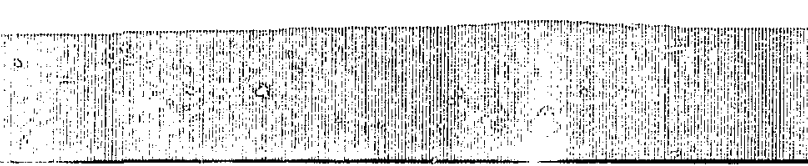
YEL, ANPL,

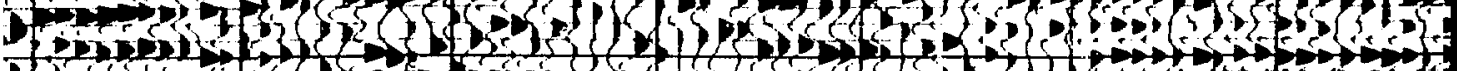

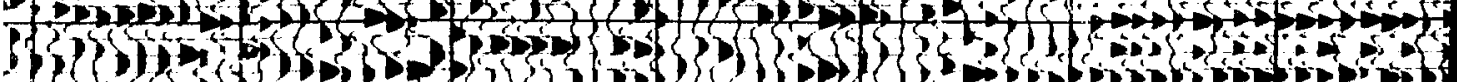

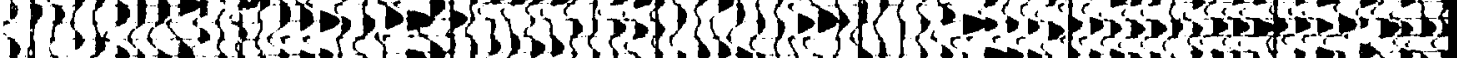

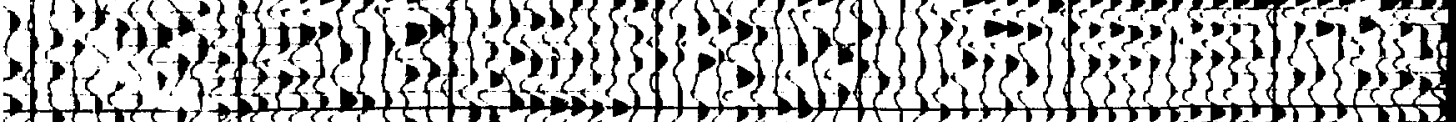

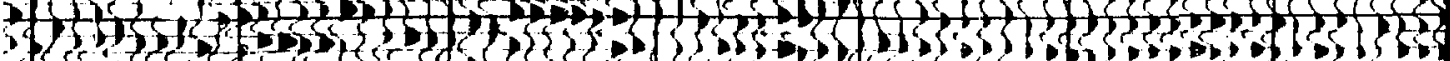

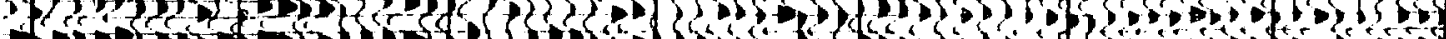

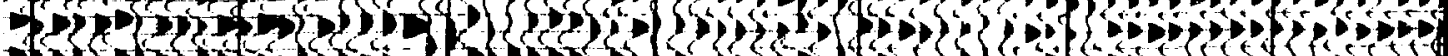

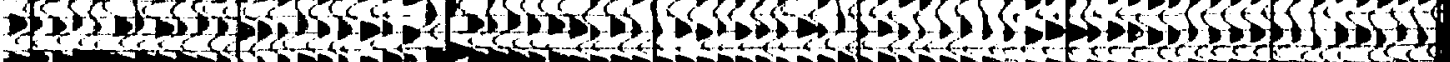

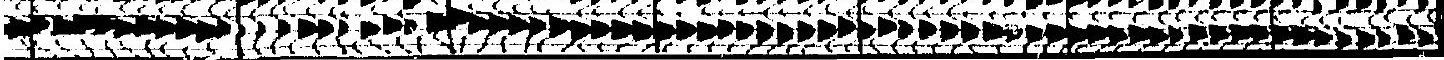



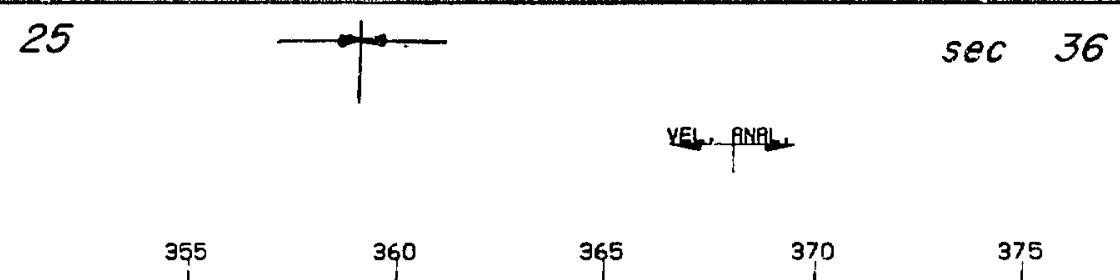

365

370

375

380

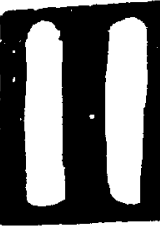

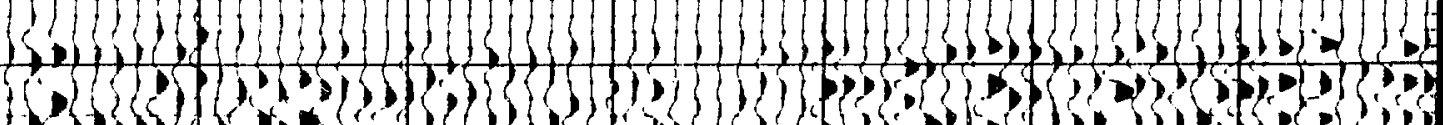

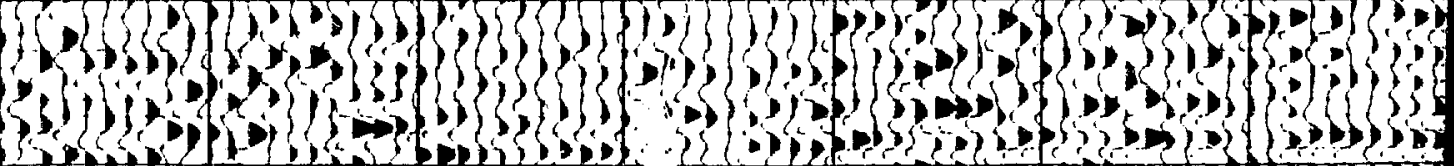

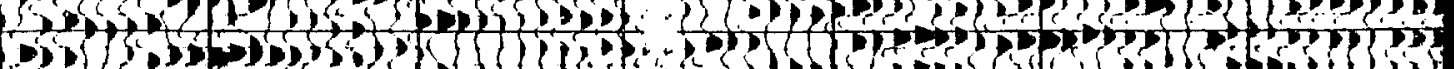

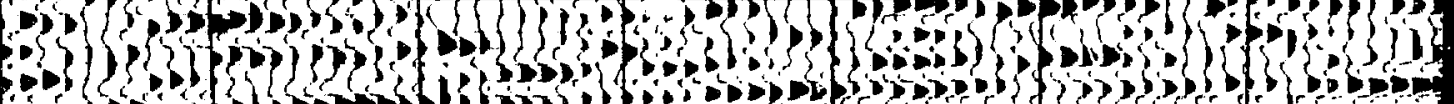

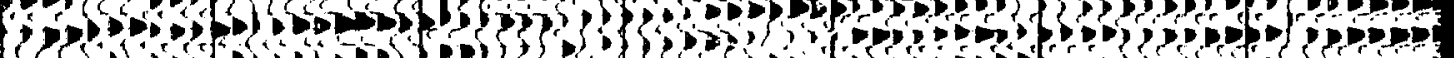

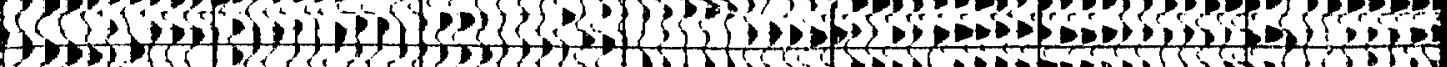

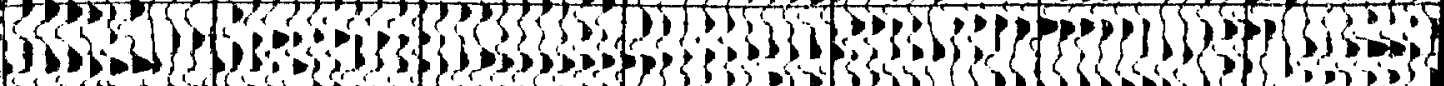
Вง 


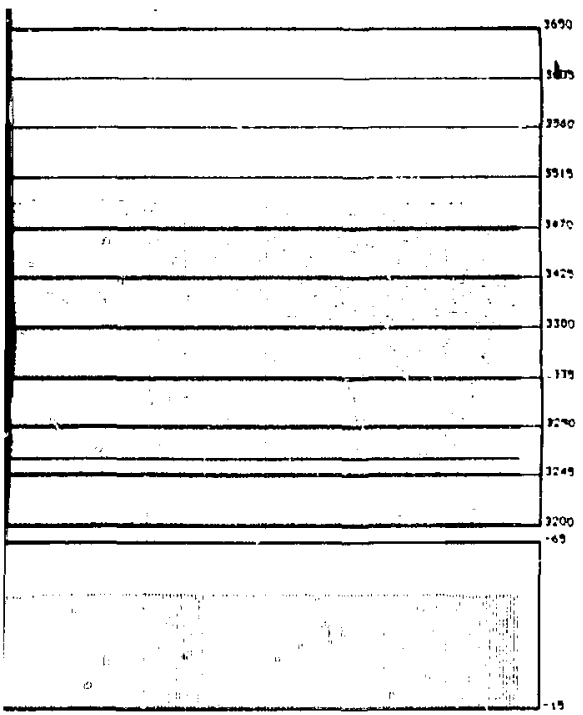

ELEVAT IONS

STAT ICS

LINE DIRECIION

GWITESI

VELOCITY FUNCTION

DIRECT ION

LINE INTERSECTION

STAT IONS
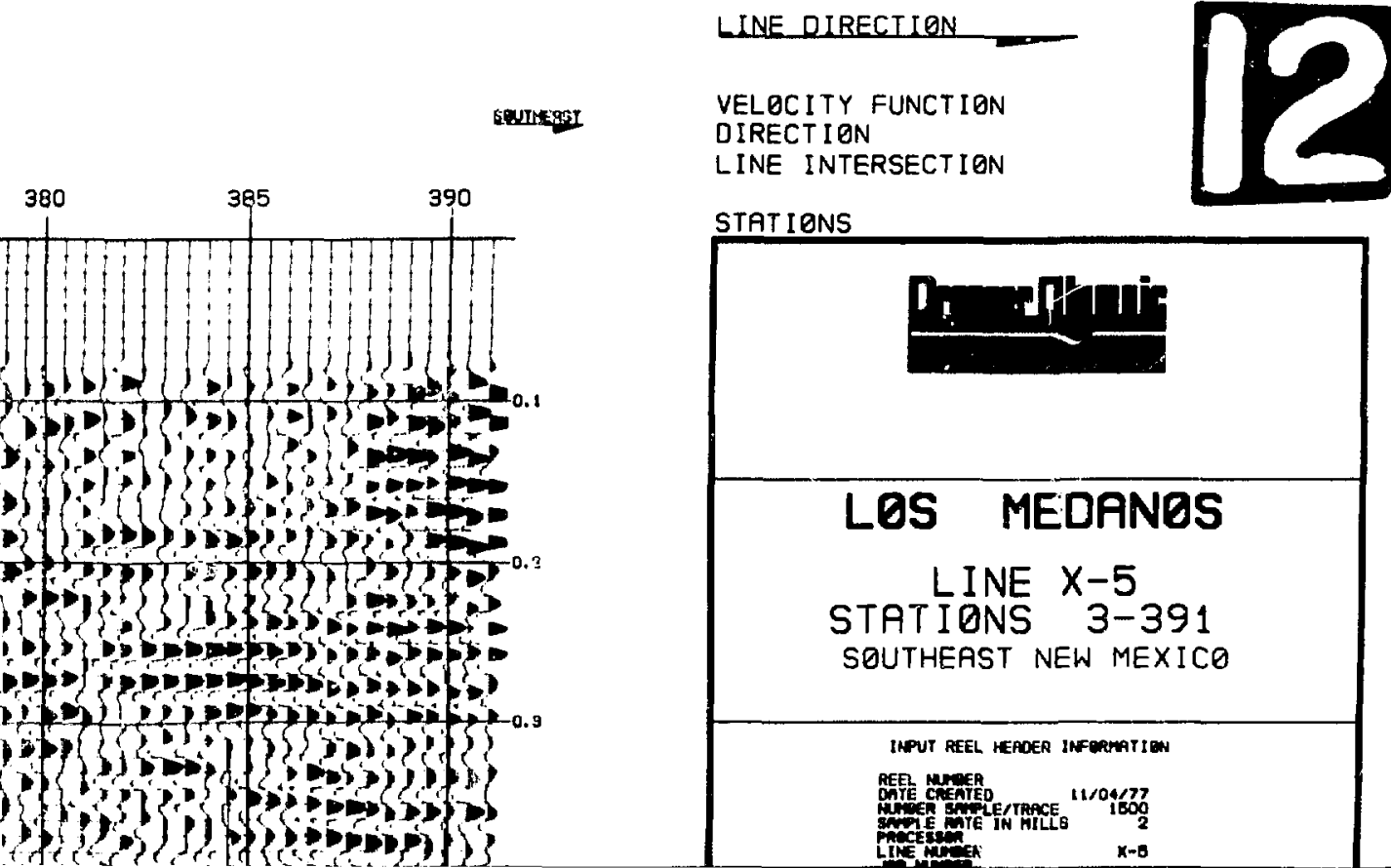

LOS MEDANOS

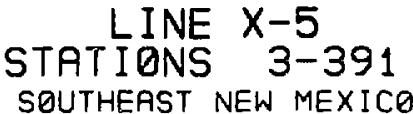

INPUT REEL HEROER INFERMTION

AEE N NEA

At

aret in

$t x=m$

$x-0$ 
75

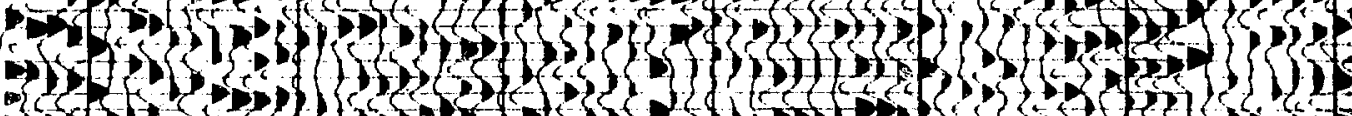

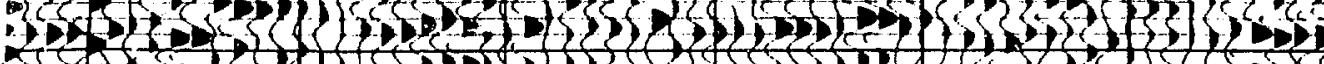

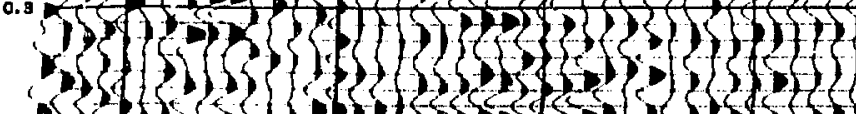

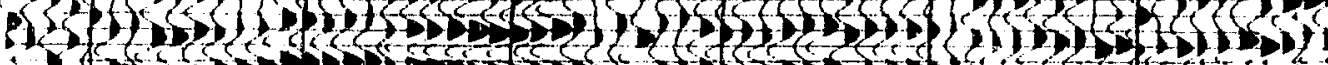

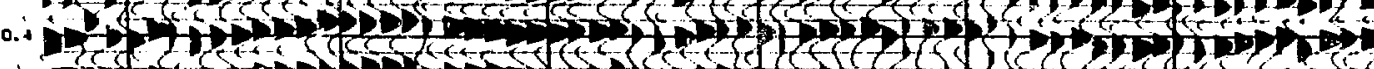

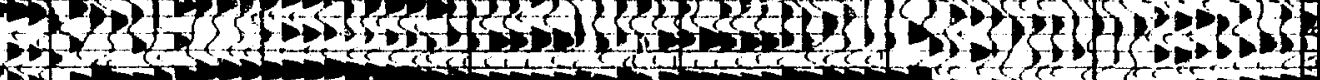

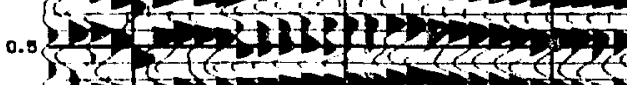

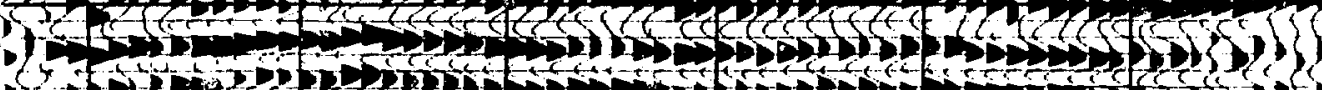

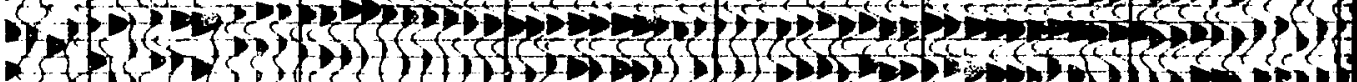
(2)

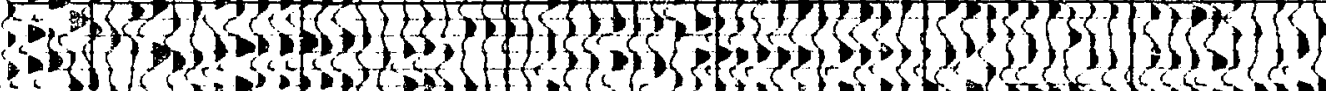

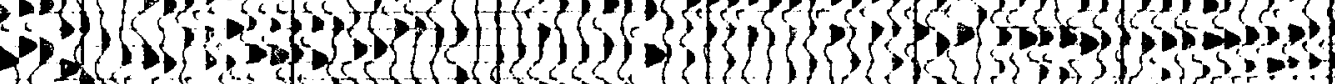

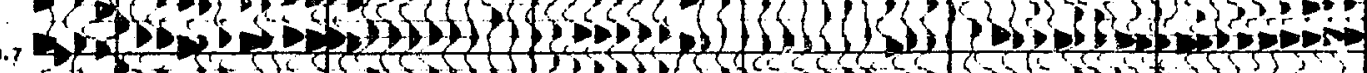

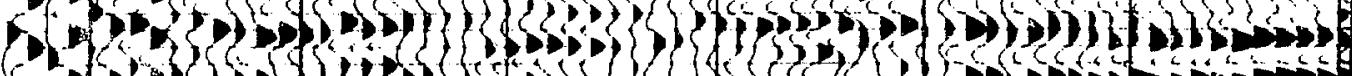

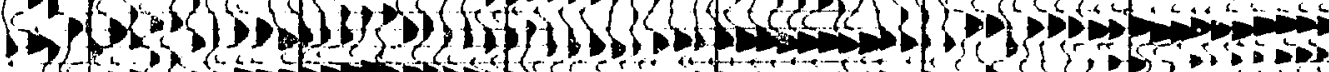

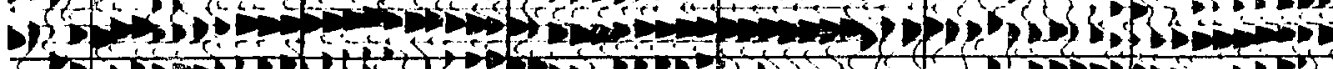

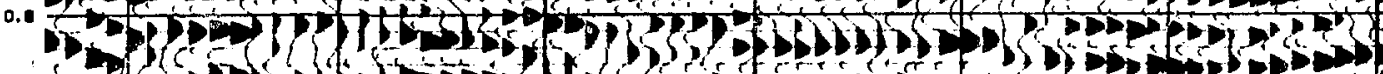

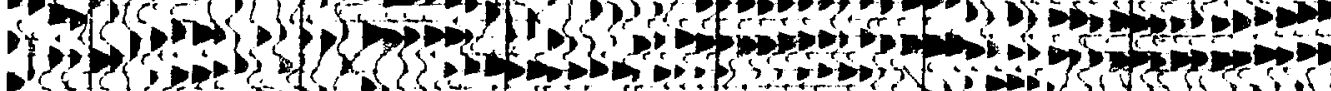

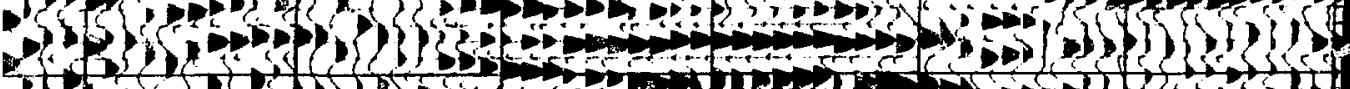

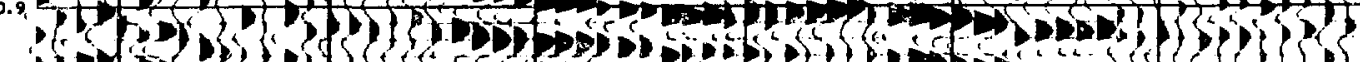

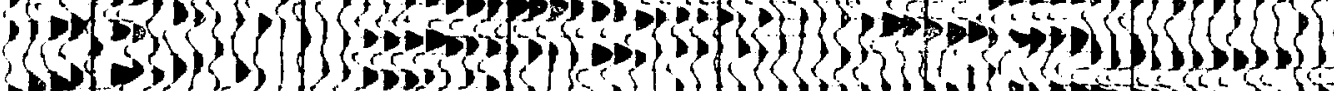

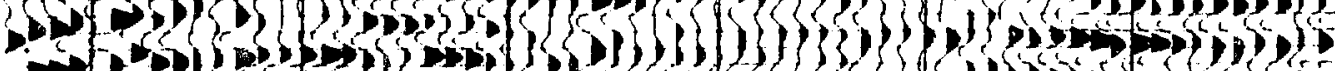

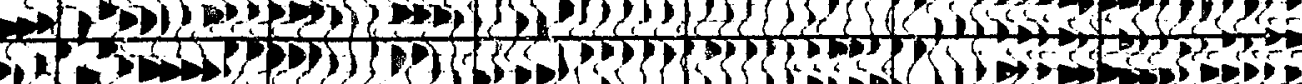

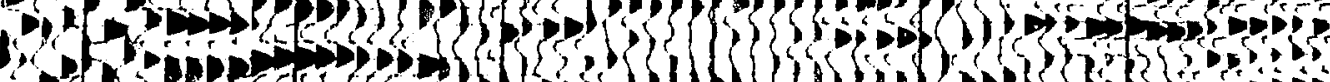

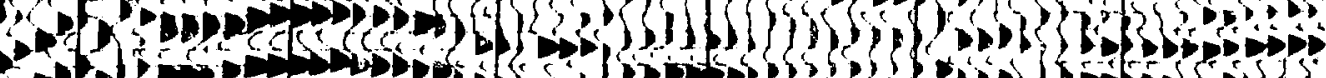

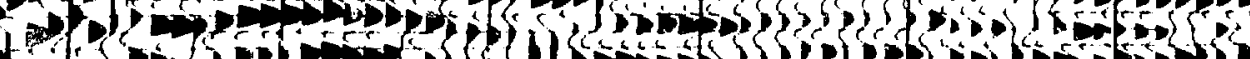

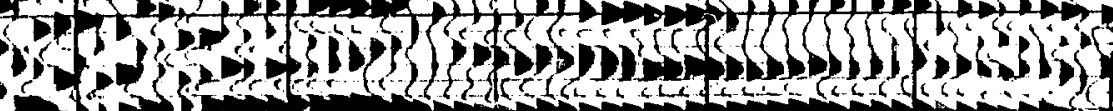

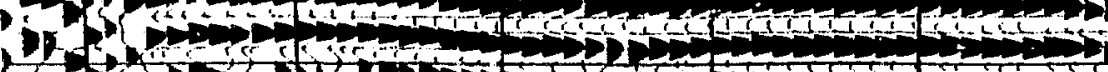

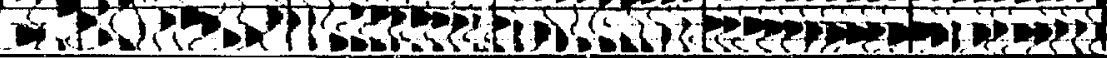




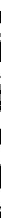

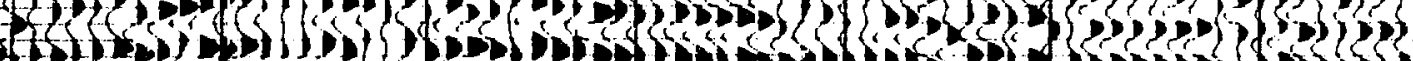

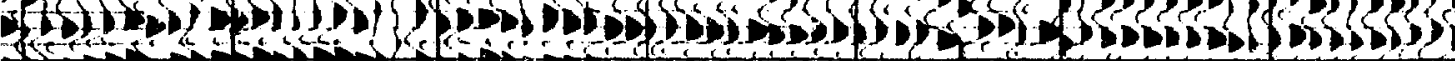

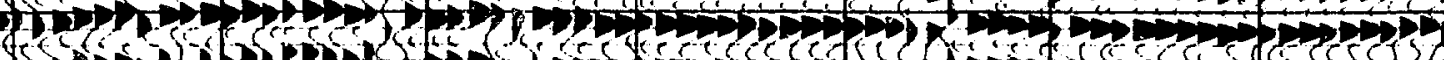

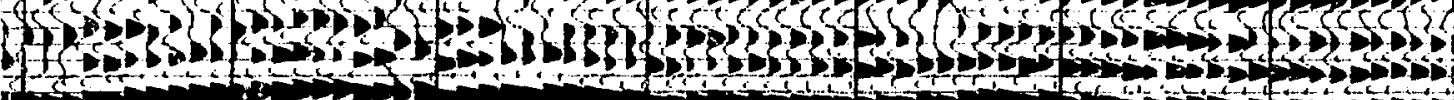

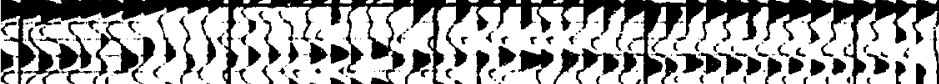

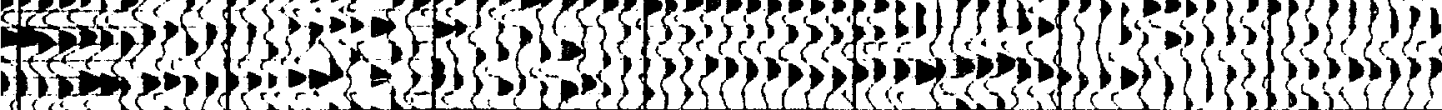

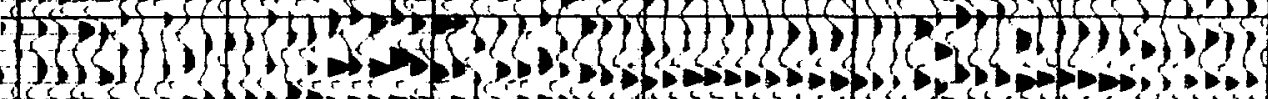
(4)

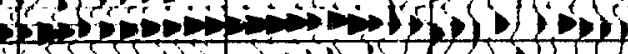

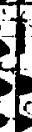
D.t?

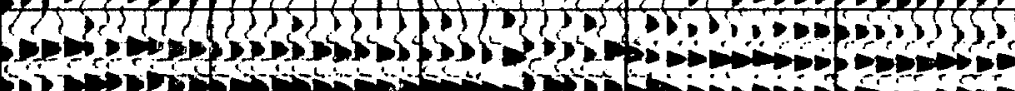
$\left\{\begin{array}{c}0 \\ 0\end{array}\right.$

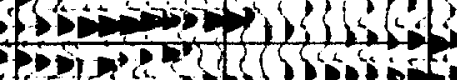

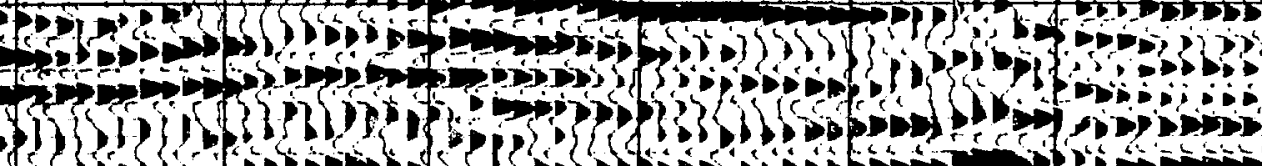

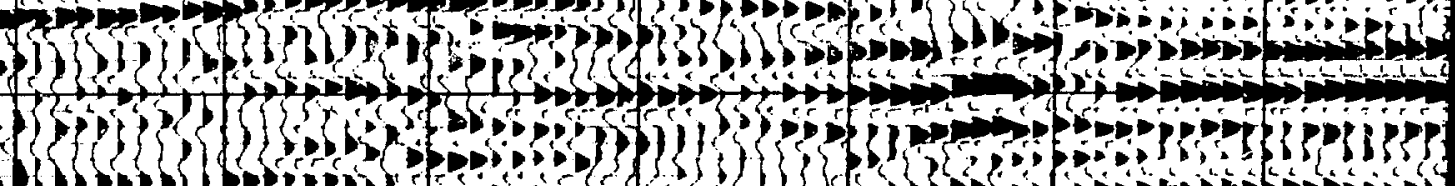

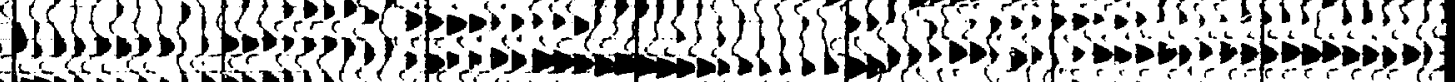

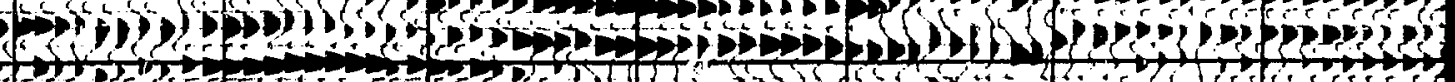

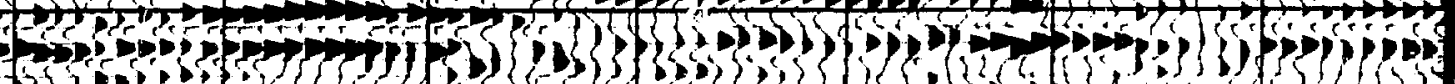

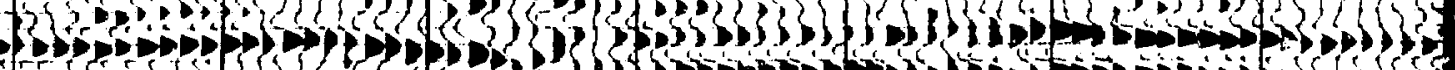

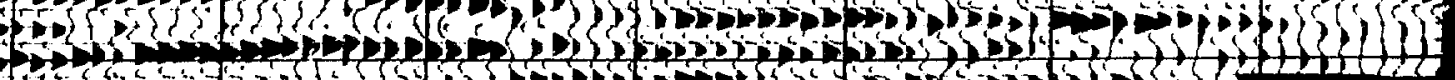

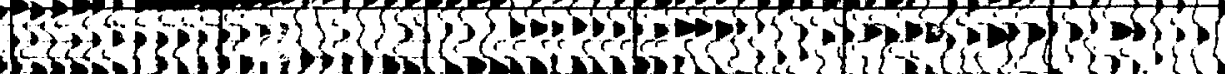

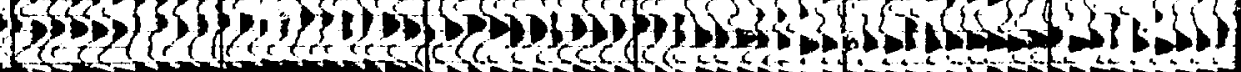




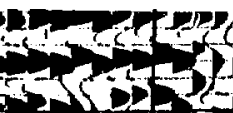

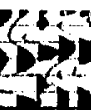

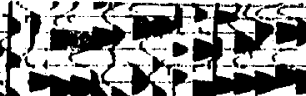

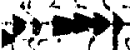

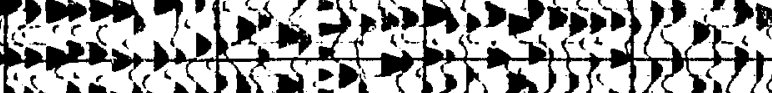




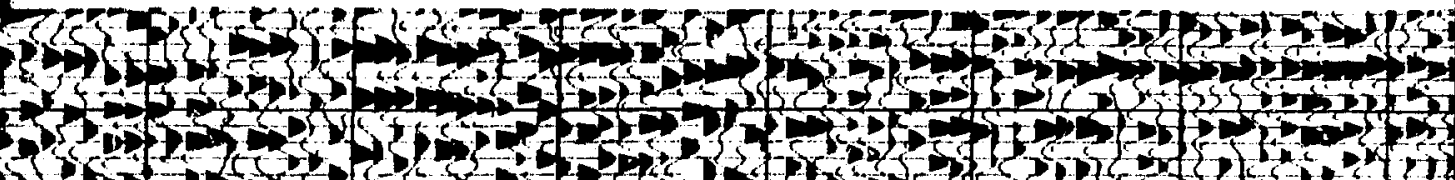

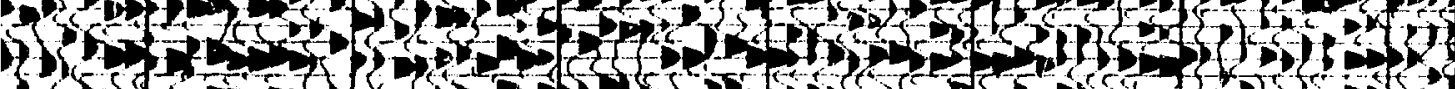

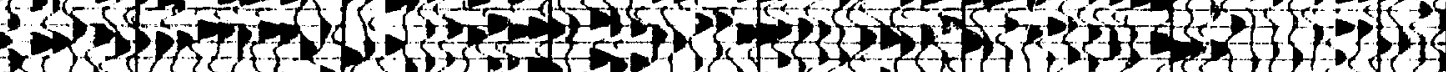

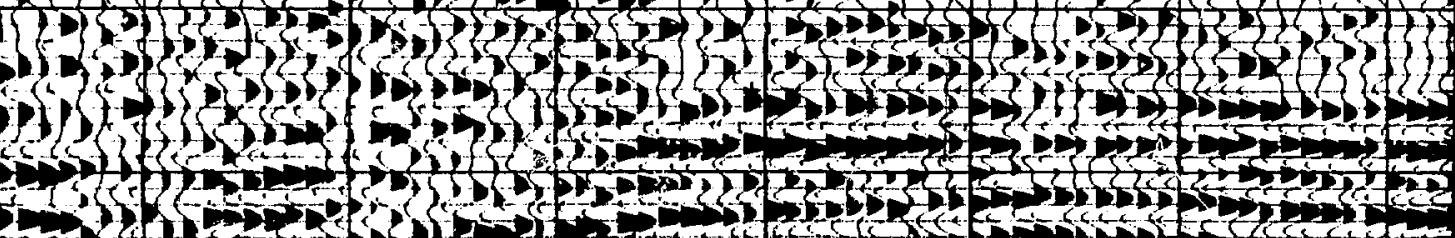

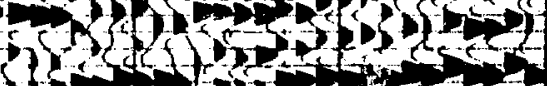

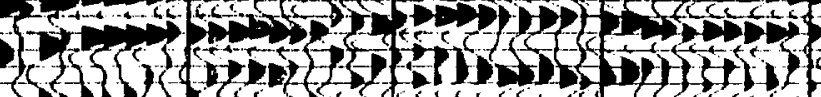

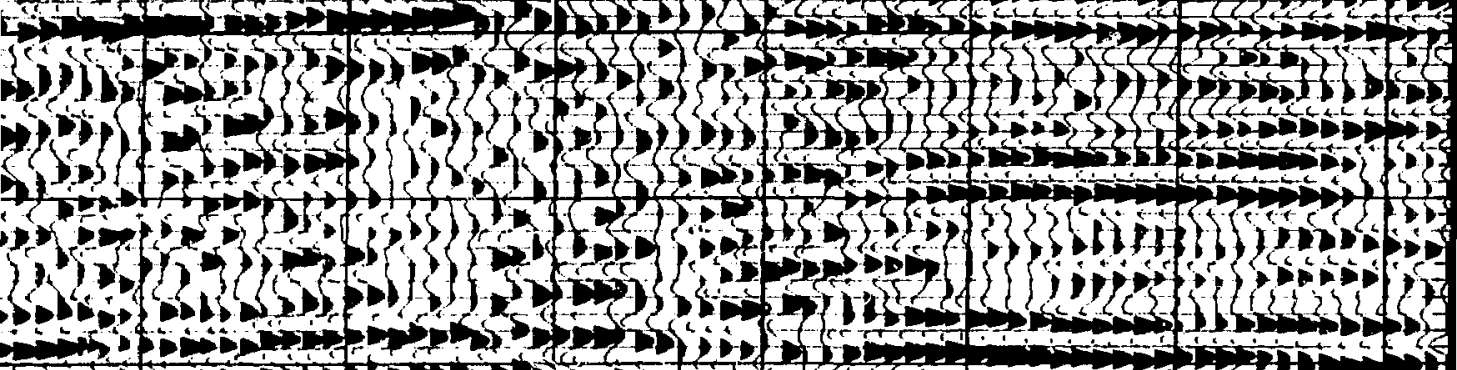

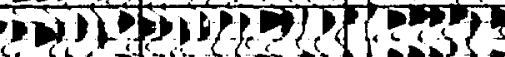

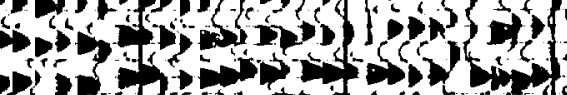
1) $\left\{\begin{array}{l}3 \\ 2\end{array}\right.$

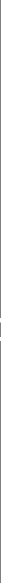




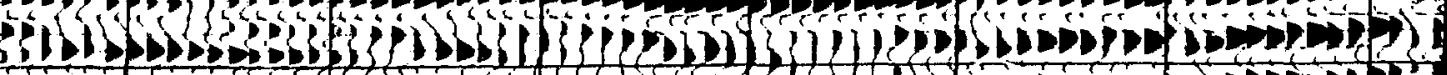

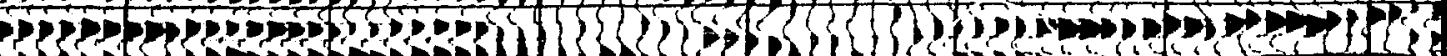

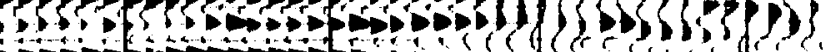





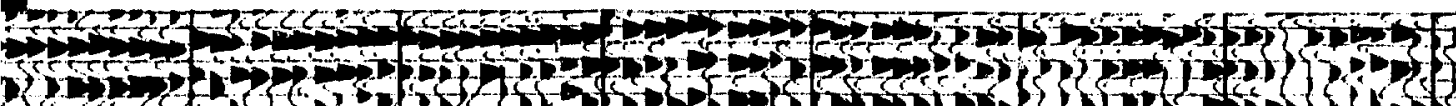

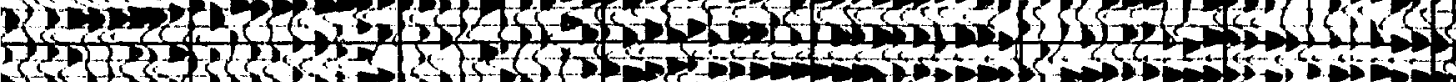

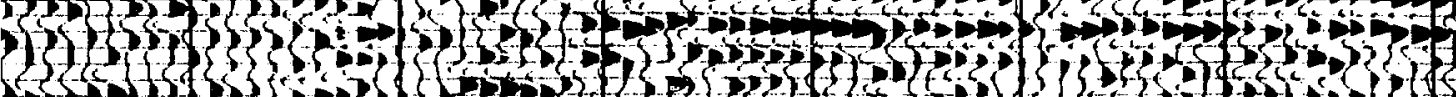

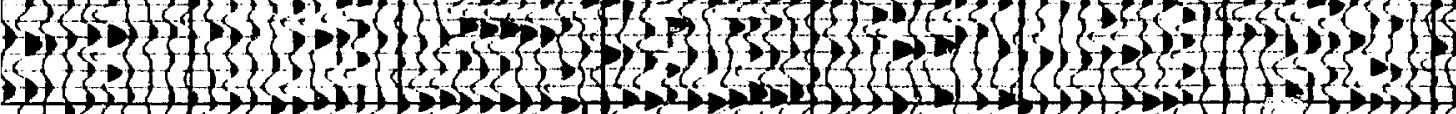

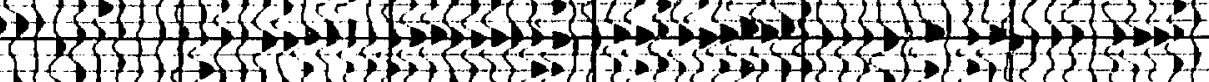

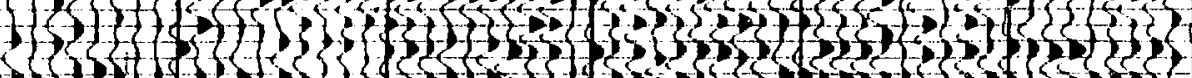

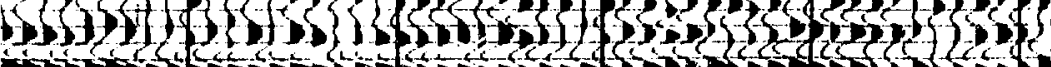

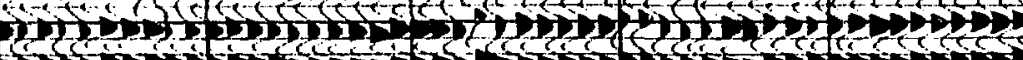

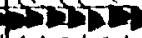




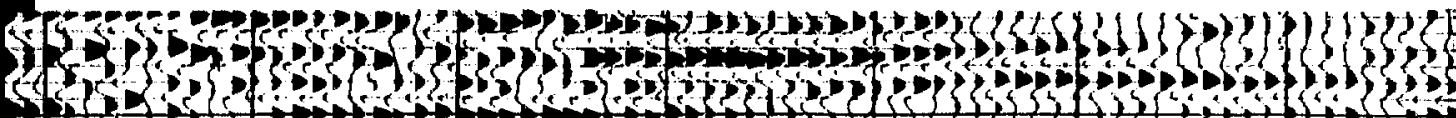

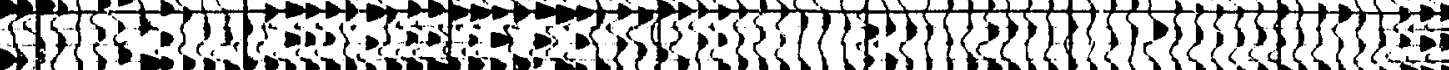

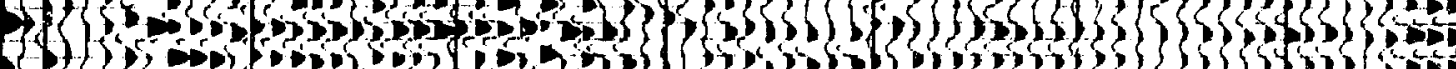

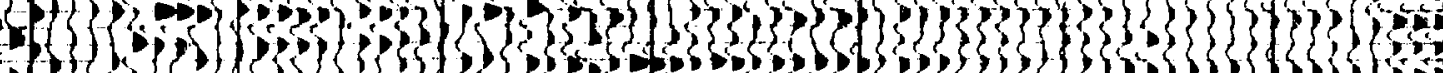

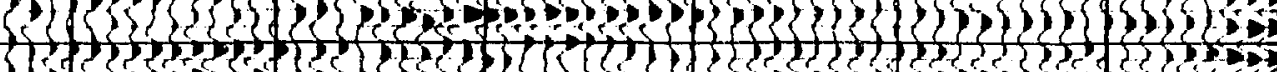

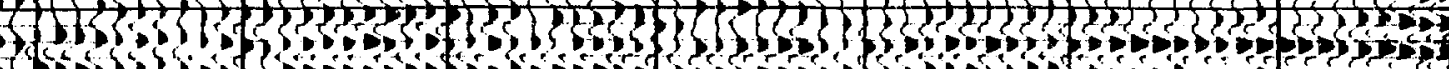

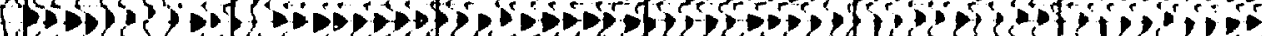

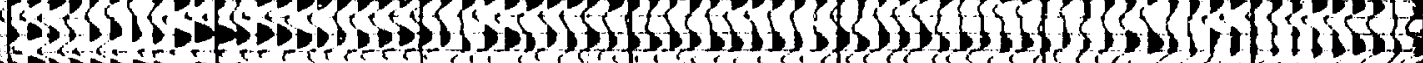

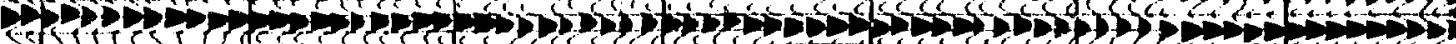

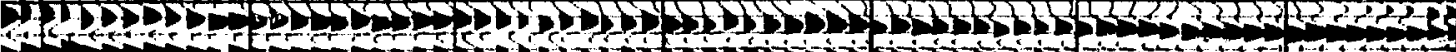

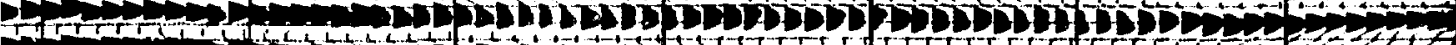

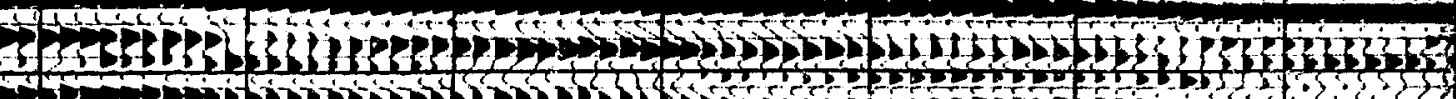

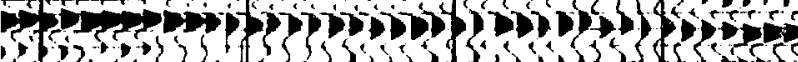

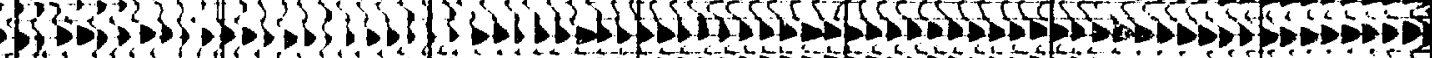

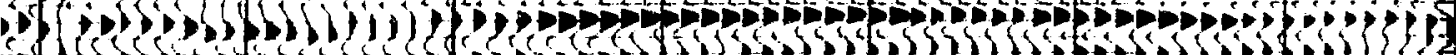
$3(2)$ 13 38 ji

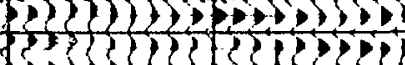

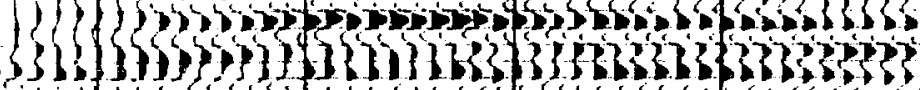

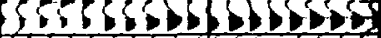

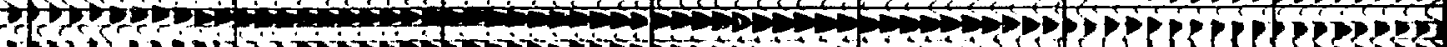

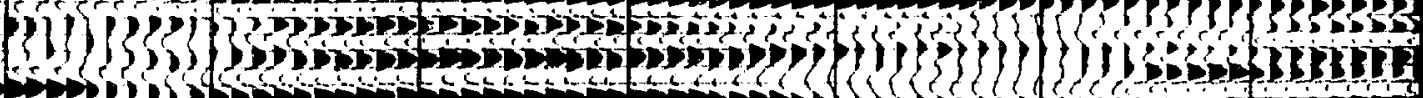

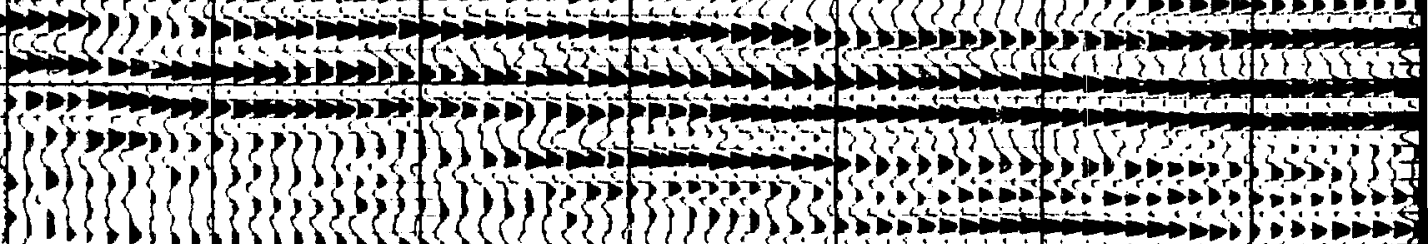

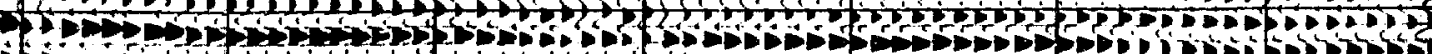
(c) (l)

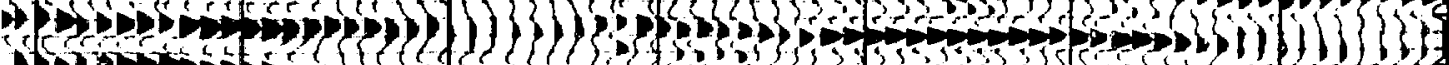

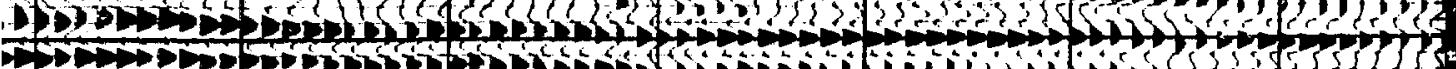

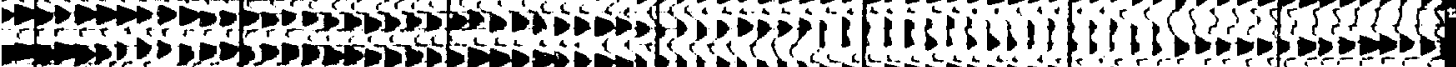

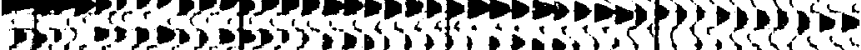

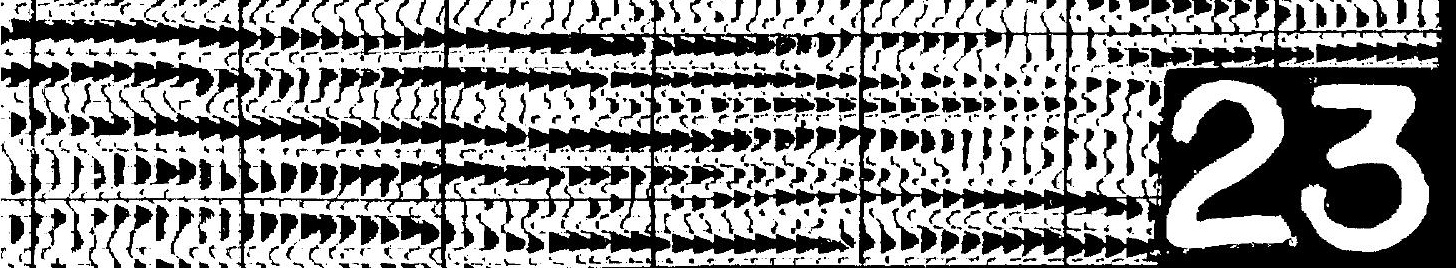




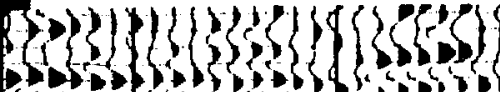

13

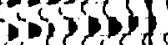

$\{\{\}\{\}$

$3\}\{\}\{3\}$

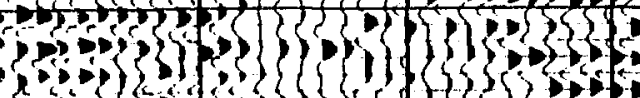

i)

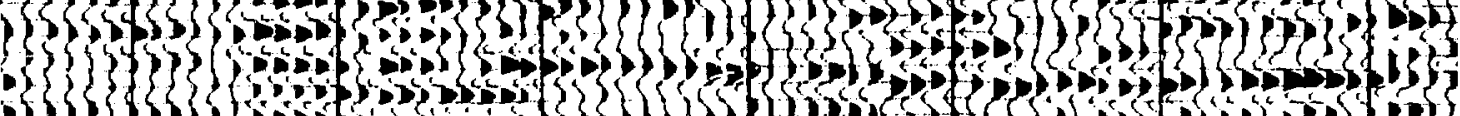

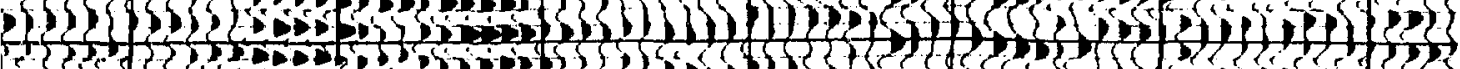

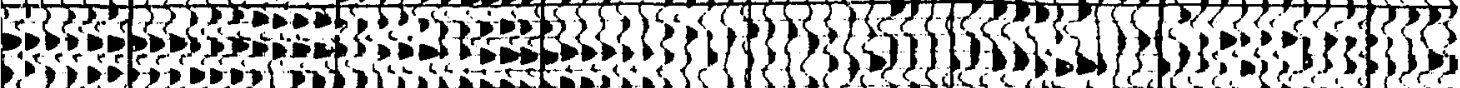

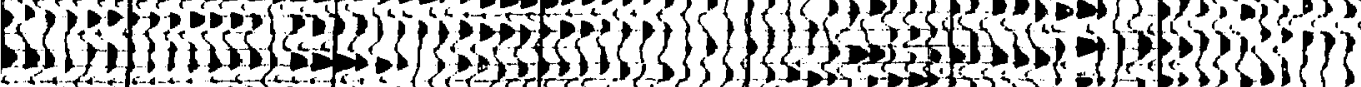

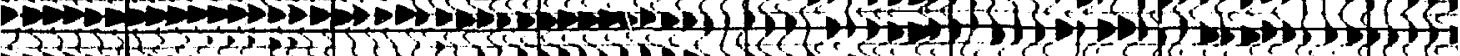

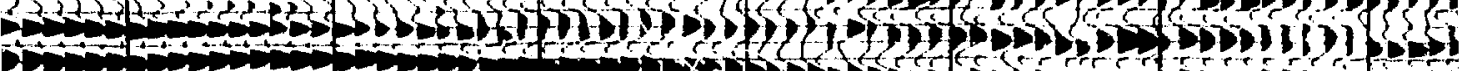

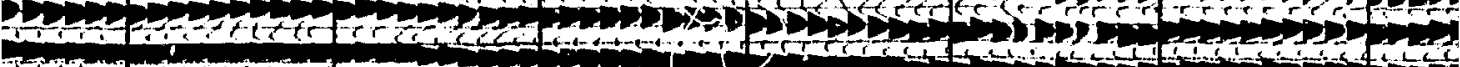

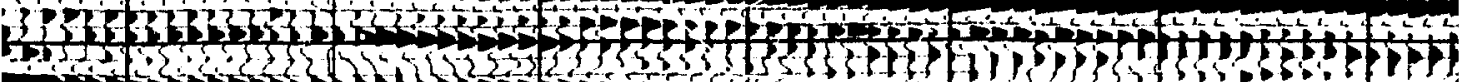

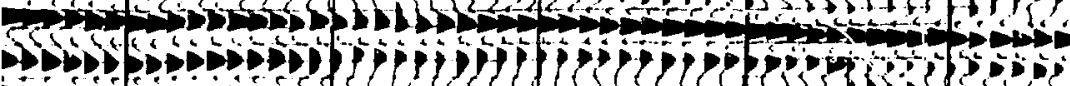

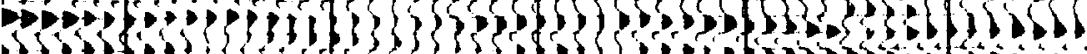
(b) 1) 3 find

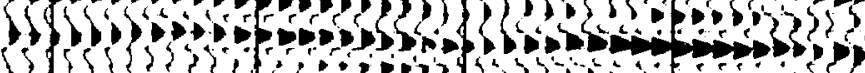

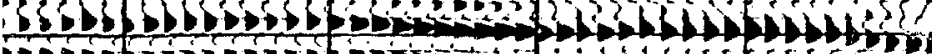

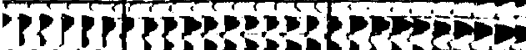

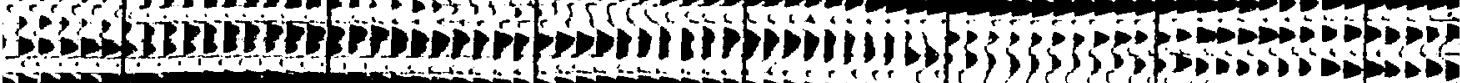
Hat

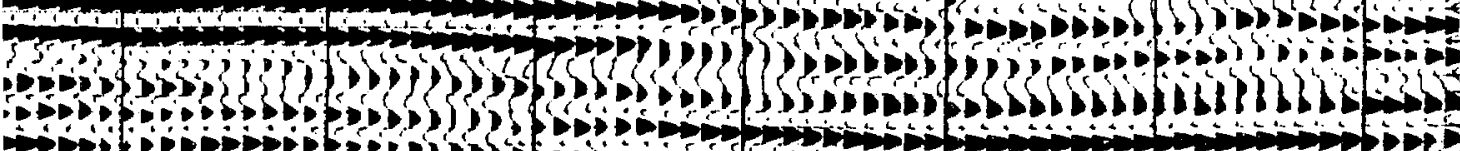

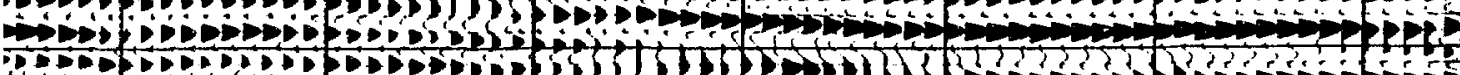

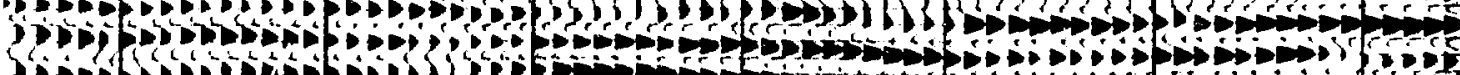

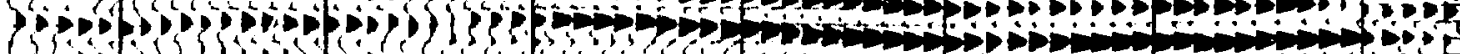

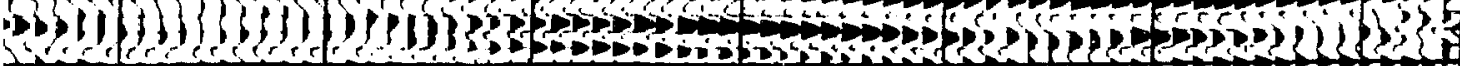

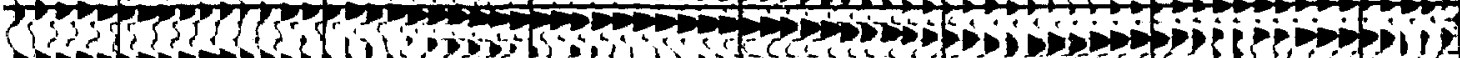

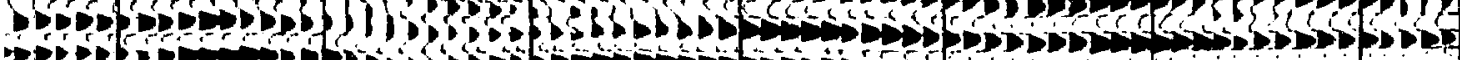
$\rightarrow-0$ \&

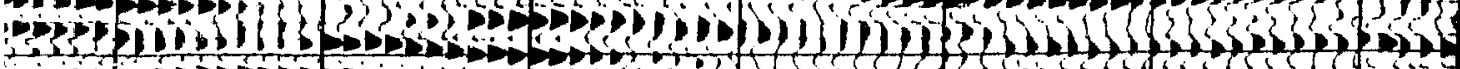

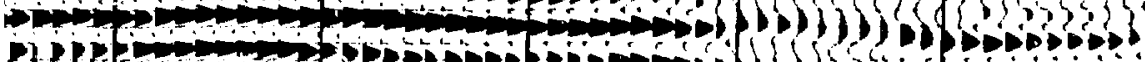

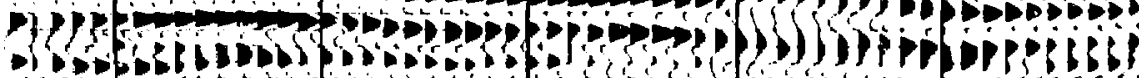

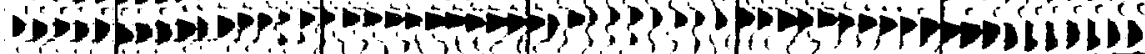
De

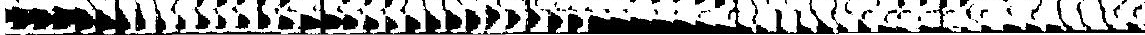

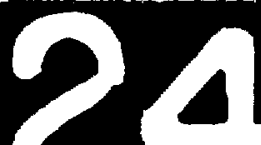




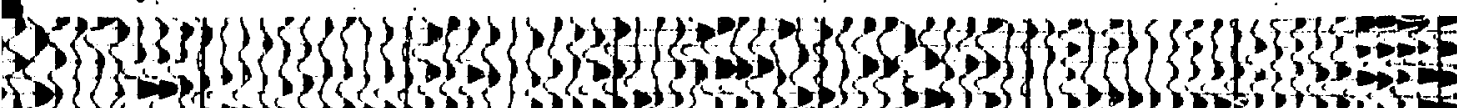

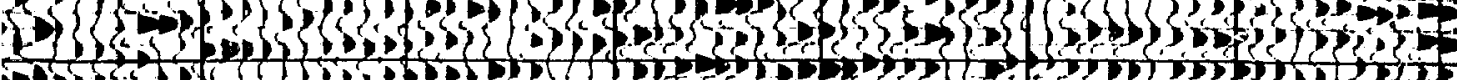

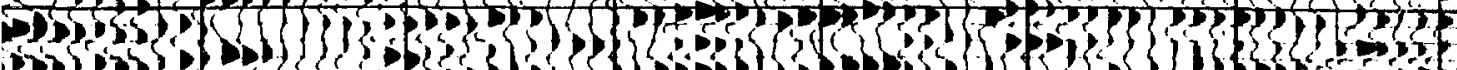

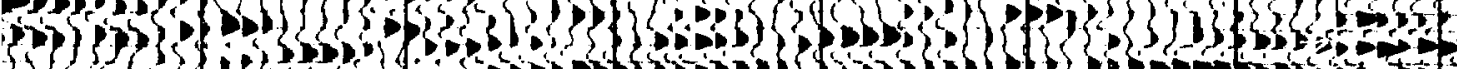

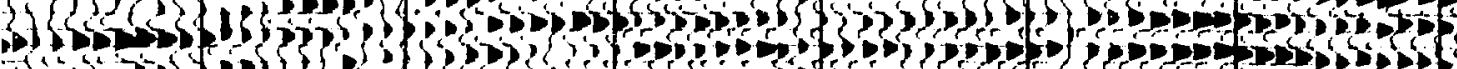

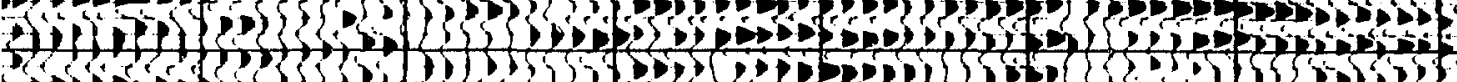

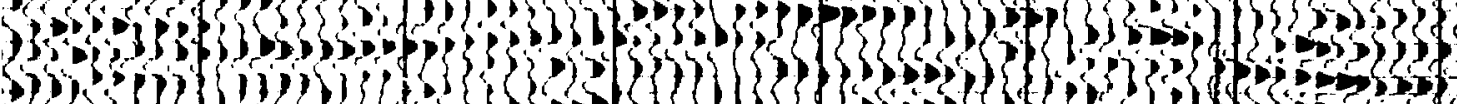

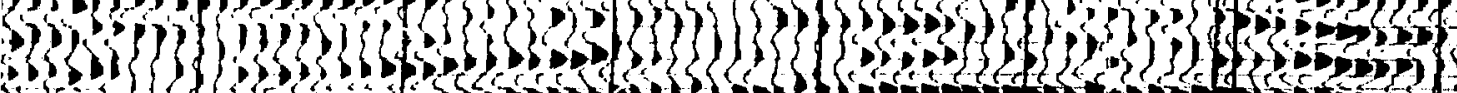

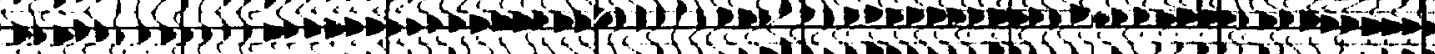

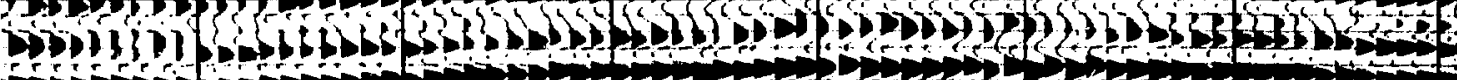

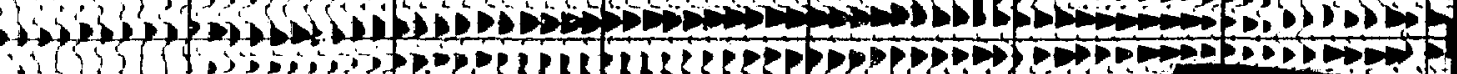

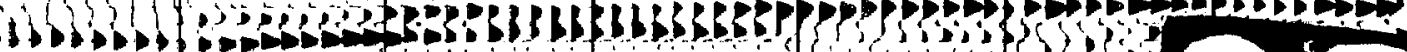

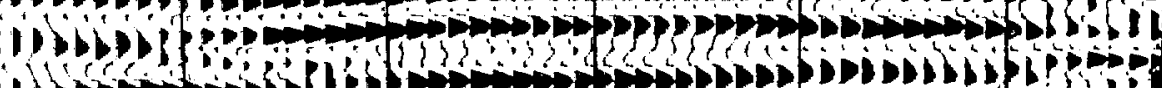

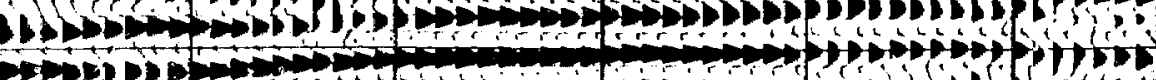




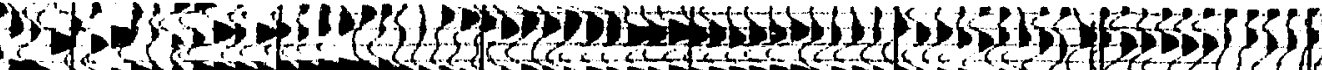
P

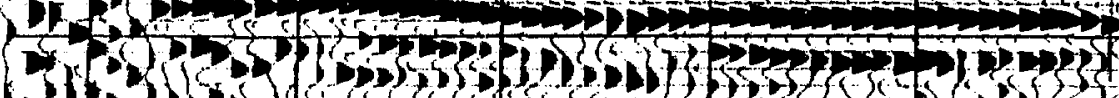

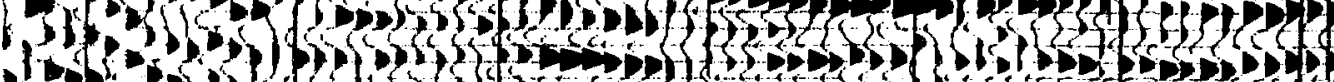

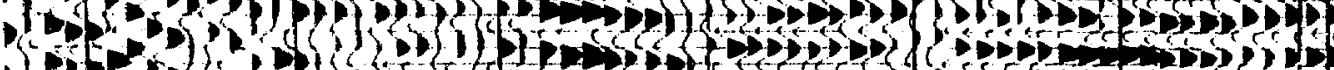

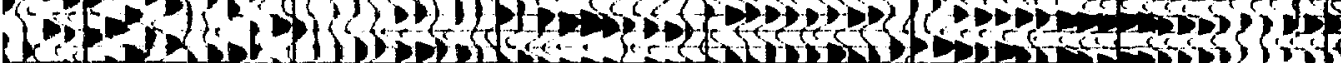

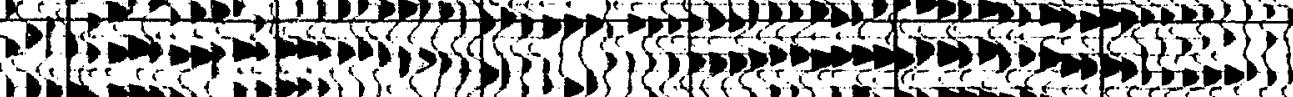

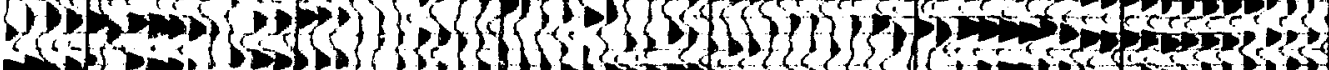

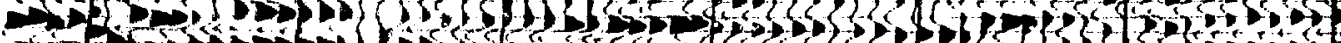

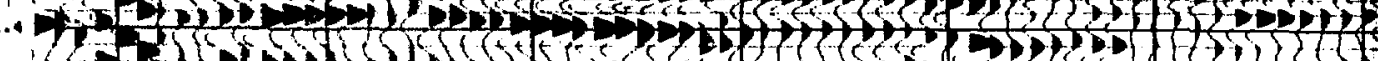

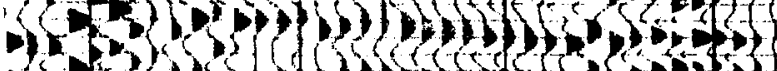

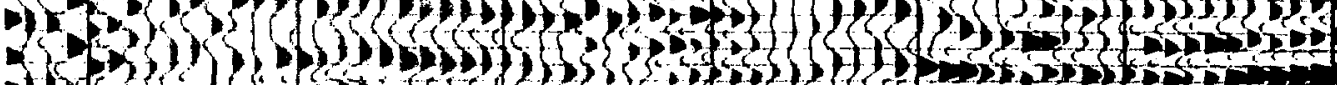

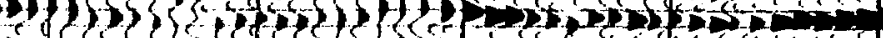

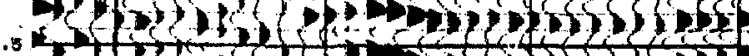

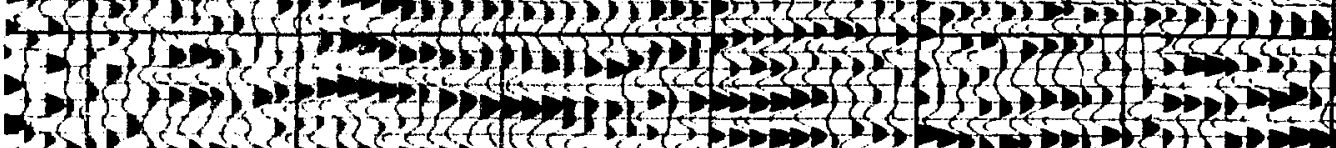

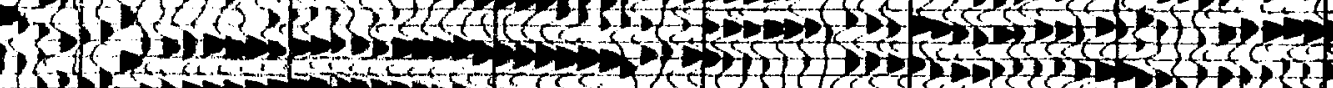

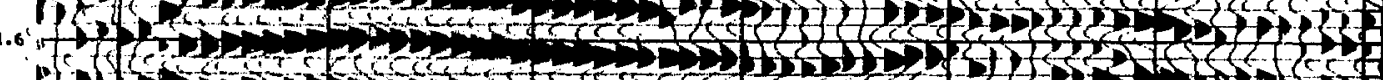

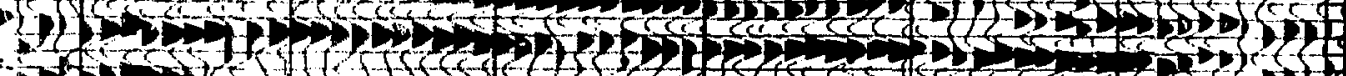

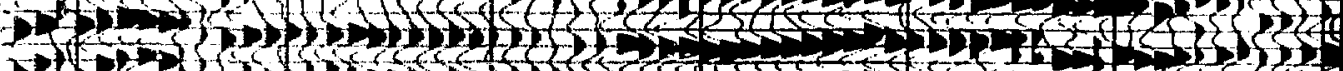

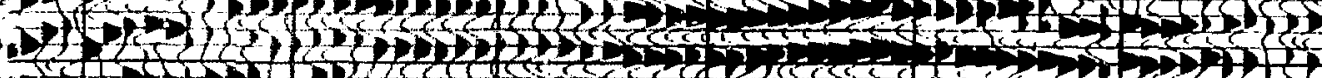

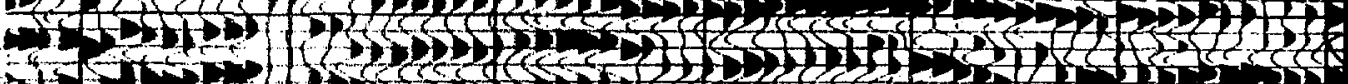

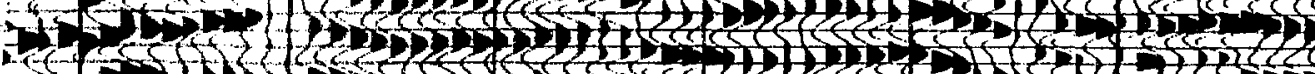

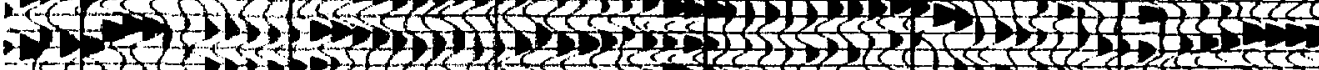

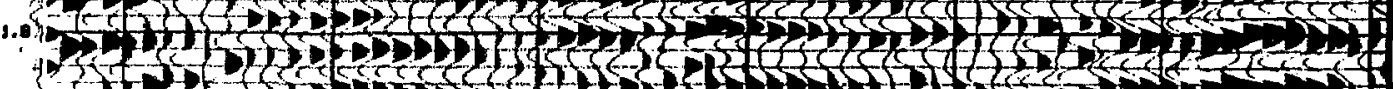

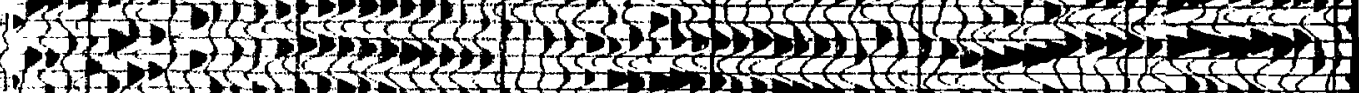

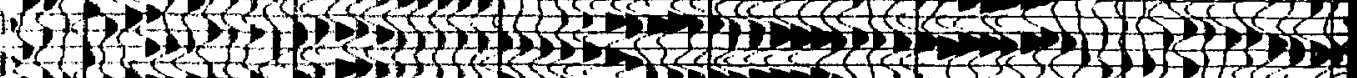

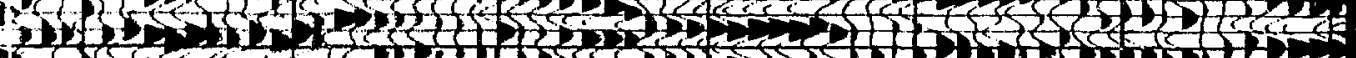

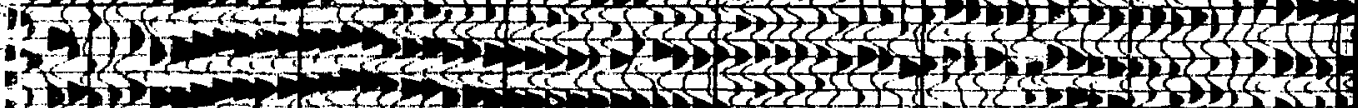

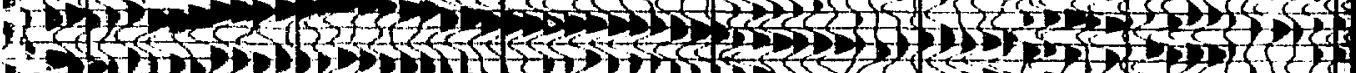

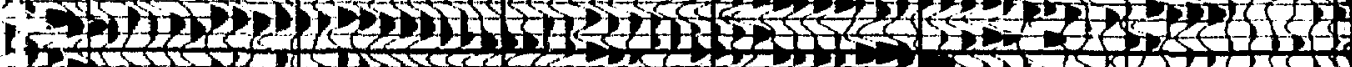

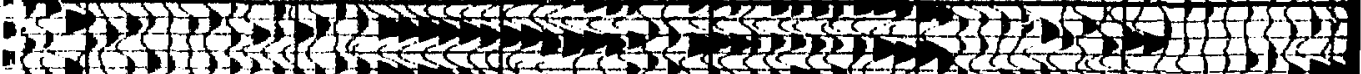

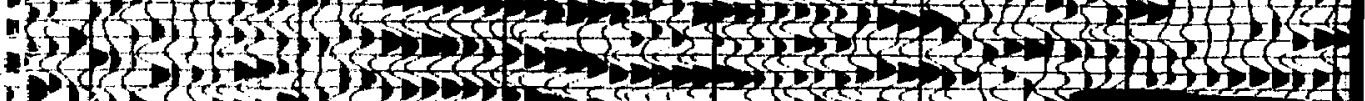

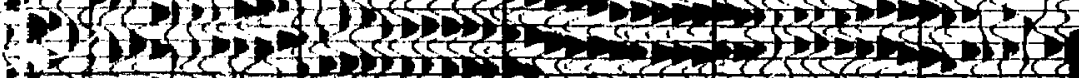

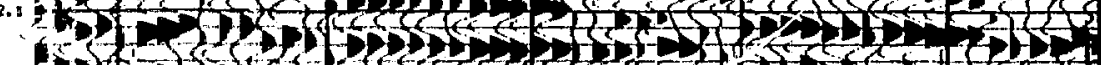
Ex 
3)

3 (1)

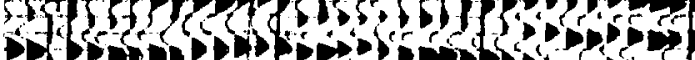

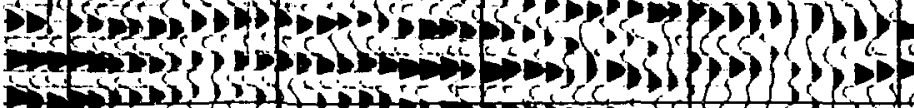

Sof

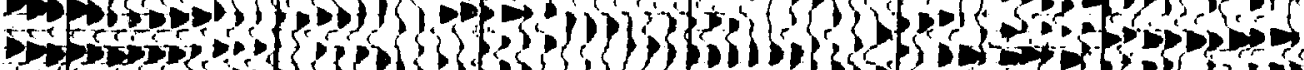

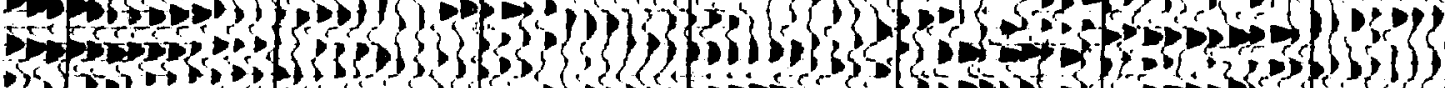

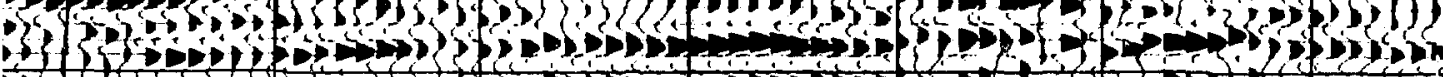

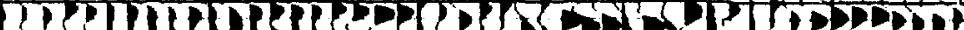

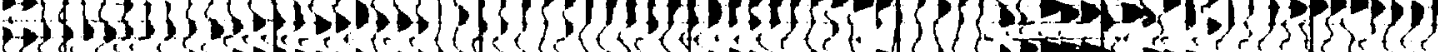

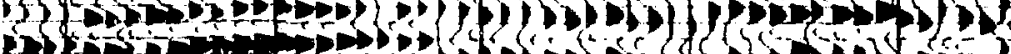

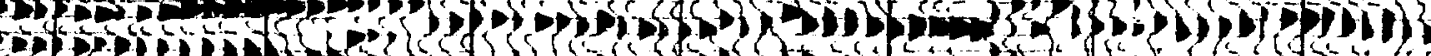

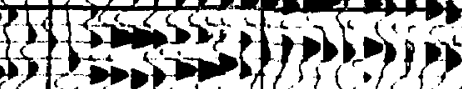

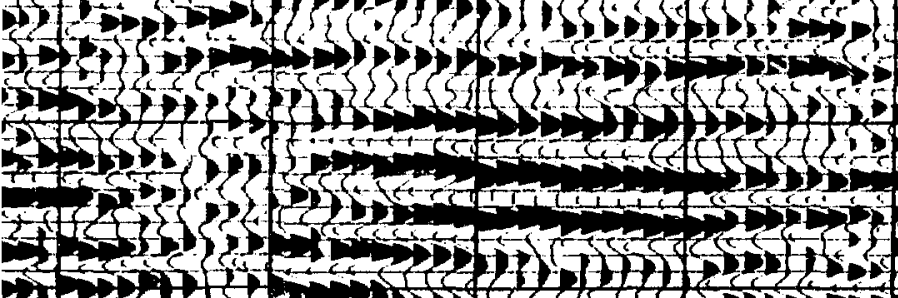

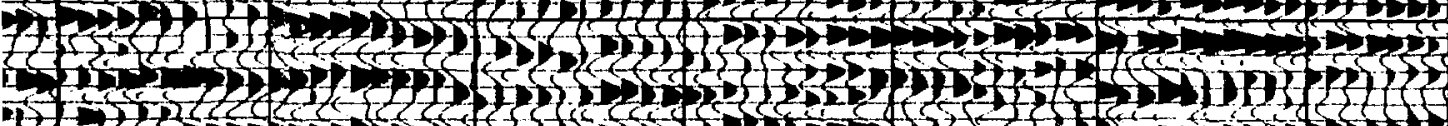

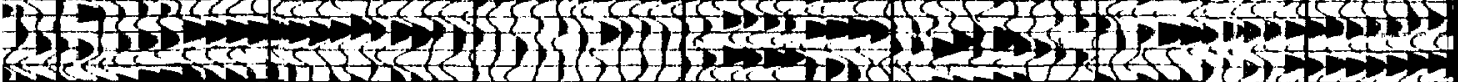

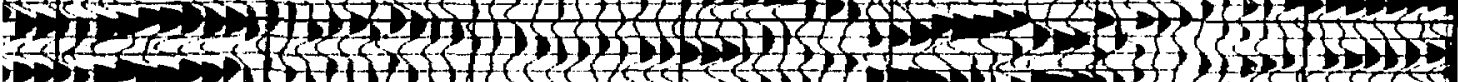

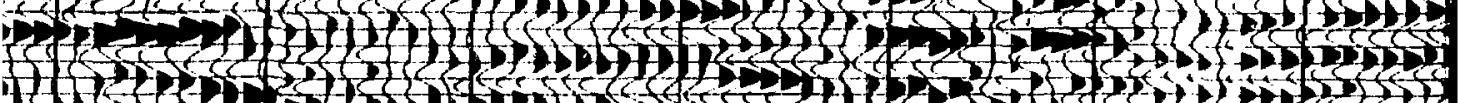

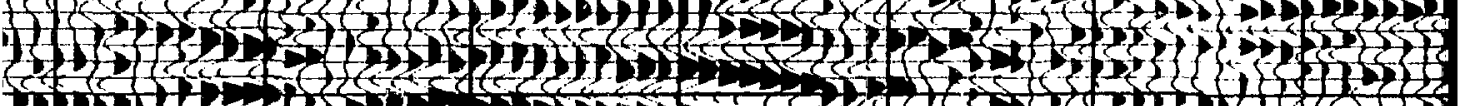

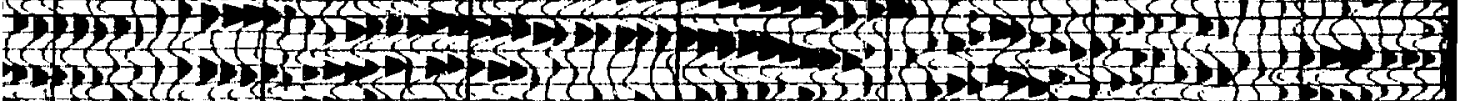

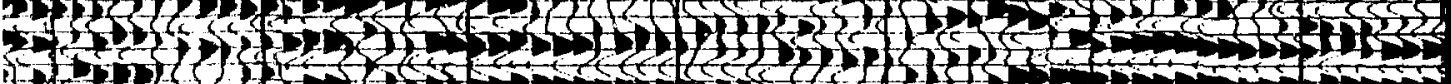

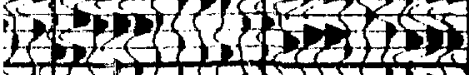

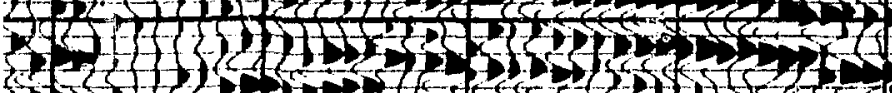

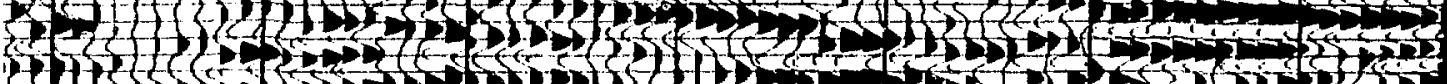

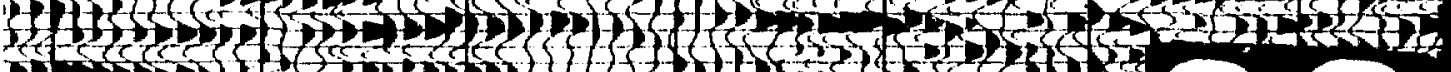

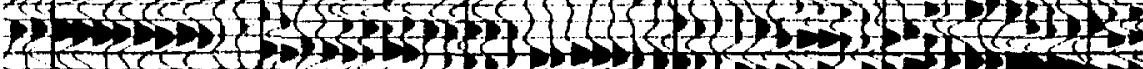

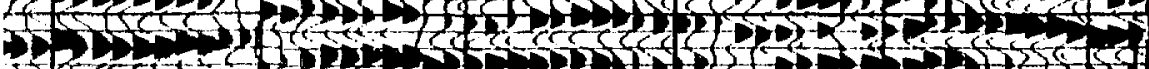

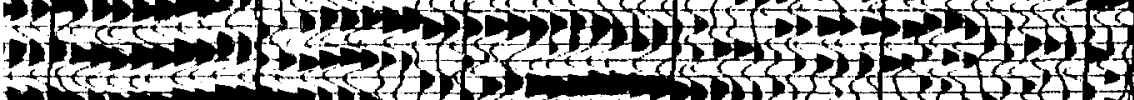

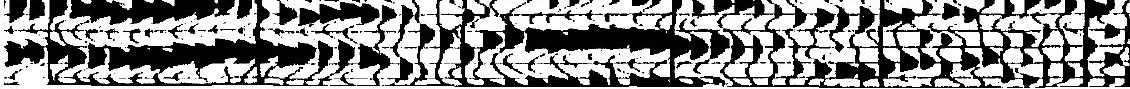




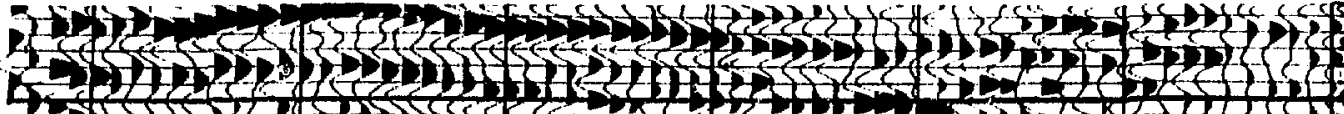

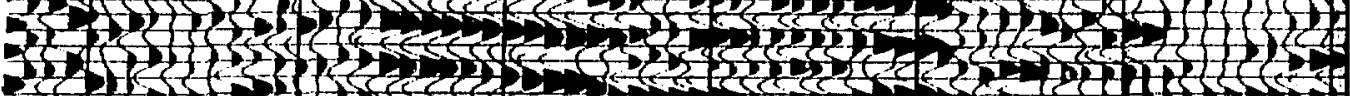

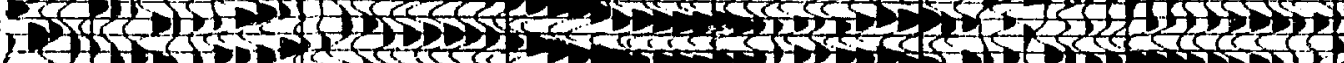

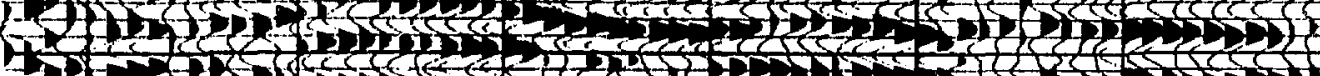

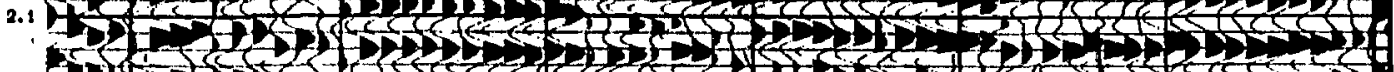

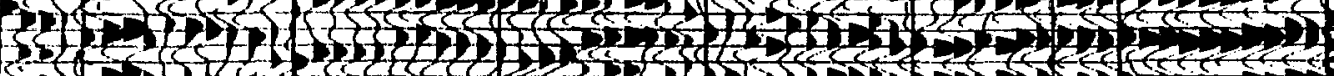

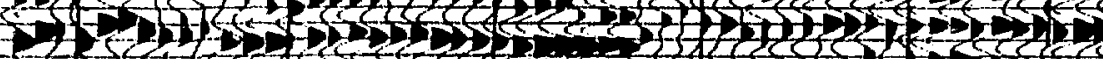

2.2

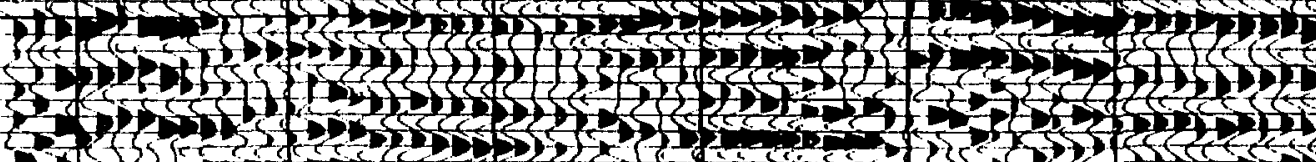

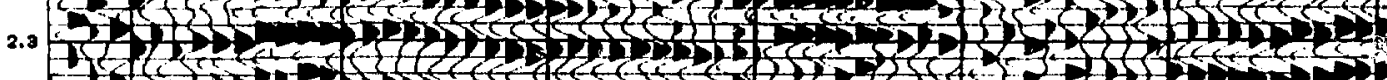

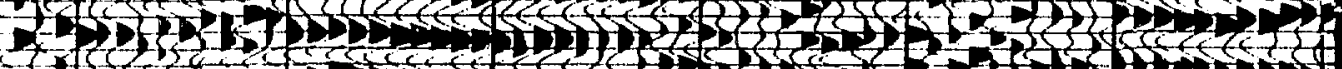

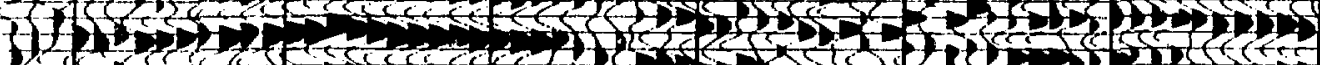

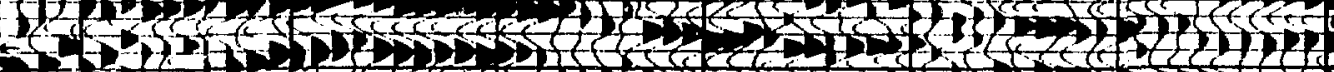

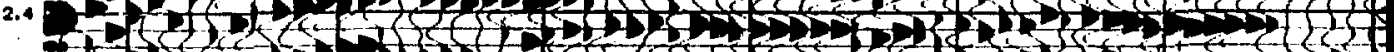

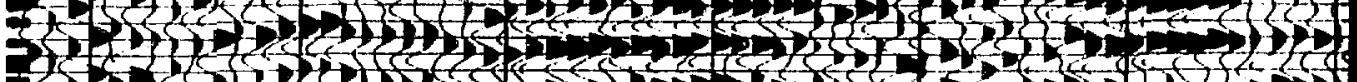

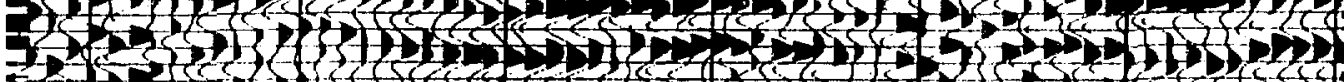

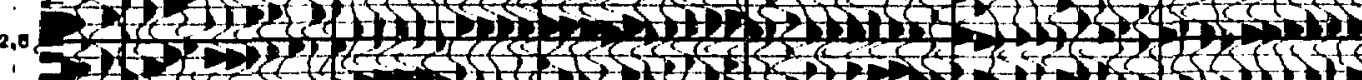

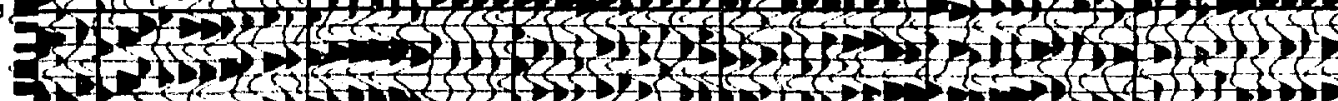

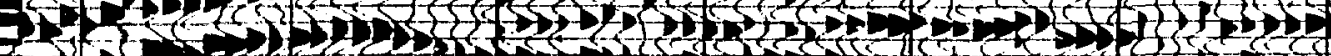

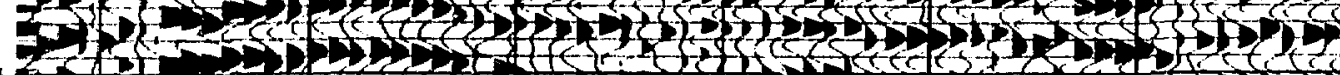

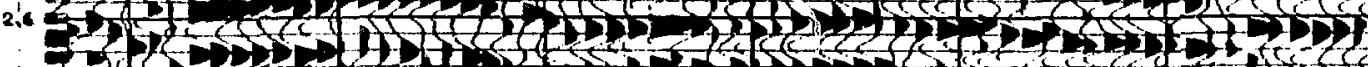

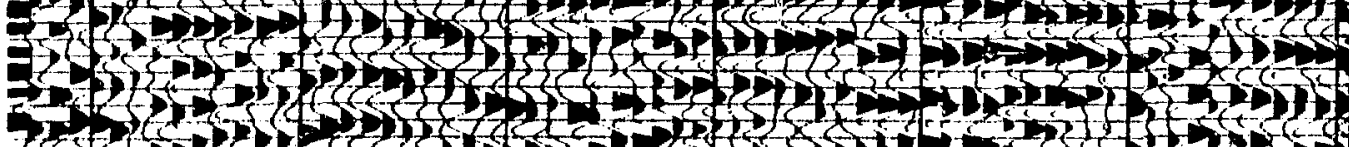
2. If

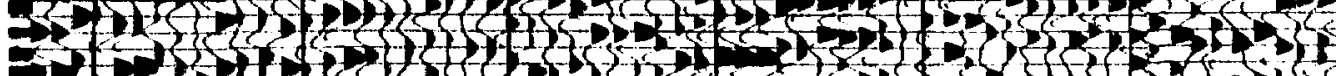

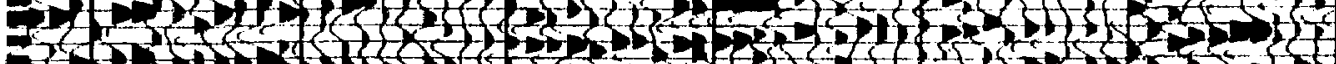

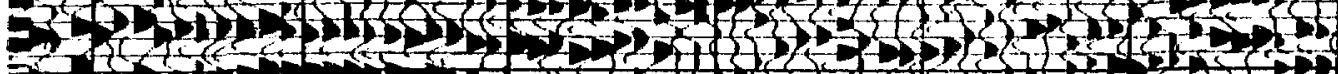

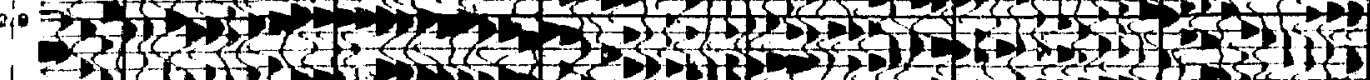

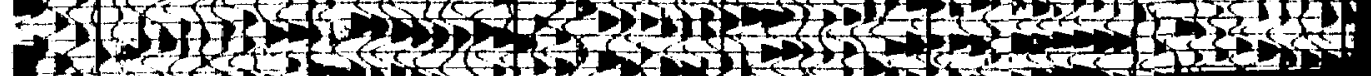

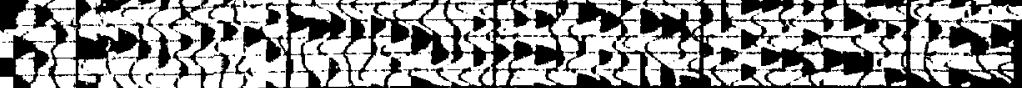

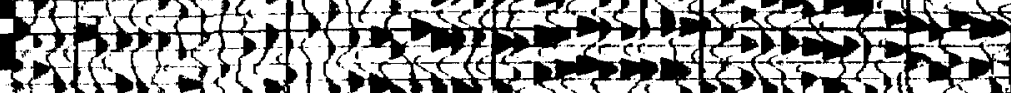

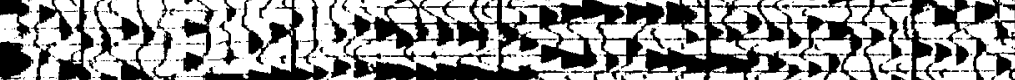
- 350 - 


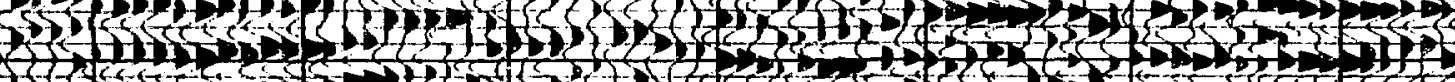

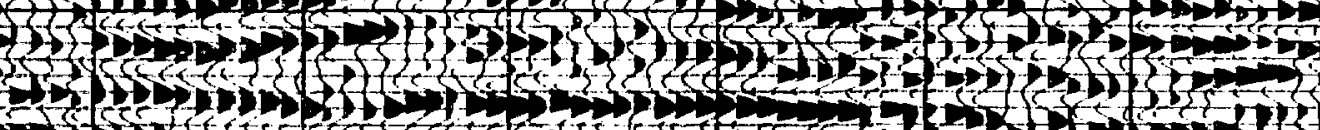




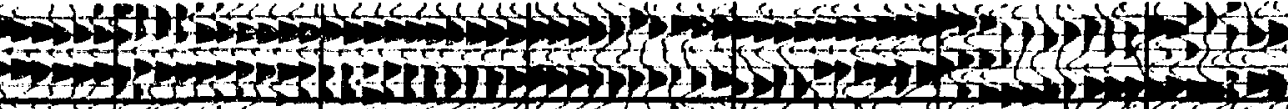

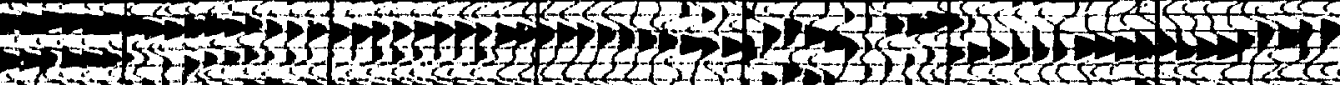

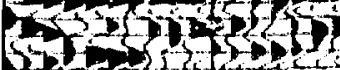

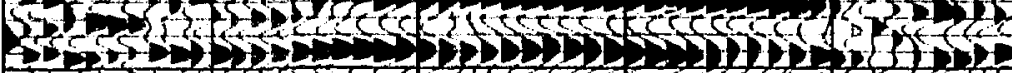

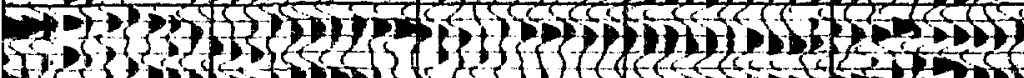

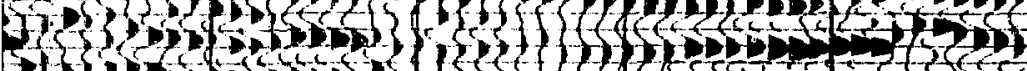

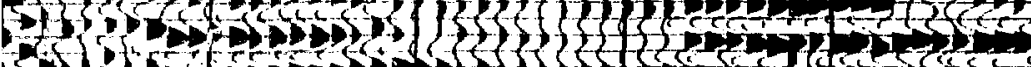
H.

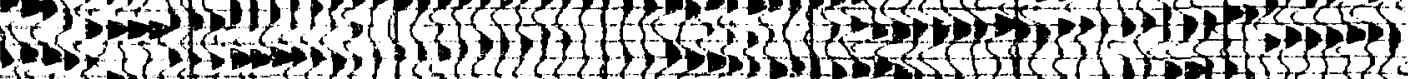

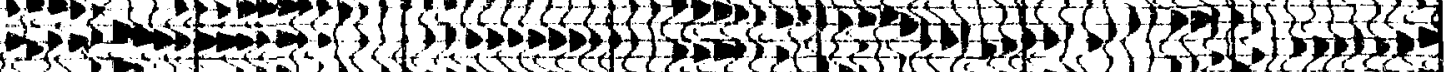

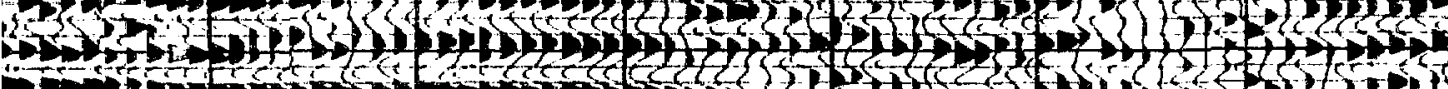

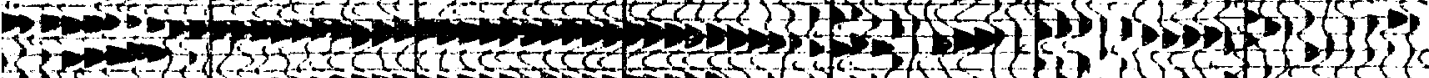

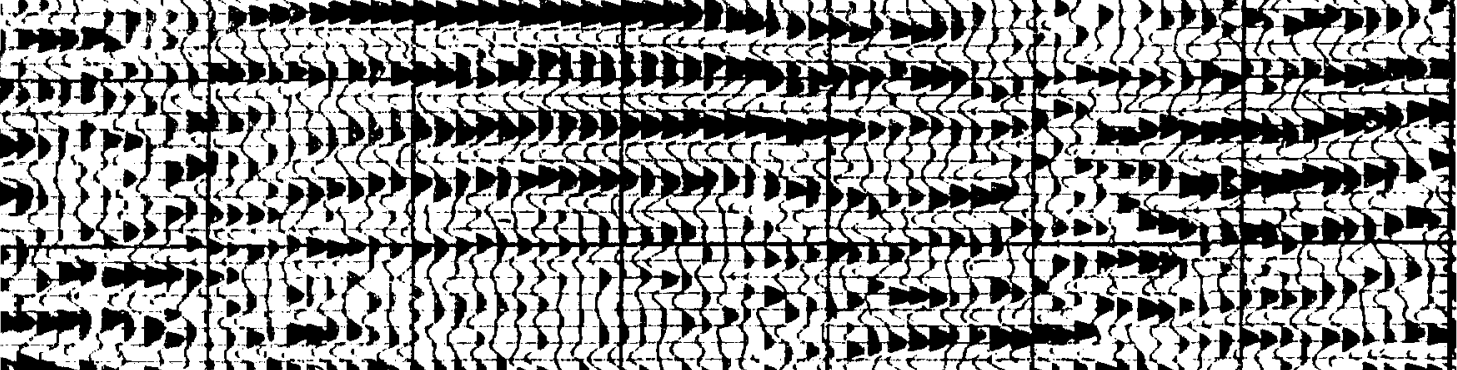
This

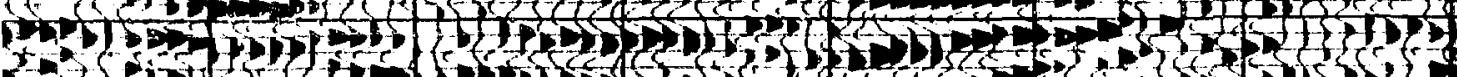
32.073.t5

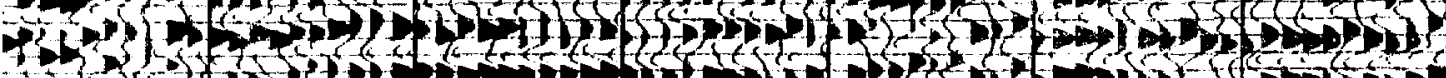

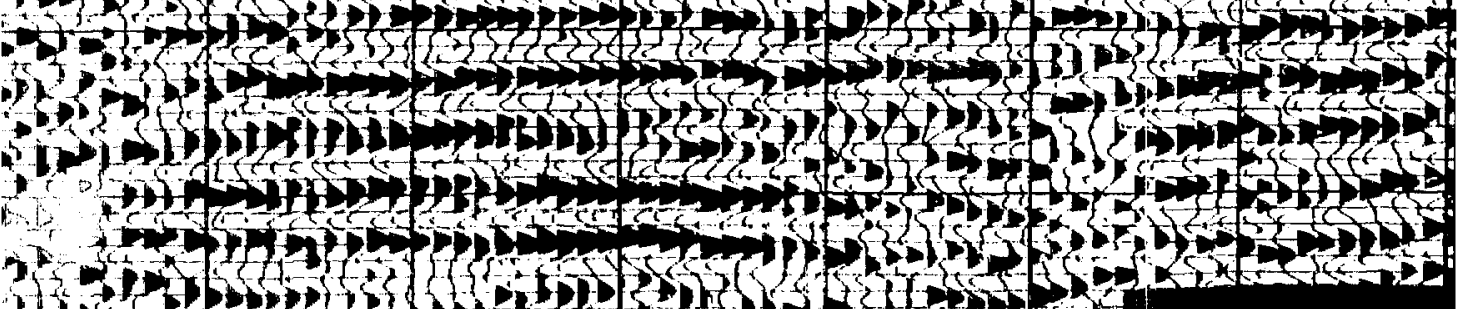

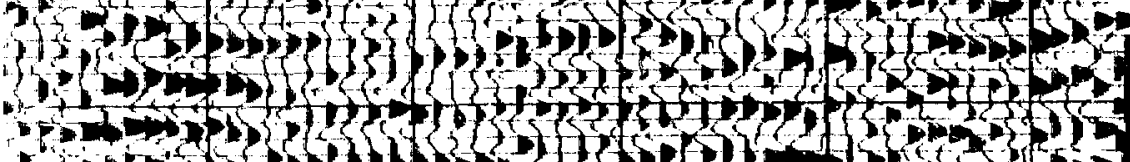

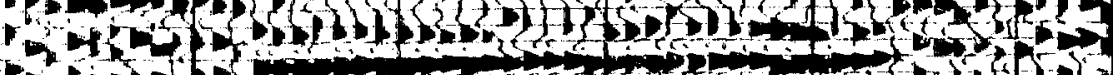


Bi) if 
13row

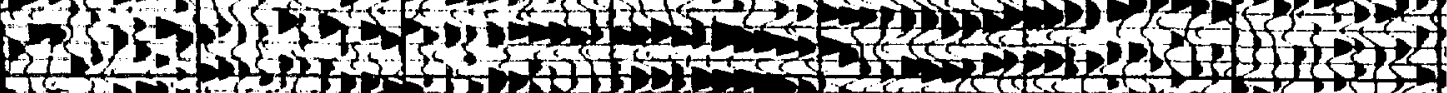

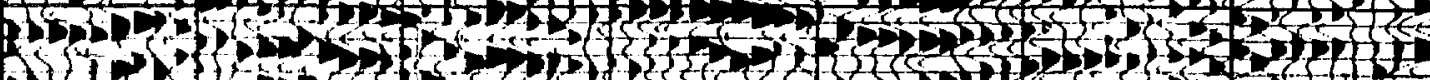

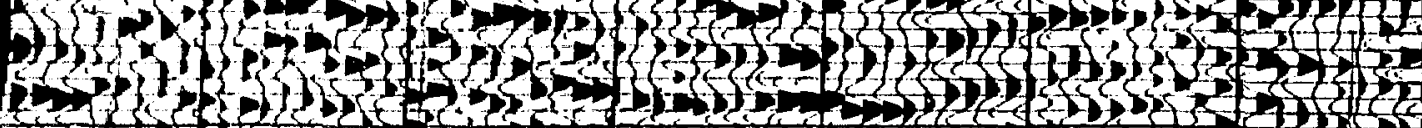

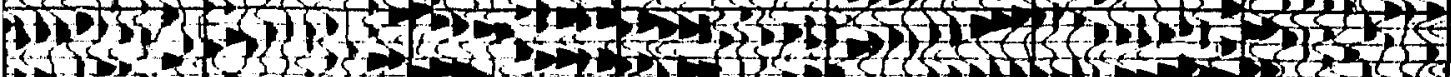

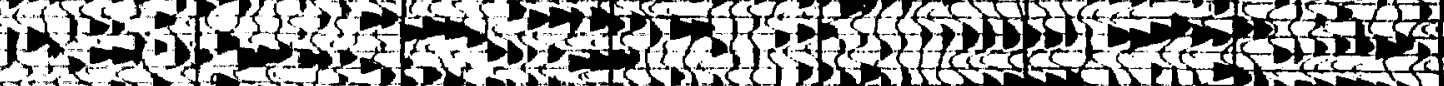

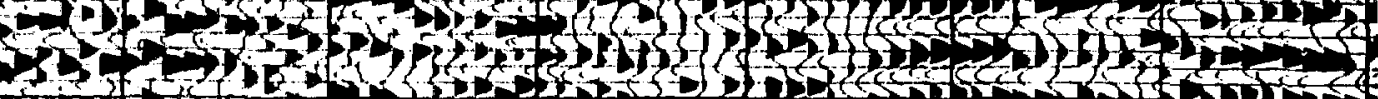

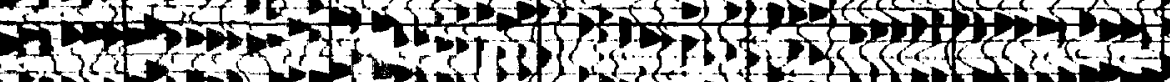

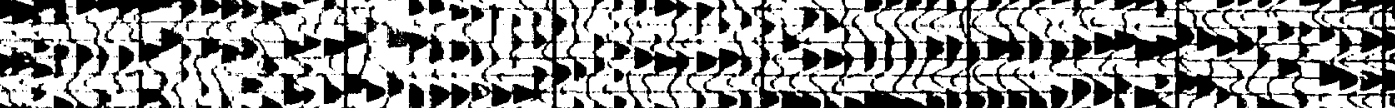

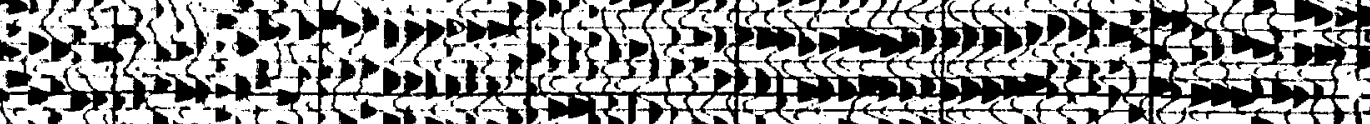

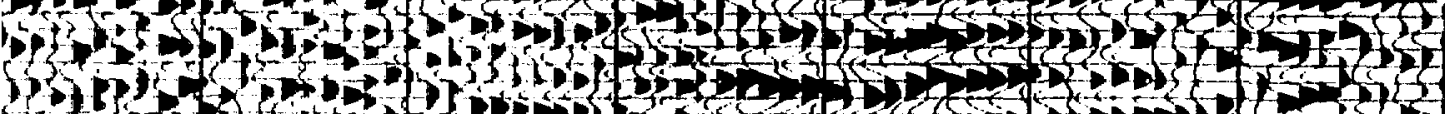

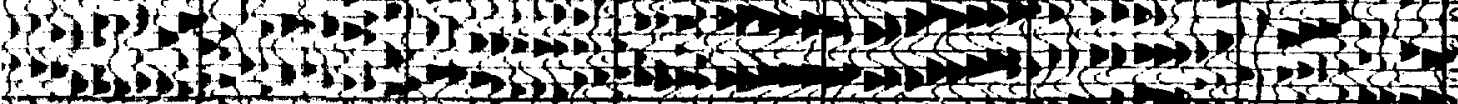

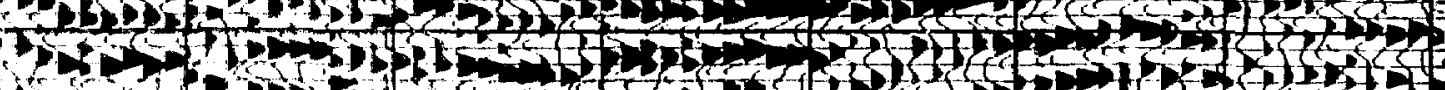

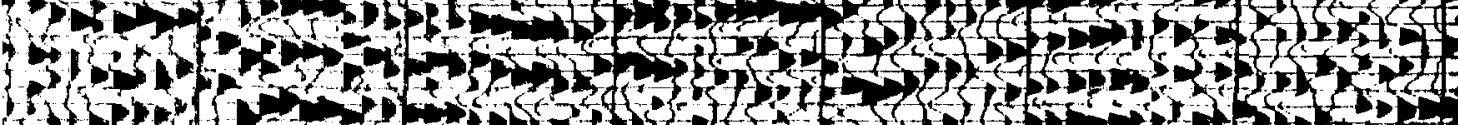

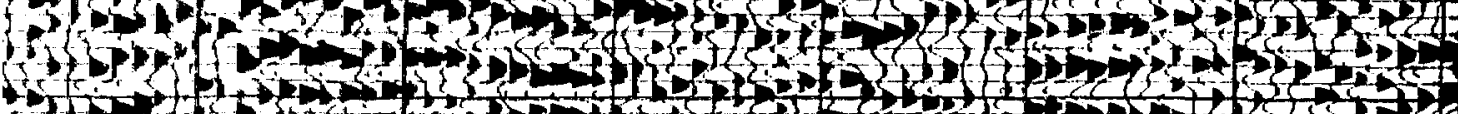

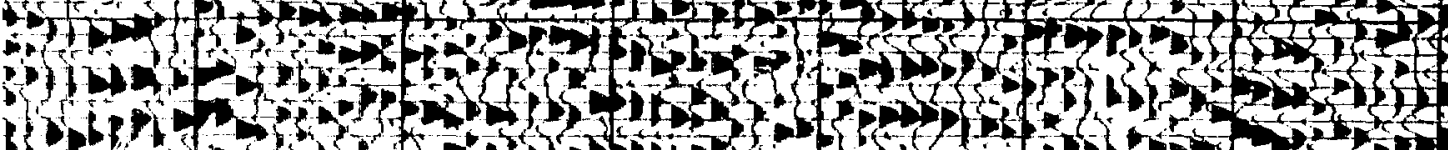

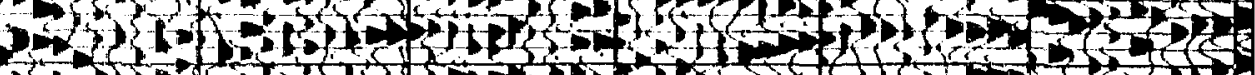

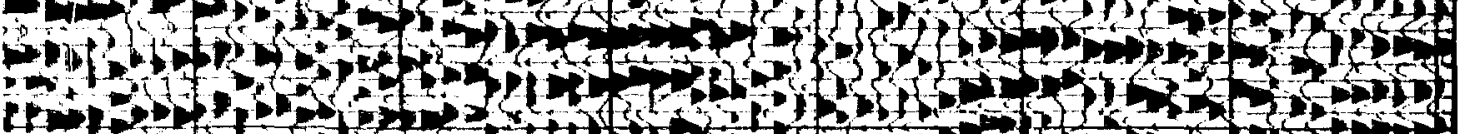

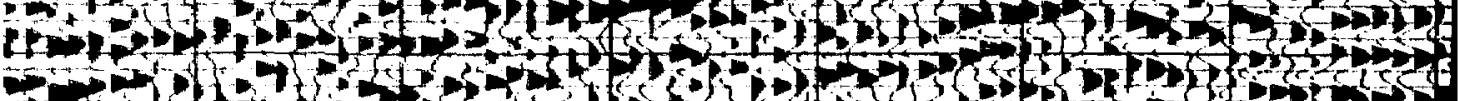

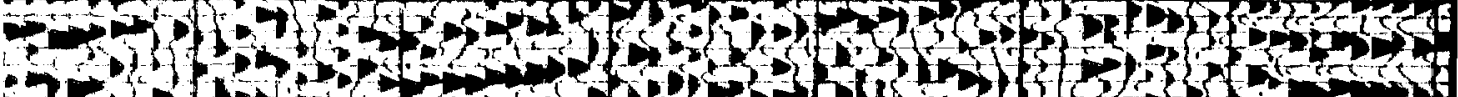

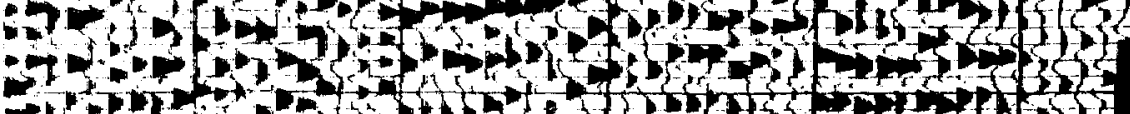

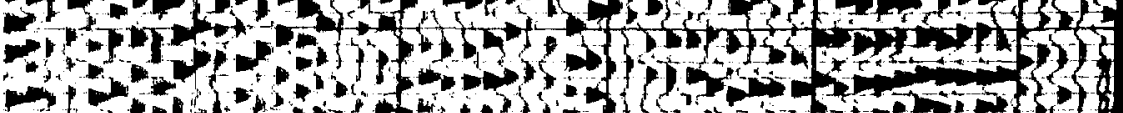


3.

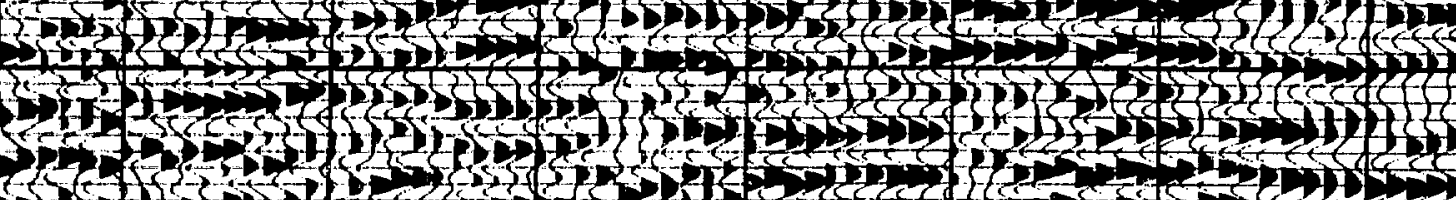
3.

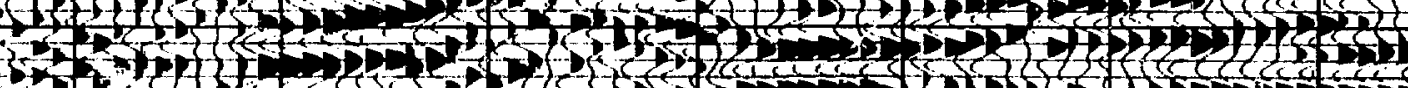

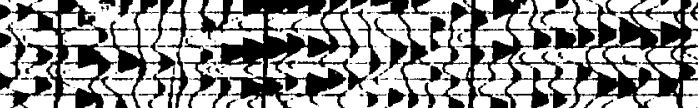

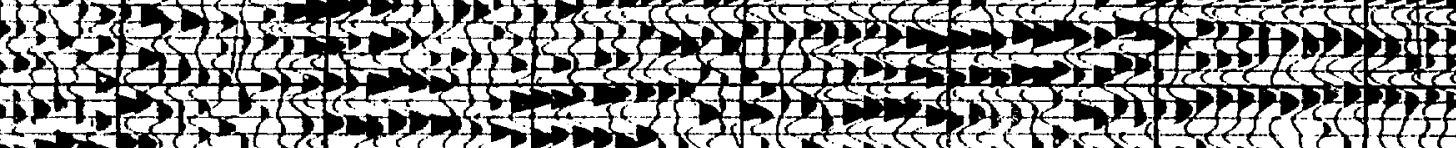

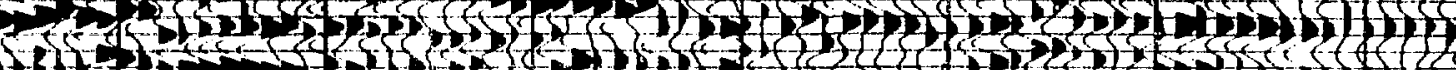

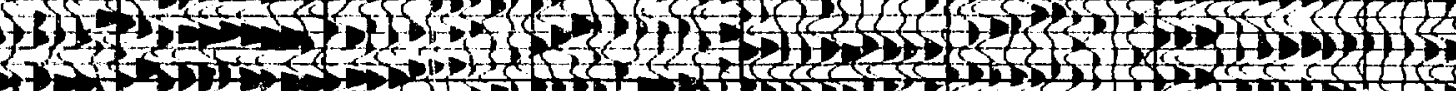
4 7.

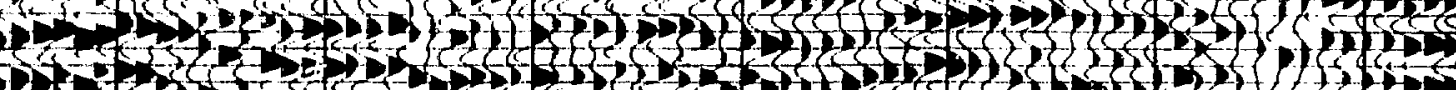

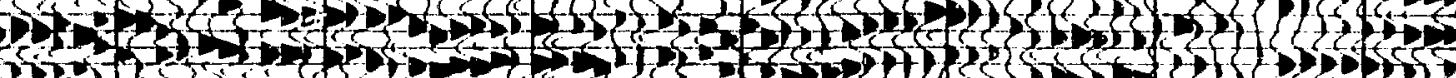

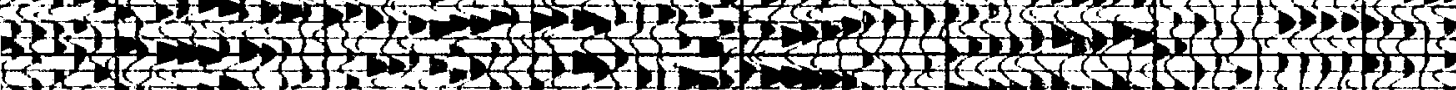

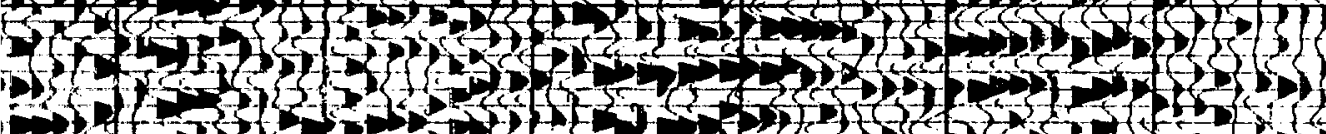

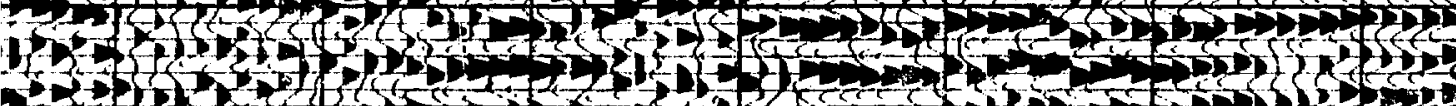

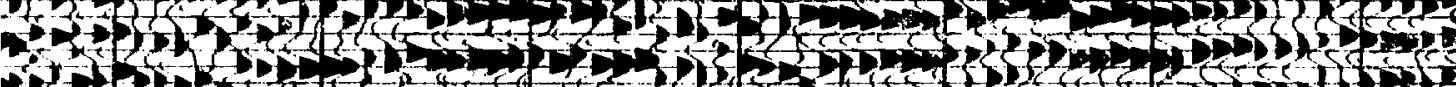
$7+3,2,1, \ldots$ S.

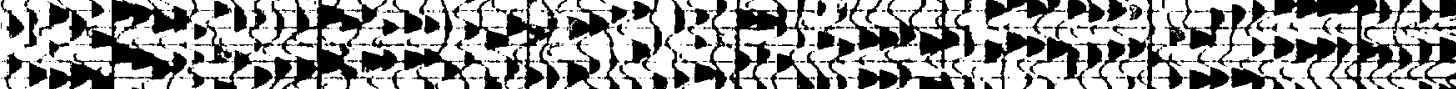

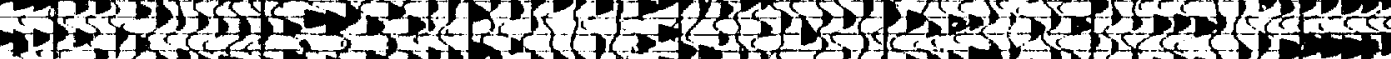

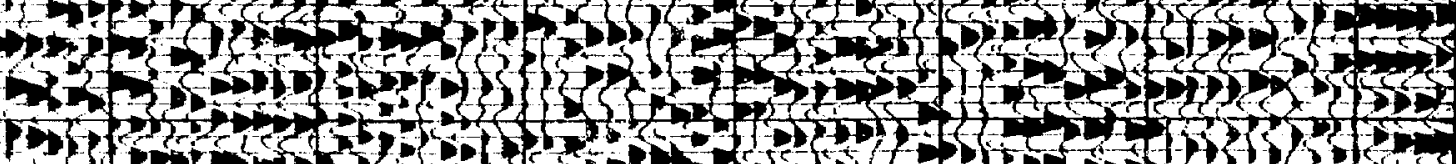

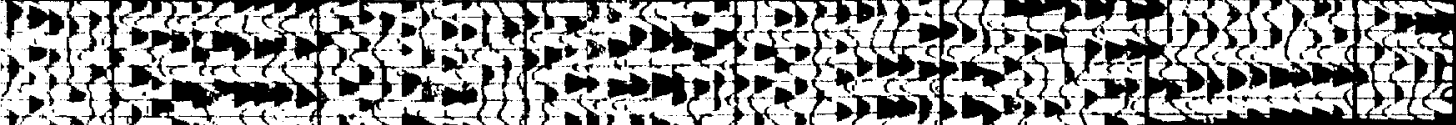

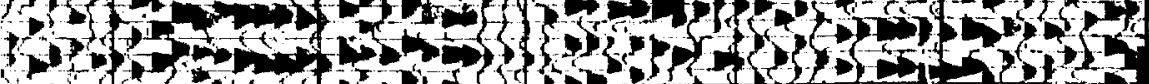



W $-\quad$ (2)

3727

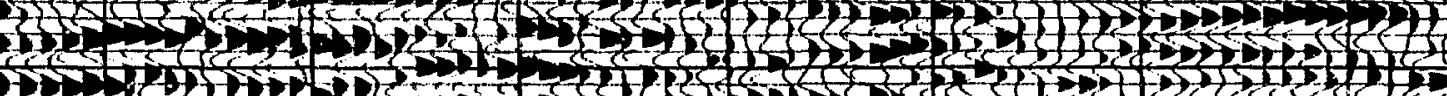

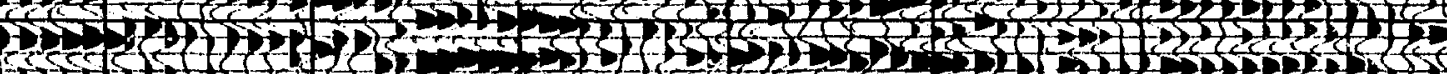

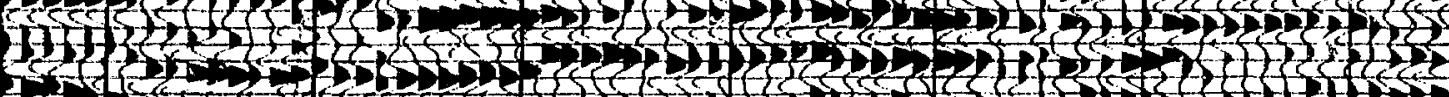
1 27.71 2.72 771013 $-2$ (1) 5.

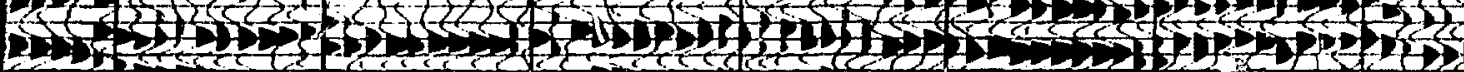
22 2.

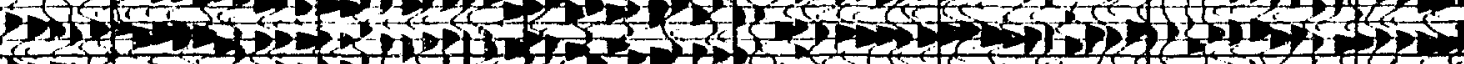

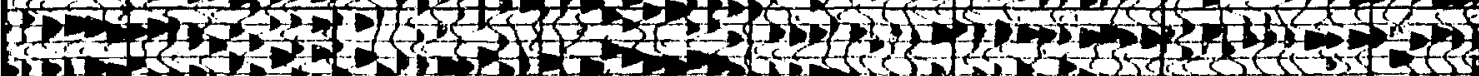

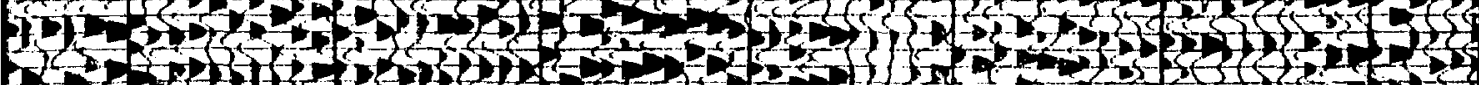

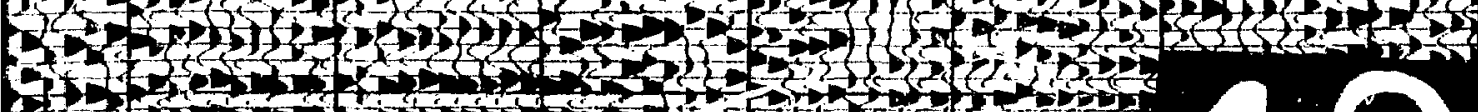

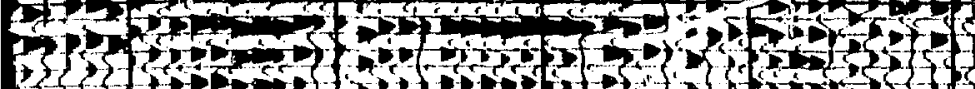
$\operatorname{lich}_{1}$ 



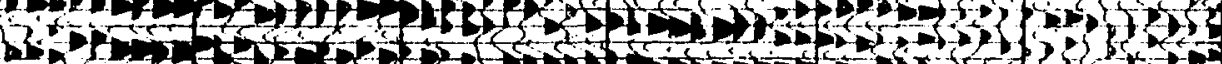

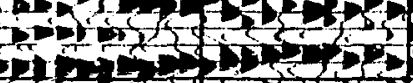

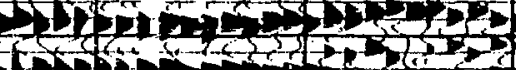

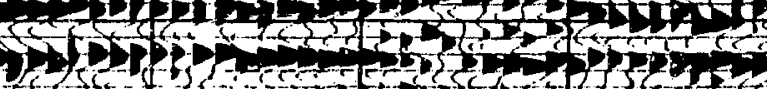

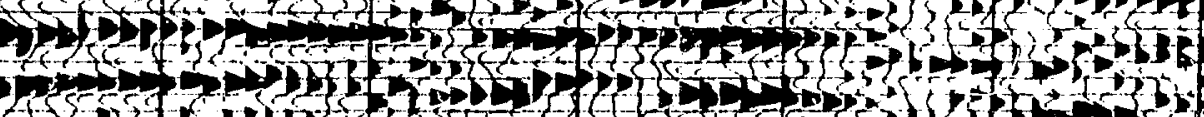

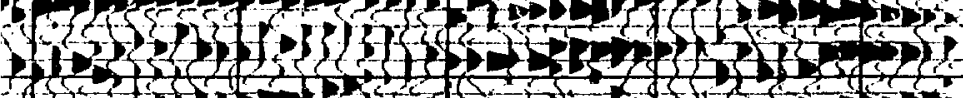

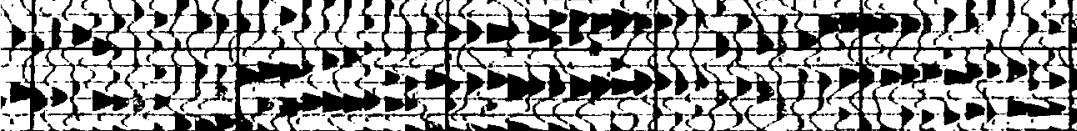
(a) -2. 



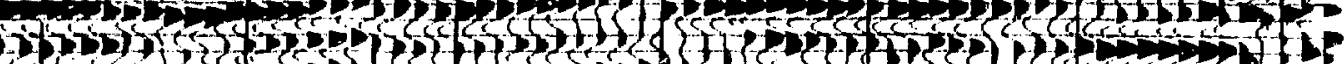

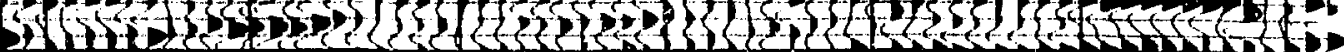

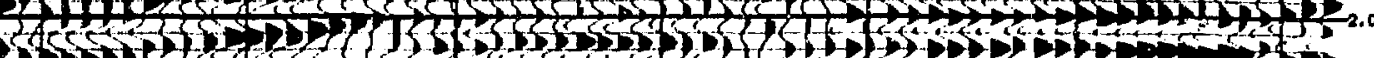

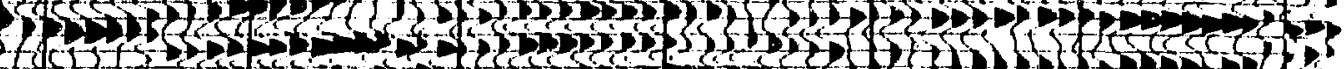

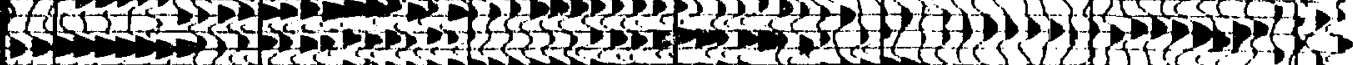

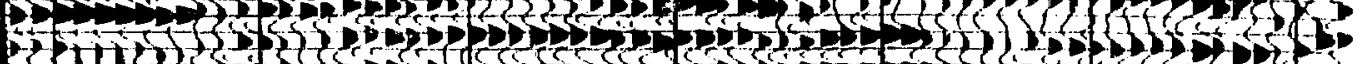

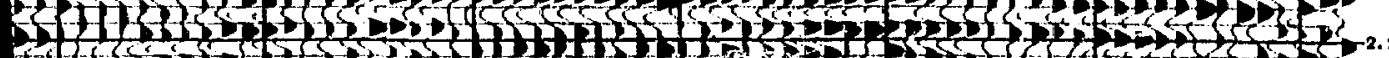

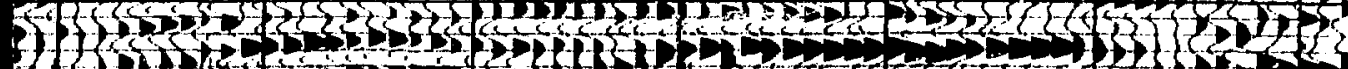

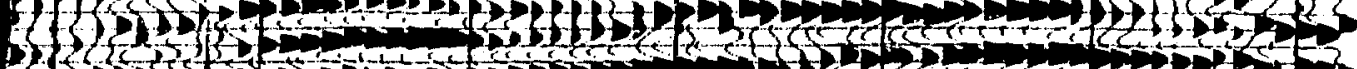

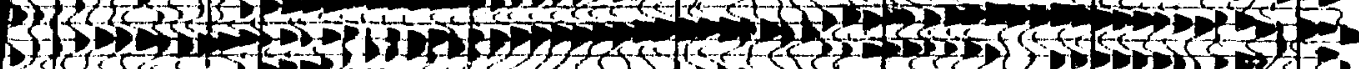

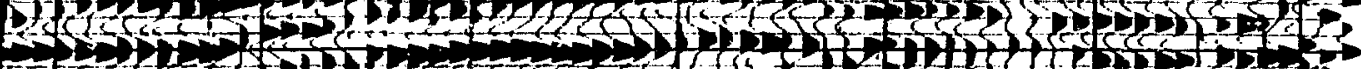

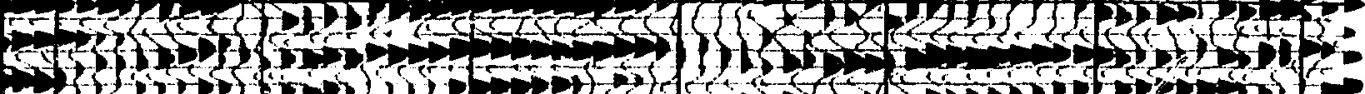

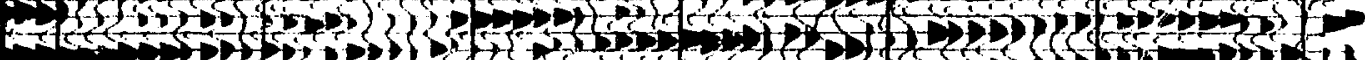

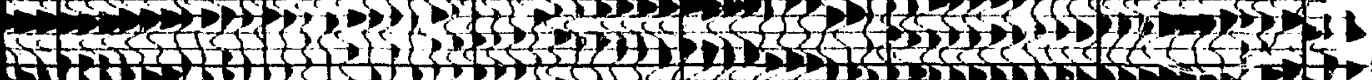

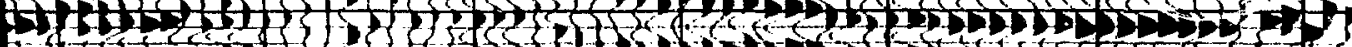

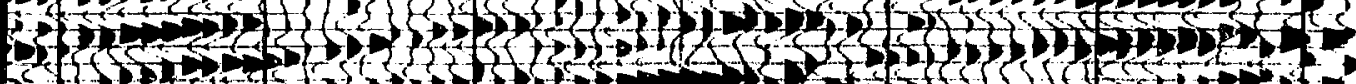

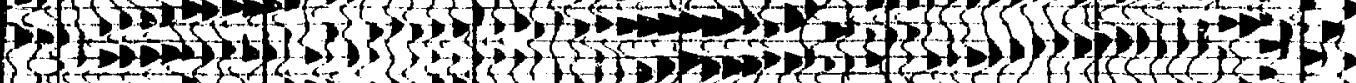

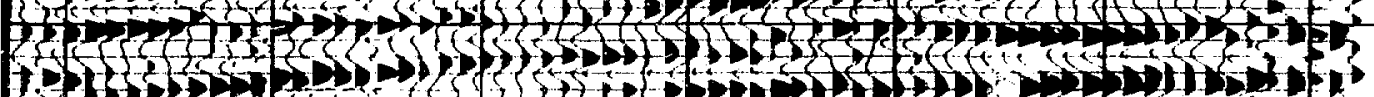

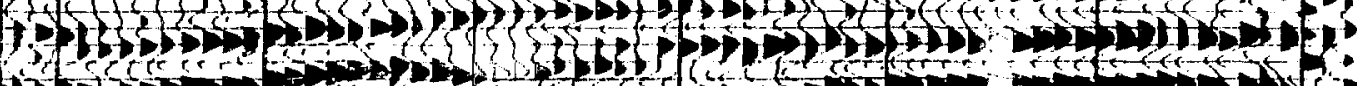

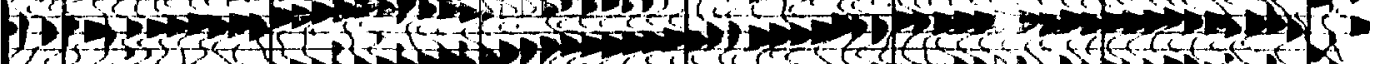

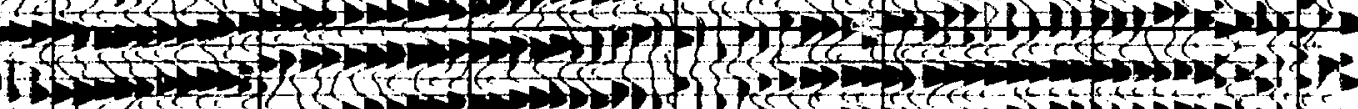

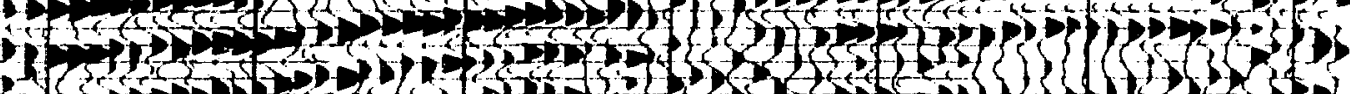

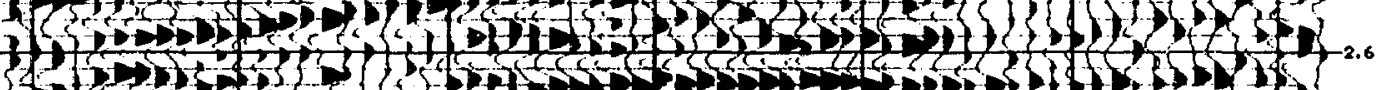

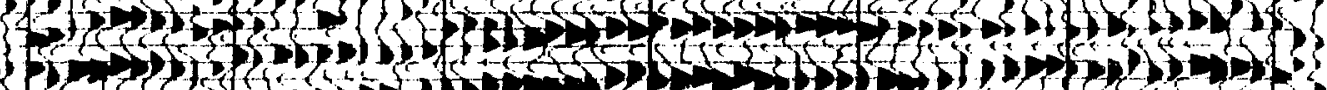

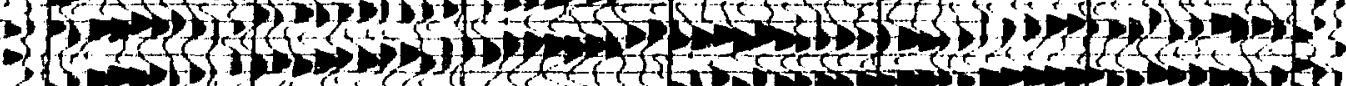

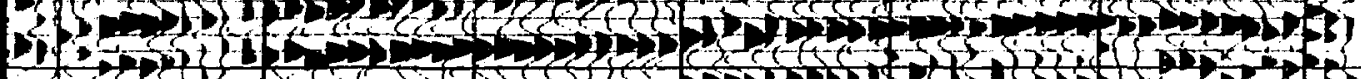

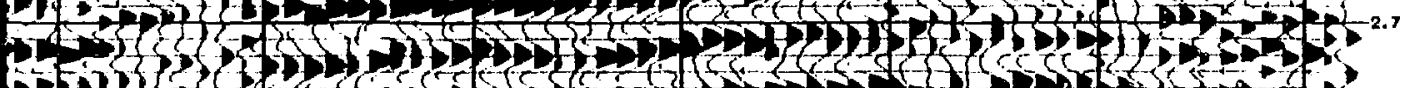

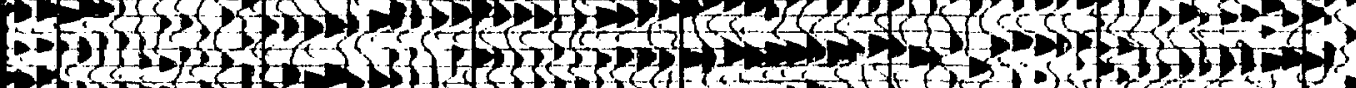

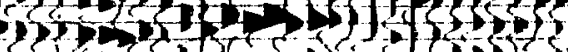
$\rightarrow\}$

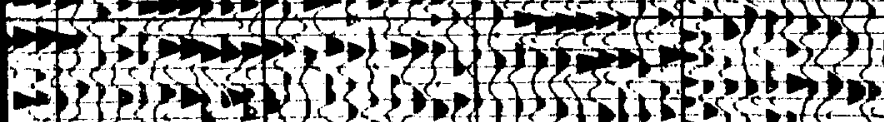
of

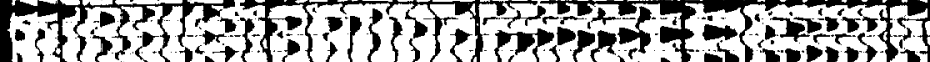

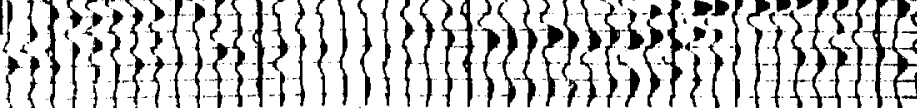




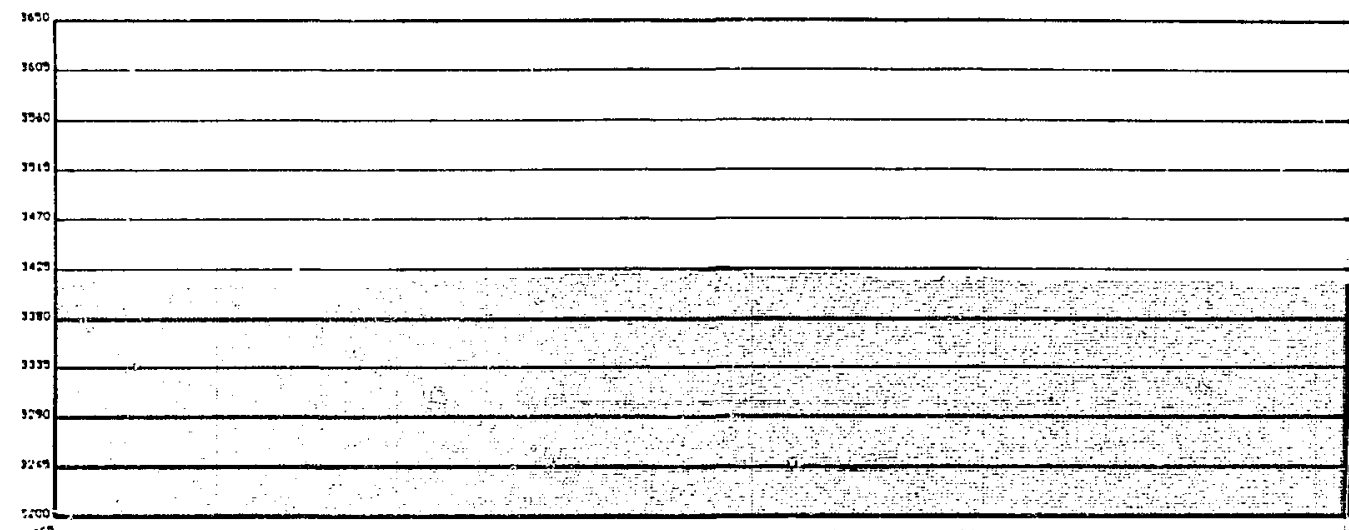

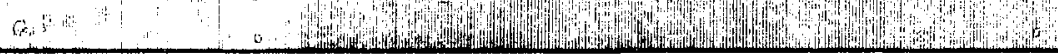

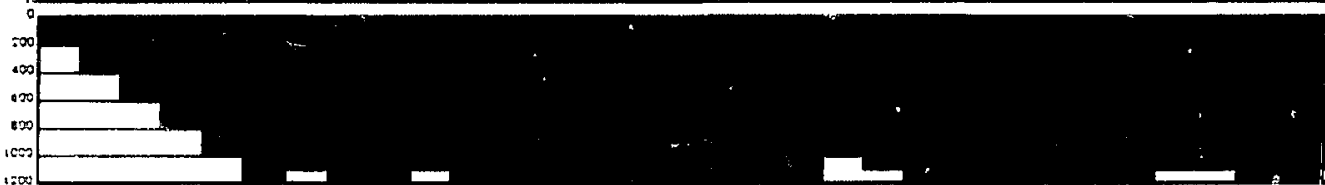

swilleasi

sec 20

YEL, PNALL

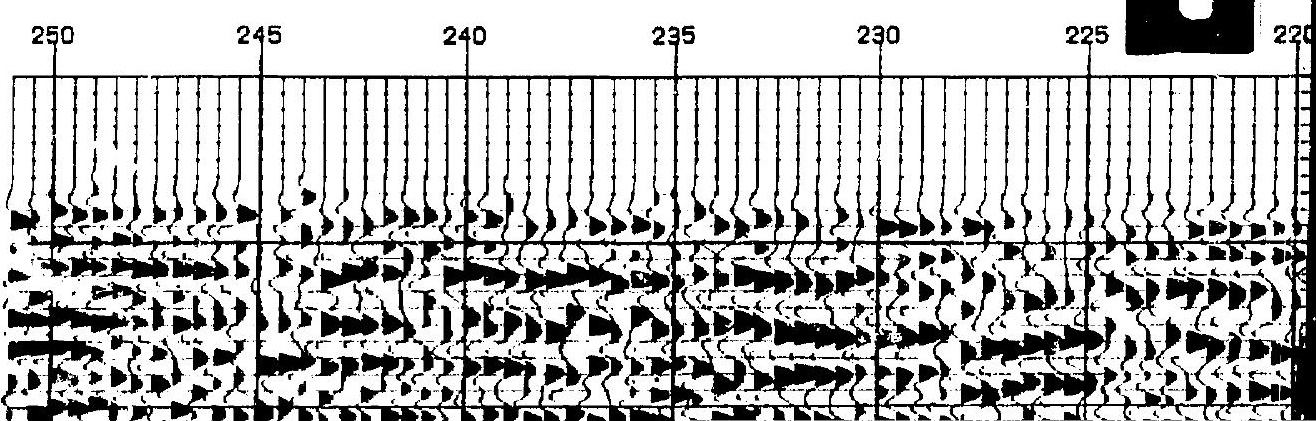




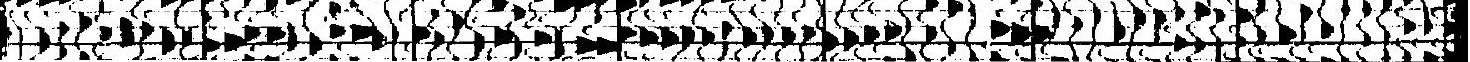




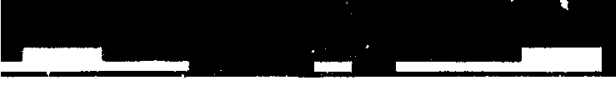

sec 28

VEL_RPBA 




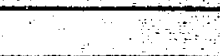

- 


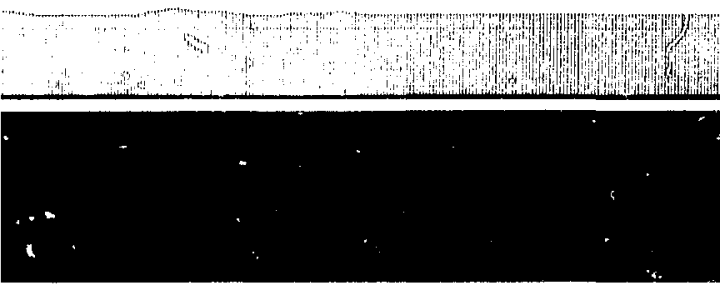

sec 34

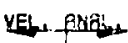

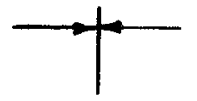

2

1

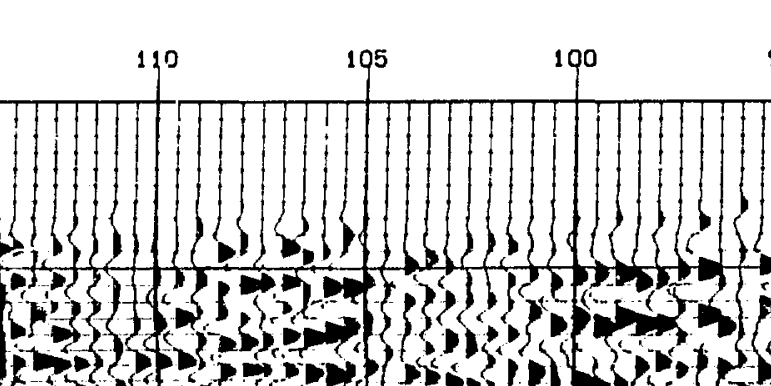

St,

VEL, PNALL,

0.123

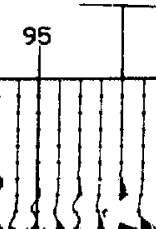

90

85
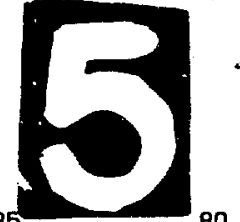


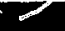

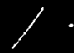

tec 35

VEILARALI
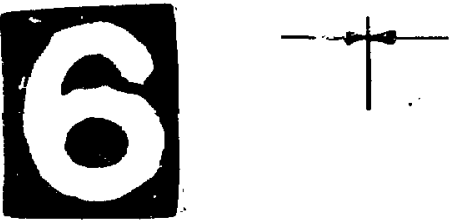

$7:$

\section{0}

65

60

55

50

45

8

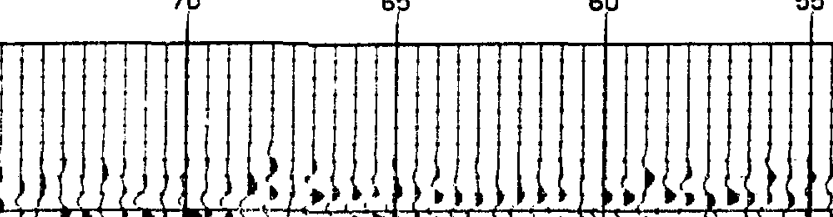

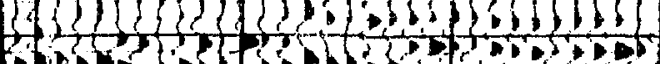



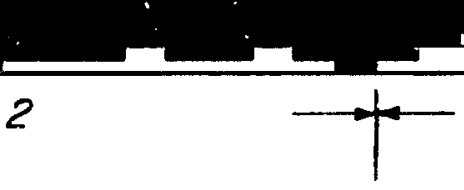

บEG. PNRIL

$\underline{A A-2}$

sec 2
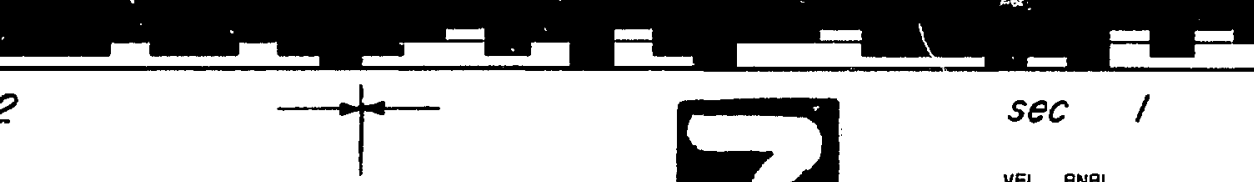

sec $/$

YEL, Finfle

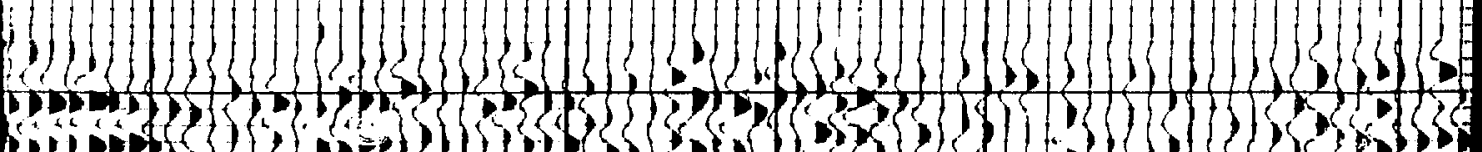
fof

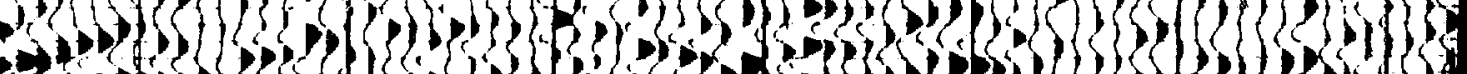
${ }_{0}$ 


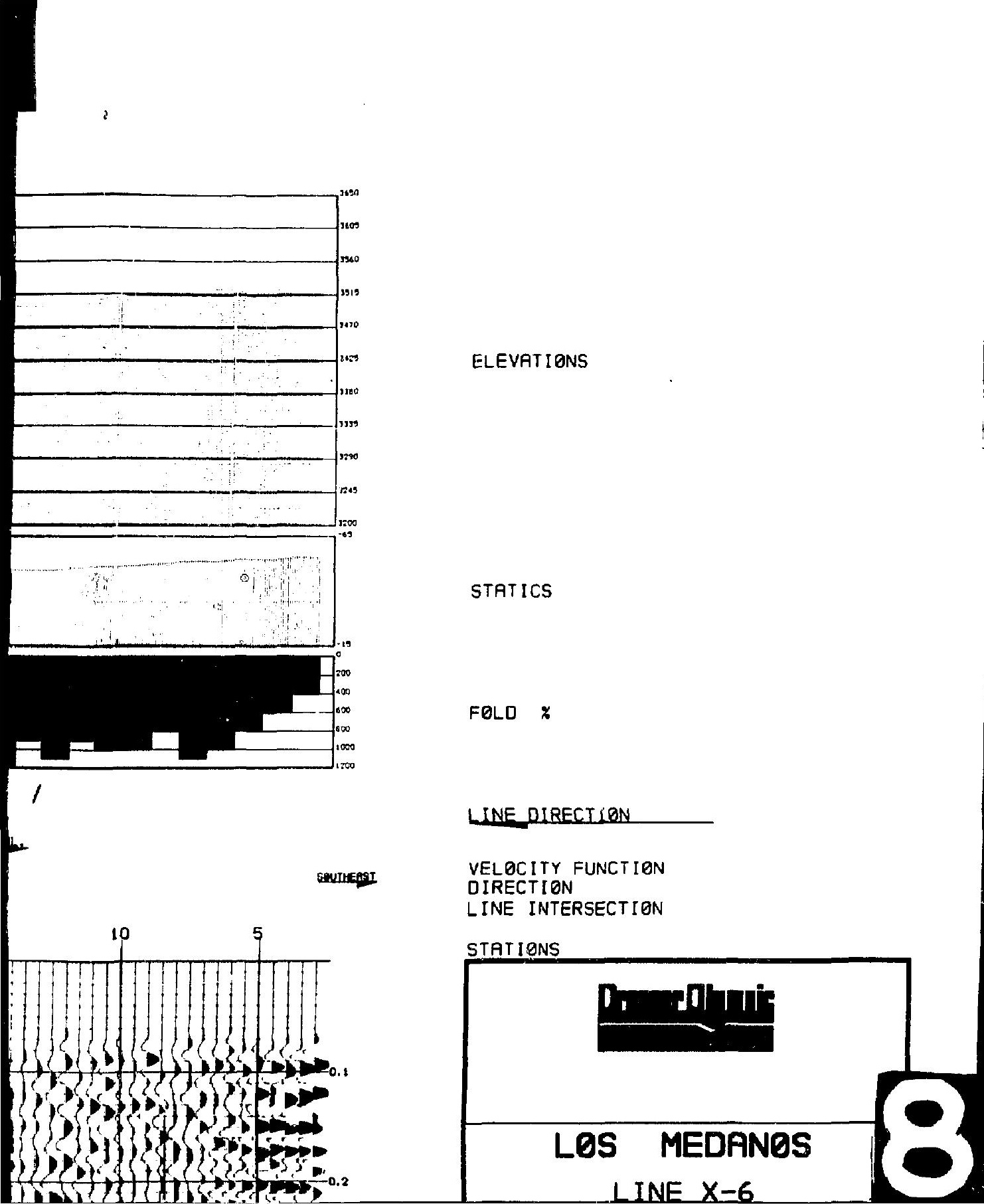


J8 $73050-25$

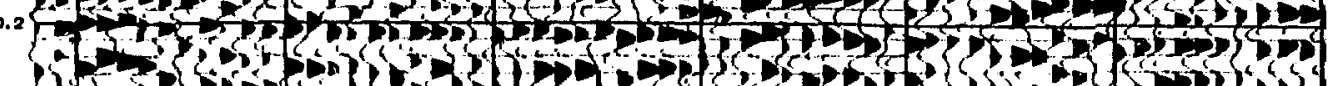

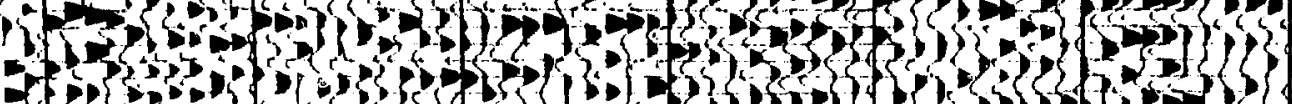

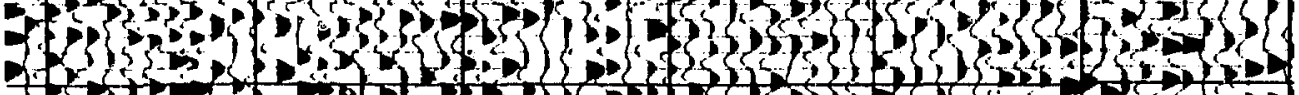

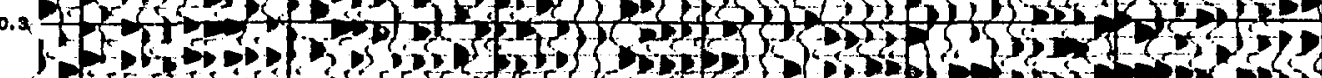

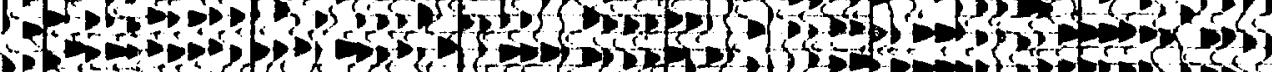

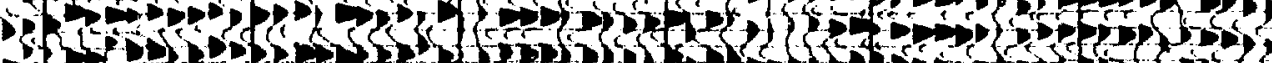

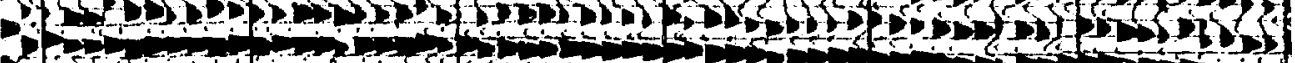

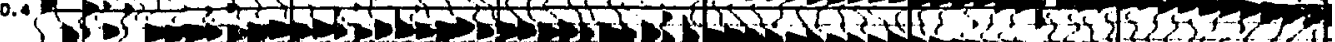

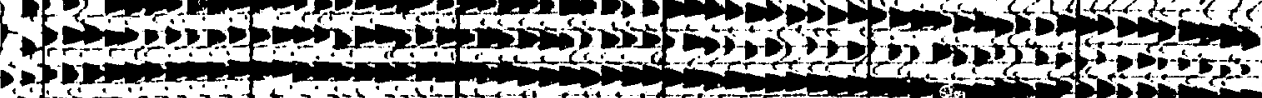

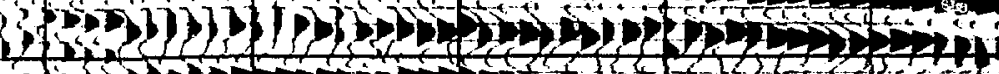

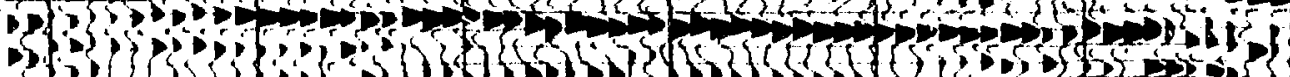

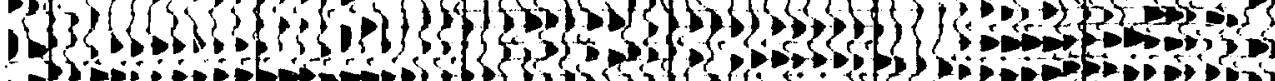

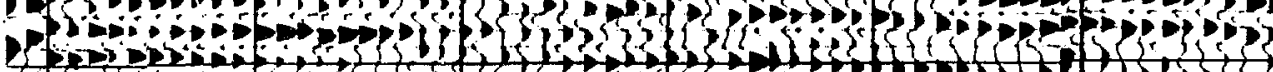

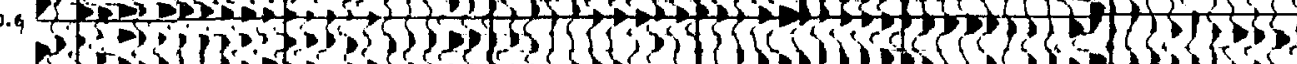

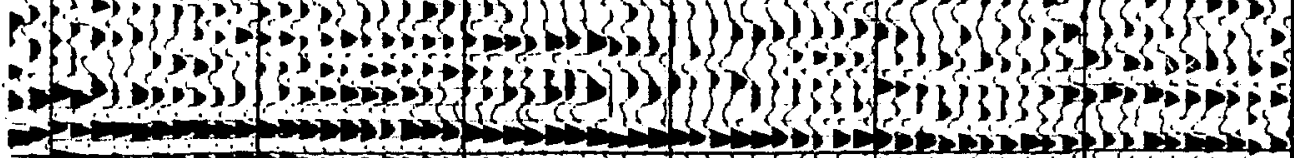

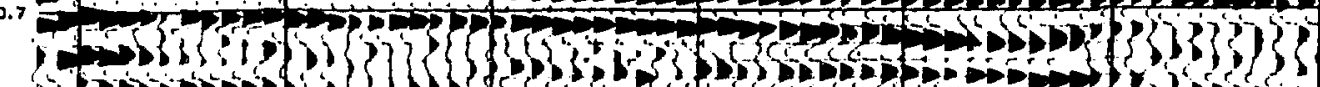

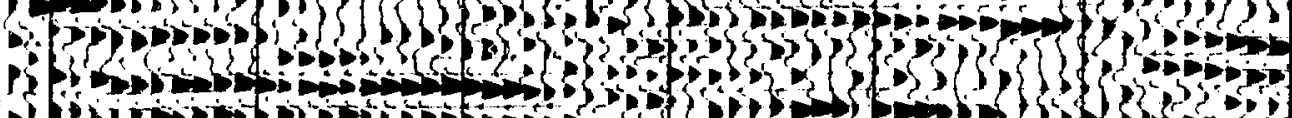

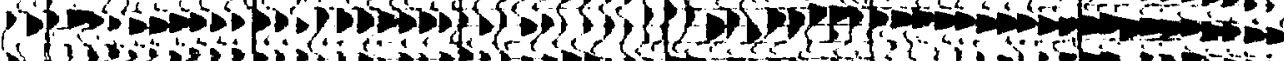

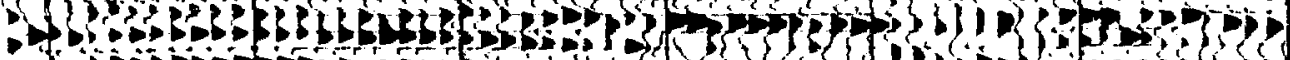
- l b.

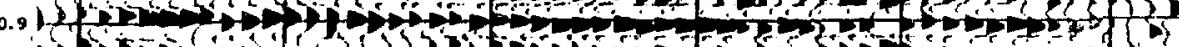

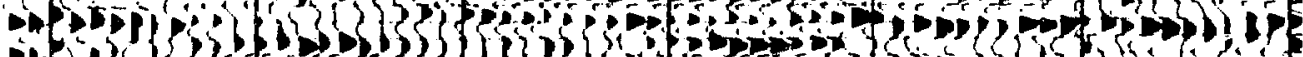

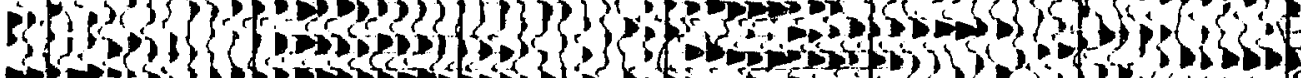

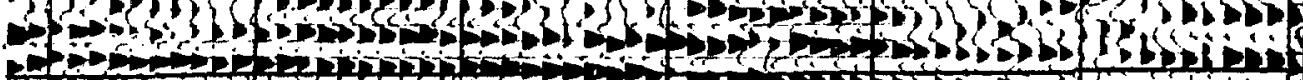

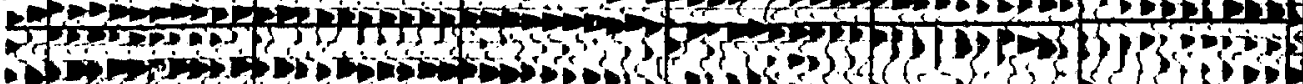

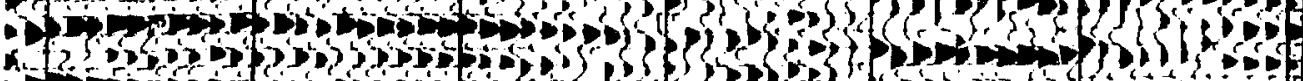

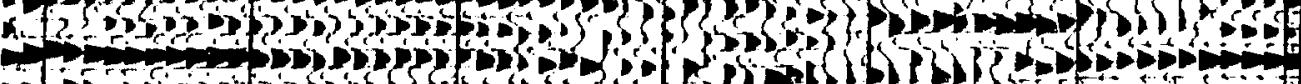

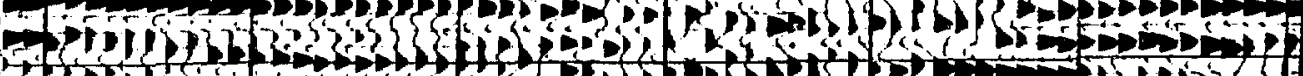
SCS

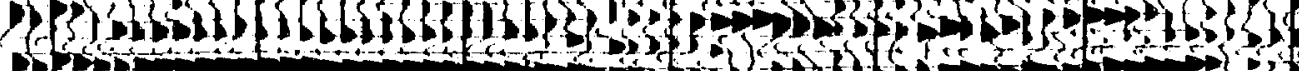

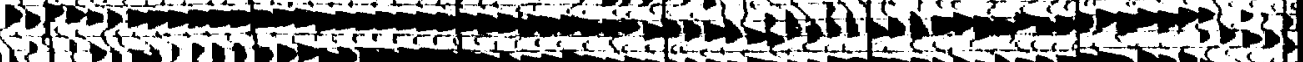

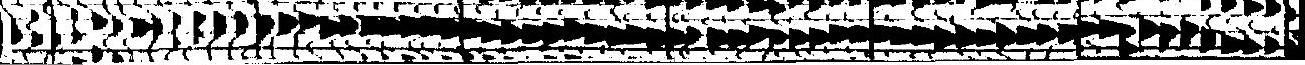




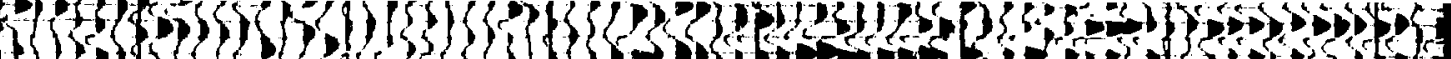

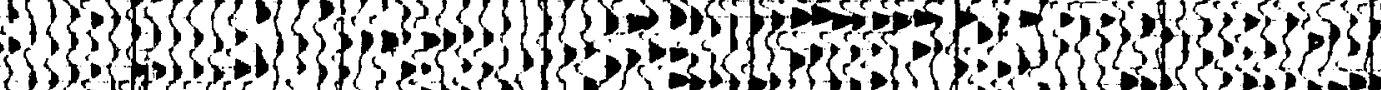

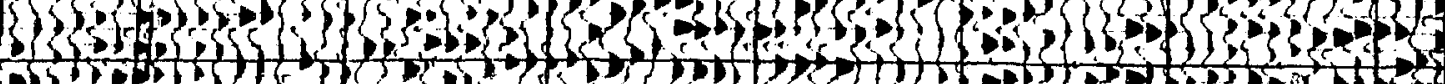

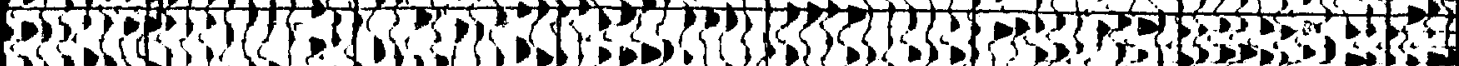

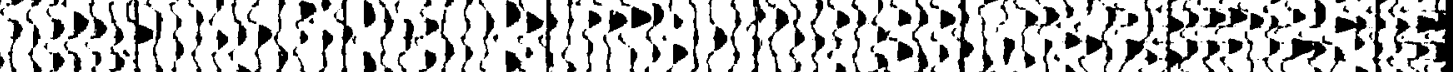

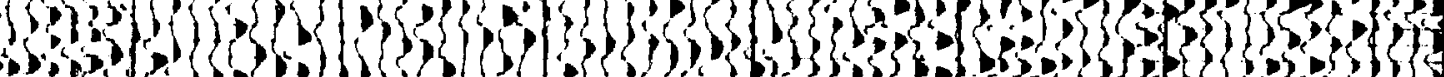

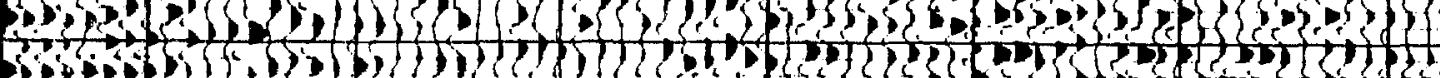

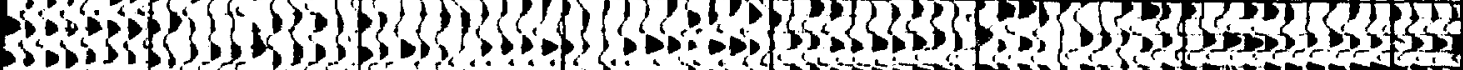

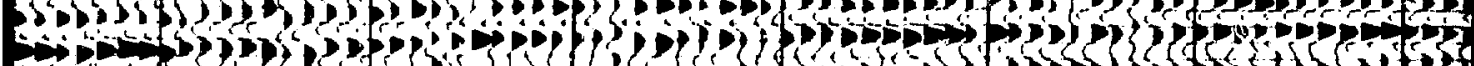

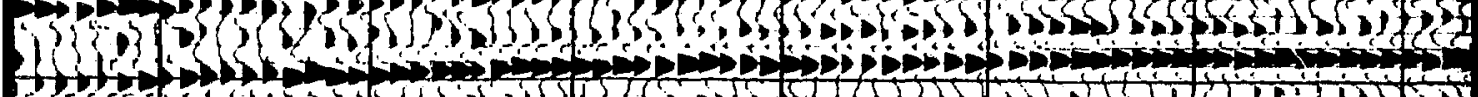

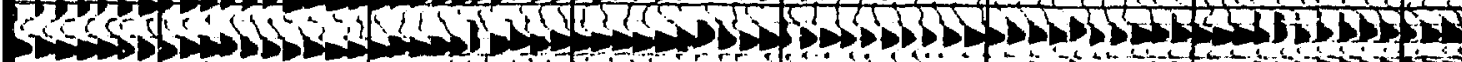

Soldidis

Gor

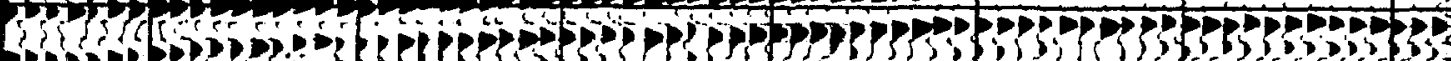

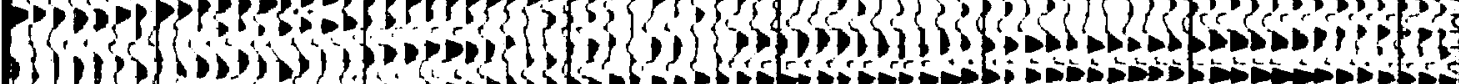

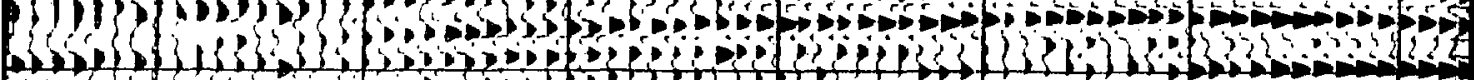

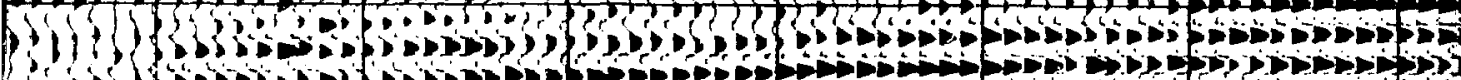

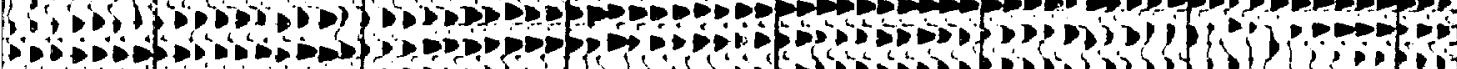

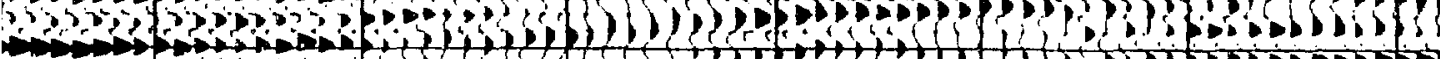

\section{stoision.}

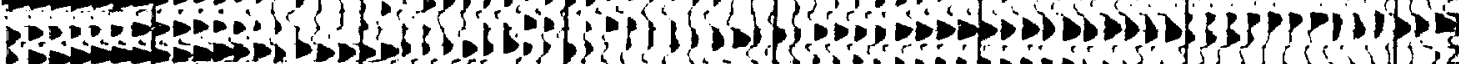

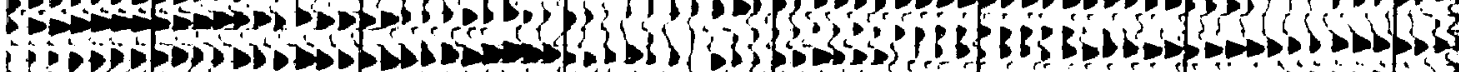
HPPRP2

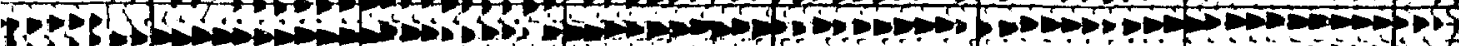

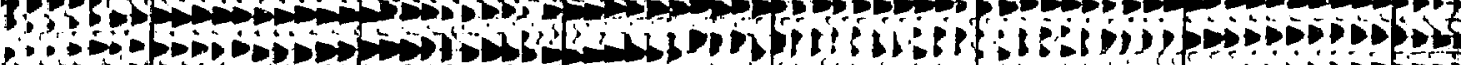

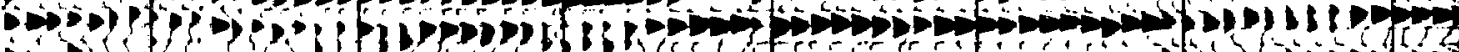

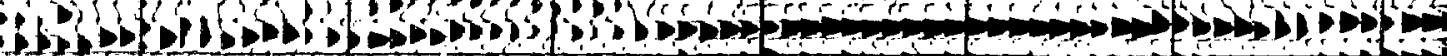

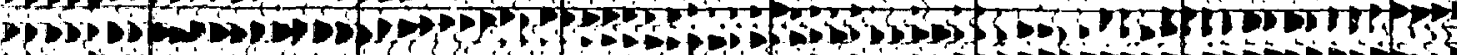

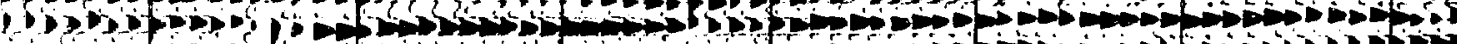

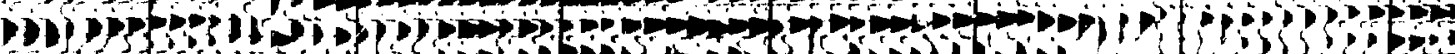

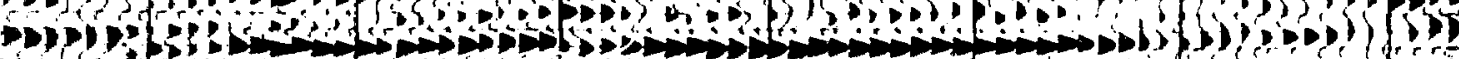

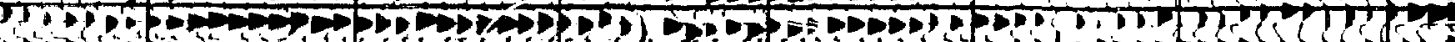

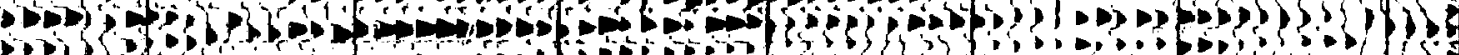

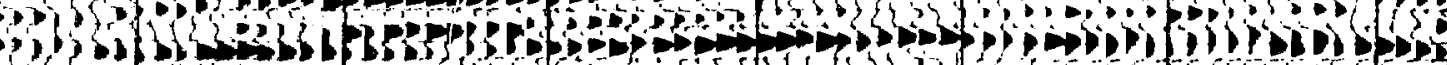

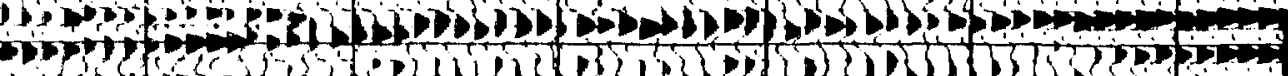

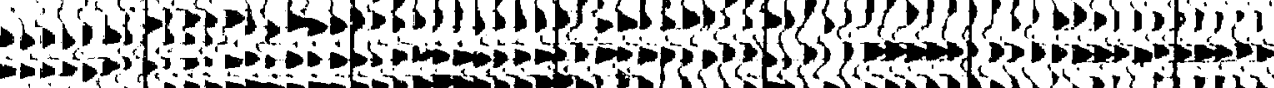

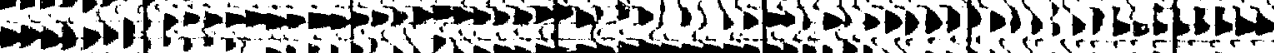

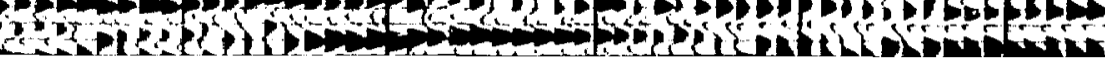




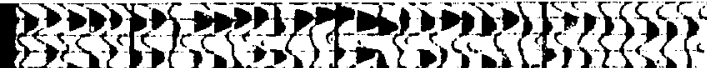

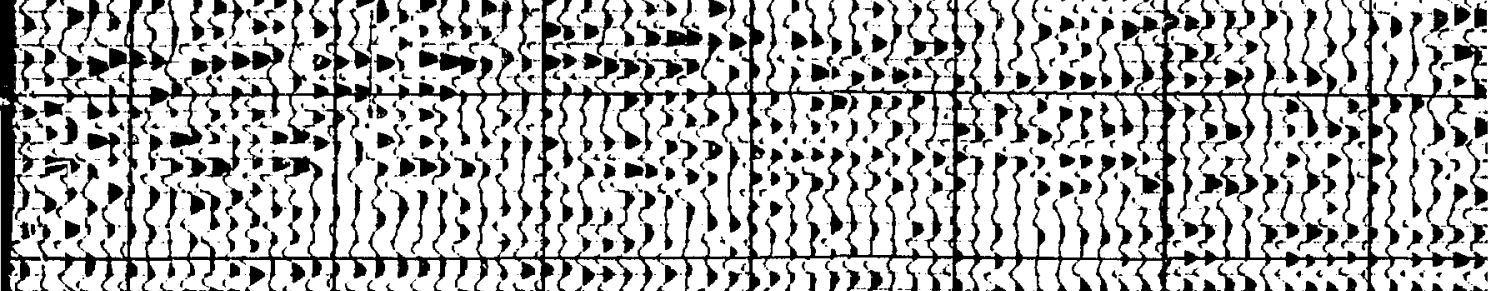

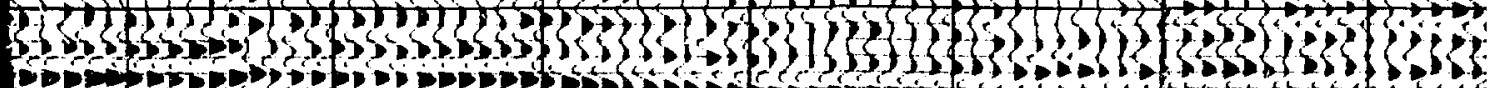

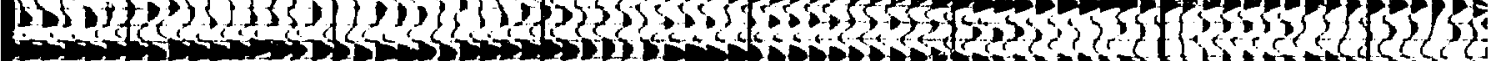

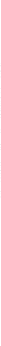

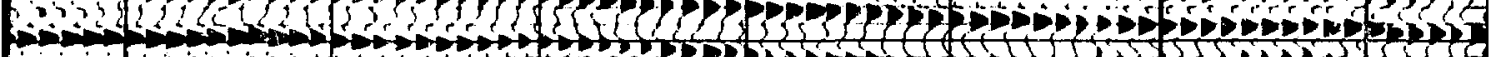

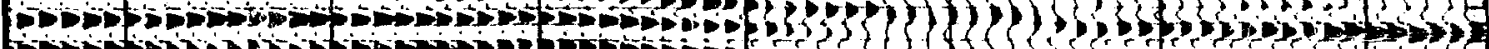

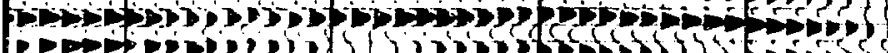

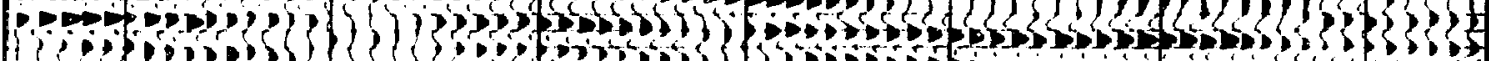

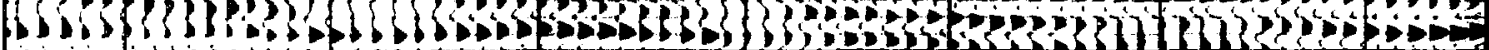

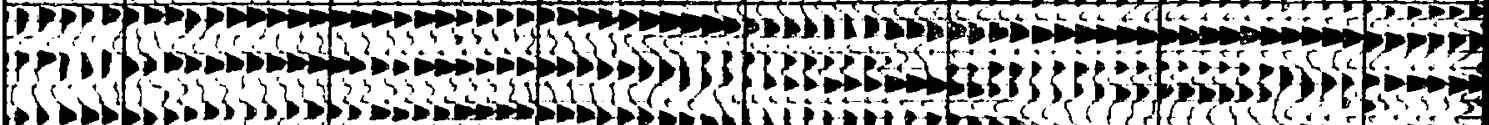

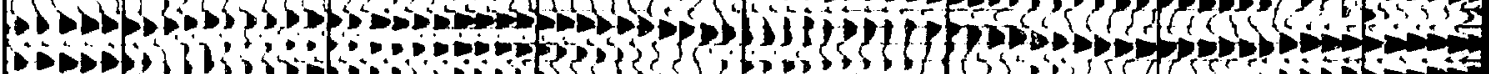

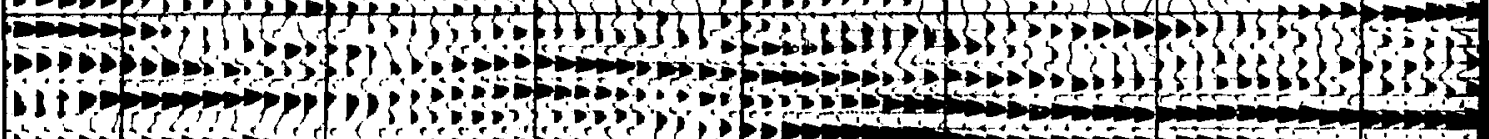

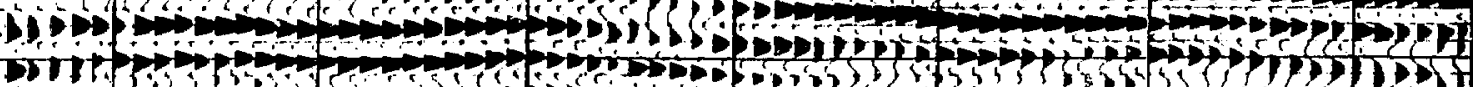

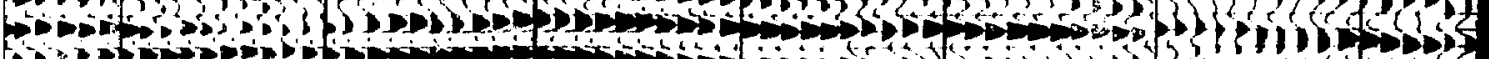

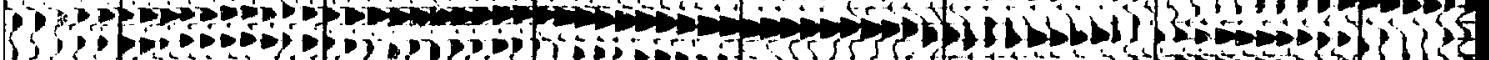

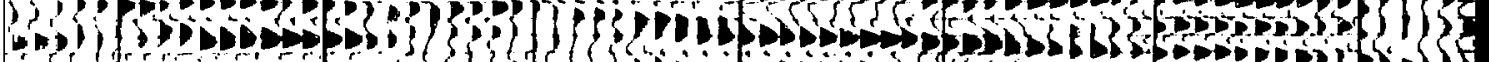

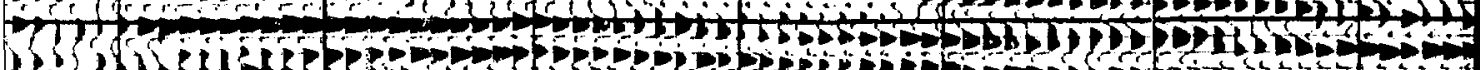
a

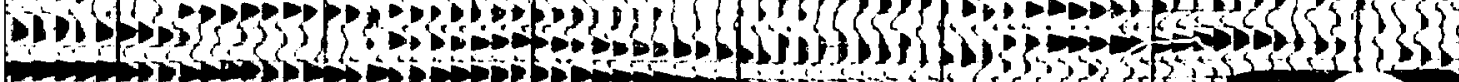

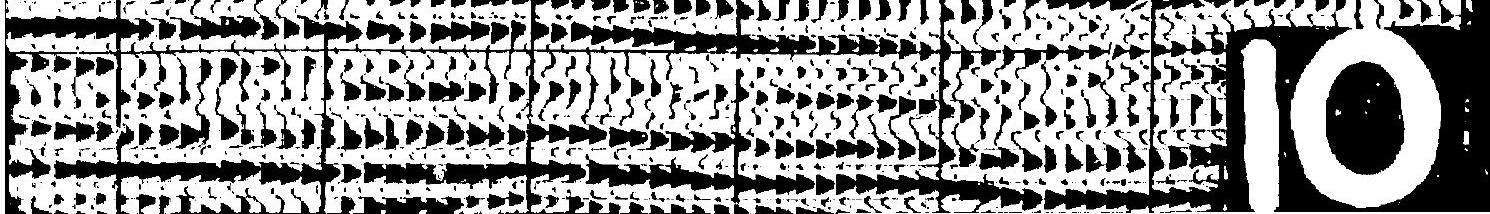


(6) ats

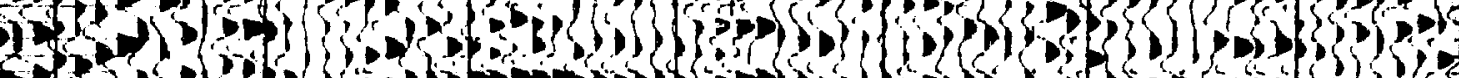

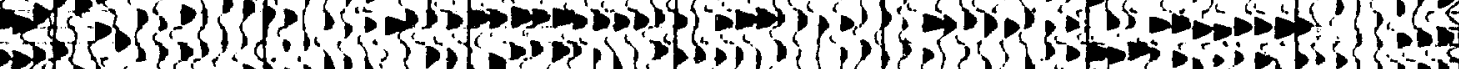

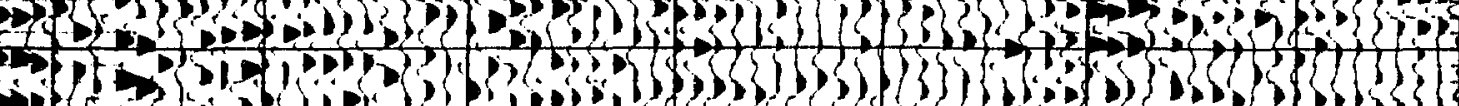

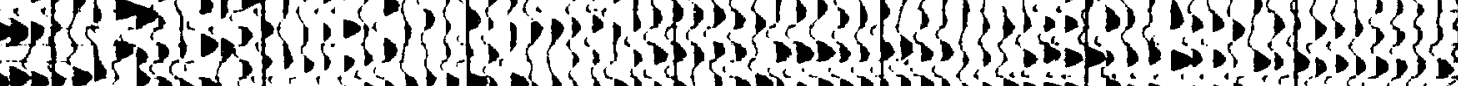

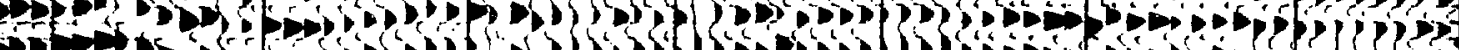

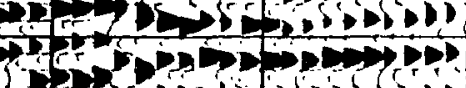

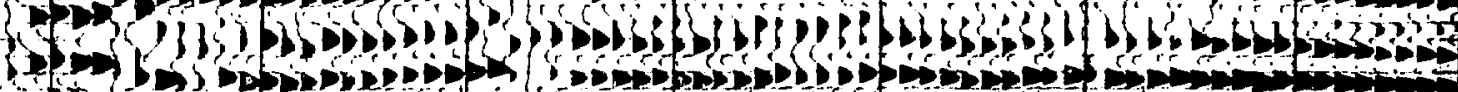

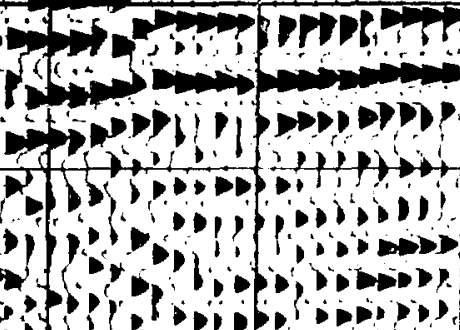

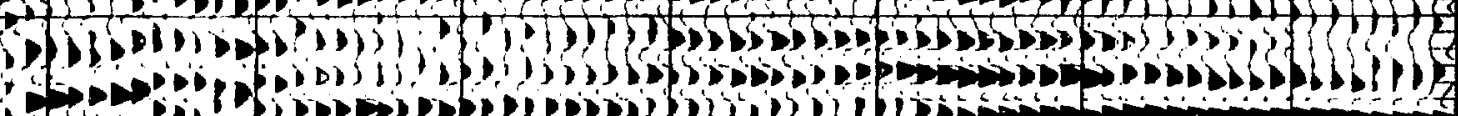

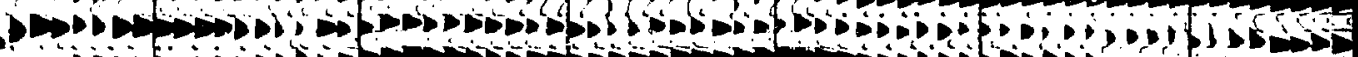

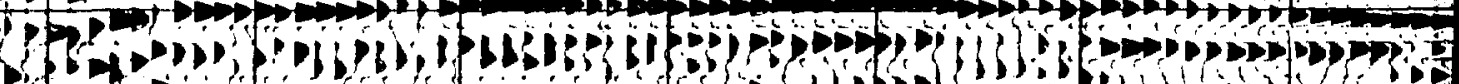

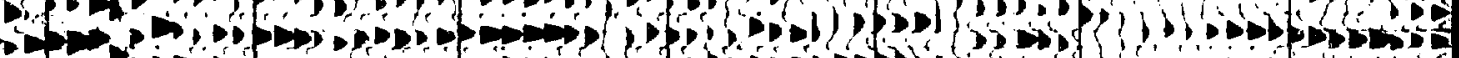

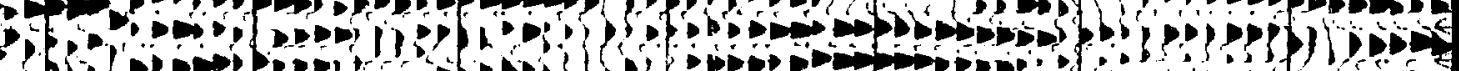
(f)

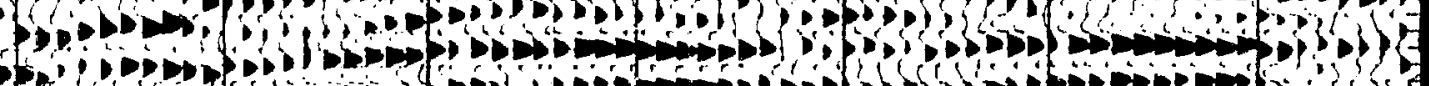

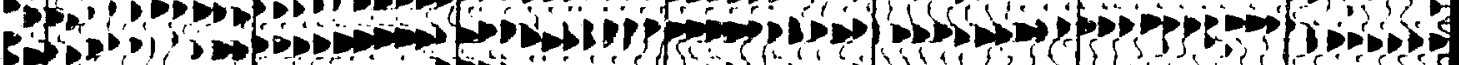

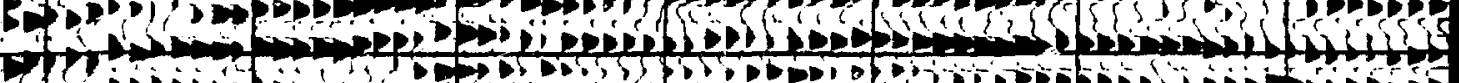

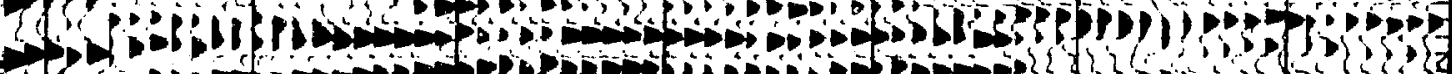

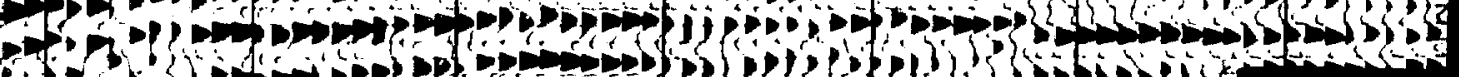

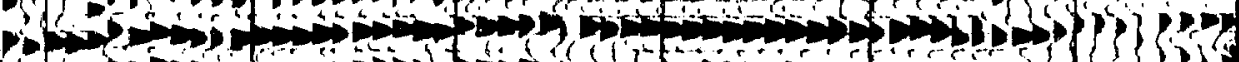
(1)

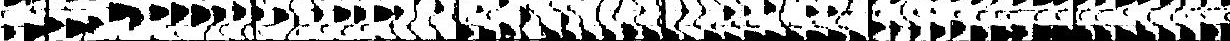



A:

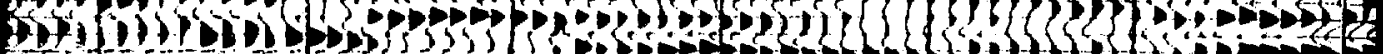
作

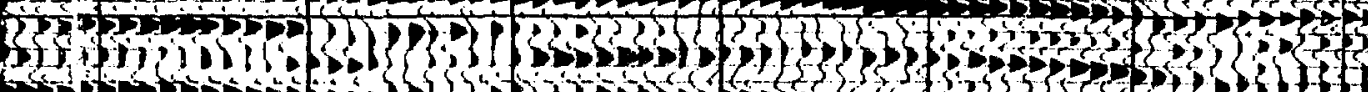

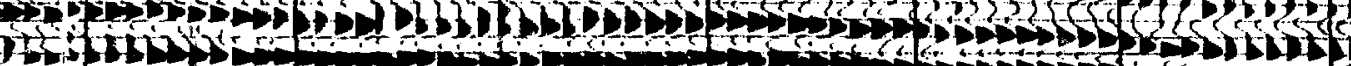

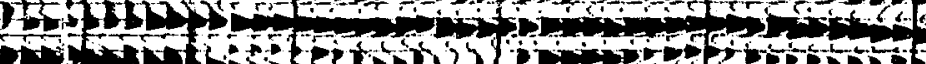

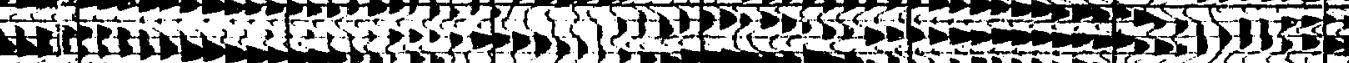

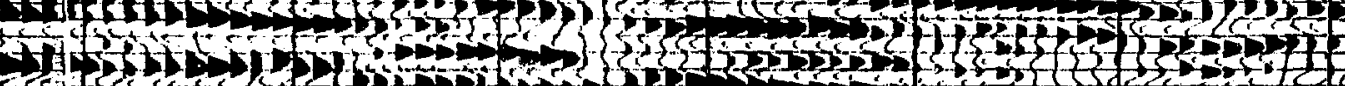

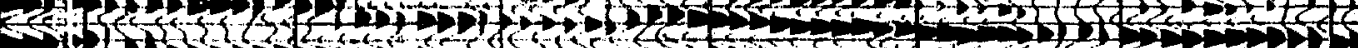

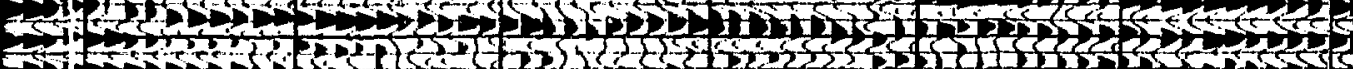

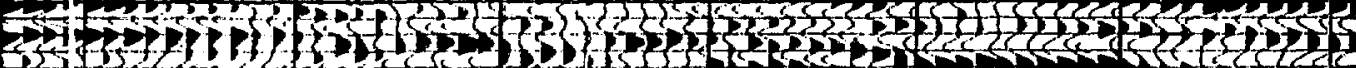

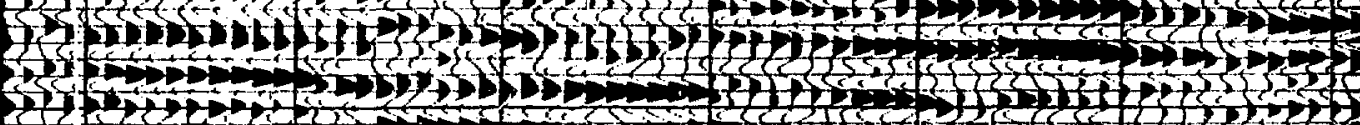

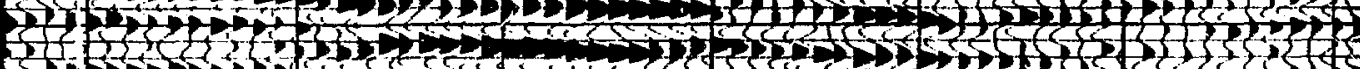

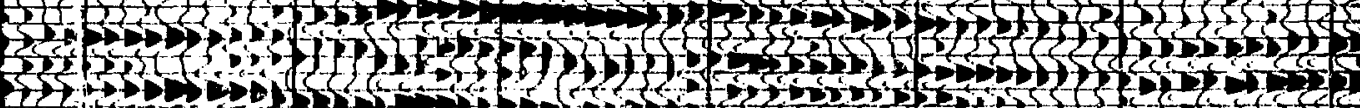

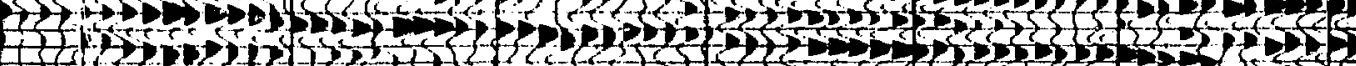

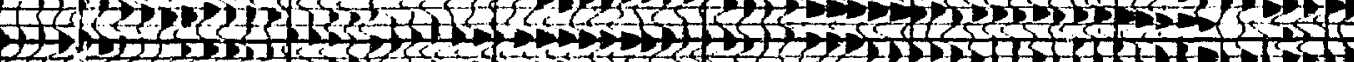

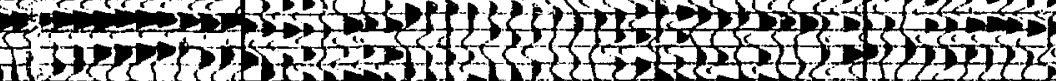

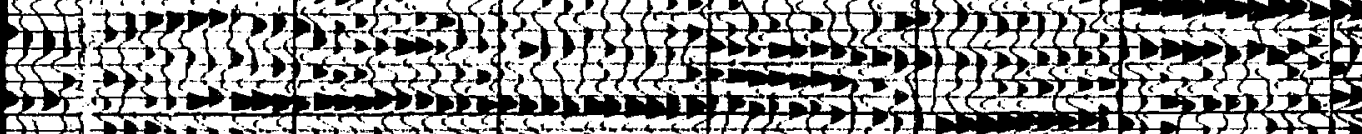

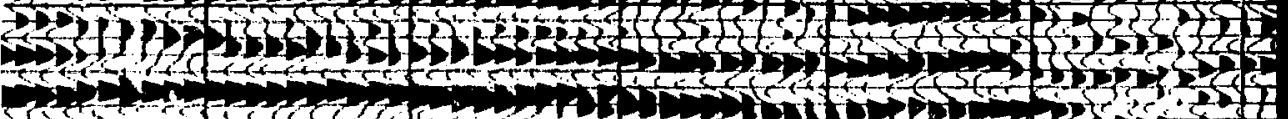

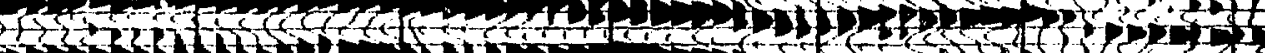

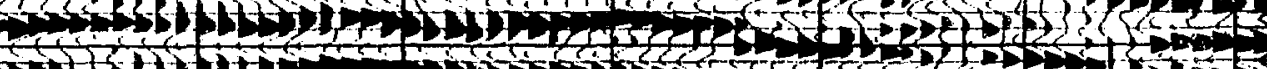

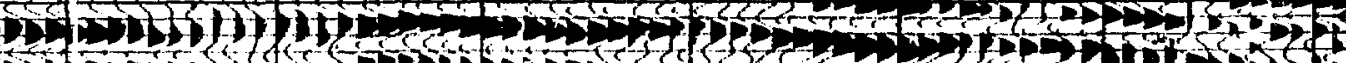

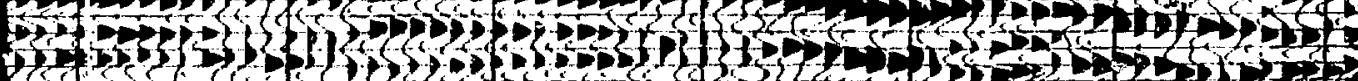

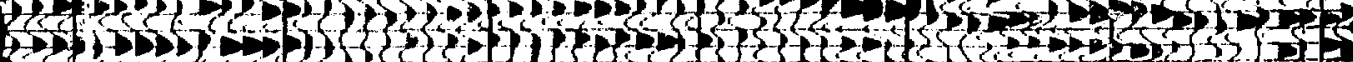

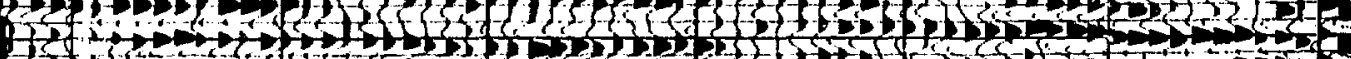

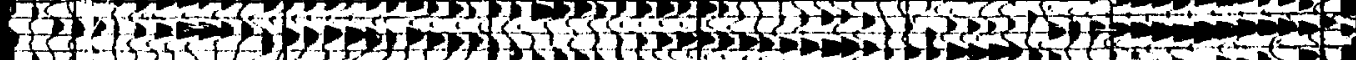

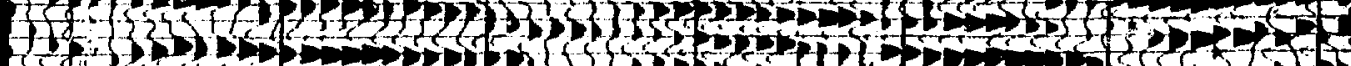
sरt

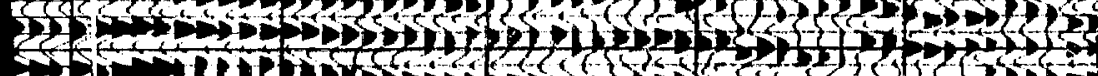

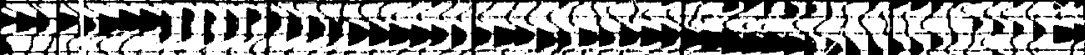

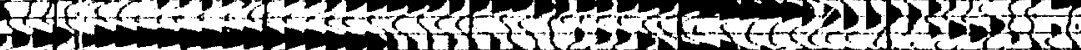

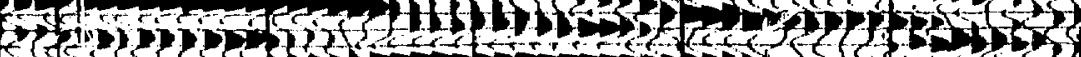

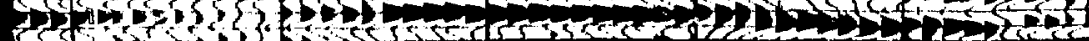

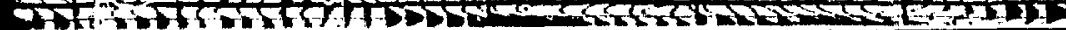


20 .

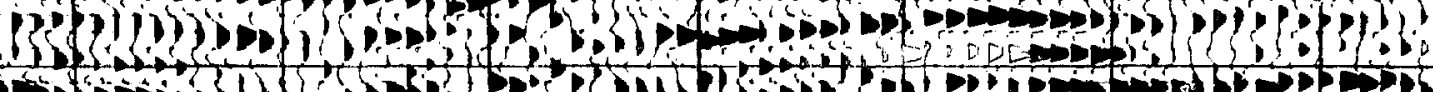

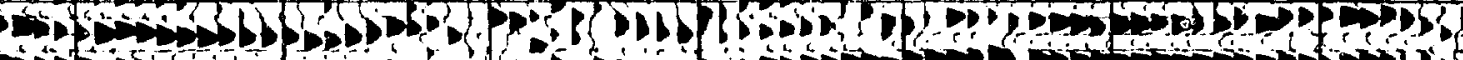

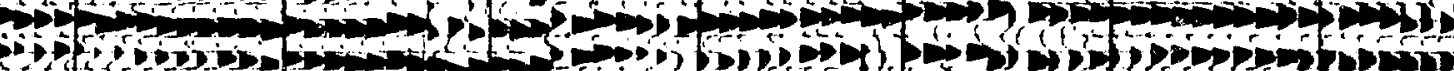

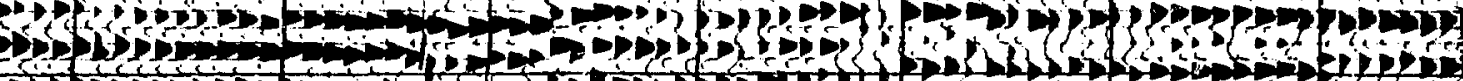

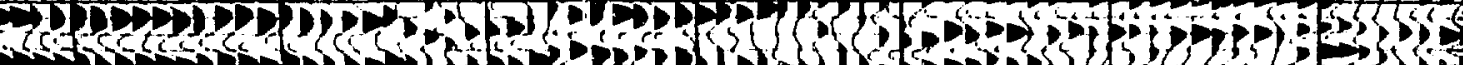

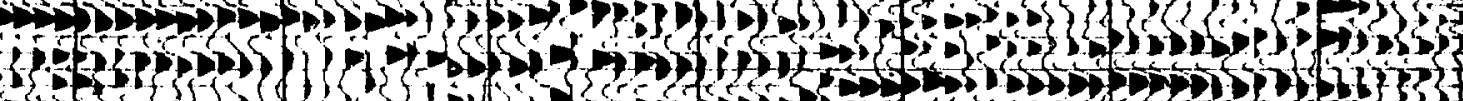

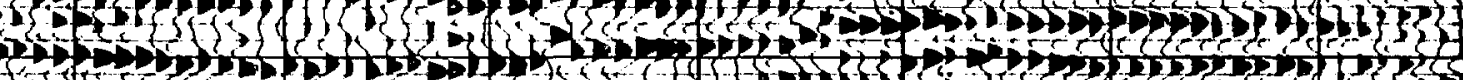

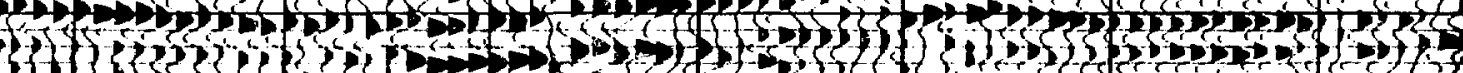

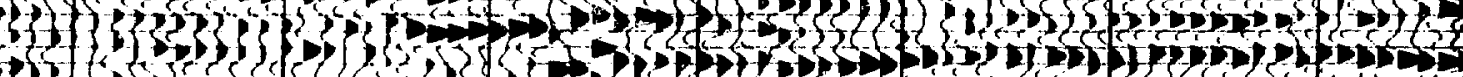

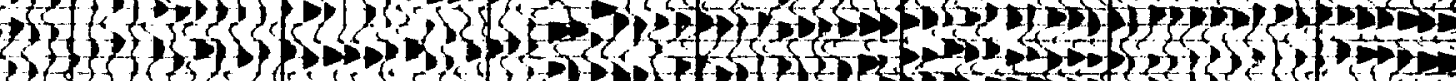

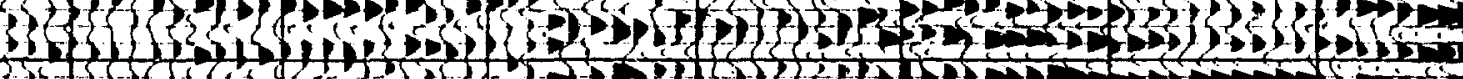

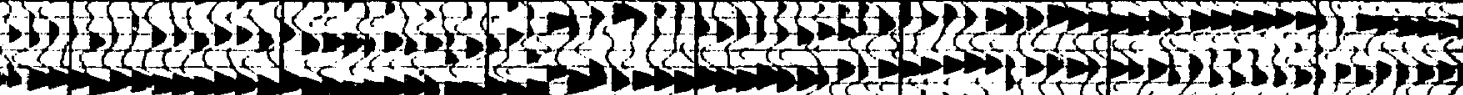

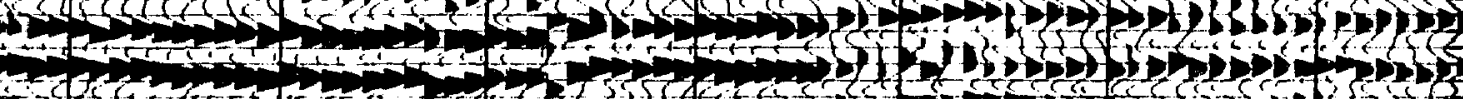

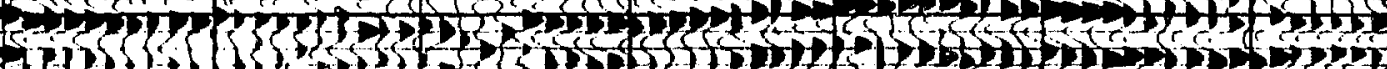

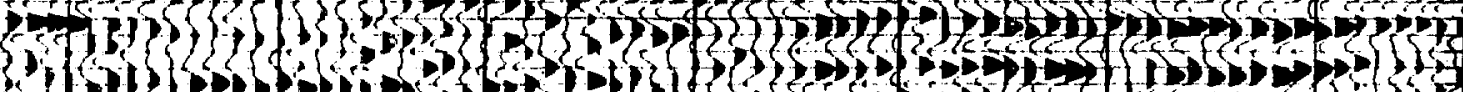

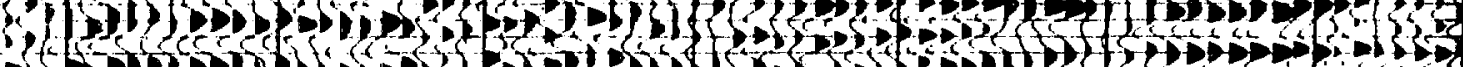

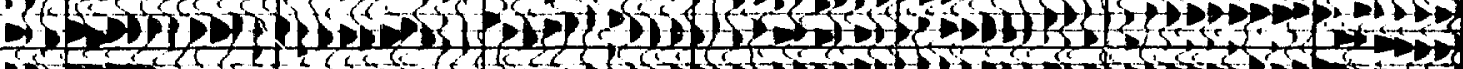

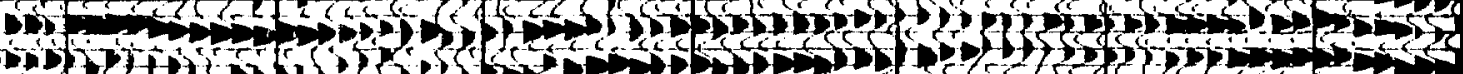

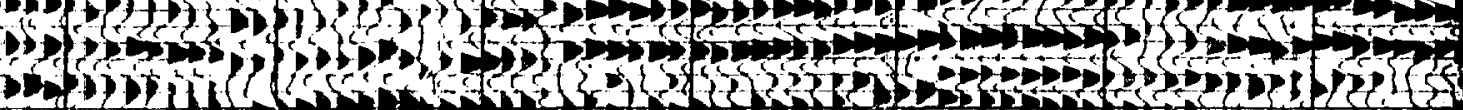

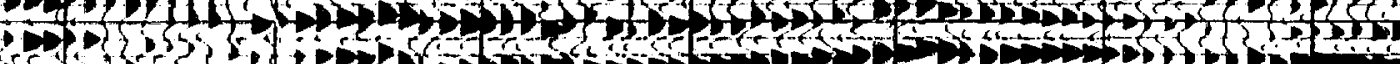

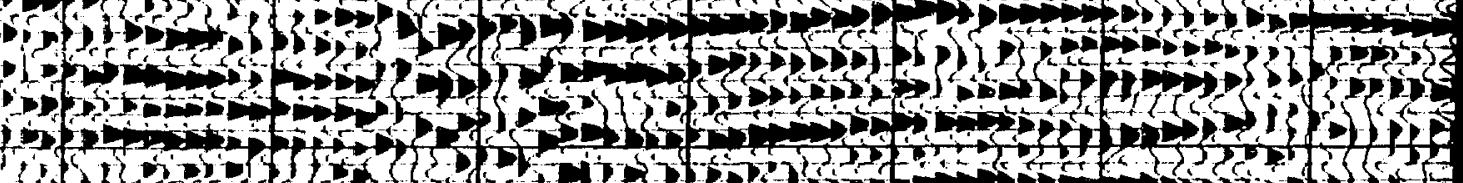

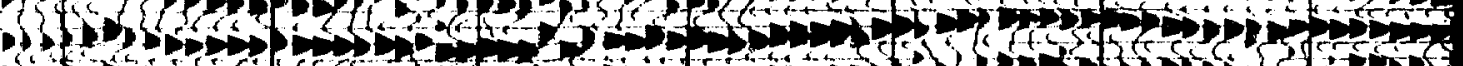

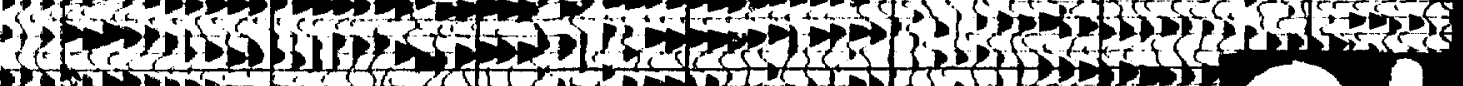

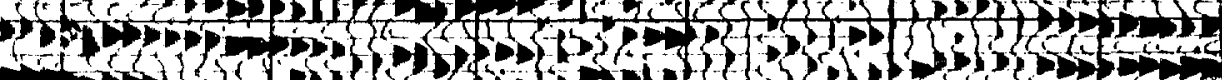

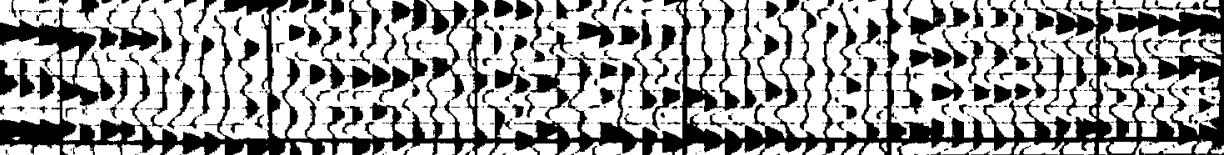




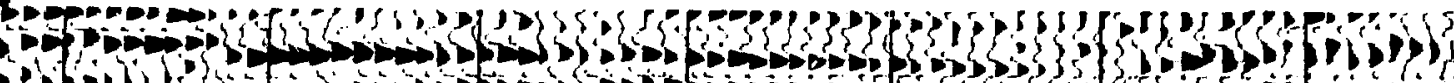
(3)

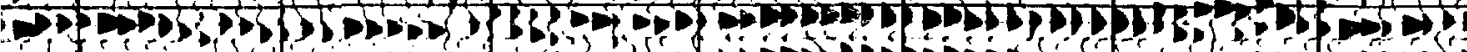

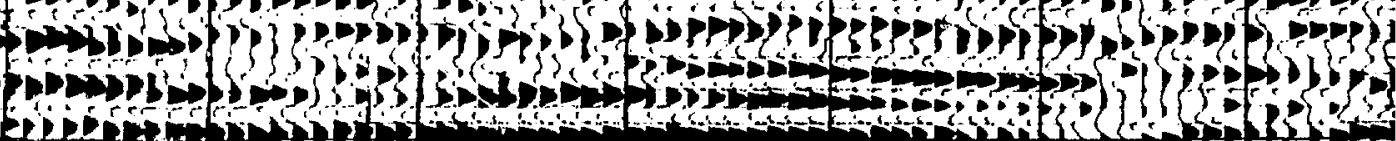

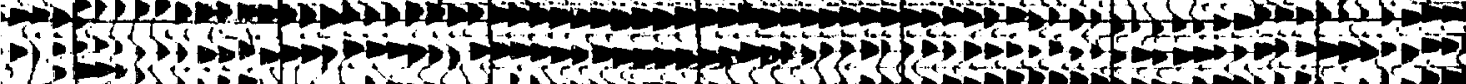

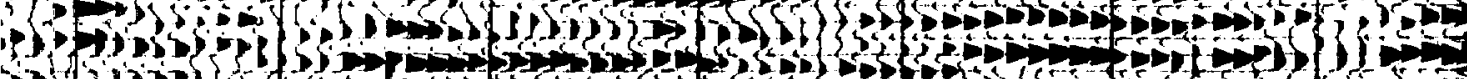

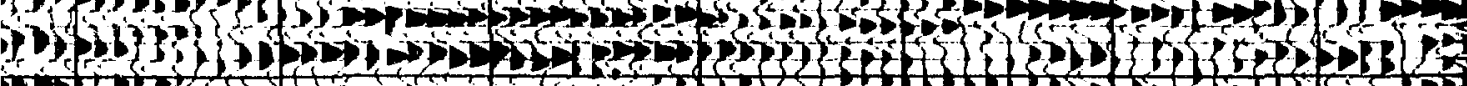

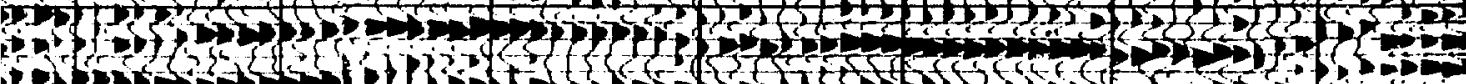

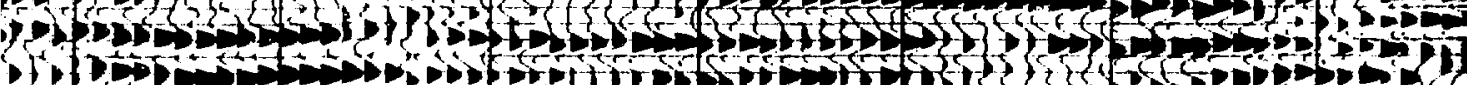

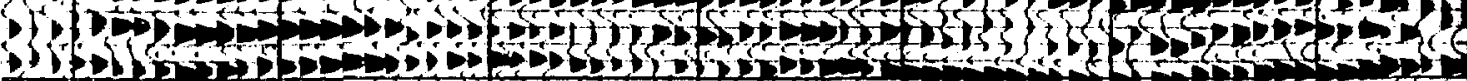

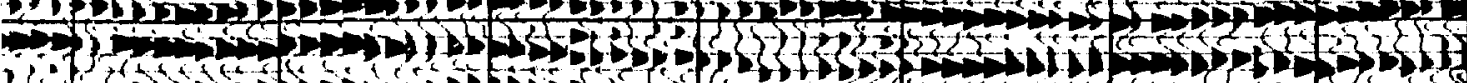

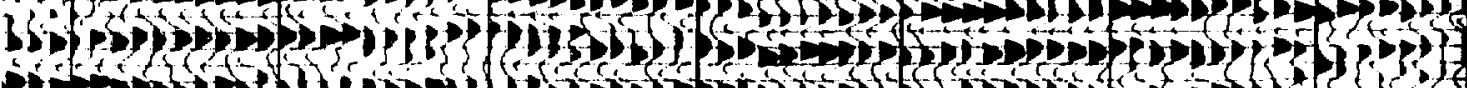

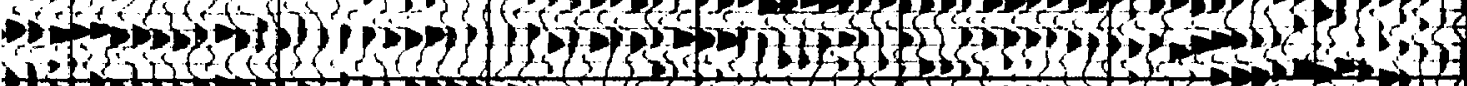

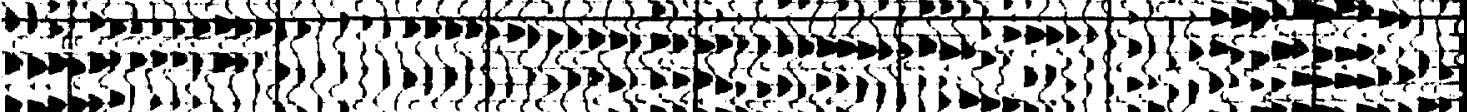
-

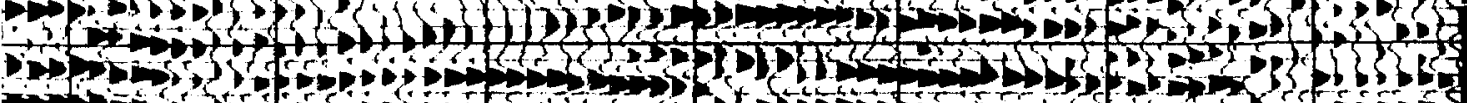

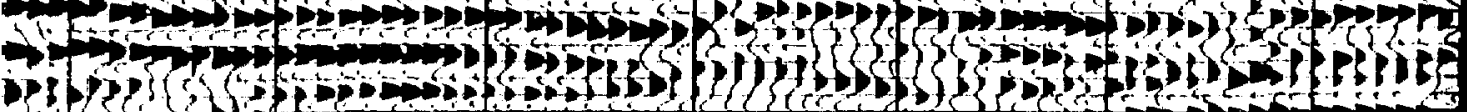

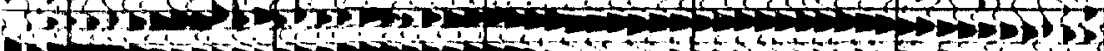

L)

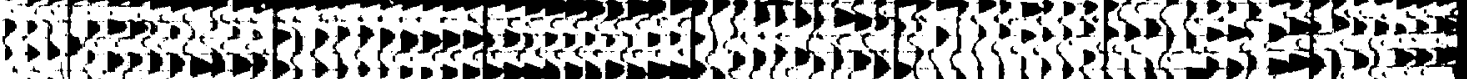

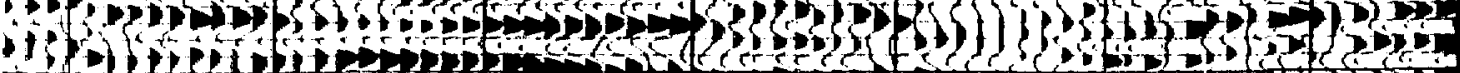

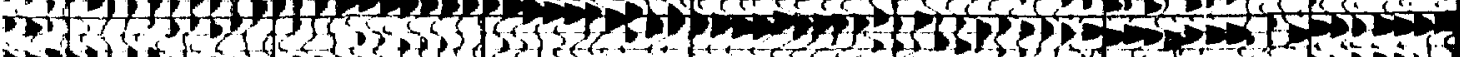

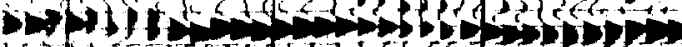

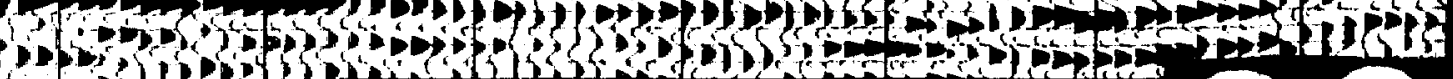
- of

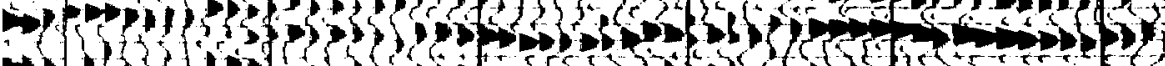

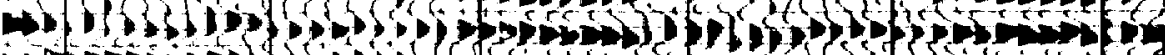

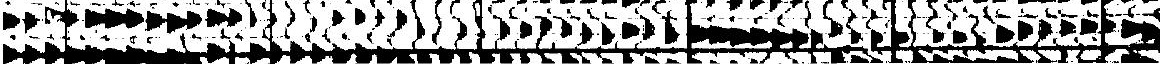

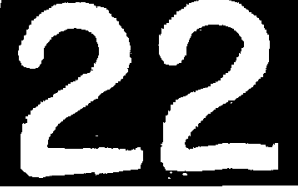




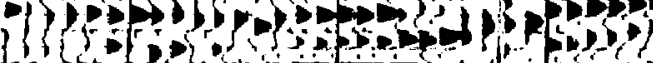

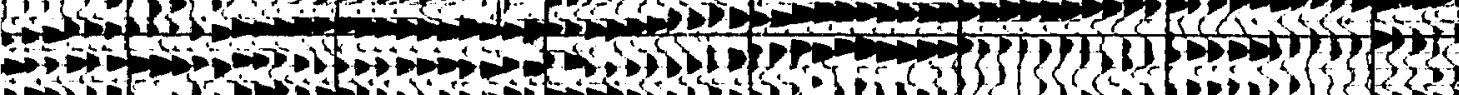

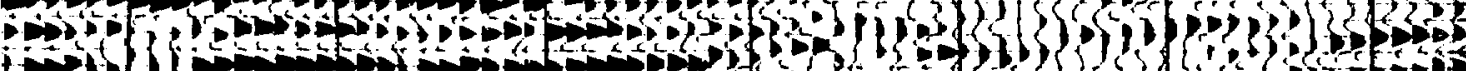

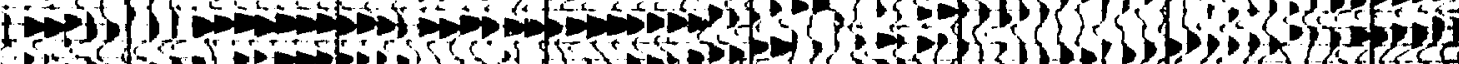

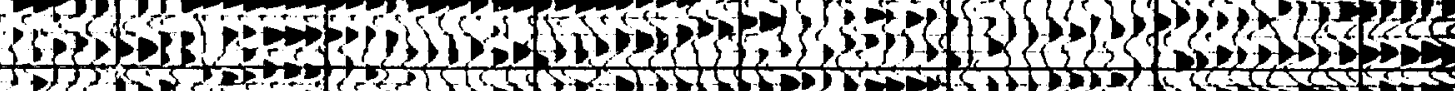

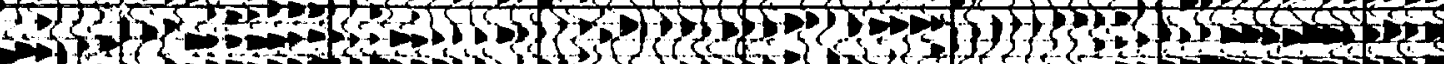

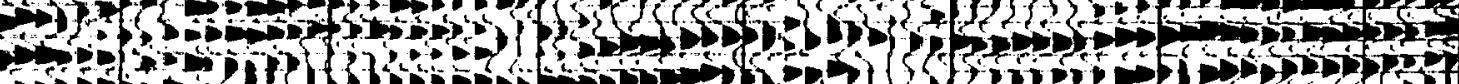

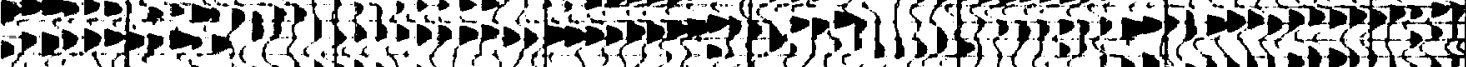

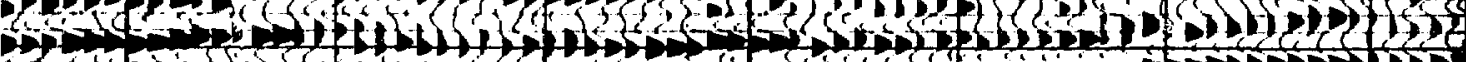

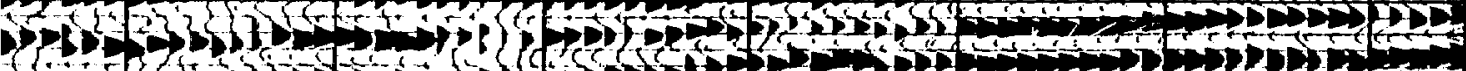

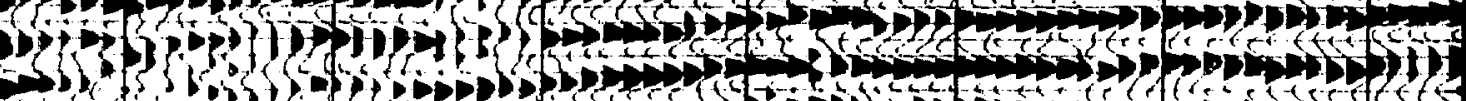

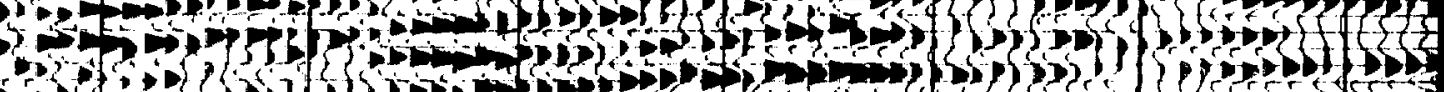

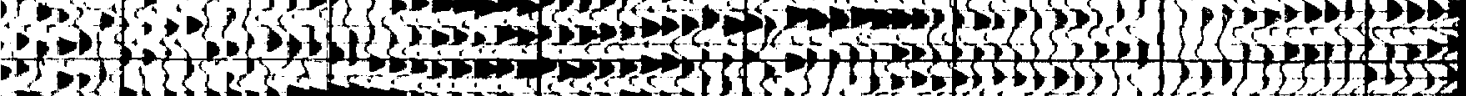

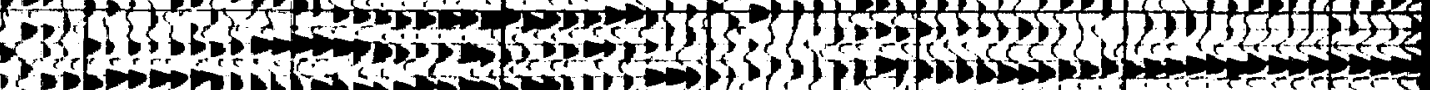

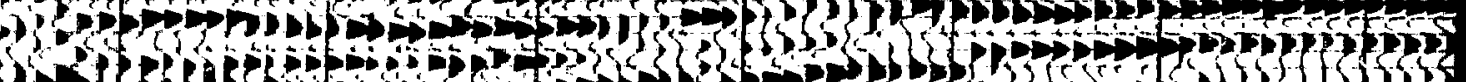

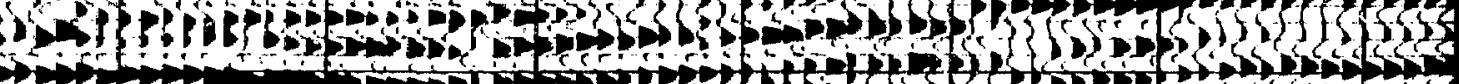

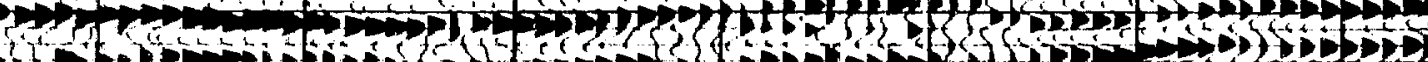

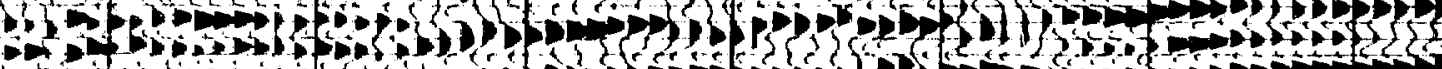

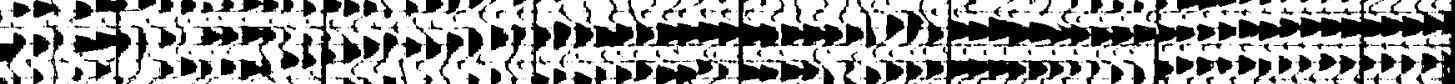

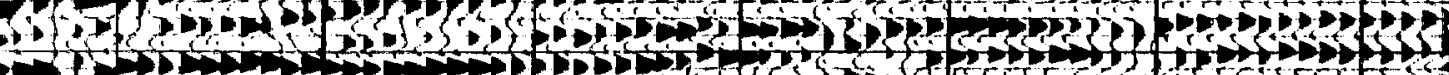

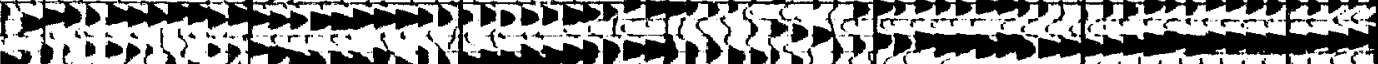

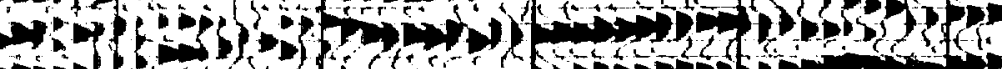

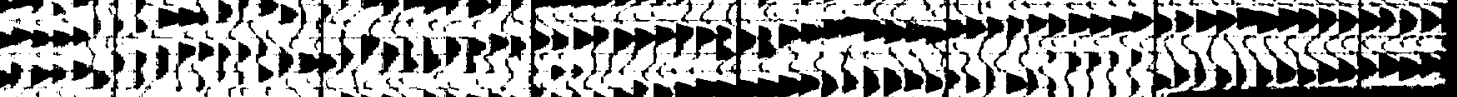

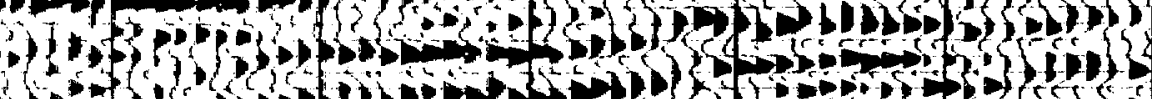

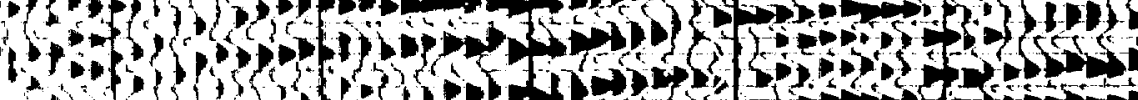




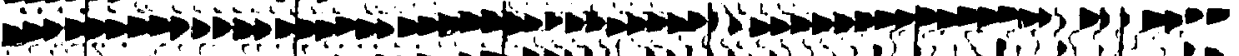

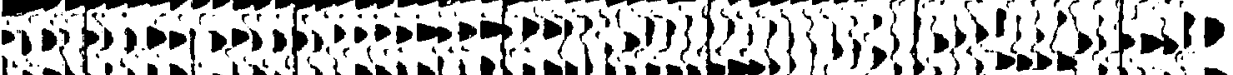
fe

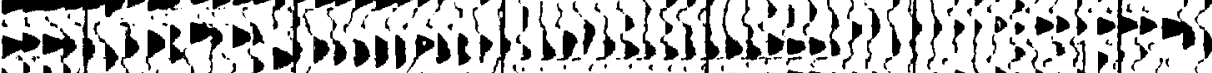

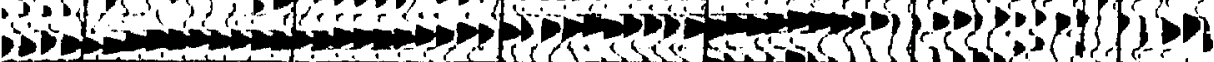

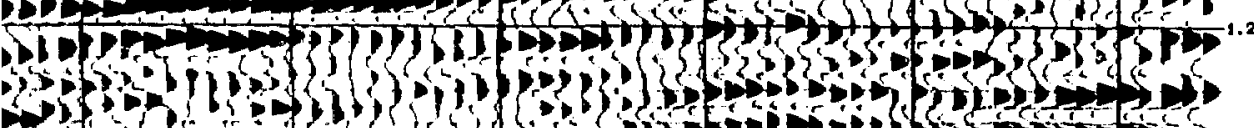

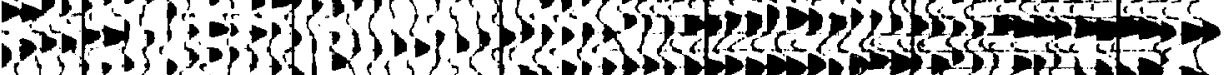

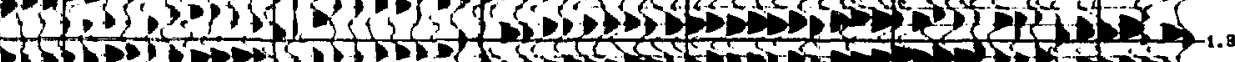

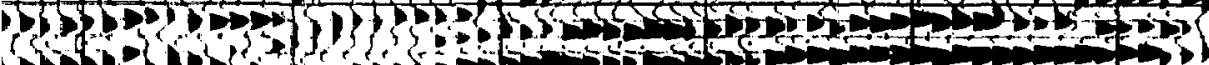

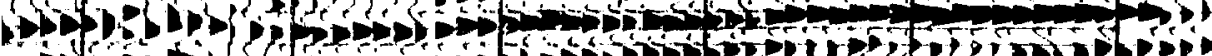

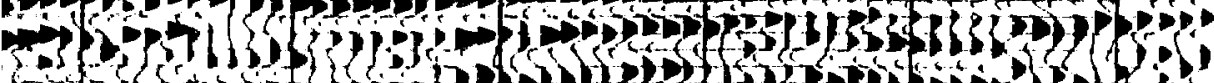

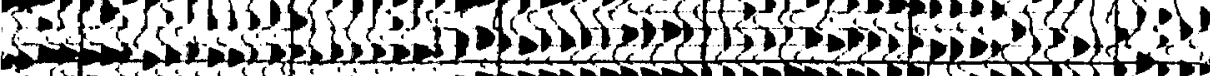

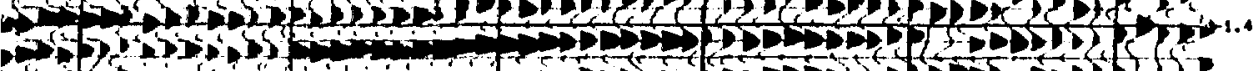

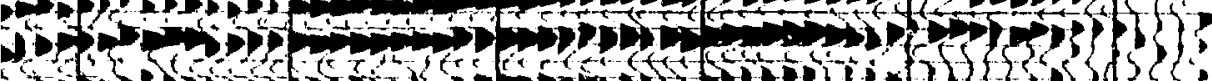

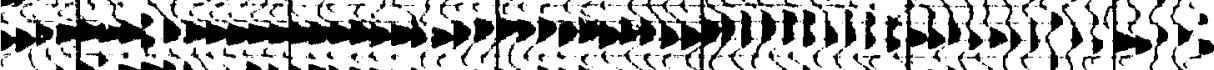

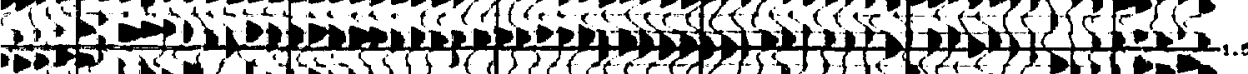

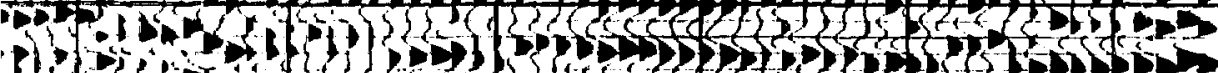

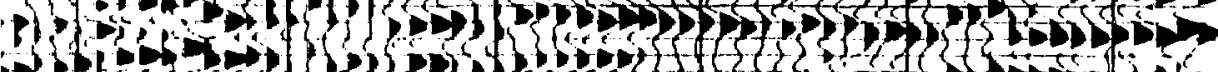

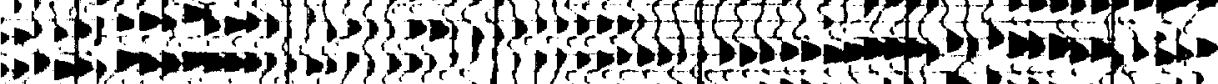

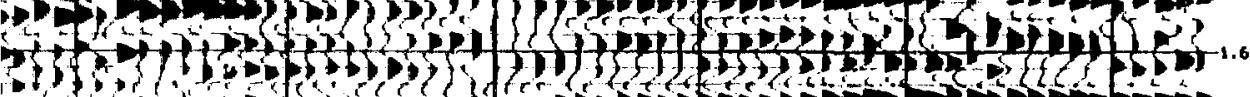

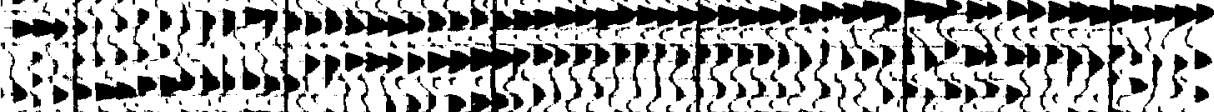

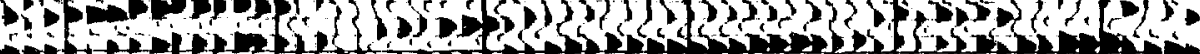

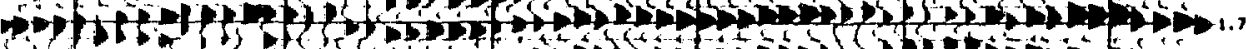

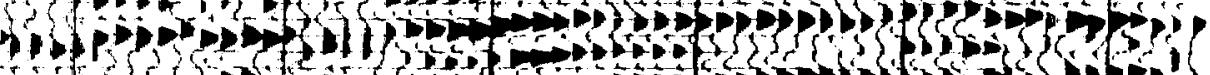

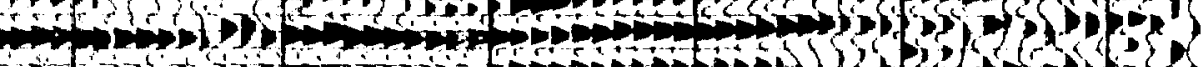

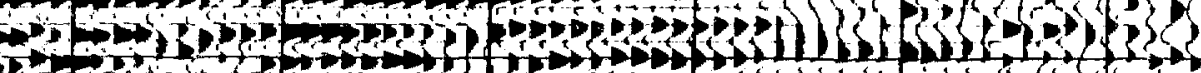

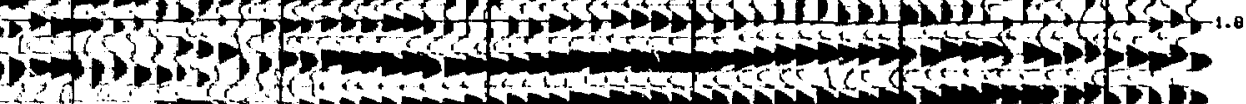

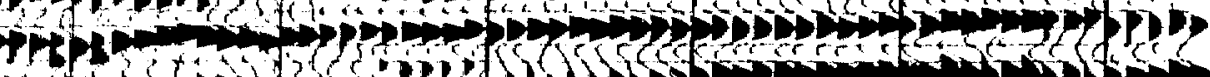

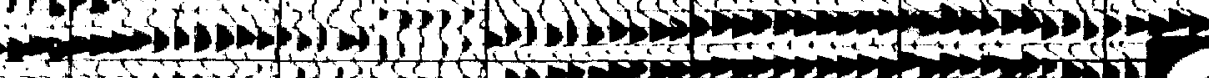

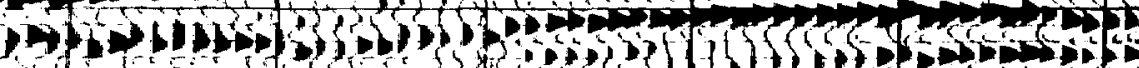

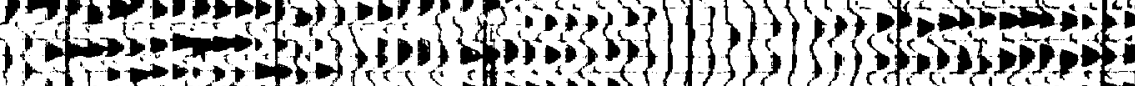

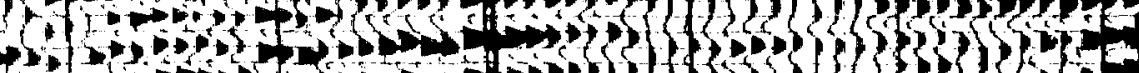

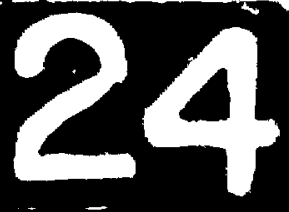


(1)

7. 7.7.723 7.35 97250.23 .

(1)

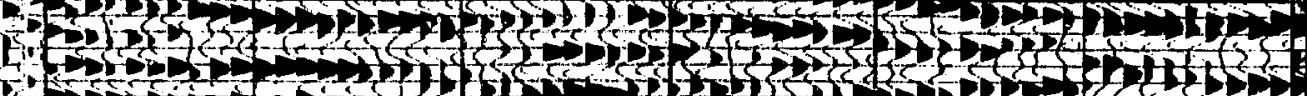

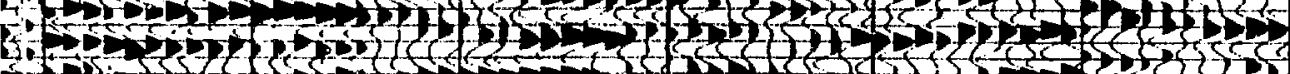

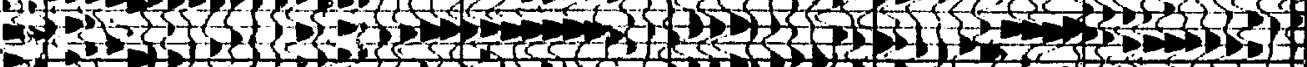

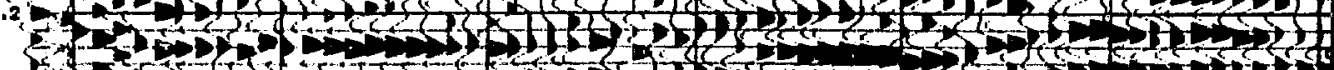

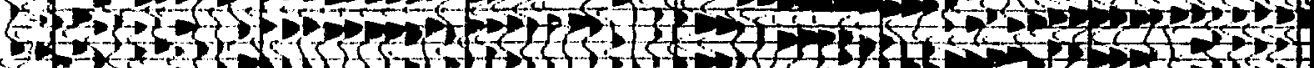

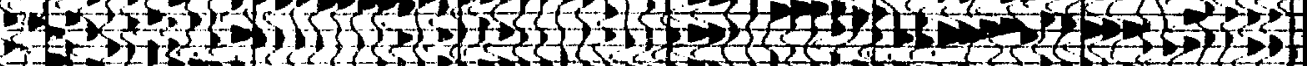

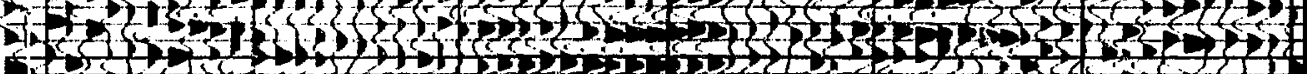

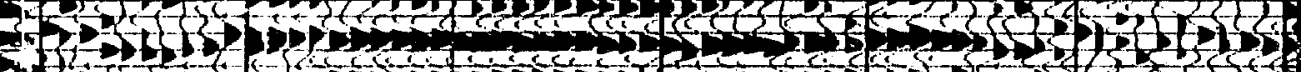

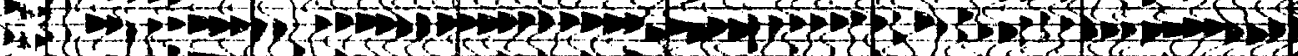

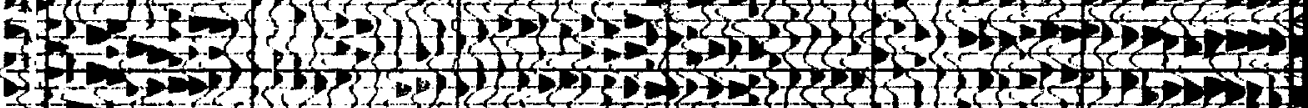

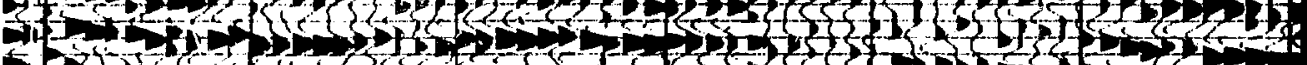

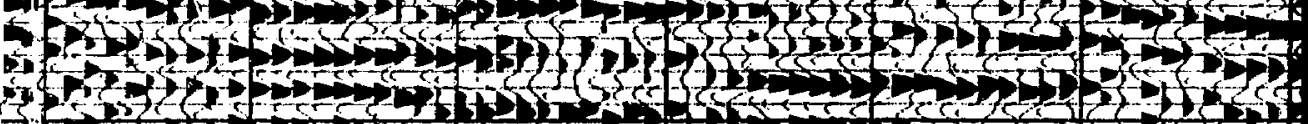

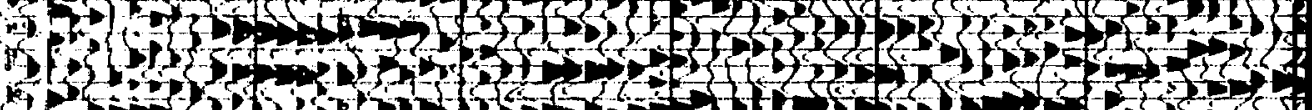

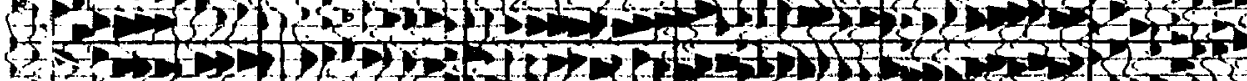

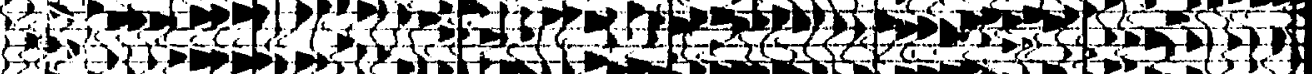

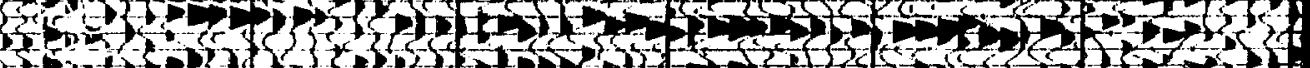

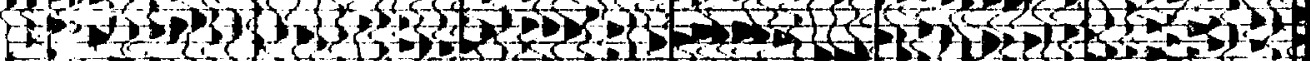

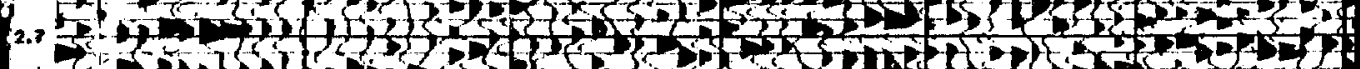

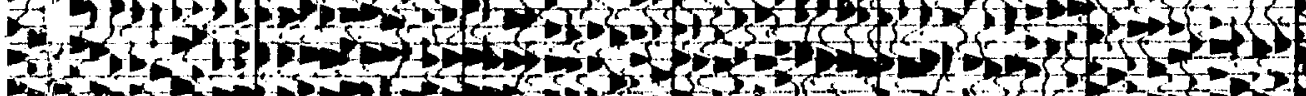

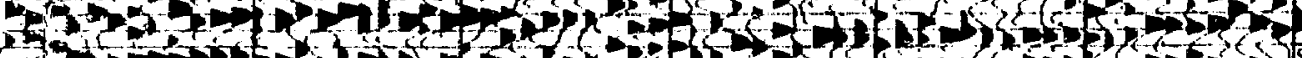

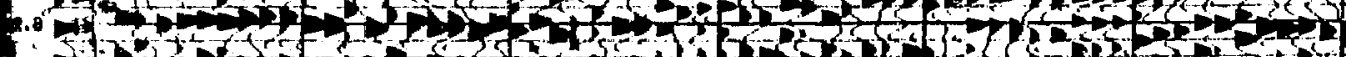

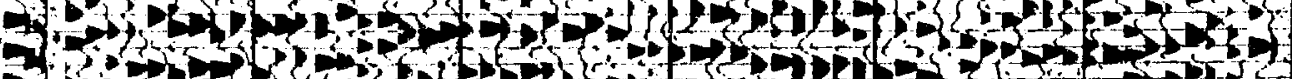

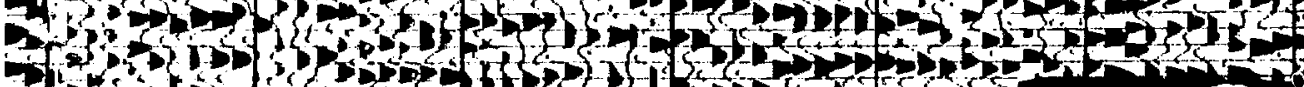

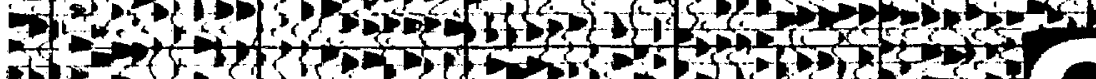

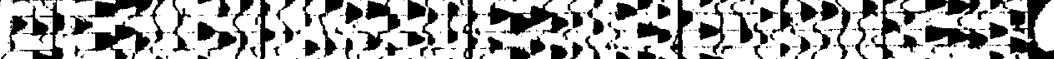

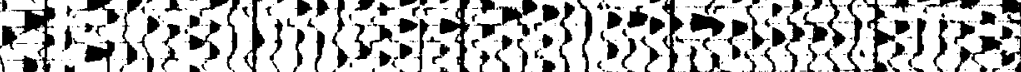




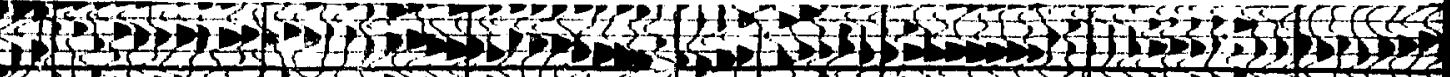

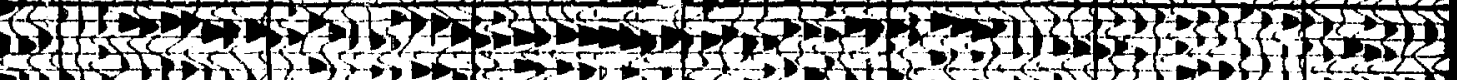

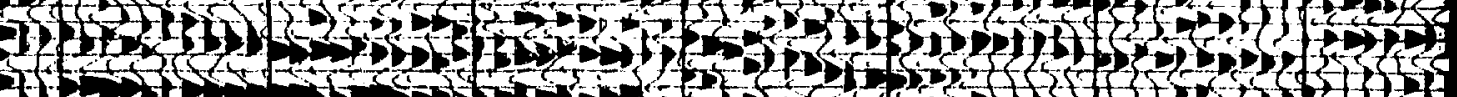

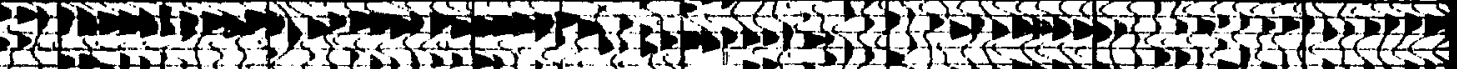

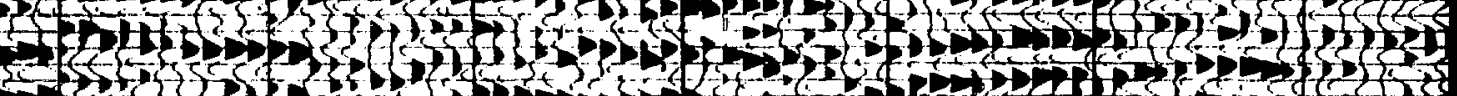

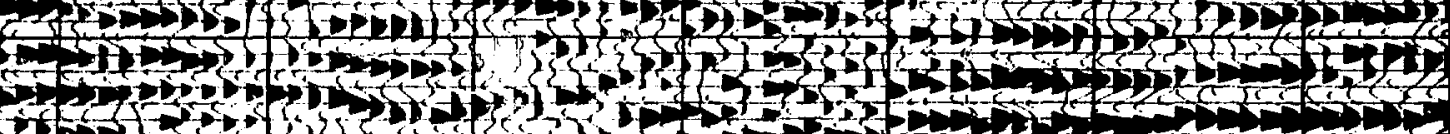

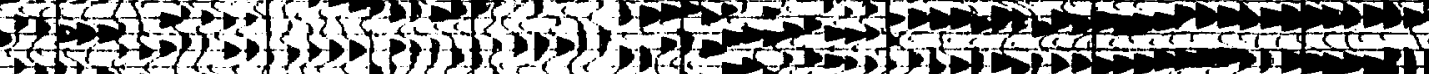

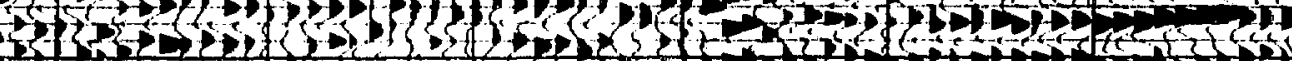

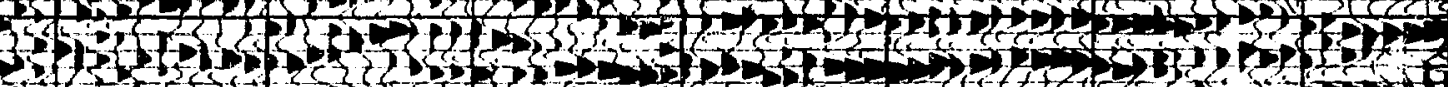

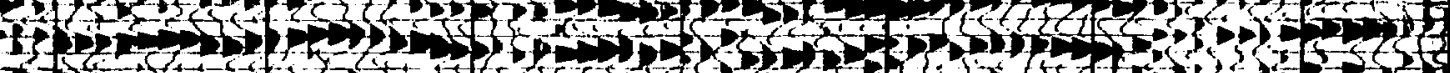

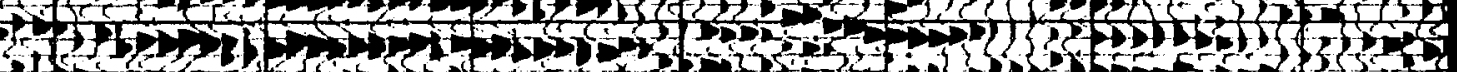

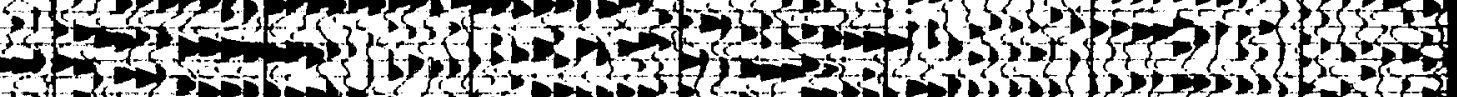

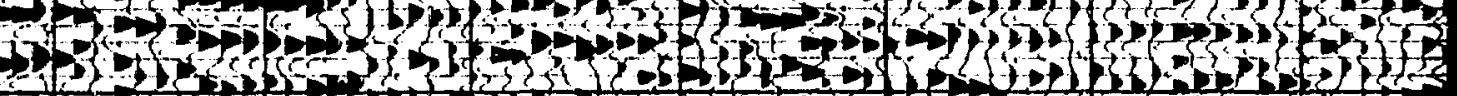

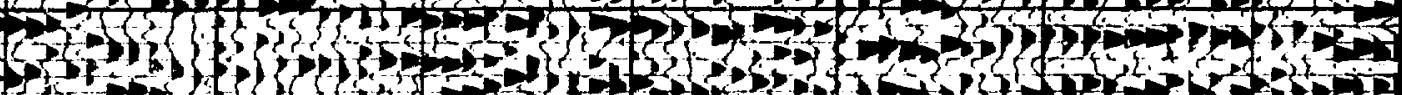

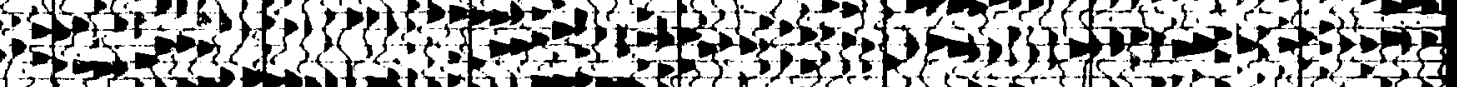

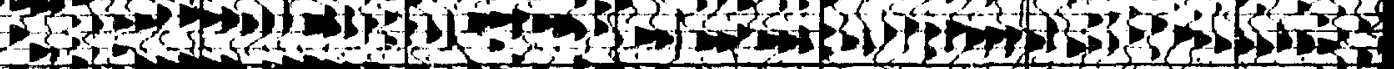

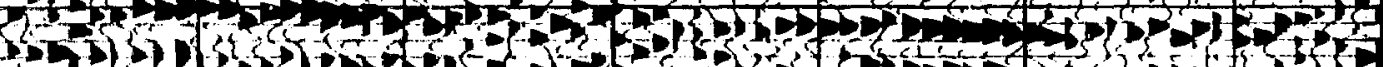

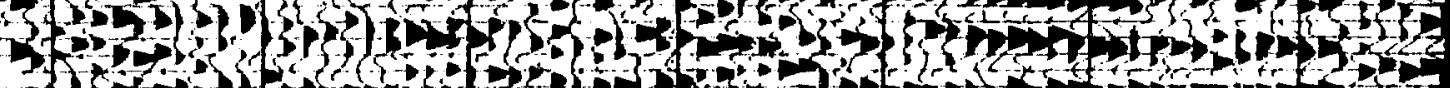

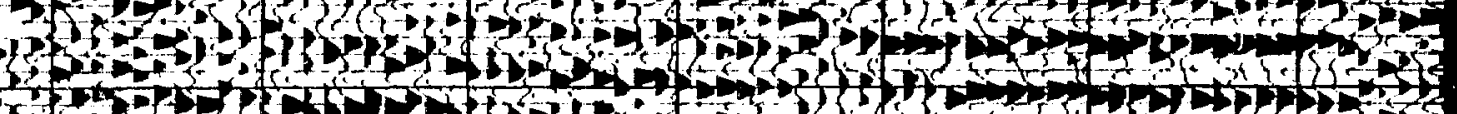

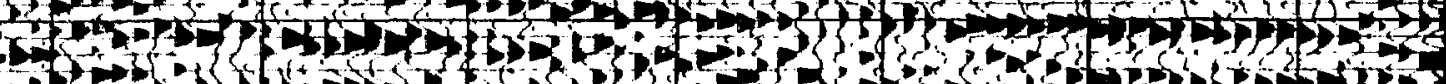

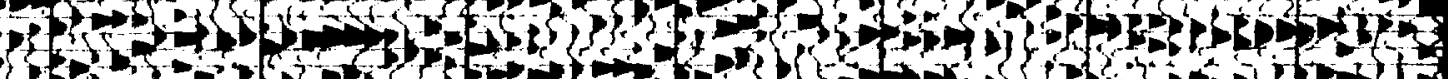

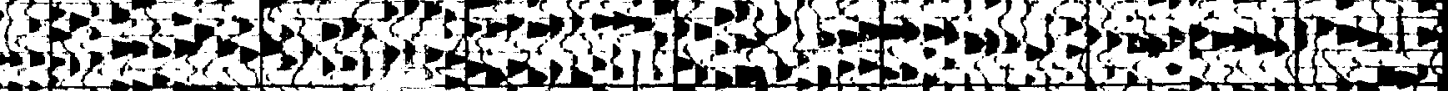
if

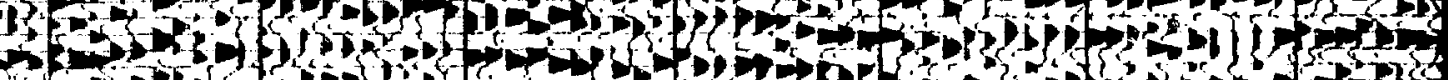

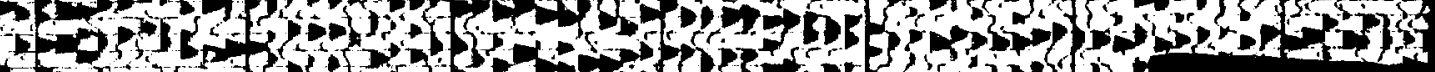

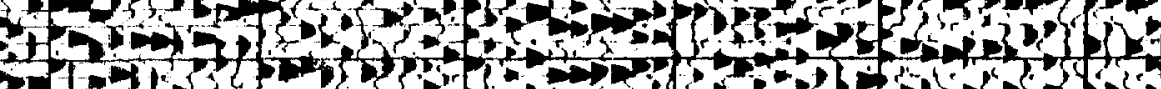

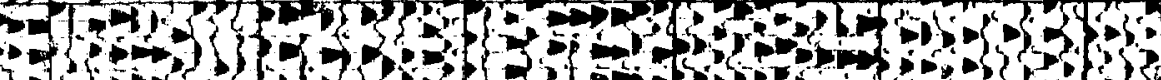

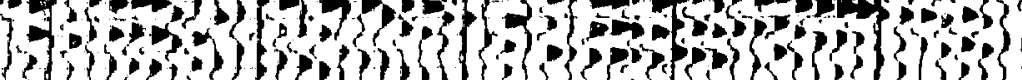




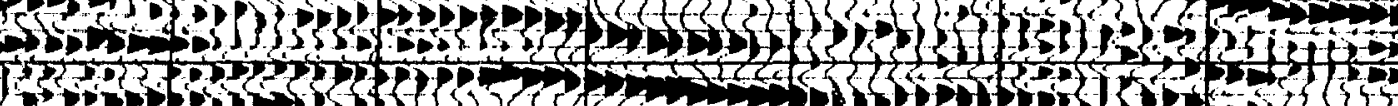

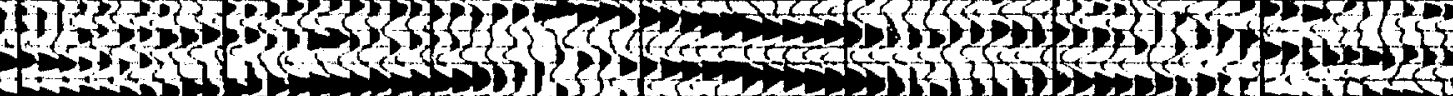
202013

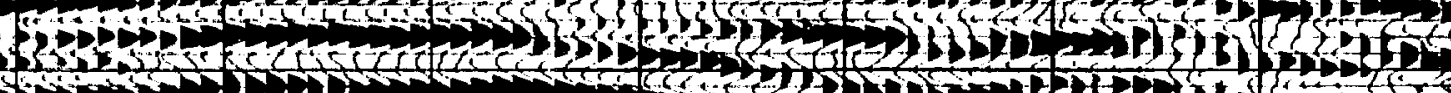
12 - 13 Hist H.

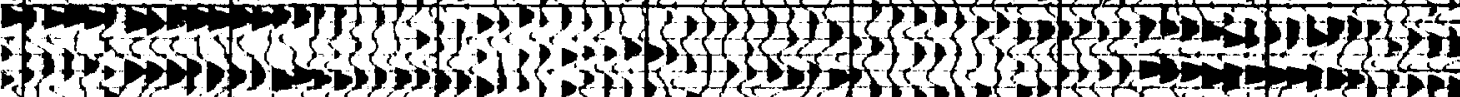

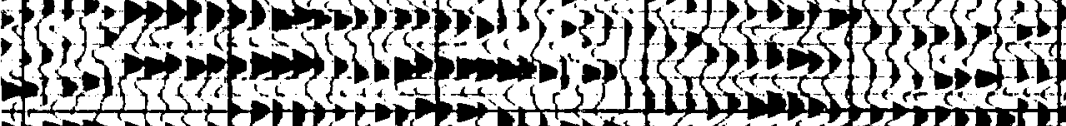

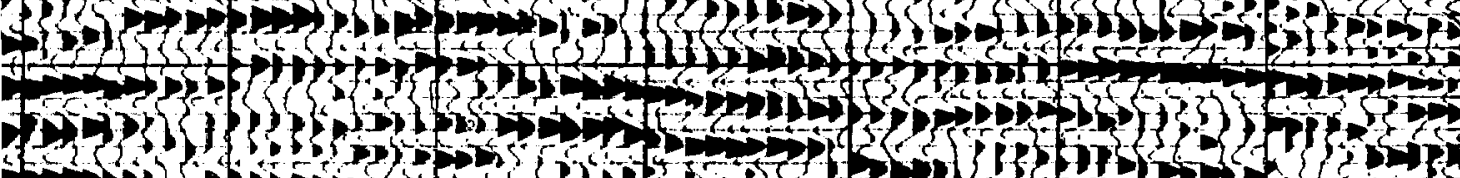

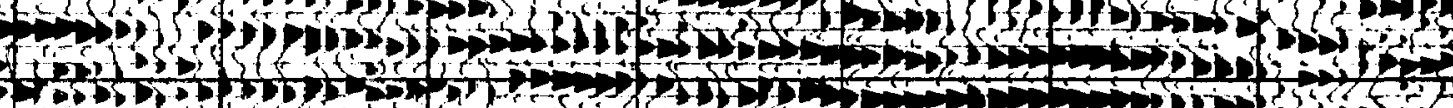

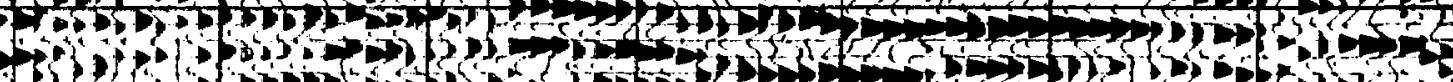

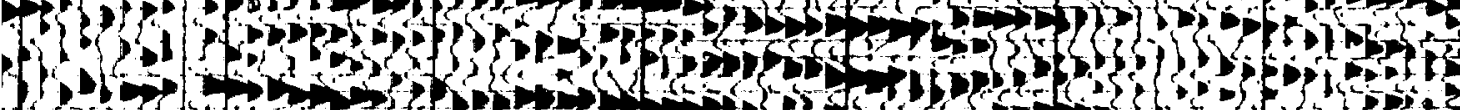

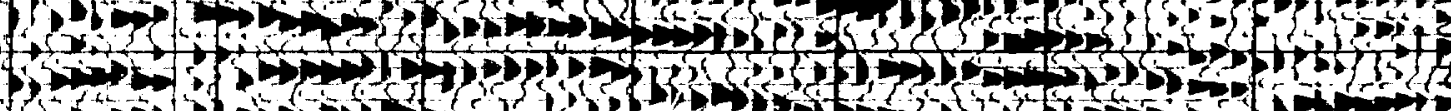
3

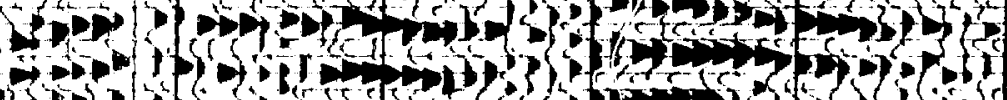

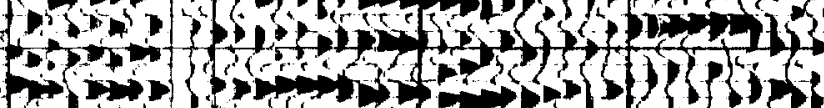

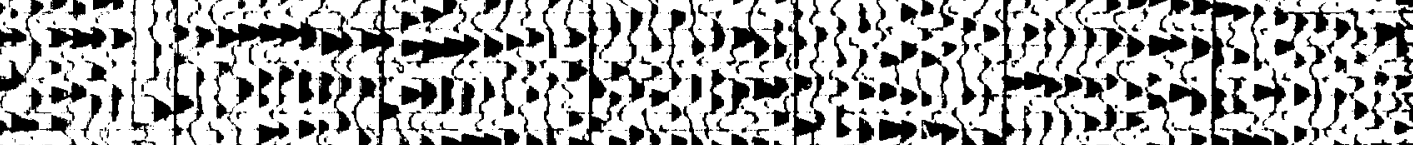

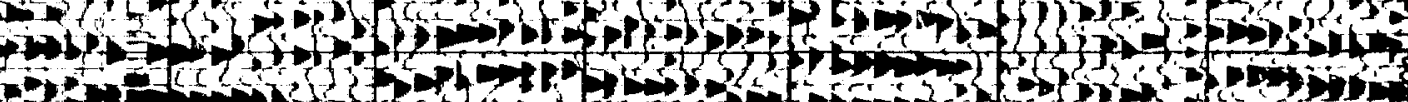

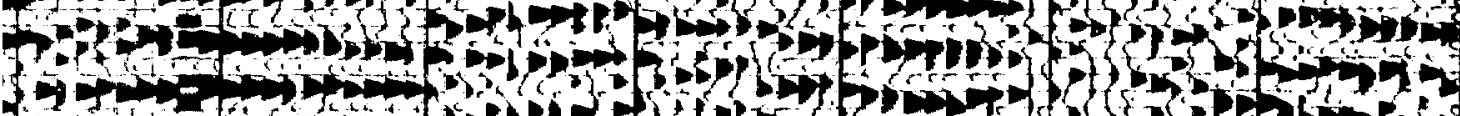

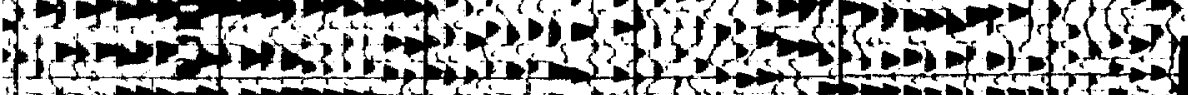

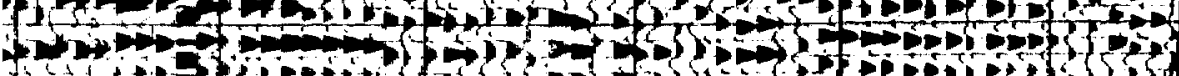

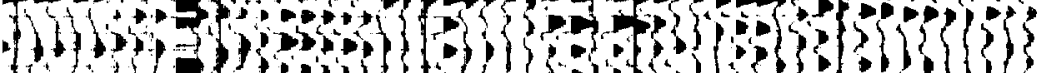




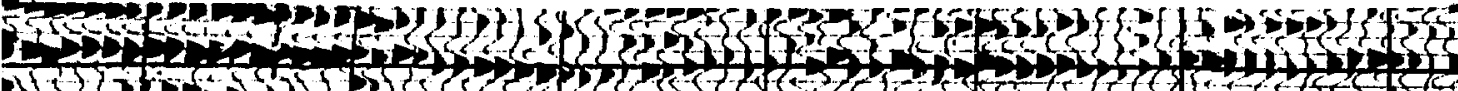

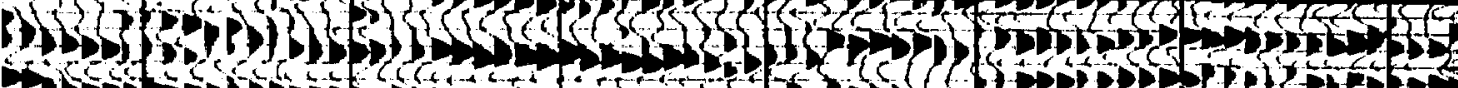

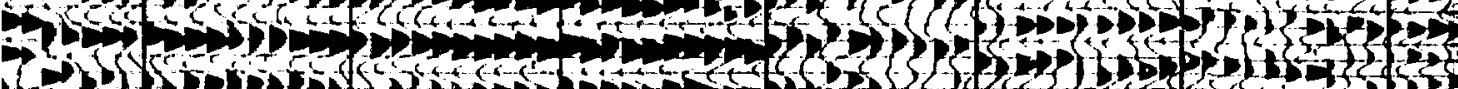
3.

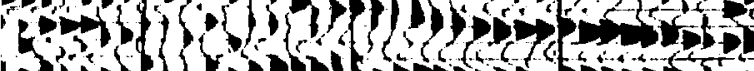

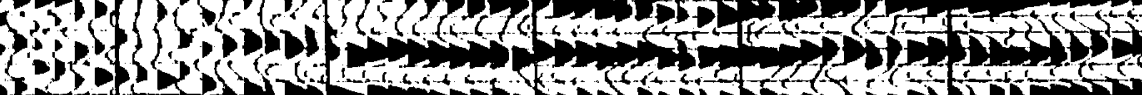
D

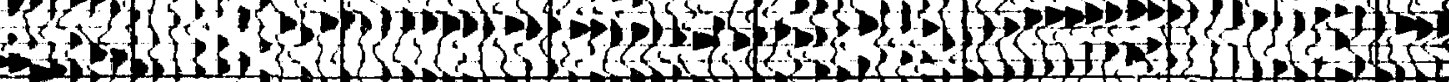
- 4 .3.

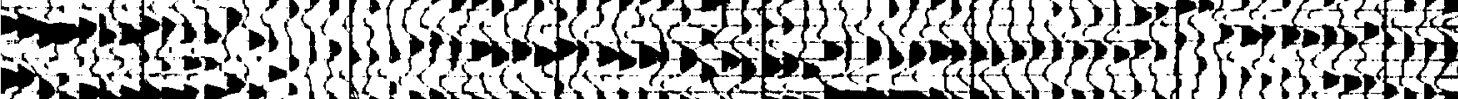

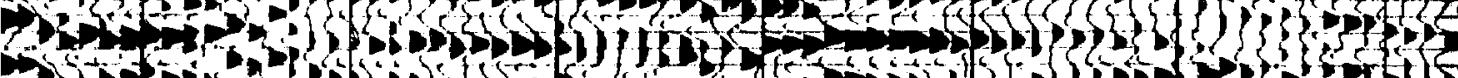

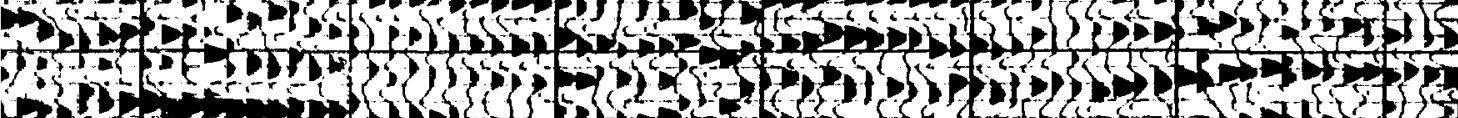
rin

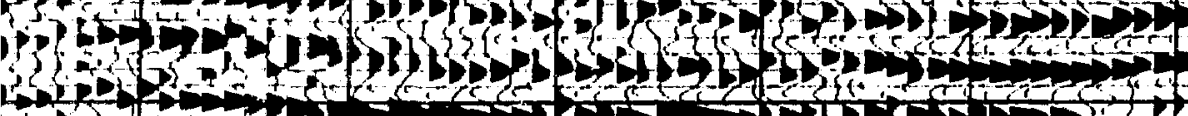

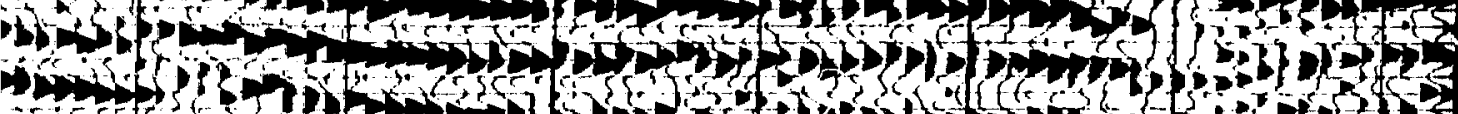

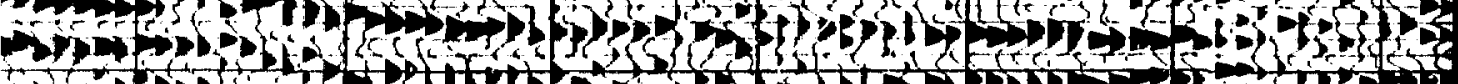

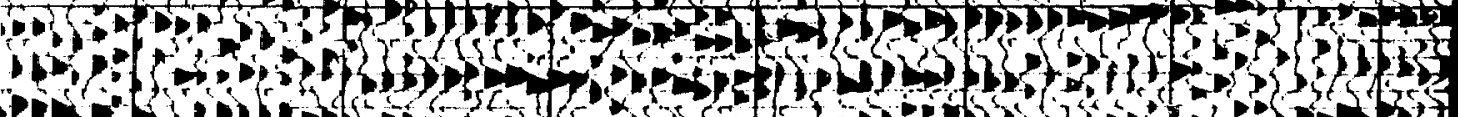

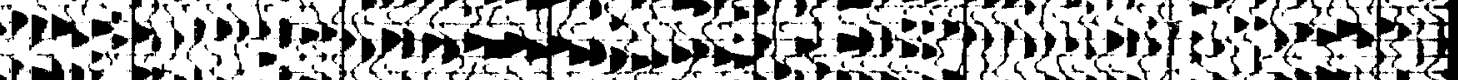

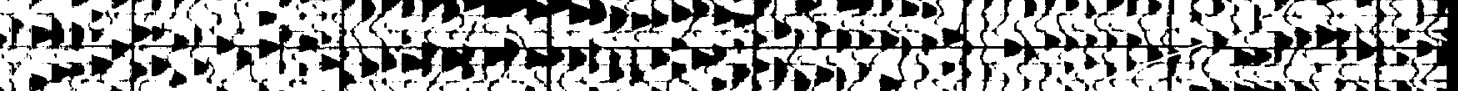
$\rightarrow+40$ in

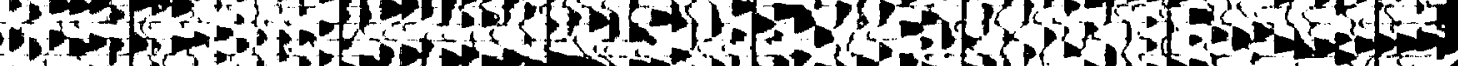
D.3.3,

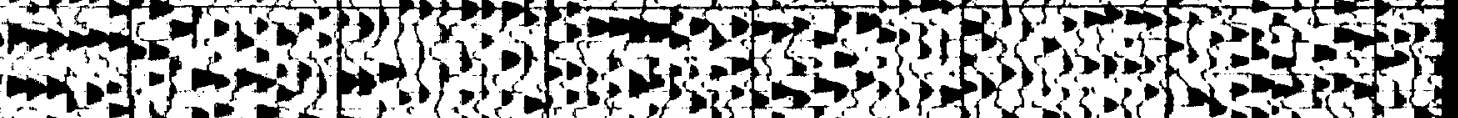

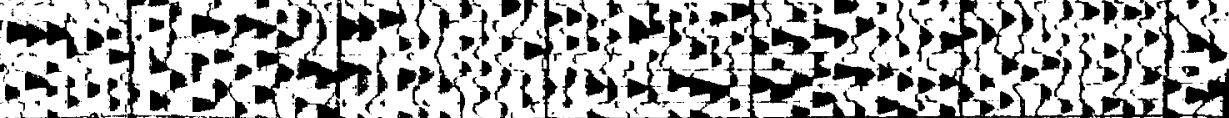
(3)

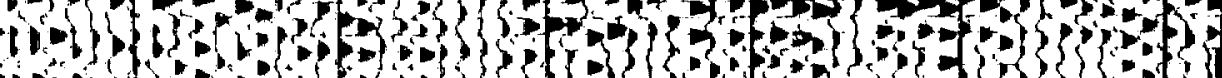

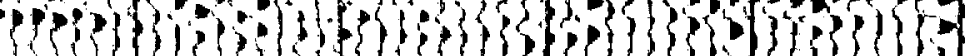




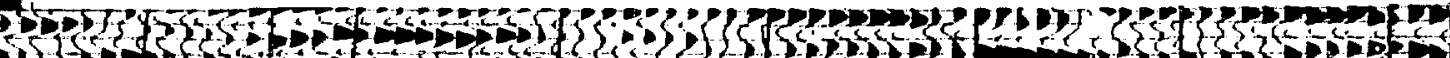

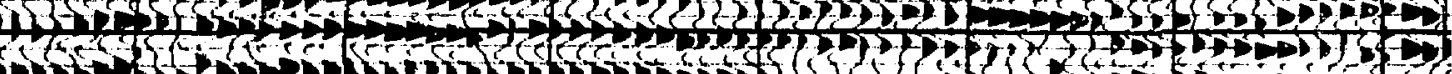

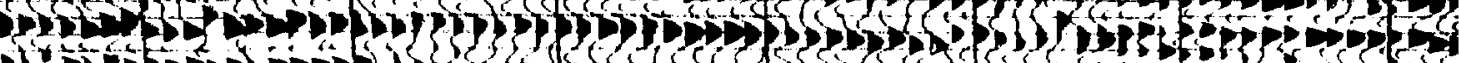

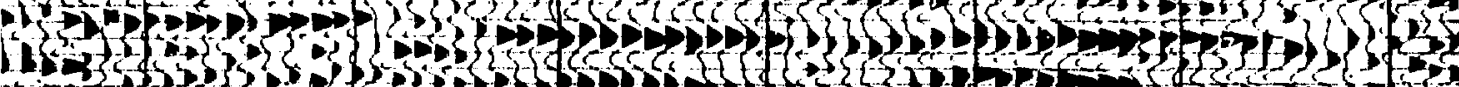

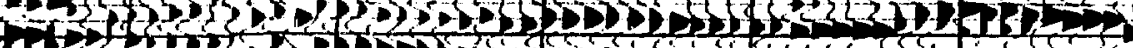

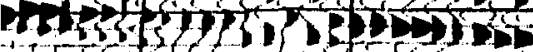

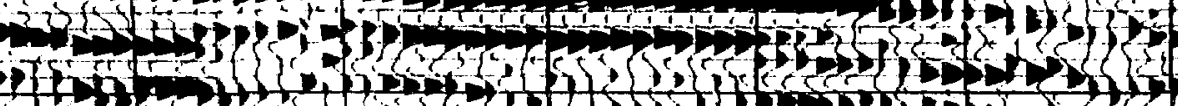
4

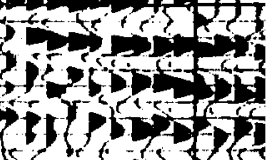

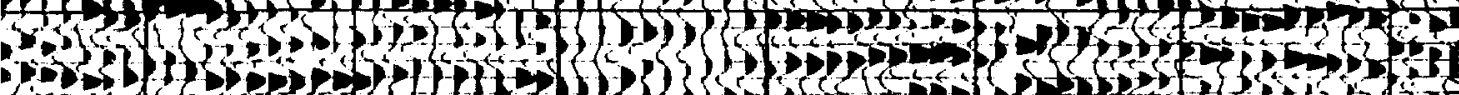

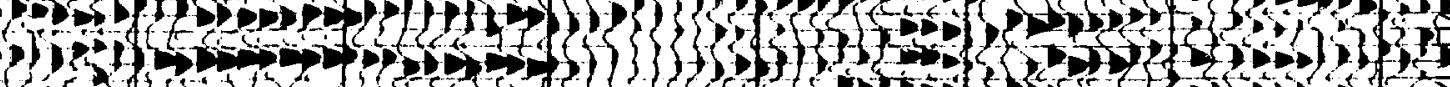

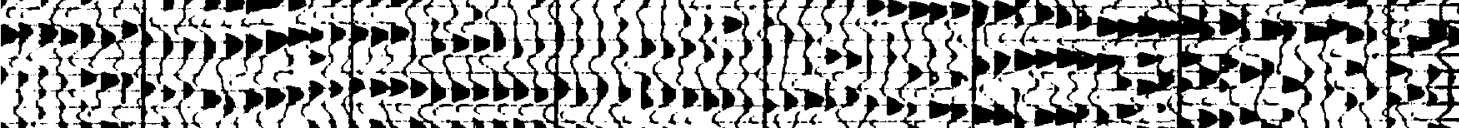

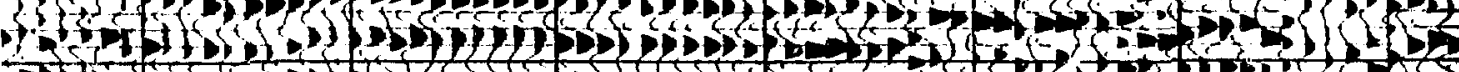
(1)

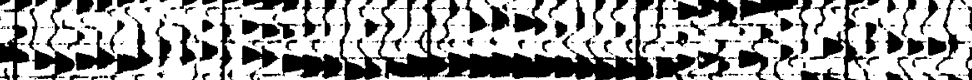

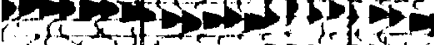

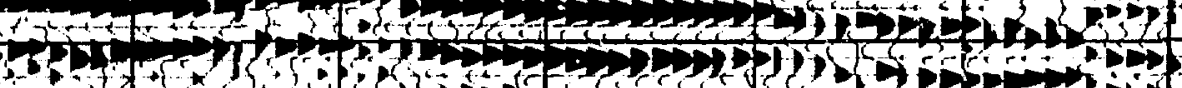

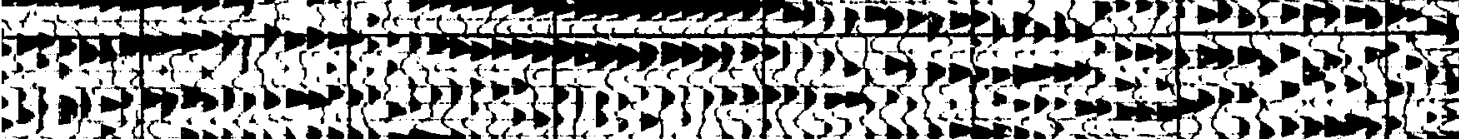

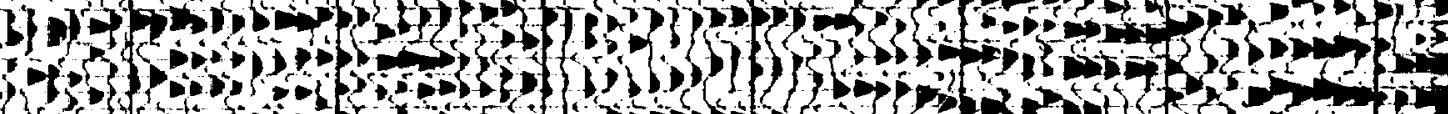
S.

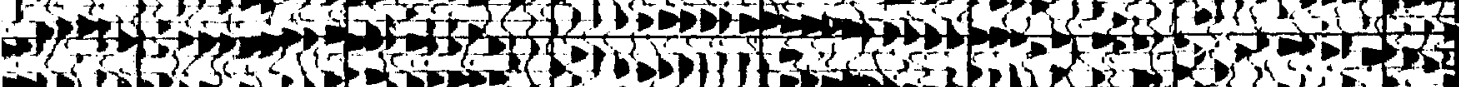

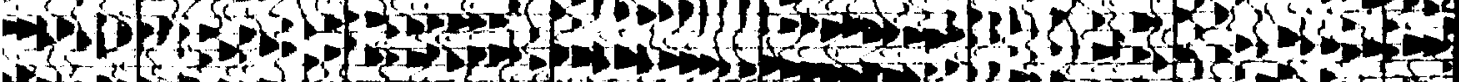

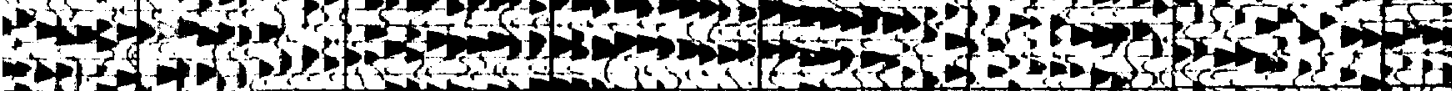

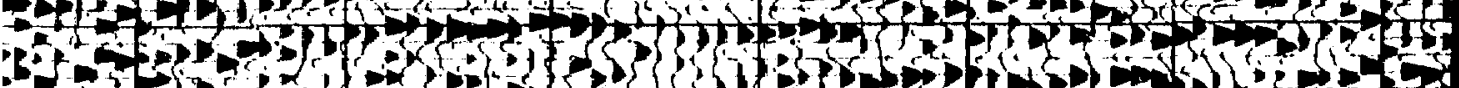

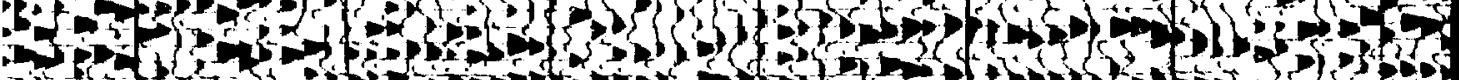

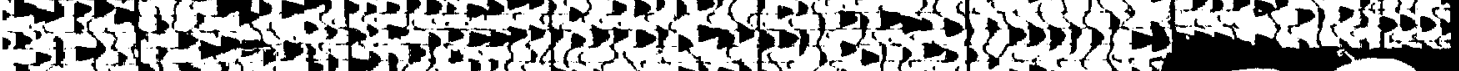

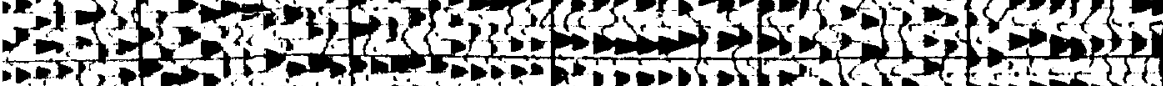
-Pos - 5 - 
D.

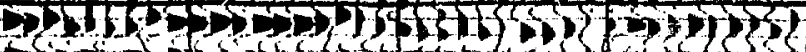

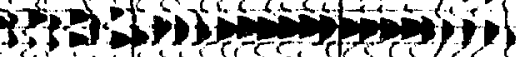

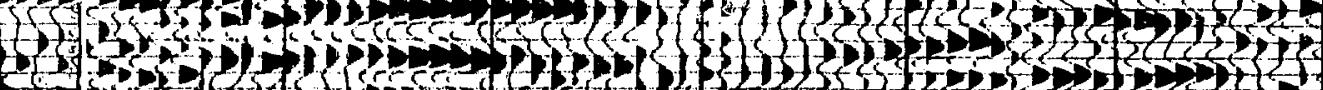

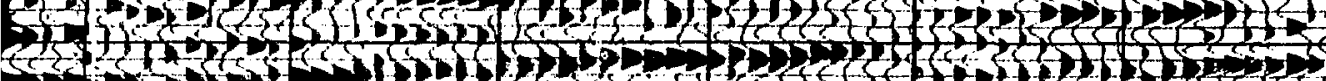

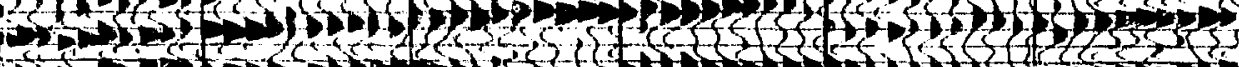
(4)

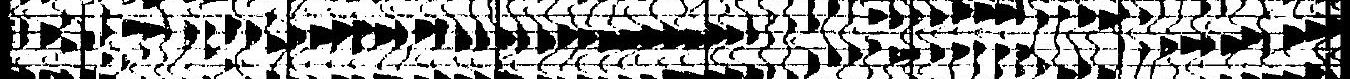

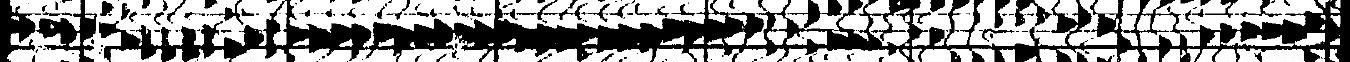

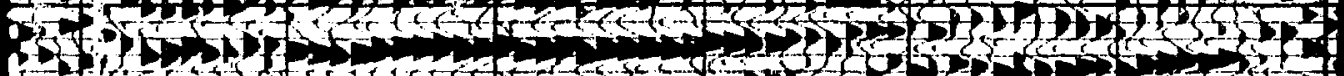

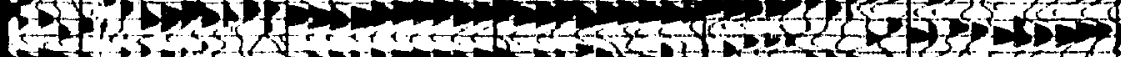

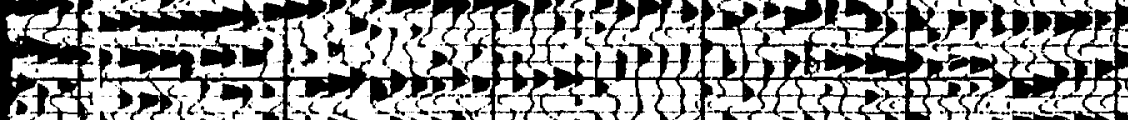

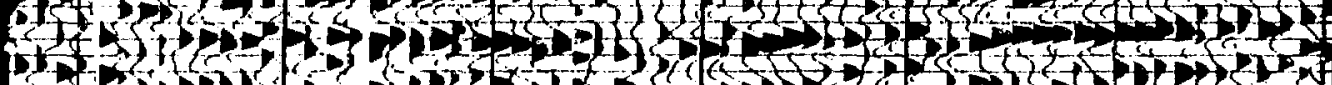

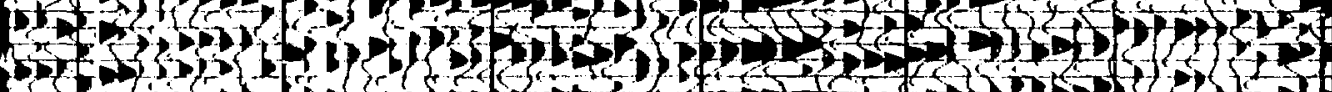

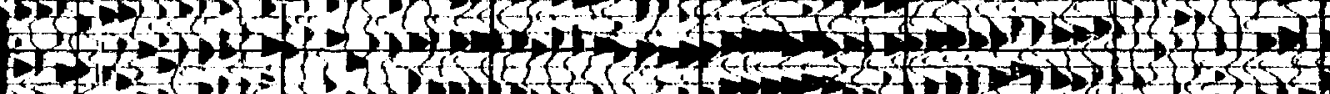

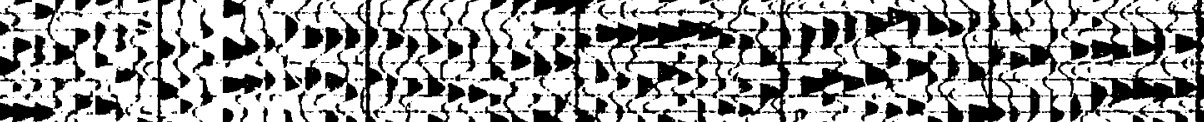

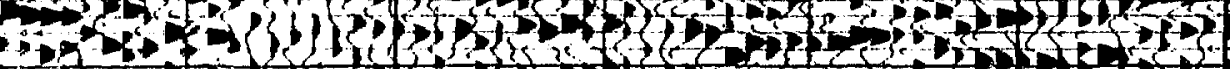

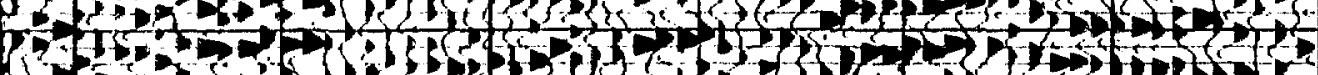

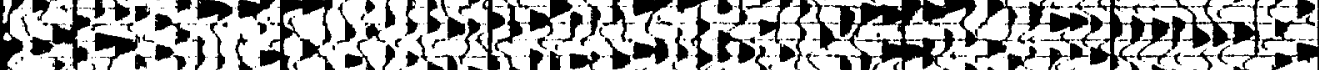

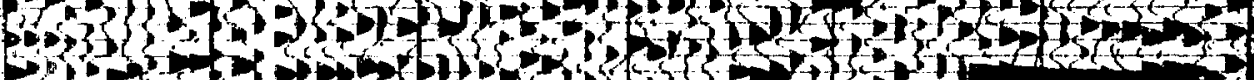

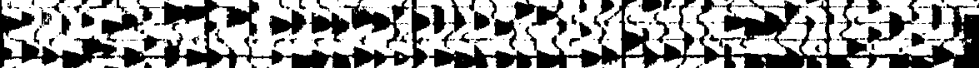

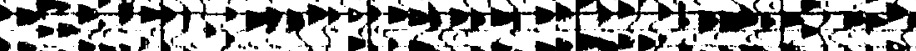

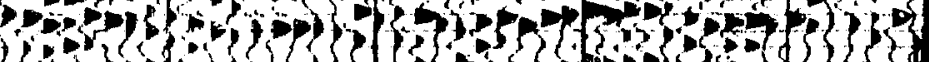

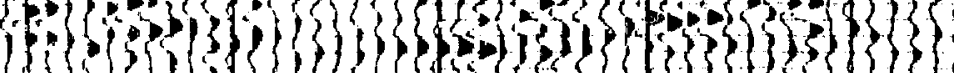


SToros

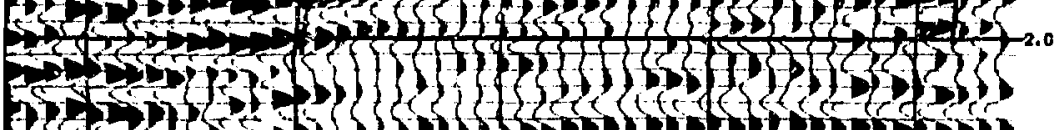
7.7. (7) (1)

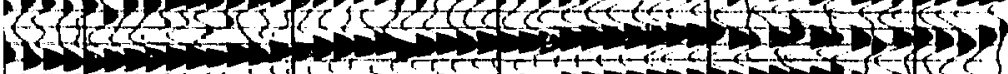
20

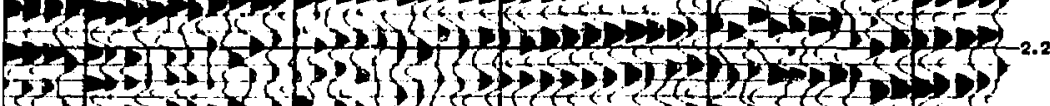

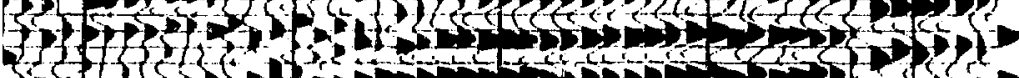
3.

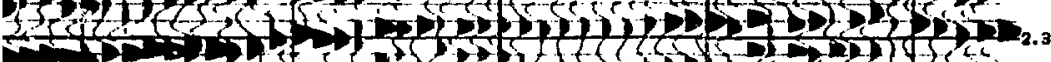

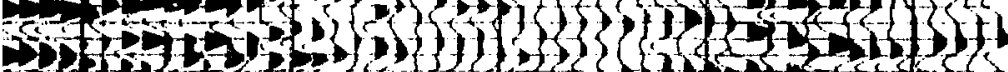

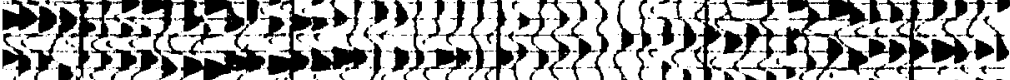

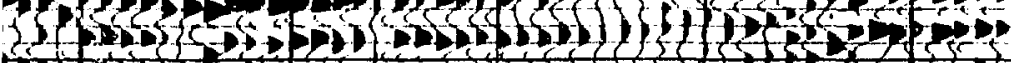

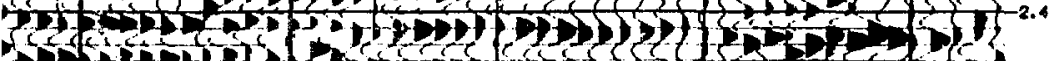

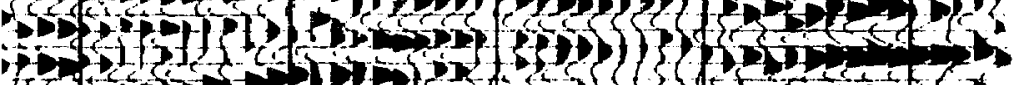

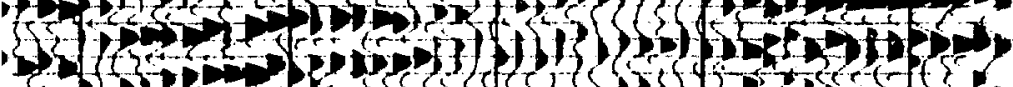

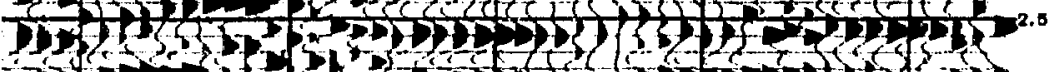

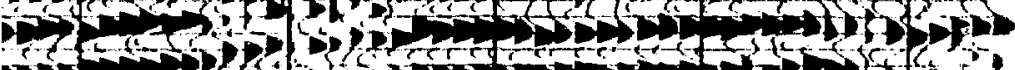

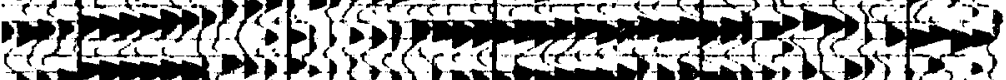

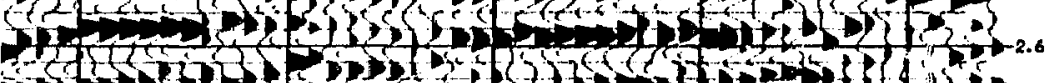

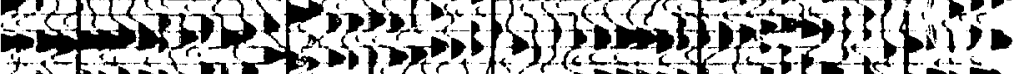

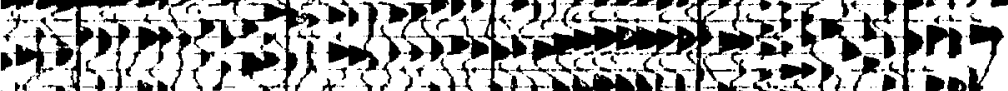

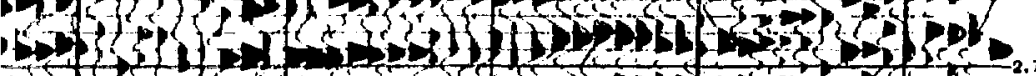

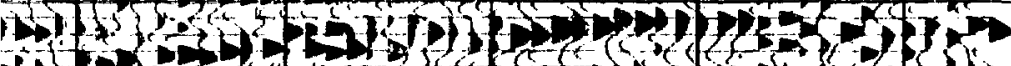
$x \rightarrow-35 x-5$ ind

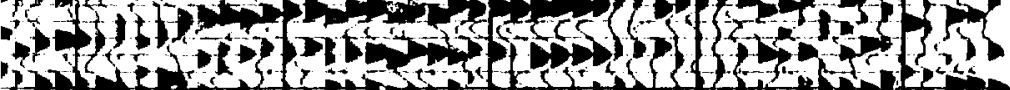

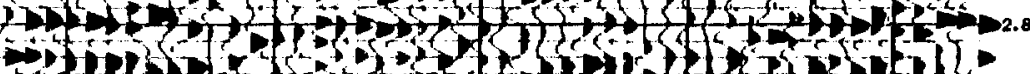

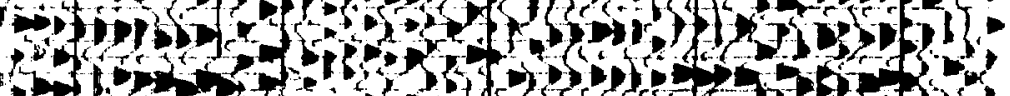

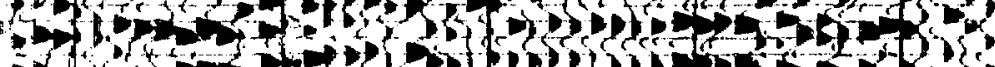

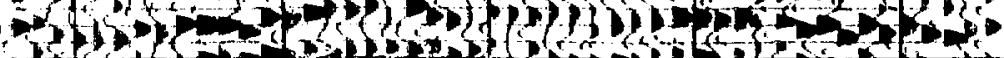
to t on

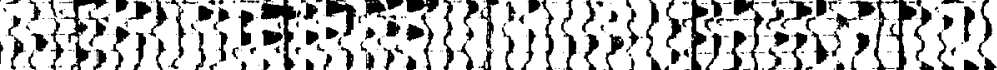

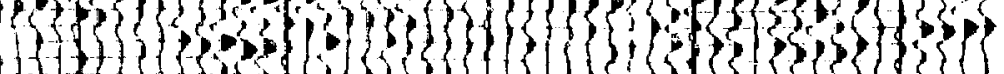




$\sec 2$

VES. ANRL

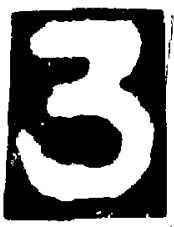

76

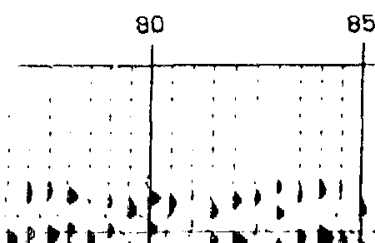

85

90

95

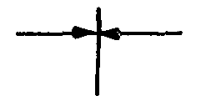

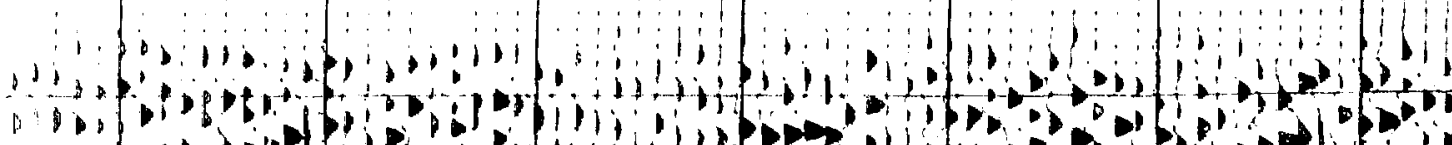

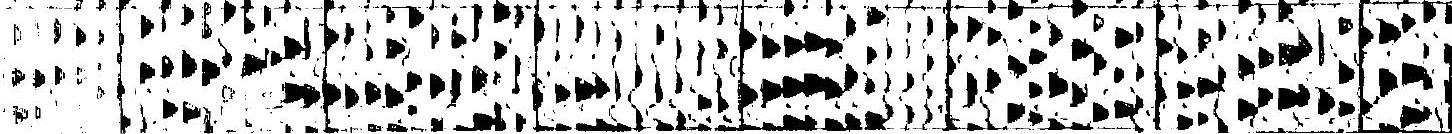






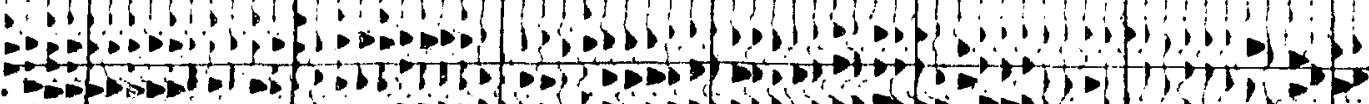

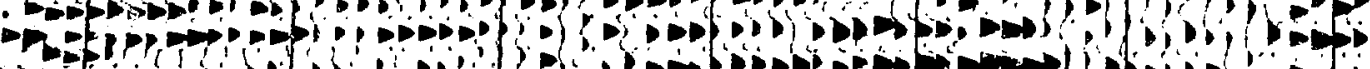

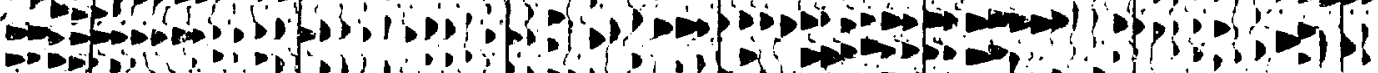

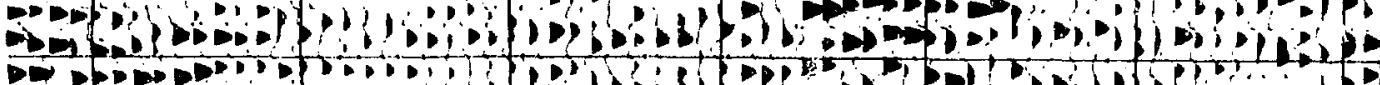

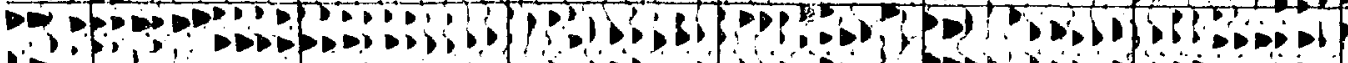

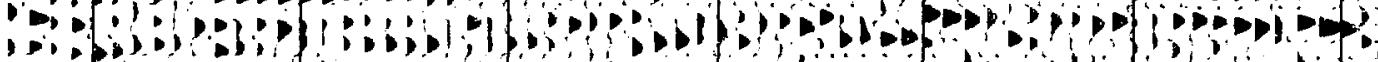
$\because$ H, of

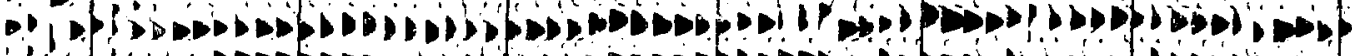
$\because$ :

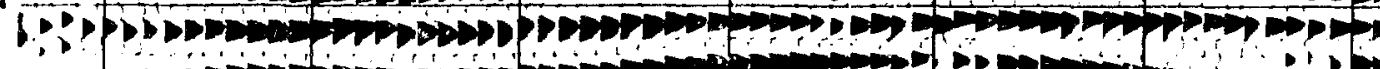

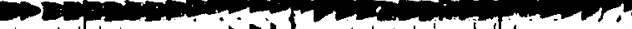

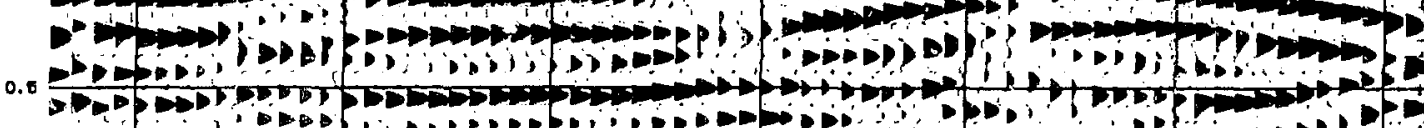

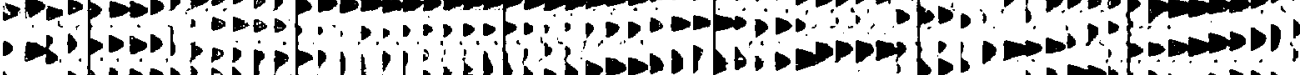

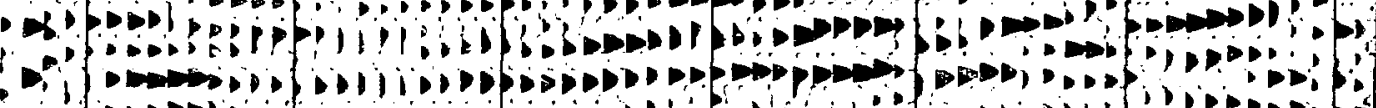

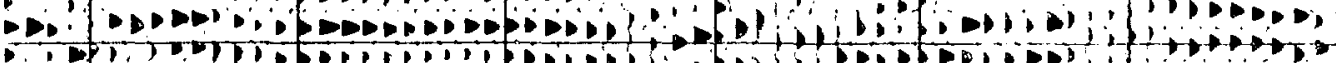

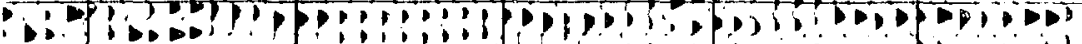

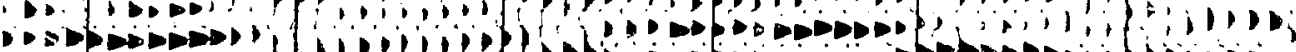

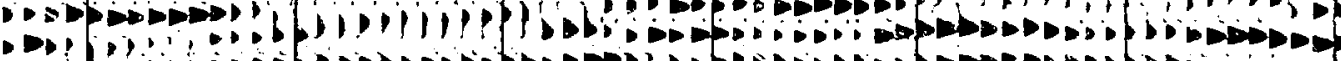

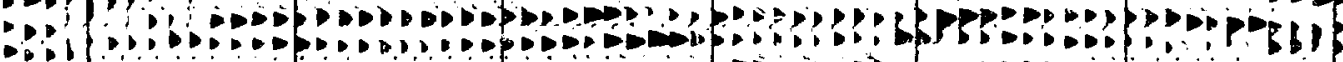
${ }^{\prime}$ IDD

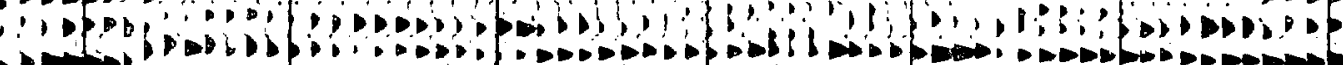

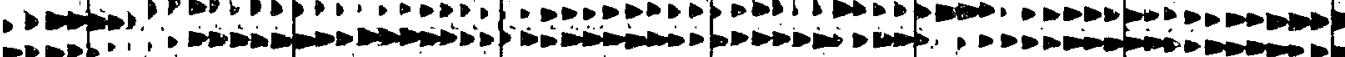

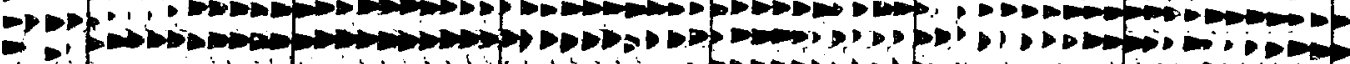

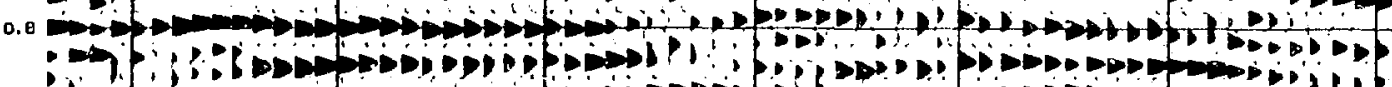

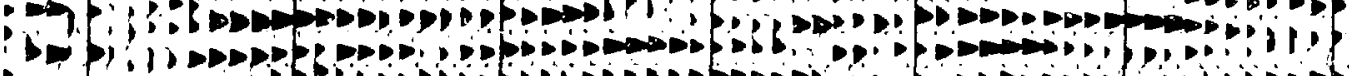

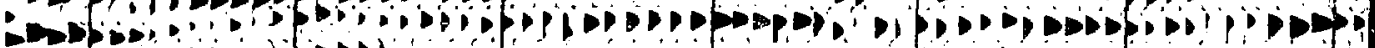

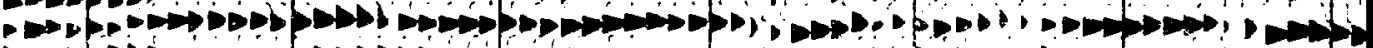

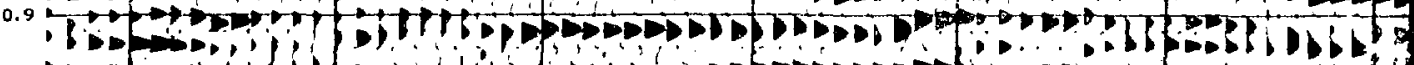
:P.

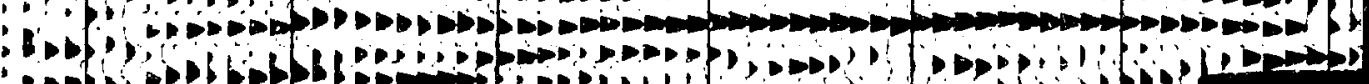

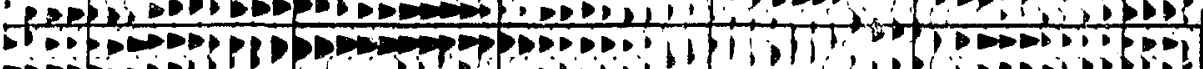

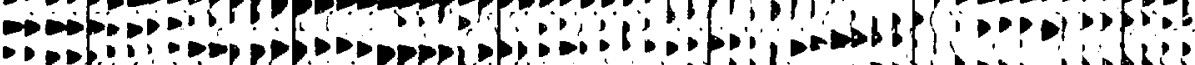

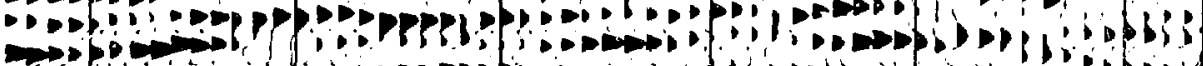

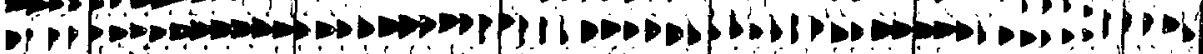


D. S.

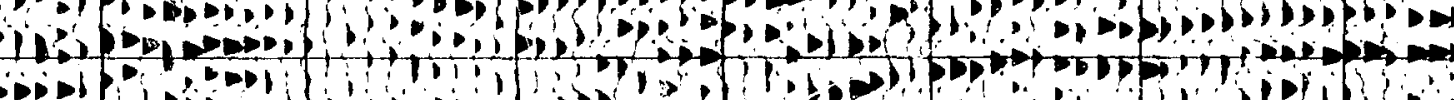
s.p. n.

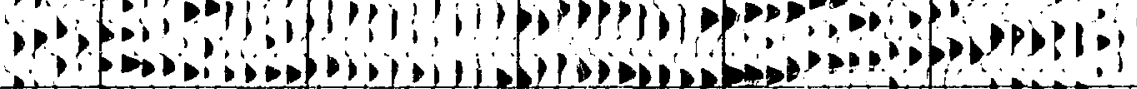
min (is:

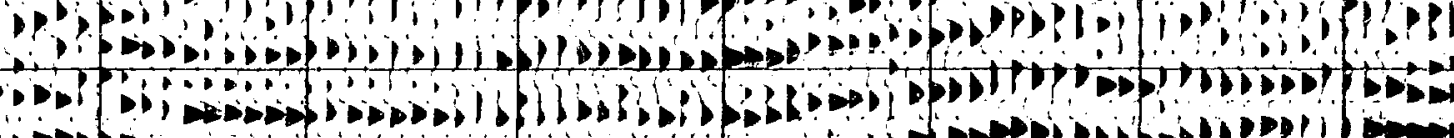

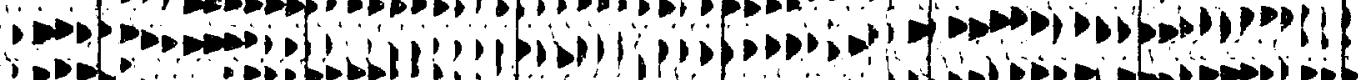

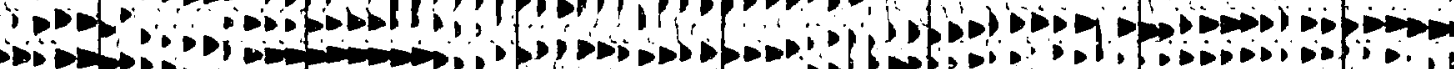

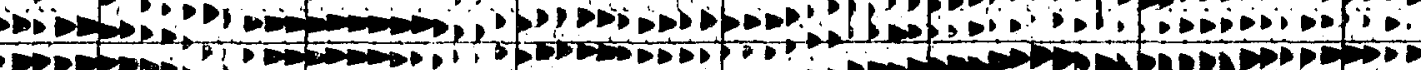

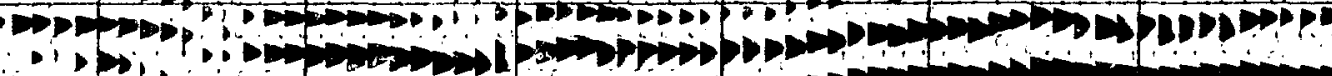

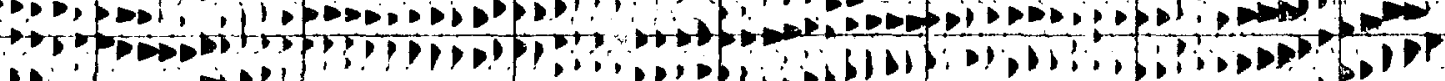

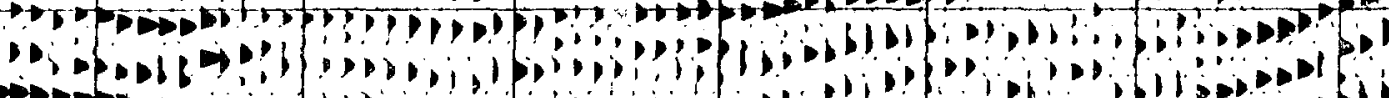

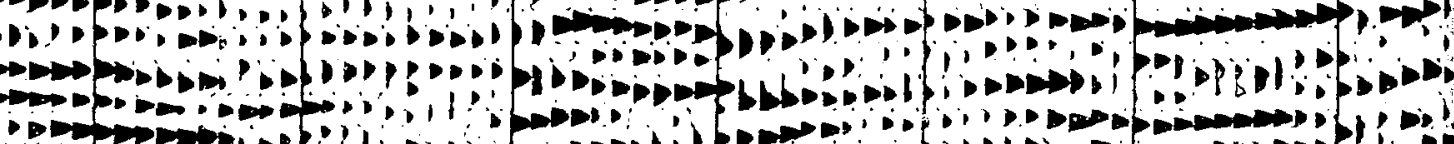
opos. I11 1.

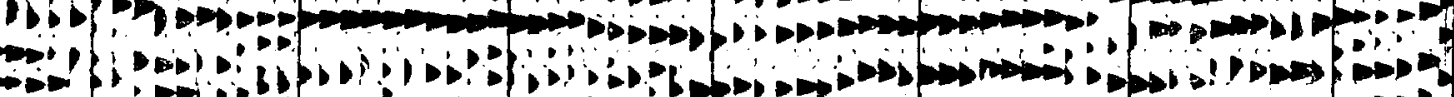

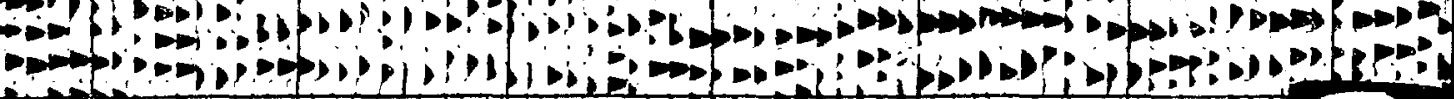
Tha

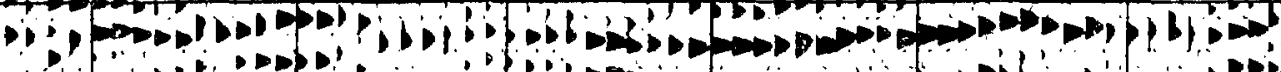

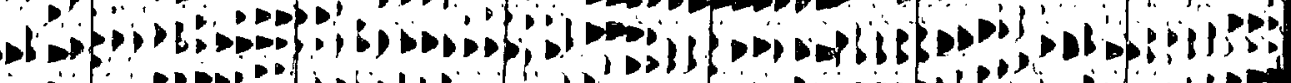

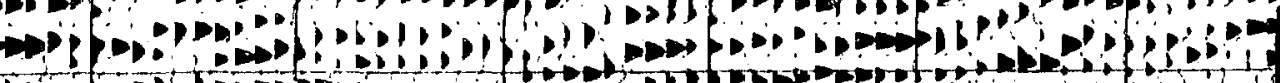




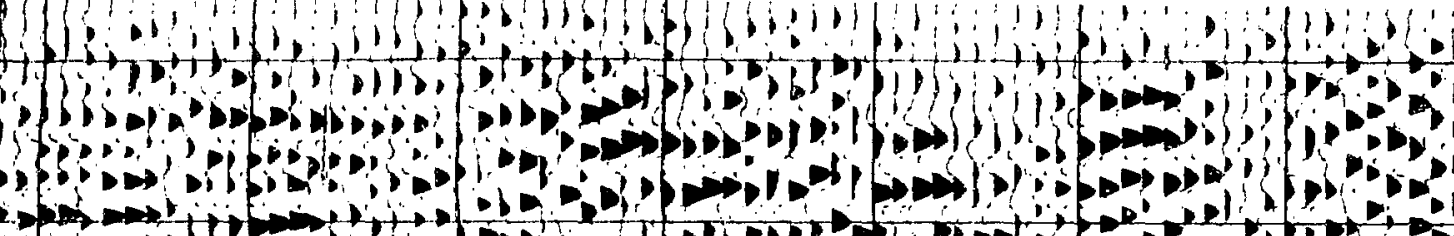
1) $\because 17$ a

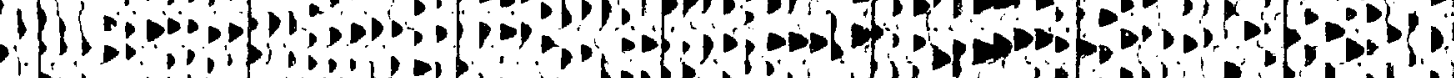

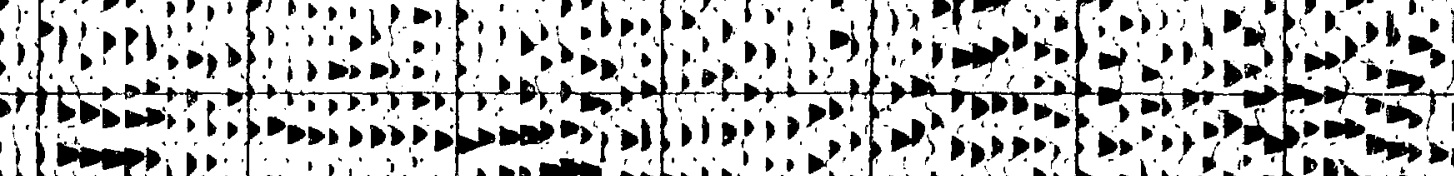

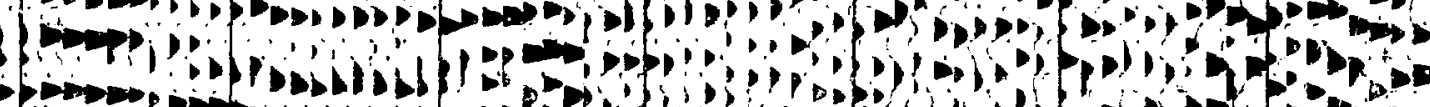

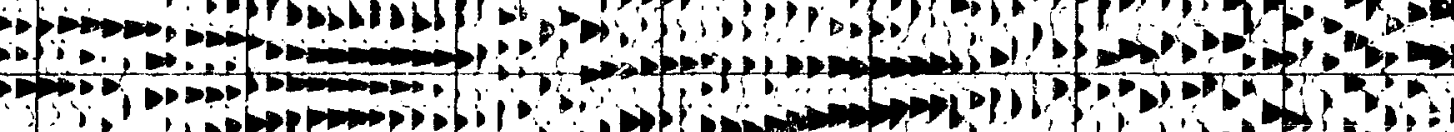

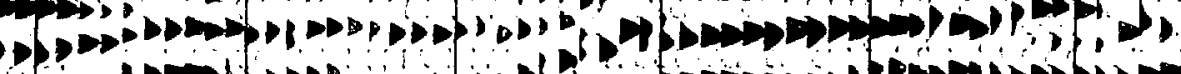
$\sin x \rightarrow 0$

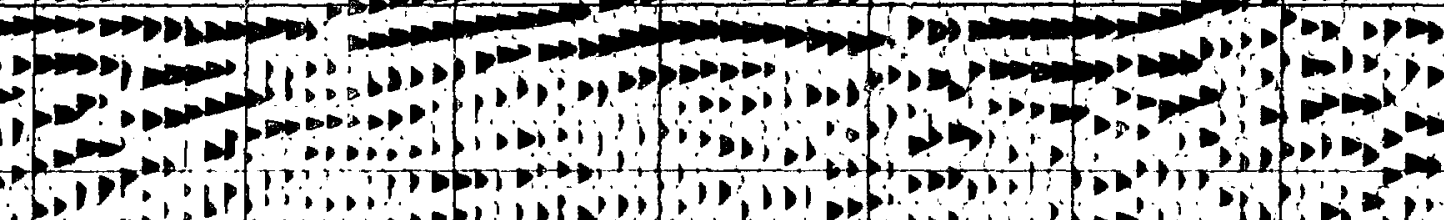

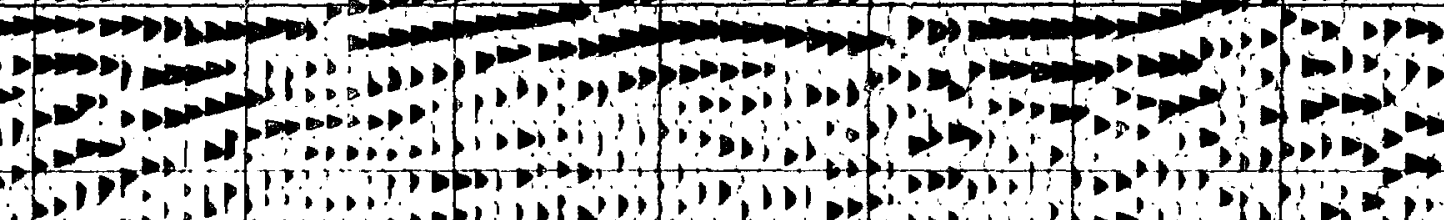
(PF, if

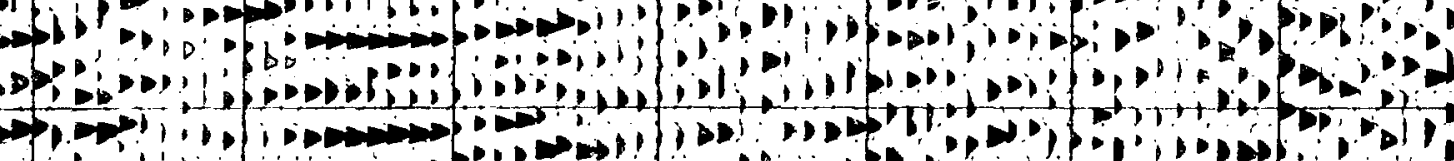

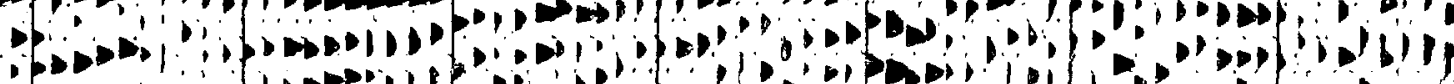

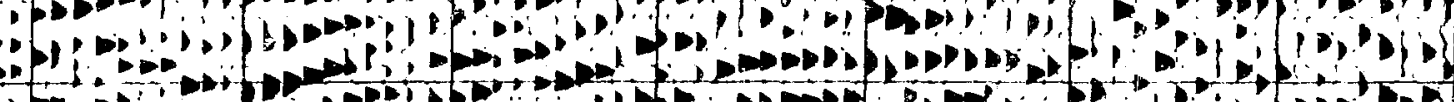

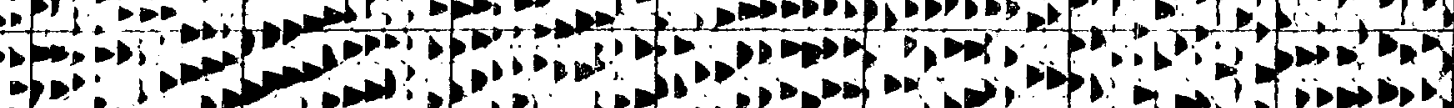

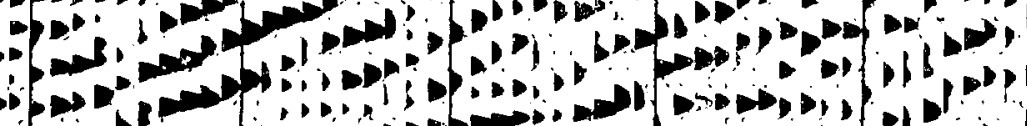

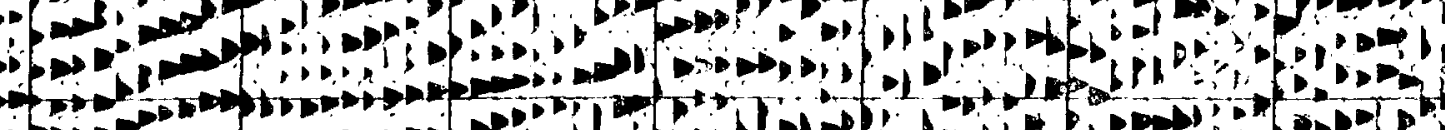

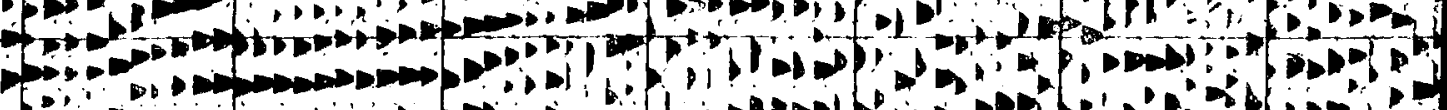

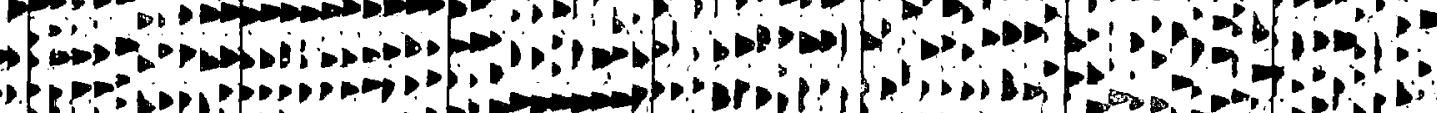

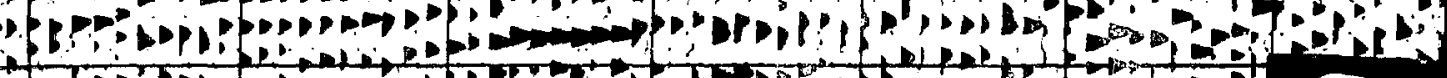

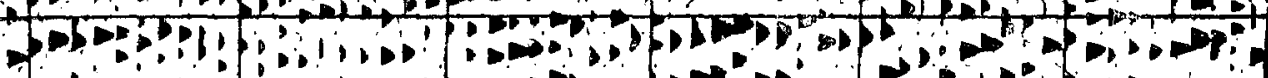

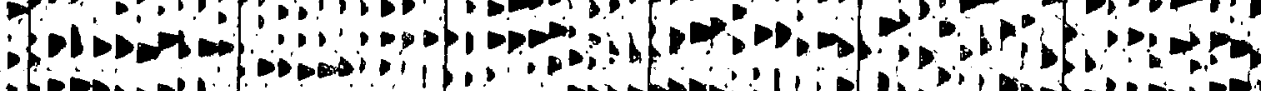

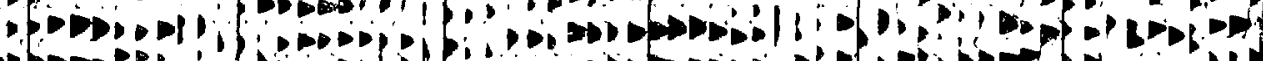

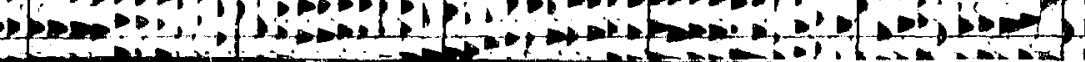




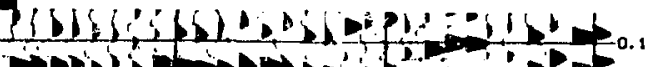

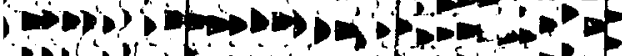
incis

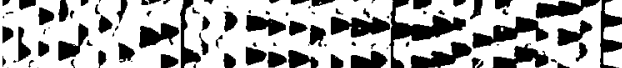

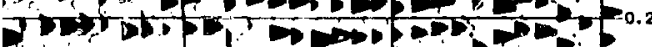

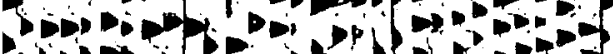

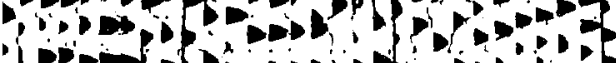

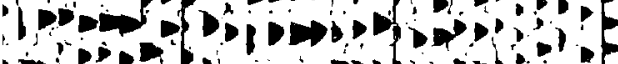

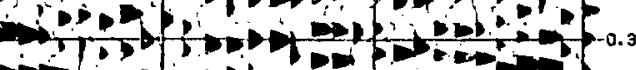

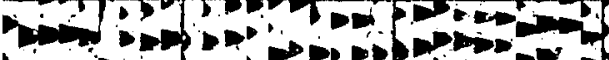

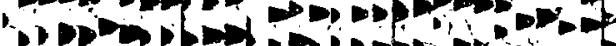

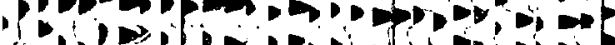

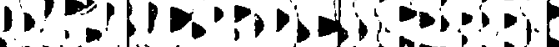
Aisi $\Rightarrow$

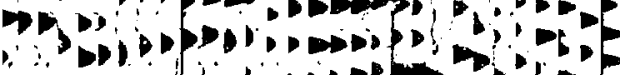

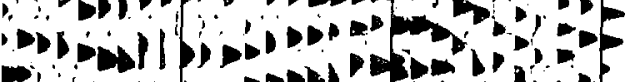

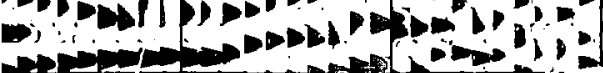

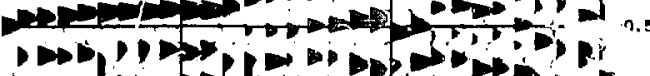

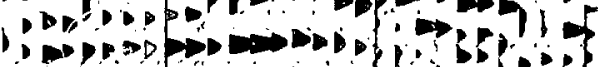

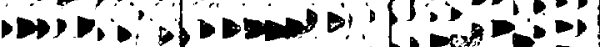

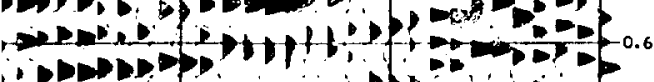

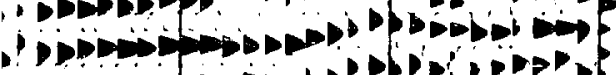

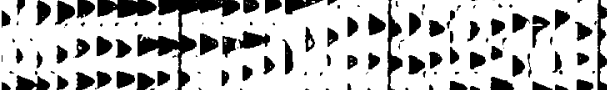
काण丁? anl $\rightarrow 10$ 1

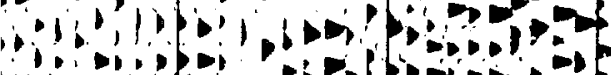

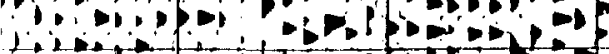

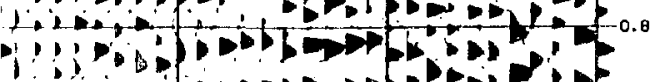

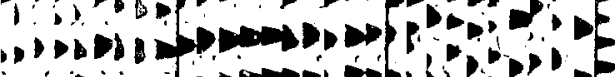

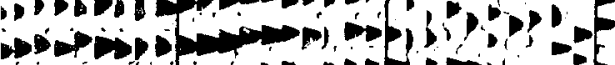

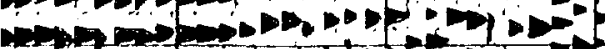

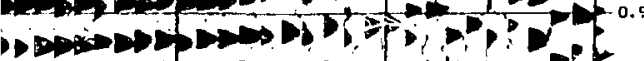

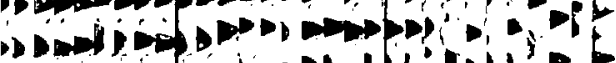

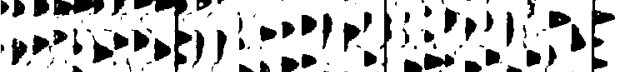
$\rightarrow \rightarrow \infty, \rightarrow P_{1}$

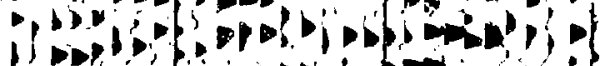

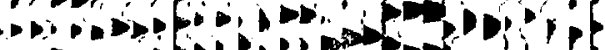

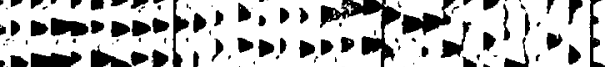
$\rightarrow \rightarrow \rightarrow 1, \rightarrow ! \rightarrow b_{1} \rightarrow b^{\prime} b_{1}$

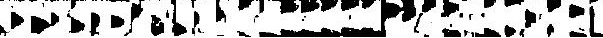

(

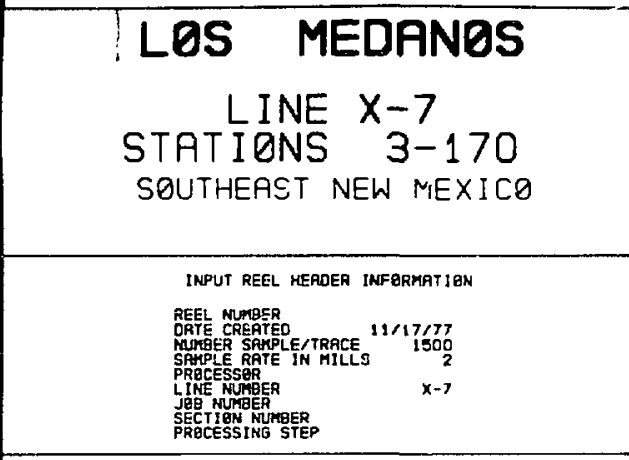

\section{FIELD INFTRMATION}

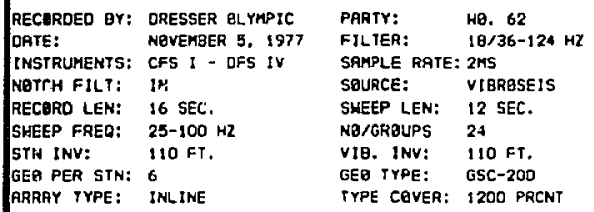

\section{PROCESSING SEQUENCE}

PRECESSSD BY ORESSER RLYMPIC

STRTICS CBMPUTATIEN

OATUM: $3200 \mathrm{FT}$.

VSH: $\quad 6000$ FIJSEC.

1) CEMULTIPLEX

2) BIMRAY GRIN RECOVER"

כ) VIERESEIS CORRELATIGH

4) COMMON OEPTH POINT GATMERS

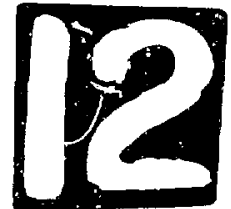

3) OECENJOLUTION

OPERATGR LENGTHa140 MILS

PREDICTION TIKE BRSEO GN 2NO ZERE CRESSING

6) TIME-INYPRIANT OIGITRL FREQUENCY FILTER 0.0-3.0 SEC. 25-80 HZ

7) RPPLY DRTUM STAIICS

日) VELOC1TY ANALYSIS

9) RPPLY NMO

10: FIRST GREAX SUPPFIESSI I SMUTE

(1) STRCK 12 FOLO

12) TIME-IKVARIANT OIGITAL FREQUENCY FILTER 0. 0-3.0 SEL. 25-80 HZ

13) DIGITAL AGC

14: OISPLAY

0 TR/?

10 IN/SEC. 


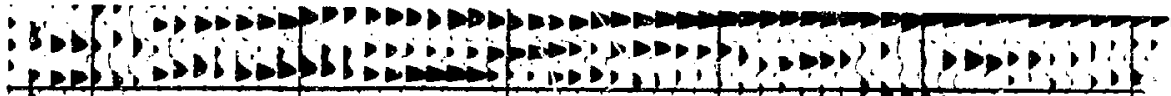

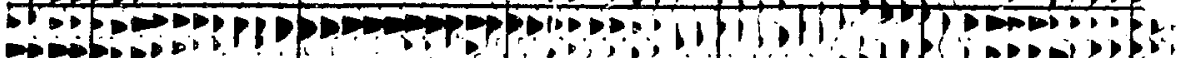

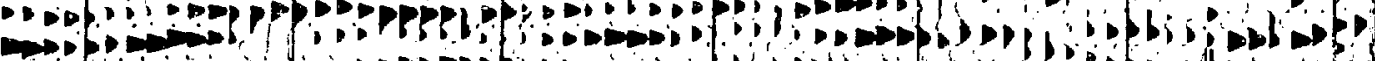

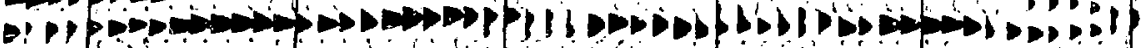

1.1 6r

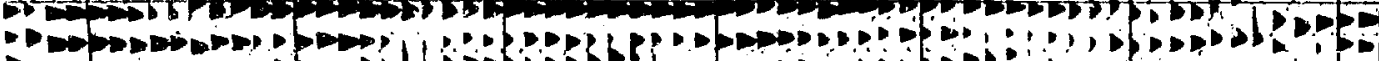

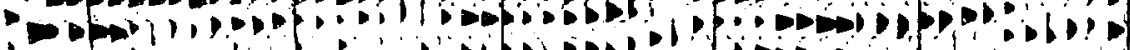

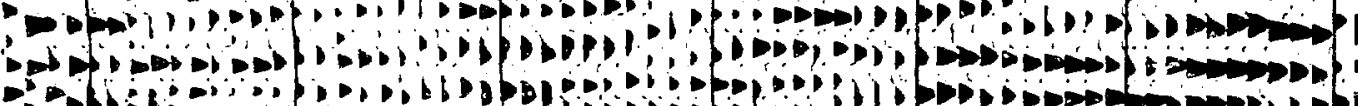

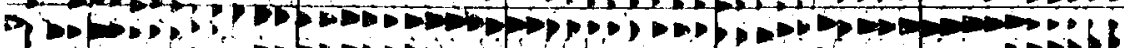

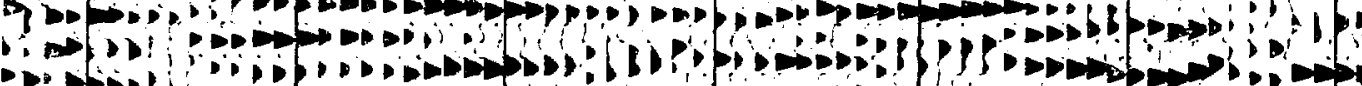

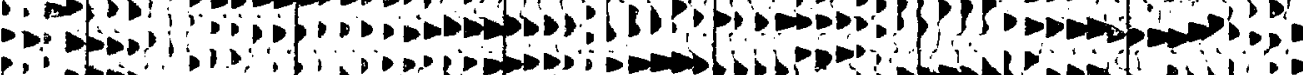

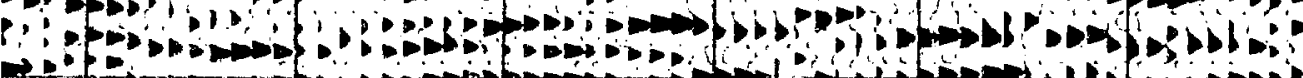

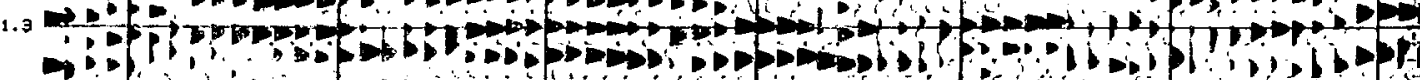

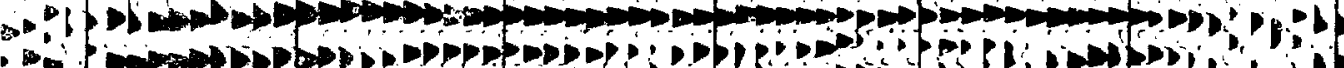

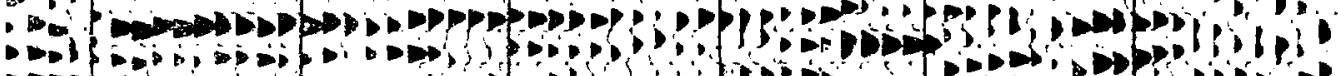
1.4 p

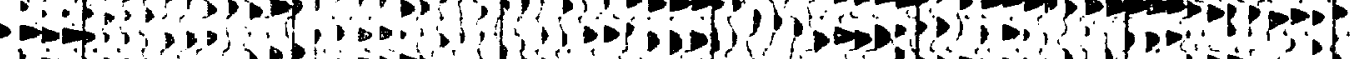

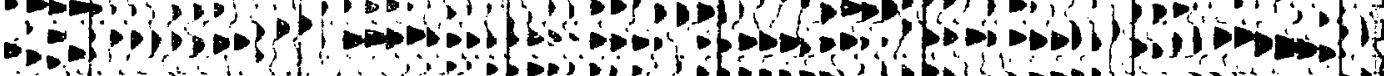

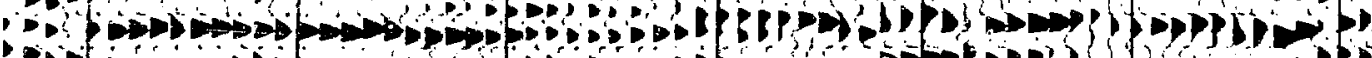

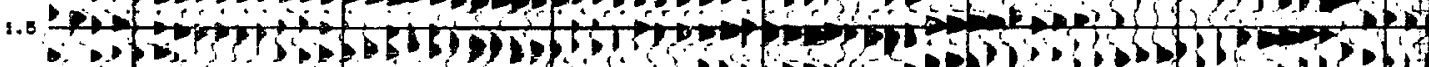

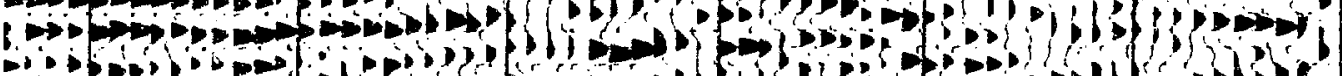

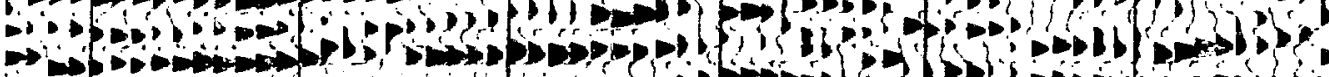

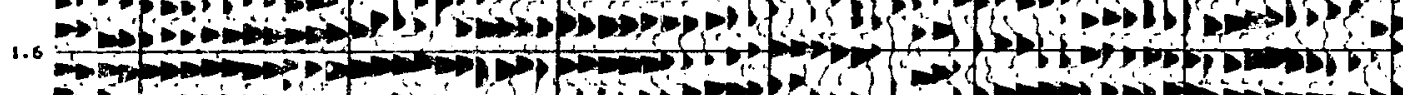

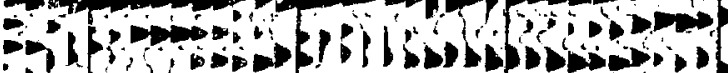

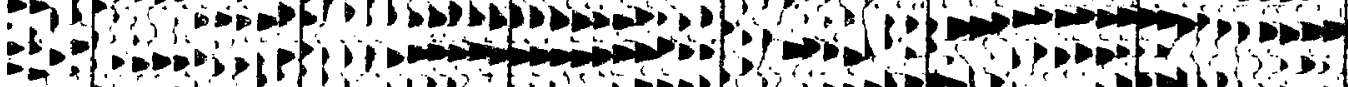

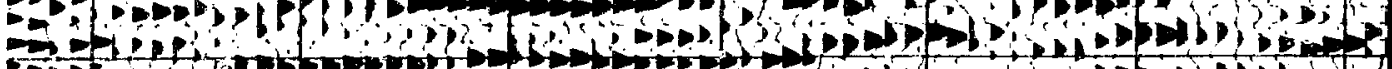

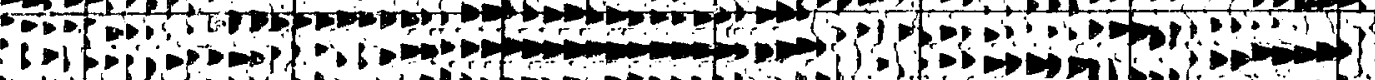

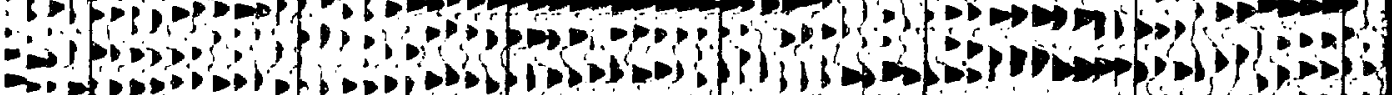

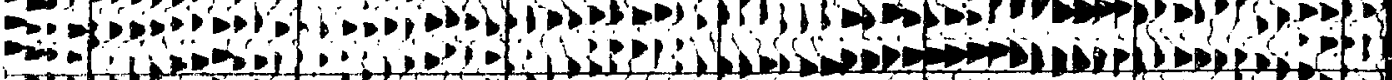
F-

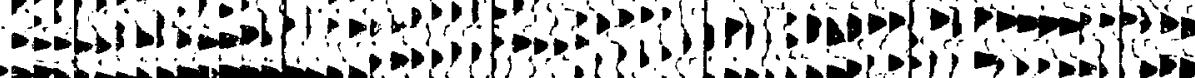

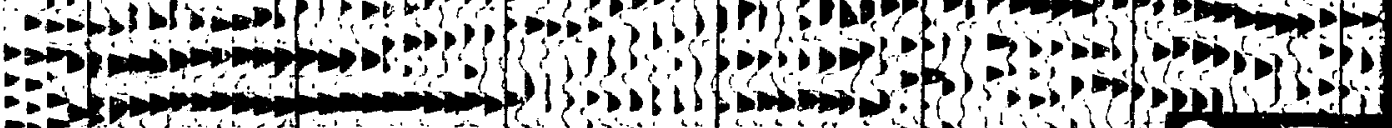

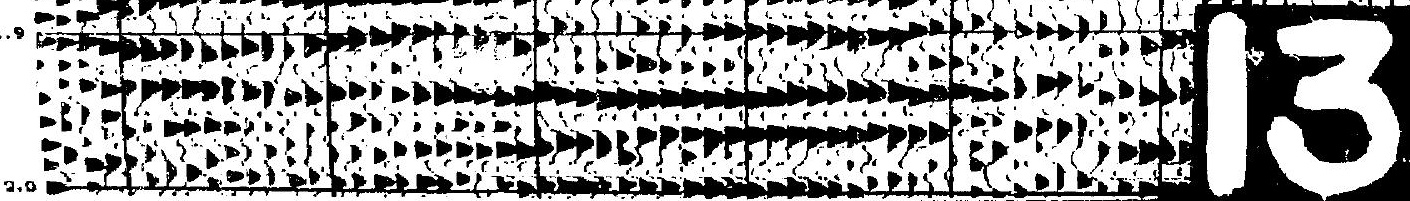




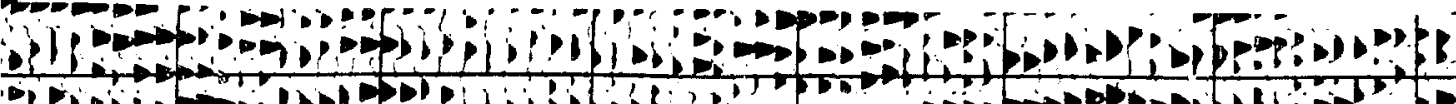

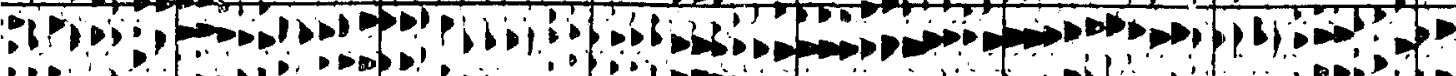

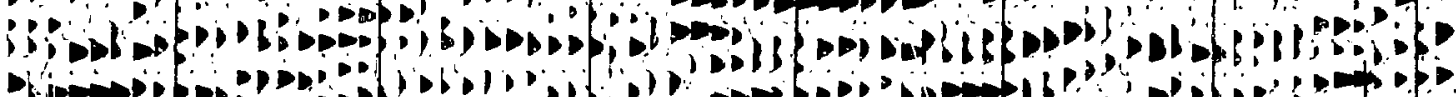

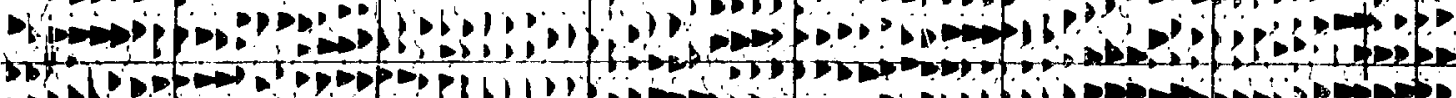
st.

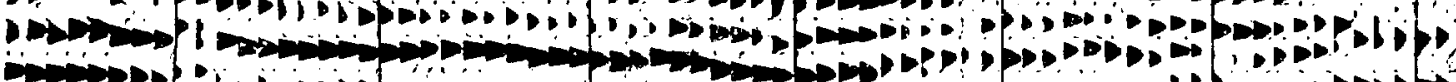

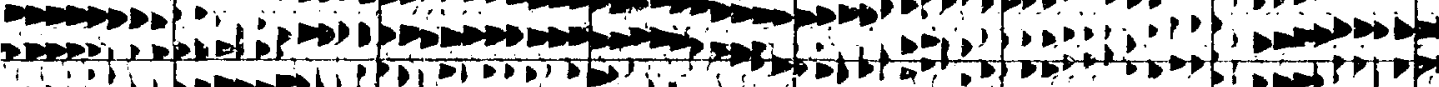
$\rightarrow$ bi bl

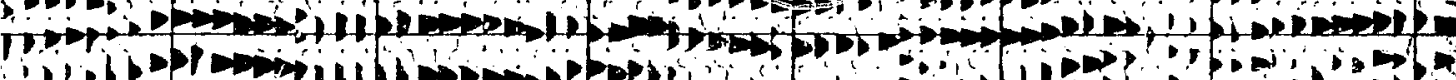
$\rightarrow 0$ -

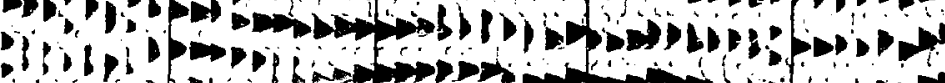

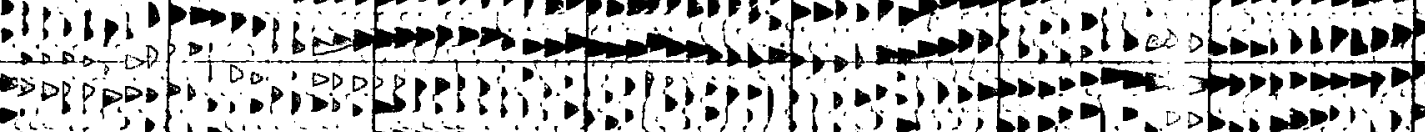

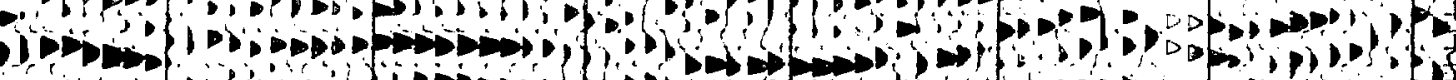

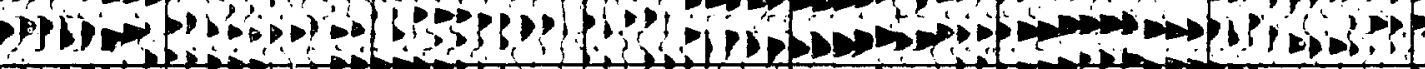

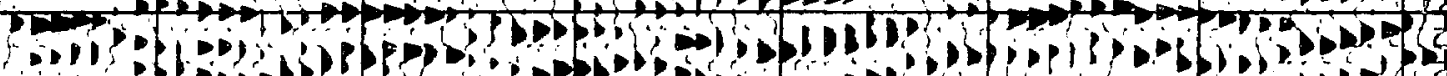

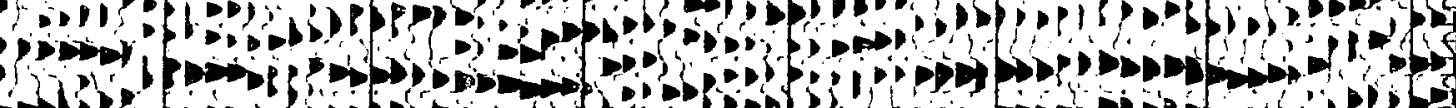
-jof

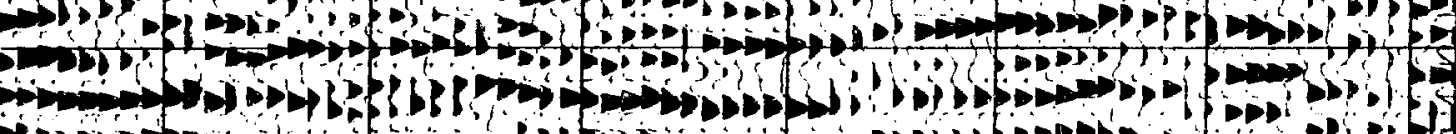

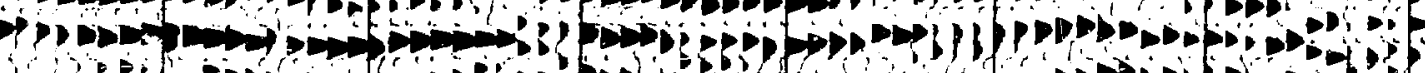

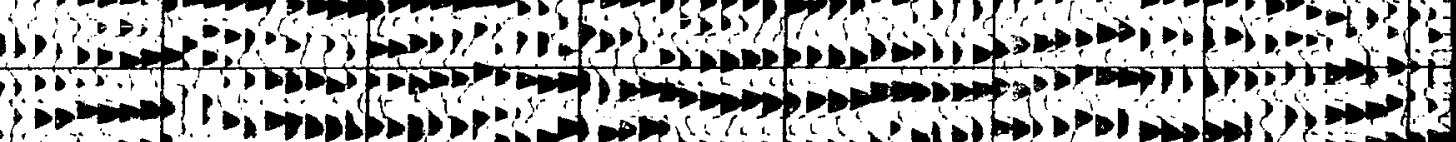

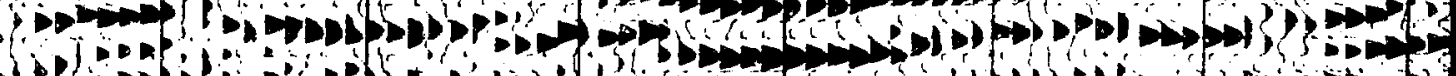

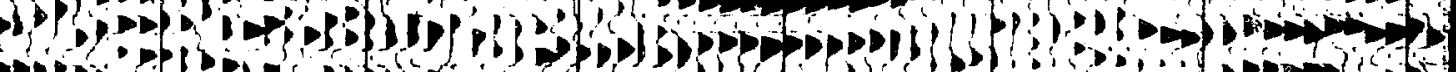

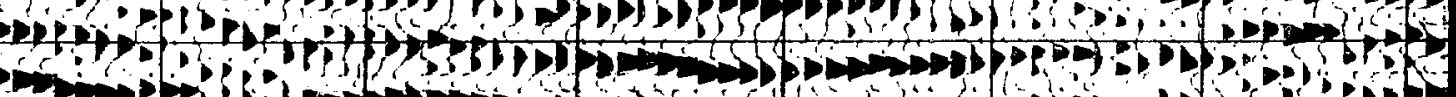

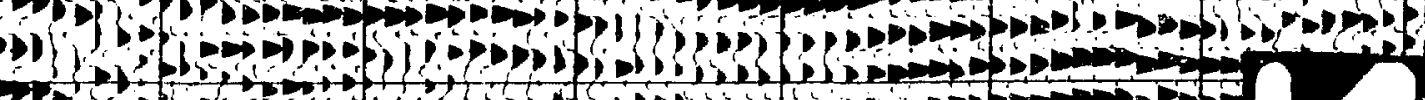
( )

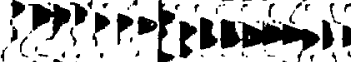


(1)

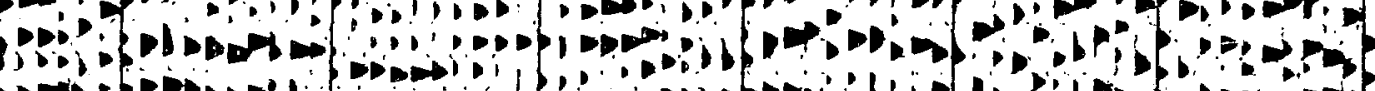

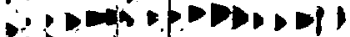

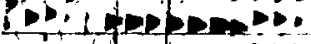

$\rightarrow \rightarrow 0 \cos$

\section{$\rightarrow \rightarrow \infty$}

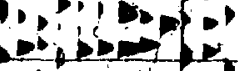

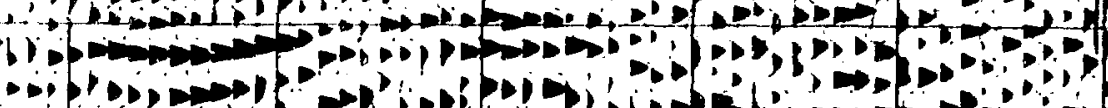

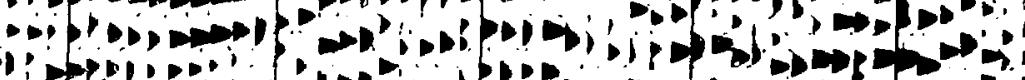

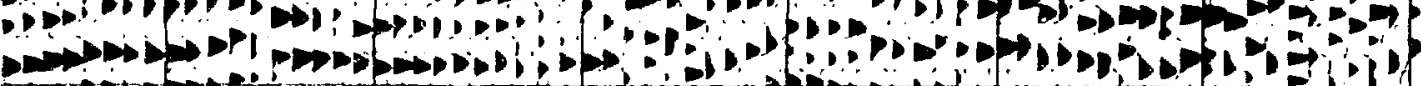
(1)

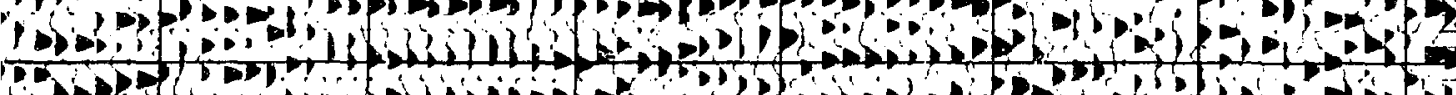

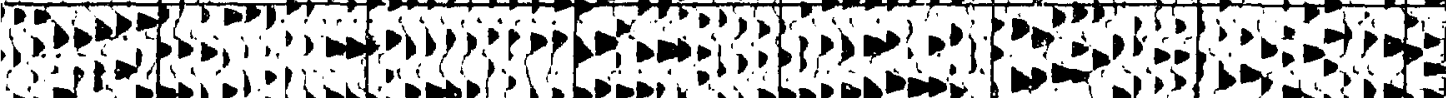

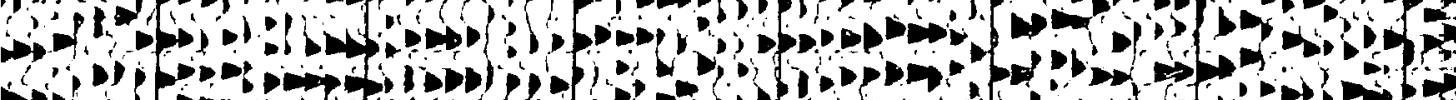

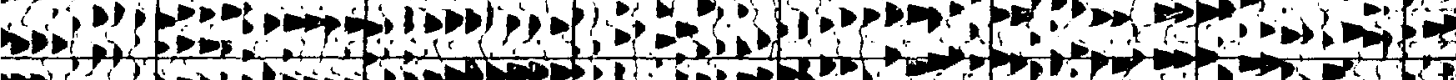

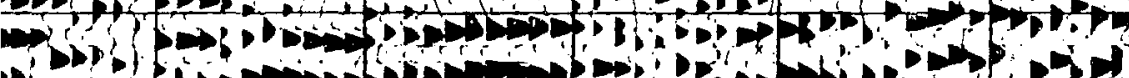

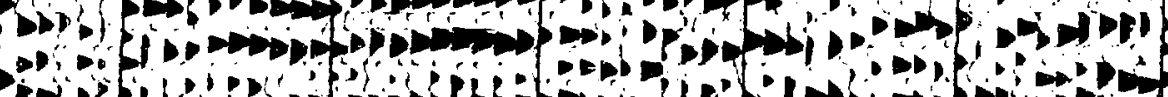
pos pos

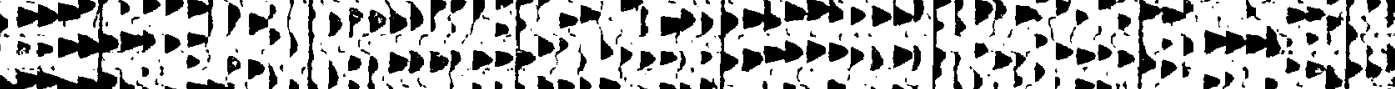
(2)

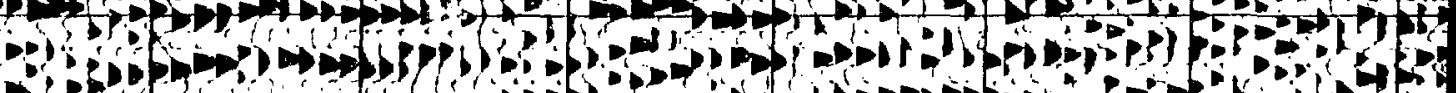

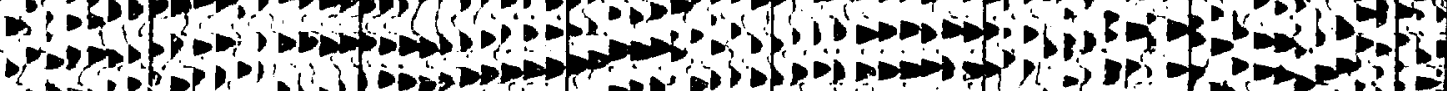

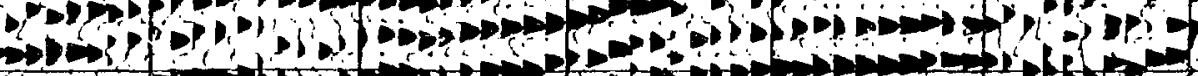

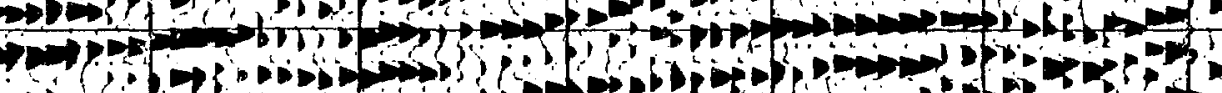

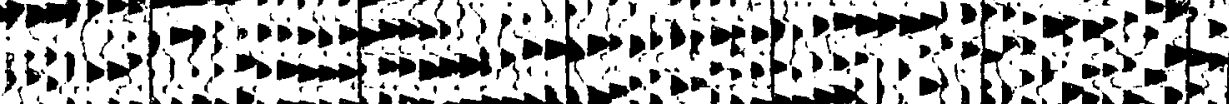

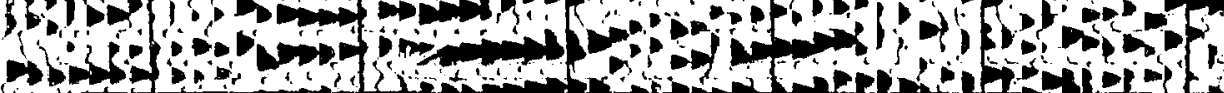




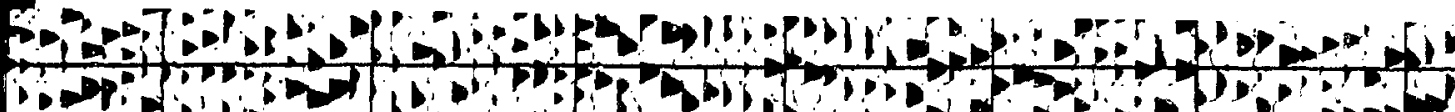
Potom

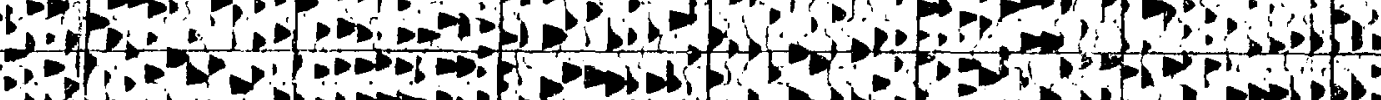
Som

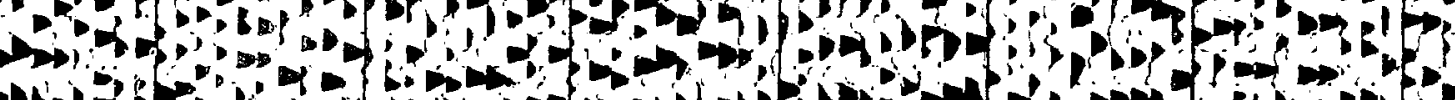

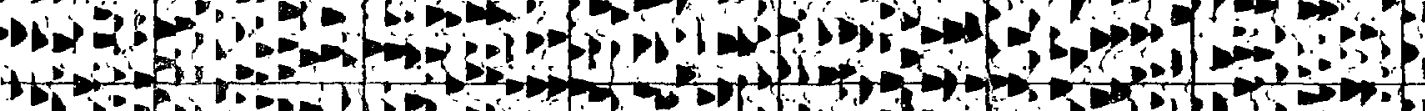

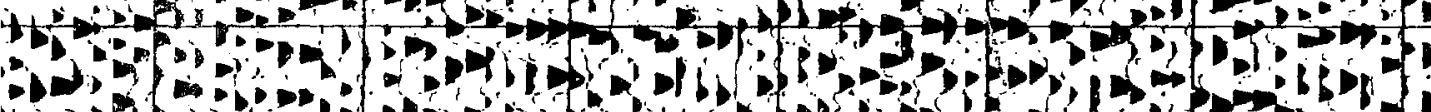
ting S.t.

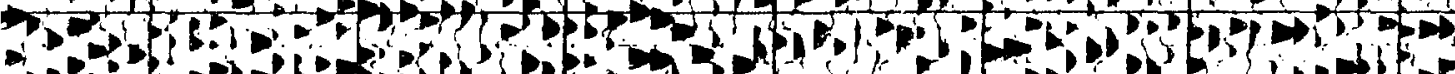

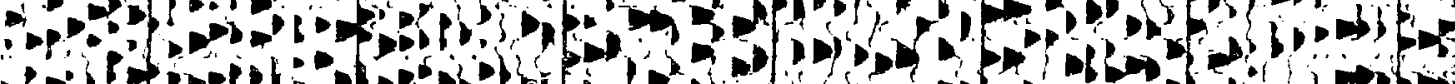
(1): $\therefore$ (b)

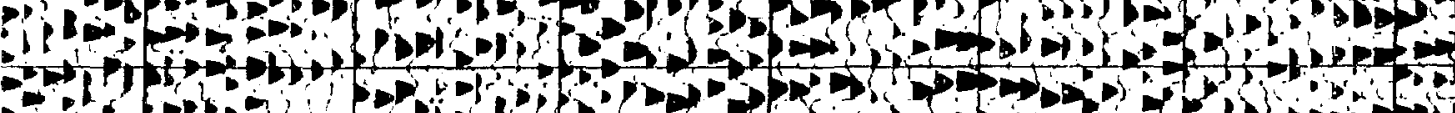
-

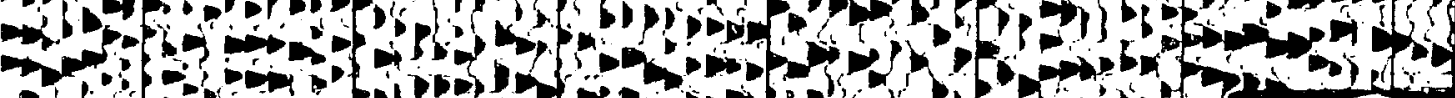

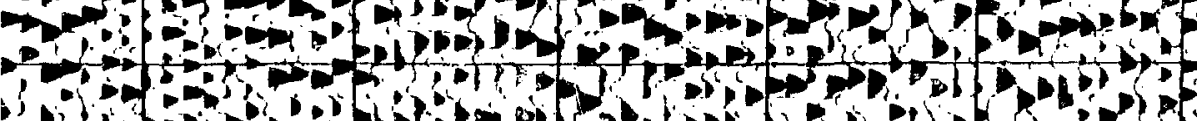

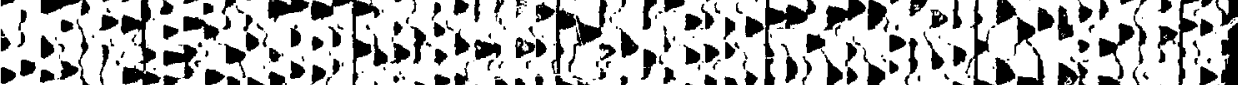

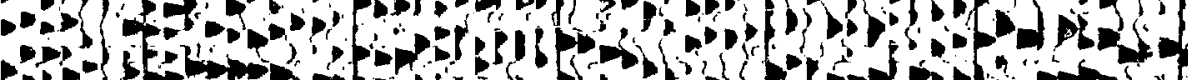


$\because$

$\because$ ind

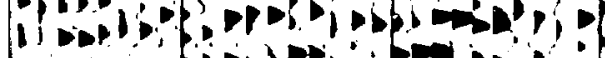

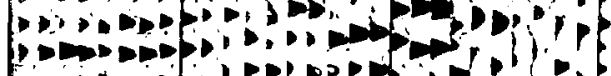

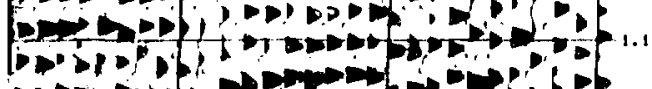
P P

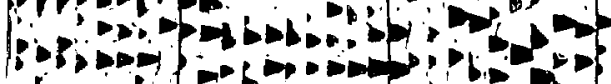

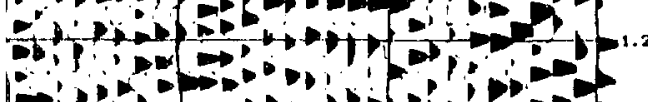
$\leftrightarrow 1 ;: \circ \rightarrow 0$

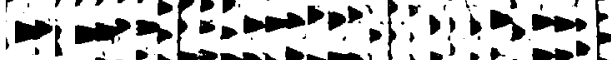

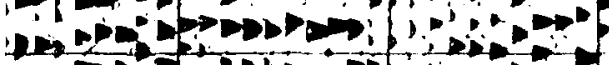

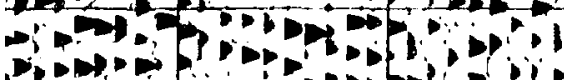

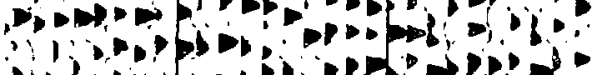
Jis:?

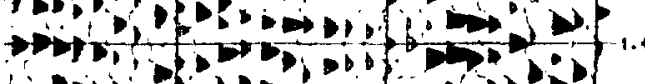

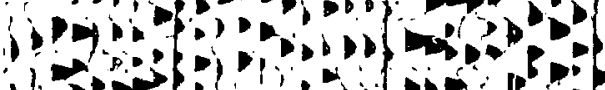

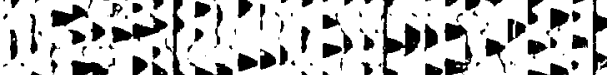

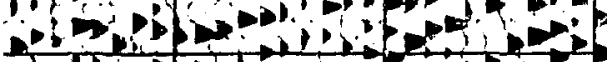

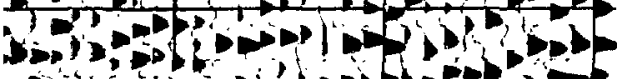

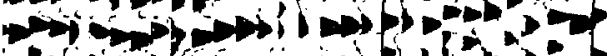
if

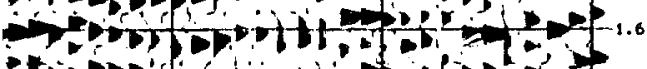

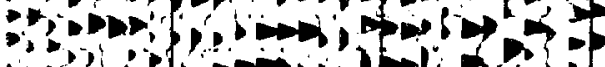

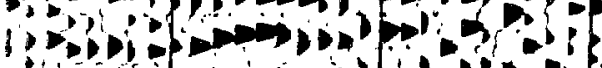

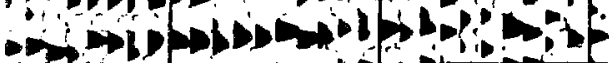

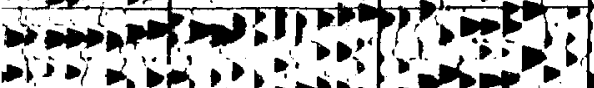

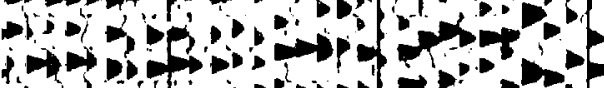
1.

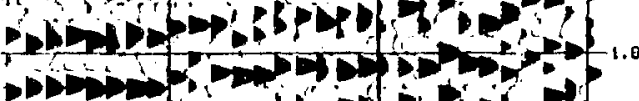
ar,

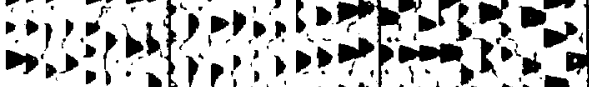

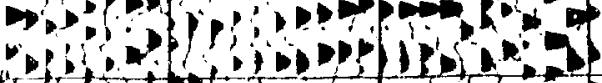

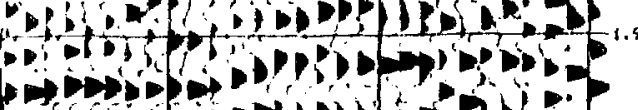

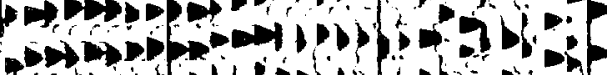

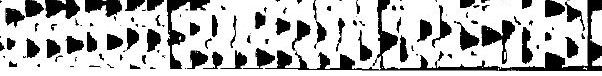

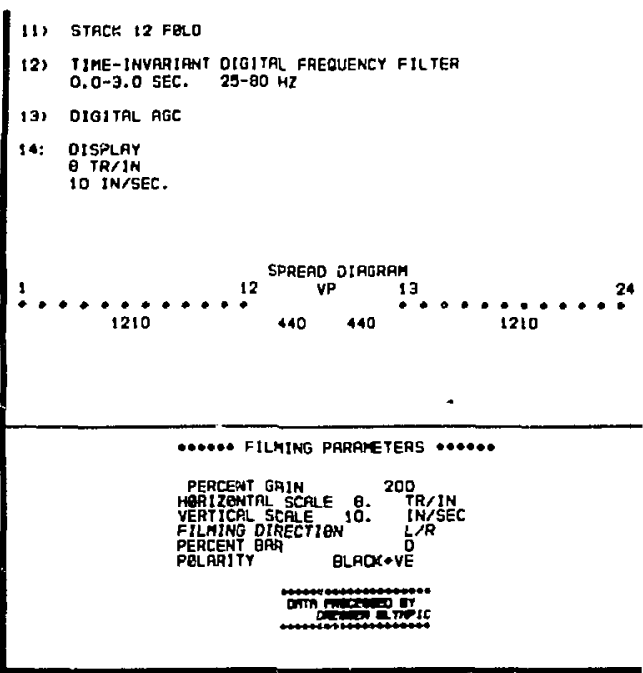




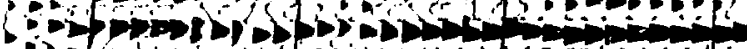

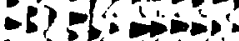

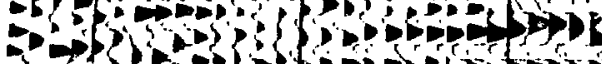

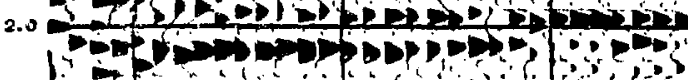

$\therefore 2$

15

$l_{3}$

Tos?

$b_{n \rightarrow \infty}$

(2)

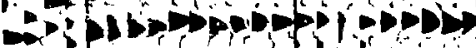

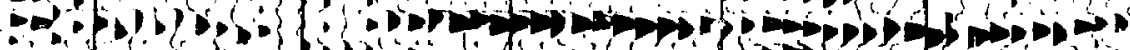

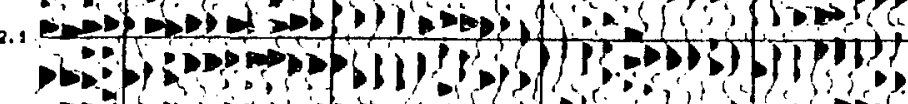

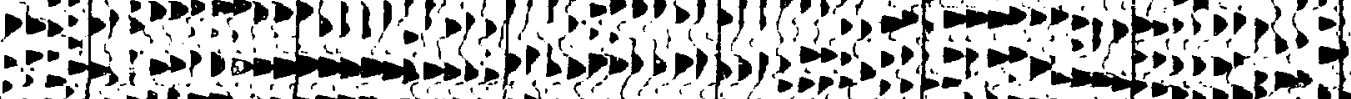

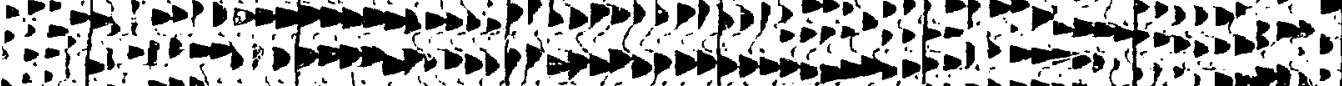

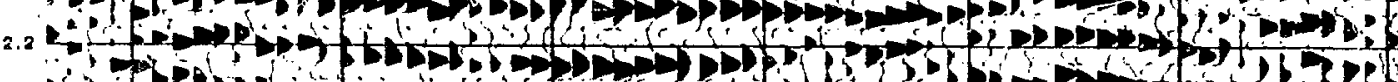

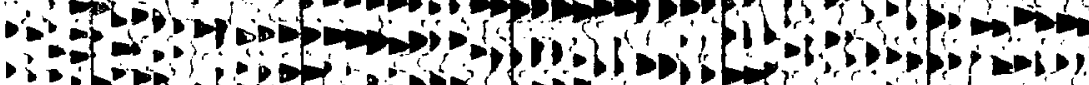

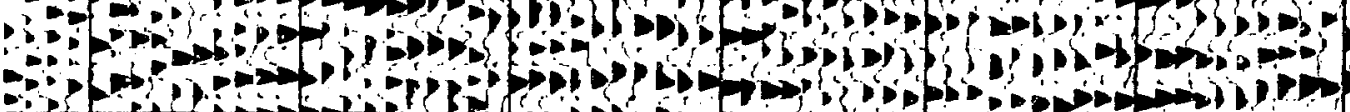

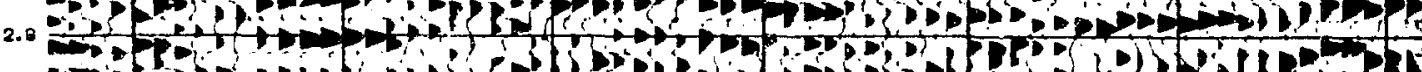

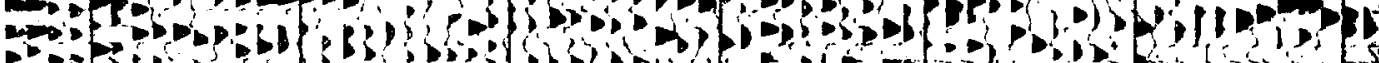

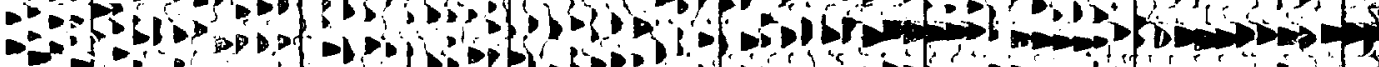

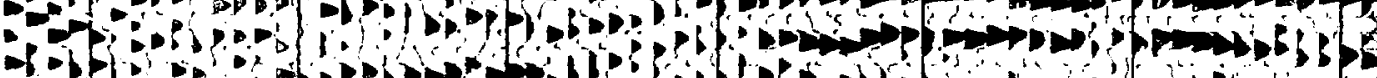

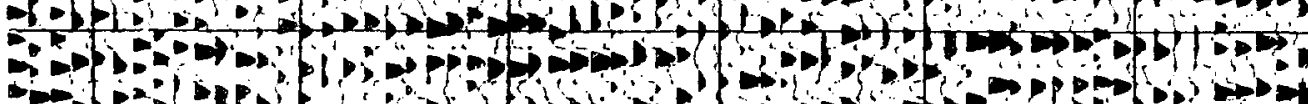

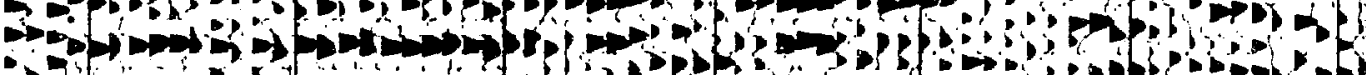

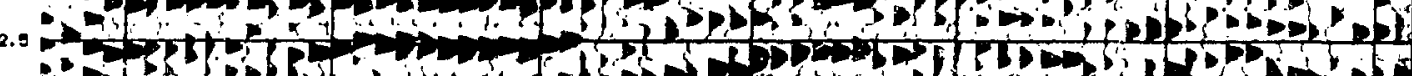

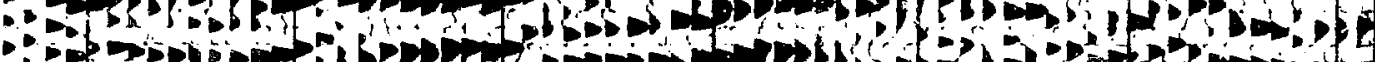

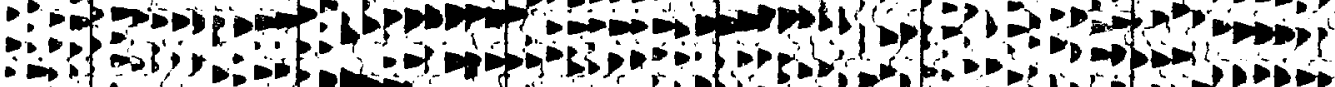

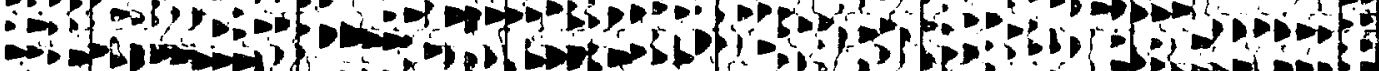

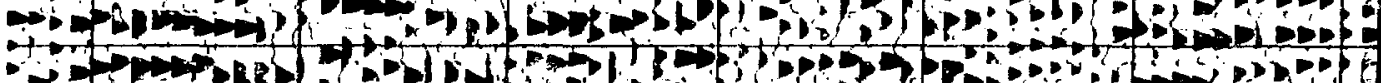

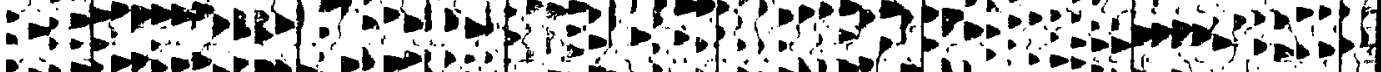

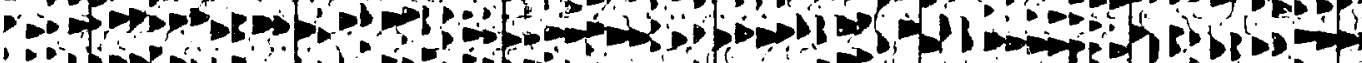

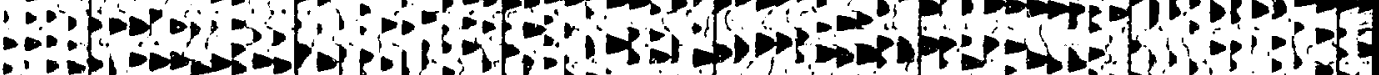

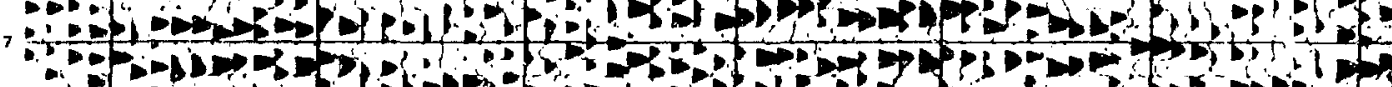

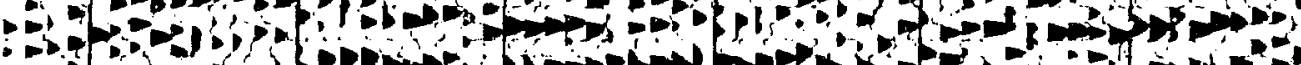

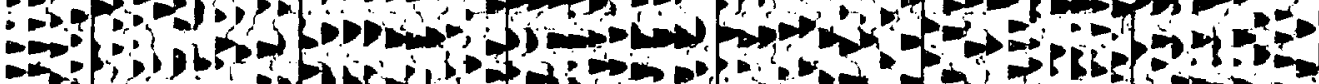

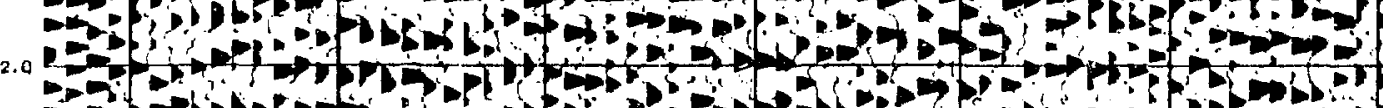

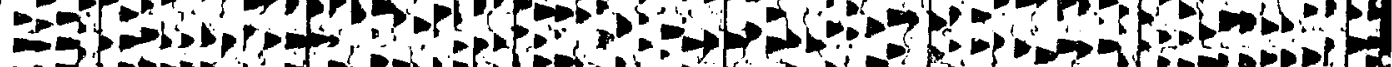

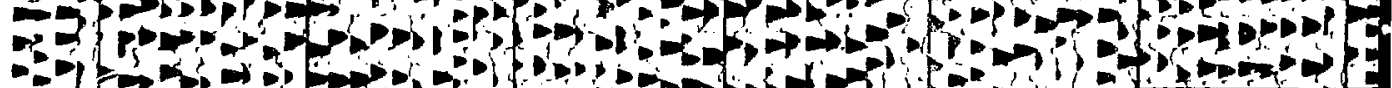
2.9 - 


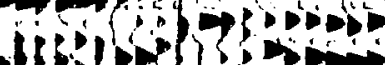

(t)

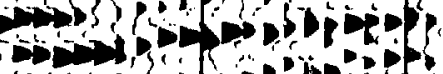

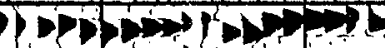

sition.

(a)

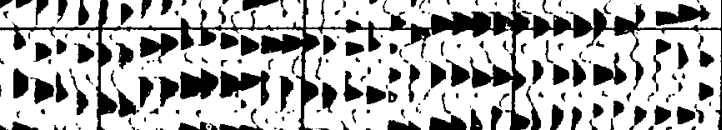

onisisipiting 


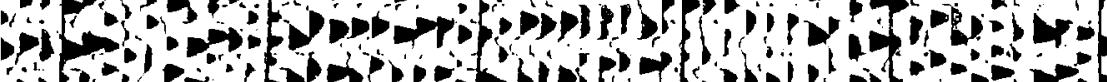

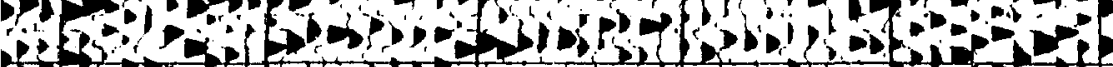

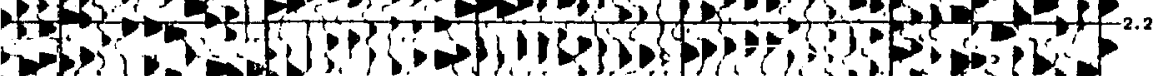

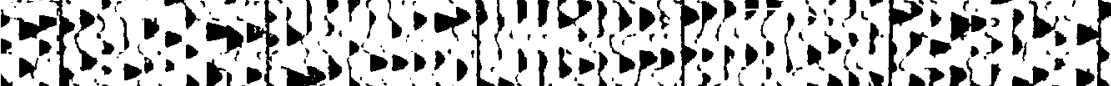

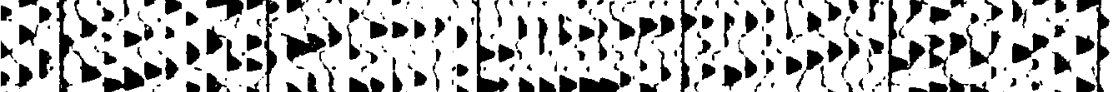

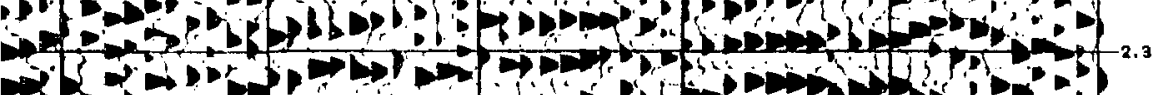

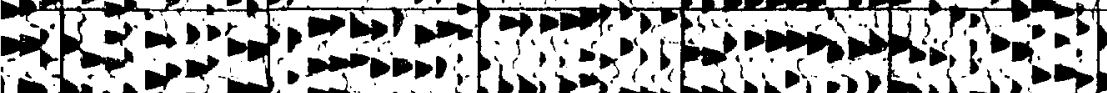

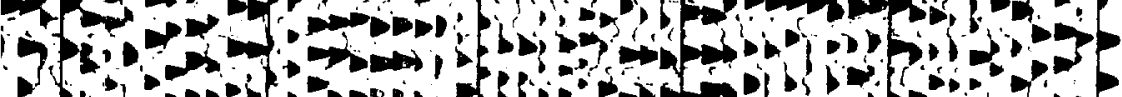

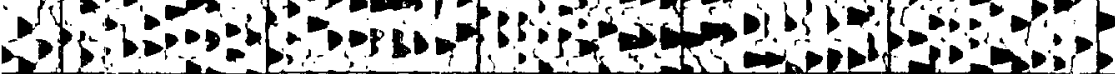

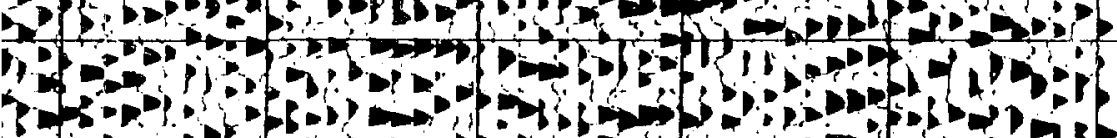
B.

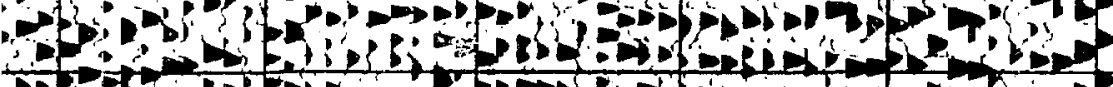

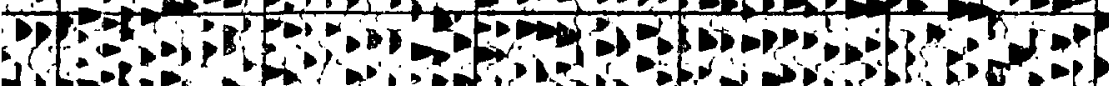

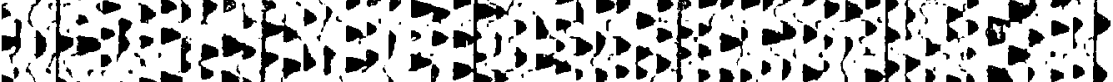

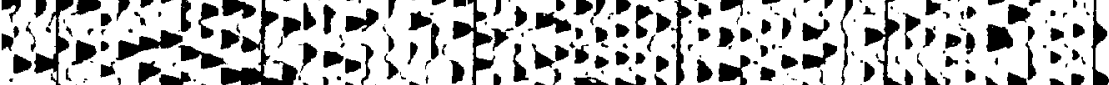

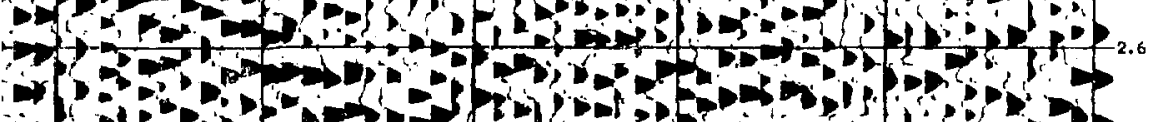

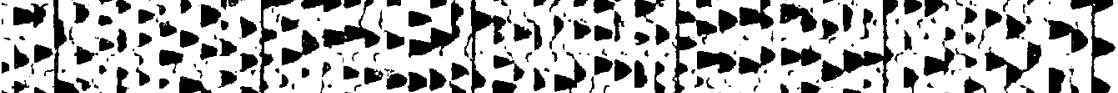

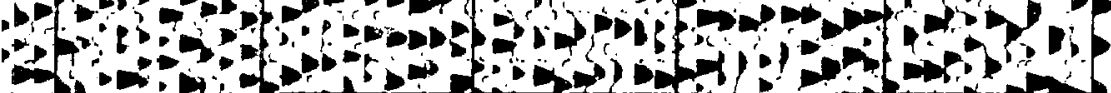
D. 2.15

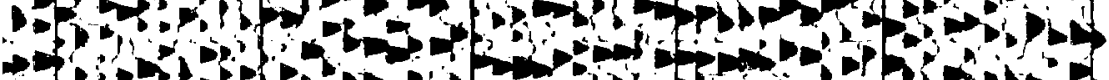

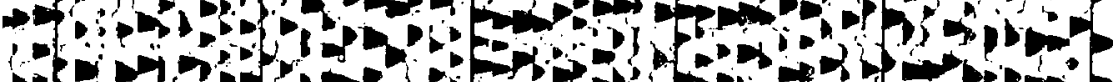

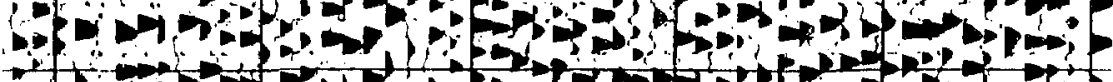

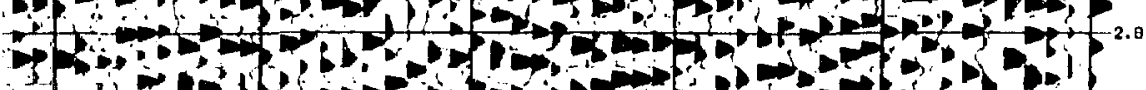

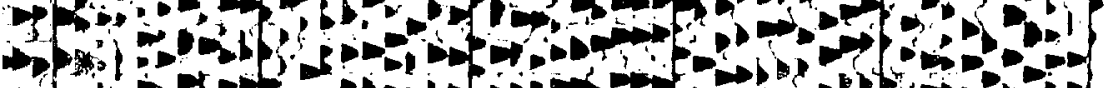

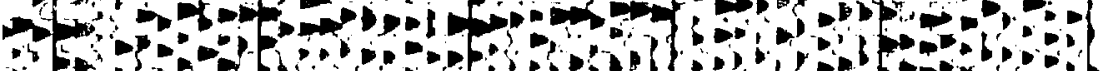
-

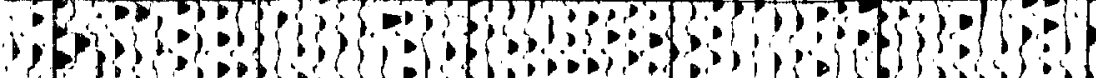

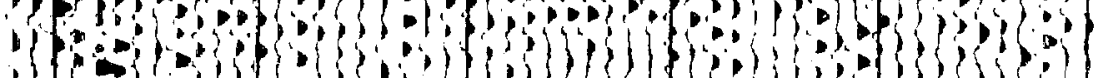
2.9 


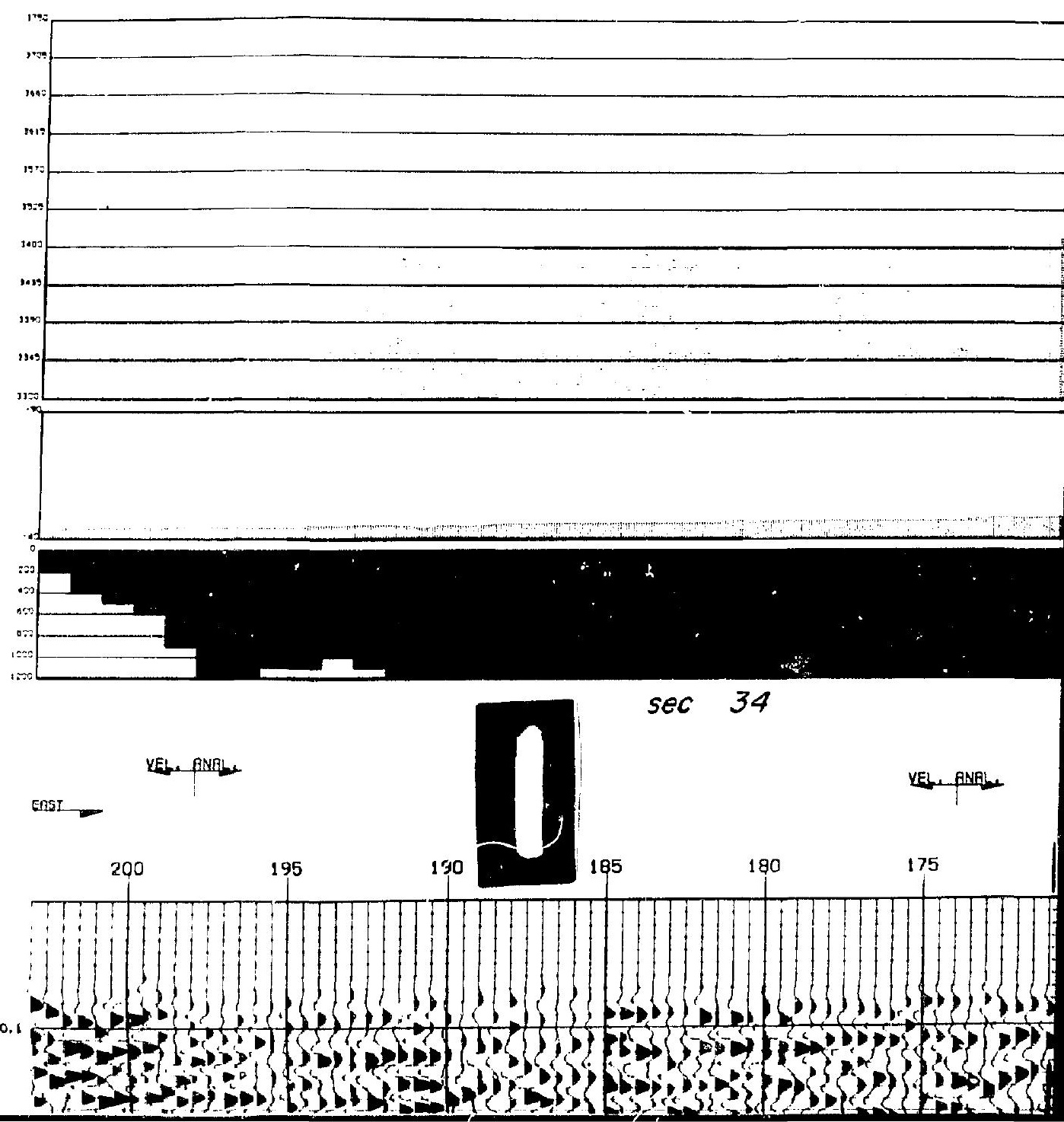




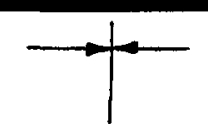

VF. BNEL

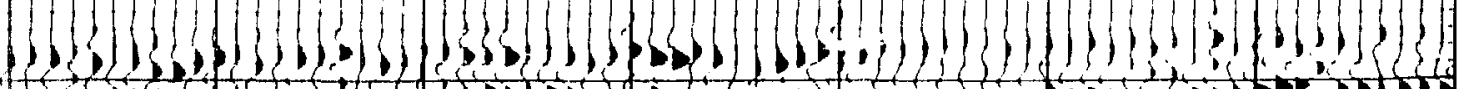
(

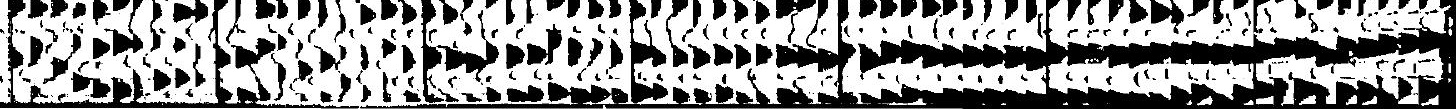




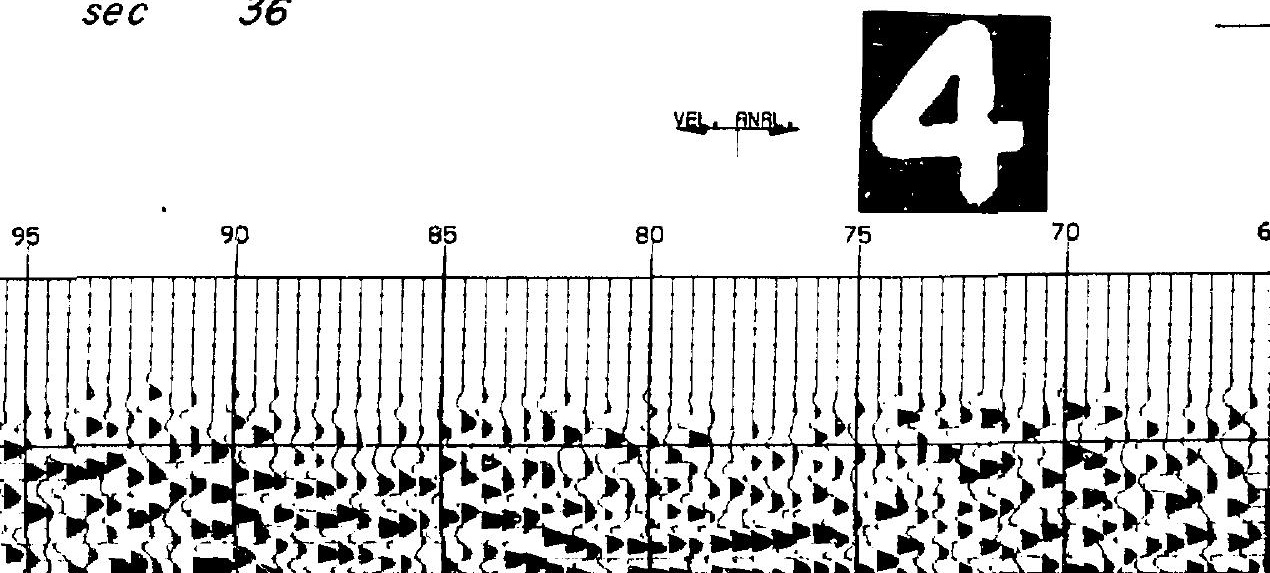

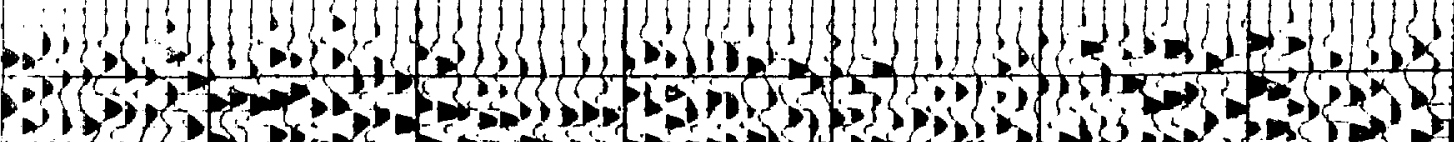

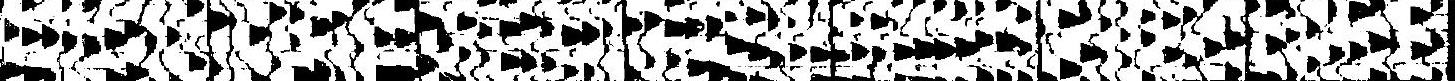


YEL ONAIL 



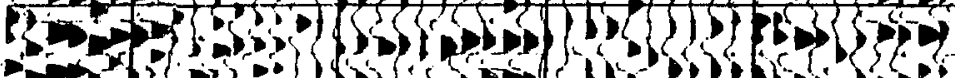

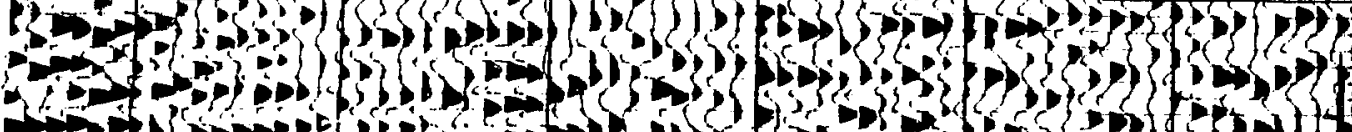

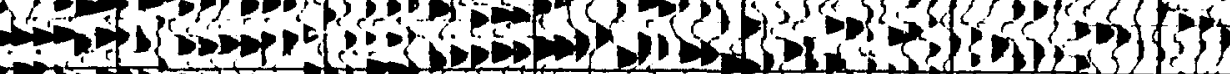

(1)

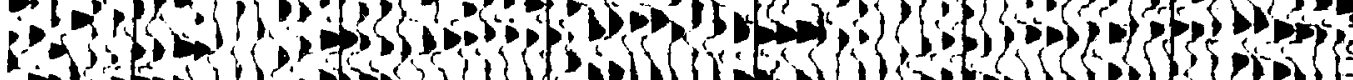

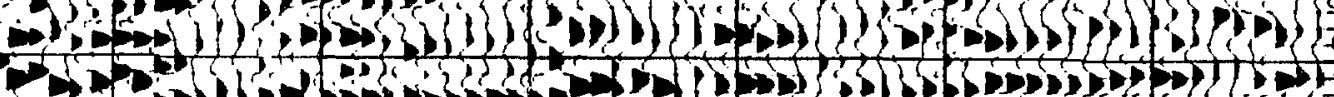

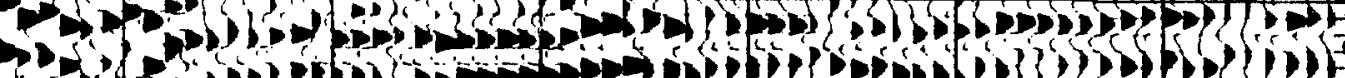
- fo. - s

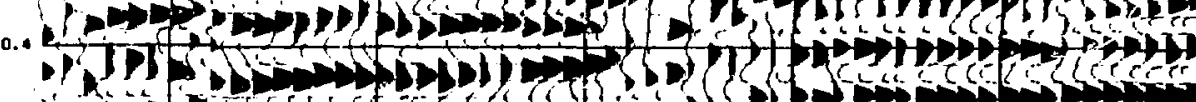

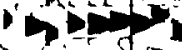

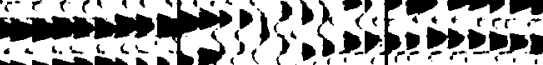

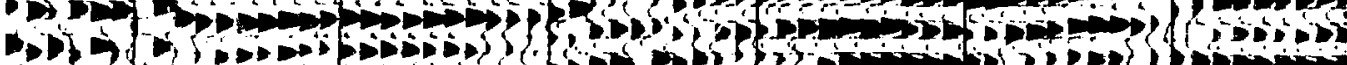

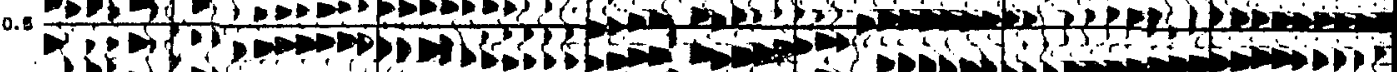

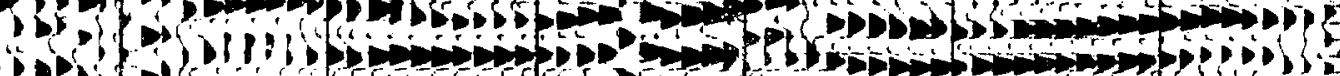

If

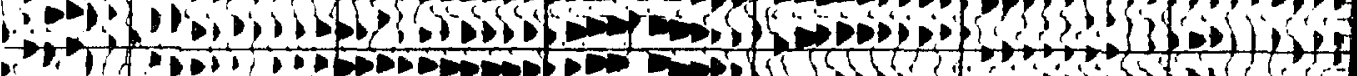

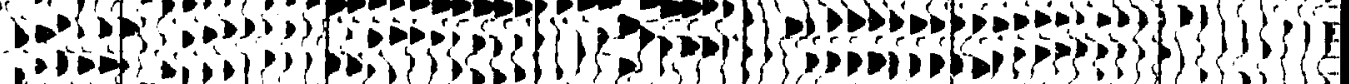

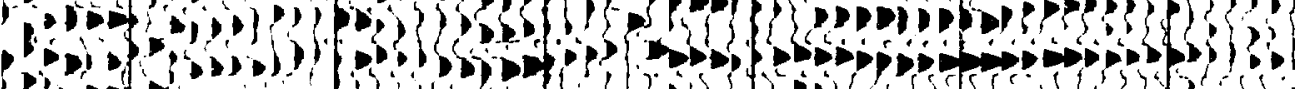

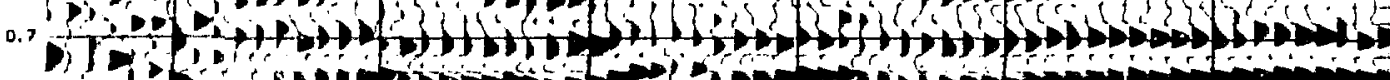

$\rightarrow 0$ -

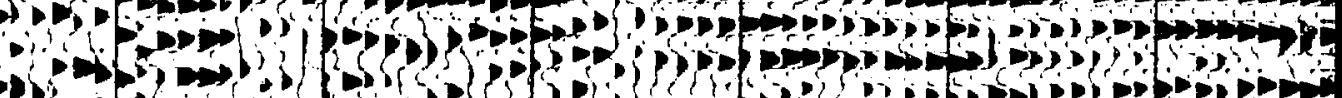

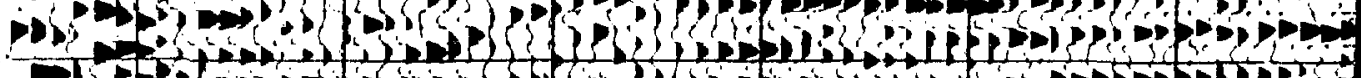

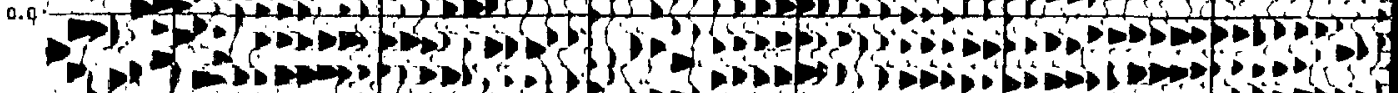

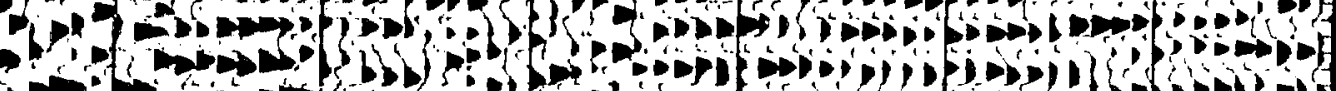

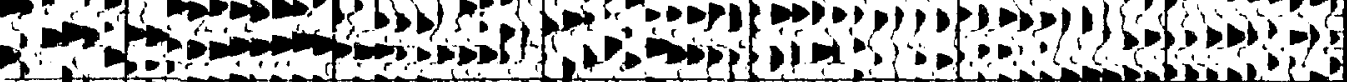

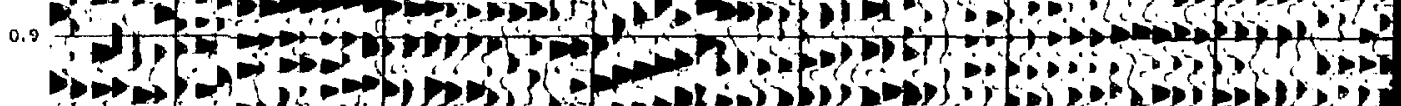

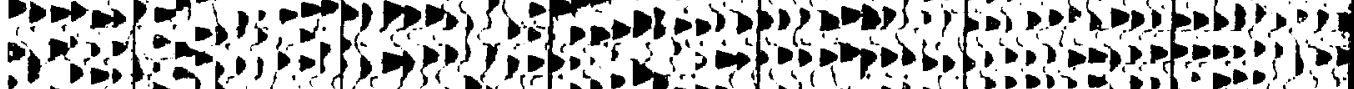
- 25 -

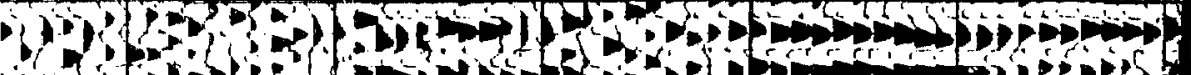

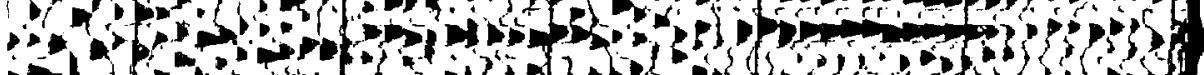
- 


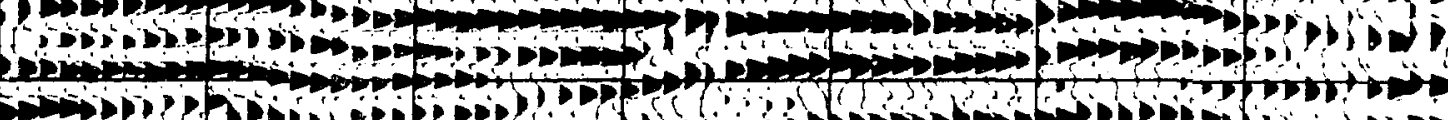

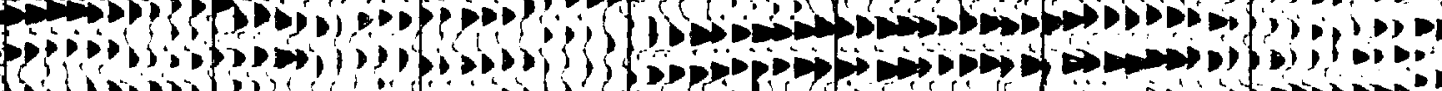
$\therefore$ (1)

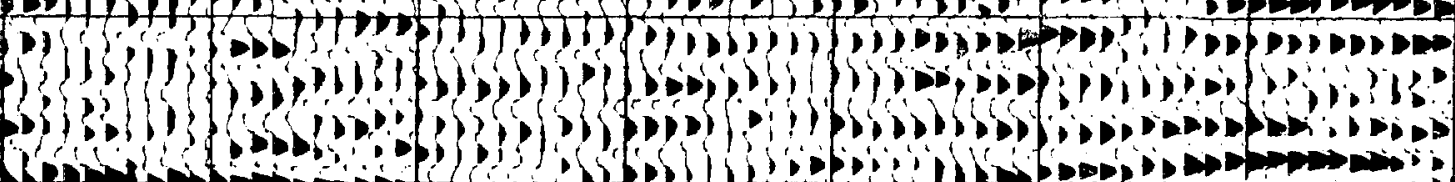

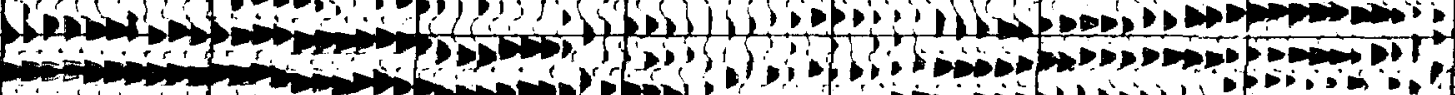

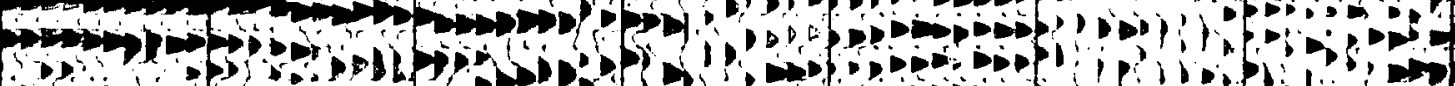

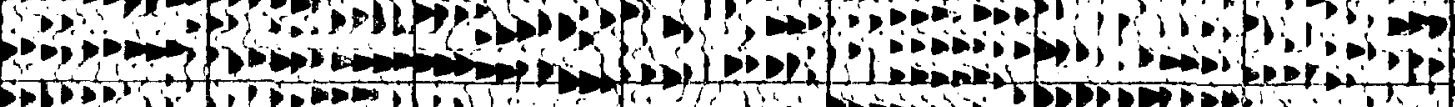

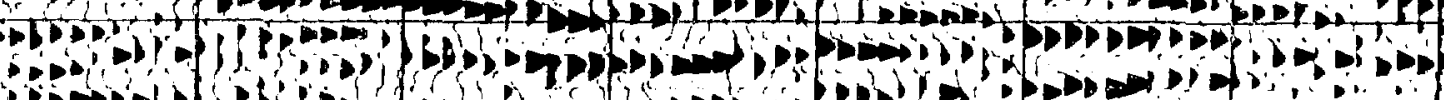

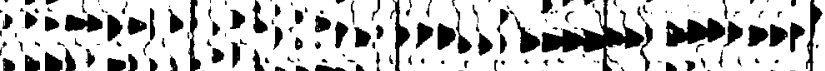

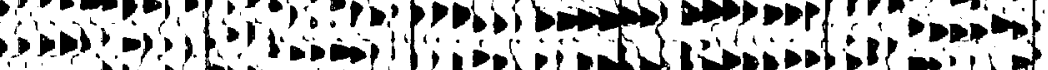

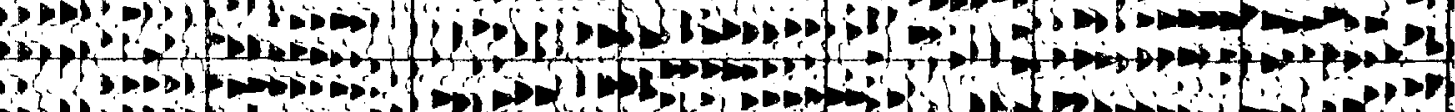

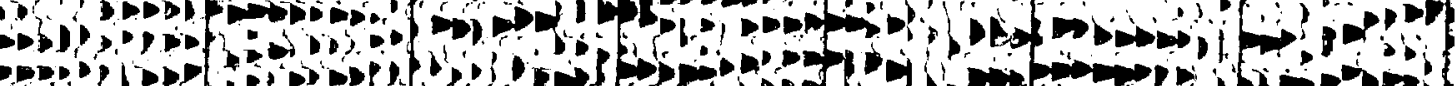

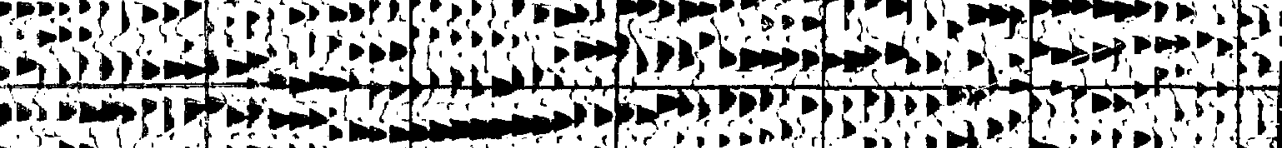

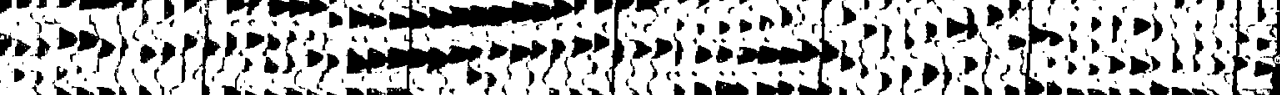

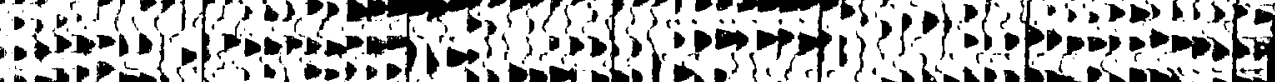
LPP 


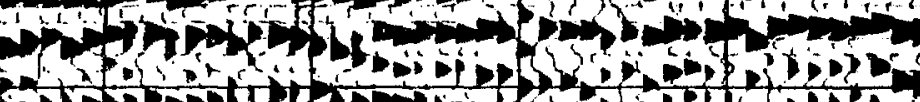

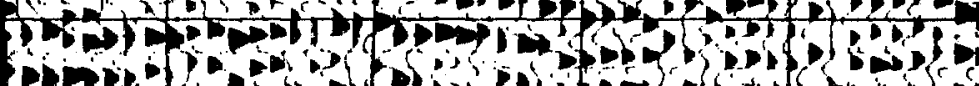

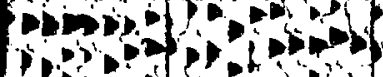
s.

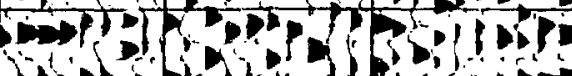

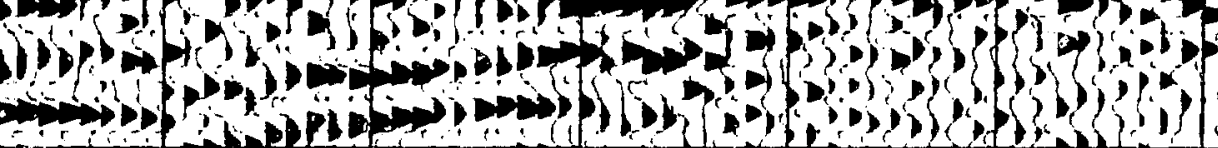
?

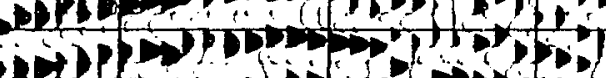
for istion. -

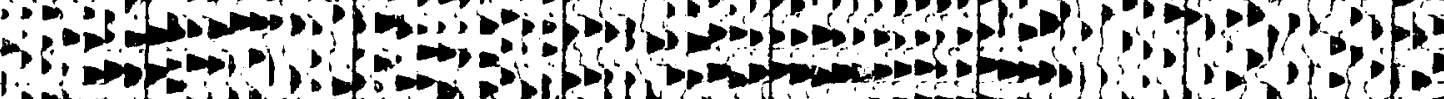
riofe

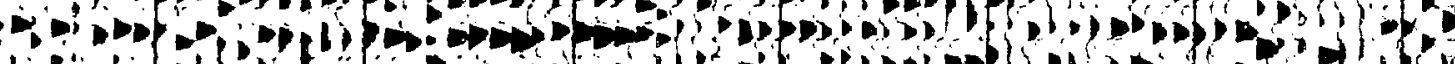
; -

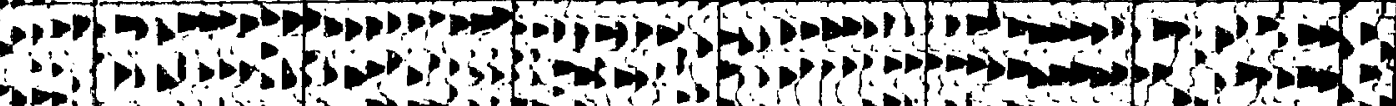

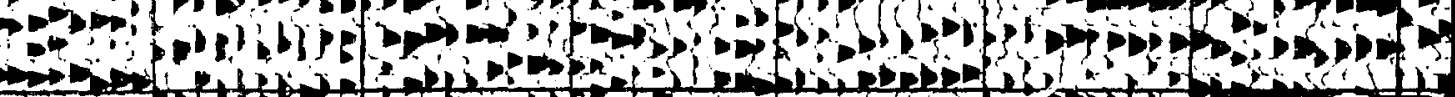
$\therefore$ is

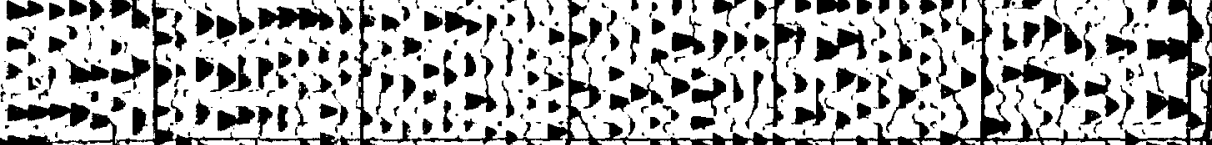




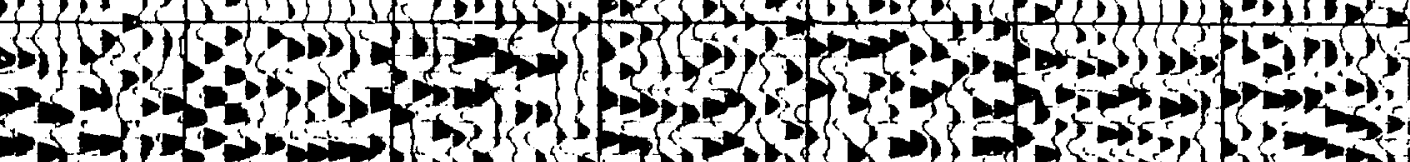
s)

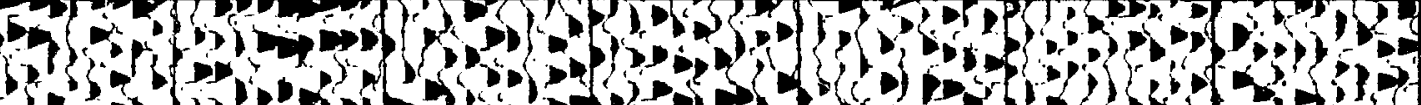

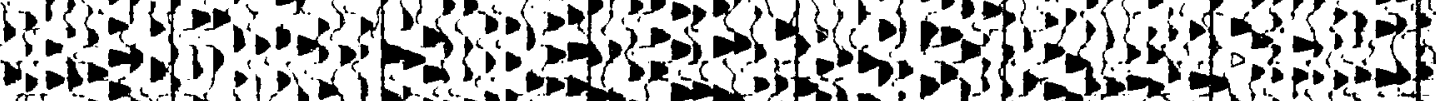

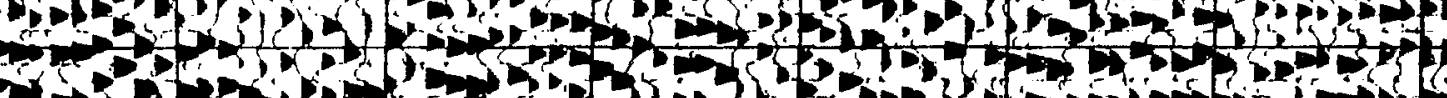

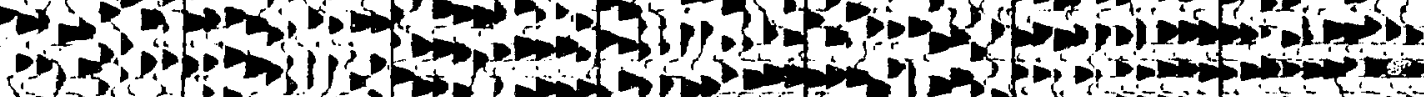

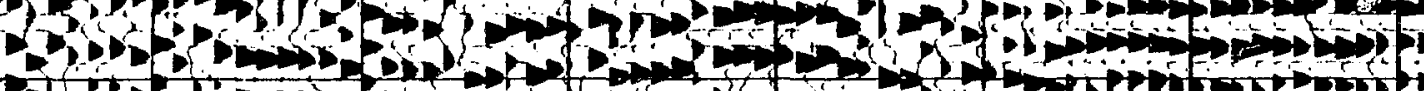

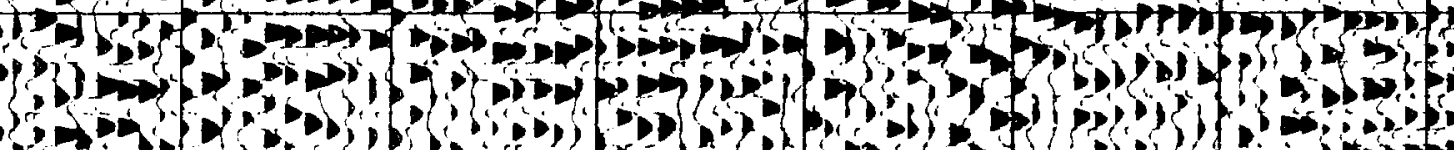

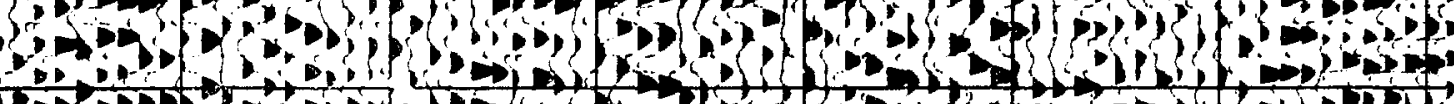

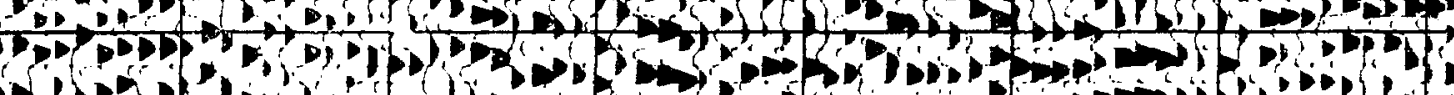

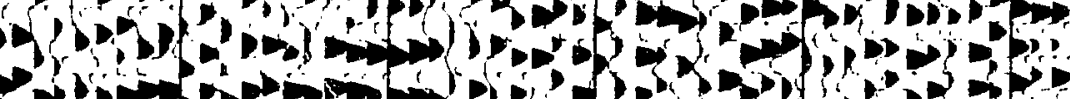

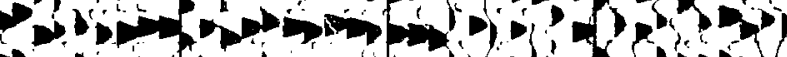

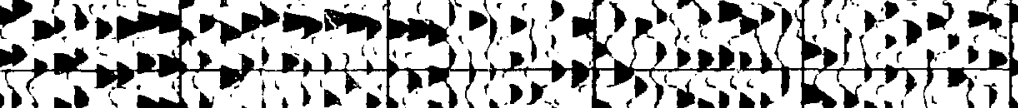
Som $\rightarrow \rightarrow$ is

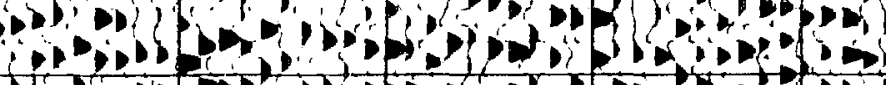
ind

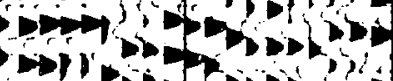
(Dipisis

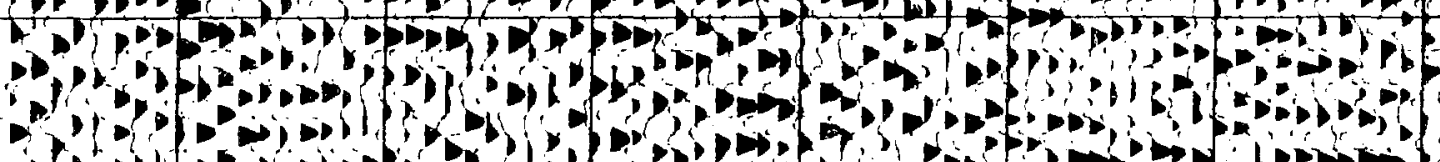

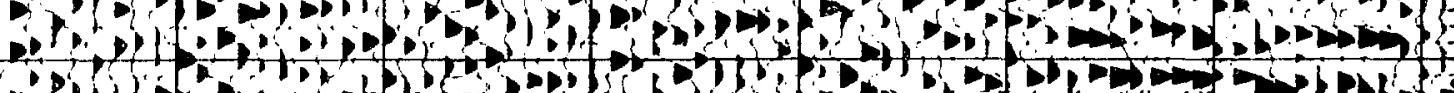

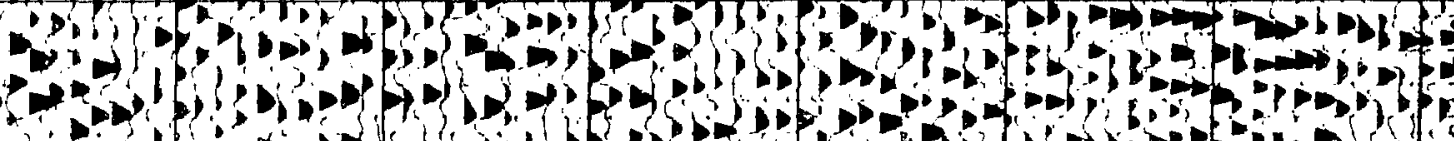

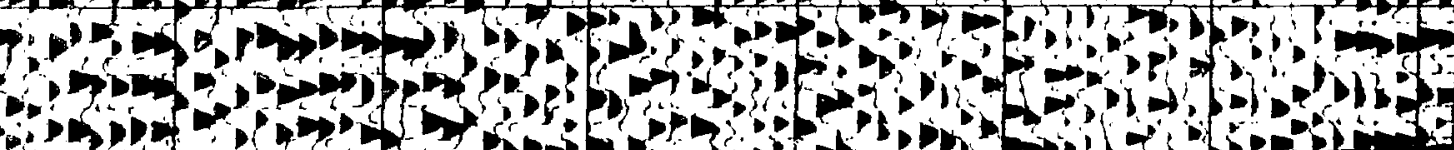

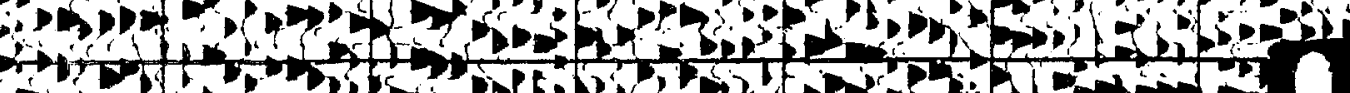

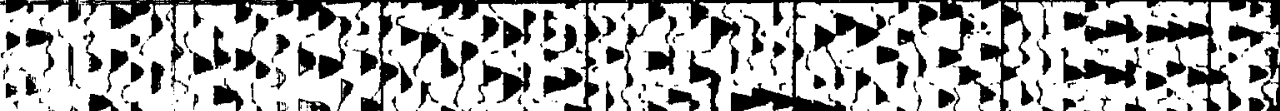

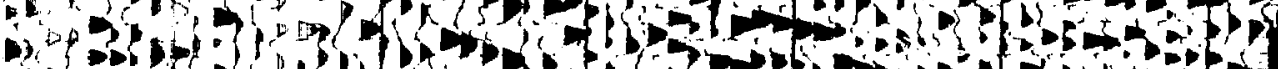

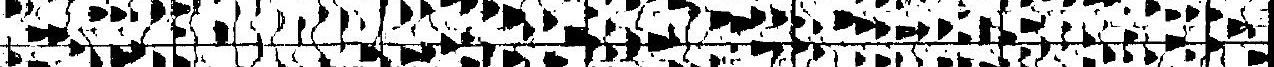




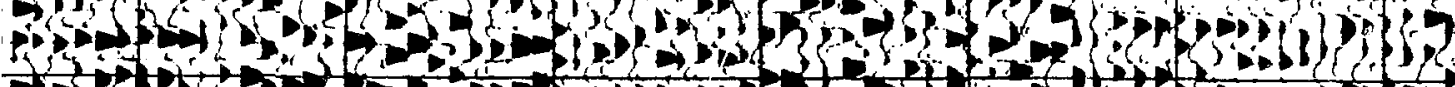

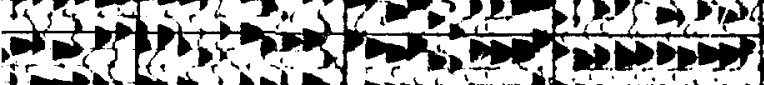

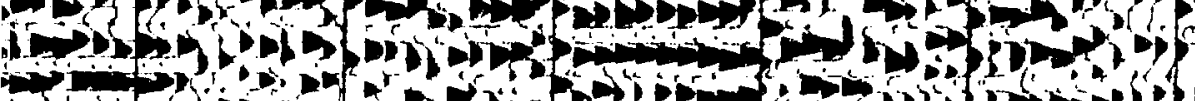

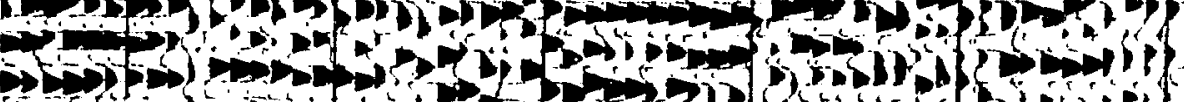
,

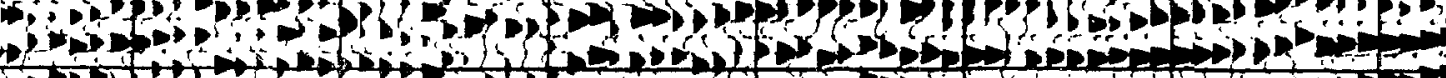

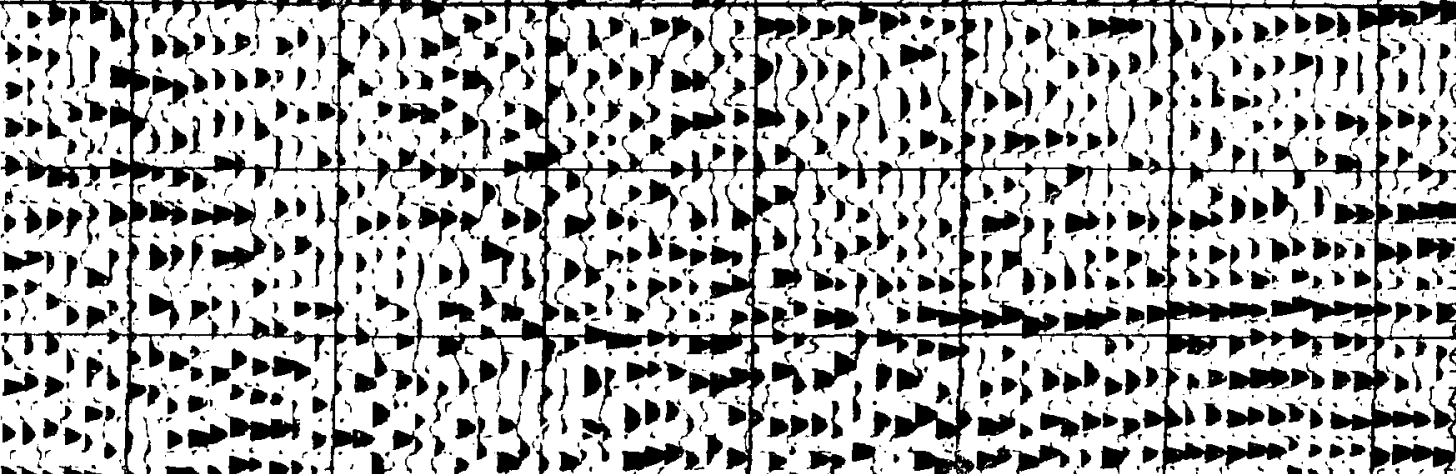

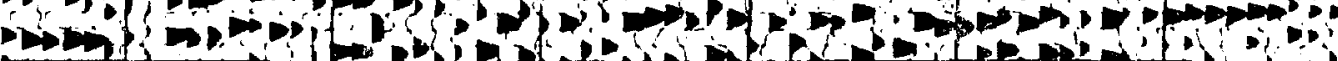

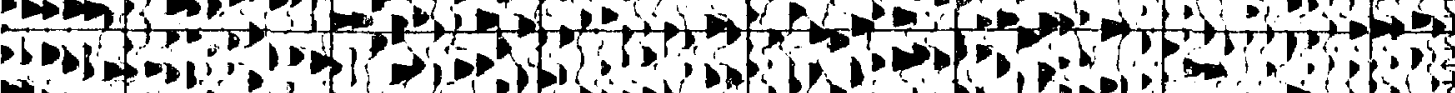

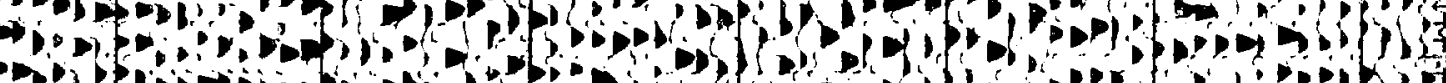

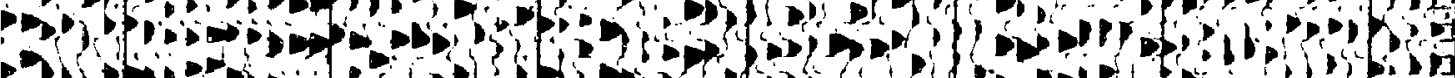

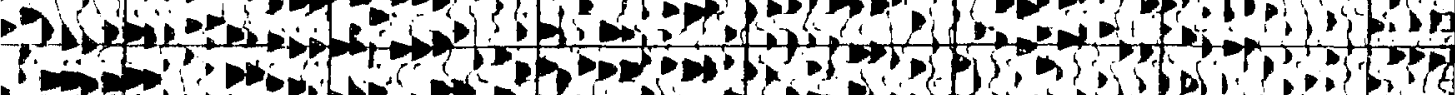

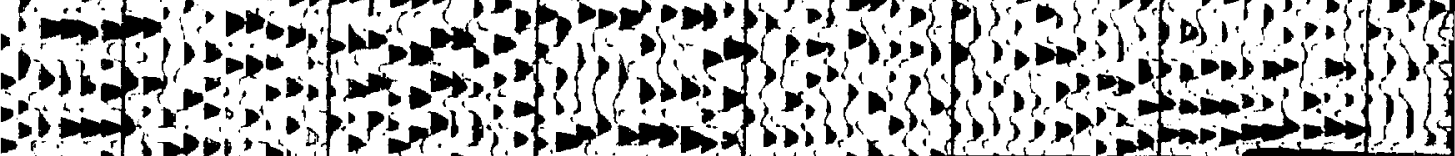
T?

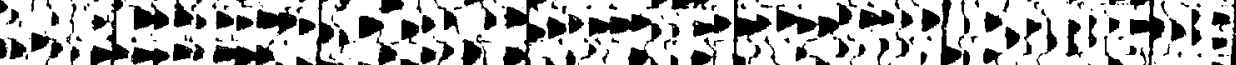

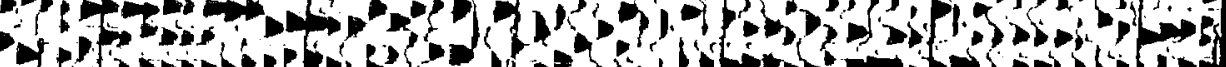

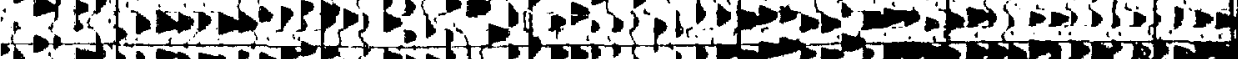




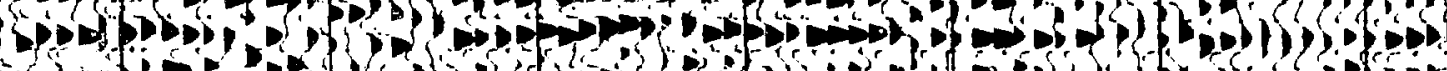

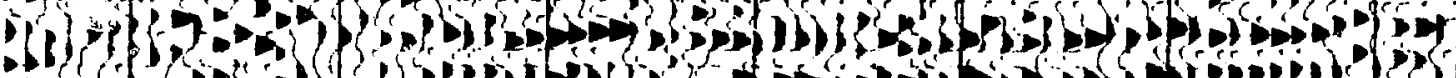

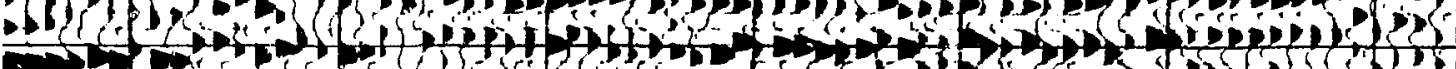

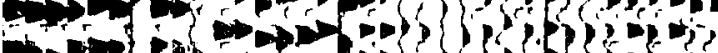
of

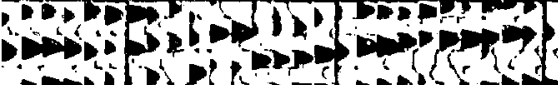

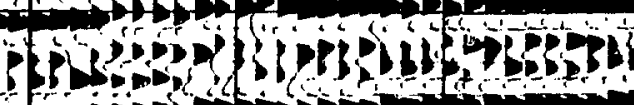

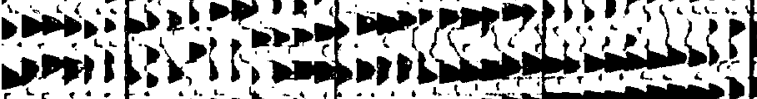

SP

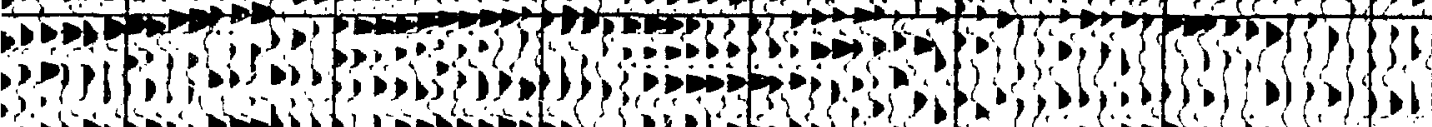

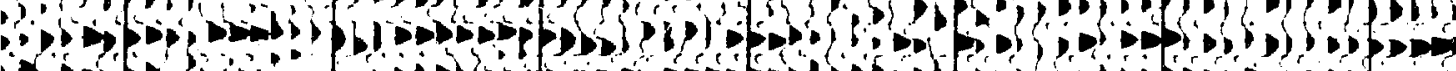
1)

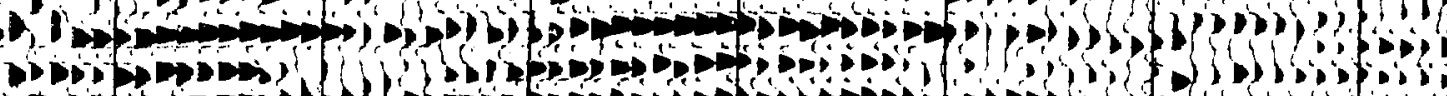

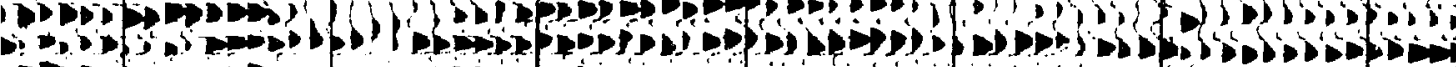
$\Leftrightarrow$ rof

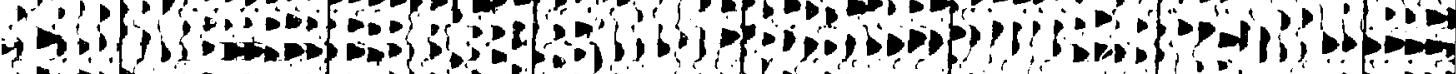

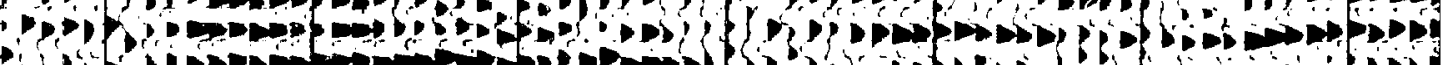

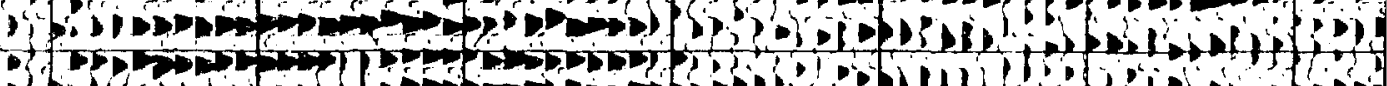

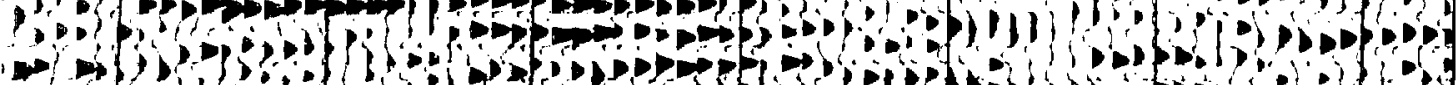
(1)

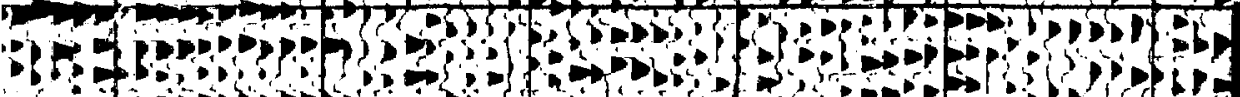

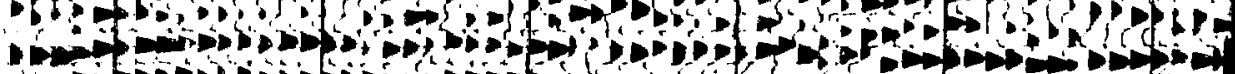

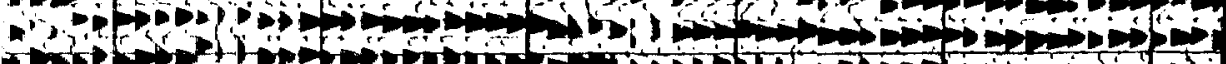

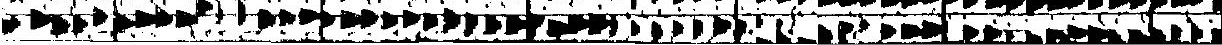




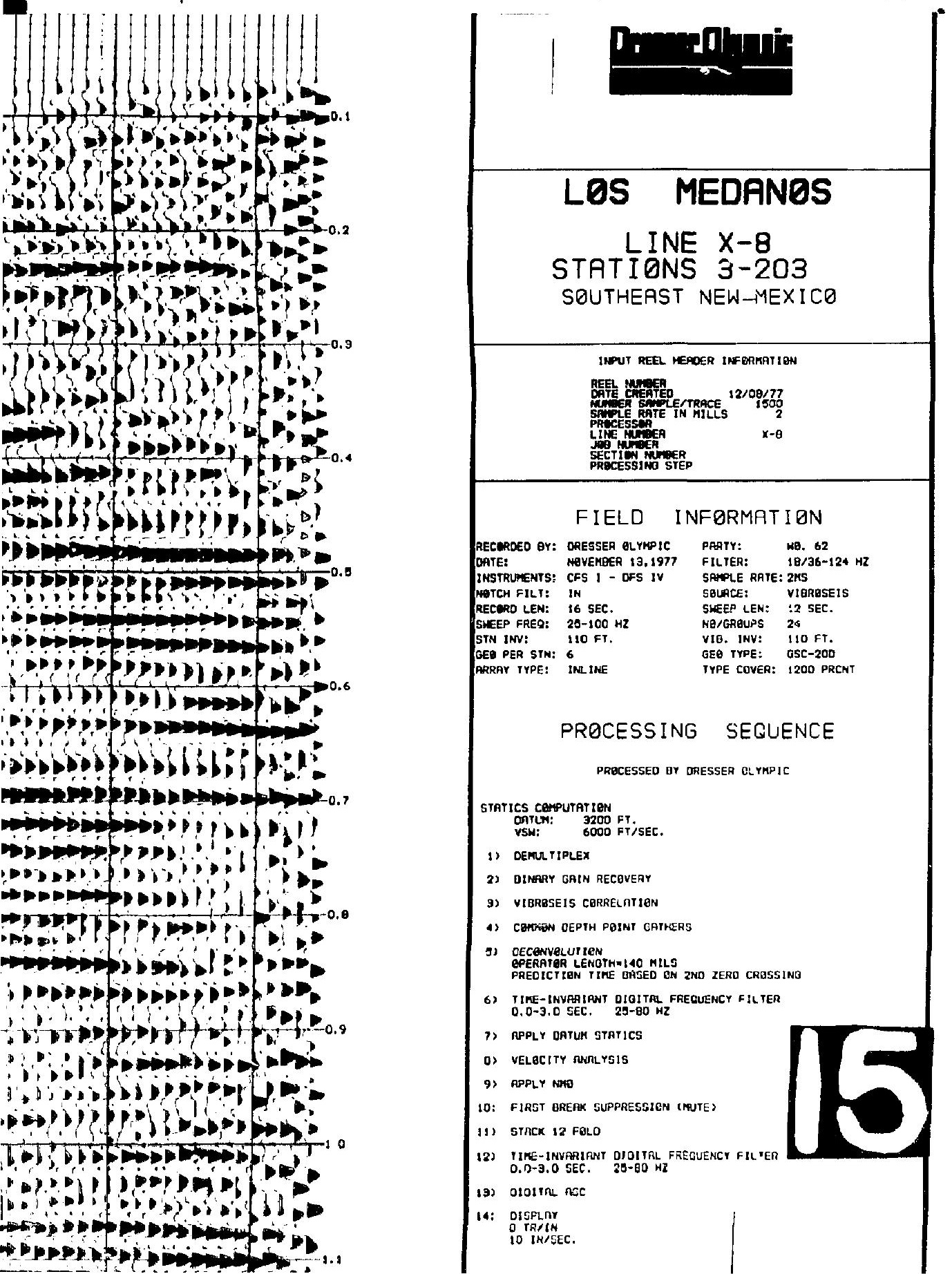




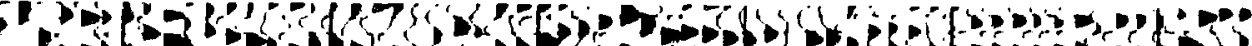

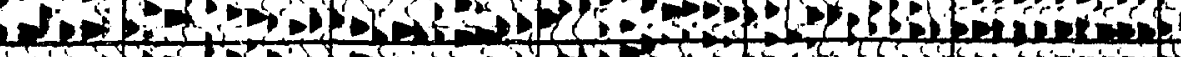

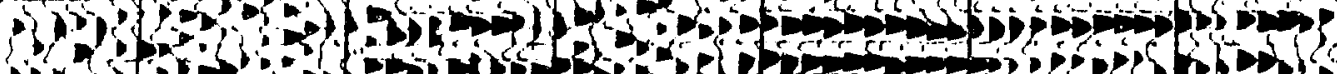

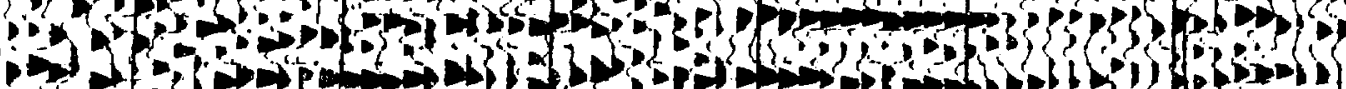

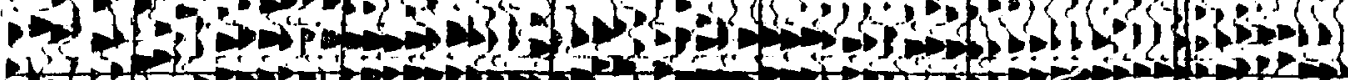

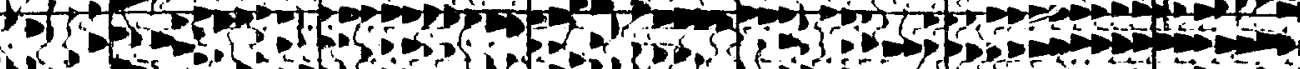

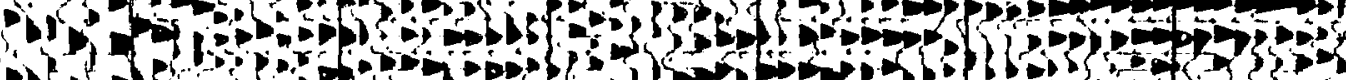

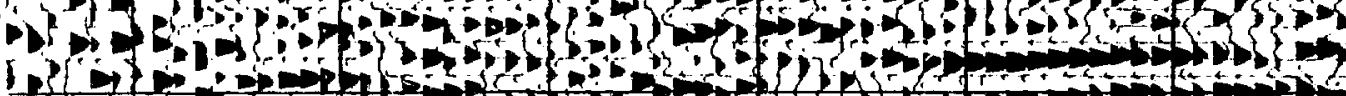
- If

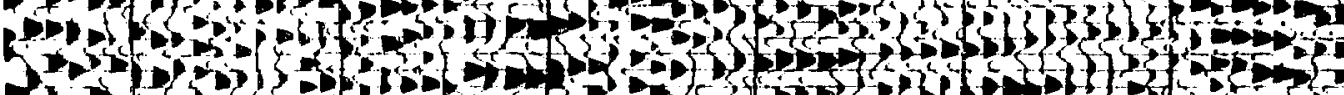

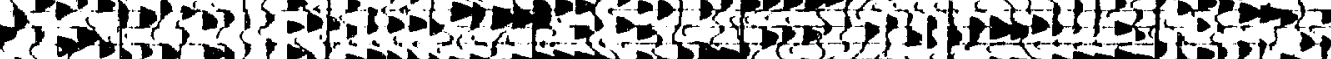

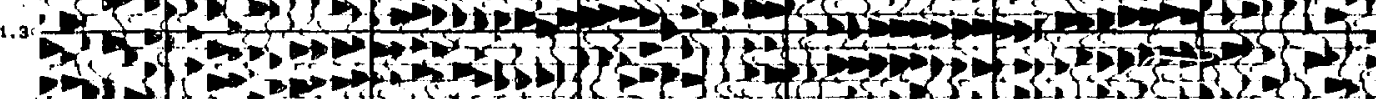

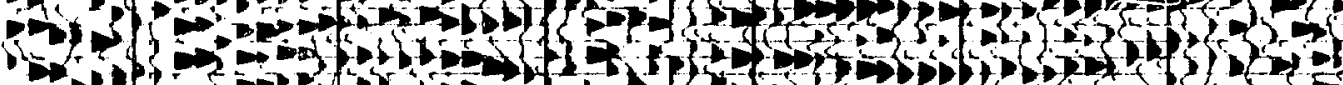

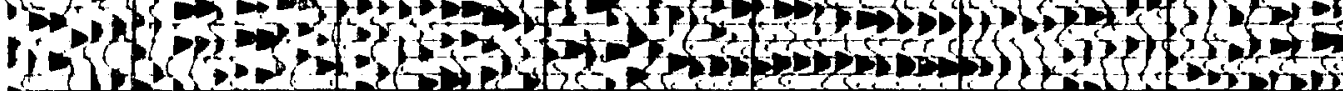

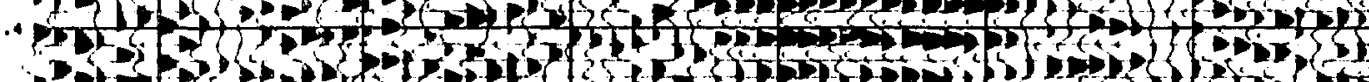

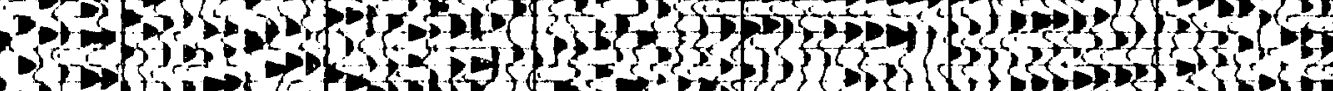

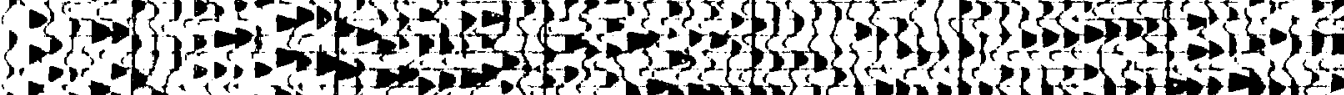

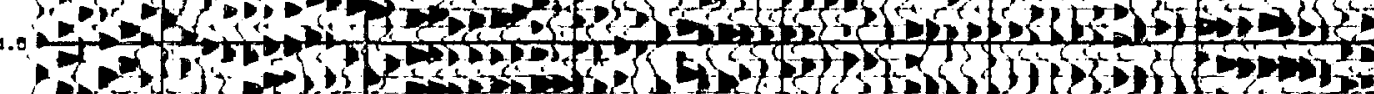

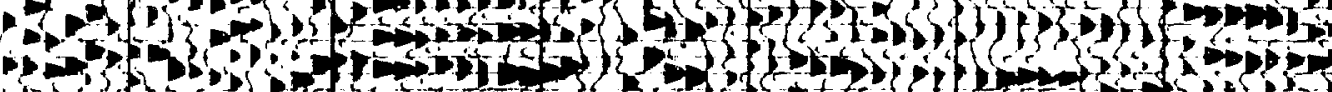

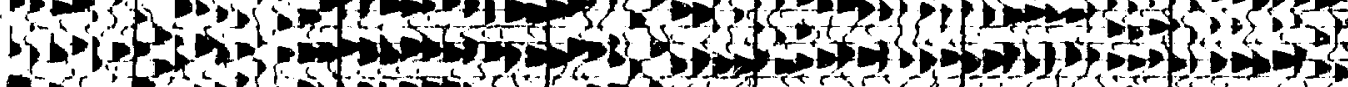

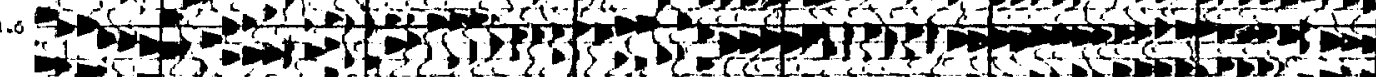

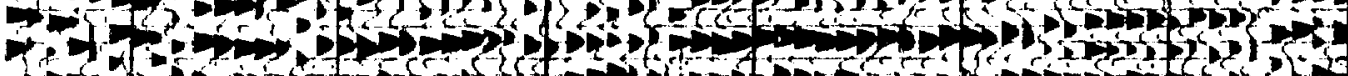

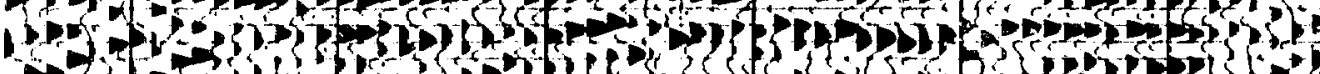

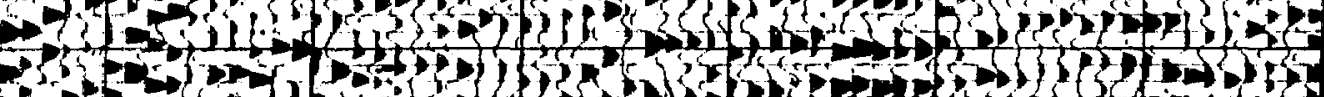

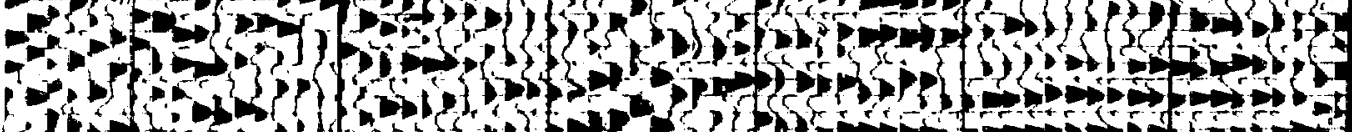

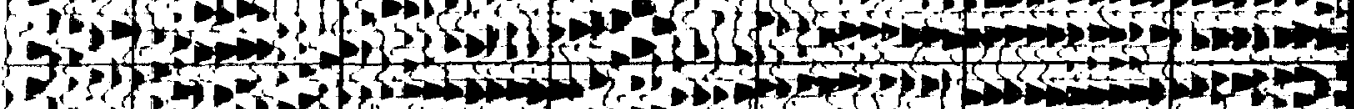
1.

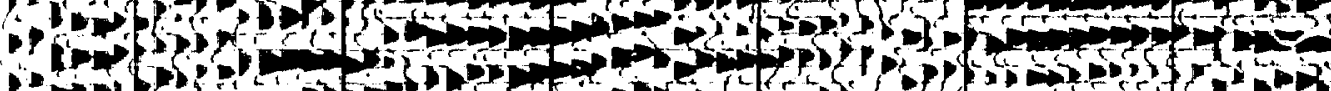

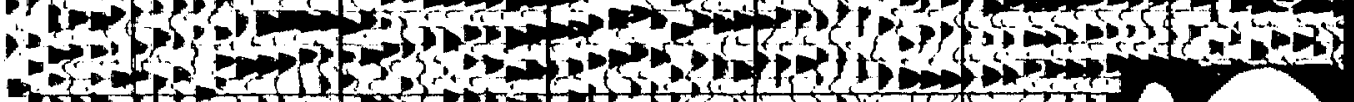

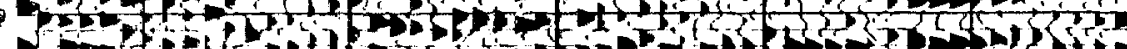

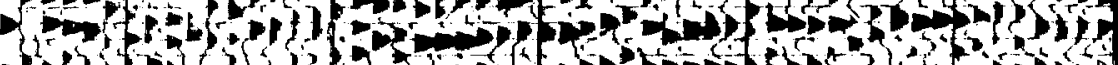

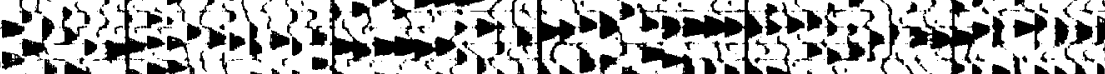

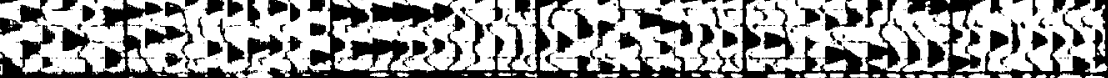




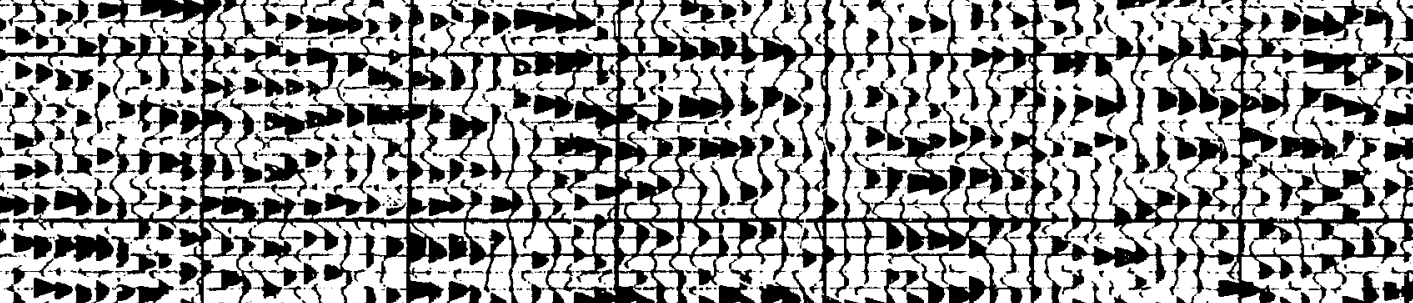

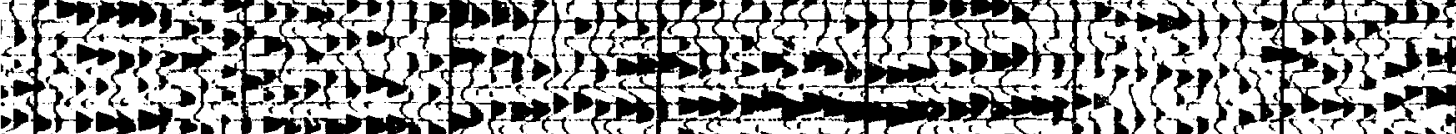

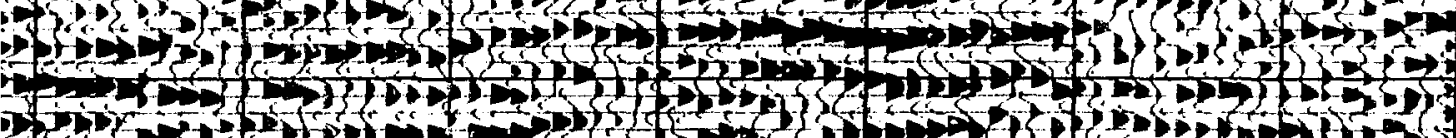

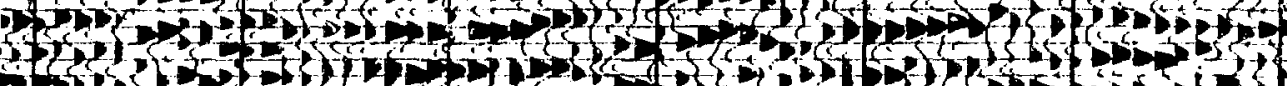

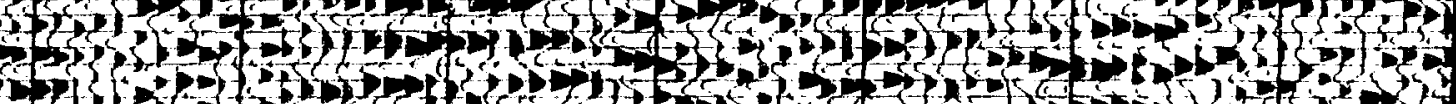

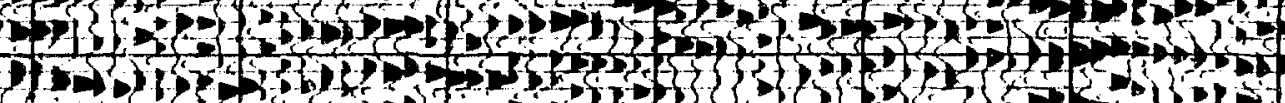

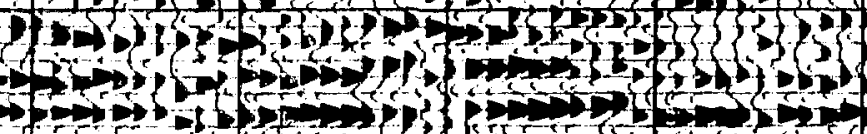

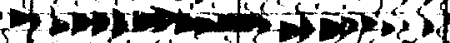

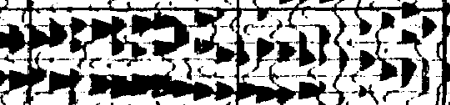

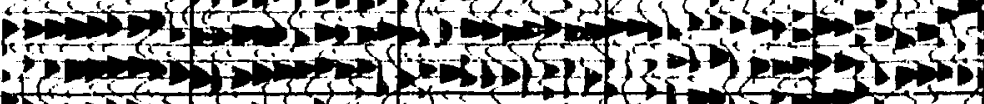

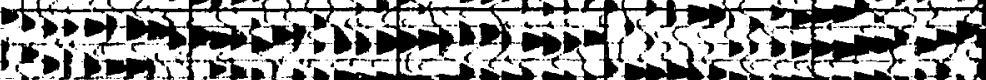

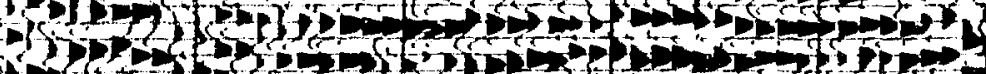

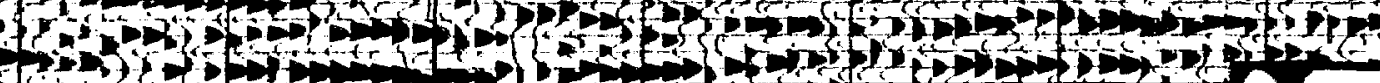

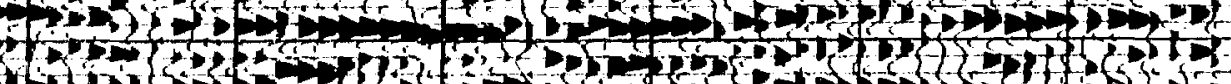

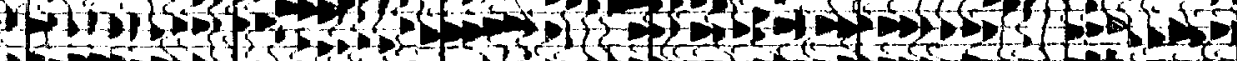

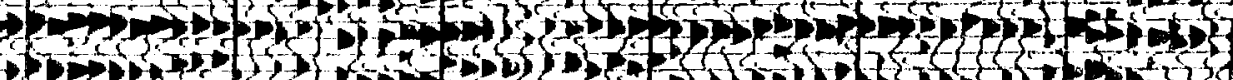

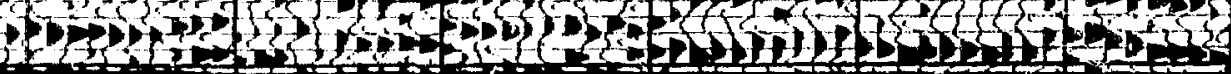




\section{morom}

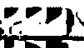

sis

song

popos

$-45+5.5$

7

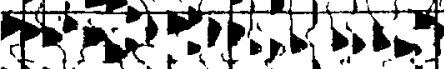

A

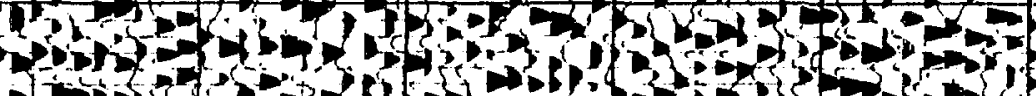

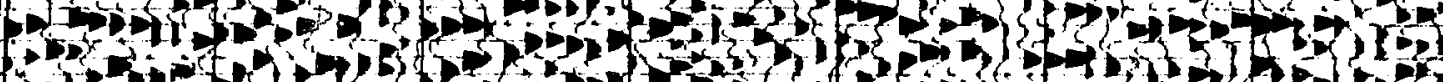

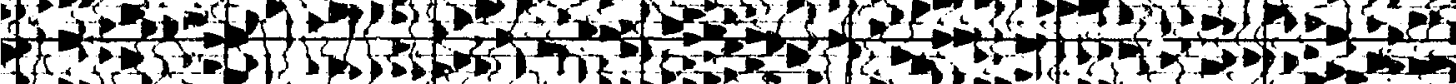

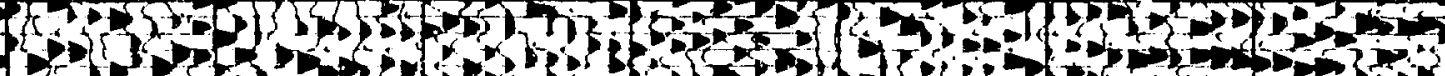

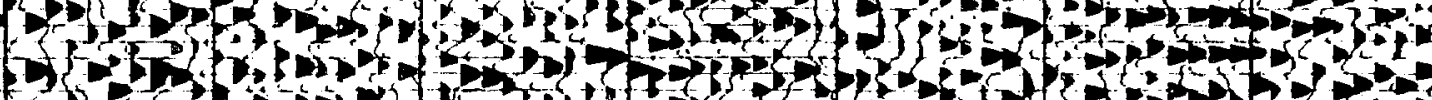

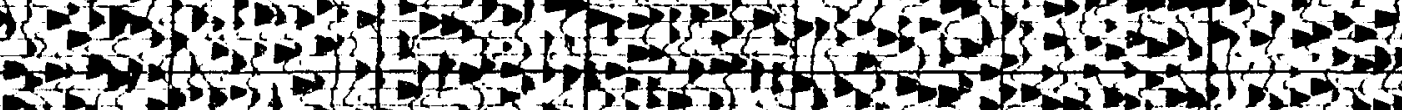

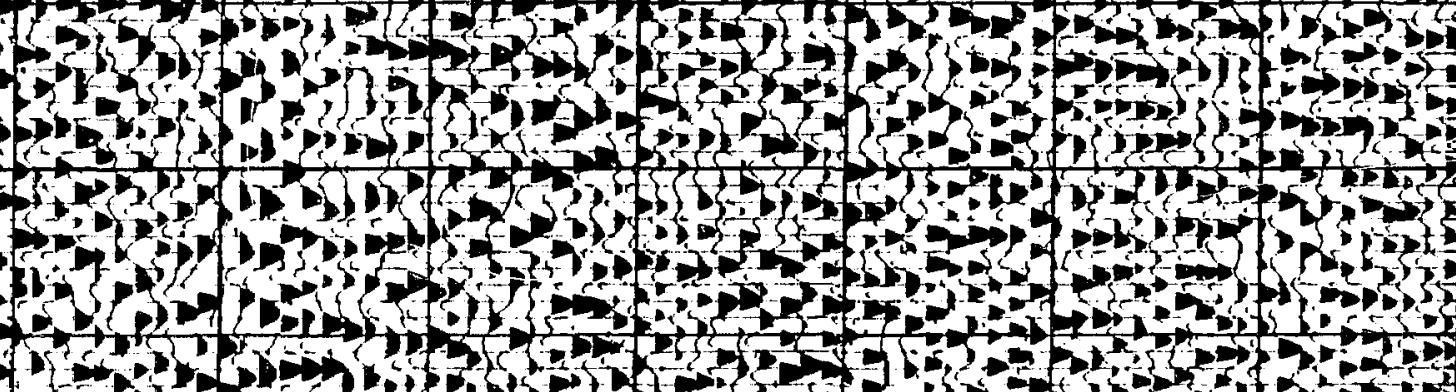

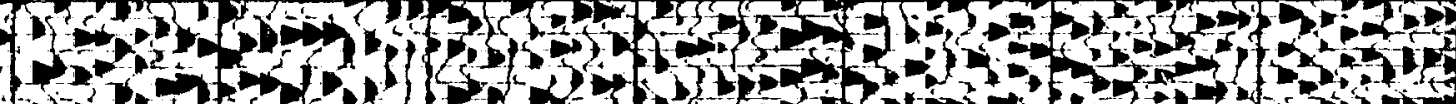

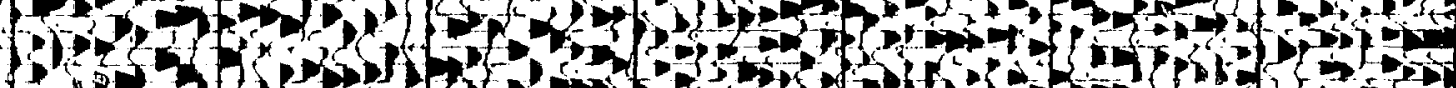

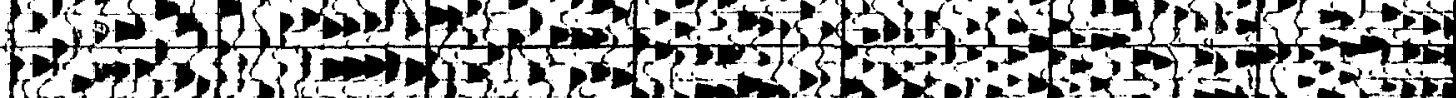

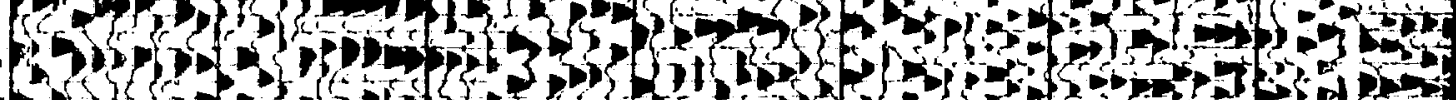

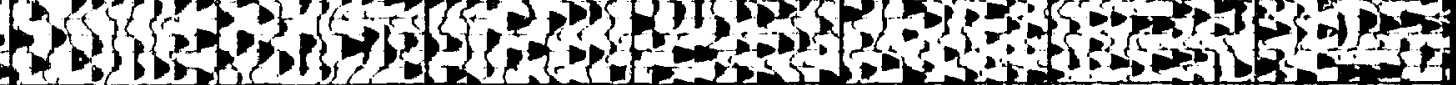

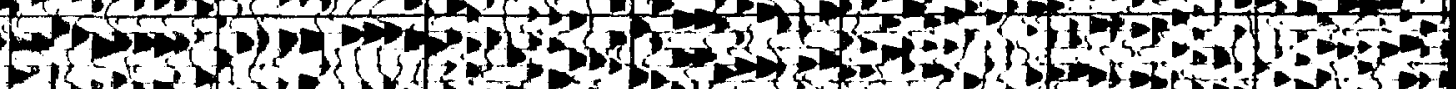

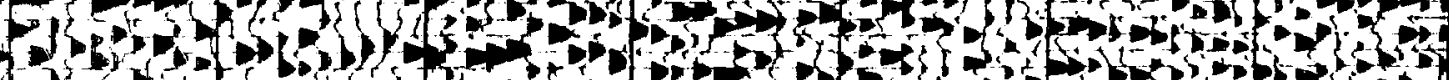

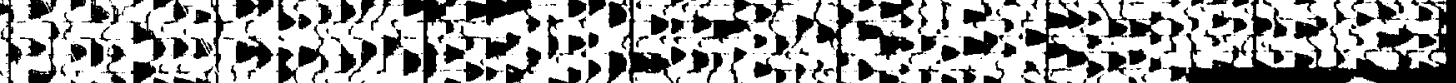

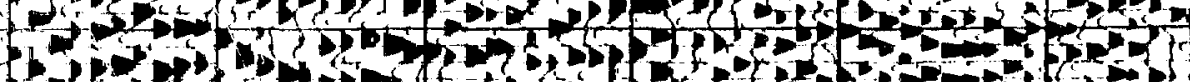

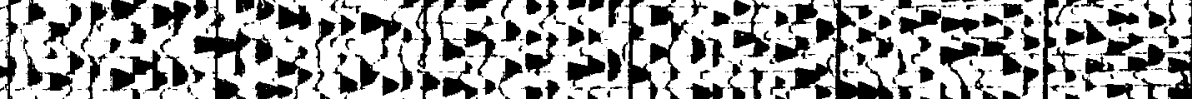

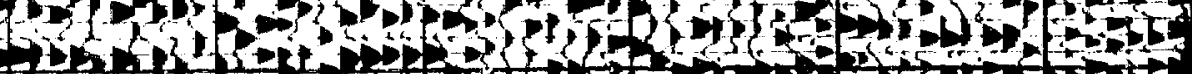




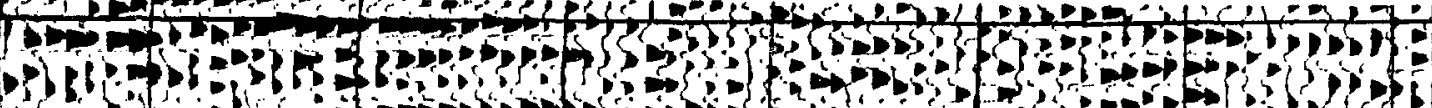

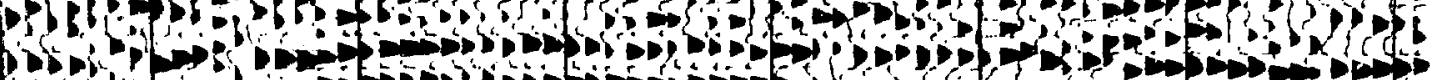

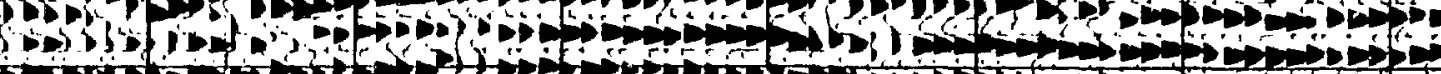

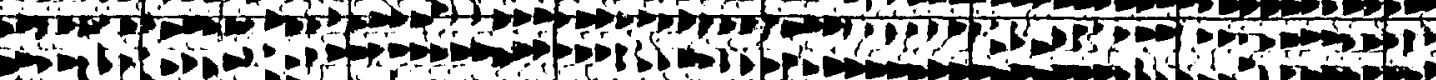

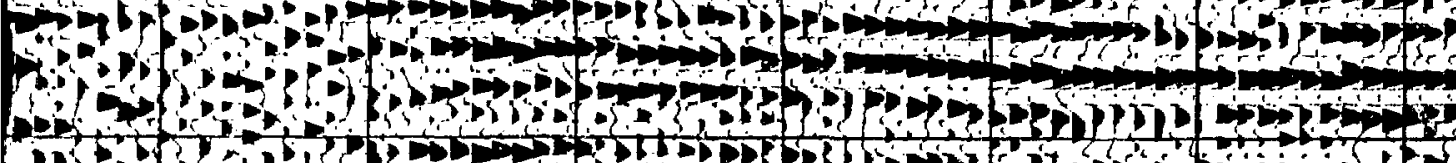

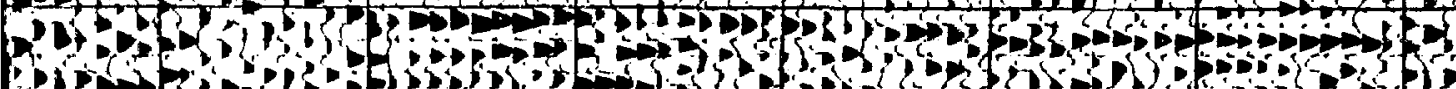

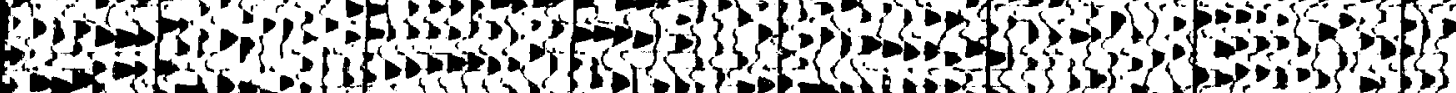

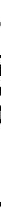

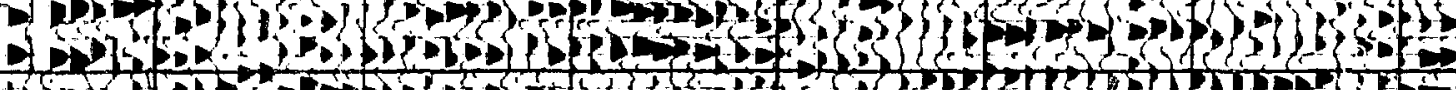

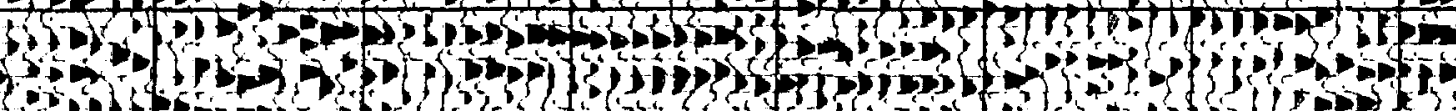
Q

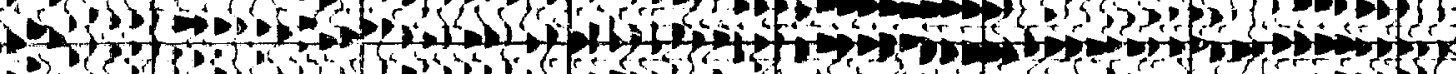

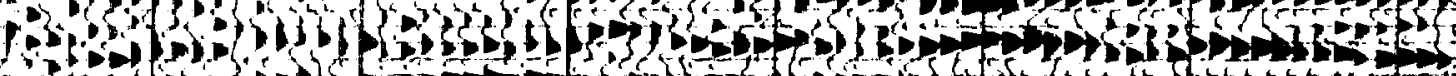
DAp

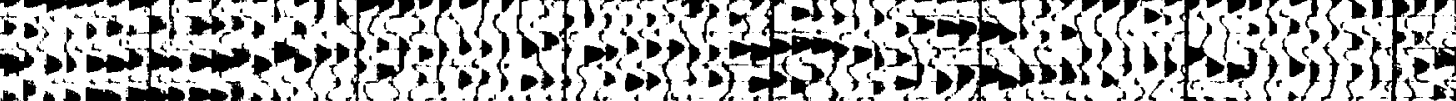

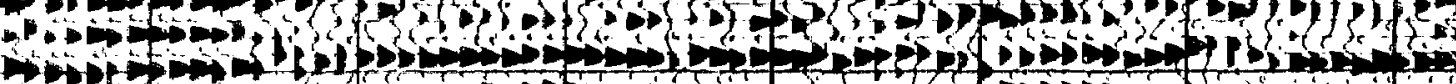

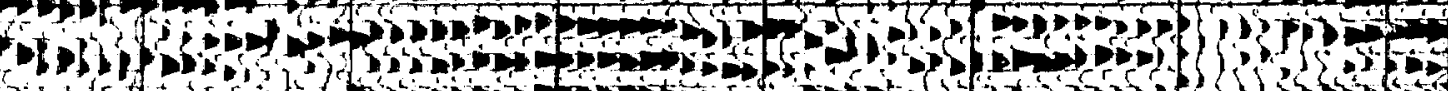

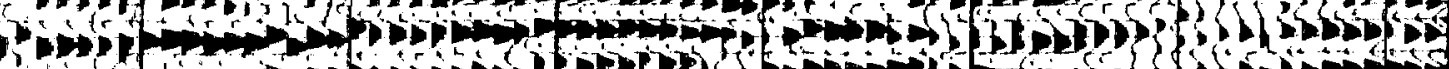

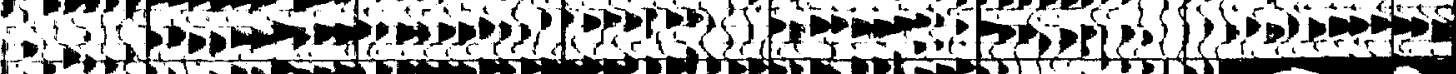
- 135 5.

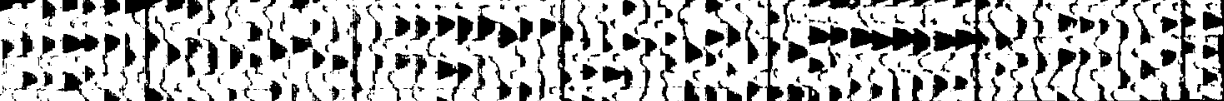

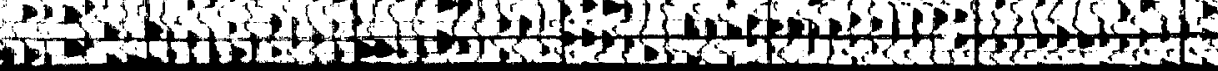




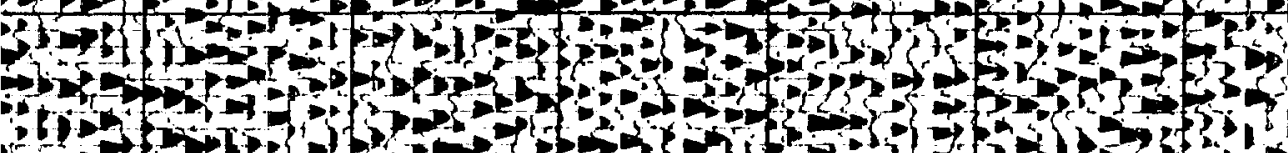




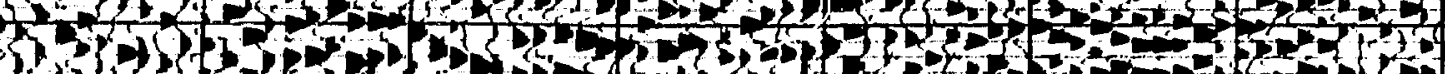

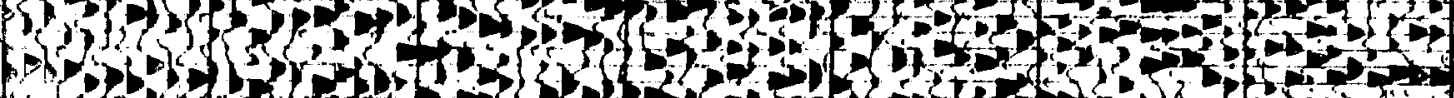

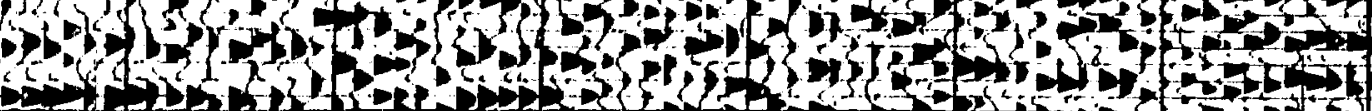

, B.t.

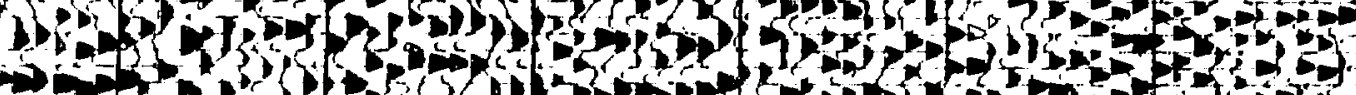
sond iopitis 5 P.m.

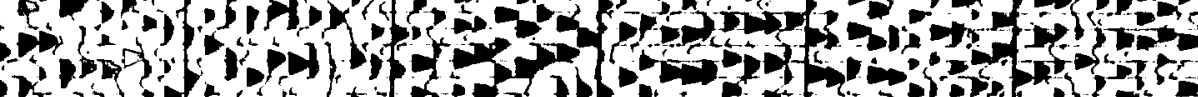

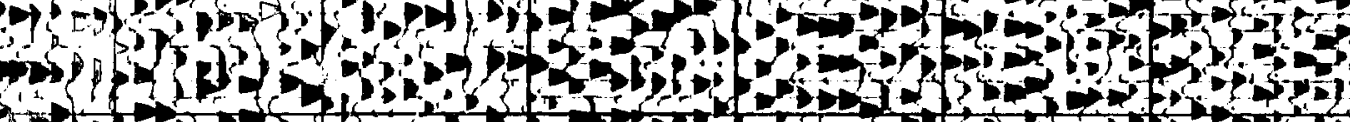

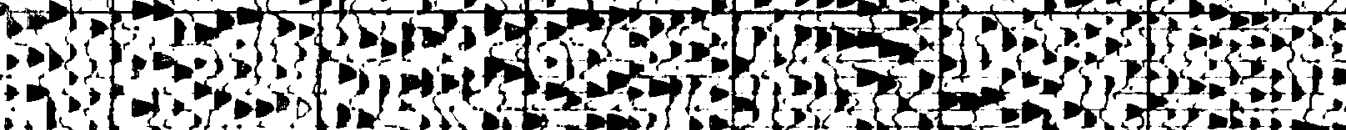

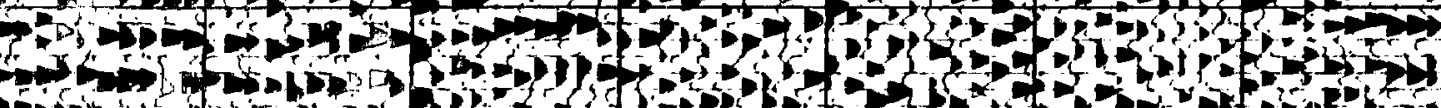

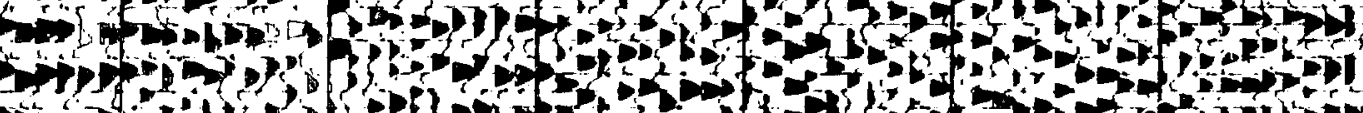

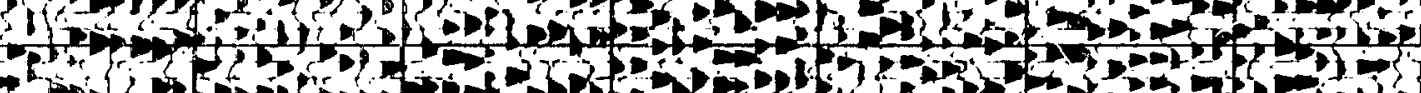

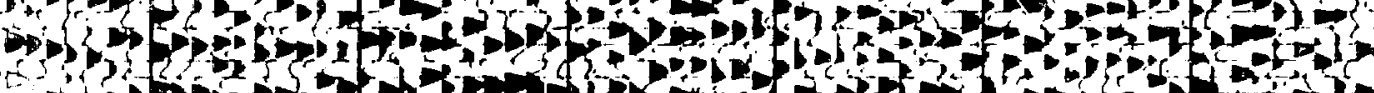

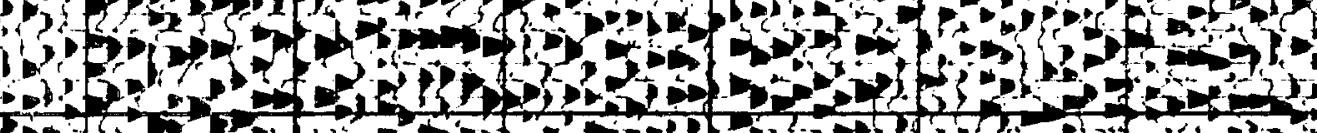

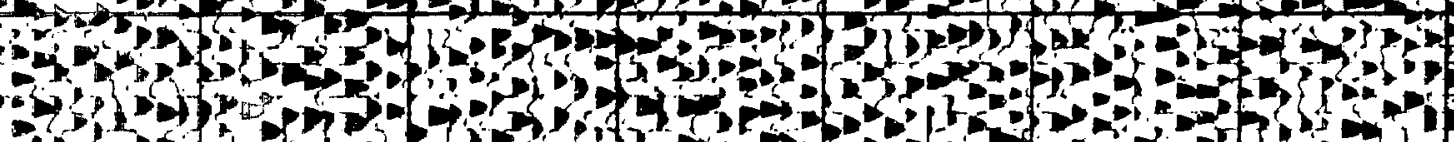
. S. .

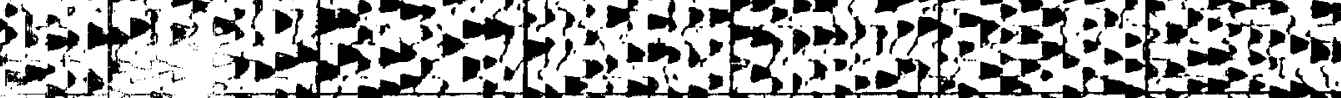
30,03035

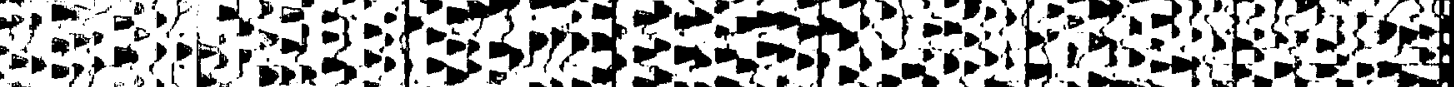

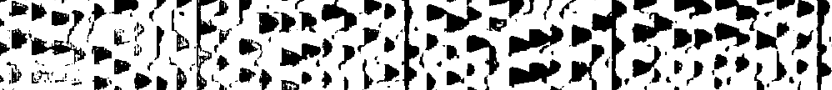
(20) $11\}$ . 



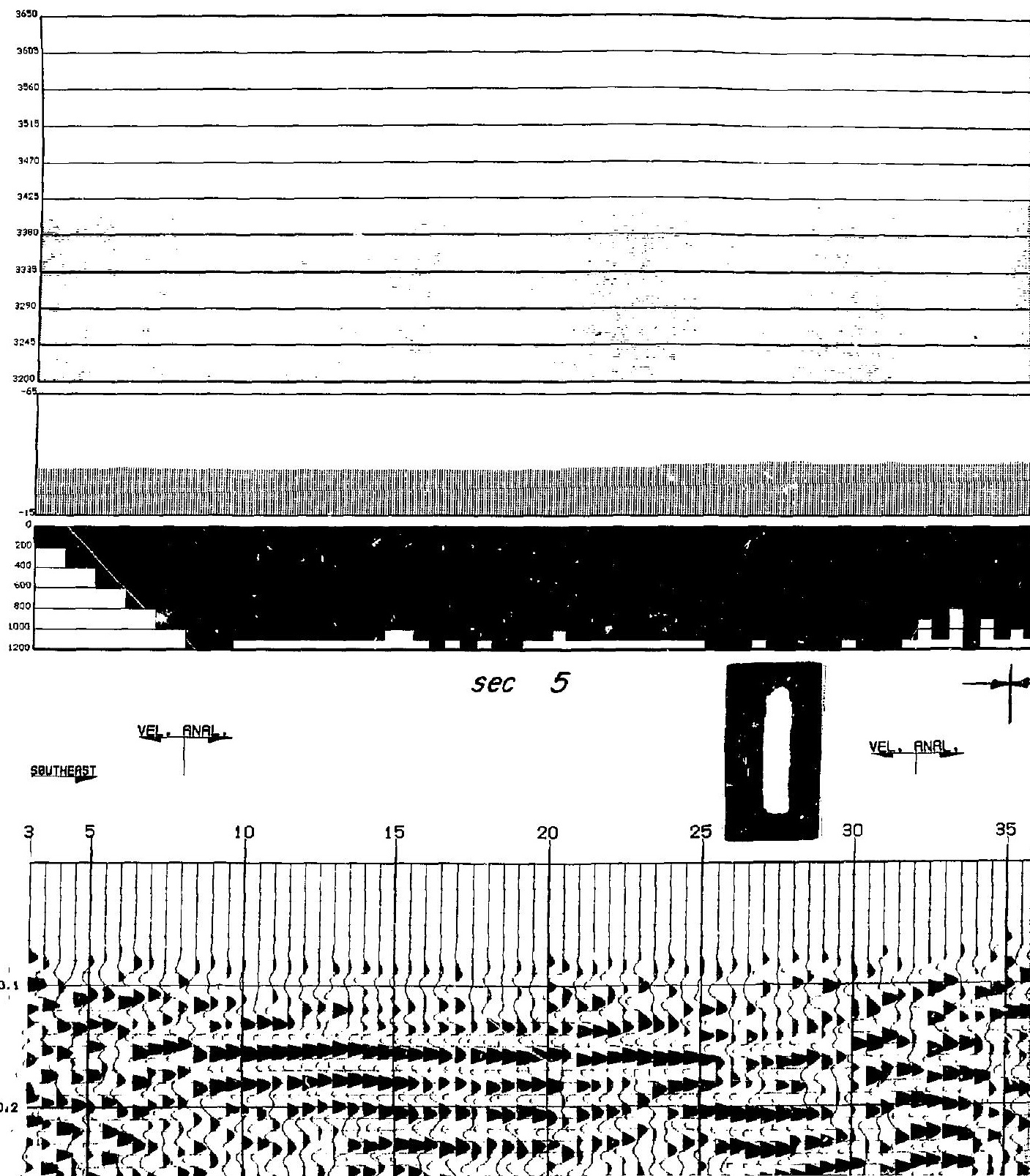




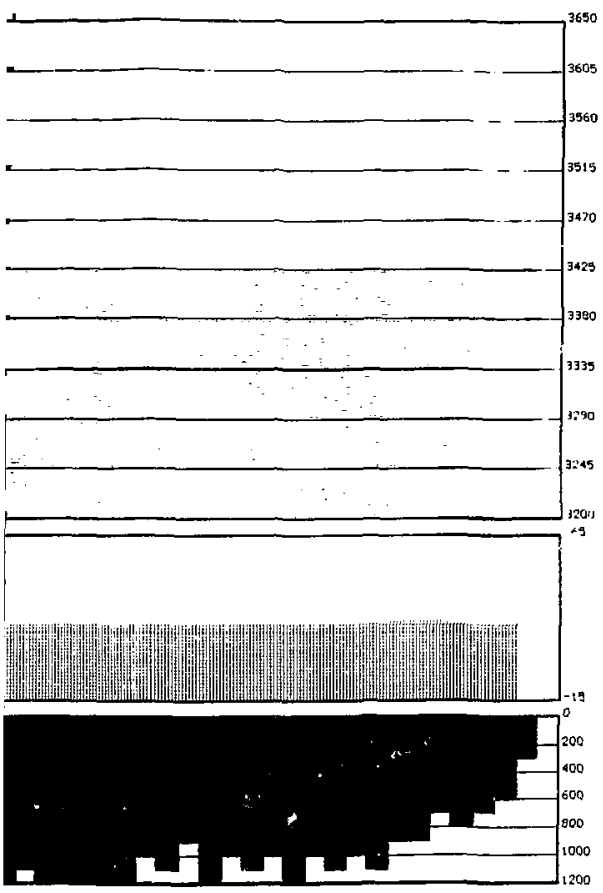

ec 9

VE1, RNAL,

SQUTHERST

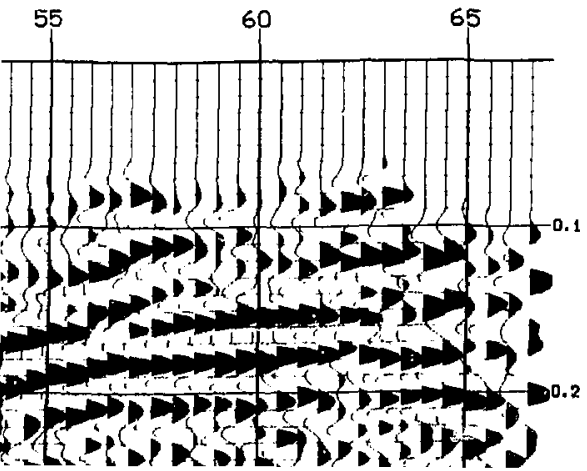

ELEVAT IONS

STAT ICS

FOLD \%

LINE UIRECTION

VELOCITY FUNCTION

DIRECTION

LINE INTERSECTION

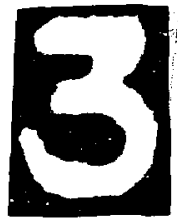

STATIONS

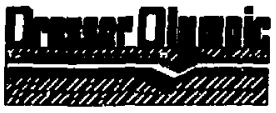

\section{LOS MEDANOS}

LINE $x-9$

STATIÖNS $3-66.5$ 


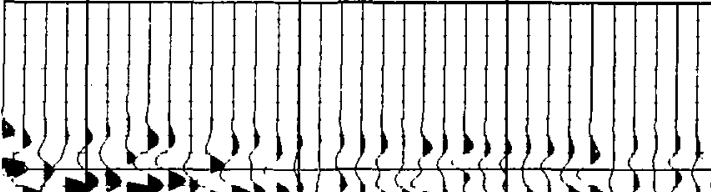

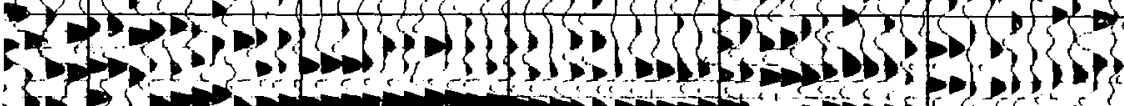

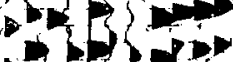

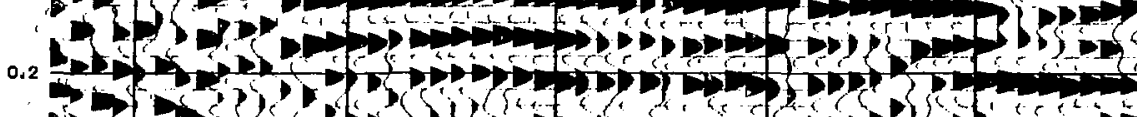

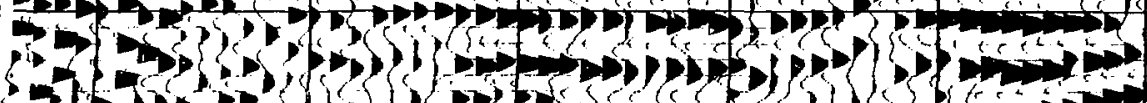

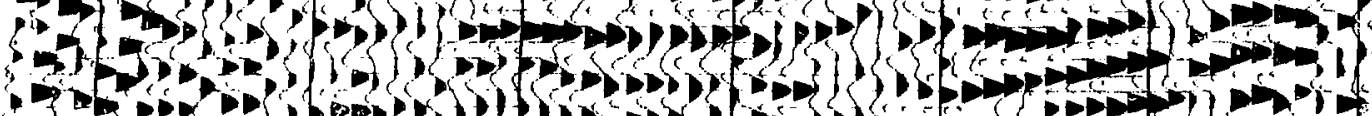

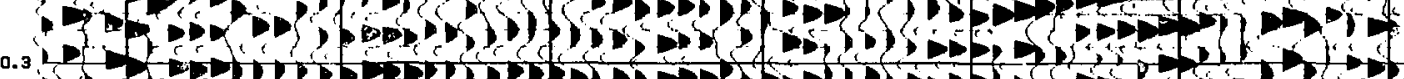

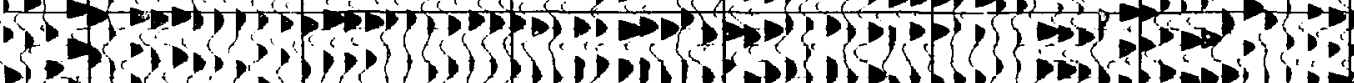

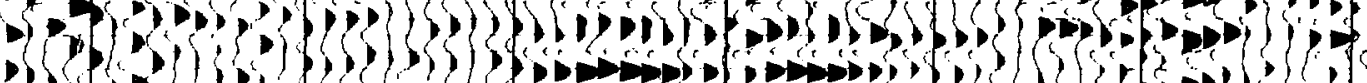

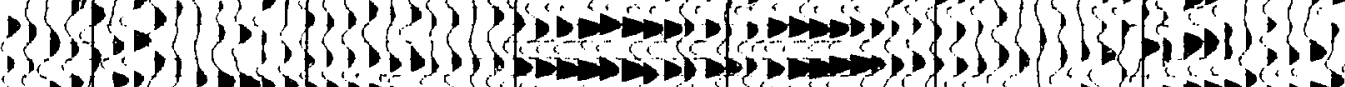

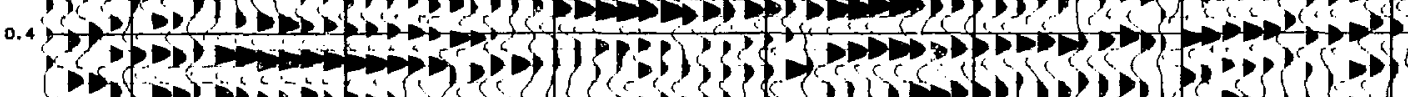

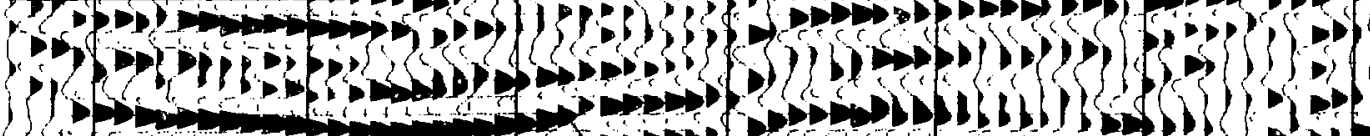

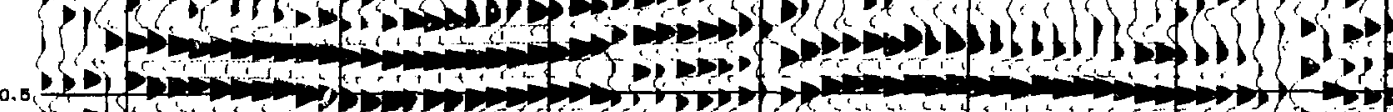

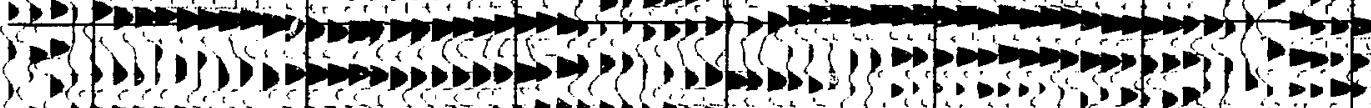

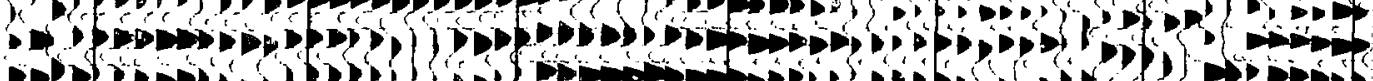
Fo 0.6 Fof $\left\{\begin{array}{l}1 \\ y\end{array}\right.$

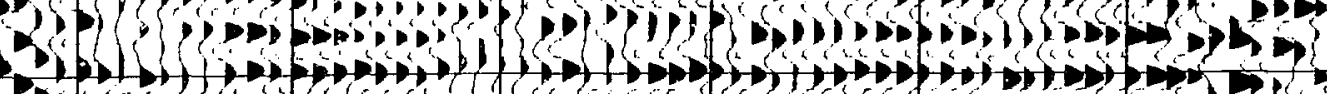

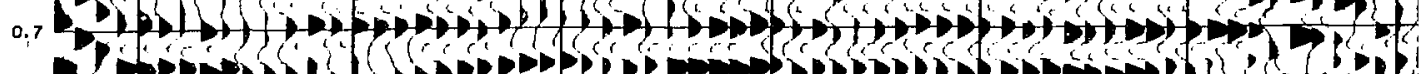

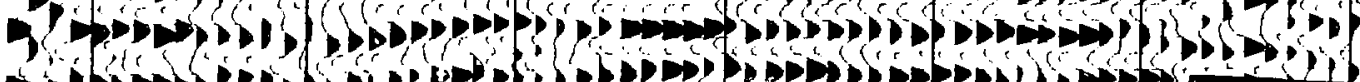

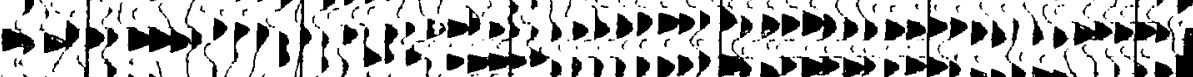

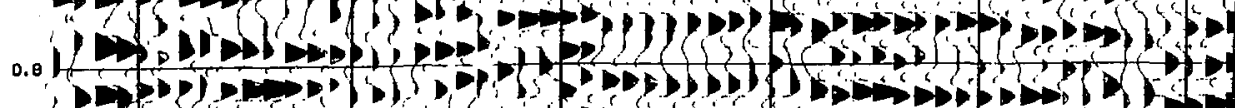

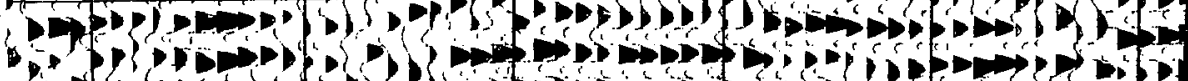

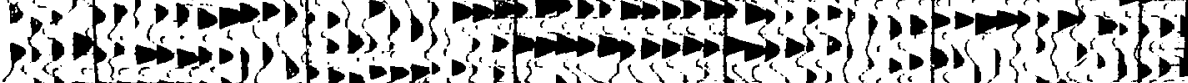


)

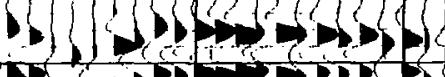

$\{\{\}, 0$,

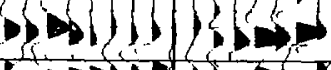

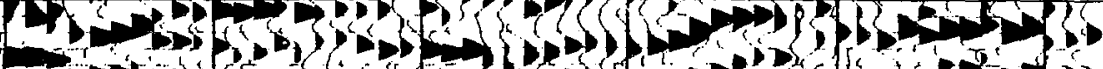

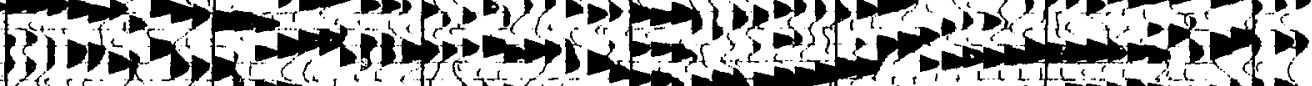

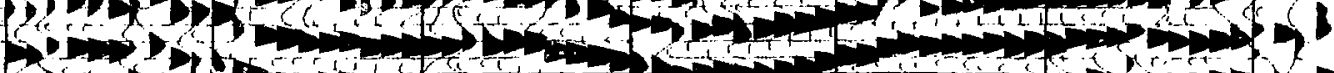

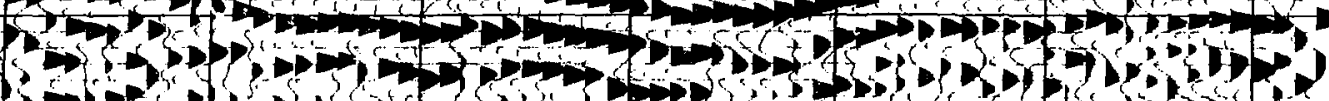

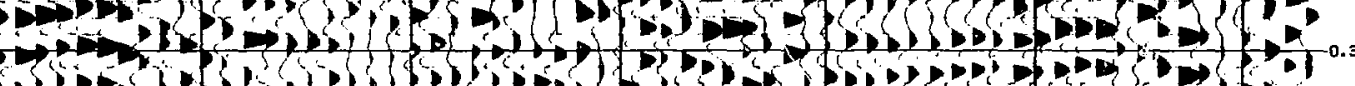

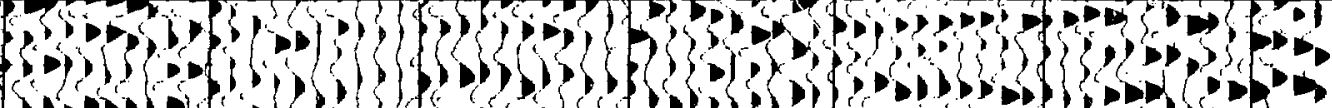

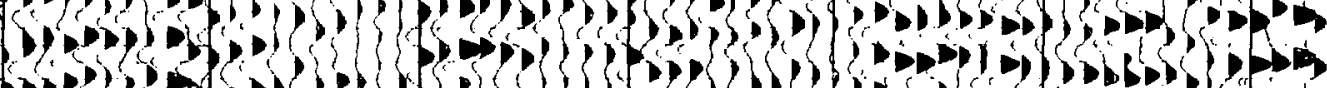

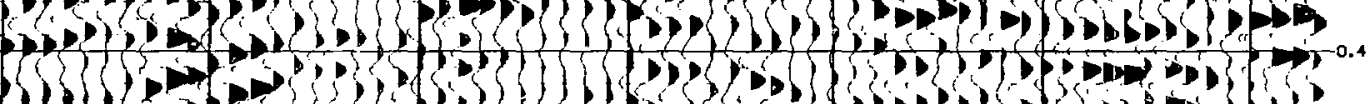

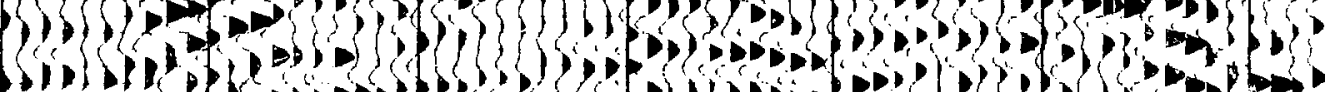

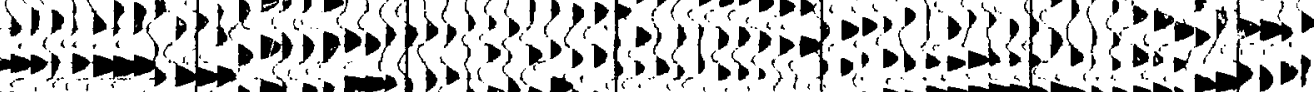

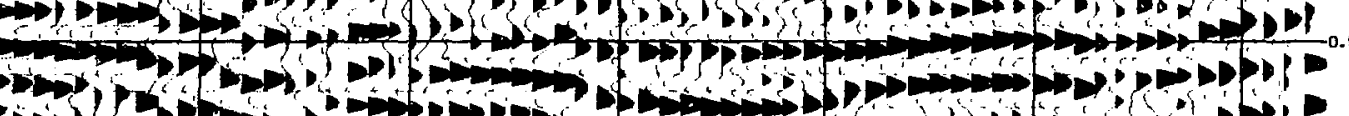

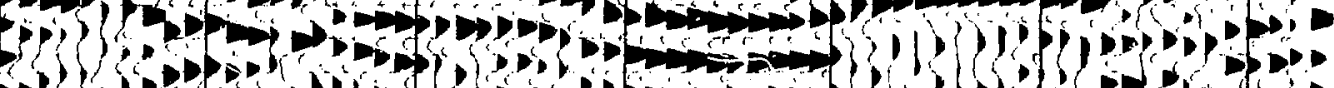

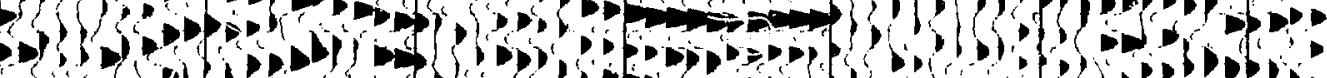
DID - tof

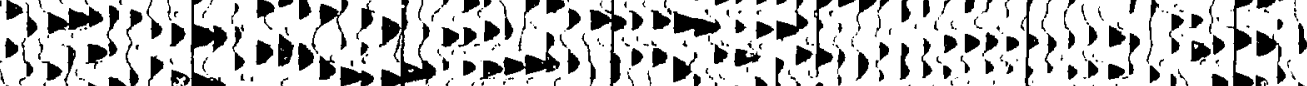

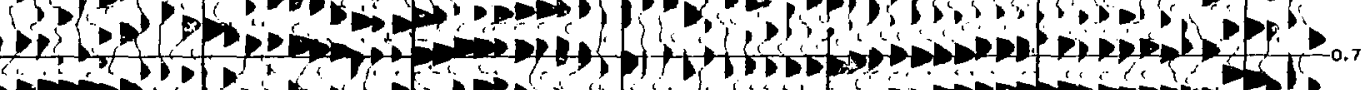

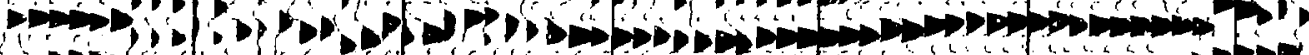

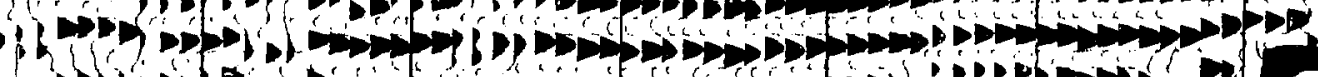

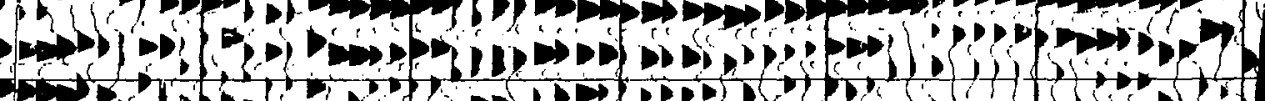

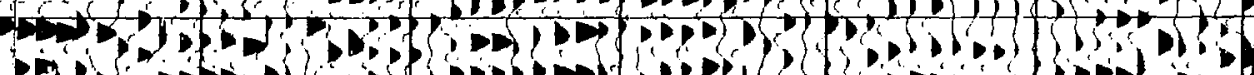

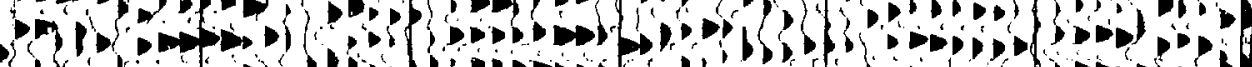

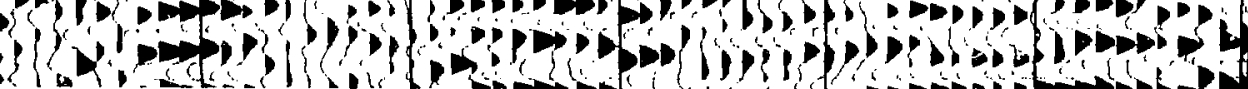

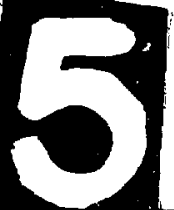




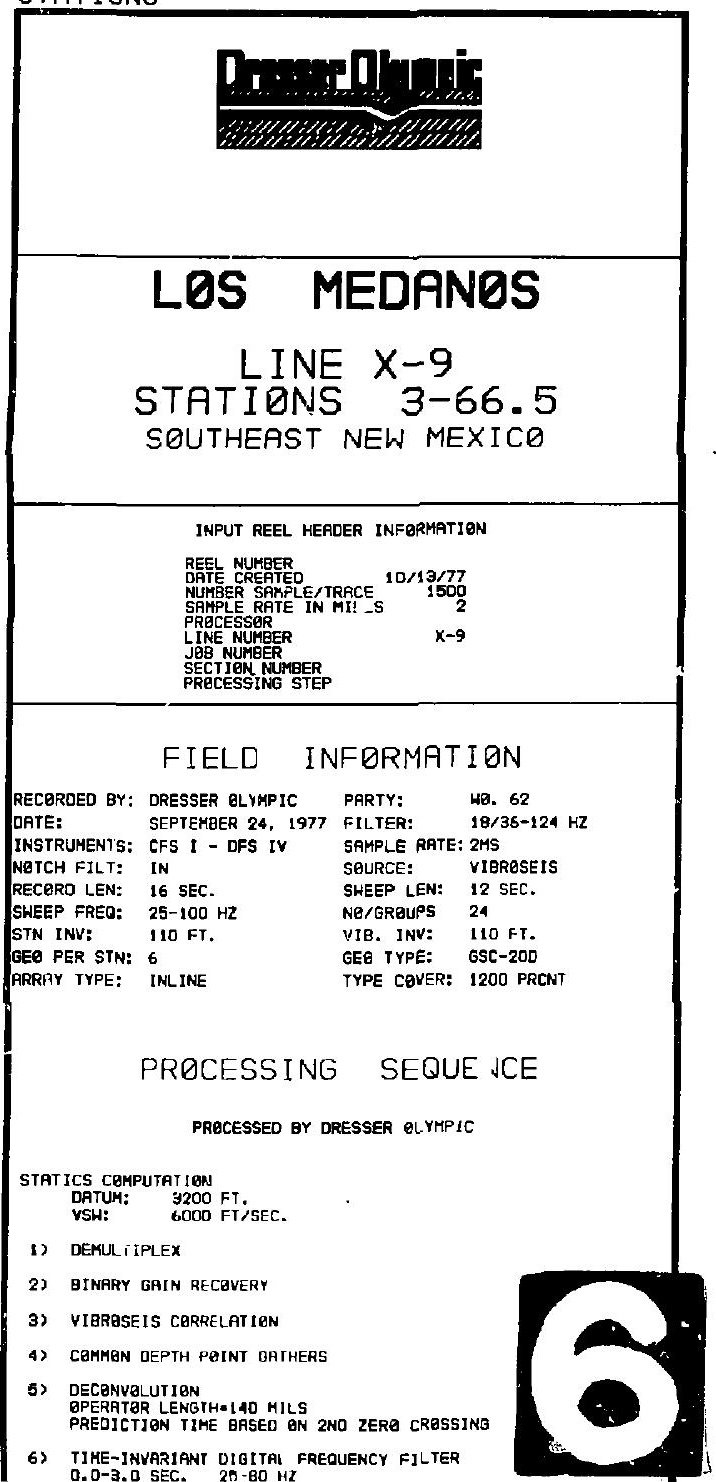




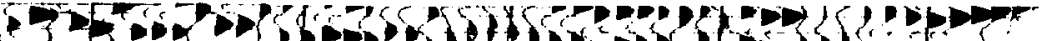

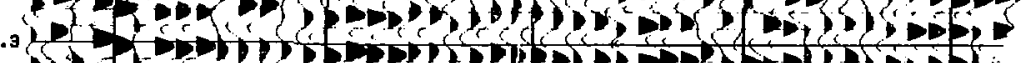

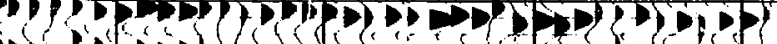

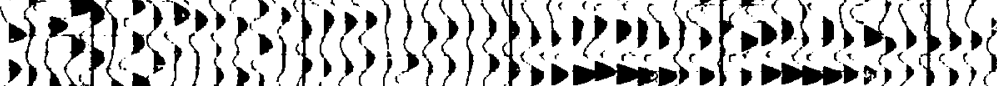

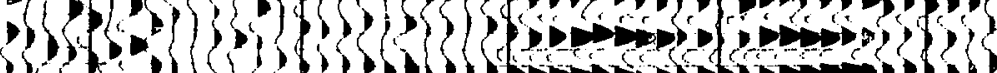

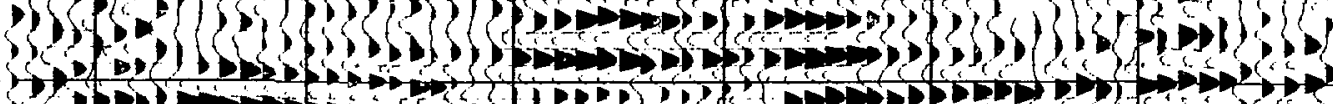

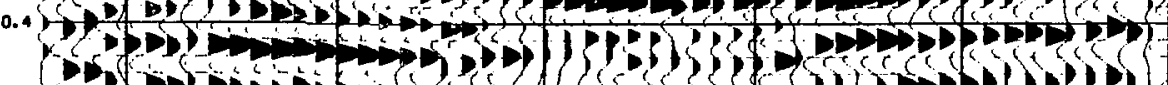

3)

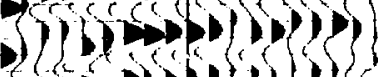
(r)

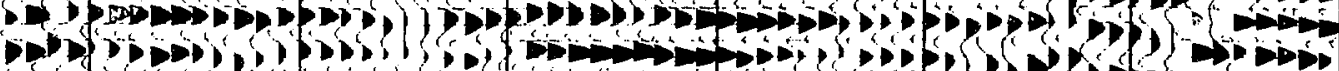

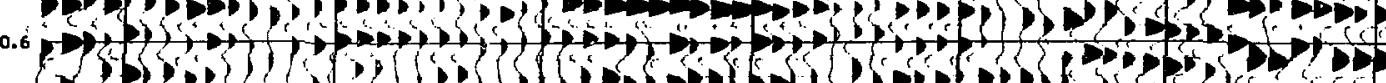

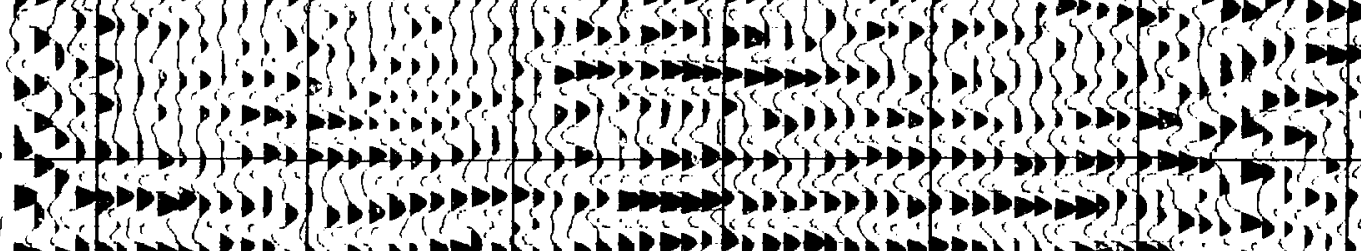

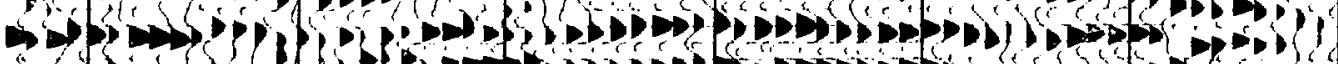

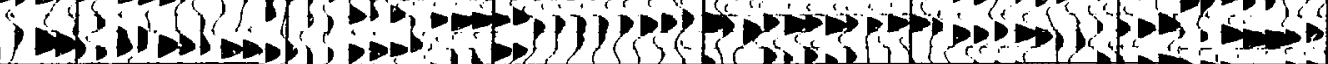

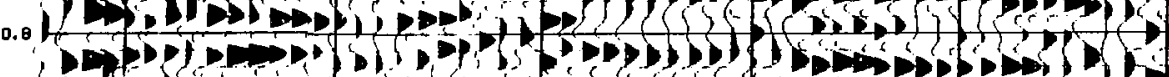

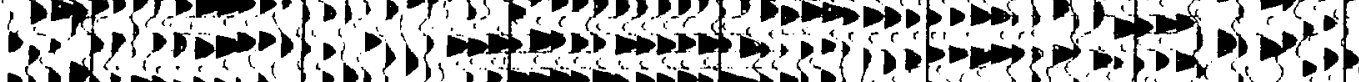

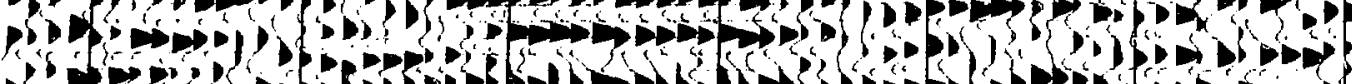

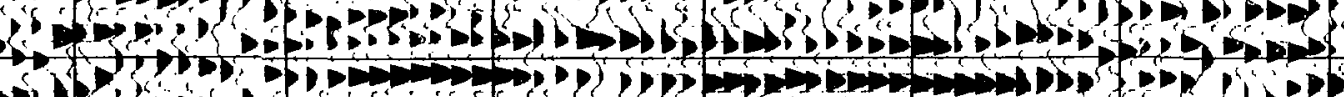

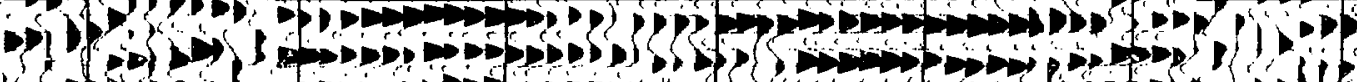

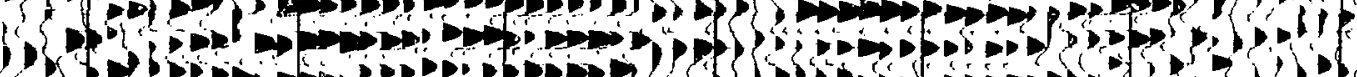

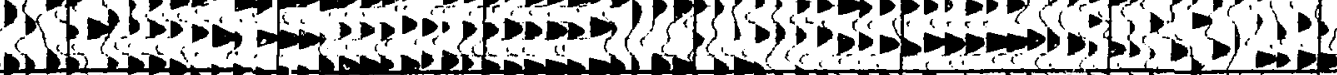

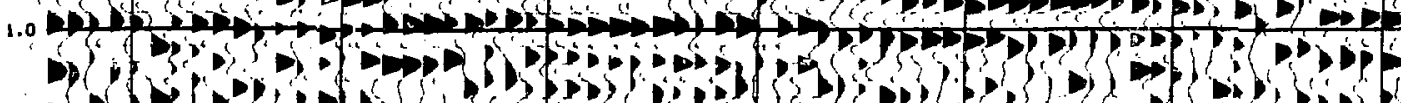

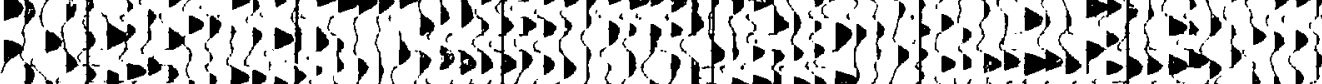

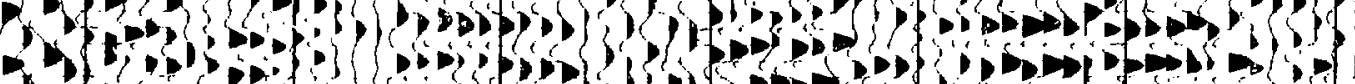

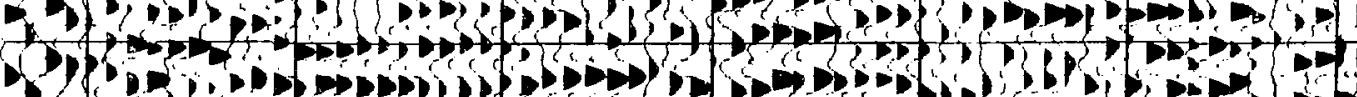

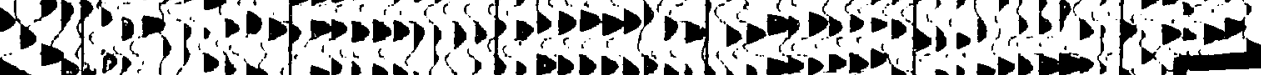

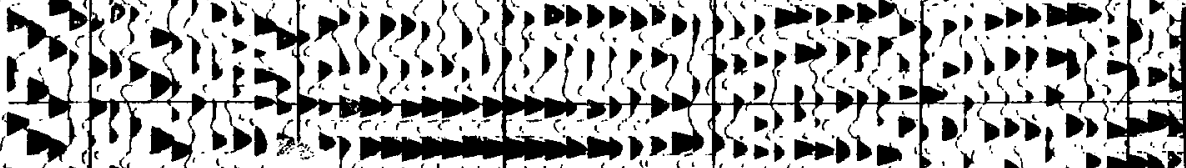
f 


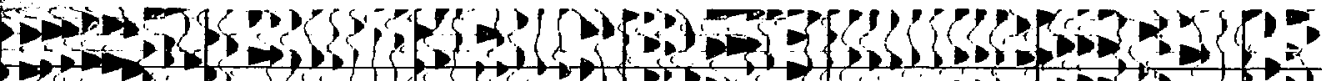

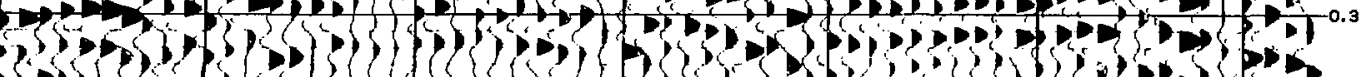

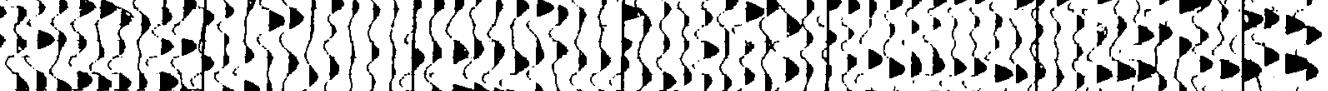

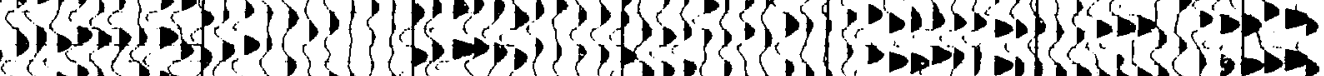

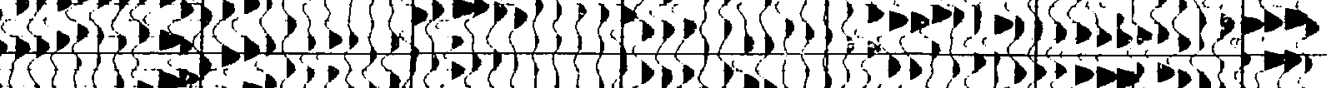
3)

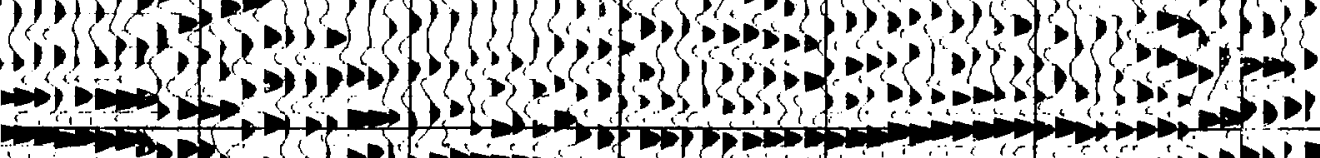
bs

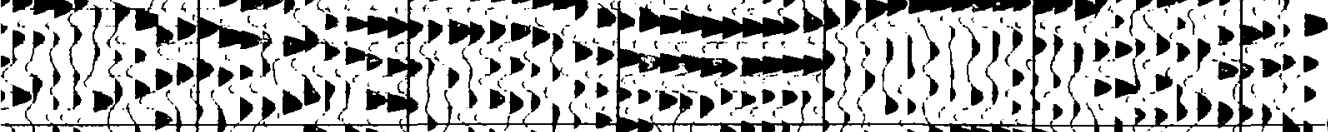

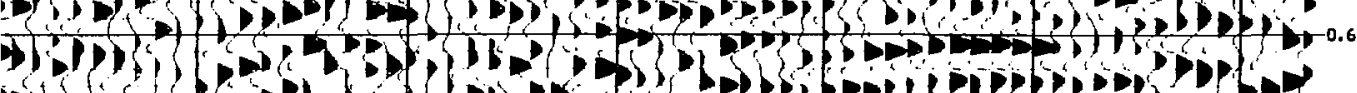

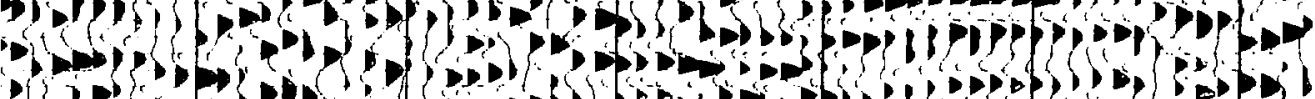

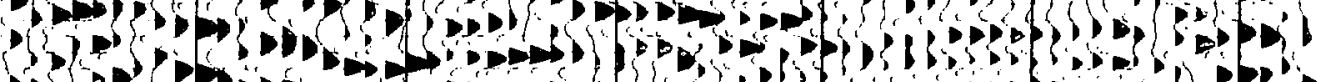

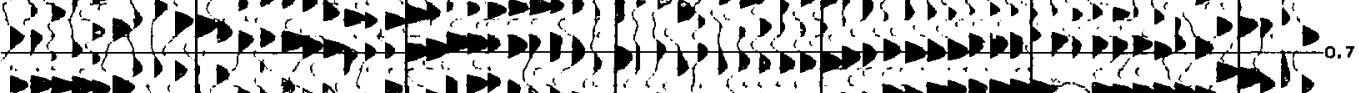

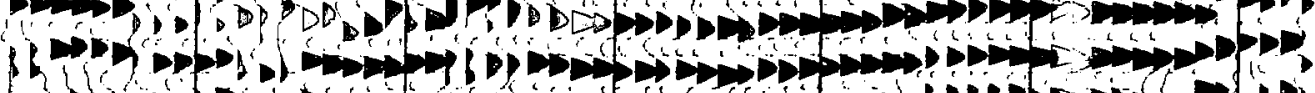

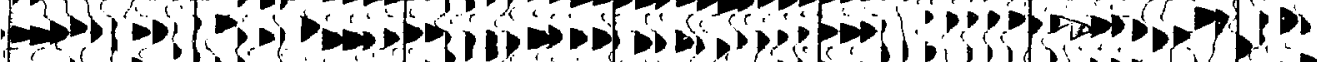
(1)

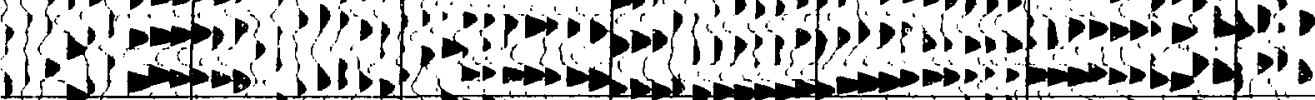

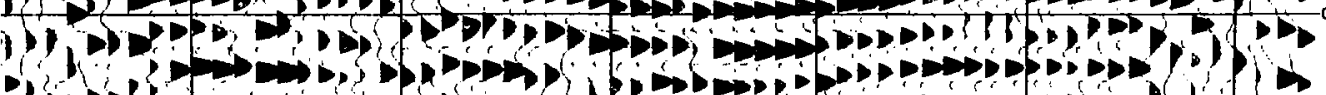

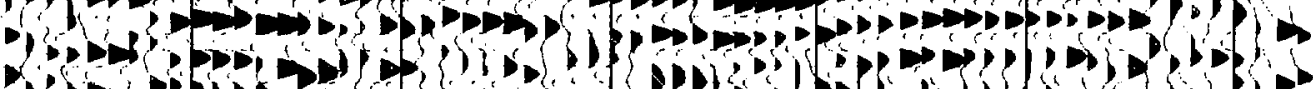

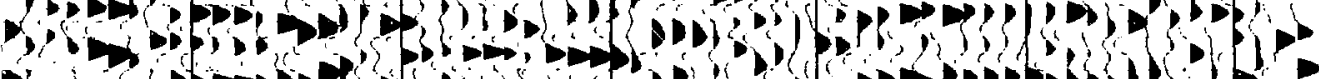
$\rightarrow$ of (5)

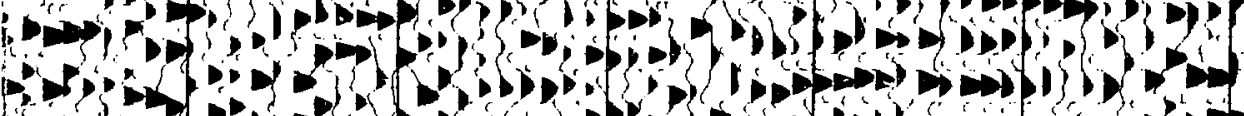

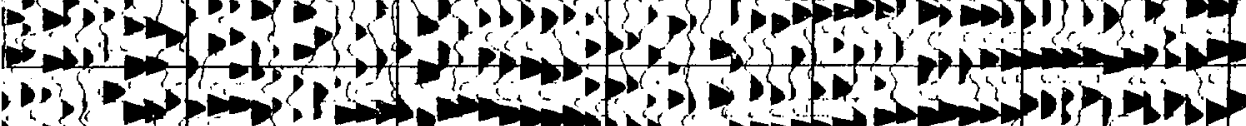




\section{i}

3 i) $\lim _{\lim }$

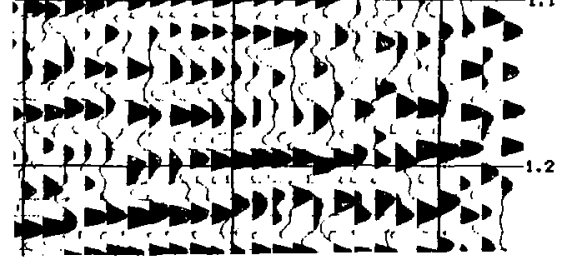

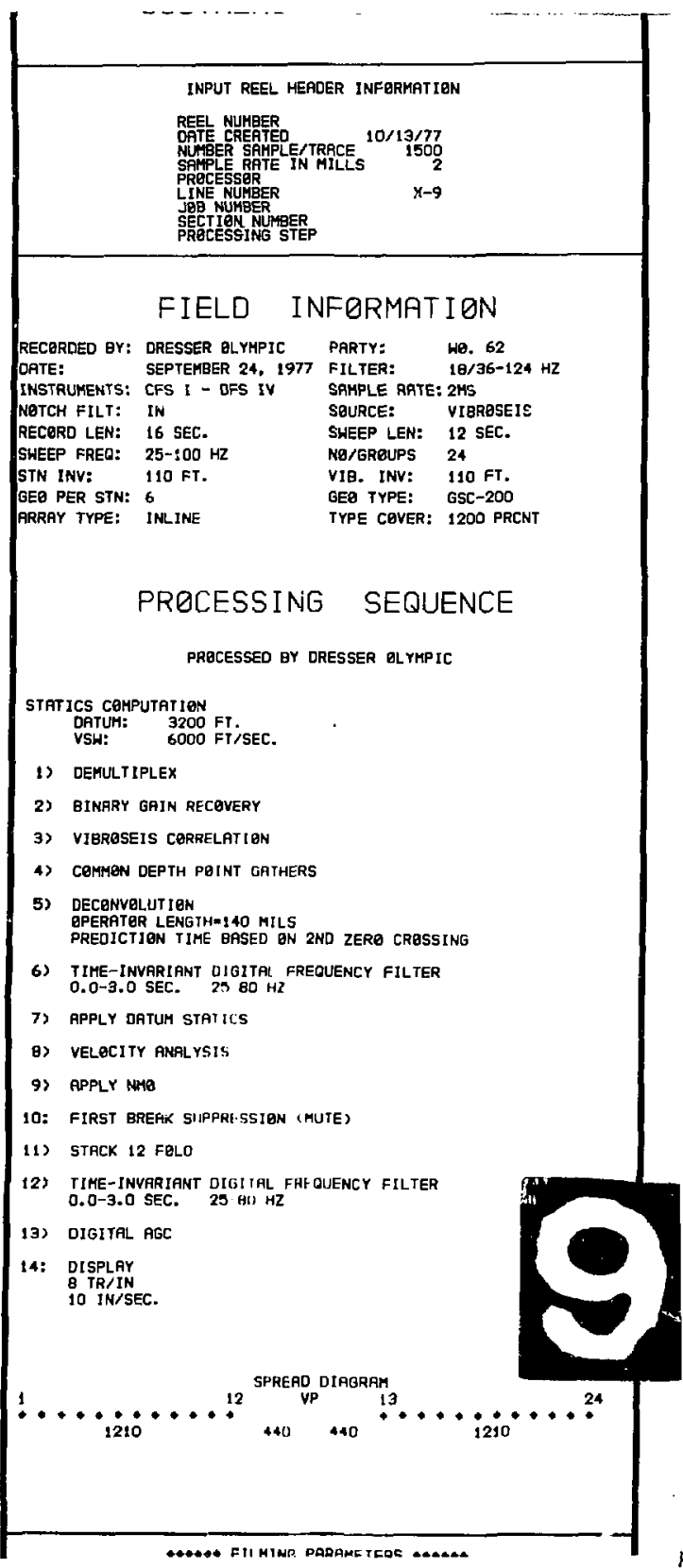




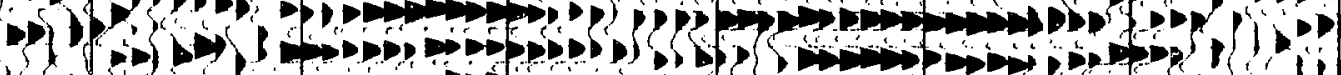

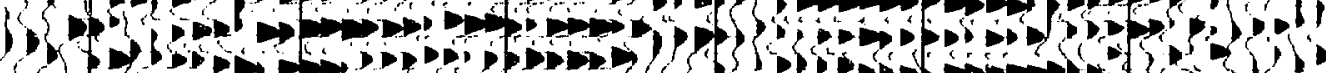

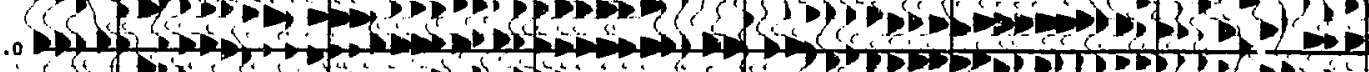
D) (1)

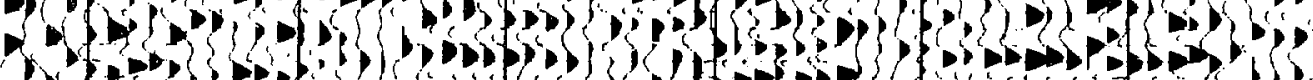

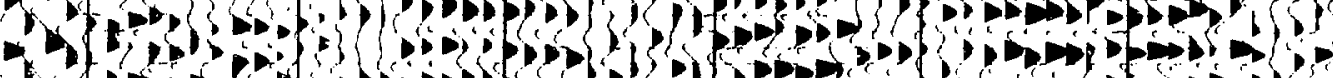
1.100 (2) 56 s.

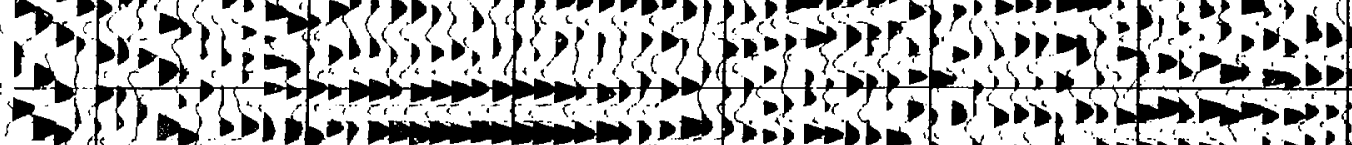
13 (1)

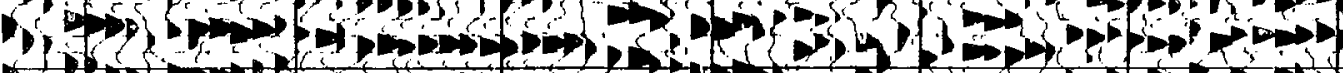
1.3 (2)

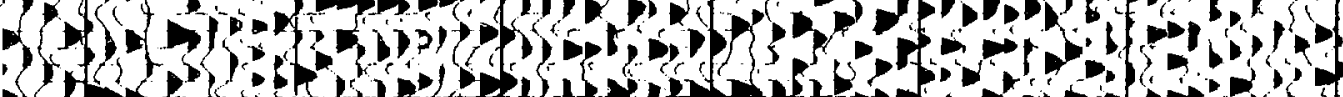

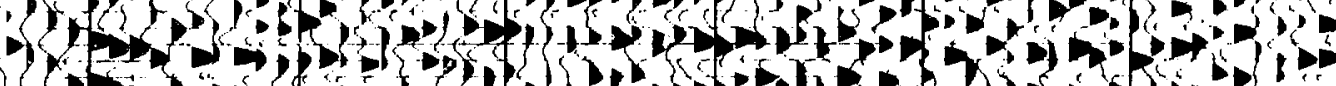

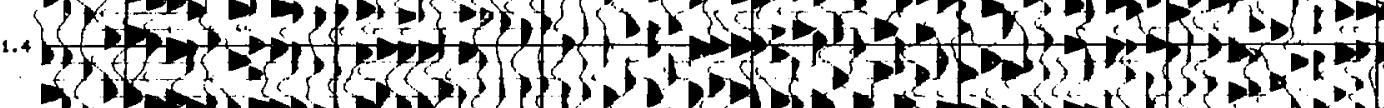

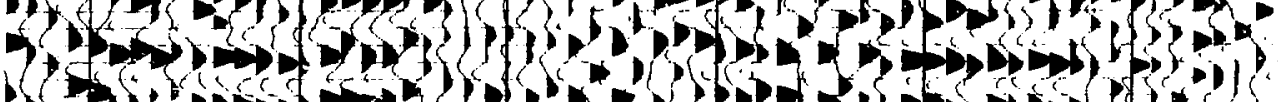

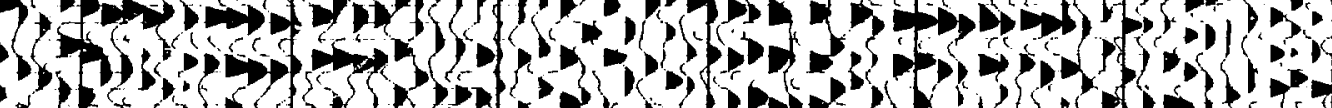

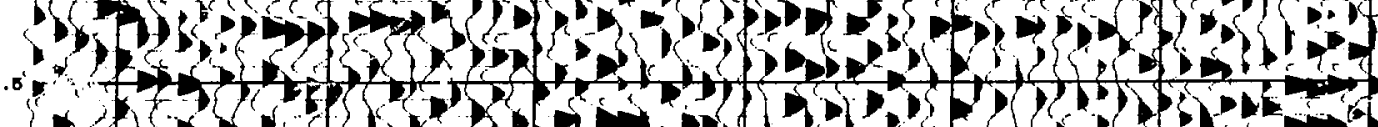

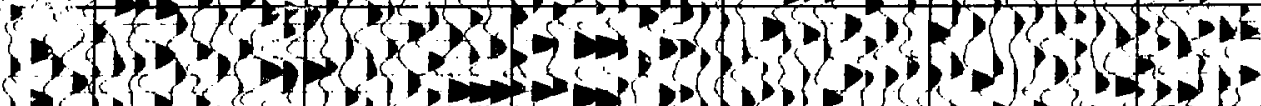

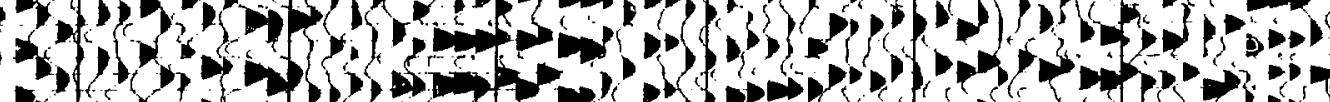

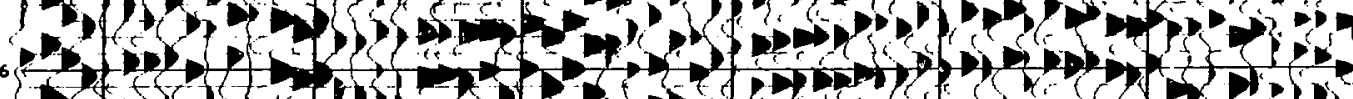

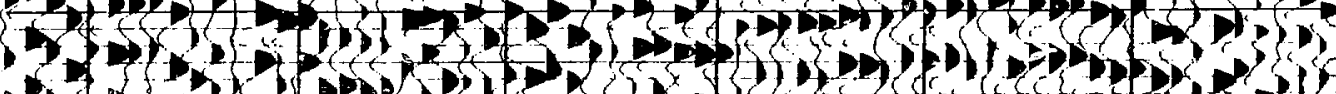

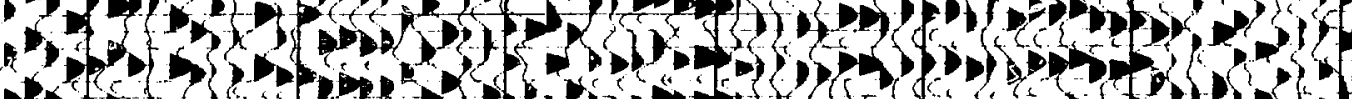

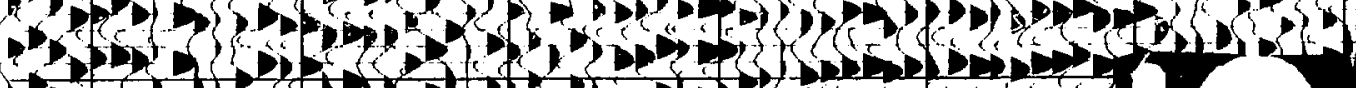

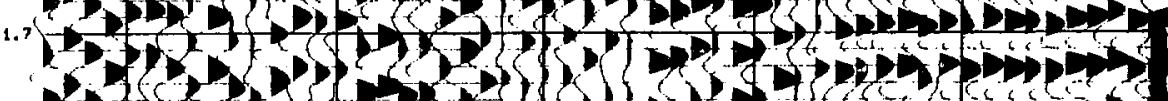
as

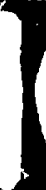




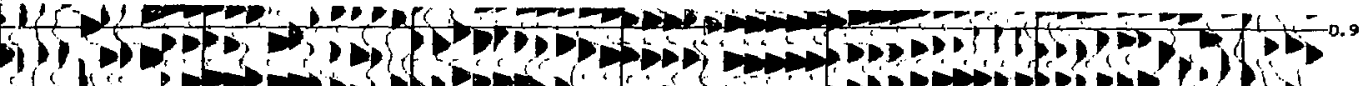

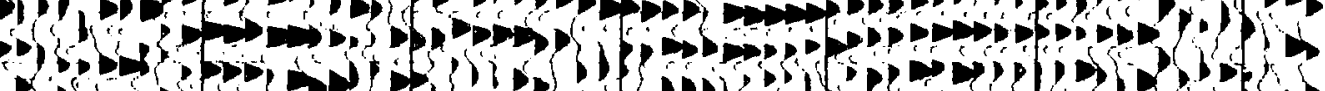

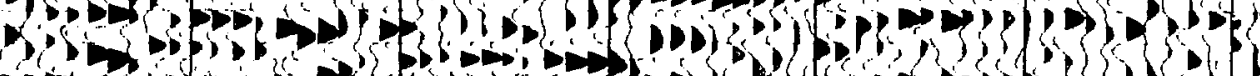

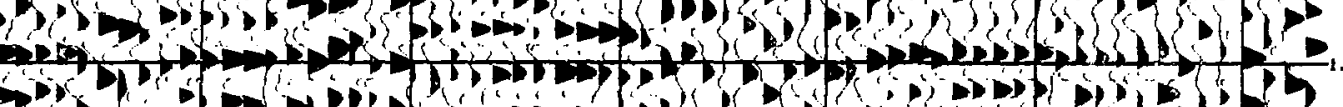
$\rightarrow$ a

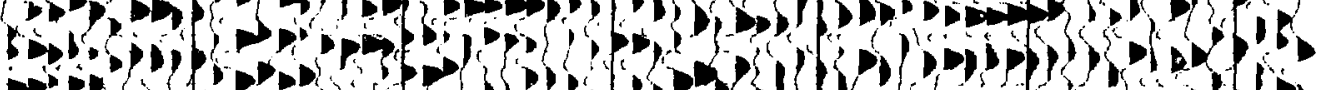

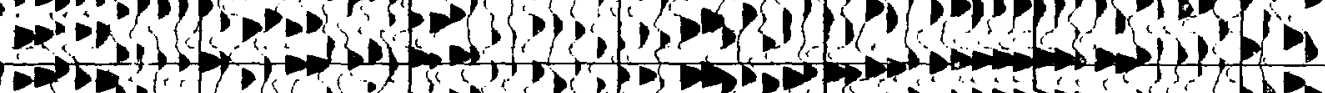

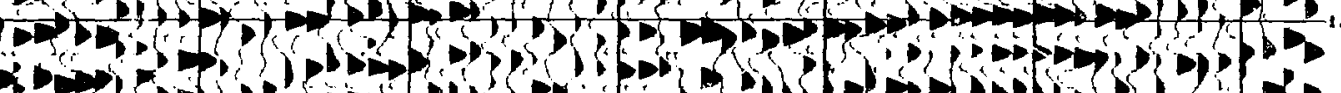
k Lap

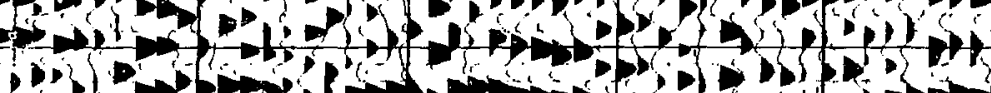

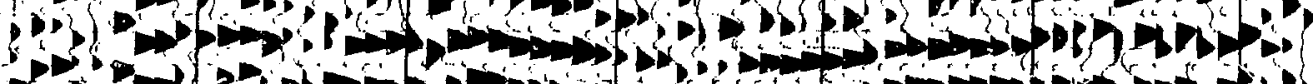

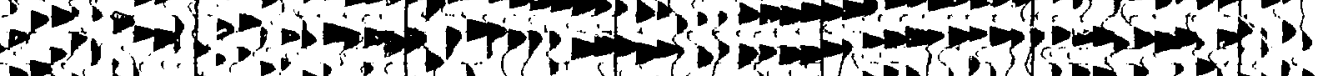

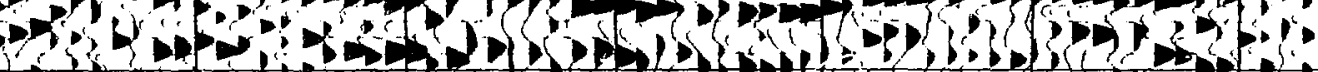

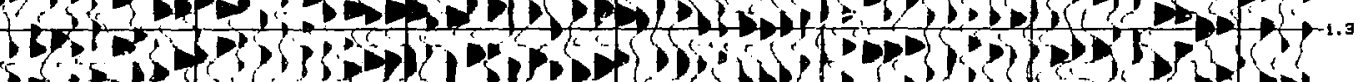

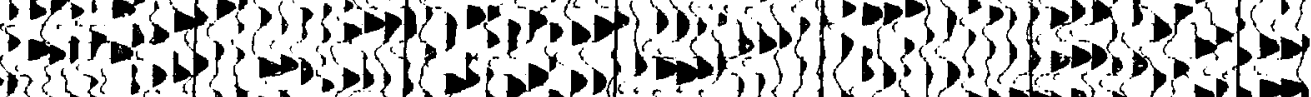

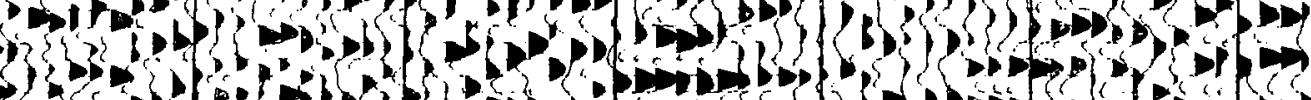

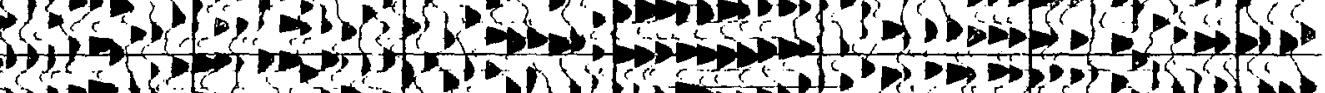

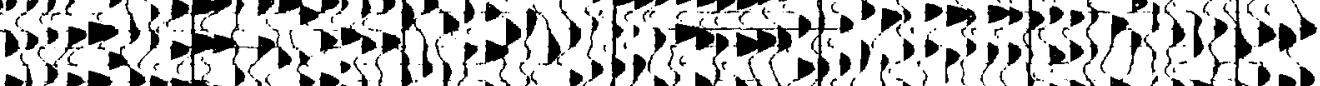

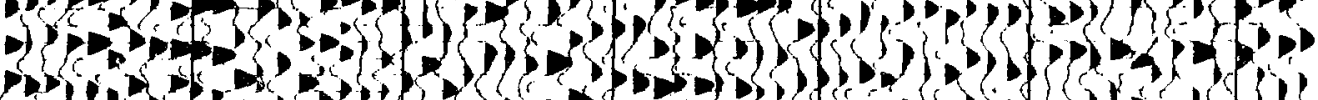

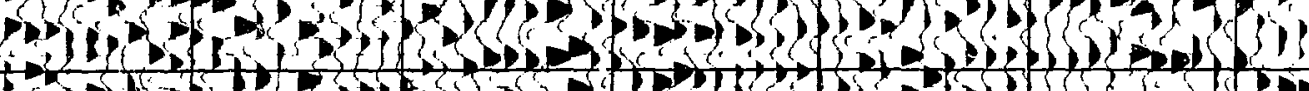
(5)

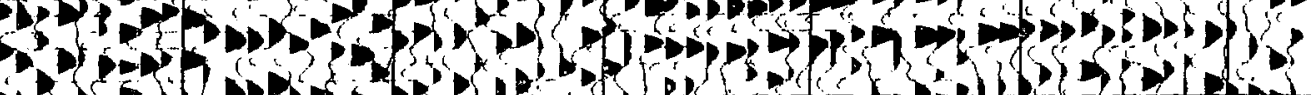
(2)

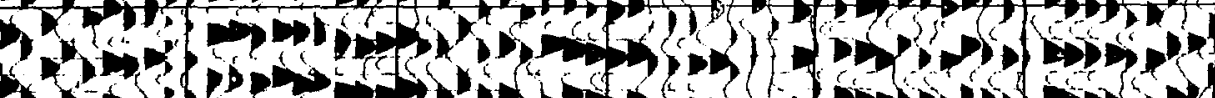

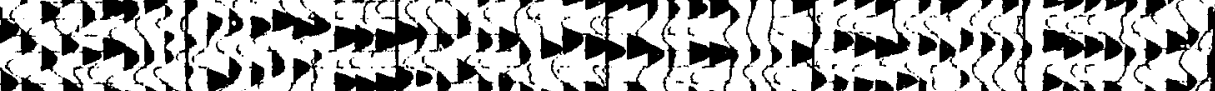

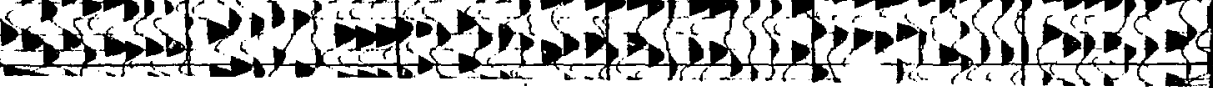




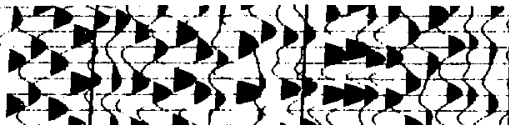

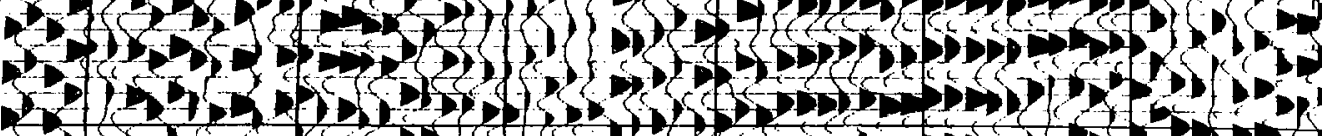

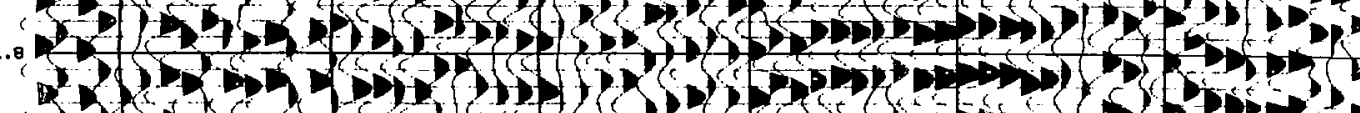

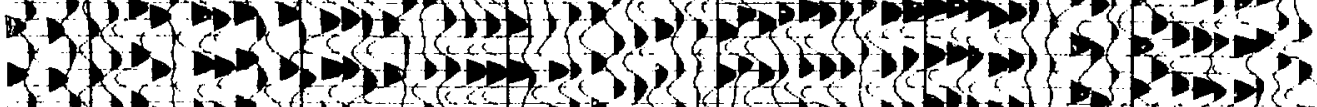

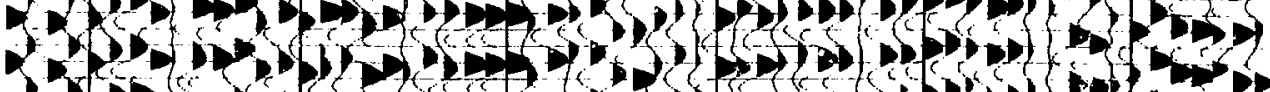

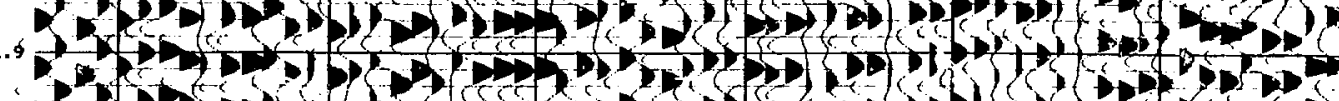

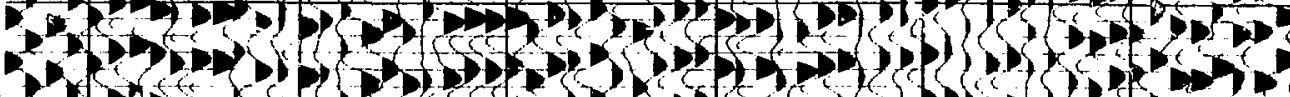

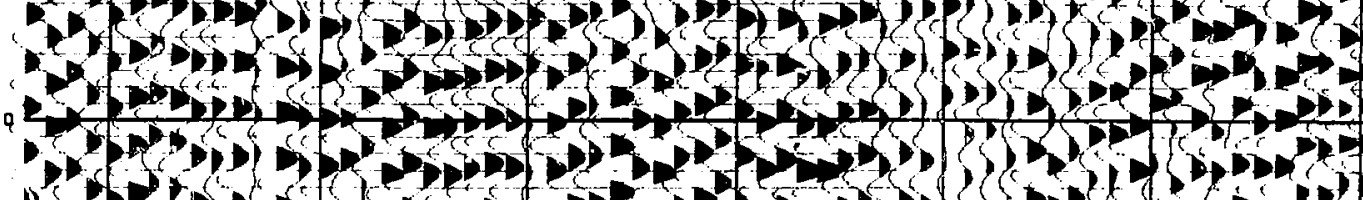

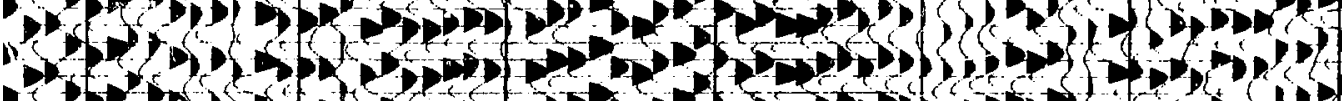

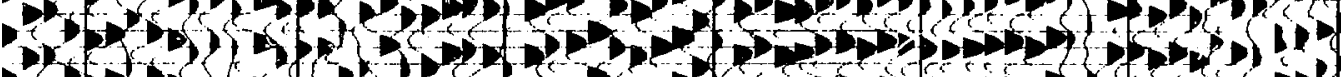

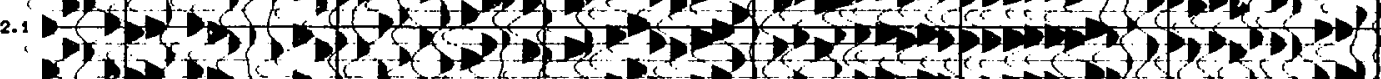

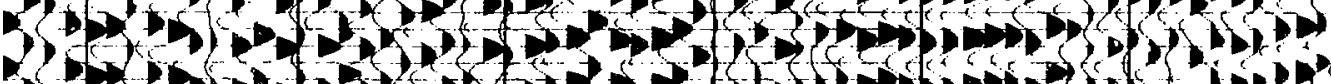

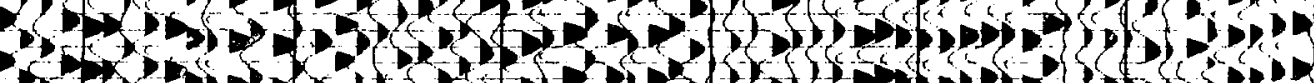

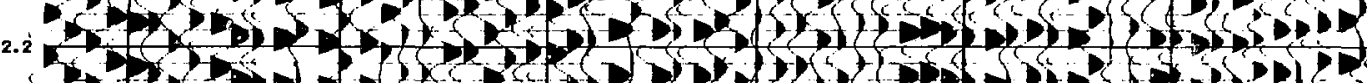

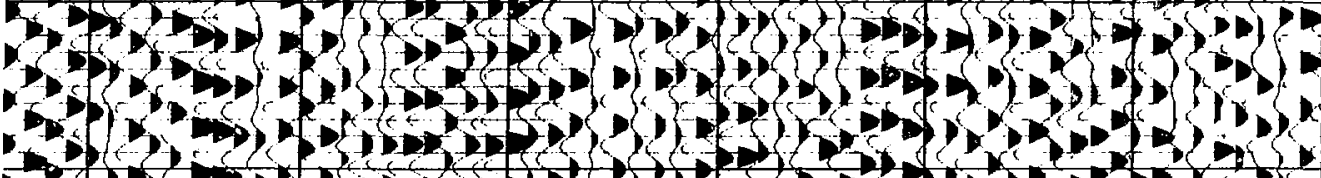

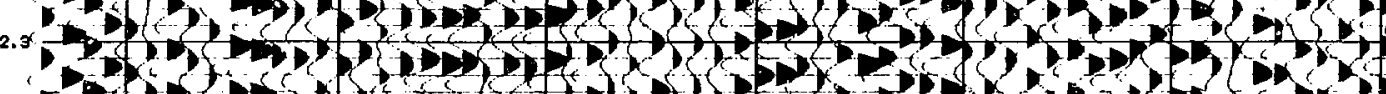

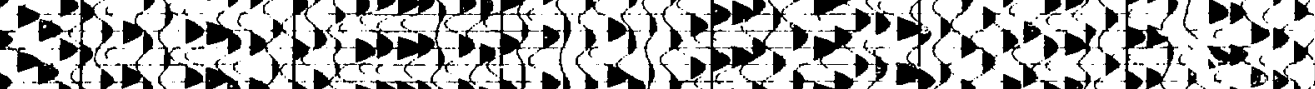

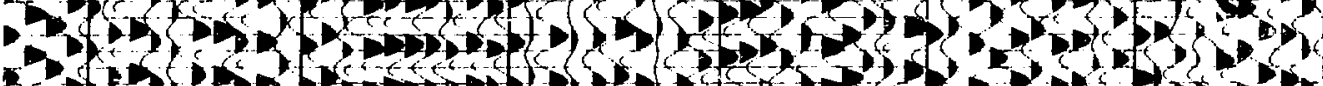

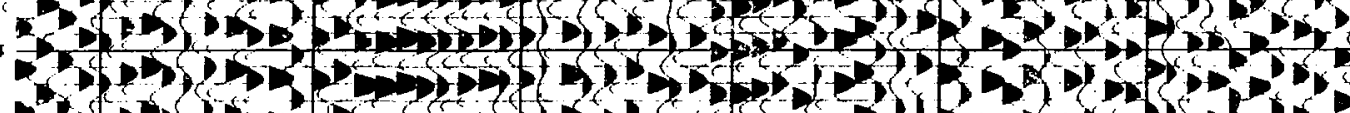

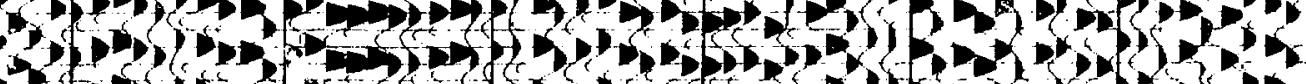

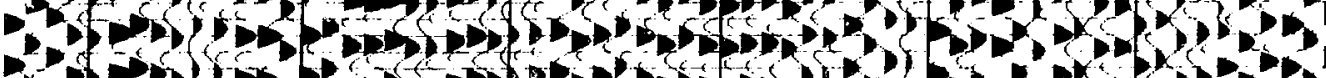

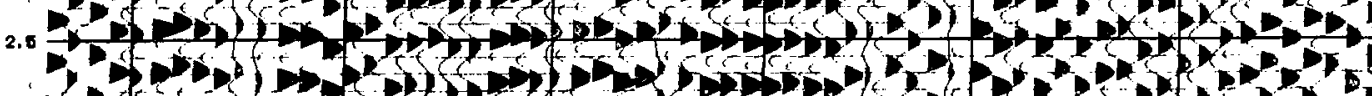

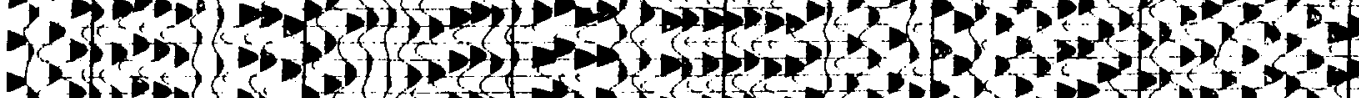

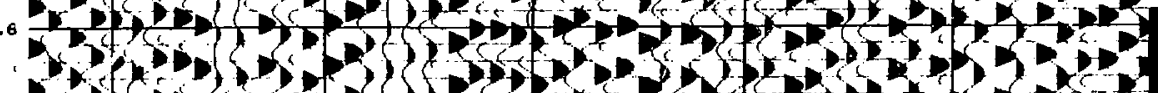

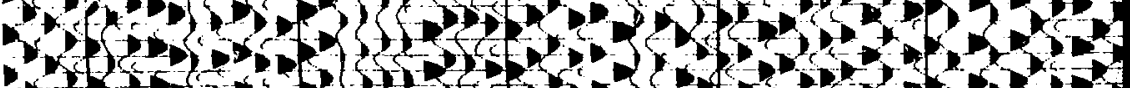

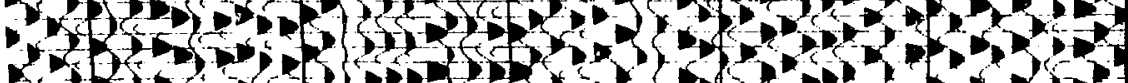

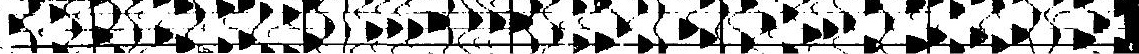




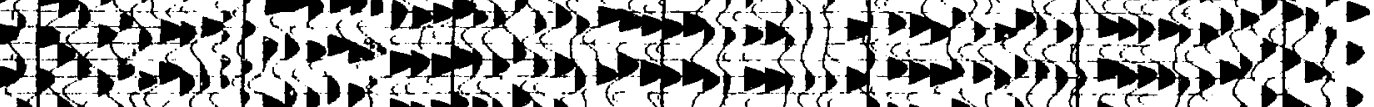

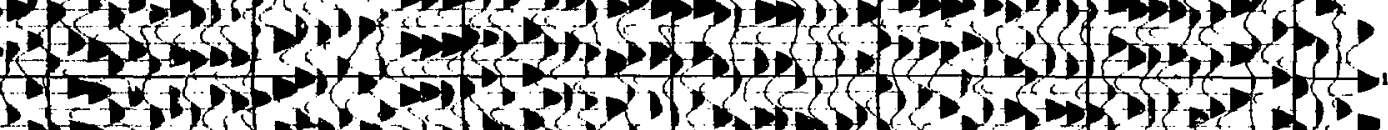

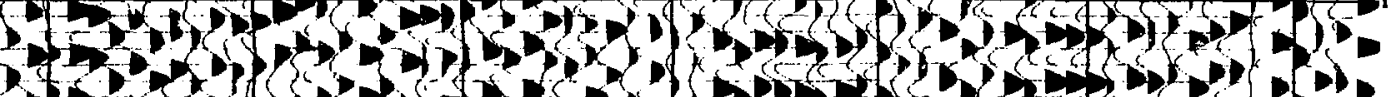

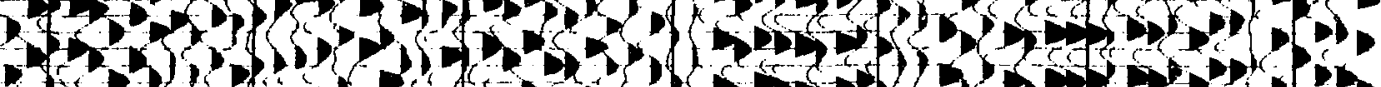

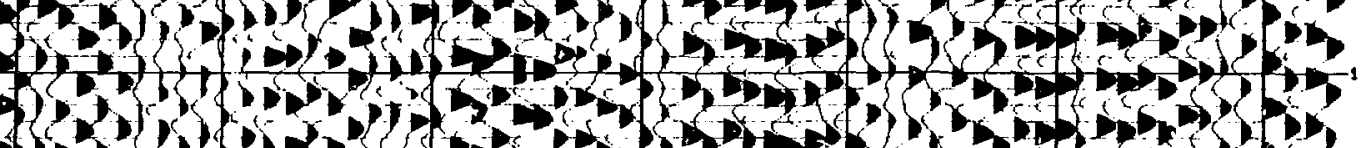

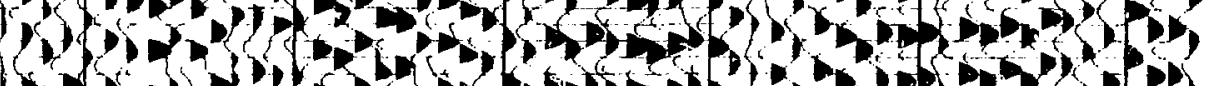

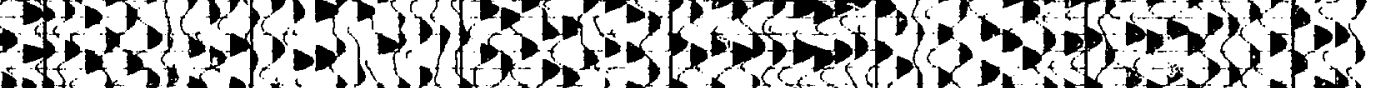
(1)

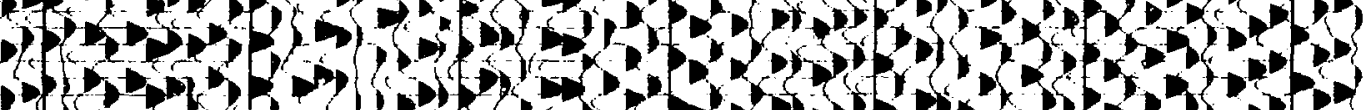

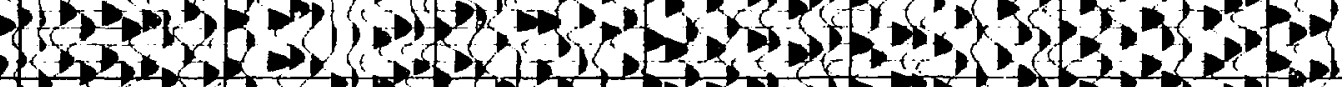

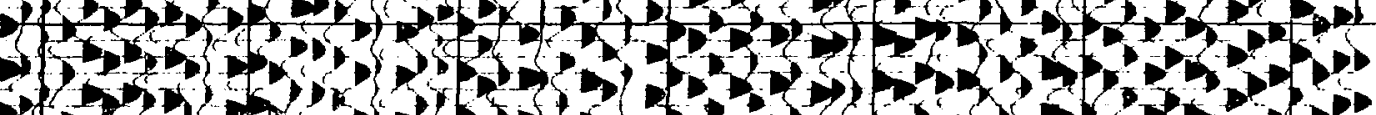

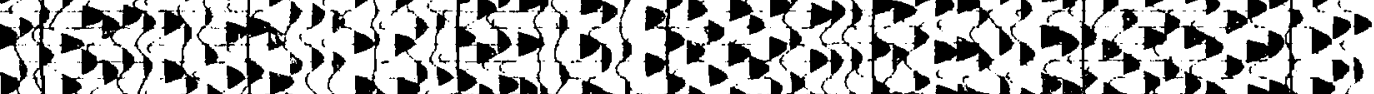

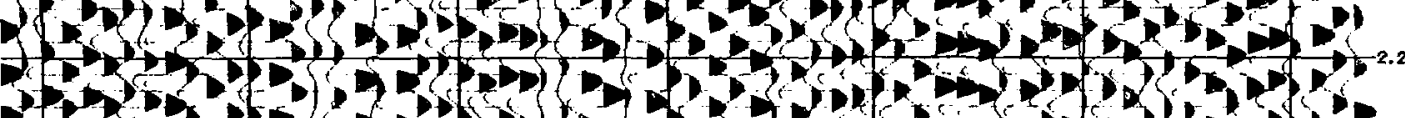

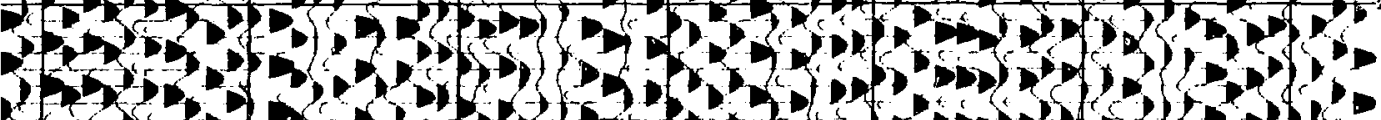

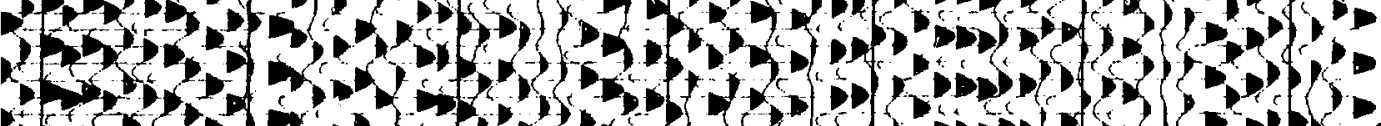
5 (1)

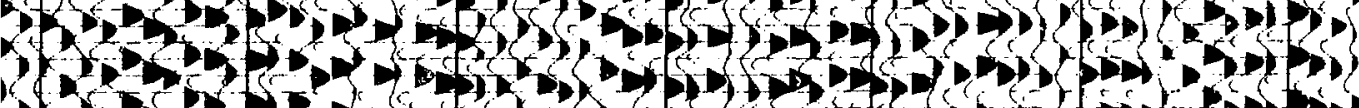

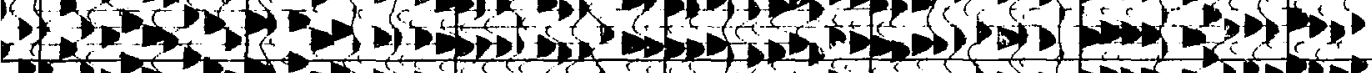

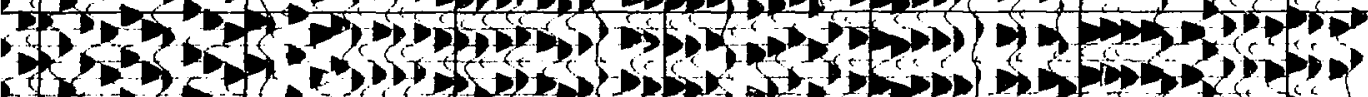

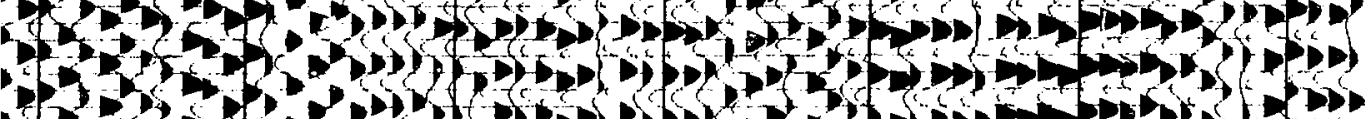

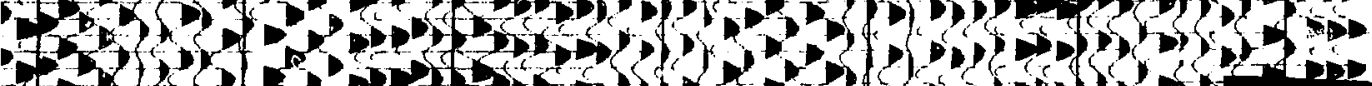

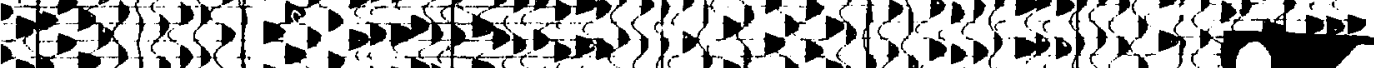

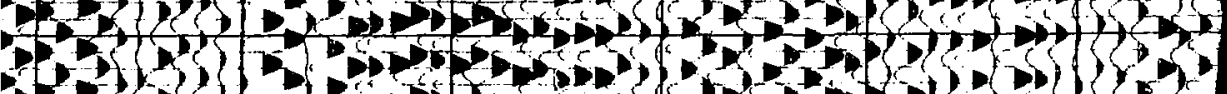

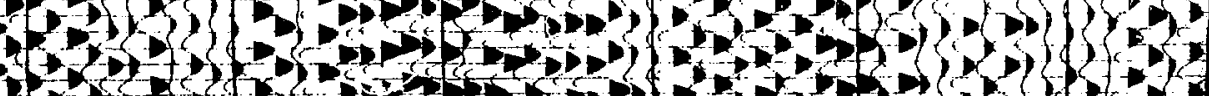
(4) 


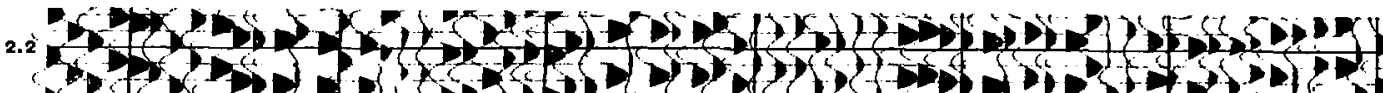

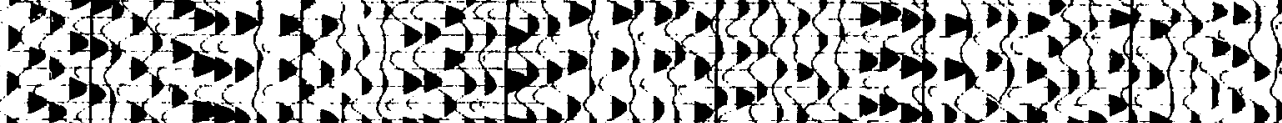

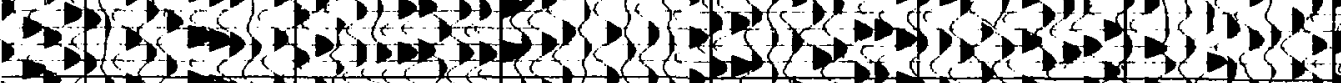

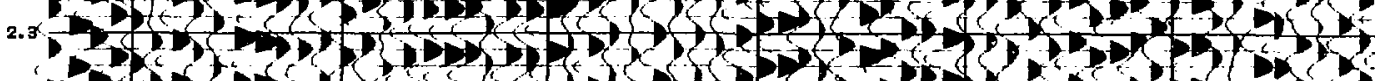

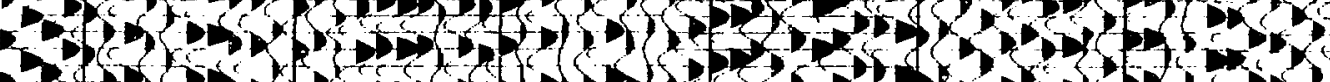

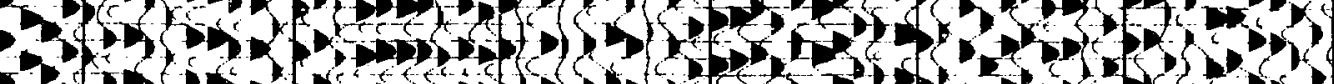

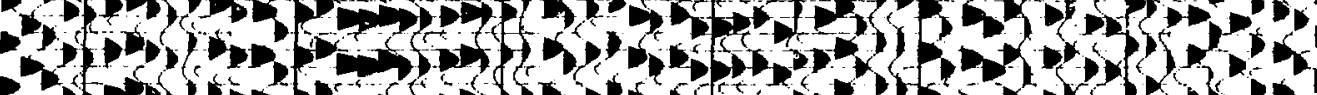

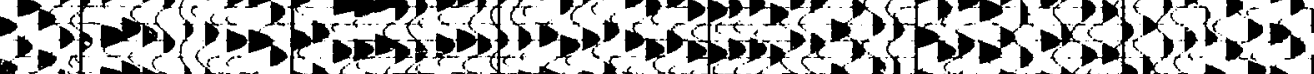

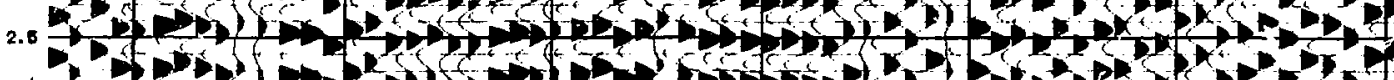

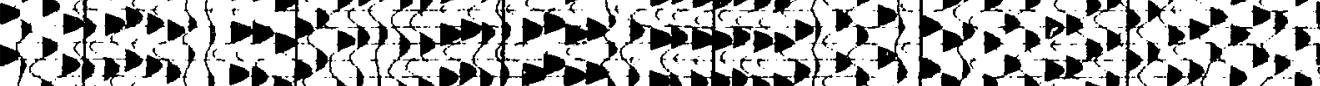

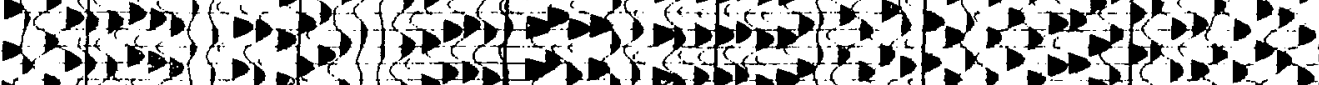

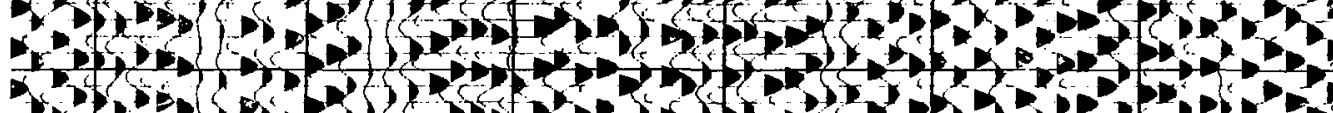
of of of

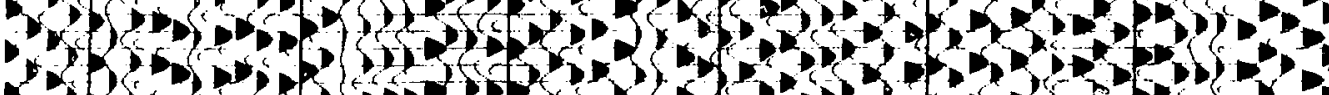

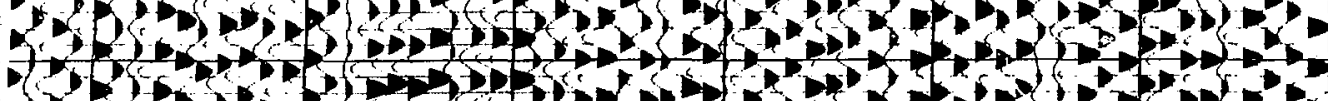

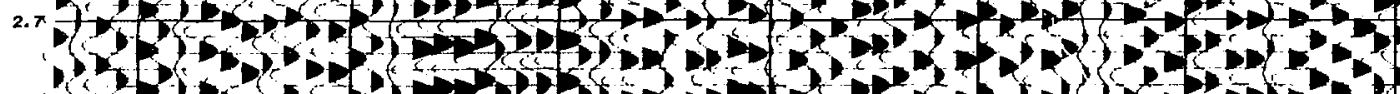
Hof

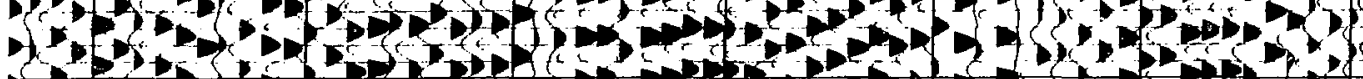
2.0

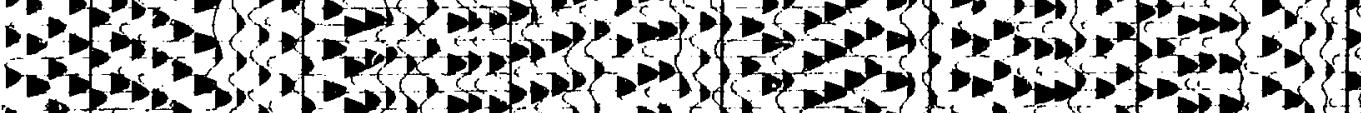

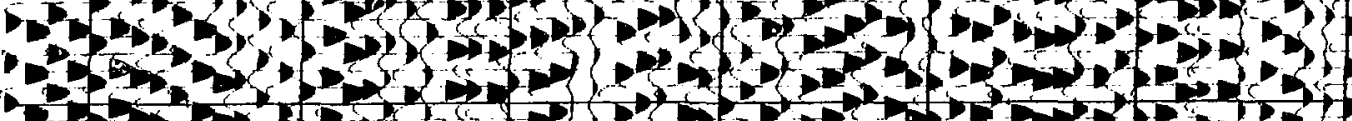

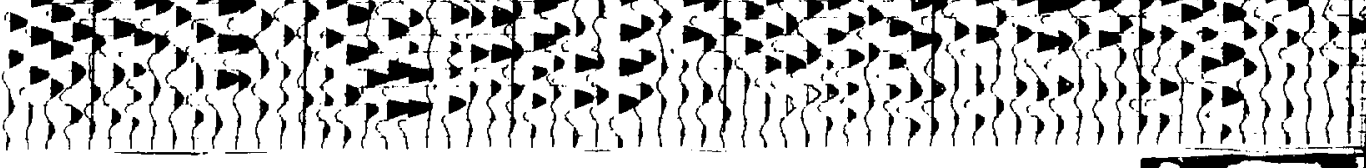




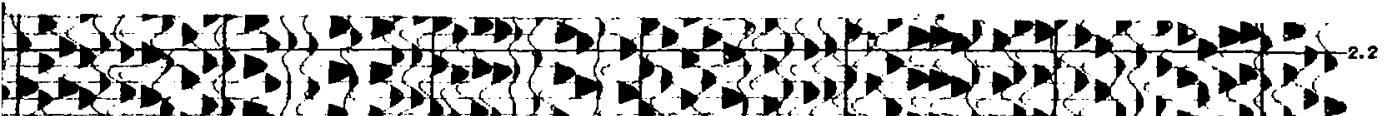

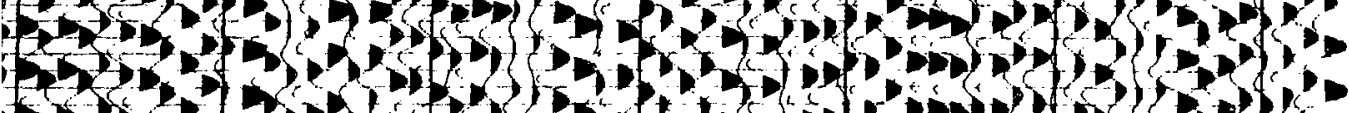

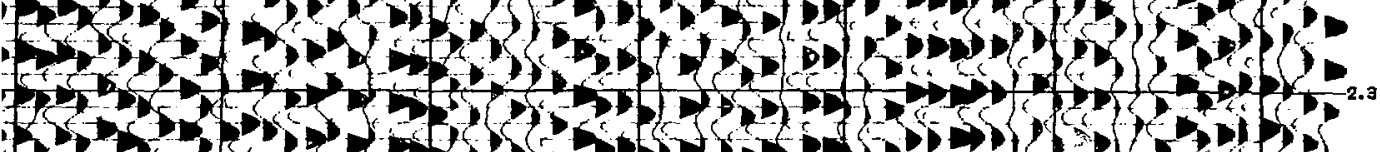

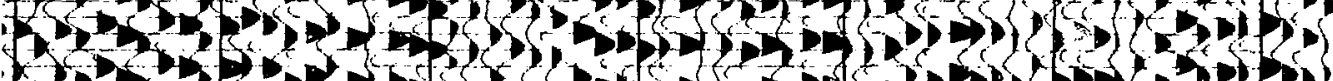

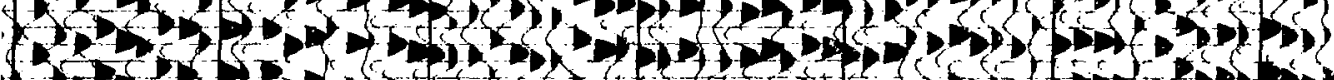

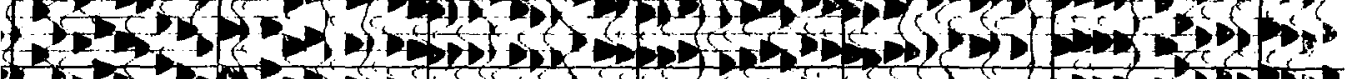

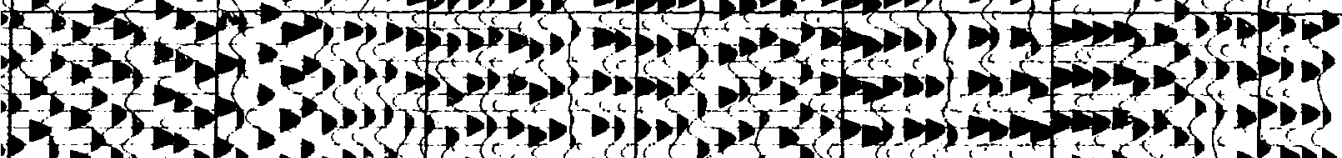

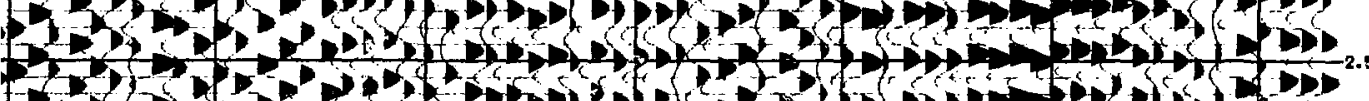

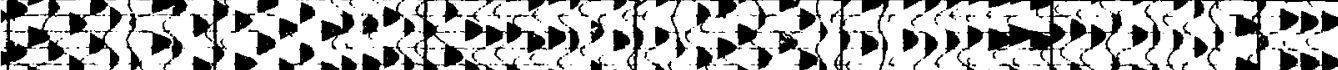

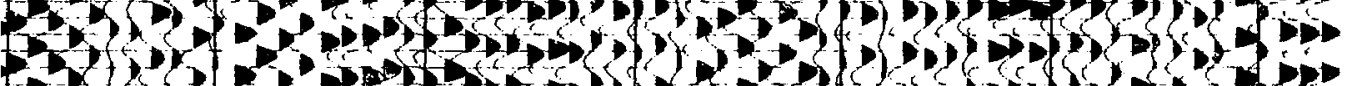

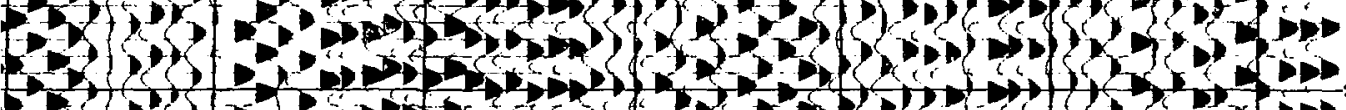

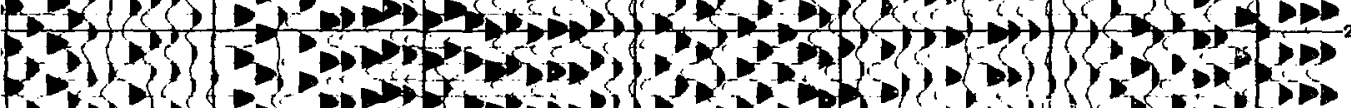

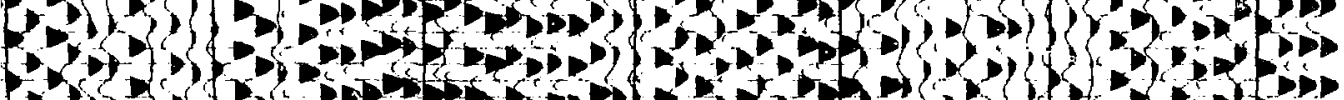

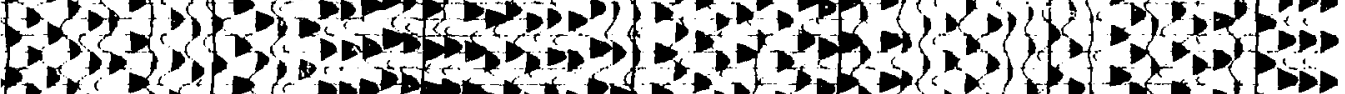

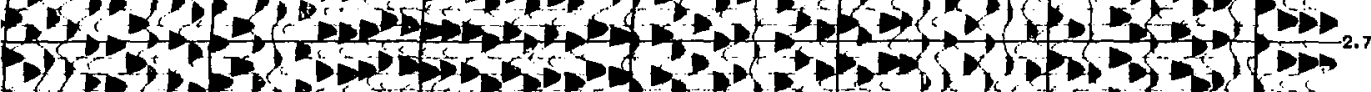

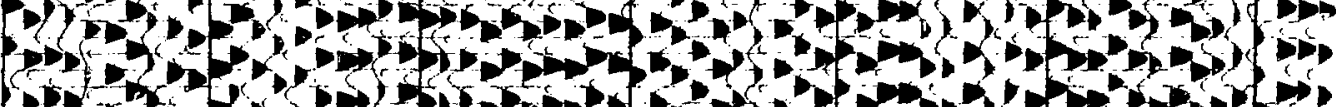

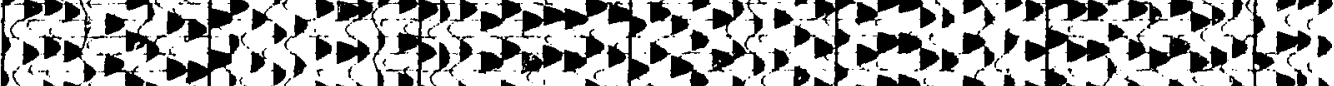

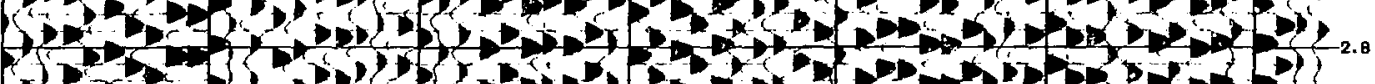

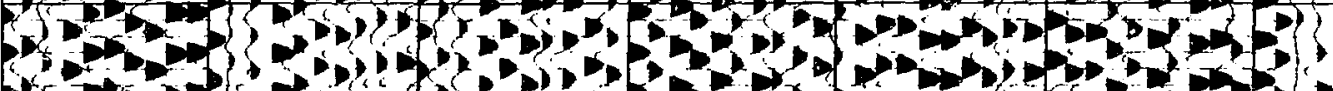

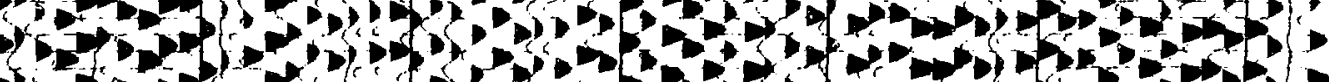

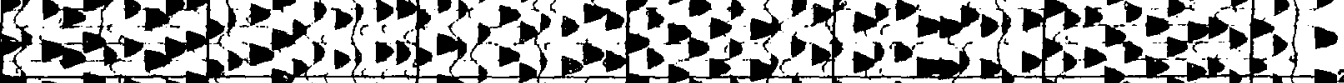

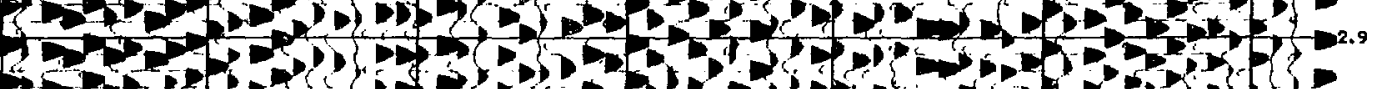

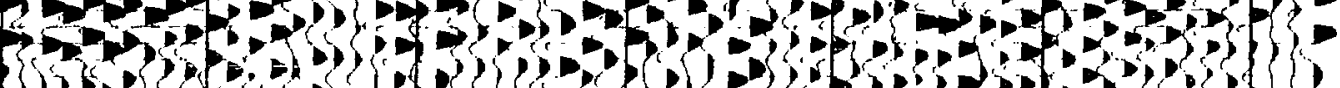

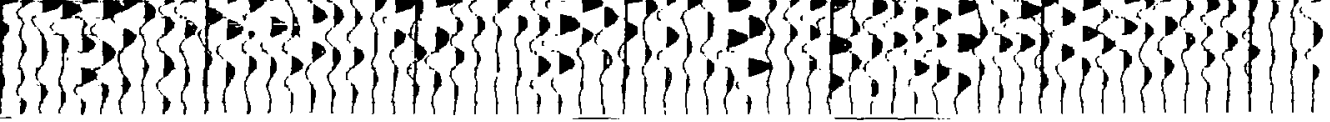




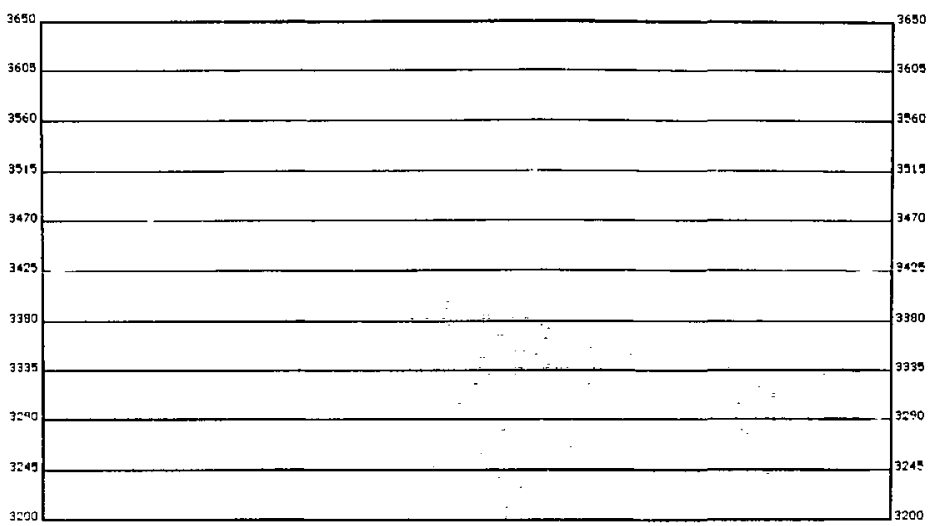

ELEVAT I ONS

STAT ICS

FOLD *

LINE DIRECIIS

VELL, FNALL

VELL, RNALLI

MERTHEASI

VELOCITY FUNO DIRECT ION

STATIONS

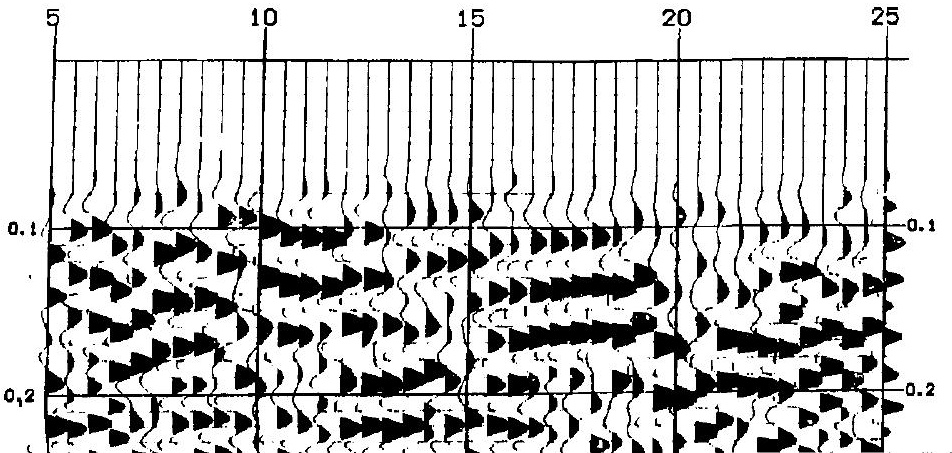




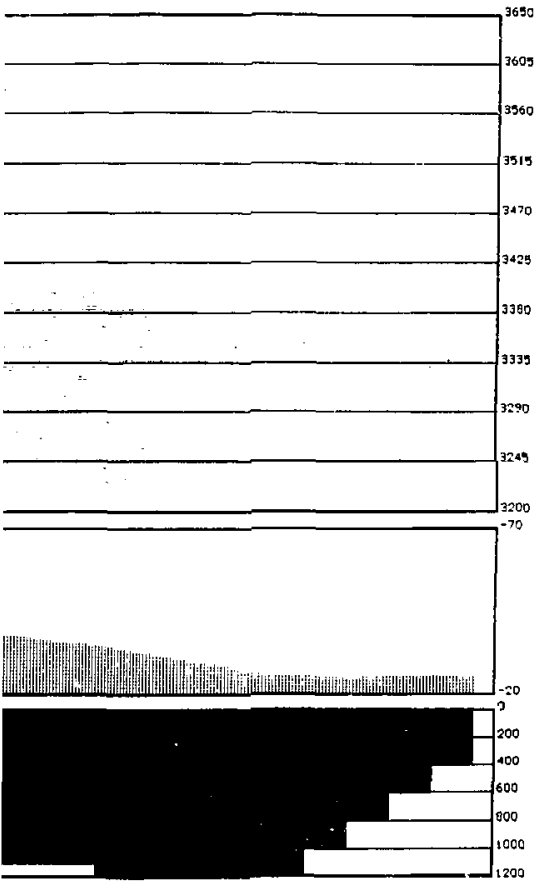

\section{ELEVAT IONS}

STATICS

FOLD *

sec 5

VEs PNA

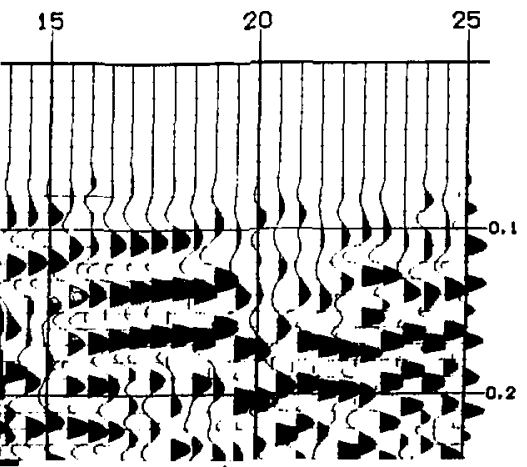

MPRIHEASI

LINE DIRECTION

VELOCITY FUNCTION DIRECTION

STATIONS
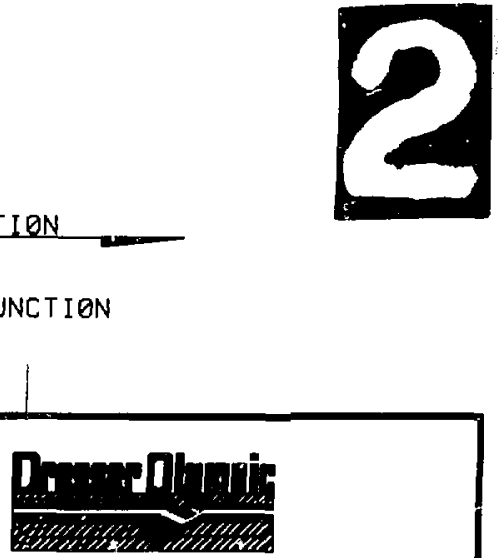

\section{LOS MEDANOS}

$$
\begin{aligned}
\text { LINI } & \times-10 \\
\text { С.TATTANS: } & 5-25
\end{aligned}
$$




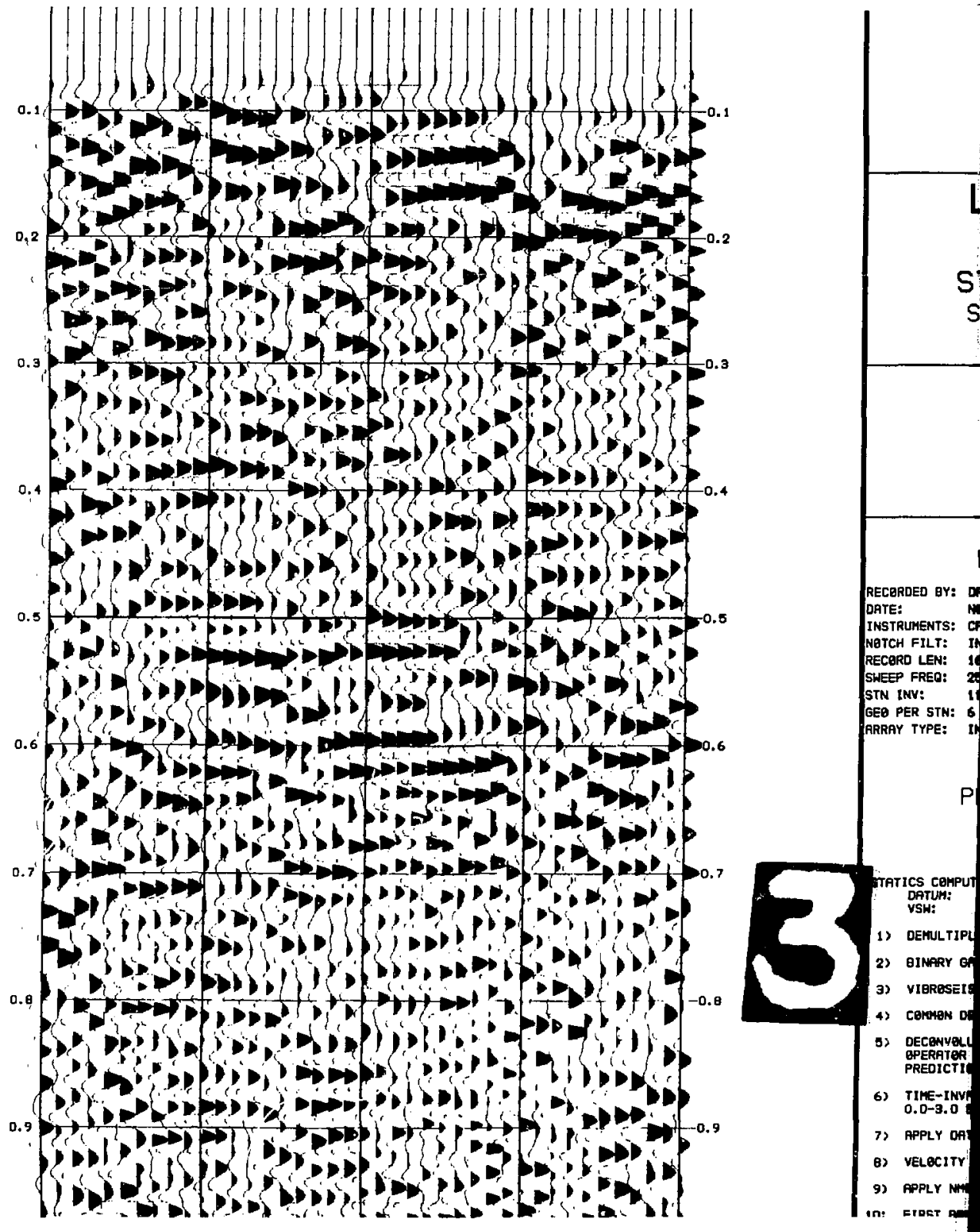


Sompopos

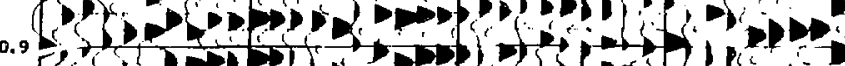

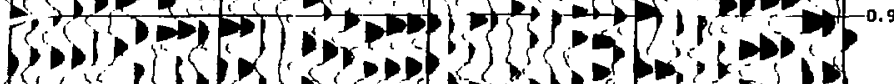

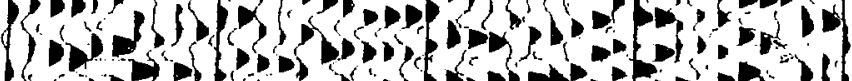

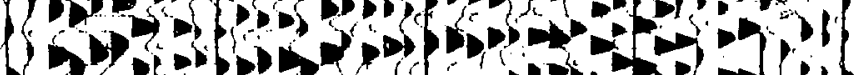
1.8

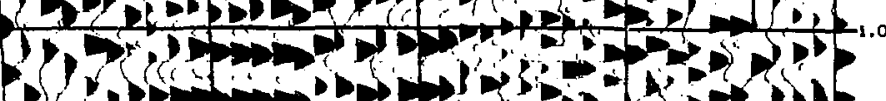

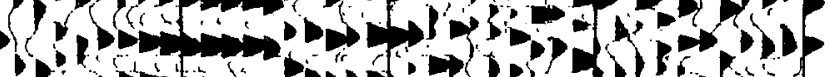
(1) 1.1

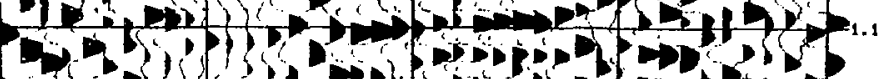

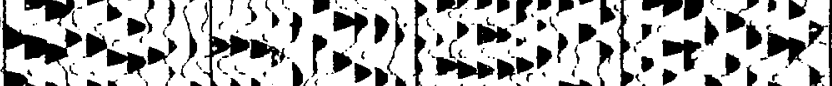

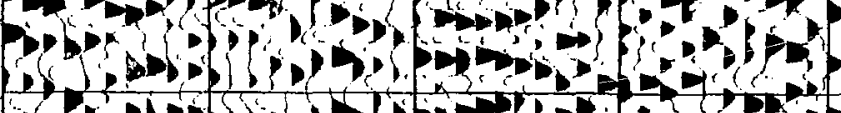

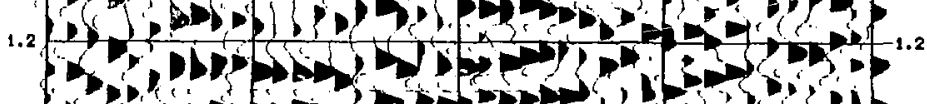

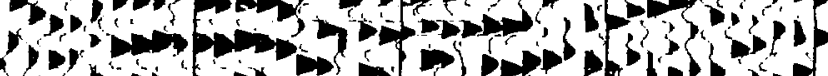

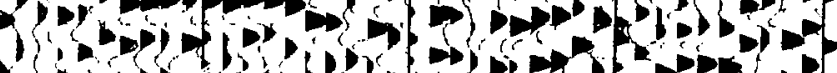

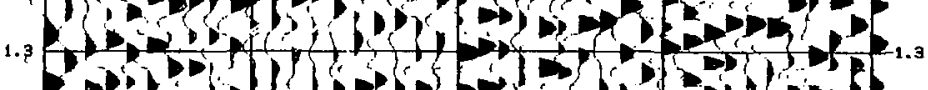

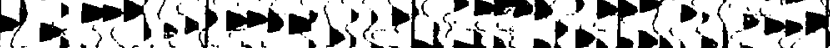

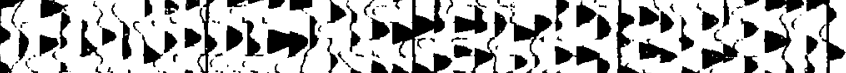

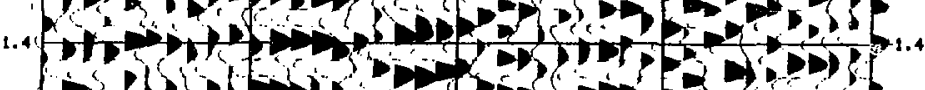

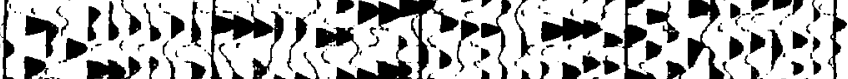

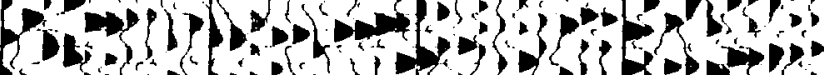
1.5 5 (s)

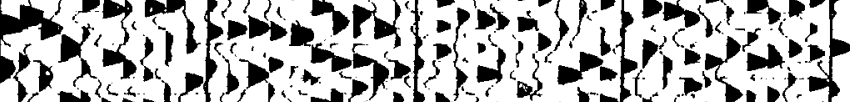

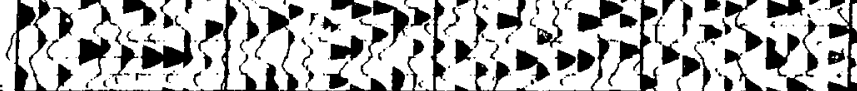

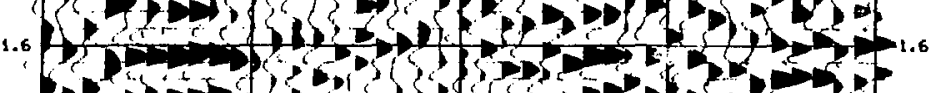

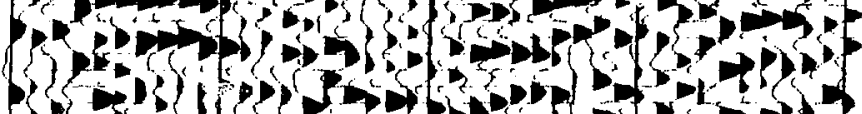

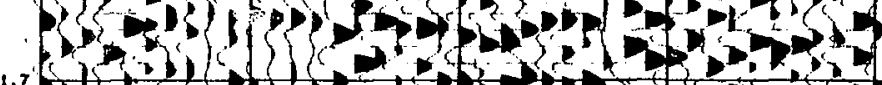

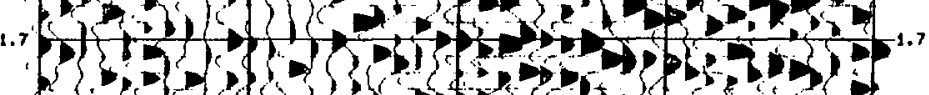

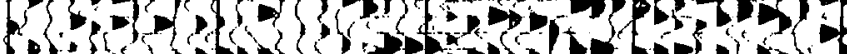

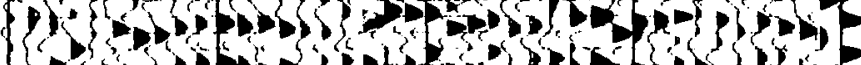

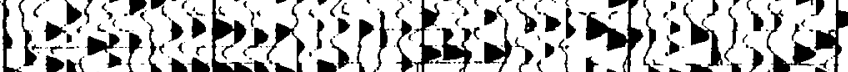

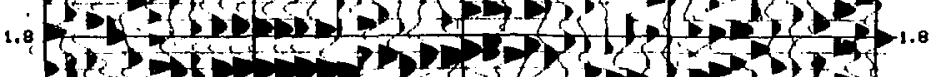

P.

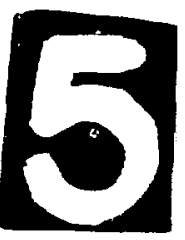




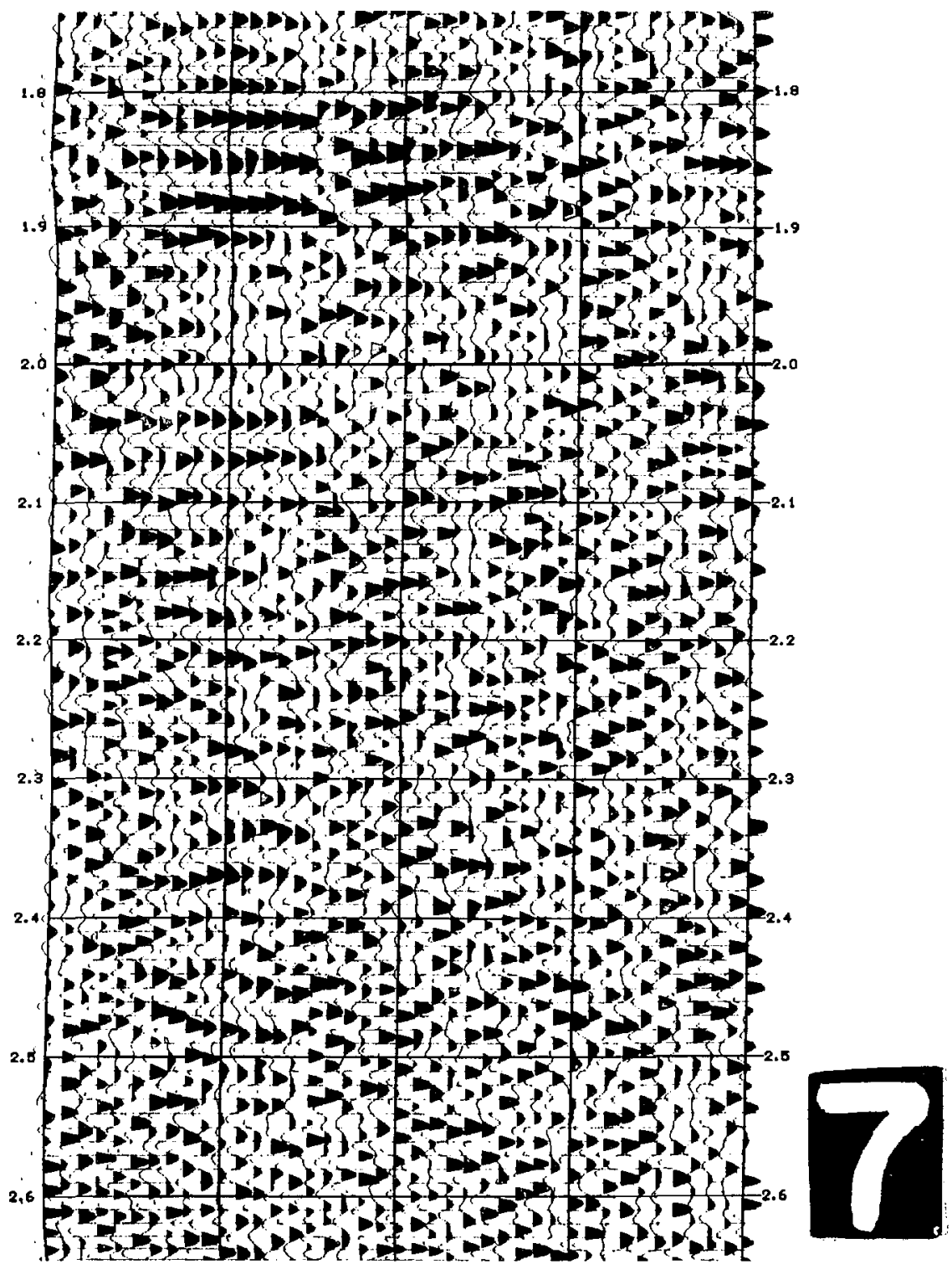


o

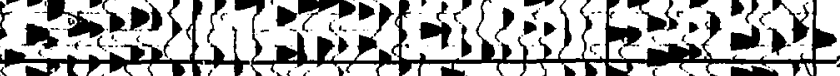

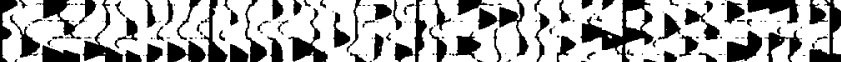

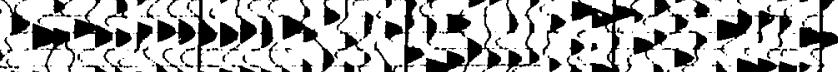

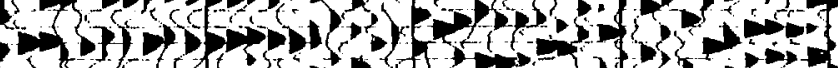

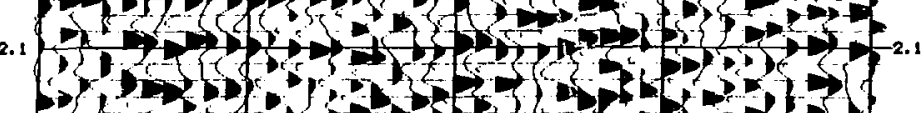

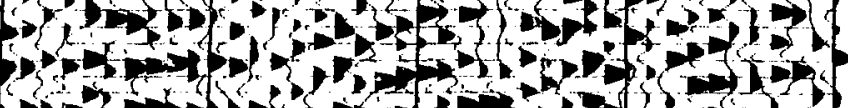

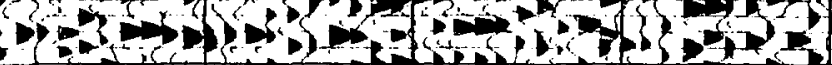

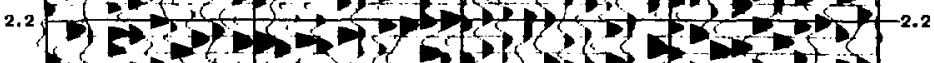

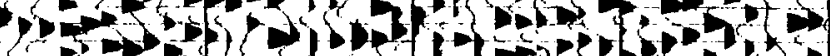

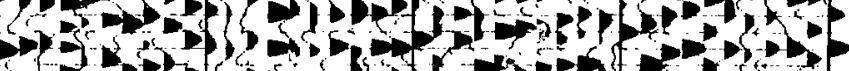

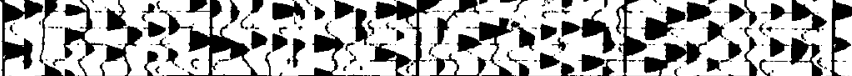

2.3

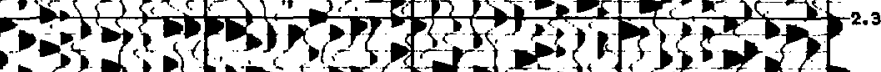

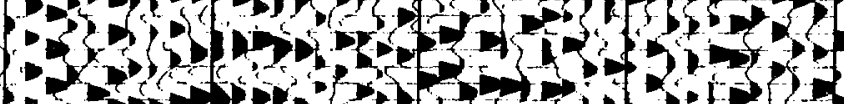

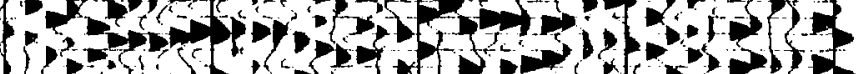

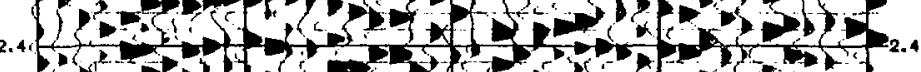

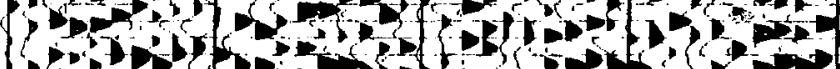

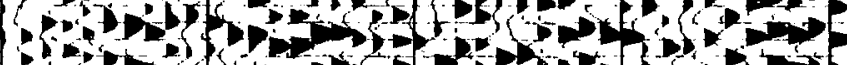

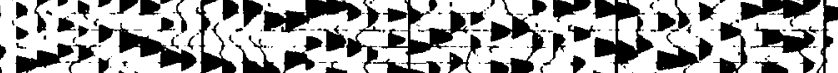

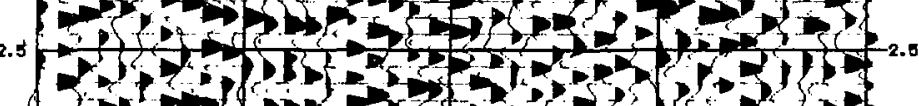

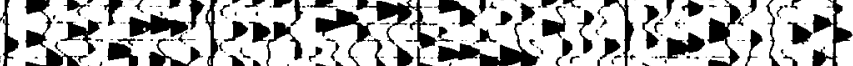

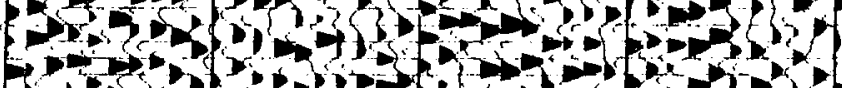

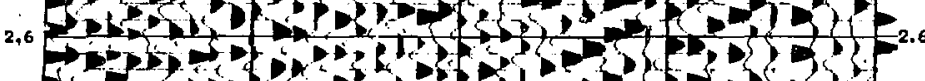

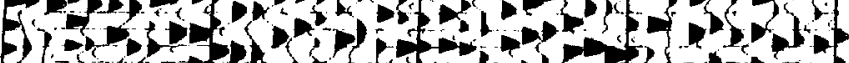

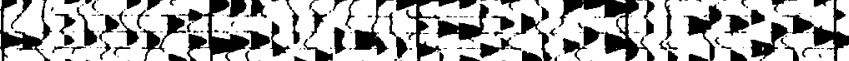

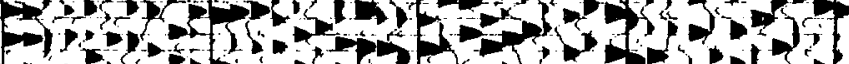

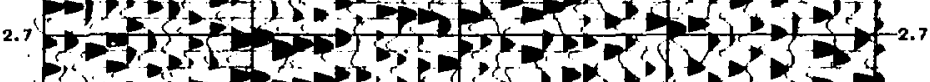

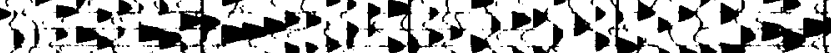

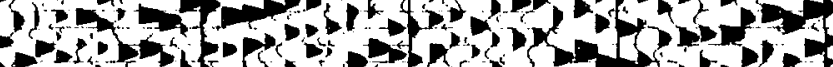

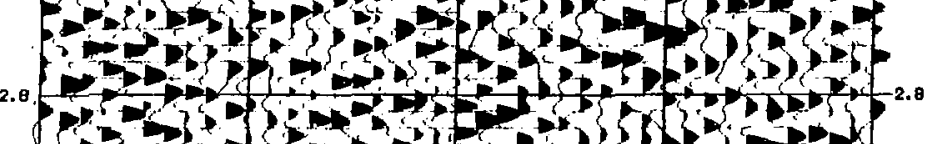

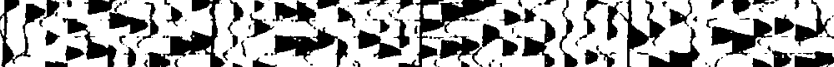

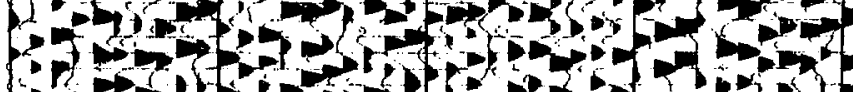

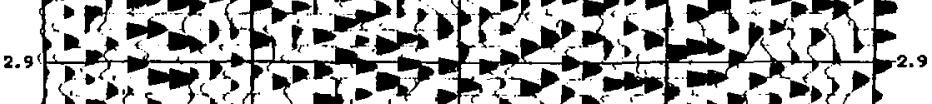

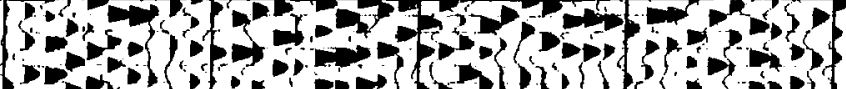

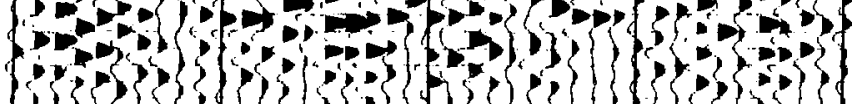




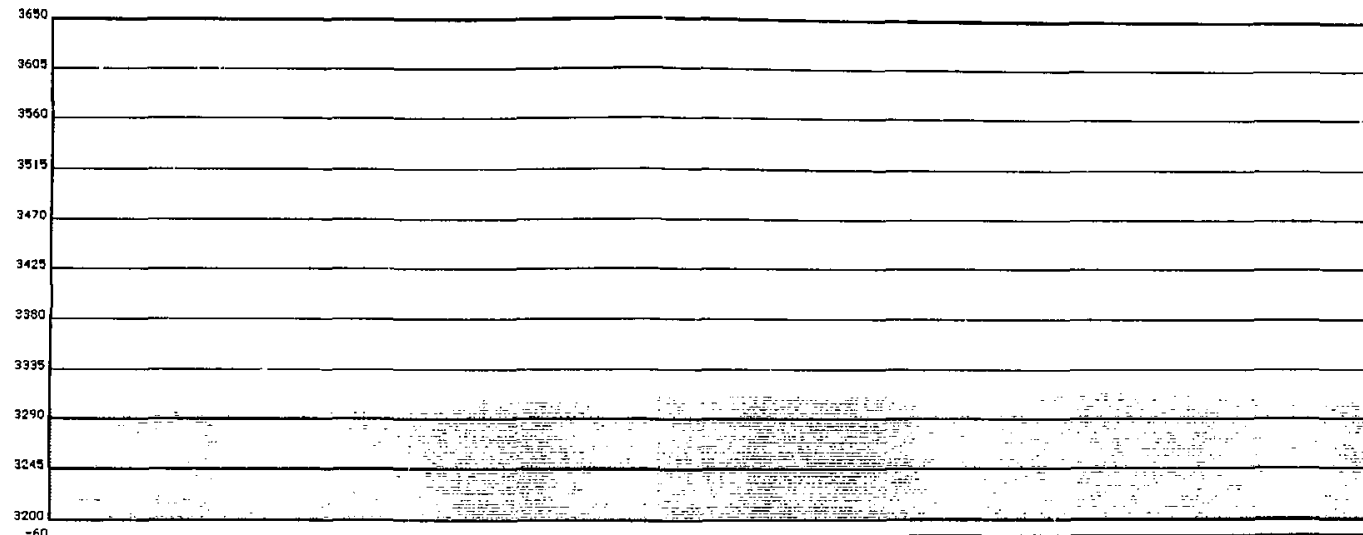

\&
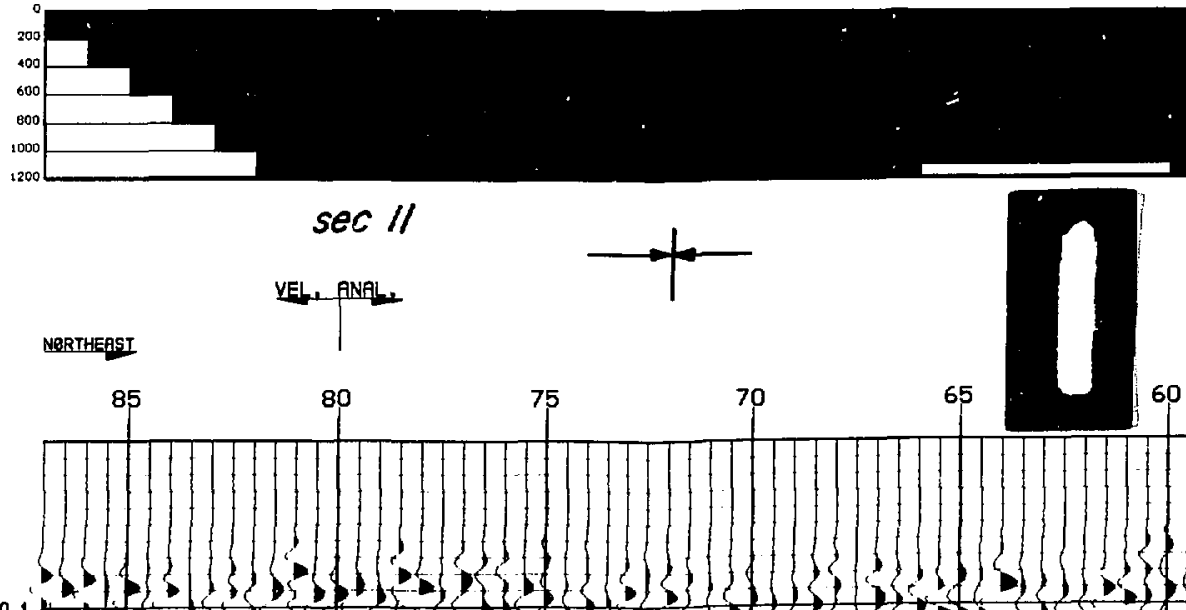
.1.5,

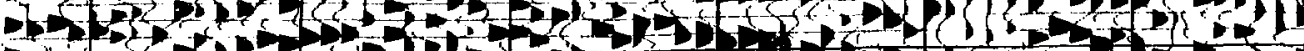

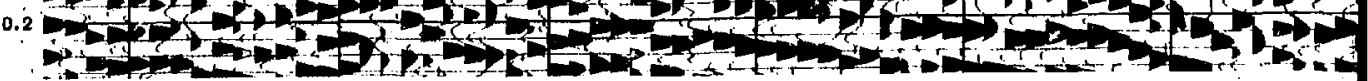


andm
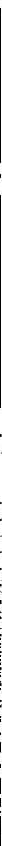


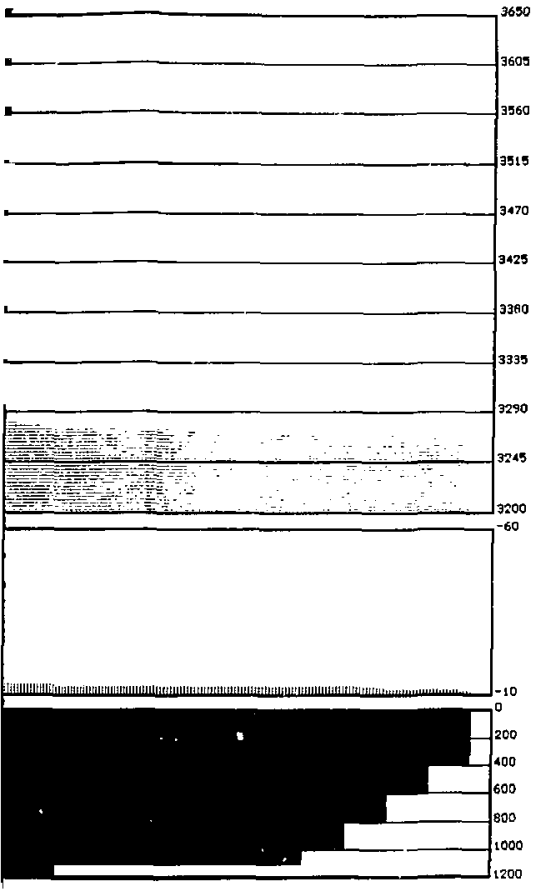

VELL. PNALL,

NQRTHEASI

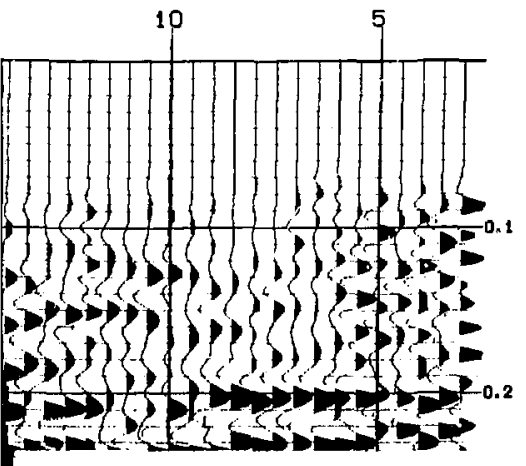

ELEVAT IONS

\section{STATICS}

FOLD $x$

\section{LINE DIRECIION}

VELOCITY FUNCTION

DIRECTION

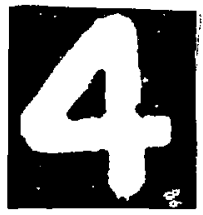

STATIONS

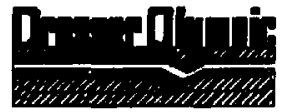

\section{LOS MEDANOS \\ LINE $X-11$ \\ CTATTANIE 2-97}




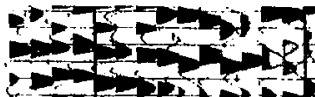

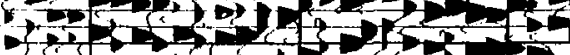

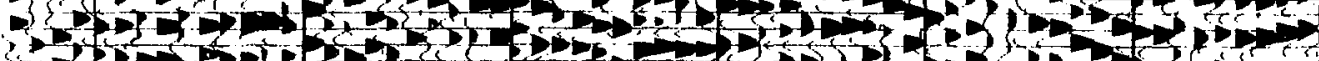

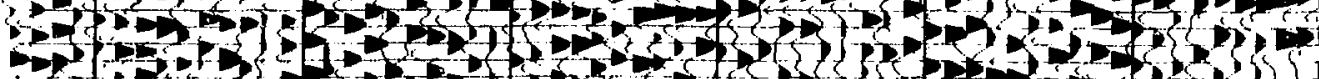

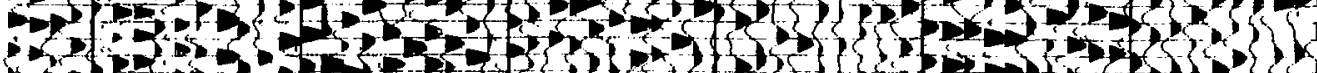

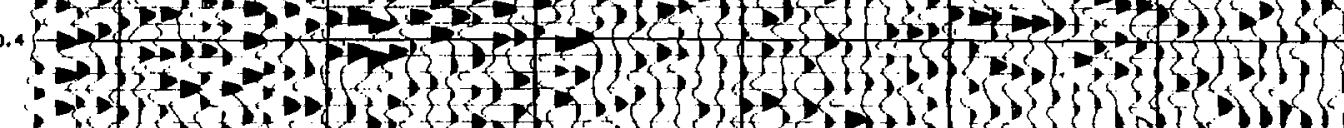

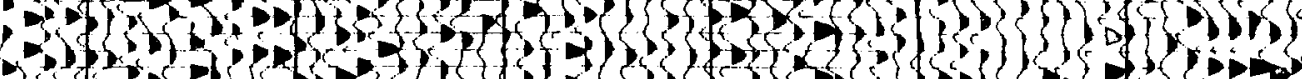

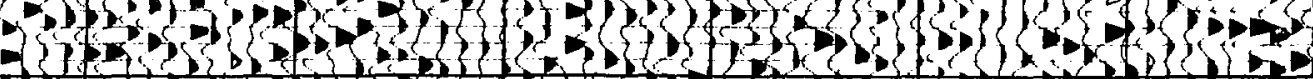

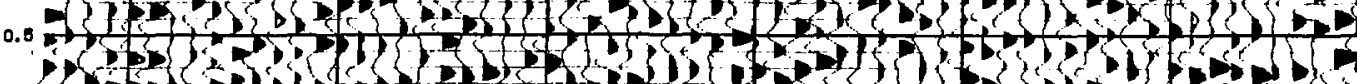

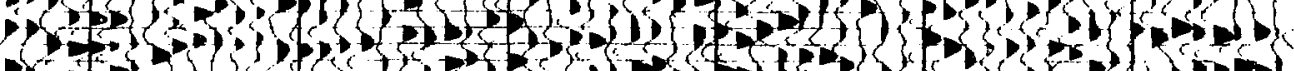

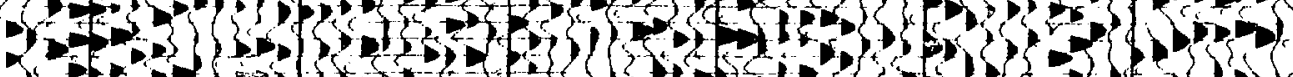

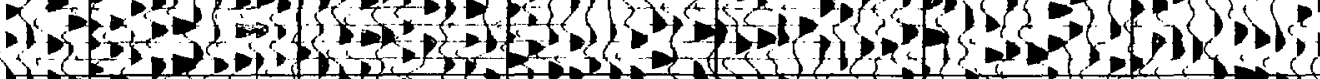

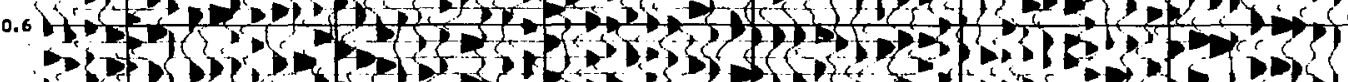

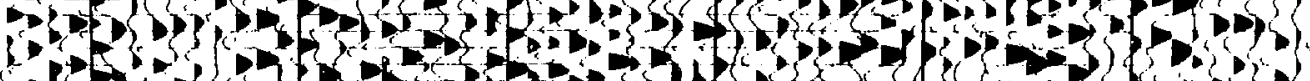

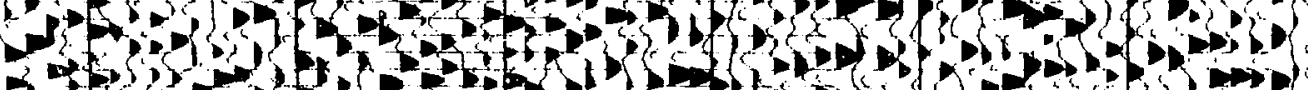

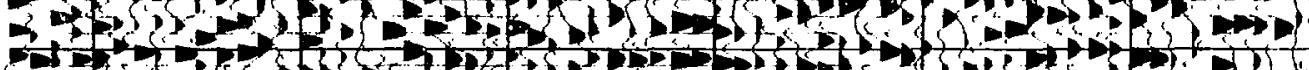

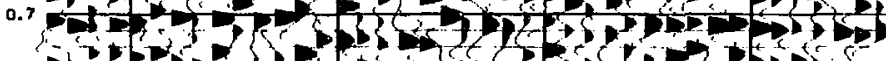

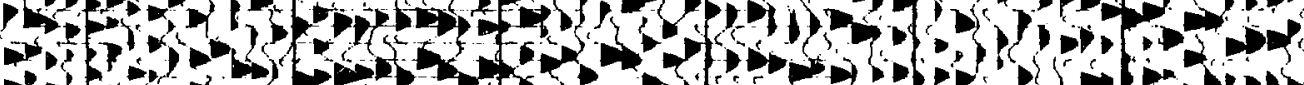

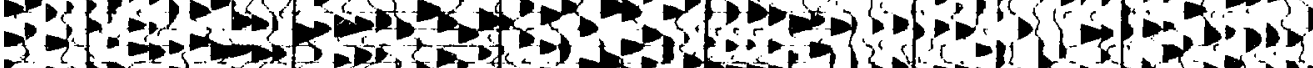

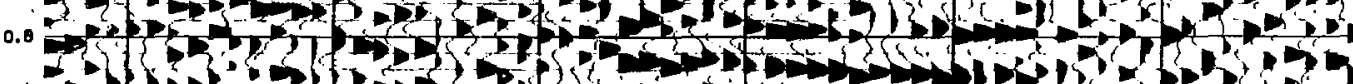

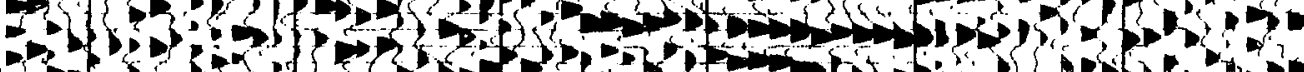

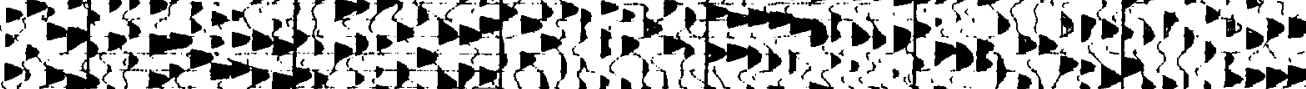

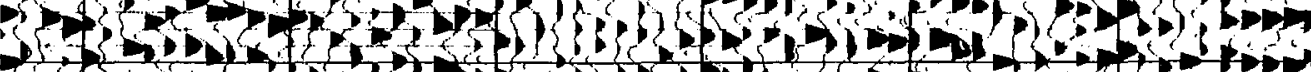

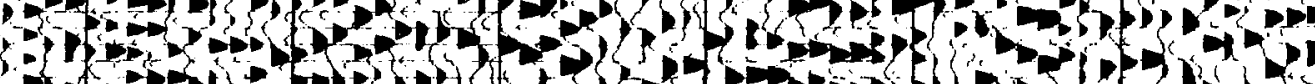

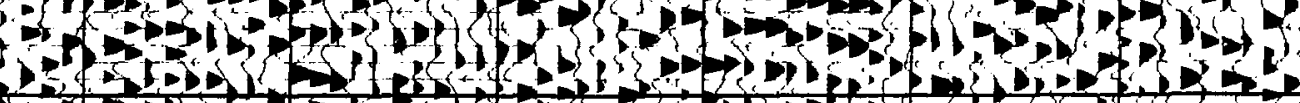
-5

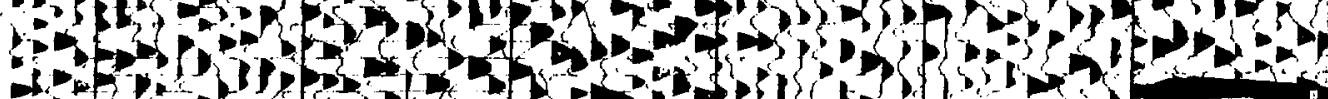

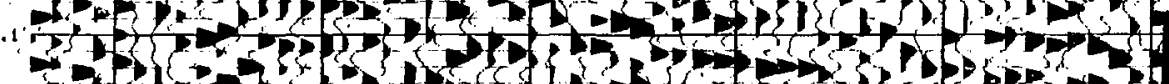

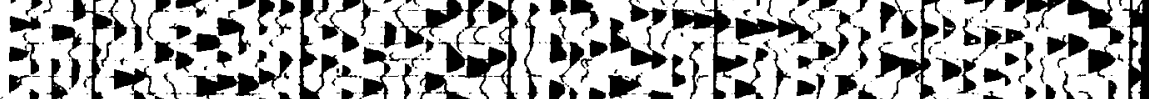

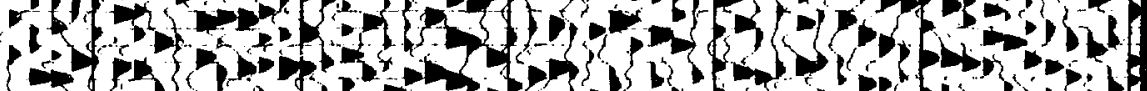




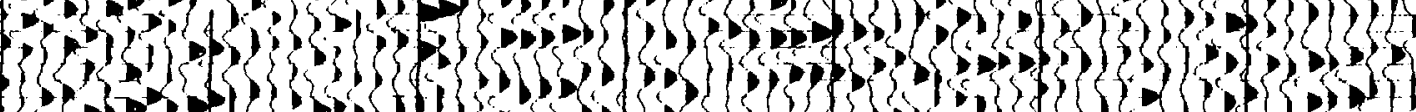

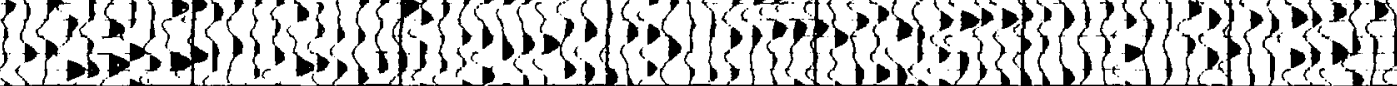

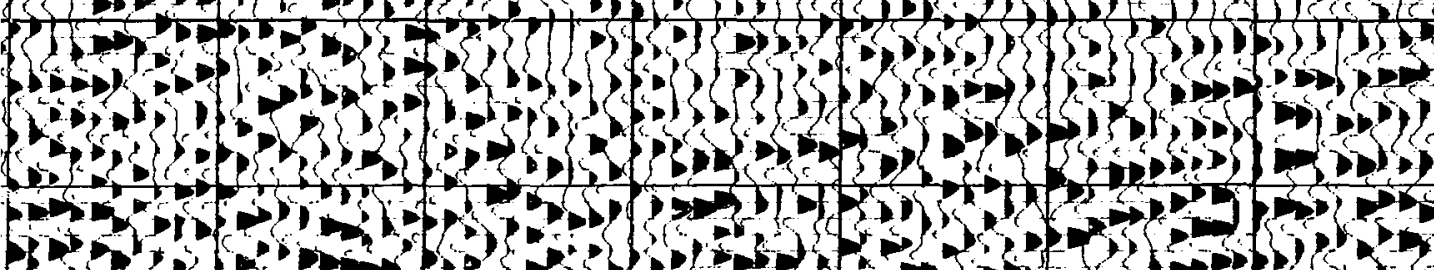

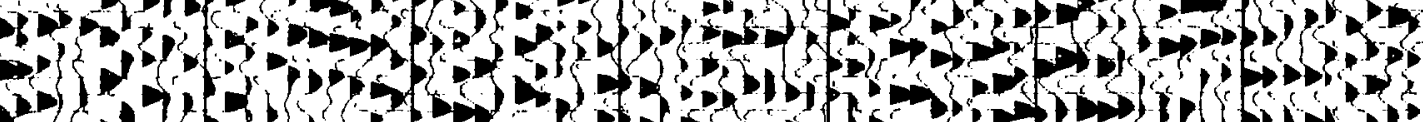

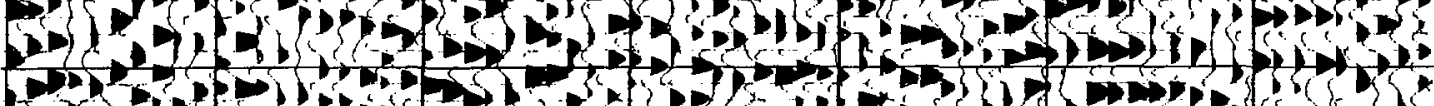

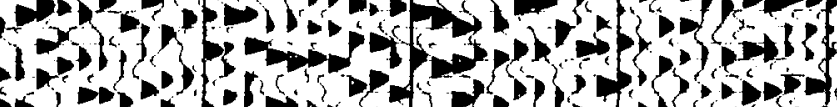

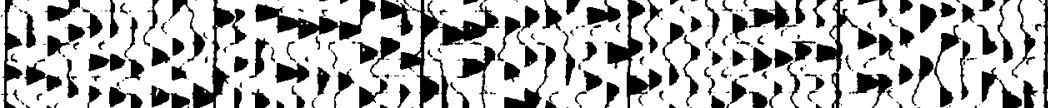
(1)

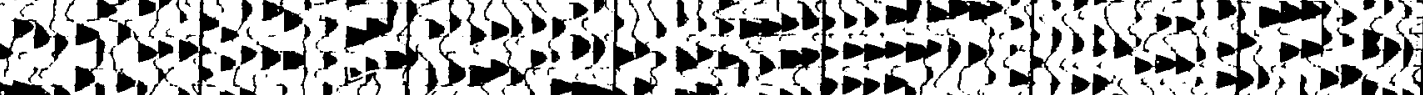
(1) a)

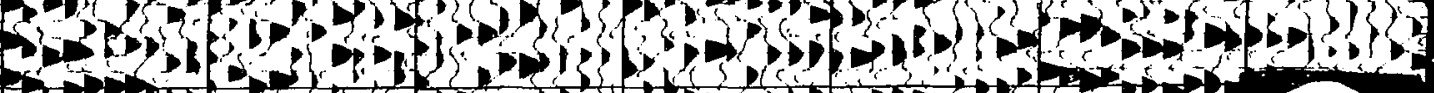

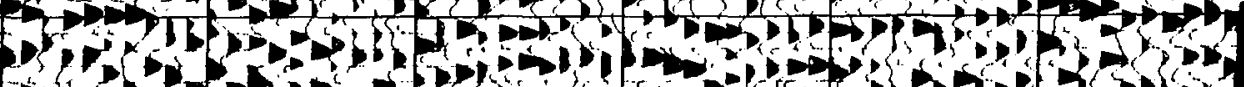

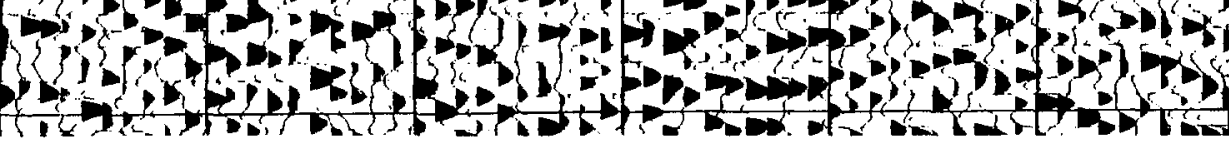


INPUT REEL HEADER INFERTATIS

REG NUWA

WHER SATPLEATRACE TSOO

PRECESSER

LTEE NUH ER

re

PHCTENANG STE

\section{F IELD}

RECERDED BY: DRESSER OLYHPIC

OATE: NOVEHEER 20.197

INSTRUHENTS: CFS I - DFS IV

NOTCH FILT: IN

RECORD LEN: 16 SEC.

SHEEP FREO: 25-100 HZ

STN INV: $110 \mathrm{FT}$.

GEQ PER STN: 6

ARRAY TYPE: INLINE
PARTY:

FILTER:

SAAPLE RATE:

SQURCE:

SHEEP LEN:

NE/GRQUPS

VIB. INV:

GEQ TYPE:

TYPE COVER:

\section{PROCESSING SEQUE}

PROCESSED GY DAESSER GLYMPIC

\section{STATICS COMPUTATIEN}

DATUM: $\quad 3200$ FT.

VSW: $\quad 6000 \mathrm{FT} / \mathrm{SEC}$.

1) DEMULTIPLEX

2) BINARY BRIN RECOVERY

3) VIBRESEIS CBRRELATIEN

4) COMHON DEPTH POINT GATHERS

5) DECONVOLUTION

OPERATOR LENGTH-140 MILS

PREOICTION TIME BASED EN 2ND ZERE CRESSII

6) TIME-INYARIANT DIOITAL FREGUENCY FILTER 0.0-3.D SEC. 25-80 HZ

7) AFPLY DATUM STATICS

e) VELECITY ANRLYSIS

9) APPLY NHO

10: FIRST GREAK SUPPRESSION (MUTE)

11) STACK 12 FOLD

12) TIME-INUARIANT DIGITAL 0.0-3.0 SEC. 25-60 H2

13) DIGITAL RBC

14: DISPLAY

G TR/IN

10 IN/SEC.

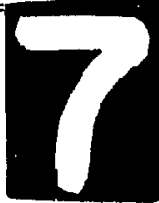

1

SPREFO DIAGRAM 12

1210

440 


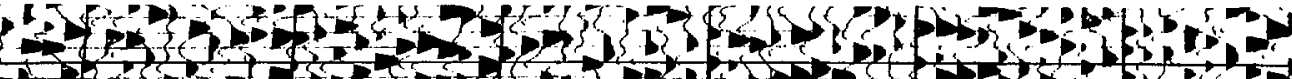

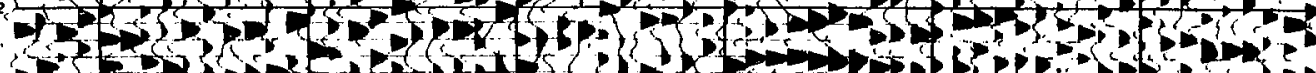

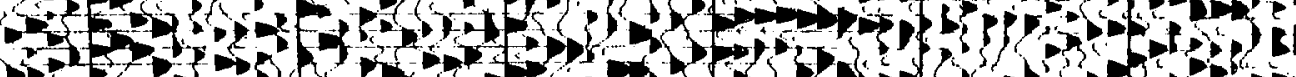

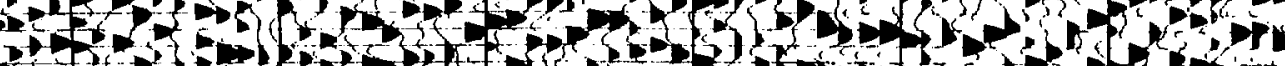
a

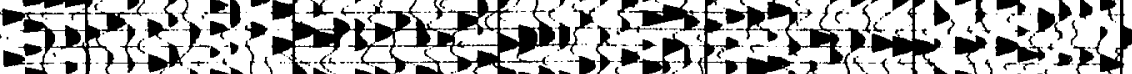

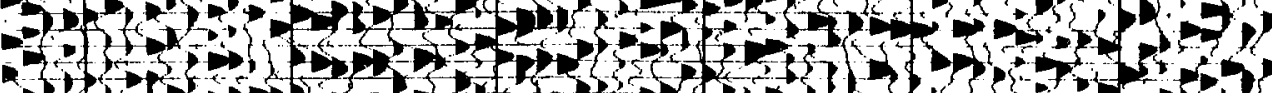

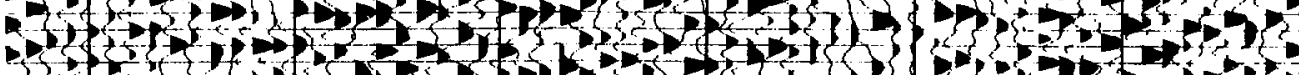

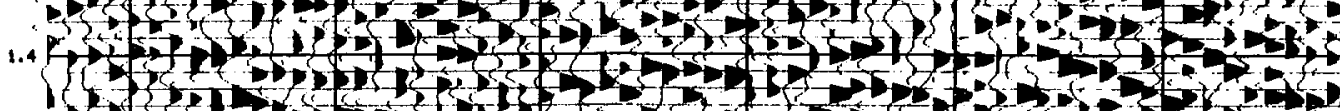

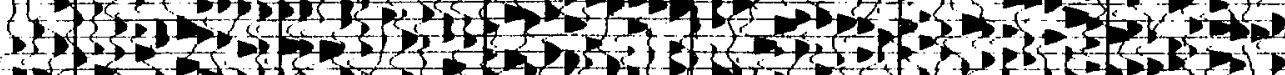

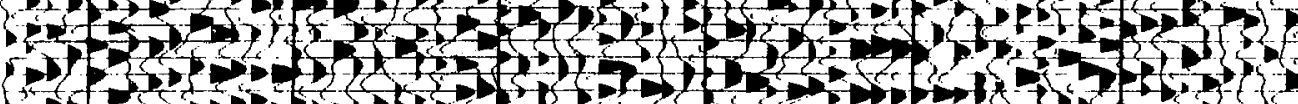

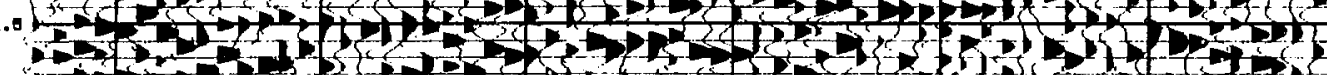

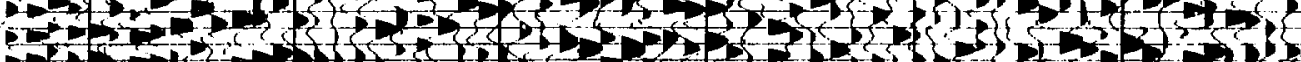

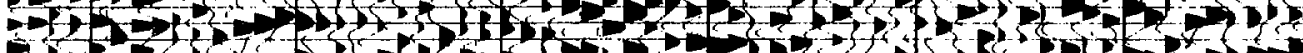
F.

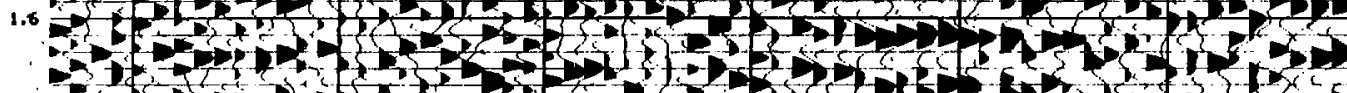

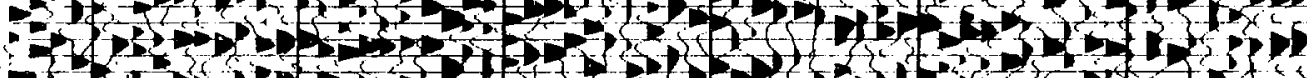

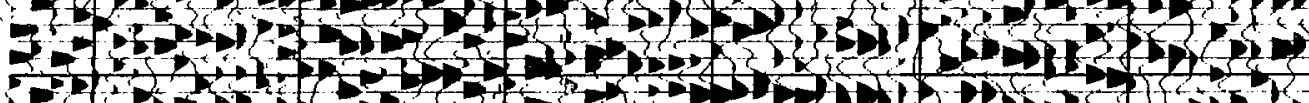

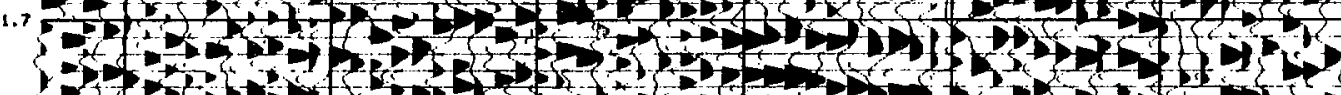

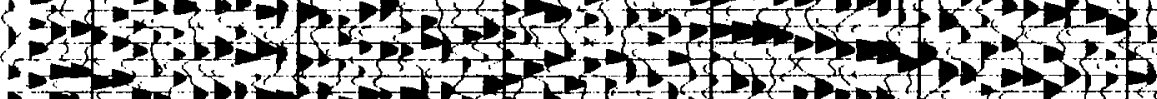

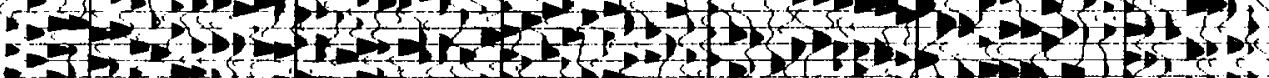

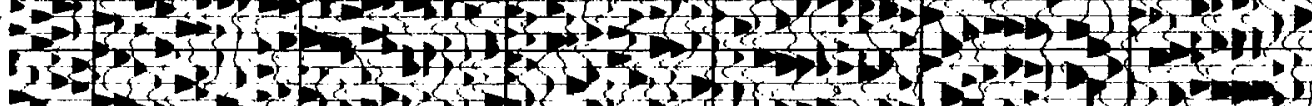

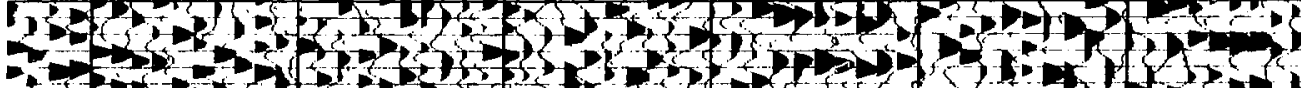

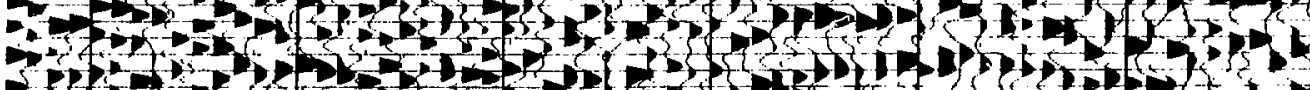

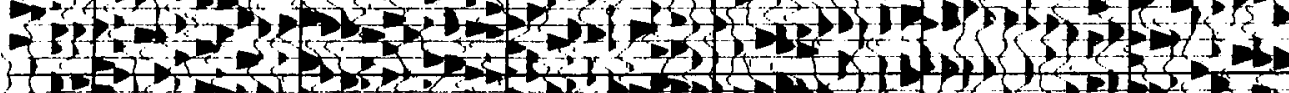

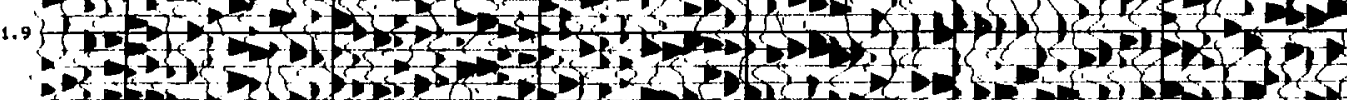

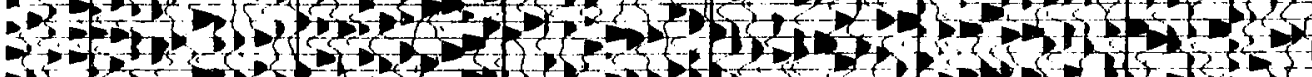

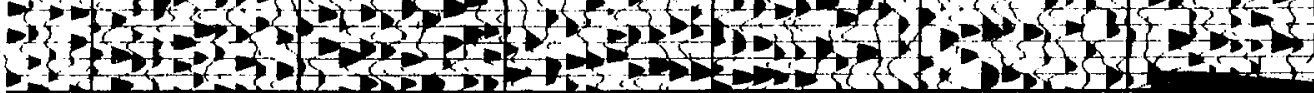

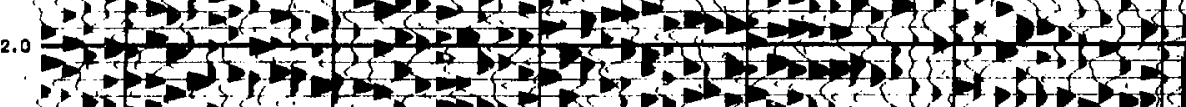

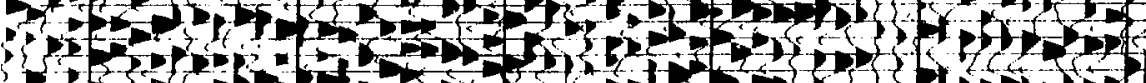

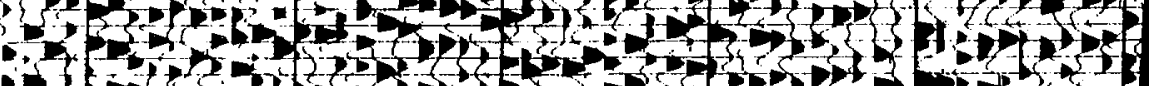

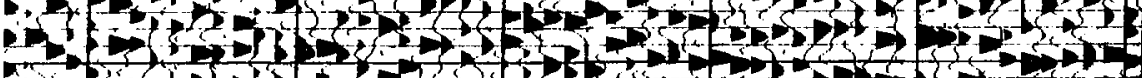

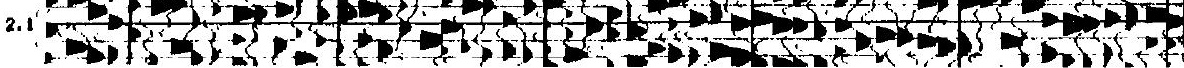




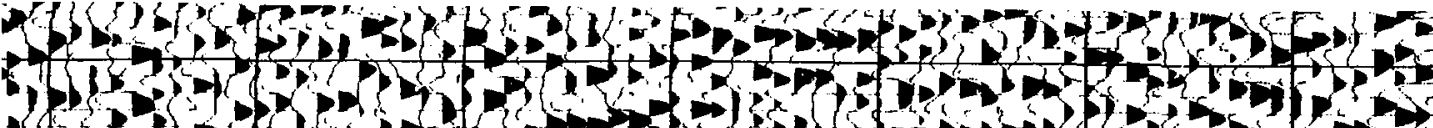

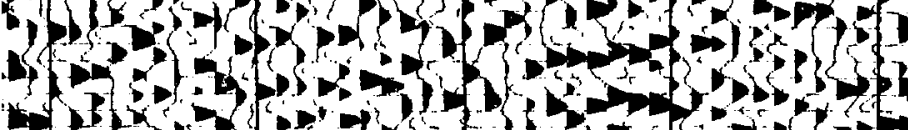




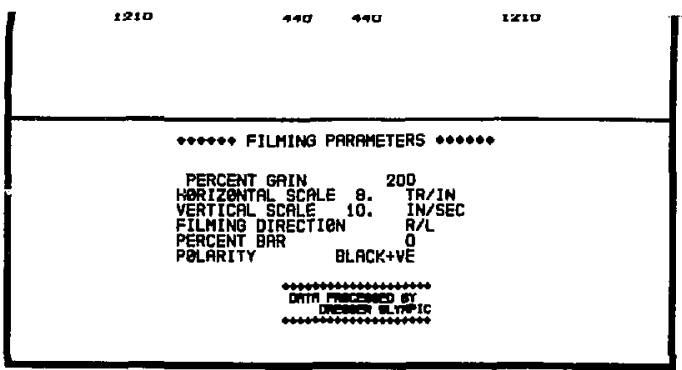




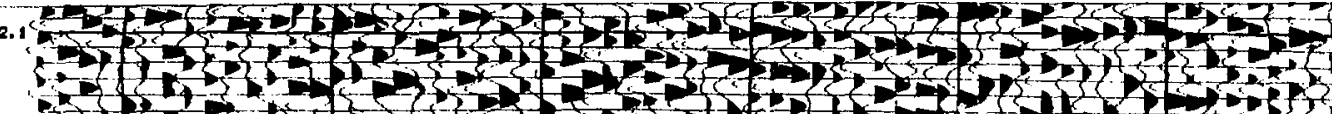

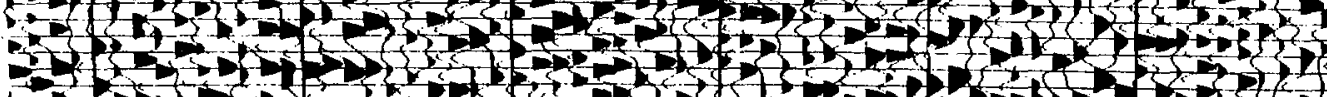

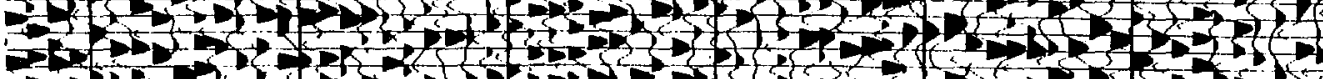

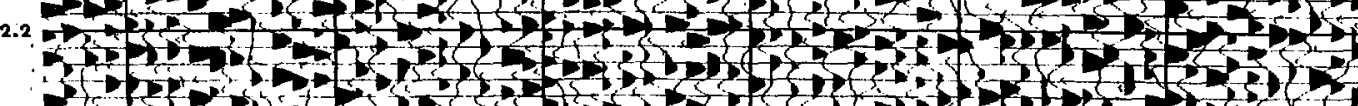

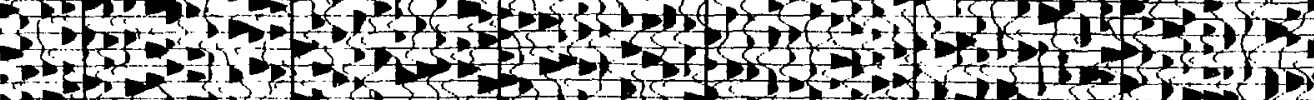

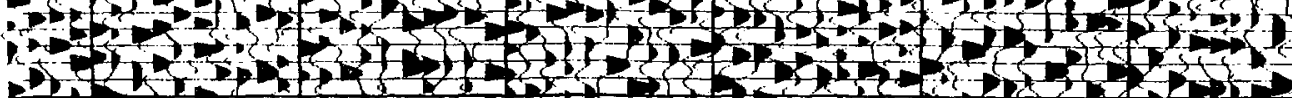

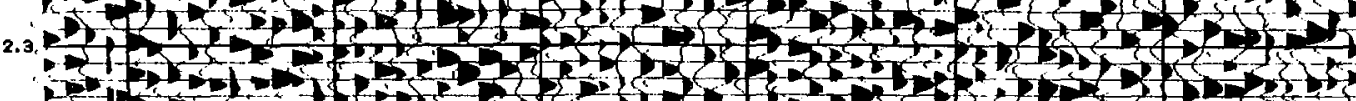

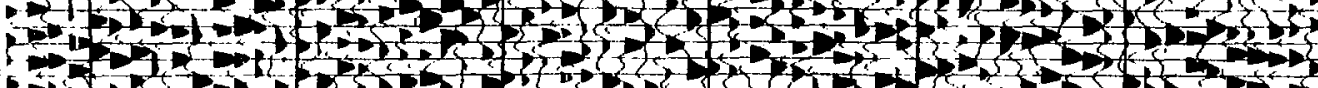

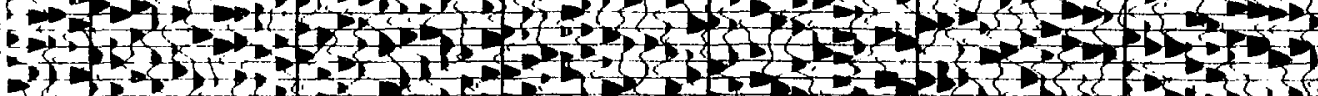

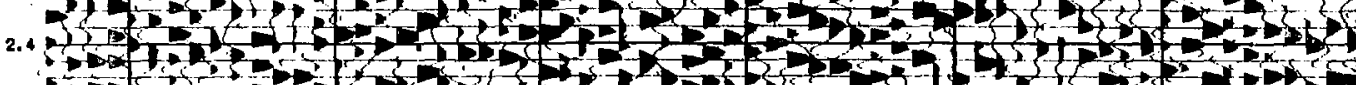

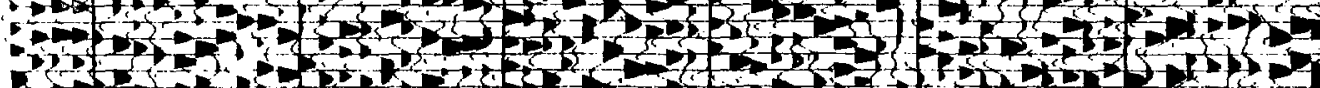

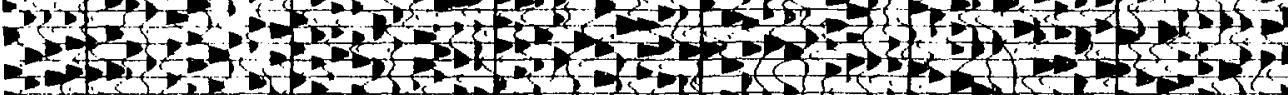

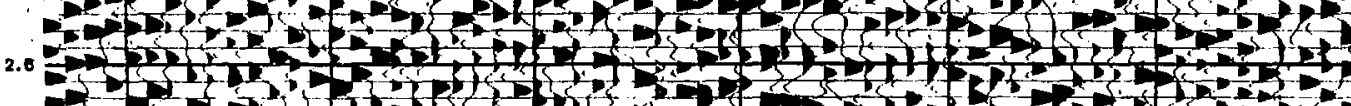

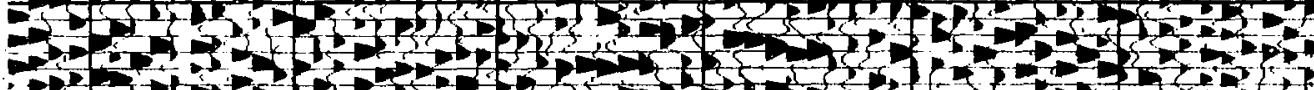

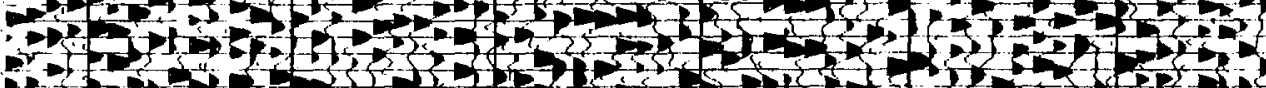

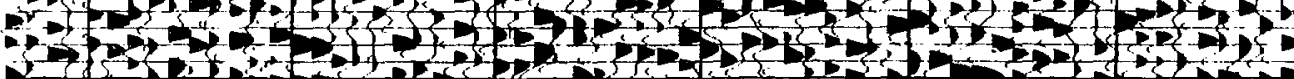

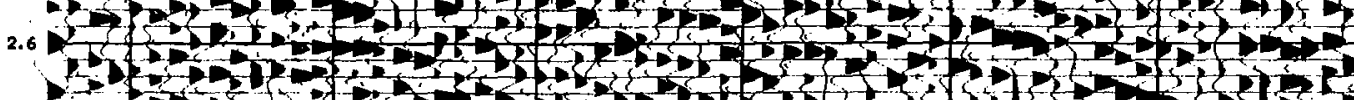

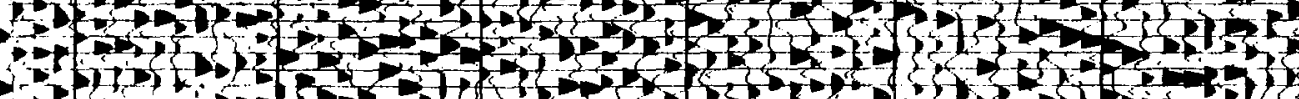

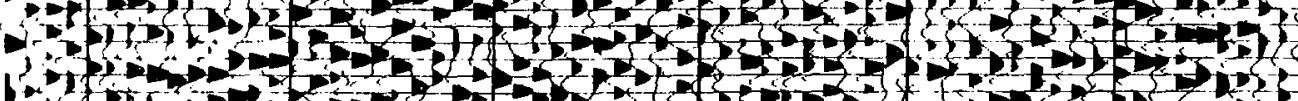

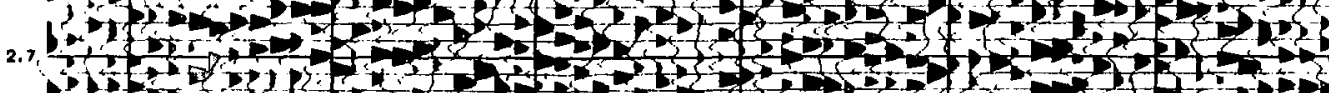

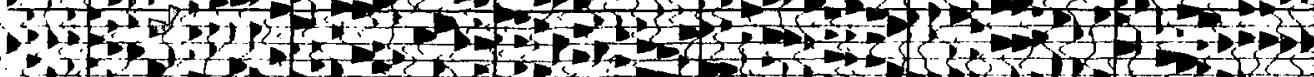

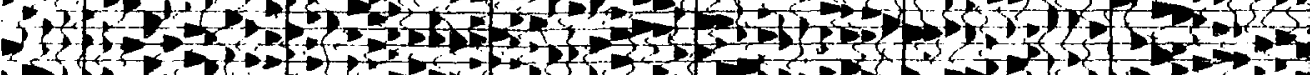

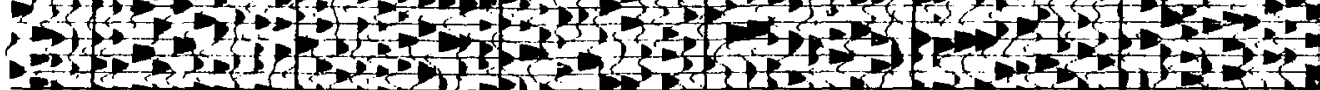

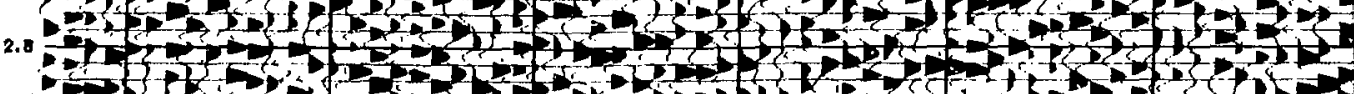

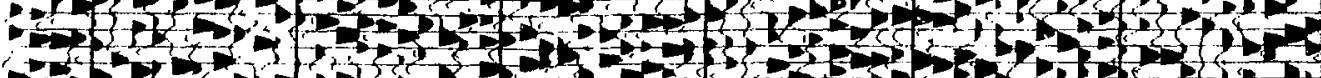

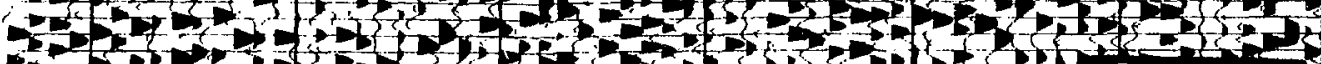

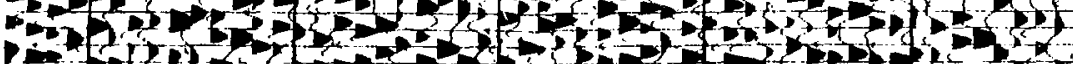

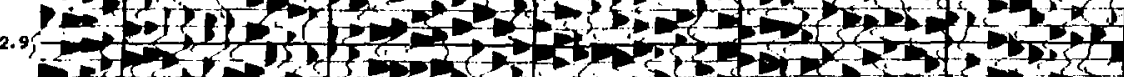

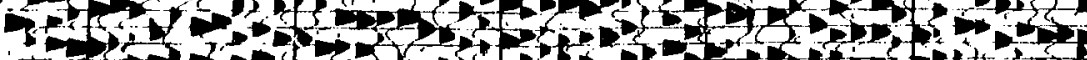

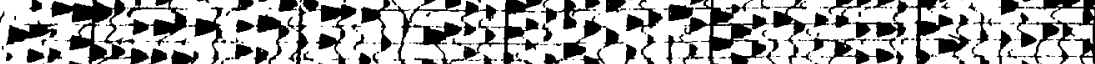

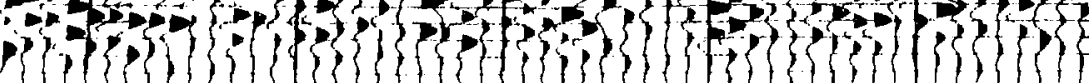




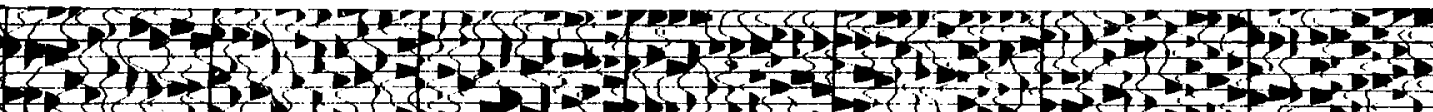
R

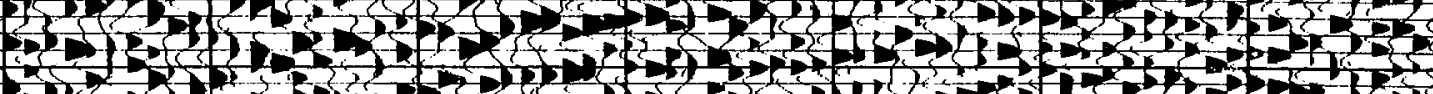

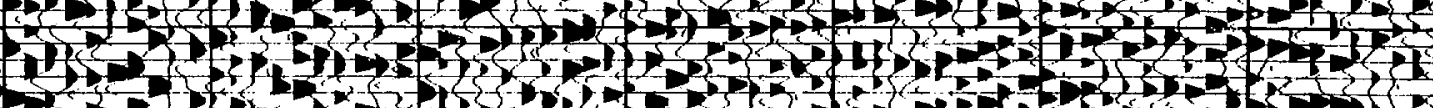

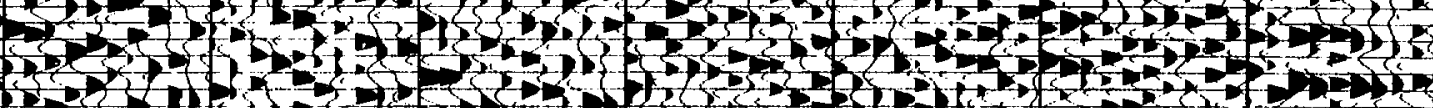

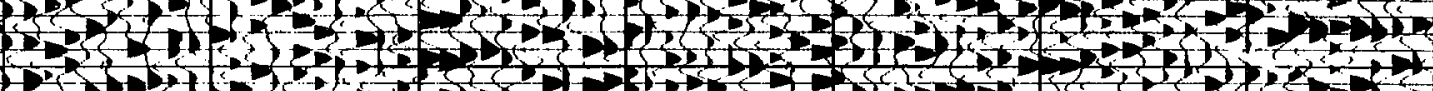

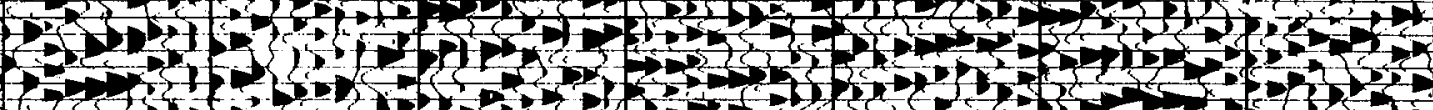

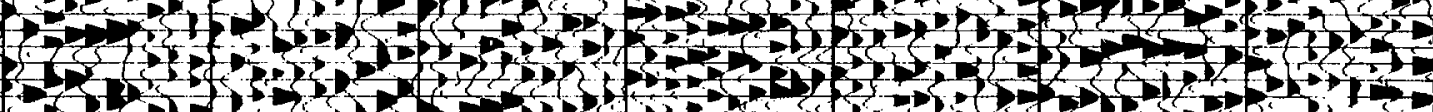

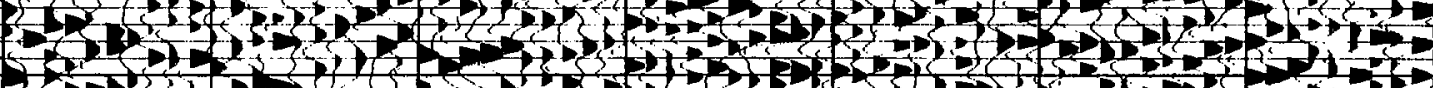

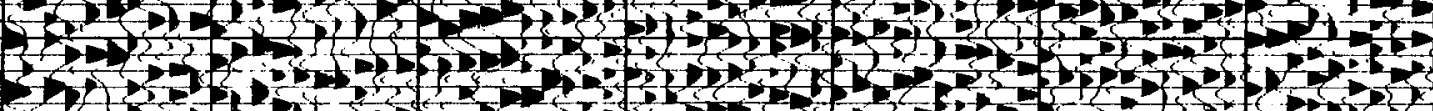

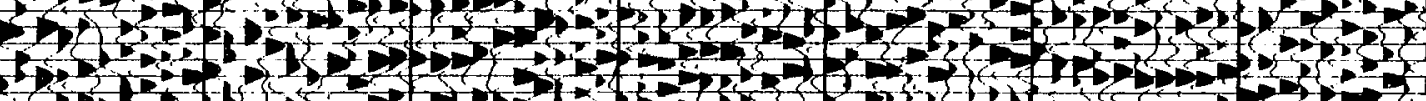

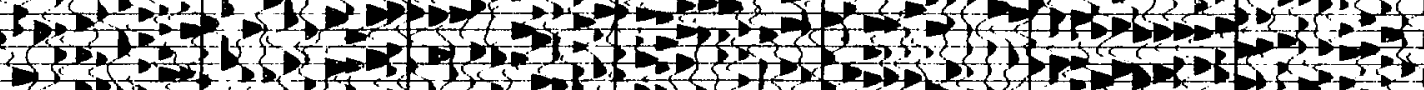

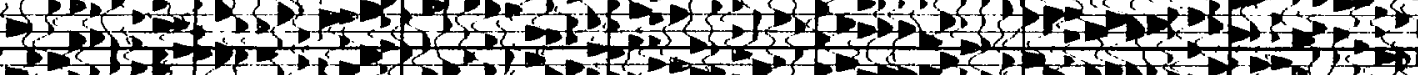

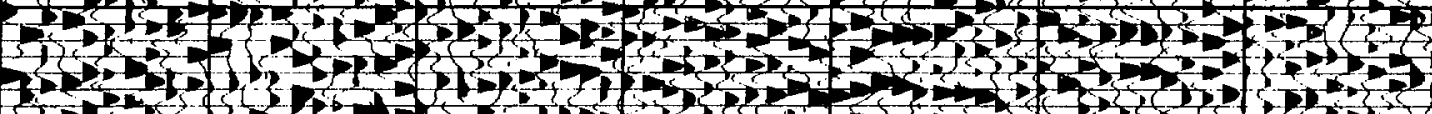

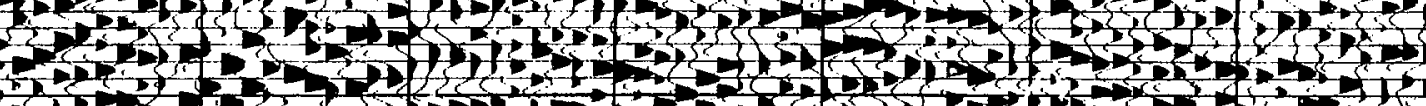

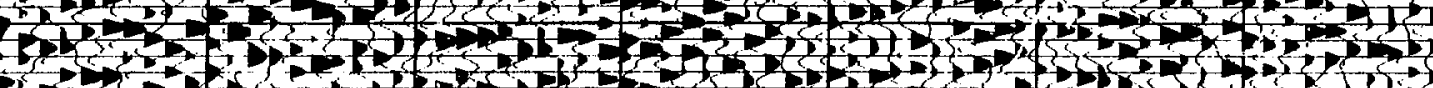

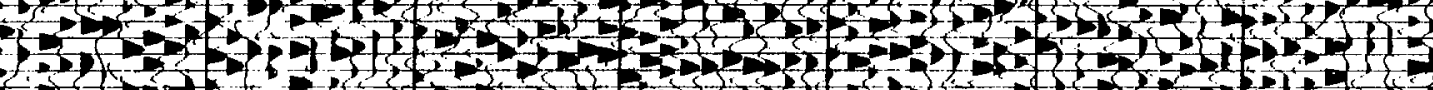

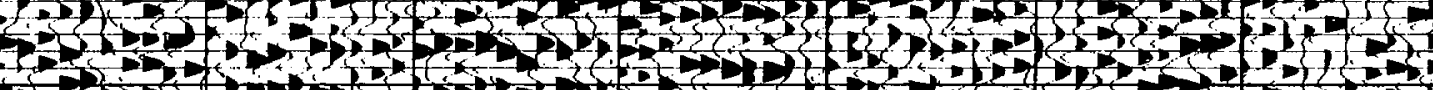

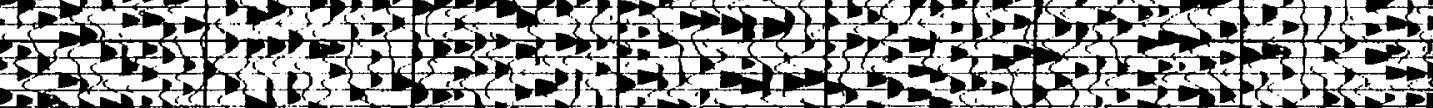

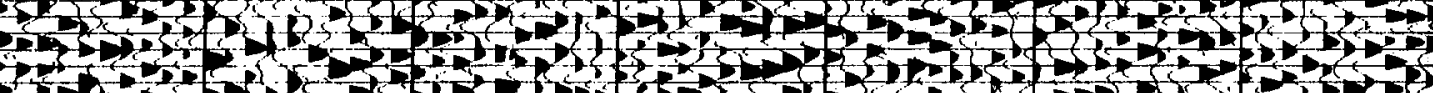

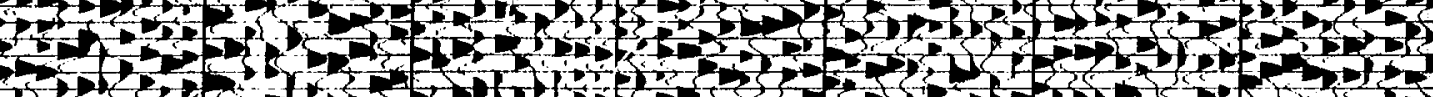

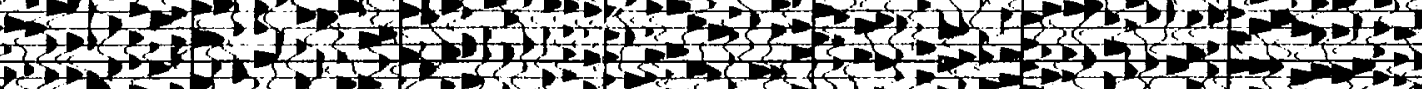

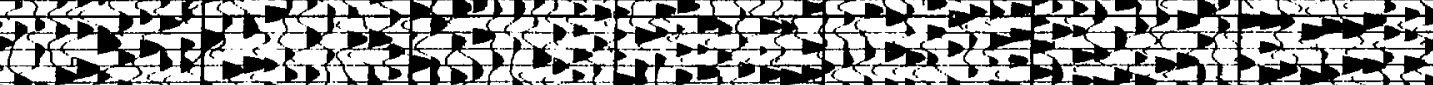

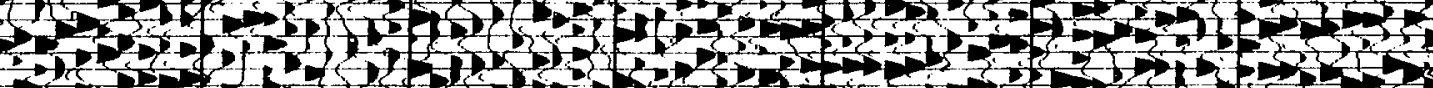

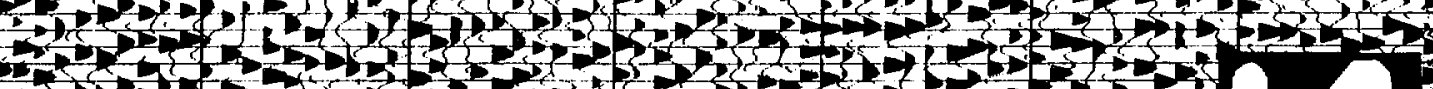

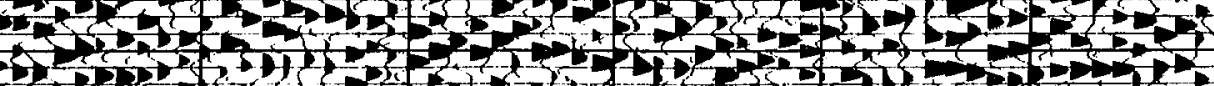

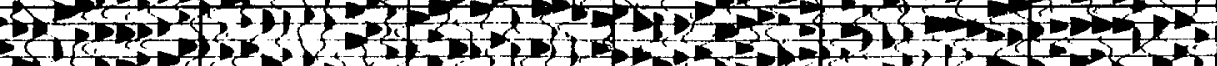

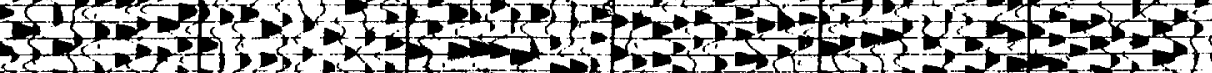

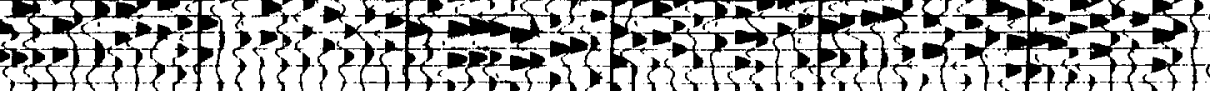




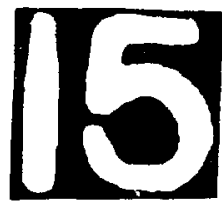




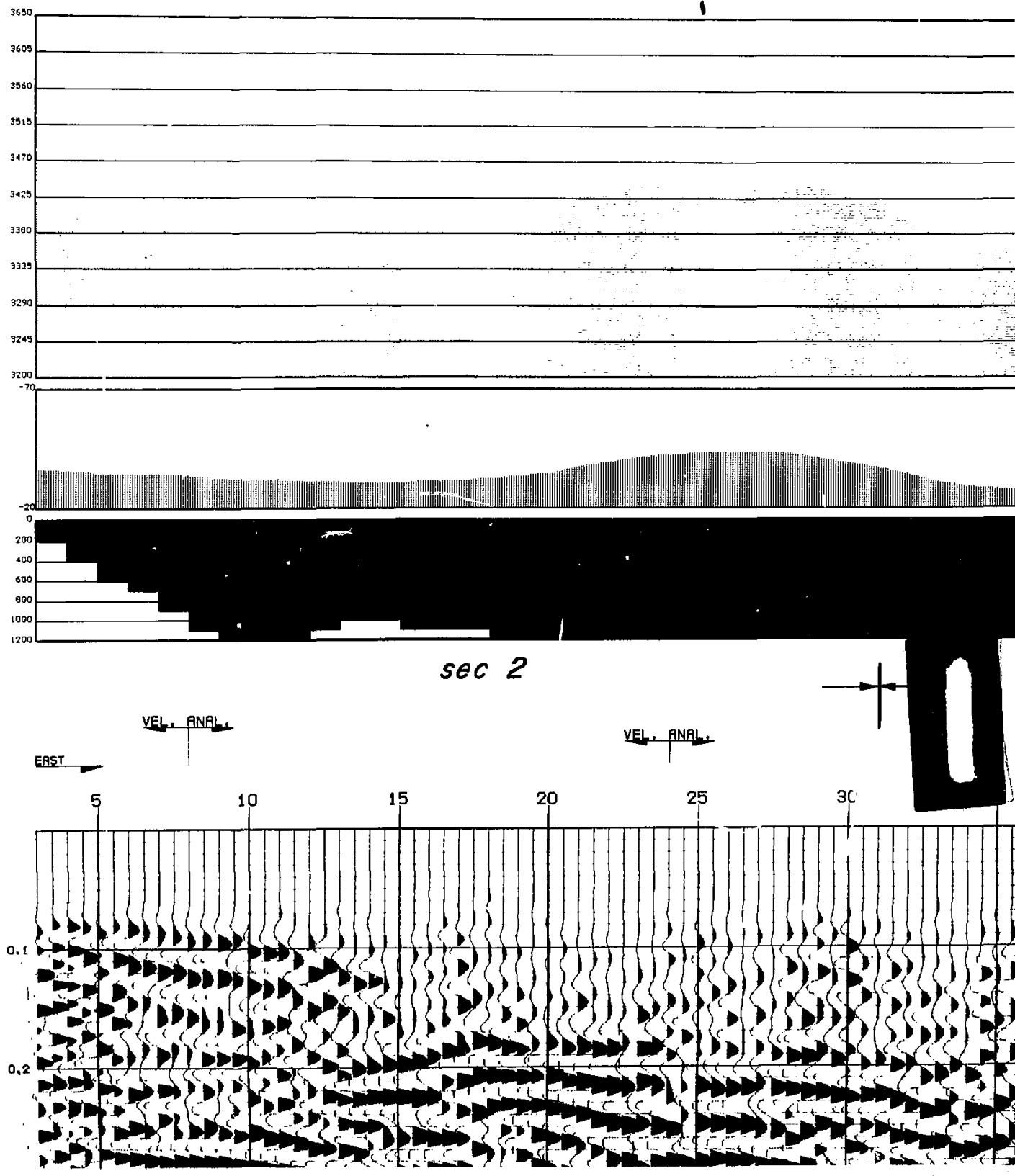




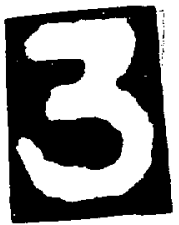

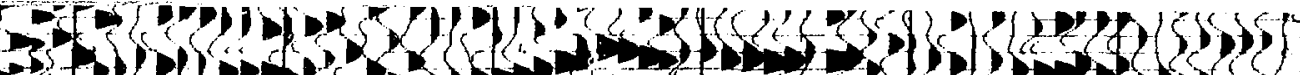

itom 0.3

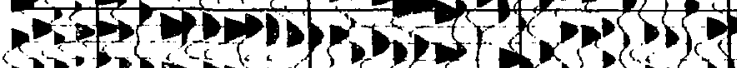

-

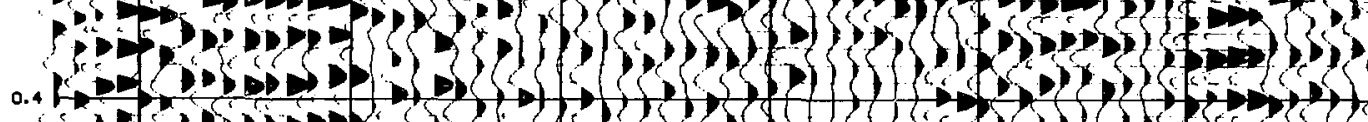

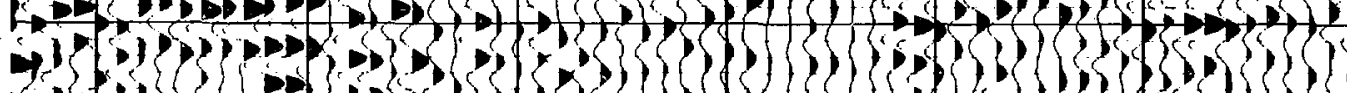

$b 3\}\{0\}\{\}\{\}\{\}$

$\because\{\{\}\{\}\{\}$

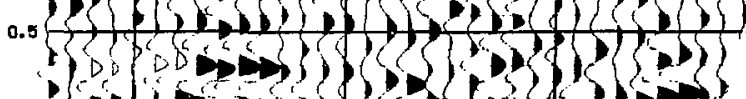

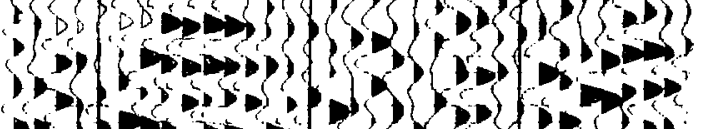

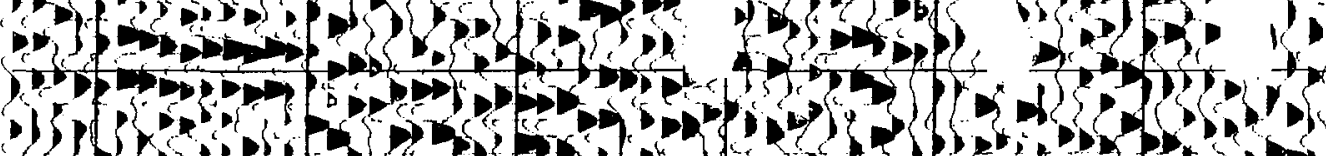

$\left\{\int\right\}\{3$

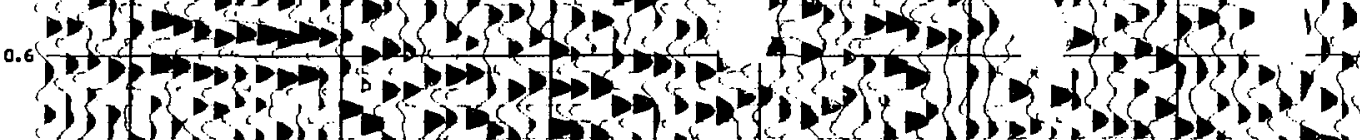

15

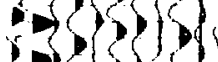

$\sum_{i \rightarrow \infty}$

15)

15) -

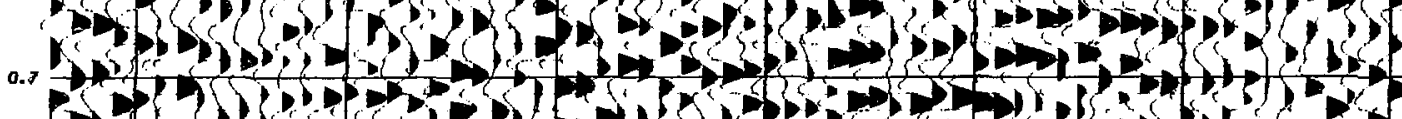

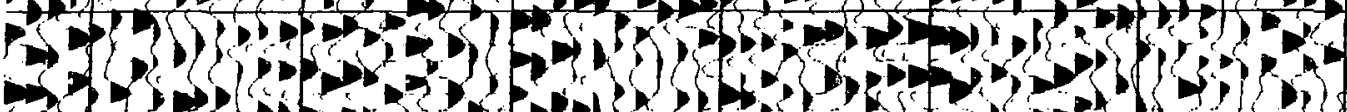

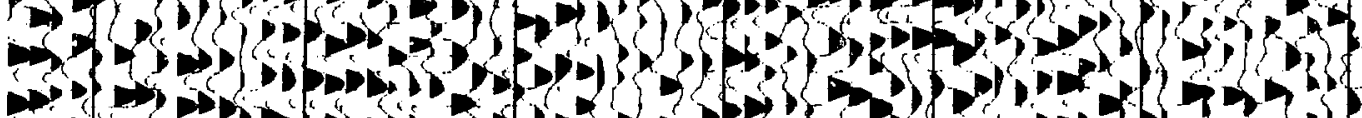

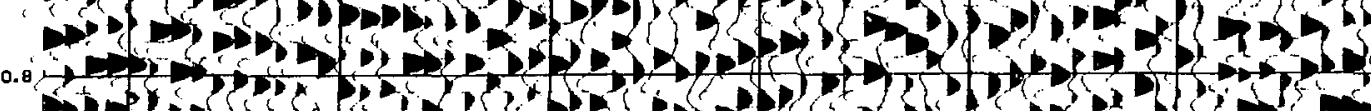

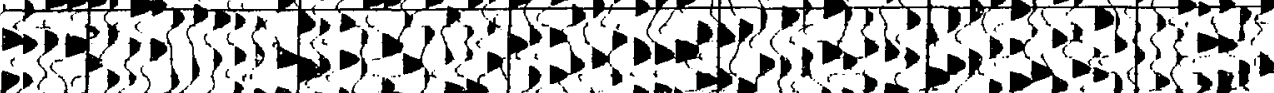
P.

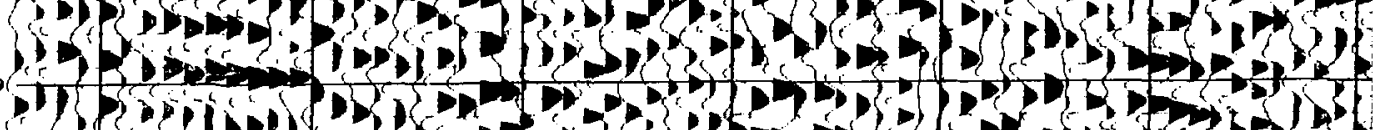

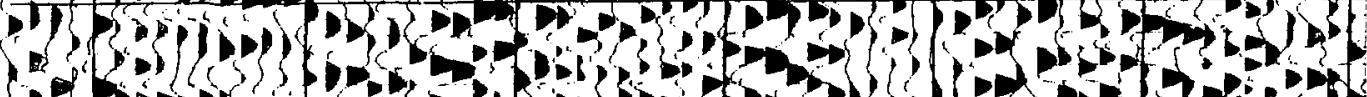

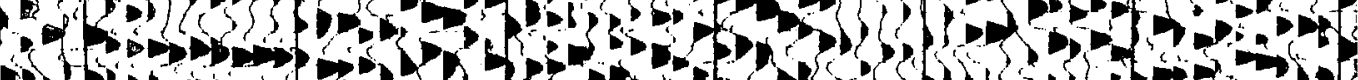

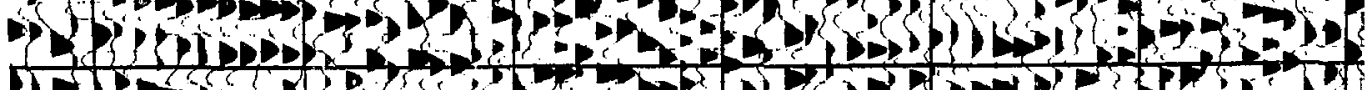
1.0 


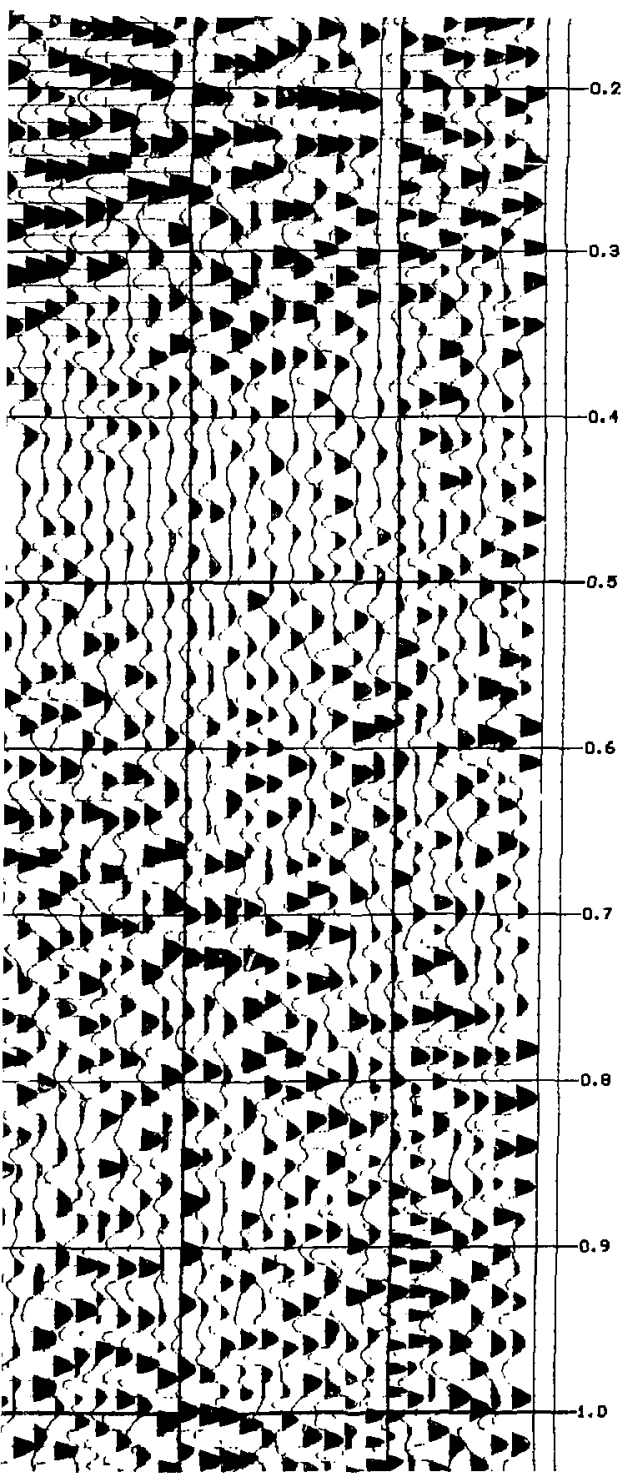

\section{LOS MEDANOS}

LINE
STATIENS -12
$3-46$
SOUTHEAST NEW MEXICO

INPL'T REEL MEADER INFGRMATION

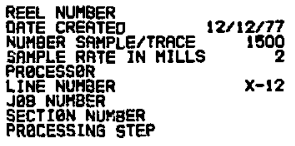

\section{FIELD INFORMAT ION}

\begin{tabular}{|c|c|c|c|}
\hline $\begin{array}{l}\text { RECORDED QY: } \\
\text { DATE: }\end{array}$ & $\begin{array}{l}\text { DRESSER BLYHPIC } \\
\text { NBVEMBER } 16,1977\end{array}$ & $\begin{array}{l}\text { PARTY: } \\
\text { FILTER: }\end{array}$ & $\begin{array}{l}\text { Ho. } 62 \\
18 / 36-124 \text { HZ }\end{array}$ \\
\hline INSTRUMENTS: & CFS I - DFS IV & SAMPLE RATE & $2 M S$ \\
\hline NВTCH FILT: & & SEURCE: & VIARBSE IS \\
\hline RECQRD LEN: & 16 SEC. & SHEEP LEN: & $12 \mathrm{SEC}$. \\
\hline SHEEP FREQ: & $25-100 \mathrm{HZ}$ & NO/GRELIPS & 24 \\
\hline STN INV: & $\begin{array}{l}110 \mathrm{FT} . \\
6 \\
\text { INLINE }\end{array}$ & $\begin{array}{l}\text { VIB, INV: } \\
\text { GEO TYPE; } \\
\text { TYPE CBVER: }\end{array}$ & $\begin{array}{l}110 \mathrm{FT} . \\
\text { GSC-2OD } \\
1200 \text { PRCNT }\end{array}$ \\
\hline
\end{tabular}

\section{PROCESSING SEQUENCE}

PROCESSED BY DRESSER OLYMPIC

STATICS COMTUTATIEN

DATUH: 3200 FT.

VSH: 6000 FT'SEC.

1) DEMULTIPLEX

2) GINARY GAIN RECOVERY

3) VIBROSEIS CORRELATION

4) CQMKEN DEPTH PEINT GATHERS

b) DEcenvelutien

GPERATAR LENGTH=140 MILS

PRET.LTIRN TIME BASED GN 2NO ZERO CROSSINE

6) TIME-INUARIANT OIOITAL FREQUENCY FILTER 0.0-3.0 SEC. 25-80 HZ

7) APPLY DATUM STATICS

B) VELOCITY ANALYSIS

9) APPLY NMO

10: FIRST BREAK SUPPRESSIEN (MUTE)

11) STACK 12 FOLD

12) TIME-INYARIANT OIGITAL FREGUENCY FILTER 0.0-3.0 SEC. 25-80 HZ

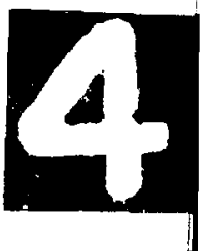




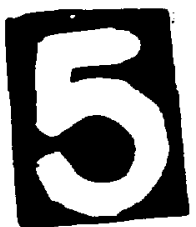

Shom 10 10 som

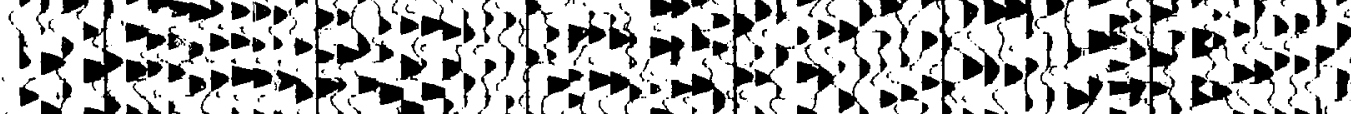
Pins

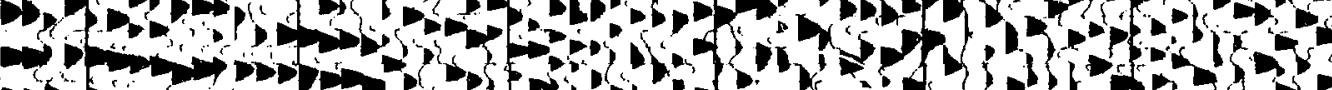

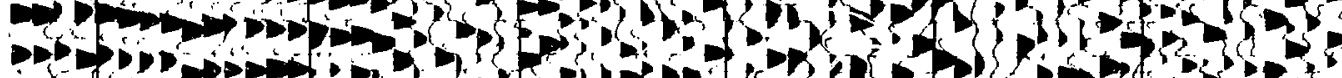

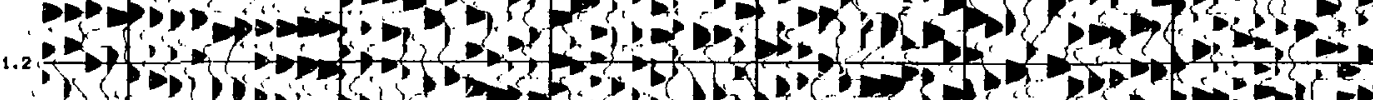

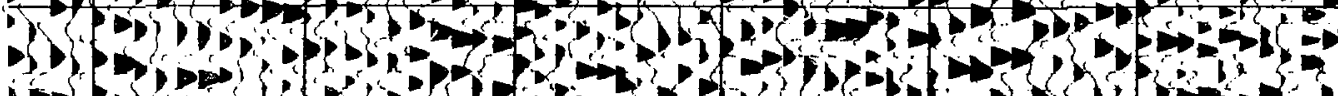

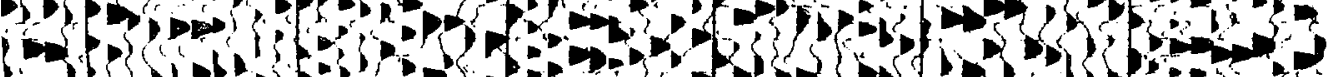

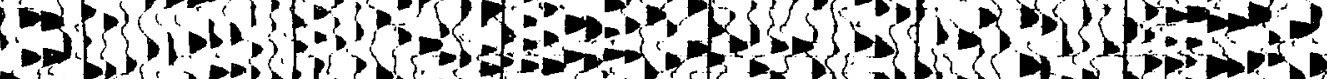

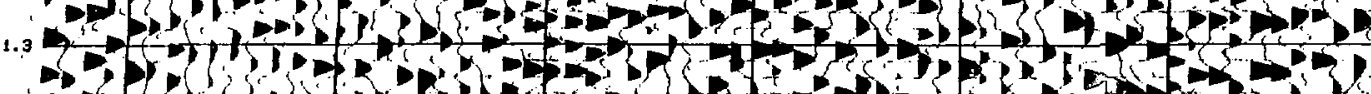

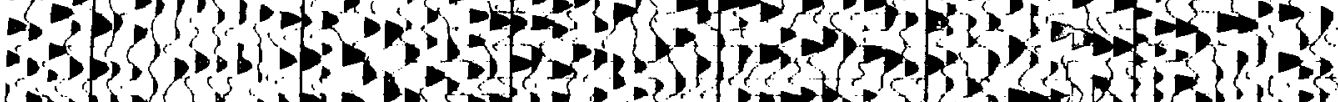

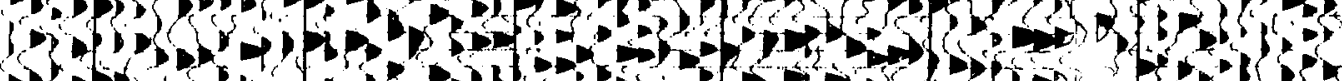
1.4

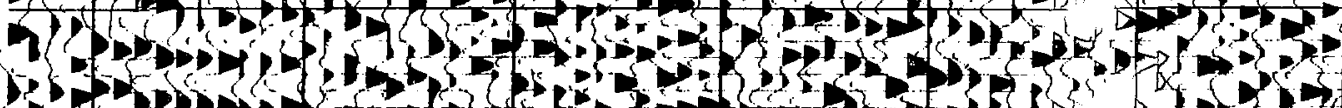
(1)

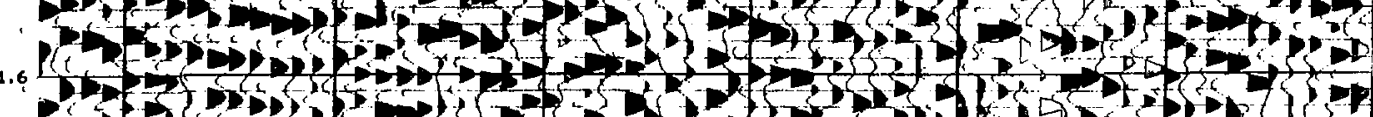

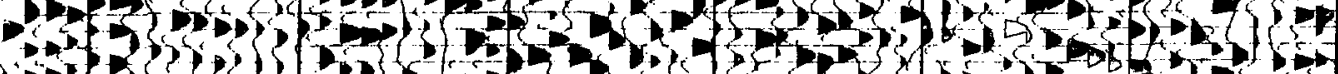

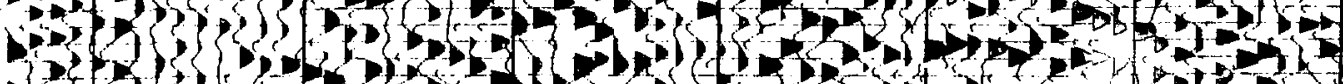

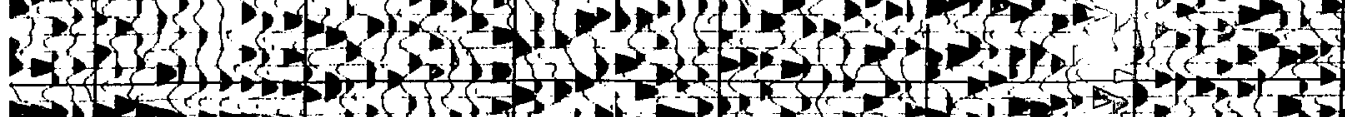

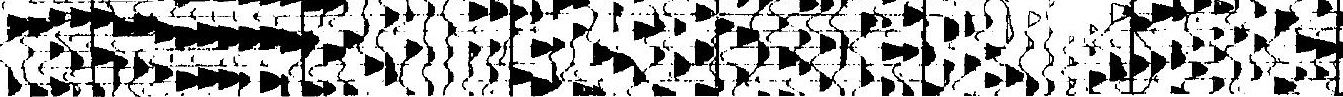




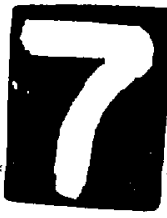

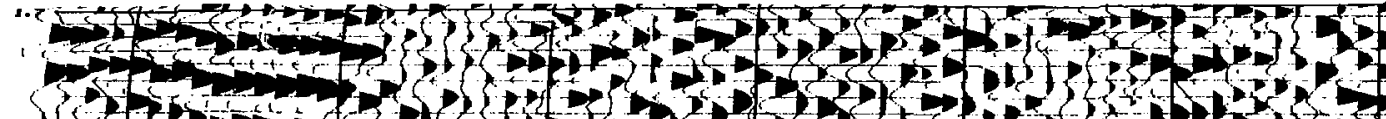

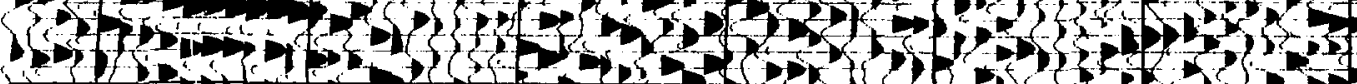
1.8 री 1 (t)

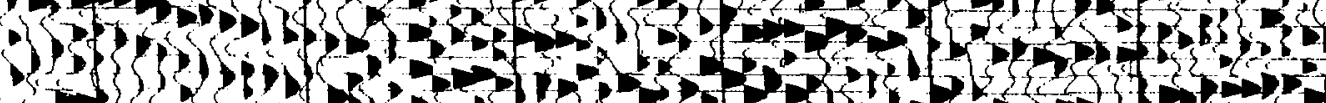

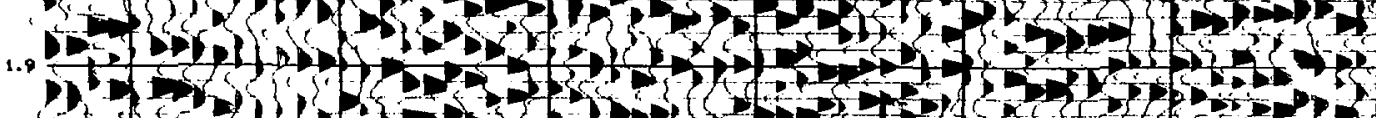

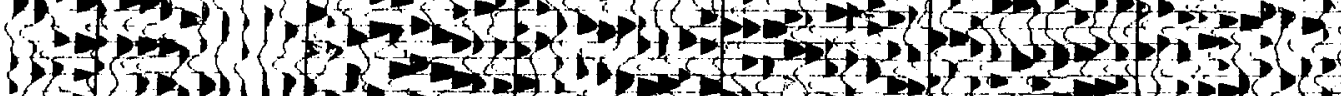
2.2.

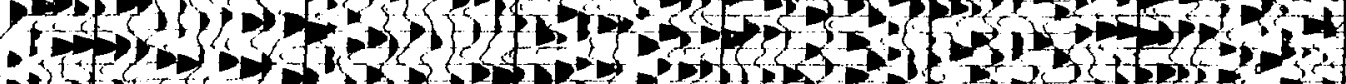

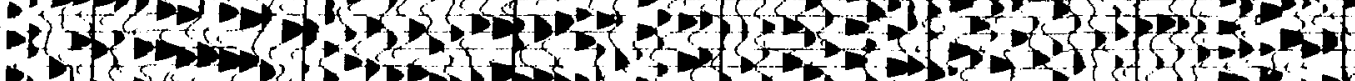

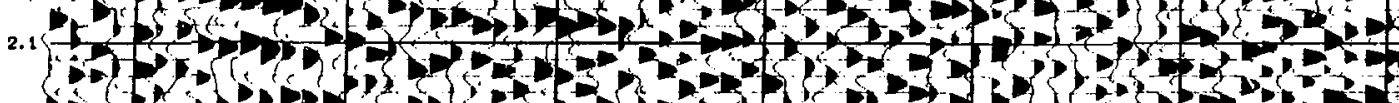

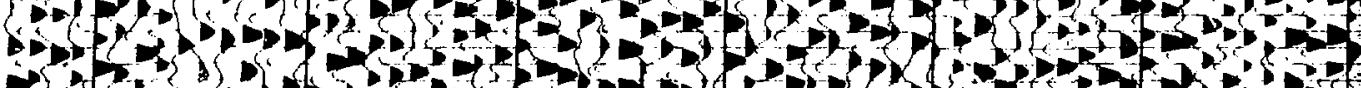

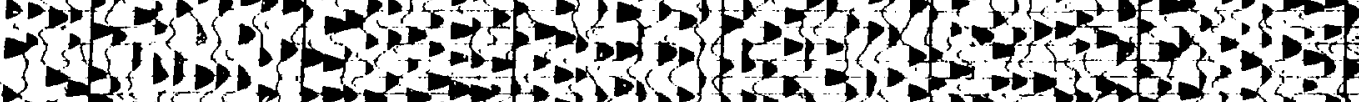

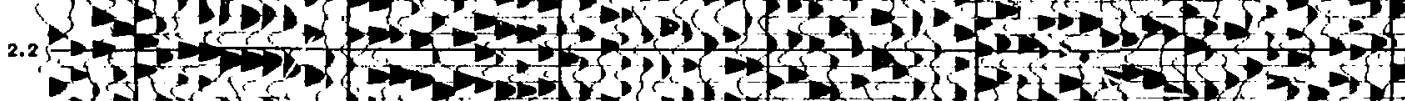

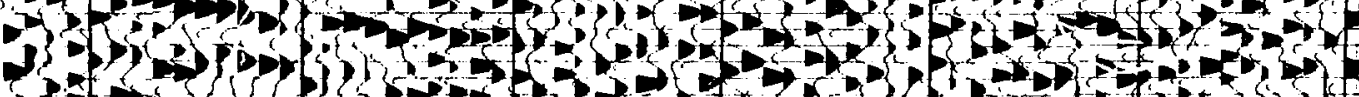

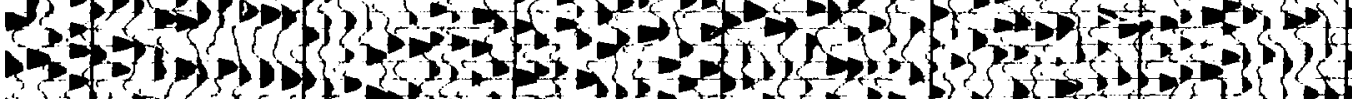

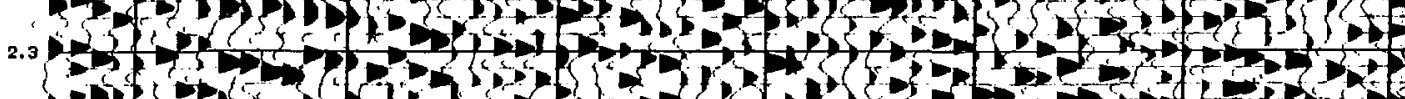

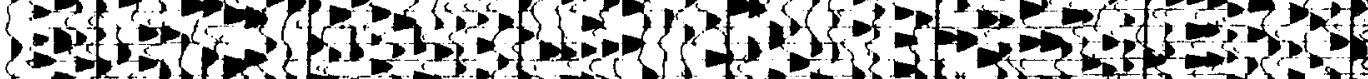

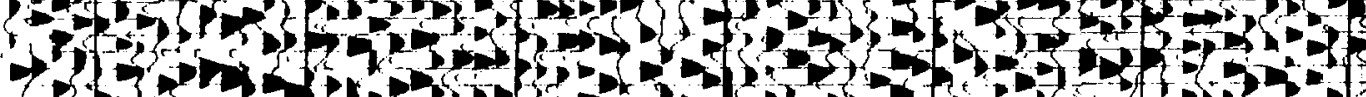

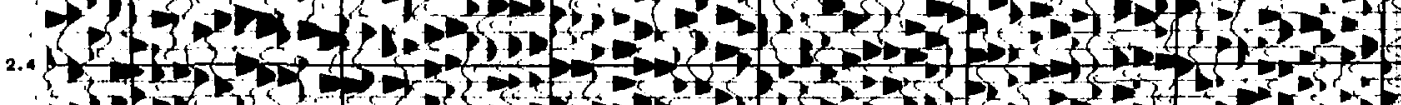

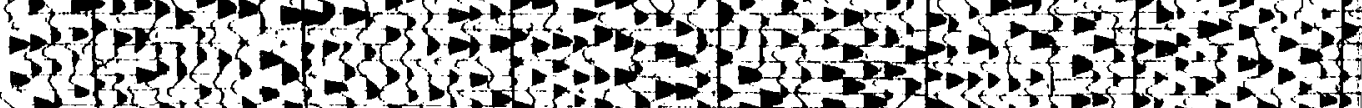

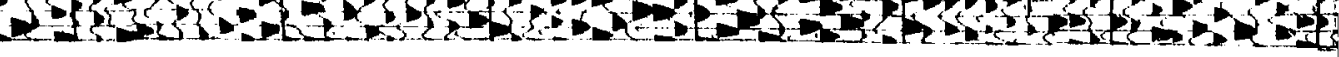




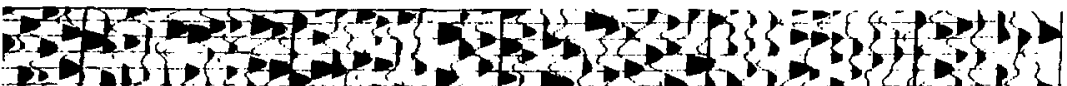

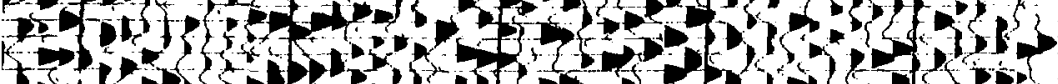

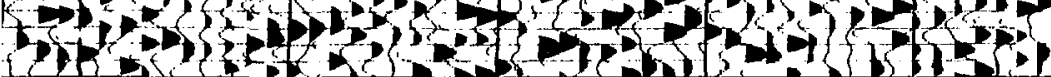

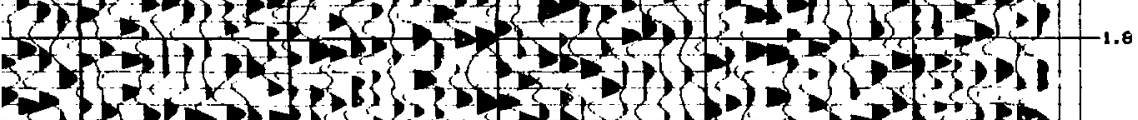

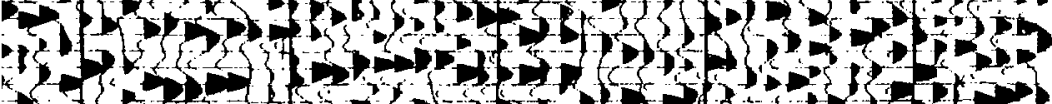

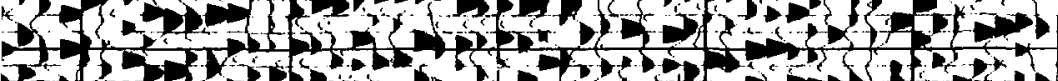

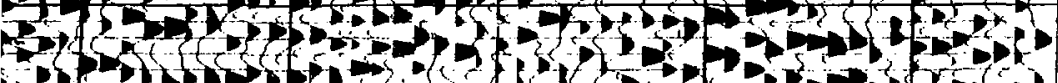

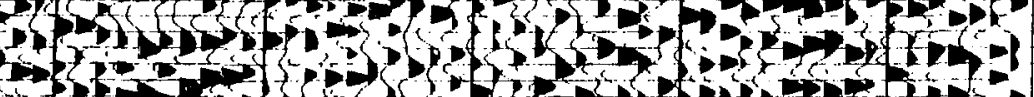

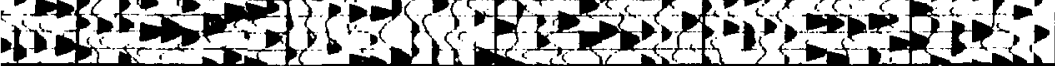

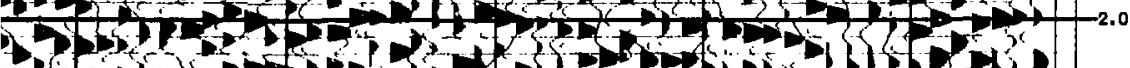

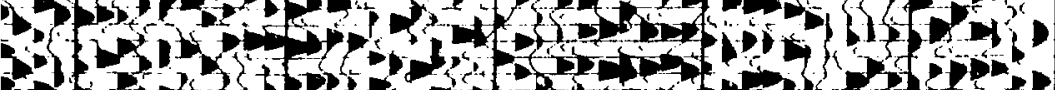

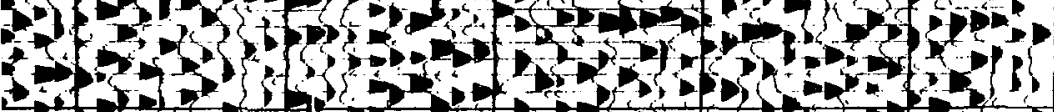

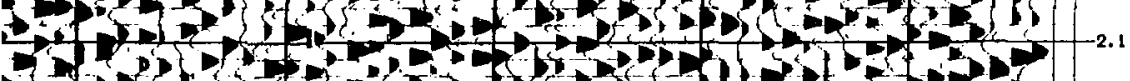

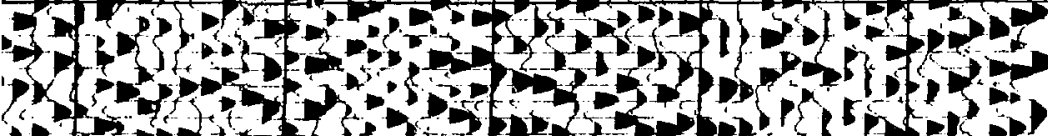

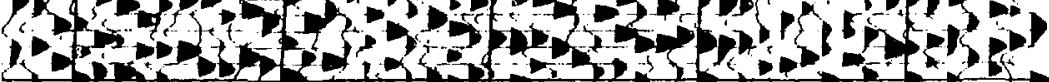

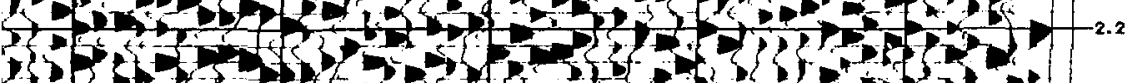

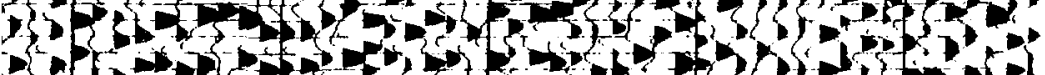

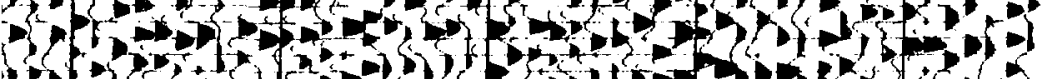

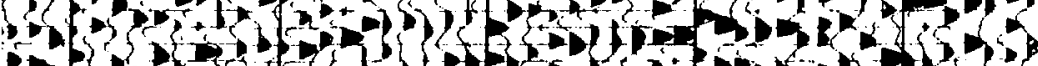

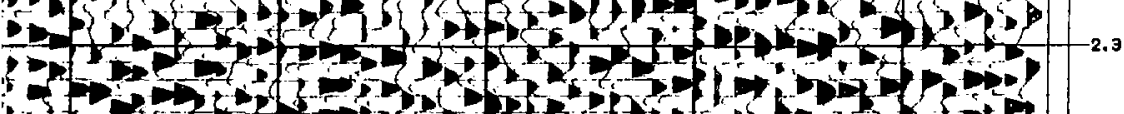
$35+325,5,5$

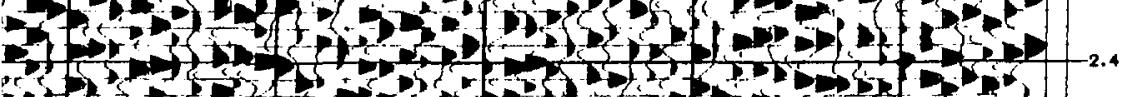

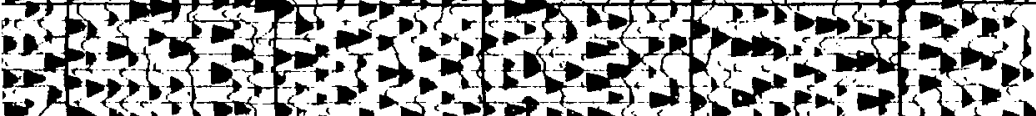

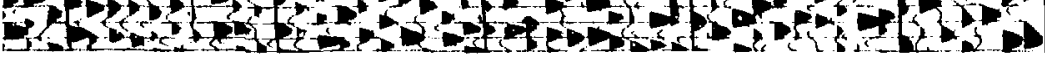

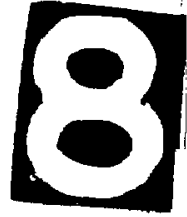




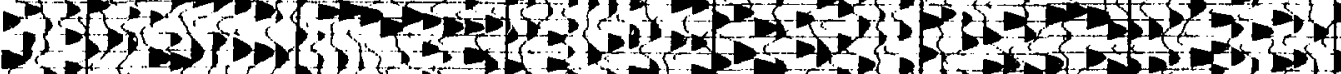

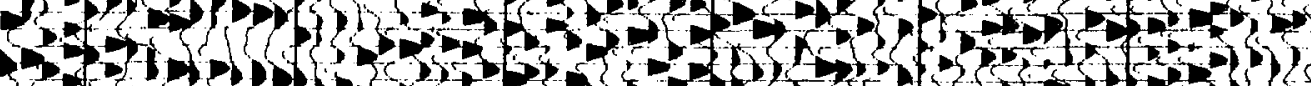

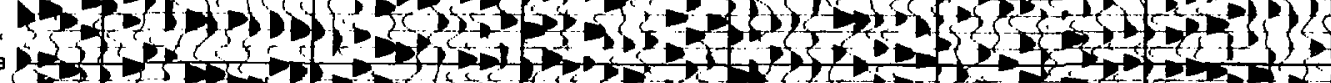
$\left\{\begin{array}{l}3 \\ 3\end{array}\right.$

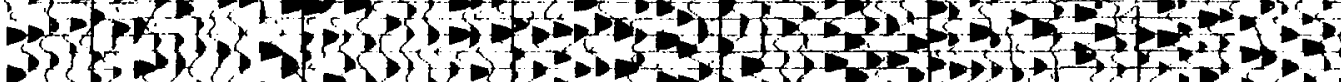

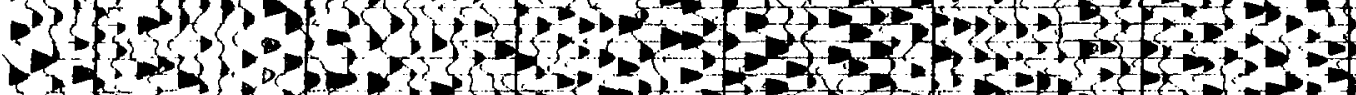
2.8

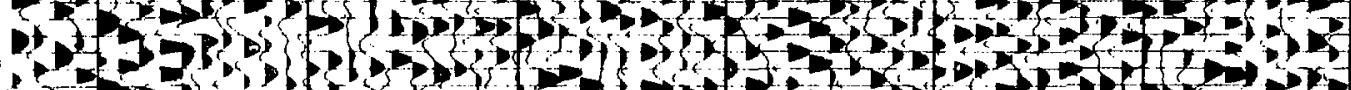
ifos

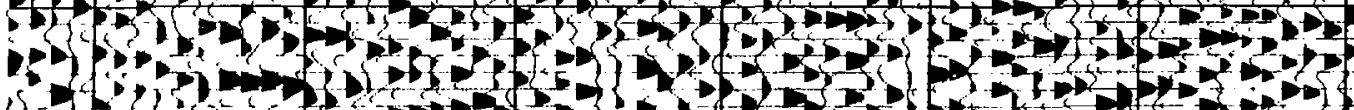

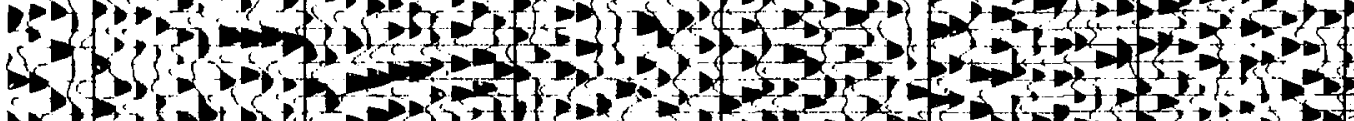

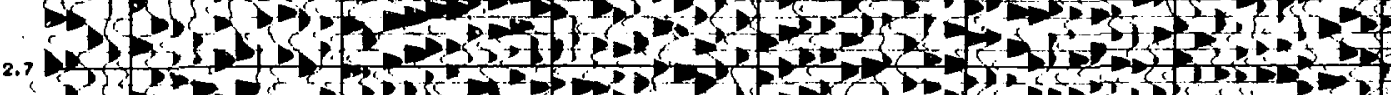

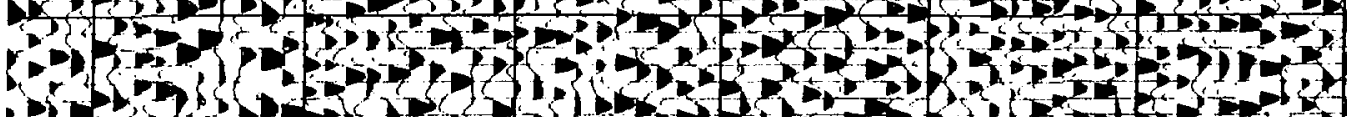

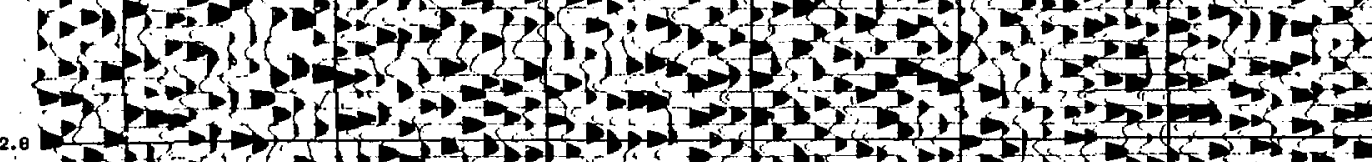

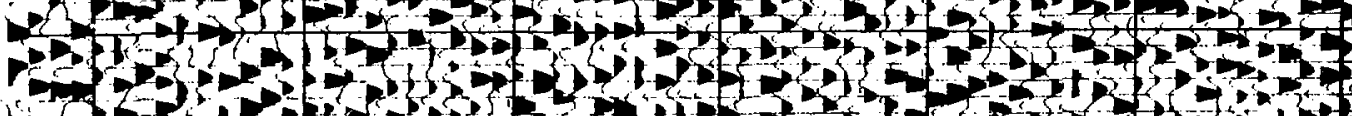

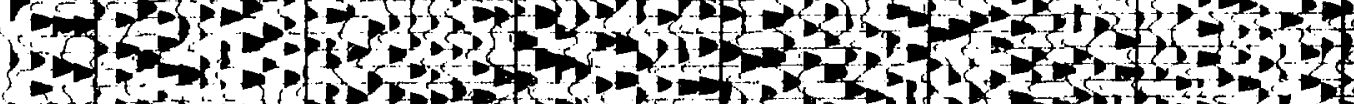

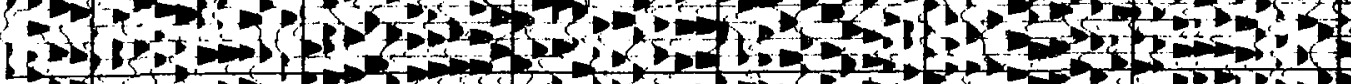

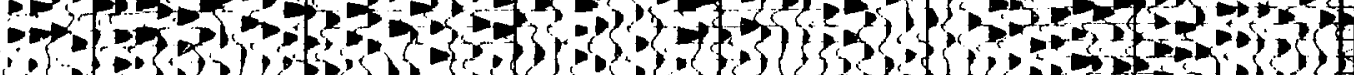

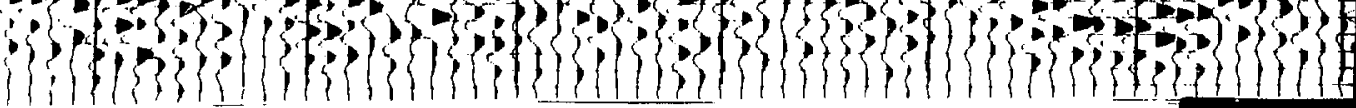


Stor

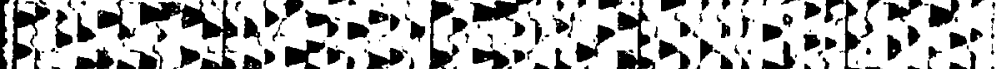
(f)

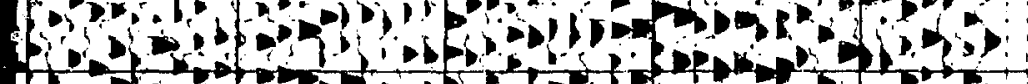

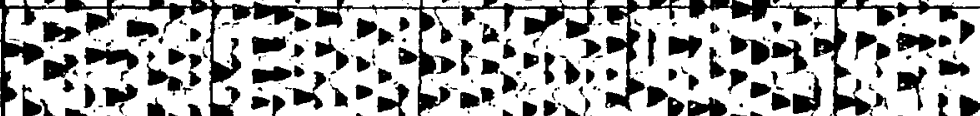

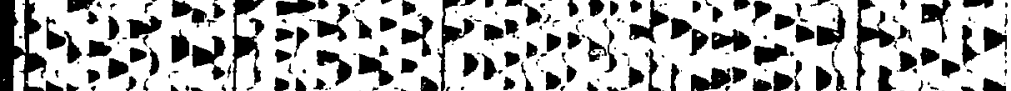

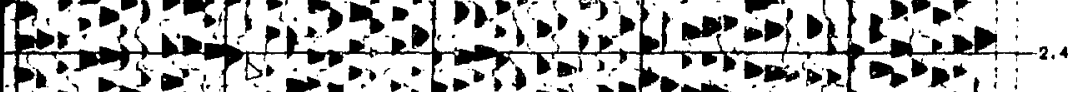
$S_{0}=\left\{\begin{array}{c}5 \\ 0\end{array}\right.$

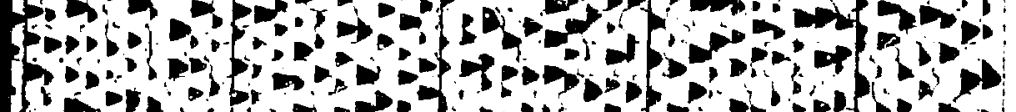
(Le)

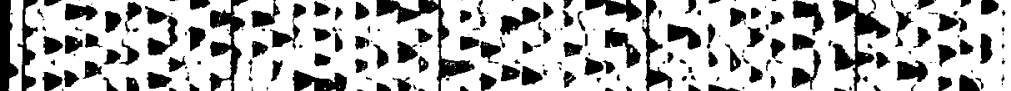

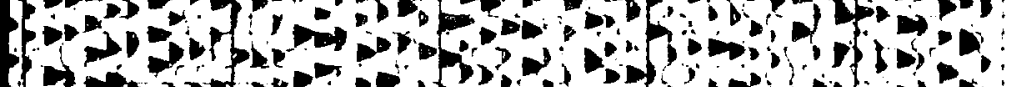

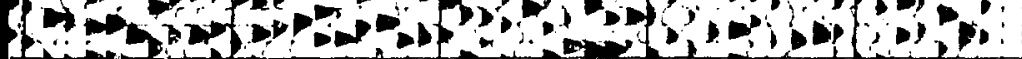

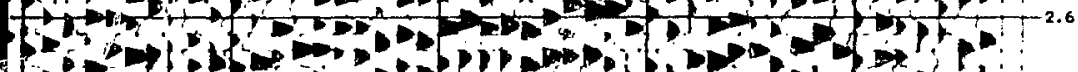

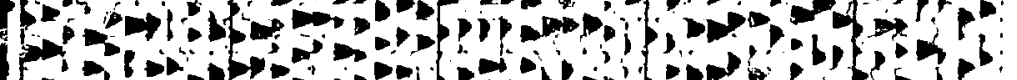

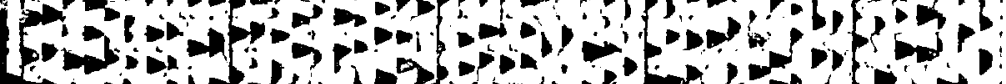

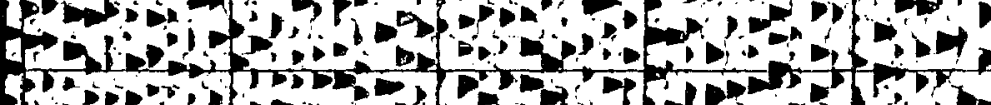
85.5.

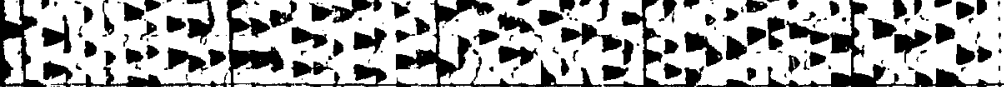

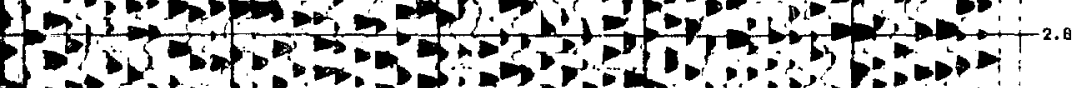

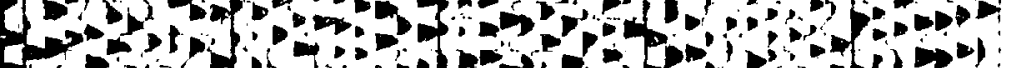

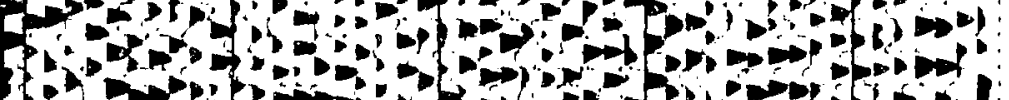
(1) 


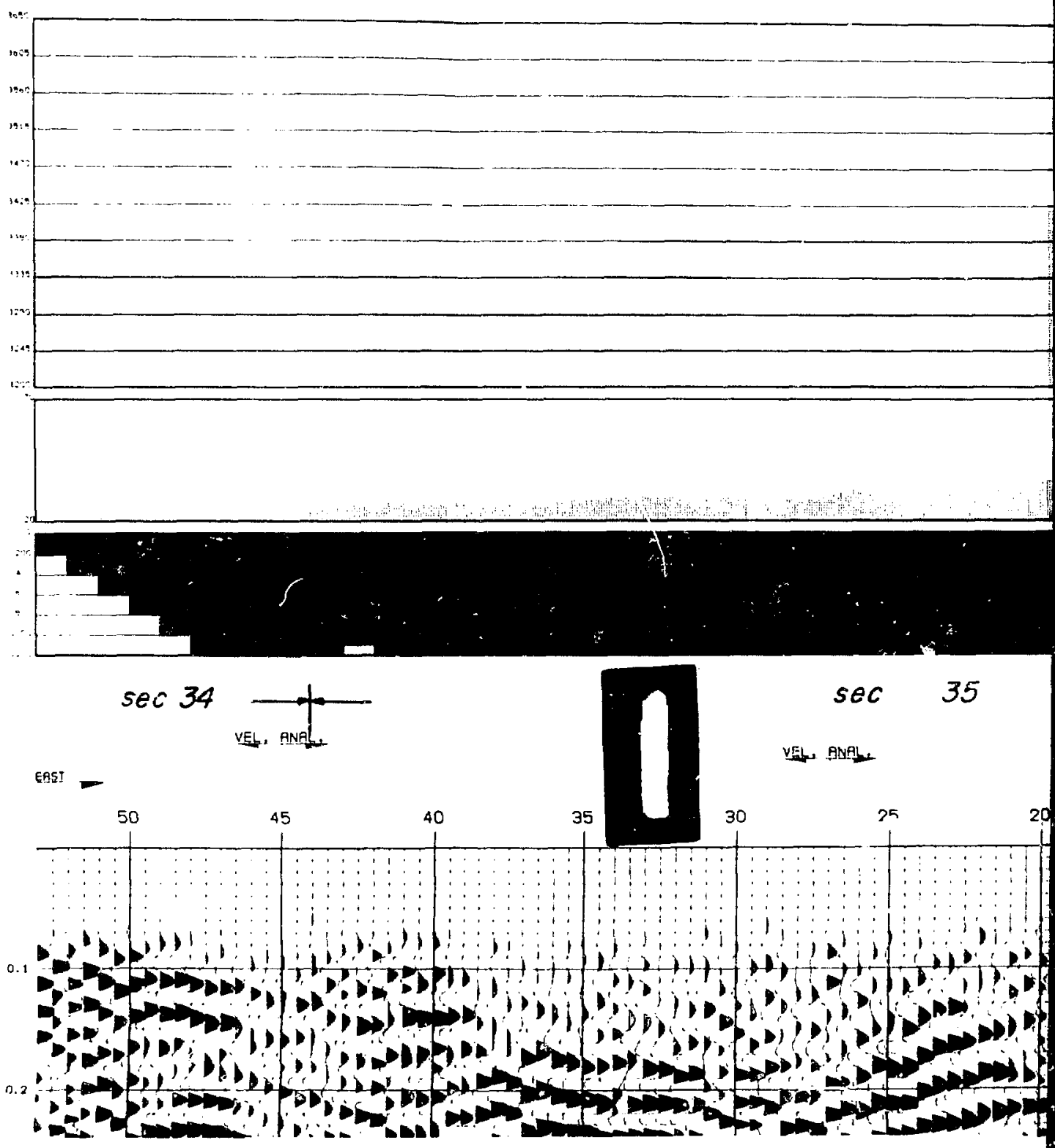


ELEVAT I ONS

STAT ICS

FOLD \%

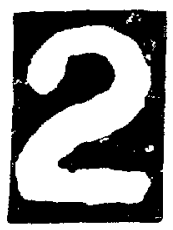

\section{LINE DIRECTION}

VEL, ANAl

VELOCITY FUNCTIBN DIRECTION

STFTIONS 


\section{$5-\infty$}

.

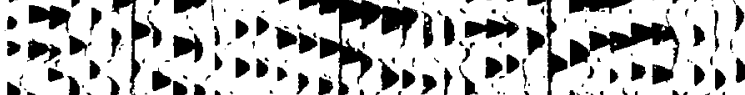

o.

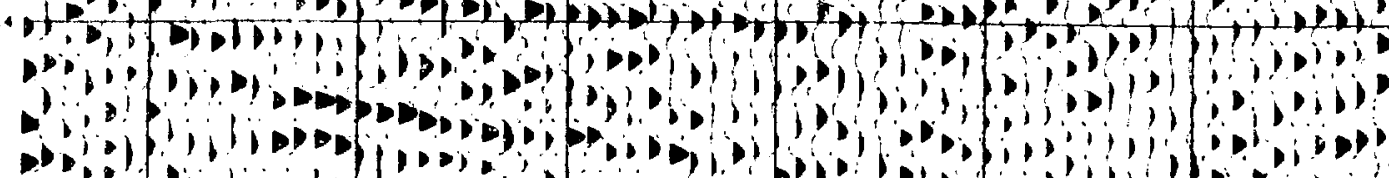

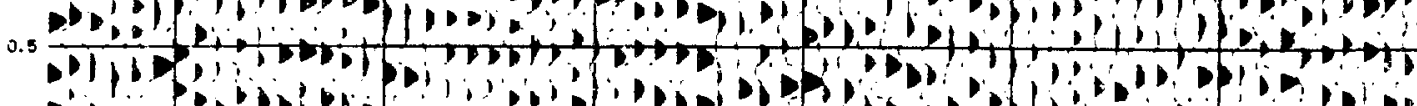
,

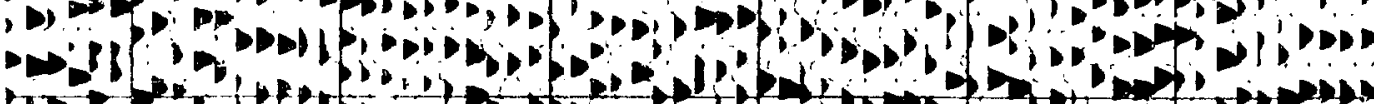

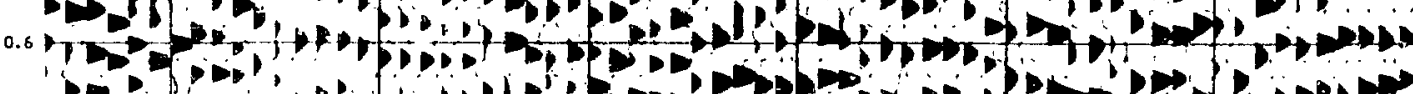

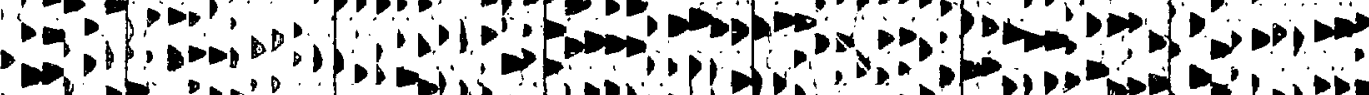

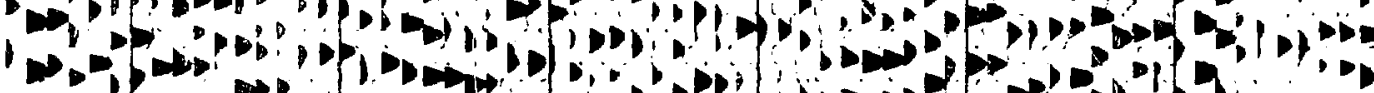

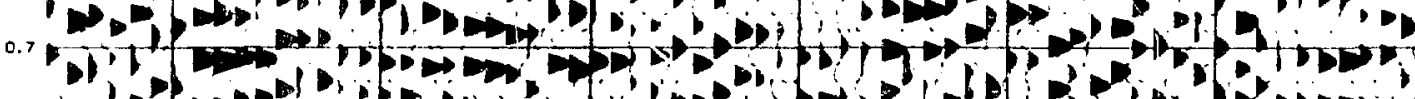

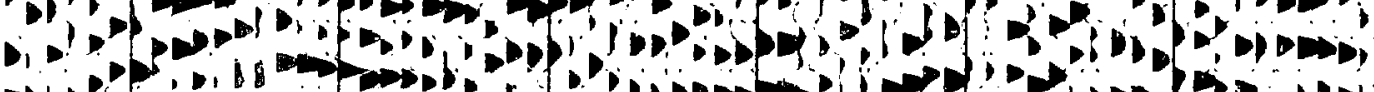

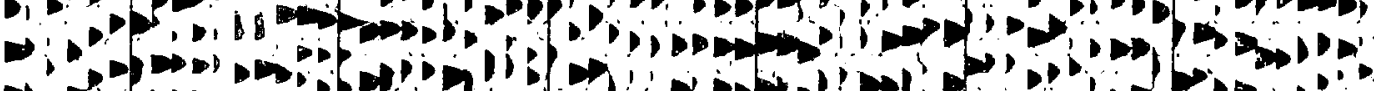

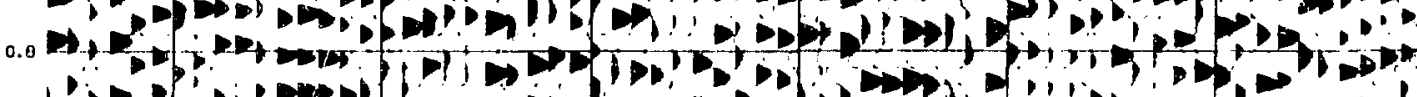
;

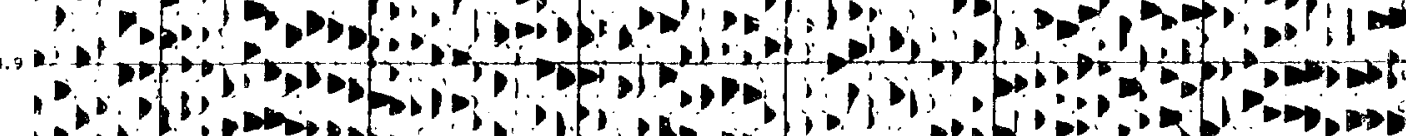

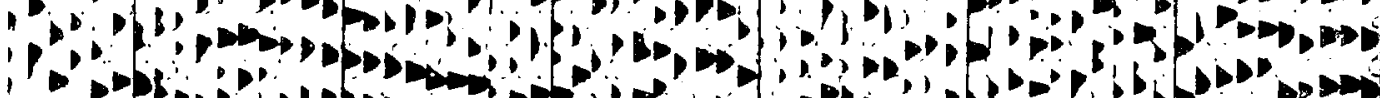

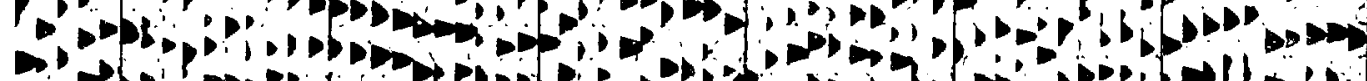

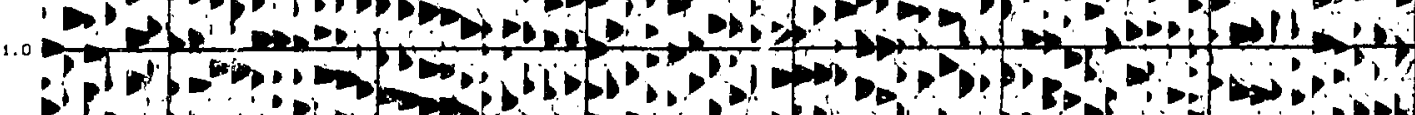

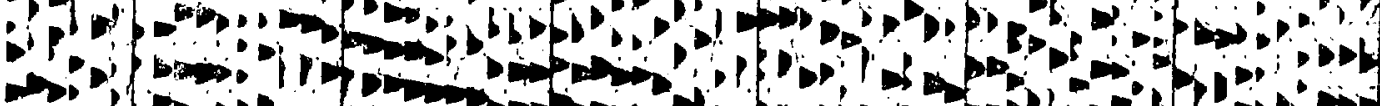

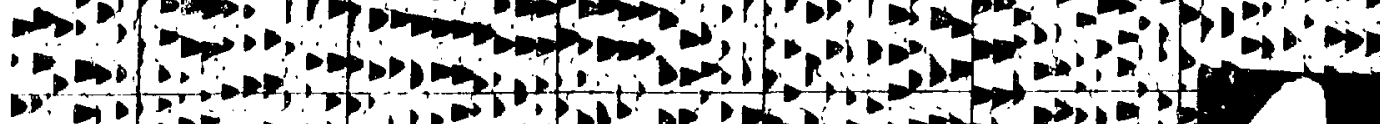

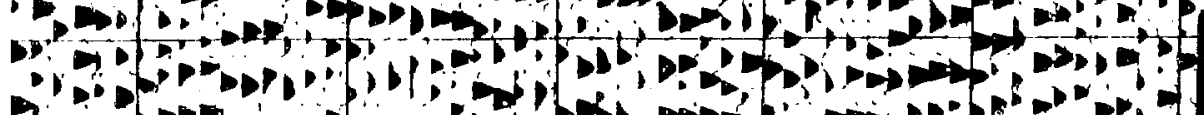

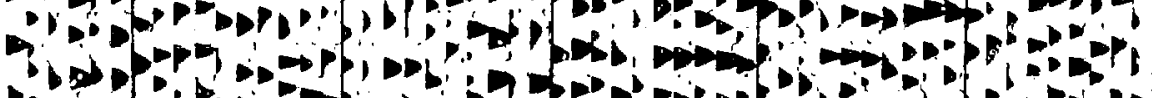

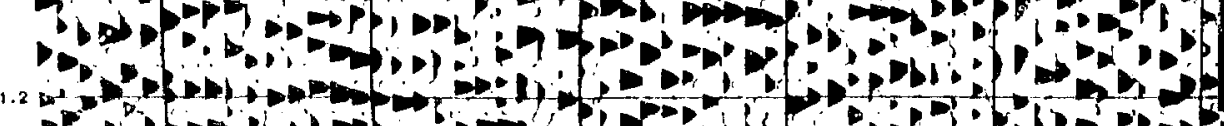

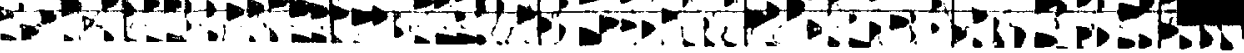



todo

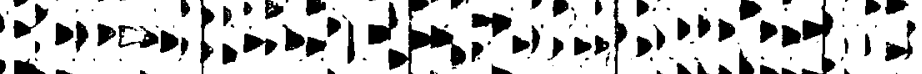

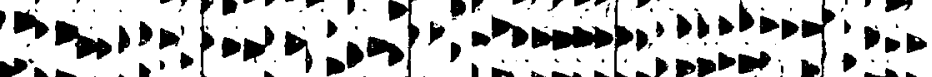

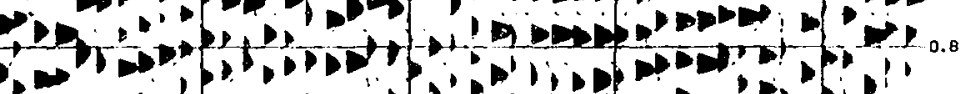

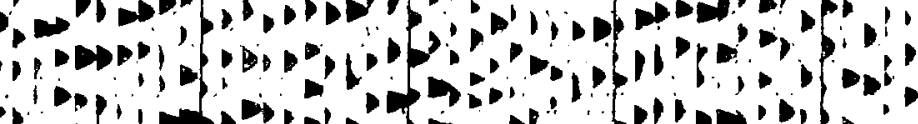

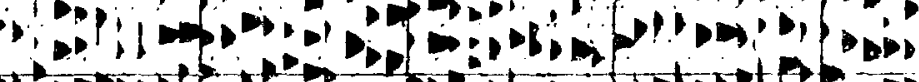

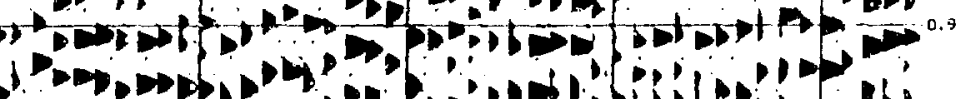

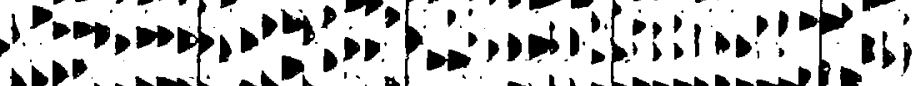

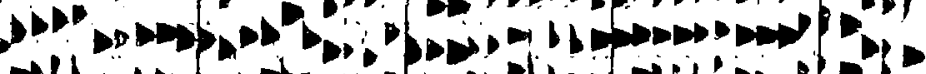

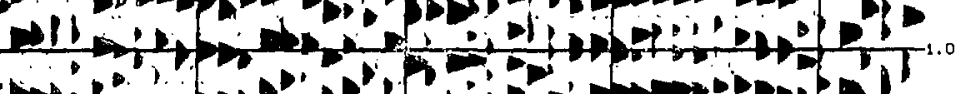

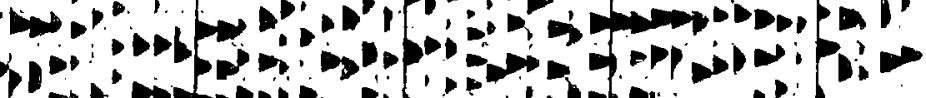

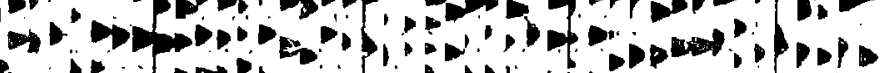

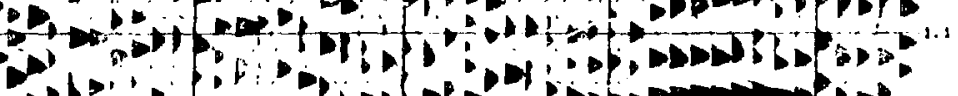

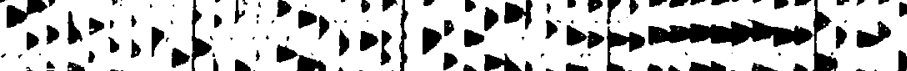

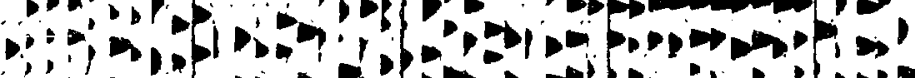

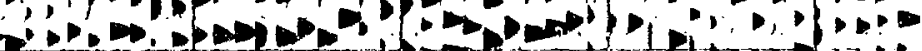

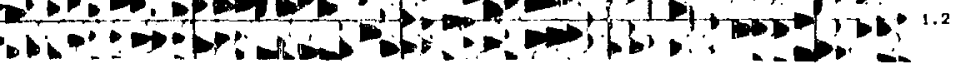

INPUT REEL HERË

REEL MUPEF

MHTER SATPLE/TR SAHLLE RATE

UIN Wunger

Jag Nutas

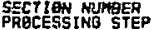

\section{FIELD}

RECBRDED BY: DRESSER QLYMPIC

DATE: NQVEHBER 15,1977

INSTRUMENTS: CFS : - OFS IV

NOTCH FILT: IN

RECBAD LEN: 16 SEC.

SWEEP FREQ: 25-100 H2

STN INY: $\quad 110 \mathrm{FT}$.

GEQ PER STN: 6

ARRAY TYPE: "ULINE

PROCESSINE

PRECESSEO aY 0

STATICS COMPUTATION

OATUK: $\quad 3200 \mathrm{FT}$.

VSW: $\quad$ GODO FT/SEC.

1) DEMULTIPLEX

2) BINARY GAIN RECQVERY

3) VIAROSEIS CBRRELRTION

42

5) OECENVRUTIEN

UPERATER LENGTHAI 40 KILS

PREDICYIEN TIKE BRSED BN

6) TIME-INVARIANT DIGITAL FR 0.0-3.0 SEC, 25-60 HZ

7) RPPLY ORTUM STATICS

B) VELBCI:-' RNALYSIS

9) RPPLY NMB

10: FIRST BREAK SUPPRESSION

11) STACK 12 FaL 0

(2) TIME-INYRRIRNT OIGITAL FA 0.0-3.0 S:-

25-60 HI

13) DIGITRL RGC

14: O1SPLAY

0 TR/IH
10 iN/SEC.

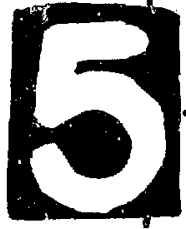




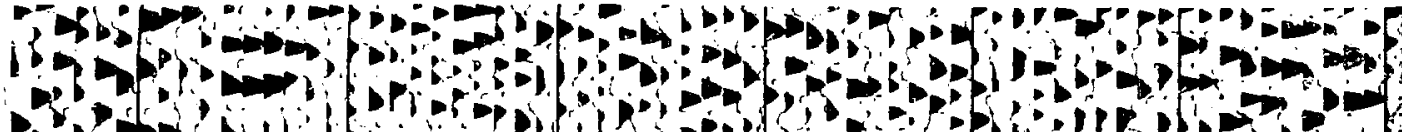

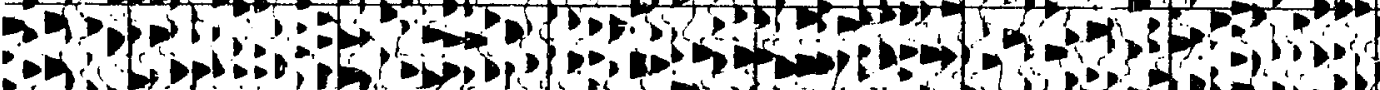

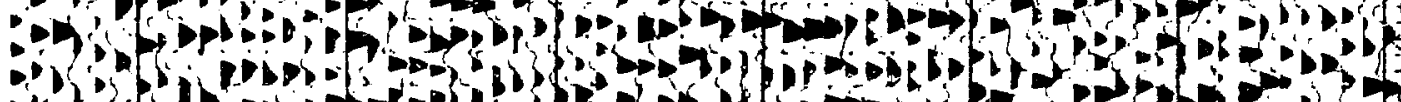

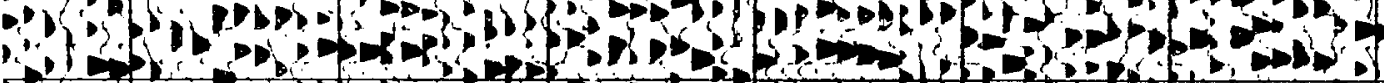

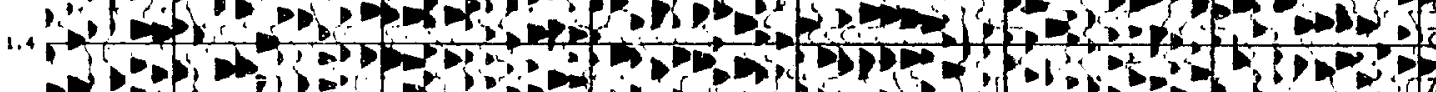

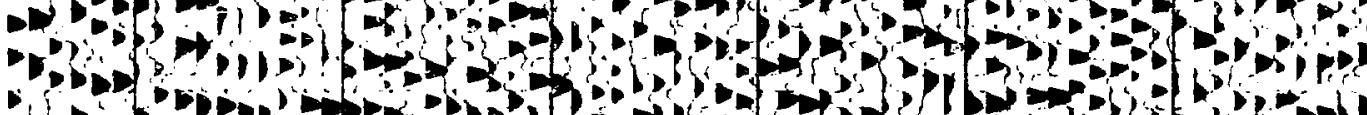

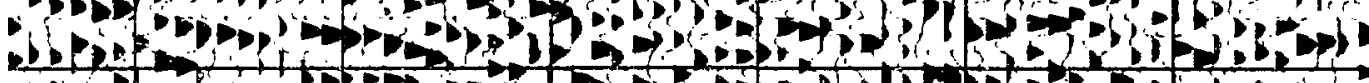

$\Rightarrow$ -

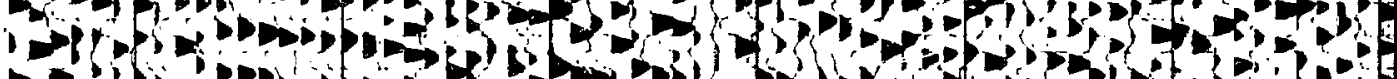
a -120 is

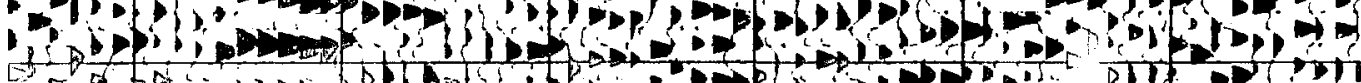

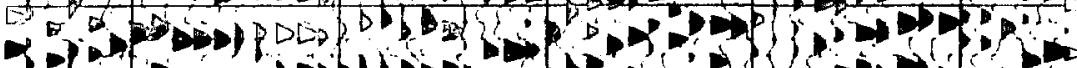

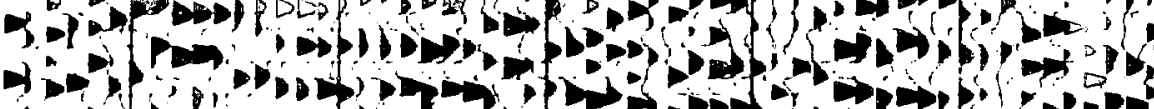

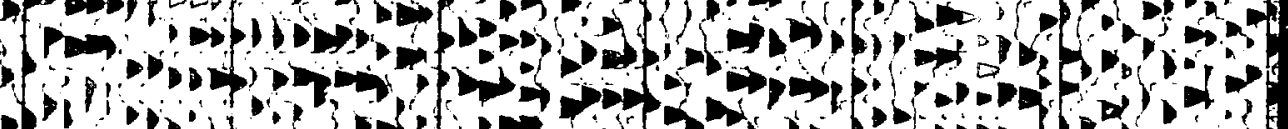

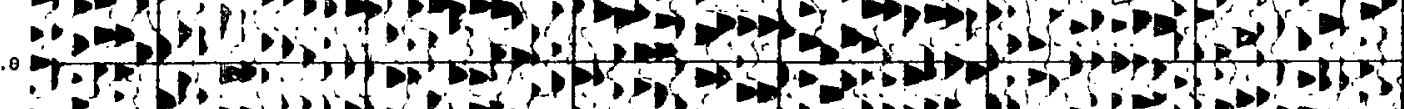

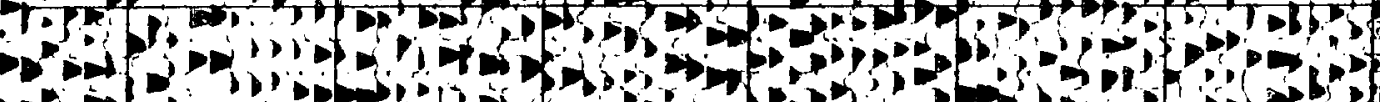

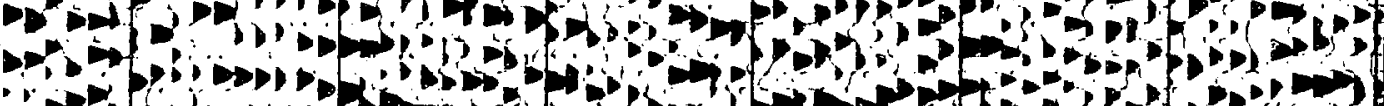

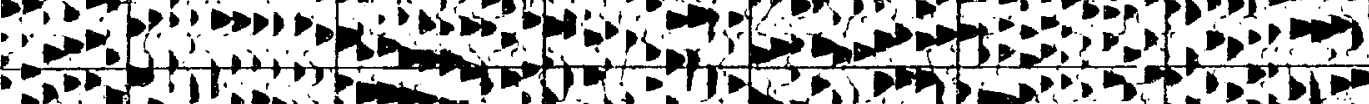

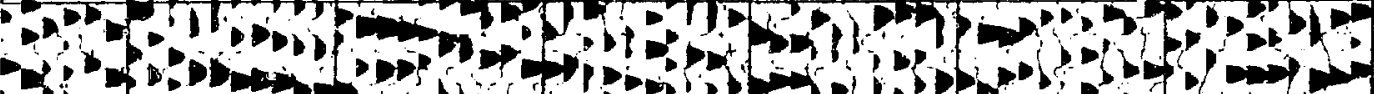

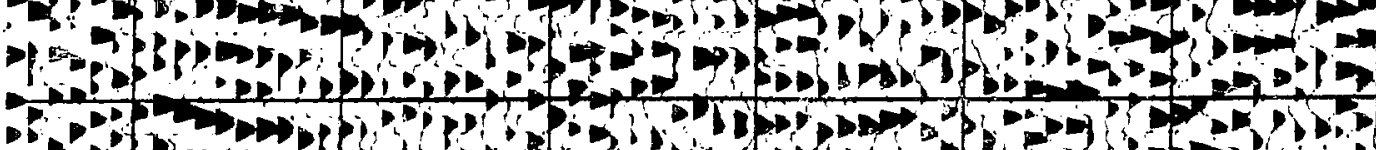

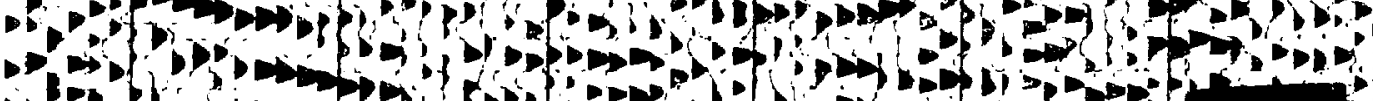

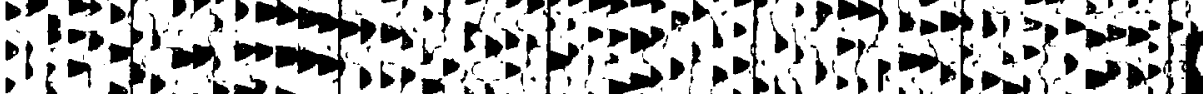

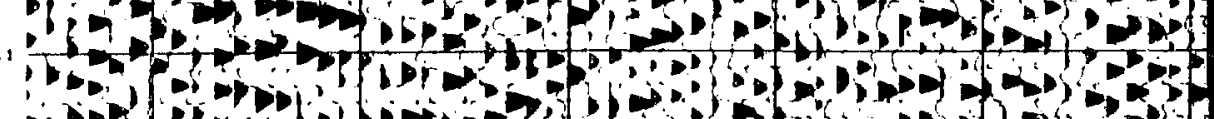

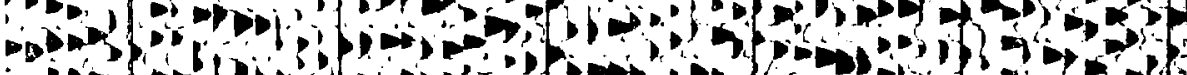

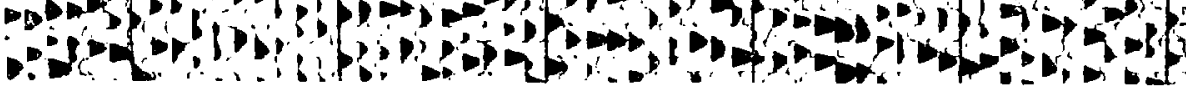




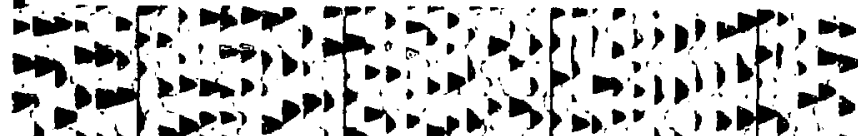

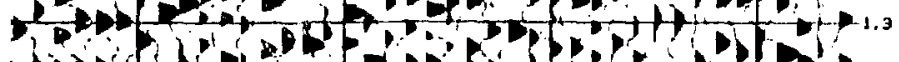

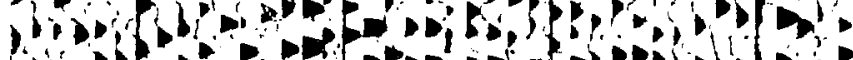

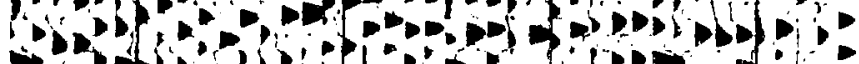
$\Leftrightarrow$ of

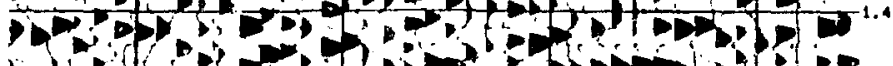

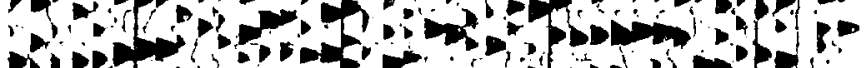
Hipl

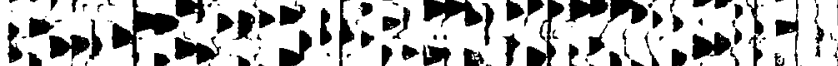

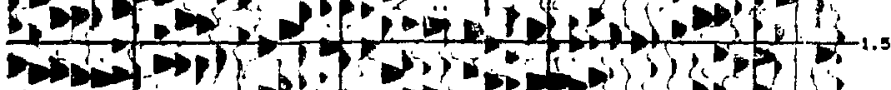
-

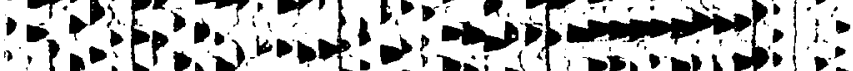

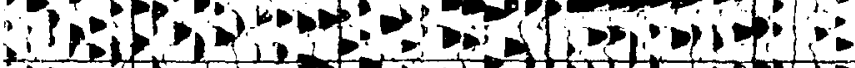

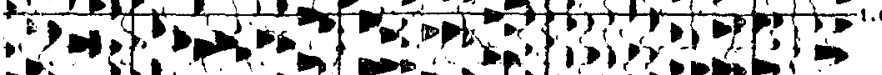

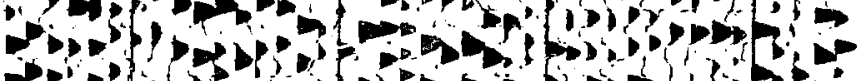

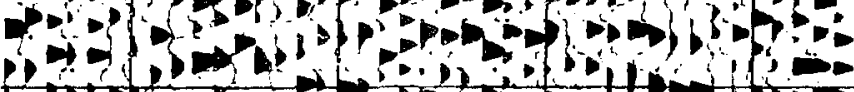
$\Rightarrow$ of

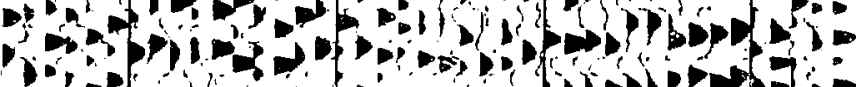

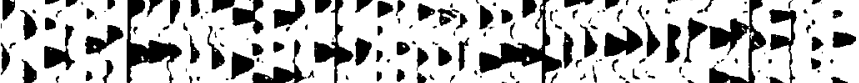
of

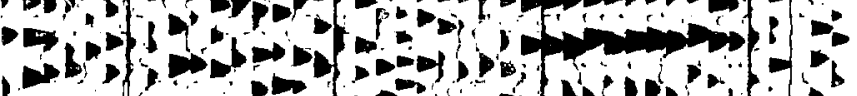

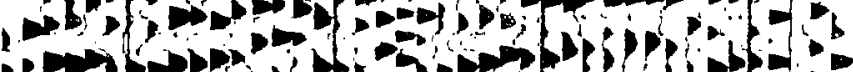
P,

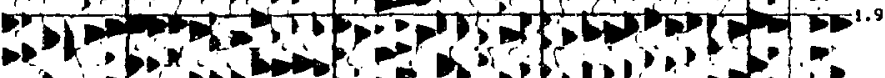

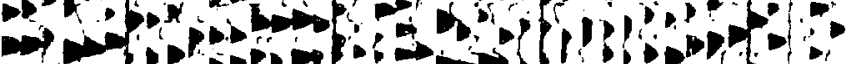

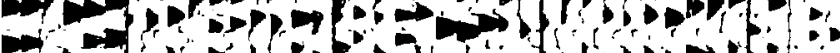

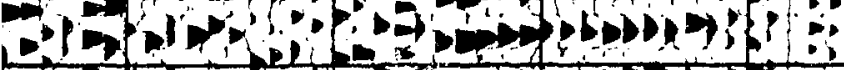

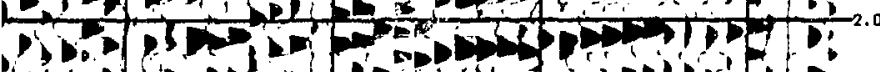

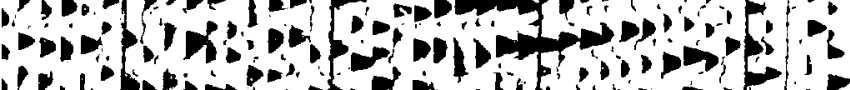

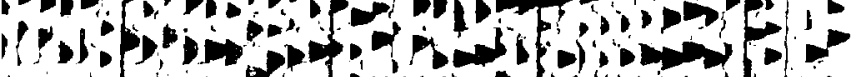

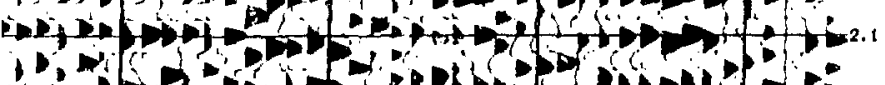

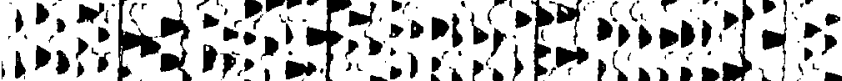

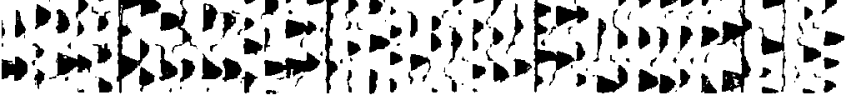

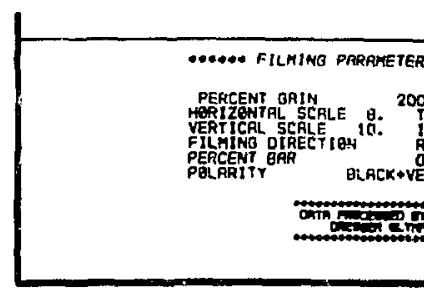


5

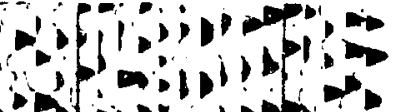

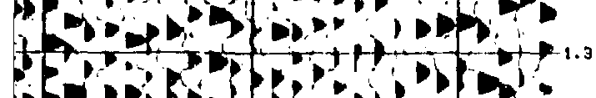

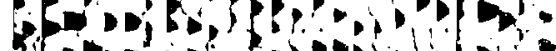

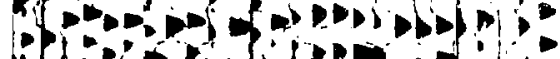

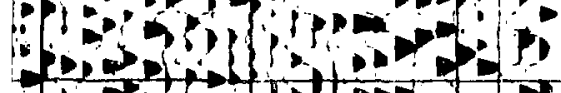

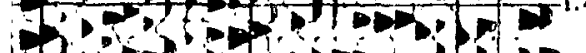

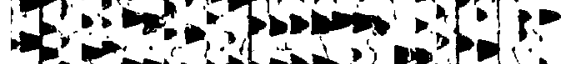

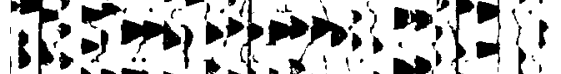

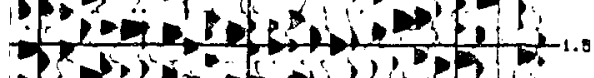
atom?

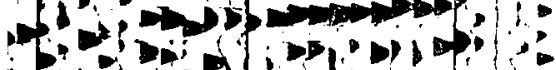
$\left\{\begin{array}{l}0 \\ -1\end{array}\right.$ -

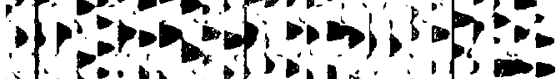
LPin

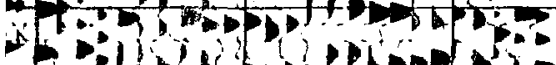

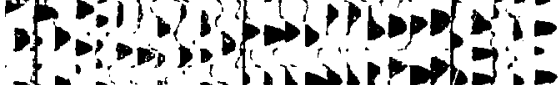

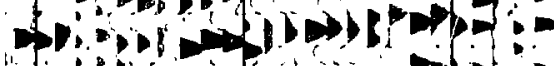
$\begin{cases}\rightarrow \infty & \end{cases}$

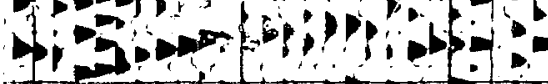

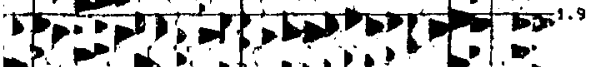
?

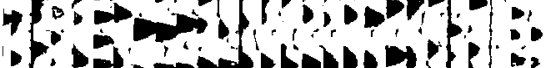

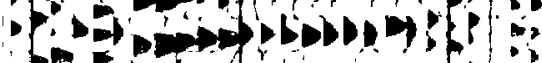

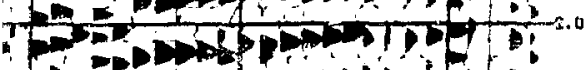

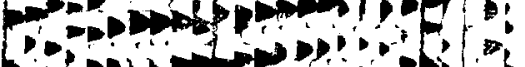

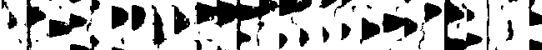

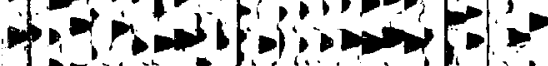

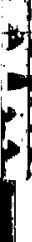




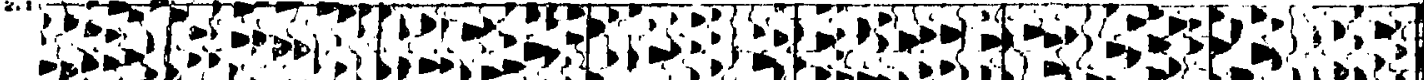

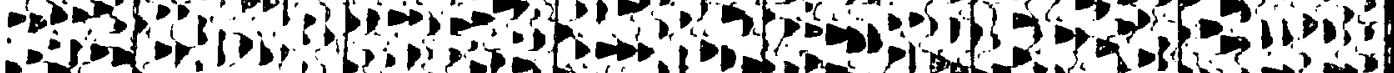

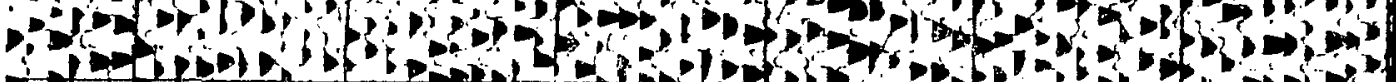

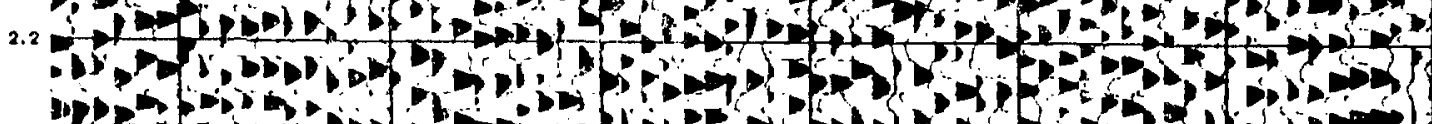

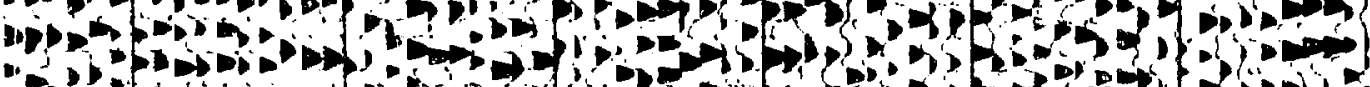

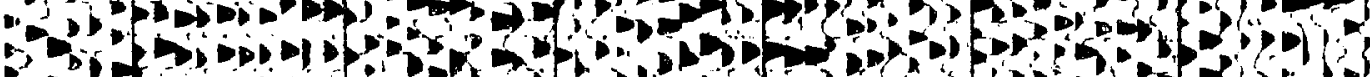

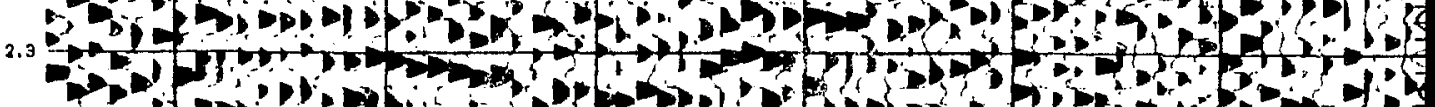

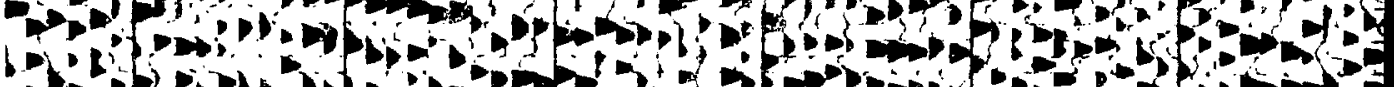

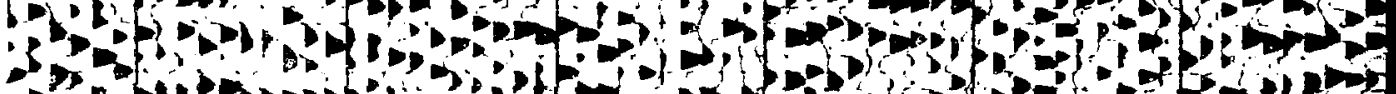

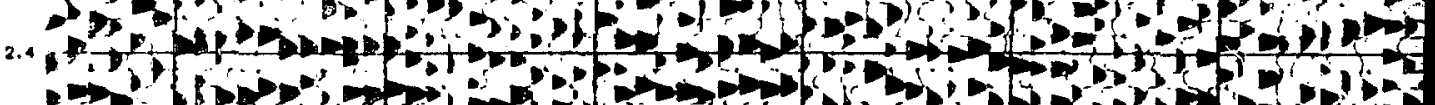
- 1

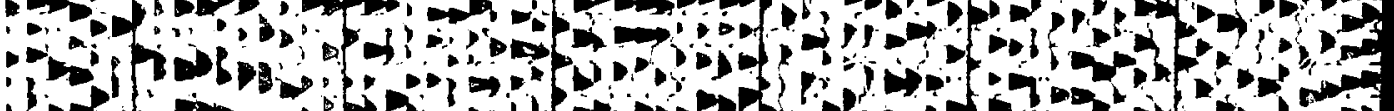

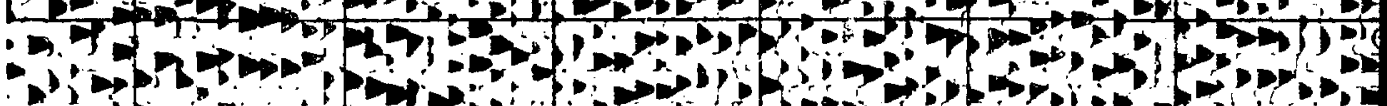

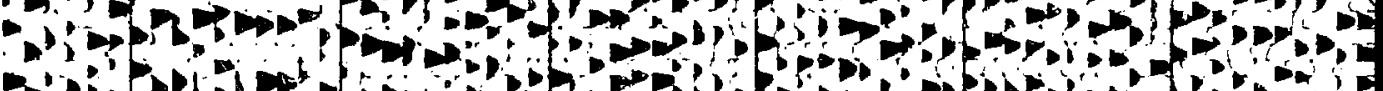

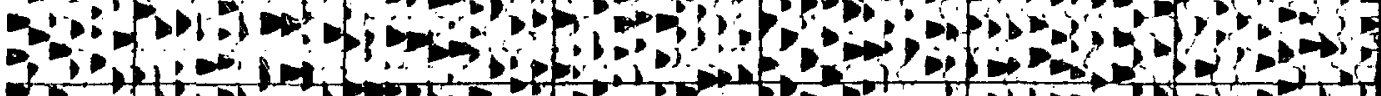

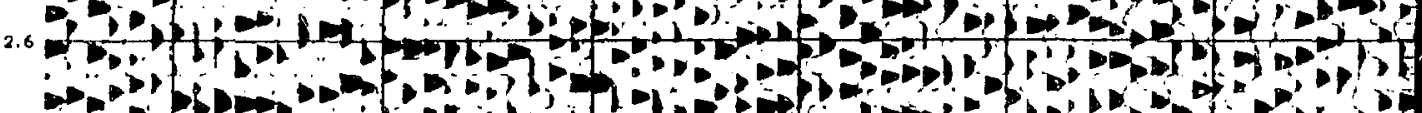
$\therefore$ :

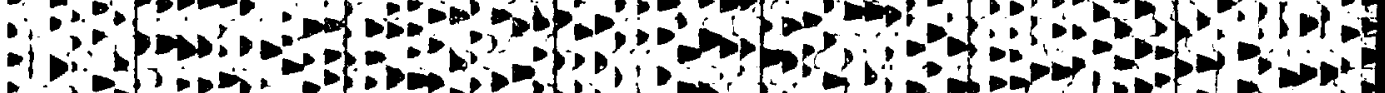

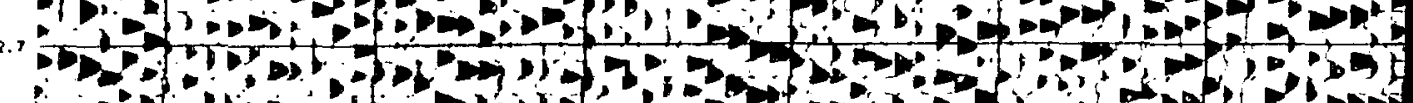

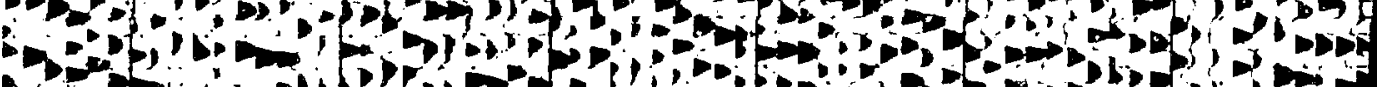

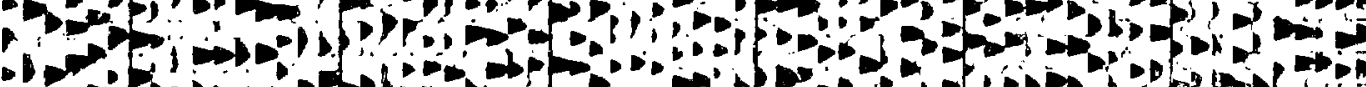

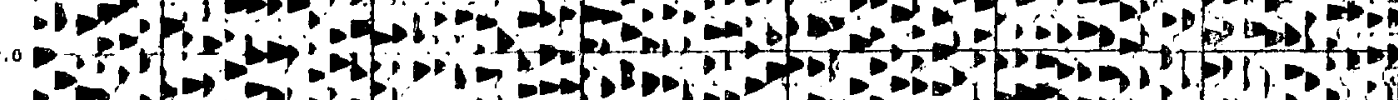

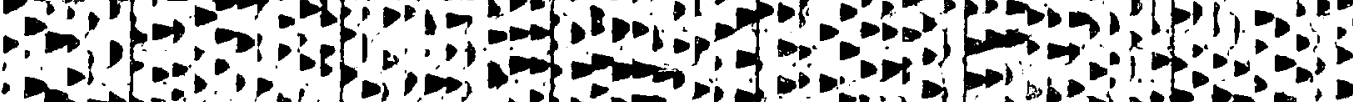

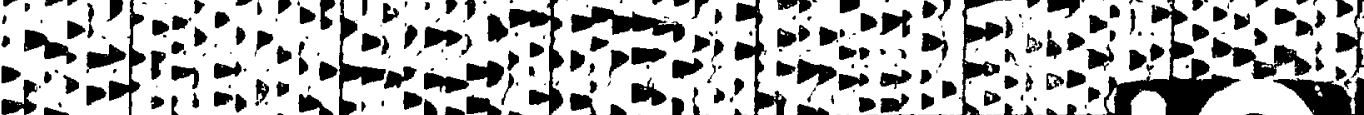

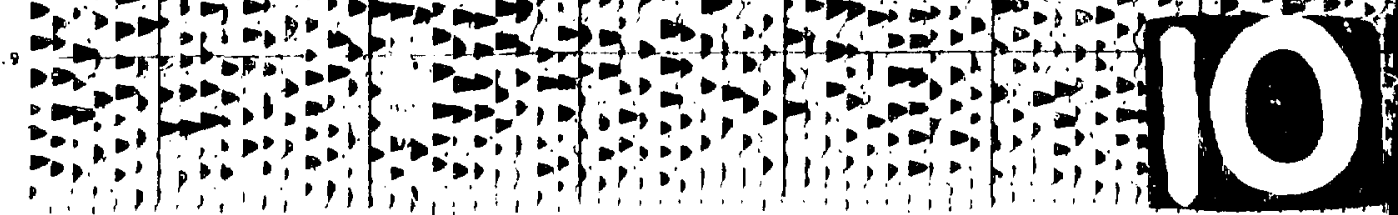




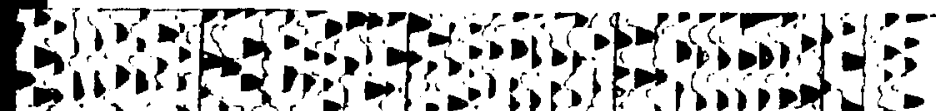

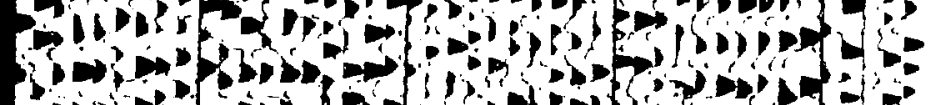

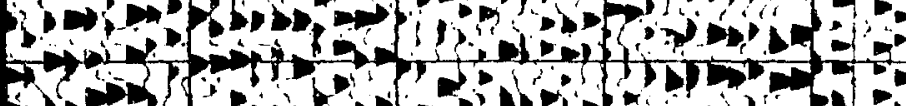
- Rons H

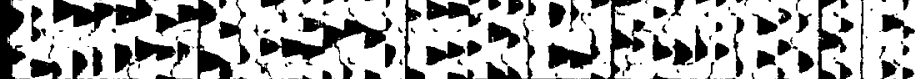

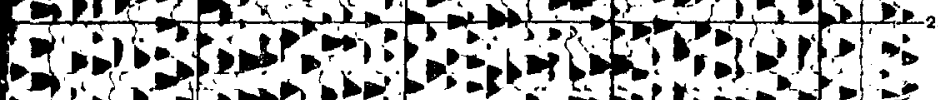

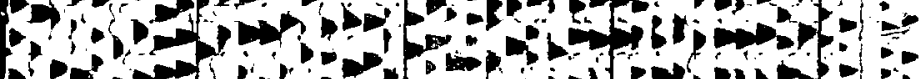

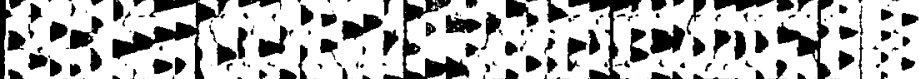
$\rightarrow 10+0$

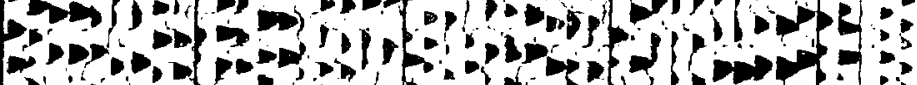

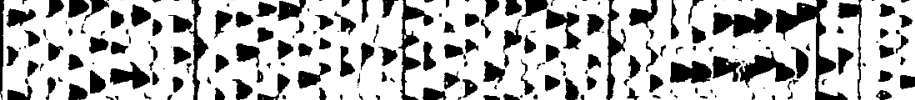

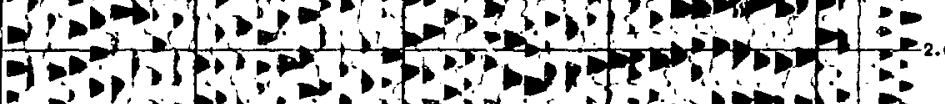

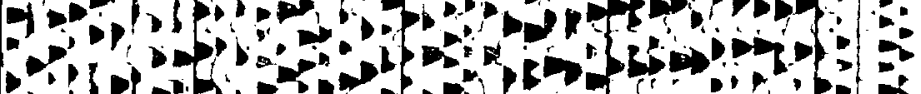
Pon

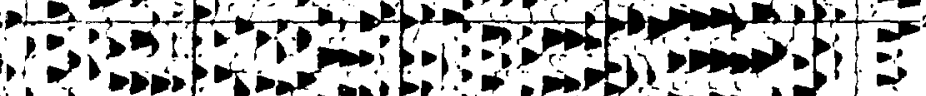

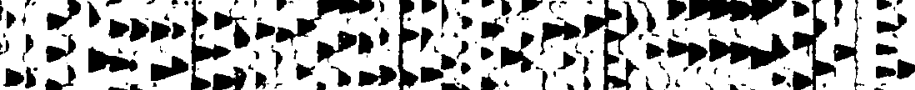

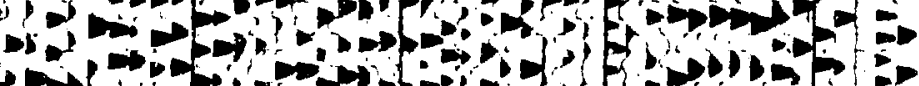
ond

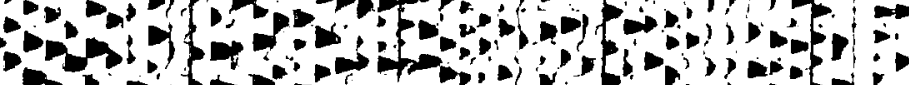
$\rightarrow \rightarrow-\infty$ - 1 (1)

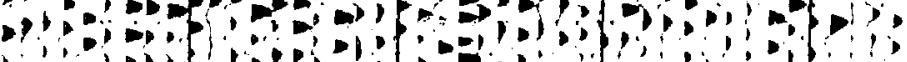

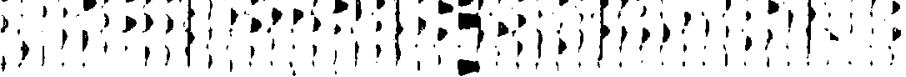

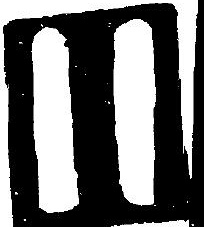




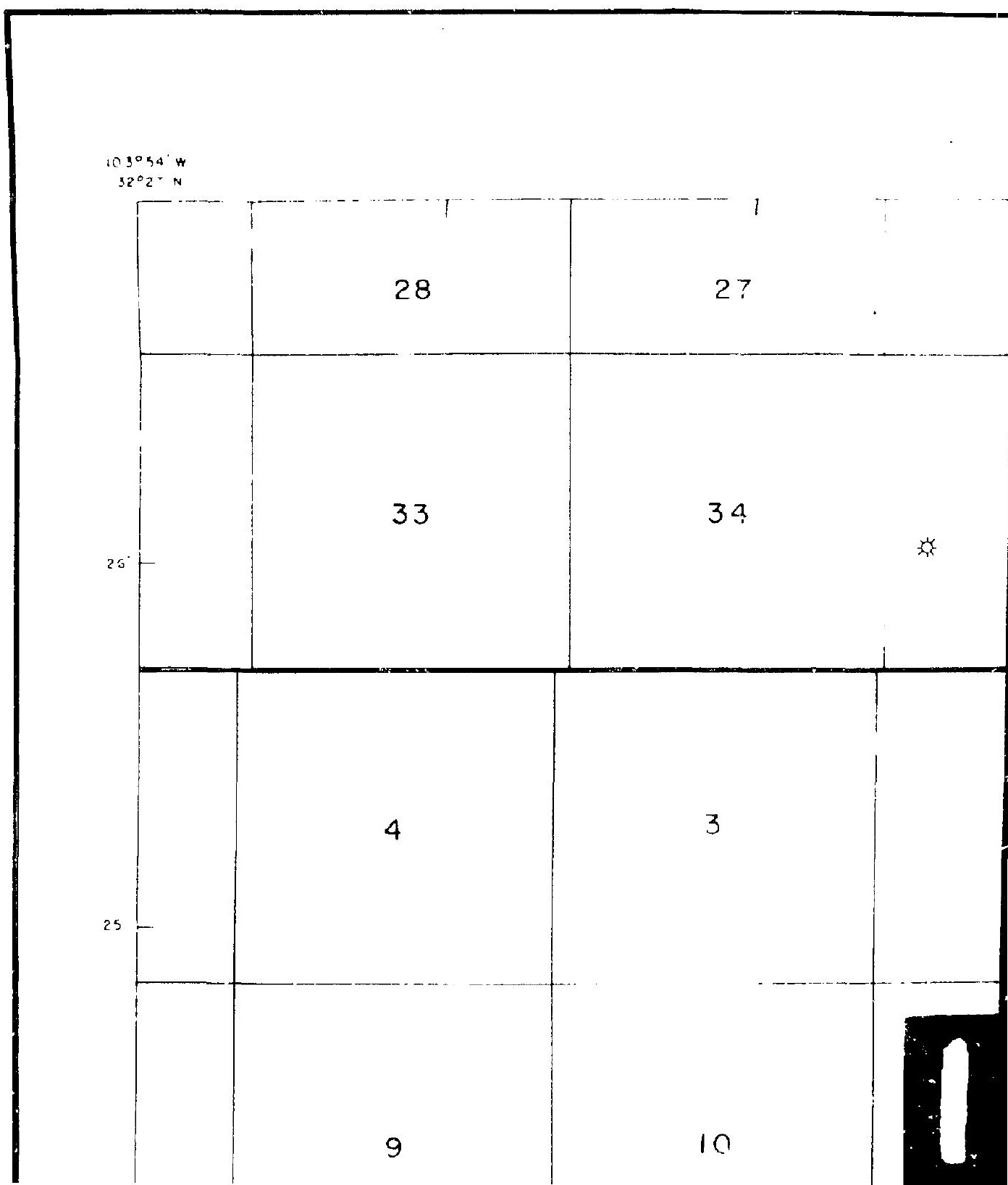


26

35

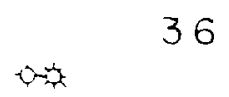

25

36

31
2

$x^{4}$

$$
z^{J-\varepsilon}
$$

11 $x x^{c-1}$

12

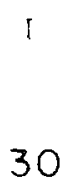

29

32
6

5 
$103^{\circ} 41^{\circ} \mathrm{W}$

$32^{\circ} 27^{\prime}$ N

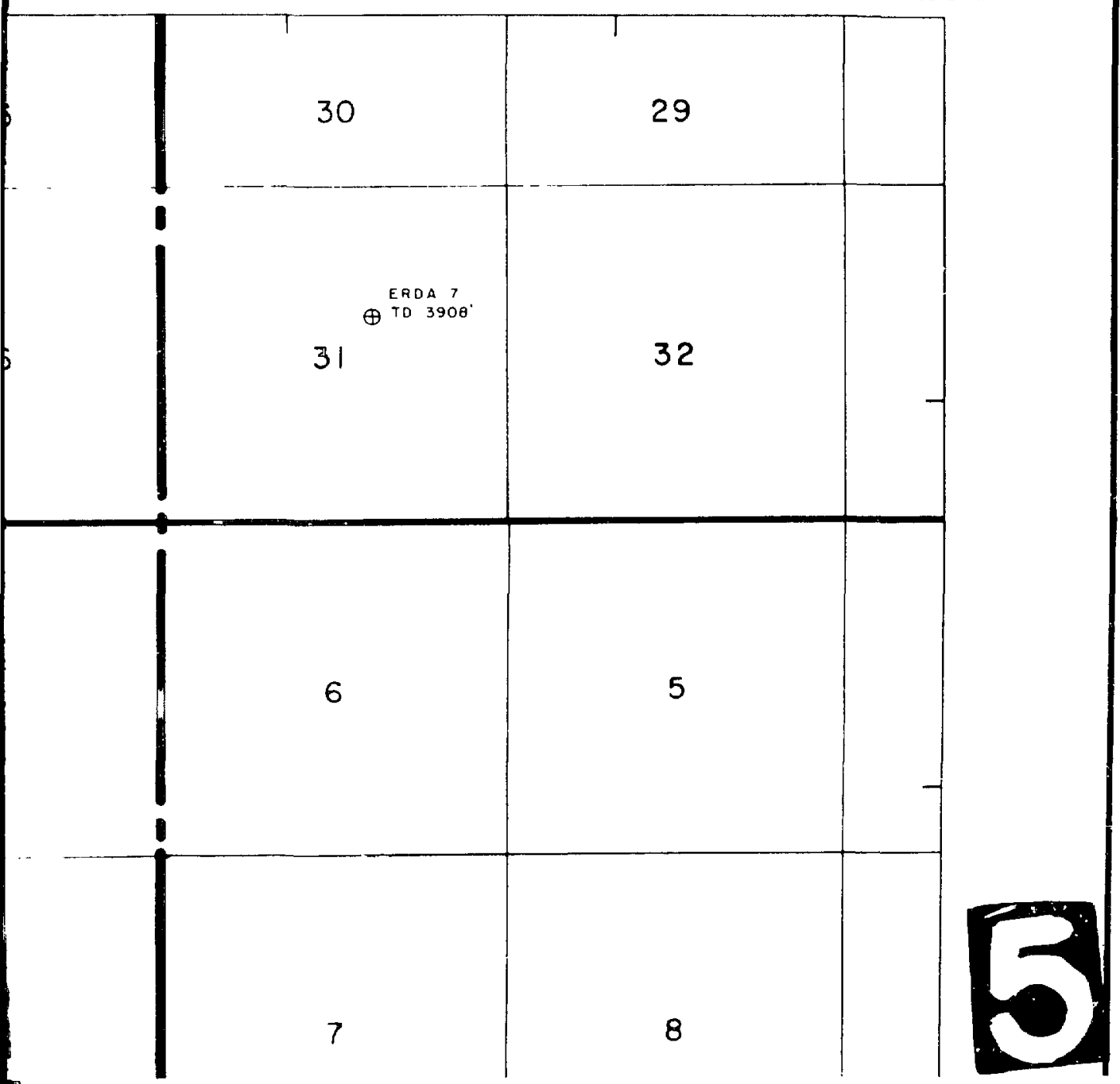




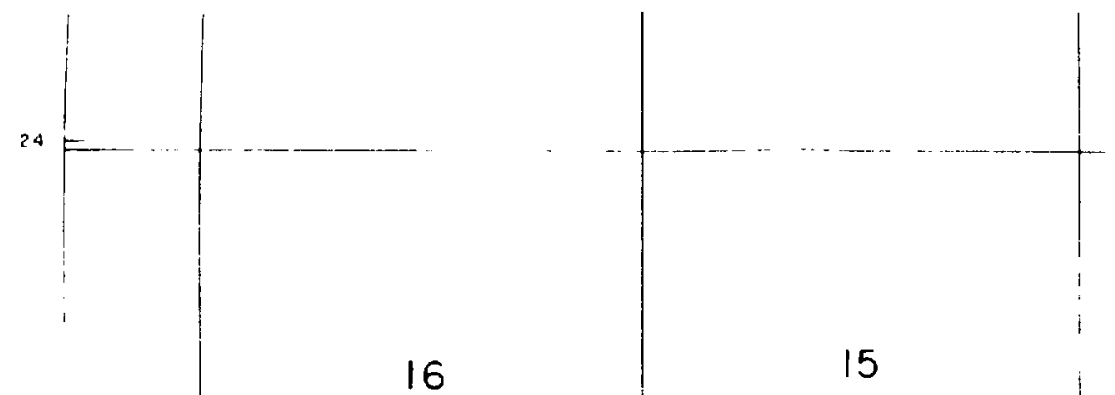

R

21

28

27

22

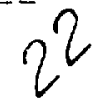

$\because 1$
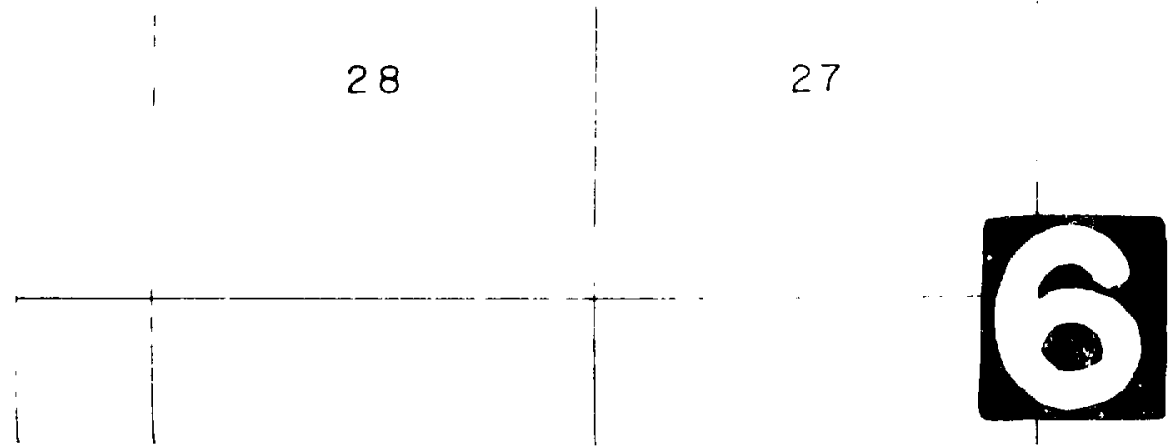
1.30

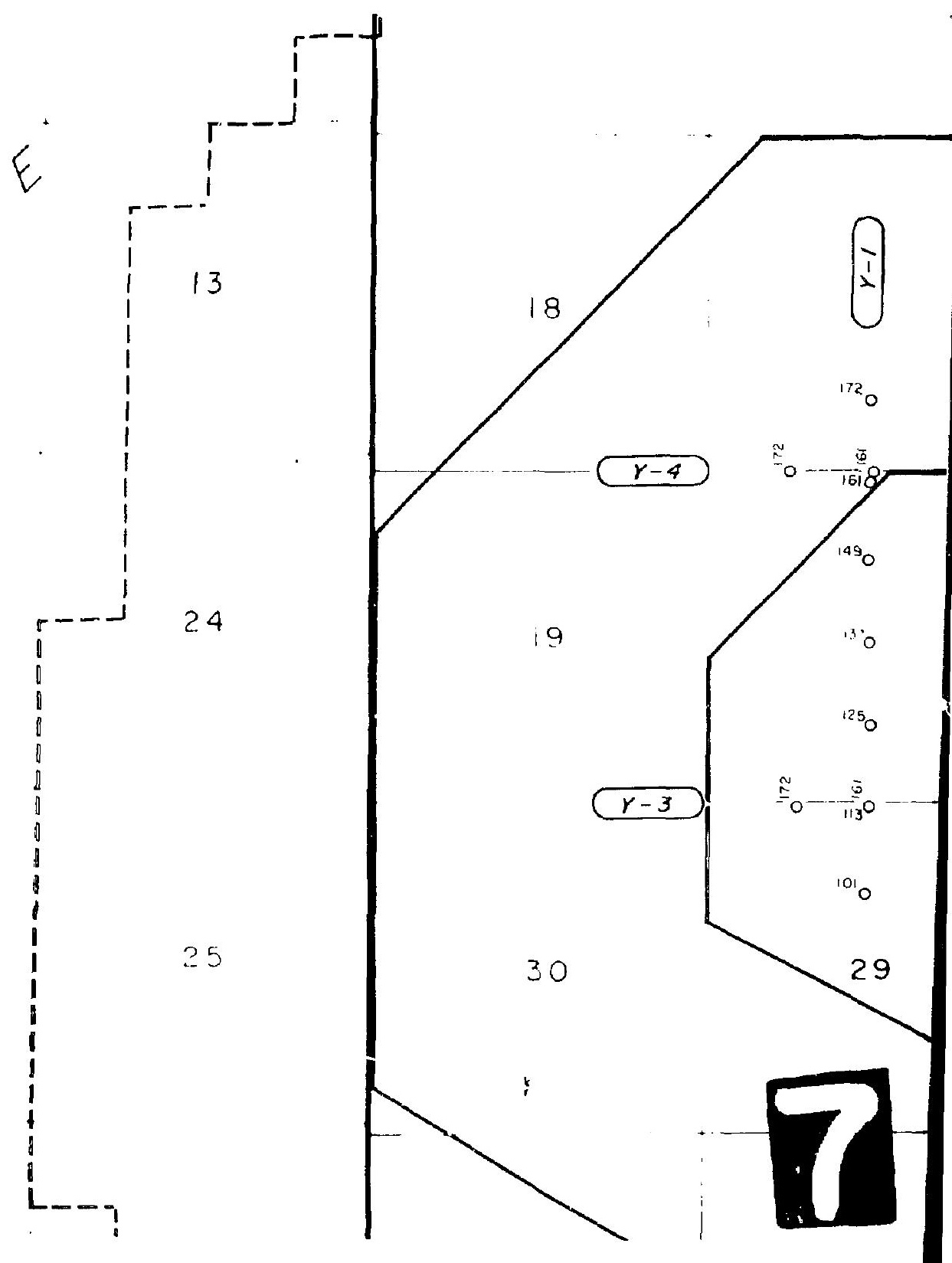




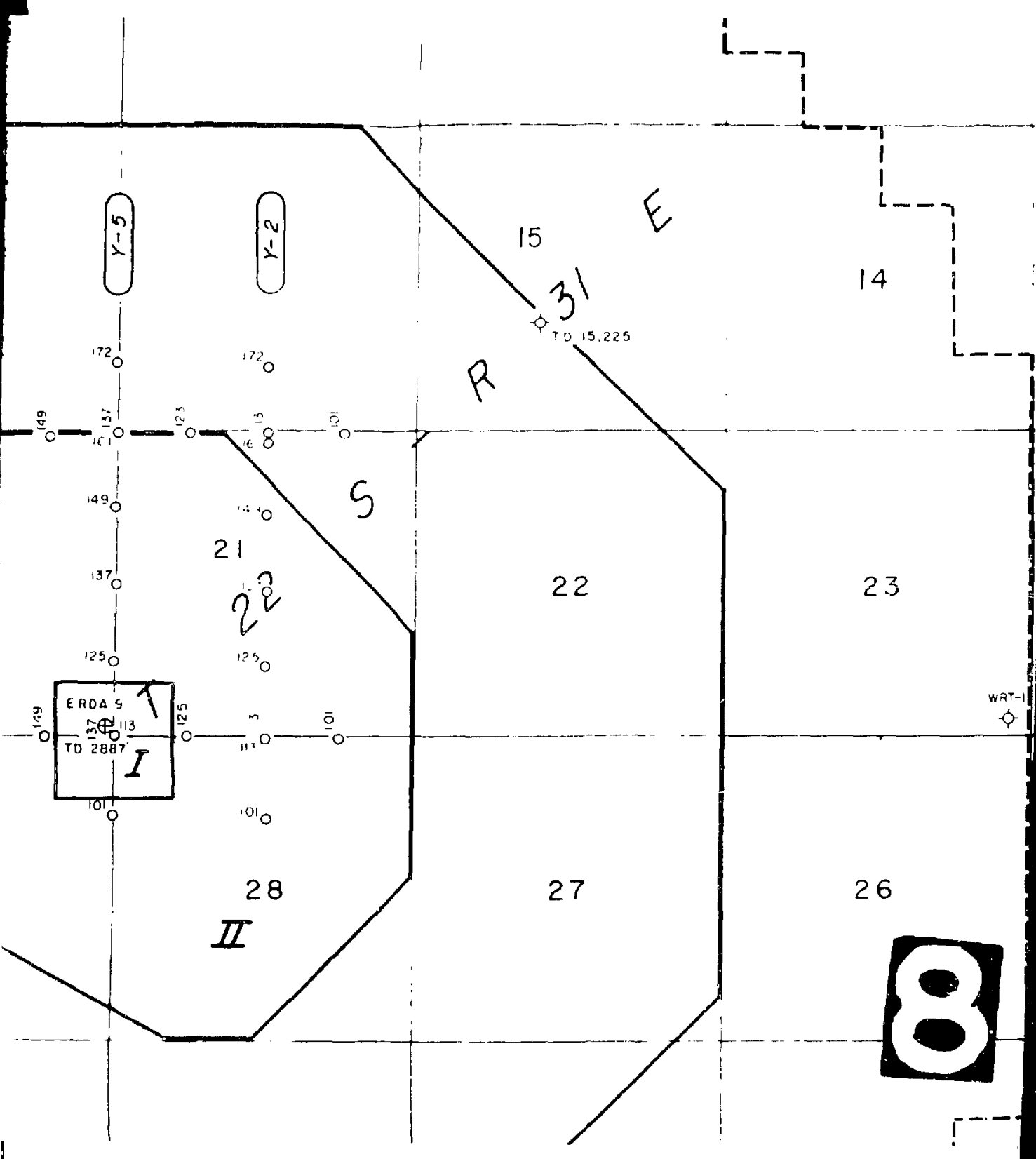




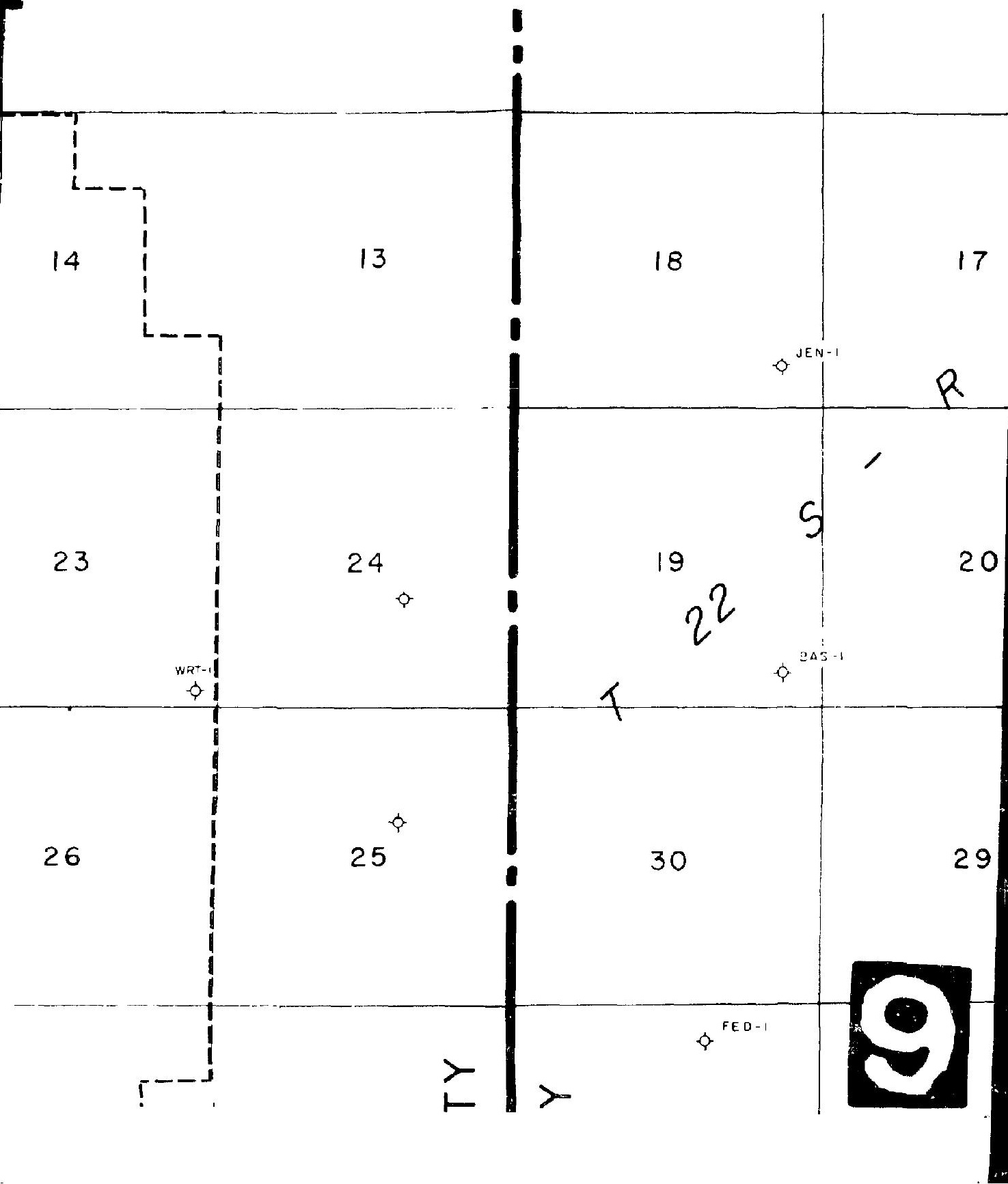




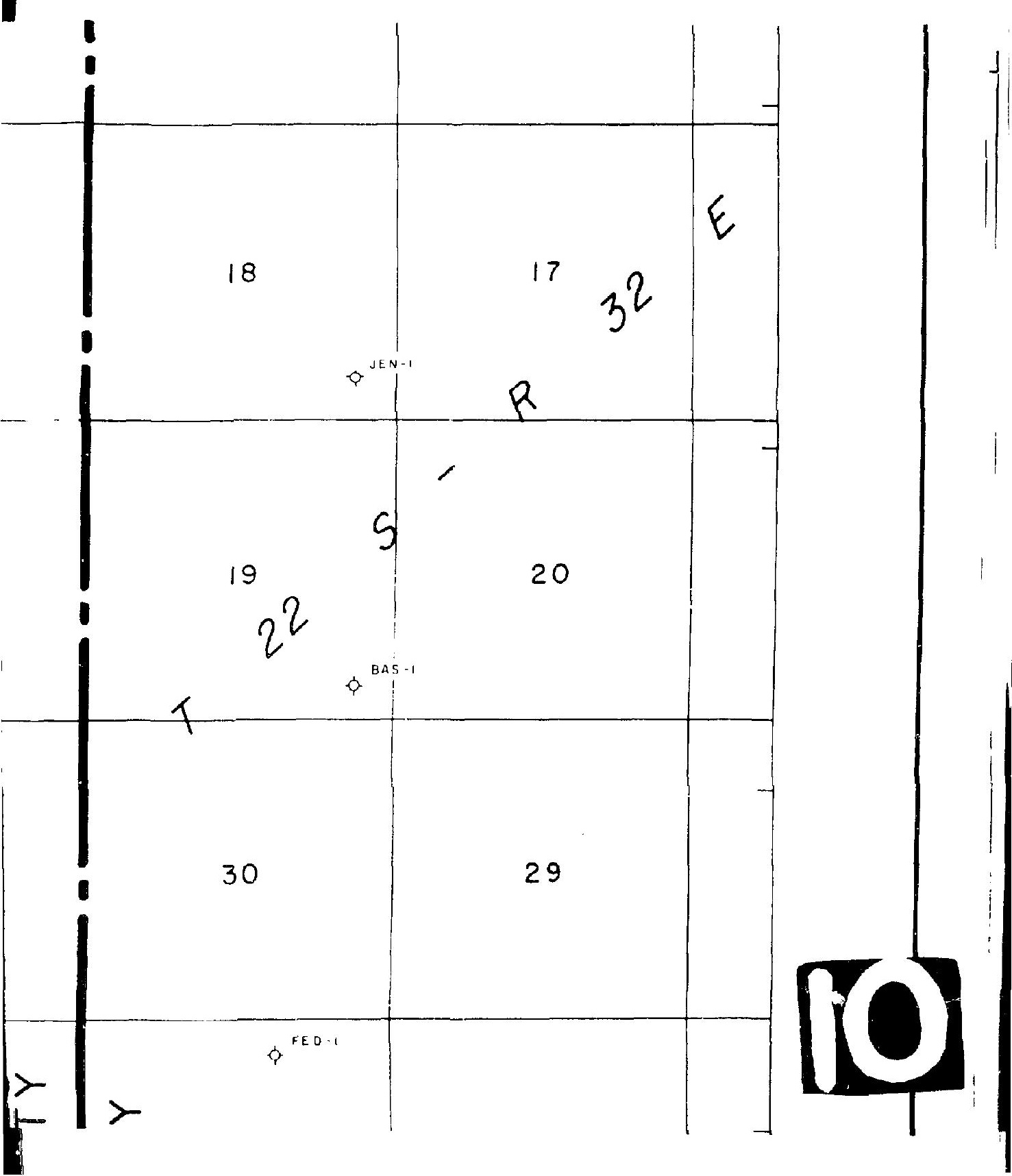




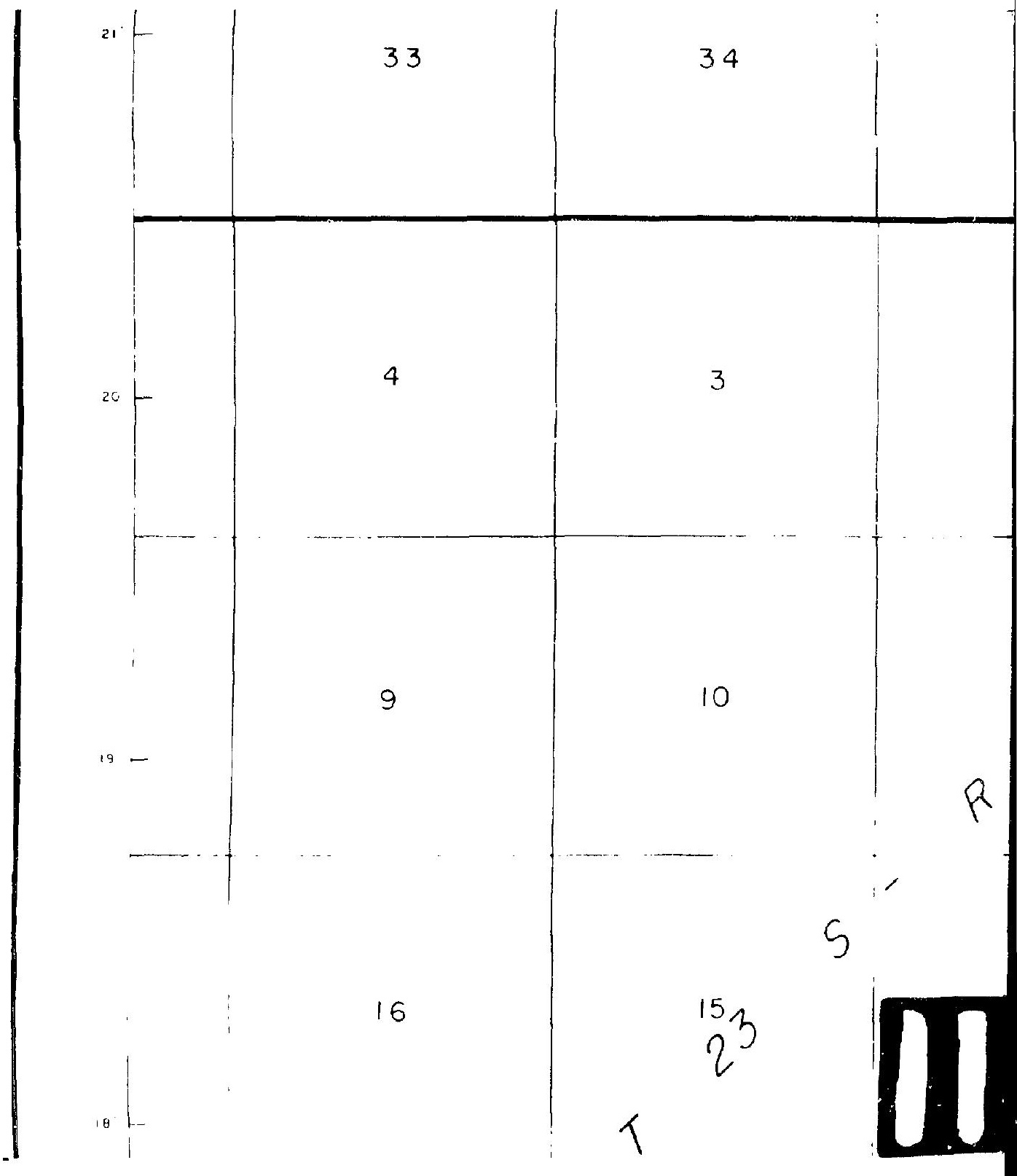


35

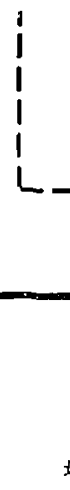

2

1130

12

14

13

31

32

$\phi^{c \theta}$

5

7

8

18

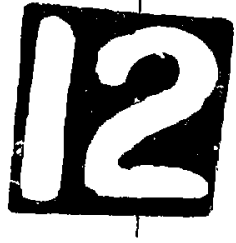




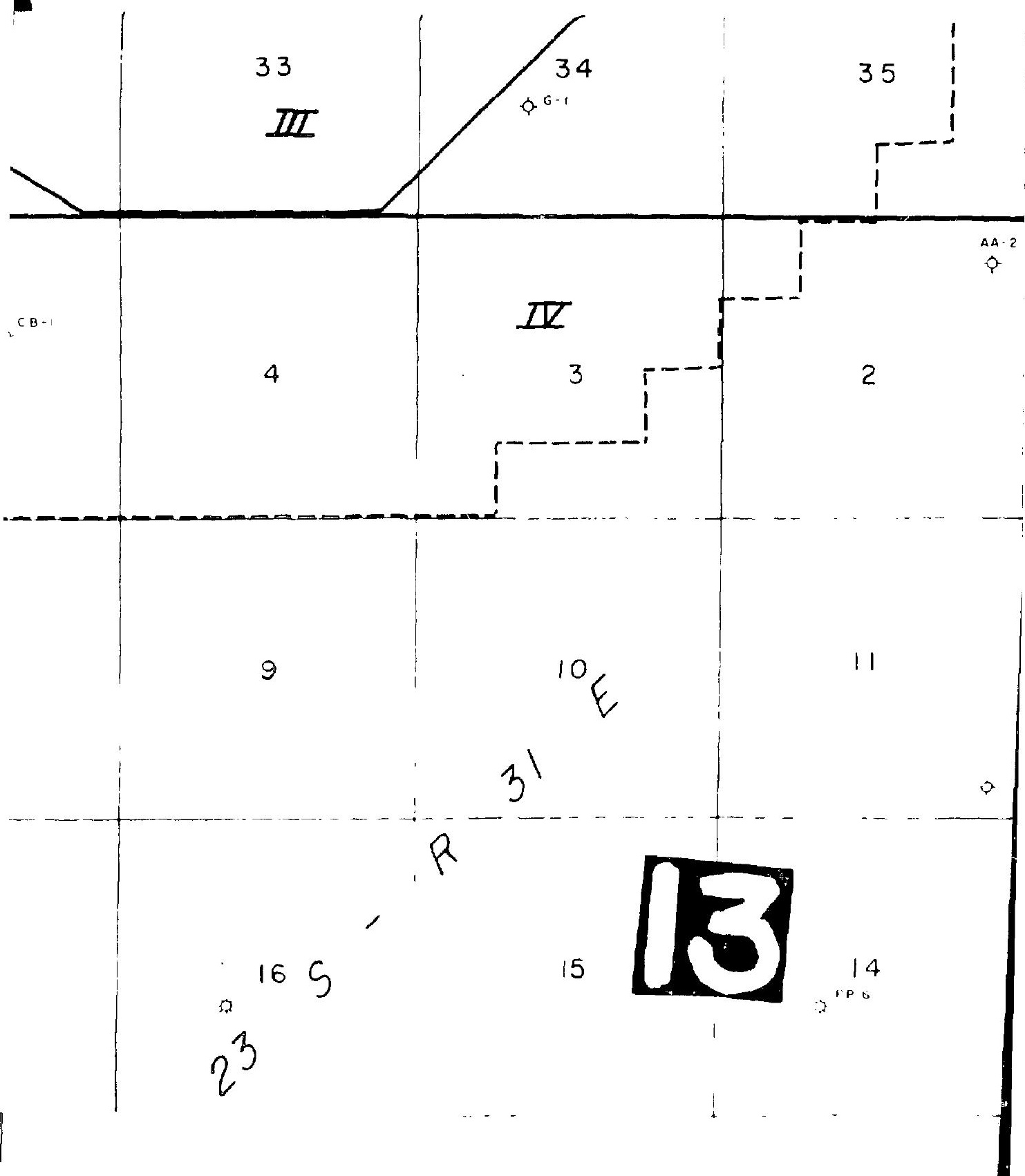




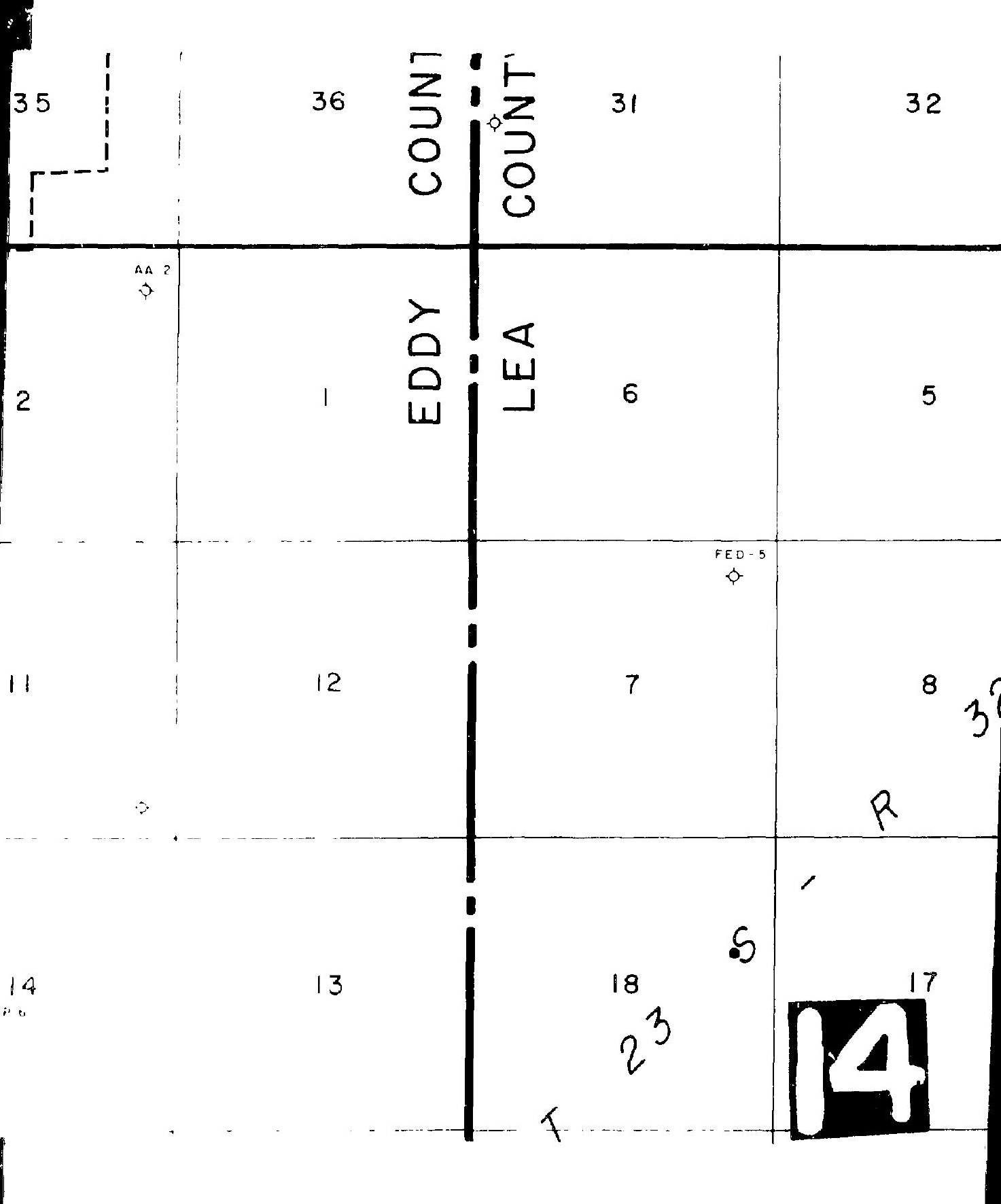




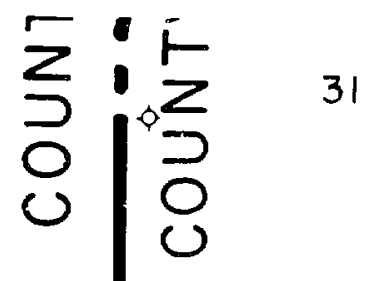

32

家。

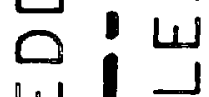

L 6

7

FED
$\phi$

I

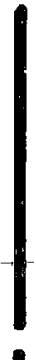

6

5

$R$

18

17

$2^{3}$

千.
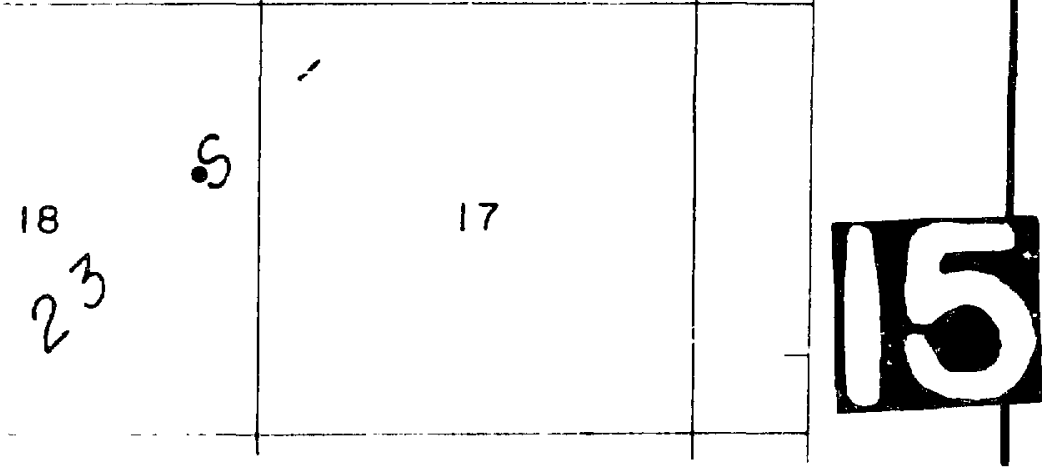


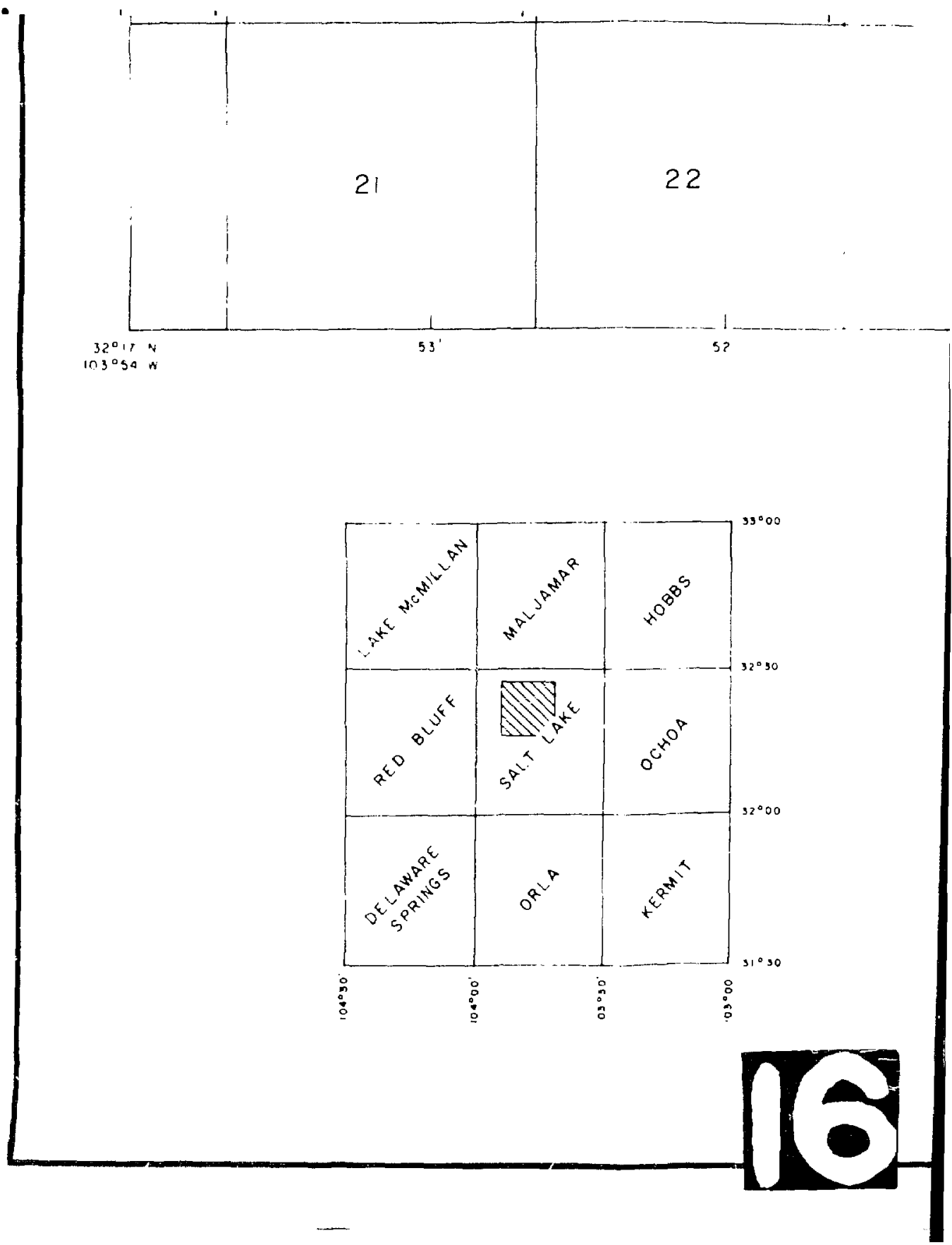





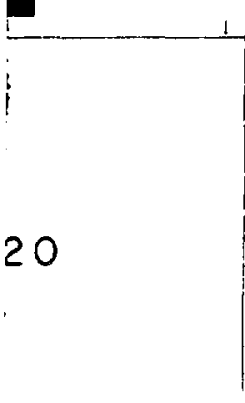

Y
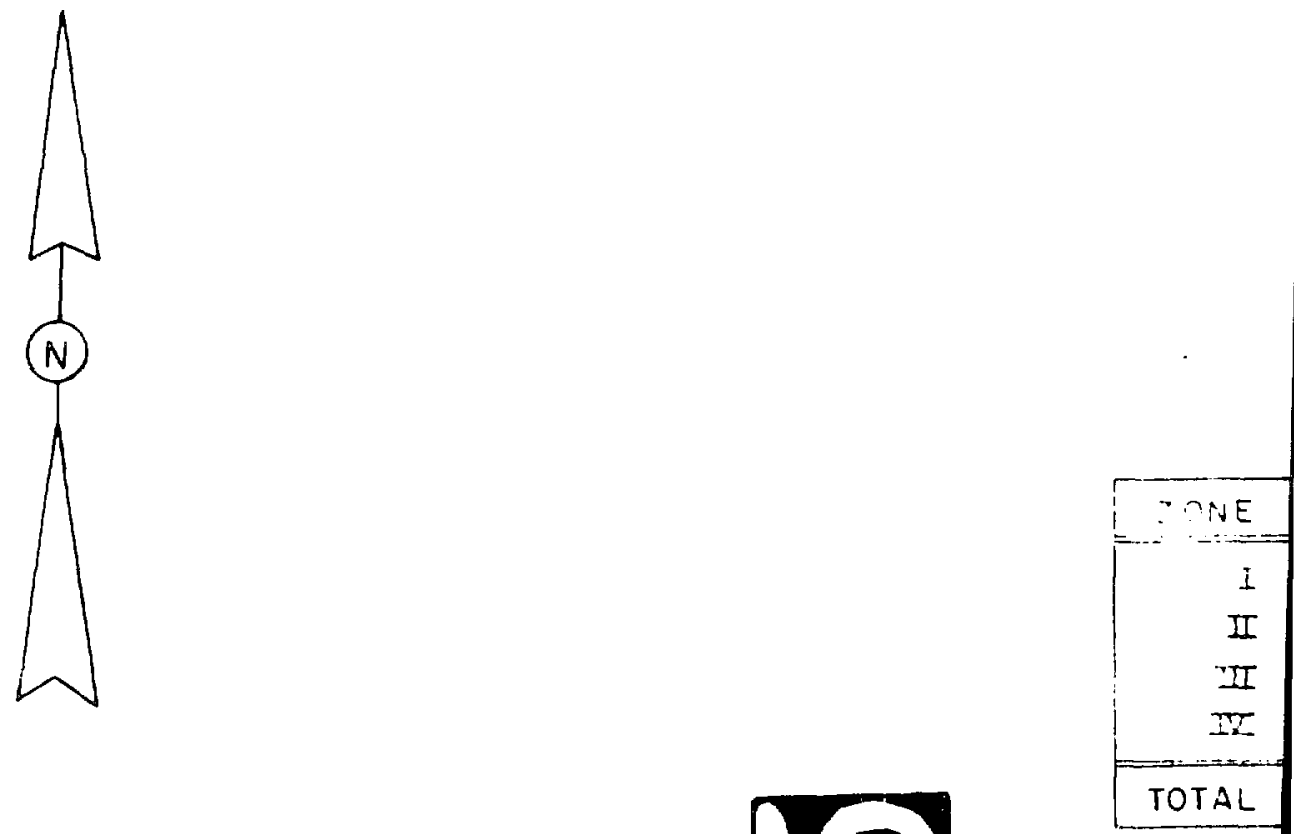


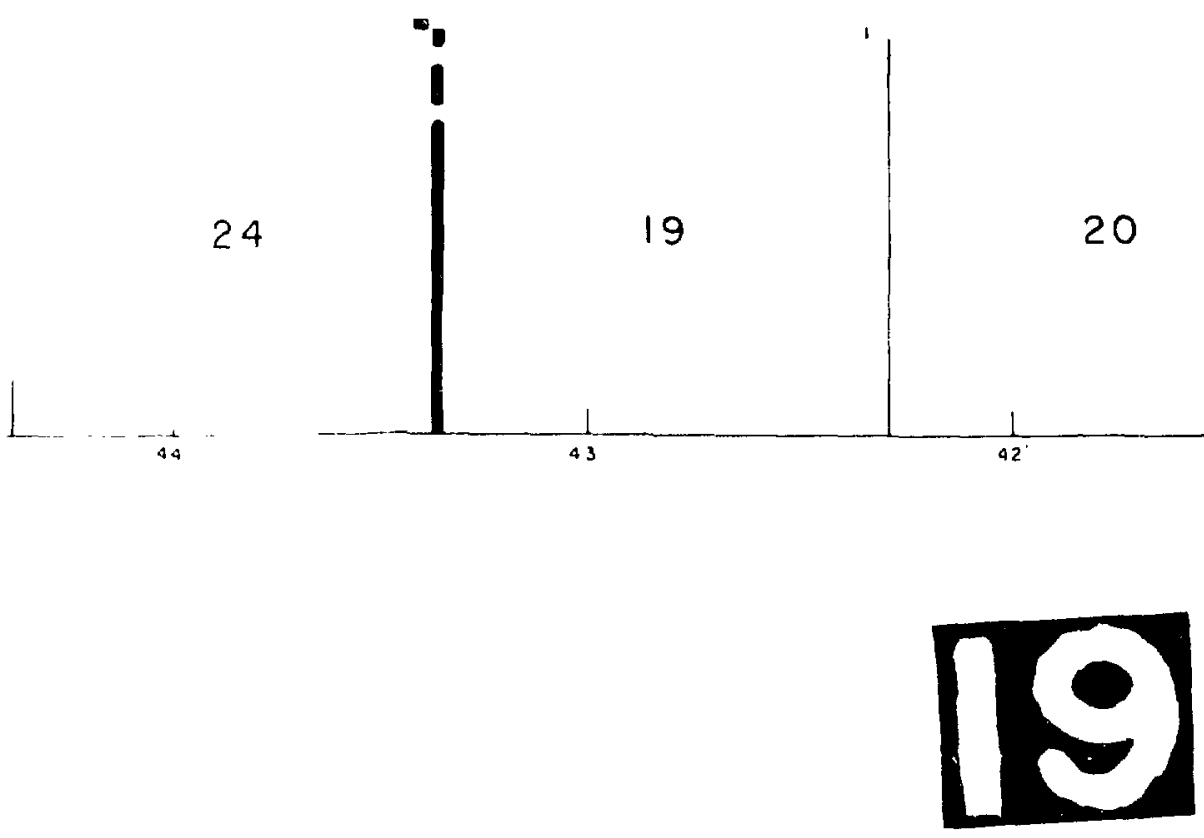

\begin{tabular}{|c|r|}
\hline ONE & AREA \\
\hline$I$ & 100 Acres \\
II & 1,818 Acres \\
III & 6,221 Azrs \\
II & 10,821 Acres \\
\hline TAL & 18,960 Acres \\
\hline
\end{tabular}

\section{SANDIA LABORAT}

SEISMIC SURVEY

LOS MEDANOS SITE A EDDY COUNTY, NEW MEX,

1978 SEISMIC PROGRA

INTERPRETATION BY

$G$ J long a associates, 

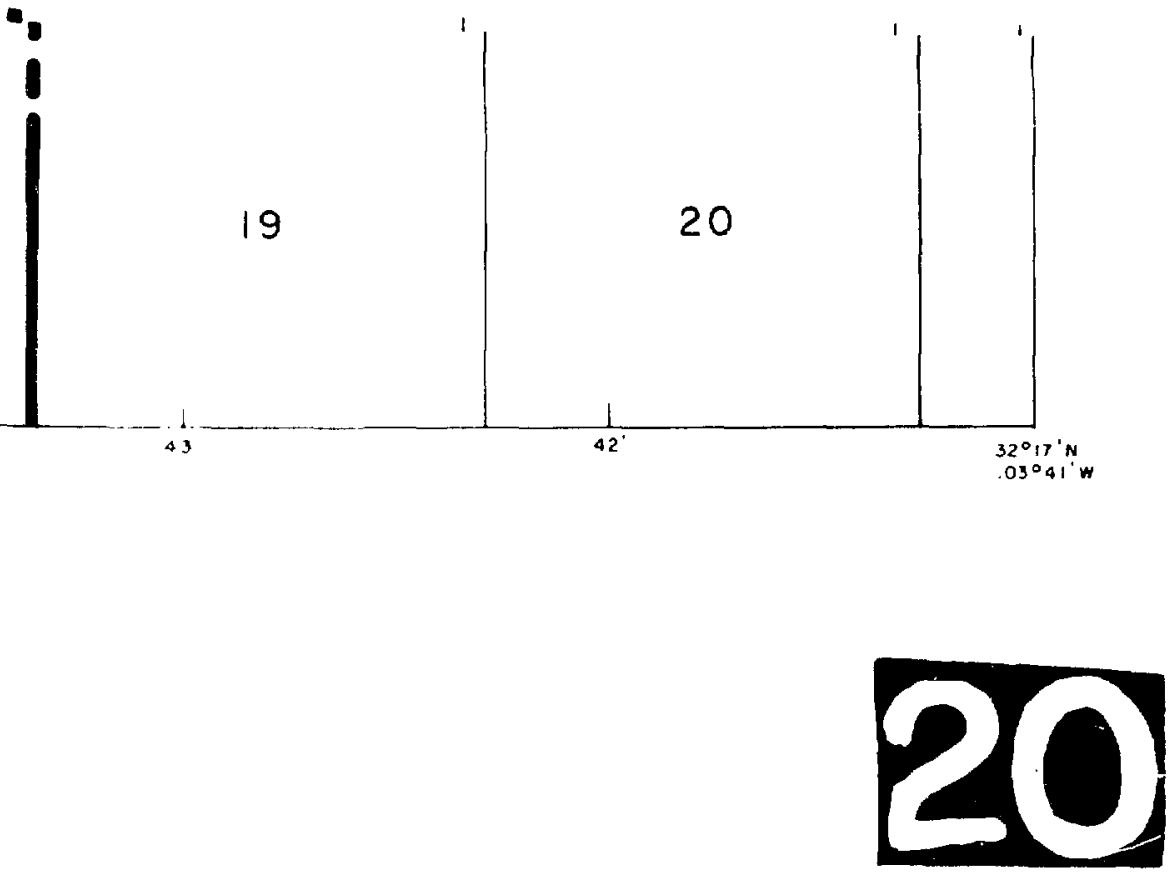

\section{SANDIA LABORATORIES}

SEISMIC SURVEY

LOS MEDANOS SITE AREA

EDDY COUNTY, NEW MEXICO

1978 SEISMIC PROGRAM MAP

INTEAPRE IATION BV

$G$ J LONG a ASSOCIATES, INC

SEISHOLOGIST $\triangle P P R O V E D$

SCALE

DATE

C 1 .




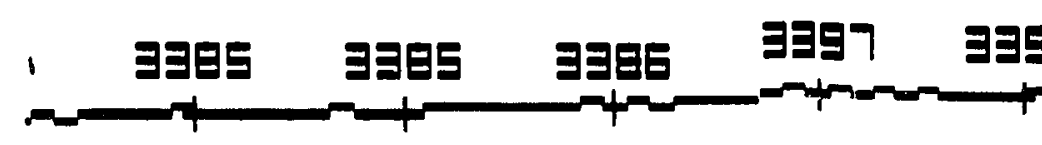

DFTUM

Bac口
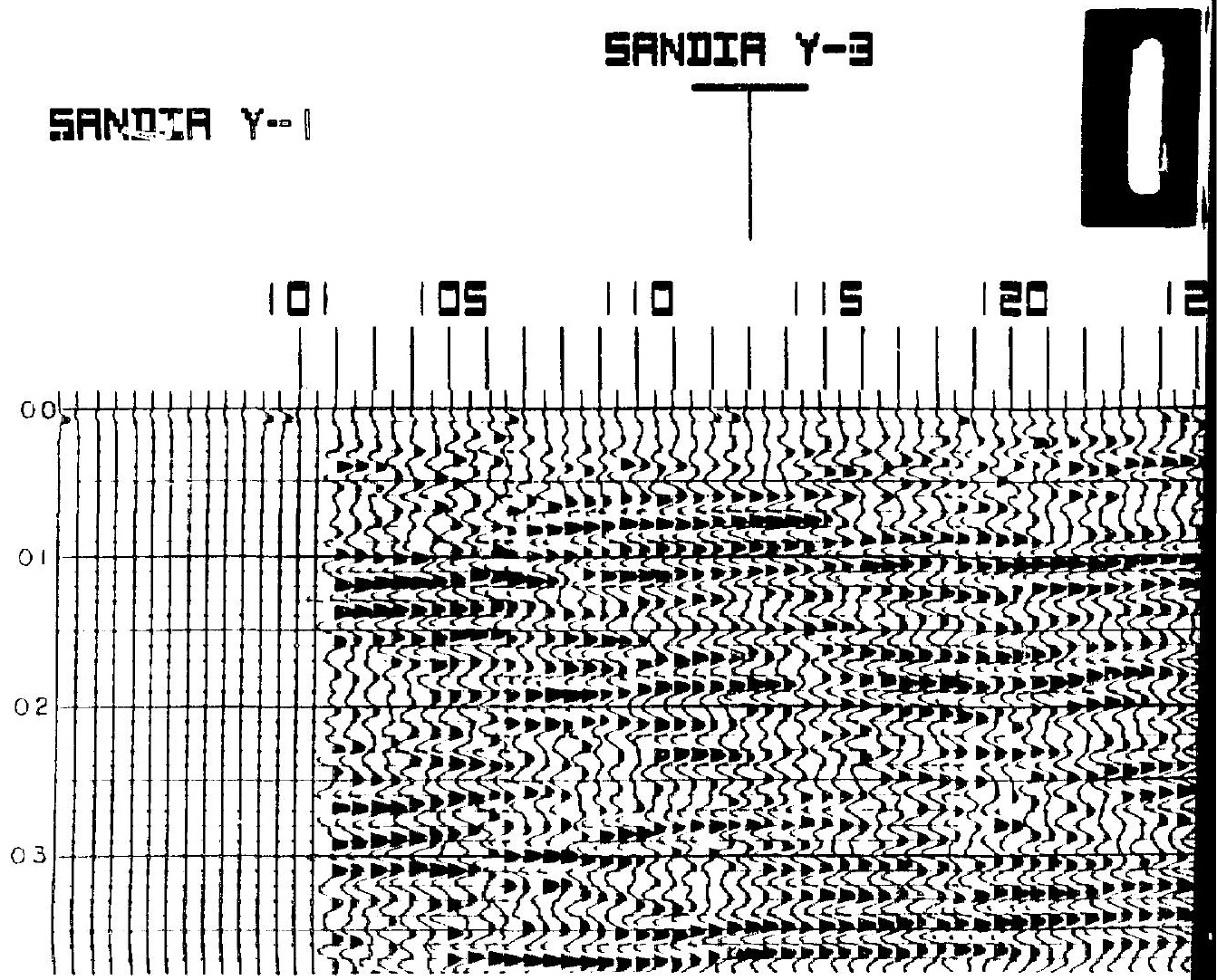
$3996 \quad 3400 \quad 3407 \quad 3412 \quad 3415 \quad 3417 \quad 3419$

\section{Vค}

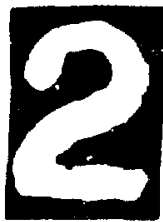

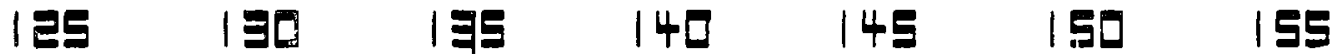

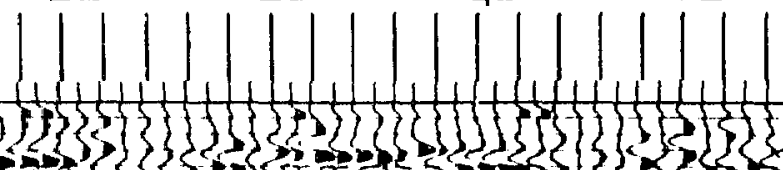
Th

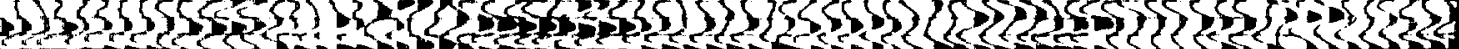

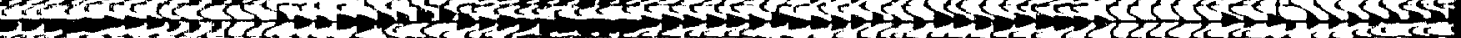

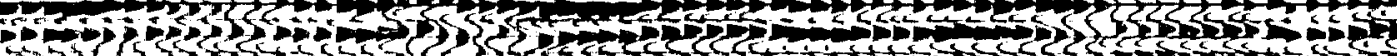

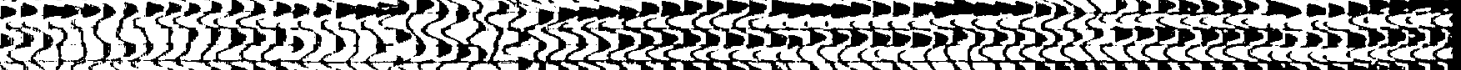

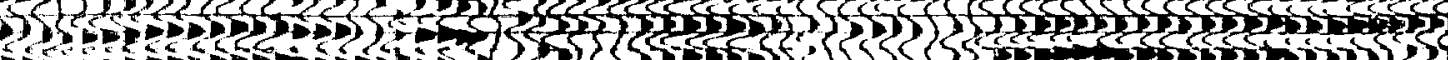

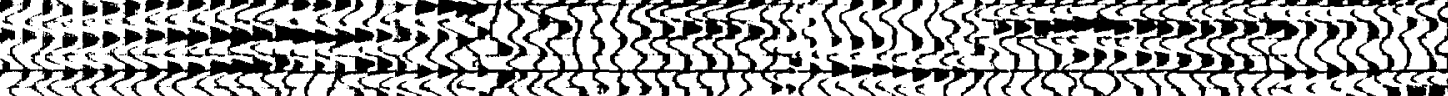

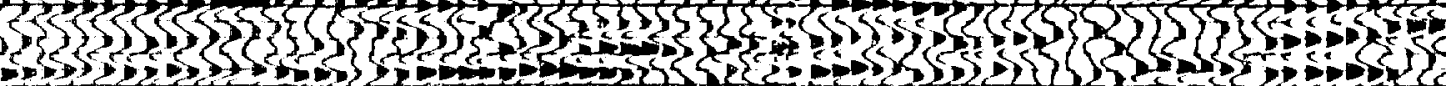

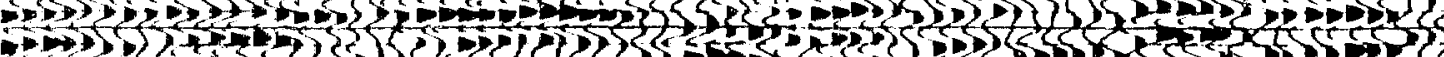

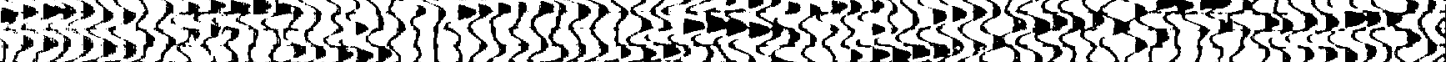

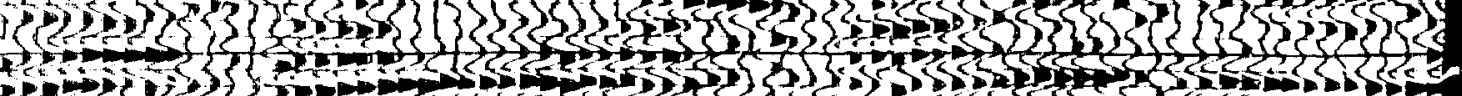

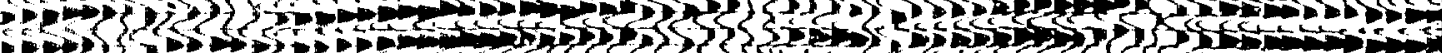

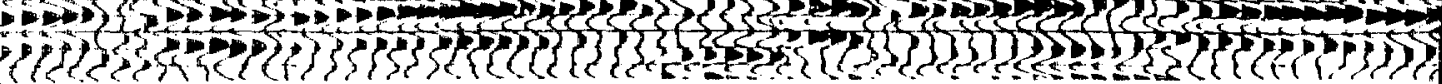




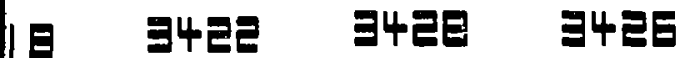

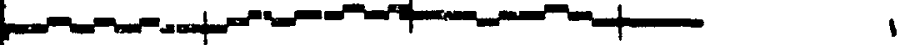

SFNDIF $Y-4$

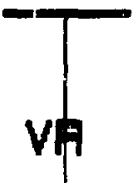

IED I I

5

\section{0}

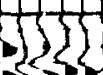

\{\}

$\{3$
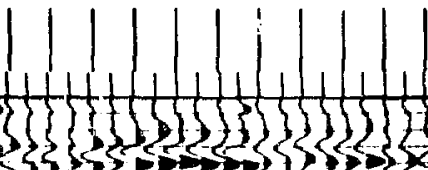

2

s.

3

रद

- 2535$\}$

f?

$30<5$

$\rightarrow 5$

$\rightarrow$

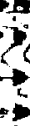

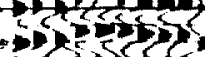

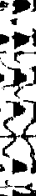

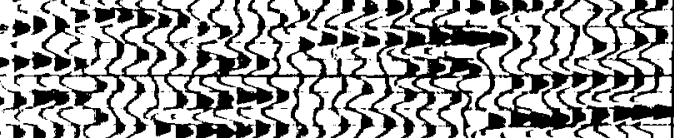

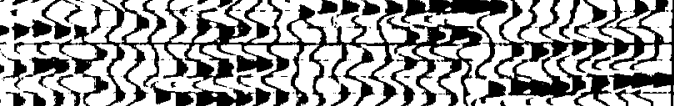

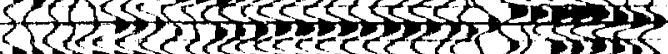

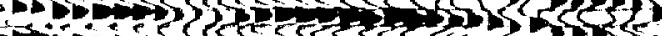

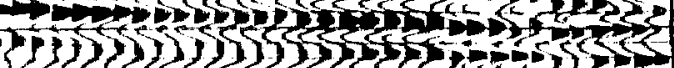

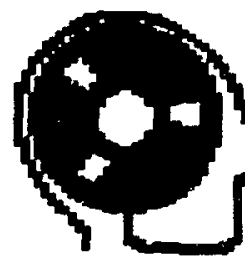

\section{AREF}

LINE

STATE:

CDUNTY

口APTE ST

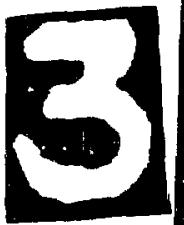

RECDRD: INISTRU1 ENERGT SWEEP SWEEP

LISTTEM SAMFLE 


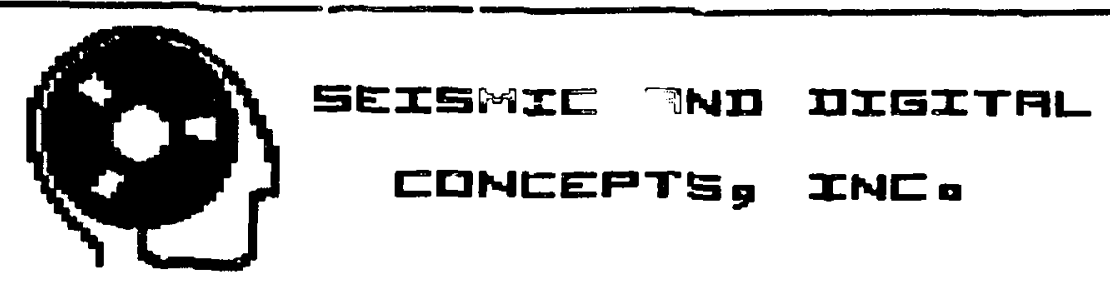

\section{SANDIA LAES.}

15 M口RTH

AREF

LINE

STATE

CDLNTY

DATE SHDT
SFMIIR

$r-1$

NEW MEXILD

LEF

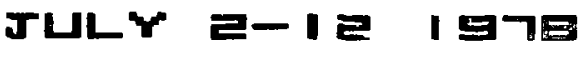

\section{FIELD PAFFMETERS}

REEDRTED Br

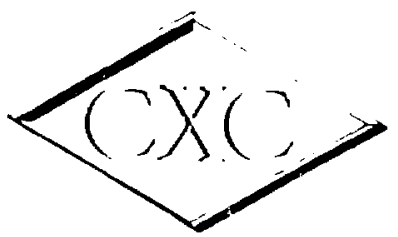

$$
\text { ( SC. I I }
$$

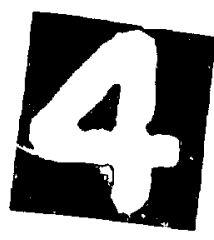

RECDRDED

IMSTRUMEMTS

EMEFER

SWIEEP LEMETH

SWEEP FRED

LISTEM TIME

SAMELE RATE
DIEITAL

HDDF-4DDD

WIgraTaR

I อ.ด SEL

25-I

4.0 S튼

$\equiv$ MS 


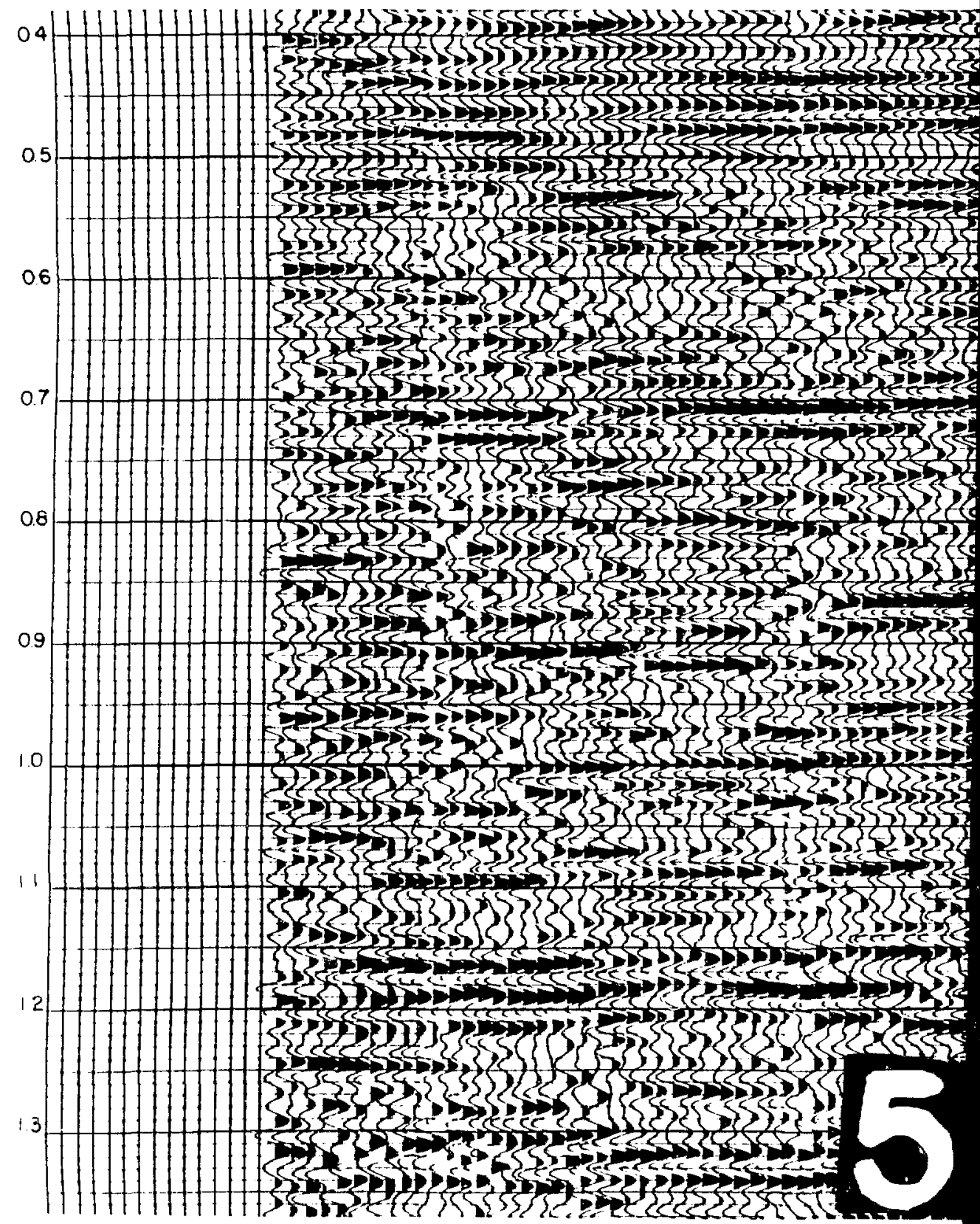


EFILE

E4 TR GPLIT

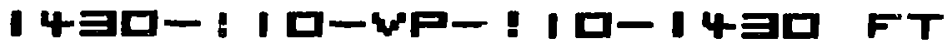

GRELU IMT

WIAFo INT

$10 F T$

EDQWEFEE

110 F

$v$ EDIF

\section{PFDEESEINE FAFAMETEFS}

PRDEEESEU

ㄹㅁ Б트 르

1 GPIKE DECGM-TIME WFFWIME

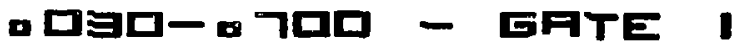

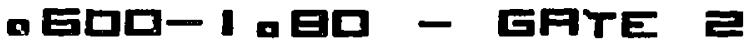

DFERFTUR IDE MS घDTH GATES

ヨgID T\& FILTEF

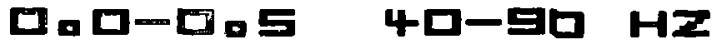

0.7-1.1

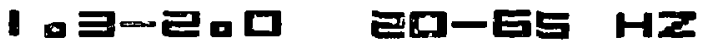

4 STFTIES gMMEgSTEF-MLTE

IFTLM झEDQ FT FEL

IFTLM WEL EDED FT.

WF-WELDEITM FMIFL WEIS

SgE FEE

E ALTE FESII БTFTIEE

7 I 1 FQLD ETAEK

9 MUISE REIECTIDM FILTEF

\section{IISPLFY PFFAMETEFE}

HORZ GEFLE E TRPIM

WEFT SEFLE 10 IMمEEE

ELEW SEFLE IQD FTمIM

PDLFRIT\& EDIE LEFT+ FIEHT-

TOUI MUMAER

CDMFLETED
트미

Fu 1979 


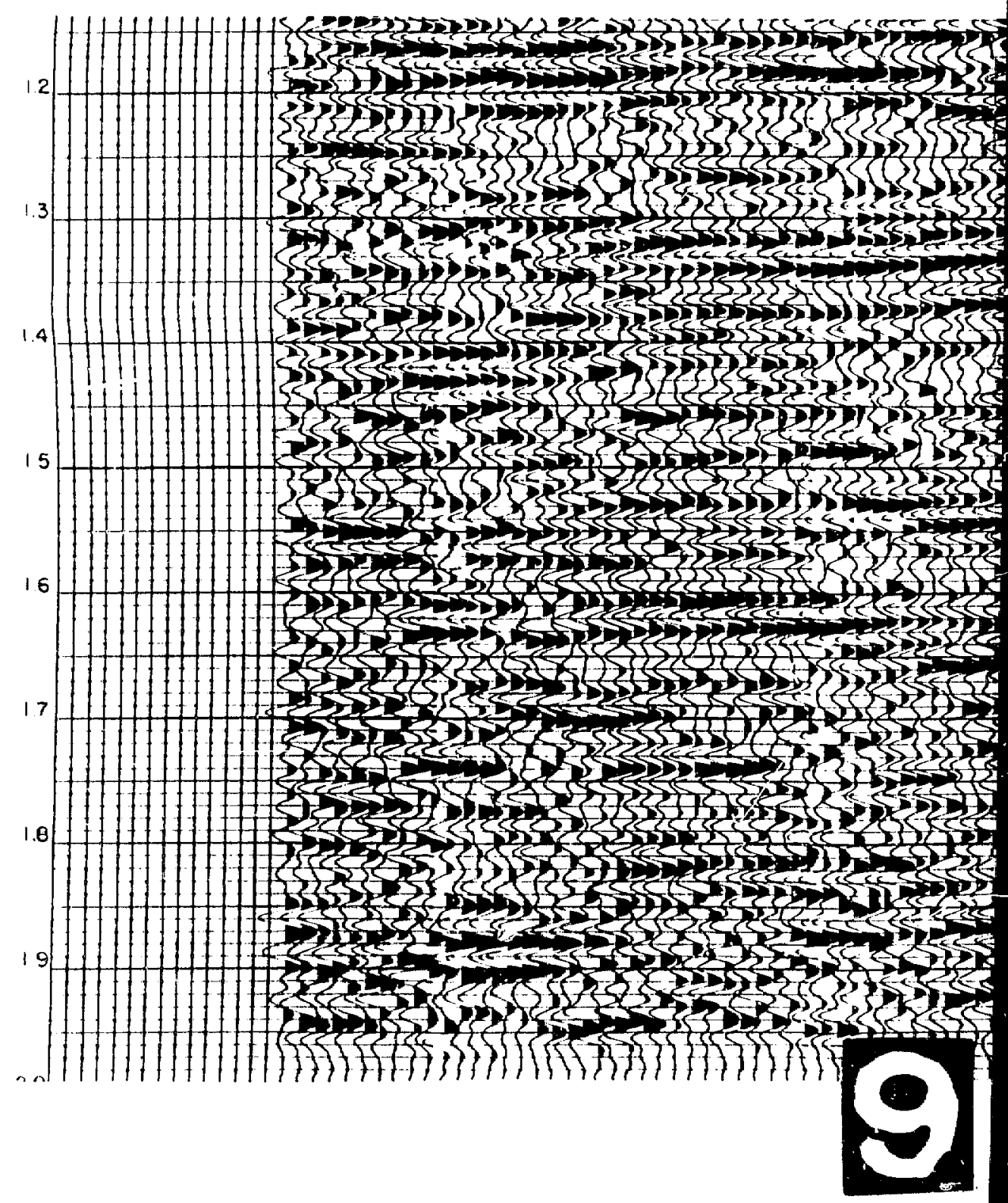




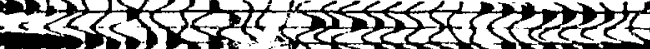

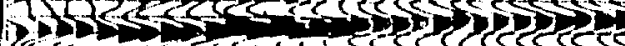

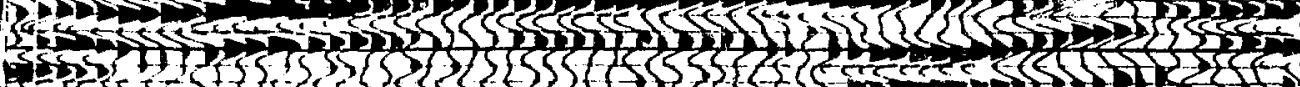

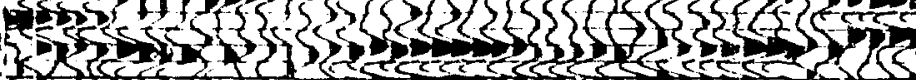

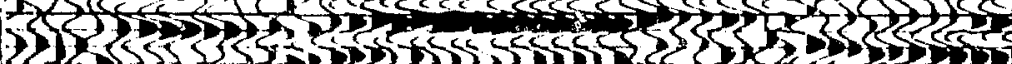

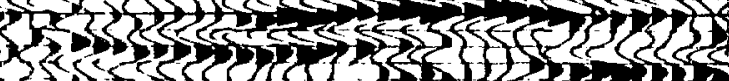

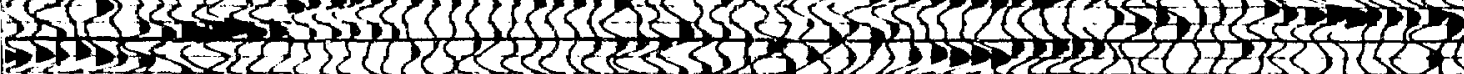

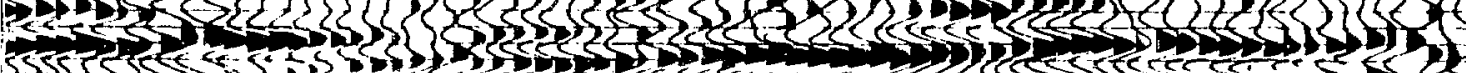

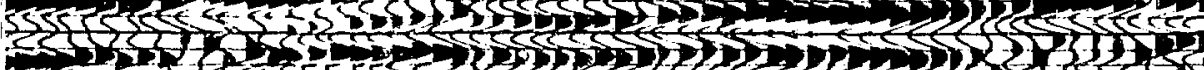

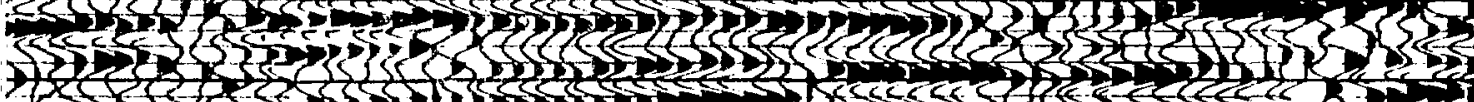

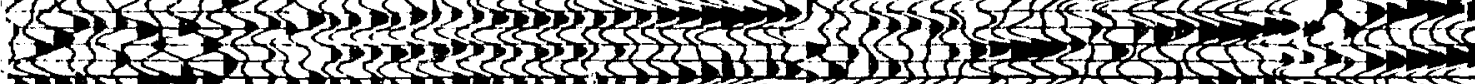

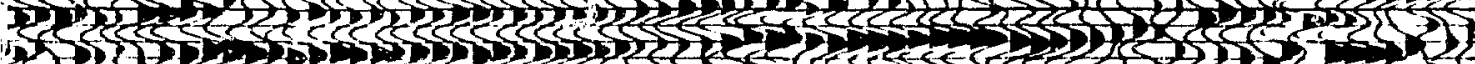

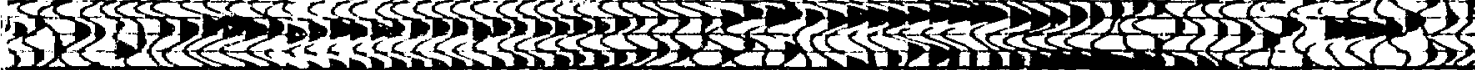

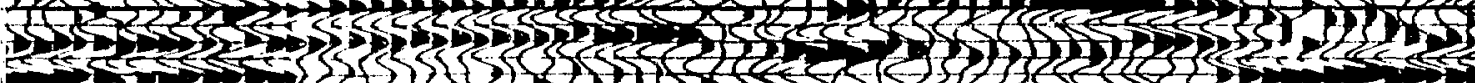

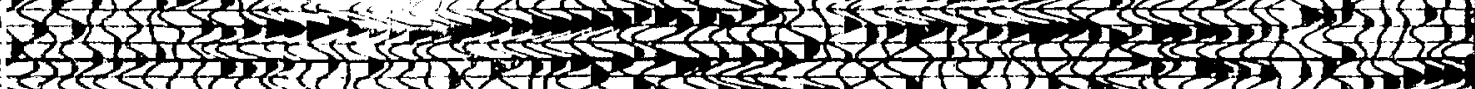

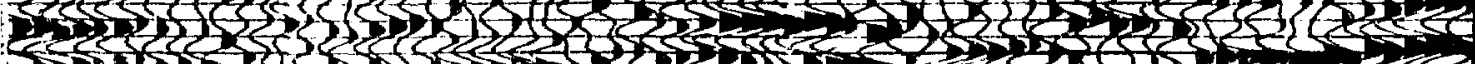

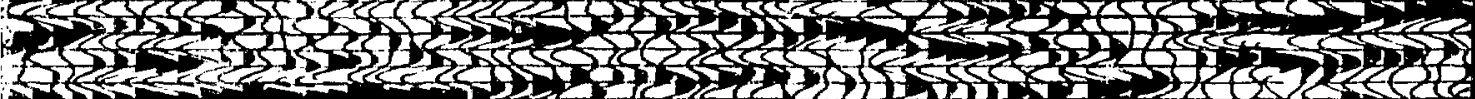

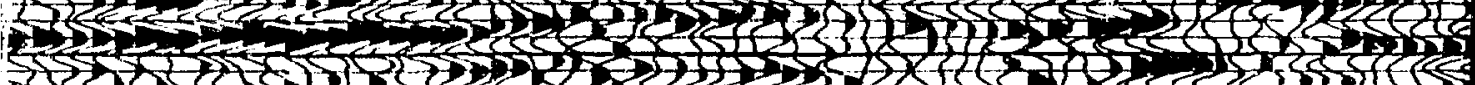

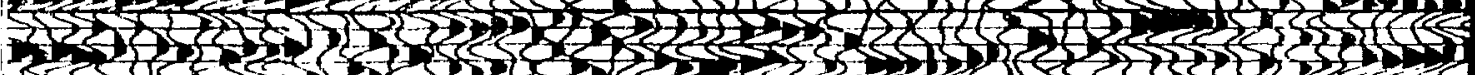

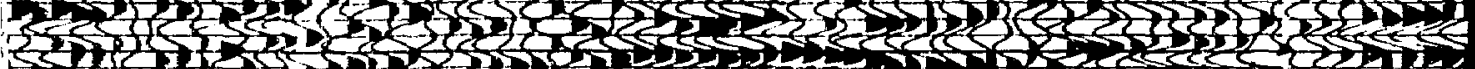

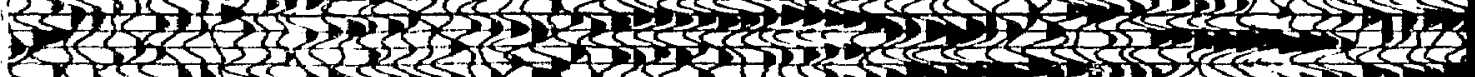
J

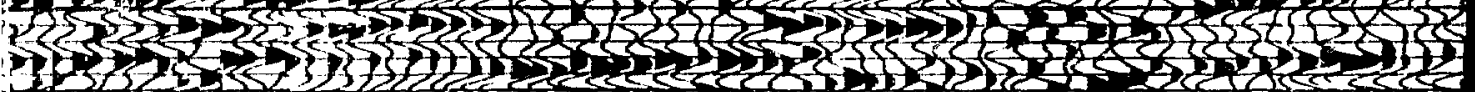

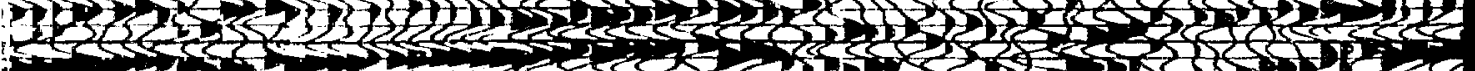

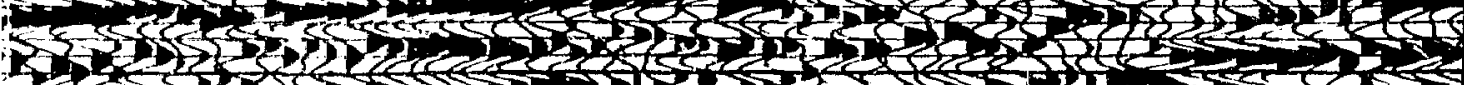

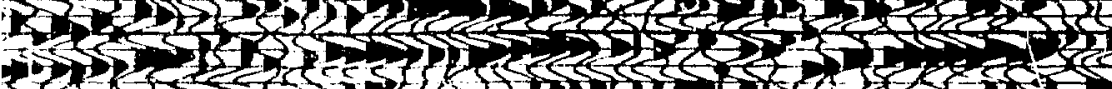

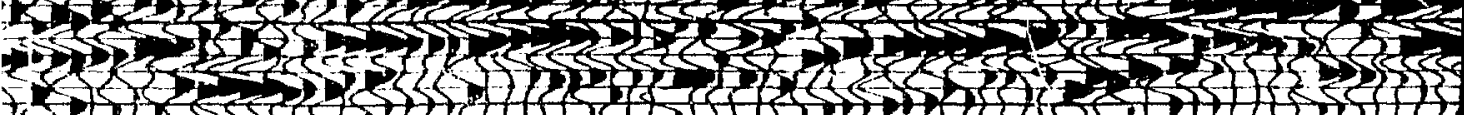

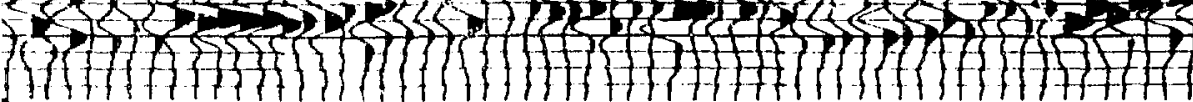




\begin{tabular}{|c|c|}
\hline $\begin{array}{l}\text { IQA WLAZEF } \\
\text { EDMFLETEL }\end{array}$ & 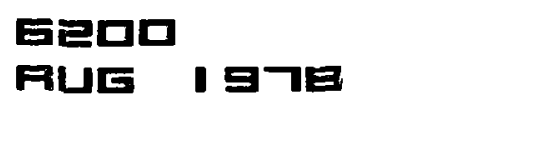 \\
\hline
\end{tabular}

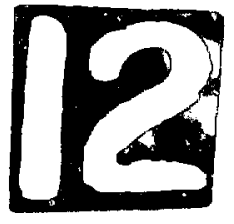




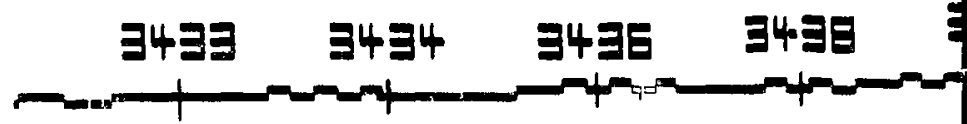

TAPU⿴囗十⺝

코름미

รคพIIค Y०อ
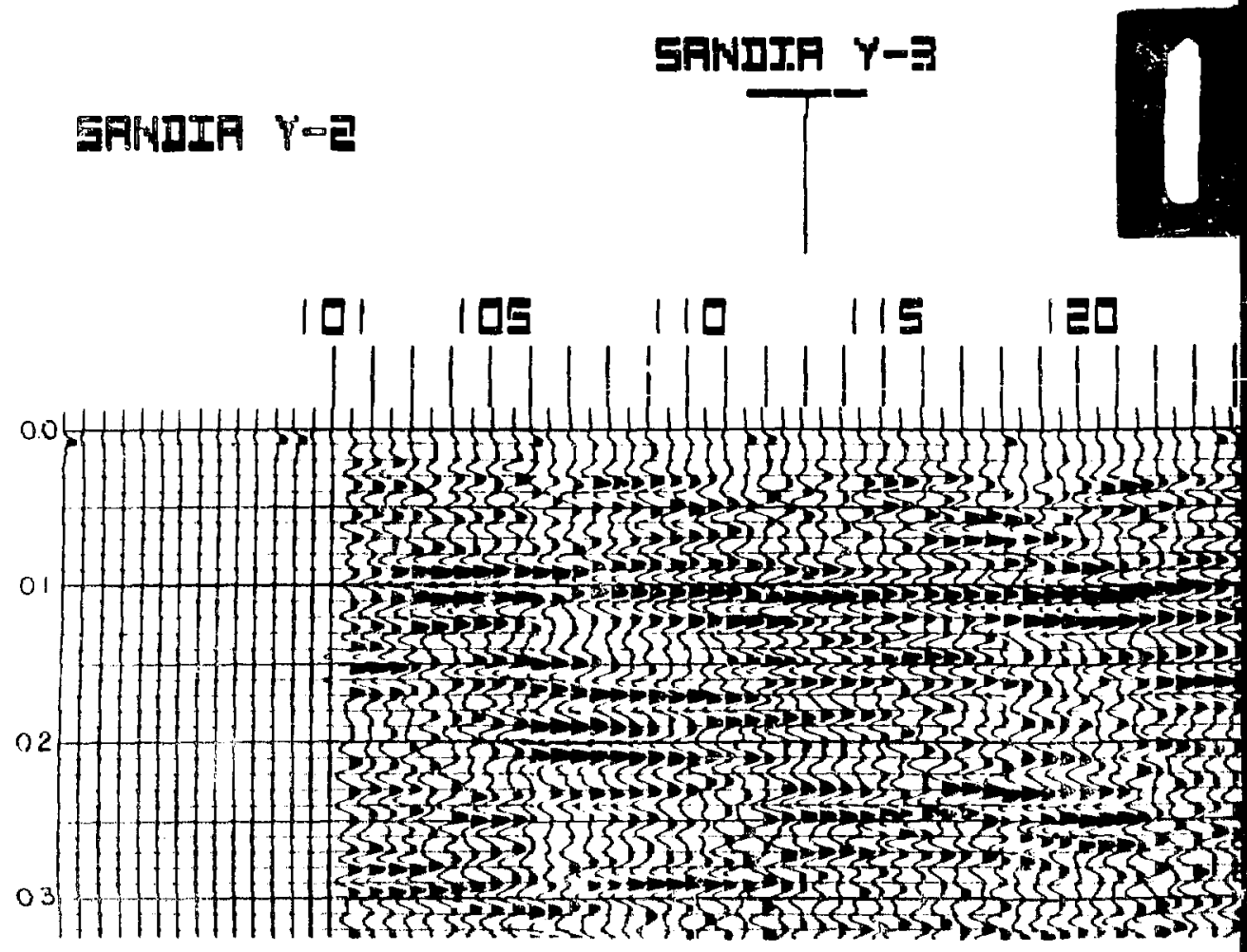
$9445 \quad 3447 \quad 3450 \quad 344 \Xi \quad 3440 \quad 3455 \quad 3464 \quad \exists$

SFN

\section{VP}

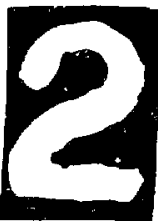

$\begin{array}{lllllll}125 & 190 & 155 & 140 & 145 & 150 & \text { ISE }\end{array}$

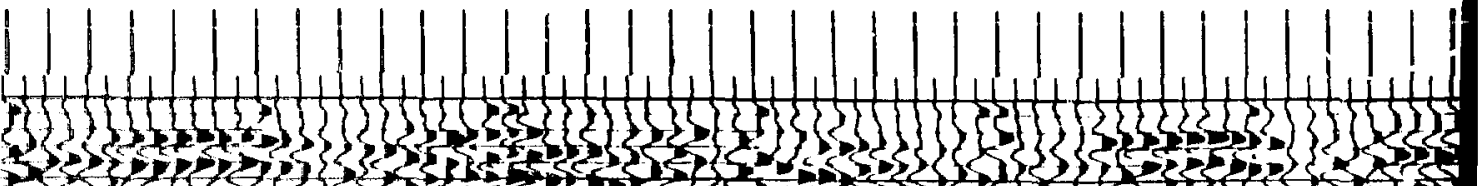

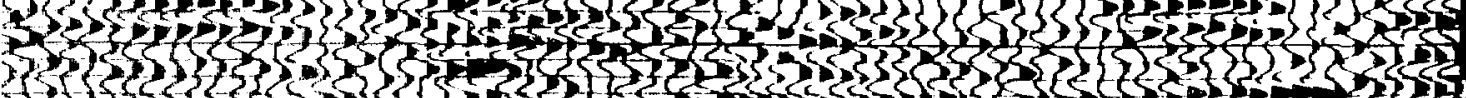

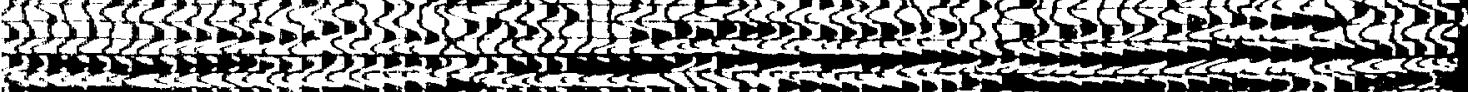

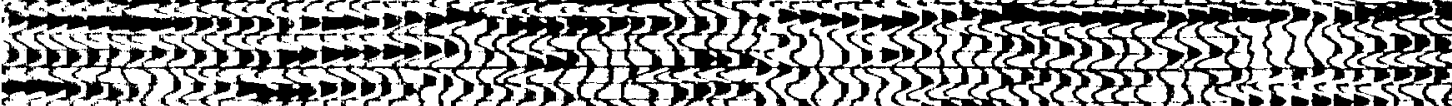
m.7.

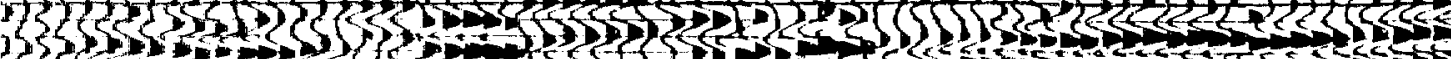
3537.45

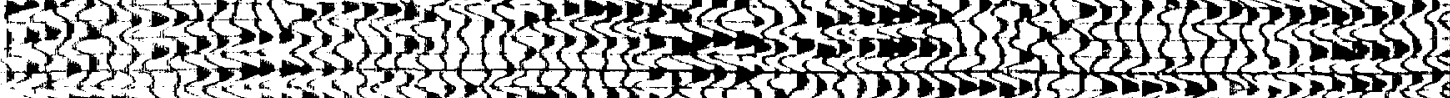

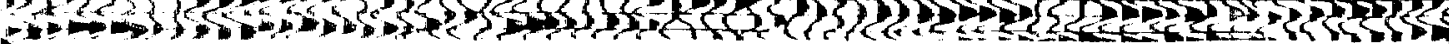




\section{5 $\quad 3480 \quad 3479$}

SANDIF $Y-4$

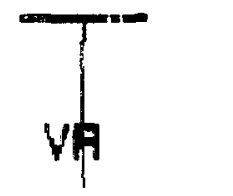

IED IEE

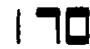

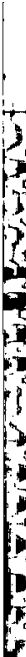


SEISMIE FND DIEITAL EDNEEPTSg IMEa

\section{SANDIA LAB.}

\section{MQDRTH}

AREA

LIME

STPTE

EDLNT'

DATE SHQT
SFWIIF

$r-2$

NEW MEXIICD

LEF

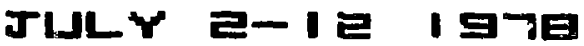

\section{FIELD FARAMETERS}

RECDRDED IY

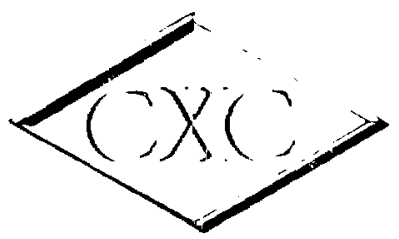

c Xis. I ic .

REEDFOED

IMETRLMENTS

EMEFE

SMIEEP LEMETH

EIVEEF FFET

LISTEM TIME

SAMALE FATE
DIEITFL

HIIF-4-DDD

MIIRFTQR

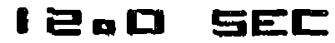

프-10日 Hב

으므드

$\Rightarrow \quad M=$

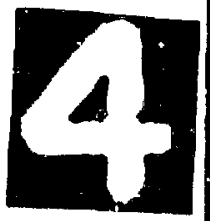




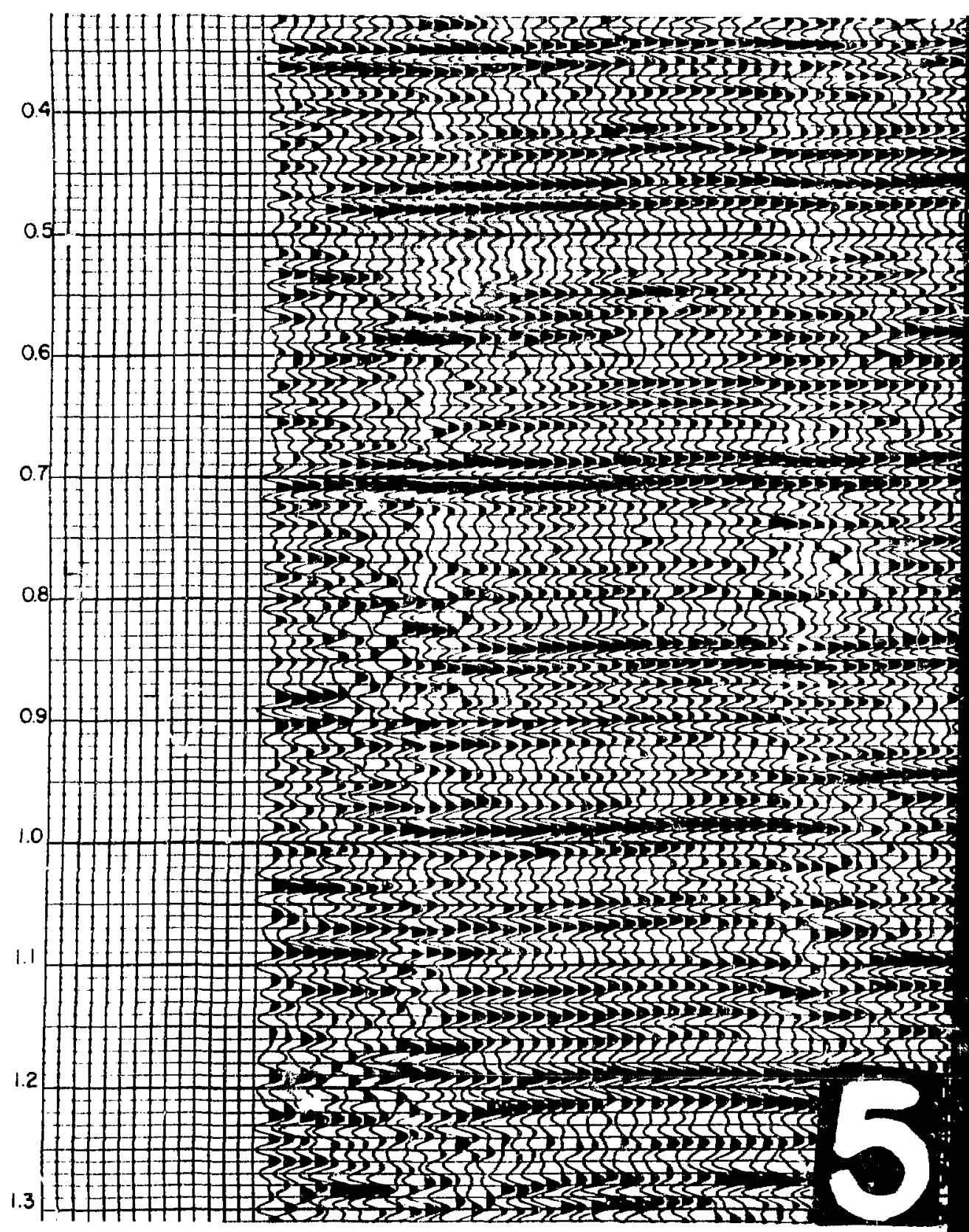




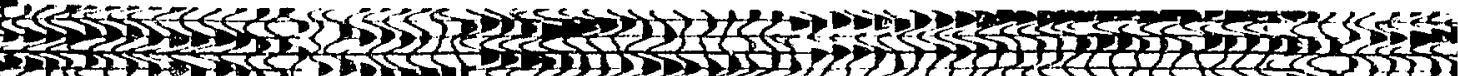

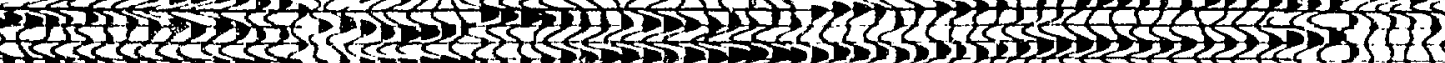

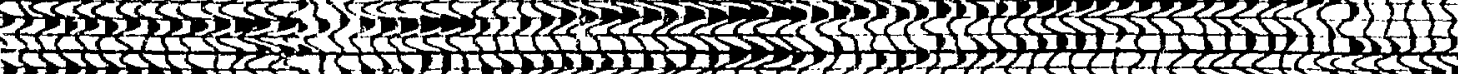

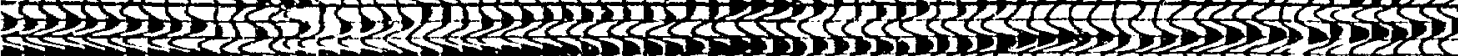

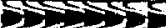

1323,05

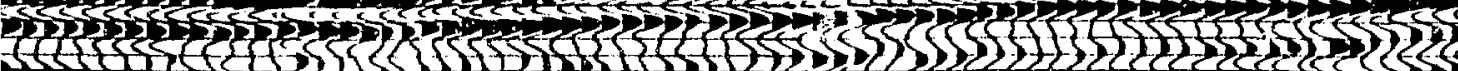

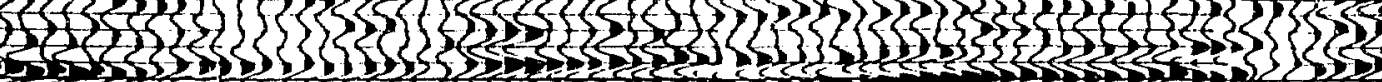

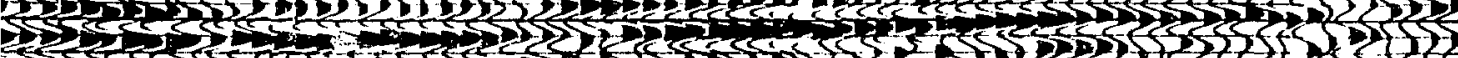

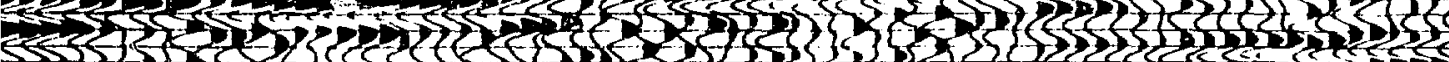

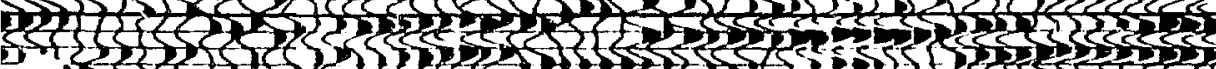

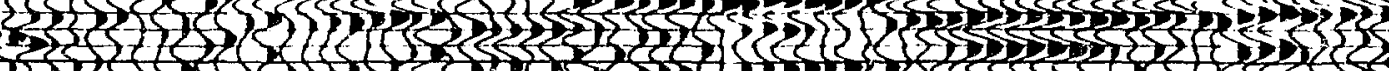

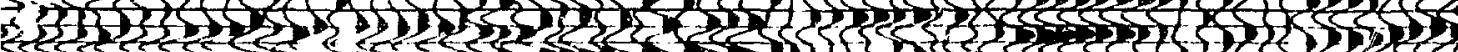

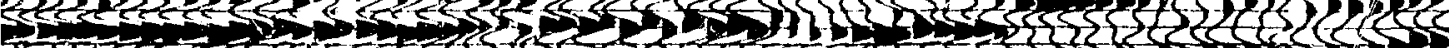

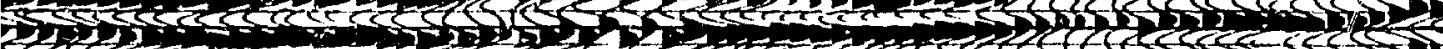

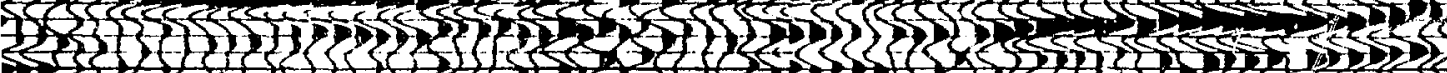

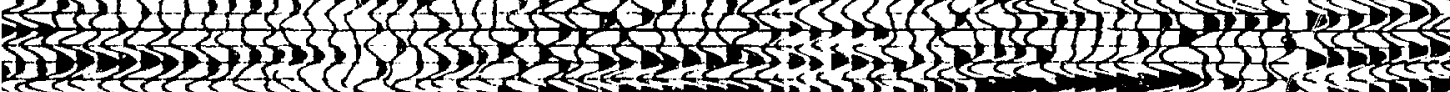

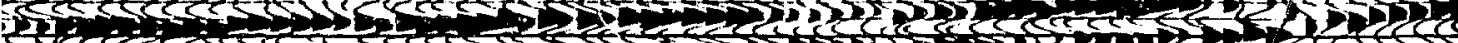

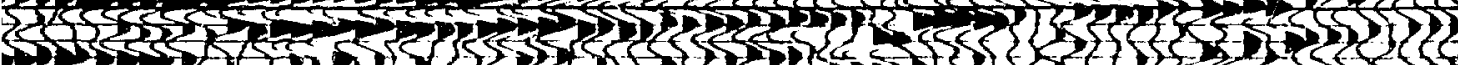

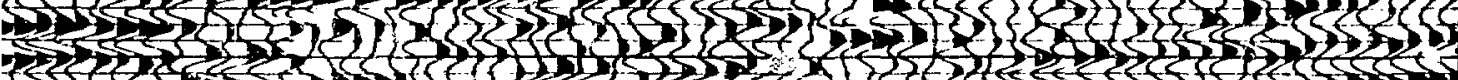

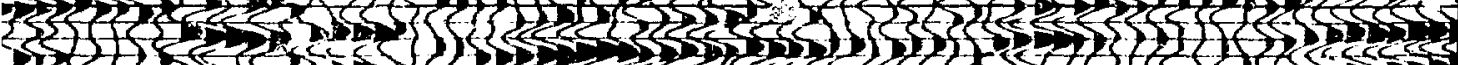

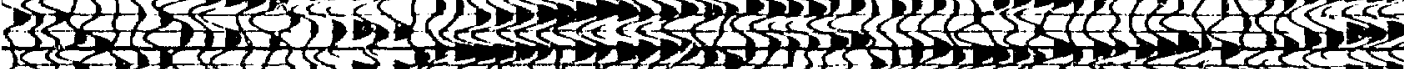

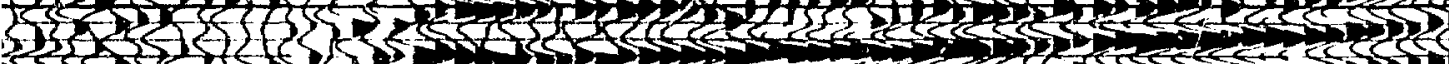

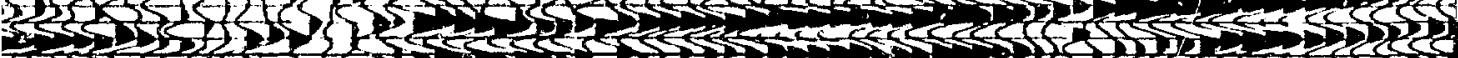

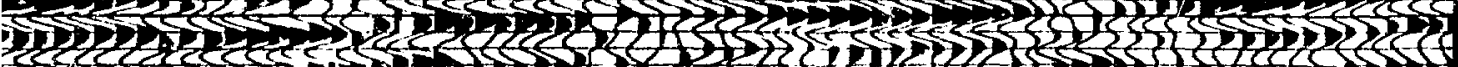

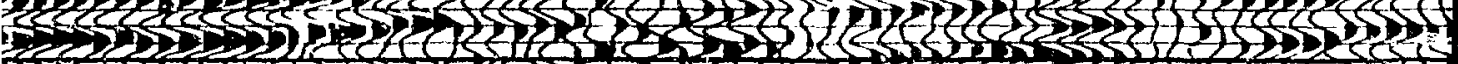

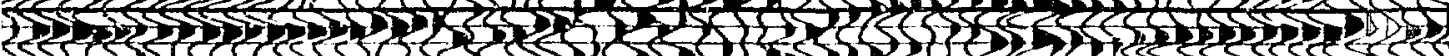

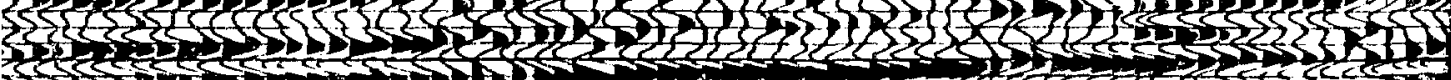

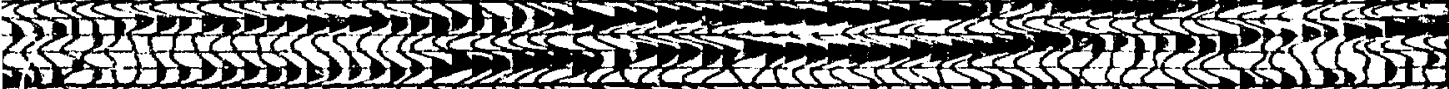

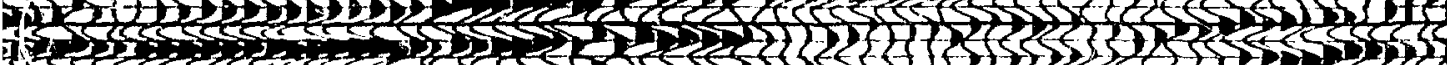

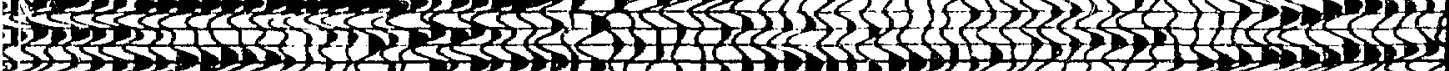

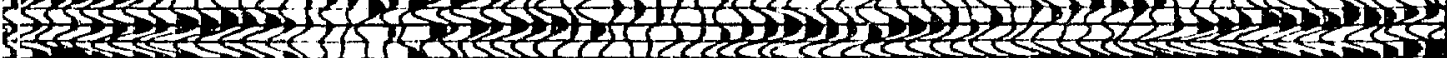

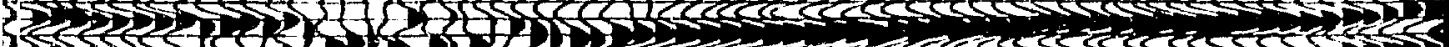

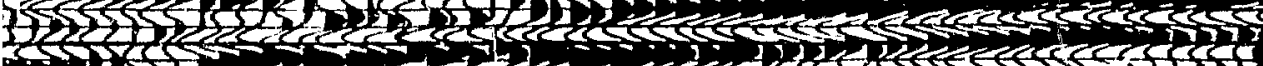

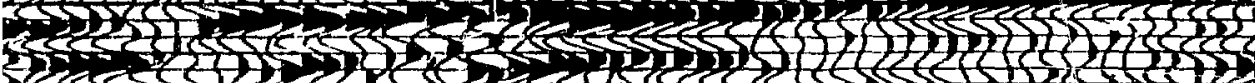
135 गी 13521 

EFILE

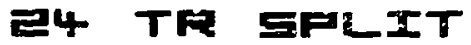

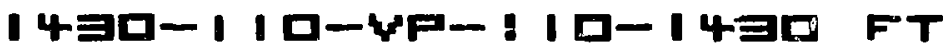
GROLE INT
$810 F T$
WIAR I IMT
I I FT
CDUERAGE
I ED曰

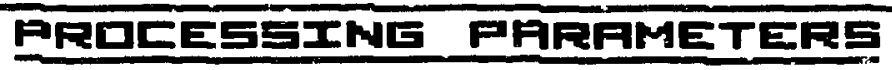

PRDCESSEX

ᄅag SEL 2 MS

1 SPIKE DEEDM-TIME WFFrIML

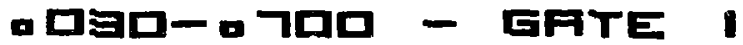

- EDD-1.ED - GATE E

DFERATLR IDQ MS ヨDTM GATES

ヨ日D T\& FILTER

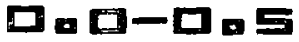
4ロ-פ口 HZ
묻ㄴ
ヨロ-ר HI

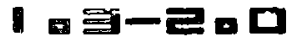
घロ-ES HZ

* STATICS FNMQgETEPーMLTE

IFTLM $\Rightarrow$ 미 FT FGL

DFTUM WEL EDQD FT PEEE

MF-צELDEITY FMAL

5 g AGL

E ALITD FESID GTATICS

ᄀ I F FDLD STAEK

9 MCISE RETELTIDN FILTER

\section{DISPLFW PARAMETERS}

HDRZ SEALE

WERT SEFLE

ELEY SEFLE

PDL FRITY CDIDE

TロX MUMEER

EDMPLETEI $\theta$ TRAIR

1 口 IMNGEC

1 10 FTم FN

LEFT+ RIEHT-

REF

ㅌㄹㅁㅗ

คuE I 


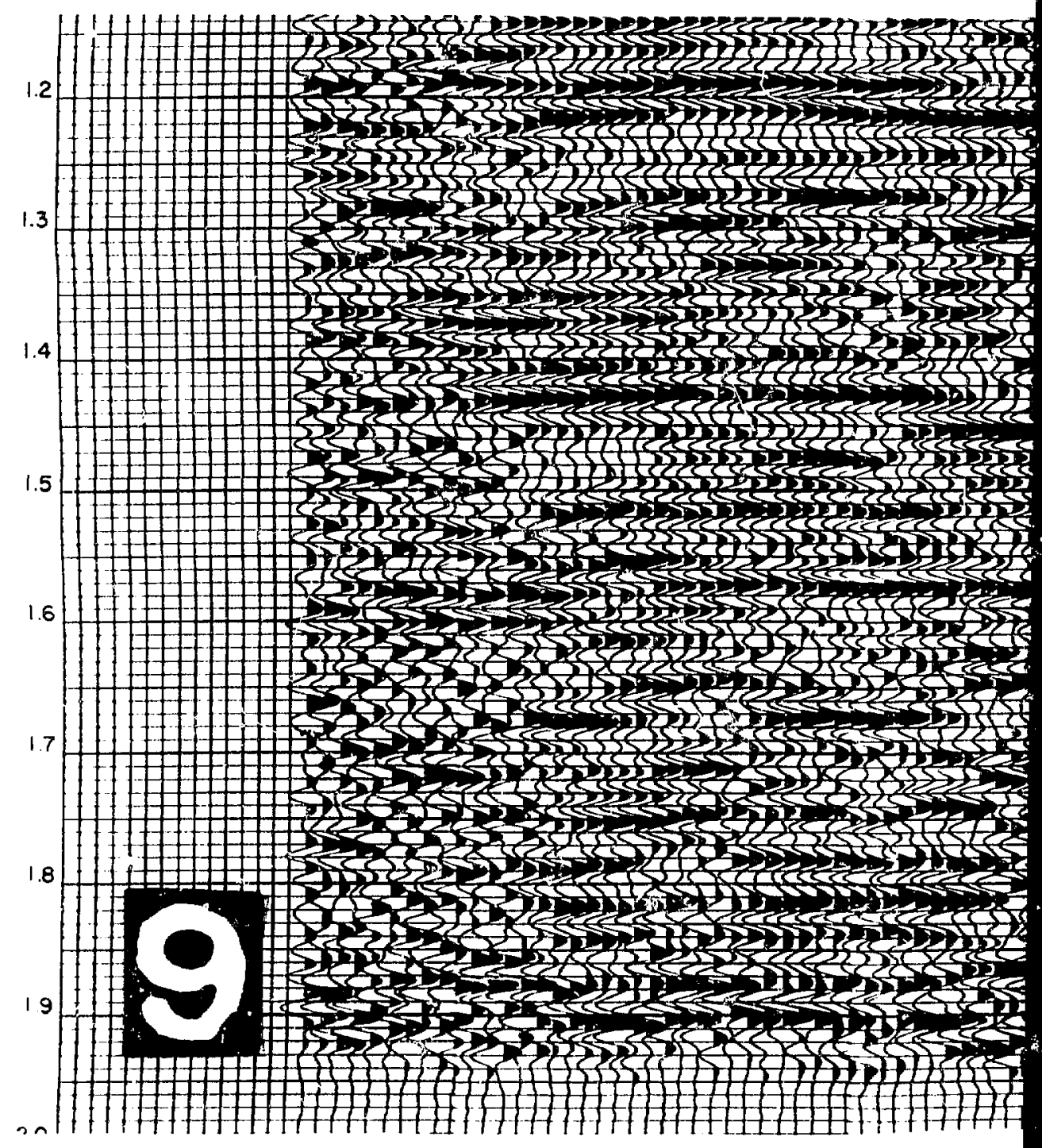




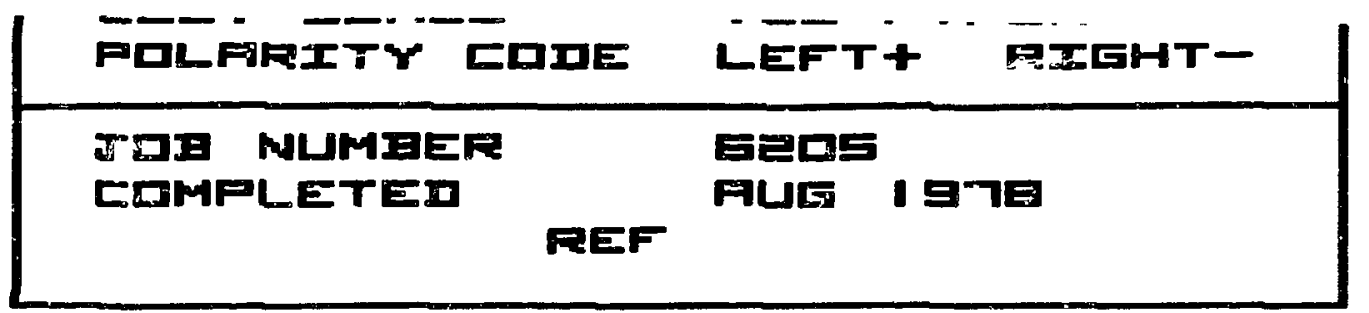

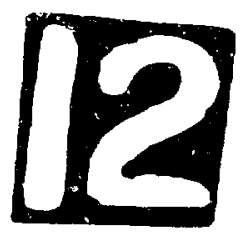




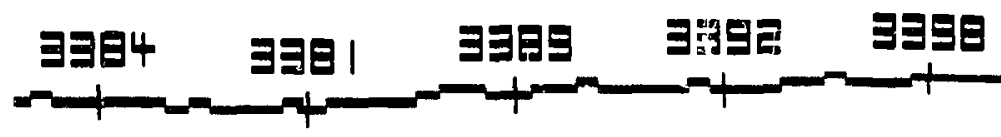

DFTUM

큽미

SANDIR $y-3$

SANDIA $Y-1$

17270 |
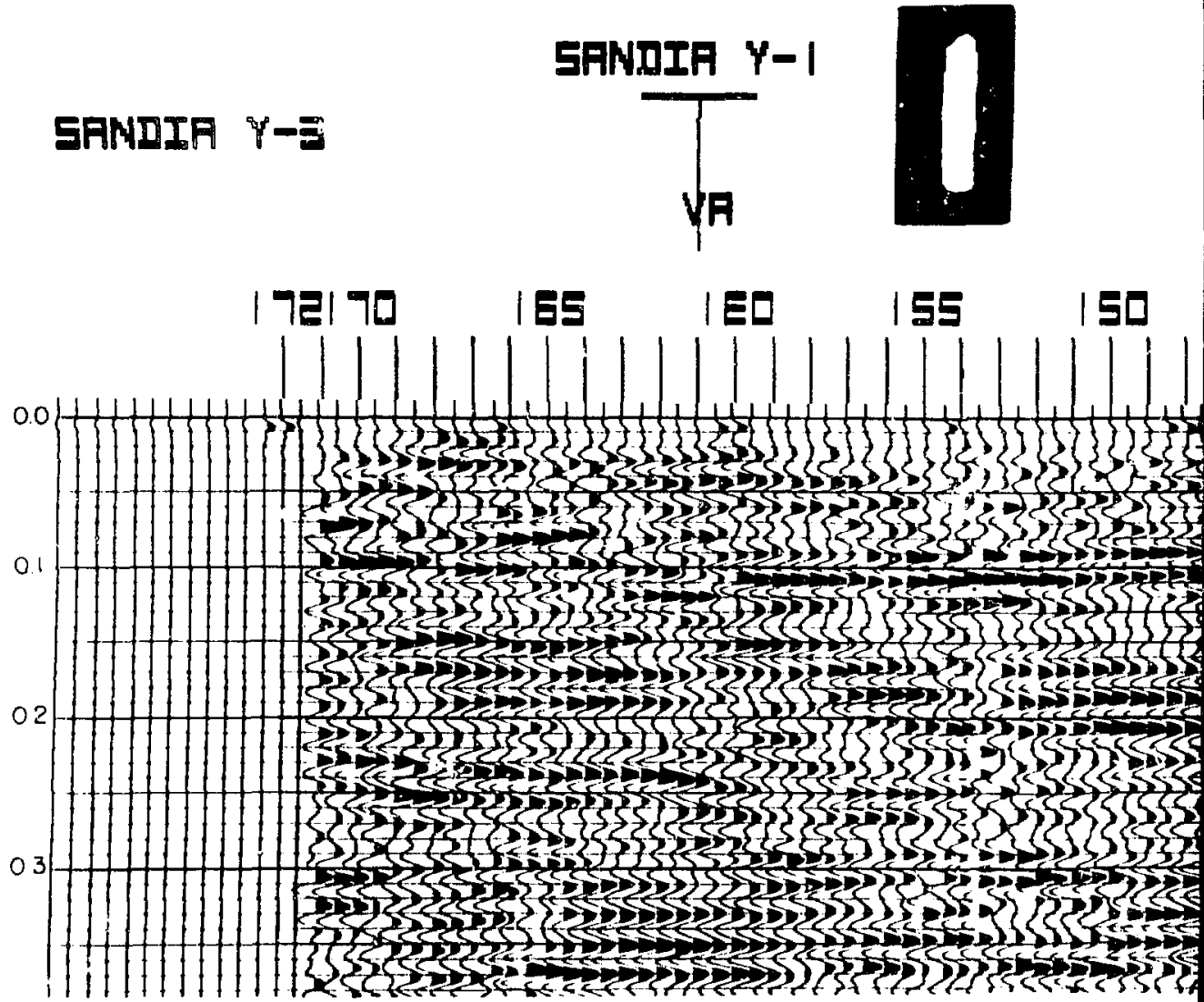


\section{SEISMIE FND DIEITAL}

CDMEPTEg IMㅁ.

\section{SANDIA LAB.}

\section{EFST}

AREF

LINE

STPTE

EDUMT \&P

DATE SGHT

\section{SANDIA}

$r-\equiv$

MEN MEXIED

LEF

エபடช ᄅ- I I

\section{FIELD PARAMETERS}

\section{REEDRDED Br}

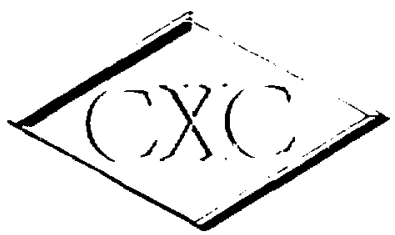

$$
C .1 \times 1.10 .
$$

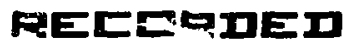

IMETRUMEMTS

EMEREY

SUNEEP LENCTH

SWEEF FFED

LISTEM TIME

SFMFLE MFTE
IIEXTFL

HมR-4D口冋

YIBRFTER

IDa

른ㅁㅁ Hב

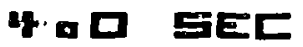

카도 


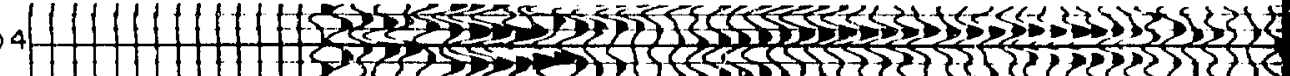

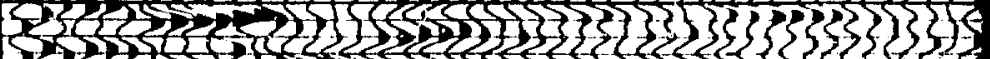

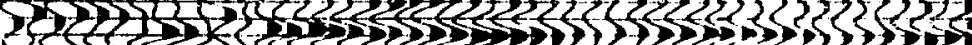

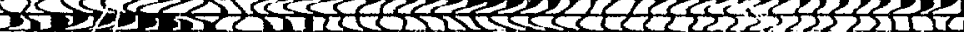

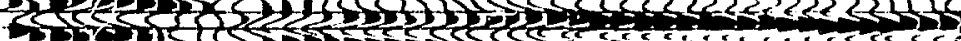
$3<2,30,5353$

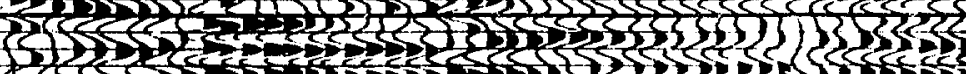

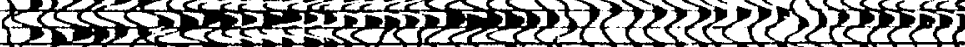

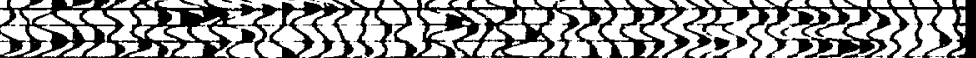

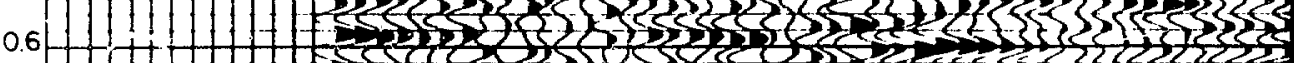

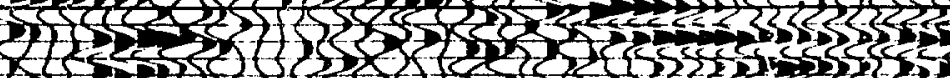

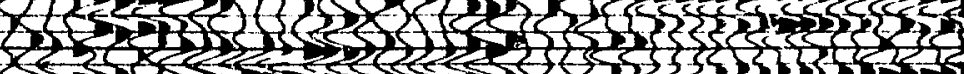

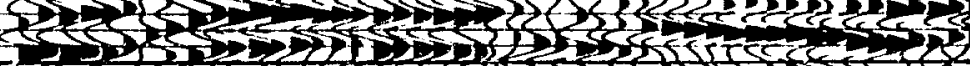

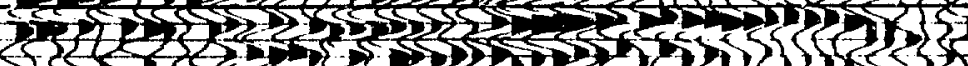

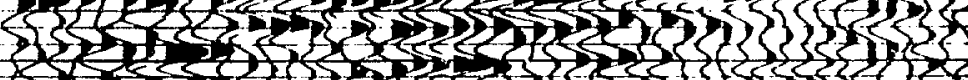

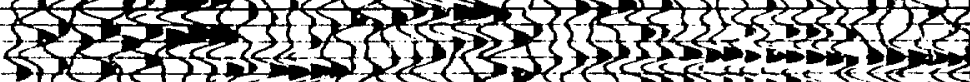

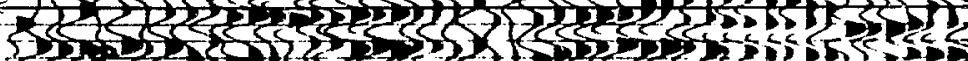

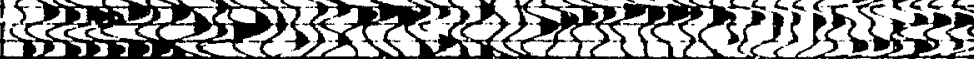

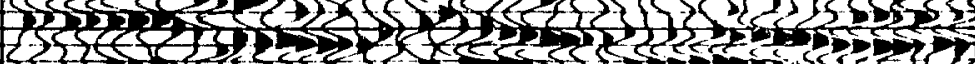

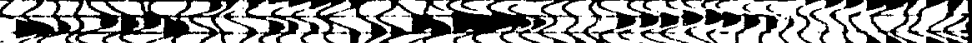

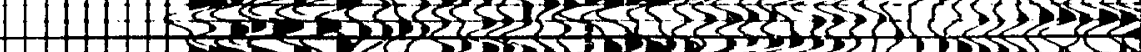

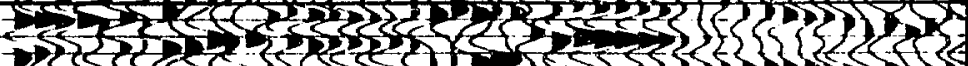

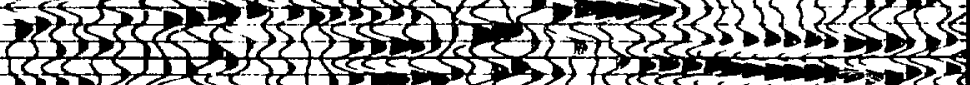

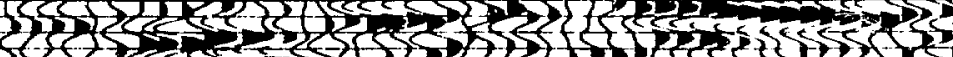

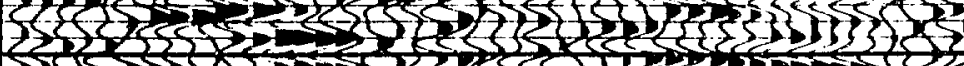

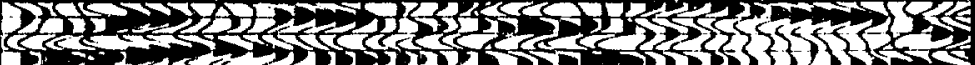

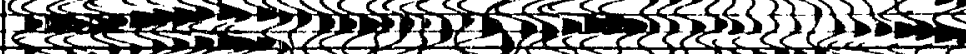

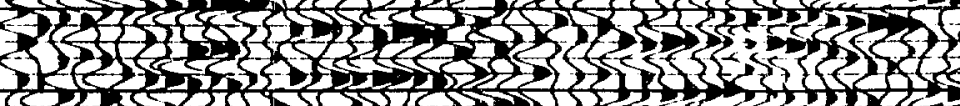

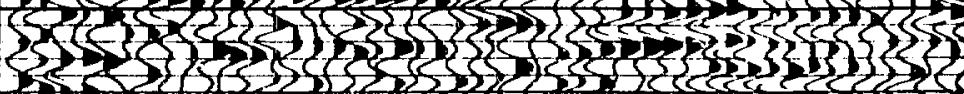

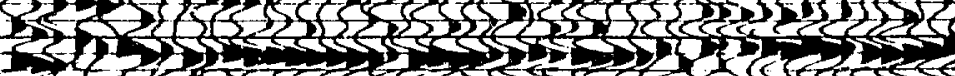

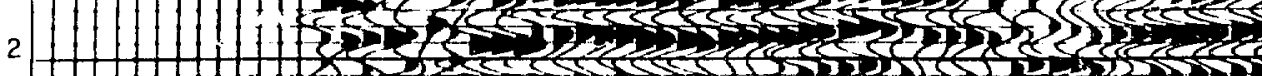

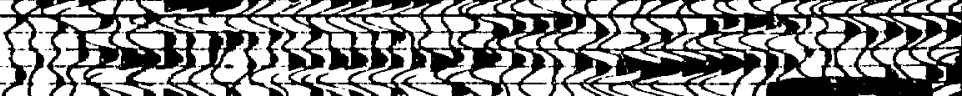

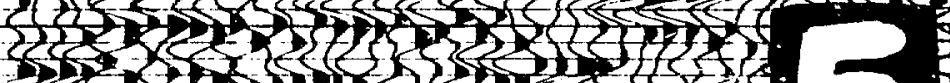

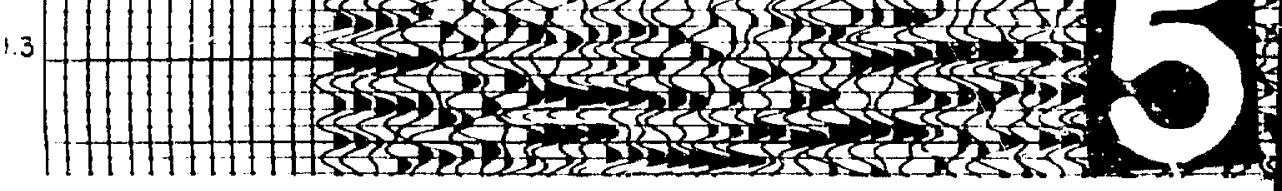




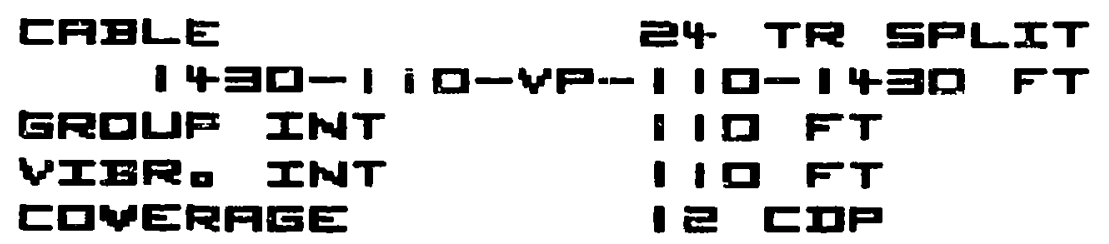

PRDEESSIMG PARAMETERS

PRDEEGSED $2 a D$ SEEC 2 MS

1 SPIKE DECDM-TIME WFRYIME

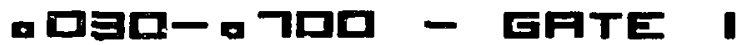

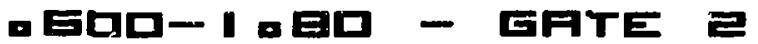

DFERATDF I D MS تDTH GATES

ヨgID TW FIL TER

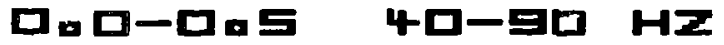

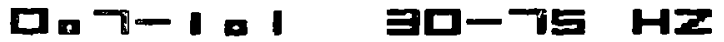

1 큼ㅁ 므-ES HZ

4. STATIES 9 NMQgSTEP-MUTE

गFTUM झᄅDᄆ FT FSL

DATUM NEL EDDD FT RSEE

\$F-WELDEITY FNAL_rSIS

SgE FEE

E RUTD RESID STATIES

7 I $\mathrm{F}$ FLU STFCK

9 MDISE RETECTIDN FILTER

\section{DISPLAY PAFAMETEFS}

HㅁZ GEFLE

WERT GLFLE

ELEW GEFLE

PDLFRIT $\mathbf{E}$ EDE

TUA MUMEER

CDMPLETED $\theta$ TRPIM

1 I INمSEE

1 DQ FT

LEFT+ FIEHT-

REF

비므

คUE I ตาย 


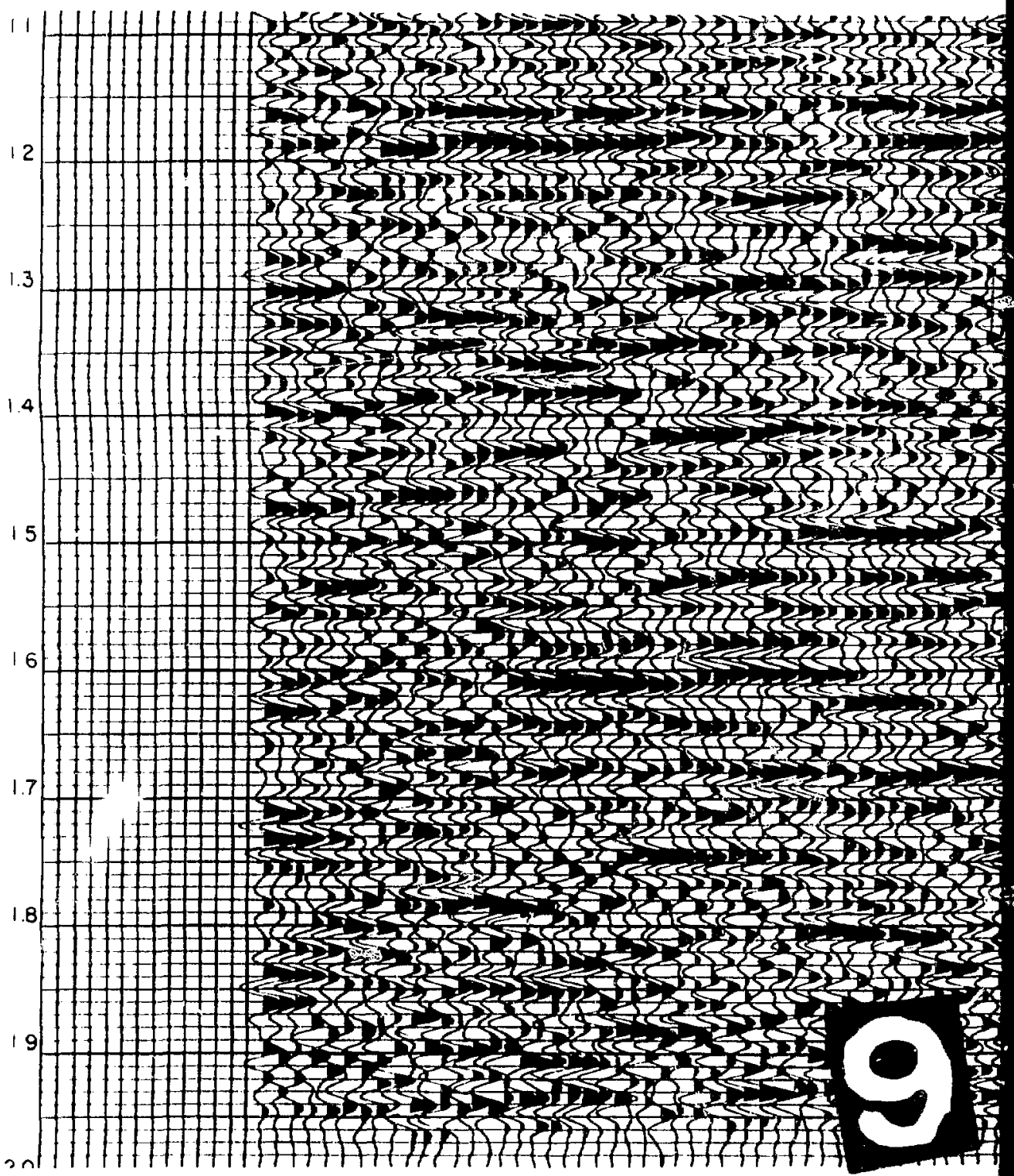




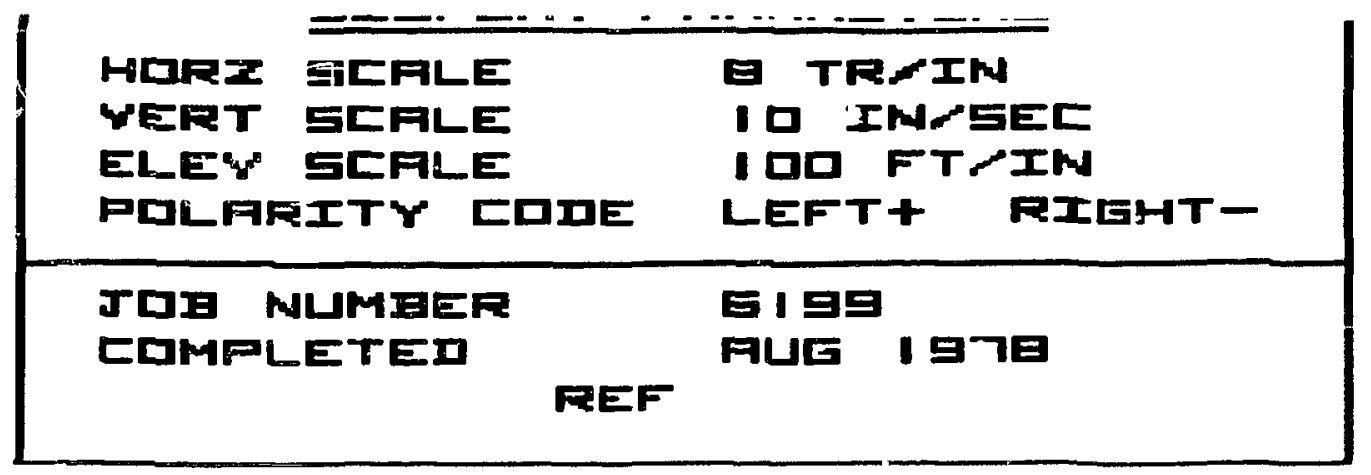

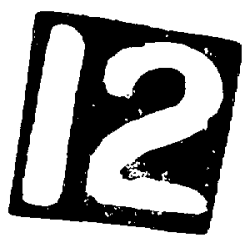


1

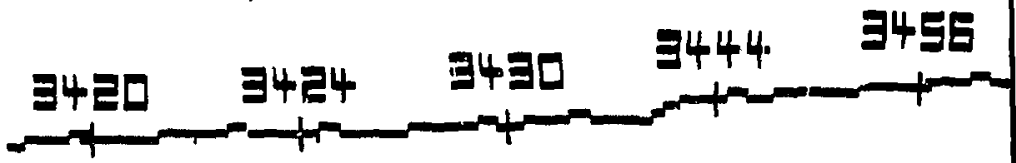

\section{DATUM}

SANDIF $\gamma=4$
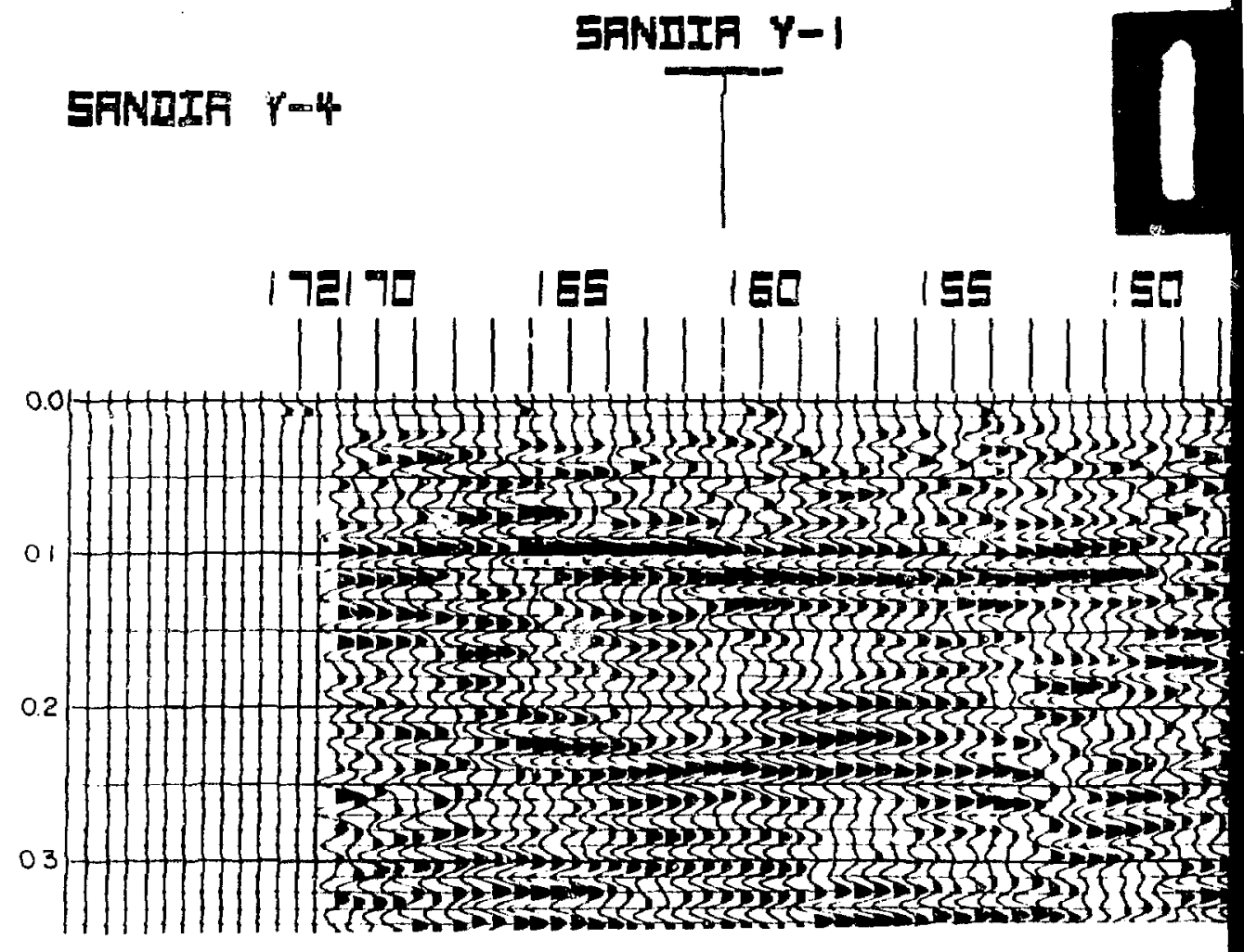
$77 \quad 3494 \quad 9475$

IDIR $Y-2$

E

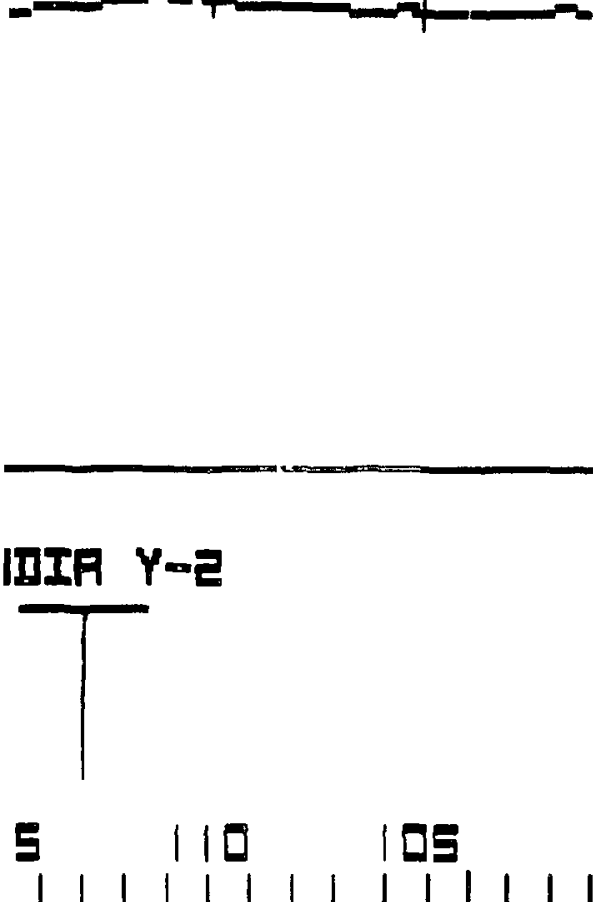

(

\section{5}

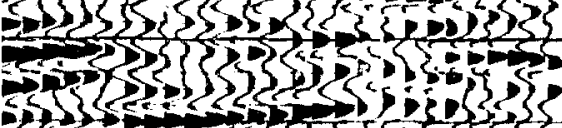

\section{\{

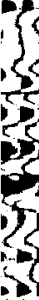

1

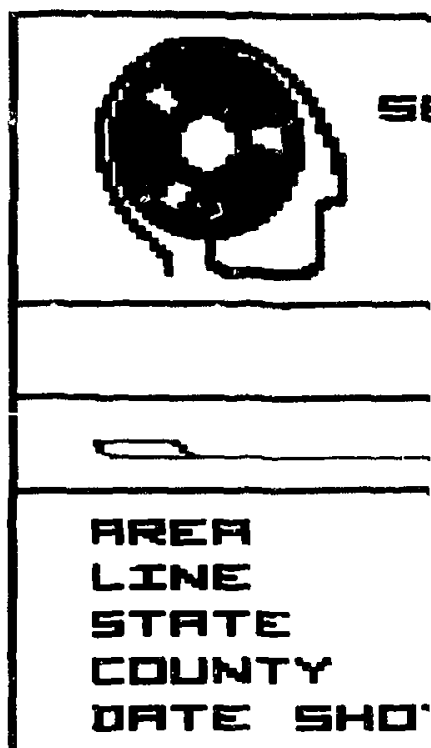

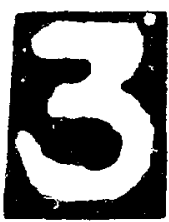

드르밀

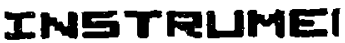
EMEEFE'

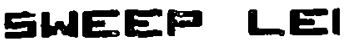
ELIEFF Fral LIEDTEW T: EFMFLE FI 


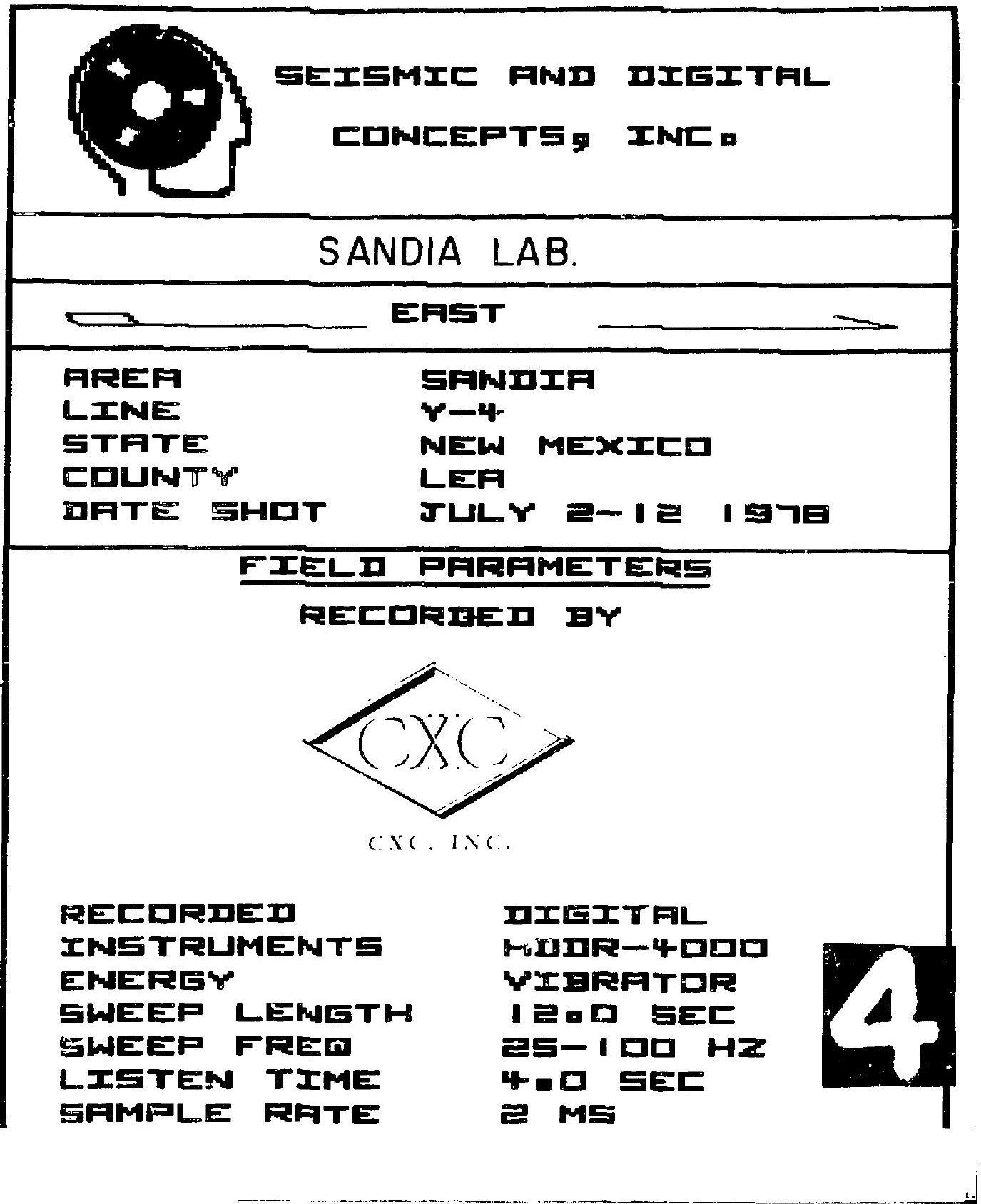




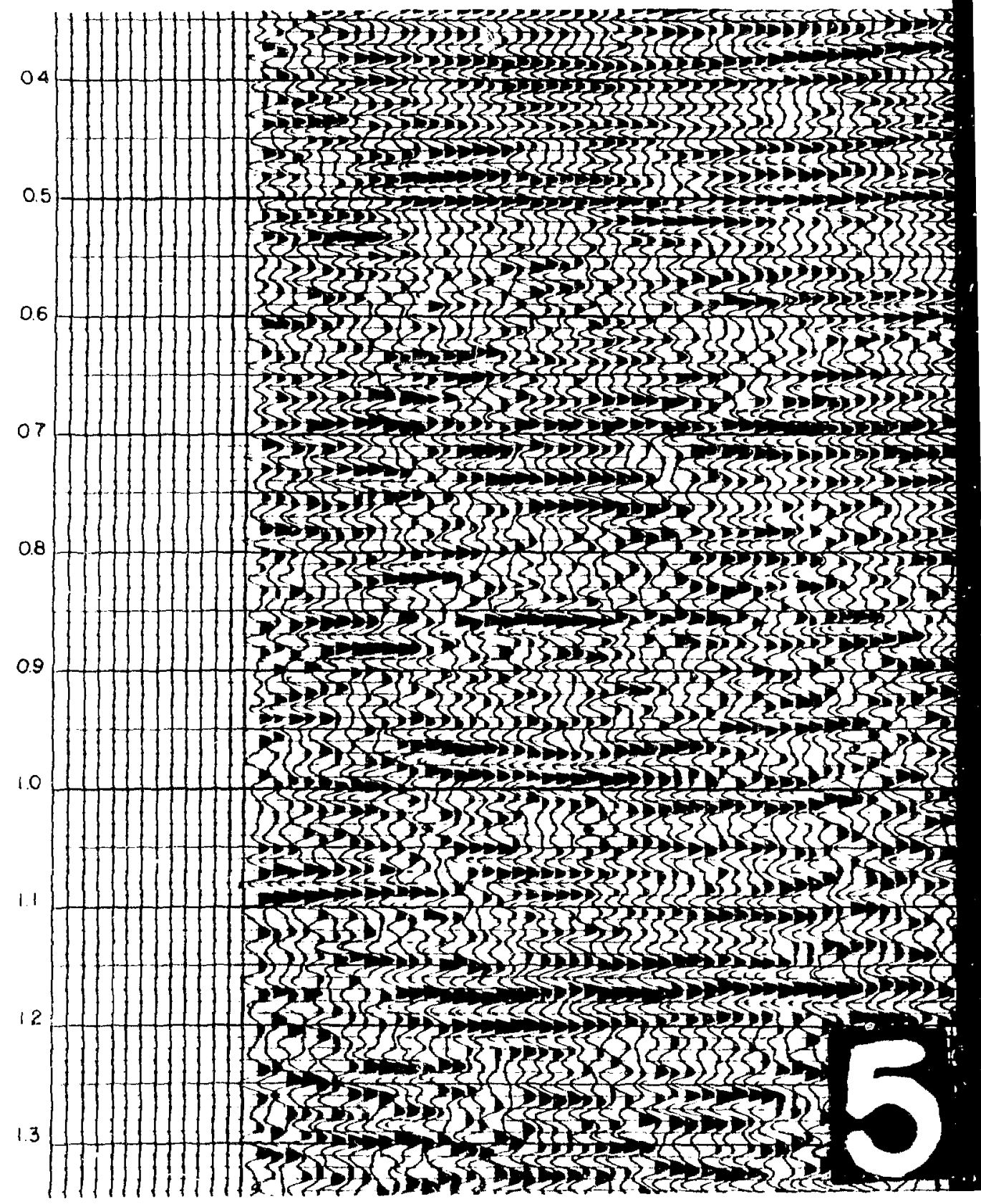




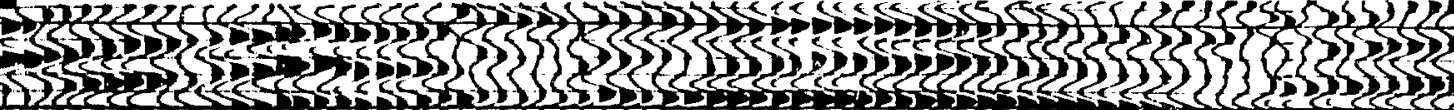

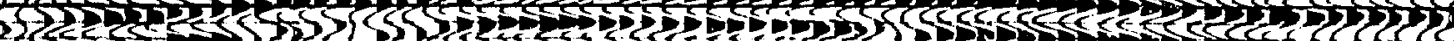

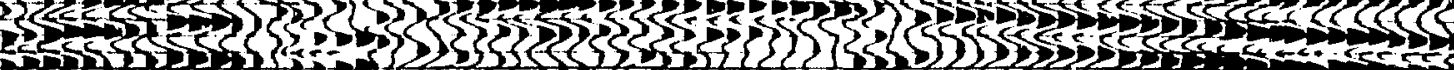

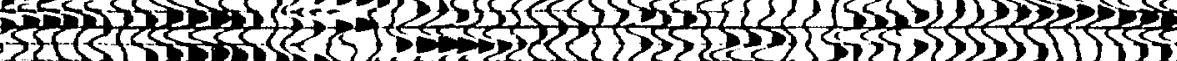

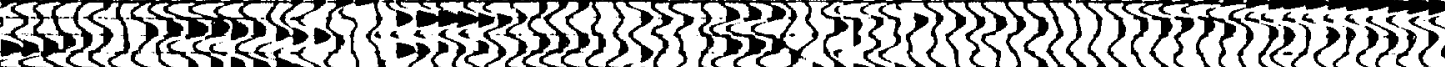

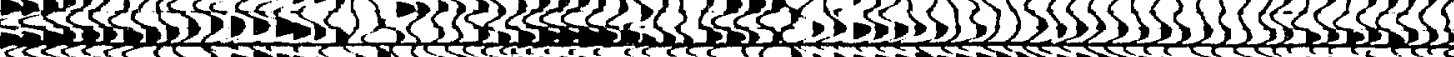

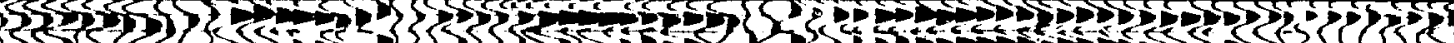

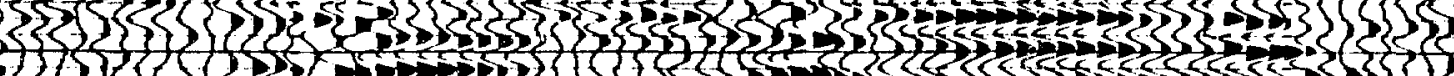

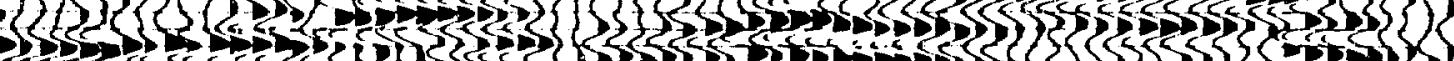

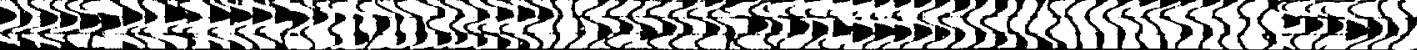

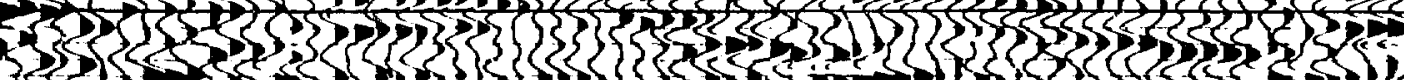

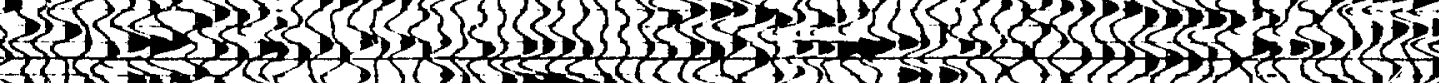

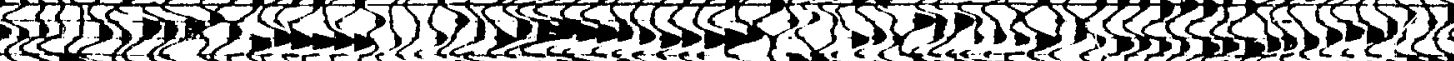

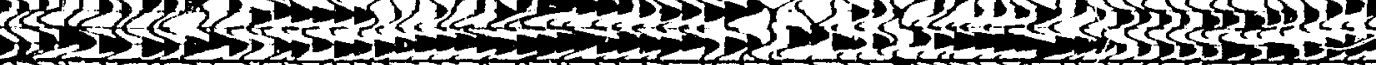

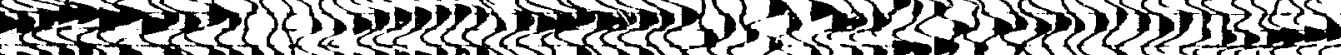

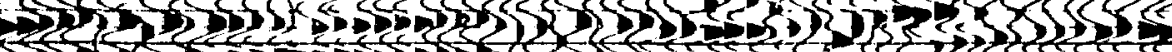

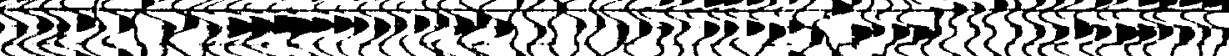

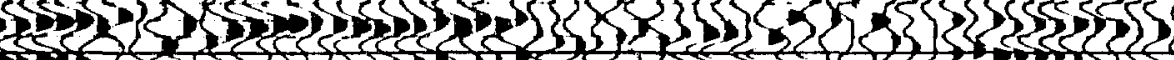

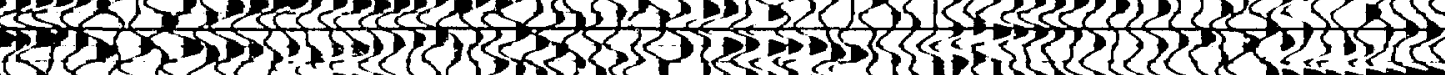

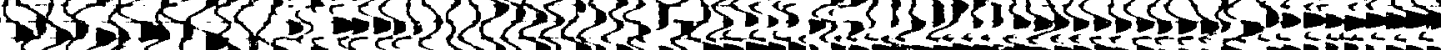

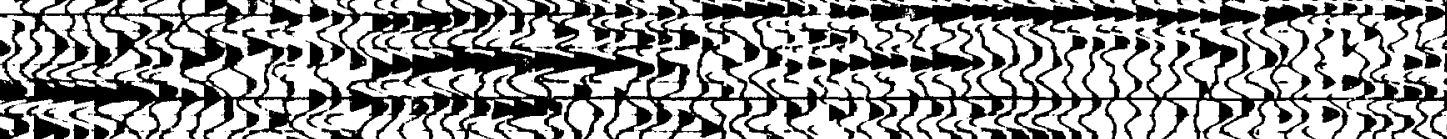

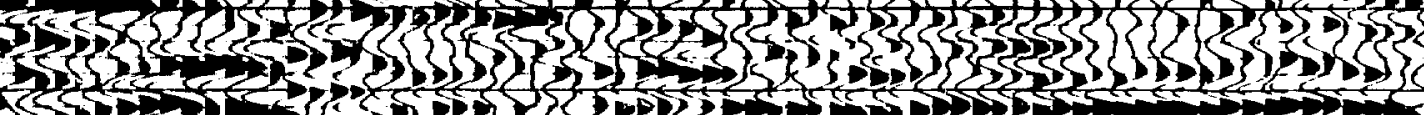

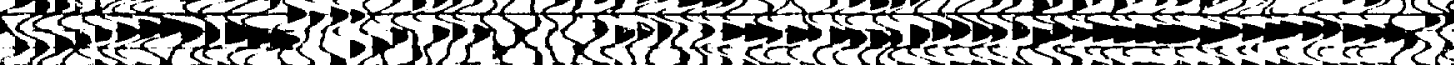

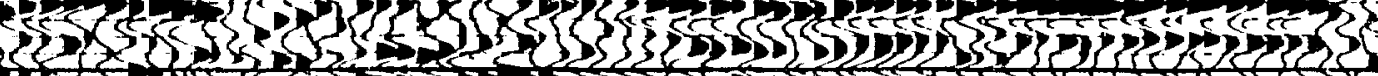

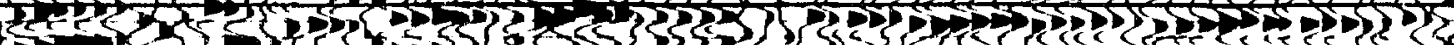

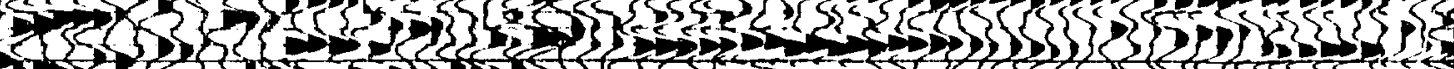

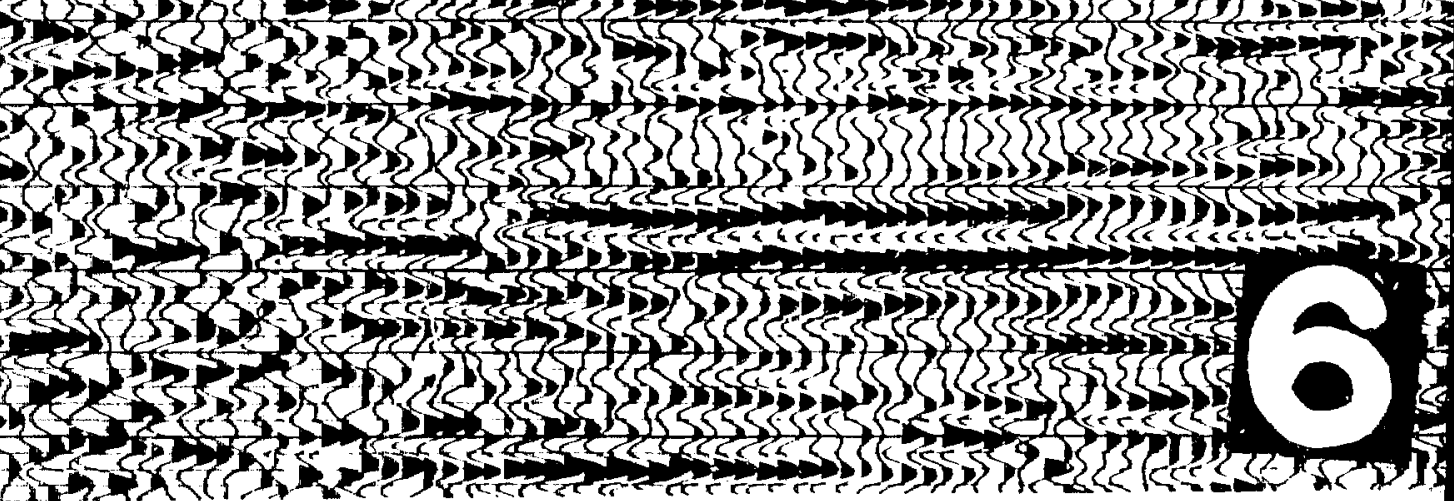


EFIL_E

루 TH EFLIT

$$
\text { | 4三E- | | }
$$

GRELF IMT

10 FT

UIIFE IMT

$110 F$

CEMARFEE

1 E EDF

PRDEESEING PARAMETERS

PRDEE틒 리 들 를

1 SPIRE DEEDM-TIME WFFHIME

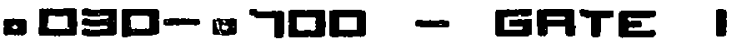

- EDE- aED - GFTE E

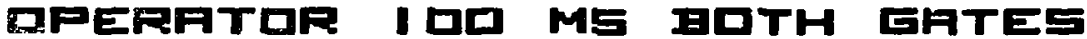

三g 10 T中 FILTEF

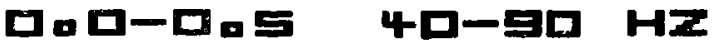

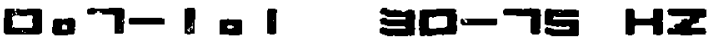

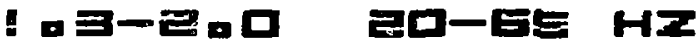

4 ETFTIEEgMMUgETEF-MUTE

IFTUM झEDE FT FEL

IFTUM भEL EDED FTمEEE

WF-WELDEITY FMFL WEIE

EgE FEE

E FUTD FEEIX ETFTIEE

7 I FELD ETAEK

wDIEE FESEETIEM FILTER

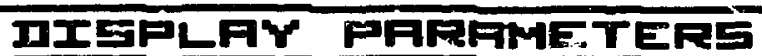

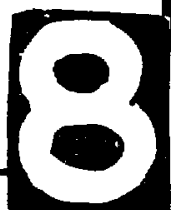

HaFI GRFLE

WEFT WEFLE

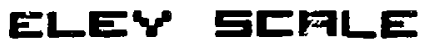

PDLFFIT H EDE

TQX MUMIEE

EUMFLETED
E TF⿵冂卄

10 IM م

10 FTم FN

LEFT+ FICEHT-
드미토

FLE \:7E 
1.2

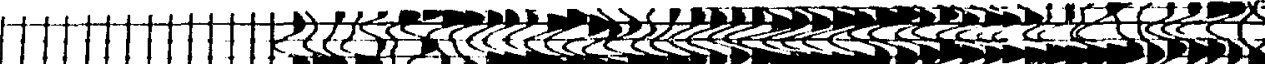

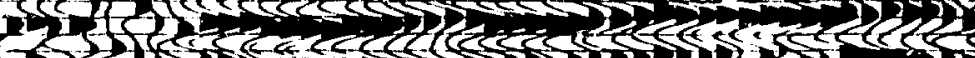

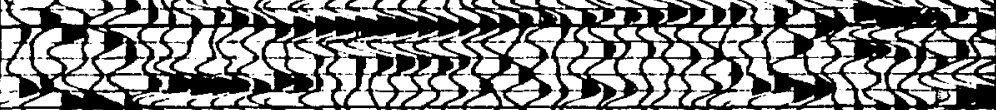

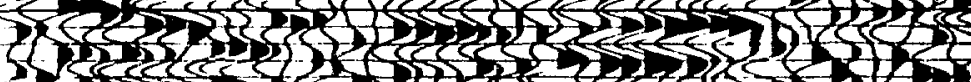

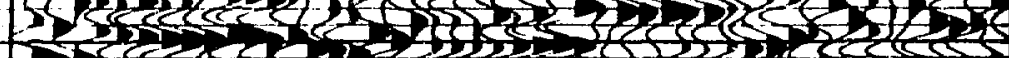

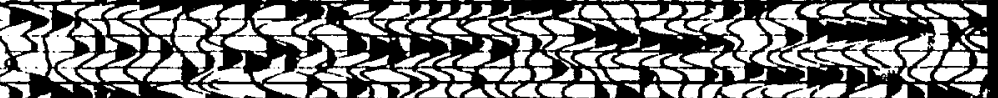

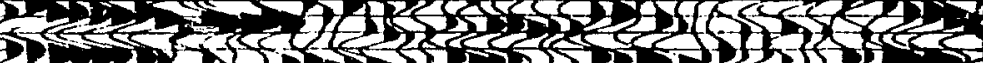

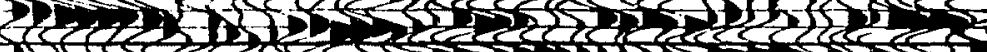

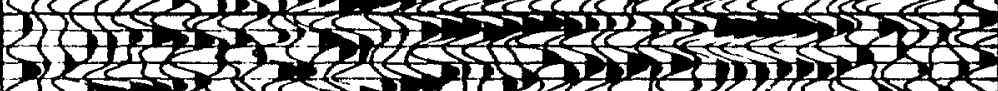

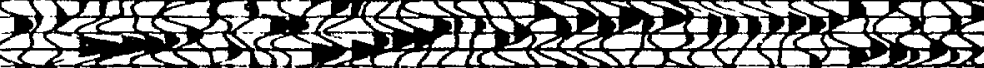

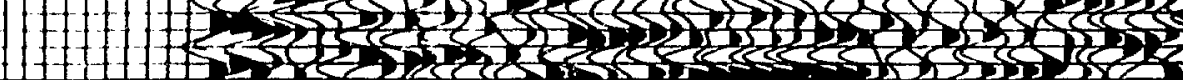

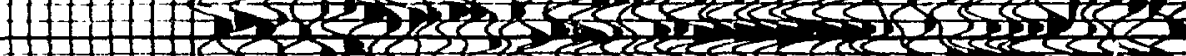
15
1

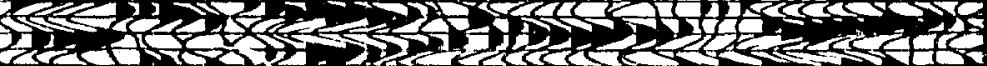

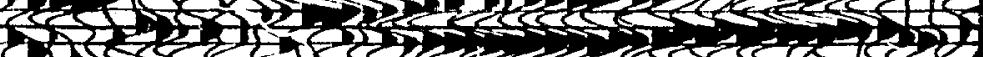

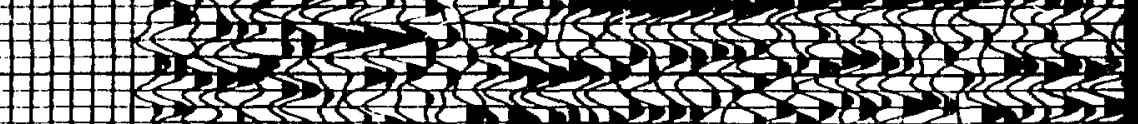

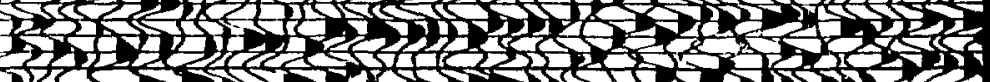

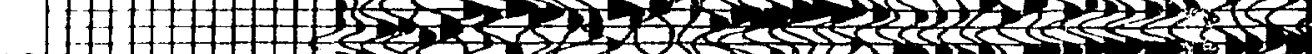
$1.8 \mathrm{H}+\mathrm{H}+\mathrm{S}$ 10 H 5 (3)

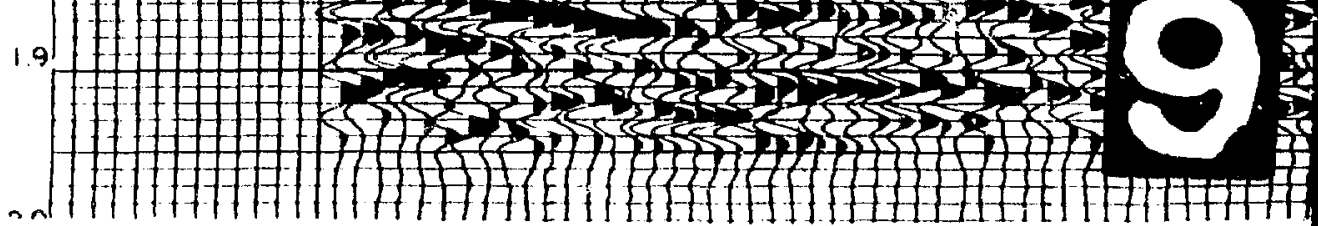


(2)

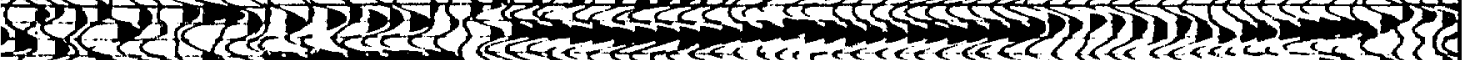

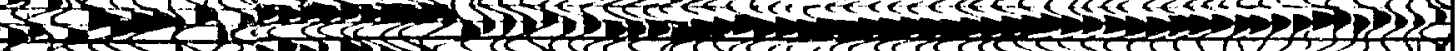

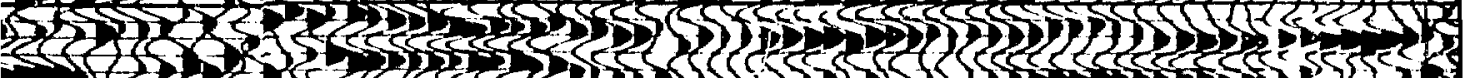

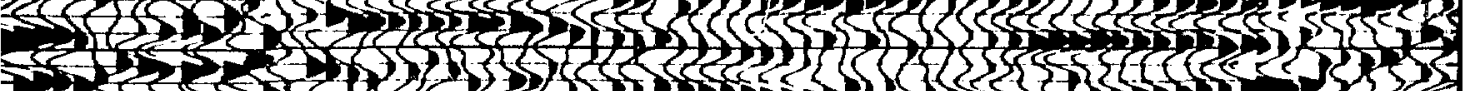

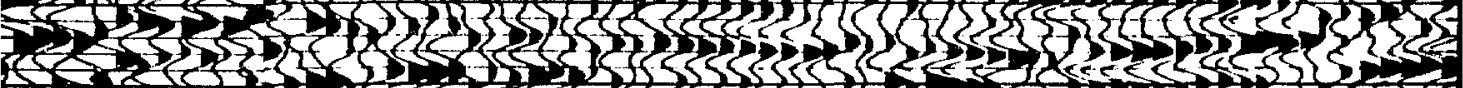
-

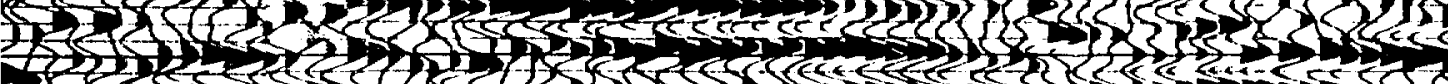

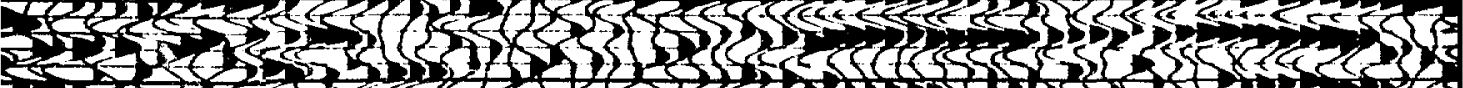

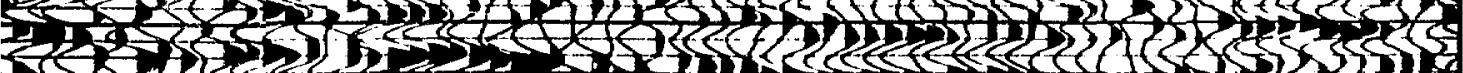

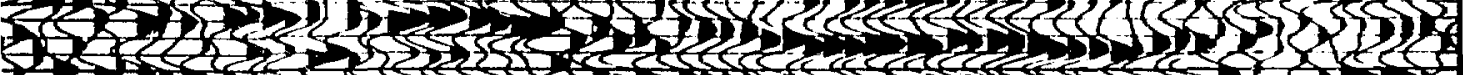
3)

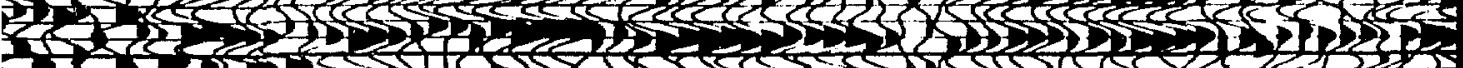
153

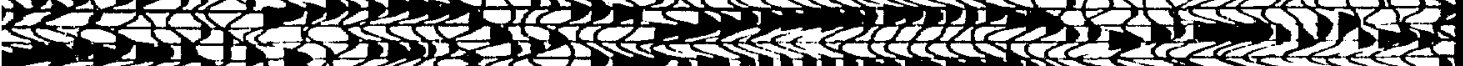
1315215

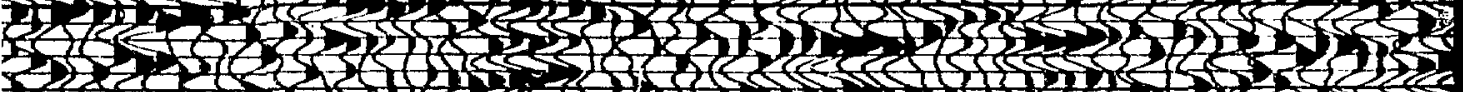

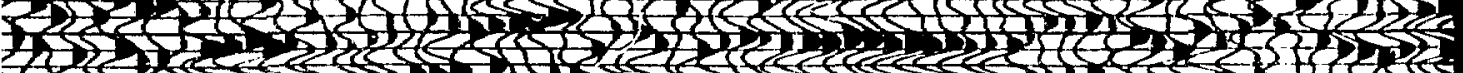
3325 131512101012 351012 3)

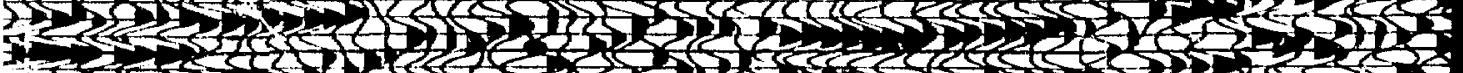
15210253 9. 7125

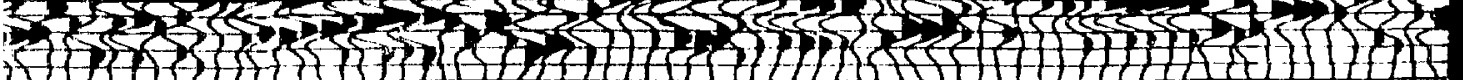




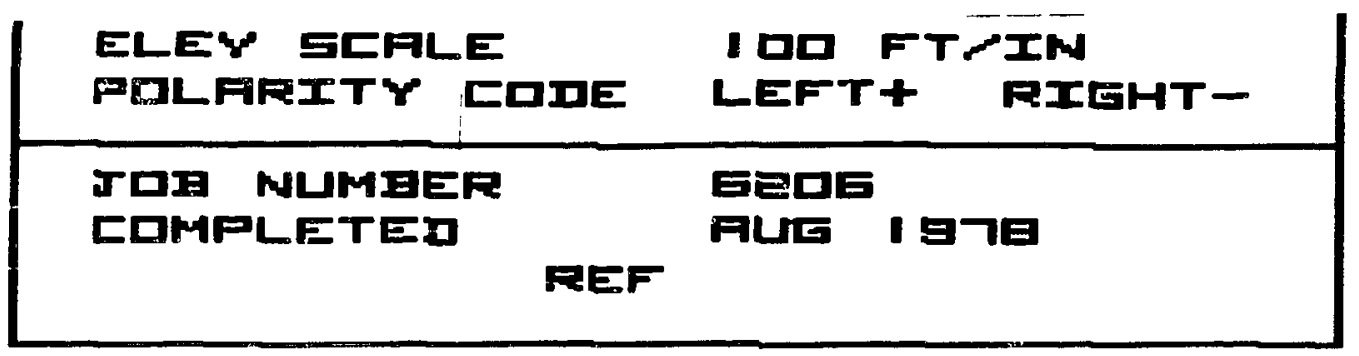

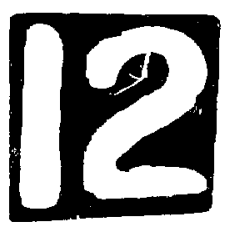




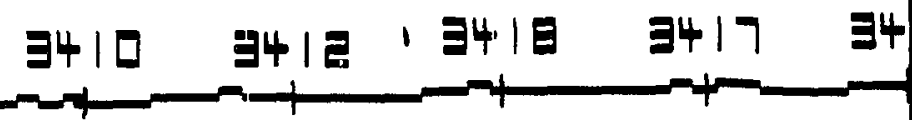

DATUM

EЕव口

SANDIR $\gamma-5$

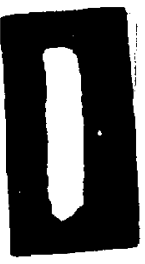

\section{SANDIA $Y-\exists$}
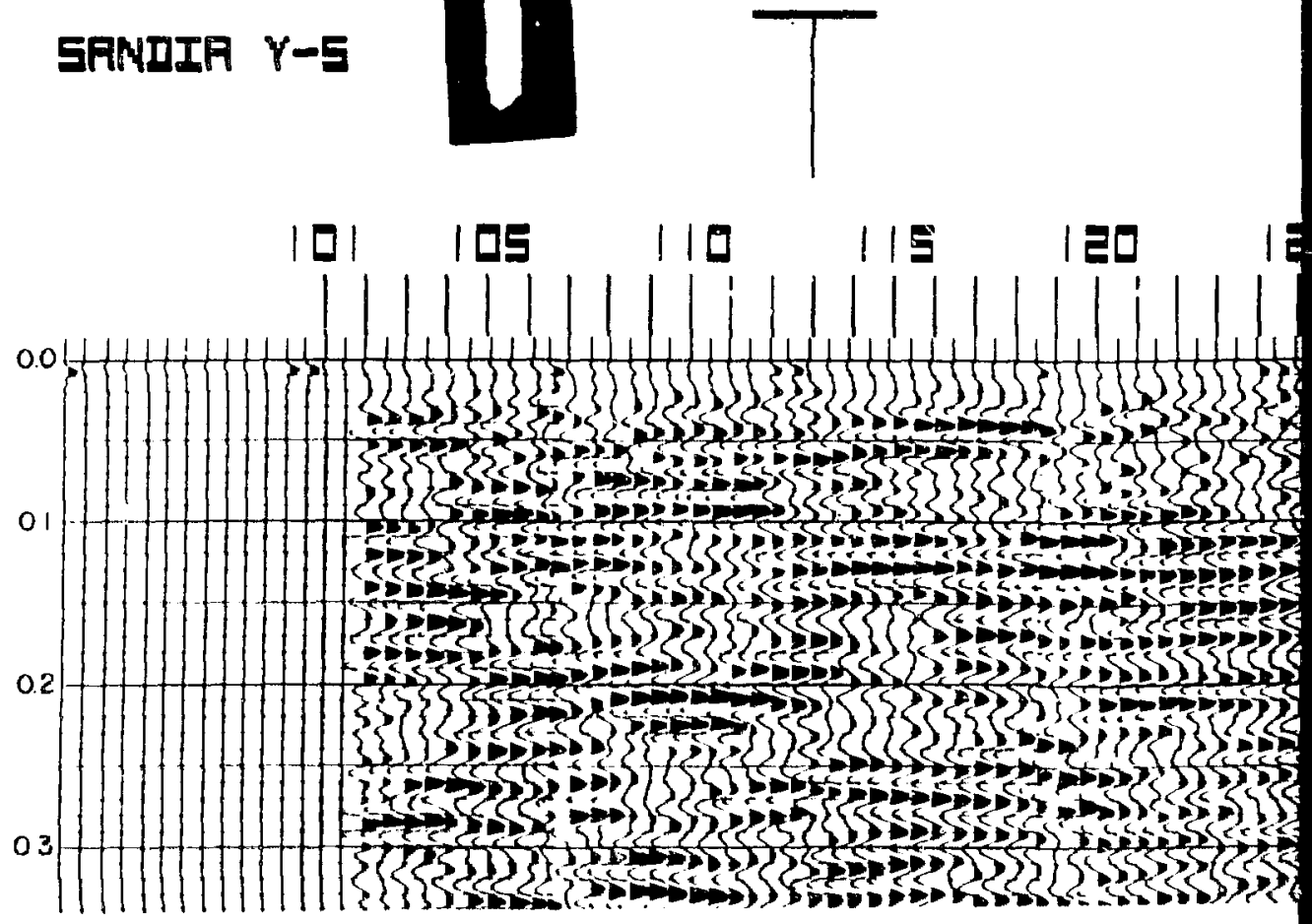


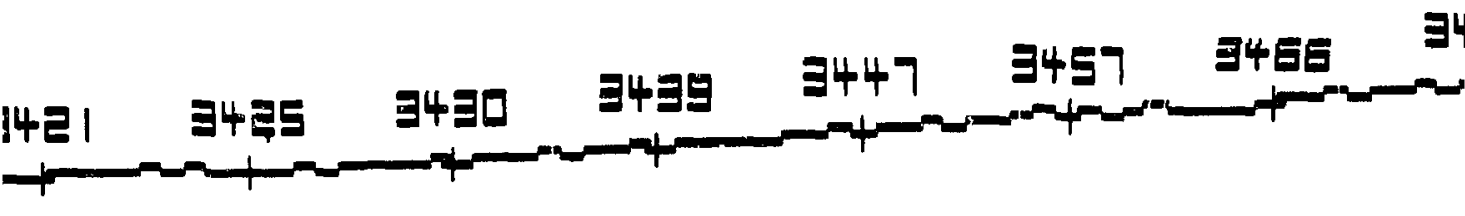

\section{Vค}

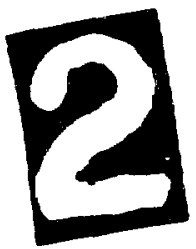

5AN.

$\begin{array}{lllllll}155 & 190 & 195 & 140 & 145 & 150 & \text { IS5 }\end{array}$

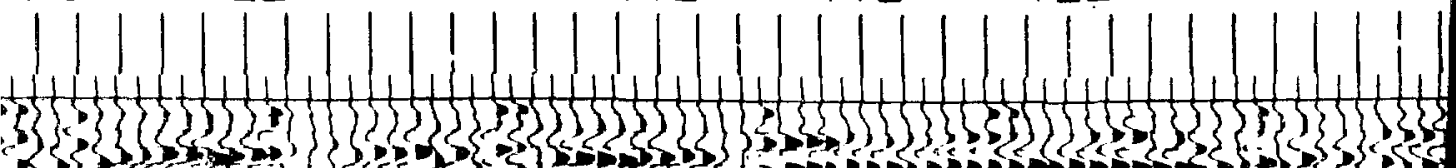

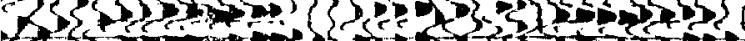

S. 5 - 250

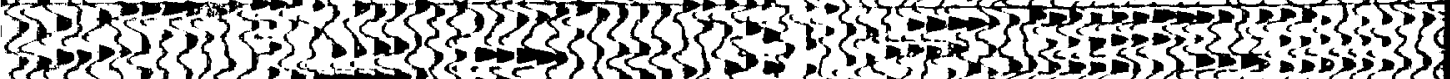

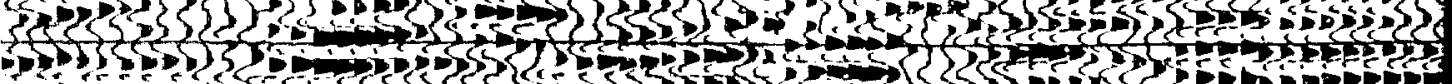

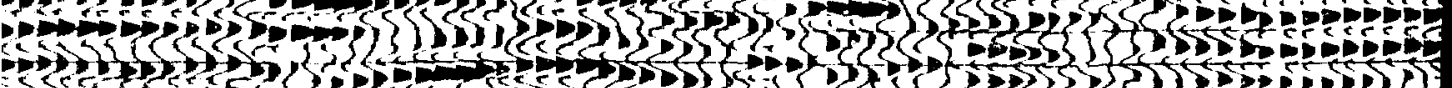

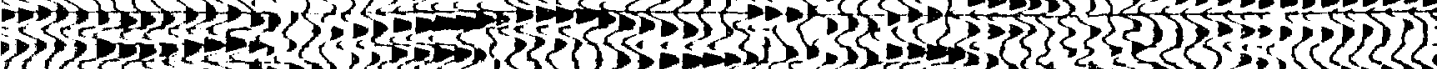

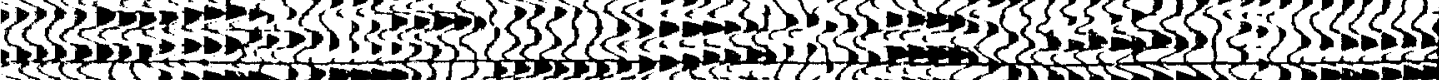

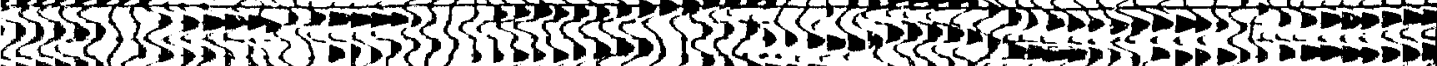

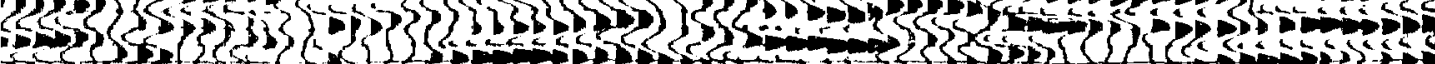

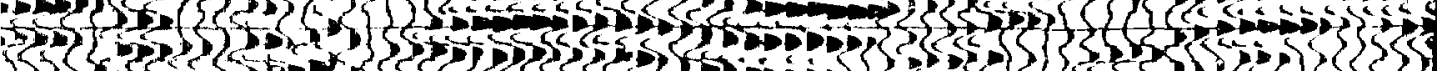

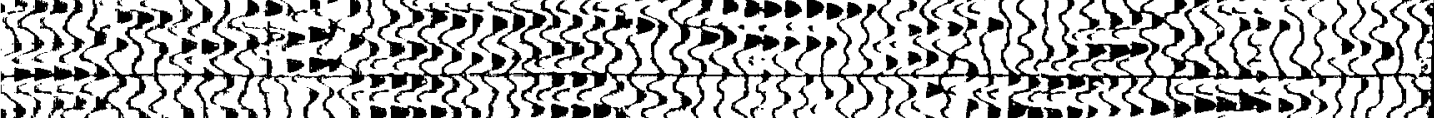

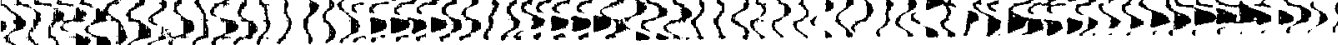




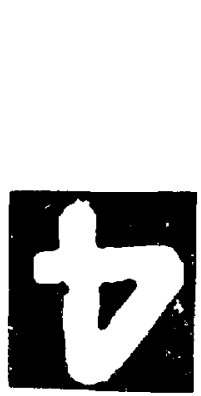

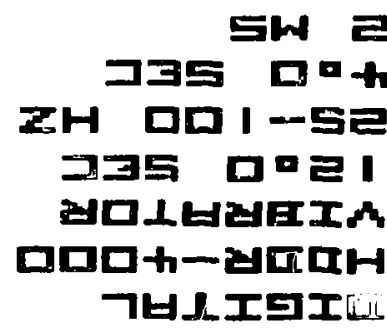

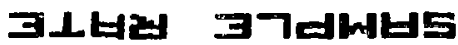

InIL ma15I?

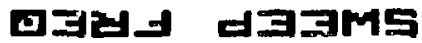

HLGME7 dIJMS

스러릴

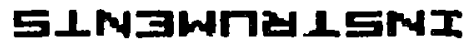

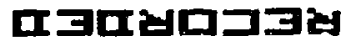

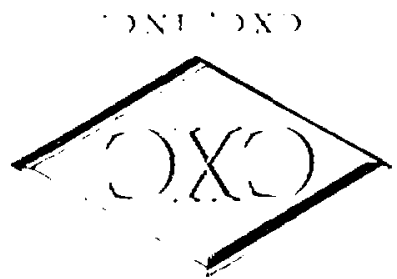

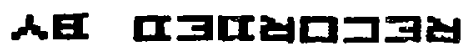

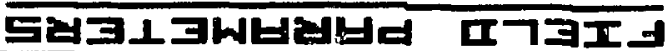

느 I $21-2$ A

$4=7$

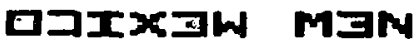

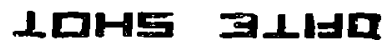

새 1 MITIEב

대

HIapula

새노

INAI

너코리버

MLider|

$\exists \forall 7 \quad \forall I O N \forall S$

- JhI Shedaコpaב

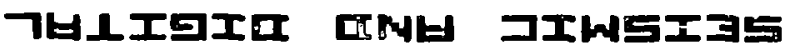

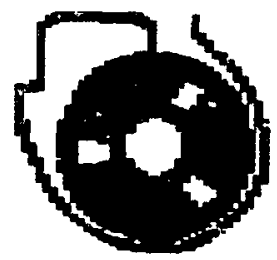




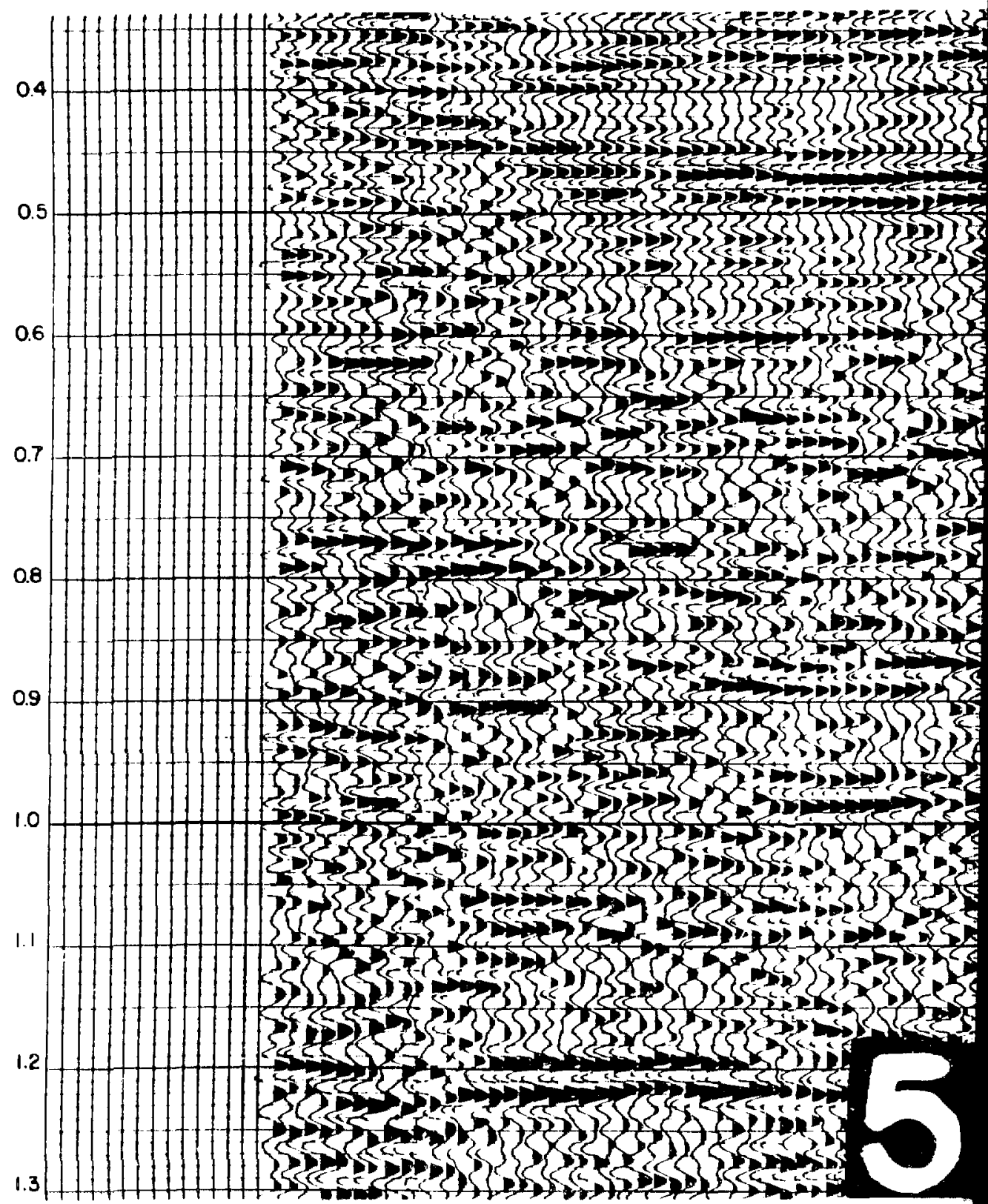




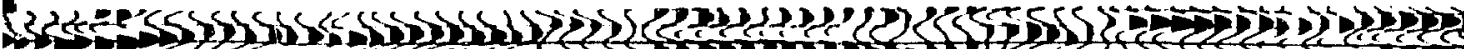

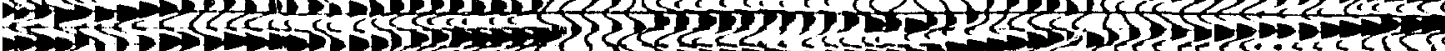

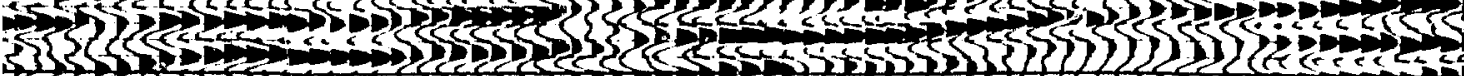

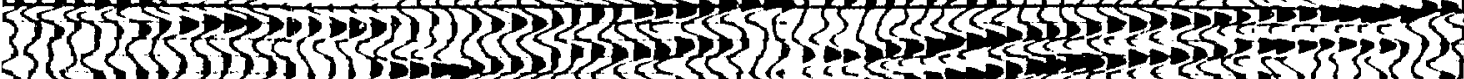

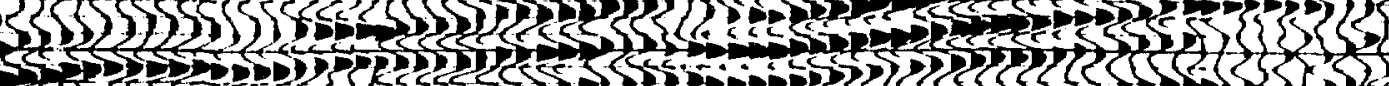

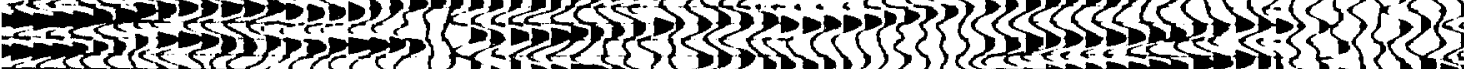

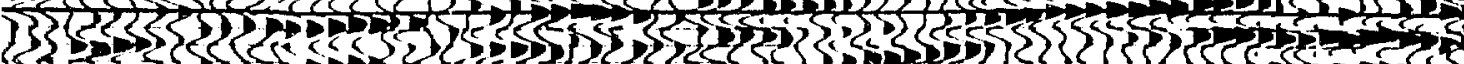

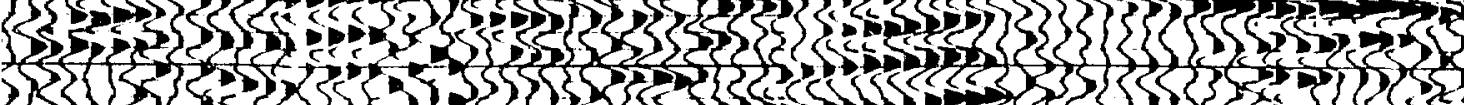

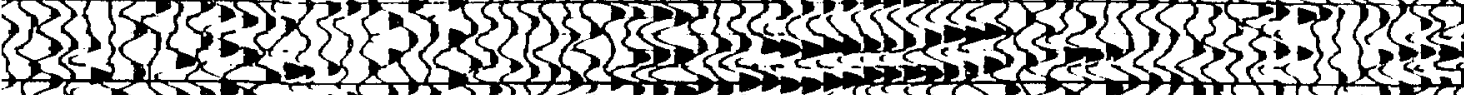

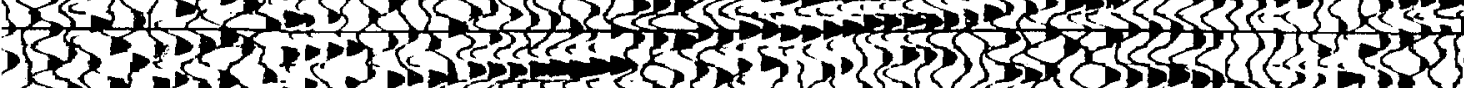

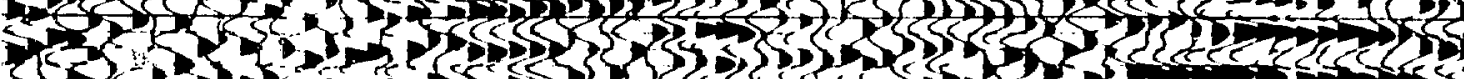

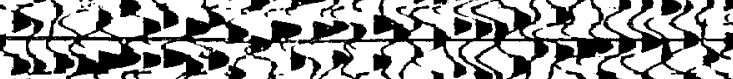

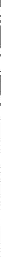

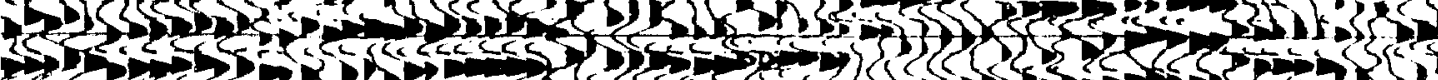

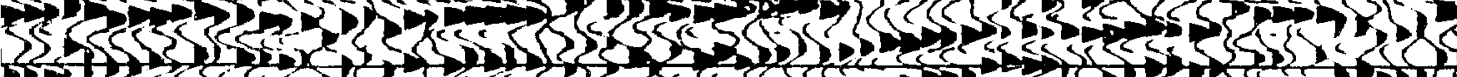

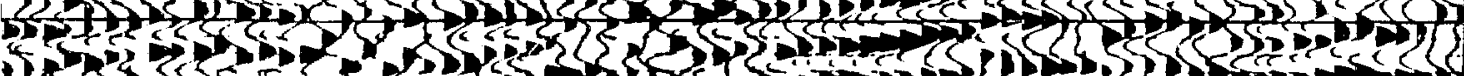

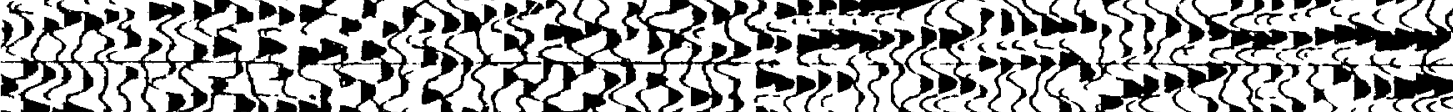

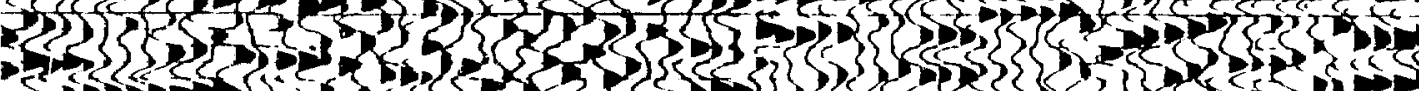

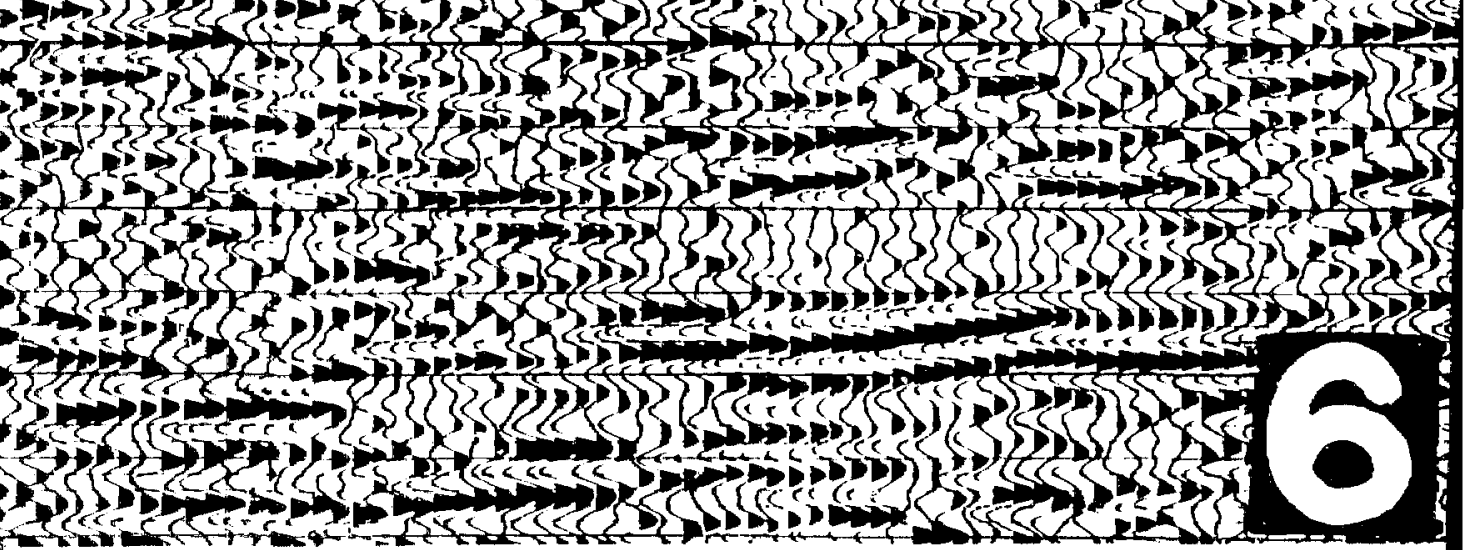




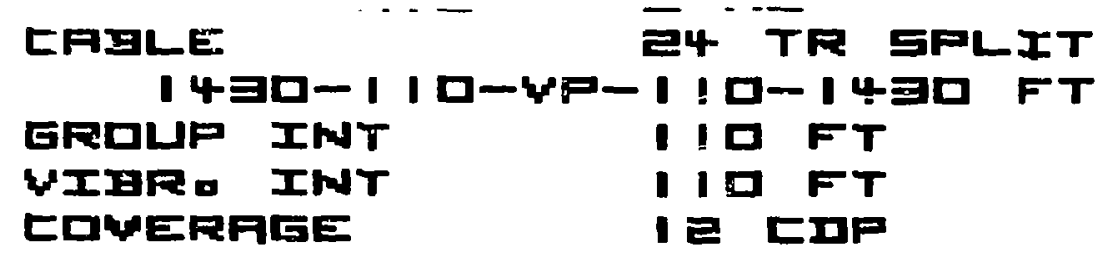

\section{PFDEESEINE PARFMETERS}

PRDEESSED

ᄅaロ SEE ᄅ $M S$

1 SPIKE DECDM-TIME WFFYIME

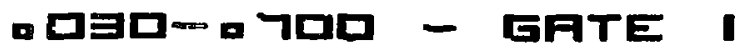

๑EםD-I.ED - GATE 2

DPEFATDF IDD MS घDTH GATES

ヨg1口 TY FILTER
口.0-口.5 4ロ-פ口 Hב

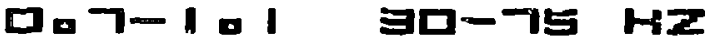
1 เ

4 STATIES gMMQэSTEF-MUTE

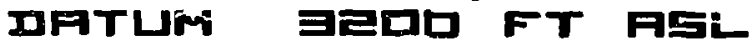

DFTUM YEL EDDD FT NSEE

WF-WELDEIT $Y$ AMFL $Y$ SIIE

Sg日 FGC

E PUTD RESID БTATIES

7 ID FDLD STAKK

9 MUISE RETEETIDN FILTER

\section{DISPLFY PAFAMETERS}

HIRZ GEALE

WERT GEFLE

ELEY TEARLE

PDLFRIT' CODE

JロB WLIMEER

COMPLETER
E TRPIN

10 IM 1 -

1 口D FT INM

LEFT+ FIEHT-
Eะロ7

FLIE I 


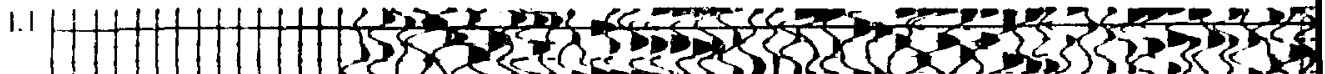

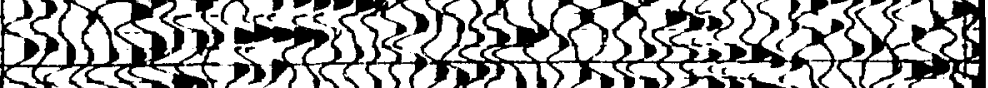

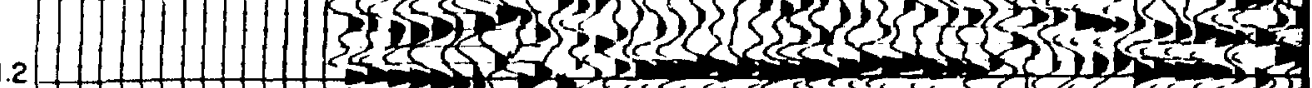

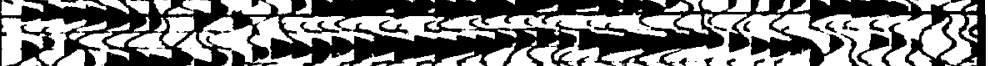

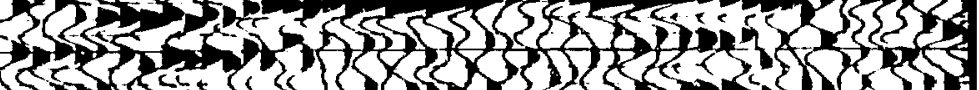

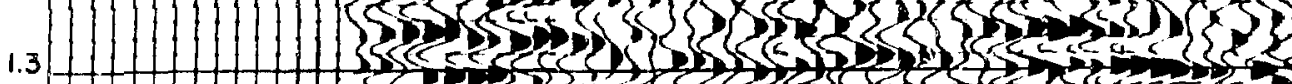

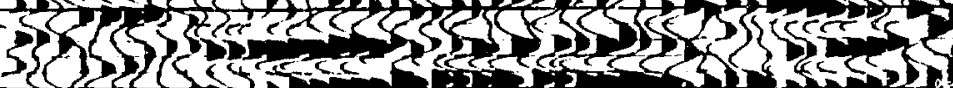
L

1.4

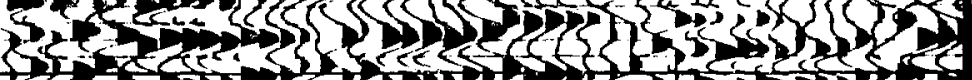

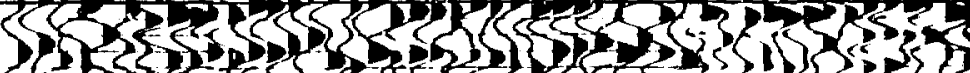

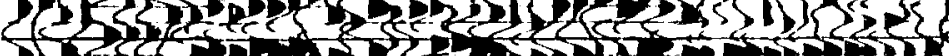

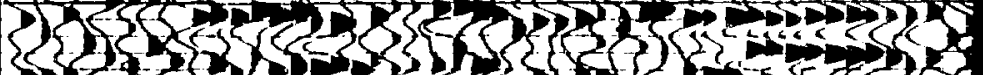

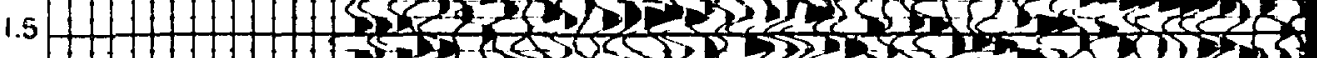

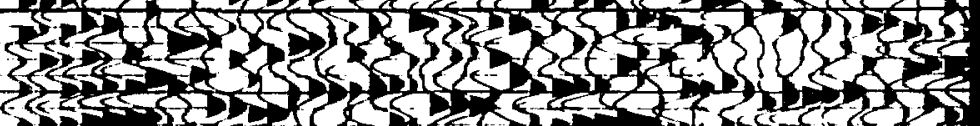

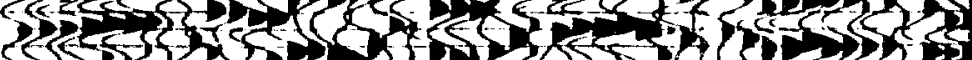

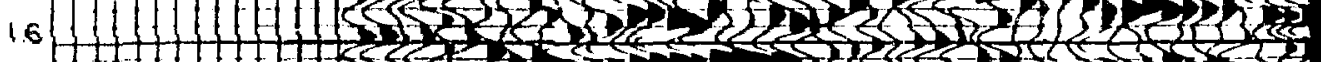

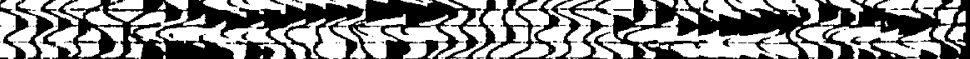

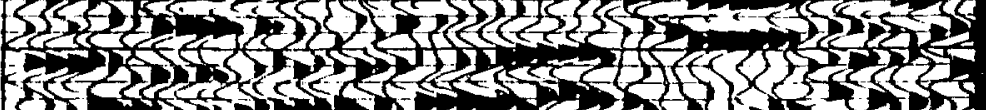

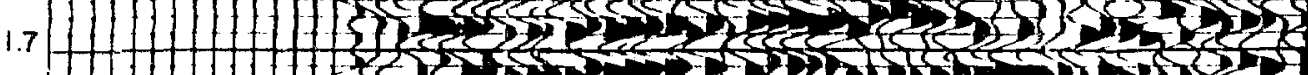

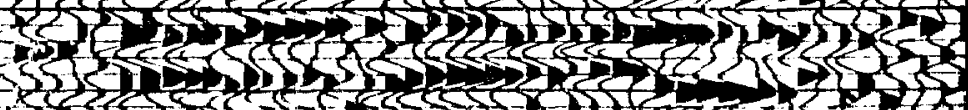

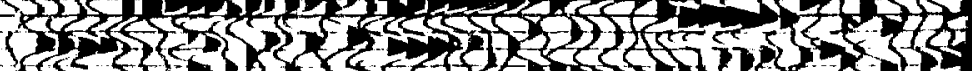

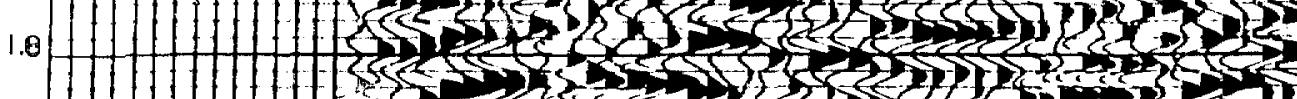

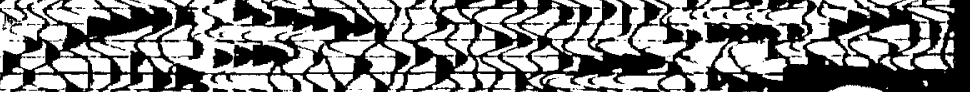

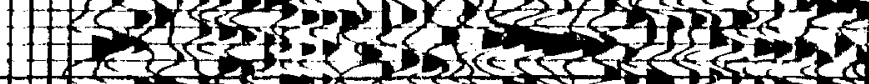
$F\left\{\begin{array}{l}5 \\ -120\end{array}\right.$ 


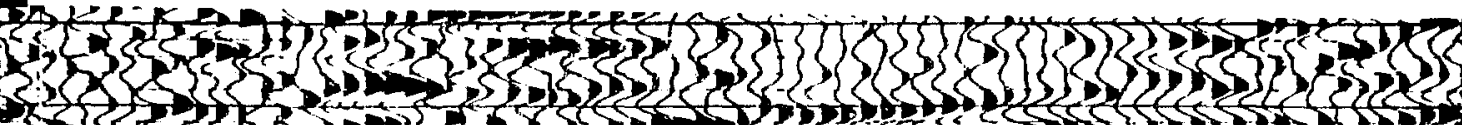

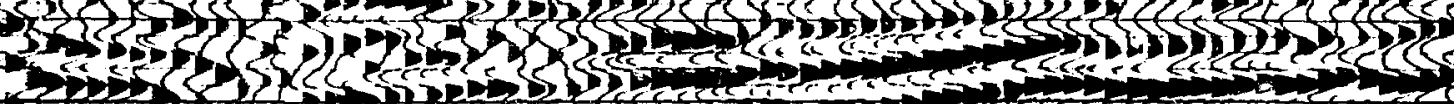

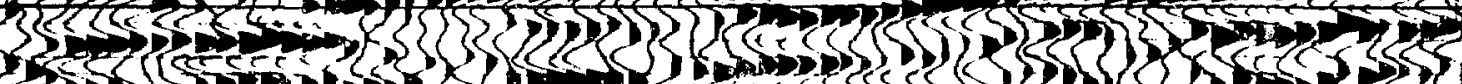

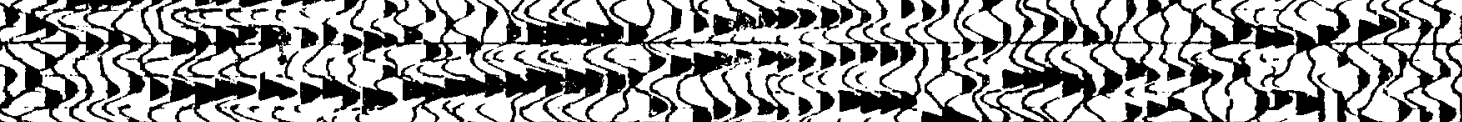

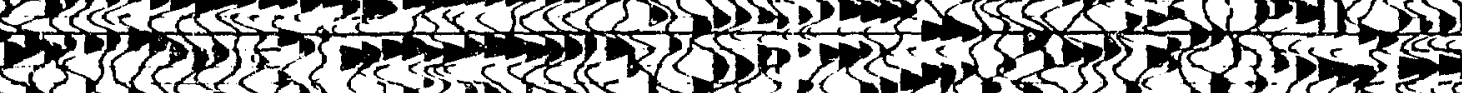

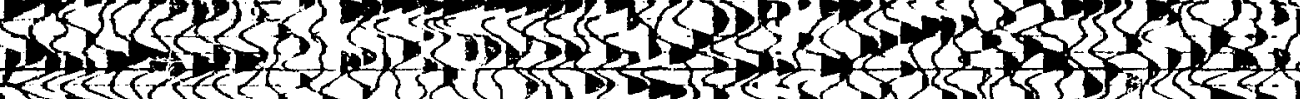

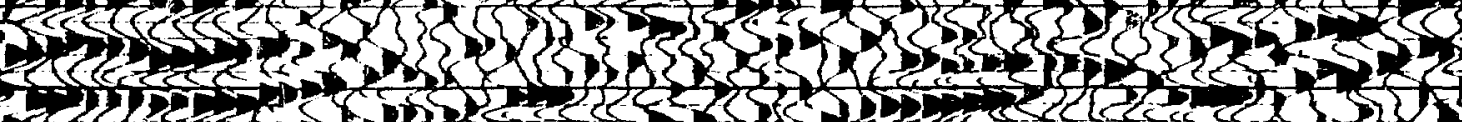

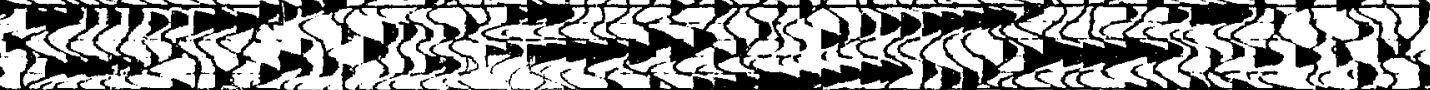

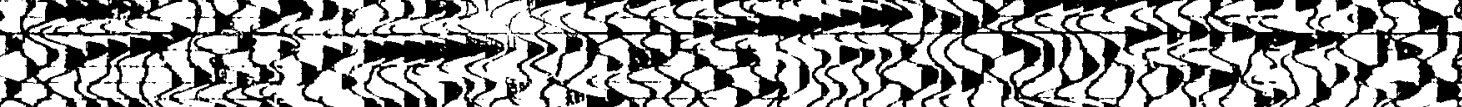

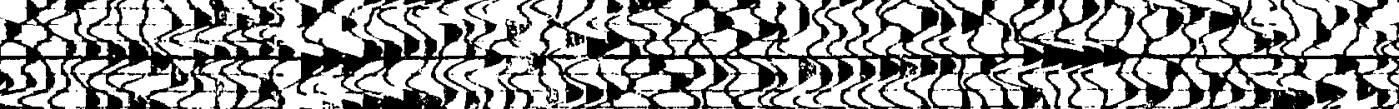

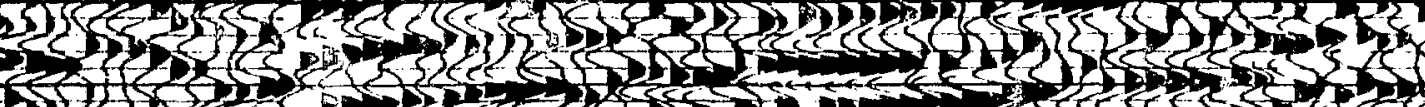

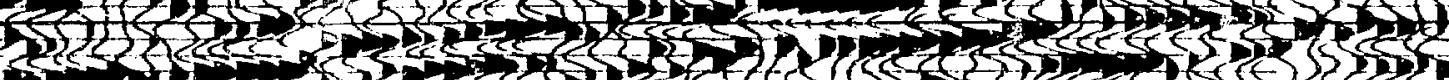

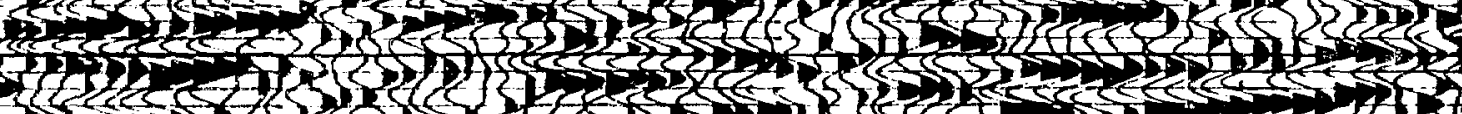

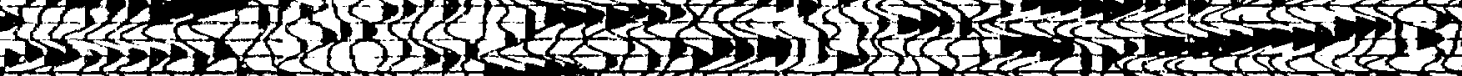

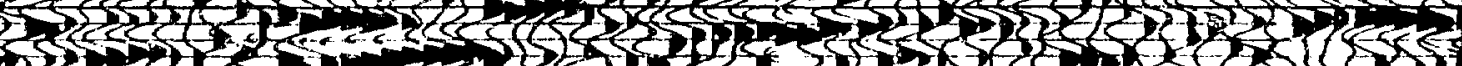

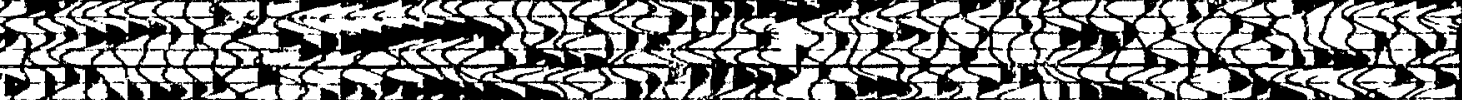

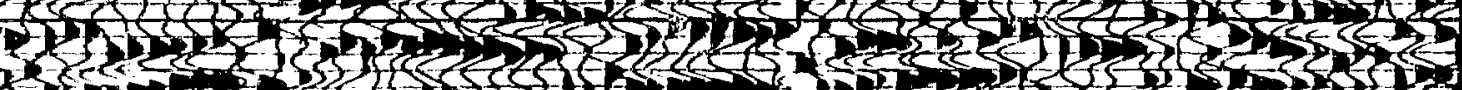

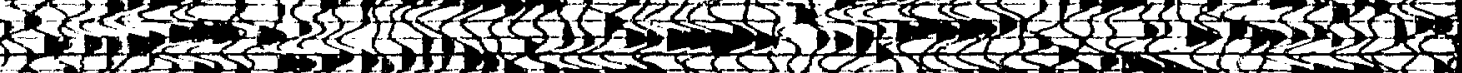

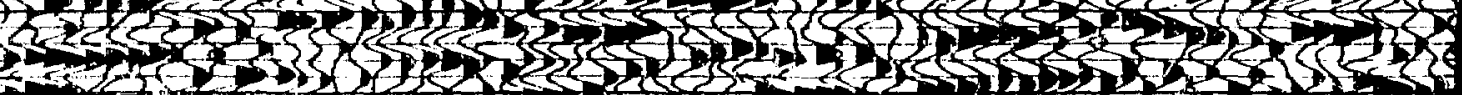

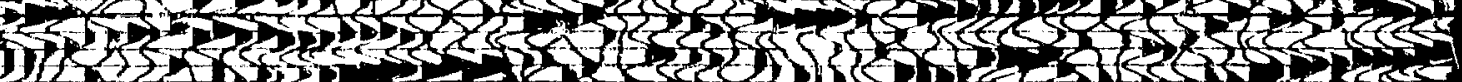

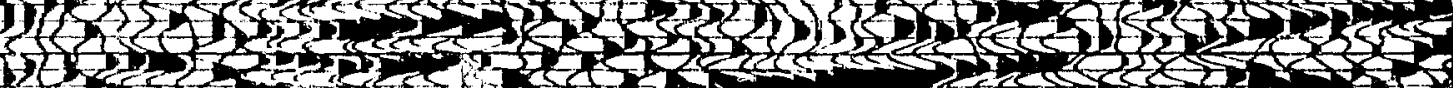

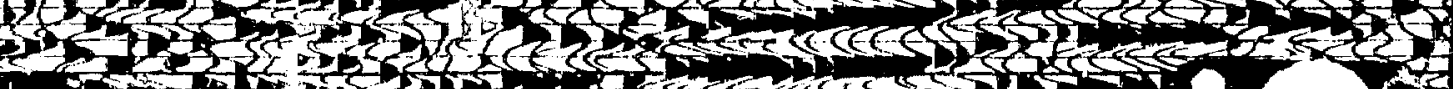

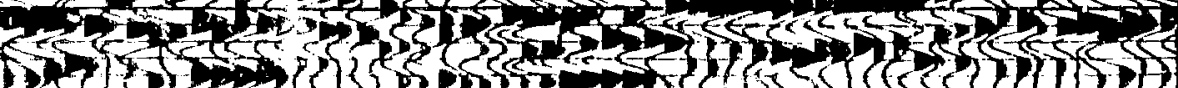

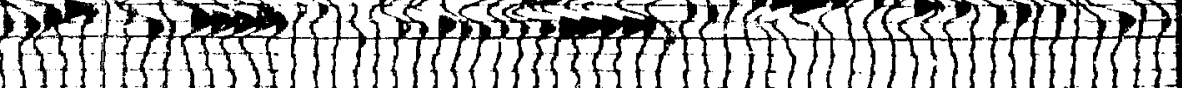


3535353 30

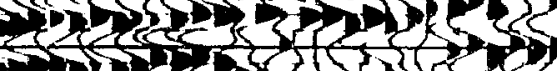

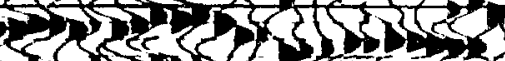
305052,150

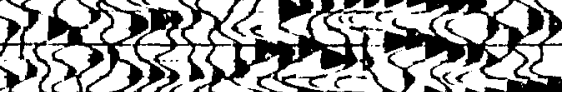
6 S SSOM

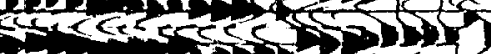
sersezensins

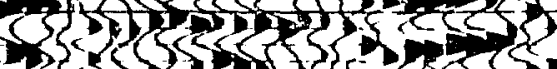

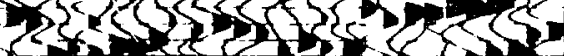
<5 270 30

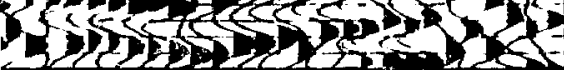
2015

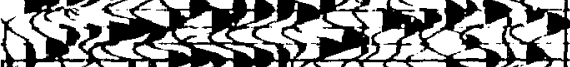
1.

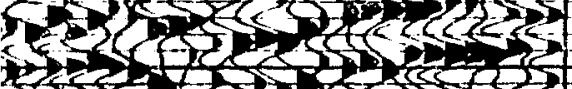
535325 2

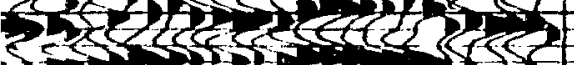

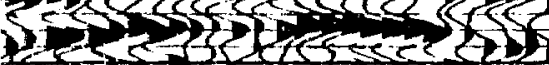
331

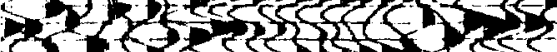

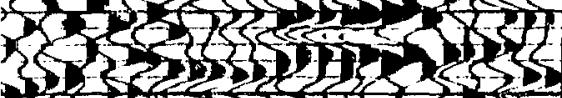

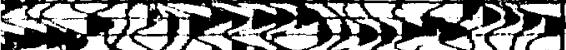

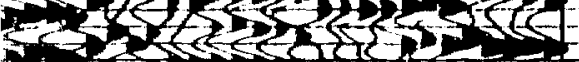

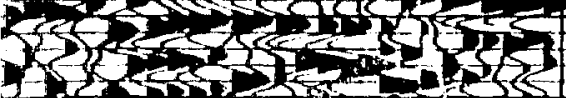

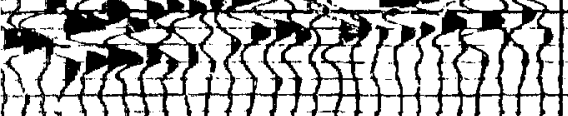

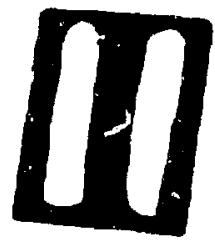

\begin{abstract}
YERT GEAL ELE\& GCFL PDLFRIT
\end{abstract}

TUב WUMAE
EDMFLETEX 

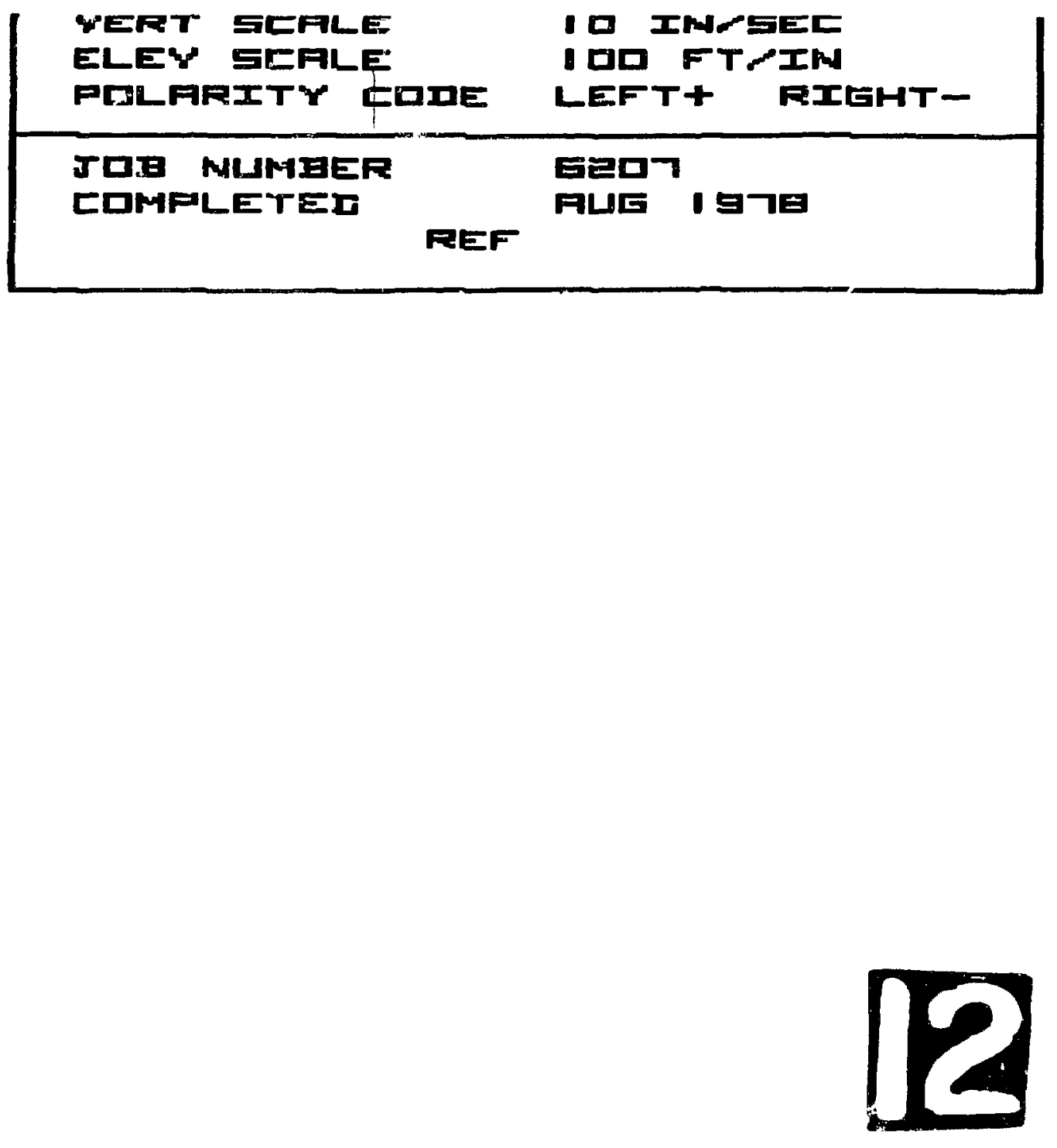